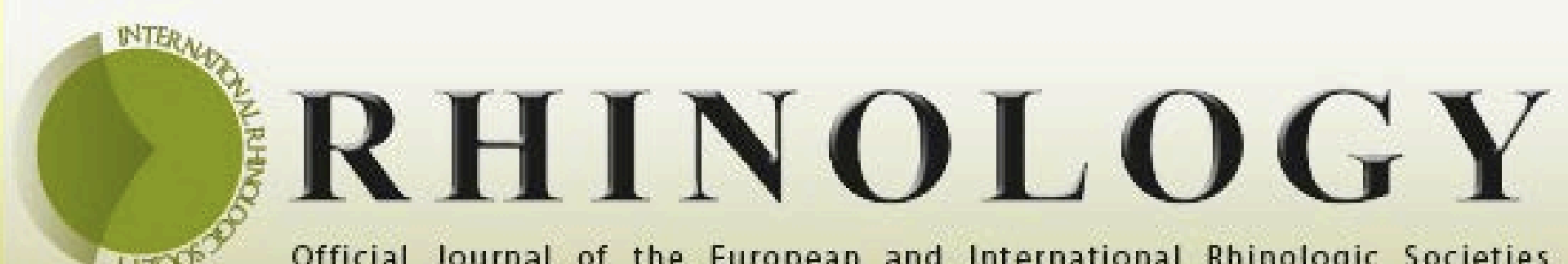
and of the Confederation of European ORL-HNS

VOLUME 58 | SUPPLENENT 29 | FEBRUARY 2020

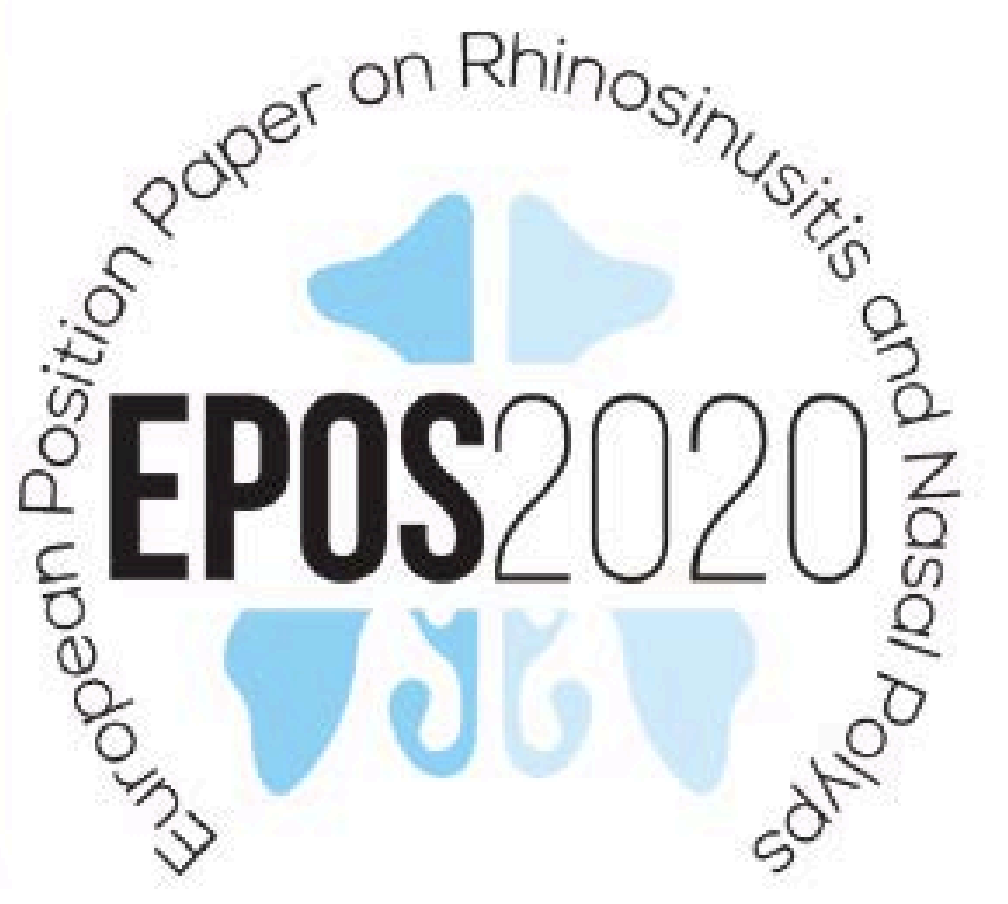

\title{
European Position Paper on \\ Rhinosinusitis and Nasal Polyps 2020
}

W.J. Fokkens, V.J.Lund, C. Hopkins, P.W. Hellings, R. Kern, S. Reitsma, M. Bernal-Sprekelsen, J. Mullol et al. 


\section{RHINOLOGY}

Official Journal of the Europesn and Intemational Fhinologic Societies and of the Confederation of European ORL-HNS

Edtor-In-Chiol

Prof W. Folbeore

Associato Edltor

Prof P.W. Halngs

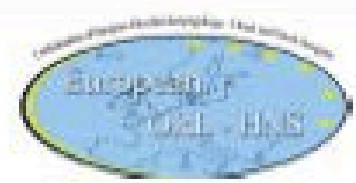

Managing Editoe

Dr.W.T.V.Gemmorad

Edtorial Assistant and Rhinology Secrotary

Mrs J. Vosman

minslogyeacestaryzamcuvani

Edtorial Production Managar

Mrs. P. Chestor

Wobmaster

Dr D. Bare

minologowabmactaregmalcom

\section{Address}

Joumal Fhinology, Ob AMC, Mrs J. Kooman / A2-234, PO Bow 22660 ,

1100 DO Amstentam, the Notheriznds

Tel: $+31-20-5664534$

Fax $+31-201-5660662$

E-mal: inhobgysecretarysamc.uval

Wreske:Www minologypumalcom

To cite this article: Fokkens WJ, Lund VJ., Hopkins C, Hellings PW, Kern R., Reltsma S, et al European Position Paper on Rhinosinur sitis and Nasal Polyps 2020 Rhinology. 2020 Suppl. 29. 1-464.

Rhbology IESN 0300-0T29 $\mathrm{k}$ the omkt Journal of the Europeen and Intemakbnal Rilndogk Socdotles and appaars bimonthly in Fobruary, Aprit June, August, Odtober and Docembar. Ched In Pubmed, Currenk Contents, index Modious, Exarpta Medca and Enbase.

Foundad in 1963 by HAE. van Dtehoeck, Fhholegy ts a workdwide non-proft. maling joumal. The joumal publthes original papers en breic reseerch as wal as dhical studles in the mapr flold of minology, nduding physiobgy, dbynostis, parthology, Immunology, medcal therapy and surgary of both the nose and peranasal shueses. Revlow artides and shert communicekbre are also publehed but no Casa reports. Al papars are peer-evilewed Lettars-to-the-edtor provide a forum for commants on putellehed papers, and are nok sutbect to adborlal rewsisn excespe for comection of Englkh language.

In-dapth studles that ara too bing to be Inchuded Into a regular lesus can be published as a supplement Suppiements are in princlple nok subjedt to peerraviow. o Rhinology, 2020.

Al rights reserwed. Ho pert of the publation may be reproducad or trensmittad h ary form or by ary meens alacironk or mechenkal, houding photocopyng. recording or any information torage and retrlevel systam whout prior pormtsslon in witting from the Publiner.

Submbsion of a maruscript for publketion Impliss the tranefor of the copyright from the authorka to the publisher and entalls the auther's imevocable and excluatve authorterkien of the publshar to coloct any sums or considarations for copying or eproduction payable by third parties. 


\section{European Position Paper on Rhinosinusitis and Nasal Polyps 2020}

Wytske J. Fokkens' ${ }^{1}$, Valerie J. Lund ${ }^{2}$, Claire Hopkins ${ }^{3}$, Peter W. Hellings ${ }^{1,4,11}$, Robert Kern ${ }^{5}$, Sietze Reitsma', Sanna Toppila-Salmi ${ }^{6}$, Manuel Bernal-Sprekelsen? Joaquim Mullol ${ }^{8}$, Isam Alobid ${ }^{9}$, Wilma Terezinha Anselmo-Lima ${ }^{10}$, Claus Bachert ${ }^{11,12}$, Fuad Baroody ${ }^{13}$, Christian von Buchwald ${ }^{14}$, Anders Cervin ${ }^{15}$, Noam Cohen $^{16}$, Jannis Constantinidis ${ }^{17}$, Ludovic De Gabory ${ }^{18}$, Martin Desrosiers ${ }^{19}$, Zuzana Diamant ${ }^{20,21,22}$, Richard G. Douglas²3, Philippe H. Gevaert24, Anita Hafner ${ }^{25}$, Richard J. Harvey ${ }^{26}$, Guy F. Joos ${ }^{27}$, Livije Kalogjera ${ }^{28}$, Andrew Knill29, Janwillem H. Kocks ${ }^{30}$, Basile N. Landis ${ }^{31}$, Jacqueline Limpens ${ }^{32}$, Sarah Lebeer ${ }^{33}$, Olga Lourenco ${ }^{34}$, Paolo M. Matricardi ${ }^{35}$, Cem Meco ${ }^{36,37}$, Liam O'Mahony ${ }^{38}$, Carl M. Philpott ${ }^{39,40}$, Dermot Ryan ${ }^{41,42}$, Rodney Schlosser ${ }^{43}$, Brent Senior ${ }^{44}$, Timothy L. Smith ${ }^{45}$, Thijs Teeling ${ }^{46}$, Peter Valentin Tomazic ${ }^{47}$, De Yun Wang ${ }^{48}$, Dehui Wang ${ }^{49}$, Luo Zhang ${ }^{50}$, Adrian M. Agius ${ }^{51}$, Cecilia Ahlström-Emanuelsson ${ }^{52}$, Rashid Alabri ${ }^{53}$, Silviu Albu ${ }^{54}$, Saied Alhabash ${ }^{55}$, Aleksandra Aleksic ${ }^{56}$, Mohammad Aloulah ${ }^{57}$, Mohannad Al-Qudah ${ }^{58}$, Saad Alsaleh" ${ }^{59}$, Muaid Aziz Baban ${ }^{60}$, Tomislav Baudoin ${ }^{61}$, Tijmen Balvers ${ }^{62}$, Paolo Battaglia ${ }^{63}$, Juan David Bedoya ${ }^{64}$, Achim Beule $^{65}$, Khaled M. Bofares ${ }^{66}$, Itzhak Braverman ${ }^{67}$, Eliza Brozek-Madry ${ }^{68}$, Richard Byaruhanga ${ }^{69}$, Claudio Callejas ${ }^{70}$, Sean Carrie ${ }^{71}$, Lisa Caulley ${ }^{72}$, Desderius Chussi ${ }^{73}$, Eugenio de Corso ${ }^{74}$, Andre Coste ${ }^{75}$, Usama El Hadi ${ }^{76}$, Ahmed Elfarouk ${ }^{77}$, Philippe H. Eloy ${ }^{78}$, Shokrollah Farrokhi ${ }^{79}$, Giovanni Felisati ${ }^{80}$, Michel D. Ferrari62, Roman Fishchuk ${ }^{81}$, Jessica W Grayson ${ }^{82}$, Paulo M. Goncalves ${ }^{83}$, Boris Grdinic ${ }^{84}$, Velimir Grgic ${ }^{28}$, Aneeza W. Hamizan ${ }^{85}$, Julio V. Heinichen ${ }^{86}$, Salina Husain ${ }^{87}$, Tang Ing Ping ${ }^{88}$, Justinas Ivaska ${ }^{89}$, Frodita Jakimovska ${ }^{90}$, Ljiljana Jovancevic ${ }^{91}$, Emily Kakande ${ }^{92}$, Reda Kame ${ }^{93}$, Sergei Karpischenko ${ }^{94}$, Harsha H. Kariyawasam ${ }^{95}$, Hideyuki Kawauchi ${ }^{96}$, Anette Kjeldsen ${ }^{97}$, Ludger Klimek ${ }^{98}$, Antoni Krzeski99, Gabriela Kopacheva Barsova ${ }^{100}$, Sung Wam Kim¹01, Devyani Lal'02 , José J. Letort $^{103}$, Andrey Lopatin ${ }^{104}$, Abdelhak Mahdjoubi ${ }^{105}$, Alireza Mesbahi ${ }^{106}$, Jane Netkovski ${ }^{107}$, Dieudonné Nyenbue Tshipukane ${ }^{108}$, Andrés Obando-Valverde ${ }^{109}$, Mitsuhiro Okano ${ }^{110}$, Metin Onerci ${ }^{111}$, Yew Kwang Ong ${ }^{112}$, Richard Orlandi ${ }^{113}$, Nobuyoshi Otori ${ }^{114}$, Kheir Ouennoughy ${ }^{115}$, Muge Ozkan ${ }^{116}$, Aleksandar Peric $^{117}$, Jan Plzak ${ }^{118}$, Emmanuel Prokopakis ${ }^{119}$, Nerayanan Prepageran ${ }^{120}$, Alkis Psaltis ${ }^{121}$, Benoit Pugin ${ }^{122}$, Marco Raftopulos ${ }^{1,123}$, Philippe Rombaux ${ }^{124}$, Herbert Riechelmann ${ }^{125}$, Semia Sahtout ${ }^{126}$, Caius-Codrut Sarafoleanu ${ }^{127}$, Kafui Searyoh ${ }^{128}$, Chae-Seo Rhee ${ }^{129}$, Jianbo Shi' ${ }^{130}$, Mahdi Shkoukani'131, Arthur K. Shukuryan ${ }^{132}$, Marian Sicak ${ }^{133}$, David Smyth ${ }^{134}$, Kornkiat Snidvongs ${ }^{135}$, Tanja Soklic Kosak ${ }^{136}$, Pär Stjärne ${ }^{137}$, Budi Sutikno ${ }^{138}$, Sverre Steinsvåg ${ }^{139}$, Pongsakorn Tantilipikorn ${ }^{140}$, Sanguansak Thanaviratananich ${ }^{141}$, Thuy Tran ${ }^{142}$, Jure Urbancic ${ }^{143}$, Arunas Valiulis ${ }^{144}$, Carolina Vasquez de Aparicio ${ }^{145}$, Dilyana Vicheva ${ }^{146}$, Paula M. Virkkula147, Gil Vicente ${ }^{148}$, Richard Voegels ${ }^{149}$, Martin Wagenmann ${ }^{150}$, Retno S. Wardani ${ }^{151}$, Antje Welge-Lussen ${ }^{152}$, Ian Witterick ${ }^{153}$, Erin Wright ${ }^{154}$, Dmytro Zabolotniy ${ }^{155}$, Bella Zsolt ${ }^{156}$, Casper P. Zwetsloot ${ }^{157}$

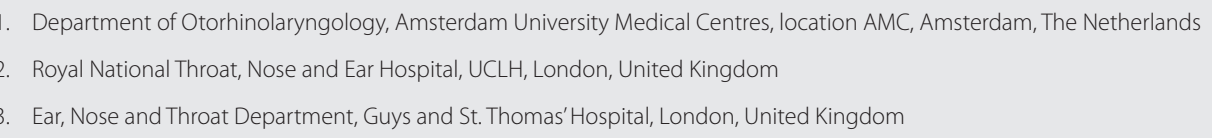


4. Department of Otorhinolaryngology, Head and Neck Surgery, University Hospitals Leuven, KU Leuven, Belgium

5. Department of Otorhinolaryngology - Head and Neck Surgery, Northwestern University, Feinberg School of Medicine, Chicago, IL, USA

6. Skin and Allergy Hospital, Helsinki University Hospital and University of Helsinki, Helsinki, Finland

7. Department Hospital Quironsalud, University of Valencia, Valencia, Spain

8. Rhinology Unit and Smell Clinic, ENT Department, Hospital Clínic, IDIBAPS, Universitat de Barcelona, CIBERES, Barcelona, Catalonia, Spain

9. Rhinology and Skull Base Unit, ENT Department, Hospital Clínic de Barcelona, Universidad de Barcelona, August Pi i Sunyer Biomedical Research Institute, Barcelona, Spain

10. Division of Otorhinolaryngology, Department of Ophthalmology, Otorhinolaryngology, Head and Neck Surgery, Ribeirao Preto Medical School-University of Sao Paulo, Sao Paulo, Brazil

11. Upper Airways Research Laboratory and ENT Department, University Hospital Ghent, Ghent, Belgium

12. Division of ENT Diseases, CLINTEC, Karolinska Institute, University of Stockholm, Stockholm, Sweden

13. Department of Otorhinolaryngology-Head and Neck Surgery, The University of Chicago Medicine and the Comer Children's Hospital, Chicago, IL, USA

14. Department of Otorhinolaryngology, Head and Neck Surgery and Audiology, Rigshospitalet, Copenhagen University, Hospital, Copenhagen, Denmark

15. Department of Otorhinolaryngology, Head and Neck Surgery, Royal Brisbane and Women's Hospital; Faculty of Medicine, University of Queensland, Brisbane, Australia

16. 16. Department of Otorhinolaryngology - Head and Neck Surgery, Perelman School of Medicine at The University of Pennsylvania, Philadelphia, PA, USA

17. 1st Department of ORL, Head and Neck Surgery, Aristotle University, AHEPA Hospital, Thessaloniki, Greece

18. Rhinology and Plastic Surgery Unit, Otorhinolaryngology, Head and Neck Surgery and Pediatric ENT Department, CHU de Bordeaux, Hôpital Pellegrin, Centre F-X Michelet, Bordeaux, France

19. Department of ORL-HNS, Université de Montréal, Montreal, Canada

20. Dept of Respiratory Medicine and Allergology, Skane University in Lund, Sweden

21. Research Director Respiratory \& Allergy, at QPS-Netherlands, Groningen, Netherlands

22. Affiliate to Charles University, Dept of Respiratory Diseases, in Prague, Czech Republic

23. Department of Surgery, The University of Auckland, Auckland, New Zealand

24. Department of Otorhinolaryngology, Ghent University, Ghent, Belgium

25. University of Zagreb Faculty of Pharmacy and Biochemistry, Zagreb, Croatia

26. Rhinology and Skull Base Department, Applied Medical Research Centre, UNSW (Conjoint) and Macquarie University (Clinical), Sydney, Australia

27. Department of Respiratory Medicine, Ghent University Hospital, Ghent, Belgium

28. ENT Department, Zagreb School of Medicine; University Hospital Center "Sestre milosrdnice", Zagreb, Croatia

29. Patient representative, Opuscomms, London, United Kingdom

30. Department of Inhalation Medicine, Observational Pragmatic Research Institute, Singapore

31. Rhinology-Olfactology Unit, Otorhinolaryngology Department, University Hospital of Geneva, Geneva, Switzerland

32. Medical Information Specialist, Medical Library, Amsterdam University Medical Centres, location AMC, Amsterdam, The Netherlands

33. Department of Bioscience Engineering, University of Antwerp, Antwerp, Belgium

34. FCS - UBI Faculty of Health Sciences, University of Beira Interior, Covilhã, Portugal

35. Department of Pediatric Pneumology and Immunology, Charité - Universitätsmedizin Berlin, Berlin, Germany

36. Department of Otorhinolaryngology, Head and Neck Surgery, Ankara University, Ankara, Turkey

37. Department of Otorhinolaryngology, Head and Neck Surgery, Salzburg Paracelsus Medical University, Salzburg, Austria

38. Departments of Medicine and Microbiology, APC Microbiome Ireland, National University of Ireland, Cork, Ireland

39. Department of Medicine, Norwich Medical School, University of East Anglia, Norwich, United Kingdom

40. ENT Department, James Paget University Hospital, Great Yarmouth, United Kingdom

41. Allergy and Respiratory Research Group, Usher Institute of Population Health Sciences and Informatics, University of Edinburgh, Edinburgh, United Kingdom

42. Optimum Patient Care, Cambridgeshire, United Kingdom

43. Department of Otorhinolaryngology - Head and Neck Surgery, Medical University of South Carolina, Charleston, USA

44. UNC Otorhinolaryngology / Head and Neck Surgery, Division of Rhinology, Allergy, and Endoscopic Skull Base Surgery and Department of Neurosurgery, University of North Carolina School of Medicine, Chapel Hill, NC, USA

45. Division of Rhinology and Sinus/Skull Base Surgery, Department of Otolaryngology-Head and Neck Surgery, Oregon Health and Science University, Portland, Oregon, USA

46. Patient representative, Task Force Healthcare, WTC Den Haag, The Netherlands 
47. Department of Otorhinolaryngology, Head and Neck Surgery, Medical University of Graz, Graz, Austria

48. Department of Otorhinolaryngology, Yong Loo Lin School of Medicine, National University of Singapore, Singapore

49. Rhinology Division, ENT Department; Eye and ENT Hospital, Fudan University, Shanghai, China

50. Department of Otorhinolaryngology Head and Neck Surgery, Beijing TongRen Hospital, Beijing, China

51. Department of Medicine and Surgery in the University of Malta

52. ENT-Department, Lund University, Sweden

53. ENT Division, Surgery Department, College of Medicine and Health and Sciences, Sultan Qaboos University, Muscat, Oman

54. Department of Otorhinolaryngology, luliu Hațieganu University of Medicine and Pharmacy, Cluj-Napoca, Romania

55. Department of ENT, Medcare UAE, Dubai

56. ENT Department, University Clinical Centre, University of Banja Luka, Bosnia and Herzegovina

57. ENT Department, King Saud University, Riyadh, Kingdom of Saudi Arabia

58. Department of Otorhinolaryngology, Jordan University of Science \& Technology, Irbid, Jordan

59. Department of Otorhinolaryngology - Head and Neck Surgery, College of Medicine, King Saud University, Riyadh, Saudi Arabia

60. Department of Otorhinolaryngology - Head and Neck Surgery, University of Sulaimani, Sulaimayniha, Iraq

61. Dept. of ORL-HNS Sisters of Mercy University Medical Center, School of Medicine University of Zagreb, Croatia

62. Department of Neurology, Leiden University Medical Center (LUMC), LEiden, the Netherlands

63. Division of Otorhinolaryngology, Department of Biotechnology and Life Sciences, University of Insubria, Varese, Italy

64. Department of Otorhinolaryngology, Universidad de Antioquia, Medellín, Colombia

65. Department of Otorhinolaryngology, University Clinic of Munster, Germany

66. Department of Otorhinolaryngology, Omar Al-Moukhtar University, Albyeda, Libya

67. Department of Otorhinolaryngology - Head and Neck Surgery, Hillel Yaffe Medical Center, Israel

68. Department of Otorhinolaryngology, Medical University of Warsaw, Poland

69. Department of ENT, Makerere University, Kampala, Uganda

70. Department of Otorhinolaryngology, Pontificia Catholic University, Santiago, Chile

71. Department of Otorhinolaryngology, Head and Neck Surgery, Newcastle University, United Kingdom

72. Department of Otorhinolaryngology, Head and Neck Surgery, University of Ottawa,Toronto, Canada

73. Department of Otorhinolaryngology, Kilimanjaro Christian Medical University College, Moshi, Tanzania

74. Department of Otorhinolaryngology, La Fondazione Policlinico Universitario Agostino Gemelli IRCCS, Università Cattolica del Sacro Cuore, Rome, Italy

75. ORL et Chirurgie Cervico-Faciale, Université Paris-Est Créteil (UPEC), France

76. Department of Otorhinolaryngology, American University of Beirut, Lebanon

77. Department of Otorhinolaryngology, Cairo University, Egypt

78. Department of ENT, CHU UCL Namur, Yvoir, Belgium

79. Department of Immunology and Allergy, The Persian Gulf Tropical Medicine Research Center; The Persian Gulf Biomedical Research Institute, Bushehr University of Medical Sciences, Bushehr, Iran

80. Department of Head and Neck, University of Milan, Italy

81. Department of ENT- Organs Microsurgery, Central city clinical hospital of Ivano-Frankivsk city council, Ivano-Frankivsk, Ukraine

82. Department of Otorhinolaryngology, Head and Neck Surgery, University of Alabama Birmingham, USA

83. ENT Department, Centro Hospitalar de Entre Douro e Vouga, Santa Maria da Feira, Portugal

84. ENT Department, General Hospital, Pula, Pula, Croatia

85. Department of Otorhinolaryngology, University Kebangsaan, Kuala Lumpur, Malasyia

86. Department of ENT of Hospital de Clínicas, Facultad de Ciencias Médicas, Universidad Nacional de Asunción, Paraguay

87. Department of Otorhinolaryngology, Head and Neck Surgery, National University of Malaysia, Kuala Lumpur, Malaysia

88. Department ORLHNS, University Malaysia Sarawak, Kuching, Malaysia

89. Clinic of Ear, Nose, Throat and Eye diseases, Vilnius University, Lithuania

90. ENT Department of Medical Faculty, St Cyril and Methodius University of Skopje, North Macedonia

91. Department of Otorhinolaryngology, Head and Neck Surgery, Clinical Centre of Vojvodina, University of Novi Sad, Faculty of Medicine, Novi Sad, Serbia

92. Department of ENT Surgery, Mulago National Referral Hospital Kampala, Uganda

93. Department of Otorhinolaryngology, Head and Neck Surgery, Cairo University, Egypt

94. ENT Department, Director of Saint Petersburg Research Institute of Ear, Throat, Nose and Speech; Professor and Chairman of First Pavlov State Medical University, 
Saint Petersburg, Russia

95. Department of Allergy and Clinical Immunology, Royal National ENT Hospital, London, United Kingdom

96. Department of Otorhinolaryngology, Shimane University, Matsue, Shimane, Japan

97. Department of Otorhinolaryngology, Head and Neck Surgery, University of Southern Denmark, Odense, Denmark

98. Center of Rhinology and Allergology, Wiesbaden, Hesse, Germany

99. Department of Otorhinolaryngology, Warsaw Medical University, Warsaw, Poland

100.Department of Otorhinolaryngology, University If Medicine, st. Ciril and Methodius, Skopje, North Macedonia

101.Department of Otorhinolaryngology, Head and Neck Surgery, Kyung Hee University, Seoul, South Korea

102.Department of Otorhinolaryngology, Head and Neck Surgery, Mayo Clinic in Arizona, Phoenix, Arizona, USA

103.Department of Otorhinolaryngology, Pontifica Catholic University of Ecuador, Quito, Ecuador

104. Department of Otorhinolaryngology, Policlinic No.1- Senior ENT Consultant and Surgeon; President of Russian Rhinologic Society, Moscow, Russia 105. Clinique Mahabi, Setif, Algeria

106. Department of Facial Surgery, Khodadoust Hospital, Ordibehesht Hospital, Shiraz, Iran

107. Department of Otorhinolaryngology-Head and Neck Surgery, St. Cyril and Methodius, Skopje, Republic of North Macedonia

108. Department of Otorhinolaryngology, University of Kinshasa, Kinshasa, Democratic Republic of Congo

109.Department of Otorhinolaryngology and Surgery, Hospital Mexico, University of Costa Rica, San Jose, Costa Rica

110.Department of Otorhinolaryngology, International University of Health and Welfare, Narita, Japan

111.Department of Otorhinolaryngology, Hacettepe, Ankara, Turkey

112.Department of Otorhinolaryngology, Head and Neck Surgery, University of Singapore, National University Hospital, Singapore

113.Department of Otorhinolaryngology, University of Utah, Salt Lake City, Utah, USA

114.Department of Otorhinolaryngology at The Jikei University School of Medicine,Tokyo, Japan

115.Department of Otorhinolaryngology-Head and Neck Surgery, Saad Dahleb Blida 1, Blida, Algeria

116.Department of Otorhinolaryngology, University of Health Sciences, Ankara City Hospital, Turkey

117.Department of Otorhinolaryngology, Military Medical Academy, Faculty of Medicine, University of Defense, Belgrade, Serbia

118.Department of Otorhinolaryngology, Head and Neck Surgery, 1st Faculty of Medicine, Charles University, Prague, Czech Republic

119.Department of Otorhinolaryngology, University of Crete School of Medicine, Heraklion, Crete, Greece

120. Department of ENT, University Malaya, Kuala Lumpur, Malaysia

121.Department of Otorhinolaryngology, Head and Neck Surgery, University of Adelaide, Adelaide, Australia

122.Department of Health Sciences and Technology, ETH Zürich, Switzerland

123. Royal Australian College of Surgeons, Trainee Representative (Australia)

124. Department of Otorhinolaryngology, University of Louvain, Brussels, Belgium

125.Department of Otorhinolaryngology, Head and Neck Surgery, University Hospital, Ulm, Baden-Wurttemberg, Germany

126. Faculty of Medicine of Tunis, Tunis El Manar University, Tunis, Tunisia

127. ENT\&H NS Department, Santa Maria Hospital, Carol Davila University of Medicine and Pharmacy, Bucharest, Romania

128. Surgery Ear, Nose and Throat Unit, School of Medicine and Dentistry, University of Ghana, Korle-Bu Teaching Hospital, Accra, Ghana

129. Department of Otorhinolaryngology, Head and Neck Surgery, Seoul, Seoul National University, Seoul, Korea

130. Department of Rhinology, The First Affiliated Hospital, Sun Yat-sen University, Guangzhou, China

131.Department of Otorhinolaryngology, Head and Neck Surgery, Cleveland Clinic Abu Dhabi, United Arab Emirates

132.Department of Otorhinolaryngology, Yerevan State Medical University, Yerevan, Armenia

133. Department of Otorhinolaryngology, Head and Neck Surgery, Central Military Hospital, Slovakia, Slovak Health University Bratislava and Catholic University, Ruzom-

berok, Slovakia

134.Department of Otorhinolaryngology, Head and Neck Surgery, Royal College of Surgeons in Ireland and University College Cork, Waterford, Ireland

135. Department of Otorhinolaryngology, Chulalongkorn University, Bangkok, Thailand

136. University Medical Centre Ljubljana, Department of Otorhinolaryngology and Cervicofacial Surgery, University of Ljubljana, Faculty of Medicine, Ljubljana, Slovenia

137. Department of Otorhinolaryngology, Karolinska University Hospital, Stockholm, Sweden

138. Department of Otorhinolaryngology, Head and Neck Surgery, University of Airlangga, Surabaya, Indonesia

139. Department of ORL, University of Bergen, Norway

140.Department of Otorhinolaryngology, Faculty of Medicine Siriraj Hospital, Mahidol University, Bangkok, Thailand

141.Department of Otorhinolaryngology, University of Khonkaen , Khonkaen, Thailand 
142. Department of ENT Hospital of Ho Chi Minh city, Faculty of medicine of Ho Chi Minh city Vietnam National University, Vietnam

143. Department of Otorhinolaryngology and cervicofacial surgery, UMC Ljubljana, University of Ljubljana, Medical Faculty, Ljubljana, Slovenia

144. Department of Children's diseases, Vilnius University Medical Faculty, Institute of Clinical Medicine, Vilnius, Lithuania

145. Department of Paediatric Surgery, National Hospital Benjamin Bloom, National University of El Salvador, San Salvador, El Salvador

146.Department of Otorhinolaryngology, Medical University Plovdiv, Bulgaria

147.Department of Otorhinolaryngology, Head and Neck Surgery, Helsinki, University Hospital, Helsinki, Finland

148. Department of Otolaryngology, St. Luke's Medical Centre, Quezon City, The Philippines

149.Department of Otorhinolaryngology, University of Sao Paulo, Sau Paulo, Brazil

150.Department of Otorhinolaryngology, Dusseldorf University Hospital, Dusseldorf, Germany

151.Department of Otorhinolaryngology Head and Neck Surgery, Dr. Cipto Mangunkusumo Hospital, University of Indonesia, Jakarta, Indonesia 152.Department of Otorhinolaryngology, University Hospital Basel, University Basel, Switzerland

153.Department of Otorhinolaryngology, Head and Neck Surgery, University of Ottawa,Toronto, Canada

154. Department of Surgery, University of Alberta, Edmonton, Alberta, Canada

155.State Institution of O.S. Kolomiychenko Institute of Othorhnilarungology of National Academy of Medical Sciences of Ukraine, Kiev, Ukraine 156.Department of Otorhinolaryngology, Head and Neck Surgery, University of Szeged, Hungary

157. Department of Neurology, Dijklander Ziekenhuis, Purmerend, The Netherlands 


\section{Summary}

The European Position Paper on Rhinosinusitis and Nasal Polyps 2020 is the update of similar evidence based position papers published in 2005 and 2007 and 2012. The core objective of the EPOS2020 guideline is to provide revised, up-to-date and clear evidence-based recommendations and integrated care pathways in ARS and CRS. EPOS2020 provides an update on the literature published and studies undertaken in the eight years since the EPOS2012 position paper was published and addresses areas not extensively covered in EPOS2012 such as paediatric CRS and sinus surgery. EPOS2020 also involves new stakeholders, including pharmacists and patients, and addresses new target users who have become more involved in the management and treatment of rhinosinusitis since the publication of the last EPOS document, including pharmacists, nurses, specialised care givers and indeed patients themselves, who employ increasing self-management of their condition using over the counter treatments. The document provides suggestions for future research in this area and offers updated guidance for definitions and outcome measurements in research in different settings.

EPOS2020 contains chapters on definitions and classification where we have defined a large number of terms and indicated preferred terms. A new classification of CRS into primary and secondary CRS and further division into localized and diffuse disease, based on anatomic distribution is proposed. There are extensive chapters on epidemiology and predisposing factors, inflammatory mechanisms, (differential) diagnosis of facial pain, allergic rhinitis, genetics, cystic fibrosis, aspirin exacerbated respiratory disease, immunodeficiencies, allergic fungal rhinosinusitis and the relationship between upper and lower airways. The chapters on paediatric acute and chronic rhinosinusitis are totally rewritten. All available evidence for the management of acute rhinosinusitis and chronic rhinosinusitis with or without nasal polyps in adults and children is systematically reviewed and integrated care pathways based on the evidence are proposed. Despite considerable increases in the amount of quality publications in recent years, a large number of practical clinical questions remain. It was agreed that the best way to address these was to conduct a Delphi exercise. The results have been integrated into the respective sections. Last but not least, advice for patients and pharmacists and a new list of research needs are included.

The full document can be downloaded for free on the website of this journal: http://www.rhinologyjournal.com.

To cite this article: Fokkens W.J., Lund V.J. , Hopkins C., Hellings P.W., Kern R., Reitsma S., et al. European Position Paper on Rhinosinusitis and Nasal Polyps 2020 Rhinology. 2020 Suppl. 29: 1-464.

MeSH keywords: Paranasal Sinus Diseases, Nasal Polyps, Therapeutics, Diagnosis, Asthma, Prevention and Control

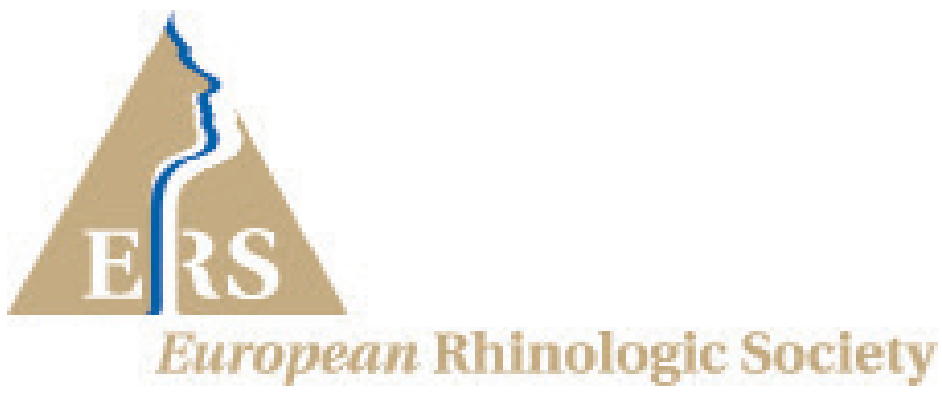

\section{Aknowledgements}

We thank Polly Chester, Wilfred Germeraad, Julija Keslere and Nina Liberda for the editing and typesetting of the document; Jacqueline Schaffer, Benoit Pugin and Sietze Reitsma for the beautiful illustrations, and Klementina Avdeeva, Bella Zsolt, Shokrollah Farrokhi, Eri Mori, Yuan Zhang, for translating papers. We particularly thank the European Rhinologic Society for their generosity in making EPOS2020 see the light. 


\section{Contents}

1. Executive Summary including management schemes

1.1. Summary

1.2. Classification and definitions of CRS

1.3. Burden of ARS and CRS

1.4. Acute rhinosinusitis including common cold and recurrent ARS in adults and children

1.5. Chronic rhinosinusitis

1.6. Management of chronic rhinosinusitis in adults

1.7. Paediatric chronic rhinosinusitis

1.8. Concomitant diseases in chronic rhinosinusitis

1.9. Patient participation, prediction, precision medicine and implementation

1.10 Pharmacist perspective on rhinosinusitis

1.11 Research priorities in rhinosinusitis

1.12 Methods used in EPOS2020

1

1

1

6

9

11

16

21

22

26

27

27

2. Classification, definitions and terminology 31

2.1. Defintions 31

2.2. Classification of CRS 32

2.3. Duration (adults and children) 34

2.4. Severity of disease 34

2.5. Exacerbation vs. recurrence 34

2.6. Control and failure 34

2.7. Phenotype 35

2.8. Endotype 35

2.9. Comorbidities 35

2.10. Medical therapy 35

2.11. Surgical therapy 35

2.12. Precision medicine vs. personalised medicine 36

2.13. Burden of rhinosinusitis 36

2.14. Age 36

2.15. Integrated Care pathways 36

2.16. Recalcitrant vs refractory to treatment 36

2.17. Nasal douche / lavage / irrigation / rinsing 36

2.18. Immunomodulation and Immunotherapy 36

2.19. Allergy 37

2.20. Duration of antibiotic courses 37

2.21 Other definitions 37

2.22 Concept of Control of CRS 37

3. Burden of acute and chronic rhinosinusitis 45

3.1. Quality of life (QOL) 45

3.2. Costs of rhinosinusitis 47

4. Acute rhinosinusitis including common cold - and recurrent ARS in adults and children 53

4.1. Epidemiology of acute rhinosinusitis (ARS) 53

4.2. Recurrent ARS (RARS) 56

4.3. Factors associated with ARS and RARS 57

4.4. Pathophysiology of ARS 59 
4.5. Diagnosis and differential diagnosis of ARS in adults and children 63

4.6 Medical management of ARS 67

4.6.1. Introduction 67

4.6.2. Management of acute viral rhinosinusitis 67

4.6.3. Oral antibiotics - short courses 73

4.6.4. Nasal corticosteroid in acute post-viral rhinosinusitis 81

4.6.5. Oral corticosteroids 87

4.6.6. Antihistamines (oral and local) 87

4.6.7. Antileukotrienes 89

4.6.8. Nasal decongestants 89

4.6.9. Saline 90

4.6.10. Steam / heated air 90

4.6.11. Physical interventions 90

4.6.12. Bacterial lysates 90

4.6.13. Homeopathy 91

4.6.14. Herbal compounds 92

4.6.15. Vaccination 94

4.6.16. Sodium hyaluronate 94

4.6.17. Mucolytics 95

4.7 Complications of acute bacterial rhinosinusitis (ABRS) 95

5. Epidemiology, predisposing factors, pathophysiology, and diagnosis of CRS 115

5.1. Epidemiology and predisposing factors 115

5.2. Pathophysiology of chronic rhinosinusitis with and without nasal polyposis 123

5.3. Diagnosis and Differential Diagnosis 147

5.3.1. Allergic and non-allergic rhinitis 147

5.3.2 Smell disorders 147

5.3.3 Facial pain 150

5.3.4. Diagnosis and differential diagnosis 152

5.3.5. Diagnostic tools 153

6. Management of chronic rhinosinusitis in adults 205

6.1 Medical Management 205

6.1.1. Short term oral antibiotics for chronic rhinosinusitis (CRS) and exacerbations of CRS 205

6.1.2. Long-term antibiotics 209

6.1.3. Topical antibiotics 213

6.1.4. Intravenous antibiotics 216

6.1.5. Intranasal corticosteroids 216

6.1.6. Corticosteroid-eluting implants 234

6.1.7. Short courses of systemic corticosteroids 236

6.1.8. Antihistamines (oral and topical) 239

6.1.9. Anti-leukotrienes 240

6.1.10. Decongestants 242

6.1.11.Saline 242

6.1.12. Aspirin treatment after desensitization (ATAD) in N-ERD 252

6.1.13. Antimycotics 256

6.1.14. Anti-lgE 259

6.1.15. Anti-IL5 261

6.1.16. Anti-IL4/IL13 263 
6.1.17. Probiotics 266

6.1.18. Muco-active drugs 266

6.1.19. Herbal treatment 267

6.1.20. Traditional Chinese medicine and acupuncture 269

6.1.21. Topical furosemide and oral verapamil 269

6.1.22. Capsaicin 271

6.1.23. Proton-pump inhibitors in patients with GORD 272

6.1.24. Bacterial lysates 272

6.1.25. Homeopathy 273

6.1.26. Phototherapy 274

6.1.27. Filgastrim (r-met-HuG-CSF) 274

6.1.28. Topical barriers e.g. algae - carrageenans 275

6.1.29. Collodial Silver 275

6.1.30. Immunotherapy 275

6.1.31. New potentials not on the market today 275

6.1.32. CRS treatment during pregnancy 278

6.2. Surgical Treatment 278

6.2.1. Primary endoscopic surgery 278

6.2.2. Revision endoscopic surgery 300

6.2.3. Indications for external surgery 305

6.2.4. Peroperative measures to improve surgical field and outcomes 308

6.2.5. Informed consent/patient information in CRS 314

6.2.6. Training in surgery 316

6.2.7. Peri-operative medication 317

7. Paediatric chronic rhinosinusitis 349

7.1. Epidemiology, predisposing factors, and comorbidities 349

7.2. Pathophysiology 354

7.3. Diagnosis and differential diagnosis 356

7.4. Management of paediatric CRS and co-morbidities 359

8. Concomitant diseases in patients with chronic rhinosinusitis 369

8.1. Role of allergy in chronic rhinosinusitis 369

8.2. Immunodeficiencies and their role in CRS 371

8.3. Lower airway disease including asthma in relation to CRS 373

8.4. Cystic fibrosis 375

8.5. Primairy ciliary dyskenesia 378

8.6. Fungal Rhinosinusitis 380

8.7. Vasculitis, granulomatous diseases and their role in CRS 392

9. Patient participation, prediction, precision medicine and implementation 413

9.1. Patient participation in CRS 413

9.2. What does EPOS 2020 mean for patients? 415

9.3. Prevention of disease 416

9.4. Prediction 423

9.5 Precision medicine 425

9.6 Implementation 426 
10. Pharmacist perspective on rhinosinusitis 433

10.1. Differentiating rhinitis and rhinosinusitis in the community pharmacy setting 433

10.2. Dispensing and use of non-prescription antibiotics in URTIs 433

10.3. Management of rhinitis and rhinosinusitis in the pharmacy 436

10.4 When and how to refer to a physician or to a specialist 439

10.5. Advice to pharmacists on explaining to patients how to use medication 439

11. Research needs and agenda for the next decade 445

11.1. Introduction 445

11.2 Classification and definitions 445

11.3 Burden of acute rhinosinusitis and chronic rhinosinusitis 445

11.4 Acute rhinosinusitis including common cold - and recurrent ARS in adults and children 445

11.5 Epidemiology, predisposing factors, pathophysiology, and diagnosis of CRS 446

11.6 Management of chronic rhinosinusitis in adults 448

11.7 Paediatric Chronic Rhinosinusitis 449

11.8 Concomitant diseases in patients with chronic rhinosinusitis 449

11.9 Patient participation, prediction, precision medicine and implementation 450

11.10 Pharmacist perspective on rhinosinusitis 450

11.11 General principles 450

12. Methods used in EPOS2020 451

12.1. Introduction 451

12.2 Search methods to identify RCTs 452

12.3 Delphi rounds 453

12.4 Dissemination and future updates 453

12.5 Editorial Independence 454

12.6 Details of search strategies used 454

13. Authors declarations 463 


\section{Abbreviations}

\begin{tabular}{|c|c|c|c|}
\hline 15LO & 15 lipoxygenase & CVID & Common Variable ImmunoDeficiency \\
\hline 15-HETE & 15-Hydroxyeicosatetraenoic acid & CXCL11 & CXC chemokine ligand \\
\hline \multirow[t]{2}{*}{ AAO-HNS } & American Academy of Otolaryngology-Head and & cysLTs & Cysteinyl leukotrienes \\
\hline & Neck Surgery & D & Desflurane \\
\hline ABPA & Allergic bronchopulmonary aspergillosis & DC & Dendritic cell \\
\hline ABRS & Acute bacterial rhinosinusitis & DBPC & Double-blind placebo-controlled \\
\hline ACE & Angiotensin converting enzyme & DBPCT & Double blind, placebo controlled trial \\
\hline ACP & Antrochoanal polyp & DC & Dendritic cell \\
\hline ACQ-7 & Asthma Control Questionnaire-7 & dd & Daily dosage \\
\hline ACTH & Adrenocorticotropic hormone & DRS & Dexarhinospray \\
\hline$A D$ & Aspirin desensitizatio & DSS & Dead Sea salt solution \\
\hline AdeV & Adenovirus & EBV & Epstein-Barr virus \\
\hline $\mathrm{AE}$ & Adverse event & EC & Epithelial cell \\
\hline AECRS & Acute exacerbation of chronic rhinosinusitis & $\mathrm{ECP}$ & Eosinophil cationic protein \\
\hline AERD & Aspirin-Exacerbated Respiratory Disease & eCRS & Eosinophilic CRS \\
\hline AFRS & Allergic fungal rhinoSinusitis & EESS & Extended ESS \\
\hline AIA & Aspirin-induced asthma & EMA & European Medicines Agency \\
\hline AIT & Allergen-specific immunotherapy & EMP1 & Epithelium membrane protein 1 \\
\hline anti lgE & Anti immunoglobulin E & EMT & Epithelial to mesenchymal transition \\
\hline APC & Antigen presenting cell & eNOS & Endothelial NOS \\
\hline AQLQ & Asthma Quality of Life Questionnaire & ENT & Ear Nose and Throat \\
\hline AR & Allergic Rhinitis & EPOS & European Position Paper on Rhinosinusitis and Nasal \\
\hline ARS & Acute Rhinosinusitis & & Polyps \\
\hline ATA & Aspirin-tolerant asthma & Eq-5D & EuroQol -5D \\
\hline ATAD & Aspirin treatment after desensitisation & ESR & Erythrocyte sedimentation rate \\
\hline B-SIT & Brief Smell Identification Test & ESS & Endoscopic Sinus Surgery \\
\hline BAFF & B-cell activating factor & EV & Enterovirus \\
\hline BAL & Bronchoalveolar lavage & $\mathrm{F}$ & Fentanyl \\
\hline $\mathrm{BCC}$ & Ballon catheter sinuplasty & FDA & Food and Drug Administration (US) \\
\hline B.i.d. & Twice daily & FESS & Functional Endoscopic Sinus Surgery \\
\hline BMT & Basement membrane thickening & FEV & Forced Expiratory Volume \\
\hline BND & Biodegradable nasal dressing & FEV1 & Forced expiratory volume \\
\hline BNO 1016 & Bionorica 1016 & FISH & Fluorescence in situ hybridization \\
\hline BP & Blood pressure & FNS & Furoate nasal spray \\
\hline BUD & Budesonide & FOXP3 & Forkhead box P3 \\
\hline СВCT & Cone beam $\mathrm{CT}$ & FP & Fluticasone propionate \\
\hline CC16 & Clara cell protein 16 & FPANS & Fluticasone propionate aqueous nasal spray \\
\hline CCAD & Central Compartment Allergic Disease & FPND & Fluticasone propionate nasal drops \\
\hline \multirow[t]{2}{*}{ CCCRC } & The Connecticut Chemosensory Clinical Research & FPNS & Fluticasone propionate nasal spray \\
\hline & Center & FVC & Forced vital capacity \\
\hline CD & Clusters of differentiation & Ga2LEN & Global Allergy and Asthma European Network \\
\hline CF & Cystic fibrosis & G-CSF & Granulocyte colony-stimulating factor \\
\hline CSS & Chronic Sinusitus Survey & GINA & Global Initiative for Asthma \\
\hline CT & Computed tomography & GM-CSF & Granulocyte macrophage colony-stimulating factor \\
\hline CTL & Cytotoxic T lymphocytes & GORD & Gastro-oesophageal reflux disease \\
\hline
\end{tabular}




\begin{tabular}{|c|c|c|c|}
\hline $\begin{array}{l}\text { GP } \\
\text { GPCRs }\end{array}$ & $\begin{array}{l}\text { General Practitioners } \\
\text { G protein-coupled receptors }\end{array}$ & KNHANES & $\begin{array}{l}\text { Korean National Health and Nutrition Examination } \\
\text { Survey }\end{array}$ \\
\hline GWAS & Genome wide association study & LAR & Local allergic rhinitis \\
\hline $\mathrm{H} 3 \mathrm{~N} 2$ & Influenza A virus subtype & LGALS3BP & Galectin-3-binding protein precursor \\
\hline (Aichi/7) & & LK & Lund-Kennedy \\
\hline HB & Hemoglobin & LKES & Lund-Kennedy endoscopy scoring \\
\hline HHV-6 & Human Herpes Virus-6 & LM & Lund-Mackay \\
\hline HLA & Human leukocyte antigen & LMS & Lund-Mackay score \\
\hline HLA-MHC & Human leukocyte antigen - major histocompatibility & LR & Likelihood ratio \\
\hline & complex & LY6E & Lymphocyte Antigen 6 Family Member E) \\
\hline HMPV & Human metapneumovirus & MAIT & Mucosal associated invariant $\mathrm{T}$ \\
\hline HNS & Hypertonic normal saline & MARS & Measurement of Acute Rhinosinusitis Instrument \\
\hline $\mathrm{HOCl}$ & Hypochlorous acid & MCA2 & Minimal cross-sectional area 1 \\
\hline HPA & Hypothalamic-Pituitary-Adrenal & MCC & Mucociliary clearance \\
\hline HPF & High powered field & MCT & Mucociliary clearance time \\
\hline Hpi & Hours post-infection & MD & Mean Difference \\
\hline HPV & Human papillomavirus & MDCT & Multi-detector CT \\
\hline$H R$ & Heart rate & MF & Mometasone furoate \\
\hline HRQL & Health-related quality-of-life & MFNS & Mometasone furoate nasal spray \\
\hline HS & Hypertonic saline & $\mathrm{M}-\mathrm{H}$ & Mantel Haenszel \\
\hline HSV1 & Herpes Simplex Virus-1 & MID & Minimal important difference \\
\hline I & Isoflurane & MII & Multichannel intraluminal impedance \\
\hline 12 & measure of heterogeneity & MIST & Minimally invasive sinus technique \\
\hline IA & Inhaled anesthesia & MM & Middle meatus \\
\hline ICAM-1 & Intercellular adhesion molecule-1 & MMP & Matrix metalloproteinase \\
\hline ICHD & International Classification of Headache Disorders & $\mathrm{MOH}$ & Medication overuse headache \\
\hline ICOR & International Consensus Statement on Rhinosinusitis & MPO & Myeloperoxidase \\
\hline ICP & Integrated care pathway & MRI & Magnetic resonance imaging \\
\hline ICU & Intensive care unit & mRNA & Messenger Ribonucleic acid \\
\hline IFI6 & Interferon Alpha Inducible Protein 6) & MRSA & Methicillin-resistant Staphylococcus aureus \\
\hline $\mathrm{IFIH} 1$ & Interferon Induced With Helicase C Domain 1 & MSCT & Multislice detector $\mathrm{CT}$ \\
\hline IFN & Interferon & MSS & Major symptom score \\
\hline IFV & Influenza virus & MUC5AC & Mucin $5 A C$ \\
\hline $\lg A$ & Immunoglobulin A & $\mathrm{mUV} / \mathrm{VIS}$ & Mixed ultraviolet and visible light \\
\hline $\lg \mathrm{E}$ & Immunoglobulin E & N-ERD & NSAID-exacerbated respiratory disease \\
\hline $\lg G$ & Immunoglobulin G & NAPT & Nasal allergen provocation test \\
\hline IL & Interleukin & NAR & Non-allergic rhinitis \\
\hline IL-5Ra & Interleukin-5 Receptor Alpha & NARES & Non-allergic rhinitis with eosinophils \\
\hline ILC & Innate lymphoid cell & NARESMA & Non-allergic rhinitis with eosinophils and Mast-Cells \\
\hline IMOS & Integrated Medicine Outcomes Scale & NARMA & Non-allergic rhinitis with Mast-Cells \\
\hline INCS & Intranasal corticosteroids spray & NARNA & Non-allergic rhinitis with neutrophils \\
\hline Inkt & Invariant natural killer T & NECs & Nasal epithelial cells \\
\hline iNOS & Inducible Nitric oxide & NF & Nuclear factor \\
\hline IOP & Intraocular pressure & $\mathrm{NIH}$ & National Institutes of Health \\
\hline IP-10 & Interferon gamma-induced protein 10 & NK & Natural killer \\
\hline IT & Immunotherapy & NO & Nitric oxide \\
\hline ITS & Internal transcribed spacer & NOSE & Nasal Obstruction Symptom Evaluation \\
\hline ITT & Intention to Treat & NP & Nasal polyps \\
\hline i.v. & Intravenous & NPS & Nasal polyp score \\
\hline
\end{tabular}




\begin{tabular}{|c|c|c|c|}
\hline NS & Nasal saline & SCCs & Solitary chemosensory cells \\
\hline NSAIDs & Non-Steroidal Anti-Inflammatory Drugs & sct & Saccharine clearance time \\
\hline NSD & no significant difference & SCUAD & Severe chronic upper airway disease \\
\hline NSS & Nasal and sinus symptom scale & SD & Standard deviation \\
\hline O.d. & Once daily & SF-12 & Short Form (12) Health Survey \\
\hline OERPs & Olfactory event-related potentials & SF-36 & Short Form (36) Health Survey \\
\hline OR & Odds ratio & SHS & Second-hand smoke \\
\hline OUT & Operational taxonomic unit & SIA & Sinus Severity Assessment \\
\hline$P$ & Propofol & slgE & Specific lgE \\
\hline PARP12 & Poly(ADP-Ribose) Polymerase & SMD & Standard mean difference \\
\hline PCD & Primary cilia dyskinesia & SNAQ & Sino-nasal questionnaire \\
\hline PCR & Polymease chain reaction & SNOT & Sino-Nasal Outcome Test \\
\hline PCV7 & Pneumococcal conjugate vaccine & SNPS & Single nucleotide polymorphisms \\
\hline PD-1 & Programmed cell death-1 & SOL IL-5Ra & Soluble Interleukin-5 Receptor Alpha \\
\hline PEA & phenyl ethyl alcohol & SPA & Surfactant protein A \\
\hline PEF & Pulmonary expiratory flow & SPGB & Sphenopalatine ganglion block \\
\hline PEFR & peak expiratory flow rate & ssDNA & Single-stranded DNA \\
\hline PG & Prostaglandin & SSS & Sinusitis Severity Score \\
\hline PIV & Parainfluenza virus & STT & Saccharin transit time \\
\hline PMN & Polymorphonuclear neutrophils & $t-P A$ & Tissue plasminogen activator \\
\hline PNAd & Peripheral lymph node addressin & TAME & P-tosyl-I-arginine methyl ester hydrochloride \\
\hline PND & Post-nasal drip & (-esterase & \\
\hline PNIF & Peak nasal inspiratory flow & activity) & \\
\hline POSE & Perioperative Sinus Endoscopy score & TGF & Transforming growth factor \\
\hline PROMS & Patient reported outcome measures & Th & Thelper \\
\hline PRRs & Pattern recognition receptors & TIVA & Total intravenous anaesthetic \\
\hline PVR & Post-viral rhinosinusitis & TLR & Toll-like receptor \\
\hline QOL & Quality of life & TNF & Tumor necrosis factor \\
\hline $\mathrm{R}$ & remifentanil & Treg & T regulatory \\
\hline RANTES & Regulated upon Activation, Normal T-cell Expressed, & TSLP & Thymic stromal lymphopoietin \\
\hline & and Secreted & TSS & Total symptom score \\
\hline RARS & Recurrent Acute Rhinosinusitis & UPSIT & University of Pennsylvania Smell Identification Test \\
\hline RAST & Radio Allergosorbent Test & URTI & Upper respiratory tract infection \\
\hline $\mathrm{RCT}$ & Randomised controlled trial & UVA & Ultraviolet A \\
\hline REAH & Respiratory epithelial adenomatoid hamartomas & UVB & Ultraviolet B \\
\hline rG-CSF & Recombinant human granulocyte colony-stimulating & VAS & Visual Analogue Scale \\
\hline & factor & VCAM & Vascular cell adhesion molecule \\
\hline RIG-1 & Retinoic acid inducible gene 1 & ZBP1 & Zipcode-binding protein 1 \\
\hline RQLQ & Rhinoconjunctivitis Quality of Life Questionnaire & ZO-1 & Zonula occludens-1 \\
\hline RR & Relative Risk & & \\
\hline RS & Rhinosinusitis & & \\
\hline RSDI & Rhinosinusitis Disability Index & & \\
\hline RSOM-31 & Rhinosinusitis Outcome Measure-31 & & \\
\hline RSV & Respiratory syncytial virus & & \\
\hline RTP4 & Receptor Transporter Protein 4 & & \\
\hline RV & Rhinovirus & & \\
\hline$S$ & Sevoflurane & & \\
\hline SBPCT & Single blind placebo controlled trial & & \\
\hline SBRT & Single blind randomised trial & & \\
\hline
\end{tabular}




\section{Executive summary including integrated care pathways}

\subsection{Summary}

The European Position Paper on Rhinosinusitis and Nasal Polyps 2020 is the update of similar evidence based position papers published in 2005 and 2007 and $2012^{(1-3)}$. The core objective of the EPOS2020 guideline is to provide revised, up-to-date and clear evidence-based recommendations and integrated care pathways in ARS and CRS. EPOS2020 provides an update on the literature published and studies undertaken in the eight years since the EPOS2012 position paper was published and addresses areas not extensively covered in EPOS2012 such as paediatric CRS and sinus surgery. EPOS2020 also involves new stakeholders, including pharmacists and patients, and addresses new target users who have become more involved in the management and treatment of rhinosinusitis since the publication of the last EPOS document, including pharmacists, nurses, specialised care givers and indeed patients themselves, who employ increasing self-management of their condition using over the counter treatments. The document provides suggestions for future research in this area and offers updated guidance for definitions and outcome measurements in research in different settings.

EPOS2020 contains chapters on definitions and classification where we have defined a large number of terms and indicated preferred terms. A new classification of CRS into primary and secondary CRS and further division into localized and diffuse disease, based on anatomic distribution is proposed. There are extensive chapters on epidemiology and predisposing factors, inflammatory mechanisms, (differential) diagnosis of facial pain, allergic rhinitis, genetics, cystic fibrosis, aspirin exacerbated respiratory disease, immunodeficiencies, allergic fungal rhinosinusitis and the relationship between upper and lower airways. The chapters on paediatric acute and chronic rhinosinusitis are totally rewritten. All available evidence for the management of acute rhinosinusitis and chronic rhinosinusitis with or without nasal polyps in adults and children is systematically reviewed and integrated care pathways based on the evidence are proposed. Despite considerable increases in the amount of quality publications in recent years, a large number of practical clinical questions remain. It was agreed that the best way to address these was to conduct a Delphi exercise which is a structured communication technique, originally developed as a systematic, interactive forecasting method which relies on a panel of experts. The EPOS2020 group firstly prioritised the areas for consideration as a result of which we have concentrated on diagnostic issues in the first instance. The results have been integrated into the respective sections. Last but not least, advice for patients and pharmacists and a new list of research needs are included.

\subsection{Classification, definitions and terminology}

\subsubsection{Introduction}

Rhinosinusitis is a common condition in most of the world, leading to a significant burden on society in terms of healthcare consumption and productivity loss ${ }^{(4-7)}$. Acute rhinosinusitis (ARS) has a one-year prevalence of $6-15 \%$ and is usually the consequence of a viral common cold. ARS is usually a selflimiting disease but serious complications leading to life threatening situations and even death have been described ${ }^{(8)}$. It is one of the most common reasons for prescription of antibiotics and proper management is extremely pertinent in the context of the global crisis of resistance to antibiotics ${ }^{(9)}$. Chronic rhinosinusitis (CRS) is a significant health problem and affects $5-12 \%$ of the general population. The major definitions are summarized here. For more definitions please refer to chapter 2.

\subsubsection{Clinical definition of rhinosinusitis}

\subsubsection{Clinical definition of rhinosinusitis in adults}

Rhinosinusitis in adults is defined as:

- inflammation of the nose and the paranasal sinuses characterised by two or more symptoms, one of which should be either nasal blockage / obstruction / congestion or nasal discharge (anterior / posterior nasal drip):

\pm facial pain/pressure

\pm reduction or loss of smell

and either

- endoscopic signs of:

- nasal polyps, and/or

- mucopurulent discharge primarily from middle meatus and/ or

- oedema / mucosal obstruction primarily in middle meatus and/or

- CT changes:

- mucosal changes within the ostiomeatal complex and/or sinuses

\subsubsection{Clinical definition of rhinosinusitis in children}

Paediatric rhinosinusitis is defined as:

- $\quad$ presence of two or more symptoms one of which should be either nasal blockage / obstruction / congestion or nasal discharge (anterior / posterior nasal drip): 
\pm facial pain/pressure

\pm cough

and either

- endoscopic signs of:

- nasal polyps, and/or

- mucopurulent discharge primarily from middle meatus and/

or

-oedema / mucosal obstruction primarily in middle meatus and/or

- $\quad$ CT changes:

-mucosal changes within the ostiomeatal complex and/or sinuses

\subsubsection{Definition of CRS epidemiology studies and General} Practice

For epidemiological studies and general practice, the definition of CRS is based on symptomatology usually without ENT examination or radiology. We are aware that this will give an over estimation of the prevalence due to overlap with allergic and non-allergic rhinitis ${ }^{(56-58)}$.

\subsubsection{Acute rhinosinusitis (ARS) in adults}

Acute rhinosinusitis in adults is defined as:

sudden onset of two or more symptoms, one of which should be either nasal blockage/obstruction/congestion or nasal discharge (anterior/posterior nasal drip):

- \pm facial pain/pressure

- $\quad \pm$ reduction or loss of smell

for $<12$ weeks;

with symptom free intervals if the problem is recurrent, with

validation by telephone or interview.

\subsubsection{Acute rhinosinusitis in children}

Acute rhinosinusitis in children is defined as: sudden onset of two or more of the symptoms:

- nasal blockage/obstruction/congestion

- or discoloured nasal discharge

- or cough (daytime and night-time)

for $<12$ weeks;

with symptom free intervals if the problem is recurrent; with validation by telephone or interview.

Questions on allergic symptoms (i.e. sneezing, watery rhinorrhoea, nasal itching, and itchy watery eyes) should be included.

\subsubsection{Recurrent acute rhinosinusitis (RARS)}

ARS can occur once or more than once in a defined time period. This is usually expressed as episodes/year but with complete resolution of symptoms between episodes.

Recurrent ARS (RARS) is defined as $\geq 4$ episodes per year with symptom free intervals ${ }^{(42,78)}$.

\subsubsection{Definition of chronic rhinosinusitis in adults}

Chronic rhinosinusitis (with or without nasal polyps) in adults is defined as:

presence of two or more symptoms, one of which should be either nasal blockage / obstruction / congestion or nasal discharge (anterior / posterior nasal drip):

- $\quad \pm$ facial pain/pressure;

- $\quad \pm$ reduction or loss of smell;

for $\geq 12$ weeks;

with validation by telephone or interview.

Figure 1.2.1. Classification of primary CRS (Adapted from Grayson et al ${ }^{(154)}$ )

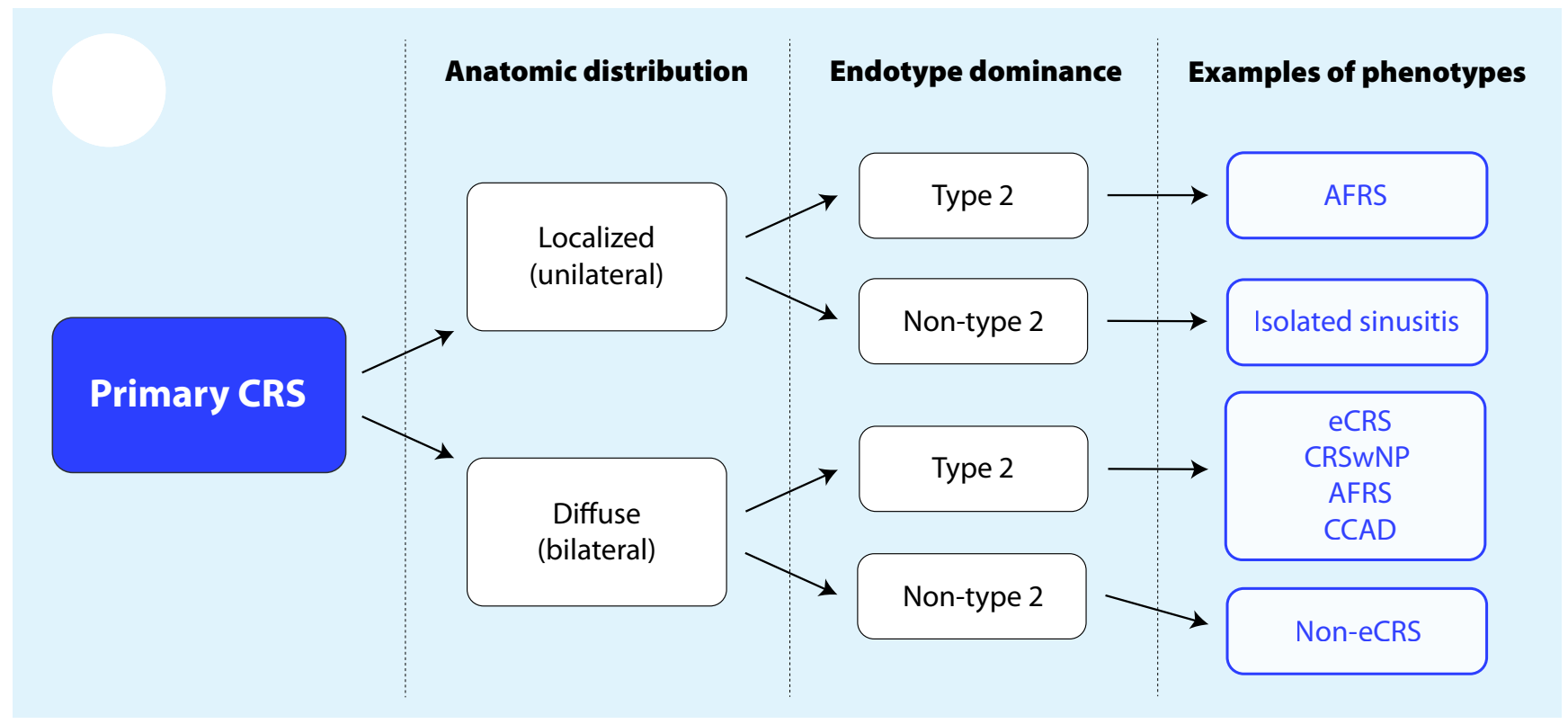

AFRS, allergic fungal rhinosinusitis; CCAD, central compartment allergic disease; CRSwNP, chronic rhinosinusitis with nasal polyps; eCRS, eosinophilic CRS. 
Questions on allergic symptoms (i.e. sneezing, watery rhinorrhoea, nasal itching, and itchy watery eyes) should be included.

\subsubsection{Definition of chronic rhinosinusitis in children}

Chronic rhinosinusitis (with or without nasal polyps) in children is defined as:

presence of two or more symptoms one of which should be either nasal blockage / obstruction / congestion or nasal discharge (anterior/posterior nasal drip):

- \pm facial pain/pressure;

- \pm cough;

for $\geq 12$ weeks;

with validation by telephone or interview.

\subsubsection{Definition of difficult-to-treat rhinosinusitis}

This is defined as patients who have persistent symptoms of rhinosinusitis despite appropriate treatment (recommended medication and surgery). Although the majority of CRS patients can obtain control, some patients will not do so even with maximal medical therapy and surgery.

Patients who do not reach an acceptable level of control despite adequate surgery, intranasal corticosteroid treatment and up to two short courses of antibiotics or systemic corticosteroids in the last year can be considered to have difficult-to-treat rhinosinusitis.

No changes have been made compared to EPOS2012 in the definition of severity or in acute versus chronic $^{(3)}$. For acute rhinosinusitis the term ARS comprises viral ARS (common cold) and post-viral ARS. In EPOS2007, the term 'non-viral ARS' was chosen to indicate that most cases of ARS are not bacterial.
However, this term apparently led to confusion and for that reason we decided in EPOS2012 to choose the term 'post-viral ARS' to express the same phenomenon. A small percentage of the patients with post-viral ARS will have acute bacterial rhinosinusitis (ABRS).Chronic rhinosinusitis has traditionally been classified into chronic rhinosinusitis with nasal polyps (CRSwNP) and without nasal polyps (CRSsNP). CRSwNP: chronic rhinosinusitis as defined above and bilateral, endoscopically visualised polyps in middle meatus; and CRSsNP: chronic rhinosinusitis as defined above and no visible polyps in middle meatus, if necessary following decongestant.

This definition accepts that there is a spectrum of disease in CRS which includes polypoid change in the sinuses and/ or middle meatus but excludes those with polypoid disease presenting in the nasal cavity to avoid overlap. Moreover, it has become progressively clear that CRS is a complex disease consisting of several disease variants with different underlying pathophysiologies ${ }^{(10,11)}$. The phenotypes do not provide full insight into all underlying cellular and molecular pathophysiologic mechanisms of CRS which becomes increasingly relevant because of the variable association with comorbidities such as asthma and responsiveness to different treatments including corticosteroids, surgery and biological agents $^{(12-15)}$. Better identification of endotypes might permit individualization of therapy that can be targeted against the pathophysiologic processes of a patient's endotype, with potential for more effective treatment and better patient outcomes.

\subsubsection{Classification of CRS}

The EPOS2020 steering group has chosen to look at CRS in terms

Figure 1.2.2. Classification of secondary CRS (Adapted from Grayson et al ${ }^{(154))}$.

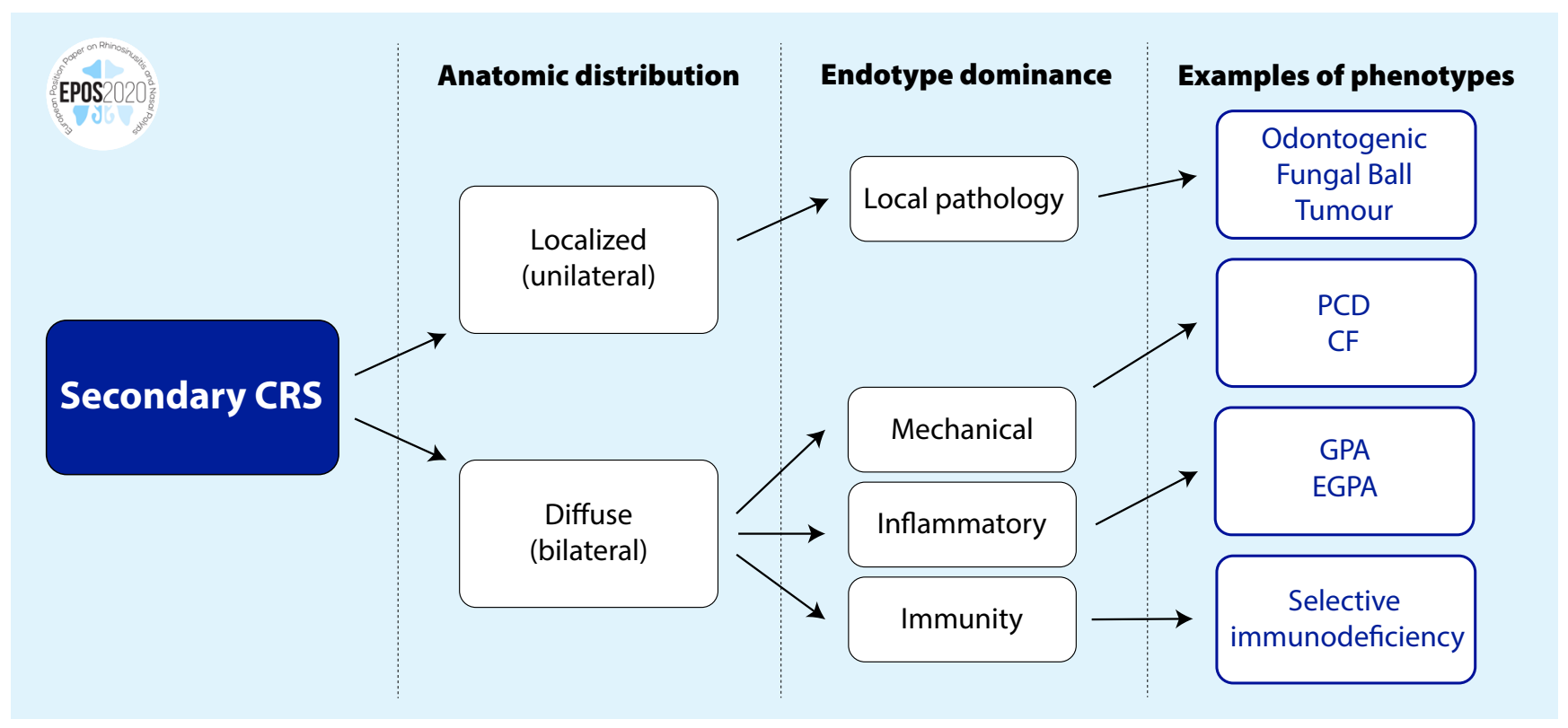

CF, cystic fibrosis; EGPA, eosinophilic granulomatosis with polyangiitis (Churg-Strauss disease); GPA, granulomatosis with polyangiitis (Wegener's disease); PCD, primary ciliary dyskinesia. 
Figure 1.2.3. Assessment of current clinical control of CRS.

\begin{tabular}{|c|c|c|c|}
\hline \multirow{2}{*}{ EPOS 2020} & \multicolumn{3}{|c|}{ EPOS 2020: Assessment of current clinical control of CRS (in the last month) } \\
\hline & $\begin{array}{l}\text { Controlled } \\
\text { (all of the following) }\end{array}$ & $\begin{array}{l}\text { Partly controlled } \\
\text { (at least } 1 \text { present) }\end{array}$ & $\begin{array}{l}\text { Uncontrolled } \\
\text { (3 or more present) }\end{array}$ \\
\hline Nasal blockage' & Not present or not bothersome ${ }^{2}$ & $\begin{array}{l}\text { Present } \\
\text { on most days of the week }\end{array}$ & $\begin{array}{l}\text { Present } \\
\text { on most days of the week }\end{array}$ \\
\hline Rhinorrhoea / Postnasal drip' & Little and mucous ${ }^{2}$ & $\begin{array}{l}\text { Mucopurulent } \\
\text { on most days of the week }\end{array}$ & $\begin{array}{l}\text { Mucopurulent } \\
\text { on most days of the week }\end{array}$ \\
\hline Facial pain / Pressure ${ }^{1}$ & $\begin{array}{l}\text { Not present } \\
\text { or not bothersome }\end{array}$ & $\begin{array}{l}\text { Present } \\
\text { on most days of the week }\end{array}$ & $\begin{array}{l}\text { Present } \\
\text { on most days of the week }\end{array}$ \\
\hline Smell' & $\begin{array}{l}\text { Normal } \\
\text { or only slightly impaired² }\end{array}$ & Impaired ${ }^{3}$ & Impaired $^{3}$ \\
\hline Sleep disturbance or fatigue ${ }^{1}$ & Not present ${ }^{2}$ & Present $^{3}$ & Present $^{3}$ \\
\hline $\begin{array}{l}\text { Nasal endoscopy } \\
\text { (if available) }\end{array}$ & $\begin{array}{l}\text { Healthy } \\
\text { or almost healthy mucosa }\end{array}$ & Diseased mucosa ${ }^{4}$ & Diseased mucosa ${ }^{4}$ \\
\hline $\begin{array}{l}\text { Rescue treatment } \\
\text { (in last } 6 \text { months) }\end{array}$ & Not needed & $\begin{array}{l}\text { Need of } 1 \text { course of } \\
\text { rescue treatment }\end{array}$ & $\begin{array}{l}\text { Symptoms (as above) persist } \\
\text { despite rescue treatment(s) }\end{array}$ \\
\hline
\end{tabular}

CRS, chronic rhinosinusitis; VAS, visual analogue scale.

of primary and secondary (Figures 1.2.1. and 1.2.2.) and to divide each into localized and diffuse disease based on anatomic distribution. In primary CRS, the disease is considered by endotype dominance, either type 2 or non-type 2 (see 1.5.2.2.).

Clinically localized primary CRS is then subdivided into two phenotypes - allergic fungal rhinosinusitis (AFRS) or an isolated sinusitis. For diffuse CRS, the clinical phenotypes are predominantly eCRS and non-eCRS, determined by the histologic quantification of the numbers of eosinophilic, i.e. number/high powered field which the EPOS panel agreed to be 10/hpf (400x) or higher.

For secondary CRS, again, the division is into localized or diffuse and then considered by four categories dependant on local pathology, mechanical, inflammatory and immunological factors. Thence a range of clinical phenotypes are included as shown.

There has been some discussion about a possible umbrella term of 'eosinophilic fungal rhinosinusitis' but it was agreed that 'allergic' fungal rhinosinusitis should be retained as the principle term due to common usage, recognising that not all cases have evidence of an allergic reaction to fungi e.g. a positive skin prick and/or specific lgE (see also chapter 8.6).

\subsubsection{Other consensus terms related to treatment}

From the many terms used regarding the sufficiency of medical treatment prior to surgery, 'appropriate medical therapy' is the preferred option of EPOS2020. Other decisions were the preferential use of the terms 'irrigation' or 'rinsing' when using saline therapy and with respect to duration of antibiotic courses, the EPOS panel also agreed that four weeks or less would be 'short-term', accepting that in general practice the duration is usually $<10$ days, and $>4$ weeks would be regarded as 'long-term'. It was also acknowledged that the aim of short-term treatment was different from long-term in that short-term courses are generally given for significant acute bacterial infection whereas long term courses are given for their immunomodulatory properties. Immunomodulation encompasses all therapeutic interventions aimed at modifying the immune response and is the preferred over-riding term by EPOS2020. In the treatment of rhinosinusitis, it encompasses the use of biological agents and macrolides as above.

With respect to surgery, functional implies restitution of physiology and is usually, though not exclusively, applied to endoscopic sinus surgery. It should fulfil the following criteria:

- Creates a sinus cavity that incorporates the natural ostium;

- Allows adequate sinus ventilation;

- Facilitates mucociliary clearance;

- Facilitates instillation of topical therapies.

In contrast, a 'Full FESS' is defined as complete sinus opening including anterior and posterior ethmoidectomy, middle meatal antrostomies (likely large), sphenoidotomy and frontal opening (e.g. Draf lla ).Extended endoscopic surgery is used in the same context as 'full' (e.g. Draf III) but could also include 
extension beyond the confines of sinuses i.e. skull base, orbit, pterygopalatine and infratemporal fossa. Finally, radical also includes significant removal of inflamed / dysfunctional mucosa.

\subsubsection{Control of disease}

In EPOS2012 we introduced the concept of control ${ }^{(3)}$. The primary goal of any treatment, especially in chronic diseases, is to achieve and maintain clinical control, which can be defined as a disease state in which the patient does not have symptoms, or the symptoms are not impacting quality of life. In the last decade some studies have been performed that attempted to validate the EPOS2012 proposed measurement of control(15-17). Based on these validation studies, the EPOS2020 steering group thinks that the current EPOS2012 control criteria might overestimate the percentage of patients being uncontrolled. For research purposes we, therefore, recommend using a VAS scale for all symptoms: "not bothersome" can be substituted by 'VAS $\leq$ 5', and 'present / impaired' by 'VAS $>5$ '. Furthermore, we want to make sure that the symptoms are related to CRS and included that in the table. For example, a typical migraine headache should not be taken into account when evaluating control in CRS. The results of the validation studies also still require further psychometric validation (including internal consistency, responsiveness and known group differences) (Figure 1.2.3.). Given the importance of the concept of disease control, from a clinical as well as from a research perspective, there still remains a need for a gold standard to assess disease control in CRS.

\subsubsection{Acute exacerbation of chronic rhinosinusitis (AECRS)} Acute exacerbation of chronic rhinosinusitis (AECRS) is defined as worsening of symptom intensity with return to baseline CRS symptom intensity, often after intervention with corticosteroids and/or antibiotics. The prevalence varies with the patient cohort being studied, season, and how the exacerbation was defined. The precise aetiology of acute exacerbation of CRS is still unclear and is likely to be multifactorial. The role of bacterial infection may have been over-emphasised in the past. Certainly, there is a lack of bacterial airway pathogens identified in the majority of patients with exacerbation. It is possible that since many of these patients have had sinus surgery in the past, postoperative changes in the microbiome create a new microbial environment and other pathogens are in play. Microbial dysbiosis in the form of an altered balance of the bacterial flora rather than a single pathogen may elicit a host inflammatory response.

Virus infections are perhaps more likely to be a key cause of exacerbation of CRS, especially with increasing evidence that rhinovirus infection can drive eosinophilic inflammation and a focus on prevention and management of virus infections may be more effective than treating secondary infections with antibiotics and eosinophilic flare ups with corticosteroids. However, this remains to be further investigated.

Firm scientific evidence is still lacking on therapy of AECRS and only treatment recommendations based on clinical experience and expert opinion are available. However, due to the cyclic and self-limiting nature of AECRS one should be mindful of the 'regression to the mean phenomena'. A patient is more likely to seek treatment when they are at their worst, the likelihood of improvement is high regardless of treatment, which may distort the doctor's clinical experience as well as rendering clinical trials lacking a placebo arm rather meaningless. In spite of this confounder, it is likely that steroids and antibiotics will remain the mainstay of treatment for the foreseeable future even though the role of antibiotics in the treatment of AECRS is not supported by the literature (see chapter 1.6 and 6.1).

Figure 1.3.1. Prevalence of cardinal symptoms of CRS 25,26$)$.

\section{Prevalence of cardinal symptoms of CRS}

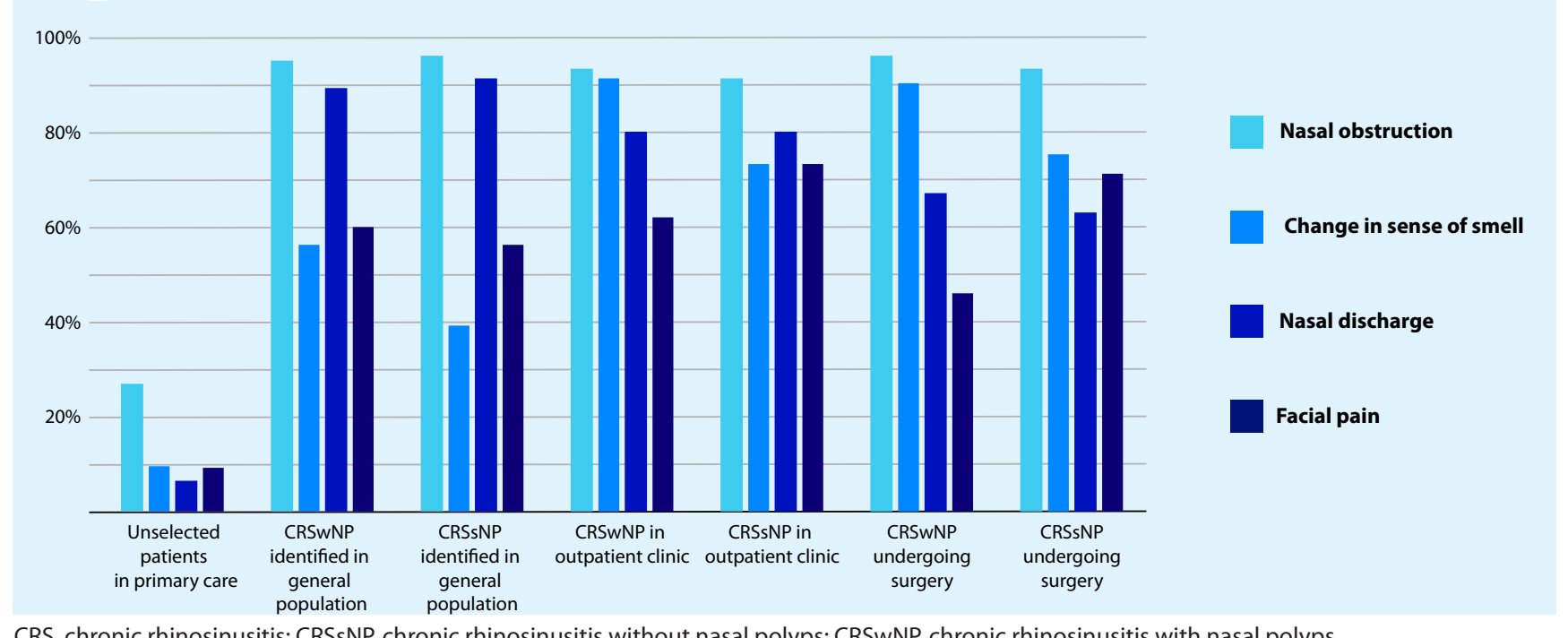

CRS, chronic rhinosinusitis; CRSsNP, chronic rhinosinusitis without nasal polyps; CRSwNP, chronic rhinosinusitis with nasal polyps. 


\section{Severity of cardinal symptoms of CRS}

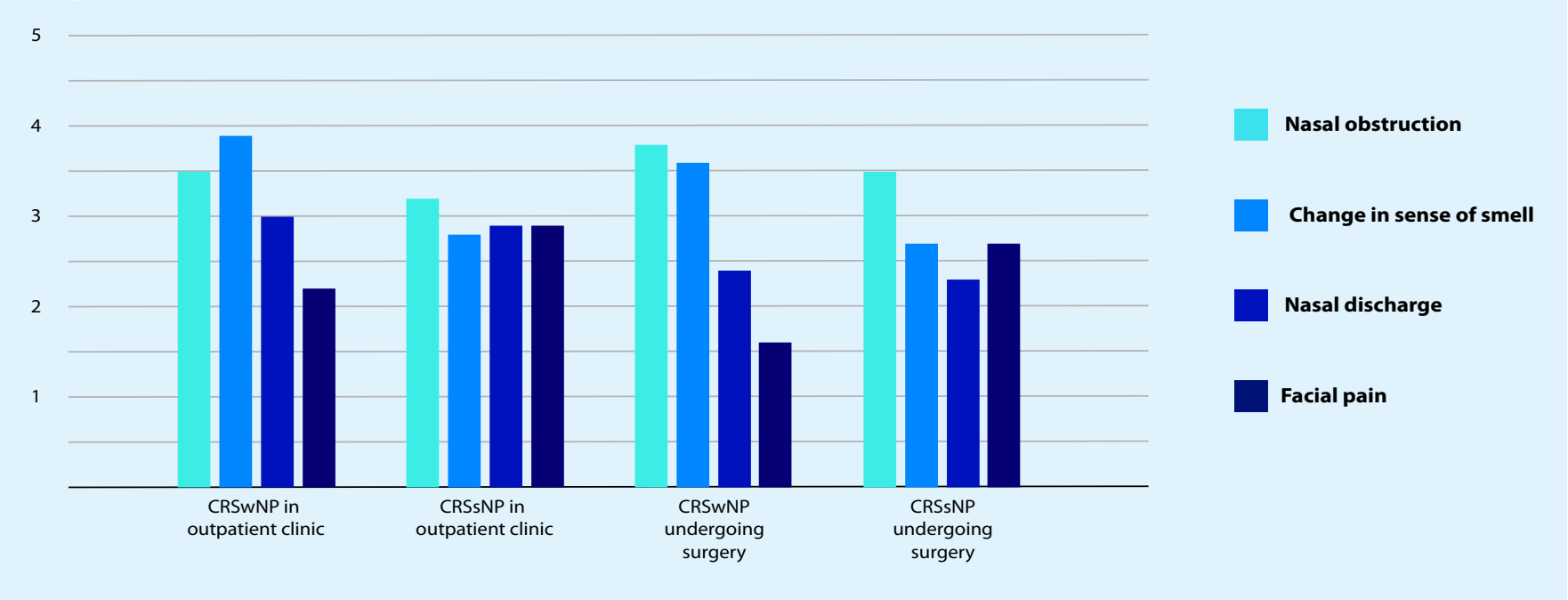

CRS, chronic rhinosinusitis; CRSsNP, chronic rhinosinusitis without nasal polyps; CRSwNP, chronic rhinosinusitis with nasal polyps.

\subsection{Burden of acute and chronic rhinosinusitis}

Chapter 3 covers the burden of rhinosinusitis, its impact on quality of life and the costs, both direct and indirect.

\subsubsection{Quality of life (QOL)}

Both ARS and CRS are associated with significant adverse effects on quality of life using a variety of validated questionnaires including the general health Eq-5D ${ }^{(18,19)}$ and SF36 $6^{(20,21)}$ and more rhinologic-specific SNOT16 $6^{(22)}$ and SNOT 22 ${ }^{(23)}$. Chronic rhinosinusitis produces greater quality of life impairment than acute $^{(24)}$. Gliklich and Metson first demonstrated the impact of CRS on global quality of life, finding that CRS had a greater impact on social functioning than angina or chronic heart failure ${ }^{(20)}$. More recently, they have shown that health utility values, measured using the EQ-5D, were lower than the general population, and comparable to other chronic diseases such as asthma ${ }^{(19)}$

In CRS, the 'cardinal' symptoms are nasal obstruction or congestion, nasal discharge (which can be anterior or posterior), alteration in sense of smell and facial pain and pressure. These may vary in prevalence between unselected patients in primary care, CRS patients in the general population, in an outpatient setting and those undergoing surgery and in severity between those seen in outpatients and those undergoing surgery (Figure 1.3.1).

Nasal obstruction and alteration in sense of smell and taste are both the most severe and prevalent symptoms in CRSwNP, while in CRSsNP, nasal obstruction is again the most severe, with facial pain and nasal discharge reported as equally severe as altered smell and taste ${ }^{(25,26)}$ (Figure 1.3.2.). In patients presenting to ENT clinics, the presence of cardinal symptoms has a positive predictive value of 39.9 , with high sensitivity but low specificity for a diagnosis of $\mathrm{CRS}^{(27)}$.

The overall severity rating of symptoms is obviously highly dependent upon the population being studied. Patients in secondary care awaiting surgery report mean symptom severity scores in the moderate to severe range, with a mean SNOT-22 score of 42.0 compared with a control group where a mean score of 9.3 was reported ${ }^{(23)}$. CRSsNP patients had higher preoperative baseline scores (44.2) compared with CRSwNP (41.0).

\subsubsection{Costs of rhinosinusitis}

Health care spending is significantly greater in rhinosinusitis than in other diseases such as peptic ulcer disease, acute asthma, and hay fever ${ }^{(28)}$. In the USA, the direct costs for the management of CRS are now between $\$ 10$ and $\$ 13$ billion per year, or $\$ 2609$ per patient per year. In Europe, Wahid et al. reported 2974 GBP on costs for primary and secondary care extrapolated for a year period compared to 555 GBP in the control group and 304 versus 51 GBP out-of-pocket expenditure ${ }^{(29)}$. Lourijsen et al. found yearly direct costs of 1501 euro per year in a group of patients with $\mathrm{CRSWNP}^{(30)}$. Overall CRS leads to an incremental direct healthcare expenditure of 2500 euro per patient per year. The highest direct costs were associated with patients who had recurrent polyposis after surgery ${ }^{(31)}$. However, whilst surgery is expensive, varying from up to $\$ 11,000$ in USA to $\$ 1100$ in India ${ }^{(32-34)}$, it results in a decrease in direct costs in the subsequent two post-operative years ${ }^{(35)}$. The indirect costs of rhinosinusitis are much greater than the direct costs. Since $85 \%$ of patients with rhinosinusitis are of working age (range: 18-65 years old), indirect costs such as missed workdays (absenteeism) and decreased productivity at work (presenteeism) significantly add to the economic burden 
Table 1.4.1. Treatment evidence and recommendations for adults and children with acute viral rhinosinusitis (common cold)*.

\begin{tabular}{|c|c|c|}
\hline Therapy & $\begin{array}{l}\text { Level of } \\
\text { evidence }\end{array}$ & GRADE recommendation \\
\hline Antibiotics & 1a $(-)$ & $\begin{array}{l}\text { There is no evidence of benefit from antibiotics for the common cold or for persisting acute purulent } \\
\text { rhinitis in children or adults. There is evidence that antibiotics cause significant adverse effects in adults } \\
\text { when given for the common cold and in all ages when given for acute purulent rhinitis. Routine use of } \\
\text { antibiotics for these conditions is not recommended. }\end{array}$ \\
\hline Nasal corticosteroid & $1 \mathrm{a}(-)$ & $\begin{array}{l}\text { The current evidence does not support the use of nasal corticosteroids for symptomatic relief from the } \\
\text { common cold }\end{array}$ \\
\hline Antihistamines & $1 \mathrm{a}$ & $\begin{array}{l}\text { Antihistamines have a limited short-term (days } 1 \text { and } 2 \text { of treatment) beneficial effect on severity of over- } \\
\text { all symptoms in adults but not in the mid to long term. There is no clinically significant effect on nasal } \\
\text { obstruction, rhinorrhoea or sneezing }\end{array}$ \\
\hline Decongestant (oral / nasal) & la & $\begin{array}{l}\text { The current evidence suggests that multiple doses of decongestants may have a small positive effect on } \\
\text { subjective measures of nasal congestion in adults with the common cold. Decongestants do not seem to } \\
\text { increase the risk of adverse events in adults in the short term. }\end{array}$ \\
\hline $\begin{array}{l}\text { Paracetamol } \\
\text { (Acetaminophen) }\end{array}$ & la & $\begin{array}{l}\text { Paracetamol may help relieve nasal obstruction and rhinorrhoea but does not appear to improve other cold } \\
\text { symptoms (including sore throat, malaise, sneezing and cough) }\end{array}$ \\
\hline NSAIDs & la & $\begin{array}{l}\text { NSAIDs do not significantly reduce the total symptom score, or duration of colds. However, for outcomes } \\
\text { related to the analgesic effects of NSAIDs (headache, ear pain and muscle and joint pain) NSAIDs produce } \\
\text { significant benefits, and malaise shows a borderline benefit, although throat irritation is not improved. Chills } \\
\text { show mixed results. For respiratory symptoms, cough and nasal discharge scores are not improved, but the } \\
\text { sneezing score is significantly improved. There is no evidence of increased frequency of adverse effects in } \\
\text { the NSAID treatment groups. }\end{array}$ \\
\hline $\begin{array}{l}\text { Antihistamine-decongestant- } \\
\text { analgesic combinations }\end{array}$ & la & $\begin{array}{l}\text { Antihistamine-analgesic-decongestant combinations have some general benefit in adults and older } \\
\text { children with common cold. These benefits must be weighed against the risk of adverse effects. There is no } \\
\text { evidence of effectiveness in young children. }\end{array}$ \\
\hline Ipratropium bromide & la & $\begin{array}{l}\text { The existing evidence suggests that ipratropium bromide is likely to be effective in ameliorating } \\
\text { rhinorrhoea. Ipratropium bromide has no effect on nasal congestion and its use is associated with more } \\
\text { side effects compared to placebo or no treatment although these appeared to be well tolerated and self- } \\
\text { limiting. }\end{array}$ \\
\hline Nasal irrigation with saline & $\mathrm{lb}$ & $\begin{array}{l}\text { Nasal saline irrigation possibly has benefits for relieving the symptoms of acute URTIs mainly in children and } \\
\text { is considered an option by the EPOS steering group. }\end{array}$ \\
\hline Steam / heated humidified air & $1 \mathrm{a}(-)$ & $\begin{array}{l}\text { The current evidence does not show any benefits or harms from the use of heated, humidified air delivered } \\
\text { for the treatment of the common cold. }\end{array}$ \\
\hline Probiotics & la & $\begin{array}{l}\text { Probiotics may be more beneficial than placebo for preventing acute URTIs. However, the quality of the } \\
\text { evidence was (very) low. }\end{array}$ \\
\hline Vitamin C & la & $\begin{array}{l}\text { Given the consistent effect of vitamin } C \text { on the duration and severity of colds in regular supplementation } \\
\text { studies, and the low cost and safety, it may be worthwhile for common cold patients to test on an } \\
\text { individual basis whether therapeutic vitamin C is beneficial for them. }\end{array}$ \\
\hline Vaccines & $1 \mathrm{~b}(-)$ & $\begin{array}{l}\text { There are no conclusive results to support the use of vaccines for preventing the common cold in healthy } \\
\text { people. This is in contrast to influenza vaccines. }\end{array}$ \\
\hline Exercise & la & Regular, moderate-intensity exercise may have an effect on the prevention of the common cold. \\
\hline Echinacea & $1 a(-)$ & $\begin{array}{l}\text { Echinacea products have not been shown to provide benefits for treating colds, although, there could be } \\
\text { a weak benefit from some Echinacea products: the results of individual prophylaxis trials consistently show } \\
\text { positive (if non-significant) trends, although potential effects are of questionable clinical relevance. }\end{array}$ \\
\hline Zinc & la & $\begin{array}{l}\text { Zinc administered as zinc acetate or zinc gluconate lozenges at a dose of }>=75 \mathrm{mg} / \text { day and taken } \\
\text { within } 24 \text { hours of onset of symptoms significantly reduces the duration of common cold. For those } \\
\text { considering using zinc it is advised to use it at this dose throughout the cold. Regarding prophylactic zinc } \\
\text { supplementation, currently no firm recommendation can be made because of insufficient data. }\end{array}$ \\
\hline $\begin{array}{l}\text { Herbal medicine (excluding } \\
\text { Echinacae) }\end{array}$ & lb & $\begin{array}{l}\text { Some herbal medicines like BNO1016, Cineole and Andrographis paniculata SHA-10 extract have significant } \\
\text { impact on symptoms of common cold without important adverse events. A formal systematic review is } \\
\text { missing. }\end{array}$ \\
\hline Fusafungine & la & $\begin{array}{l}\text { Fusafungine is an effective treatment of common cold especially when administered early. However, } \\
\text { serious allergic reactions involving bronchospasm although rare have occurred after the use of fusafungine. } \\
\text { For that reason, the medication is no longer on the market. }\end{array}$ \\
\hline
\end{tabular}


Table 1.4.2. Treatment evidence and recommendations for adults with acute post-viral rhinosinusitis.

\begin{tabular}{|c|c|c|}
\hline Therapy & $\begin{array}{l}\text { Level of } \\
\text { evidence }\end{array}$ & GRADE recommendation \\
\hline Antibiotics & $1 \mathrm{a}(-)$ & $\begin{array}{l}\text { There is no benefit from prescribing antibiotics for post viral ARS in adults. There is no effect on } \\
\text { cure or duration of disease and there are more adverse events. Based on the moderate level of } \\
\text { evidence and the fact that acute post-viral rhinosinusitis is a self-limiting disease, the EPOS2020 } \\
\text { steering group advises against the use of antibiotics for adults in this situation. }\end{array}$ \\
\hline Nasal corticosteroids & $1 \mathrm{a}$ & $\begin{array}{l}\text { Nasal corticosteroids are effective in reducing total symptom score in adults suffering from } \\
\text { acute post-viral rhinosinusitis. However, the effect is small. Nasal corticosteroids have not been } \\
\text { shown to have an effect on QOL. Acute post-viral rhinosinusitis is a self-limiting disease. Based } \\
\text { on the moderate quality of the evidence and the small effect size the EPOS2020 steering group } \\
\text { advises only to prescribe a nasal corticosteroid when reduction of the symptoms of the acute } \\
\text { post-viral rhinosinusitis is considered necessary. }\end{array}$ \\
\hline Systemic corticosteroids & $1 \mathrm{a}$ & $\begin{array}{l}\text { Systemic corticosteroids, with or without antibiotics do not have a positive effect on recovery } \\
\text { at 7-14 days. There is a small but significant effect of systemic corticosteroids versus placebo } \\
\text { on facial pain at days } 4-7 \text { after start of the treatment. There are no studies comparing systemic } \\
\text { corticosteroids to nasal corticosteroids. The quality of the evidence is low. Based on the } \\
\text { evidence, the numbers needed to treat and the potential harm of systemic corticosteroids, } \\
\text { the EPOS2020 steering group advises against the use of systemic corticosteroids in patients } \\
\text { suffering from acute post-viral rhinosinusitis. }\end{array}$ \\
\hline Decongestant (oral / nasal) & $\mathrm{lb}$ & $\begin{array}{l}\text { Nasal decongestants may be effective in improving mucociliary clearance throughout the } \\
\text { acute phase of the disease. No studies have been performed evaluating the effect on resolution } \\
\text { or reduction of symptoms of postviral ARS. Based on the absence of clinically relevant data, } \\
\text { the EPOS2020 steering group cannot advise on the use of decongestants in acute post-viral } \\
\text { rhinosinusitis. }\end{array}$ \\
\hline Nasal irrigation with saline & $\mathrm{lb}$ & $\begin{array}{l}\text { One small study did not find a difference between saline nasal spray versus no treatment. } \\
\text { One very small study found a larger effect of high volume versus low volume saline rinsing } \\
\text { on purulent rhinorrhoea and post-nasal drip. Based on the very low quality of the evidence } \\
\text { no strong advice can be given about the use of nasal saline irrigation although on theoretical } \\
\text { grounds saline can be expected to be beneficial rather than harmful. }\end{array}$ \\
\hline Homeopathy & $\mathrm{lb}$ & $\begin{array}{l}\text { We found one study evaluating the effect of homeopathy (sinfrontal) showing a significant } \\
\text { reduction of symptoms and radiographic improvement versus placebo. Based on the limited } \\
\text { evidence the EPOS2020 steering group cannot give clear advice on the use of homeopathy in } \\
\text { acute post-viral rhinosinusitis. }\end{array}$ \\
\hline Herbal medicine & $\mathrm{lb}$ & $\begin{array}{l}\text { Some herbal medicines like BNO1016 tablets and Pelargonium sidoides drops and Myrtol } \\
\text { (and other essential oil) capsules have significant impact on symptoms of acute postviral } \\
\text { rhinosinusitis without significant adverse events. }\end{array}$ \\
\hline
\end{tabular}

ARS, acute rhinosinusitis; QOL, quality of life.

Table 1.4.3. Treatment evidence and recommendations for children with acute post-viral rhinosinusitis.

\begin{tabular}{|c|c|c|}
\hline Therapy & $\begin{array}{l}\text { Level of } \\
\text { evidence }\end{array}$ & GRADE recommendation \\
\hline Antibiotics & $1 \mathrm{a}(-)$ & $\begin{array}{l}\text { The use of antibiotics in children with acute post-viral rhinosinusitis is not associated with greater } \\
\text { cure/significant improvement. Based on the moderate level of evidence and the fact that acute } \\
\text { post-viral rhinosinusitis is a self-limiting disease, the EPOS2020 steering group advises against the } \\
\text { use of antibiotics for children in this situation. }\end{array}$ \\
\hline Nasal corticosteroids & $1 \mathrm{a}$ & $\begin{array}{l}\text { Nasal corticosteroids seem to be effective in reducing total symptom score in children } \\
\text { suffering from acute post-viral rhinosinusitis on top of (ineffective) antibiotics. Acute post- } \\
\text { viral rhinosinusitis is a self-limiting disease. Based on the very low quality of the evidence the } \\
\text { EPOS2020 steering group cannot advise on the use of nasal corticosteroids in children with acute } \\
\text { post-viral rhinosinusitis. }\end{array}$ \\
\hline Antihistamines & $1 b(-)$ & $\begin{array}{l}\text { There is one study evaluating antihistamines versus placebo in addition to (ineffective) antibiotics } \\
\text { in children with post-viral ARS showing no additive effect of antihistamines over the treatment } \\
\text { given. Based on the very low quality of the evidence, the EPOS2020 steering group cannot advise } \\
\text { on the use of antihistamines in post-viral ARS. }\end{array}$ \\
\hline Bacterial lysates & $\mathrm{lb}$ & One study has shown benefit in the use of OM-85-BV for shortening the duration of illness. \\
\hline
\end{tabular}

ARS, acute rhinosinusitis. 
Figure 1.4.1. Integrated care pathway of acute rhinosinusitis.

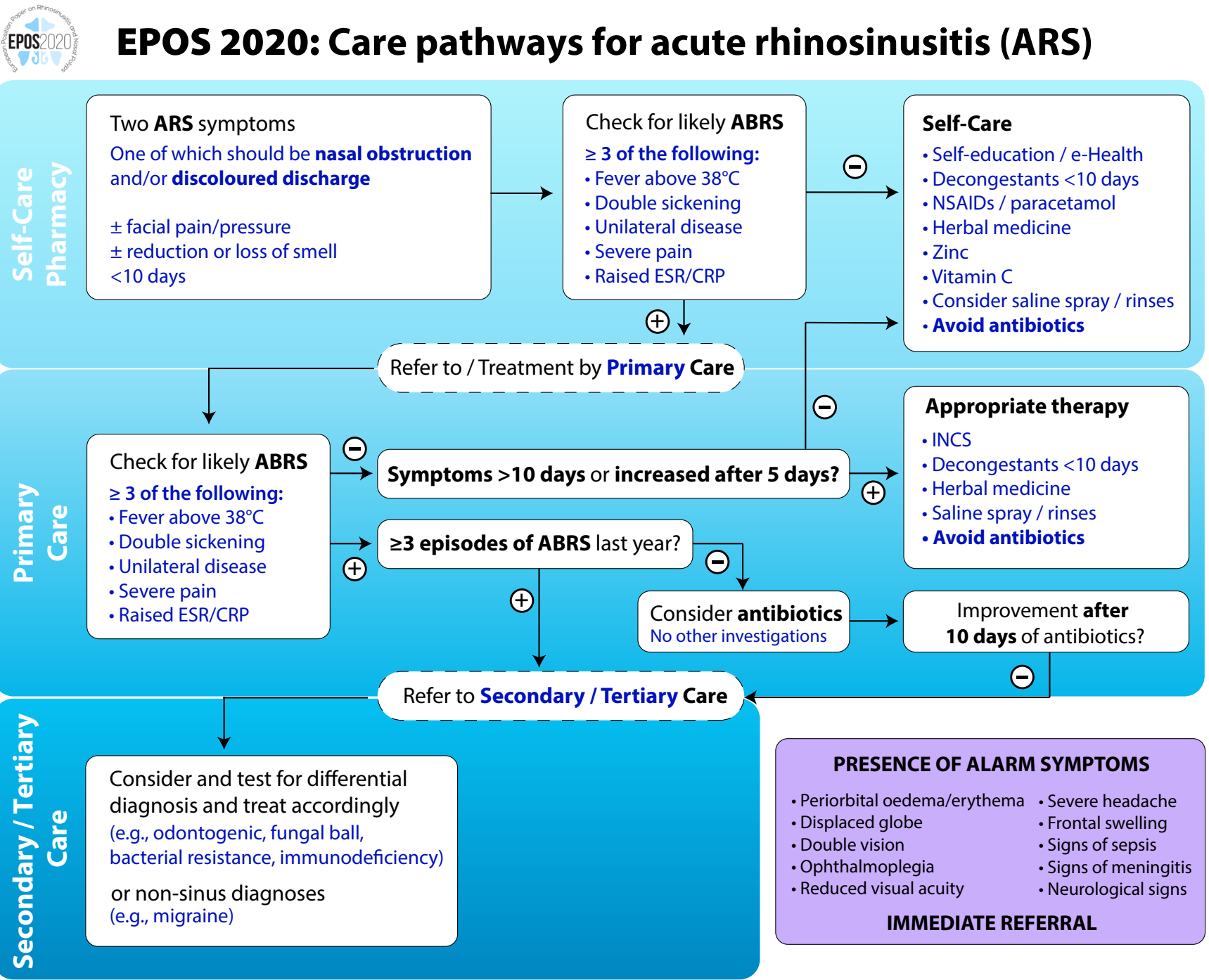

ABRS, acute bacterial rhinosinusitis; INCS, intranasal corticosteroids.

of the disease $\mathrm{e}^{(35)}$. As a consequence, rhinosinusitis is one of the top 10 most costly health conditions to US employers ${ }^{(36)}$. Overall, the total indirect costs of CRS were estimated to be in excess of $\$ 20$ billion per year in the USA ${ }^{(37)}$ mainly due to presenteeism.

\subsection{Acute rhinosinusitis including common cold and recurrent ARS in adults and children}

Chapter 4 describes the epidemiology, pathophysiology, diagnosis and differential diagnosis, and management of ARS in adults and children. Also, a new integrated care pathway based on all the evidence is proposed.

\subsubsection{Epidemiology}

In EPOS2012 the division of ARS into viral ARS (common cold), post-viral ARS and ABRS (acute bacterial rhinosinusitis) was proposed. In the last decade studies have been performed using this classification. In a recent Dutch paper using the GA2LEN questionnaire a prevalence of $18 \%$ (17-21\%) was found for symptoms pointing to post-viral ARS in three different cities in the Netherlands ${ }^{(38)}$. ABRS is a rare disease with an incidence of $0.5-2 \%$ of viral ARS (common cold) ${ }^{(2,39)}$. RARS is defined as $\geq 4$ episodes per year with symptom free intervals ${ }^{(40-43)}$. Each episode must meet the criteria for acute post-viral (or bacterial) rhinosinusitis. The EPOS2020 steering group advises to have at least one proven diagnosis of post-viral ARS with endoscopy and/or CT scan before a diagnosis of RARS is considered.

\subsubsection{Predisposing factors for ARS and RARS}

Predisposing factors for ARS are seldom evaluated. There is some indication that anatomical abnormalities may predispose to recurrent acute rhinosinusitis (RARS) ${ }^{(44-47)}$. Active and passive smoking predisposes to ARS and there is some evidence that concomitant chronic disease may increase the chance of getting ARS following an influenza infection ${ }^{(48-50)}$.

Other potential factors like allergy and GORD do not seem to 
Table 1.4.4. Treatment evidence and recommendations for adults with acute bacterial rhinosinusitis (ABRS).

\begin{tabular}{|c|c|c|}
\hline Therapy & $\begin{array}{l}\text { Level of } \\
\text { evidence }\end{array}$ & GRADE recommendation \\
\hline Antibiotics & $1 a$ & $\begin{array}{l}\text { Antibiotics are effective in a select group of patients with symptoms and signs suggestive of ABRS. From the limited } \\
\text { data available (two studies versus one) it seems that amoxicillin/penicillin (beta-lactams) especially are effective } \\
\text { and moxifloxacin (fluoroquinone) is not. The efficacy of beta-lactams is evident at day three where patients } \\
\text { already experience better symptom improvement and continues with a higher number of cures at completion of } \\
\text { treatment. However, careful patient selection for those with ABRS is needed to avoid unnecessary use of antibiotics } \\
\text { and side effects. }\end{array}$ \\
\hline Antihistamines & $1 \mathrm{~b}(-)$ & $\begin{array}{l}\text { There is one study evaluating antihistamines versus placebo in adults with allergic rhinitis and ABRS showing no } \\
\text { effect. Based on the very low quality of the evidence, the EPOS2020 steering group cannot advise on the use of } \\
\text { antihistamines in post-viral ARS and ABRS. }\end{array}$ \\
\hline $\begin{array}{l}\text { Nasal irrigation with } \\
\text { saline }\end{array}$ & $1 \mathrm{~b}(-)$ & $\begin{array}{l}\text { One study comparing hypertonic saline nasal spray, isotonic saline nasal spray and no treatment in addition to } \\
\text { antibiotics did not find a difference between the groups. Based on the very low quality of the evidence no advice } \\
\text { can be given about the use of nasal saline irrigation. }\end{array}$ \\
\hline Sodium Hyaluronate & $\mathrm{lb}$ & $\begin{array}{l}\text { One study evaluating sodium hyaluronate compared to placebo in a nebulizer ampoule for nasal douching in } \\
\text { addition to levofloxacin and prednisone showed significantly fewer symptoms and better smell threshold in the } \\
\text { sodium hyaluronate group. Based on the very low quality of the evidence no advice can be given about the use of } \\
\text { sodium hyaluronate. }\end{array}$ \\
\hline
\end{tabular}

ABRS, acute bacterial rhinosinusitis; ARS, acute rhinosinusitis.

Table 1.4.5. Treatment evidence and recommendations for children with acute bacterial rhinosinusitis (ABRS).

\begin{tabular}{|c|c|c|}
\hline Therapy & $\begin{array}{l}\text { Level of } \\
\text { evidence }\end{array}$ & GRADE recommendation \\
\hline Antibiotics & $1 \mathrm{a}(-)$ & $\begin{array}{l}\text { Data on the effect of antibiotics on the cure/improvement of symptoms in ABRS in children are very limited. There } \\
\text { are only two studies with limited numbers that do not show a significant difference over placebo but do show a } \\
\text { significant higher percentage of adverse events. Larger trials are needed to explain the difference between adults } \\
\text { where antibiotics in ABRS has been shown to be effective and this outcome. }\end{array}$ \\
\hline Mucolytics & $1 \mathrm{~b}(-)$ & Erdosteine as an adjunct to antibiotic was not more effective than placebo \\
\hline
\end{tabular}

ABRS, acute bacterial rhinosinusitis.

predispose to $\operatorname{ARS}^{(51,52)}$.

\subsubsection{Pathophysiology of ARS}

The pathophysiology of ARS is systematically evaluated, again trying to organize the literature based on the different categories of ARS. Since EPOS2012, there have been increasing experimental data supporting the fact that nasal epithelium is the primary portal of entry for respiratory viruses as well as an active component of initial host responses against viral infection. The cascade of inflammation initiated by nasal epithelial cells will lead to damage by the infiltrating cells, causing oedema, engorgement, fluid extravasation, mucus production and sinus obstruction in the process, eventually leading to ARS or exacerbating ARS (see chapter 4.2.).

\subsubsection{Diagnosis and differential diagnosis of ARS in adults and children}

Post-viral ARS is a common condition in the community, usually following viral URTI. Most acute viral URTI infections are selflimiting, thus post-viral ARS should not be diagnosed before 10 days' duration of symptoms unless there is a clear worsening of symptoms after five days.

Subjective assessment should take into account the severity and the duration of symptoms (see above). The recommended method of assessing severity of symptoms is with a visual analogue scale (VAS) recorded by the patient on a $10 \mathrm{~cm}$ line giving a score on a measurable continuum of 1 to 10 . Bacterial infection may occur in ARS, but in most cases antibiotics have little effect on the course of the illness (see 1.4.5.). A number of studies have attempted to provide clinicians with combinations of symptoms and signs predicting more severe disease, particularly of a bacterial infection and the likelihood of a response to antibiotics ${ }^{(53)}$. The EPOS2020 steering group decided to maintain suggestions made in the earlier EPOS versions: at least three of five symptoms of discoloured discharge, severe local pain, fever, elevated ESR/CRP and double sickening.

\subsubsection{Treatment of $A R S$ in adults and children}

For EPOS2020 a systematic review was performed evaluating treatment of the different categories of ARS (viral, post-viral or ABRS) separately. For acute viral rhinosinusitis we found many excellent systematic reviews and report on them. For post-viral rhinosinusitis and ABRS a systematic review of the literature has been performed for children and adults. The different treatments, levels of evidence and GRADE recommendations are reported in Tables 1.4.1-1.4.5. For medication not mentioned in these tables, we could not find RCTs. 
Based on the systematic review, a new integrated care pathway is proposed (Figure 1.4.1.). In this figure it is emphasized that the treatment of almost all patients with ARS should be symptomatic, if needed, combined with local corticosteroids. The place for antibiotics is very limited and they should only be given in situations pointing to severe disease with symptoms and signs such as high fever, double sickening, severe pain and elevated $\mathrm{ESR}^{(3)}$.

Finally, in chapter 4 the complications of ABRS are discussed. Complications of bacterial rhinosinusitis are rare but potentially serious. However, a number of studies have shown that they are not prevented by routine prescribing of antibiotics. A low threshold of suspicion must always be maintained for their early diagnosis.

\subsection{Epidemiology, predisposing factors, pathophysiology, and diagnosis of CRS}

\subsubsection{Epidemiology and predisposing factors}

The overall prevalence of symptom-based CRS in the population has been found to be between $5.5 \%$ and $28 \%(4,5,54,55), C R S$ is more common in smokers than in non-smokers ${ }^{(4)}$. The prevalence of self-reported physician-diagnosed CRS is highly correlated with the prevalence of EPOS-diagnosed CRS ${ }^{(4)}$. When symptoms are combined with endoscopy or CT scan prevalence is reduced to $3-6 \%{ }^{(56-58)}$.

CRS is associated with asthma, with a prevalence of asthma around $25 \%$ in patients with CRS compared to $5 \%$ in the general population. CRS is also associated with COPD,

$\mathrm{N}$-ERD, hypogammaglobulinemia, and GORD (see chapter 5.1). Smoking, air-pollution and occupational exposure are negatively correlated with CRS (symptoms).

The prevalence of allergy in CRS may vary by phenotype, with CCAD and AFRS having a stronger association than CRSWNP and
CRSsNP(59, 60). An important percentage of subjects diagnosed with chronic upper airway disease report alcohol-induced worsening of their symptoms ${ }^{(61)}$.

\subsubsection{Genetics}

The current knowledge base on the genomics of CRS disease offers the promise of identifying new mechanisms of disease development and markers predicting optimal response to available therapies. However, for the moment, genetics do not allow prediction of disease or outcome and its uses are currently restricted to extreme cases to understand the molecular underpinning of pathologies. It is probable that over the coming years we will identify individual or complex genetic traits conferring susceptibility to CRS, evolution of disease, and response to medical or surgical treatment ${ }^{(62,63)}$.

\subsubsection{The emerging clinical relevance of CRS pathophysiology}

Research into the aetiology and pathogenesis of chronic rhinosinusitis has been largely irrelevant to the clinician, with minimal impact on management. Historically, CRS has been divided into two groups based on the presence or absence of polyps and, in rough overview, corticosteroids were commonly used for CRSwNP and antibiotics for CRSsNP. The rationale for these regimens was based on decades-old presumptions that CRSsNP was the result of an incompletely treated acute bacterial infection that then became 'chronic' and CRSwNP had some relationship to local or systemic 'allergy'. Surgery was the only option for failures. It has been clear for at least 20 years that this assessment was simplistic at best. The emerging view was that CRS was a syndrome with a multifactorial aetiology resulting from a dysfunctional interaction between various environmental factors and the host immune system. It was, however, very unclear which environmental and host factors were important

Figure 1.5.1. Aetiology and pathogenesis of CRS.

EPSS2020 Aetiology and Pathogenesis of CRS

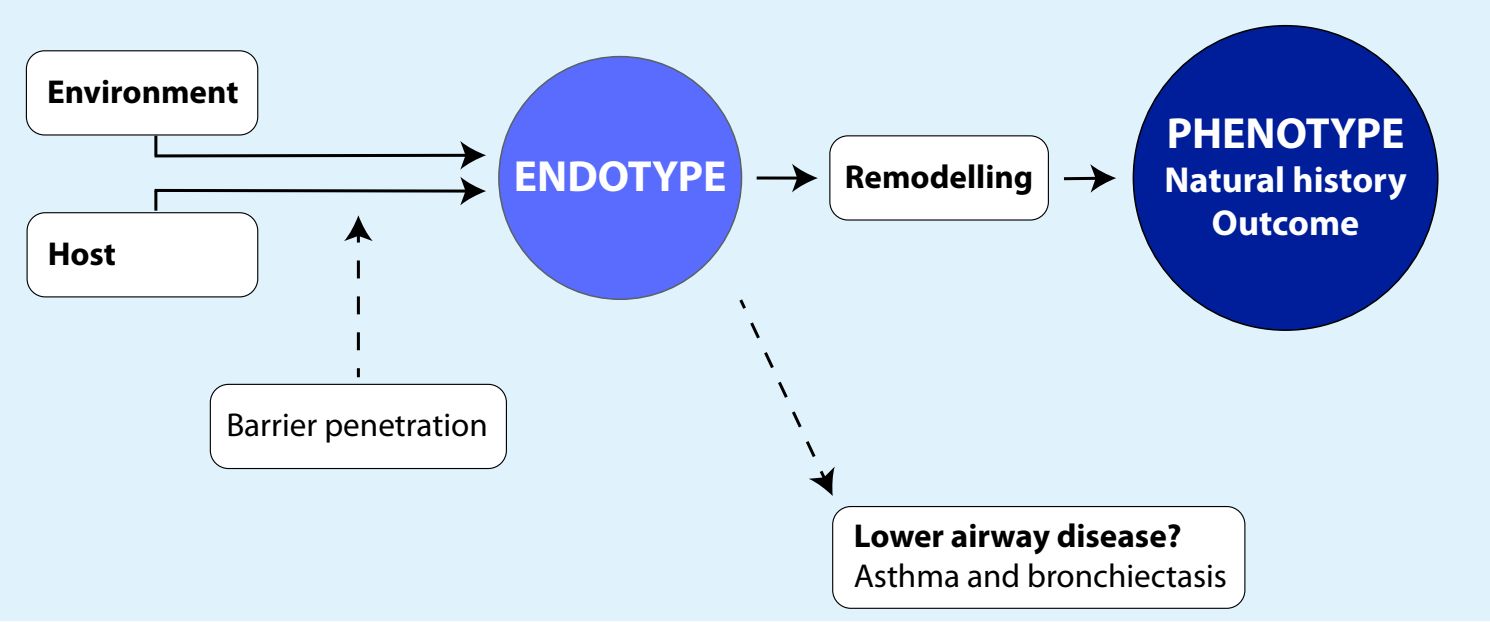

CRS, chronic rhinosinusitis. 
even in the population at large, let alone in an individual CRS patient. Nevertheless, research was undertaken with the initial goal of examining causation of CRS as a route to therapy. Later, the results of these efforts shifted emphasis toward the tissue effects generated by those causative factors and away from the factors themselves. The following brief synopsis describes how that 20-year journey is finally beginning to impact how we treat patients with CRS.

Research into the aetiology and pathogenesis of CRS was first energized by the work on fungus, which was proposed as the key aetiologic agent, at least in patients with recalcitrant CRS. This was followed shortly after with Staphylococcus aureus being proposed as a rival pathogen, perhaps in biofilm format to enable greater resistance. Later, the more general hypothesis of microbial dysbiosis was proposed, wherein the collective microbial community was abnormal and pathogenic, propagating sinonasal inflammation occurred at anatomically vulnerable sites. Unfortunately, therapies directed at fungi, staphyloccus aureus and even the microbiome as a whole have been, at best, underwhelming. This suggested the opposite therapeutic tactic: shift attention away from antimicrobials and towards the goal of correcting any immune dysfunction in the individual CRS patient. By then it was understood that both the nose and sinuses were not sterile: a process which begins at birth with the rapid colonization by viruses, bacteria and fungi. In healthy individuals, the mucosa serves as a relative barrier modulating interaction with the host immune system, promoting tolerance and symbiosis as well as preventing or limiting inflammation. In patients with CRS, the barrier is penetrated with resultant chronic inflammation leading to, in many cases, tissue remodeling and clinical symptoms. In theory, identification of specific genetic or epigenetic variations in the host immune system that permit CRS to develop should be possible, providing targets for future therapies. Unfortunately, outside of cystic fibrosis and CFTR, the genetics of CRS appear to be quite complex for the typical patient, involving multiple genes, each with a small effect size. Moreover, genetic studies on the large populations necessary to identify these genes would be very expensive and have generally not been undertaken. Effectively, this approach was rendered impractical and therapeutic approaches to manage CRS based on putative aetiologies - either host or environment based - have made relatively little clinical impact. Nevertheless, this entire body of work revealed a great deal about the nature of the inflammation present in the tissue of CRS patients.

The failure of aetiology-based treatments for CRS is, in retrospect, not surprising since CRS is typically an adult onset disorder with diagnosis most commonly in the fifth decade of life. This extended premorbid time course suggests a complex host-environmental interaction, with great variability in nature, sequence and intensity of exogenous stressors including superimposed stochastic events. Dissecting out the process in an individual patient would be a daunting, if not impossible task that might still not lead to any therapeutic path forward. By analogy, identifying smoking as carcinogenic may help prevent future cancers through avoidance, but it will not significantly affect treatment recommendations for a patient who has already acquired the problem. The accompanying line drawing (Figure
1.5.1.) illustrates a contemporary model of CRS pathogenesis. Rather than analysis of the complex and usually unknown factors that cause CRS in an individual patient, interest now is centred on the resulting inflammation that develops in the sinus tissue. The focus is toward the identification of the molecular pathway(s) or endotypes that have been activated. This effort has been aided by recent advances in our understanding of the physiologic immune response against pathogens across mucosal barriers. When the barrier is breached, a self-limited immunodefensive response is generated, characterized by a cellular and cytokine repertoire targeting one of the three classes of pathogens: type 1 immune responses target viruses; type 2 responses target parasites and type 3 target extracellular bacteria and fungi, all of which resolve with elimination of the pathogens and restoration of barrier integrity. In cases of CRS, barrier penetration results in a chronic inflammatory response that fails to resolve, but still typically utilizes the type 1, 2 or 3 pathways alone, or in combinations. Type 2 inflammation is characterized by cytokines IL-4, IL-5 and IL-13 as well as activation and recruitment of eosinophils and mast cells. CRS research has revealed that patients with a pure or mixed type 2 endotype tend to be much more resistant to current therapies, exhibiting a high recurrence rate when compared with pure type 1 or 3 endotypes. Furthermore, while type 2 CRS clearly varies between patients by intensity of inflammation, subtypes may exist wherein discrete aspects of the pathway are relatively enhanced (e.g. mast cell activation, eosinophil activation, and plasma cells activity). Most importantly, biologic agents have now become available that target specific aspects of type 2 inflammation. In the very near future, it may be possible to offer personalized medicine for CRS patients where treatment is based on molecular biomarkers for the endotype or subendotype activated in an individual patient.

Remodelling of sinonasal tissues in CRS consists most prominently of polyp formation, goblet cell hyperplasia and epithelial barrier abnormalities, which in aggregate, may account for many or most of the CRS symptoms. In the case of the barrier remodelling, the result is greater permeability, likely facilitating persistence or recurrence of CRS. All of these changes are most apparent in type 2 CRS, possibly accounting for the observed greater symptomatology and higher rate of treatment failure. The precise relationship between the endotype and the remodelling pattern is not completely clear but recent evidence suggests that it may be cause and effect as depicted in Figure 1.5.1. Specifically, the use of biologic agents that suppress the type 2 endotype, also shrink polyps. Reversal of goblet cell hyperplasia has not yet been documented, but in vitro studies suggest that barrier-related remodelling is driven directly, in large measure, by canonical type 2 cytokines. Biologic agents that suppress type 2 inflammation may, therefore, suppress the inflammation, reverse the remodelling and limit recurrence, thereby altering the clinical course of the most severe CRS phenotypes. Further research into type 2 inflammation will be extremely helpful in the use of these powerful drugs, which have the potential to revolutionize CRS treatment ${ }^{(64)}$. 
Figure 1.6.1. Treatment evidence and recommendations for adults with chronic rhinosinusitis.

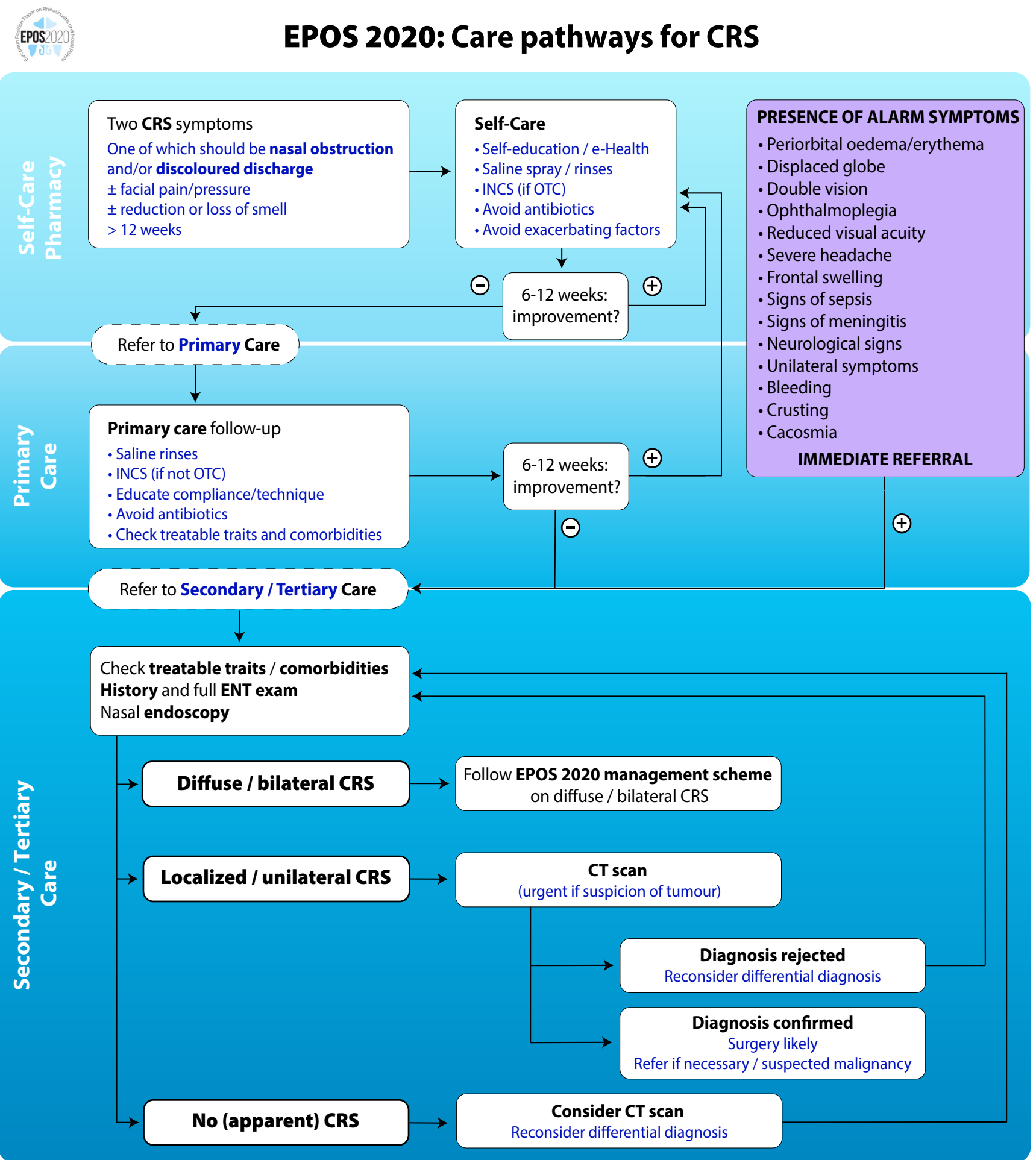

CRS: chronic rhinosinusitis; CT, computed tomography; INCS, intranasal corticosteroids spray; OTC, over-the-counter.

\subsubsection{Differential diagnosis and diagnostic tools}

\subsubsection{Differential diagnosis}

It was decided to include more information in EPOS2020 to better allow differential diagnosis of rhinosinusitis from certain other conditions and common symptoms, notably allergic and non-allergic rhinitis, olfactory loss and facial pain. We also include an updated and expanded range of diagnostic tools, though many have not substantially changed since 2012. Upper airway diseases present with a variable pattern of common symptoms such as nasal obstruction and discharge, making the epidemiological diagnosis of CRS difficult to differentiate from allergic and nonallergic rhinitis based on symptomatic grounds. Combining data from different studies 
Figure 1.6.2. EPOS2020 management scheme on diffuse CRS.

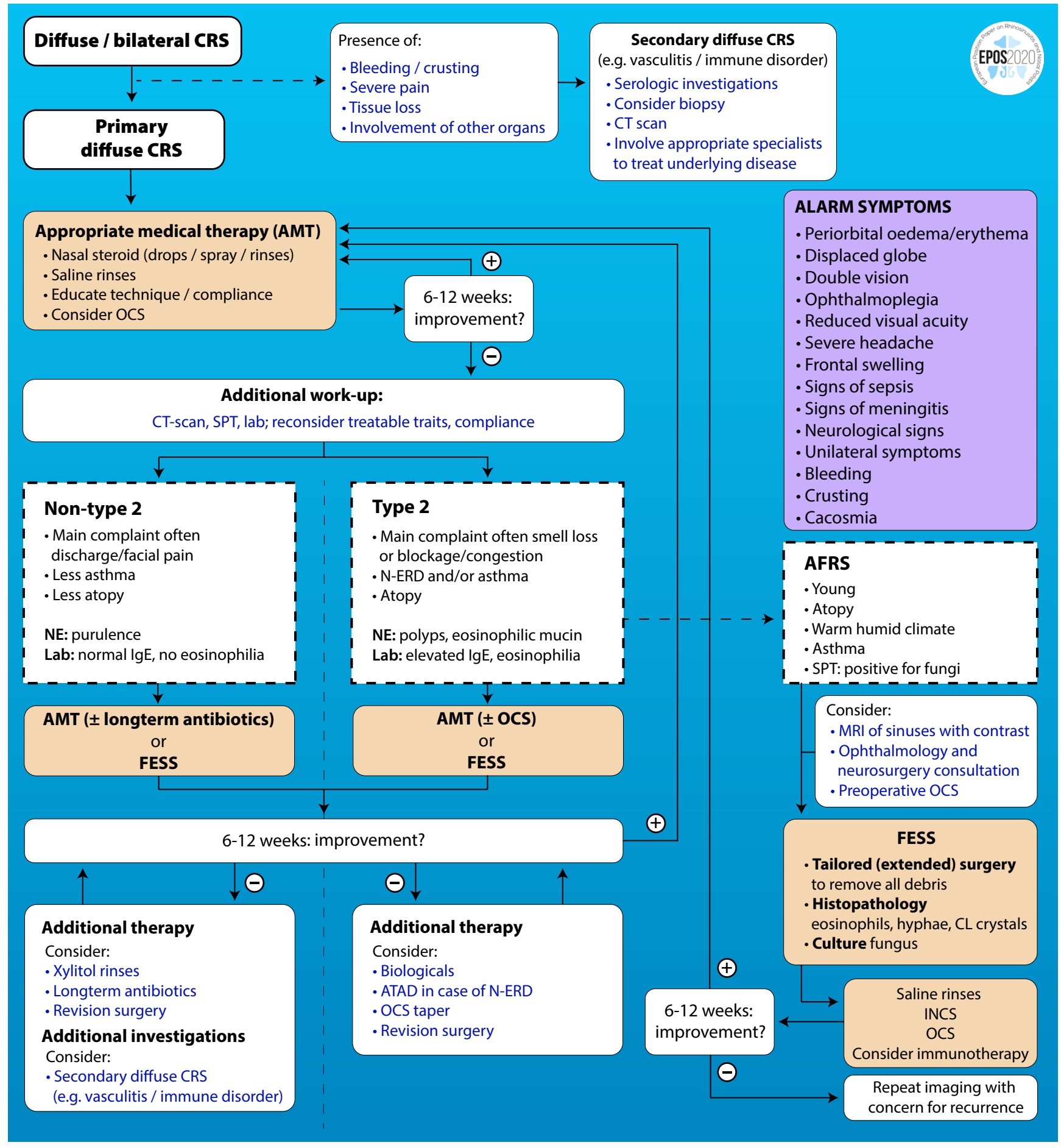

For explanation of (primary and secondary) diffuse CRS see 1.2.3.

AMT, appropriate medical therapy; ATAD, Aspirin treatment after desensitisation; $C L$, Charcot Leyden; CRS, chronic rhinosinusitis; $C T$, computed tomography; FESS, functional endoscopic sinus surgery; INCS, intranasal corticosteroid spray; MRI, magnetic resonance imaging; NE, nasal endoscopy; N-ERD, NSAID-exacerbated respiratory disease; OCS, Oral corticosteroids; SPT, Skin prick test. 
leads to a picture of significant overlap in prevalence and severity of symptomatology. However, as there are generally less inflammatory changes seen on CT sinuses in AR and NAR than $\mathrm{CRS}^{(65)}$ a combination of symptoms, CT scan and nasal endoscopy can point in the right direction.

Olfactory loss is one of the cardinal symptoms of CRS but has a wide differential diagnosis ${ }^{(66)}$. The prevalence of olfactory disorders in the general population is estimated to be 3-5\% for total smell loss (anosmia) and 15-25\% for partial impairment (hyposmia) ${ }^{(67,68)}$. In CRS the mechanism leading to olfactory impairment is twofold: inflammatory and purely mechanical due to obstruction of the olfactory cleft $^{(69,70)}$, which explains why not all patients have an olfactory benefit from surgical removal of polyps alone but also require subsequent anti-inflammatory treatment. However, CRS-related olfactory loss has a good success rate of improvement if the CRS is treated even if not always sustained in the long-term.

Facial pain is another cardinal symptom of CRS which can occur in many other conditions ${ }^{(71)}$. However, facial pain when it occurs alone is rarely caused by CRS and, therefore, when it occurs without other nasal complaints or abnormalities on examination, it should not (primarily) be addressed surgically.

\subsubsection{Diagnostic tools}

The different imaging modalities in diagnosing rhinosinusitis [conventional X-ray, computerized tomography (CT), cone beam CT and magnetic resonance imaging (MRI)] have been evaluated $^{(72)}$. Overall CT scan remains the gold standard in the radiologic evaluation of rhinologic disease, notably $\mathrm{CRS}^{\text {(73-75). }}$ However, in acute rhinosinusitis, the diagnosis is made on clinical grounds and CT is not recommended ${ }^{(3)}$ unless the condition persists despite treatment, or a complication is suspected $^{(76)}$. Conventional sinus X-rays are no longer indicated in either ARS or CRS.

The most commonly used and validated scoring system of sinonasal inflammatory change remains the Lund-Mackay score (LMS) which gives a maximum score of 24 or $12 /$ side $^{(77)}$. An LMS of 2 or less has an excellent negative predictive value, and an LMS of 5 or more has an excellent positive predictive value, strongly indicating true disease. In CRS, CT was not normally recommended until after an appropriate course of medical therapy had failed ${ }^{(3,78)}$ and without an intervening acute episode but more recent studies suggest that early $\mathrm{CT}$ scanning may be more cost-effective as compared to extended courses of antibiotics given empirically and is preferred by patients ${ }^{(79-81)}$.Multi-detector CT (MDCT) scanners and conebeam $C T$ are reducing the radiation dose whilst preserving image quality by shortening the scan time and using postprocessing techniques ${ }^{(82,83)}$ without compromising anatomical accuracy ${ }^{(84)}$, making them increasingly attractive ${ }^{(85,86)}$.

In the measurement of health-related quality-of-life (HRQL), a wide range of validated patient reporting outcome measures (PROMS) are available but currently none of the established PROMS capture all the desired aspects of CRS; the SNOT-22 fails to capture disease duration or medication usage. Current recommendations include the use of SNOT-22 scores repeated over time, Lund Kennedy endoscopic scores, and additional questions to evaluate the need for systemic medications or progression to surgery, compliance with and side effects of treatment, additional information on symptom frequency, and impact on ability to perform normal activities ${ }^{(87)}$.

Nasal endoscopy remains an essential part of the rhinological examination. A recent systematic review analysed the accuracy of nasal endoscopy in diagnosing chronic rhinosinusitis (CRS) compared with paranasal sinus computed tomography (CT). Sixteen observational or retrospective studies were included resulting in a high correlation $(r=0.85 ; 95 \%$ confidence interval [CI][0.78-0.94], p<0.0001, $\left.\right|^{2} 77 \%$ ) between endoscopy and CT in terms of the diagnostic accuracy for $\mathrm{CRS}^{(88)}$.

A clinical history supported with a skin prick test or serum IgE measuremernt will probably remain the gold standard of the upper airway allergy diagnosis but advances are expected from the molecular in vitro diagnosis which may change this trend, due to improved technology which enables faster diagnosis on a broader panel of allergens ${ }^{(89,90)}$.

As CRS patients are commonly not fully aware of their olfactory impairment, or are unable to estimate the severity of the loss, the use of smell tests is recommended in order to objectively evaluate this disorder ${ }^{(91,92)}$. The most widely used remain the North American UPSIT ${ }^{(93)}$, its short version (SIT, B-SIT) and the European Sniffin'Sticks ${ }^{(94)}$. Although there are many others, all have cultural bias and there have been recent advances to overcome this with culturally unbiased, universally usable smell tests $^{(95)}$.

Nasal obstruction is the most significant of the cardinal symptoms of rhinosinusitis and nasal patency may be objectively evaluated with peak nasal inspiratory flow (PNIF), (active anterior) rhinomanometry (AAR), and acoustic rhinometry (AR) Newer methods such as computational fluid dynamics ${ }^{(96)}$ are presently mainly used for research purposes ${ }^{(97,98)}$ but may be of value in the future.

In addition to confirming diagnosis, histopathology is becoming more important to assist in endotyping of inflammatory disease, thereby directing potential therapies, e.g. biologics. Eosinophilic CRS (eCRS) requires quantification of the numbers of eosinophils, i.e. number/high powered field (HPF (400x) and EPOS2020 supports 10 or >/HPF. Further stratification may be made between those with 10-100 eosinophils per HPF in two or more areas and those with $>100$ eosinophils per HPF in two or more areas ${ }^{(99)}$. The amount of eosinophilic infiltration and the overall intensity of the inflammatory response are closely related to the prognosis and severity of disease ${ }^{(100)}$. Until recently most blood tests in patients with CRS were performed to diagnose immunodeficiencies and vasculitis. However, recently the options to treat with biologicals has put more emphasis on markers of type 2 disease, although as it stands we are not aware of biomarkers that can predict response to biologicals in CRS $^{(101)}$.

For microbiology, in addition to the standard culture-dependant tests, newer culture-independent techniques including next generation sequencing may provide significant insight into CRS pathophysiology. This could include sequencing of all DNA (metagenomics) or all transcribed RNA (metatranscriptomics) or identification of proteins (metaproteomics) or metabolites 
(metabolomics), showing not only the true diversity and structure, but also the full genetic potential and in situ activity of the mucosa-associated microbiota(102).

EPOS2020 also includes an update on mucociliary testing and other tests for primary ciliary dyskinesia (PCD), sweat testing and other tests for cystic fibrosis and advances in genetic testing as well as new diagnostic tools for N-ERD. Finally, the lower respiratory tract is not forgotten and the full range of available investigations are covered from peak expiratory flow to provocation tests and expired nitric oxide measurement.

\subsection{Management of chronic rhinosinusitis in adults}

\subsubsection{Introduction}

An important difference compared to EPOS2012 is that we have decided to move away from differentiating between the management of CRSsNP and CRSwNP per se. The understanding of the last decade of endotyping of CRS and the consequences of endotypes for the management of disease has led to the decision to describe management of CRS based on endotyping and phenotyping.

We propose a new clinical classification based on the disease being localized (often unilateral) or diffuse (always bilateral). Both these groups can be further divided into type 2 or nontype 2 disease (Figure 1.2.1.). The major challenge is to find reliable biomarkers that define type 2 inflammation and predict reaction to medication. Unfortunately, recent large studies with monoclonal antibodies directed at type 2 endotypes have not found reliable biomarkers to predict response to treatment ${ }^{(103,104)}$. For the moment the combination of phenotype (e.g. CRSwNP, N-ERD), response to treatment (systemic corticosteroids) and possibly also markers like eosinophils, periostin and IgE either in blood or tissue lead us to the best estimation of the endotype and reaction to treatment. This is a rapidly evolving field at the moment and we expect that frequent updates will be necessary.

\subsubsection{Management of CRS: an integrated care pathway}

For the management of CRS, a full systematic review of the literature has been performed (see chapter 6 and Table 1.6.1.). Many forms of localised CRS (Figure 1.2.1.) in general, either type 2 or non-type 2, are not responsive to medical treatment and need surgery. For that reason, we advise patients with unilateral disease to be referred to secondary care for further diagnosis. Many studies do not make a clear differentiation between CRSsNP and CRSwNP. Very few studies further define CRS phenotypes or endotypes in the disease. CRS research has revealed that patients with a pure or mixed type 2 endotype tend to be more resistant to current therapies, exhibiting a high recurrence rate when compared with pure type 1 or 3 endotypes.

For diffuse, bilateral CRS, local corticosteroids and saline remain the mainstay of the treatment (Figure 1.6.1.).

Furthermore, the integrated care pathway (ICP) advises to check treatable traits, to avoid exacerbating factors and advises against the use of antibiotics. In secondary care, nasal endoscopy can confirm disease, point to secondary CRS (e.g. vasculitis) and further differentiate between localized and diffuse disease (Figure 1.6.2.).

In addition, emphasis is put on optimum techniques of medication delivery and compliance. If treatment with nasal steroid and saline is insufficient, an additional work-up with CT scan and endotyping is relevant. Depending on the endotype indication, treatment can be tailored to a more type 2 or nontype 2 profile. International guidelines differ regarding whether long-term antibiotics and oral steroids should be included as part of adequate medical therapy (AMT), reflecting conflicting evidence in the current literature $\mathrm{e}^{(3,78,105)}$, and concerns with regard to side-effects. There is a lot of debate on the appropriate moment for surgery for $\mathrm{CRS}^{(105)}$. In a recent study for adult patients with uncomplicated CRS, it was agreed that ESS could be appropriately offered when the CT Lund-Mackay score was $\geq 1$ and there had been a minimum trial of at least eight weeks' duration of a topical intranasal corticosteroid plus a short-course of systemic corticosteroid (CRSwNP) or either a short-course of a broad spectrum / culture-directed systemic antibiotic or the use of a prolonged course of systemic low-dose anti-inflammatory antibiotic (CRSsNP) with a post-treatment total SNOT-22 score $\geq 20$. These criteria were considered the minimal threshold, and clearly not all patients who meet the criteria should have surgery, but their application should reduce unnecessary surgery and practice variation. A subsequent study applied these criteria retrospectively to patients recruited to a multicentre cohort study and found that patients where surgery was deemed 'inappropriate' reported significantly less improvement in their quality of life postoperatively ${ }^{(106)}$.

It is important to emphasize that CRS is a chronic disease and ESS a step in the management that is primarily aimed at creating better conditions for local treatment. After surgery continuous appropriate medical treatment is mandatory.

If surgery in combination with appropriate medical treatment fails, additional therapy can be considered. Options are the use of aspirin treatment after aspirin desensitisation (ATAD) ${ }^{(107)}$, longer (tapering) treatment with OCS, long term antibiotics ${ }^{(108)}$ and/or biologicals when indicated.

\subsubsection{New treatment options with biologicals (monoclonal} antibodies)

The acceptance of dupilumab (anti IL-4Ra) for the treatment of CRSwNP by the US Food and Drug Administration(FDA) and European Medicines Agency (EMA) in 2019 has significantly changed the treatment options in type 2 type CRS and it is expected that other monoclonal antibodies will follow. Until 2019 monoclonal antibodies could only be prescribed in patients with concomitant (severe) asthma. Within the EUFOREA setting, the positioning of biologics in the ICP of CRS with criteria for use and stopping of biologics have been published $^{(101)}$. The EPOS2020 steering group made some modifications and tightening of these criteria. They concluded that biologicals are indicated in a patient with bilateral polyps, who had had sinus surgery or was not fit for surgery and who had three of the following characteristics: evidence of type 2 disease (tissue eosinopils $\geq 10 / \mathrm{HPF}$ or blood eosinophils $\geq 250$ OR total lgE $\geq 100$ ), need for at least two courses of systemic 
Figure 1.6.3. Indications for biological treatment in CRS.

\section{Indications for biological treatment in CRSwNP}

\section{Presence of bilateral polyps in a patient who had ESS*}

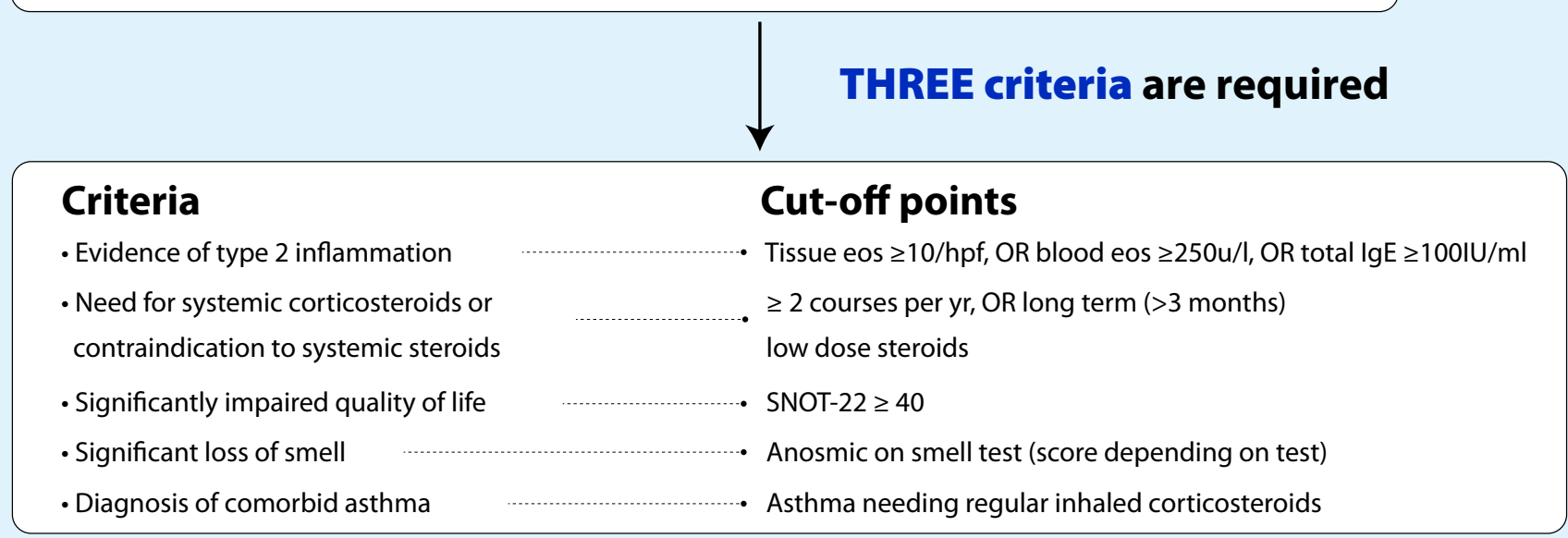

*exceptional circumstances excluded (e.g., not fit for surgery)

CRS, chronic rhinosinusitis; CRSwNP: chronic rhinosinusitis with nasal polyps; ESS, endoscopic sinus surgery; hpf: high power field (x400); SNOT-22, sino-nasal outcome test-22.

Figure 1.6.4. Response criteria for biologicals in the treatment of CRS.

\section{Defining response to biological treatment in CRSwNP}

\section{Evaluation of 5 criteria}

- Reduced nasal polyp size

- Reduced need for systemic corticosteroids

- Improved quality of life

- Improved sense of smell

- Reduced impact of co-morbidities
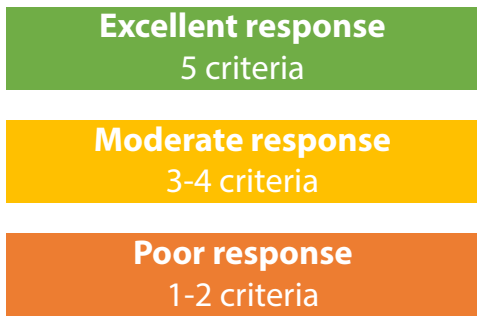

No response 0 criteria

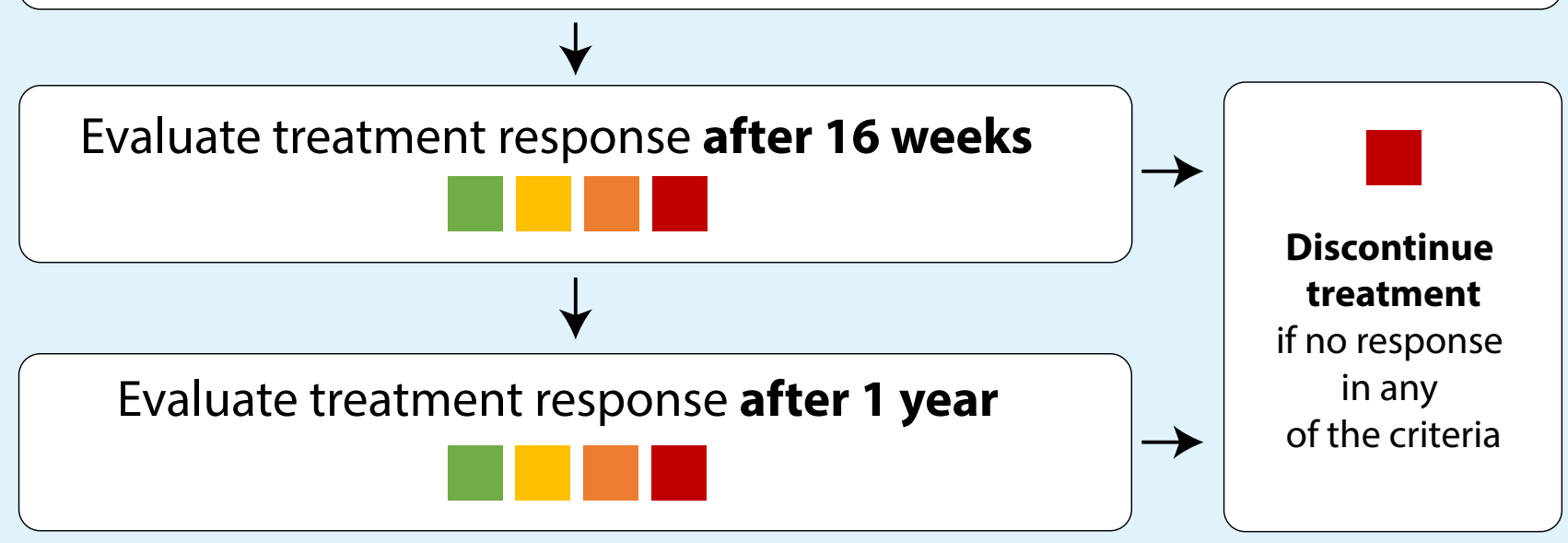


Table 1.6.1. Treatment evidence and recommendations for adults with chronic rhinosinusitis.

\begin{tabular}{|c|c|c|}
\hline Therapy & $\begin{array}{l}\text { Level of } \\
\text { evidence }\end{array}$ & GRADE recommendation \\
\hline Short term antibiotics for CRS & $1 \mathrm{~b}(-)$ & $\begin{array}{l}\text { There are only two small placebo-controlled studies, one in CRS and one in acute exacerbation of CRS. Both show no } \\
\text { effect on symptomatology apart from significantly reduced postnasal drip symptom scores at week } 2 \text { in the CRS study. } \\
\text { Seven studies evaluated two different antibiotics regimes, of which only one was placebo-controlled. One out of seven } \\
\text { studies in patients with CRS showed a significant effect on SNOT at } 2 \text { and } 4 \text { weeks and also one study a significant } \\
\text { improvment in symptoms of infection at day } 3 \text { to } 5 \text { in one antibiotic versus another in a mixed group of patients with } \\
\text { CRS and with acute exacerbation. The other } 5 \text { studies showed no difference in symptomatology. Only two of these seven } \\
\text { studies, both of which were negative, evaluated the effect after one month. } \\
\text { The EPOS2020 steering group, is uncertain, due to the very low quality of the evidence, whether or not the use of a short } \\
\text { course of antibiotics has an impact on patient outcomes in adults with CRS compared with placebo. Also, due to the very } \\
\text { low quality of the evidence, it is uncertain whether or not the use of a short course of antibiotics has an impact on patient } \\
\text { outcomes in adults with acute exacerbations of CRS compared with placebo. Gastrointestinal-related adverse events } \\
\text { (diarrhoea and anorexia) are frequently reported. }\end{array}$ \\
\hline $\begin{array}{l}\text { Short term antibiotics for acute } \\
\text { exacerbation of CRS }\end{array}$ & $1 \mathrm{~b}(-)$ & $\begin{array}{l}\text { The EPOS2020 steering group, is uncertain, due to the very low quality of the evidence, whether or not the use of a } \\
\text { short course of antibiotics has an impact on patient outcomes in adults with acute exacerbations of CRS compared with } \\
\text { placebo. Gastrointestinal-related adverse events (diarrhoea and anorexia) are frequently reported. }\end{array}$ \\
\hline Longterm antibiotics for CRS & $1 \mathrm{a}(-)$ & $\begin{array}{l}\text { The EPOS2020 steering group, due to the low quality of the evidence, is uncertain whether or not the use of long-term } \\
\text { antibiotics has an impact on patient outcomes in adults with CRS, particularly in the light of potentially increased risks of } \\
\text { cardiovascular events for some macrolides. Further studies with larger population sizes are needed and are underway . }\end{array}$ \\
\hline Topical antibiotics & $1 \mathrm{~b}(-)$ & $\begin{array}{l}\text { Topical antibacterial therapy does not seem to be more effective than placebo in improving symptoms in patients } \\
\text { with CRS. However, it may give a clinically non-relevant improvement in symptoms, SNOT- } 22 \text { and LK endoscopic score } \\
\text { compared to oral antibiotics. The EPOS } 2020 \text { steering group, due to the very low quality of the evidence, is uncertain } \\
\text { whether or not the use of topical antibiotic therapy has an impact on patient outcomes in adults with CRS compared with } \\
\text { placebo. }\end{array}$ \\
\hline Nasal corticosteroids & $1 \mathrm{a}$ & $\begin{array}{l}\text { There is high-quality evidence that long term use of nasal corticosteroids is effective and safe for treating patients with } \\
\text { CRS. They have impact on nasal symptoms and quality of life improvement, although the effect on SNOT- } 22 \text { is smaller } \\
\text { than the minimal clinically important difference. The effect size on symptomatology is larger in CRSwNP (SMD - } 0.93 \text {, } \\
95 \% \mathrm{CI}-1.43 \text { to -0.44) than in CRSsNP (SMD - } 0.30,95 \% \mathrm{CI}-0.46 \text { ). The meta-analysis did not show differences between } \\
\text { different kinds of nasal corticosteroids. Although in meta-analysis higher dosages and some different delivery methods } \\
\text { seem to have a larger effect size on symptomatology, direct comparisons are mostly missing. For CRSwNP, nasal } \\
\text { corticosteroids reduce nasal polyp size. When administered after endoscopic sinus surgery, nasal corticosteroids prevent } \\
\text { polyp recurrence. Nasal corticosteroids are well tolerated. Most adverse events reported are mild to moderate in severity. } \\
\text { Nasal corticosteroids do not affect intraocular pressure or lens opacity. The EPOS2020 steering group advises to use nasal } \\
\text { corticosteroids in patients with CRS. Based on the low to very low quality of the evidence for higher dosages or different } \\
\text { delivery methods and the paucity of direct comparisons the steering committee cannot advise in favour of higher } \\
\text { dosages or certain delivery methods. }\end{array}$ \\
\hline Corticosteroid-eluting implants & $1 \mathrm{a}$ & $\begin{array}{l}\text { The placement of corticosteroid-eluting sinus implants in the ethmoid of patients with recurrent polyposis after sinus } \\
\text { surgery has a significant but small ( } 0.3 \text { on a } 0-3 \text { scale) impact on nasal obstruction but significantly reduces the need } \\
\text { for surgery and reduces nasal polyp score. Based on the moderate to high quality of the evidence the steering group } \\
\text { considered the use of corticosteroid-eluting sinus implants in the ethmoid an option. }\end{array}$ \\
\hline Systemic corticosteroids & $1 \mathrm{a}$, & $\begin{array}{l}\text { A short course of systemic corticosteroid, with or without local corticosteroid treatment results in a significant reduction } \\
\text { in total symptom score and nasal polyp score. Although the effect on the nasal polyp score remains significant up to } \\
\text { three months after the start of treatment by that time there is no longer an effect on the symptom score. The EPOS2020 } \\
\text { steering group felt that } 1-2 \text { courses of systemic corticosteroids per year can be a useful addition to nasal corticosteroid } \\
\text { treatment in patients with partially or uncontrolled disease. A short course of systemic corticosteroid postoperatively does } \\
\text { not seem to have an effect on quality of life. Systemic corticosteroids can have significant side effects. }\end{array}$ \\
\hline Antihistamines & $\mathrm{lb}$ & $\begin{array}{l}\text { There is one study reporting on the effect of antihistamines in partly allergic patients with CRSwNP. Although there was no } \\
\text { difference in total symptom score, the days with a symptom score } \leq 1 \text { was higher in the treated group. The quality of the } \\
\text { evidence comparing antihistamines with placebo was very low. There is insufficient evidence to decide on the effect of } \\
\text { the regular use of antihistamines in the treatment of patients with CRS. }\end{array}$ \\
\hline Anti-leukotrienes & $1 \mathrm{~b}(-)$ & $\begin{array}{l}\text { Based on the very low quality of the available evidence, the EPOS2020 steering group is unsure about the potential } \\
\text { use of montelukast in CRS and does not recommend its use unless in situations where patients do not tolerate nasal } \\
\text { corticosteroids. Also, the quality of the evidence comparing montelukast with nasal corticosteroid is low. Based on the } \\
\text { evidence, the steering group does not advise adding montelukast to nasal corticosteroid but studies evaluating the effect } \\
\text { of montelukast in patients that failed nasal corticosteroids are missing. }\end{array}$ \\
\hline Decongestant & $\mathrm{lb}$ & $\begin{array}{l}\text { There is one small study in CRSwNP patients showing a significantly better effect of oxymetazoline combined with MFNS } \\
\text { than MFNS alone without inducing rebound swelling. There was no effect of xylometazoline compared to saline in the } \\
\text { early postoperative period. This review found a low level of certainty that adding a nasal decongestant to intranasal } \\
\text { corticosteroids improves symptomatology in CRS. Although the risk of rebound swelling was not shown in this study, the } \\
\text { EPOS2020 steering group suggests in general not to use nasal decongestants in CRS. In situations where the nose is very } \\
\text { blocked, the temporary addition of a nasal decongestant to nasal corticosteroid treatment can be considered. }\end{array}$ \\
\hline
\end{tabular}


Table 1.6.1. Cont.

\begin{tabular}{|c|c|c|}
\hline Therapy & $\begin{array}{l}\text { Level of } \\
\text { evidence }\end{array}$ & GRADE recommendation \\
\hline Nasal irrigation with saline & la & $\begin{array}{l}\text { There are a large number of trials evaluating the efficacy of nasal irrigation. However, the quality of the studies is not } \\
\text { always very good which makes it difficult to give a strong recommendation. However, the data show: } \\
\text { Nasal irrigation with isotonic saline or Ringer's lactate has efficacy in CRS patients. } \\
\text { There is insufficient data to show that a large volume is more effective than a nasal spray. } \\
\text { The addition of xylitol, sodium hyaluronate, and xyloglucan to nasal saline irrigation may have a positive effect. } \\
\text { The addition of baby shampoo, honey, or dexpanthenol as well as higher temperature and higher salt concentration do } \\
\text { not confer additional benefit. } \\
\text { The steering group advises the use of nasal saline irrigation with isotonic saline or Ringer's lactate with or without the } \\
\text { addition of xylitol, sodium hyaluronate, and/or xyloglucan and advises against the use of baby shampoo and hypertonic } \\
\text { saline solutions due to side effects. }\end{array}$ \\
\hline $\begin{array}{l}\text { Aspirin treatment after } \\
\text { desensitization (ATAD) with oral } \\
\text { aspirin in N-ERD }\end{array}$ & la & $\begin{array}{l}\text { Oral ATAD has been shown to be significantly more effective and clinically relevant than placebo in improving QOL } \\
\text { (measured with SNOT) and total nasal symptom score in patients with N-ERD. However, the change in SNOT from treating } \\
\text { with oral ATAD compared to placebo did not reach the clinically important mean difference. ATAD reduced symptoms } \\
\text { after six months compared to placebo. However, ATAD is associated with significant adverse effects, and the risks of not } \\
\text { taking the medication strictly on a daily basis puts a burden on patient and caregiver. } \\
\text { Based on these data, the EPOS } 2020 \text { steering group suggests that ATAD can be a treatment for N-ERD patients with } \\
\text { CRSwNP whenever there is confidence in the patient's compliance. }\end{array}$ \\
\hline $\begin{array}{l}\text { Aspirin treatment after } \\
\text { desensitization (ATAD) with nasal } \\
\text { lysine aspirin in N-ERD }\end{array}$ & $1 \mathrm{~b}(-)$ & $\begin{array}{l}\text { ATAD with lysine aspirin and platelet inhibitors (like Pradugrel) have not been shown to be an effective treatment in } \\
\text { CRSwNP patients with N-ERD and are not advised. }\end{array}$ \\
\hline Low salicylate diet & $\mathrm{lb}$ & $\begin{array}{l}\text { Diets, like low salicylate diet have been shown to improve endoscopic scores and may improve symptoms compared to } \\
\text { a normal diet in patients with N-ERD. However, the quality of the evidence at this moment is not enough to draw further } \\
\text { conclusions. }\end{array}$ \\
\hline
\end{tabular}

Local and systemic antifungal $\quad$ 1a (-) Local and systemic antifungal treatments do not have a positive effect of QOL, symptoms and signs of disease in patients treatments with CRS. The EPOS2020 steering group advises against the use of anti-mycotics in CRS.

\begin{tabular}{|c|c|c|}
\hline Anti-lgE & $\mathrm{lb}$ & $\begin{array}{l}\text { Anti-IgE (amalizumab) therapy has been proposed as a promising biologic therapy for CRS. Two RCTs that evaluated anti- } \\
\text { IgE monoclonal antibody (omalizumab) did not show impact on disease specific QOL but one study did show an effect } \\
\text { on the physical domain of SF-36 and AQLQ. One study demonstrated lower symptom scores (change from baseline in anti } \\
\text { IgE group) for nasal congestion, anterior rhinorrhoea, loss of sense of smell, wheeze and dyspnoea, a significant reduction } \\
\text { of NPS on endoscopic examination, and Lund-MacKay scores on radiologic imaging. Due to the small study population } \\
\text { in the existing studies, further studies with larger population sizes are needed and are underway. The available data are } \\
\text { insufficient to advise on the use of anti-IgE in CRSwNP at this moment. }\end{array}$ \\
\hline Anti-II-5 & $\mathrm{Ib}$ & $\begin{array}{l}\text { There is only one large sufficiently powered study with mepolizumab that showed a significant reduction in patients' need } \\
\text { for surgery and an improvement in symptoms. Unlike in CRS, there is a significant experience with anti-II5 in other type } \\
2 \text { driven diseases like asthma that do show a favourable safety profile so far. The EPOS2020 steering group advises use of } \\
\text { mepolizumab in patients with CRSwNP fulfilling the criteria for treatment with monoclonal antibodies (when approved). }\end{array}$ \\
\hline Anti IL-4/IL-13 (IL-4 receptor a) & la & $\begin{array}{l}\text { At the moment the only anti-II-4 treatment studied in CRS is dupilumab. Dupilumab is the only monoclonal antibody } \\
\text { that is approved for the treatment of CRSwNP so far. When evaluating all trials with dupilumab, the drug seems to induce } \\
\text { conjunctivitis in trials in patients with atopic dermatitis but not in trials with asthma and CRSwNP. No other adverse } \\
\text { events have been reported in the literature until now. The EPOS steering group advises to use dupilumab in patients with } \\
\text { CRSwNP fulfilling the criteria for treatment with monoclonal antibodies. }\end{array}$ \\
\hline Probiotics & $1 \mathrm{~b}(-)$ & $\begin{array}{l}\text { Although probiotic therapies show theoretical promise, the two studies performed so far did not show any differences } \\
\text { compared to placebo. For this reason, the EPOS2020 steering group advises against the use of probiotics for the treatment } \\
\text { of patients with CRS. }\end{array}$ \\
\hline Muco-active agents & $1 b$ & $\begin{array}{l}\text { Data on the effect of muco-active agents in CRS are very limited. The only DBPCT evaluating the addition of } \\
\text { S-carboxymethylcysteine to clarithromycin showed a significantly higher percentage of patients with effective response } \\
\text { and improved characteristics of nasal discharge at } 12 \text { weeks. The EPOS2020 steering group considered the quality of the } \\
\text { data insufficient to advise on the use of muco-active agents in the treatment of patients with CRS. }\end{array}$ \\
\hline Herbal treatment & $1 b$ & $\begin{array}{l}\text { Of five RCTs evaluating herbal treatment, a large DBPCT, using tablets, showed overall no effect, although a post-hoc } \\
\text { sensitivity analysis, showed a significant benefit in major symptom score at } 12 \text { weeks of treatment over placebo in patients } \\
\text { with a diagnosis of CRS for }>1 \text { year and a baseline MSS }>9 \text { (out of max 15). Of the four studies evaluating different local } \\
\text { herbal treatment, three showed a favourable effect. However, not all studies were blinded and the quality of the studies } \\
\text { was variable. } \\
\text { The treatment does not show significantly more adverse events than placebo. The quality of the evidence for the local } \\
\text { treatment is low. Based on the available data, the EPOS2020 group cannot advise on the use of herbal medicine in CRS. }\end{array}$ \\
\hline $\begin{array}{l}\text { Acupuncture and traditional } \\
\text { Chinese medicine }\end{array}$ & $1 \mathrm{~b}(-)$ & $\begin{array}{l}\text { There is no evidence that traditional Chinese medicine or acupuncture is more effective than placebo in the treatment of } \\
\text { CRS. The safety of Chinese medicine is unclear because most of the papers are not (easily) accessible. Minor and serious } \\
\text { adverse events can occur during the use of acupuncture and related modalities, contrary to the common impression } \\
\text { that acupuncture is harmless. For this reason, the EPOS2020 steering group advises against the use of traditional Chinese } \\
\text { medicine or acupuncture. }\end{array}$ \\
\hline
\end{tabular}


Table 1.6.1. Cont.

\begin{tabular}{|c|c|c|}
\hline Therapy & $\begin{array}{l}\text { Level of } \\
\text { evidence }\end{array}$ & GRADE recommendation \\
\hline Oral verapamil & $1 b$ & $\begin{array}{l}\text { A very small pilot study showed significant improvement in QOL (SNOT-22), polyp score (VAS), and CT scan (LM-score) of } \\
\text { oral verapamil over placebo. (Potential) side effects limited the dosage. } \\
\text { The quality of the evidence for oral verapamil is very low. Based on the potential side effects the EPOS2020 steering group } \\
\text { advises against the use of oral verapamil. }\end{array}$ \\
\hline Nasal furosemide & $1 b$ & $\begin{array}{l}\text { A recent DBPCT study showed significantly reduced QOL (SNOT-22) scores and polyp score (VAS), and significantly } \\
\text { more patients with an NPS of } 0 \text { in the furosemide nasal spray treated group versus placebo. There was no indication of a } \\
\text { difference in adverse events between topical furosemide and placebo. However, the quality of the evidence is very low. } \\
\text { The EPOS2020 steering group cannot advise on the use of nasal furosemide. }\end{array}$ \\
\hline Capsaicin & $1 b$ & $\begin{array}{l}\text { Capsaicin showed a significant decrease in nasal obstruction and nasal polyp score in two small studies, however data on } \\
\text { other symptoms like rhinorrhea and smell are either non-significant or unreported. The quality of the evidence is low and } \\
\text { the EPOS steering group concludes that capsaicin may be an option in treatment of CRS in patients with CRSwNP but that } \\
\text { larger studies are needed. }\end{array}$ \\
\hline Proton-pump inhibitors & $1 b(-)$ & $\begin{array}{l}\text { Proton-pump inhibitors have been shown in one study to be not effective. Moreover, long term use of proton pump } \\
\text { inhibitors has been associated with increased risk of cardiovascular disease. The EPOS2020 steering group therefore does } \\
\text { advise against the use of proton pump inhibitors in the treatment of CRS. }\end{array}$ \\
\hline Bacterial lysate & $1 b$ & $\begin{array}{l}\text { There is one DBPCT from } 1989 \text { comparing the bacterial lysate Broncho-Vaxom to placebo in a large group of CRS patients } \\
\text { resulting in a significant decrease in purulent nasal discharge and headache over the full six month period compared to } \\
\text { placebo and reduced opacification of the sinus X-ray. Based on this limited evidence, the EPOS2020 steering group cannot } \\
\text { advise on the use of Broncho-Vaxom in the treatment of CRS. }\end{array}$ \\
\hline Phototherapy & $1 b(-)$ & $\begin{array}{l}\text { We identified two trials with opposing findings. The quality of the evidence for the use of phototherapy in patients with } \\
\text { CRS is very low. Based on the evidence, the EPOS2020 steering group cannot make a recommendation on the use of } \\
\text { phototherapy in patients with CRS. }\end{array}$ \\
\hline Filgastrim (r-met-HuG-CSF) & $1 \mathrm{~b}(-)$ & $\begin{array}{l}\text { There is one study evaluating Filgastrim compared to placebo in CRS. There was no significant difference in effect on QOL } \\
\text { between the two groups. Based on the evidence, the EPOS2020 steering group cannot make a recommendation on the } \\
\text { use of Filgastrim in patients with CRS. }\end{array}$ \\
\hline Collodial silver nasal spray & $1 b(-)$ & $\begin{array}{l}\text { One very small study did not find differences between nasal colloidal silver spray and placebo. Based on the evidence, the } \\
\text { EPOS2020 steering group cannot make a recommendation on the use of collodial silver nasal spray in patients with CRS. }\end{array}$ \\
\hline
\end{tabular}

ATAD, Aspirin treatment after desensitisation ; $\mathrm{Cl}$, confidence interval; CRS, chronic rhinosinusitis; CRSsNP, chronic rhinosinusitis without nasal polyps; CRSwNP, chronic rhinosinusitis with nasal polyps; DBPCT, double blind placebo controlled trial; LK, Lund Kennedy; LM, Lund-Mackay score; MFNS, mometasone fuorate nasal spray; MSS, major symptom score; N-ERD, NSAID-exacerbated respiratory disease; NPS, nasal polyp score; QOL, quality of life; RCT, randomised controlled trial; SNOT-22, sino-nasal outcome test-22; SMD, standard mean difference.

corticosteroids or continuous use of systemic corticosteroids ( $\geq 2$ courses per year OR long term ( $>3$ months) low dose steroids OR contraindication to systemic steroids), significantly impaired quality of life ( SNOT-22 $\geq 40$ ), anosmic on smell test and/or a diagnosis of comorbid asthma needing regular inhaled corticosteroids (Figure 1.6.3.).

The response criteria for biologicals have been taken from the EUFOREA paper (Figure 1.6.4.), although the EPOS2020 group also discussed whether there was an indication to repeat surgery in patients on biologicals to give them a better starting point. It was decided that we had insufficient data to advise on surgery whilst on biologicals before deciding that they are not effective and that this is a research need.

\subsubsection{Conclusion}

EPOS2020 provides a full evidence based systematic review of the management of CRS that has been incorporated into an integrated care pathway (Figures 1.6.1. and 1.6.2.). A significant shift in the management of CRS has occurred since EPOS2012.
The options of biologicals in the treatment of type 2 CRS will be a paradigm shift in the management of the disease. The exact positioning of this presently very expensive treatment needs to be determined. (Figures 1.6.3. and 1.6.4.).EPOS2020 further emphasizes the criteria for (revision) surgery in the disease.

\subsection{Paediatric chronic rhinosinusitis}

\subsubsection{Epidemiology and predisposing factors}

This section has been considerably expanded, reflecting new literature. The prevalence of CRS in paediatric patients is now estimated to be up to $4 \%{ }^{(109)}$. Both passive and active cigarette smoking are associated with chronic rhinitis and rhinosinusitis in children ${ }^{(110)}$ though a clear and definitive causal relationship between allergic rhinitis and CRS has not been established ${ }^{(111)}$. Evidence suggests that the adenoids may act as a reservoir for pathogenic bacteria, rather than a source of obstruction ${ }^{(112,}$ 
${ }^{113)}$ whilst the relationship between GORD and CRS in children remains controversial ${ }^{(114)}$. A large database study suggests a significant familial risk associated with paediatric $\mathrm{CRS}^{(115)}$ but studies on monozygotic twins have not shown that both siblings always develop polyps, indicating that environmental factors are as likely as genetic ones to influence the occurrence of nasal polyps.

\subsubsection{Inflammatory mechanisms}

Multiple studies suggest upregulation of different inflammatory substances important in adaptive and innate immunity as well as tissue remodelling in sinus tissues, adenoids, nasal lavage, mucus and serum in children with CRS. Although the evidence is still scarce, these studies suggest a role for inflammatory mechanisms in paediatric CRS. Although many of the markers parallel those seen in adults, the data is very heterogeneous and does not yet lend itself to endotyping. Inflammatory cytokines are present in sinus tissues of children with CRS and are more abundant when concomitant asthma is present ${ }^{(116)}$. Although more evidence is emerging to support upregulation of inflammatory markers in paranasal sinus tissues and nasal lavages of children with CRS, the data is also relatively limited and heterogeneous and again does not yet lend itself to endotyping.

\subsubsection{Management of paediatric CRS including integrated care pathway}

Medical therapy remains the mainstay of management of paediatric chronic rhinosinusitis (Table 1.7.1.). Saline nasal irrigation is recommended for the treatment of CRS in children. Addition of nasal antibiotics to saline irrigations is not recommended. There is currently no evidence to support treatment of children with CRS with either oral or intravenous antibiotics. There is also no evidence to support the utilization of prolonged macrolide therapy in children with CRS. Intranasal steroids are recommended for use in children with CRS despite the absence of good level evidence. This is based on safety in children and favourable efficacy data in adults with CRS (see chapter 6) and children with rhinitis ${ }^{(117)}$.

There is hardly any scientific support for other ancillary therapies such as antihistamines (intranasal or oral), leukotriene modifiers, decongestants (intranasal or oral), or mucus thinners and these treatments are not recommended. Exceptions are using ancillary therapies when indicated for concomitant disease such as allergic rhinitis or GORD.

Surgical intervention is considered for patients with CRS who have failed appropriate medical therapy (and, less commonly, in complicated acute rhinosinusitis). It seems that adenoidectomy with / without antral irrigation is certainly the simplest and safest first procedure to consider in younger children with symptoms of CRS. Evidence suggests that antral irrigation should be considered in addition to an adenoidectomy in children with asthma who have more severe disease on preoperative CT scans. FESS is a safe and possibly effective surgical modality in children with CRS and can be used as primary modality or after failure of adenoidectomy in older children. Decisions on use depend on severity of disease, age and existing co-morbidities. The rate of major complications following paediatric FESS was $0.6 \%$, and the rate of minor complications $2 \%$.

The systematic review of the literature resulted in an integrated care pathway for paediatric CRS (Figure 1.7.1.). The differential diagnosis in primary care is broad with the most essential diagnosis in young children being adenoid hypertrophy / adenoiditis. In secondary and tertiary care, the ICP also advises saline irrigation and INCS as first line treatment followed by adenoidectomy with or without sinus irrigation if insufficient. FESS is reserved for older children who fail adenoidectomy (with

Table 1.7.1. Evidence supporting therapy of CRS in children.

\begin{tabular}{|c|c|c|}
\hline Therapy & $\begin{array}{l}\text { Level of } \\
\text { evidence }\end{array}$ & GRADE recommendation \\
\hline Antibiotics & $1 \mathrm{~b}(-)$ & $\begin{array}{l}\text { There is no high level evidence to support the efficacy of either short or long term antibiotics for } \\
\text { CRS in children. }\end{array}$ \\
\hline Nasal corticosteroids & 5 & $\begin{array}{l}\text { There is no evidence regarding the efficacy of intranasal steroids in the treatment of CRS in } \\
\text { children. Nevertheless the EPOS steering group is supportive of their use in light of their anti- } \\
\text { inflammatory effects and excellent safety record in children. }\end{array}$ \\
\hline Systemic Steroids & $1 \mathrm{~b}(+)$ & $\begin{array}{l}\text { Adding a taper course of systemic steroids to an antibiotic (not effective on its own) is more } \\
\text { effective than placebo in the treatment of paediatric CRS. Judicious use of this regimen is } \\
\text { advised considering systemic side effects. }\end{array}$ \\
\hline Saline Irrigation & $\mathrm{lb}(+)$ & $\begin{array}{l}\text { There are a few clinical trials demonstrating the efficacy of saline irrigations in paediatric patients } \\
\text { with CRS. The EPOS steering group is supportive of the use of saline in light of the excellent } \\
\text { safety record in children. }\end{array}$ \\
\hline Adenoidectomy & 4 & $\begin{array}{l}\text { Adenoidectomy is effective in younger children with symptoms of CRS. The EPOS steering group } \\
\text { supports adenoidectomy in young children refractory to appropriate medical therapy. }\end{array}$ \\
\hline FESS & 4 & $\begin{array}{l}\text { FESS is safe and effective for the treatment of older children with CRS refractory to medical } \\
\text { therapy or previous adenoidectomy. }\end{array}$ \\
\hline
\end{tabular}

CRS, chronic rhinosinusitis; FESS, functional endoscopic sinus surgery. 
Figure 1.7.1. Integrated care pathway in paediatric CRS.

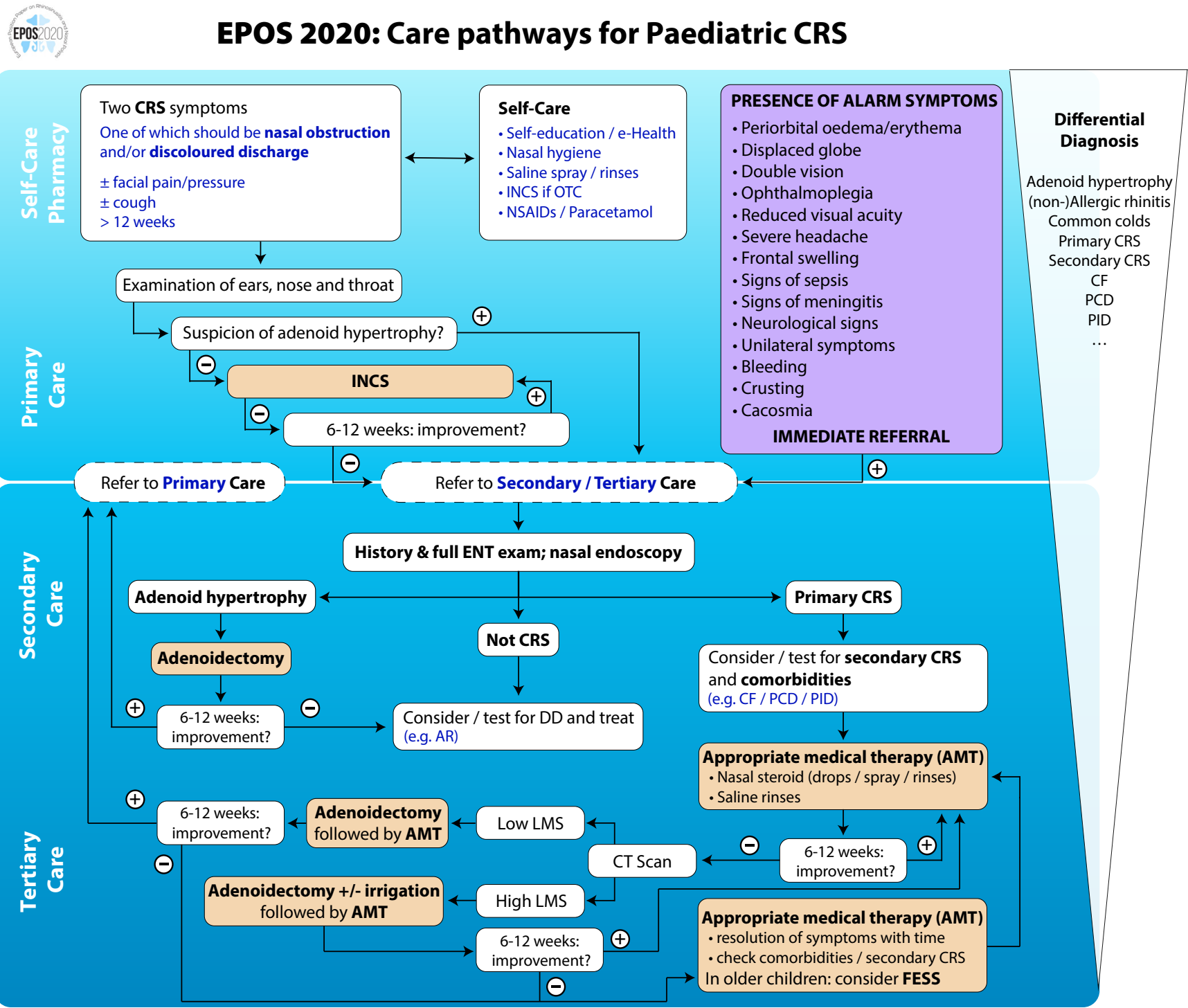

AMT, appropriate medical treatment; CF, cystic fibrosis; CRS, chronic rhinosinusitis; CT, computed tmography; DD, differential diagnosis ; INCS, intranasal corticsteroids; LMS, Lund-Mackay score; NSAIDs, non-steroidal anti-inflammatory drugs; OTC, over the counter; PCD, primary ciliary dyskinesia; PID, primary immune deficiencies.

sinus irrigation). CRS in children may be an indication of severe diseases such as immunodeficiencies, cystic fibrosis or primary ciliary dyskinesia. Practitioners should be aware of these and also of serious complications needing immediate referral.

\subsection{Concomitant diseases in chronic rhinosinusitis}

Chapter 8 discusses the role of concomitant diseases in CRS. The role of allergy, including central compartment atopic disease, immunodeficiencies and their role in CRS, a work-up for ENTs before referring to immunologists, lower airway disease including asthma, cystic fibrosis and PCD, fungal rhinosinusitis, vasculitis and granulomatous diseases and their role in CRS are all discussed.

\subsubsection{Role of allergy and chronic rhinosinusitis}

It has become clear in recent years that the role of allergy in CRS depends on different phenotypes / endotypes of CRS. In some phenotypes / endotypes such as AFRS or central compartment atopic disease, allergy seems to play an important role whilst in others the prevalence does not seem to be higher than in the general population, although even in these patient groups, allergy can be an aggravating factor. Allergic rhinitis (AR) is a highly prevalent disease and there is a significant overlap in symptomatology between CRS and AR. It is not always easy to evaluate the role of sensitization to allergens in patients with CRS especially in perennial sensitisations. Optimal treatment of the allergic rhinitis seems advisable.

\subsubsection{Immunodeficiencies and their role in CRS}

Conditions that are associated with immunodeficiency are 


\section{Fungi and the human immune response} Allergic fungal
rhinosinusitis
Fungal ball
Invasive fungal rhinosinusitis

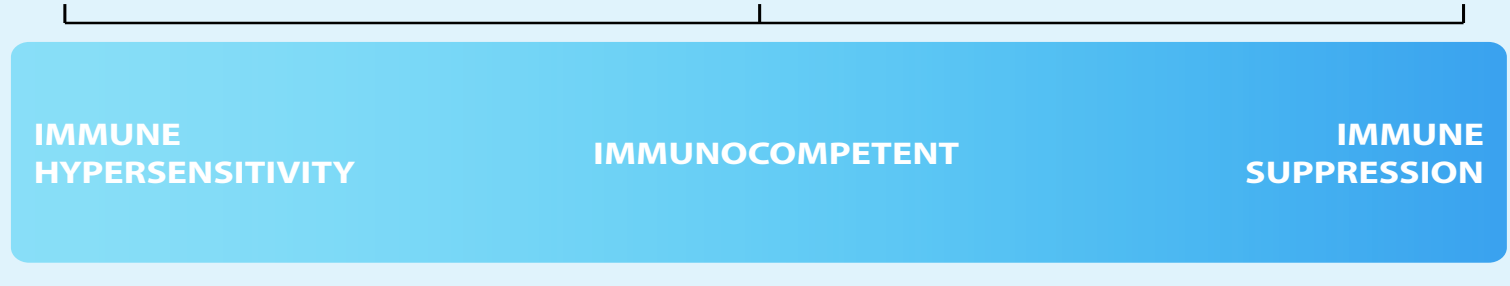

of clinical importance to rhinologists because some patients who present with CRS are predisposed to their condition by an underlying immunodeficient state. Immunodeficiency conditions may cause CRS patients to respond less favourably to standard therapies, and some patients require specific treatment for their immunodeficiencies in order for their CRS to be optimally treated.

Testing of immune function in all patients who present with CRS is almost certainly unwarranted as it is likely to produce more false positive results than true positives. However, it is recommended that recalcitrance to standard treatments (and particularly rapid recurrence of symptoms after stopping antibiotics) and association of CRS with lower respiratory tract infections (pneumonia, particularly if recurrent, or bronchiectasis) are used to identify those patients who warrant some form of immune testing.

For CRS patients suspected of having humoral immunodeficiency because of the characteristics of their presentation or their response to treatment, measurement of serum immunoglobulin levels is the key investigation. If the levels are normal, but the suspicion of humoral immunodeficiency is high, referral to a clinical immunologist is recommended.

The best approach for confirming a diagnosis of an antibodydeficiency disorder is the measurement of serum-specific antibody titres (usually lgG) in response to vaccine antigens. This approach involves immunizing a patient with protein antigens (e.g. tetanus toxoid) and polysaccharide antigens (e.g. pneumococcus) and assessing pre- and post-immunization antibody levels.

Treatment of patients with primary immune deficiency may consist of long-term antibiotics, often at half dose, pneumococcal vaccinations and immunoglobulin replacement therapy.

The prevalence of secondary immune deficiency is rising due to the increased use of immunosuppressive agents such as rituximab, corticosteroids and other drugs and otorhinolaryngologists need to directly ask about immunosuppressive agents in their history taking.

\subsubsection{Lower airway disease including asthma in relation to CRS}

Given the epidemiologic and pathophysiologic connection between CRS and lower respiratory airway disease $\mathrm{e}^{(11,118)}$ the concept of global airway disease has gained more interest, leading to better diagnosis and therapeutic approaches in patients with global airway disease. Lower airway inflammation often co-exists in CRS, with up to two thirds of patients with CRS affected by comorbid asthma, COPD or bronchiectasis. Endoscopic sinus surgery in asthma has been reported to improve multiple clinical asthma parameters with improved overall asthma control, reduced frequency of asthma attacks and number of hospitalizations, as well as decreased use of oral and inhaled corticosteroids.

\subsubsection{Cystic fibrosis}

Cystic fibrosis (CF) is a life-shortening genetic condition caused by a mutation in the cystic fibrosis transmembrane conductance regulator (CFTR) gene leading to defective chloride channels, which results in secretions with more than double the viscosity of secretions of a non-CF individuals. In the Western world national screening programs on specific genetic disorders including CF have been implemented for newborns. Bilateral nasal polyposis in children may be a clinical indicator of CF. A major goal in the treatment of patients with $\mathrm{CF}$ is thus to prevent or delay chronic lung infections. There is a high concordance of bacteria cultured from the paranasal sinuses (based on irrigations, swabs, or mucosal biopsies) and from the lungs.

The treatment of CF is currently symptomatic whilst the treatment of the underlying genetic defect, thus curing the disease, has not yet been possible. However, new treatment options such as (the combination of) Ivacaftor, a CFTR potentiator, and Tezacaftor, a selective CFTR corrector, have shown promising results in improving rhinologic QOL in patients with CF. 
Several studies have evaluated the effect of sinus surgery on pulmonary function with divergent conclusions. Sinus surgery is recommended in CF patients without chronic lung infection or with a transplanted lung in an attempt to eradicate gramnegative bacteria in the paranasal sinuses, thereby avoiding or preventing re-colonisation of the lungs. Detecting gramnegative sinus bacteria at an early stage is an important step towards eradicating the bacteria and avoiding a chronic bacterial sinus infection. The use of topical antibiotics correlates with improvement in symptom and endoscopic scoring and is safe.

\subsubsection{Primary ciliary dyskinesia}

Primary ciliary dyskinesia (PCD) is a collection of rare inherited disorders that affects motile cilia and is primarily inherited in an autosomal recessive manner. Situs inversus (i.e. Kartagener syndrome) exists in approximately half of all PCD cases. Both men and women diagnosed with PCD commonly present with fertility disorders as the reproductive process is largely dependent on ciliary function. PCD has a strong association with history of CRS, being associated with CRSwNP in 15-30\% of patients, and is commonly seen in children with CRS. PCD also predisposes to bacterial infections commonly including $\mathrm{H}$. influenza, S. pneumoniae and P. aeruginosa. In the absence of hard clinical and paraclinical criteria for diagnosing PCD, confirming the diagnosis with clinical exam alone is a challenge. An electron microscopic analysis of cilia can yield valuable information about ciliary ultrastructure and function. However, it should be noted that cilia may appear normal in patients that present with symptoms strongly suggestive of PCD due to mutations that can result in normal structure.

A number of studies have shown that exhaled nitric oxide (NO), particularly nasal NO production levels, are low in PCD patients. An nNO cut-off value of $<77 \mathrm{nl} / \mathrm{min}$ can allow detection of PCD with a sensitivity and specificity of $98 \%$ and $>99 \%$, respectively, after excluding CF and acute viral respiratory infections. Prolonged macrolide therapy has been shown to produce marked improvement in symptomatology of PCD due to the anti-inflammatory and immune-mediating properties of the antibiotic. Surgical intervention (ESS) may be required when medical therapy has failed.

\subsubsection{Fungal rhinosinusitis}

Fungi are ubiquitous in our environment and with dedicated assessments they can be found in nasal mucus from almost all healthy and diseased sinuses. However, there are several forms of sinus disease that are associated with fungi as pathogens. In these situations, rather than the fungi determining the disease process, it is usually the host immune state that determines the clinical presentation (Figure 1.8.1).

There was much prior debate regarding the role of fungi in CRSwNP. Some authors had proposed that a response to fungi might be the basis for most type 2 dominated polypoid forms of CRS. However, subsequent research has not supported this ${ }^{(119,}$ ${ }^{120)}$. Thus, this chapter will discuss these three phenotypes of 'fungal' related CRS but an intentional focus is made on AFRS as a unique phenotype, and its treatment, within the broader definition of CRS.

A fungal ball is a non-invasive collection of fungal debris. Recent studies indicate that anatomic variants are not major contributors to their formation, which in the maxillary sinus is more often related to dental interventions ${ }^{(121-123)}$. Neoosteogenesis of the maxillary sinus wall is common with fungal balls compared to normal patients and is independent of bacterial coinfection ${ }^{(124)}$. Isolated maxillary or sphenoid sinus opacification is a marker of neoplasia in $18 \%$ and malignancy in $7-10 \%$ of patients presenting with these radiologic findings so clinicians should be wary of conservative management and have a low threshold for early surgical intervention ${ }^{(125)}$. Little has changed in the management of fungal balls since 2012 which remains surgical, consisting of removal via an adequate antrostomy. However, persistent dysfunction of the sinus cavity with mucostasis was reported to be as high as $18 \%$ and, therefore, some authors have proposed a medial maxillectomy for some maxillary cases ${ }^{(126)}$. Invasive fungal rhinosinusitis (IFRS) is almost always associated with immunocompromise, of which diabetes (50\%) and haematologic malignancy (40\%) account for $90 \%$ of the immunosuppression reported ${ }^{(127)}$. IFRS is defined as any state in which fungal hyphae can be seen 'within' the mucosal tissue, demonstrating classic angio-invasion or other infiltrative patterns ${ }^{(128)}$ which result in thrombosis, tissue infarction and necrosis. Although originally several forms of invasive disease were described: granulomatous, chronic and fulminant, they all potentially represent an immunocompromised host reaction to the fungus ${ }^{(129)}$. The most common causative pathogens remain the Zygomycetes (Rhizopus, Mucor, Rhizomucor) and the Aspergillus species. Unilateral disease on radiology is typical ${ }^{(130,131)}$ but loss of contrast enhancement on MRI is more sensitive (86\%) than CT $(69 \%)$ in detecting invasive fungal disease ${ }^{(132)}$. Serum analysis via PCR (serum or whole blood) and/or galactomannan for invasive aspergillosis can be useful ${ }^{(133)}$.

There are three principles for treatment:

1. Systemic antifungals therapy should be started;

2. Patients should undergo, at least, endoscopic surgical debridement of necrotic sinonasal tissue, which may need to be repeated;

3. The patient's immune suppression should be reduced when feasible.

Allergic fungal rhinosinusitis (AFRS) is a subset of polypoid chronic rhinosinusitis that is characterized by the presence of eosinophilic mucin with non-invasive fungal hyphae within the sinuses and a type I hypersensitivity to fungi. The EPOS2020 steering group discussed whether the term 'eosinophilic fungal rhinosinusitis' would be a better umbrella term but it was agreed that 'allergic fungal rhinosinusitis' should be retained as the principle term due to common usage, recognising that not all cases have evidence of an allergic reaction to fungi. AFRS accounts for about $5-10 \%$ of CRS cases $^{(134)}$. Ideally all five of the major criteria in the original Bent-Kuhn diagnostic criteria should be met to make the diagnosis as three 
of the five are common in most cases of CRSwNP. These major criteria consist of the following ${ }^{(135)}$ :

1. Nasal polyposis;

2. Fungi on staining;

3. Eosinophilic mucin without fungal invasion into sinus tissue;

4. Type I hypersensitivity to fungi and;

5. Characteristic radiological findings with soft tissue differential densities on CT scanning and unilaterality or anatomically discrete sinus involvement.

The minor criteria include bone erosion, Charcot Leyden Crystals, unilateral disease, peripheral eosinophilia, positive fungal culture and the absence of immunodeficiency or diabetes $^{(136)}$. CT shows densely packed hyperdensities in the sinuses with expansion and erosion of the bony walls whereas on MRI signal voids occur on both T1 and T2 sequences ${ }^{(137)}$. The mainstay of treatment remains surgery as medical treatment alone is usually ineffective. However, oral steroids both pre- and postoperatively are of benefit ${ }^{(138)}$. Nebulised topical corticosteroids reduce recurrence ${ }^{(139)}$ and allergen immunotherapy was also helpful in atopic individuals but studies are retrospective and underpowered. There is some evidence that oral antifungals may reduce recurrence but do not improve symptoms.

Fungal rhinosinusitis remains an important phenotype of CRS in its invasive and non-invasive forms. Clinicians should have a low threshold for seeking its diagnosis, especially in the presence of the immunocompromised. The mainstay of treatment remains surgical though may be combined with medical therapies in invasive and allergic forms. See Figure 1.6.2. which includes an integrated care pathway for AFRS although the steering group realized that diagnosis in primary and secondary care can be difficult.

\subsubsection{Vasculitis}

ANCA-associated vasculitis includes GPA, EGPA and microscopic polyangiitis (MPA) and frequently affect the upper respiratory tract and specifically the sinonasal region where they may be mistaken for more common forms of chronic rhinosinusitis. Classically GPA affects the nose, lungs and kidneys but can present in any system and limited forms of the disease are recognised. Two thirds of patients initially present with an ENT-related symptom, of which the majority are rhinological. During the course of the disease, the majority of GPA patients experience nasal symptoms with patients experiencing crusting (75\%), discharge (70\%), nasal stuffiness (65\%), bleeding (59\%), reduced sense of smell $(52 \%)$ and facial pain $(33 \%)^{(140,141)}$. ANCA tests have become the mainstay of diagnosis in vasculitis. A positive c-ANCA test and proteinase-3 (PR3) will confirm the clinical diagnosis of GPA in up to $95 \%$ of patients with active systemic disease. An ANCA test should be considered in any patient with suspicious clinical manifestations, in particular nasal crusting and bleeding, especially if they feel disproportionally unwell ${ }^{(142)}$.

Cocaine abuse in the form of nasal 'snorting' can resemble the sinonasal symptoms of GPA and can give c-ANCA and PR-3 positivity, making differentiation between the conditions difficult ${ }^{(143)}$. Without treatment the mean survival of systemic GPA is five months. Modern immunosuppressive treatment following a strategy of combined remission, induction and maintenance has markedly improved this to a mean survival of 21.7 years from diagnosis assisted by higher awareness and earlier diagnosis. Nasal irrigation, topical intranasal corticosteroid sprays or creams e.g. triamcinolone and/or a nasal lubricant such as $25 \%$ glucose and glycerine drops, honey ointment or an aqueous gel are usually recommended together with regular debridement of the crusts. The possible aetiological role of Staphylococcus aureus has led to the use of long-term oral co-trimoxazole (trimethoprim-sulfamethoxazole) and topical anti-staphylococcal creams in the nose. Reconstructive surgery has a very limited role and is associated with poor outcomes, increased scarring and adhesions so should be a last resort. Eosinophilic granulomatosis with polyangiitis (EGPA) (previously Churg Strauss Syndrome) is a rare form of vasculitis characterised by adult onset asthma, severe rhinitis, nasal polyps and other systemic manifestations as a result of widespread eosinophilic granulomatous infiltration of tissues ${ }^{(144)}$. EGPA should be considered in any patient with severe nasal polyposis who is not responding to conventional therapy. Active EGPA is characterised by marked peripheral eosinophilia (usually $>1500$ cells/ul or $>10 \%$ ) and ANCA-positivity is found in a proportion of the patients. In most patients, disease control is achieved with immunosuppressant therapy, usually oral prednisolone +/cytotoxic drugs such as pulsed cyclophosphamide, azathioprine, mycophenolate mofetil and methotrexate dependent on the severity of the disease at presentation. Sarcoidosis is a chronic multi-system inflammatory disease of unknown aetiology characterised by non-caseating granuloma. There is no definitive test for sarcoidosis other than a positive biopsy. Blood tests may include raised serum and urinary calcium levels, raised alkaline phosphatase and raised serum angiotensinconverting enzyme (SACE) but none are diagnostic (sensitivity $60 \%$; specificity $70 \%$ ). Systemic steroids remain the mainstay of treatment in sarcoidosis, though hydroxychloroquine, steroidsparing cytotoxic agents such as methotrexate and TNF-alpha antagonists such as infliximab are being used.

\subsection{Patient participation, prediction, precision medicine and implementation}

\subsubsection{Patient participation in CRS}

Patient participation in rhinosinusitis can relate on an individual basis to participation of the patient in the design and/or discussion of the treatment plan, or to participation in the follow-up after medical or surgical treatment ${ }^{(145)}$. There is limited research on the impact of patient participation on outcomes of treatment in CRS.

Patient involvement, moreover, is recognized as a key 
component of clinical practice guideline development with important implications for guideline implementability ${ }^{(146)}$. Aspects of patient participation are covered for the first time in EPOS2020 because patient involvement is essential in the development of their future care. Patients were actively involved in the development of EPOS2020. Recent mobile health initiatives to educate patients on CRS, on correct medication use and treatment options have been implemented in certain areas in Europe with success ${ }^{(147)}$. Whilst they allow a more proactive follow-up of patients with remote monitoring of symptoms by physicians ${ }^{(147,148)}$ the impact of e-health on CRS outcomes still needs to be defined and proven.

For individual patients, shared decision-making is one of the four cardinal principles of Precision Medicine ${ }^{(149)}$. In order to improve compliance, it is important to explain the aim of ongoing usage or any maintenance treatments to both control symptoms and reduce need for recurrent interventions. Information on the safety of treatment and instructions for use must be provided in all necessary languages. While physicians are likely to understand the chronic nature of sinus disease in many patients and the need for ongoing treatment, it is essential to share this with the patient from the outset. The aim of treatment is to achieve adequate control of symptoms with as little need for intervention as possible; for many this will involve ongoing usage of intranasal treatments and in some, repeated need for systemic treatments or surgical interventions. Some patients will remain inadequately controlled despite receiving optimum current evidence-based care. Cure, with an absence of symptoms in the setting of no ongoing medication usage, is unusual in CRS with the exception of localized sinus disease where there has been a curable cause, such as an odontogenic source.

\subsubsection{Primary, secondary and tertiary disease prevention in CRS}

Prevention may be considered as primary, secondary and tertiary ${ }^{(150)}$. Primary prevention aims to reduce incidence of disease by reducing exposure to risk factors or triggers. CRS is a heterogeneous disease, where inflammation, mucociliary dysfunction and changes in the microbial community interact with differing influences to cause disease; the aetiology is likely multifactorial, and opportunities to prevent targeting specific causes will likely vary between subgroups. Occupational and environmental factors, especially exposure to tobacco smoke, are of increasing importance in primary prevention and the effects of global warming should be carefully monitored. Co-morbidities such as allergy, asthma and GORD should be considered. Genetic and microbiological factors will likely become of greater importance. Early diagnosis and selection of the optimal treatment is central to secondary prevention. Optimising medical treatment and consideration of the timing and extent of surgery can improve outcomes. In tertiary prevention, a careful review of ongoing treatment, technique and compliance with medication should be undertaken. Growth in digital healthcare and patient apps may encourage self-management and increase compliance. There are a small number of studies using big data sets that suggest that endoscopic sinus surgery for CRS reduces the yearly incidence of new asthma diagnoses. Those patients who have later surgery may develop higher rates of asthma than those who undergo surgery at an earlier timepoint. Finally, the prevention of recurrent disease is important. Continued use of intranasal corticosteroids after surgery has been shown to improve postoperative endoscopic scores in all CRS patients and, in those with CRSwNP, reduce risk of recurrence. Adherence with prescribed postoperative medications dropped to only $42 \%$ at 12 months after surgery in one study, despite regular telephone contact; strategies to improve this such as utilizing digital technology will likely be important in future. One can also imagine that other forms of ensuring the application of postoperative medication, e.g. by drug eluting stents, may solve the problem of compliance. A small number of studies have found that ongoing occupational exposure to irritants may increase risk of recurrence. Any factors thought to be involved in the underlying aetiology of CRS in each individual patient should be addressed where possible to reduce risk of recurrence. In contrast to the large number of studies evaluating changes in $\mathrm{HRQOL}$ after treatment, few studies have evaluated patient satisfaction with outcomes of treatment, and only following surgical interventions. Although data is limited, it appears that pre-treatment counselling to ensure that a patient has realistic expectations of treatment outcomes is important to avoid a dissatisfied patient. This is in respect to improvement overall and in those symptoms deemed to be most important to the patient, as well as optimizing outcomes with respect to their nasal symptoms.

\subsubsection{Prediction}

There are no studies evaluating the natural history of untreated CRS although there is some evidence for the adverse effects of delayed surgical treatment ${ }^{(151)}$. Notwithstanding ethical considerations, there is clearly an urgent need for more research in this area. Similarly, there are very few studies predicting outcomes of medical treatment. When predicting outcomes following sinus surgery, a number of studies have shown that the preoperative symptom score such as SNOT-22 is the best predictor of outcome ${ }^{(152,153)}$. Primary surgery has better outcomes than revision. When loss of smell is a major symptom, response in olfactory function to oral corticosteroids (OCS) predicts the outcome of surgery. Prediction of recurrent disease involves many factors including age, gender, ethnicity, co-morbidities, and duration of disease. Both blood and tissue eosinophil levels can be measured with little additional expense and may be used to help predict risk of recurrence and need for targeted postoperative care.

\subsubsection{Precision medicine}

In 2015 President Obama launched the precision medicine initiative: "delivering the right treatment at the right time, every time, to the right person". The principles of precision medicine can be implemented within existing adult treatment algorithms for $\mathrm{CRS}^{(149)}$. At the time of diagnosis, prediction of success of the initiated treatment as well as patient participation in decisions regarding the treatment plan can be undertaken. Precision 
medicine allows real-time clinical decision support at the point of care with implementation of harmonized care based on quality criteria and allows patients to be treated and monitored more precisely and effectively to better meet their individual needs. It brings together clinicians from many inter-related specialities, scientists and above all patients in a collaborative effort to provide the most efficient and effective management.

\subsubsection{Implementation}

The implementation of high-quality guidelines and position papers is essential to improve clinical practice and public health. We tried to make EPOS2020 implementable by writing a clear and concise executive summary with extensive chapters with all the evidence behind it. We hope that the executive summary will be translated in all necessary languages. Furthermore, we reached out to many key opinion leaders all over the world to review and comment on the document and included their suggestions in the final text. We do realize that not all advice in EPOS2020 can be followed in all health care systems and social circumstances. A full implementation plan will be written separately to the EPOS2020 document in the near future.

\subsection{Pharmacist perspective on rhinosinusitis}

Chapter 10 gives the pharmacist's perspective on rhinosinusitis and offers specific advice to pharmacists on how to differentiate and treat the various forms of ARS (common cold, post-viral rhinosinusitis and acute bacterial rhinosinusitis) and CRS in contradistinction to allergic rhinitis. Special emphasis has been placed on the avoidance of antibiotics in the treatment of rhinosinusitis and the role that the pharmacist can have in advising patients on the correct use of nasal sprays.

\subsection{Research priorities in rhinosinusitis}

Chapter 11 gives an overview of research priorities. In many areas of rhinosinusitis, evidence is still of low quality and most subchapters in EPOS2020 originally ended with:'more research is needed to provide high quality evidence'. We decided, therefore, to remove the majority of these exortations and to collate the most urgent questions in this final chapter.

\subsection{Methods used in EPOS2020}

In chapter 12 the methods used in EPOS2020 are discussed. We describe the development strategy used in EPOS2020, which has been published before we started the work ${ }^{(155)}$. We did a full systematic review of the literature and used GRADE methodology for recommendations. On a large number of practical clinical questions with no or or very low level of evidence we conducted a Delphi exercise.

\section{References}

1. Fokkens W, Lund V, Mullol J. European position paper on rhinosinusitis and nasal polyps 2007. Rhinol Suppl. 2007:1-136.

2. Fokkens W, Lund V, Bachert C, et al. European position paper on rhinosinusitis and nasal polyps. Rhinol Suppl. 2005:1-87.

3. Fokkens WJ, Lund VJ, Mullol J, et al. European Position Paper on Rhinosinusitis and Nasal Polyps 2012. Rhinol Suppl. 2012;23:3 p preceding table of contents, 1-298.

4. Hastan D, Fokkens WJ, Bachert C, et al. Chronic rhinosinusitis in Europe--an underestimated disease. A GA(2)LEN study. Allergy 2011;66:121623.

5. Hirsch AG, Stewart WF, Sundaresan AS, et al. Nasal and sinus symptoms and chronic rhinosinusitis in a population-based sample. Allergy 2017;72:274-81.

6. Obaseki D, Potts J, Joos G, et al. The relation of airway obstruction to asthma, chronic rhinosinusitis and age: results from a population survey of adults. Allergy 2014;69:1205-14.

7. Sundaresan AS, Hirsch AG, Storm M, et al. Occupational and environmental risk factors for chronic rhinosinusitis: a systematic review. International Forum of Allergy \& Rhinol. 2015;5:996-1003.

8. Piatt Jr. JH. Intracranial suppuration complicating sinusitis among children: an epidemiological and clinical study. J Neurosurg Pediatr 2011;7:567-74.

9. Jaume F, Quintó L, Alobid I, Mullol J. Overuse of diagnostic tools and medications in acute rhinosinusitis in Spain: a population-based study (the PROSINUS study). BMJ open 2018;8:e018788.

10. Wei B, Liu F, Zhang J, et al. Multivariate analysis of inflammatory endotypes in recurrent nasal polyposis in a Chinese population. Rhinology 2018;56:216-26.

11. Tomassen $P$, Vandeplas $G$, Van Zele $T$, et al. Inflammatory endotypes of chronic rhinosinusitis based on cluster analysis of biomarkers. J Allergy Clin Immunol. 2016;137:1449-56.e4.

12. Jarvis $D$, Newson R, Lotvall J, et al. Asthma in adults and its association with chronic rhinosinusitis: the GA2LEN survey in Europe. Allergy. 2012;67:91-8.

13. Hakansson $\mathrm{K}$, Thomsen SF, Konge L, Mortensen J, Backer V, von Buchwald C. A comparative and descriptive study of asthma in chronic rhinosinusitis with nasal polyps. Am J Rhinol Allergy. 2014;28:383-7.

14. Samitas K, Carter A, Kariyawasam HH, Xanthou G. Upper and lower airway remodelling mechanisms in asthma, allergic rhinitis and chronic rhinosinusitis: The one airway concept revisited. Allergy 2018;73:993-1002.

15. van der Veen J, Seys SF, Timmermans M, et al. Real-life study showing uncontrolled rhinosinusitis after sinus surgery in a tertiary referral centre. Allergy 2017;72:282-90.

16. Snidvongs K, Heller GZ, Sacks R, Harvey RJ.Validity of European position paper on rhinosinusitis disease control assessment and modifications in chronic rhinosinusitis. Otolaryngol Head Neck Surg. 2014;150:479-86.

17. Calus L, Van Bruaene N, Bosteels C, et al. Twelveyear follow-up study after endoscopic sinus surgery in patients with chronic rhinosinusitis with nasal polyposis. Clin and Transl Allergy. 2019;9:30.

18. Stjärne P, Odebäck P, Ställberg B, Lundberg J,
Olsson P. High costs and burden of illness in acute rhinosinusitis: real-life treatment patterns and outcomes in Swedish primary care. Primary Care Resp Journal. 2012;21:174-9.

19. Remenschneider AK, Scangas G, Meier JC, et al. EQ-5D-derived health utility values in patients undergoing surgery for chronic rhinosinusitis. Laryngoscope 2015;125:1056-61.

20. Gliklich RE, Metson R. The health impact of chronic sinusitis in patients seeking otolaryngologic care. Otolaryngol Head Neck Surg. 1995;113:104-9.

21. Teul I, Zbislawski W, Baran S, Czerwinski F, Lorkowski J. Quality of life of patients with diseases of sinuses. J Physiol Pharmacol. 2007;58 Suppl 5:691-7.

22. Garbutt J, Spitznagel E, Piccirillo J. Use of the modified SNOT-16 in primary care patients with clinically diagnosed acute rhinosinusitis. Arch of Otolaryng--Head \& Neck Surg 2011;137:792-7.

23. Hopkins C, Browne JP, Slack R, et al. The national comparative audit of surgery for nasal polyposis and chronic rhinosinusitis. Clinical Otolaryngol. 2006;31:390-8.

24. Teul I, Baran S, Zbislawski W. Upper respiratory tract diseases in self-evaluation of health status of Polish students based on the SF-36 questionnaire. J Physiol Pharmacol. 2008;59 Suppl 6:697-707.

25. Dietz de Loos DA, Hopkins C, Fokkens WJ. Symptoms in chronic rhinosinusitis with and without nasal polyps. Laryngoscope 2013;123:57-63.

26. Abdalla S, Alreefy H, Hopkins C. Prevalence of sinonasal outcome test (SNOT-22) symptoms in patients undergoing surgery for chronic 
rhinosinusitis in the England and Wales National prospective audit. Clin Otolaryngol 2012;37:27682.

27. Bhattacharyya N, Lee LN. Evaluating the diagnosis of chronic rhinosinusitis based on clinical guidelines and endoscopy. Otolaryngol Head Neck Surg. 2010;143:147-51.

28. Bhattacharyya N. Contemporary assessment of the disease burden of sinusitisThe economic burden and symptom manifestations of chronic rhinosinusitis. Am J Rhinol Allergy. 2009;23:392-5.

29. Wahid NW, Smith R, Clark A, Salam M, Philpott C. The Socioeconomic Cost of Chronic Rhinosinusitis Study. Rhinology 2020;58,2:112 125.

30. Lourijsen ES, Fokkens WJ, Reitsma S. Direct and indirect costs of Dutch adult patients with Chronic Rhinosinusitis with nasal polyps. Rhinology 2020;3:213-217.

31. Bhattacharyya N. Assessing the additional disease burden of polyps in chronic rhinosinusitis. The Annals of otology, rhinology, and laryngology 2009;118:185-9.

32. Gross Mark;Burgess LP, Rick; Sheridan. Endoscopic Sinus Surgery Complications in Residency. Laryngoscope 1997;107:1080-5.

33. Gliklich RE, Metson R. Economic implications of chronic sinusitis. Otolaryngol Head Neck Surg. 1998;118:344-9.

34. Bhattacharyya N, Orlandi RR, Grebner J, Martinson M. Cost burden of chronic rhinosinusitis: a claims-based study. Otolaryngol Head Neck Surg. 2011;144:440-5

35. Blackwell DL, Collins JG, Coles R. Summary health statistics for U.S. adults: National Health Interview Survey, 1997. Vital Health Stat. 10 2002:1-109.

36. Goetzel RZ, Hawkins K, Ozminkowski RJ, Wang S. The health and productivity cost burden of the "top 10 " physical and mental health conditions affecting six large U.S. employers in 1999. J Occup Environ Med. 2003;45:5-14.

37. Rudmik L. Economics of Chronic Rhinosinusitis. Current Allergy and Asthma Reports 2017;17:20.

38. Hoffmans R, Wagemakers A, van Drunen C, Hellings $P$, Fokkens W. Acute and chronic rhinosinusitis and allergic rhinitis in relation to comorbidity, ethnicity and environment. PloS one 2018;13:e0192330.

39. Revai K, Dobbs LA, Nair S, Patel JA, Grady JJ, Chonmaitree T. Incidence of acute otitis media and sinusitis complicating upper respiratory tract infection: the effect of age. Pediatrics 2007;119:e1408-12.

40. Benninger MS, Ferguson BJ, Hadley JA, et al. Adult chronic rhinosinusitis: Definitions, diagnosis, epidemiology, and pathophysiology. Otolaryngol - Head \& Neck Surg. 2003;129:S1-32.

41. Shapiro DJ, Gonzales R, Cabana MD, Hersh AL. National trends in visit rates and antibiotic prescribing for children with acute sinusitis. Pediatrics. 2011;127:28-34.

42. Rosenfeld RM, Piccirillo JF, Chandrasekhar SS, et al. Clinical practice guideline (update): Adult sinusitis. Otolaryngology - Head and Neck Surgery (United States) 2015;152:S1-S39.
43. Leung R, Almassian S, Kern R, Conley D, Tan $B$, Chandra R. Patient level decision making in recurrent acute rhinosinusitis: a costbenefit threshold for surgery. Laryngoscope. 2013;123:11-6.

44. Alkire BC, Bhattacharyya N. An assessment of sinonasal anatomic variants potentially associated with recurrent acute rhinosinusitis. Laryngoscope. 2010;120:631-4.

45. Jain R, Stow N, Douglas R. Comparison of anatomical abnormalities in patients with limited and diffuse chronic rhinosinusitis. Int Forum Allergy Rhinol. 2013;3:493-6.

46. Loftus PA, Lin J, Tabaee A. Anatomic variants of the paranasal sinuses in patients with recurrent acute rhinosinusitis. Int Forum of Allergy \& Rhinol. 2016;6:328-33.

47. Costa ML, Psaltis AJ, Nayak JV, Hwang PH. Medical therapy vs surgery for recurrent acute rhinosinusitis. Int Forum Allergy Rhinol. 2015;5:667-73.

48. De Sario M, Katsouyanni K, Michelozzi P. Climate change, extreme weather events, air pollution and respiratory health in Europe. Eur Resp Journal. 2013, 826-43.

49. Kuiper JR, Hirsch AG, Bandeen-Roche K, et al. Prevalence, severity, and risk factors for acute exacerbations of nasal and sinus symptoms by chronic rhinosinusitis status. Allergy. 2018;73:1244-53.

50. Eyigor $\mathrm{H}$, Basak $\mathrm{S}$. [Evaluation of predisposing factors and bacteriologic agents in pediatric rhinosinusitis]. Kulak Burun Bogaz Ihtis Derg 2005; 15:49-55.

51. Pant $H$, Ferguson BJ, Macardle PJ. The role of allergy in rhinosinusitis. Curr Opin Otolaryngol Head Neck Surg. 2009;17:232-8.

52. Flook EP, Kumar BN. Is there evidence to link acid reflux with chronic sinusitis or any nasal symptoms? A review of the evidence. Rhinology. 2011;49:11-6.

53. Seresirikachorn K, Snidvongs K, Chitsuthipakorn W, et al. EPOS2012 has better specificity compared to IDSA2012 for diagnosing acute bacterial rhinosinusitis. Rhinology. 2018;56:241-4.

54. Pilan RR, Pinna FR, Bezerra TF, et al. Prevalence of chronic rhinosinusitis in Sao Paulo. Rhinology. 2012;50:129-38

55. Shi JB, Fu QL, Zhang H, et al. Epidemiology of chronic rhinosinusitis: results from a crosssectional survey in seven Chinese cities. Allergy. 2015;70:533-9.

56. Dietz de Loos D, Lourijsen ES, Wildeman MAM, et al. Prevalence of chronic rhinosinusitis in the general population based on sinus radiology and symptomatology. J Allergy Clin Immunol. 2019;143:1207-14.

57. Tomassen P, Newson RB, Hoffmans R, et al. Reliability of EP3OS symptom criteria and nasal endoscopy in the assessment of chronic rhinosinusitis--a GA(2) LEN study. Allergy. 2011;66:556-61.

58. Hirsch AG, Nordberg C, Bandeen-Roche K, et al. Radiologic sinus inflammation and symptoms of chronic rhinosinusitis in a population-based sample. Allergy. 2019, 10.1111/all.14106.
59. Hamizan AW, Loftus PA, Alvarado R, et al. Allergic phenotype of chronic rhinosinusitis based on radiologic pattern of disease. Laryngoscope. 2018;128:2015-21.

60. Philpott CM, Erskine S, Hopkins C, et al. Prevalence of asthma, aspirin sensitivity and allergy in chronic rhinosinusitis: data from the UK National Chronic Rhinosinusitis Epidemiology Study. Respiratory research 2018;19:129.

61. De Schryver E, Derycke L, Campo P, et al. Alcohol hyper-responsiveness in chronic rhinosinusitis with nasal polyps. Clin Exp Allergy 2016, 10.1111/ cea. 12836 .

62. Endam LM, Filali-Mouhim A, Boisvert P, Boulet L-P, Bosse Y, Desrosiers M. Genetic variations in taste receptors are associated with chronic rhinosinusitis: a replication study. Int forum All \& Rhinology. 2014;4:200-6.

63. Purnell PR, Addicks BL, Zalzal HG, et al. Single Nucleotide Polymorphisms in Chemosensory Pathway Genes GNB3, TAS2R19, and TAS2R38 Are Associated with Chronic Rhinosinusitis. Int Arch Allergy Immunol. 2019;180:72-8.

64. Stevens WW, Peters AT, Tan BK, et al. Associations Between Inflammatory Endotypes and Clinical Presentations in Chronic Rhinosinusitis. The Jof Allergy and Clin Immunol In Practice. 2019, 10.1016/j.jaip.2019.05.009.

65. Brook CD, Kuperstock JE, Rubin SJ, Ryan MW, Platt MP. The association of allergic sensitization with radiographic sinus opacification. Am J Rhinol Allergy 2017;31:12-5.

66. Hummel T, Whitcroft KL, Andrews P, et al. Position paper on olfactory dysfunction. Rhinology Supplement 2017;54:1-30.

67. Stogbauer J, Wirkner K, Engel C, et al. Prevalence and risk factors of smell dysfunction - a comparison between five German populationbased studies. Rhinology. 2019, 10.4193/ Rhin19.181.

68. Landis BN, Hummel T. New evidence for high occurrence of olfactory dysfunctions within the population. Am J Med. 2006;119:91-2.

69. Lane AP, Turner J, May L, Reed R. A genetic model of chronic rhinosinusitis-associated olfactory inflammation reveals reversible functional impairment and dramatic neuroepithelial reorganization. J Neurosci. 2010;30:2324-9.

70. Pfaar O, Landis BN, Frasnelli J, Huttenbrink $\mathrm{KB}$, Hummel T. Mechanical obstruction of the olfactory cleft reveals differences between orthonasal and retronasal olfactory functions. Chem Senses. 2006;31:27-31.

71. Jones NS. Sinogenic facial pain: Diagnosis and management. Otolaryngol Clin North Am. 2005;38:1311-25.

72. Kirsch CFE, Bykowski J, Aulino JM, et al. ACR Appropriateness Criteria((R)) Sinonasal Disease. J Am Coll Radiol 2017;14:S550-9.

73. Younis RT, Anand VK, Davidson B. The role of computed tomography and magnetic resonance imaging in patients with sinusitis with complications. Laryngoscope 2002;112:224-9.

74. Bhattacharyya N. A comparison of symptom scores and radiographic staging systems in chronic rhinosinusitis. American J of Rhinology 
2005;19:175-9.

75. Lund VJ, Kennedy DW. Staging for rhinosinusitis. Otolaryng - Head \& Neck Surg. 1997;117:S35-40.

76. Bayonne E, Kania R, Tran P, Huy B, Herman P. Intracranial complications of rhinosinusitis. A review, typical imaging data and algorithm of management*. Rhinology. 2009;47:59-65.

77. Lund VJ, Mackay IS. Staging in rhinosinusitus. Rhinology. 1993;31:183-4.

78. Orlandi RR, Kingdom TT, Hwang PH, et al. International Consensus Statement on Allergy and Rhinology: Rhinosinusitis. Int Forum Allergy Rhinol. 2016;6 Suppl 1:S22-S209.

79. Amine M, Lininger L, Fargo KN, Welch KC. Outcomes of endoscopy and computed tomography in patients with chronic rhinosinusitis. Int Forum Allergy Rhinol. 2013;3:73-9.

80. Daramola OO, Lidder AK, Ramli R, et al. Patient knowledge and perception of computed tomography scan in the management of chronic rhinosinusitis symptoms. Laryngoscope. 2015;125:791-5.

81. Leung RM, Chandra RK, Kern RC, Conley DB, Tan BK. Primary care and upfront computed tomography scanning in the diagnosis of chronic rhinosinusitis: A cost-based decision analysis. Laryngoscope. 2014;124:12-8.

82. Bulla S, Blanke P, Hassepass F, et al. Reducing the radiation dose for low-dose $\mathrm{CT}$ of the paranasal sinuses using iterative reconstruction: Feasibility and image quality. Eur J of Radiology. 2012;81:2246-50.

83. Sodickson A. CT radiation risks coming into clearer focus. BMJ. 2013;346:f3102-f.

84. Fraczek M, Guzinski M, Morawska-Kochman M, Krecicki T. Investigation of sinonasal anatomy via low-dose multidetector $\mathrm{CT}$ examination in chronic rhinosinusitis patients with higher risk for perioperative complications. Eur Arch of OtoRhino-Laryngol. 2017;274:787-93.

85. Gevaert P, Van Bruaene N, Cattaert T, et al. Mepolizumab, a humanized anti-IL-5 mAb, as a treatment option for severe nasal polyposis. J of Allergy and Clin. Imm. 2011;128:988-9.

86. Pinto JM, Mehta N, DiTineo M, Wang J, Baroody FM, Naclerio RM. A randomized, double-blind, placebo-controlled trial of anti-lgE for chronic rhinosinusitis. Rhinology. 2010;48:318-24.

87. Hopkins C, Hettige R, Soni-Jaiswal A, et al. CHronic Rhinosinusitis Outcome MEasures (CHROME), developing a core outcome set for trials of interventions in chronic rhinosinusitis. Rhinology. 2018;56:22-32.

88. Kim DH, Seo Y, Kim KM, Lee S, Hwang SH. Usefulness of Nasal Endoscopy for Diagnosing Patients With Chronic Rhinosinusitis: A MetaAnalysis. Am J Rhinol Allergy. 2019, 10.1177/1945 892419892157:1945892419892157.

89. Flores Kim J, McCleary N, Nwaru BI, Stoddart A, Sheikh A. Diagnostic accuracy, risk assessment, and cost-effectiveness of component-resolved diagnostics for food allergy: A systematic review. Allergy. 2018;73:1609-21.

90. Eiringhaus K, Renz H, Matricardi P, Skevaki C.
Component-Resolved Diagnosis in Allergic Rhinitis and Asthma. J Appl Lab Med 2019;3:88398.

91. Lotsch J, Hummel T. A data science-based analysis of seasonal patterns in outpatient presentations due to olfactory dysfunction. Rhinology. 2019, 10.4193/Rhin 19.099.

92. Rimmer J, Hellings $\mathrm{P}$, Lund VJ, et al. European position paper on diagnostic tools in rhinology. Rhinology. 2019;57:1-41.

93. Kern RC. Chronic sinusitis and anosmia: pathologic changes in the olfactory mucosa. Laryngoscope. 2000;110:1071-7.

94. Apter AJ, Gent JF, Frank ME. Fluctuating olfactory sensitivity and distorted odor perception in allergic rhinitis. Arch Otolaryngol Head Neck Surg. 1999;125:1005-10.

95. Hsieh JW, Keller A, Wong M, Jiang R-S, Vosshall LB. SMELL-S and SMELL-R: Olfactory tests not influenced by odor-specific insensitivity or prior olfactory experience. Proc of the Nat Academy of Sciences. 2017;114:11275-84.

96. Leite SHP, Jain R, Douglas RG. The clinical implications of computerised fluid dynamic modelling in rhinology. Rhinology. 2019;57:2-9.

97. Wong E, Inthavong K, Singh N. Comment on the European position paper on diagnostic tools in rhinology - computational fluid dynamics. Rhinology. 2019, 10.4193/Rhin19.269.

98. Rimmer J. Reply to the letter by Wong et al. Rhinology. 2019, 10.4193/Rhin19.296.1.

99. Snidvongs K, Lam M, Sacks R, et al. Structured histopathology profiling of chronic rhinosinusitis in routine practice. Int Forum All \& Rhinology. 2012;2:376-85.

100. Jiang N, Kern RC, Altman KW. Histopathological evaluation of chronic rhinosinusitis: a critical review. Am J of Rhinol \& Allergy. 2013;27:396402.

101. Fokkens WJ, Lund V, Bachert C, et al. EUFOREA consensus on biologics for CRSWNP with or without asthma. Allergy. 2019;74:2312-9.

102. Hoggard M, Zoing M, Biswas K, Taylor MW, Douglas RG. The sinonasal mycobiota in chronic rhinosinusitis and control patients. Rhinology. 2019;57:190-9.

103. Bachert C, Han JK, Desrosiers M, et al. Efficacy and safety of dupilumab in patients with severe chronic rhinosinusitis with nasal polyps (LIBERTY NP SINUS-24 and LIBERTY NP SINUS-52): results from two multicentre, randomised, double-blind, placebo-controlled, parallel-group phase 3 trials. Lancet. 2019;394:1638-50.

104. Bachert C, Sousa AR, Lund VJ, et al. Reduced need for surgery in severe nasal polyposis with mepolizumab: Randomized trial. J Allergy Clin Immunol. 2017;140:1024-31.e14.

105. Rudmik L, Soler ZM, Hopkins C, et al. Defining appropriateness criteria for endoscopic sinus surgery during management of uncomplicated adult chronic rhinosinusitis: a RAND/UCLA appropriateness study. Int Forum Allergy Rhinol. 2016;6:557-67.

106. Beswick DM, Mace JC, Rudmik L, Soler ZM, DeConde AS, Smith TL. Productivity changes following medical and surgical treatment of chronic rhinosinusitis by symptom domain. Int Forum Allergy Rhinol. 2018;8:1395-405.

107. Kowalski ML, Agache I, Bavbek S, et al. Diagnosis and management of NSAID-Exacerbated Respiratory Disease (N-ERD)-a EAACI position paper. Allergy. 2018, 10.1111/all.13599.

108. Oakley GM, Christensen JM, Sacks R, Earls P, Harvey RJ. Characteristics of macrolide responders in persistent post-surgical rhinosinusitis. Rhinology. 2018;56:111-7.

109. Sidell D, Shapiro NL, Bhattacharyya N. Obesity and the risk of chronic rhinosinusitis, allergic rhinitis, and acute otitis media in school-age children. Laryngoscope. 2013;123:2360-3.

110. Reh DD, Higgins TS, Smith TL. Impact of tobacco smoke on chronic rhinosinusitis: A review of the literature. Int. Forum of Allergy and Rhinol. 2012, 362-9.

111. Georgalas C, Vlastos I, Picavet V, van Drunen C, Garas G, Prokopakis E. Is chronic rhinosinusitis related to allergic rhinitis in adults and children? Applying epidemiological guidelines for causation. Allergy. 2014;69:828-33.

112. Neff $L$, Adil EA. What is the role of the adenoid in pediatric chronic rhinosinusitis? Laryngoscope. 2015;125:1282-3.

113. Belcher R, Virgin F. The Role of the Adenoids in Pediatric Chronic Rhinosinusitis. Med Sci. (Basel) 2019;7.

114. Brietzke SE, Shin JJ, Choi S, et al. Clinical consensus statement: pediatric chronic rhinosinusitis. Otolaryngol - Head \& Neck Surgery. 2014;151:542-53.

115. Orb Q, Curtin K, Oakley GM, et al. Familial risk of pediatric chronic rhinosinusitis. Laryngoscope. 2016;126:739-45.

116. Skoner DP, Anfuso A, Ramadan $\mathrm{H}$, et al. Sinus and adenoid inflammation in children with chronic rhinosinusitis and asthma. Am J of Resp and Crit Care Med. 2015;191.

117. Schenkel EJ, Skoner DP, Bronsky EA, et al. Absence of growth retardation in children with perennial allergic rhinitis after one year of treatment with mometasone furoate aqueous nasal spray. Pediatrics. 2000;105:E22.

118. Yoo KH, Ahn HR, Park JK, et al. Burden of Respiratory Disease in Korea: An Observational Study on Allergic Rhinitis, Asthma, COPD, and Rhinosinusitis. Allergy Asthma Immunol Res. 2016;8:527-34.

119. Orlandi RR, Marple BF. The role of fungus in chronic rhinosinusitis. Otolaryngol Clin North Am 2010;43:531-7, viii.

120. Fokkens WJ, Lund VJ, Mullol J, et al. EPOS 2012: European position paper on rhinosinusitis and nasal polyps 2012. A summary for otorhinolaryngologists. Rhinology. 2012;50:1-12.

121. Oshima H, Nomura K, Sugawara M, Arakawa K, Oshima T, Katori Y. Septal deviation is associated with maxillary sinus fungus ball in male patients. The Tohoku J of exp medicine. 2014;232:201-6.

122. Yoon YH, Xu J, Park SK, Heo JH, Kim YM, Rha KS. A retrospective analysis of 538 sinonasal fungus ball cases treated at a single tertiary medical 
center in Korea (1996-2015). Int Forum Allergy Rhinol. 2017;7:1070-5.

123. Park GY, Kim HY, Min JY, Dhong HJ, Chung SK. Endodontic treatment: a significant risk factor for the development of maxillary fungal ball. Clin Exp Otorhinolaryngol. 2010;3:136-40.

124. Jun YJ, Shin JM, Lee JY, Baek BJ. Bony Changes in a Unilateral Maxillary Sinus Fungal Ball. J Craniofac Surg. 2018;29:e44-e7.

125. Knisely A, Holmes T, Barham H, Sacks R, Harvey R. Isolated sphenoid sinus opacification: A systematic review. Am J Otolaryngol. 2017;38:237-43.

126. Nomura K, Asaka D, Nakayama T, et al. Sinus fungus ball in the Japanese population: clinical and imaging characteristics of 104 cases. Int J Otolaryngol. 2013;2013:731640.

127. Turner JH, Soudry E, Nayak JV, Hwang PH. Survival outcomes in acute invasive fungal sinusitis: a systematic review and quantitative synthesis of published evidence. Laryngoscope. 2013;123:1112-8.

128. Trief D, Gray ST, Jakobiec FA, et al. Invasive fungal disease of the sinus and orbit: a comparison between mucormycosis and Aspergillus. Br J Ophthalmol 2016;100:184-8.

129. deShazo RD, O'Brien M, Chapin K, Soto-Aguilar M, Gardner L, Swain R. A new classification and diagnostic criteria for invasive fungal sinusitis. Arch. of Otolaryngol. -- Head \& Neck Surgery. 1997;123:1181-8.

130. Wandell GM, Miller C, Rathor A, et al. A multiinstitutional review of outcomes in biopsyproven acute invasive fungal sinusitis. Int Forum Allergy Rhinol. 2018;8:1459-68.

131. Payne SJ, Mitzner R, Kunchala S, Roland L, McGinn JD. Acute Invasive Fungal Rhinosinusitis: A 15-Year Experience with 41 Patients. Otolaryngol Head Neck Surg. 2016;154:759-64.

132. Groppo ER, El-Sayed IH, Aiken AH, Glastonbury $\mathrm{CM}$. Computed tomography and magnetic resonance imaging characteristics of acute invasive fungal sinusitis. Arch Otolaryngol Head Neck Surg. 2011;137:1005-10.

133. Arvanitis M, Anagnostou T, Mylonakis E. Galactomannan and Polymerase Chain ReactionBased Screening for Invasive Aspergillosis
Among High-Risk Hematology Patients: A Diagnostic Meta-analysis. Clin Infect Dis. 2015;61:1263-72.

134. Bakhshaee M, Fereidouni M, Nourollahian M, Movahed R. The presence of fungal-specific $\lg E$ in serum and sinonasal tissue among patients with sinonasal polyposis. Eur. Archives of Oto-rhinolaryng. 2014;271:2871-5.

135. Bent 3rd JP, Kuhn FA, Bent JP, Kuhn FA. Diagnosis of allergic fungal sinusitis. Otolaryngol Head Neck Surg. 1994;111:580-8.

136. deShazo RD, Swain RE. Diagnostic criteria for allergic fungal sinusitis. J of Allergy \& Clin. Immunology. 1995;96:24-35.

137. Aribandi M, McCoy VA, Bazan C, 3rd. Imaging features of invasive and noninvasive fungal sinusitis: a review. Radiographics. 2007;27:128396.

138. Landsberg R, Segev Y, DeRowe A, Landau T, Khafif A, Fliss DM. Systemic corticosteroids for allergic fungal rhinosinusitis and chronic rhinosinusitis with nasal polyposis: a comparative study. Otolaryngol Head Neck Surg. 2007;136:252-7.

139. Dai Q, Duan C, Liu Q, Yu H. Effect of nebulized budesonide on decreasing the recurrence of allergic fungal rhinosinusitis. Am J of otolaryngol. 2017;38:321-4.

140. Gottschlich S, Ambrosch P, Kramkowski D, et al. Head and neck manifestations of Wegener's granulomatosis. Rhinology. 2006;44:227-33.

141. Srouji IA, Andrews P, Edwards C, Lund VJ. Patterns of presentation and diagnosis of patients with Wegener's granulomatosis: ENT aspects. The J of Laryngol and otology. 2007;121:653-8.

142. Bossuyt $X$, Cohen Tervaert J-W, Arimura Y, et al. Position paper: Revised 2017 international consensus on testing of ANCAs in granulomatosis with polyangiitis and microscopic polyangiitis. Nature reviews Rheumatol. 2017;13:683-92.

143. Trimarchi M, Bertazzoni G, Bussi M. Cocaine induced midline destructive lesions. Rhinology. 2014;52:104-11.

144. Greco A, Marinelli C, Fusconi M, et al. Clinic manifestations in granulomatosis with polyangiitis. Int. J of immunopath pharmacol. 2016;29:151-9.
145. Hellings PW. Joint action with European CRSwNP Patients for better outcomes. Rhinology. 2019;57:321.

146. Pugin B, Deneyer L, Bachert C, et al. Patient Advisory Board for Chronic Rhinosinusitis - A EUFOREA initiative. Rhinology. 2019;57:331-5.

147. Seys SF, Bousquet J, Bachert C, et al. mySinusitisCoach: patient empowerment in chronic rhinosinusitis using mobile technology. Rhinology. 2018;56:209-15.

148. Khanwalkar AR, Shen J, Kern RC, et al. Utilization of a novel interactive mobile health platform to evaluate functional outcomes and pain following septoplasty and functional endoscopic sinus surgery. Int Forum Allergy Rhinol. 2019;9:345-51.

149. Hellings PW, Fokkens WJ, Bachert C, et al. Positioning the principles of precision medicine in care pathways for allergic rhinitis and chronic rhinosinusitis - A EUFOREA-ARIA-EPOS-AIRWAYS ICP statement. Allergy. 2017;72:1297-305.

150. Hopkins C, Surda P, Bast F, Hettige R, Walker A, Hellings PW. Prevention of chronic rhinosinusitis. Rhinology. 2018;56:307-15.

151. Hopkins C, Rimmer J, Lund VJ. Does time to endoscopic sinus surgery impact outcomes in Chronic Rhinosinusitis? Prospective findings from the National Comparative Audit of Surgery for Nasal Polyposis and Chronic Rhinosinusitis. Rhinology. 2015;53:10-7.

152. Hopkins C, Rudmik L, Lund VJ. The predictive value of the preoperative Sinonasal Outcome Test-22 score in patients undergoing endoscopic sinus surgery for chronic rhinosinusitis. Laryngoscope. 2015;125:1779-84.

153. Rudmik L, Soler ZM, Hopkins C. Using postoperative SNOT-22 to help predict the probability of revision sinus surgery. Rhinology. 2016;54:111-6.

154. Grayson JW, Hopkins C, Mori E, Senior B, Harvey RJ. Contemporary Classification of Chronic Rhinosinusitis Beyond Polyps vs No Polyps: A Review. JAMA Otolaryngol Head Neck Surg. 2020; doi: 10.1001/jamaoto.2020.1453.

155. Fokkens W, Desrosiers M, Harvey R, et al. EPOS2020: development strategy and goals for the latest European Position Paper on Rhinosinusitis. Rhinology 2019;57:162-8. 


\section{Classification, definitions and terminology}

\subsection{Defintions}

\subsubsection{Sinusitis vs. rhinosinusitis}

Rhinosinusitis recognises that rhinitis and sinusitis co-exist and that physiologically and pathophysiologically it is difficult to make a distinction between the nose and sinuses although one area may be more evidently affected than another. This term was first used in the early 1990s and has been widely adopted internationally ${ }^{(1-9)}$.

In primary care, GPs may distinguish between rhinosinusitis and rhinitis, in secondary care ENT surgeons may distinguish between phenotypes of rhinosinusitis and in tertiary care, rhinologists may distinguish between rhinosinusitis endotypes. In primary care, patients with symptoms of nasal obstruction, discharge, pressure, pain, lack of barotrauma and often retained sense of smell are likely to have rhinitis only ${ }^{(10)}$. Additionally, those who are younger ( $<35$ years) with seasonality, distinct allergen exacerbations and involvement of other sites (conjunctiva, lung, skin) are likely to have rhinitis ${ }^{(11-13)}$.

\subsubsection{Clinical definition}

\subsubsection{Adults}

Rhinosinusitis = inflammation of the nose and paranasal sinuses characterised by two or more symptoms*, one of which should be either nasal blockage/obstruction/congestion or nasal discharge (anterior/posterior nasal drip):

- \pm facial pain/pressure

- $\quad \pm$ reduction or loss of sense of smell

and either

- endoscopic signs of:

- nasal polyps and/or

- mucopurulent discharge primarily from middle meatus and/or

- oedema/mucosal obstruction primarily in middle meatus

and/or

- CT changes:

- mucosal changes within the ostiomeatal complex and/or sinuses

[Minimal thickening, involving only 1 or 2 walls and not the ostial area is unlikely to represent rhinosinusitis ${ }^{(14,15)}$ ]
[*It is recognised that symptoms have high sensitivity, but low specificity hence the need for objective findings.]

\subsubsection{Children}

Rhinosinusitis = inflammation of the nose and paranasal sinuses characterised by two or more symptoms, one of which should be either nasal blockage/obstruction/congestion or nasal discharge (anterior/posterior nasal drip):

- \pm facial pain

- $\pm \operatorname{cough}^{(16)}$

and either

- endoscopic signs of:

- nasal polyps and/or

- mucopurulent discharge primarily from middle meatus and/or

- oedema/mucosal obstruction primarily in middle meatus

and/or

- CT changes:

- mucosal changes within the ostiomeatal complex and/or sinuses

\subsubsection{Definition for epidemiology studies and General Practice}

For epidemiological studies and general practice, the definition is based on symptomatology usually without ENT examination or radiology.

\subsubsection{Acute rhinosinusitis (ARS) in adults}

Acute rhinosinusitis in adults is defined as: sudden onset of two or more symptoms, one of which should be either nasal blockage/obstruction/congestion or nasal discharge (anterior/posterior nasal drip):

- \pm facial pain/pressure,

- $\quad \pm$ reduction or loss of smell

for $<12$ weeks;

with symptom free intervals if the problem is recurrent, with validation by telephone or interview.

\subsubsection{Acute rhinosinusitis in children}

Acute rhinosinusitis in children is defined as: 
sudden onset of two or more of the symptoms:

- nasal blockage/obstruction/congestion

- or discoloured nasal discharge

- or cough (daytime and night-time)

for < 12 weeks;

with symptom free intervals if the problem is recurrent;

with validation by telephone or interview.

Questions on allergic symptoms (i.e. sneezing, watery rhinorrhoea, nasal itching, and itchy watery eyes) should be included.

\subsubsection{Recurrent acute rhinosinusitis (RARS)}

ARS can occur once or more than once in a defined time period. This is usually expressed as episodes/year but with complete resolution of symptoms between episodes.

Recurrent ARS (RARS) is defined as $\geq 4$ episodes per year with symptom free intervals ${ }^{(3,17)}$.

\subsubsection{Definition of chronic rhinosinusitis in adults}

Chronic rhinosinusitis (with or without nasal polyps) in adults is defined as:

presence of two or more symptoms, one of which should be either nasal blockage / obstruction / congestion or nasal discharge (anterior / posterior nasal drip):

- $\quad \pm$ facial pain/pressure;

- $\quad \pm$ reduction or loss of smell;

for $\geq 12$ weeks;

with validation by telephone or interview.

Questions on allergic symptoms (i.e. sneezing, watery rhinorrhoea, nasal itching, and itchy watery eyes) should be included.

\subsubsection{Definition of chronic rhinosinusitis in children}

Chronic rhinosinusitis (with or without nasal polyps) in children is defined as:

presence of two or more symptoms one of which should be either nasal blockage / obstruction / congestion or nasal discharge (anterior/posterior nasal drip):

- \pm facial pain/pressure;

- \pm cough;

for $\geq 12$ weeks;

with validation by telephone or interview.

\subsubsection{Definition for research}

For research purposes acute rhinosinusitis is defined as per the clinical definition. Bacteriology (antral tap, middle meatal culture) and/or radiology (CT) are advised, but not obligatory. For research purposes chronic rhinosinusitis is defined as per the clinical definition and should be based on phenotypes and endotypes, with and without previous surgery. It may include sub-analysis for other co-morbidities.

\subsubsection{Definition of chronic rhinosinusitis when no earlier sinus surgery has been performed \\ Chronic rhinosinusitis with nasal polyps: bilateral, endoscopi- cally visualised in middle meatus. \\ Chronic rhinosinusitis without nasal polyps: no visible polyps in middle meatus, if necessary following decongestant. \\ This definition accepts that there is a spectrum of disease in CRS which includes polypoid change in the sinuses and/or middle meatus but excludes those with polypoid disease presenting in the nasal cavity to avoid overlap.}

\subsubsection{Definition of chronic rhinosinusitis when sinus sur- gery has been performed}

Once surgery has altered the anatomy of the lateral wall, the presence of polyps is defined as bilateral pedunculated lesions as opposed to cobblestoned mucosa $>6$ months after surgery on endoscopic examination. Any mucosal disease without overt polyps should be regarded as chronic rhinosinusitis without nasal polyps.

\subsubsection{Co-morbidities for sub-analysis in research}

The following conditions should be considered for sub-analysis:

1. NSAID-exacerbated respiratory disease (N-ERD). Aspirin sensitivity based on positive oral, bronchial, or nasal provocation or an obvious history;

2. Asthma / bronchial hyper-reactivity / chronic obstructive pulmonary disease (COPD) / bronchiectasies based on symptoms, respiratory function tests;

3. Allergy based on specific serum specific immunoglobulin $E$ (IgE) or Skin Prick Test (SPT);

4. Total lgE in serum (treatment effects may be influenced by IgE level);

5. Eosinophil levels in blood and tissue.

\subsubsection{Exclusion from general studies}

Patients with the following diseases should be excluded from general studies, but may be the subject of a specific study depending on phenotype:

1. Cystic fibrosis based on positive sweat test or DNA alleles;

2. Gross immunodeficiency (congenital or acquired);

3. Congenital mucociliary problems (e.g. primary ciliary dyskinesia (PCD));

4. Non-invasive fungal balls and invasive fungal disease;

5. Systemic vasculitis and granulomatous diseases;

6. Cocaine abuse;

7. Neoplasia.

\subsection{Classification of CRS}

The EPOS2020 steering group have chosen to look at CRS in terms of primary and secondary (Figures 2.2.1. and 2.2.2.) and to 
Figure 2.2.1. Classification of primary CRS (Adapted from Grayson et $\left.\mathrm{al}^{(154)}\right)$

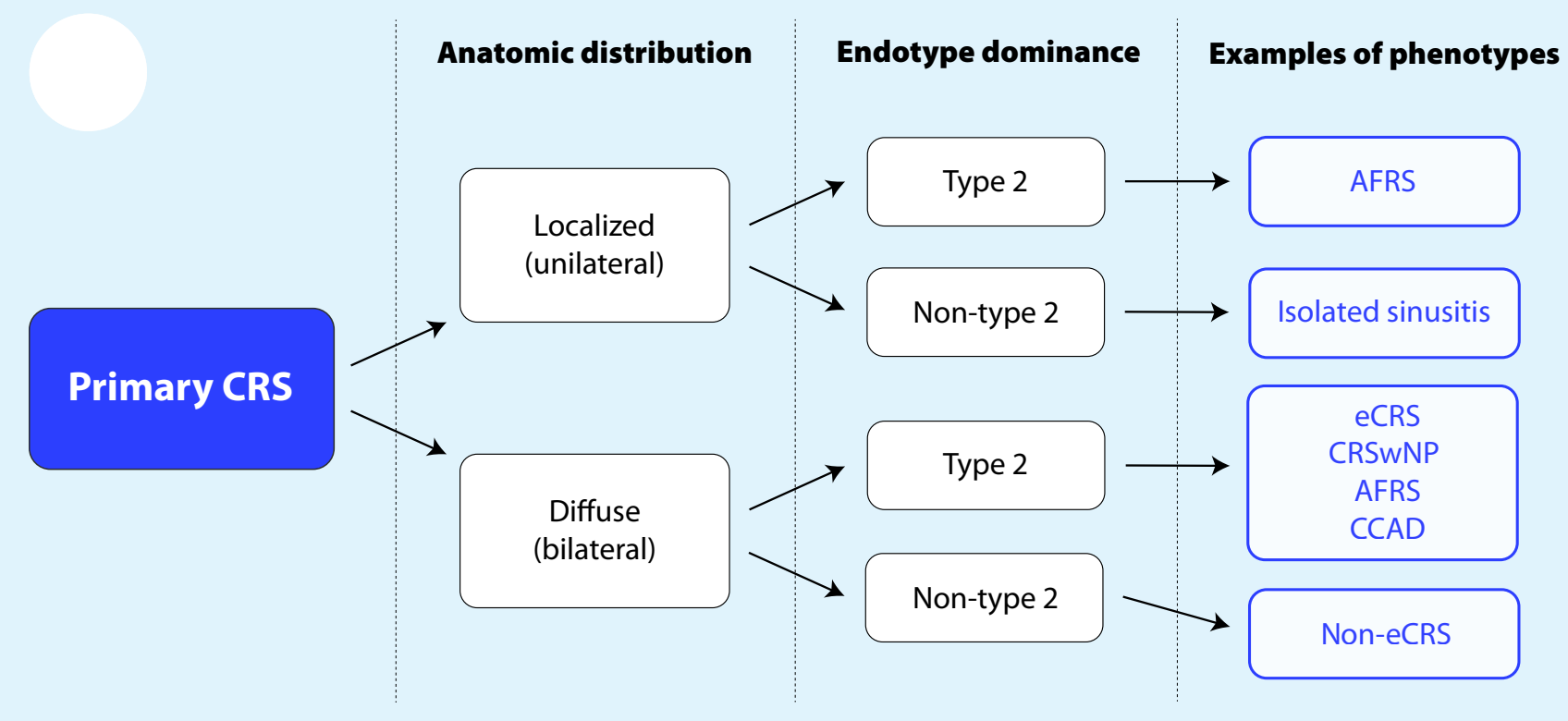

AFRS, allergic fungal rhinosinusitis; CCAD, central compartment allergic disease; CRS, chronic rhinosinusitis; CRSwNP, chronic rhinosinusitis with nasal polyps; eCRS, eosinophilic CRS; OMC, ostiomeatal complex.

Figure 2.2.2. Classification of secondary CRS (Adapted from Grayson et al $\left.{ }^{(154)}\right)$.

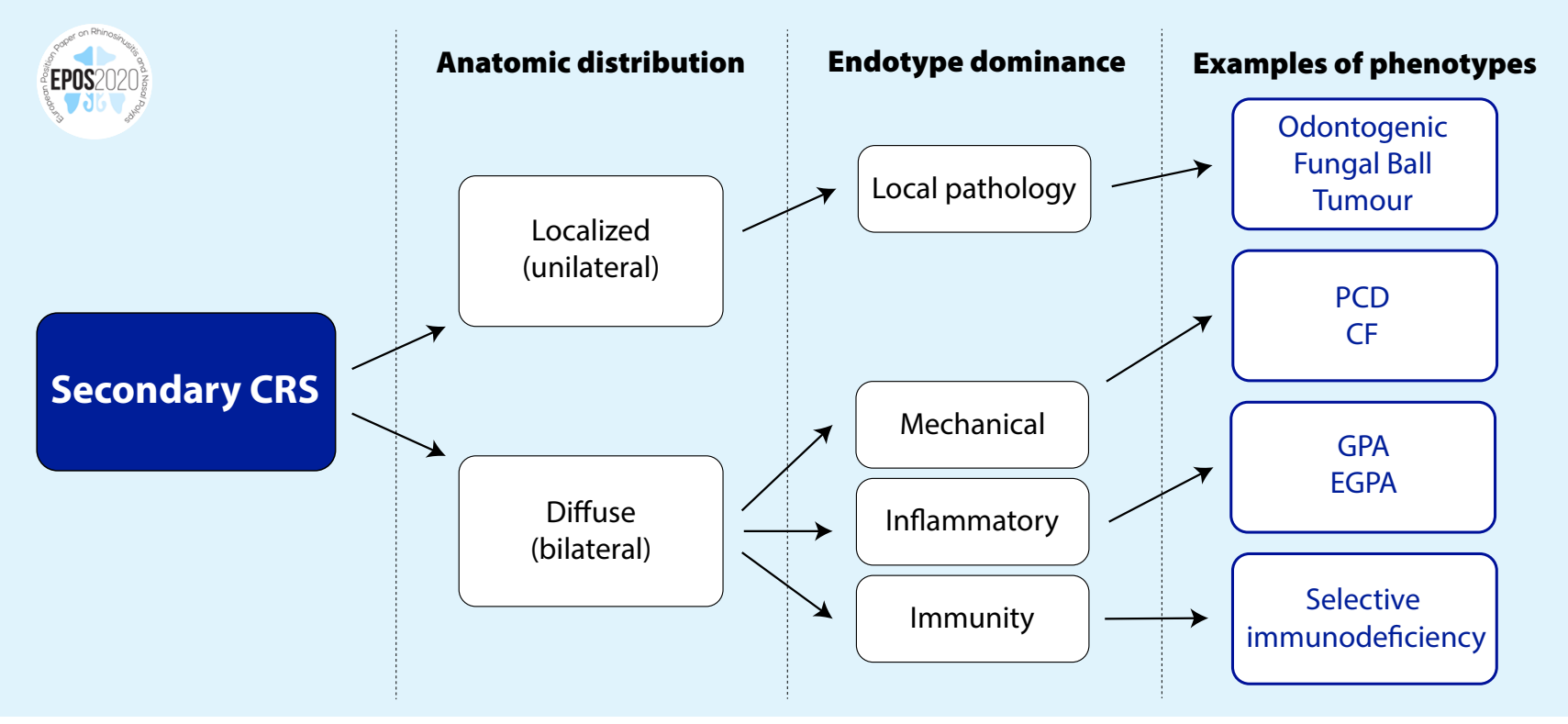

CRS, chronic rhinosinusitis; PCD, primary ciliary dyskinesia.; CF, cystic fibrosis; GPA, granulomatosis with polyangiitis (Wegener's disease); EGPA, eosinophilic granulomatosis with polyangiitis (Churg-Strauss disease).

divide each into localized and diffuse disease based on anatomic distribution. In primary CRS, the disease is considered by endotype dominance, either type 2 or non-type 2 (see 1.5.2.2.). Clinically localized primary CRS is then subdivided into two phenotypes - allergic fungal rhinosinusitis (AFRS) or an isolated sinusitis. For diffuse CRS, the clinical phenotypes are predo- minantly eCRS and non-eCRS, determined by the histologic quantification of the numbers of eosinophilic, i.e. number/high powered field which the EPOS panel agreed to be 10/hpf or higher.

For secondary CRS, again, the division is into localized or diffuse and then considered by four categories dependant on local pa- 
thology, mechanical, inflammatory and immunological factors. Thence a range of clinical phenotypes are included as shown.

\subsection{Duration (adults and children)}

2.3.1. Acute $<12$ weeks with sudden onset and complete resolution of symptoms (<4 weeks in ICOR) ${ }^{(4,7-9)}$ (Figure 2.3.1.)

\section{EPOS recognises acute viral, acute post-viral and acute bacterial rhinosinusitis.}

\subsubsection{Common cold}

Acute viral rhinosinusitis: duration of symptoms $<10$ days

\subsubsection{Acute post-viral}

Increase in symptoms $>5$ days or persistent symptoms $>10$ days with $<12$ weeks duration

\subsubsection{Acute bacterial}

Defined by at least three symptoms / signs:

- discoloured mucus;

- $\quad$ severe local pain;

- fever $>38^{\circ} \mathrm{C}$.

- raised $\mathrm{CRP} / \mathrm{ESR}$;

- 'double' sickening.

It was noted that in many cases of acute bacterial rhinosinusitis, the disease is unilateral ${ }^{(18)}$.

(See chapter 4 for extensive discussion.)

\subsubsection{Prolonged acute rhinosinusitis}

We recognise that in general, acute rhinosinusitis will usually last a maximum of a few weeks. In the literature a number of different classifications have been proposed. In the past the term 'subacute' was sometimes used to fill the gap between acute and chronic rhinosinusitis. However, the EPOS group felt that a

Figure 2.3.1. Definition of acute rhinosinusitis.

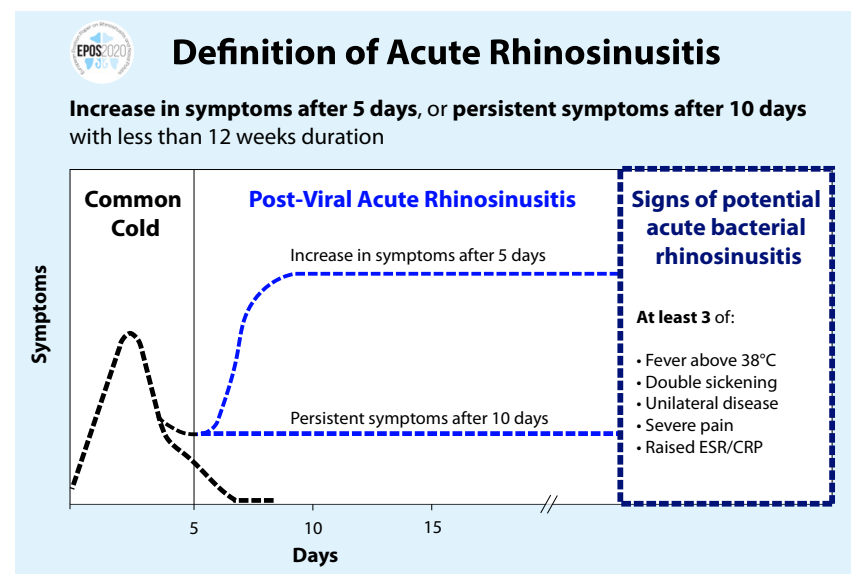

separate term to describe patients with prolonged acute rhinosinusitis was not necessary because the number of patients who have such a prolonged course is small and there are very little data on which to offer evidence based recommendations on how to manage these patients.

\subsection{Severity of disease}

\subsubsection{Severity \\ - $\quad$ Mild $=$ VAS 0-3 \\ - $\quad$ Moderate $=\mathrm{VAS}>3-7$ \\ - $\quad$ Severe $=$ VAS $>7-10$}

Based on VAS 0-10 (not troublesome to worst thinkable troublesome) ${ }^{(19)}$

VAS $>5$ affects patient QOL (validated in adult CRS only)

VAS degree of severity correlates with SNOT $22^{(20,21)}$

\subsubsection{SCUAD: Severe Chronic Upper Airway Disease ${ }^{(22)}$}

Patients whose symptoms are inadequately controlled despite adequate (i.e. effective, safe, acceptable) pharmacologic treatment based on guidelines. Includes severe uncontrolled allergic rhinitis, nonallergic rhinitis, chronic rhinosinusitis, N-ERD or occupational airway diseases. Defined by impaired quality of life (QoL), social functioning, sleep, school/work performance.

\subsubsection{Acute complications}

Sudden onset of disease beyond local site.

\subsection{Exacerbation vs. recurrence}

Exacerbation: aggravation [Oxford English Dictionary (OED)] implies increase of a problem against background of disease $\mathrm{e}^{(23)}$ as in acute exacerbation of chronic rhinosinusitis (AECRS) (See also 5.1.3.) Recurrence: come back, return, repeat, occurring again (OED), - implies a disease episode after a period without the problem.

Also, in the literature the term 'acute on chronic' can be found.

\section{The EPOS steering group felt that the term 'exacerbation of $C R S^{\prime}$ was more appropriate and also consistent with the term used in other respiratory diseases, such as asthma.}

\subsection{Control and failure}

2.6.1. Control: dominate, command, hold in check, regulate (OED) (see section 2.22.)

A disease state in which the patients do not have symptoms, or the symptoms do not adversely affect quality of life, if possible combined with a healthy or almost healthy mucosa and only need for local medication. 
In asthma, the Global Initiative for Asthma (GINA) guidelines have defined the term 'control' as effective management of the clinical characteristics of the disease, including symptoms, nocturnal awakening, reliever use, limitation of activity, and lung function, as well as future risk of adverse outcomes. Three levels of asthma control have been established (well controlled, partially controlled, and uncontrolled) ${ }^{(24)}$.

\subsubsection{Failure: uncontrolled}

Using a VAS of 0-10, partially or uncontrolled patients will have symptoms of nasal blockage, discharge, facial pain/pressure, reduced sense of smell and sleep disturbance of $>5$ in addition to nasal endoscopy findings and the need for rescue medication.

\subsubsection{Difficult-to-treat rhinosinusitis}

Patients who have persistent symptoms of rhinosinusitis despite appropriate treatment (recommended medication and surgery). Although the majority of CRS patients can obtain control, some patients will not do so even with the maximal medical therapy and surgery.

Patients who do not reach an acceptable level of control despite adequate surgery, intranasal corticosteroid treatment and up to two short courses of antibiotics or systemic corticosteroids in the last year can be considered to have difficult-to-treat rhinosinusitis.

\subsection{Phenotype}

An organism distinguishable from others by clinical features e.g. $\mathrm{N}$-ERD using symptoms, endoscopy $\pm \mathrm{NPs}, \pm \mathrm{CT}$.

\subsection{Endotype}

Features within an individual e.g. raised IgE, IL-5, eosinophilia, periostin and based on a pathophysiological mechanism.

\subsection{Comorbidities}

\subsubsection{Comorbidity}

Comorbidity is the presence of one or more additional diseases or disorders co-occurring with a primary disease or disorder or any distinct additional entity that has existed or may occur during the clinical course of a patient who has the index disease under study. In chronic rhinosinusitis these are divided into respiratory and other systemic conditions.

\subsubsection{United airway disease}

A pathological continuum due to the interaction between upper and lower airways in allergy, asthma, infection and inflammation ${ }^{(25)}$.

\subsection{Medical therapy}

\subsubsection{Maximal}

The most possible, greatest.

\subsubsection{Appropriate}

The most suitable in the circumstances.

\subsubsection{Adequate}

Satisfactory or suitable in amount, just enough to produce the desired effect.

\subsubsection{Sufficient}

The same as adequate.

\subsubsection{Tailored}

Specific or adapted for a particular condition or person (as in precision / personalised medicine).

\subsubsection{Best}

Finest, greatest, top, foremost, leading, pre-eminent, premier, prime, first, chief, principal, supreme, of the highest quality, superlative, unrivalled, second to none, without equal, nonpareil, unsurpassed, unsurpassable, peerless, matchless, unparalleled, unbeaten, unbeatable, unexcelled, optimum, optimal, ultimate, surpassing, incomparable, ideal, perfect (OED).

\subsubsection{Optimal}

As for 'best'

\section{Of these terms, 'appropriate medical therapy' is the preferred option of the EPO2020 steering group.}

\subsection{Surgical therapy}

\subsubsection{Polypectomy}

Removal of polyps from the nose or post-surgical cavity without altering the bone anatomy.

\subsubsection{Minimal}

Least tissue removal compatible with clinical improvement, conservation of mucosa.

\subsubsection{Full as in 'Full FESS'}

Complete sinus opening including anterior and posterior ethmoidectomy, middle meatal antrostomies (likely large), sphenoidotomy and frontal opening (e.g. Draf lla ).

\subsubsection{Extended}

Used in same context as 'full' (e.g. Draf III) but could also include extension beyond confines of sinuses i.e. skull base, orbit, pte- 
rygopalatine and infratemporal fossa.

\subsubsection{Radical}

Also used in same context as 'full' but could include significant removal of inflamed /dysfunctional mucosa'.

\subsubsection{Functional}

Implies restitution of physiology and is usually, though not exclusively, applied to endoscopic sinus surgery. It should fulfil the following criteria:

- Creates a sinus cavity that incorporates the natural ostium;

- Allows adequate sinus ventilation;

- Facilitates mucociliary clearance;

- Facilitates instillation of topical therapies.

\subsection{Precision medicine vs. personalised medi- cine}

\subsubsection{Precision medicine}

Medical care designed to optimize efficiency or therapeutic benefit for particular groups of patients, especially by using genetic or molecular profiling by tailoring therapy to the individual.

\subsubsection{Personalised medicine}

A type of medical care in which treatment is customized for an individual patient.

\subsection{Burden of rhinosinusitis}

\subsubsection{Quality of life}

The standard of health, comfort, and happiness experienced by an individual or group.

\subsubsection{Outcomes}

Results - subjective / objective; patient / provider; generic / disease-specific.

\subsubsection{Cost}

Direct and Indirect (costs that are directly or not directly accountable to the treatment itself (can be fixed or variable)).

\subsection{Age}

\subsubsection{Child}

A young human being below the age of puberty or below the legal age of majority i.e. a minor.

\subsubsection{Paediatric}

Medical care of infants, children and adolescents. Maximum age varies e.g. up to 21 years in USA.

\subsubsection{Adult}

A legal adult is a person who has attained the age of majority and is therefore regarded as independent, self-sufficient, and responsible, e.g. $>18$ years in UK.

\subsection{Integrated care pathways}

An integrated care pathway (ICP) is a multidisciplinary outline of anticipated care, placed in an appropriate timeframe, to help a patient with a specific condition or set of symptoms move progressively through a clinical experience to positive outcomes.

\subsection{Recalcitrant vs. refractory to treatment}

\subsubsection{Recalcitrant}

Difficult to manage or operate; not responsive to treatment.

\subsubsection{Refractory}

Resistant to cure.

According to the OED, recalcitrant and refractory are synonymous.

\section{The EPOS steering group prefers 'recalcitrant'.}

\subsection{Nasal douche / lavage / irrigation / rinsing}

\subsubsection{Douche}

A stream of water applied for cleansing purposes.

\subsubsection{Lavage}

The therapeutic washing out of an organ.

\subsubsection{Irrigation}

Washing out or flushing a wound or body opening with a stream of water.

\subsubsection{Rinsing}

To cleanse by washing with fluid.

$$
\begin{gathered}
\text { The EPO2020 steering group prefers 'irrigation' } \\
\text { or 'rinsing'. }
\end{gathered}
$$

\subsection{Immunomodulation and immunotherapy}

\subsubsection{Immunomodulation}

Immunomodulation encompasses all therapeutic interventions aimed at modifying the immune response and is the preferred over-riding term by the EPO2020 steering group. In the treatment of rhinosinusitis, it encompasses the use of biological agents and macrolides. 


\subsubsection{Immunotherapy}

Treatment to stimulate or restore the ability of the immune system to fight infection and disease OR treatment or prevention of disease (such as an autoimmune disorder, allergy, or cancer) that involves the stimulation, enhancement, suppression, or desensitization of the immune system. Generally, this term is used in relation to the treatment of allergy.

\subsubsection{Biological therapy}

A type of treatment that uses substances made from living organisms to treat disease.

\subsection{Allergy}

\subsubsection{Allergy}

A damaging immune response by the body to a substance, especially a particular food, pollen, fur, or dust, to which it has become hypersensitive.

\subsubsection{Allergic rhinitis}

A symptomatic IgE-driven inflammation of the nasal mucosa ${ }^{(26)}$. An IgE-mediated inflammatory nasal condition resulting from allergen introduction in a sensitized individual(27).

\subsubsection{Entopy or local allergic rhinitis (LAR)}

A phenotype of allergic rhinitis characterised by a localised nasal allergic response in patients with negative skin prick testing to inhalant allergens and non-detectable serum specific IgE antibodies. Diagnosis is based on a positive response to nasal allergen provocation $^{(28-30)}$.

\subsubsection{Atopy}

Atopy refers to the genetic tendency to develop allergic diseases such as allergic rhinitis, asthma and atopic dermatitis (eczema). Atopy is typically associated with heightened immune responses to common allergens, especially inhaled allergens and food allergens ${ }^{(26)}$.

A genetic disposition to develop an allergic reaction (as allergic rhinitis, asthma, or atopic dermatitis) and produce elevated levels of IgE upon exposure to an environmental antigen and especially one inhaled or ingested.

An inherited predisposition to produce $\lg$ E antibody ${ }^{(27)}$.

\subsubsection{Atopic march}

The progression from atopic dermatitis in infants and children to allergic rhinitis and/or asthma ${ }^{(31)}$.

\subsection{Duration of antibiotic courses}

\subsubsection{Short-term}

Applied to anything from 2-3-5-7-10-14 days in the literature.

\subsubsection{Long-term}

$>2$ weeks i.e. $4,6,8,10,12$, etc. up to years.

The EPO2020 steering group agreed that four weeks or less would be 'short-term', accepting that in general practice the duration is usually $<10$ days, and $>4$ weeks would be regarded as 'long-term.'

They also acknowledged that the aim of shortterm treatment was different from long-term. Short-term courses are generally given for acute bacterial infection whereas long term courses are given for their immunomodulatory properties.

\subsection{Other definitions}

2.21.1. Eosinophilic fungal rhinosinusitis vs. 'allergic' fungal rhinosinusitis

The EPO2020 steering group discussed this umbrella term for fungal rhinosinusitis but it was agreed that 'allergic' fungal rhinosinusitis should be retained as the principle term due to common usage, recognising that not all cases have evidence of an allergic reaction to fungi e.g. a positive skin prick and/or specific lgE.

\section{It was agreed that 'allergic' fungal rhinosinusitis should be retained as the principle term due to common usage.}

\subsubsection{Eosinophilic rhinosinusitis (eCRS)}

Requires quantification of the numbers of eosinophils, i.e. number / high powered field which varies in the literature [8-12/hpf $(400 x)]^{(32,33)}$.

\section{The EPO2020 steering group prefers 10/hpf.}

\subsubsection{Central compartment disease}

$A$ variant of $C R S$ with polypoid changes of the entire central sinonasal compartment (i.e. the middle and superior turbinates, and the posterosuperior nasal septum), while the lateral sinus mucosa remains relatively normal, likely due to allergy ${ }^{(34)}$.

\subsection{Concept of Control of CRS}

\subsubsection{Introduction}

The primary goal of any treatment, especially in chronic diseases, is to achieve and maintain clinical control, which can be defined as a disease state in which the patient does not have symptoms, or the symptoms are not impacting quality of life $(\mathrm{QoL})^{(35)}$. 
Figure 2.22.1. Assessment of current clinical control of CRS.

\begin{tabular}{|c|c|c|c|}
\hline \multirow{2}{*}{ EPOS 2020} & \multicolumn{3}{|c|}{ EPOS 2020: Assessment of current clinical control of CRS (in the last month) } \\
\hline & $\begin{array}{l}\text { Controlled } \\
\text { (all of the following) }\end{array}$ & $\begin{array}{l}\text { Partly controlled } \\
\text { (at least } 1 \text { present) }\end{array}$ & $\begin{array}{l}\text { Uncontrolled } \\
\text { ( } 3 \text { or more present) }\end{array}$ \\
\hline Nasal blockage' & Not present or not bothersome ${ }^{2}$ & $\begin{array}{l}\text { Present } \\
\text { on most days of the week }\end{array}$ & $\begin{array}{l}\text { Present } \\
\text { on most days of the week }\end{array}$ \\
\hline Rhinorrhoea / Postnasal drip' & Little and mucous ${ }^{2}$ & $\begin{array}{l}\text { Mucopurulent } \\
\text { on most days of the week }\end{array}$ & $\begin{array}{l}\text { Mucopurulent } \\
\text { on most days of the week }\end{array}$ \\
\hline Facial pain / Pressure' & $\begin{array}{l}\text { Not present } \\
\text { or not bothersome }{ }^{2}\end{array}$ & $\begin{array}{l}\text { Present } \\
\text { on most days of the week }\end{array}$ & $\begin{array}{l}\text { Present } \\
\text { on most days of the week }\end{array}$ \\
\hline Smell 1 & $\begin{array}{l}\text { Normal } \\
\text { or only slightly impaired }{ }^{2}\end{array}$ & Impaired ${ }^{3}$ & Impaired ${ }^{3}$ \\
\hline Sleep disturbance or fatigue ${ }^{1}$ & Not present ${ }^{2}$ & Present $^{3}$ & Present $^{3}$ \\
\hline $\begin{array}{l}\text { Nasal endoscopy } \\
\text { (if available) }\end{array}$ & $\begin{array}{l}\text { Healthy } \\
\text { or almost healthy mucosa }\end{array}$ & Diseased mucosa ${ }^{4}$ & Diseased mucosa ${ }^{4}$ \\
\hline $\begin{array}{l}\text { Rescue treatment } \\
\text { (in last } 6 \text { months) }\end{array}$ & Not needed & $\begin{array}{l}\text { Need of } 1 \text { course of } \\
\text { rescue treatment }\end{array}$ & $\begin{array}{l}\text { Symptoms (as above) persist } \\
\text { despite rescue treatment(s) }\end{array}$ \\
\hline
\end{tabular}

${ }^{1}$ Symptoms of CRS; ${ }^{2}$ For research VAS $\leq 5 ;{ }^{3}$ For research VAS $>5 ; \quad{ }^{4}$ Showing nasal polyps, mucopurulent secretions or inflamed mucosa

A number of tools are currently being used in daily clinical practice and research context, to evaluate different aspects of disease control in chronic rhinosinusitis (CRS). These include QoL and symptom severity questionnaires, but also more objective measurements such as endoscopic scoring systems.

However, the concept of control of disease is relatively new in the field of chronic rhinosinusitis (CRS). The European Position Paper on Rhinosinusitis (EPOS) 2012 incorporated criteria for the assessment of CRS control, to address the lack of uniformity in the application and interpretation of existing tools in the context of disease control (Figure 2.22.1). Such staging systems can be useful in clinical practice to evaluate burden of disease, to guide management and to assess quality of care, especially since there is still a significant group of patients with CRS who remain uncontrolled despite receiving a combination of adequate medical treatment and endoscopic sinus surgery (ESS) following evidence-based guidelines ${ }^{(35,36)}$. A variety of factors can be associated with inadequate disease control and it is important to first define this group of patients in order to identify and address these contributing factors and to optimize CRS management ${ }^{(36)}$.

Furthermore, the concept of control can be used in a research context to better characterize patient populations or as an outcome measurement for preventive or therapeutic interventions. The primary goal of any treatment, especially in chronic diseases, is to achieve and maintain clinical control, which can be defined as a disease state in which the patient does not have symptoms, or the symptoms are not impacting quality of life.
The EPOS expert committee proposed to combine the severity of patients' symptoms, aspect of nasal mucosa and medical intake as parameters of control. The proposed CRS control test takes into account the presence and severity of the four major sinonasal symptoms, sleep disturbance and/or fatigue, nasal endoscopic evaluation and need for oral medication. Based on the presence of none, one or more items from this list, patients are divided into those with controlled, partly controlled and uncontrolled rhinosinusitis.

\subsubsection{Validation of the EPOS 2012 criteria for disease control}

Since the criteria for CRS control proposed by the EPOS expert panel in 2012 are largely based on opinion rather than datadriven, further validation was required.

A systematic literature search on control in CRS produced three papers summarized in Table 2.22.1.

In a study of van der Veen et al. $19.5 \%$ of patients $(n=389)$ met the criteria of well controlled CRS when being evaluated 3-5 years after ESS, whereas $36.8 \%$ had partly controlled and $43.7 \%$ had uncontrolled $\mathrm{CRS}^{(37)}$. Very stable results were recorded in the smaller $(n=47)$, prospective 12 year follow-up study by Calus et al., where $40 \%$ of patients was uncontrolled 6 years after ESS, $44 \%$ was partially controlled and only $16 \%$ was controlled ${ }^{(38)}$. Although the percentage of uncontrolled patients after surgery was similar in both studies, it was surprisingly high as the perception of success of FESS is currently estimated higher with reported success rates of up to $80 \%{ }^{(39)}$. It is important to 
Table 2.22.1. Overview of clinical studies that used the EPOS control criteria (March 2012 - June 2019).

\begin{tabular}{|c|c|c|}
\hline Study & Objectives & Methods \\
\hline $\begin{array}{l}\text { Snidvongs, } \\
2014^{(41)}\end{array}$ & $\begin{array}{l}\text { To develop a chronic rhinosinusitis (CRS) } \\
\text { disease control staging system that predicts } \\
\text { patient and physician opinion. This involved } \\
\text { exploring the predictive capacity of the } \\
\text { proposed EPOS- } 2012 \text { staging system and } \\
\text { other potential scoring systems based on } \\
\text { patient symptoms and objective criteria. }\end{array}$ & $\begin{array}{l}\text { Design: Prospective study } \\
\text { Population: Adult CRS patients undergoing ESS were recruited from a tertiary referral } \\
\text { clinic. } \\
\text { Method: Patients ( } \mathrm{n}=106 \text { ) returned at } 6 \text { months and } 12 \text { months after ESS. } \\
\text { Symptoms, endoscopy score, and systemic medication used were collected at each } \\
\text { visit along with physician's and patient's report of their condition as either "controlled, } \\
\text { "partly controlled," or "uncontrolled". Ordinal regression was used for modelling a sta- } \\
\text { ging system. The EPOS criteria and various combinations were assessed. Kappa agree- } \\
\text { ments between the staging systems and patient/physician reports were analysed. }\end{array}$ \\
\hline $\begin{array}{l}\text { van der } \\
\text { Veen, } \\
2017^{(37)}\end{array}$ & $\begin{array}{l}\text { To study the degree of CRS control using } \\
\text { novel EPOS control criteria at 3-5 years } \\
\text { after a functional endoscopic sinus } \\
\text { surgery (FESS) and correlate these data to } \\
\text { symptoms scores (VAS \& SNOT-22). } \\
\text { To study the influence of performing nasal } \\
\text { endoscopy on defining patients' level of } \\
\text { control based on EPOS criteria. }\end{array}$ & $\begin{array}{l}\text { Design: Cross-sectional study } \\
\text { Population: Adult CRS patients who had undergone bilateral FESS for chronic inflam- } \\
\text { matory sinonasal disease } 3-5 \text { years prior to the study were included. } \\
\text { Method: Patients received a postal questionnaire asking for control items according } \\
\text { to EPOS control criteria, visual analogue scale (VAS) scores for total and individual } \\
\text { sinonasal symptoms, sinonasal outcome test (SNOT)-22 and Short Form (SF)-36 } \\
\text { questionnaires. } \\
389 \text { of the } 560 \text { patients included in the study returned a filled questionnaire }(69,0 \% \\
\text { response rate). Among patients who responded, } 81 \text { ( } 20.8 \% \text { ) accepted the invitation } \\
\text { for a voluntary outpatient visit where nasal endoscopy was performed. }\end{array}$ \\
\hline $\begin{array}{l}\text { Calus, } \\
2019^{(38)}\end{array}$ & $\begin{array}{l}\text { To monitor recurrence and revision surgery } \\
\text { over } 12 \text { years after endoscopic sinus sur- } \\
\text { gery in CRSwNP patients. } \\
\text { To validate EPOS } 2012 \text { control criteria }\end{array}$ & $\begin{array}{l}\text { Design: Prospective study, however EPOS control criteria were evaluated retrospecti- } \\
\text { vely. } \\
\text { Population: Adult patients ( } n=47 \text { ) with CRSwNP, undergoing primary or revision } \\
\text { extended endoscopic sinus surgery, were followed. } \\
\text { Method: Clinical symptoms, total nasal endoscopic polyp score and inflammatory } \\
\text { markers in tissue, nasal secretions and serum were evaluated before, } 6 \text { years and } 12 \\
\text { years after surgery. }\end{array}$ \\
\hline
\end{tabular}

note that patients included in the studies by van der Veen et al. and Calus et al. had been treated in a tertiary referral centre for rhinologic diseases, causing a bias towards the more severe spectrum of disease. Also, the success rate in other studies was defined as symptomatic improvement after FESS and belonging to the uncontrolled group does of course not exclude a beneficial effect of surgery. This is also shown in the study of van der Veen et al., where 10 out of 21 patients (47.6\%) who were telephoned and asked how they perceived CRS control after FESS, regarded themselves as having controlled CRS. When EPOS criteria were applied on these patients, only four of them (19.1\%) met the criteria of being controlled ${ }^{(37)}$. Calus et al. also focused on how patients appraised their condition. Twelve years after FESS, 97.4\% of patients reported general therapeutic relief (21.1\% reported a complete, $36.8 \%$ a marked, $26.3 \%$ a moderate and $13.2 \%$ a slight relief over time) ${ }^{(38)}$. Regarding the distribution of patients over the 3 EPOS categories of control, a significant change towards more control was found $6(p=0.001)$ and 12 years $(p<0.001)$ after surgery, when compared to the distribution prior to $\mathrm{FESS}^{(38)}$. Due to its cross-sectional design, improvement of disease control could not be evaluated by van der Veen et al., since there were no data available from before surgery. They did see a significant higher proportion of women compared to men in the uncontrolled group. Patients that had revision FESS were less frequently controlled compared to the ones that had primary FESS, suggesting that they might form a more difficult-to-treat group of patients. Aspirin intolerance was also associated with lower percentages of CRS control after FESS ${ }^{(37)}$. A first comparison was performed between EPOS assessment of CRS control with both VAS global symptom scores and SNOT22 scores $^{(37)}$. The average VAS total nasal symptom scores of controlled, partly and uncontrolled groups were $0.8,2.7$ and 5.7 respectively. The average SNOT-22 scores were 9.7, 22.2 and 44.8 , respectively ${ }^{(37)}$.

Van der Veen et al. also evaluated the added value of nasal endoscopy for defining disease control in CRS patients. In $95.1 \%$ of the cases, performing a nasal endoscopy did not cause a shift in the control category that was defined by just the presence of symptoms and use of systemic medication ${ }^{(37)}$. Although nasal endoscopy is described as optional ('if available') in the EPOS control criteria, this might have been a barrier to apply them in some study protocols, as this was also explicitly mentioned by the authors of one of the studies reviewed ${ }^{(40)}$. This is especially the case in large-scale studies and/or studies involving non-ENT practitioners.

Snidvongs et al. conducted a prospective trial in which 106 patients undergoing ESS were evaluated at 6 and 12 months after surgery to investigate if the EPOS 2012 CRS control staging system, or any modification of this system, correlates with both patient and physician reports of disease ${ }^{(41)}$. They selected a modified staging system using Nasal Obstruction, Systemic medication and Endoscopic inflammation ('NOSE') based on predictive 
strength. More specifically, they evaluated which symptoms were, as a single factor, associated with patient's report (nasal obstruction $p=0.02$ ) and which nasal endoscopy features were, as a single factor, associated with physician's report of disease progression (endoscopic mucosal inflammation $p<0.001$ and thick and/or purulent discharge $p=0.01$ ). Unfortunately, data concerning the use of EPOS control criteria is rather limited and no comparison can be made with results from the other studies, e.g. on patients' distribution in the different EPOS control categories before and (six and 12 months) after surgery.

After analysis of kappa agreements between the staging systems and the patient's and physician's reports, they arrived to the conclusion that both the EPOS and the NOSE criteria for control had significant agreement with these reports. They propose the NOSE system, as a modified version of the EPOS criteria, since it has fewer criteria (i.e. fewer symptoms) to assess and they found better agreement with physician and patient rated control in their study. It is not entirely clear from the article how the criteria used in these patients' and physicians' reports of disease control were established. This is an important consideration to make, since they largely determine the primary outcome of this study, namely the kappa agreement between these reports and the different investigated disease control staging systems.

Two other studies mentioned the EPOS control criteria but did not use them in real-life studies with CRS patients and are for that reason not listed in Table 2.22.1.

The first was an article published by Hellings et al. in $2013^{(36)}$. They reviewed the state of the art on control of both allergic rhinitis and CRS, emphasizing the importance of this concept to define those patients with poorly controlled disease. They propose a treatment algorithm for CRS in relation to the categories of control provided in EPOS 2012, with proposed treatments based on the EPOS 2012 treatment algorithms. Secondly, they describe the wide variety of factors that can contribute to a lack of control and divide them into four categories: disease-related factors, diagnosis-related factors, treatment-related factors and patient-related factors.

Another article published by Doulaptsi et al. in 2018 correlates VAS and SNOT-22 scores in 180 CRS patients ${ }^{(40)}$. They mention the EPOS 2012 criteria for CRS control, but decided that applying them was not feasible in their postal questionnaire survey. Instead they used VAS total nasal symptom score to assess disease control, using the following cut-off points: well controlled (VAS $\leq 2$ ), partly controlled (VAS $>2$ and $\leq 5$ ), uncontrolled (VAS $>5$ ). These cut-off values were based on the study of van der Veen et al. and have also recently been used in a mobile app for patients with CRS, developed by the European Forum for Research and Education in Allergy and Airway diseases (EUFOREA) ${ }^{(37,42)}$.

\subsubsection{Alternative tools for (indirect) assessment of CRS control}

All clinicians involved in the treatment of CRS aim to achieve clinical control in their patients. Nevertheless, the methods used to assess CRS control in daily practice are still very heterogeneous and the idea of controlled disease can differ between physicians. Uniformity in the routine application and interpretation of the existing tools for assessment of CRS control is lacking as a consensus on assessment criteria has not yet been reached. This is in contrast to asthma control assessment criteria in the GINA (Global Initiative for Asthma) guidelines, which are widely accepted and are recommended as good clinical practice ${ }^{(43)}$.

Apart from the CRS control criteria proposed by the EPOS 2012, numerous other tools for assessing (elements of) control, disease severity and/or Quality of Life are already being used in clinical practice and research. In 2017, a Core Outcome Set for four key domains of CRS was selected by the CHROME-study. For the domain 'control of disease' they proposed three measurement tools: need for systemic medication (steroid or antibiotic), progression to surgery and the Lund-Kennedy endoscopic score $^{(44)}$.

The SNOT-22 and VAS for total as well as individual sinonasal symptoms are both validated tools that are widely known among clinicians and researchers in the field of CRS and used for assessing Quality of Life and symptom severity respectively ${ }^{145}$, ${ }^{46)}$. More recently, in this age of big data and precision medicine, mobile health technology has been emerging and mobile applications are being developed for numerous diseases, including $\mathrm{CRS}^{(42)}$. Sedaghat et al. investigated chronic rhinosinusitis control from the patient and physician perspectives in 209 patients. Participants were asked to rate their global level of CRS control as "not at all," "a little," "somewhat," "very," and "completely"(47). This 5 scale control scores by patients and physicians were compared to the SNOT-22(48) and also reported the number of sinus infections, CRS-related antibiotic courses taken, CRS-related oral corticosteroid courses taken, and missed days of work or school due to CRS, all in the last 3 months. While both patients and physicians rely on the burden of CRS symptomatology, patients consider primarily nasal symptoms while physicians include nasal and extra-nasal symptoms of CRS in determining CRS control. Physicians also independently consider CRS-related antibiotic use, as a reflection of acute bacterial CRS exacerbations, and CRS-related oral corticosteroid use in the determination of global CRS control.

\subsubsection{SNOT-22}

The SNOT-22 questionnaire is a 22-item, disease-specific, healthrelated questionnaire assessing quality of life in CRS patients, that has been validated in multiple languages (see 5.3.4.2). Van der Veen et al. showed significant differences in SNOT-22 scores between the three stages of control based on the EPOS 
control criteria ${ }^{(37)}$. More prospective studies comparing these different scoring systems are needed to validate these results. Considering its ability to predict CRS control status, it is important to note that some variables of SNOT-22, e.g. ear symptoms and emotional disorders, are not disease-specific.

\subsubsection{VAS}

The VAS is widely used by rhinologists both in research and in daily practice. Patients quantify the severity of their symptoms on a $10-\mathrm{cm}$ scale, with 0 meaning total absence of symptom(s) and 10 being the worst thinkable severity ${ }^{(46)}$ (see 5.3.4.2).

VAS for total nasal symptoms is already being used in clinical practice, based on the EPOS guidelines, to classify CRS into mild, moderate and severe disease ${ }^{(49)}$ and has also been incorporated and validated in several mHealth apps ${ }^{(42,50)}$.

Van der Veen et al. compared VAS scores to the EPOS control criteria and the three categories of CRS control showed significant differences in mean total and individual VAS scores, as was also the case with the SNOT-22 scores ${ }^{(37)}$. Another interesting finding in this study, was that only uncontrolled patients had VAS scores higher than 5 . The mean VAS score for total nasal symptoms in this uncontrolled group was 5.5, which is relatively low when comparing to the cut-off values used to classify CRS severity ${ }^{(35)}$. Based on these findings, Doulaptsi et al. created new cut-off points for VAS TNSS to define the level of disease control: well controlled (VAS $\leq 2$ ), partly controlled (VAS $>2$ and $\leq 5$ ), uncontrolled $(\text { VAS }>5)^{(7)}$. Using these cut-off points, $10 \%$ of patients were classified as well controlled, $28.3 \%$ as partly controlled, and $61.7 \%$ as uncontrolled ${ }^{(40)}$.

Recently, these same cut-off points have also been used in the mySinusitisCoach app to assess CRS control ${ }^{(42)}$. Taking into account its ease of use and its applicability in mHealth tools, the role of VAS in assessment of disease severity, monitoring disease and maybe also assessment of disease control might become even more prominent in coming years.

However, regarding its use in assessment of CRS control, it is important to consider that these VAS scores are patientreported outcomes, lacking any form of objective support such as medication use or nasal endoscopic evaluation. Also, VAS scores for individual symptoms might not all be equally useful in predicting disease control, as e.g. rhinorrhea, facial pain or hyposmia may also be caused by numerous other conditions.

\subsubsection{Other questionnaires}

Over the course of years, many other questionnaires have been used to evaluate CRS symptoms and/or their impact on QoL and general health status (see 5.3.4.2) These include the Sinus Control Test (SCT) the 31-Item Rhinosinusitis Outcome Measure (RSOM-31), the 20-Item Sino-Nasal Outcome Test (SNOT-20), the Sinonasal questionnaire (SNAQ-11) and the Rhinosinusitis Disability Index (RSDI) 15.
In addition, the 36-item Short Form (SF-36), the 12-item Short Form (SF-12) and the EuroQol-5Dimension-5Level (EQ-5D-5L) are health questionnaires that are designed to assess general, health-related QoL and to be applied to all health conditions. The SF-36 was also included in the study of van der Veen et al. and, as was the case for VAS and SNOT-22 scores, SF-36 scores were significantly different when comparing the 3 categories of CRS control based on EPOS criteria ${ }^{(37)}$.

\subsubsection{Recommendations and future needs}

Based on the results of van der Veen et al., showing a mean VAS of 5.5 for total nasal symptoms, the EPOS2020 steering group think that the current EPOS2012 control criteria might overestimate the number of patients being uncontrolled. For research purposes we therefore recommend using a VAS scale for all symptoms: "not bothersome" can be substituted by 'VAS $<5$ ', and 'present/impaired' by 'VAS $\geq 5$. This is in keeping with the VAS score of 5 or more that has already been proposed to evaluate symptom control in allergic rhinitis ${ }^{(51)}$.

The fact that only one feature has to be present for a patient to be classified as partly controlled, is something else that we think should be reconsidered. This is especially important since sleep disturbance and/or fatigue and also, but to lesser extent, rhinorrhoea, facial pain, loss of smell and even nasal obstruction can all possibly be attributed to other medical conditions. Based on the results of van der Veen et al., we have changed the criteria so that these symptoms must be related to CRS ${ }^{(37)}$. For example, a typical migraine headache should not be taken into account. Generally, there is a need for additional, large-scale studies, preferably with long follow up to confirm the high percentage of uncontrolled patients after surgery as shown by Calus et al. and van der Veen et al. and to further evaluate the responsiveness of the EPOS criteria to treatment ${ }^{(37,38)}$.

These studies will help to explore differences in disease control (based on EPOS criteria) between men and women, patients undergoing primary or revision FESS, and between different phenotypes e.g. with or without nasal polyps, AR, asthma, AERD, and endotypes based on inflammatory patterns. Such data will likely prove to be valuable in predicting patients at risk of having uncontrolled disease.

\subsubsection{Conclusion}

Since the third EPOS update was published in 2012, only a few studies have applied the proposed criteria for assessment of current disease control and the results of these studies still require further psychometric validation (including internal consistency, responsiveness and known group differences).

Given the importance of the concept of disease control, from a clinical as well as from a research perspective, there still remains a need for a gold standard to assess disease control in CRS. 
The results of previous studies and the recommendations for future research described in this document can hopefully facilitate this process of validation in the coming years, together with the arrival of mHealth technologies.

- The criteria have been revised in EPOS2020 for defining controlled, partly controlled and uncontrolled CRS.

- $\quad$ Since the third EPOS update was published in 2012, only a few studies have applied the proposed criteria for assessment of current disease control and the results of these studies still require further psychometric validation (including internal consistency, responsiveness and known group differences).

- Given the importance of the concept of disease control, from a clinical as well as from a research perspective, there still remains a need for a gold standard to assess disease control in CRS.

- The results of previous studies and the recommendations for future research can hopefully facilitate validation in future years, together with the arrival of mHealth technologies.

\section{References}

1. Lund VJ, Holmstrom M, Scadding GK Functional endoscopic sinus surgery in the management of chronic rhinosinusitis. An objective assessment. J Laryngol Otol 1991;105:832-5.

2. Lund VJ, Kennedy DW. Staging for rhinosinusitis. Otolaryngol-Head Neck Surg. 1997;117:S35-40.

3. Orlandi RR, Kingdom TT, Hwang PH. International Consensus Statement on Allergy and Rhinology: Rhinosinusitis Executive Summary. Int Forum Allergy Rhinol 2016;6:S3-S21

4. Meltzer EO, Hamilos DL, Hadley JA, et al. Rhinosinusitis: establishing definitions for clinical research and patient care. J Allergy Clin Immunol 2004;114:155-212.

5. Meltzer EO, Hamilos DL, Hadley JA, et al. Rhinosinusitis: Developing guidance for clinical trials. Otolaryngol Head Neck Surg 2006;135:S31-80.

6. Gwaltney JM, Phillips CD, Miller RD, Riker DK. Computed Tomographic Study of the Common Cold. N Engl J Med 1994;330:2530 .

7. Bhattacharyya N. Chronic rhinosinusitis: is the nose really involved? Am J Rhinol 2001;15:169-73.

8. Van Crombruggen K, Van Bruaene N, Holtappels G, Bachert C. Chronic sinusitis and rhinitis: clinical terminology "Chronic Rhinosinusitis" further supported. Rhinology 2010;48:54-8.

9. Fokkens WJ, Lund VJ, Mullol J, et al. European Position Paper on Rhinosinusitis and Nasal Polyps 2012. Rhinol Suppl 2012;23:3 p preceding table of contents, 1-298.

10. Hsueh WD, Conley DB, Kim H, et al. Identifying clinical symptoms for improving the symptomatic diagnosis of chronic rhinosinusitis.Int Forum Allergy Rhinol 2013;3:307-14.

11. Hamizan AW, Azer M, Alvarado R, et al. The Distinguishing Clinical Features of Nonallergic Rhinitis Patients. Am J Rhinol
Allergy 2019;33:524-30.

12. Brandt D, Bernstein JA. Questionnaire evaluation and risk factor identification for nonallergic vasomotor rhinitis. Ann Allergy Asthma Immunol 2006;96:526-32.

13. Habib A-R, Campbell R, Kalish L, et al. The burden of chronic upper airway disorders in Australia: a population-based cross-sectional study. Aus J Otolaryngol; 2019, 228

14. Barham HP, Zhang AS, Christensen JM, Sacks R, Harvey RJ. Acute radiology rarely confirms sinus disease in suspected recurrent acute rhinosinusitis. Int Forum Allergy Rhinol 2017;7:726-33.

15. Dietz de Loos D, Lourijsen ES, Wildeman MAM, et al. Prevalence of chronic rhinosinusitis in the general population based on sinus radiology and symptomatology. J Allergy Clin Immunol 2019;143:1207-14.

16. Rachelefsky GS, Goldberg M, Katz RM, et al. Sinus disease in children with respiratory allergy. J Allergy Clin Immunol 1978;61:3104.

17. Rosenfeld RM, Piccirillo JF, Chandrasekhar SS, et al. Clinical practice guideline (update): Adult sinusitis. Otolaryngol - Head Neck Surg 2015;152:S1-S39.

18. Seresirikachorn K, Snidvongs K Chitsuthipakorn W, et al. EPOS2012 has better specificity compared to IDSA2012 for diagnosing acute bacterial rhinosinusitis. Rhinology 2018;56:241-4.

19. Lim M, Lew-Gor S, Darby Y, Brookes N Scadding G, Lund VJ. The relationship between subjective assessment instruments in chronic rhinosinusitis. Rhinology 2007:45:144-7.

20. Remenschneider AK, D'Amico L, Gray ST, Holbrook EH, Gliklich RE, Metson R. The EQ-5D: A new tool for studying clinical outcomes in chronic rhinosinusitis. Laryngoscope 2015;125:7-15.

21. van Oene CM, van Reij EJ, Sprangers MA Fokkens WJ. Quality-assessment of diseasespecific quality of life questionnaires for rhinitis and rhinosinusitis: a systematic review. Allergy 2007;62:1359-71.
22. Bousquet J, Bachert C, Canonica GW, et al. Unmet needs in severe chronic upper airway disease (SCUAD). J Allergy Clin Immunol 2009;124:428-33.

23. Wise SK, Lin SY, Toskala E, et al. International Consensus Statement on Allergy and Rhinology: Allergic Rhinitis. Ilnt Forum Allergy Rhinol 2018;8:108-352.

24. Global Initiative for Asthma. Global Strategy for Asthma Management and Prevention (2018 update). 2018. www.ginasthma.org.

25. Hens G, Hellings PW. The nose: gatekeeper and trigger of bronchial disease. Rhinology 2006:44:179-87.

26. Global Atlas of Allergic Rhinitis and Chronic Rhinosinusitis. 2015. http://www.eaaci.org/ globalatlas/ENT_Atlas_web.pdf.

27. Bousquet J, Van Cauwenberge P, Khaltaev N, Group AW, Organization WH. Allergic rhinitis and its impact on asthma. The Journal of allergy and clinical immunology 2001;108:S147-334.

28. Powe DG, Jagger C, Kleinjan A, Carney AS, Jenkins D, Jones NS. 'Entopy': localized mucosal allergic disease in the absence of systemic responses for atopy. Clin Exp Allergy: 2003;33:1374-9.

29. Rondon C, Campo P, Togias A, et al. Local allergic rhinitis: concept, pathophysiology, and management. J Allergy Clin Immunol 2012;129:1460-7.

30. Hellings PW, Klimek L, Cingi C, et al. Nonallergic rhinitis: Position paper of the European Academy of Allergy and Clinical Immunology. Allergy 2017;72:1657-65.

31. Spergel JM, Paller AS. Atopic dermatitis and the atopic march. J Allergy Clin Immunol 2003;112:S118-27.

32. Ho J, Hamizan AW, Alvarado R, Rimmer J, Sewell WA, Harvey RJ. Systemic Predictors of Eosinophilic Chronic Rhinosinusitis. Am J Rhinol Allergy 2018;32:252-7.

33. Nakayama T, Sugimoto $N$, Okada $N$, et al. JESREC score and mucosal eosinophilia can predict endotypes of chronic rhinosinusitis with nasal polyps. Auris Nasus Larynx 2019;46:374-83 
34. DelGaudio JM, Loftus PA, Hamizan AW, Harvey RJ, Wise SK. Central Compartment Atopic Disease. Am J Rhinol Allergy 2017;31:228-34

35. Fokkens WJ, Lund VJ, Mullol J, et al. EPOS 2012: European position paper on rhinosinusitis and nasal polyps 2012. A summary for otorhinolaryngologists. Rhinology 2012;50:1-12.

36. Hellings PW, Fokkens WJ, Akdis C, et al. Uncontrolled allergic rhinitis and chronic rhinosinusitis: where do we stand today? Allergy 2013;68:1-7.

37. van der Veen J, Seys SF, Timmermans M, et al. Real-life study showing uncontrolled rhinosinusitis after sinus surgery in a tertiary referral centre. Allergy 2017;72:282-90.

38. Calus L, Van Bruaene N, Bosteels C, et al. Twelve-year follow-up study after endoscopic sinus surgery in patients with chronic rhinosinusitis with nasal polyposis. Clin Transl Allergy 2019;9:30.

39. Vlaminck S, Vauterin T, Hellings PW, et al. The importance of local eosinophilia in the surgical outcome of chronic rhinosinusitis: a 3-year prospective observational study. Am J Rhinol Allergy 2014;28:260-4.

40. Doulaptsi M, Prokopakis E, Seys S, Pugin B, Steelant B, Hellings P. Visual analogue scale for sino-nasal symptoms severity correlates with sino-nasal outcome test 22: paving the way for a simple outcome tool of CRS burden. Clin Transl Allergy 2018;8:32.

41. Snidvongs K, Heller GZ, Sacks R, Harvey RJ. Validity of European position paper on rhinosinusitis disease control assessment and modifications in chronic rhinosinusitis. Otolaryngol Head Neck Surg 2014;150:47986

42. Seys SF, Bousquet J, Bachert C, et al. mySinusitisCoach: patient empowerment in chronic rhinosinusitis using mobile technology. Rhinology 2018;56:209-15.

43. Bousquet J, Clark TJH, Hurd S, et al. GINA guidelines on asthma and beyond. Allergy: Eur J Allergy Clin Immunol 2007;62:102-12.

44. Hopkins C, Hettige R, Soni-Jaiswal A, et al. CHronic Rhinosinusitis Outcome MEasures (CHROME), developing a core outcome set for trials of interventions in chronic rhinosinusitis. Rhinology 2018;56:22-32.

45. Hopkins C, Gillett S, Slack R, Lund VJ, Browne JP. Psychometric validity of the 22-item Sinonasal Outcome Test. Clin Otolaryngol 2009;34:447-54.

46. Klimek L, Bergmann K-C, Biedermann T, et al. Visual analogue scales (VAS): Measuring instruments for the documentation of symptoms and therapy monitoring in cases of allergic rhinitis in everyday health care.
Allergo J Int 2017;26:16-24

47. Sedaghat AR, Hoehle LP, Gray ST. Chronic rhinosinusitis control from the patient and physician perspectives. Laryngoscope Investig Otolaryngol. 2018;3:419-33.

48. Toma S, Hopkins C. Stratification of SNOT-22 scores into mild, moderate or severe and relationship with other subjective instruments. Rhinology 2016;54:129-33.

49. Fokkens WJJ, Lund VJJ, Mullol J, et al. EPOS 2012: European position paper on rhinosinusitis and nasal polyps 2012. A summary for otorhinolaryngologists. Rhinology 2012;50:1-12.

50. Caimmi D, Baiz N, Tanno LK, et al. Validation of the MASK-rhinitis visual analogue scale on smartphone screens to assess allergic rhinitis control. Clin Exp Allergy 2017;47:1526-33.

51. Brozek JL, Bousquet J, Agache I, et al. Allergic Rhinitis and its Impact on Asthma (ARIA) guidelines-2016 revision. J Allergy Clin Immunol 2017;140:950-8.

52. Grayson JW, Hopkins C, Mori ES, Senior B, Harvey RJ. Contemporary Classification of Chronic Rhinosinusitis Beyond Polyps vs No Polyps: A Review. JAMA Otolaryngol Head Neck Surg. doi: 10.1001/jamaoto.2020.1453. 


\section{Burden of acute and chronic rhinosinusitis}

\subsection{Quality of life (QOL)}

\subsubsection{The burden of ARS}

Few studies have measured the impact of ARS on quality of life, in contrast to the myriad of studies reporting on both direct and indirect costs. This may reflect the short-lived duration of illness, with patients usually returning to their pre-morbid health status. Of studies that do report on symptoms, many fail to differentiate between ARS and ABRS.

In a prospective study of 150 adult patients with ARS, 88\% patients reported pain and discomfort, and $43 \%$ had difficulties performing normal daily activities at the onset of an episode of ARS, measured using the Eq-5D ${ }^{(1)}$. By day 15 only $31.5 \%$ reported pain or discomfort and all but $1.4 \%$ had fully returned to normal daily activities. A study of 1585 adults diagnosed with acute rhinosinusitis, found the most common presenting symptoms were moderate-to-severe nasal obstruction (80.4\%), facial pain (74.5\%), rhinorrhoea (70.4\%), and headache (63.6\%)

(2). Symptoms were indicated as having a moderate to very significant effect on activities of daily living (71.6\% of patients), leisure (63.1\%), and professional/school activities (59.2\%). In contrast, in children, cough is the most prevalent symptom in both presumed URTIs and ARS ${ }^{(3)}$.

A prospective study of 2610 patients diagnosed with ARS according to the EPOS $2012^{(4)}$ criteria separated patients into viral ARS (36\%) and post-viral ARS (63\%). Assessment of the severity of symptoms using a VAS found that $2 \%$ reported mild symptoms at baseline, $51 \%$ moderate symptoms and $44 \%$ reported symptoms to be severe. There was no significant difference in the frequency of nasal obstruction (98 vs. $97 \%$ ), rhinorrhoea (95 vs $94 \%$ ) facial pain and pressure (76 vs. $77 \%$ ) or loss of smell (59 vs. $63 \%$ ) comparing viral and post-viral groups. Disease severity measured using a VAS did not differ at baseline between groups (6.98 for viral vs. 7.13 for post viral ARS). Disease specific QOL measured with the SNOT-16 found statistically higher baseline scores in the post-viral group (38.7 vs. 36.0 in the viral ARS group), however, this difference is not clinically significant, A comparative study performed using the SF-36 found that patients with acute rhinosinusitis (which was not clearly defined) reported impaired quality of life compared with a control population, but were not as severely affected as patients with chronic rhinosinusitis ${ }^{(5)}$.
There are two validated disease-specific quality of life instruments for use in ARS. The Measurement of Acute Rhinosinusitis Instrument (MARS) demonstrates significantly higher scores in patients with ARS than healthy controls. The SNOT-16, a derivative of the RSOM-31 instrument originally developed and validated in a chronic rhinosinusitis cohort, has also been validated for use in $\mathrm{ARS}^{(6)}$.

\subsubsection{The burden of CRS}

CRS is associated with a wide range of symptoms, across sinonasal, aural, sleep and general domains. The 'cardinal' symptoms are considered to be nasal obstruction or congestion, nasal discharge (which can be anterior or posterior), alteration in sense of smell and facial pain and pressure.

Population studies show that symptoms of nasal obstruction and discharge are common. In a survey of unselected patients in primary care (Figure 3.1.1.) ${ }^{(7)}, 11.9 \%$ of adults sampled fulfilled the EPOS criteria for rhinosinusitis. With repeated review at six months, symptoms were persistent in $4.7 \%$ of the cohort ${ }^{(8)}$, suggesting the presence of CRS.

Using EPOS criteria to identify CRS in responses from a random sample of the general population, thereby preferentially selecting those with nasal obstruction and /or discharge shows a high prevalence of cardinal symptoms but provides only limited insight into the severity of patients with CRS successfully managed in a primary care setting ${ }^{(9)}$, as selection bias will likely identify more severe patients in secondary care.

The majority of studies evaluating the prevalence and severity of symptoms analyse responses in patients referred to secondary care, or those undergoing sinus surgery, therefore selecting those with symptoms more resistant to treatment in primary care. Surveys of patients seen in ENT outpatient clinics ${ }^{(10)}$ and those electing sinus surgery ${ }^{(11)}$ show that the cardinal symptoms are reported as the most severe and prevalent. Nasal obstruction and alteration in sense of smell and taste are both the most severe and prevalent symptoms in CRSwNP, while in CRSsNP, facial pain and nasal discharge are reported as equally severe as altered smell and taste, with nasal obstruction again being the most severe (Figures 3.1.1. and 3.1.2.). Fatigue and waking up 
Figure 3.1.1. Prevalence of cardinal symptoms of CRS in unselected patients in primary care, and CRS patients in the general population, in an outpatient setting and those undergoing surgery.

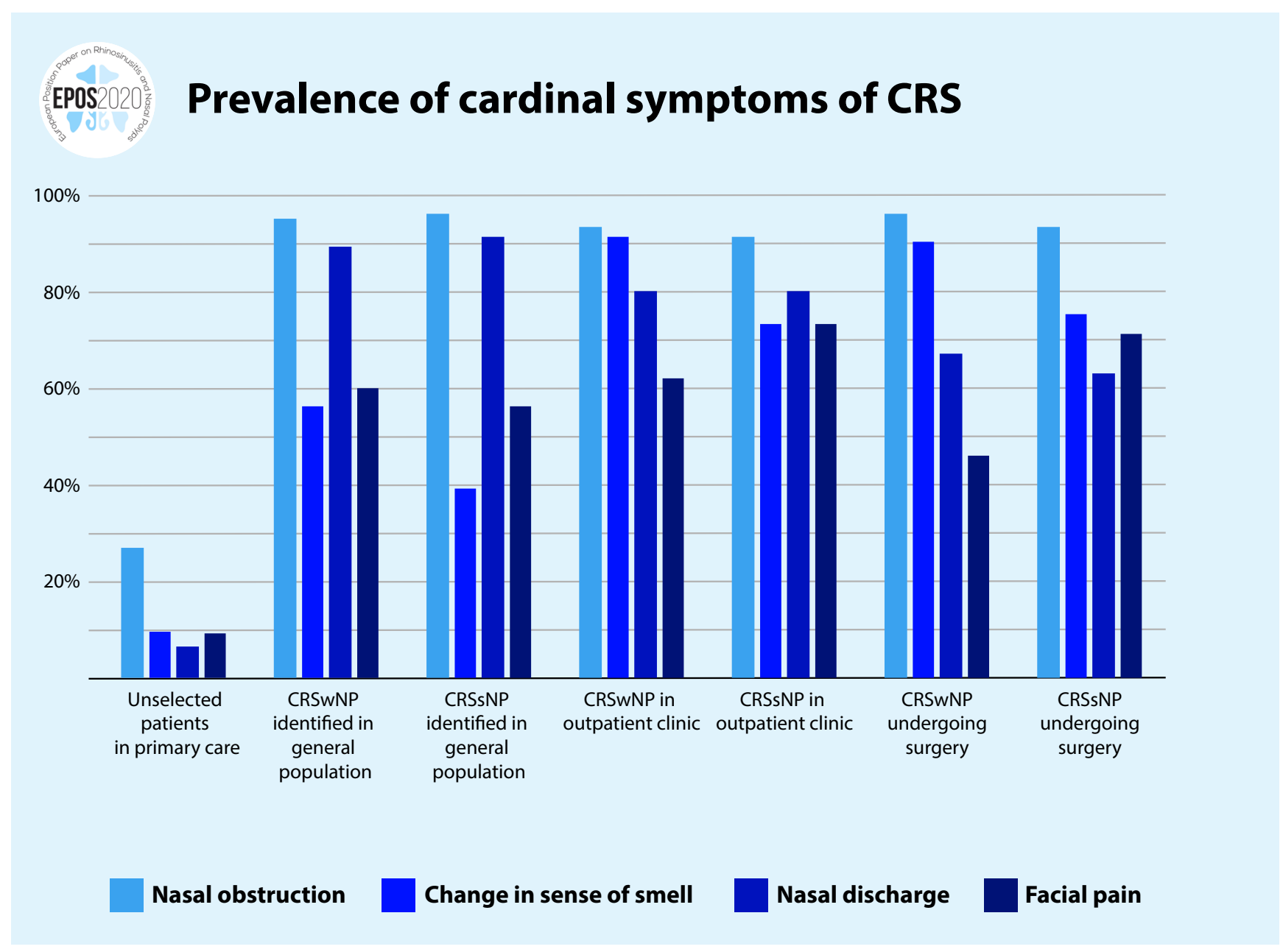

tired are also highly prevalent and bothersome. When a group of CRS patients (not differentiated by polyp status) were asked what symptoms they felt were the most important to experience improvement in after surgery, nasal obstruction was rated as "extremely" or "very" important by $93 \%$ of patients, followed by smell/taste, thick nasal discharge, need to blow nose, postnasal discharge, and sleep symptoms (range 61-72\%) $)^{(12)}$.

In patients presenting to ENT clinics, the presence of cardinal symptoms has a positive predictive value of 39.9, with high sensitivity but low specificity ${ }^{(13)}$ for a diagnosis of CRS. Similarly, when patients undergoing CT imaging for non-sinogenic conditions were surveyed, $50 \%$ of those who reported CRS symptoms were found to have normal CT scans (Lund-Mackay $=0)^{(14)}$. Asymptomatic changes are commonplace on CT imaging ${ }^{(14)}$. Individual symptoms cannot be used to reliably differentiate between CRS and other conditions, although the presence of hyposmia is predictive of $\mathrm{CRS}(15)$, while facial pain is negatively predictive $^{(16)}$.

The overall severity rating of symptoms is obviously highly dependent upon the population being studied. When asked how bothersome their symptoms were overall, the CRS patients identified in the general population study reported mean scores of 8.2 and 7.8 for CRSWNP and CRSsNP respectively, on a VAS scale of range $0-10$. Patients in secondary care awaiting surgery report mean symptom severity scores in the moderate to severe range, with a mean SNOT-22 score of $42.0^{(17)}$, compared with a control group where a mean score of 9.3 was reported. The median score of 7.0 was proposed as a threshold for normal scores ${ }^{(18)}$; CRSsNP patients had higher pre-operative baseline scores (44.2) compared with CRSwNP (41.0).

CRS has been shown to impact on patients' health related quality of life. Significant differences are found in all domains of the SF-36 compared with healthy controls ${ }^{(19)}$. In a landmark paper, Gliklich and Metson first demonstrated the impact of CRS on global quality of life, finding that CRS had a greater impact on social functioning than angina or chronic heart failure ${ }^{(20)}$. More recently, they have shown that health utility values, measured using the EQ-5D, were lower than the general population, and 


\section{EPSS2020 Severity of cardinal symptoms of CRS}

$$
5
$$

4

3

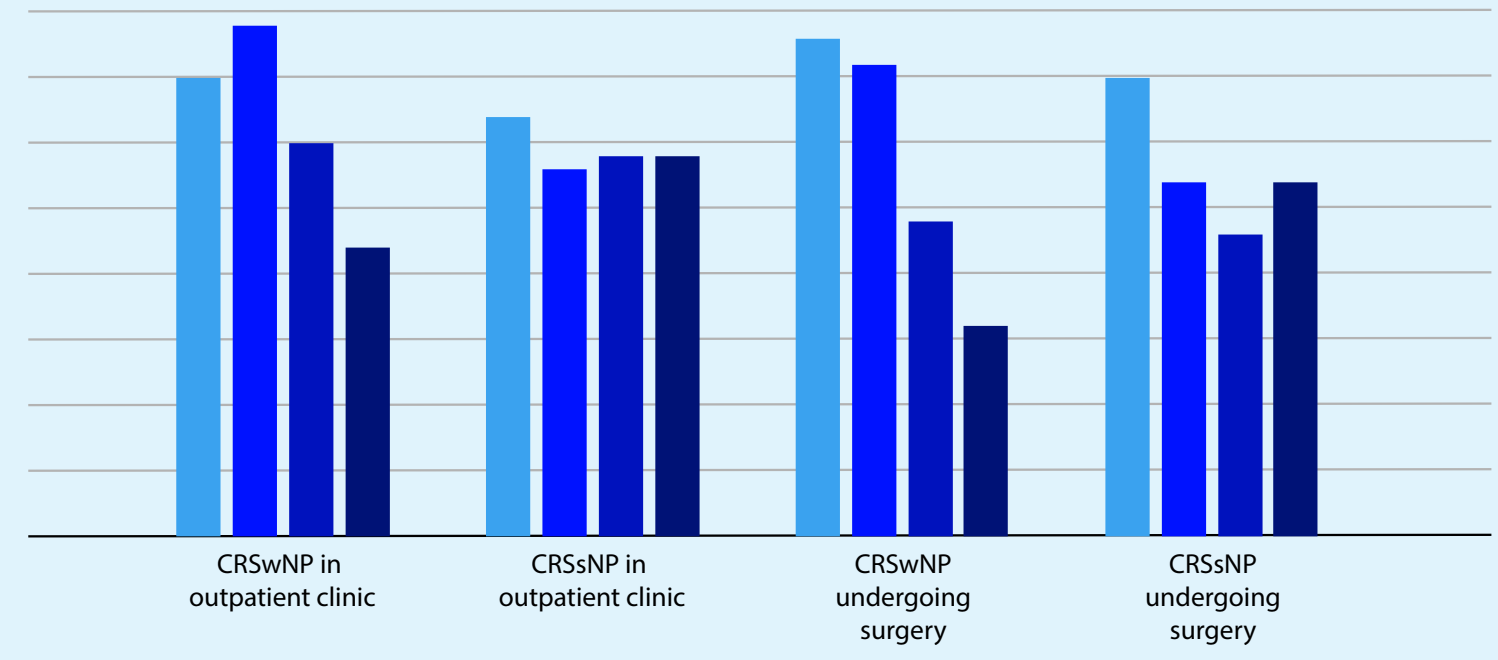

Nasal obstruction

Change in sense of smell

Nasal discharge

Facial pain

comparable to other chronic diseases such as asthma ${ }^{(21)}$. This is further discussed in the section on PROMS (see section 5.3.1.4.). Symptom severity has been shown to be influenced by gender, with females reporting greater symptom severity and impact on their quality of life, when measured with disease specific instruments or with global measures, such as the SF-36 or Eq-5D ${ }^{(19,21)}$. Co-morbid depressive illness is associated with worse CRS-specific quality of life ${ }^{(22)}$. Symptom severity may be in part determined by severity of disease but is further modified by intrinsic patient features (gender, ethnicity, religious and cultural beliefs), coexisting illnesses and extrinsic features such as socio-economic factors, and support systems. This likely explains the mismatch commonly found between objective and patient rated disease severity scales, such as has been found with radiological staging and symptom scores ${ }^{(23)}$.

\subsection{Costs of rhinosinusitis}

Research concerning the socioeconomic impact of rhinosinusitis is a nascent field. In particular, CRSw/sNP is a common condi- tion with long-term ramifications with regard to both medical and surgical treatments, associated total cost, as well as the impact of disease on patient productivity and quality-adjusted life years ${ }^{(24)}$. Total costs are often divided between direct and indirect costs where direct costs primarily refer to traditional healthcare costs such as physician visits, prescription medical therapy and surgery while indirect costs refer primarily to lost productivity in those suffering rhinosinusitis ${ }^{(25)}$. Conservative estimates suggest that for CRS, total costs are in excess of $\$ 30$ billion per year in the USA with $\$ 20$ billion accounted for in indirect costs ${ }^{(26)}$.

\subsubsection{Direct costs}

\subsubsection{Direct costs of chronic rhinosinusitis}

In 2009, Bhattacharyya published an assessment of the economic disease burden of rhinosinusitis ${ }^{(27)}$. Data were extracted from the National Health Interview Survey over a 10-year period of 1997-2006 in the USA. One-year disease prevalence showed 
that one quarter (23\%) of patients with CRS visited an emergency department, one third (34\%) saw a medical specialist, more than half (56\%) spent $\$ 500$ or more per year on health care. Health care spending was significantly greater in rhinosinusitis than that of other diseases such as peptic ulcer disease, acute asthma, and hay fever. In Europe two studies reported on direct costs of CRS, Wahid et al. reported $£ 2974$ on costs for primary and secondary care extrapolated for a year period compared to $£ 555$ in the control group and $£ 304$ versus $£ 51$ out-of-pocket expenditure (28). Lourijsen et al. found yearly direct costs of $€ 1501$ per year in a group of patients with CRSwNP(29).

\section{Health care spending was significantly greater in rhinosinusitis than in other diseases such as peptic ulcer disease, acute asthma, and hay fever.}

Direct costs of CRS, over and above inflation, appear to be increasing in the USA where most estimates originate. In 1999, Ray et al. estimated the total direct cost in the USA at nearly $\$ 6$ billion per year ${ }^{(30)}$. In 2011, Bhattacharyya estimated direct costs to be $\$ 8.6$ billion $^{(31)}$. In 2017, Rudmik established that the overall direct costs of CRS had increased to between $\$ 10$ and $\$ 13$ billion $^{(26)}$.

\section{In the USA, the direct costs for the management of $C R S$ have risen in recent years and are now between $\$ 10$ and $\$ 13$ billion per year, or $\$ 2609$ per patient per year.}

While total direct costs are important, in 2002, Murphy et al. examined the direct costs of an individual patient with CRS in the USA per year ${ }^{(32)}$. These patients accounted for $43 \%$ more outpatient and $25 \%$ more urgent care visits than patients without CRS. Patients with CRS filed $43 \%$ more prescriptions but had fewer inpatient hospital stays. The direct cost of treating an individual patient with CRS was $\$ 2,609$ per year, $6 \%$ more than the average adult. In Europe, a study executed by van Agthoven established that the direct costs of a patient treated in a university hospital for severe chronic rhinosinusitis were $€ 1861 /$ year $^{(33)}$.

The impact of nasal polyps on direct costs has also been a subject of interest. In Sweden, Berggren evaluated the costs of a scenario treating CRSWNP with budesonide following by sinus surgery when indicated versus direct surgery followed by budesonide ${ }^{(34)}$. For the surgical intervention a comparison was made between in-patient FESS, in the office polypectomy under sedation or in the office polypectomy under local anaesthesia. The scenario of treating CRSwNP with budesonide following by sinus surgery was due to the high success of the nasal corticosteroid treatment alone (yearly costs $€ 409-602$ ) , significantly less expensive than the scenarios that started with surgery: from $€ 67$ for the polypectomy in local treatment to $€ 976$ for the in-patient FESS. In the US, Bhattacharyya's 2009 study provided an assessment of the additional disease burden of nasal polyps in $\mathrm{CRS}^{(35)}$. Patients were included according to the Rhinosinusitis Symptom Inventory (Task Force on Rhinosinusitis criteria) and by findings with nasal endoscopy and on CT (Lund MacKay score). Three groups were composed: one with CRS without nasal polyps (CRSsNP), a second group with CRS with nasal polyps (CRSwNP) and a third with CRS with recurrent nasal polyps after surgery. While the groups with and without nasal polyps showed a clear difference in phenotype, this did not translate into a difference in expenditures for physician visits and medication costs between the first two groups. However, there was a difference in total medication costs for the last group with recurrent polyps after surgery with a higher cost for this group of $\$ 866$ compared to the $\$ 570$ for Group 1 and $\$ 565$ for Group 2. Further study of the differences in cost in patients with polyps were studied by Bhattacharyya et al. in 2019 using the Truven Health MarketScan US claims database ${ }^{(36)}$. Annual incremental costs were $\$ 11,507$ higher for patients with CRSwNP versus those without CRS. Costs were higher in subgroups of patients with CRSwNP undergoing functional endoscopy sinus surgery (FESS), with a comorbid diagnosis of asthma, receiving oral corticosteroids, or macrolides versus the overall CRSwNP group. This study did not include, generally speaking, patients treated with biologics. The authors concluded that patients with CRSwNP with high clinical burden had higher overall costs than CRSwNP patients without.

\section{The highest direct costs were associated with patients who had recurrent polyposis after surgery.}

Evaluating incremental direct costs is important to determine the cost directly attributable to the management of CRS. In 2011, an evaluation calculated the incremental health care utilization and expenditure for CRS in the USA ${ }^{(31)}$. Patient data were extracted from the Medical Expenditure Panel Survey. For utilization of health care, data show that CRS patients incurred 3.5 additional office visits and 5.5 additional prescriptions compared to patients without CRS. This extra utilization of healthcare evokes higher expenditures; a patient with CRS would have a substantial incremental increase in health care expenditures of approximately $\$ 800$ per year consisting of $\$ 346( \pm \$ 130)$ for office-based expenditures, $\$ 397( \pm \$ 88)$ for prescription expenditures and $\$ 90( \pm \$ 24)$ for self-expenditures.

\section{CRS leads to an incremental direct healthcare expenditure of 2500 euro per patient per year.}

Direct costs associated with severe CRS that eventually requires surgery have received additional attention. Bhattacharyya et al. reported the costs of managing CRS in the year prior to and fol- 
lowing endoscopic sinus surgery (ESS) ${ }^{(37)}$. Data from the Market Scan Commercial Claims and Encounters Database from 2003 to 2008 were evaluated. Patients with nasal polyps were excluded from this study. All sinus-related healthcare utilization costs were rolled into the study (medication, office visits, diagnostic assessment with radiology and endoscopy). Results show that in the year prior to ESS, costs increase to approximately $\$ 2,500$ with a clear increase in the six months directly preceding ESS; the first three months account for $\$ 361$ and final three months account for $\$ 1,965$. This is due to an increase in office visits, diagnostic investigations and medication use. The increase in prescription medication is primarily due to a higher antibiotic use; from $\$ 75$ in the first three months to $\$ 225$ in the second three months. The ESS-procedure and the 45-day post procedure period account for $\$ 7,726(\$ 7,554-\$ 7,898)$. In the first year following ESS, costs drop by $\$ 885$ to an average of $\$ 1,564$ per year. In the second year post procedure they drop an additional $\$ 446$ to $\$ 1,118$ per year. This decrease was mostly due to fewer physician visits; there was only a minor change in the costs of anti-inflammatory medication.

\section{Endoscopic sinus surgery is expensive but results in a decrease in direct costs in the subsequent two post-operative years.}

Surgery for CRS represents a substantial direct cost and depends on geography ${ }^{(37-39)}$. Based on the literature, the direct costs of ESS in the USA range between $\$ 8,500$ and $\$ 11,000$. However, direct costs are lower in Canada $(\$ 3,700)$, Taiwan $(\$ 1,900)$, and India $(\$ 1,100)$, all of these direct costs measured in 2016 USD.

\section{Patients with recurrent acute rhinosinusitis have average direct health care costs of $\$ 1,091 /$ year}

$$
\text { (USA 2012). }
$$

\subsubsection{Direct costs of acute rhinosinusitis}

While most ENT specialists spend much of their clinical time treating CRS, acute rhinosinusitis also presents in both non-recurrent (acute bacterial rhinosinusitis, ABRS) and recurrent forms (recurrent acute rhinosinusitis, RARS) ${ }^{(40)}$. Much less is known about the costs related to RARS. In 2004, Anand estimated that there were approximately 20 million cases of ABRS yearly in the USA ${ }^{(41)}$. However, RARS is more commonly treated by the rhinologist and it is estimated that 1 in 3,000 adults suffer from RARS $^{(42)}$. In 2012, Bhattacharyya found that RARS required an average of 5.6 health care visits/year and 9.4 prescriptions filled ( $40 \%$ antibiotic). Only $20 \%$ of patients had either nasal endoscopy or CT scan annually, indicating that it is likely that only a small proportion of these patients are referred for ENT-specialist evaluation. The individual patient annual direct healthcare costs of recurrent acute rhinosinusitis (RARS) averaged \$1,091/year:
\$210 for antibiotics, \$452 for other sinus-related prescriptions, $\$ 47$ for imaging and $\$ 382$ for other visit costs.

\subsubsection{Indirect costs for ARS and CRS}

The studies of direct medical costs of rhinosinusitis demonstrate a tremendous socioeconomic burden. Interestingly, the indirect costs of rhinosinusitis are much greater than the direct costs. Since $85 \%$ of patients with rhinosinusitis are of working age (range: 18-65 years old), indirect costs such as missed workdays (absenteeism) and decreased productivity at work (presenteeism) significantly add to the economic burden of the disease ${ }^{(43)}$.

\section{Rhinosinusitis is one of the top 10 most costly health conditions to US employers.}

Goetzel et al. attempted to quantify the indirect costs of rhinosinusitis ${ }^{(44)}$. Their 2003 study resulted in rhinosinusitis being named one of the top 10 most costly health conditions to US employers. A large multi-employer database was used to track insurance claims through employee health insurance, absentee days, and short-term disability claims. Episodes of illness were linked to missed workdays and disability claims, accurately correlating absenteeism to a given disease. In a large sample size ( 375,000 employees), total healthcare payments per employee per year for rhinosinusitis (both acute and chronic) were found to be $\$ 60.17,46 \%$ of which came from the cost of absenteeism and disability. These figures approximate the cost to employers, disregarding the cost incurred by other parties, and therefore tremendously underestimate the entire economic burden of the disease.

\section{Indirect costs account for a majority of the total costs of rhinosinusitis.}

Ray et al. estimated by the 1994 National Health Interview Survey that the number of missed workdays due to rhinosinusitis was 12.5 million and restricted activity days was 58.7 million days ${ }^{(45)}$. In his 2003 study, Bhattacharyya used patientcompleted surveys from 322 patients to estimate the direct and indirect costs of chronic rhinosinusitis ${ }^{(46)}$. Patients completed a survey assessing symptoms of disease, detailing medication use, and quantifying missed workdays attributable to CRS. The report concluded that the cost of treating CRS per patient totalled $\$ 1,539$ per year with $40 \%$ of these costs due to indirect costs of missed work; the mean number of missed workdays in this sample of 322 patients was 4.8 days/year ( $95 \% \mathrm{Cl}$ : $3.4-6.1$ ). The study was followed up in 2009 and 2012 using data from the National Health Interview Survey between 1997 and 2006 encompassing nearly 315,000 individuals and reported that patients with rhinosinusitis missed on average 5.7 days of work per year ${ }^{(27)}$. These cohorts report on all patients with CRS and 
therefore include less severe forms of the disease that are likely never referred for ENT-specialist management. Stankiewicz et al. reported on the rates of absenteeism and presenteeism in a population of 56 patients undergoing surgical intervention for chronic rhinosinusitis. Prior to surgery, they reported a $6.5 \%$ rate of absenteeism (i.e. $6.5 \%$ of work time missed) and $36 \%$ rate of presenteeism (reduction of on-the-job effectiveness). When combined, the rate of absenteeism and presenteeism yielded a $38 \%$ work productivity loss in the study population, but no dollar value was placed on this figure ${ }^{(47}$. Supporting this, Stull et al. reported that nasal congestion alone resulted in poor sleep, increased fatigue, and daytime sleepiness contributing to decreased work productivity ${ }^{(48)}$. In 2014, Rudmik et al. specifically evaluated recalcitrant CRS in 55 patients and found that patients with this more severe form of CRS had mean annual presenteeism and absenteeism rates of 25 - 39 days per patient per year equating to an average indirect cost of over $\$ 10,000$ per patient per year ${ }^{(49)}$. Overall, the total indirect costs of CRS were estimated to be in excess of $\$ 20$ billion per year in the USA ${ }^{(26)}$.
Yip reported in the patients in a Canadian tertiairy care centre an average of 20.6 workdays missed over a 12-month period ${ }^{(50)}$. In Europe Wahid reported a total number of missed workdays of 18.7 per patient per year ${ }^{(28)}$. Lourijsen found a total of missed workdays of 10.6, work-related productivity loss of 30.4 days and unpaid work productivity loss of 23.7 days leading to total indirect costs of $€ 5659$ per patient/year ${ }^{(29)}$.

\section{A major component of the indirect costs results from absenteeism and presenteeism and is in excess of $\$ 20$ billion per year in the USA.}

More recent data has emerged which demonstrate changes in productivity costs after treatment of chronic rhinosinusitis, with differential changes across symptom domain and severity ${ }^{(51-56)}$. While patients who were considered candidates for ESS who elected to continue medical therapy showed no improvement in average measures of productivity, patients who elected ESS showed substantial improvement in productivity.

\section{References}

1. Stjärne P, Odebäck P, Ställberg B, Lundberg J, Olsson P. High costs and burden of illness in acute rhinosinusitis: real-life treatment patterns and outcomes in Swedish primary care. Primary Care Respiratory Journal 2012;21:174-9.

2. Klossek JM, Mesbah K. Presentation and treatment of acute maxillary sinusitis in general practice: a French observational study. Rhinology 2011;49:84-9.

3. Shaikh N, Hoberman A, Kearney DH, et al. Signs and symptoms that differentiate acute sinusitis from viral upper respiratory tract infection. Pediatr Infect Dis J 2013;32:1061-5

4. Jaume F, Quintó L, Alobid I, Mullol J. Overuse of diagnostic tools and medications in acute rhinosinusitis in Spain: a population-based study (the PROSINUS study). BMJ open 2018;8:e018788.

5. Teul I, Zbislawski W, Baran S, Czerwinski F, Lorkowski J. Quality of life of patients with diseases of sinuses. J Physiol Pharmacol 2007:58 Suppl 5:691-7.

6. Garbutt J, Spitznagel E, Piccirillo J. Use of the modified SNOT-16 in primary care patients with clinically diagnosed acute rhinosinusitis. Arch Otolaryngol Head Neck Surg. 2011;137:792-7.

7. Hirsch AG, Stewart WF, Sundaresan AS, et al. Nasal and sinus symptoms and chronic rhinosinusitis in a population-based sample. Allergy 2017;72:274-81.

8. Sundaresan AS, Hirsch AG, Young AJ, et al. Longitudinal Evaluation of Chronic Rhinosinusitis Symptoms in a PopulationBased Sample. J Allergy Clin Immunol Pract 2018;6:1327-35.e3.
9. Palmer JN, Messina JC, Biletch R, Grosel K, Mahmoud RA. A cross-sectional, population-based survey of U.S. adults with symptoms of chronic rhinosinusitis. Allergy Asthma Proc 2019;40:48-56.

10. Dietz de Loos DA, Hopkins C, Fokkens WJ. Symptoms in chronic rhinosinusitis with and without nasal polyps. Laryngoscope 2013;123:57-63.

11. Abdalla S, Alreefy $H$, Hopkins C. Prevalence of sinonasal outcome test (SNOT-22) symptoms in patients undergoing surgery for chronic rhinosinusitis in the England and Wales National prospective audit. Clin Otolaryngol 2012;37:276-82.

12. Mattos JL, Rudmik L, Schlosser RJ, et al. Symptom importance, patient expectations, and satisfaction in chronic rhinosinusitis. Int Forum Allergy Rhinol 2019;9:593600.

13. Bhattacharyya N, Lee LN. Evaluating the diagnosis of chronic rhinosinusitis based on clinical guidelines and endoscopy. Otolaryngol Head Neck Surg 2010;143:14751.

14. Dietz de Loos D, Lourijsen ES, Wildeman MAM, et al. Prevalence of chronic rhinosinusitis in the general population based on sinus radiology and symptomatology. J Allergy Clin Immunol 2019;143:1207-14.

15. Bhattacharyya N. Clinical and symptom criteria for the accurate diagnosis of chronic rhinosinusitis. Laryngoscope 2006;1 16:1-22.

16. Hsueh WD, Conley DB, Kim H, et al. Identifying clinical symptoms for improving the symptomatic diagnosis of chronic rhinosinusitis. Int Forum Allergy Rhinol 2013;3:307-14.

17. Hopkins C, Browne JP, Slack R, et al. The national comparative audit of surgery for nasal polyposis and chronic rhinosinusitis. Clin Otolaryngol 2006;31:390-8.

18. Gillett S, Hopkins C, Slack R, Browne JP. A pilot study of the SNOT 22 score in adults with no sinonasal disease. Clin Otolaryngol 2009;34:467-9.

19. Fu C-H, Huang C-C, Chen Y-W, Chang $\mathrm{P}-\mathrm{H}$, Lee T-J. Nasal Nitric Oxide in Relation to Quality-of-life Improvements after Endoscopic Sinus Surgery. Am J Rhinol Allergy 2015;29:e187-e91.

20. Gliklich RE, Metson R. The health impact of chronic sinusitis in patients seeking otolaryngologic care. Otolaryngol Head Neck Surg 1995;113:104-9.

21. Remenschneider AK, Scangas G, Meier JC, et al. EQ-5D-derived health utility values in patients undergoing surgery for chronic rhinosinusitis. Laryngoscope 2015;125:105661.

22. Schlosser RJ, Gage SE, Kohli P, Soler ZM. Burden of illness: A systematic review of depression in chronic rhinosinusitis. Am J Rhinol Allergy. 2016;30:250-6.

23. Hopkins C, Browne JP, Slack R, Lund V, Brown P. The Lund-Mackay staging system for chronic rhinosinusitis: How is it used and what does it predict? Otolaryngol Head Neck Surg 2007;137:555-61.

24. Rudmik L, Smith TL. Economic Evaluation of a Steroid-Eluting Sinus Implant following Endoscopic Sinus Surgery for Chronic Rhinosinusitis. Otolaryngol Head Neck Surg 2014;151:359-66.

25. Caulley L, Thavorn K, Rudmik L, Cameron C, Kilty SJ. Direct costs of adult chronic rhinosinusitis by using 4 methods of estimation: Results of the US Medical Expenditure 
Panel Survey. I Allergy Clin Immunol 2015;136:1517-22.

26. Rudmik L. Economics of Chronic Rhinosinusitis. Curr Allergy Asthma Rep 2017;17:20

27. Bhattacharyya N. Contemporary assessment of the disease burden of sinusitis. Am J Rhinol Allergy 2009;23:392-5.

28. WahidNW, Smith R, Clark A, Salam M, Philpott CM. The socioeconomic cost of chronic rhinosinusitis study. Rhinology 2020, 58,2:112-125.

29. Lourijsen ES, Fokkens WJ, Reitsma S. Direct and indirect costs of adult patients with chronic rhinosinusitis with nasal polyps. Rhinology 2020; 58,3:213-217.

30. Ray NF, Baraniuk JN, Thamer M, et al. Healthcare expenditures for sinusitis in 1996: contributions of asthma, rhinitis, and other airway disorders. J Allergy Clin Immunol 1999;103:408-14.

31. Bhattacharyya N. Incremental health care utilization and expenditures for chronic rhinosinusitis in the United States. Ann Otol Rhinol Laryngol 2011;120:423-7.

32. Murphy MP, Fishman P, Short SO, et al. Health care utilization and cost among adults with chronic rhinosinusitis enrolled in a health maintenance organization Healthcare expenditures for sinusitis in 1996: contributions of asthma, rhinitis, and other airway disorders. Otolaryngol Head Neck Surg 2002;127:367-76.

33. van Agthoven $M$, Uyl-de Groot CA, Fokkens WJ, van de Merwe JP, Busschbach JJV, Agthoven V. Cost analysis of regular and filgrastim treatment in patients with refractory chronic rhinosinusitis. Rhinology 2002;40:69-74.

34. Berggren F, Johansson L. Cost effectiveness of nasal budesonide versus surgical treatment for nasal polyps. Pharmacoeconomics 2003;21:351-6.

35. Bhattacharyya N. Assessing the additional disease burden of polyps in chronic rhinosinusitis. Ann Otol Rhinol Laryngol 2009;1 18:185-9.

36. Bhattacharyya N, Villeneuve $\mathrm{S}$, Joish VN, et al. Cost burden and resource utilization in patients with chronic rhinosinusitis and nasal polyps. Laryngoscope 2019, 10.1002/ lary.27852:lary.27852.

37. Bhattacharyya N, Orlandi RR, Grebner J, Martinson M. Cost burden of chronic rhinosinusitis: a claims-based study. Otolaryngol Head Neck Surg 2011;144:4405.

38. Gross RD, Sheridan MF, Burgess LP. Endoscopic Sinus Surgery Complications in Residency. Laryngoscope 1997;107:1080-5.

39. Gliklich RE, Metson R. Economic implications of chronic sinusitis. Otolaryngol Head Neck Surg 1998;118:344-9.

40. Orlandi RR, Kingdom TT, Hwang PH, et al. International Consensus Statement on Allergy and Rhinology: Rhinosinusitis. Int Forum Allergy Rhinol 2016;6:S22-S209.

41. Anand VK. Epidemiology and economic impact of rhinosinusitis. Ann Otol Rhinol Laryngol 2004;113:3-5.

42. Bhattacharyya N, Grebner J, Martinson NG. Recurrent Acute Rhinosinusitis: Epidemiology and Health Care Cost Burden. Otolaryngol Head Neck Surg 2012;146:30712.

43. Blackwell DL, Collins JG, Coles R. Summary health statistics for U.S. adults: National Health Interview Survey, 1997. Vital Health Stat 10 2002:1-109.

44. Goetzel RZ, Hawkins K, Ozminkowski RJ, Wang S. The health and productivity cost burden of the "top 10" physical and mental health conditions affecting six large U.S. employers in 1999. J Occup Environ Med 2003:45:5-14

45. Ray NF. Healthcare expenditures for sinusitis in 1996: contributions of asthma, rhinitis, and other airway disorders. J Allergy Clin Immunol 1999;103:408-14.

46. Bhattacharyya N. The economic burden and symptom manifestations of chronic rhinosinusitis. Am J Rhinol 2003;17:27-32.

47. Stankiewics J, Stankiewicz J, Tami T, Truitt T, Atkins J, Winegar B, et al. Impact of chronic rhinosinusitis on work productivity through one-year follow-up after baloon dilation of the ethmoid infundibulum. Int Forum Allery Rhinol. 2011;1:38-45.

48. Stull DE, Roberts L, Frank L, Heithoff $K$. Relationship of nasal congestion with sleep, mood, and productivity. Curr Med Res Opin 2007;23:811-9.

49. Rudmik L, Smith TL, Schlosser RJ, Hwang $\mathrm{PH}$, Mace JC, Soler ZM. Productivity costs in patients with refractory chronic rhinosinusitis. Laryngoscope 2014;124:2007-12.

50. Yip J, Vescan AD, Witterick IJ, Monteiro E. The personal financial burden of chronic rhinosinusitis: A Canadian perspective. Am J Rhinol Allergy 2017;31:216-21.

51. Rudmik L, Soler ZM, Smith TL, Mace JC, Schlosser RJ, DeConde AS. Effect of Continued Medical Therapy on Productivity Costs for Refractory Chronic Rhinosinusitis. JAMA Otolaryngol Head Neck Surg 2015;141:969-73.

52. Rudmik L, Smith TL, Mace JC, Schlosser RJ, Hwang PH, Soler ZM. Productivity costs decrease after endoscopic sinus surgery for refractory chronic rhinosinusitis. Laryngoscope 2016;126:570-4.

53. Luk L, Mace JC, Bhandarkar ND, Sautter NB. Comparison of electrosurgical plasma coagulation and potassium-titanyl-phosphate laser photocoagulation for treatment of hereditary hemorrhagic telangiectasiarelated epistaxis. Int Forum Allergy Rhinol 2014;4:640-5.

54. Beswick DM, Mace JC, Rudmik L, Soler ZM, DeConde AS, Smith TL. Productivity changes following medical and surgical treatment of chronic rhinosinusitis by symptom domain. Int Forum Allergy Rhinol 2018:8:1395-405.

55. Chowdhury NI, Mace JC, Smith TL, Rudmik L. What drives productivity loss in chronic rhinosinusitis? A SNOT-22 subdomain analysis. Laryngoscope 2018;128:23-30.

56. Smith KA, Rudmik L. Medical therapy, refractory chronic rhinosinusitis, and productivity costs. Curr Opin Allergy Clin Immunol 2017;17:5-11. 


\section{Acute rhinosinusitis including common cold - and recurrent $A R S$ in adults and children}

\subsection{Epidemiology of acute rhinosinusitis (ARS)}

\subsubsection{Common cold, post-viral rhinosinusitis and acute bacterial rhinosinusitis}

Many patients consult their primary care clinician or pharmacist with problems associated with upper respiratory infections. The majority are self-limiting and can be classified as common colds. Common colds are defined as acute viral rhinosinusitis with a duration of symptoms of $<10$ days (but less than 12 weeks) (Figure 4.1.1).

When symptoms increase after five days, or when symptoms are persistent for more than 10 days, with less than 12 weeks duration, then EPOS classifies this as acute post-viral rhinosinusitis. Only a minority of acute rhinosinusitis is from bacterial origin. Acute bacterial rhinosinusitis if defined by EPOS by at least three symptoms/signs of the five below:

- Discoloured mucus

- $\quad$ Severe local pain (often unilateral)

- Fever $>\underline{38^{\circ} \mathrm{C}}$

- $\quad$ Raised CRP/ESR

- 'double' sickening

This chapter describes the epidemiology and predisposing factors for different forms of acute rhinosinusitis (ARS).

\subsubsection{Incidence of ARS in the population}

ARS is a common problem, the precise incidence of which is difficult to estimate. The incidence of acute viral rhinosinusitis (common cold) is very high, as previously described ${ }^{(1)}$ and as summarised in Table 4.1.1. It has been estimated that adults suffer two to five episodes of viral ARS (or colds) per year and school children may suffer seven to 10 colds per year ${ }^{(1,2)}$. In a recent Dutch paper using the GA2LEN questionnaire a prevalence of $18 \%$ (17-21\%) was found for symptoms pointing to post-viral/ ABRS ARS in three different cities in the Netherlands ${ }^{(3)}$. Approximately $0.5-2 \%$ of viral upper respiratory tract infections are complicated by bacterial infection ${ }^{(1,4)}$ (Figure 4.1.2.).

\subsubsection{Incidence of acute bacterial rhinosinusitis (ABRS)}

The precise incidence of ABRS is not known. This has been placed at $0.5-2 . \%$ of all viral infections ${ }^{(5)}$. To develop acute bacterial rhinosinusitis it is reasonable to assume that this is a compli-
Figure 4.1.1. Definition of acute rhinosinusitis.

\section{(Bos}

Increase in symptoms after 5 days, or persistent symptoms after 10 days with less than 12 weeks duration

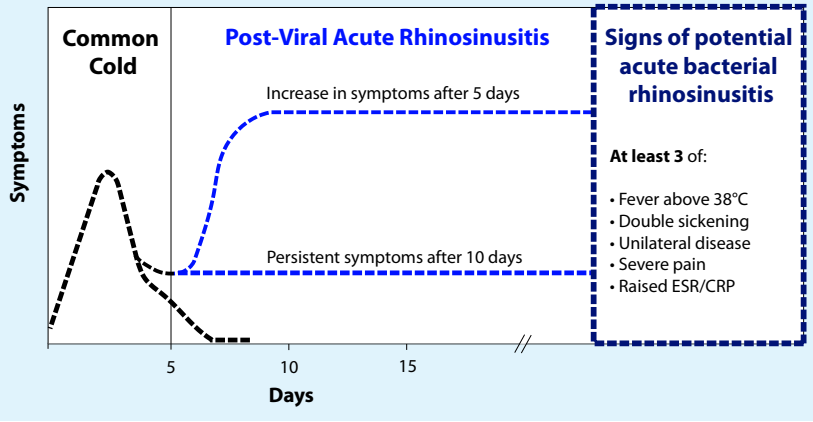

CRP, C-reactive protein; ESR, erythrocyte sedimentation rate.

Fig 4.1.2. The incidence of different forms of ARS: Common cold, postviral rhinosinusitis and acute bacterial rhinosinusitis (ABRS). Antibiotics are only indicated in a small part of the patients with ABRS.

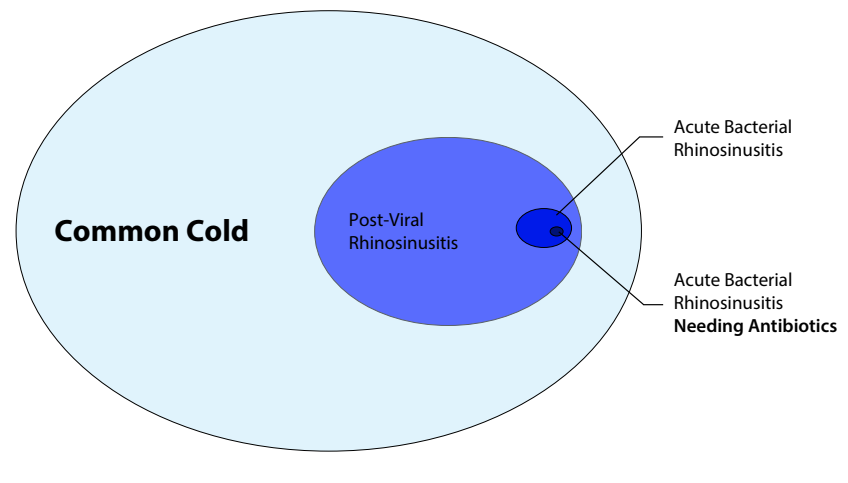

cation of (post)viral ARS, a recent review described a collection of factors which make this outcome more likely (Table 4.1.2.) ${ }^{(6)}$. It is also important to understand the natural history of a bout of ARS and the spectrum of symptoms which accompany it (Figure 4.1.3). It can be seen that the majority of the symptom complex has resolved by day 7 but that nasal discharge and cough may last for a further three or four days (Figure 4.1.3.). It is clear that most symptoms, however, resolve by day 5 and that in general it 
Table 4.1.1. Acute rhinosinusitis (ARS) incidence and prevalence in primary care studies.

\begin{tabular}{|c|c|c|}
\hline Study Author, year & Evidence & Type of study \\
\hline $\begin{array}{l}\text { Hoffmans } \\
2018^{(3)}\end{array}$ & $\begin{array}{l}\text { Prevalence of (post-viral and ABRS) ARS based on the EPOS criteria 18\% (17- } \\
21 \%)\end{array}$ & Prospective population study \\
\hline $\begin{array}{l}\text { Hoffmans } \\
2015^{(17)}\end{array}$ & $\begin{array}{l}\text { Incidence of (acute) rhinosinusitis in primary care in the Netherlands } \\
18.8 / 1000 \text { patient years }\end{array}$ & $\begin{array}{l}\text { Retrospective primary care morbidity } \\
\text { registration }\end{array}$ \\
\hline $\begin{array}{l}\text { Uijen } \\
2011^{(374)}\end{array}$ & $\begin{array}{l}\text { Incidence of acute rhinosinusitis during } 2002 \text { to } 2008 \text { : } \\
0-4 \text { years: } 2 / 1000 \text { per year in all years. } \\
\text { 5-14 years: } 7 / 1000 \text { in } 2002 \text { reducing to } 4 / 1000 \text { in } 2008(p<0.001) \\
12-17 \text { years: } 18 / 1000 \text { per year in all years. }\end{array}$ & Retrospective, population study \\
\hline $\begin{array}{l}\text { Oskarsson } \\
2011^{(375)}\end{array}$ & $\begin{array}{l}\text { Incidence of ARS is } 3.4 \text { cases per } 100 \text { inhabitants per year, or } 1 \text { in } 29.4 \text { patients } \\
\text { visit their GP due to acute rhinosinusitis. }\end{array}$ & Retrospective population study \\
\hline $\begin{array}{l}\text { Wang } \\
2011^{(176)}\end{array}$ & $\begin{array}{l}\text { 6-10\% of patients present at GP, otolaryngologist or paediatric out-patient } \\
\text { practices with ARS }\end{array}$ & Multi-national questionnaire survey \\
\hline $\begin{array}{l}\text { Bhattacharyya } \\
2011^{(31)}\end{array}$ & $\begin{array}{l}\text { Point prevalence of } 0.035 \% \text { for recurrent acute rhinosinusitis during } 2003 \text { - } \\
2008 \text {. }\end{array}$ & Retrospective cohort study \\
\hline $\begin{array}{l}\text { Meltzer, Kaliner, Kaliner } \\
2011,1997,1997^{(168,376)}\end{array}$ & 1 in 7 adults affected by rhinosinusitis in USA & Guidelines \\
\hline $\begin{array}{l}\text { Neumark } \\
2009^{(45)}\end{array}$ & $\begin{array}{l}7.5 \% \text { of consultations for respiratory tract infections (or } 1 \text { in every } 13.3 \text { ) were } \\
\text { attributable to sinusitis. Expanding to all primary care consultations, } 19.3 \\
\text { consultations/1000 patients were attributable to sinusitis. }\end{array}$ & Prospective population study \\
\hline $\begin{array}{l}\text { Bhattacharyya } \\
2009^{(50,377)}\end{array}$ & For 1997-2006, 1-year prevalence of sinusitis (all forms) was $15.2 \%$ & Retrospective cohort study \\
\hline $\begin{array}{l}\text { Fokkens } \\
2007^{(378)}\end{array}$ & $\begin{array}{l}\text { For } 1999,8.4 \% \text { of the Dutch population reported at least one episode of acute } \\
\text { rhinosinusitis. }\end{array}$ & Guideline \\
\hline $\begin{array}{l}\text { van Gageldonk-Lafeber } \\
2005^{(15)}\end{array}$ & $\begin{array}{l}\text { Incidence of acute respiratory tract infection (including ARS) during 2000- } \\
2003 \text { was } 54.5 \text { cases / } 1000 \text { patient-years, or } 1 \text { in every } 18.3 \text { consultations }\end{array}$ & Prospective case-control study \\
\hline $\begin{array}{l}\text { Cherry } \\
2005^{(379)}\end{array}$ & $\begin{array}{l}\text { In the USA, upper respiratory tract infection is third most common cause of a } \\
\text { primary care consultation, of which a third is attributable to ARS. }\end{array}$ & National Survey \\
\hline $\begin{array}{l}\text { Louie } \\
2005^{(380)}\end{array}$ & $\begin{array}{l}\text { In US study conducted during January to March } 2002,9 \% \text { of previously heal- } \\
\text { thy patients presented with acute sinusitis. }\end{array}$ & Prospective study \\
\hline $\begin{array}{l}\text { Varonen, Rautakorpi } \\
2004,2001^{(381,382)}\end{array}$ & $\begin{array}{l}\text { During } 1998-1999,12 \% \text { of patients were diagnosed with ARS. } 12 \% \text { of consulta- } \\
\text { tions for infection (all cause) over this time period were attributable to ARS. }\end{array}$ & $\begin{array}{l}\text { Cross-sectional multi-centre } \\
\text { epidemiological survey }\end{array}$ \\
\hline $\begin{array}{l}\text { Bachert } \\
2003^{(2)}\end{array}$ & $\begin{array}{l}\text { Between July } 2000 \text { and June } 20016.3 \text { million separate diagnoses of acute } \\
\text { sinusitis were identified in Germany, resulting in } 8.3 \text { million prescription }\end{array}$ & Review \\
\hline
\end{tabular}

ABRS, acute bacterial rhinosinusitis; ARS, acute rhinosinusitis; GP, general practitioner.

is impossible to differentiate between bacterial and non-bacterial before this time, although the possibility of bacterial infection increases if there is deterioration in symptoms after day $5^{(6)}$. However, some guidelines state that symptoms should go on for longer before bacteria are implicated ${ }^{(7)}$. A recent review and meta-analysis suggests that the percentage of bacterial infection is somewhat higher than has previously been thought, but recognises the complexity of the diagnosis in both radiographical diagnosis, with abnormal findings being associated with the presence of non-pathogenic bacteria and bacterial sampling techniques which may suggest differing levels of infection or contamination. They found that even when the strictest clinical and radiologic criteria are applied, only $53 \%$ of cultures are positive for pathogenic bacteria. They recommend further research is needed ${ }^{(8)}$.

What is very clear is that bacterial rhinosinusitis is greatly over-diagnosed with concomitant overuse of both diagnostic tools and of antibiotics, with up to $60 \%$ receiving a course of antibiotics on day 1 of an event ${ }^{(9-11)}$. Furthermore, early administration of antibiotics appears to have little or no bearing on the development of complications of ARS (12-14).

\subsubsection{Health seeking in ARS}

Patients with common cold and post-viral rhinosinusitis will often seek help from their GP.

In a three-year case-control study of the Dutch population, van Gageldonk-Lafeber estimated that annually, 900,000 individual patients (545/10,000 patient years) consulted their primary care physician for acute respiratory tract infection and that the most common aetiology was viral infection ${ }^{(15)}$.

In the USA, between 2000-2009, acute rhinosinusitis was diagnosed in $0.5 \%$ (95\% confidence interval $(\mathrm{Cl}), 0.4 \%-0.5 \%$ ) of 
Table 4.1.2. Predisposing factors for acute bacterial rhinosinusitis.

\section{Dental: infections and procedures}

latrogenic causes: sinus surgery, nasogastric tubes, nasal packing, mechanical ventilation

Immunodeficiency: human immunodeficiency virus infection, immunoglobulin deficiencies Impaired ciliary motility: smoking, cystic fibrosis, Kartagener syndrome, immotile cilia syndrome

Mechanical obstruction: deviated nasal septum, nasal polyps, hypertrophic middle turbinates, tumour, trauma, foreign body, granulomatosis with polyangiitis

Mucosal oedema: preceding viral upper respiratory infection, allergic rhinitis, vasomotor rhinitis

all outpatient visits among adults, averaging 19.4 visits $(95 \% \mathrm{Cl}$, 16.5-22.3) per 1000 adults and this did not change during the study period ${ }^{(16)}$. A recent Dutch paper found a comparable figure using two different Dutch GP registries; an incidence of consultations for acute rhinosinusitis of 18.8-28.7 per 1000 patient years. Because these registries do not make a clear differentiation between ARS and CRS a proportion of these consultation might have been for CRS. Medication was prescribed in over $90 \%$ of cases $^{(17)}$.

A global analysis reveals that unspecified Upper Respiratory Tract Infection (URTI) is the most common cause for consultation in the developing world and the second most common reason for consultation. In contrast, acute rhinosinusitis was not specified by clinicians although patients rated it as the thirteenth most common cause for seeking medical help ${ }^{(18)}$. A very detailed study of activity in Australian primary care demonstrated that acute /chronic rhinosinusitis accounted for $0.8 \%$ of total problems but was encountered in $1.3 \%$ of consultations (in primary care patients frequently consult with more than one clinical problem) $)^{(19)}$.

It is, however, difficult to be precise concerning prevalence:

Flgure 4.1.3. Common cold symptoms.

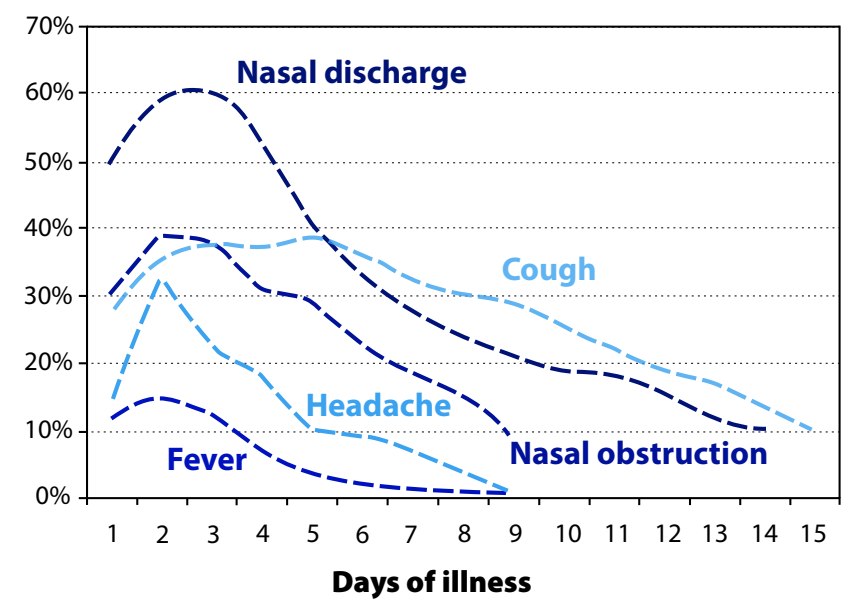

diagnostic criteria differ from place to place and environment. Primary care, by its very nature sees the vast majority of patients with acute respiratory infections, however, diagnostic labelling and subsequent coding is not always accurate with failure to differentiate between acute and chronic rhinosinusitis ${ }^{(17)}$. Similarly patients presenting to academic centres of excellence appear to have lower diagnostic rates, probably due to a more rigorous diagnostic approach ${ }^{(20)}$. Thus any estimation of incidence and prevalence has to be largely inferred from examining data collected retrospectively while providing routine clinical care, or collected prospectively.

A recent study suggests that both prevalence of viruses and climatic factors influence the expression of influenza type illness, suggesting that fluctuations in variability of numbers of presentations are to be expected ${ }^{(21)}$. Studies of prescribing habits may also shed light as to what the real prevalence of patients with ARS seeking medical attention might be in the general population. A retrospective study of 3.7 million patients revealed that 74,359 patients had had a consultation for ARS over a two-year time span suggesting a consultation rate of $1 \%$ of the population per annum ${ }^{(22)}$. A longitudinal study of 856 primary care trainees in Australia (108.759 individual consultations with 169,303 problems/diagnoses) resulted in a prevalence of $0.9 \%$ of acute rhinosinusitis of all problems or $1.39 \%$ of individual consultations ${ }^{(23)}$.

A cross-sectional study including GPs from two Nordic countries, two Baltic countries and two Hispano-American countries registered patients with respiratory tract infections (RTIs) during three weeks in January 2008. In total, 618 participating GPs registered 33.273 patients with RTIs, of whom 1150 (3.46\%) were considered to have acute post-viral or acute bacterial rhinosinusitis as defined by EPOS. Over $50 \%$ of the patients with acute rhinosinusitis had symptoms for $<5$ days and most had no fever ${ }^{(24)}$. The wide variability in diagnostic rates may also indicate geographical factors, the cost or ease of accessing healthcare, diagnostic norms in differing countries, patient expectations, patient worries, concerns or beliefs, and clinical expertise ${ }^{(24)}$.

This problem is further compounded and confounded with misdiagnosis especially regarding migraine headaches ${ }^{(25-27)}$.

\subsubsection{Conclusion}

In summary, it is difficult to give a precise estimate of the prevalence of ARS. Viral ARS (common cold) is very common and it has been estimated that adults suffer two to five and school children may suffer seven to 10 episodes per year. The only available prospective population study evaluating EPOS defined post-viral ARS (and ABRS) points to a prevalence of $18 \%{ }^{(3)}$ and (post-viral and ABRS) ARS is likely to be responsible for $1-2 \%$ of consultations in primary care. Carefully constructed prospective epidemiological studies with valid diagnoses of ARS may give a clearer picture of the real burden of ARS. 
Figure 4.2.1. Delphi: In the diagnosis of recurrent acute rhinosinusitis do you rely on: History alone?

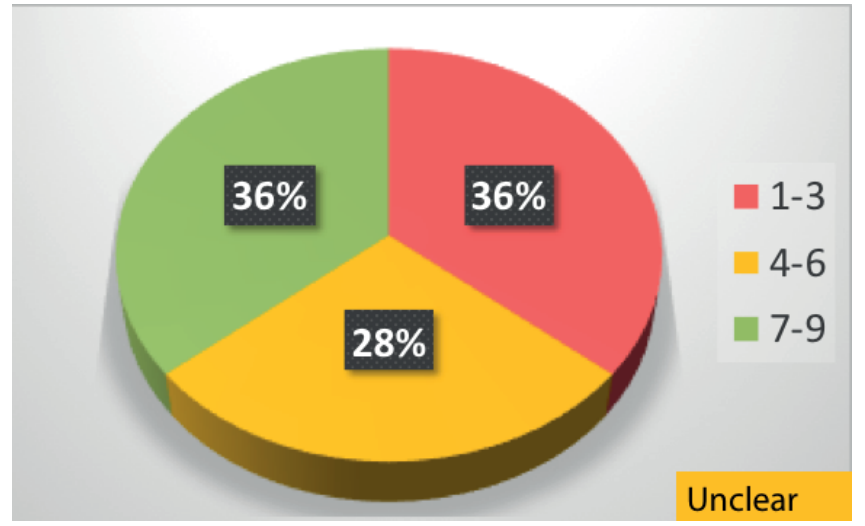

Figure 4.2.2. Delphi: In the diagnosis of recurrent acute rhinosinusitis do you rely on: History + positive objective findings, i.e. endoscopy and/or CT?

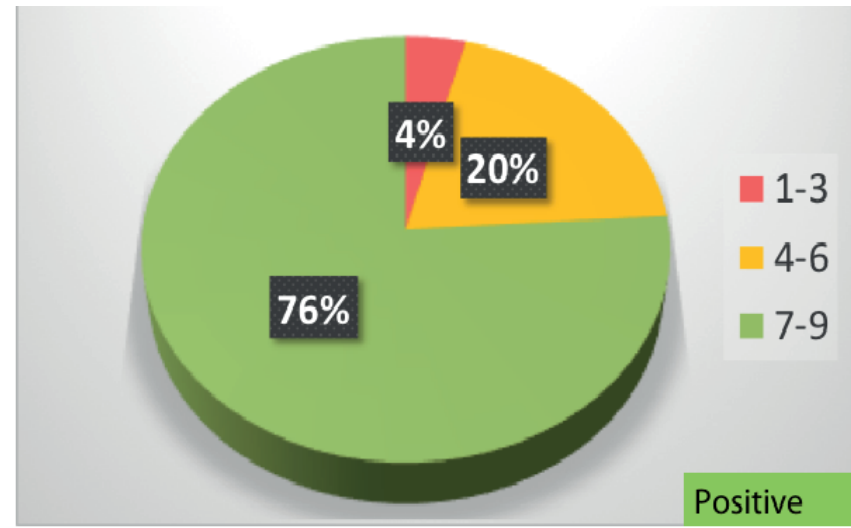

Figure 4.2.3. Delphi: In the diagnosis of recurrent acute rhinosinusitis: Is it essential to examine the patient with a CT-sinuses during (or shortly after) an acute episode if endoscopy has not been performed?

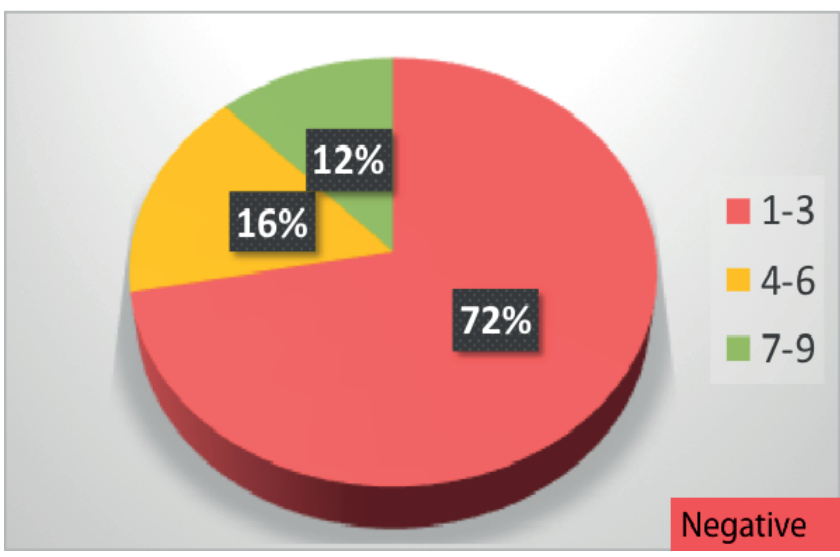

Figure 4.2.4. Delphi: In the diagnosis of recurrent acute rhinosinusitis: Is it essential to examine the patient with a CT sinuses during (or shortly after) an acute episode if endoscopy has been performed and is normal?

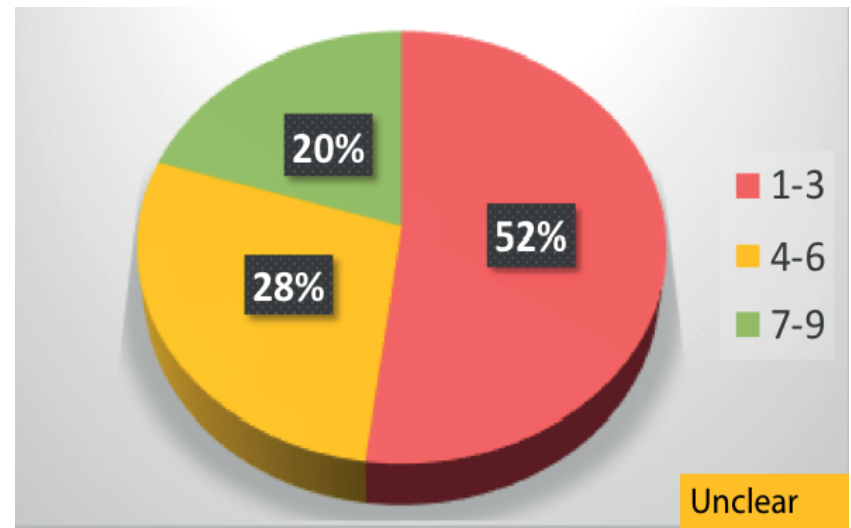

Figure 4.2.5. Delphi: In the diagnosis of recurrent acute rhinosinusitis: Is it essential to examine the patient with a CT of sinuses during (or shortly after) an acute episode if endoscopy has been performed and is abnormal?

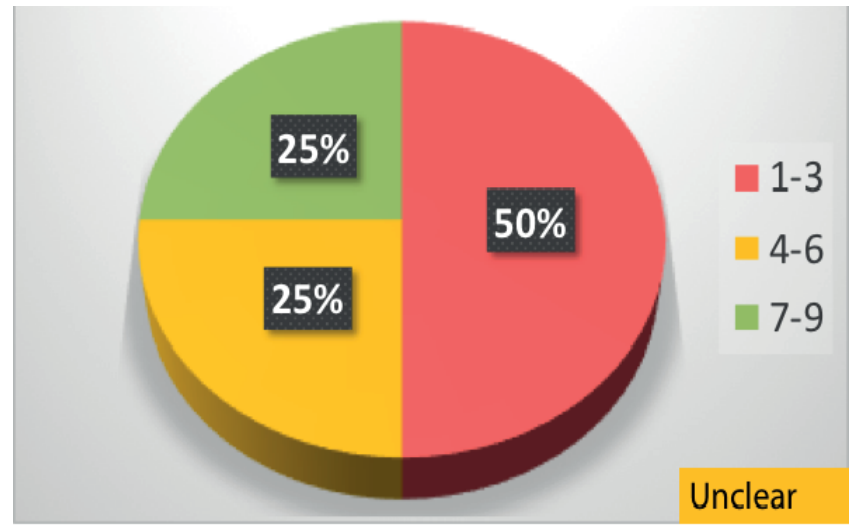

\subsection{Recurrent ARS (RARS)}

RARS is defined as $\geq 4$ episodes per year with symptom free intervals ${ }^{(7,28-30)}$. Each episode must meet the criteria for acute postviral (or bacterial) rhinosinusitis. Recurrent ARS may be considered distinct from ARS and CRS. Using data from a medical claims database for 13.1 million patients from 2003 to 2008, the point prevalence of recurrent ARS has been reported to be $0.035 \%$, considerably lower than that of $\mathrm{ARS}^{(31)}$. However, in the study by Hoffmans et al. only four out of 5574 ARS patients visiting their GP met this categorisation ${ }^{(17)}$. Whether RARS should be considered a form of acute or CRS requires further discussion. Lee et al. ${ }^{(32)}$ underlined the diagnostic challenge when it comes to RARS, and this is due to the relative lack of awareness and also the sporadic diagnosis by different healthcare providers. Most of the time, endoscopy is clear and CT sinuses do not show 
any significant mucosal thickening ${ }^{(33)}$. The EPOS2020 steering group advises to have at least one proven diagnosis of post-viral ARS with endoscopy and/or CT scan before a diagnosis of RARS is considered (Figures 4.2.1.-4.2.5.).

\subsection{Factors associated with ARS and RARS}

There appear to be many factors which impact upon ARS, and it is likely that many people suffering from a bout of ARS have more than one contributing factor. In a recent questionnairebased study from the Netherlands, the factors which predisposed to increased likelihood of ARS were: a doctor-diagnosis of CRS, allergic rhinitis, female gender, eczema, itchy rash and smoking. Increasing age was a protective factor ${ }^{(3)}$. In the Netherlands non-Caucasian ethnicity was also a protective factor, but this clearly depends on other factors as, for example, in a study from the USA, being Caucasian was protective ${ }^{(34)}$, suggesting that risk factors have different impacts in different locations. There is limited evidence on predisposing factors in RARS ${ }^{(35)}$ though it appears that the majority of episodes follow viral ARS and some patients are immunodeficient ${ }^{(36,37)}$, mainly lgA deficient and common variable immunodeficient ${ }^{(38-40)}$.

\subsubsection{Environmental exposures}

Using a matched case-control study design conducted in a Dutch population over the period 2000 to 2003, van GageldonkLafeber et al. ${ }^{(41)}$ reported that exposure to an individual(s) with respiratory complaints, inside or outside of the immediate household was an independent risk factor for attending their GP with an acute respiratory tract infection (adjusted odds ratio $(\mathrm{OR})=1.9$ and adjusted $\mathrm{OR}=3.7$, respectively). In contrast, patients with children in secondary education, who had dampness or mould at home, or had exposure to passive smoking were less likely to visit their GP compared to those without children, mould or dampness or passive smoking exposure respectively. Increased levels of dampness, but not mould, in the home has been associated with rhinosinusitis ${ }^{(42)}$. Seasonal trends in occurrences of ARS have been reported. This is of course to be expected and is common knowledge that does not need to be over-elaborated as acute viral infections are the most common cause of upper respiratory tract infections including acute rhinosinusitis $^{(43)}$. What has become clear is that weather patterns are increasingly variable which will impact on many of the currently identified risk factors ${ }^{(21,44)}$.

In a study of respiratory tract infections, Neumark et al. reported seasonal variation in the incidence rate of rhinosinusitis from 1999 through to 2005, with increased incidence in the first quarter of each year ${ }^{(45)}$. For acute respiratory illnesses in 2000 to 2003 , van Gageldonk-Lafeber et al. reported similar seasonal trends to those of Neumark ${ }^{(15)}$. Compared to July to September, van Gageldonk-Lafeber et al. reported that the relative risk of acquiring an acute respiratory illness was 2.9 (95\% Cl: 2.8- 3.0) in January to March, 1.8 (95\% Cl: 1.7-1.9) in October to December and 1.4 (95\% Cl: 1.3-1.5) in April to June. In an audit of complications of ARS, Babar-Craig et al. reported that $69 \%$ of patients were admitted during the winter months of November to April $^{(12)}$. Similar patterns have been reported in acute exacerbations of $\mathrm{CRS}^{(46)}$ and upper respiratory tract infections ${ }^{(47)}$. Climate variations have been reported to induce facial pain similar to ARS. Chinook or Föhn winds are weather events in which a rapidly moving warm, high-pressurised wind enters a specific location. The pressure changes that occur during the Chinook may induce facial pain similar to that experienced in rhinosinusitis pain. Rudmik et al. report that compared to controls, the presence of concha bullosa and spheno ethmoidal cell (Onodi cell; $p=0.004$ ), and larger maxillary sinus size (right, $p=0.015$; left, $\mathrm{p}=0.002$ ) are all associated with complaints of Chinook headache ${ }^{(47,48)}$. However, as the Lund-Mackay (LM) score was higher in the control group, the authors conclude that CRS is unlikely to be associated with the Chinook induced facial pain. Exposure to air pollution ${ }^{(49-51)}$, irritants used in the preparation of pharmaceutical products ${ }^{(52)}$, during photocopying ${ }^{(53)}$ and forest fire smoke ${ }^{(54)}$ have all been associated with an increase in the prevalence of symptoms of ARS. Of importance in seasonality of ARS is the presence of CRS which seems to predispose to ARS especially in the winter months, whereas ARS was more common in those without CRS in the spring months ${ }^{(55)}$. This study also quantified the effect of no previous past history of CRS compared to those who had a previous and current history of CRS, demonstrating an increasing incidence gradient also shown for never, former (1.01) and current smokers (1.53), the presence of asthma symptoms, a diagnosis of hay fever (1.36), migraine (1.55), anxiety (0.96-1.29), sinus surgery (1,46-1.74), being Caucasian (1.5) and female gender (1.35). Broadly similar results were found in a study from the Netherlands ${ }^{(3)}$. A review of the literature by De Sario et al. described how the role of our changing climate, outdoor pollution, temperature, wild-fires, and desert storms may act synergistically to present a challenge to those suffering from respiratory disease ${ }^{(44)}$.

\subsubsection{Anatomical factors}

Abnormalities on radiology are often seen in the healthy population. Two recent studies, one in Japan and one in the Netherlands reported on radiology in healthy populations and found significant percentages that could be misinterpreted as abnormalities pointing to ARS or CRS. The Dutch study showed some abnormality $(L M>0)$ in $43 \%$ of the population and $14 \%$ had a $L M$ score $\geq 4$ pointing to ARS or CRS ${ }^{(56)}$. The Japanese study was in an elderly population and found $17 \% \mathrm{LM}>0$ and $7.4 \% \mathrm{LM}$ score $\geq 4$ respectively ${ }^{(57)}$.

It has been suggested that anatomical variation is more likely to be of causal significance in patients with $\operatorname{RARS}^{(33,58-60)}$ with 
infraorbital cells and concha bullosa most often cited. In a small retrospective study on CT in 36 patients with RARS versus 42 controls, the LM score was 2.25 in the RARS group compared with 1.27 in the controls, notably related to the presence of infraorbital cells ${ }^{(58)}$. However, the presence of an anatomic variant did not correlate with the pattern of inflammation in a small retrospective study ${ }^{(60)}$. Endoscopy and CT were shown to be undertaken less frequently than in CRS, 9.2\% in the first three years and $40 \%$ in the first four years, respectively ${ }^{(31)}$. In conclusion, there are limited data correlating anatomical factors to (recurrent) ARS. Abnormalities seen on radiology are also common in a healthy population.

\section{It has been suggested that anatomical variation is more likely to be of causal significance in patients with RARS patients.}

\subsubsection{Odontogenic infections}

Odontogenic infections, or infections arising from dental sources, causing acute maxillary sinusitis have been reported in the literature. Bomeli et al. reported that oroantral fistula and periodontal disease plus either a projecting tooth root or periapical abscess were significantly identified as sources of acute maxillary sinusitis ${ }^{(61)}$. Furthermore, they demonstrated that the greater the extent of fluid opacification and mucosal thickening, the greater the likelihood of an identifiable dental infective source. In a computed tomography (CT) radiological study of the maxillary sinus in elderly dentate and edentulous patients, Mathew et al. reported an increased prevalence of mucosal thickenings ( 74.3 versus $25.6 ; \mathrm{p}<0.05$ ) and mucous cysts $(2.1 \%$ versus 0 ) in dentate patients compared to edentate controls, but most of these abnormalities can be considered chronic ${ }^{(62)}$. A recent retrospective analysis from Finland suggests that some $15 \%$ of ARS may be odontogenic in nature ${ }^{(63)}$.

\section{In patients with (recurrent) ARS, odontogenic sources of infections should be considered.}

\subsubsection{Allergy}

The role of allergy in ARS is the subject of much debate with literature both supporting and disputing a role for allergy in predisposing for $\mathrm{ARS}^{(64)}$. Schatz et al. reported that the odds of developing an episode of ARS was 4.4 times higher in patients with rhinitis than in healthy controls ${ }^{(65)}$. The major difficulty is the high prevalence of around 30\% depending on the location of allergy in the population worldwide ${ }^{(66-70)}$. In 1989, Savolainen reported that $25 \%$ of 224 patients with acute maxillary sinusitis had allergy, as verified by allergy questionnaire, skin testing and nasal smears, with a further $6.5 \%$ of patients having probable allergy ${ }^{(71)}$. However, upon comparison of those with and without allergy, no differences were found in the number of previous episodes of ARS, or bacteriological and radiological findings suggesting that the presence of allergy may be incidental. In a comparative case-control study of Israeli air force pilots, Ulanovski reported that $33 \%$ of pilots with a history of AR and $21 \%$ of the control group had one or more episodes of ARS $(p=0.09)$ (72). In 2009, Pant et al. undertook a review of allergy in rhinosinusitis. In agreement with the above literature, they concluded that insufficient evidence exists to confirm seasonal or perennial AR as a significant predisposing factor for ARS ${ }^{(73)}$. Lin et al. reported that children with atopy were more likely to develop $\mathrm{ARS}^{(74)}$. They reported that atopic children with ARS reported significantly higher symptoms (including dizziness, sneeze, snore, itchy or burning eyes, eye congestion and tearing) as well as significantly higher levels of anxiety, dyspnoea, chest tightness, and lower nasal peak inspiratory flow than non-atopic children with ARS. A more recent study in children demonstrated that although ARS was common in the studied population, there was no difference in incidence between those sensitised to grass pollen and those not sensitised but that the most common risk factor was an acute viral infection ${ }^{(75)}$. We are not aware of studies evaluating the role of allergy in RARS.

In summary, there appears to be little to support the presence of allergic rhinitis as a risk factor for developing ARS.

\section{There appears to be little to support the presence of allergic rhinitis as a risk factor for developing ARS.}

\subsubsection{Ciliary impairment}

Ciliary impairment has been demonstrated to be a feature of both viral and bacterial rhinosinusitis ${ }^{(1)}$. This includes both the loss of cilia and ciliated cells as well as a disruption of normal mucociliary flow. Smoking and allergy have been implicated in the disruption of cilia function. Indeed, impaired mucociliary clearance in AR patients predisposes patients to ARS ${ }^{(76)}$. Ciliary impairment has also been associated with cigarette smoking. In vitro studies have demonstrated that cigarette smoke condensate and cigarette smoke extract impair ciliogenesis in a dose-dependent manner ${ }^{(77)}$. Clinical studies have also reported that exposure to passive smoking increases the levels of matrix metalloproteinase 9 (MMP-9), a gelatinase associated with tissue modelling which is significantly increased in nasal secretions of children ${ }^{(78)}$ exposed to passive smoking. As increased production of MMP-9 has been found in the acute allergic response in the nose and lungs, the implications for the involvement of MMP-9, ciliary function, allergic response, and smoking in ARS needs further exploration.

\subsubsection{Smoking}

Limited research exists on the impact of smoking on ARS. Using data from the 1970 US National Health Interview Survey, and 
after excluding families with children with chronic respiratory illness, Bonham and Wilson reported that children from households with one or more adult cigarette smokers had significantly more restricted activity and bed-disability days than children from families with non-smoking adults ${ }^{(79)}$. This difference was found to be due to children from families with active smokers having more episodes of acute respiratory illness (including ARS). Comparable significant results were found when families in which 45 cigarettes or more were consumed per day were compared to families with non-smoking adults. The authors concluded that higher cigarette consumption was associated with increased predisposition for acute respiratory illness. In a paediatric characterisation study of 76 patients with acute rhinosinusitis aged 4-18 years, Eyigör and Başak reported that $51.3 \%$ (39 patients) were exposed to second-hand smoke and $2.6 \%$ (two patients) were active smokers ${ }^{(80)}$. Based on their population, the authors concluded that exposure to primary or second hand smoke were predisposing factors for ARS. In a study characterising the respiratory symptoms of adult postal workers in Zagreb, Croatia, the prevalence of rhinosinusitis in active smokers was $53.1 \%$ compared to $26.4 \%$ in non-smokers, although no information was available on whether the rhinosinusitis was recurrent acute or chronic in nature ${ }^{(81)}$.

Active and passive smoking has been shown to alter the normal bacterial flora present in the nasopharyngeal spaces, resulting in the colonisation of more potential pathogens than in non-smokers ${ }^{(82)}$. Following smoking cessation, the microbial population has been shown to revert back to that found in non-smokers ${ }^{(83)}$. The impact of smoking cessation programmes on the incidence and prevalence of ARS is unknown. In vitro and in vivo studies have recently shown increased MMP-9 production in children exposed to passive smokers ${ }^{(78)}$ and increased complement activation in human respiratory epithelial cells and mice exposed to cigarette smoke extract ${ }^{(84)}$. Whether increased MMP-9 production or complement activation due to exposure to cigarette smoke predisposes to ARS is unknown and requires further investigation. More recent studies confirm the negative impact of smoking ${ }^{(55)}$.

\section{Active and passive smoking predisposes to (R)ARS.}

\subsubsection{Laryngopharyngeal reflux}

Little is known about the association of ARS and laryngopharyngeal reflux. As reviewed by Pacheco-Galván et al., epidemiological studies conducted between 1997 and 2006 have shown significant associations between gastro-oesophageal reflux disease (GORD) and rhinosinusitis ${ }^{(85)}$. However, in a recent systematic review, Flook and Kumar showed only a poor association between acid reflux, nasal symptoms, and ARS ${ }^{(86)}$. The role of reflux in ARS remains unclear ${ }^{(87)}$.

\subsubsection{Anxiety and depression}

Poor mental health or anxiety and depression have been significantly associated with ARS. In a study of 47,202 college students aged 18 to 24 years, Adams et al. reported that the prevalence of acute infectious illness, which included bronchitis, ear infection, rhinosinusitis, and strep throat, ranged from $8 \%$ to $29 \%$, while the prevalence of anxiety and depression ranged from $12 \%$ to $20 \%$, respectively ${ }^{(88)}$. It is important to recognise the confounding impact of smoking as smoking contributes to ARS but also to anxiety/depression. Those who stop smoking demonstrate an improvement in mood and quality of life with reduced levels of anxiety and depression ${ }^{(89)}$.

\subsubsection{Concomitant chronic disease}

Concomitant chronic disease (bronchitis, asthma, cardiovascular disease, diabetes mellitus, or malignant cancer) in children has been associated with an increased risk of developing ARS secondary to influenza. Loughlin et al. reported that the overall incidence rate of developing ARS following influenza ranged from 0.9 to 1.3 in children aged 0 to 14 years. While the incidence of ARS subsequent to influenza in healthy children aged 5-14 years was 1.2 ( $95 \% \mathrm{Cl}: 0.9-1.5)$, this increased to 3.1 (95\% $\mathrm{Cl}: 1.5$ - 5.8) in children with chronic disease (rate ratio: 2.7 (95\% $\mathrm{Cl}: 1.5$ - 5.4). Increased monitoring of children with chronic disease who develop influenza may be necessary ${ }^{(90)}$.

\subsubsection{Conclusion}

Predisposing factors for ARS are seldom evaluated. There is some indication that anatomical abnormalities may predispose for RARS. Active and passive smoking predisposes to ARS and there is some indication that concomitant chronic disease may increase the chance of getting ARS following an influenza infection. Other potential factors like allergy and GORD do not seem to predispose to ARS.

\subsection{Pathophysiology of ARS}

ARS can be divided into acute viral rhinosinusitis, post-viral rhinosinusitis and acute bacterial rhinosinusitis. ABRS is frequently preceded by acute viral rhinosinusitis or common cold $^{(91)}$. In addition to the strain and virulence of individual viruses, the severity and pathogenesis of ARS is highly dependent on the host factors or predisposing conditions, such as age, host defence parameters or immune deficiency, previous infection or immunization, pre-existing mucosal inflammation caused by exposure to allergens, pathogens or other environmental risk factors, and anatomic deformity of the nose and sinuses.

The pathogenesis and pathophysiology of ARS is incompletely understood. This is mainly due to the lack of prospective clinical and laboratory studies in patients being performed during the natural course of ARS. In the literature, most reported studies 
were carried out using human volunteers, in vitro study of human tissue or cell lines, and experimental animals. These results have yet to be validated in human patients with naturally acquired viral infection and ARS.

ARS can theoretically be divided into viral (common cold) and post-viral rhinosinusitis. A small subgroup of ARS is caused by bacteria (ABRS). The pathogenesis and inflammatory mechanisms of viral and post-viral infection, and ABRS (if it occurs) can considerably overlap as can their clinical presentation.

\section{ARS can theoretically be divided into viral (common cold), post-viral rhinosinusitis and a small subgroup of acute bacterial rhinosinusitis.}

\subsubsection{Viral rhinosinusitis}

Viral rhinosinusitis (or the common cold) is, by definition, an acute rhinitis induced by respiratory viruses, such as rhinovirus (RV), respiratory syncytial virus (RSV), influenza virus (IFV), coronavirus (CorV), parainfluenza virus (PIV), adenovirus (AdeV) and enterovirus $(E V)^{(35,91,92)}$. RV and CorV are the most common viruses isolated from adult ARS, accounting for approximately $50 \%$ of viral ARS diagnosis ${ }^{(93)}$. In children, there is a wider variety of responsible viruses, i.e. besides rhinoviruses and coronaviruses, one can also expect to find RSV, parainfluenza viruses and adenoviruses. Geographically, there are also other viruses isolated from patients with ARS, e.g. human bocavirus is frequently isolated from ARS cases $^{(94)}$. With the new development of more sensitive and high-throughput viral detecting and screening techniques, multiple viruses can be detected. It is, however, relatively difficult to identify key viruses that cause or exacerbate ARS in clinical practice.

\section{Post-viral ARS and ABRS are frequently preceded by acute viral rhinosinusitis or common cold.}

\subsubsection{Nasal epithelium \\ Receptors}

The nasal epithelium is the primary portal of entry for respiratory viruses and immediate target for viral replication in the airway ${ }^{(95-98)}$. It is also an active component of initial host responses against viral infection. Nasal epithelial cells express various receptors recognizing specific viruses, such as intercellular adhesion molecule-1 (ICAM-1) ${ }^{(99,100)}$, toll-like receptor 3 (TLR3) $)^{(101)}$, a-2,3-linked sialic acid (a-2,3-SA)/a-2,6-SA containing receptor (102), retinoic acid inducible gene 1 (RIG-1, also known as DDX58) $(101,103)$, and MDA4 (also known as IFIHI) ${ }^{(103)}$. Upon infection the virus enters by receptor-mediated endocytosis, followed by expression and replication of the viral genome within hours after infection $^{(99,104,105)}$ (Figure 4.4.1.).
Figure 4.4.1. Nasal epithelium is the primary portal of entry for respiratory viruses as well as an active component of initial host responses against viral infection. The cascade of inflammation initiated by nasal epithelial cells will lead to damage by the infiltrating cells, causing oedema, engorgement, fluid extravasation, mucus production and sinus obstruction in the process, eventually leading to postviralARS or even ABRS.

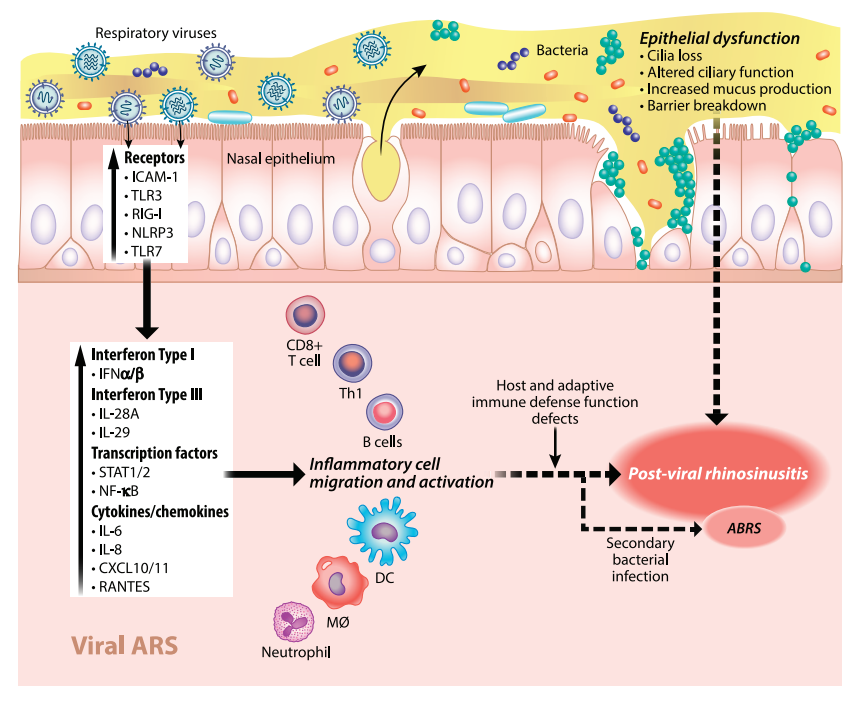

The nasal epithelium is the primary portal of entry for respiratory viruses and immediate target for viral replication in the airway. It serves as a mechanical barrier to protect from environmental factors, microorganisms, and toxins, but also participates in both innate and adaptive immune responses.

RV infection can upregulate the expression of ICAM- 1 via IL-1 $\beta$ and nuclear factor (NF)-KB-dependent mechanisms, directly enhancing infectivity and promoting inflammatory cell infiltration $^{(100,106,107)}$. In nasal polyp epithelium, higher expression of $a-2,3-S A$ and $a-2,6-S A$ will lead to the entry of more avian and seasonal influenza virus infection than normal nasal mucosa ${ }^{(108)}$. In in vitro culture of differentiated nasal, tracheal and bronchial cells, the a-2,3-SA and a-2,6-SA receptors were located in ciliated and non-ciliated cells, respectively ${ }^{(109)}$. Therefore, influenza virus likely targets non-ciliated cells in the nose, as the a-2,6-SA receptor is expressed in both the nose and trachea ${ }^{(110)}$.

\section{Immune responses}

The nasal epithelium not only serves as a mechanical barrier to protect from environmental factors, microorganisms, and toxins, but also participates in both innate (non-specific) and adaptive immune responses. The pseudostratified airway surface epithelium can be damaged in different degrees depending on the type of viruses and can also regenerate to restore its 
defence functions. Therefore, the interactions between the nasal epithelium and invading pathogens play key roles in the disease progression and subsequent immune responses against the virus, thus contributing to both disease burden and combating infection of the nasal epithelium.

Many studies indicate that the nasal epithelium actively triggers innate immune responses and also modulates adaptive immunity against these viruses ${ }^{(95,96,111)}$. Nasal epithelial-specific transcriptomic signatures may significantly influence the downstream immune responses and homeostasis that define the pathology of respiratory infection and complications ${ }^{(97,112-114)}$. In addition, respiratory viruses are also implicated to disrupt cilia and tight junction integrity in airway epithelial cells through the modulation of ZO-1, claudin-1 and occludin in the airway epithelial barrier ${ }^{(115-117)}$.

Nasal epithelial cells elicit their own repertoire of immune responses and actively prevent pathogens from damaging the airway ${ }^{(111,118)}$. Upon infection, they not only release anti-microbial surfactants and mucus to delay pathogen transmission in the airway ${ }^{(14,119)}$, but also express and secrete various cytokines and chemokines to drive immune responses against invading pathogens in the airways ${ }^{(120,121)}$. In an in vitro study, H3N2 (Aichi/7) infection of human primary nasal epithelial cells, showed: 1) highly efficient viral replication dynamics starting as early as four hours post-infection (hpi); 2) upregulation of four main pathogen recognition receptors (PRRs) RIG-I, NLRP3, TLR3 and TLR7; 3) exponentially elevated IFN- $\alpha 2$, IFN- $\beta$, IL-28A and IL-29 (8 to $72 \mathrm{hpi}$ ) at both intracellular mRNA and secreted protein levels; 4) a rapid production and release of chemokines IP-10, CXCL11, and RANTES and inflammasome markers including IL- $1 \mathrm{a}$, IL$1 \beta$, IL-6, IL-8, TNFa and TGF $\beta$ after 24-72 hpi; 5) indices of cell damage and death showed a steady decline in viability, integrity and survival rate from 16 to $72 \mathrm{hpi}$. These findings demonstrated quantitatively virus-host relationship, transmission capacity and virulence of the respiratory viruses in the upper airway ${ }^{(111)}$.

\section{Transcriptomic signatures}

The transcriptomic changes of infected nasal epithelial cells revealed differential regulation of 11 targets (CD38, HERC5, HERC6, IFI6, IFIH1, LGALS3BP, LY6E, MX1, PARP12, RTP4, ZBP1) creating influenza-specific signatures ${ }^{(122)}$. Hence, these key transcriptomic signatures during influenza are nasal-initiated, underscoring the potential application of nasal epithelial responses in rapid and sensitive molecular-based diagnostics to improve influenza detection ${ }^{(98)}$. In addition, recent studies have highlighted the strong expression of interferon lambda (IFN- $\lambda$, including IL-28A and IL29) as an important factor for limiting influenza viral spread, and potentially other viruses ${ }^{(97,98,111,123)}$. On the other hand, while other viruses express highly similar transcriptomic signatures exerting antiviral effects, the magnitude and response-time of the signatures may differ between viral infections. For example, rhinoviruses tend to express much weaker signatures compared to influenza infection ${ }^{(97)}$; and infections such as RSV tend to elicit a sustained response following infection compared to other viruses ${ }^{(124)}$. Therefore, studies to differentiate the pathogenic mechanism of different respiratory viruses are pivotal to understand their differential symptoms and severity in ARS, further highlighting the need for viral detection for symptom management of ARS.

\section{Motile cilia}

In an early study, a significant and long-lasting (up to 32 days) impairment of nasal mucociliary clearance functions such as a fall in the number of ciliated cells and a moderate and shortlasting change in beating frequency and intracellular synchrony were observed in patients with common cold ${ }^{(125)}$. More recent studies have further confirmed that impaired ciliogenesis was prominent following viral infections ${ }^{(126)}$, consistently leading to loss of cilia and ciliated cell ultrastructural abnormalities (i.e., cytoplasmic blebbing, swollen mitochondria) ${ }^{(97,111,127,128)}$. In vitro studies from human nasal epithelial cell models demonstrated that downregulation of ciliogenesis marker Foxj1 and upregulation of goblet cell marker Mucin5AC indicated the altered muco-ciliary function due to RV infection (clone RV16) ${ }^{(97)}$. In another study, RSV was found to infect preferentially the ciliated cells in human primary nasal epithelium. A portion of the RSV proteins ( $F$ and G) were trafficked into the cilia between 24 and 48 hour-post-infection followed by extensive cilia loss at five days-post-infection ${ }^{(129)}$. For influenza, the infection was followed by apoptotic and necrotic cell death causing the loss of epithelium including ciliated cells, impacting ciliary function ${ }^{(111)}$.

\section{Goblet cells}

The mucosal lining of the nasal cavity is coated by a mucus layer 10 to $15 \mu \mathrm{m}$ thick. Mucus is supplied by goblet cells in the epithelium and submucous seromucous glands. Sinus secretions are a mixture of glycoproteins, other glandular products, and plasma proteins. Secretions are rich in lysozyme, lactoferrin, albumin, secretory leukoprotease inhibitors, and mucoproteins ${ }^{(130)}$. In an ideal scenario, immediately after viral infection, a timely immune response is elicited, culminating in early viral elimination with minimal damage to the host. However, the cascade of inflammation initiated by the epithelial cells normally leads to damage by the infiltrating cells, causing oedema, engorgement, fluid extravasation, mucus production and sinus obstruction, eventually leading to ARS or exacerbating ARS (131). It has been reported that common cold symptoms may result from release of inflammatory mediators, such as bradykinin and TAME-esterase activity (but not histamine), into the nasal mucosa and secretions ${ }^{(132)}$. There is a luminal entry of plasma, including large binding proteins such as fibrinogen and a2 -macroglobulin, which may bind and transport a variety of cytokines 
in both the common cold and allergic rhinitis(133). In addition, it has been shown that rhinovirus infection induces mucus hypersecretion, which may contribute to a progress from watery rhinorrhoea to mucoid discharge during the common cold ${ }^{(134)}$. Viral interaction with goblet cells may also contribute to symptoms and aggravation of ARS. For example, MUC5AC production from goblet cells increased following RV and RSV infection ${ }^{(97,135)}$, while MUC5B was found to increase following infection with human metapneumovirus (hMPV) in epithelial cell lines ${ }^{(136)}$. With influenza virus, goblet cells produce sialic acid rich glycoprotein decoys in the mucus layer to prevent influenza binding to the epithelial cells ${ }^{(137,138)}$. However, the virus circumvents the sialic acid rich mucus layer via neuraminidase mediated cleavage of the sialic acids ${ }^{(138)}$. This interaction also contributes to secondary bacterial aggravation of ARS with cleaved sialic acid serving as additional nutrient sources for bacteria such as $\mathrm{S}$. pneumoniae ${ }^{(139)}$. Hence, the role of goblet cells in the pathogenesis of ARS is complex and multifaceted requiring controlled studies with appropriate models to establish their roles with different viruses.

\section{Other factors}

Other factors such as soluble chemical factors, kinins, nitric oxide, nerve stimulation and neuromediators, may play important roles in the pathophysiology or pathogenesis of viral rhinosinusitis have been previously reviewed in EPOS $2012^{(91)}$. Additionally, membrane tethered mucins (including MUC1, MUC3A, MUC3B, MUC4, MUC12, MUC13, MUC15, MUC16, MUC17, MUC20, and MUC21) that are expressed by the mucosal epithelium unlike secreted mucins expressed by goblet cells, may also play a role in viral ARS $(140,141)$. For example, MUC1 has been implicated in influenza infection and its subsequent inflammatory responses (142, 143); while MUC4 and MUC16 may also play a role in forming a protective barrier against invading pathogens $^{(141)}$.

\subsubsection{Post-viral rhinosinusitis}

In EPOS 2012 the term 'post-viral ARS' has been recommended in order to express the phenomenon of increase of symptoms after 5 days or persistent symptoms after 10 days with less than 12 weeks duration ${ }^{(91)}$. It is not an indicator of development of bacterial infection as only a small percentage of the patients with ARS will have ABRS.

The pathophysiology and pathogenic mechanisms of post-viral rhinosinusitis remain unclear. Viral infection of the nose and sinuses induces multiple changes, which include infiltration and activation of various inflammatory cells in the sinonasal mucosa and defects in the host and adaptive immune defence functions, as well as increase the risk of bacterial superinfection. Therefore, in most patients, this is a time frame for recovery from a single episode of ARS to complete resolution.

\section{Post-viral rhinosinusitis is not an indicator of development of bacterial infection as only a small percentage of the patients with ARS will have ABRS.}

\subsubsection{Acute bacterial rhinosinusitis}

Acute bacterial rhinosinusitis (ABRS) is an uncommon complication of viral upper respiratory tract infections that may cause mucosal damage and bacterial super-infection. Damage or disruption of mucociliary function due to viral infection is probably a major cause of super- or secondary bacterial infection. Bacterial and fungal infections are normally accompanied by viral infections, as observed in the common cold (RV-infection), and recurrent or chronic rhinosinusitis ${ }^{(144-146)}$. Streptococcus pneumoniae, Hemophilus influenzae and Moraxella catarrhalis are the most frequent bacteria in rhinosinusitis(146). RV-1b infection can promote the internalization of Staphylococcus aureus into non-fully permissive cultured pneumocytes with a mechanism that involves the virus-induced release of IL- 6 and IL-8, and the overexpression of ICAM-1 ${ }^{(145)}$. RV infection also promotes expression of cell adhesion molecules and bacterial adherence to primary human respiratory epithelial cells $s^{(147-149)}$. Furthermore, in RV-infected nasal epithelial cells (NECs) from the nasopharynx, TNF-a expression was increased by Aspergillus infection ${ }^{(150)}$. Viral infection of the nasal mucosa may trigger an inflammatory cascade thought to be responsible for the cold symptoms, but also forming the basis for immunological defence. The process of clearing the virus generates dead epithelial and infiltrating cells that contribute to the pathology of ARS. Such nasal epithelial-specific transcriptomic alterations may significantly influence the downstream immune responses and homeostasis that define the pathology of respiratory infection and complications ${ }^{(97 .}$ $98,112,114)$. It also creates an environment suitable for secondary bacterial infections (such as Staphylococcus aureus and Streptococcus pneumoniae), representing another factor exacerbating

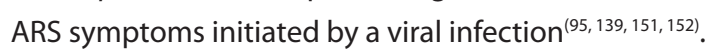

\section{Viral infection of the nasal mucosa may trigger an inflammatory cascade thought to be responsible for the cold symptoms, but also forming the basis for immunological defence.}

It has been suggested that respiratory virus infection induces the production of type I interferons (IFNs), inhibiting both the recruitment of circulating neutrophils and macrophages to the lung following bacterial challenge and the differentiation of antibacterial T helper 17 (TH17) cells from naive T cells or other Thelper (TH) cell types (such as TH1 and TH2 cells). This then potentiates host susceptibility to secondary bacterial infection ${ }^{(95,}$ ${ }^{153)}$. Interleukin-10 (IL-10) production by influenza virus-specific effector T cells may inhibit the ability of innate immune cells, 
in particular macrophages, to kill bacteria. Finally, the direct interaction and/or infection of innate immune cells — such as macrophages, neutrophils and natural killer (NK) cells - with influenza virus suppresses the ability of these cells to take up and kill bacteria(95).

S. pneumoniae infection is commonly associated with the aggravation of viral infections ${ }^{(154)}$. Studies have shown that influenza infection alters the gene expression of S. pneumoniae promoting dispersal from biofilms on the nasal mucosa ${ }^{(154,155)}$. As respiratory viruses induce a similar antiviral repertoire in the nasal epithelium, these viruses can cause similar dispersal of S. pneumoniae into the airway mucosa.

\subsubsection{Host defence mechanism}

The anti-viral immune response involves innate and specific components and requires the coordinated actions of many different cell types including neutrophils, macrophages, eosinophils, dendritic cells, epithelial cells, mast cells, natural killer cells and B- and T-lymphocytes. Coordination of this response involves numerous cytokines and chemokines.

It is often speculated that the T-helper 1 (Th1) response is initiated from the epithelial innate immune response via tolllike receptors 3, 7 and 9 (TLR 3, TLR7 and TLR9) due to the virus infection $^{(111,156)}$. Depending on the type of virus, the pathogensensing molecules in turn activate the production and secretion of nuclear factor- $\mathrm{KB}$ (NF-kB), interferon- $\beta$ (IFN- $\beta$ ), tumour necrosis factor- $\alpha$ (TNFa) and interleukins- $1 \beta, 6$ and 8 (IL-1 $\beta$, IL- 6 and IL-8), which are potent inducers or recruiters of neutrophils and macrophages ${ }^{(92,95)}$. The initial action of neutrophils against virus-infected cells usually contributes to the early symptoms of an acute respiratory virus infection. Following this, the further secretion of TNFa and interferon- $\gamma$ (IFN- $\gamma$ ) increases the recruitment of Th1 cells and cytotoxic T-cells leading to the clearance of the viral pathogens and viral-infected cells.

IFNY production by influenza virus-specific effector $T$ cells decreases the expression of macrophage receptor with collagenous structure (MARCO) by alveolar macrophages and inhibits the ingestion of bacteria by these cells. It is known that through secretion of type I IFNs (IFN- $\alpha / \beta$ ), the first line innate immunity defence in the infected cells, the neighbouring uninfected cells would be alerted to trigger its antiviral mechanisms. IFN- $\beta$ is involved in the upregulation of antiviral proteins, induction of apoptosis to inhibit the viral replication, and removal of infected cells in the normal airways upon RV infection ${ }^{(157)}$.

In addition, type III IFNs (IL-28A and IL-29) are shown to be strongly responsive to $\mathrm{H} 3 \mathrm{~N} 2$ infection, particularly their responses were found as early as $4 \mathrm{hpi}$ and peaked at $24-48 \mathrm{hpi}^{(111)}$. It is, therefore, important to look at the antiviral responses shown by IFNs where we will be able to assess viral pathogenesis differences in the nasal epithelium.

Naïve epithelial cells evoke an appropriate type-1 inflammatory response against invading viruses, especially in the context of influenza(111). Furthermore, the nasal cells could initiate cross-talk between innate and adaptive immunity via strong production of adaptive immune-activating cytokines and chemokines. A recent study showed that $\mathrm{H} 3 \mathrm{~N} 2$ infection of the nasal epithelium was associated with significant increase in interferons (IFN- $a$, IFN- $\gamma$, IL-29), pro-inflammatory cytokines (TNF- $a$, BDNF, IL-3) and viral-associated chemokines (IP-10, MCP-3, I-TAC, MIG), detectable as early as $24 \mathrm{~h}$ post infection ${ }^{(158)}$. This translates into rapid monocyte, NK-cell and innate T-cell (MAIT and $\gamma \delta$ T-cells) activation, evident with $\mathrm{CD} 8^{+}$and/or $\mathrm{CD} 69^{+}$upregulation $^{(158)}$. Therefore, an understanding of the predominant type and underlying mechanisms of mucosal inflammation triggered by common viral infections will allow us to identify targets for better management of chronic airway inflammatory diseases.

There are also critical down-regulated functions in nasal epithelium related to multiple metabolic and DNA damage responses against influenza that are not observed in blood or serum samples ${ }^{(98,159-163)}$. Such reductions in metabolic function and related metabolites at the primary influenza infection site may be an interesting area for future investigation to understand their relationships with viral replication and immune functions. In addition, these changes in the metabolic and homeostatic pathways are unique to the nasal epithelium.

Key points | What's new since EPOS 2012

Since EPOS 2012, there has been increasing experimental data supporting the fact that nasal epithelium is the primary portal of entry for respiratory viruses as well as an active component of initial host responses against viral infection. The cascade of inflammation initiated by nasal epithelial cells will lead to damage by the infiltrating cells, causing oedema, engorgement, fluid extravasation, mucus production and sinus obstruction in the process, eventually leading to postviral ARS or even ABRS.

\subsection{Diagnosis and differential diagnosis of ARS in adults and children}

\subsubsection{Update from EPOS 2012}

Although the diagnosis and differential diagnosis of ARS has not changed substantially, there is more evidence on the prevalence of symptoms and the predictive value of individual and combined symptoms on the diagnosis of (bacterial) acute rhinosinusitis. This update reflects that new information. 


\section{Post-viral ARS is a common condition in the community, usually following viral URTI.}

\subsubsection{Introduction}

ARS is a common condition and is usually self-limiting. Many patients will self-manage or use over the counter remedies, so will not seek medical care or have a formal diagnosis made. When medical care is sought, most patients will consult with a primary care physician, although in some health systems may directly access specialist services. Although educational efforts have been made to familiarize General Practitioners (GPs) with the concepts of rhinosinusitis and the diagnostic criteria for the diagnosis of $\mathrm{ARS}^{(164)}$, 'sinusitis' is commonly used as a diagnostic label, and as this is frequently considered by GPs an acute bacterial rather than inflammatory condition ${ }^{(165)}$, antibiotics are extensively prescribed ${ }^{(166,167)}$. The dissemination of the EP3OS(1) and other recent guidelines ${ }^{(168,169)}$ emphasizing the inflammatory nature of ARS and providing standardization of diagnostic criteria and use of investigations has led to more rational diagnosis and management in some ${ }^{(167,170)}$ but not all ${ }^{(171,172)}$ settings. In addition to misunderstandings concerning the inflammatory nature of ARS ${ }^{(171)}$, concern over the risk of septic complications from untreated bacterial disease may be a factor in the ongoing high use of antibiotics in ARS. Observational evidence indicates, however, that complications are rare ${ }^{(13,173)}$ usually manifest early in the course of the illness with severe symptoms ${ }^{(174,175)}$, and that antibiotic treatment of ARS in general practice does not prevent complications ${ }^{(12,13)}$

\section{Observational evidence indicates that antibiotic treatment of ARS in general practice does not prevent complications.}

Guidelines agree that in uncomplicated cases, ARS is diagnosed on clinical criteria and supplementary investigations are not required $^{(168)}$. In particular patient groups and in those with severe or atypical symptoms, additional diagnostic procedures may be needed, as discussed below. ARS is frequently an isolated clinical event and a self-limiting condition, although may be recurrent in some cases.

\subsubsection{Clinical diagnosis in primary care}

\section{ARS is diagnosed by the acute onset of typical symptoms that include nasal blockage, discharge, facial pain or pressure and reduction in smell.}

In the primary care setting (and for epidemiological research), ARS is defined by symptomatology without detailed ENT examination or imaging. ARS is defined by the presence of relevant symptoms for up to 12 weeks (see Chapter 2).
ARS is sub-divided into 'acute viral rhinosinusitis' (synonymous with the 'common cold'), in which the duration of symptoms is less than 10 days, usually a self-limiting condition that frequently does not present to clinicians, 'acute post-viral rhinosinusitis', defined by an increase in symptoms after five days or persistence beyond 10 days ${ }^{(176)}$ and 'acute bacterial rhinosinusitis' defined by at least three symptoms/signs - discoloured mucus, severe local pain, fever $>38$ ', raised CRP/ESR, 'double' sickening.

\subsubsection{Assessment of ARS symptoms}

\section{Most acute viral URTI infections are self-limiting, thus post-viral ARS should not be diagnosed before 10 days duration of symptoms unless there is a clear worsening of symptoms after five days.}

The subjective assessment of ARS in adults is based on the presence and severity of symptoms.

- Nasal blockage, congestion or stuffiness

- Nasal discharge or postnasal drip, often mucopurulent

- Facial pain or pressure, headache, and

- Reduction/loss of smell

Besides these local symptoms, distant and systemic symptoms may occur. Associated symptoms are pharyngeal, laryngeal, and tracheal irritation causing sore throat, dysphonia, and cough, and general symptoms including drowsiness, malaise, and fever. There is little reliable evidence of the relative frequency of different symptoms in ARS in community practice. Individual variations of these general symptom patterns are many ${ }^{(177-181)}$. Only a small proportion of patients with purulent rhinosinusitis, without coexisting chest disease, complain of cough $^{(178)}$. In patients with a suspicion of infection, facial or dental pain (especially if unilateral) have been found to be predictors of acute maxillary sinusitis, when validated by maxillary antral aspiration ${ }^{(178)}$ or paranasal sinus radiographs ${ }^{(179)}$. The symptoms of ARS occur abruptly without a history of recent nasal or sinus symptoms. A history of sudden worsening of pre-existing symptoms suggests an acute exacerbation of chronic rhinosinusitis, which should be diagnosed by similar criteria and treated in a similar way to ARS. In children acute rhinosinusitis is defined as a sudden onset of two or more of the following symptoms: nasal blockage/obstruction/congestion or discoloured nasal discharge or cough (daytime and night-time) for $<12$ weeks.

Subjective assessment should take into account the severity and the duration of symptoms (see above). The recommended method of assessing severity of symptoms is with the use of a visual analogue scale (VAS) recorded by the patient on a $10 \mathrm{~cm}$ line giving a score on a measurable continuum of 1 to 10 . Diseasespecific questionnaires measuring quality of life impairment are available ${ }^{(182,183)}$ but not commonly used in clinical practice; a 
good clinician will, however, informally assess the impact of ARS on their patient as part of a full clinical assessment. The VAS can be used to assess overall and individual symptom severity (see below) or patients can be asked to simply rate their symptoms as absent, mild, moderate or severe.

Patient reported purulence of nasal discharge has been recommended as a diagnostic criterion for acute bacterial rhinosinusi$\mathrm{tis}^{(169)}$, and is prioritized by GPs as a feature indicating the need for antibiotics ${ }^{(167)}$. However, the positive likelihood ratio for (purulent) nasal discharge as a symptom ( $L R+1.3$ ) and on physical examination ( $L R+0.88$ ) do not support using purulent discharge to identify bacterial origin ${ }^{(184)}$.

Facial or dental pain, especially when unilateral, has been found to be a predictor of acute maxillary sinusitis. Retained sinus secretions in patients with suspected bacterial infection can be confirmed by maxillary antral aspiration ${ }^{(178)}$ or paranasal sinus radiographs ${ }^{(179)}$. Pain on bending forwards and toothache in the upper jaw, particularly when unilateral, are often interpreted by GPs as indicative of more severe disease and the need for antibiotics ${ }^{(167)}$, although with limited supporting evidence. The presence of toothache in the upper jaw has a positive likelihood ratio for the presence of acute bacterial rhinosinusitis of 2.0, which ranks this symptom as one of the highest predictors ${ }^{(184)}$.

\section{Bacterial infection may occur in ARS, but in most cases antibiotics have little effect on the course of the illness.}

\subsubsection{Clinical rules for the prediction of bacterial disease}

A number of studies have attempted to provide clinicians with combinations of symptoms and signs predicting more severe disease, particularly of a bacterial infection and the likelihood of a response to antibiotics. A recent meta-analysis tried to combine all these studies ${ }^{(184)}$. However, in such a meta-analysis it is very important for the prediction to choose the right population (primary care patients with at least 10 days of symptoms or increase of symptoms after five days in which the GP felt antibiotics were needed) and the right gold standard. The EPOS group in 2007 decided to base their advice on the study of Lindbaek ${ }^{(186)}$ who chose an air fluid level or total opacification at CT scan as the gold standard in a population of primary care patients in which the doctor suspected ARS requiring antibiotics. We do not believe abnormalities on CT scan in general or abnormalities at sinus X-ray or ultrasound are good gold standards, neither do we consider patients sent in for an antral puncture to the ENT the ideal population. For that reason, we decided to stick to suggestions as made in the earlier EPOS versions: at least three of five symptoms of discoloured discharge, severe local pain, fever, elevated ESR/CRP. We appreciate that a study is required to determine the best clinical predictors in a carefully selected population.

\subsubsection{Clinical examination}

\section{Anterior rhinoscopy}

Although anterior rhinoscopy alone is a very limited investigation, it should be performed in the primary care setting as part of the clinical assessment of suspected ARS as it may reveal supportive findings such as nasal inflammation, mucosal oedema and purulent nasal discharge, and can sometimes reveal previously unsuspected findings such as polyps or anatomical abnormalities.

\section{Temperature}

The presence of a fever of $>38^{\circ} \mathrm{C}$ indicates the presence of a more severe illness and the possible need for more active treatment, particularly in conjunction with more severe symptoms. A fever of $>38^{\circ} \mathrm{C}$ is significantly associated with the presence of a positive bacteriologic culture, predominantly S. pneumoniae and H. influenzae, obtained by sinus aspiration or lavage ${ }^{(185)}$.

\section{Inspection and palpation of sinuses}

Acute rhinosinusitis does not lead to swelling or redness of maxillofacial area unless there is a dental origin when the diagnostic odds ratio is 0.97 . Data on local tenderness are inconclusive ${ }^{(184)}$

\section{Nasal endoscopy}

Nasal endoscopy is not generally available in routine primary care settings and is not required in the clinical diagnosis of ARS in these circumstances.

\section{C-reactive protein (CRP)}

CRP is a haematological biomarker (available as rapid assay near-patient testing kits) and is raised in bacterial infection. Its use has been advocated in respiratory tract infection ${ }^{(186)}$ as an aid to targeting bacterial infection and thus limiting unnecessary antibiotic use. A low or normal CRP may identify patients with a low likelihood of bacterial infection and who are unlikely to need or benefit from antibiotics. CRP guided treatment has been associated with a reduction in antibiotic use without any impairment of outcomes ${ }^{(187)}$ and CRP levels are significantly correlated with changes in CT scans ${ }^{(188)}$ and a raised CRP is predictive of a positive bacterial culture on sinus puncture or lavage ${ }^{(185,189)}$.

\section{Procalcitonin}

Procalcitonin has also been advocated as a potential haematological biomarker indicating more severe bacterial infection and investigated as a tool for guiding antibiotic prescribing in respiratory tract infections in the community. A recent review revealed two randomised controlled trials (RCTs) aiming to reduce antibiotic prescription with procalcitonin as guiding marker ${ }^{(190)}$. These studies did show reduced antibiotic prescriptions without detrimental effects on outcomes. 


\section{Erythrocyte sedimentation rate (ESR) and plasma viscosity}

Markers of inflammation such as ESR and plasma viscosity are raised in $A B R S$, may reflect disease severity and can indicate the need for more aggressive treatment in a similar way to CRP. ESR levels are correlated with CT changes in $\mathrm{ARS}^{(251)}$ with an ESR of $>10$ predictive of sinus fluid levels or sinus opacity on CT scans $^{(189)}$. Raised ESR has a positive likelihood ratio for rhinosinusitis of 2,61 and a negative likelihood ratio of $0,68^{(187)}$, with higher ERS levels increasing the likelihood of the presence of rhinosinusitis. Additional investigations such as imaging, microbiology and nasal nitric oxide measures are not required in the diagnosis of ARS in routine practice.

\subsubsection{Differential diagnosis of ARS in clinical practice}

The symptoms of ARS are non-specific and may overlap with a number of other conditions, from which it should be differentiated.

\section{Viral upper respiratory tract infection (URTI)}

The symptoms of the common cold and of self-limiting viral URTIs overlap with those of post-viral ARS. Indeed, all episodes of postviral ARS will start as a viral URTI but manifest a prolonged illness beyond 10 days or with worsening symptoms after five days. Most common colds are associated with rhinovirus infection with symptoms peaking by three days ${ }^{(191)}$, and the majority of patients not seeking medical care. The diagnosis is clinical and supportive advice, symptomatic treatment and reassurance are generally the only interventions required.

\section{Allergic rhinitis (AR)}

AR is a common global condition affecting at least $10-20 \%$ of the adult population ${ }^{(192)}$. AR is the most common form of non-infectious rhinitis and is associated with an IgE-mediated immune response against allergens. Since the nasal mucosa is continuous with that of the paranasal sinuses, congestion of the ostia may result in rhinosinusitis, which does not exist without rhinitis, so AR may be part of an allergic rhinosinusitis with similar symptoms to those of ARS (and CRS). Symptoms of AR include rhinorrhoea (non-purulent), nasal obstruction, nasal itching, and sneezing, which are reversible spontaneously or with treatment. AR is subdivided into "intermittent" or "persistent" disease. Intermittent rhinitis may occur suddenly in response to exposure to a specific allergen, and so cause diagnostic confusion between AR and ARS. Seasonal AR is related to a wide variety of outdoor allergens such as pollens or moulds, and sudden exposure to such aeroallergens or to others (e.g. cat and dog dander in sensitized individuals) can cause acute onset of symptoms. In AR, there will usually be a history of similar symptoms in response to similar exposures, often with a seasonal pattern. Non-specific irritants such as air pollution and viral infection may aggravate symptoms in symptomatic AR patients.
The differentiation of AR from ARS is made mainly on the basis of a prior history of allergy and atopy, and exposure to an allergen (usually an aeroallergen) to which the patient is sensitized. Ocular symptoms are common in AR, in particular in patients allergic to outdoor allergens, but not in ARS. Mucopurulent rhinorrhoea, pain, nasal obstruction without other symptoms and anosmia are uncommon in AR. Diagnostic tests for AR are based on the demonstration of allergen-specific $\lg E$ in the skin (skin tests) or the blood (specific lgE), and may be considered to clarify the diagnosis, particularly in those with severe or persistent symptoms.

\section{Orodontal disease}

Patients with orodontal disease may present to primary care physicians with ill-defined facial pain, with or without fever and toothache. The absence of other ARS-associated symptoms such as rhinorrhoea, nasal discharge and smell disturbance will make ARS a less likely diagnosis, although in some cases doubt may persist. A dental assessment and dental radiography may be required to clarify the diagnosis. ARS may occur more frequently and have overlapping symptoms in patients with orodontal disease ${ }^{(61)}$.

\section{Rare diseases}

\section{Facial pain syndromes}

A number of conditions can present acutely with facial pain and nasal symptoms, including migraine and cluster headaches. The differential diagnosis of facial pain is discussed in Chapter 5.3.3.

\section{Vasculitis}

Autoimmune vasculitides such as granulomatosis with polyangiitis, eosinophilic granulomatosis with polyangiitis or sarcoidosis may involve the nose and sinuses and on rare occasions may present acutely. The presence of other suggestive symptoms and an atypical clinical course can alert the clinician to alternative diagnoses. (See Chapter 8.7.)

\section{Acute invasive fungal rhinosinusitis}

In immunosuppressed patients and in (uncontrolled) diabetics, acute invasive fungal rhinosinusitis may present in a similar way to ARS, but with severe and rapidly progressive symptoms ${ }^{(193,194)}$. When this diagnosis is suspected, a more aggressive diagnostic approach is required as a delay in diagnosis worsens the prognosis. (See Chapter 8.6.)

\section{CSF leak}

Unilateral watery rhinorrhoea is uncommon and should raise suspicion of cerebrospinal fluid leakage ${ }^{(195)}$.

\subsubsection{Warning signs of complications of ABRS}

Septic complications of ABRS represent a potential medical 
emergency and require prompt recognition by generalists and immediate referral to secondary care for assessment (Chapter 4.7; Table 4.5.1.). Observational surveys suggest that these complications occur rarely but early in the course of the disease, and that outcomes are not influenced by the use or non-use of antibiotics in primary care $\mathrm{c}^{(13,175)}$

\section{Complications of ARS are uncommon, but vital} to identify early. They occur early in the course of the illness and primary care clinicians need to be vigilant for the danger signs and symptoms.

\subsubsection{Diagnosis of ARS in specialist care}

Although uncomplicated ARS is more likely to present to primary care physicians, in some health systems patients may present acutely to specialists or may be referred early for a specialist assessment, usually to a rhinologist or ENT specialist. Generally, the diagnosis may be made clinically using the same criteria outlined above, but sometimes more detailed diagnostic investigations may be applied. Immediate referral and/or hospitalization are indicated for any of the symptoms listed in Table 4.5.1. These investigations include nasal endoscopy and imaging (see Chapters 5.3.4.1., 5.3.4.3.).

\subsubsection{Diagnosis of ARS in specific settings}

\subsubsection{Diagnosis for research}

In research settings, a more formal diagnosis may be required. In such settings, a variable combination of symptoms, imaging findings, examination findings, and bacteriology samples (obtained from middle meatus or from sinus puncture) may be required for confirmation of the diagnosis as specified in the study protocol. The diagnostic criteria used must be specified in research studies to allow comparison of results between studies.

\subsubsection{Diagnosis of ARS in the intensive care unit}

ABRS is common in ICU (with risk factors including naso-gastric tubes, mechanical ventilation, failure of defence mechanisms and pronged supine posture), and is associated with poor outcomes. Sepsis may involve multiple sinuses ${ }^{(196)}$. As a consequence, more aggressive diagnostic processes may be appropriate such as CT scanning to confirm the diagnosis ${ }^{(197)}$, and sinus puncture which is safe in skilled hands and can provide important microbiological information to confirm the diagnosis and guide therapy ${ }^{(196)}$.

\subsubsection{Diagnosis of ARS in immunosuppressed patients}

Immunosuppressed patients are much more vulnerable to complications of ABRS, and a more aggressive diagnostic approach is required. Acute invasive fungal rhinosinusitis ${ }^{(290)}$ is a serious disease with high mortality and morbidity and requires prompt
Table 4.5.1. Warning symptoms of complications in ARS requiring immediate referral / hospitalization.

Periorbital oedema/erythema

Displaced globe

Double vision

Ophthalmoplegia

Reduced visual acuity

Severe unilateral or bilateral frontal headache

Frontal swelling

Signs of meningitis

Neurological signs

Reduced consciousness

diagnosis and treatment with open or endoscopic sinus surgery (see Chapter 8.6.). The diagnosis is usually histopathological, so early endoscopic evaluation is indicated, with open biopsy if doubt remains ${ }^{(193,194)}$.

\subsubsection{Conclusion}

Acute rhinosinusitis may be divided into a viral common cold, post-viral or acute bacterial rhinosinusitis depending on the duration and severity of the symptoms. There have been some recent studies supporting the symptom base, but more are required to reduce the inappropriate use of antibiotics.

\subsection{Medical management of ARS}

\subsubsection{Introduction}

As mentioned before, ARS is divided into acute viral rhinosinusitis, acute post-viral rhinosinusitis and acute bacterial rhinosinusitis. For the definitions see Chapters 2 and 3.

In recent years a large number of systematic reviews and metaanalyses have covered the significant parts of the management of acute viral rhinosinusitis (often called common cold and/or upper respiratory tract infection). For that reason, this chapter contains a short overview of these systematic reviews and metaanalysis. For acute post-viral rhinosinusitis and acute bacterial rhinosinusitis new systematic reviews and meta-analysis are performed in this chapter.

\subsubsection{Management of acute viral rhinosinusitis (common cold)}

In recent years a large number of systematic reviews and metaanalyses have covered the significant parts of the management of acute viral rhinosinusitis. For that reason, this chapter contains a short overview of the systematic reviews and metaanalysis published after 2012. For the search performed please see Chapter 11. 


\subsubsection{Antibiotics}

Eleven randomized controlled trials compared any antibiotic therapy against placebo in people with symptoms of common cold $^{(198)}$. Participants receiving antibiotics for the common cold did no better in terms of lack of cure or persistence of symptoms than those on placebo (risk ratio (RR) $0.95,95 \% \mathrm{Cl} 0.59$ to 1.51 , (random-effects)), based on a pooled analysis of six trials with a total of 1047 participants. The RR of adverse effects in the antibiotic group was $1.8,95 \% \mathrm{Cl} 1.01$ to 3.21 , (random effects). Adult participants had a significantly greater risk of adverse effects with antibiotics than with placebo (RR $2.62,95 \% \mathrm{Cl} 1.32$ to 5.18) (random effects) while there was no greater risk in children (RR $0.91,95 \% \mathrm{Cl} 0.51$ to 1.63). The pooled RR for persisting acute purulent rhinorrhoea with antibiotics compared to placebo was 0.73 ( $95 \% \mathrm{Cl} 0.47$ to 1.13 ) (random effects), based on four studies with 723 participants. There was an increase in adverse effects in the studies of antibiotics for acute purulent rhinitis (RR 1.46, 95\% $\mathrm{Cl} 1.10$ to 1.94). The authors concluded that there is no evidence of benefit from antibiotics for the common cold or for persisting acute purulent rhinitis in children or adults. There is evidence that antibiotics cause significant adverse effects in adults when given for the common cold and in all ages when given for acute purulent rhinitis. Routine use of antibiotics for these conditions is not recommended.

\subsubsection{Nasal corticosteroids}

The anti-inflammatory effects of nasal corticosteroids may be beneficial in the common cold. In 2013, a Cochrane review was published to compare nasal corticosteroids versus usual care for the common cold on measures of symptom resolution and improvement in children and adults. Three trials (353 participants) were included. Two trials compared nasal corticosteroids to placebo and one trial compared nasal corticosteroids to usual care. In the two placebo-controlled trials, no benefit of nasal corticosteroids was demonstrated for duration or severity of symptoms. The authors concluded that the current evidence does not support the use of nasal corticosteroids for symptomatic relief from the common cold ${ }^{(199)}$.

\subsubsection{Antihistamines}

To assess the effects of antihistamines on the common cold the authors of a Cochrane review included 18 RCTs, which were reported in 17 publications (one publication reports on two trials) with 4342 participants (of which 212 were children) suffering from the common cold, both naturally occurring and experimentally induced. The interventions consisted of an antihistamine as monotherapy compared with placebo. In adults there was a short-term beneficial effect of antihistamines on severity of overall symptoms: on day 1 or 2 of treatment $45 \%$ had a beneficial effect with antihistamines versus $38 \%$ with placebo (odds ratio (OR) $0.74,95 \% \mathrm{Cl} 0.60$ to 0.92 ). However, there was no difference between antihistamines and placebo in the mid-term (three to four days) to long term (six to 10 days). When evaluating individual symptoms such as nasal congestion, rhinorrhoea and sneezing, there was some beneficial effect of the sedating antihistamines compared to placebo (e.g. rhinorrhoea on day 3: mean difference (MD) $-0.23,95 \% \mathrm{Cl}-0.39$ to -0.06 on a four- or five-point severity scale; sneezing on day 3: MD - 0.35 , $95 \% \mathrm{Cl}-0.49$ to -0.20 on a four-point severity scale), but this effect is clinically non-significant. Adverse events such as sedation were more commonly reported with sedating antihistamines although the differences were not statistically significant. Only two trials included children and the results were conflicting. The authors concluded that antihistamines have a limited short-term (days 1 and 2 of treatment) beneficial effect on severity of overall symptoms in adults but not in the mid to long term. There is no clinically significant effect on nasal obstruction, rhinorrhoea or sneezing ${ }^{(200)}$.

\subsubsection{Decongestants}

In 2016, Deckx et al. published a Cochrane review on the efficacy, and short- and long-term safety, of topical and/or oral decongestants used in monotherapy to alleviate symptoms of the common cold in adults and children ${ }^{(201)}$. The authors included 15 trials with 1838 participants. In six studies the intervention was a single dose and in nine studies multiple doses were used. Eleven studies used oral decongestants; four studies used topical decongestants. Nine studies used pseudoephedrine and three studies used oxymetazoline. Nine studies compared multiple doses of topical or oral decongestants with placebo. Subjective measures of congestion were significantly better for the treatment group compared with placebo approximately three hours after the last dose (SMD 0.49, $95 \% \mathrm{Cl} 0.07$ to 0.92; $\mathrm{p}=0.02$ ); seven studies reported adverse events (six oral and one topical decongestant); meta-analysis showed that there was no statistical difference between the number of adverse events in the treatment group compared to the placebo group. The authors concluded that the current evidence suggests that multiple doses of decongestants may have a small positive effect on subjective measures of nasal congestion in adults with the common cold. Due to the small number of studies that used a topical nasal decongestant, they were unable to draw conclusions on the effectiveness of oral versus topical decongestants. Decongestants do not seem to increase the risk of adverse events in adults in the short term.

\subsubsection{Paracetamol (acetaminophen)}

To investigate the effectiveness of paracetamol RCTs comparing paracetamol to placebo or no treatment in adults with the common cold four RCTs involving 758 participants were evaluated in a Cochrane review ${ }^{(202)}$. Participants treated with paracetamol had significant improvements in nasal obstruction in two of the 
four studies. One study showed that paracetamol was superior to placebo in decreasing rhinorrhoea severity but was not superior for treating sneezing and coughing. Paracetamol did not improve sore throat or malaise in two of the four studies. Results were inconsistent for some symptoms. Two studies showed that headache and achiness improved more in the paracetamol group than in the placebo group, while one study showed no difference between the paracetamol and placebo group. None of the included studies reported the duration of common cold symptoms. Minor side effects in the paracetamol group were reported in two of the four studies. One of them used a combination of pseudoephedrine and paracetamol. The authors concluded that paracetamol may help relieve nasal obstruction and rhinorrhoea but does not appear to improve some other cold symptoms (including sore throat, malaise, sneezing and cough).

\subsubsection{NSAIDs}

Non-steroidal anti-inflammatory drugs (NSAIDs) have been widely used for the treatment of pain and fever associated with the common cold. A Cochrane review performed in 2009 includes nine RCTs, describing 37 comparisons: six were NSAIDs versus placebo, and three were NSAIDs versus NSAIDs ${ }^{(203)}$. A total of 1064 patients with the common cold were included. The authors reported that NSAIDs did not significantly reduce the total symptom score, or duration of colds. However, for outcomes related to the analgesic effects of NSAIDs (headache, ear pain and muscle and joint pain) NSAIDs produced significant benefits, and malaise showed a borderline benefit, although throat irritation was not improved. Chills showed mixed results. For respiratory symptoms, cough and nasal discharge scores were not improved, but the sneezing score significantly improved. They found no evidence of increased frequency of adverse effects in the NSAID treatment groups. The authors recommended NSAIDs for relieving discomfort or pain caused by the common cold.

\subsubsection{Antihistamine-decongestant-analgesic combinations}

To assess the effectiveness of antihistamine-decongestant-analgesic combinations in reducing the duration and alleviating the symptoms of the common cold in adults and children 27 trials (5117 participants) of common cold treatments were evaluated in a Cochrane review ${ }^{(204)}$. Fourteen trials studied antihistaminedecongestant combinations; two antihistamine-analgesic; six analgesic-decongestant; and five antihistamine-analgesicdecongestant combinations. In 21 trials the control intervention was placebo and in six trials an active substance. The evidence in this systematic review suggests that antihistamine-analgesicdecongestant combinations have some general benefit in adults and older children. These benefits must be weighed against the risk of adverse effects. There is no evidence of effectiveness in young children.

\subsubsection{Ipratropium bromide}

A Cochrane review was published in 2013 to determine the effect of ipratropium bromide versus placebo or no treatment on severity of rhinorrhoea and nasal congestion in children and adults with the common cold ${ }^{(205)}$. Seven trials with a total of 2144 participants were included. Four studies (1959 participants) addressed subjective change in severity of rhinorrhoea. All studies were consistent in reporting statistically significant changes in favour of ipratropium bromide. Nasal congestion was reported in four studies and was found to have no significant difference between the groups. Two studies found a positive response in the ipratropium bromide group for the global assessment of overall improvement. Side effects were more frequent in the ipratropium bromide group, OR 2.09 ( $95 \% \mathrm{Cl} 1.40$ to 3.11 ). Commonly encountered side effects included nasal dryness, blood tinged mucus and epistaxis. The authors concluded that for people with the common cold, the existing evidence suggests that ipratropium bromide is likely to be effective in ameliorating rhinorrhoea. Ipratropium bromide had no effect on nasal congestion and its use was associated with more side effects compared to placebo or no treatment although these appeared to be well tolerated and self-limiting.

\subsubsection{Nasal irrigation with saline}

Nasal irrigation with saline is often employed as an (adjunct) treatment for URTI symptoms. The Cochrane review published in 2015 identified five RCTs that randomised 544 children (three studies) and 205 adults (exclusively from two studies). All included studies compared saline irrigation to routine care or other nose sprays, rather than placebo. Most outcome measures differed greatly between included studies and, , could not be pooled. Most results showed no difference between nasal saline treatment and control. However, one larger trial, conducted with children, did show a significant reduction in nasal secretion score (MD $-0.31,95 \% \mathrm{Cl}-0.48$ to -0.14 ) and nasal breathing (obstruction) score (MD $-0.33,95 \% \mathrm{Cl}-0.47$ to -0.19 ) in the saline group. The trial also showed a significant reduction in the use of decongestant medication by the saline group. Minor nasal discomfort and/or irritation was the only side effect reported by a minority of participants. The authors concluded that nasal saline irrigation possibly has benefits for relieving the symptoms of acute URTIs ${ }^{(206)}$.

\subsubsection{Steam / heated humidified air}

Background: Heated, humidified air has long been used by common cold sufferers. The theoretical basis is that steam may help congested mucus drain better and heat may destroy cold virus as it does in vitro. The Cochrane review in 2017 included six trials from five publications involving a total of 387 participants(207). The authors reported that it is uncertain whether heated, humidified air provides symptomatic relief for the common cold, 
as the fixed-effect analysis showed evidence of an effect (OR $0.30,95 \% \mathrm{Cl} 0.16$ to 0.56 ; two studies, 149 participants), but the random-effects analysis showed no significant difference in the results (OR $0.22,95 \% \mathrm{Cl} 0.03$ to 1.95). No studies demonstrated an exacerbation of clinical symptom scores. One study conducted in the USA demonstrated worsened nasal resistance, but an earlier Israeli study showed improvement. One study examined viral shedding in nasal washings, finding no significant difference between treatment and placebo groups (OR $0.47,95 \% \mathrm{Cl}$ 0.04 to 5.19 ). As judged by the subjective response to therapy the number of participants reporting resolution of symptoms was not significantly higher in the heated humidified group (OR $0.58,95 \% \mathrm{Cl} 0.28$ to 1.18 ; two studies, 124 participants). There was significant heterogeneity in the effects of heated, humidified air on different outcomes, therefore, the authors graded the quality of the evidence as low. Some studies reported minor adverse events (including discomfort or irritation of the nose). The authors concluded that the current evidence does not show any benefits or harms from the use of heated, humidified air delivered for the treatment of the common cold.

\subsubsection{Probiotics}

In 2015, a Cochrane review assessing the effectiveness and safety of probiotics (any specified strain or dose), compared with placebo, in the prevention of acute URTIs in people of all ages, who are at risk of acute URTIs was published ${ }^{(208)}$.

The authors included 13 RCTs, although they could only extract data to meta-analyse 12 trials, which involved 3720 participants including children, adults (aged around 40 years) and older people. We found that probiotics were better than placebo when measuring the number of participants experiencing episodes of acute URTI (at least one episode: OR: 0.53; 95\% Cl=0.37-0.76, $\mathrm{p}<.001$, low quality evidence; at least three episodes: OR: 0.53 ; $95 \% \mathrm{Cl}=0.36-0.80, \mathrm{p}=.002$, low quality evidence); the mean duration of an episode of acute URTI (MD: $-1.89 ; 95 \% \mathrm{Cl}=-2.03$ to $-1.75, \mathrm{p}<.001$, low quality evidence); reduced antibiotic prescription rates for acute URTIs (OR: $0.65 ; 95 \% \mathrm{Cl}=0.45-0.94$, moderate quality evidence) and cold-related school absence (OR: 0.10 ; $95 \% \mathrm{Cl}=0.02-0.47$, very low quality evidence). Probiotics and placebo were similar when measuring the rate ratio of episodes of acute URTI (rate ratio: $0.83 ; 95 \% \mathrm{Cl}=0.66-1.05, \mathrm{p}=.12$, very low quality evidence) and adverse events (OR: $0.88 ; 95 \% \mathrm{Cl}=0.65$ $1.19, p=.40$, low quality evidence). Side effects of probiotics were minor and gastrointestinal symptoms were the most common. The authors concluded that probiotics were better than placebo in reducing the number of participants experiencing episodes of acute URTI, the mean duration of an episode of acute URTI, antibiotic use and cold-related school absence. This indicates that probiotics may be more beneficial than placebo for preventing acute URTIs. However, the quality of the evidence was low or very low ${ }^{(208)}$.

\subsubsection{Vitamin C}

In 2013, a Cochrane review on the use of vitamin C for preventing and treating the common cold appeared ${ }^{(209)}$. In the review 29 placebo-controlled trials evaluating 11,306 participants contributed to the meta-analysis on the RR of developing a cold whilst taking vitamin $C$ regularly over the study period. In the general community trials involving 10,708 participants, the pooled RR was 0.97 ( $\mathrm{Cl}$ interval 0.94 to 1.00). Five trials involving a total of 598 marathon runners, skiers and soldiers on subarctic exercises yielded a pooled RR of 0.48 ( $95 \% \mathrm{Cl} 0.35$ to 0.64 ). Thirty-one comparisons examined the effect of regular vitamin C on common cold duration (9745 episodes). In adults the duration of colds was reduced by $8 \%$ (3\% to $12 \%$ ) and in children by $14 \%$ ( $7 \%$ to $21 \%)$. In children, $1-2 \mathrm{~g}$ /day vitamin C shortened colds by $18 \%$. The severity of colds was also reduced by regular vitamin $\mathrm{C}$ administration. Seven comparisons examined the effect of therapeutic vitamin C (3249 episodes). No consistent effect of vitamin $C$ was seen on the duration or severity of colds in the therapeutic trials. The authors concluded that the failure of vitamin C supplementation to reduce the incidence of colds in the general population indicates that routine vitamin C supplementation is not justified, yet vitamin C may be useful for people exposed to brief periods of severe physical exercise. Regular supplementation trials have shown that vitamin $C$ reduces the duration of colds, but this was not replicated in the few therapeutic trials that have been carried out. Nevertheless, given the consistent effect of vitamin $\mathrm{C}$ on the duration and severity of colds in the regular supplementation studies, and the low cost and safety, it may be worthwhile for common cold patients to test on an individual basis whether therapeutic vitamin $C$ is beneficial for them. Further therapeutic RCTs are warranted. In 2018 and 2019, four systematic reviews investigated effects of vitamin C on common cold ${ }^{(210-213)}$. However, none of these systematic reviews included newer studies than included in the Cochrane review ${ }^{(209)}$. So these reviews were not considered.

\subsubsection{Vaccines}

In 2017, a Cochrane review was published to assess the clinical effectiveness and safety of vaccines for preventing the common cold in healthy people ${ }^{(214)}$. The development of vaccines for the common cold has been difficult because of antigenic variability of the common cold virus and the indistinguishable multiple other viruses. There is uncertainty regarding the efficacy and safety of interventions for preventing the common cold in healthy people.

The review includes only one RCT dating from the 1960s with an overall high risk of bias. The authors found no conclusive results to support the use of vaccines for preventing the common cold in healthy people compared with placebo. 


\subsubsection{Exercise}

In 2014, Lee et al. published a systematic review and meta-analysis to determine the effects of exercise on prevention of the common cold ${ }^{(215)}$. Four randomized controlled trials with a total of 281 participants were included. The effect of exercise on the prevention of the common cold had a relative risk (RR) of 0.73 $(95 \% \mathrm{Cl}, 0.56$ to $0.95 ; \mathrm{I}(2)=7 \%)$. The mean difference of mean illness days between exercise group and control group was -3.50 $(95 \% \mathrm{Cl},-6.06$ to $-0.94 ; \mathrm{I}(2)=93 \%)$. The authors concluded that regular, moderate-intensity exercise may have an effect on the prevention of the common cold.

\subsubsection{Echinacea}

To assess whether there is evidence that Echinacea preparations are effective and safe compared to placebo in the prevention and treatment of the common cold Karsch-Volk et al. included 24 double-blind trials with 4631 participants including a total of 33 comparisons of Echinacea preparations and placebo in a Cochrane review $^{(216)}$. Ten trials with 13 comparisons investigated prevention and 15 trials with 20 comparisons investigated treatment of colds (one trial addressed both prevention and treatment). None of the 12 prevention comparisons reporting the number of patients with at least one cold episode found a statistically significant difference. However, a post hoc pooling of their results, suggests a relative risk reduction of $10 \%$ to $20 \%$. Of the seven treatment trials reporting data on the duration of colds, only one showed a significant effect of Echinacea over placebo. The authors concluded that Echinacea products have not shown to provide benefits for treating colds, although, there could be a weak benefit from some Echinacea products: the results of individual prophylaxis trials consistently show positive (if non-significant) trends, although potential effects are of questionable clinical relevance.

\subsubsection{Zinc}

To assess whether zinc (irrespective of the zinc salt or formulation used) is efficacious in reducing the incidence, severity and duration of common cold symptoms placebo-controlled trials using zinc for at least five consecutive days to treat, or for at least five months to prevent the common cold. included 16 therapeutic trials (1387 participants) and two preventive trials (394 participants). Intake of zinc was associated with a significant reduction in the duration (days) (MD -1.03, 95\% Cl-1.72 to -0.34$)(p=0.003)(\mathrm{l}<$ sup $>2</$ sup $>$ statistic $=89 \%)$ but not the severity of common cold symptoms (MD $-1.06,95 \% \mathrm{Cl}-2.36$ to $0.23)(p=0.11)(\mathrm{l}<$ sup $>2</$ sup $>$ statistic $=84 \%)$. The proportion of participants who were symptomatic after seven days of treatment was significantly smaller (OR $0.45,95 \% \mathrm{Cl} 0.20$ to 1.00 ) $(p=0.05)$ than those in the control, $(\mid<$ sup $>2</$ sup $>$ statistic $=$ $75 \%)$. The incidence rate ratio (IRR) of developing a cold (IRR $0.64,95 \% \mathrm{Cl} 0.47$ to 0.88$)(\mathrm{p}=0.006)(\mathrm{l}<\mathrm{sup}>2</$ sup $>$ statistic $=$
$88 \%)$, school absence $(p=0.0003)$ and prescription of antibiotics $(p<0.00001)$ was lower in the zinc group. Overall adverse events (OR 1.58, 95\% Cl 1.19 to 2.09 ) ( $p=0.002$ ), bad taste (OR 2.31, 95\% $\mathrm{Cl} 1.71$ to 3.11$)(\mathrm{p}<0.00001)$ and nausea (OR $2.15,95 \% \mathrm{Cl} 1.44$ to 3.23) $(p=0.002)$ were higher in the zinc group. The very high heterogeneity means that the averaged estimates must be viewed with caution. Authors' conclusions: Zinc administered within 24 hours of onset of symptoms reduces the duration of common cold symptoms in healthy people, but some caution is needed due to the heterogeneity of the data. As the zinc lozenges formulation has been widely studied and there is a significant reduction in the duration of cold at a dose of $>=75 \mathrm{mg} /$ day, for those considering using zinc it would be best to use it at this dose throughout the cold. Regarding prophylactic zinc supplementation, currently no firm recommendation can be made because of insufficient data. When using zinc lozenges (not as syrup or tablets) the likely benefit has to be balanced against side effects, notably a bad taste and nausea ${ }^{(217)}$. Based on the same studies additional meta-analysis were performed. Hemila et al. evaluated whether the allergy status and other characteristics of common cold patients modify the effects of zinc acetate lozenges and concluded that since the effects of zinc acetate lozenges were consistent between the compared subgroups, the overall estimates for effect seemed applicable over a wide range of common cold patients ${ }^{(218)}$. Hemila furthermore compared the efficacy of zinc acetate lozenges with zinc gluconate and examined the dose-dependency of the effect and concluded that properly composed zinc gluconate lozenges may be as effective as zinc acetate lozenges. Moreover, he found no evidence that zinc doses over $100 \mathrm{mg} /$ day might lead to greater efficacy in the treatment of the common cold. Finally, the same group estimated using individual patient data (IPD) meta-analysis the effect of zinc acetate lozenges on the rate of recovery from colds and found a three-fold increase in the rate of recovery from the common cold ${ }^{(219)}$. From these meta-analyses it can be concluded that zinc administered as zinc acetate or zinc gluconate lozenges at a dose of $>=75 \mathrm{mg} /$ day and taken within 24 hours of onset of symptoms significantly reduces the duration of common cold. It is advised for those considering using zinc to use it at this dose throughout the cold. Regarding prophylactic zinc supplementation, currently no firm recommendation can be made because of insufficient data.

\subsubsection{Herbal medicine (excluding Echinacae)}

We are not aware of a systematic review analysing the effectiveness of herbal medicine in common cold. A recent review of Koch et al. included patients with symptoms and signs indicative of viral rhinosinusitis (common cold), post-viral rhinosinusitis and maybe a few patients with ABRS although in most studies high fever and/or severe illness were excluded ${ }^{(220)}$ and, therefore, could not be used. However, studies including large 
number of patients have been done evaluating the effectiveness of herbal medicine for common cold and, therefore, a short summary is given here. Four papers describe the effectiveness of BNO1016 (Sinupret) in patients with symptoms indicative of common cold ${ }^{(221-224)}$. The two papers by Jund are both double blind placebo controlled trials (DBPCTs) and the study described in the 2012 papers has also been included in the 2015 paper. BN01016 is an extract of five herbal drugs (gentian root, primula flower, sorrel herb, elder flower, and verbena herb) that has demonstrated antimicrobial and antiviral activity. The herbal drug combination was assessed for efficacy and tolerability in 600 patients with acute viral rhinosinusitis in two DBPCT trials ${ }^{(221,}$ ${ }^{222)}$. A significant higher response to treatment, and significantly more improvement in SNOT-20, major symptoms score, rhinorrhoea, postnasal drip headache and facial pain were found at day 14 in favour of BNO1016. No serious adverse events were reported in either trial. In a study of 64 patients with common cold Sinupret for eight days on top of antibiotic was evaluated (223). The Sinupret group had shorter duration of headache, impaired breathing, nasal discharge and olfactory impairment and faster resolution of clinical findings at rhinoscopy. Finally, a recent trial evaluated Sinupret syrup three times daily in a group of 184 children (mean age 8.4 (6-11)) on top of saline and symptomatic medication ${ }^{(224)}$. The children's self-assessment of their condition during the first 10 days of the treatment for three symptoms (rhinorrhoea, facial pain, and headache) was significantly better in the Sinupret group than placebo on days 5-8. The physicians had a similar assessment.

Two studies investigated cineole, an extract from eucalyptus oil with anti-inflammatory properties, in 302 patients with symptoms and signs indicative of viral rhinosinusitis, one versus placebo $^{(225)}$ and one versus an alternative herbal preparation ${ }^{(226)}$. Both studies found a greater reduction in symptom scores, individual symptom scores and rhinoscopy findings in the cineole group than in the control group after seven days of treatment. It can be concluded that the herbal medicine preparations BNO1016 and possibly also cineole have a significant impact on symptoms of common cold without important adverse events. Two studies evaluated the effectiveness of andrographis paniculata SHA-10 extract (1200 mg/day) for five days in one study ${ }^{(227)}$ and Kan Jang (85 mg standardized extract of andrographis paniculata SHA-10 and $10 \mathrm{mg}$ of eleutherococcus senticosus extract) four tablets, three times daily, for five days ${ }^{(228)}$ in reducing the prevalence and intensity of symptoms and signs in respectively 158 and 200 patients with common cold as compared with a placebo. A significant decrease in the intensity of symptoms was observed for the Andrographis paniculate group compared to placebo in both studies. No adverse effects were observed or reported. Finally, Hawkins et al. published a systematic review about the potential use of black elderberry (sambucus nigra) for common cold. But they combined three studies with patients with influenza with one study with patient with common cold. The study evaluating patients with common cold did not show a significant effect ${ }^{(229)}$.

In conclusion, some herbal medicines like BNO1016, cineole and andrographis paniculata SHA-10 extract have significant impact on symptoms of common cold without important adverse events. A formal systematic review is missing.

\subsubsection{Fusafungine}

Fusafungine displays bacteriostatic activity and has antiinflammatory properties. Lund et al. described a pooled analysis of three randomised double-blind placebo-controlled parallelgroup studies in 532 patients with identical objectives design and dosage ${ }^{(230)}$. The percentage of responders (patients with nasal symptom score improvement from day 0 to day 4) was $61.5 \pm 2.9 \%$ with fusafungine versus $46.8 \pm 3.1 \%$ with placebo $(p=0.009)$ with an odds ratio of $1.8(p=0.01)$ in favour of fusafungine. The nasal symptom score distribution at day 4 showed an odds ratio of $1.56(p=0.011)$ also in favour of fusafungine. For patients treated early the percentage of responders was $65.9 \pm 4.1 \%$ with fusafungine versus $38.3 \pm 4.0 \%$ with placebo $(p=0.022)$ with an OR of 3.08 ( $p=0.033)$ in favour of fusafungine. The authors concluded that fusafungine is an effective treatment of common cold especially when administered early. However, serious allergic reactions involving bronchospasm although rare have occurred after the use of fusafungine (https:// www.ema.europa.eu). For that reason, the medication is no longer on the market.

\subsubsection{Homeopathy}

A systematic review by Hawke aimed at assessing the effectiveness and safety of oral homoeopathic medicinal products compared with placebo or conventional therapy to prevent and treat acute respiratory infections in children ${ }^{(402)}$. After the analysis of eight RCTs including 1562 children, the authors reported no significant benefit of homoeopathic products compared to placebo on infection recurrence or cure rates in children. No similar study was found for the use of homoeopathic products in adults with respiratory tract infections.

\subsubsection{Conclusion}

In recent years a number of systematic reviews, many performed within the Cochrane collaboration have been performed evaluating the effectiveness of medication for the prevention and treatment of acute viral rhinosinusitis (common cold). The authors of these reviews concluded that to prevent common colds probiotics were better than placebo in reducing the number of participants experiencing episodes of acute URTI and that also moderate-intensity exercise may have an effect on the prevention of the common cold.

It was concluded that the failure of vitamin $\mathrm{C}$ supplementation 
to reduce the incidence of colds in the general population indicates that routine vitamin C supplementation is not justified, yet vitamin $C$ may be useful for people exposed to brief periods of severe physical exercise. And finally, no conclusive results supported the use of vaccines for preventing the common cold in healthy people compared with placebo.

For the treatment of acute viral rhinosinusitis, the authors of these reviews concluded that there is no evidence of benefit from antibiotics for the common cold or for persisting acute purulent rhinitis in children or adults and that there is evidence that antibiotics cause significant adverse effects in adults when given for the common cold. Although the treatment has no significant adverse effect it was also concluded that the current evidence does not support the use of nasal corticosteroids for symptomatic relief from the common cold. There is however some low / very low-quality evidence that probiotics were better than placebo in reducing the mean duration of an episode of acute URTI, antibiotic use and cold-related school absence.

For symptomatic medication, it was concluded that antihistamines have a limited short-term (days one and two of treatment) beneficial effect on severity of overall symptoms in adults but not in the mid to long term. There is no clinically significant effect on nasal obstruction, rhinorrhoea or sneezing, that multiple doses of decongestants may have a small positive effect on subjective measures of nasal congestion in adults with the common cold without increasing the risk of adverse events in adults in the short term. Moreover, it was concluded that paracetamol may help relieve nasal obstruction and rhinorrhoea but does not appear to improve some other cold symptoms (including sore throat, malaise, sneezing and cough), NSAIDs relieve discomfort or pain caused by the common cold and it was suggested that antihistamine-analgesic-decongestant combinations have some general benefit in adults and older children. These benefits must be weighed against the risk of adverse effects. The authors also concluded that ipratropium bromide is likely to be effective in ameliorating rhinorrhea in common cold but had no effect on nasal congestion and its use was associated with more side effects compared to placebo or no treatment although these appeared to be well tolerated and self-limiting.

It was also concluded that because there is a consistent effect of vitamin $\mathrm{C}$ on the duration and severity of colds in supplementation studies, and the low cost and safety, it may be worthwhile for common cold patients to test on an individual basis whether therapeutic vitamin $C$ is beneficial for them. The same holds for zinc administered as zinc acetate or zinc gluconate lozenges at a dose of $>=75 \mathrm{mg} /$ day and taken within 24 hours of onset of symptoms significantly reduces the duration of common cold. When using zinc lozenges the likely benefit has to be balanced against side effects, notably a bad taste and nausea. Also, nasal saline irrigation has possible benefits for relieving the symptoms of acute URTIs contrary to steam which does not show any be- nefits or harms for the treatment of the common cold. Finally, it was concluded that Echinacea products have not been shown to provide benefits for treating colds, although, there could be a weak benefit from some Echinacea products. However, some herbal medicine like BN1016, Cineole and Andrographis paniculata SHA-10 extract seem to have a significant impact on symptoms of common cold without important adverse events.

\subsubsection{Oral antibiotics - short courses}

\subsubsection{Short courses of oral antibiotics in adult patients with acute} bacterial rhinosinusitis (ABRS)

\subsection{Summary of the evidence}

ABRS involves a small portion of patients with ARS. ABRS is considered to be present when three or more of the following criteria are present: discoloured discharge, severe (unilateral dominance) facial pain, high fever $\left(>38^{\circ} \mathrm{C}\right)$, double sickening or raised ESR / CRP(231). Antibiotics are thought to be useful in at least part of the patients with ABRS but poor patient selection increases the risk of unwanted antibiotics resistance. There is a need to assess the efficacy of antibiotics among patients who fulfil the criteria for ABRS. In this section, studies which compared antibiotics with placebo for the treatment ABRS were assessed.

Studies which involved adults and adolescents ( 12 years and above) with symptoms that fulfilled the criteria for ABRS were included. Studies without a placebo arm, involving children (under 12 years old), participants with other respiratory tract infection (such as otitis media, tonsillitis or pharyngitis) or including participants with symptoms lasting more than 12 weeks, were excluded. Only randomized controlled trials were considered. The outcomes evaluated were the percentage of patients that achieved cure or improvement during or at the end of the treatment period.

Three studies were included ${ }^{(232-234)}$, two double blinded placebo controlled randomized trials $s^{(232,233)}$ and one open labelled randomized trial(234) (Table 4.6.1.). All studies involved participants who had symptoms and/or signs suggestive of ABRS. Lindbaek et al. ${ }^{(232)}$ recruited patients with ABRS symptoms and abnormalities at CT scan (total opacification or fluid level in any sinus). A score of one point was given for: symptoms lasting longer than seven days before the first visit, unilateral facial pain, pain in upper teeth, pain worsening on bending forward, two phases of disease, rhinorrhoea, nasal obstruction, sinus pain, malaise, fever above $38^{\circ} \mathrm{C}$ and hyposmia or anosmia and two extra points for purulent secretion resulting in a maximum severity score of 13 points. The participants in this study had a severity score between 7.8 to 8.8 which meant they would have at least six of the 11 symptoms assessed, and, therefore, would fulfil the criteria for ABRS. Hadley et al. ${ }^{(233)}$ included adults diagnosed by 
Table 4.6.1. Antibiotics vs. placebo in acute post-viral rhinosinusitis.

\begin{tabular}{|c|c|c|c|c|c|}
\hline Study & Methods & Participants & Interventions & Outcomes & Results \\
\hline $\begin{array}{l}\text { Garbutt } \\
2012^{(5)}\end{array}$ & DBPCRT & $\begin{array}{l}\text { Adults with maxillary pain/ } \\
\text { sinus tenderness, purulent } \\
\text { secretions and rhinosinusitis } \\
\text { symptoms which did not } \\
\text { improve/worsened after } \\
\text { 7-28 days. }\end{array}$ & $\begin{array}{l}\text { - Amoxicillin } 1500 \mathrm{mg} \\
3 \text { times daily for } 10 \text { days } \\
(\mathrm{n}=85) \\
\text { - Placebo }(n=81)\end{array}$ & $\begin{array}{l}\text { - Mean difference between } \\
\text { groups (SNOT-16) day } 3,7 \text {, } \\
10 \text { and } 28 \\
\text { - Symptom improvement/ } \\
\text { cure at day } 3,7,10 \text { and } 28 \\
\text { - Days unable to perform } \\
\text { usual activity } \\
\text { - Relapse or recurrence at } \\
\text { day } 28 \\
\text { - Adverse event }\end{array}$ & $\begin{array}{l}\text { - SNOT- } 16 \text { score was lower } \\
\text { at day } 7 \text { in antibiotics group } \\
\text { but no difference at day } 3 \\
\text { and } 28 \\
\text { - More patients in antibiotic } \\
\text { group had symptoms } \\
\text { improvement at day } 7 \text { but } \\
\text { not at day } 3,10 \text { or } 28 \\
\text { - No difference in other } \\
\text { outcomes }\end{array}$ \\
\hline $\begin{array}{l}\text { Meltzer } \\
2005^{(6)}\end{array}$ & DBPCRT & $\begin{array}{l}\text { Adults ( } \geq 12 y \text { ), clinical } \\
\text { diagnosis acute sinusitis } \\
\text { (7-28 days) }\end{array}$ & $\begin{array}{l}\text { - Amoxicillin } 500 \mathrm{mg} \text { three } \\
\text { times daily for } 10 \text { days } \\
(\mathrm{n}=251) \\
\text { - Placebo ( } n=252) \\
\text { - MFNS } 200 \mathrm{mg} \text { once daily } \\
\text { with placebo spray in the } \\
\text { evening ( } n=243 \text { ) } \\
\text { - MFNS } 200 \mathrm{mg} \text { twice daily } \\
\text { ( } n=235)\end{array}$ & $\begin{array}{l}\text { - Mean symptoms score } \\
\text { (diary)between day } 2 \text { to } 15 \\
\text { - Global response at day } 15 \\
\text { - Treatment failure during } \\
\text { treatment } \\
\text { - Adverse event }\end{array}$ & $\begin{array}{l}\text { - No difference in symptom } \\
\text { score between antibiotics } \\
\text { and placebo } \\
\text { - No difference in global } \\
\text { response } \\
\text { - Treatment failure in } 7.2 \% \\
\text { antibiotics and } 10.7 \% \\
\text { placebo } \\
\text { - No difference in adverse } \\
\text { event }\end{array}$ \\
\hline $\begin{array}{l}\text { Merenstein } \\
2005^{(7)}\end{array}$ & DBPCRT & $\begin{array}{l}\text { Adult (18y) with at least } \\
1 \text { symptom of sinusitis } \\
\text { (purulent } d / c \text {, facial pain, } \\
\text { pus in nasal cavity) for at } \\
\text { least } 7 \text { days }\end{array}$ & $\begin{array}{l}\text { - Amoxicillin } 1 \mathrm{~g} \text { twice daily } \\
\text { for } 10 \text { days }(\mathrm{n}=67) \\
\text { - Placebo twice daily for } 10 \\
\text { days }(\mathrm{n}=68)\end{array}$ & $\begin{array}{l}\text { - Patient reported improve- } \\
\text { ment at day } 14 \\
\text { - Likert scale for feeling sick } \\
(0-10) \text { at day } 3,7 \text { and } 14 \\
\text { - Days to improvement } \\
\text { - Adverse event }\end{array}$ & $\begin{array}{l}\text { - No difference in improve- } \\
\text { ment at day } 14 \\
\text { - No difference in likert } \\
\text { score at day } 3,7 \text { and } 14 \\
\text { - Antibiotic group improved } \\
\text { earlier ( } 8.1 \text { vs. } 10.7 \text { days) }\end{array}$ \\
\hline $\begin{array}{l}\text { Haye } \\
1998^{(8)}\end{array}$ & DBPCRT & $\begin{array}{l}\text { Adults (28-70y), GP practice, } \\
\text { purulent nasal secretion } \\
\text { (10-30 days) AND maxillary } \\
\text { sinus pain/tenderness }\end{array}$ & $\begin{array}{l}\text { - Azithromycin } 500 \mathrm{mg} \text { once } \\
\text { daily for } 3 \text { days }(n=87) \\
\text { - Placebo }(n=82)\end{array}$ & $\begin{array}{l}\text { - Cure or improvement, at } \\
\text { day } 3-5 \text {, day } 10-12 \text { days and } \\
\text { day } 23-27 \\
\text { - Relapse at day } 27 \\
\text { - Adverse event }\end{array}$ & $\begin{array}{l}\text { - No difference in overall } \\
\text { cure or improvement at day } \\
23-27 \text { but more patients } \\
\text { with improvement in antibi- } \\
\text { otics group at day } 10-12 \\
\text { - No difference in relapse } \\
\text { - No difference in adverse } \\
\text { effect }\end{array}$ \\
\hline $\begin{array}{l}\text { Lindbaek } \\
1998^{(9)}\end{array}$ & DBPCRT & $\begin{array}{l}\text { Adults with clinically } \\
\text { diagnosed acute sinusitis } \\
\text { AND mucosal thickening } \\
\text { ( } \geq 5 \mathrm{~mm} \text { ) on CT scan (exclude } \\
\text { opacification). (Duration } \\
\text { illness median 10-13 days } \\
\text { from results.) }\end{array}$ & $\begin{array}{l}\text { - Penicillin } \vee 1,320 \mathrm{mg} 3 \\
\text { times daily for } \\
10 \text { days }(n=20) \\
\text { - Amoxicillin } 500 \mathrm{mg} 3 \text { times } \\
\text { daily for } 10 \text { days }(n=22) \\
\text { - Placebo }(n=21)\end{array}$ & $\begin{array}{l}\text { - Number restored or much } \\
\text { better at day } 10 \\
\text { - Mean VAS score for sinus } \\
\text { symptoms at day } 10 \\
\text { - Duration of illness (feeling } \\
\text { sick) }\end{array}$ & $\begin{array}{l}\text { - No difference in number } \\
\text { restored or much better } \\
\text { between groups } \\
\text { - No difference in VAS } \\
\text { between groups at day } 10 \\
\text { - No difference in duration } \\
\text { of illness }\end{array}$ \\
\hline $\begin{array}{l}\text { Stalman } \\
1997^{(10)}\end{array}$ & DBPCRT & $\begin{array}{l}\text { Adults (15y) with symptoms } \\
\text { of URTI at least } 5 \text { days. Pres- } \\
\text { ence of } 2 \text { out of } 3 \text { symptoms } \\
\text { (common cold, purulent } \\
\text { discharge, pain in maxillary } \\
\text { sinus when bending) }\end{array}$ & $\begin{array}{l}\text { - Doxycycline } 100 \mathrm{mg} \text { once } \\
\text { daily for } 10 \text { days }(n=98) \\
\text { - Placebo }(n=94)\end{array}$ & $\begin{array}{l}\text { - Days to cure (no pain) } \\
\text { - Cure or improvement day } \\
10 \text { and day } 42 \\
\text { - Side effect }\end{array}$ & $\begin{array}{l}\text { - No difference in time to } \\
\text { recover } \\
\text { - No difference in numbers } \\
\text { cures or improvement at } \\
\text { day } 10 \text { or day } 42 \\
\text { - } 17 \text { in antibiotics and } 2 \text { in } \\
\text { placebo group reported } \\
\text { side effects }\end{array}$ \\
\hline $\begin{array}{l}\text { Van Bu- } \\
\text { chem } \\
1997^{(11)}\end{array}$ & DBPCRT & $\begin{array}{l}\text { Patients with maxillary } \\
\text { sinusitis (acute onset of a } \\
\text { common cold with sickness, } \\
\text { headache, nose obstruction, } \\
\text { discharge and tapping pain } \\
\text { of the maxillary sinus) AND } \\
\text { abnormal radiograph on } \\
\text { Caldwell or water's view } \\
\text { (mucosal thickness }>5 \mathrm{~mm} \text {, } \\
\text { air fluid level, opacification). } \\
\text { Mean symptom duration } 2.2 \\
\text { weeks. }\end{array}$ & $\begin{array}{l}\text { - Amoxicillin } 750 \mathrm{mg} 3 \text { times } \\
\text { daily for } 7 \text { days }(n=108) \\
\text { - Placebo }(n=106)\end{array}$ & $\begin{array}{l}\text { - Cure or greatly decreased } \\
\text { symptoms at } 2 \text { weeks } \\
\text { - Mean change symptom } \\
\text { score after } 2 \text { weeks (scale } \\
1-5 \text { ) } \\
\text { - Normal radiograph at } 2 \\
\text { weeks } \\
\text { - Relapse after } 1 \text { year } \\
\text { - Side effects }\end{array}$ & $\begin{array}{l}\text { - No difference in cure } \\
\text { - No difference in symptoms } \\
\text { score } \\
\text { - Normal radiographs at } 2 \\
\text { weeks were similar in both } \\
\text { groups } \\
\text { - More side effects in antibi- } \\
\text { otics group } \\
\text { - No difference in relapse } \\
\text { after } 1 \text { year }\end{array}$ \\
\hline
\end{tabular}

$\mathrm{CT}$, computed tomography; DBPCRT, double blind placebo controlled randomised trial; dc, discharge; GP, general practitioner; MFNS, mometasone furoate nasal spray; SNOT-16, Sino-nasal Outcome Test-16; URTI, upper respiratory tract infection; VAS, visual analogue scale; $y$, years. 
clinical symptoms of rhinosinusitis [two major (purulent anterior or posterior nasal discharge and unilateral facial pain or malar tenderness) or one major and one minor symptom (frontal headache or fever $>37.5^{\circ} \mathrm{C}$ axillary temp)] lasting between seven and 28 days and presence of air-fluid levels and/or opacification on Water's view of a sinus X-ray and positive bacterial culture from a sinus puncture. Høsøien et al. ${ }^{(234)}$ included patients with facial pain, purulent discharge, double sickening within 10 days initial improvement and neutrophilia.

Three different types of antibiotics were used (moxifloxacin $400 \mathrm{mg}$ daily) ${ }^{(233)}$, penicillin V $1320 \mathrm{mg}$ three times daily ${ }^{(232)}$ and amoxicillin $500 \mathrm{mg}$ three times daily ${ }^{(232,234)}$ for the duration of five to 10 days. Two studies used matched placebos while one study used ultrasound as comparison ${ }^{(234)}$. Cure was defined as resolution of signs or symptoms after completion of treatment which did not need further intervention ${ }^{(233)}$, or self-rated as either feeling restored or much better ${ }^{(232)}$. Improvement was defined as improved clinical symptoms during the treatment phase. Hadley et al. ${ }^{(233)}$ did not report any difference in improvement or cure between antibiotics and placebo while Lindbaek et al. ${ }^{(232)}$ reported significantly better improvement and cure rate for the antibiotics group. Høsøin et al. ${ }^{(234)}$ compared antibiotics with ultrasound therapy and found little or no difference in the pain reduction between groups. Although this study reported a larger decrease in pain score at day 4 in the ultrasound group, the difference in pain reduction between the groups was small (1.5 out of 10).

Two studies could be combined into a meta-analysis. In the study of Lindbaek two antibiotics were assessed and evaluated separately ${ }^{(232)}$. At completion of treatment (day 6 to 10), the antibiotics group (two studies (three arms), 289 patients) showed a higher cure rate than placebo RR 1.36 (95\% Cl 1.16-1.59, 12=23\%) (Figure 4.6.1.). The antibiotics group (two studies, 289 patients) also had more patients with improved symptoms at day three of treatment compared to placebo (RR 1.68 95\% Cl 1.04-2.71, I2=84\%) (Figure 4.6.2.). Meta-analysis of adverse events did not quite reach significance in terms of more adverse events in the antibiotics group in these studies (RR 1.27 95\% Cl 0.87-1.86, 12 64\%) (Figure 4.6.3.).

\subsection{Conclusion}

Antibiotics are effective in a select group of patients with symptoms and signs suggestive of ABRS. From the limited data available (two studies versus one) it seems that especially amoxicillin / penicillin (beta-lactams) are effective and moxifloxacin (fluoroquinolone) is not. The efficacy of beta-lactams is evident at day three where patients already experience better symptom improvement and continues to have a higher number of cures at completion of treatment. However, careful patient selection for those with ABRS is needed to avoid unnecessary use of antibiotics and side effects.

\subsubsection{Short courses of oral antibiotics in children with ABRS}

\subsection{Summary of the evidence}

Studies which involved children ( $<12$ years of life) with symptoms that fulfilled the criteria for ABRS were included. Only randomized controlled trials were considered. The outcomes evaluated were the percentage of patients that achieved cure or improvement during or at the end of the treatment period. Two small double blinded placebo controlled randomized trials were included $^{(235,236)}$. Both studies involved at least partly children who had symptoms and/or signs suggestive of ABRS (Table 4.6.2.).

Wald et al. ${ }^{(235)}$ included 56 children from one to 10 years of age (average five years) with ARS including one of three features (persistent symptoms $>10$ days, acutely worsening symptoms after day 5 , or severe symptoms with fever $\geq 102 \mathrm{~F}$ and purulent discharge for three consecutive days). In 24 (43\%) children, the illness was classified as mild, whereas in the remaining 32 (57\%) children it was severe. Children were randomly assigned to receive either amoxicillin $(90 \mathrm{mg} / \mathrm{kg}$ ) with potassium clavulanate $(6.4 \mathrm{mg} / \mathrm{kg})$ or placebo. Children's conditions were rated as cured, improved, or failed according to scoring rules. Of the 28 children who received the antibiotic, 14 (50\%) were cured, four (14\%) were improved, four (14\%) experienced treatment failure, and six (21\%) withdrew. Of the 28 children who received placebo, four (14\%) were cured, five (18\%) improved, and 19 (68\%) experienced treatment failure. Children receiving the antibiotic were more likely to be cured (50\% versus $14 \%$ ) and less likely to have treatment failure ( $14 \%$ versus $68 \%$ ) than children receiving the placebo. There was no division between the mild and severe patients. Adverse events were reported in $44 \%$ children receiving antibiotics and in $414 \%$ children receiving the placebo $(p=.014)$. The most common adverse event was diarrhoea, which, in general, was self-limited.

Ragab $^{(236)}$ included 53 children ( $<12$ years, average five years) with URTI symptoms $>10$ days $<28$ days with at least three symptoms/signs pointing to ABRS (discharge (with unilateral predominance) and purulent secretion in the middle meatus, severe local pain (with unilateral predominance), fever $\left(>38^{\circ} 8 \mathrm{C}\right.$ ), and double sickening) in a DBPCT comparing amoxicillin 100 $\mathrm{mg} / \mathrm{kg} /$ day to placebo. All patients were on saline irrigation. In the amoxicillin group clinical cure was observed in $83.9 \%$ in comparison to $71 \%$ patients in the placebo group ( $p=0.22$ ). There were also no differences between both groups in the reported nasal symptom scores, total symptoms scores improvements at day $7(p=0.09$ and 0.65$)$ and day $14(p=0.29$ and 0.14$)$, and the mean total PRQLQ values after the two weeks of treatment $(p=0.06)$. Saline with placebo had less reported adverse effects than amoxicillin and nasal saline irrigations $(p=0.005)$.

The studies could be combined into a meta-analysis considering cure at 14 days. Although both studies favoured antibiotics, there was no significant difference over placebo (RR $1.4595 \% \mathrm{Cl}$ 
Figure 4.6.1. Forest plot of the effect of antibiotic versus placebo for cure at completion of intervention (day 6-10) in adult patients with acute bacterial rhinosinusitis.

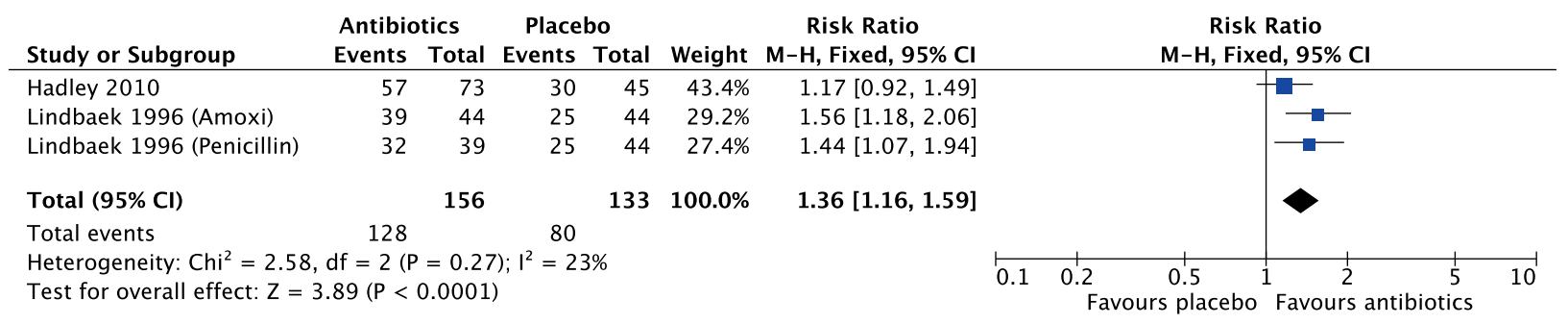

Figure 4.6.2. Forest plot of the effect of antibiotic versus placebo to assess improvement at day 3 of treatment of adult patients with acute bacterial rhinosinusitis

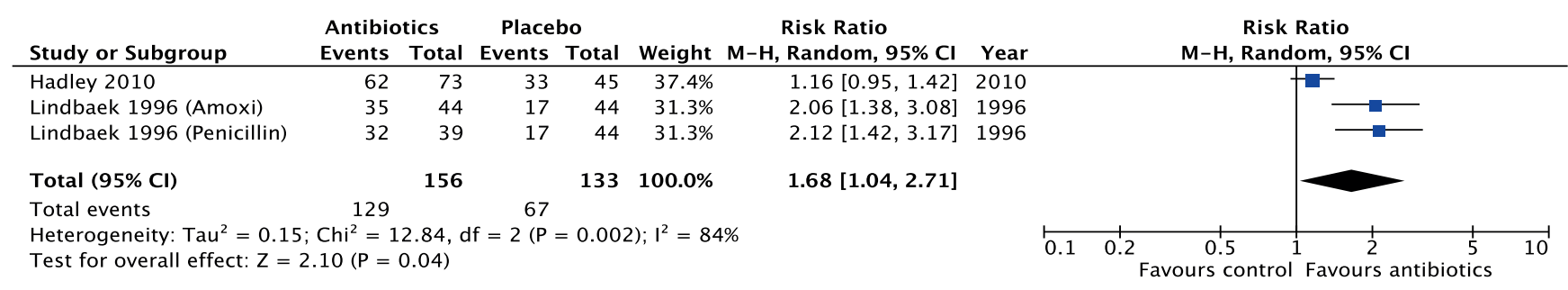

$\mathrm{Cl}$, confidence interval; $\mathrm{M}-\mathrm{H}$, Mantel Haenszel.

Figure 4.6.3. Forest plot of the effect of antibiotic versus placebo for adverse effects during treatment of adult patients with acute bacterial rhinosinusitis

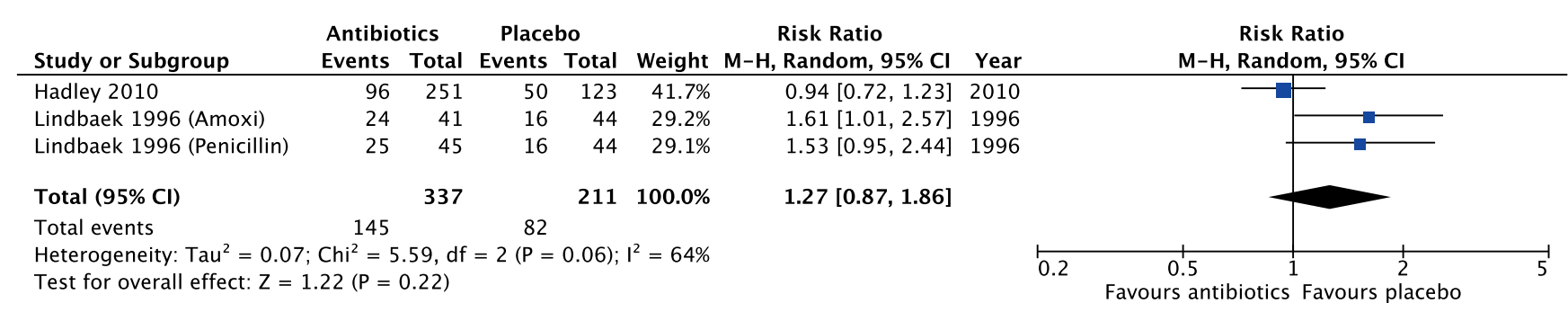

$\mathrm{Cl}$, confidence interval; $\mathrm{M}-\mathrm{H}$, Mantel Haenszel.

Figure 4.6.4. Forest plot of the effect of antibiotic vs placebo for improvement at completion of intervention (day 14) in children with acute bacterial rhinosinusitis

Antibiotics Placebo

Study or Subgroup Events Total Events Total Weight $\mathrm{M}-\mathrm{H}, \mathrm{Random}, \mathbf{9 5 \%} \mathrm{Cl}$

$\begin{array}{lllllll}\text { Ragab } 2015 & 26 & 31 & 22 & 31 & 61.2 \% & 1.18[0.90,1.55]\end{array}$

Wald 2009

Total $(95 \% \mathrm{Cl})$

Total events

59

44

$59 \quad 100.0 \%$

31

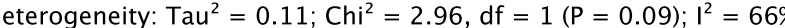

Test for overall effect: $Z=1.31(P=0.19)$
$2.00[1.09,3.66]$

$1.45[0.83,2.53]$

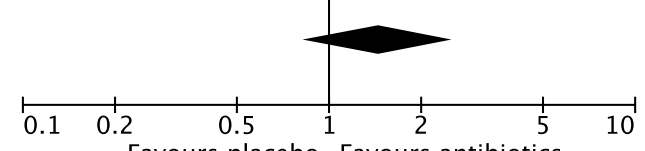

$\mathrm{Cl}$, confidence interval; $\mathrm{M}-\mathrm{H}$, Mantel Haenszel. 
Table 4.6.2. Double blind placebo controlled RCT on antibiotics in presumed ABRS in children.

\begin{tabular}{|c|c|c|c|c|c|}
\hline Study & Methods & Participants & Interventions & Outcomes & Results \\
\hline $\begin{array}{l}\text { Ragab } \\
2015^{(236)}\end{array}$ & DBPCRT & $\begin{array}{l}\text { Children }(<12 y \text { average } 5 y \text { ) with URTI } \\
\text { symptoms } \\
>10 \text { days }<28 \text { days. At least } 3 \\
\text { symptoms/signs (discharge (with } \\
\text { unilateral } \\
\text { predominance) and purulent secretion } \\
\text { in the middle meatus, severe local pain } \\
\text { (with unilateral predominance), fever } \\
\left(>38,8^{\circ} \mathrm{C}\right) \text {, and double sickening). }\end{array}$ & $\begin{array}{l}\text { - Amoxicillin } 100 \mathrm{mg} / \\
\mathrm{kg} / \text { day three times } \\
\text { daily with normal sa- } \\
\text { line irrigation ( } \mathrm{n}=41) \\
\text { - Placebo with normal } \\
\text { saline irrigation only } \\
(\mathrm{n}=43)\end{array}$ & $\begin{array}{l}\text { - Clinical cure at day } \\
14 \\
\text { - Mean total nasal } \\
\text { symptoms score } \\
\text { (TNSS) day 0-7 and } 7 \\
\text { - PRQOL score } \\
\text { - Neutrophil counts on } \\
\text { cytology } \\
\text { - Adverse event }\end{array}$ & $\begin{array}{l}\text { - No difference in clinical } \\
\text { cure } \\
\text { - No difference in TNSS } \\
\text { - No difference in PRQOL } \\
\text { score at day } 7 \text { and } 14 \\
\text { - No difference in neutrophil } \\
\text { count at day } 7 \text { and day } 14 \\
\text { - More adverse event in } \\
\text { antibiotic group }\end{array}$ \\
\hline $\begin{array}{l}\text { Wald } \\
2009^{(235)}\end{array}$ & DBPCRT & $\begin{array}{l}\text { Children } 1 \text { to } 10 y \text { (average } 5 y \text { ) ( } n=56 \text {, } \\
50 \text { evaluated) with a clinical presen- } \\
\text { tation compatible with ABRS. One of } \\
\text { three features (persistent symptoms } \\
>10 \text { days, acutely worsening } \\
\text { symptoms after day } 5 \text {, or severe } \\
\text { symptoms with fever } \geq 102 \mathrm{~F} \text { and puru- } \\
\text { lent discharge for } 3 \text { consecutive days). }\end{array}$ & $\begin{array}{l}\text { - Amoxicillin ( } 90 \mathrm{mg} / \\
\mathrm{kg} \text { ) with potassium } \\
\text { clavulanate ( } 6.4 \mathrm{mg} / \\
\mathrm{kg})(\mathrm{n}=22) \text { (unclear } \\
\text { how many days) } \\
\text { - Placebo }(\mathrm{n}=28)\end{array}$ & $\begin{array}{l}\text { - Cured (defined } \\
\text { as clinical severity } \\
\text { score }<2 \text { ) or improved } \\
\text { (score decrease at } \\
\text { least } 50 \% \text { ) at day } 14 \\
\text { - Adverse event }\end{array}$ & $\begin{array}{l}\text { - Children receiving the anti- } \\
\text { biotic were more likely to be } \\
\text { cured }(50 \% \text { vs. } 14 \%, p=0.01) \\
\text { - More adverse event in } \\
\text { antibiotics group }\end{array}$ \\
\hline
\end{tabular}

ABRS, acute bacterial rhinosinusitis; DBPCRT, double blind placebo controlled randomised trial; PRQOL, patient rated quality of life; TNSS, total nasal symptoms score; $y$, years.

0.83-2.53, $\mathrm{p}=0.19,2 \mathrm{RCT}, \mathrm{n}=118, \mathrm{I} 2=66 \%$ ) (Figure 4.6.4.).

The children on antibiotics had significantly more adverse events compared to the placebo (RR $2.5095 \% \mathrm{Cl}$ 1.43-4.37, $\mathrm{p}=0.001,2 \mathrm{RCT}, \mathrm{n}=118, \mathrm{I}=0 \%$ ) (Figure 4.6.5.).

\subsection{Conclusion}

Data on the effect of antibiotics on the cure/improvement of symptoms in ABRS in children are very limited. There are only two studies with limited numbers that do not show a significant difference over placebo but do show a significant higher percentage of adverse events. Larger trials are needed to explain the difference between adults where antibiotics in ABRS has been shown to be effective (see paragraph 4.6.3.1.) and this outcome. Systematic reviews or meta-analysis of antibiotic use need to pay careful consideration to the different classes of antibiotics that target different bacterial molecular mechanisms, similarly to how different biologics will target different host molecular mechanisms (Figure 4.6.6.).
4.6.3.3. Short course of oral antibiotics in adult patients with acute post viral rhinosinusitis

\subsection{Summary of the evidence}

Acute post viral rhinosinusitis is defined as having symptoms of acute rhinosinusitis (ARS) persisting for more than 10 days or worsening after five days ${ }^{(231)}$. This section aims to assess the efficacy of antibiotics in the post viral ARS group. Studies were included which involved adults with ARS with symptoms of at least five days but less than 12 weeks (to exclude chronic rhinosinusitis). Only double-blind placebo-controlled randomized trials were included.

Studies were excluded if the study population fulfilled the criteria for acute bacterial rhinosinusitis (ABRS) or if more than $20 \%$ of the study population had symptoms for less than five days. The minimum duration of five days was used as a cutoff to exclude patients with common cold. Studies without a placebo arm, involving children (under 12 years old) or involving participants with other respiratory tract infection (such as otitis media, tonsillitis or pharyngitis) were also excluded. The primary

Figure 4.6.5. Forest plost of percentage of patients on antibiotics vs placebo for adverse effects during treatment for children with acute bacterial rhinosinusitis.

\begin{tabular}{|c|c|c|c|c|c|c|c|}
\hline \multirow[b]{2}{*}{ Study or Subgroup } & \multicolumn{2}{|c|}{ Antibiotics } & \multicolumn{2}{|c|}{ Placebo } & \multicolumn{3}{|c|}{ Risk Ratio } \\
\hline & Events & Total & Events & Total & Weight & M-H, Fixed, $95 \% \mathrm{Cl}$ & Yea \\
\hline Wald 2009 & 12 & 28 & 4 & 28 & $33.3 \%$ & $3.00[1.10,8.18]$ & 2009 \\
\hline Ragab 2015 & 18 & 31 & 8 & 31 & $66.7 \%$ & $2.25[1.15,4.39]$ & 2015 \\
\hline Total $(95 \% \mathrm{Cl})$ & & 59 & & 59 & $100.0 \%$ & $2.50[1.43,4.37]$ & \\
\hline $\begin{array}{l}\text { Total events } \\
\text { Heterogeneity: } \mathrm{Chi}^{2} \\
\text { Test for overall effec }\end{array}$ & $\begin{array}{r}30 \\
0.22, \mathrm{df} \\
Z=3.2\end{array}$ & $\begin{array}{l}=1(P= \\
(P=0 .\end{array}$ & $\begin{array}{l}12 \\
=0.64) \\
001)\end{array}$ & $=0 \%$ & & & \\
\hline
\end{tabular}

$\mathrm{Cl}$, confidence interval; $\mathrm{M}-\mathrm{H}$, Mantel Haenszel. 
Figure 4.6.6. Antibiotic classes and molecular targets.

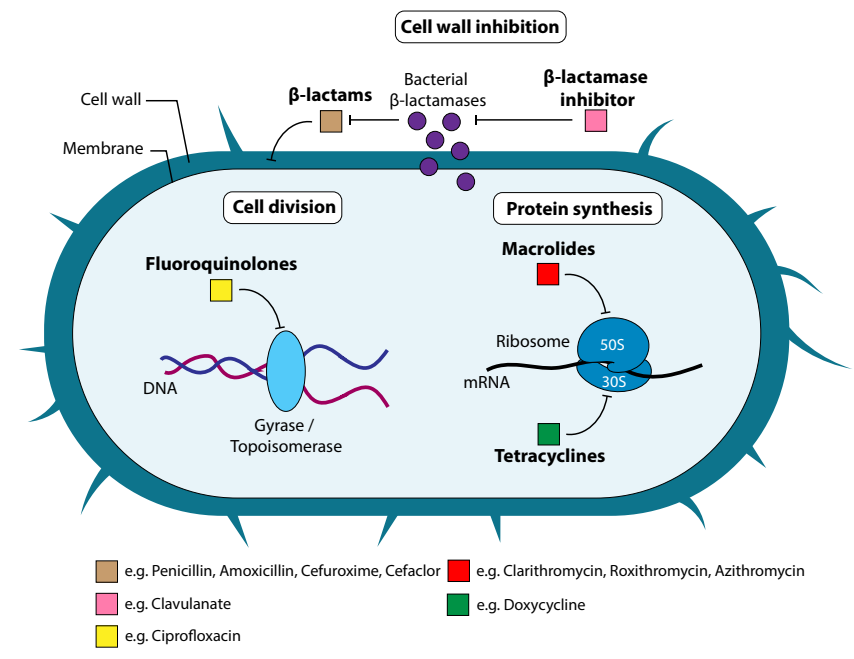

Beta-lactams (e.g. Penicillin, Amoxicillin, Cefuroxime, Cefaclor) target peptidoglycan crosslinking in the cell wall. Beta-lactamase inhibitors (e.g. Clavulanate) target bacterial beta-lactamases that break down beta-lactam antibiotics. Fluoroquinolones (e.g. Ciprofloxacin) target DNA gyrase and Topoisomerase IV, thereby blocking DNA separation and cell division. Tetracylines (e.g. Doxycycline) target the 30s ribosomal submit thereby blocking protein synthesis. Similarly, macrolides (e.g. Clarithromycin, Roxithromycin, Azithromycin) target the 50s ribosomal submit thereby blocking protein synthesis.

outcome is number of patients who obtained cure. Cure was defined as clinical resolution or significant improvement of symptoms which did not require any further intervention. The secondary outcomes assessed were number of days to cure and adverse effects.

Seven studies were included ${ }^{(237-243)}$ (Table 4.6.1). Most studies evaluated amoxicillin ${ }^{(237-239,241,243)}$ and three studies evaluated penicillin $\mathrm{V}^{(241)}$, doxycycline ${ }^{(242)}$ or azithromycin ${ }^{(240)}$, respectively. The antibiotics were taken for seven to ten days except for azithromycin (three days) ${ }^{(240)}$. All participants had symptoms of acute rhinosinusitis which fulfilled the EPOS criteria for at least at least five days. There were three studies which required participants to have symptoms for at least seven days prior to randomization ${ }^{(237-239)}$ while one study required 10 days ${ }^{(240)}$. Lindbaek et al. ${ }^{(241)}$ did not specify duration of symptoms prior to recruitment but the median duration of illness for participants after randomization was 10 to 13.5 days after treatment indicating post viral ARS. Garbutt et al. ${ }^{(237)}$ reported higher improvement in the antibiotics group on day seven of treatment compared to the placebo group. However, this difference was not significant at completion of treatment at day 10. Haye et al. ${ }^{(240)}$ assessed azithromycin for three days and reported more improvement in the antibiotics group at day 10-12. However, this difference was not seen at day 3 or 28 . The other five studies ${ }^{(238,239,241-243)}$ did not find any difference in improvement of symptoms or achievement of cure between groups at any time point.

All but one of the studies could be combined into a metaanalysis to determine the cure rate at day 10-14 (931 patients in seven studies) $)^{(237,239-243)}$. The use of antibiotics was not associated with greater cure at day 10-14 (RR $1.0695 \% \mathrm{Cl} .98-1.14$, I2=0\%) (Figure 4.6.7).

There were three studies ${ }^{(239,241,243)}$ which reported on the number of days needed to achieve cure after treatment. Only Merenstein et al. ${ }^{(239)}$ reported an earlier cure for the antibiotics group (median: eight versus 12 days, $\mathrm{p}=0.04$ ). The other two studies did not report significant differences in the number of days to cure between the antibiotics and the placebo group ${ }^{(241,243)}$. Two studies ${ }^{(239,242)}$ including 233 patients could be pooled to assess the number of days to achieve cure. The use of antibiotics in post-viral ARS was not associated with earlier cure (SMD -0.43, $95 \% \mathrm{Cl}-1.02-0.16,12=73 \%$ ) (Figure 4.6.8.).

Improvement of symptoms was assessed at day 3 of treatment in four studies. This was defined as complete/partial disappearance of symptoms ${ }^{(240)}$, patient reporting feeling a lot better/no symptoms $^{(237)}$, not feeling ill at day 3 of treatment ${ }^{(241)}$ or without

Figure 4.6.7. Forest plot of the effect of antibiotic versus placebo for cure at completion of the intervention (days 10-14) in adult patients with acute post-viral acute rhinosinusitis.

\author{
Garbutt 2012 \\ Haye 2000 \\ Lindbaek 1998 (Amoxy) \\ Lindbaek 1998 (pen V) \\ Merenstein 2005 \\ Stalman 1997 \\ Van Buchem 1997
}

Total $(95 \% \mathrm{Cl})$

Total events

Heterogeneity: $\mathrm{Chi}^{2}=2.75, \mathrm{df}=6(\mathrm{P}=0.84) ; \mathrm{I}^{2}=0 \%$

Test for overall effect: $Z=1.50(P=0.13)$

$\begin{array}{rrrrr}63 & 81 & 57 & 71 & 18.8 \% \\ 80 & 86 & 72 & 82 & 22.9 \% \\ 17 & 22 & 14 & 21 & 4.4 \% \\ 15 & 20 & 14 & 21 & 4.2 \% \\ 32 & 67 & 25 & 68 & 7.7 \% \\ 56 & 94 & 55 & 92 & 17.2 \% \\ 87 & 105 & 78 & 101 & 24.7 \% \\ & 475 & & 456 & 100.0 \% \\ 350 & \\ f=6(P=0.84) ; I^{2}=0 \% & \\ 50(P=0.13) & \end{array}$

$0.97[0.82,1.14]$

$1.06[0.96,1.17]$

$1.16[0.79,1.69]$

$1.13[0.76,1.67]$

$1.30[0.87,1.94]$

$1.00[0.79,1.26]$

$1.07[0.94,1.23]$

$1.06[0.98,1.14]$

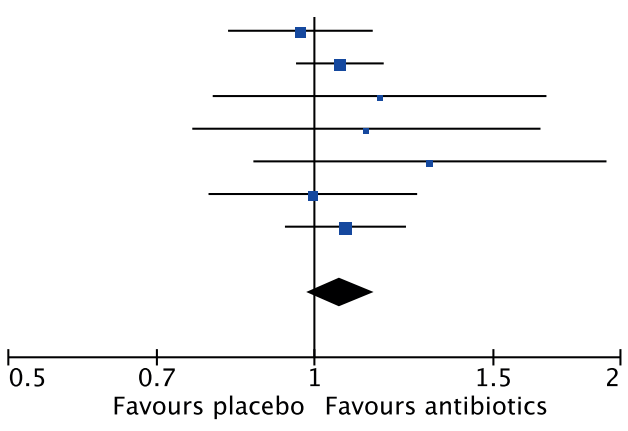


treatment failure (worsening or no improvement of symptoms) at day 3 of treatment ${ }^{(238)}$.

Four studies, including 907 patients could be pooled to assess improvement at day 3-4 of treatment ${ }^{(237,238,240,241)}$. There was a trend that the use of antibiotics was associated with greater improvement at day 3-4 of treatment compared to placebo but it just did not reach significance (RR 1.06, 95\% Cl 1.00-1.12, I2=0\%) (Figure 4.6.9).

There were five studies ${ }^{(237-241)}$ which used patient reported outcome measures such as SNOT-16 (237), questionnaire or symptoms score ${ }^{(238-241)}$ to measure severity of nasal symptoms either scored daily in a diary, or upon baseline and follow up visits. Garbutt et al. ${ }^{(237)}$ found that the SNOT-16 score was lower in the antibiotics group at day 7 of treatment, but this was not significant upon completion of treatment. Studies which assessed symptoms of rhinosinusitis ${ }^{(238,239,241,243)}$ assessed for common nasal symptoms such as rhinorrhea, nasal congestion, facial pain and feeling sick. These symptoms were either scored on a visual analogue scale or on an ordinal scale. There were no differences in the mean overall change in symptoms score ${ }^{(238,241)}$ from baseline between antibiotics and placebo. There was also no difference in the mean individual symptoms score between antibiotics and placebo ${ }^{(239,243)}$. It was not possible to combine data into a meta-analysis. This indicates that the use of antibiotics does not significantly reduce the symptoms burden of post viral rhinosinusitis.

Figure 4.6.8. Forest plot of the effect of antibiotic versus placebo to assess the difference (mean difference) in the number of days to achieve cure after treatment in adult patients with acute post-viral acute rhinosinusitis

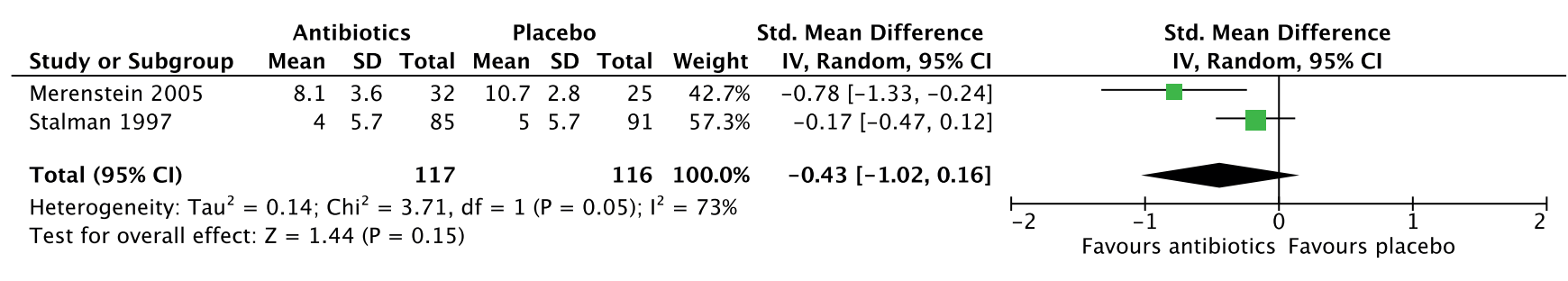

$\mathrm{Cl}$, confidence interval; $\mathrm{M}-\mathrm{H}$, Mantel Haenszel.

Figure 4.6.9. Forest plot of the effect of antibiotic versus placebo to assess improvement at day 3 of treatment of adult patients with acute post-viral acute rhinosinusitis.

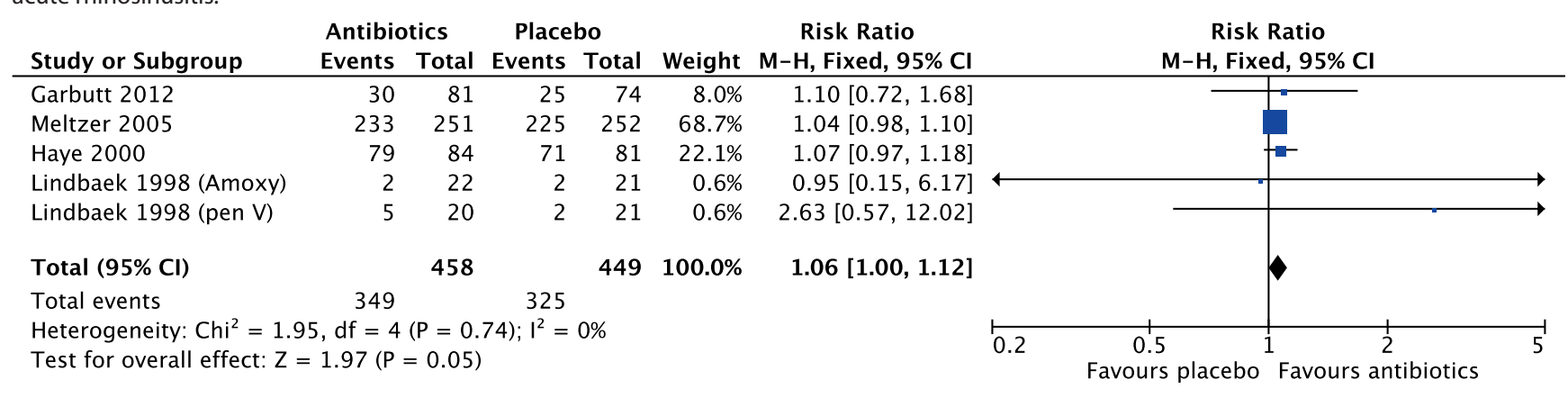

$\mathrm{Cl}$, confidence interval; $\mathrm{M}-\mathrm{H}$, Mantel Haenszel.

Figure 4.6.10. Forest plot of the effect of antibiotics vs. placebo for adverse effects during treatment of adult patients with acute post-viral acute rhinosinusitis

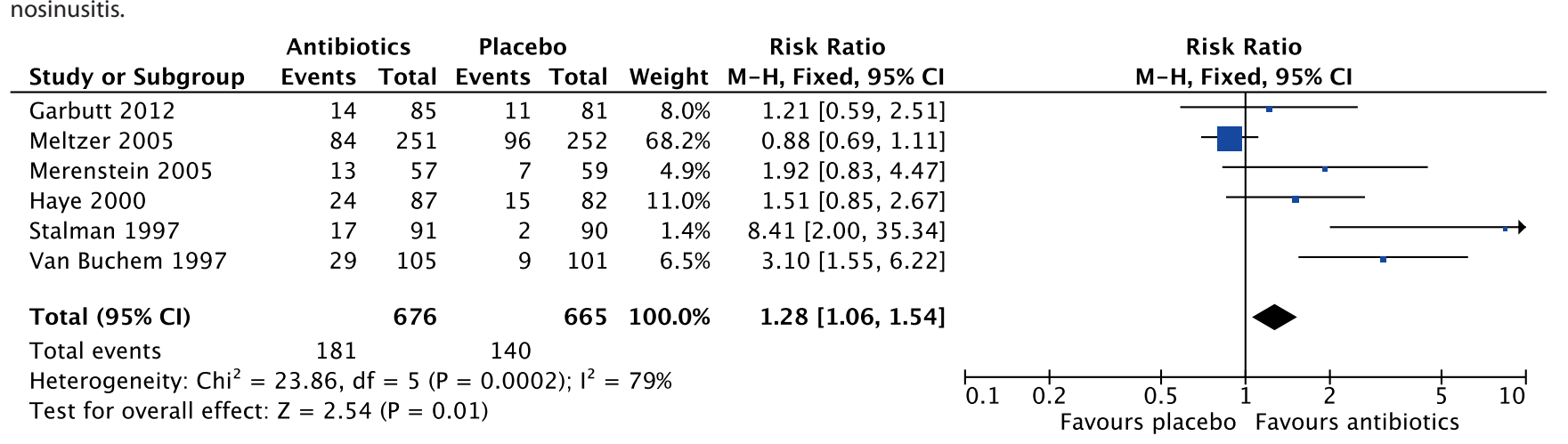

$\mathrm{Cl}$, confidence interval; $\mathrm{M}-\mathrm{H}$, Mantel Haenszel. 
Table 4.6.3. Double blind placebo controlled RCT on antibiotics in post-viral ARS in children.

\begin{tabular}{|c|c|c|c|c|c|}
\hline Study & Methods & Participants & Interventions & Outcomes & Results \\
\hline $\begin{array}{l}\text { Khoshdel } \\
2014^{(246)}\end{array}$ & RCT & $\begin{array}{l}\text { Children ( } 4-15 y \text {, mean age } \\
7.6 \pm 2.86) \text { with a clinical presen- } \\
\text { tation of mild - moderate post- } \\
\text { viral acute rhinosinusitis (recent } \\
\text { upper respiratory infection, } \\
\text { postnasal discharge and/or nasal } \\
\text { congestion) for } 10-30 \text { days. }\end{array}$ & $\begin{array}{l}\text { - Amoxicillin ( } 80 \mathrm{mg} / \mathrm{kg} / \text { day) in } \\
3 \text { divided doses for } 14 \text { days with } \\
\text { saline nasal irrigation (for } 5 \text { days) } \\
\text { and } 0.25 \% \text { phenylephrine (for } 2 \\
\text { days) ( } \mathrm{n}=50 \text { ) } \\
\text { - Saline nasal irrigation (for } 5 \\
\text { days) and } 0.25 \% \\
\text { phenylephrine (for } 2 \text { days) (no } \\
\text { placebo tablet) ( } n=50 \text { ) }\end{array}$ & $\begin{array}{l}\text { - Cure at day } 3 \text { and } 14 \\
\text { (Children visited at home } \\
\text { and examined for com- } \\
\text { plete absence of sinusitis } \\
\text { signs and symptoms). }\end{array}$ & $\begin{array}{l}\text { - Cure better in amoxi- } \\
\text { cillin group at day } 3 \\
\text { ( } 85 \text { vs. } 37.5 \%, p<0.01 \text { ) } \\
\text { but no difference at } \\
\text { other time points. }\end{array}$ \\
\hline $\begin{array}{l}\text { Kristo } \\
2005^{(245)}\end{array}$ & DBPCRT & $\begin{array}{l}\text { Children (4-10y) with acute } \\
\text { respiratory symptoms }<3 \text { weeks } \\
\text { (average } 8.4 \pm 5.6 \text { days) sugges- } \\
\text { tive of post-viral rhinosinusitis } \\
\text { (nasal discharge and obstruc- } \\
\text { tion, sneezing, cough) and an } \\
\text { abnormal ultrasound finding } \\
\text { of at least one maxillary sinus } \\
\text { (mucosal thickening or fluid) }\end{array}$ & $\begin{array}{l}\text { - Cefuroxime axetil in } 125 \mathrm{mg} \\
\text { capsules twice daily for } 10 \text { days } \\
(\mathrm{n}=41) \\
\text { - Placebo twice daily for } 10 \text { days } \\
(\mathrm{n}=41)\end{array}$ & $\begin{array}{l}\text { - Cure, improve, unchan- } \\
\text { ged or deteriorate based } \\
\text { on clinician assessment } \\
\text { and parents' opinion at } \\
\text { day } 14 \\
\text { - } 14 \text {-day diary nasal } \\
\text { symptoms score (total } \\
0-6 \text { ) } \\
\text { - Adverse effect }\end{array}$ & $\begin{array}{l}\text { - No difference in } \\
\% \text { cure or improve } \\
\text { between groups at } \\
\text { day } 14 \\
\text { - No difference in } \\
\text { symptom score } \\
\text { between groups at } \\
\text { from day } 1 \text { to } 14 \\
\text { - No difference in } \\
\text { adverse effect }\end{array}$ \\
\hline $\begin{array}{l}\text { Garbutt } \\
2001^{(244)}\end{array}$ & DBPCRT & $\begin{array}{l}\text { Children ( } 1 \text { to } 18 \text { years, average } \\
\text { age } 8 y \text { ) with persistent sinus } \\
\text { symptoms and a clinical diagno- } \\
\text { sis of acute post-viral rhinosinu- } \\
\text { sitis for } 10 \text { to } 28 \text { days }\end{array}$ & $\begin{array}{l}\text { - Amoxicillin } 40 \mathrm{mg} / \mathrm{kg} / \mathrm{d} \text { three } \\
\text { times daily }(\mathrm{n}=58 \text { ) for } 14 \text { days } \\
\text { - Amoxicillin-clavulanate } 45 \mathrm{mg} / \\
\mathrm{kg} / \mathrm{d} \text { twice daily }(\mathrm{n}=48 \text { ) for } 14 \\
\text { days } \\
\text { - Placebo twice daily for } 14 \text { days } \\
(\mathrm{n}=55)\end{array}$ & $\begin{array}{l}\text { - Improvement (little or a } \\
\text { lot better) at day } 14 \\
\text { - Change in sinus } \\
\text { symptoms score using S5 } \\
\text { score }(0-3) \text { at day } 14 \\
\text { - Adverse effects of } \\
\text { treatment }\end{array}$ & $\begin{array}{l}\text { - No difference in im- } \\
\text { provement at day } 14 \\
\text { - No difference in S5 } \\
\text { sinus symptoms score } \\
\text { at day } 14 \\
\text { - No difference in } \\
\text { adverse event }\end{array}$ \\
\hline
\end{tabular}

DBPCRT, double blind placebo controlled randomised trial; RCT, randomised controlled trial; y, years.

Figure 4.6.11. Forest plot of the effect of antibiotic versus placebo to assess improvement at day 10-14 of treatment of children with acute post-viral acute rhinosinusitis

\begin{tabular}{|c|c|c|c|c|c|c|c|c|c|}
\hline Study or Subgroup & \multicolumn{2}{|c|}{ Antibiotics } & \multicolumn{2}{|c|}{ Placebo } & \multicolumn{3}{|c|}{ Risk Ratio } & \multicolumn{2}{|l|}{$\begin{array}{c}\text { Risk Ratio } \\
\mathrm{M}-\mathrm{H}, \text { Random, } 95 \% \mathrm{Cl} \\
\end{array}$} \\
\hline Khoshdel 2014 & 39 & 40 & 38 & 40 & $52.1 \%$ & $1.03[0.94,1.12]$ & 2014 & & \\
\hline Kristo 2005 & 32 & 35 & 31 & 35 & $16.0 \%$ & $1.03[0.88,1.21]$ & 2005 & & \\
\hline Garbutt (Amoxi) 2001 & 46 & 58 & 47 & 55 & $13.4 \%$ & $0.93[0.78,1.10]$ & 2001 & & \\
\hline Garbutt (Amoxi-clavulanic) 2001 & 43 & 48 & 47 & 55 & $18.5 \%$ & $1.05[0.91,1.21]$ & 2001 & - & \\
\hline Total $(95 \% \mathrm{Cl})$ & & 181 & & 185 & $100.0 \%$ & $1.02[0.96,1.08]$ & & \rangle & \\
\hline Total events & 160 & & 163 & & & & & & \\
\hline $\begin{array}{l}\text { Heterogeneity: } \mathrm{Tau}^{2}=0.00 ; \mathrm{Chi}^{2} \\
\text { Test for overall effect: } \mathrm{Z}=0.54(\mathrm{P}\end{array}$ & $\begin{array}{l}1.52, \mathrm{df} \\
=0.59)\end{array}$ & $=3(\mathrm{P}$ & $=0.68)$ & $I^{2}=0 \%$ & & & & $\begin{array}{ccccc} & 1 & 1 & 1 & 1 \\
0.1 & 0.2 & 0.5 & 1 & 2 \\
& & \text { Favours placebo } & \text { Favours }\end{array}$ & $\begin{array}{c}5 \\
5 \\
t i c s\end{array}$ \\
\hline
\end{tabular}

$\mathrm{Cl}$, confidence interval; $\mathrm{M}-\mathrm{H}$, Mantel Haenszel.

Figure 4.6.12. Forest plot of the effect of antibiotics vs. placebo for adverse effects during treatment of children with acute post-viral acute rhinosinusitis.

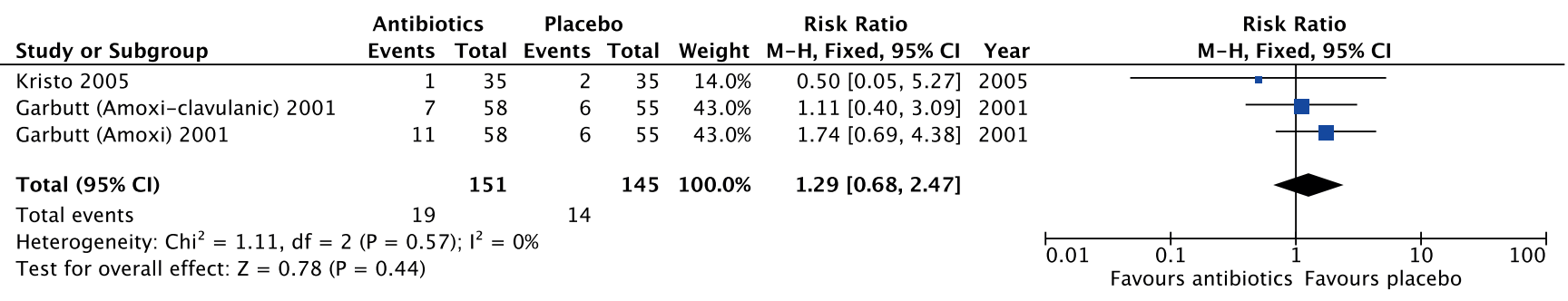

$\mathrm{Cl}$, confidence interval; $\mathrm{M}-\mathrm{H}$, Mantel Haenszel. 
Finally, the antibiotics group had significantly more adverse events compared to the placebo group although there was a significant heterogeneity (1341 patients, RR 1.28 95\% Cl 1.061.54, 12=79\%) (Figure 4.6.10).

\subsection{Conclusion}

In conclusion, there is no benefit of prescribing antibiotics for post viral ARS in adults. There is no effect on cure or duration of disease and there are more adverse events. Based on the moderate level of evidence and the fact that acute post-viral rhinosinusitis is a self-limiting disease, the EPOS2020 steering group advises against the use of antibiotics for adults in this situation.

\subsubsection{Short course of oral antibiotics in children with acute post viral rhinosinusitis}

\subsection{Summary of the evidence}

This section aims to assess the efficacy of antibiotics in the post viral ARS group in children. Studies were included which involved children with ARS with symptoms of at least five days but less than 12 weeks (to exclude chronic rhinosinusitis). Only DBPCTs were included.

Studies were excluded if a significant proportion of the study population fulfilled the criteria for ABRS or if more than $20 \%$ of the study population had symptoms for less than five days. The minimum duration of five days was used as a cut-off to exclude patients with common cold. Studies without a placebo arm, and/or involving adults were also excluded. The primary outcome is number of patients who obtained cure/significant improvement at 10-14 days. The secondary outcomes assessed were number of days to cure and adverse effects.

Three studies were included: Garbutt, 2001; Kristo, 2005; and Khoshdel, 2014(244-246). The Garbutt study contained three arms. Two studies evaluated amoxicillin ${ }^{(244,246)}$, one amoxicillin with clavulanic acid $^{(24)}$ and one cefuroxime ${ }^{(245)}$. The antibiotics were taken for 10-14 days. All participants had symptoms of acute post-viral rhinosinusitis which fulfilled the EPOS criteria for at least at least five days (Table 4.6.3.).

Khoshdel et al. ${ }^{(246)}$ found a higher cure rate in the amoxicillin group at day 3 ( 85 vs. $37.5 \%, p<0.01$ ) but no difference at other time points. The other two studies did not find a significant difference between antibiotics and placebo ${ }^{(244,245)}$.

The studies could be combined into a meta-analysis to determine the cure rate at day 10-14 (366 patients in three studies) (244-246). The use of antibiotics was not associated with greater cure/significant improvement at day 10-14 (RR 1.02 95\% Cl .961.08, I2=0\%) (Figure 4.6.11.).

Two studies could be combined and could not show that the patients on antibiotics did have significantly more adverse events compared to the placebo group (RR 1.29 95\% Cl 0.69-4.38, $\mathrm{p}=0.44,2 \mathrm{RCT}$ (3 arms) , $\mathrm{n}=296, \mathrm{I}^{2}=0 \%$ ) (Figure 4.6.12.).

\subsection{Conclusion}

The use of antibiotics in children with acute post-viral rhinosinusitis is not associated with greater cure/significant improvement. Although the two studies that could be combined did not show significantly more side effects than placebo, studies in adults (see 4.6.3.3) did show significantly more side effects. Based on the moderate level of evidence and the fact that acute post-viral rhinosinusitis is a self-limiting disease, the EPOS2020 steering group advises against the use of antibiotics for children in this situation.

\subsubsection{Methods that could change inappropriate prescription of antibiotics}

Antibiotic resistance is a major public health problem. A major risk factor is irrational antibiotic use, e.g., in patients with common respiratory tract infections ${ }^{(247-250)}$. Prescription of antibiotics in in Europe varies greatly; the highest rate in 2016 was in Greece (36.3 DDD per 1000 inhabitants daily ${ }^{(251)}$ ) and the lowest was in the Netherlands (10.4 DDD per 1000 inhabitants daily) ${ }^{(247,}$ ${ }^{248)}$. Higher rates of antibiotic resistance are shown in high antibiotic consuming countries (Figures 4.6.13 and 4.6.14). It has been shown that individuals prescribed an antibiotic in primary care for a respiratory or urinary infection develop bacterial resistance to that antibiotic. The effect is greatest in the month immediately after treatment but may persist for up to 12 months ${ }^{(252)}$. But even in countries in Northwest Europe substantially higher proportions of consultations result in an antibiotic prescription than was deemed appropriate according to what has been advised in guidelines, and presumed acute rhinosinusitis is one of the diseases where discrepancies are largest. In a study in the UK $88 \%$ of the consultations for rhinosinusitis resulted in an antibiotic prescription where experts deemed $11 \%$ appropriate ${ }^{(22)}$. The same was true in the Netherlands where $34 \%$ of the interviewed primary care physicians chose an antibiotic as treatment for a patient with moderate severe acute rhinosinusitis ${ }^{(171)}$. Substantial variation between practices is found ${ }^{(22,253)}$.

A number of studies have evaluated measurements that could help to decrease the inappropriate prescription of antibiotics for acute rhinosinusitis ${ }^{(254-259)}$. Most studies emphasize training of physician communication skills on the use of antibiotics ${ }^{(255,}$ 256), accountable justification and peer comparison ${ }^{(255,256,259)}$ and training of physicians ${ }^{(256,258)}$. The use of a point-of-care C-reactive protein testing did not reduced antibiotic prescription in children with (non-severe) acute respiratory infections ${ }^{(254,257)}$.

\subsubsection{Nasal corticosteroid in acute post-viral rhinosinusitis}

4.6.4.1. Nasal corticosteroid in acute post-viral rhinosinusitis in adults

4.6.4.1.1. Summary of the evidence 
Figure 4.6.13. Consumption of antibiotics for systemic use in the community by antibiotic group in 30 EU/EEA countries, 2013 (expressed in DDD per 1000 inhabitants and per day(251).

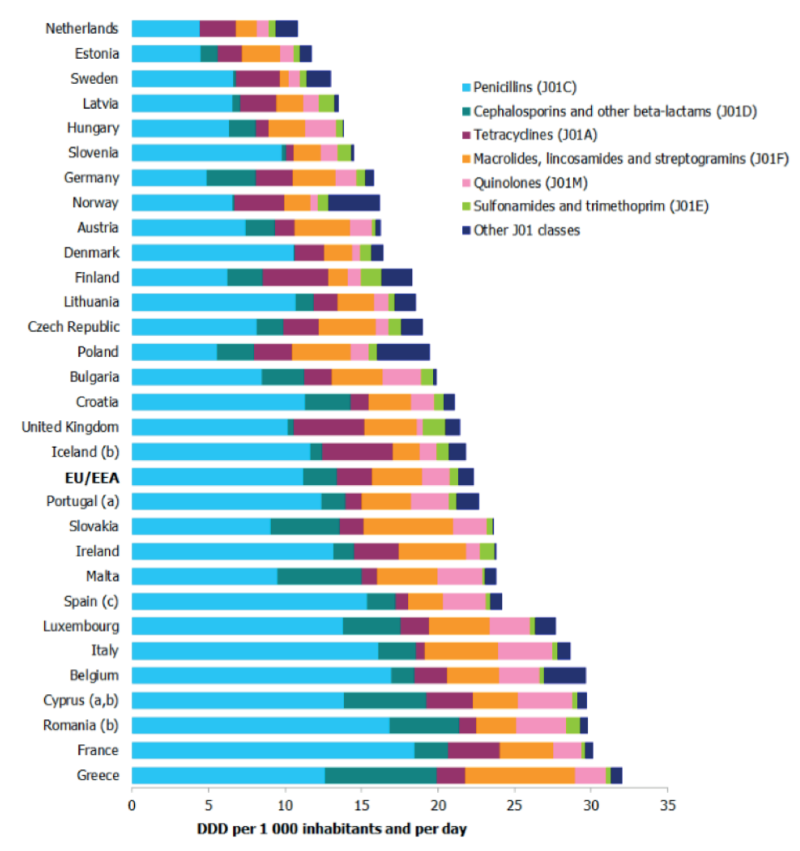

This section aims to assess the efficacy of nasal corticosteroids in adults with post-viral ARS. There are no studies evaluating the effect of nasal corticosteroids on ABRS. Studies were included if they involved adults with ARS with symptoms of at least five days but less than 12 weeks (to exclude chronic rhinosinusitis). Only double-blind placebo-controlled randomized trials published after the year 1990, assessing the effects of nasal corticosteroids for patients who fulfilled the criteria of post-viral acute rhinosinusitis are included. Patients with common cold and acute bacterial rhinosinusitis (ABRS), are excluded. Nasal corticosteroids delivered into the nose and the paranasal sinuses by any delivery methods generally available in clinical practice are included. Outcomes included in this analysis are time to cure, quality of life, symptoms and adverse events.

Of the eight studies included in this section, five studies(238, 260-263) were placebo-controlled trials assessing the effects of nasal corticosteroids alone (Table 4.6.4.) and three were studies ${ }^{(264)}$ evaluating the effect of the addition of nasal corticosteroids to treatment with antibiotics (Table 4.6.5.). The papers of Bachert ${ }^{(263)}$ and Meltzer $2012^{(261)}$ are post-hoc analyses of the data from Meltzer $2005^{(238)}$. All studies mainly evaluated patients meeting the EPOS criteria for acute post-viral rhinosinusitis although in the study of Dolor ${ }^{(264)}$ duration of disease was not indicated. A number of studies evaluated more than one dosage of nasal corticosteroids. These are analysed separately in the meta-analysis.

Five studies ${ }^{(260-262,264,265)}$ reported on time to cure or reduction in symptoms. Three studies reported a reduced time to cure/
Figure 4.6.14. Proportion of macrolide Resistant (R) Streptococcus pneumonia isolates in participating countries in 2013(250).

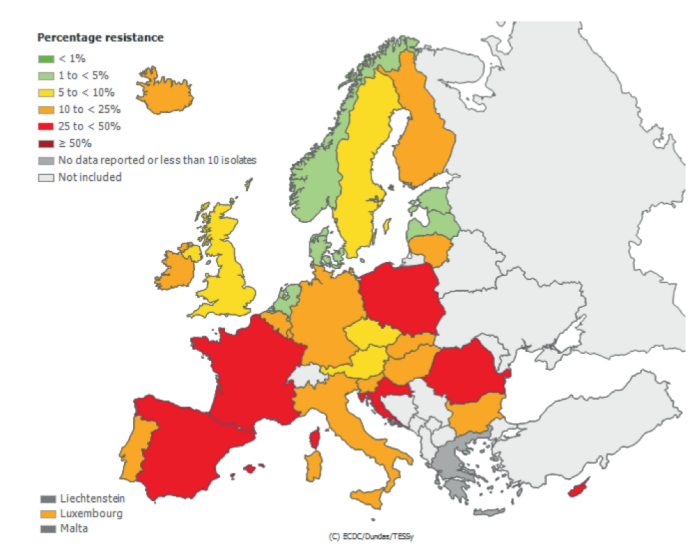

reduced symptoms $s^{(261,264,265)}$, the other two did not. The data were insufficiently reported to meta-analyse. Three studies ${ }^{(263,264)}$ evaluated the SNOT-20 score. The SNOT-20 is not validated for ARS but may give some impression of quality of life also in acute disease. The meta-analysis showed no significant difference between treatment with nasal corticosteroids and placebo although the data showed significant heterogeneity (MD 0.13, 95\% $\mathrm{Cl}-0.04$ to $0.31, \mathrm{p}=0.14 ; 4 \mathrm{RCTs}, \mathrm{n}=1120$; Figure 4.6.15.). Four studies reported on total symptom score $238,260,265,266)$. The studies could be combined into a meta-analysis. Both the analyses of the studies where nasal corticosteroid was the single treatment (SMD 0.32, 95\% Cl 0.15 to $0.50, p=0.0003$; two (two arms) RCTs, $n=1860$; Figure 4.6.16.) and where nasal corticosteroid was added to an antibiotic (that on its own has shown to be not effective (see paragraph 4.6.4.3.)) showed a significant effect over placebo (SMD $0.21,95 \% \mathrm{Cl} 0.11$ to $0.30, \mathrm{p}<0.00001$; two RCTs, $n=1699$; Figure 4.6.16.).

Some studies reported on separate symptoms where nasal congestion was often found to be significantly improved in the nasal corticosteroid group compared to placebo. No meta-analysis was performed.

There was no difference in adverse events and/or in patients needing antibiotics in the studies that evaluated that parameter (Table 4.6.4.).

Finally, Svensson et al. ${ }^{(267)}$ used a cost-effectiveness model to analyse the cost-effectiveness of mometasone furoate nasal spray (MFNS) compared with amoxicillin or placebo in the treatment of post-viral rhinosinusitis. Costs were reduced and quality-adjusted life years were increased with MFNS $200 \mu \mathrm{g}$ twice daily compared with amoxicillin $500 \mathrm{mg}$ three times daily. MFNS was cost-saving or cost-effective compared with amoxicillin or non-active treatment in the sensitivity analyses regardless of the HRQOL measurement used. 
Table 4.6.4. Nasal corticosteroids compared to placebo in adult patients with acute post-viral rhinosinusitis.

\begin{tabular}{|c|c|c|c|c|c|}
\hline Study & Methods & Participants & Interventions & Outcomes & Results \\
\hline $\begin{array}{l}\text { Keith } \\
2012^{(260)}\end{array}$ & DBPCT & $\begin{array}{l}737 \text { patients (age }>12 \mathrm{y} \text { ) } \\
\text { with acute post-viral } \\
\text { rhinosinusitis }\end{array}$ & $\begin{array}{l}\text { - FFNS } 110 \mu \mathrm{g} \text { once daily } \\
\text { ( } \mathrm{n}=240) \text { for } 2 \text { weeks } \\
\text { - FFNS } 110 \mu \mathrm{g} \text { twice daily } \\
(\mathrm{n}=252) \text { for } 2 \text { weeks } \\
\text { - Placebo }(\mathrm{n}=245) \text { for } 2 \\
\text { weeks }\end{array}$ & $\begin{array}{l}\text { During } 1 \text { month: } \\
\text { - SNOT-20 } \\
\text { - Daily major symptom } \\
\text { score (a composite } \\
\text { score of three indivi- } \\
\text { dual symptoms (nasal } \\
\text { congestion/stuffiness, } \\
\text { sinus headache/pressure } \\
\text { or facial pain/pressure, } \\
\text { postnasal drip), each } \\
\text { using a scale of 0-3. } \\
\text { These assessments were } \\
\text { conducted twice daily, } \\
\text { before the morning and } \\
\text { evening dose) } \\
\text { - Individual symptoms } \\
\text { - The first time to } \\
\text { symptom improvement } \\
\text { (defined as reduction } \\
\text { of individual symptom } \\
\text { scores of nasal con- } \\
\text { gestion/stuffiness, sinus } \\
\text { headache/pressure or } \\
\text { facial pain/pressure, and } \\
\text { postnasal drip) } \\
\text { - Need of antibiotics } \\
\text { - Adverse events }\end{array}$ & $\begin{array}{l}\text { Treatment with FFNS vs. } \\
\text { placebo resulted in: } \\
\text { - No significant reduction } \\
\text { in SNOT-20 } \\
\text { - Significant reduction in } \\
\text { major symptom score } \\
\text { - Significant reduction in } \\
\text { congestion } \\
\text { - No significant difference } \\
\text { in time to symptom } \\
\text { improvement } \\
\text { - No difference in need } \\
\text { for antibiotics } \\
\text { - No difference in adverse } \\
\text { events }\end{array}$ \\
\hline $\begin{array}{l}\text { Meltzer } \\
2012^{(261)}\end{array}$ & DBPCT & $\begin{array}{l}728 \text { patients (age }>12 y) \\
\text { with post-viral ARS } \\
\text { Post hoc analysis of } \\
\text { Meltzer 2005(238) }\end{array}$ & $\begin{array}{l}\text { - MFNS } 200 \mu \mathrm{g} \text { twice daily } \\
\text { for } 15 \text { days }(\mathrm{n}=234) \\
\text { - MFNS } 200 \mu \mathrm{g} \text { once daily } \\
\text { for } 15 \text { days ( } \mathrm{n}=243 \text { ) } \\
\text { - Amoxicillin } 500 \mathrm{mg} 3 \\
\text { times daily for } 15 \text { days } \\
\text { ( } \mathrm{n}=248 \text { ) } \\
\text { - Placebo for } 15 \text { days } \\
(\mathrm{n}=246)\end{array}$ & $\begin{array}{l}\cdot \text { MSS } \\
\text { - The percentage of } \\
\text { days with minimal MSS } \\
\text { (defined as average am / } \\
\text { pm MSS } \leq 4 \text { ) for } 1 \text { month } \\
\text { - The percentage of days } \\
\text { with minimal congestion } \\
(\leq 1) \text { for } 1 \text { month } \\
\text { - Time to first day with } \\
\text { minimal symptoms }\end{array}$ & $\begin{array}{l}\text { Treatment with MFNS } \\
\text { compared to placebo } \\
\text { resulted in: } \\
\text { - Significantly reduced } \\
\text { mean rhinosinusitis } \\
\text { major symptom score } \\
\text { - Significantly reduced } \\
\text { percentage of days with } \\
\text { minimal MSS } \\
\text { - Significantly reduced } \\
\text { percentage of days with } \\
\text { minimal congestion } \\
\text { - Significantly reduced } \\
\text { time to first day with } \\
\text { minimal symptoms }\end{array}$ \\
\hline $\begin{array}{l}\text { Williamson } \\
2007^{(262)}\end{array}$ & DBPCT & $\begin{array}{l}240 \text { adults post-viral ARS } \\
\text { ( } 207 \text { analysed) }\end{array}$ & $\begin{array}{l}\cdot 500 \mathrm{mg} \text { of amoxicillin } 3 \\
\text { times per day for } 7 \text { days } \\
\text { and } 200 \mu \mathrm{g} \text { budesonide in } \\
\text { each nostril once per day } \\
\text { for } 10 \text { days }(n=46) \\
\text { - } 500 \mathrm{mg} \text { of amoxicillin } 3 \\
\text { times per day for } 7 \text { days } \\
(\mathrm{n}=54) 200 \mu \mathrm{g} \text { budeso- } \\
\text { nide in each nostril } \\
\text { once per day for } 10 \text { days } \\
(\mathrm{n}=56) \\
\text { - Placebo }(n=51)\end{array}$ & $\begin{array}{l}\text { - Proportion of patients } \\
\text { having symptoms lasting } \\
10 \text { days or more } \\
\text { - Time to cure (graph) } \\
\text { - \% cured at day } 10 \\
\text { - Total symptom score }\end{array}$ & $\begin{array}{l}\text { No significant effect in } \\
\text { any treatment group } \\
\text { compared to placebo for } \\
\text { all outcomes }\end{array}$ \\
\hline $\begin{array}{l}\text { Bachert } \\
2007^{(263)}\end{array}$ & 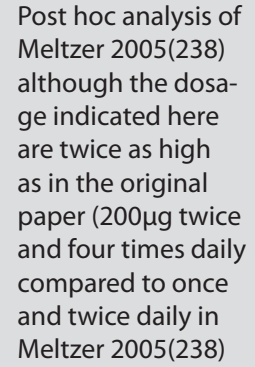 & $\begin{array}{l}340 \text { patients ( } 331 \text { ana- } \\
\text { lysed) with post-viral ARS }\end{array}$ & $\begin{array}{l}\text { - MFNS } 200 \mu \mathrm{g} \text { twice daily } \\
\text { for } 15 \text { days }(\mathrm{n}=81 \text { ) } \\
\text { - MFNS } 200 \mu \mathrm{g} \text { four times } \\
\text { daily for } 15 \text { days }(\mathrm{n}=84) \\
\text { - Amoxicillin } 500 \mathrm{mg} 3 \\
\text { times daily for } 10 \text { days } \\
\text { ( } \mathrm{n}=84 \text { ) } \\
\text { - Placebo nasal spray and } \\
\text { tablets ( } \mathrm{n}=82 \text { ) }\end{array}$ & $\begin{array}{l}\text { - SNOT-20 } \\
\text { - Global scores }\end{array}$ & $\begin{array}{l}\text { Treatment with MFNS } \\
\text { compared to placebo } \\
\text { resulted in: } \\
\text { - Significant improve- } \\
\text { ment in SNOT-20 } \\
\text { - Significant improve- } \\
\text { ment in global response } \\
\text { to treatment }\end{array}$ \\
\hline
\end{tabular}


Table 4.6.4. Nasal corticosteroids compared to placebo in adult patients with acute post-viral rhinosinusitis. (continued)

\begin{tabular}{|c|c|c|c|c|c|}
\hline Study & Methods & Participants & Interventions & Outcomes & Results \\
\hline $\begin{array}{l}\text { Meltzer } \\
2005^{(238)}\end{array}$ & DBPCT & Post-viral ARS ( $n=981)$ & $\begin{array}{l}\text { - MFNS } 200 \mu \mathrm{g} \text { once daily } \\
\text { for } 15 \text { days }(\mathrm{n}=243 \text { ) } \\
\text { - MFNS } 200 \mu \mathrm{g} \text { twice daily } \\
\text { for } 15 \text { days }(\mathrm{n}=235) \\
\text { - Amoxicillin } 500 \mathrm{mg} 3 \\
\text { times daily for } 10 \text { days } \\
\text { ( } \mathrm{n}=251 \text { ) } \\
\text { - Placebo nasal spray and } \\
\text { tablets ( } \mathrm{n}=252 \text { ) }\end{array}$ & $\begin{array}{l}\text { - Mean am / pm MSS over } \\
\text { days } 2 \text { to } 15 \text { of the treatment } \\
\text { phase } \\
\text { - Mean major symptom score, } \\
\text { total symptom score, and } \\
\text { individual scores (average } \\
\text { of am / pm scores) for each } \\
\text { symptom averaged weekly } \\
\text { and daily } \\
\text { - Safety assessments included } \\
\text { disease recurrence during } \\
\text { follow-up and adverse event } \\
\text { monitoring }\end{array}$ & $\begin{array}{l}\text {-MFNS } 200 \text { mg twice daily } \\
\text { produced significant symp- } \\
\text { tom improvements versus } \\
\text { amoxicillin and placebo } \\
\text { - MFNS } 200 \text { mg once daily pro- } \\
\text { duced significant symptom } \\
\text { improvements versus placebo } \\
\text { - The incidence of treatment- } \\
\text { emergent adverse events was } \\
\text { similar among the treatment } \\
\text { groups }\end{array}$ \\
\hline
\end{tabular}

ARS, acute rhinosinusitis; DBPCT, double blind placebo controlled trial; FFNS, fluticasone furoate nasal spray; MFNS, mometasone furoate nasal spray; MSS, Mean Rhinosinusitis Major Symptom Score; SNOT-20, Sino-nasal Outcome Test-20; y, years.

Table 4.6.5. Nasal corticosteroid as addition to oral antibiotics in adult patients with acute post-viral rhinosinusitis.

\begin{tabular}{|c|c|c|c|c|c|}
\hline Study & Methods & Participants & Interventions & Outcomes & Results \\
\hline $\begin{array}{l}\text { Nayak } \\
2002^{(265)}\end{array}$ & DBPCT & $\begin{array}{l}967 \text { post-viral rhinosinusitis } \\
\text { patients }\end{array}$ & 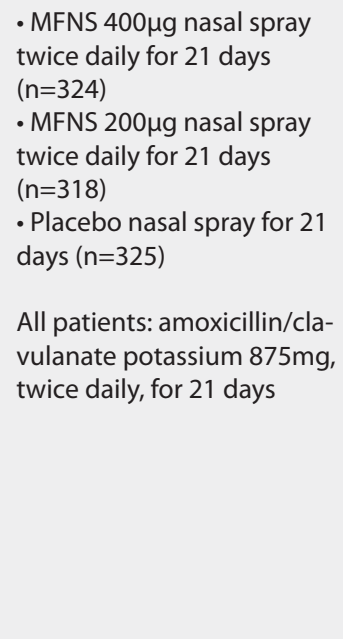 & $\begin{array}{l}\text { - Mean improvement in } \\
\text { daily symptom scores (0-3) } \\
\text { for total symptoms, heada- } \\
\text { che, facial pain, congestion, } \\
\text { purulent rhinorrhoea, } \\
\text { postnasal drip, and cough } \\
\text { over } 15 \text { days } \\
\text { - Overall therapeutic } \\
\text { response to treatment (0-5) } \\
\text { at day } 21 \\
\text { - Onset of relief } \\
\text { - CT scan at day } 21 \\
\text { - Adverse events } \\
\text { - Co-syntropin-stimulation } \\
\text { plasma cortisol }\end{array}$ & $\begin{array}{l}\text { Treatment with MFNS } 400 \\
\text { and } 200 \text { twice daily compa- } \\
\text { red to placebo resulted in a } \\
\text { significantly: } \\
\text { - Larger mean reduction in } \\
\text { total symptom scores and } \\
\text { congestion than placebo } \\
\text { - Larger mean reduction in } \\
\text { facial pain, rhinorrhoea and } \\
\text { post-nasal drip than placebo } \\
\text { - Significantly better overall } \\
\text { therapeutic response at day } \\
21 \text { compared to placebo } \\
\text { - Significant faster onset of } \\
\text { relief } \\
\text { - Comparable adverse events } \\
\text { No differences in CT scans, } \\
\text { adverse events and plasma } \\
\text { cortisol }\end{array}$ \\
\hline $\begin{array}{l}\text { Dolor } \\
2001^{(264)}\end{array}$ & DBPCT & $\begin{array}{l}95 \text { ARS patients with acute } \\
\text { sinonasal symptoms and } \\
\text { history of recurrent rhino- } \\
\text { sinusitis or chronic rhinitis } \\
\text { ( } 92 \text { analyzed) (duration of } \\
\text { disease unclear) }\end{array}$ & $\begin{array}{l}\text { - FPNS } 2 \text { puffs twice daisy } \\
\text { - Placebo nasal spray in } \\
\text { each nostril once daily for } \\
21 \text { days ( } n=48 \text { ) } \\
\text { All received } 250 \mathrm{mg} \text { of ce- } \\
\text { furoxime axetil twice daily } \\
\text { for } 10 \text { days and } 2 \text { puffs of } \\
\text { xylometazoline hydrochlo- } \\
\text { ride in each nostril twice } \\
\text { daily for } 3 \text { days }\end{array}$ & $\begin{array}{l}\text { - Time to clinical success } \\
\text { (patient reported cured or } \\
\text { much improved) } \\
\text { - Sinus symptoms score } \\
\text { - SNOT-20 } \\
\text { - SF-12 } \\
\text { - Mean work performance }\end{array}$ & $\begin{array}{l}\text { Patients using FPNS had: } \\
\text { - Significantly shortened time } \\
\text { to clinical success } \\
\text { - Significantly shorter time to } \\
\text { clinical success } \\
\text { - Improved work performance } \\
\text { - No significant differences in } \\
\text { rhinosinusitis symptom }\end{array}$ \\
\hline $\begin{array}{l}\text { Meltzer } \\
2000^{(266)}\end{array}$ & DBPCT & 407 post-viral ARS patients & $\begin{array}{l}\text { - MFNS } 400 \mu \mathrm{g} \text {, twice daily } \\
\text { ( } \mathrm{n}=200) \\
\text { - Placebo spray twice daily } \\
\text { for } 21 \text { days ( } \mathrm{n}=207 \text { ) } \\
\text { All patients: } \\
\begin{array}{l}\text { Augmentin, } 875 \mathrm{mg} \text { twice } \\
\text { daily }\end{array}\end{array}$ & $\begin{array}{l}\text { - Daily symptom scores } \\
(0-3) \text { for headache, facial } \\
\text { pain, congestion, purulent } \\
\text { rhinorrhoea, postnasal drip, } \\
\text { and cough } \\
\text { - Overall therapeutic } \\
\text { response to treatment }(0-5) \\
\text { at day } 21 \\
\text { - CT scan at day } 21 \\
\text { - Adverse events }\end{array}$ & $\begin{array}{l}\text { - Significantly greater decre- } \\
\text { ase in total symptom score } \\
\text { and headache, congestion, } \\
\text { and facial pain compared } \\
\text { with placebo. } \\
\text { - No differences in other } \\
\text { outcomes } \\
\text { - No difference in therapy- } \\
\text { related local adverse events }\end{array}$ \\
\hline
\end{tabular}

CT, computed tomography; DBPCT, double blind placebo controlled trial; FPNS: fluticasone propionate nasal spray; MFNS, mometasone furoate nasal spray; SF-12, Short Form-12 (12-item short form QOL survey; SNOT-20, Sino-nasal Outcome Test-20. 
Figure 4.6.15. Forest plot of the effect of intranasal corticosteroids versus placebo on change from baseline SNOT-20 score in acute post-viral rhinosinusitis.

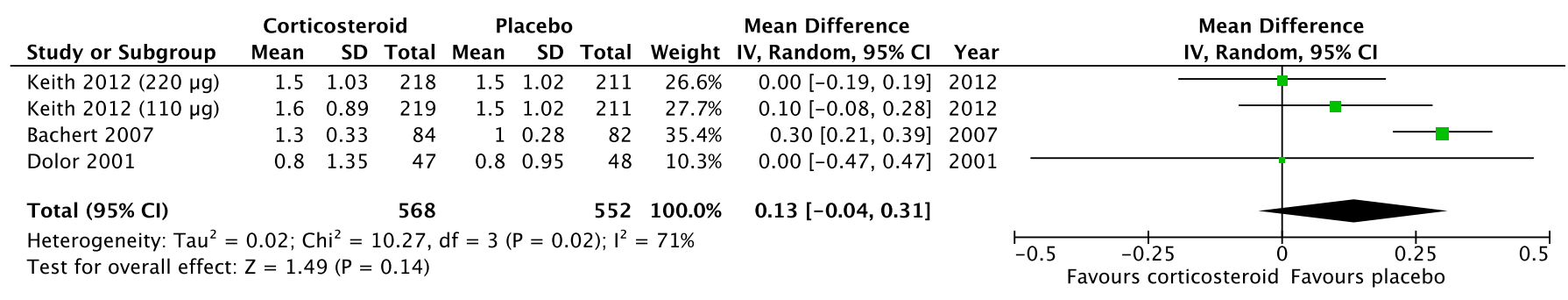

$\mathrm{Cl}$, confidence interval; $\mathrm{M}-\mathrm{H}$, Mantel Haenszel.

Figure 4.6.16. Forest plot of the effect of intranasal corticosteroids versus placebo on change from baseline of total symptom score in acute post-viral rhinosinusitis.

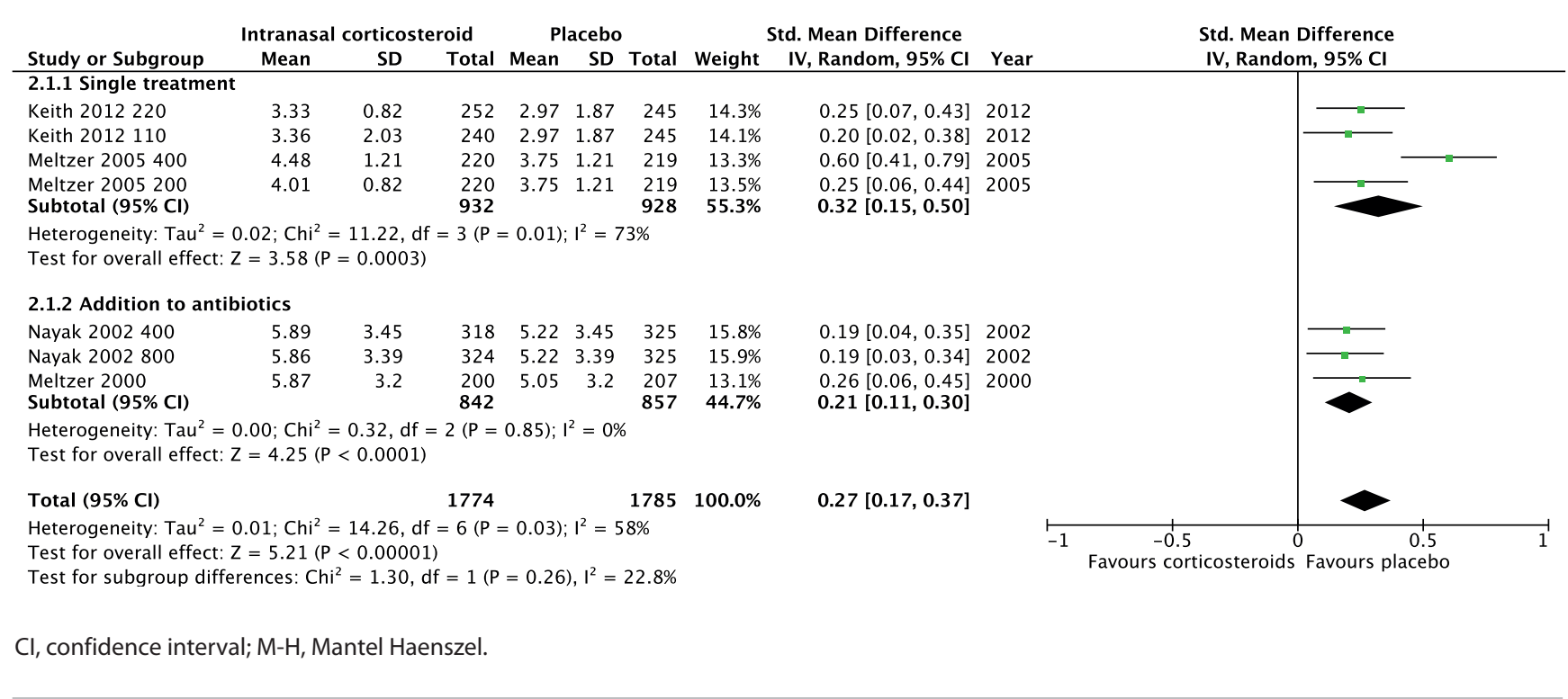

\subsection{Conclusion}

Nasal corticosteroids are effective in reducing total symptom score in adults suffering from acute post-viral rhinosinusitis. However, the effect is small. Nasal corticosteroids have not been shown to have an effect on QOL. We downgraded the evidence because of high heterogeneity. Acute post-viral rhinosinusitis is a self-limiting disease. Based on the moderate quality of the evidence and the small effect size the EPOS2020 steering committee advises only to prescribe a nasal corticosteroid when reduction of the symptoms of the acute post-viral rhinosinusitis is considered necessary.

\subsubsection{Nasal corticosteroid in acute post-viral rhinosinusitis in children}

\subsection{Summary of the evidence}

This section aims to assess the efficacy of nasal corticosteroids in children with post-viral ARS. There are no studies evaluating the effect of nasal corticosteroids on ABRS. Studies were included if they involved children with ARS with symptoms of at least five days but less than 12 weeks (to exclude chronic rhinosinusitis). We identified two studies, one DBPCT and one single blind ${ }^{(268,}$ 269). Both evaluated the use of nasal corticosteroids on top of antibiotics. Both studies found a significantly larger decrease in symptoms and in one study more children were cured $(21 / 50$ versus $2 / 50$ ) (Table 4.6.8.).

\subsection{Conclusion}

Nasal corticosteroids seem to be effective in reducing total symptom score in children suffering from acute post-viral rhinosinusitis on top of (ineffective) antibiotics. Acute post-viral rhinosinusitis is a self-limiting disease. Based on the very low quality of the evidence the EPOS2020 steering committee cannot advise on the use of nasal corticosteroids in children with 
Table 4.6.6. Bacterial lysates in children with acute post-viral rhinosinusitis.

\begin{tabular}{|c|c|c|c|c|c|}
\hline Study & Methods & Participants & Interventions & Outcomes & Results \\
\hline $\begin{array}{l}\text { Barreto } \\
1998^{(283)}\end{array}$ & DBPCT & $\begin{array}{l}\text { Children aged } 18 m-9 y \text { with } \\
\text { ARS }\end{array}$ & $\begin{array}{l}\text { - OM-85-BV }(3.5 \mathrm{mg})(\mathrm{n}=26) \\
\text { - Placebo }(\mathrm{n}=30) \text { daily for } \\
10 \text { days } \\
\text { Both groups received } \\
\text { amoxicillin/clavulanic acid } \\
(40 / 10 \mathrm{mg} / \mathrm{kg}) \text { daily in } 3 \\
\text { doses for } 21 \text { days. }\end{array}$ & $\begin{array}{l}\text { - Improvement } \\
\text { - Convalescence }\end{array}$ & $\begin{array}{l}-\mathrm{OM}-85-\mathrm{BV} \text { group improved } \\
\text { sooner than the placebo group } \\
(5.56 \pm 4.98 \text { vs. } 10 \pm 8.49 ; \mathrm{p}<0.05) \text {. } \\
- \text { OM- } 85-\mathrm{BV} \text { had significantly shor- } \\
\text { ter convalescence time compared } \\
\text { to the placebo group }(15.38 \pm 8.91 \\
\text { vs. } 20.28 \pm 7.17 ; p<0.05)\end{array}$ \\
\hline
\end{tabular}

DBPCT, double blind placebo controlled trial; $m$, months; OM-85-BV, oral bacterial extract; $y$, years.

Table 4.6.7. Homeopathy for acute post-viral rhinosinusitis.

\begin{tabular}{|c|c|c|c|c|c|}
\hline Study & Methods & Participants & Interventions & Outcomes & Results \\
\hline $\begin{array}{l}\text { Zabolotnyi } \\
2007^{(285)}\end{array}$ & DBPCT & $\begin{array}{l}\text { Adults with radio- } \\
\text { graphic confirmed } \\
\text { acute maxillary sinusitis } \\
(\mathrm{n}=133) \text {. }\end{array}$ & $\begin{array}{l}\text { Sinfrontal ( } n=57 \text { ) vs. } \\
\text { placebo ( } n=56 \text { ); one tablet } \\
\text { every hour until first im- } \\
\text { provement (max } 12 \text { tablets } \\
\text { per day). After first sign of } \\
\text { improvement or day } 3, \text { two } \\
\text { tablets three times daily. } \\
\text { Tablets had to be melted } \\
\text { slowly in the mouth at least } \\
30 \text { minutes before or after } \\
\text { meals. }\end{array}$ & $\begin{array}{l}\text { Assessed at day } 0,7,14, \\
\text { and } 21 \\
\text { - SSS } \\
\text { - Radiographic cure } \\
\text { - Clinical cure } \\
\text { - Improvement in health } \\
\text { state (EQ-VAS) } \\
\text { - Ability to work or do } \\
\text { usual activities } \\
\text { - Treatment outcome }\end{array}$ & $\begin{array}{l}\text { - There was a significant reduction } \\
\text { in the SSS compared to placebo } \\
(5.8 \pm 2.3[6.0] \text { point vs. } 2.3 \pm 1.8[2.0] \\
\text { points; } \mathrm{p}<0.0001) \text {. } \\
\text { - At } 3 \text { weeks, } 68.4 \% \text { of those on me- } \\
\text { dication had complete remission } \\
\text { of symptoms compared to } 8.9 \% \text { of } \\
\text { placebo patients. } \\
\text { - Substantial radiographic impro- } \\
\text { vement was noted in significantly } \\
\text { more patients in the Sinfrontal } \\
\text { group compared to placebo ( } 77.2 \% \\
\text { vs. } 21.4 \%, p<0.0001) \text {. } \\
\text { - By day } 7,52.6 \% \text { of patients in the } \\
\text { Sinfrontal group were able to carry } \\
\text { out daily activities compared to the } \\
17.9 \% \text { in the placebo group. } \\
\text { - The mean change for the EQ-VAS } \\
\text { was significantly different for the } \\
\text { Sinfrontal group compared to } \\
\text { the placebo group (17.3 } 9.1 \text { vs. } \\
6.2 \pm 8.1, p<0.01 \text { ). }\end{array}$ \\
\hline
\end{tabular}

DBPCT, double blind placebo controlled trial; EQ-VAS: Euroqol visual analoque score; SSS, sinusitis severity score.

Table 4.6.8. Nasal corticosteroid as addition to oral antibiotics in children with acute post-viral rhinosinusitis.

\begin{tabular}{|c|c|c|c|c|c|}
\hline Study & Methods & Participants & Interventions & Outcomes & Results \\
\hline $\begin{array}{l}\text { Rahmati } \\
2013^{(268)}\end{array}$ & SBRT & $\begin{array}{l}100 \text { children with mostly } \\
\text { post-viral ARS ( } 2 \text { to } 14 \\
\text { years) }\end{array}$ & $\begin{array}{l}\text { - Amoxicillin } 80 \text { to } 100 \mathrm{mg} / \\
\mathrm{kg} / \text { day with FPNS } 50 \mu \mathrm{g} \\
\text { per nostril twice daily for } 14 \\
\text { days ( } \mathrm{n}=50 \text { ) } \\
\text { - Amoxicillin } 80 \text { to } 100 \mathrm{mg} / \\
\mathrm{kg} / \text { day for } 14 \text { days }(\mathrm{n}=50)\end{array}$ & $\begin{array}{l}\text { At } 10-14 \text { days: } \\
\cdot \text { Cure } \\
\cdot \text { Total symptom score } \\
(0-50)\end{array}$ & $\begin{array}{l}\text { In the group receiving am- } \\
\text { oxicillin and FPNS compared } \\
\text { to the groups with amoxicil- } \\
\text { lin alone: } \\
\text { - Significantly more patients } \\
\text { were cured } \\
\text { - Symptom score was signifi- } \\
\text { cantly lower }\end{array}$ \\
\hline $\begin{array}{l}\text { Barlan } \\
1997^{(269)}\end{array}$ & DBPCT & $\begin{array}{l}151 \text { ( } 89 \text { evaluated) children } \\
\text { with mostly post-viral ARS } \\
\text { (mean age } 7 y \text { ) }\end{array}$ & $\begin{array}{l}\text { - Budesonide nasal spray } \\
\text { bid, } 50 \mu \mathrm{g} \text {, to each nostril for } \\
3 \text { weeks ( } \mathrm{n}=43 \text { ) } \\
\text { - Placebo nasal spray bid, } \\
50 \mu \mathrm{g} \text {, to each nostril for } 3 \\
\text { weeks ( } \mathrm{n}=46 \text { ) } \\
\text { All patients received amoxi- } \\
\text { cillin-clavulanate potassium, } \\
40 \mathrm{mg} / \mathrm{kg} / \mathrm{d} \text { three times daily } \\
\text { for } 3 \text { weeks }\end{array}$ & $\begin{array}{l}\text { Evaluation in first } 3 \text { weeks: } \\
\text { - Daily record card (filled } \\
\text { in by parents) symptom } \\
\text { scores including purulent } \\
\text { nasal discharge, cough } \\
\text { and presence of fever and } \\
\text { headache (0-3). }\end{array}$ & $\begin{array}{l}\text { The budesonide group com- } \\
\text { pared to the placebo group } \\
\text { showed: } \\
\text { - Significant larger impro- } \\
\text { vement in cough and nasal } \\
\text { discharge scores at the end } \\
\text { of second week but not } 1 \mathrm{st} \\
\text { or 3rd week. }\end{array}$ \\
\hline
\end{tabular}

ARS, acute rhinosinusitis; DBPCT, double blind placebo controlled trial; FPNS, fluticasone propionate nasal spray; SBRT, single blind randomised trial; $y$, years. 
acute post-viral rhinosinusitis.

\subsubsection{Oral corticosteroids}

\subsubsection{Summary of the evidence}

Systemic corticosteroids could be effective by attenuating the inflammatory response. Systemic administration of corticosteroids may have advantages over nasal use, such as quicker onset of action, higher therapeutic levels and no risk of poor deliverance because of nasal congestion/sinus blockage. In 2014 a Cochrane review of systemic corticosteroid therapy for acute rhinosinusitis suggested that oral corticosteroids in combination with antibiotics may be modestly beneficial for short-term relief of symptoms in acute rhinosinusitis ${ }^{(270)}$.

We analyzed four DBPC studies evaluating the effect of 3-7 days of systemic corticosteroids (varying dose) in patients with main-

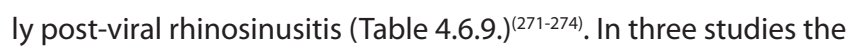
systemic corticosteroid was combined with antibiotics ${ }^{(272-274)}$, in one it was given without antibiotics(271).

The inclusion criteria were not very clear in most studies but most resembled post-viral rhinosinusitis, although the study of Klossek included patients with less than five days of symptoms (common cold).

Two studies looked at recovery at 7-14 days and found no difference $^{(271,274)}$.

Three studies evaluated the proportion of patients with resolution of facial pain at day 4-7 after start of the treatment, these studies could be combined into a meta-analysis, showing a RR of 1.17 (95\% Cl 1.05-1.30), RD of 0.11 (0.03-0.18), 633 patients (Figure 4.6.17.) and showed a significant higher resolution of facial pain 4-7 days after start of systemic corticosteroid treatment than placebo. The differences were however, small and notably almost two-thirds of the patients had no symptoms after placebo treatment (197/316). The effect of systemic corticosteroids compared to placebo was not found at 10-14 days after start of the treatment.

Two studies could be combined to evaluate the proportion of patients with resolution of nasal discharge at day 4-7 after start of the treatment showing no significant differences in resolution of nasal discharge at day 4-7 ( RR of 1.24 (95\% Cl 0.88-1.74), 387 patients (Figure 4.6.18.)). Also here, spontaneous recovery was high (72\%).

\subsubsection{Conclusion}

In conclusion, post-viral rhinosinusitis is a self-limiting disease. Systemic corticosteroids, with or without antibiotics do not have a positive effect on recovery at 7-14 days. There is a small but significant effect of systemic corticosteroids versus placebo on facial pain at days 4-7 after start of the treatment. There are no studies comparing systemic corticosteroids to nasal corticoste- roids. The quality of the evidence is low. Based on the evidence, the numbers needed to treat and the potential harm of systemic corticosteroids, the EPOS2020 steering group advises against the use of systemic corticosteroids in patients suffering from acute post-viral rhinosinusitis. There is insufficient data in patients with $A B R S$ to advise on treatment of ABRS.

\subsubsection{Antihistamines (oral and local)}

\subsubsection{Summary of the evidence}

While there has been some data showing efficacy of antihistamines in common cold (see 4.6.2.), we found one study in children evaluating the addition of nasal oxymetazolone and oral syrup containing brompheniramine and phenylpropanolamine versus placebo to oral amoxicillin for 14 days. Responses to treatment were similar between the two groups. The authors concluded that decongestant antihistamine need not be given to the child with acute maxillary sinusitis ${ }^{(275)}$. In addition, one such study in adults has been completed in acute bacterial rhinosinusitis. Braun and colleagues ${ }^{(276)}$ evaluated the use of antihistamines in acute bacterial rhinosinusitis (Table 4.6.10.). This study involved 139 patients with a history of allergic rhinitis who were diagnosed with acute bacterial rhinosinusitis. This diagnosis was made upon visualization of purulent rhinorrhoea or purulence noted from the middle meatus on exam. These patients were randomized to receive either Loratadine $(10 \mathrm{mg})$ or placebo for 28 days. Each group was also given the following: 14 days of antibiotics and 10 days of corticosteroids. Outcomes were evaluated at the end of treatment, and the addition of antihistamines significantly decreased nasal obstruction $(\mathrm{MD}=-0.58 ; 95 \%$ $\mathrm{Cl}=-0.85--0.31, \mathrm{p}<0.01)$, but did not reduce total symptom scores $(M D=-1.25 ; 95 \% C l=-2.77-0.27, p=0.11)$, or rhinorrhoea symptoms $(\mathrm{MD}=-0.06 ; 95 \% \mathrm{Cl}=-0.37-0.25, \mathrm{p}=0.71)$. Similar results were noted at day 14 with the addition of antihistamines, there was significantly decreased nasal obstruction $(\mathrm{MD}=-0.34$; $95 \% \mathrm{Cl}=-0.64--0.04, \mathrm{p}=0.02)$, but did not reduce total symptom scores $(\mathrm{MD}=-0.26 ; 95 \% \mathrm{Cl}=-3.11-0.59, \mathrm{p}=0.18)$ or rhinorrhoea symptoms $(\mathrm{MD}=-0.12 ; 95 \% \mathrm{Cl}=-0.39-0.15, \mathrm{p}=0.39)$.

\subsubsection{Conclusion}

In conclusion, there are two studies evaluating antihistamines versus placebo in addition to antibiotics; one in adults with ABRS the other in children with post-viral ARS. Both studies showed no additive effect of antihistamines over the treatment given. Based on the very low quality of the evidence, the EPOS2020 steering group cannot advise on the use of antihistamines in post-viral ARS and ABRS.

\subsubsection{Antileukotrienes}

There is no evidence from RCTs for the use of anti-leukotrienes 


\begin{tabular}{|c|c|c|c|c|c|}
\hline Study & Methods & Participants & Interventions & Outcomes & Results \\
\hline $\begin{array}{l}\text { Venekamp } \\
\text { et al. } \\
2012^{(271)}\end{array}$ & DBPCT & $\begin{array}{l}185 \text { patients with post- } \\
\text { viral rhinosinusitis ( } 174 \\
\text { evaluated) }\end{array}$ & $\begin{array}{l}\text { - Prednisolone } 30 \mathrm{mg} / \mathrm{d} \text { for } 7 \\
\text { days }(\mathrm{n}=88) \\
\text { - Placebo for } 7 \text { days }(\mathrm{n}=86)\end{array}$ & $\begin{array}{l}\text { - The proportion of patients } \\
\text { with resolution of symptoms } \\
\text { (facial pain or pressure, nasal } \\
\text { congestion or blockage, post- } \\
\text { nasal discharge, runny nose, } \\
\text { poor sleep, cough and redu- } \\
\text { ced productivity) on day } 7 \\
\text { - SNOT-20 on days } 1,7 \text { and } 14 \\
\text { - Median duration of symp- } \\
\text { tom }\end{array}$ & $\begin{array}{l}\text { No clinically relevant bene- } \\
\text { ficial effects }\end{array}$ \\
\hline $\begin{array}{l}\text { Ratau } \\
2004^{(272)}\end{array}$ & DBPCT & $\begin{array}{l}42 \text { patients with post-viral } \\
\text { rhinosinusitis }\end{array}$ & $\begin{array}{l}\text { - Betamethasone } 1 \mathrm{mg} \text { orally } \\
\text { once a day for } 5 \text { days }(\mathrm{n}=21) \\
\text { - Placebo tablets for } 5 \text { days } \\
(\mathrm{n}=21) \\
\text { All patients received } \\
\text { amoxicillin-clavulanic acid } \\
625 \mathrm{mg} \text { orally, three times } \\
\text { daily for } 5 \text { days }\end{array}$ & $\begin{array}{l}\text { - Change in treatment effects } \\
\text { for the duration of therapy } \\
\text { expressed as slope values } \\
\text { based on a daily symptom } \\
\text { score card (headache, facial } \\
\text { pain, nasal congestion and } \\
\text { dizziness) for } 5 \text { days } \\
\text { - Percentage of patients with } \\
\text { percussion tenderness and } \\
\text { nasal secretions on day } 6 \\
\text { - Use of paracetamol for } 6 \\
\text { days }\end{array}$ & $\begin{array}{l}\text { Bethamethasone versus } \\
\text { placebo resulted in: } \\
\text { - Significantly larger reduc- } \\
\text { tion in headache (none/ } \\
\text { mild versus moderate se- } \\
\text { vere) and facial pain, nasal } \\
\text { congestion and dizziness } \\
\text { (none versus symptoms) } \\
\text { - Significantly less patients } \\
\text { with percussion tenderness } \\
\text { and nasal secretions on } \\
\text { day } 6 \\
\text { - No significant difference } \\
\text { in paracetamol taken }\end{array}$ \\
\hline $\begin{array}{l}\text { Klossek } \\
2004^{(273)}\end{array}$ & DBPCT & $\begin{array}{l}289 \text { patients with common } \\
\text { cold/acute rhinosinusitis } \\
\text { with symptoms for }<5 \\
\text { days (prednisone } n=142 \text {, } \\
\text { placebo } n=147 \text { ) }\end{array}$ & $\begin{array}{l}\text { - Prednisone }(40-60 \mathrm{~kg} \\
\text { bodyweight: } 40 \mathrm{mg}, 60-80 \mathrm{~kg} \\
\text { bodyweight: } 60 \mathrm{mg}>80 \mathrm{~kg} \\
\text { bodyweight: } 80 \mathrm{mg}) \text {, for } 3 \\
\text { days }(n=142) \\
\text { - Placebo }(n=147) \\
\text { Both groups treated with } \\
\text { cefpodoxime } 100 \mathrm{mg} \text { twice } \\
\text { daily for } 10 \text { days }\end{array}$ & $\begin{array}{l}\text { - Mean pain intensity dif- } \\
\text { ference, nasal obstruction } \\
\text { (VAS) compared to baseline } \\
\text { on day } 1-3 \\
\text { - Paracetamol use in day } 1-3\end{array}$ & $\begin{array}{l}\text { Prednisone versus placebo } \\
\text { resulted in: } \\
\text { - Significant reduction in } \\
\text { mean pain intensity dif- } \\
\text { ference, nasal obstruction } \\
\text { and use of paracetamol at } \\
\text { day } 1-3\end{array}$ \\
\hline $\begin{array}{l}\text { Gehanno } \\
2000^{(274)}\end{array}$ & DBPCT & $\begin{array}{l}417 \text { adult patients with } \\
\text { mainly post-viral rhinosi- } \\
\text { nusitis }\end{array}$ & $\begin{array}{l}\text { - } 8 \text { mg of methylprednisolone } \\
3 \text { times daily ( } n=208 \text { ) for } 5 \\
\text { days } \\
\text { - Placebo } 3 \text { times daily } \\
\text { ( } n=209 \text { ) for } 5 \text { days } \\
\text { All patients received } 500 \mathrm{mg} \\
\text { of amoxicillin-clavulanate } \\
3 \text { times daily for } 5 \text { days and } \\
\text { half received } 10 \text { days }\end{array}$ & $\begin{array}{l}\text { - Clinical and/or radiological } \\
\text { recovery at day } 14 \\
\text { - Craniofacial pain, nasal } \\
\text { discharge at day } 4\end{array}$ & $\begin{array}{l}24 \mathrm{mg} \text { methylprednisolone } \\
\text { versus placebo resulted in: } \\
\text { - No difference in recovery } \\
\text { - Significant reduction in } \\
\text { craniofacial pain but not in } \\
\text { nasal discharge at day } 4\end{array}$ \\
\hline
\end{tabular}

DBPCT, double blind placebo controlled trial; VAS, visual analogue scale..

Figure 4.6.17. Proportion of patients with resolution of facial pain after 4-7 days of systemic corticosteroid treatment versus placebo.

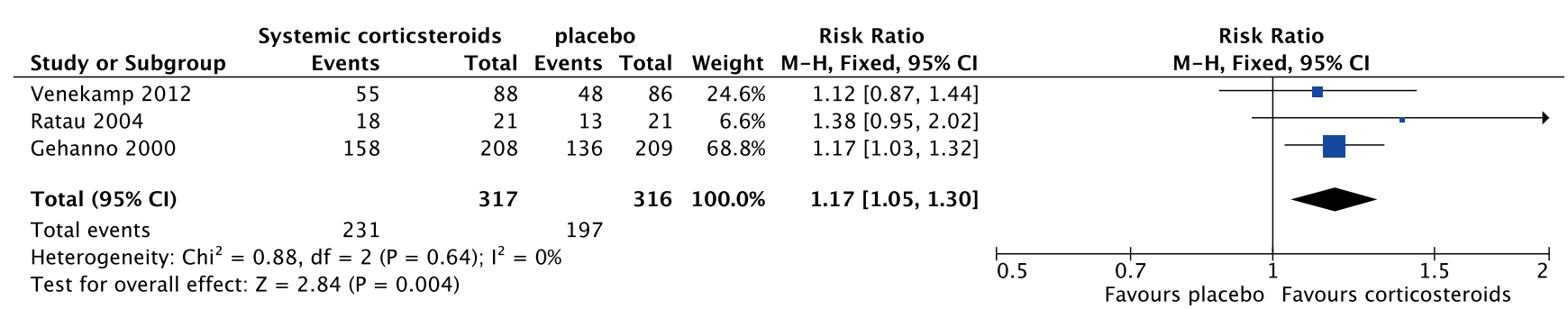


Figure 4.6.18. Proportion of patients with resolution of nasal discharge after 4-7 days of systemic corticosteroid treatment versus placebo.

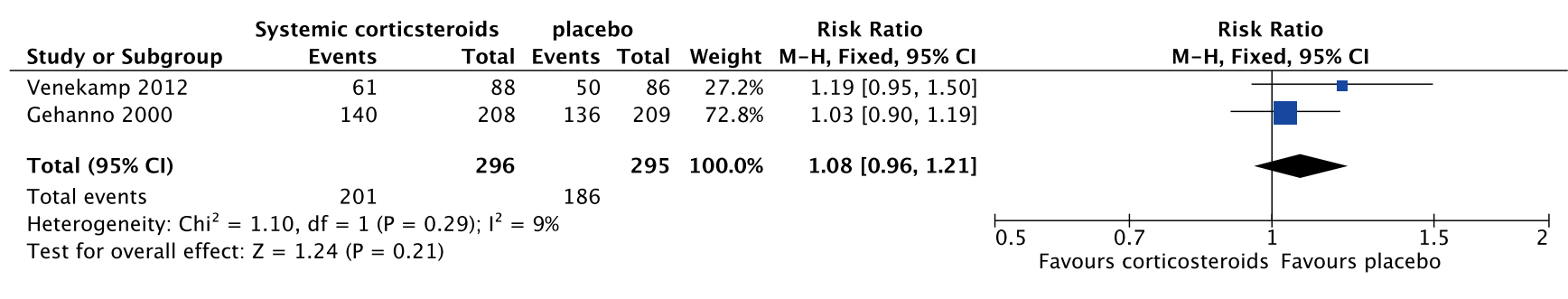

$\mathrm{Cl}$, confidence interval; $\mathrm{M}-\mathrm{H}$, Mantel Haenszel.

in common colds, post-viral ARS or ABRS.

\subsubsection{Nasal decongestants}

\subsubsection{Summary of the evidence}

Decongestants are commonly recommended by primary health care physicians, as well as otorhinolaryngologists, as part of the treatment for ARS. Although the current evidence suggests that multiple doses of decongestants may have a small positive effect on subjective measures of nasal congestion in adults with the common cold ${ }^{(201)}$, their use in ARS has had limited investigation. We systematically reviewed nasal decongestants/vasoconstrictors in ARS. Studies that included either ABRS or post-viral rhinosinusitis were included. The common cold was excluded. We identified one randomised controlled (RCT) study that evaluated the impact of nasal decongestants on the clinical outcome of adult acute post-viral ARS. Inanli et al.(277) examined the effect of several different topical agents in 60 patients with post-viral ARS (as described in the paper although the authors speak about ABRS) on mucociliary clearance (MCC). The final
MCC score of the oxymetazoline group, taken after three weeks, was not significantly different to any other intervention. In the oxymetazoline group, however, there was a significant improvement in MCC scores throughout the duration of treatment. Interestingly, regardless of intervention, patients with post-viral ARS continued to have significantly poorer MCC scores compared to healthy controls after three weeks of treatment. This study was limited in that the baseline MCC scores between groups were significantly different. The baseline MCC score in the oxymetazoline group was significantly higher than the other groups, therefore, any improvements noted in this group could be attributed to a type 1 error.

\subsubsection{Conclusion}

In adult acute post-viral rhinosinusitis nasal decongestants may be effective in improving mucociliary clearance throughout the acute phase of the disease but further RCTs are required. No studies are performed evaluating the effect of resolution or reduction of symptoms of ARS. Based on the absence of clinically relevant data, the EPOS2020 steering group cannot advise on

Table 4.6.10. Antihistamines in ABRS and post-viral ARS.

\begin{tabular}{|c|c|c|c|c|c|}
\hline Study & Methods & Participants & Interventions & Outcomes & Results \\
\hline $\begin{array}{l}\text { Braun } \\
1997^{(276)}\end{array}$ & $\begin{array}{l}\mathrm{RCT}, \\
\text { placebo- } \\
\text { control }\end{array}$ & $\begin{array}{l}130 \text { adults with allergic } \\
\text { rhinitis and } A B R S\end{array}$ & $\begin{array}{l}\text { - Loratadine ( } 10 \mathrm{mg} \text { ) four times } \\
\text { daily) for } 28 \text { days }(\mathrm{n}=71) \\
\text { - Placebo four times daily for } \\
28 \text { days ( } \mathrm{n}=68) \\
\text { All patients received: Aug- } \\
\text { mentin ( } 2 \mathrm{~g} / \text { day) } \times 14 \text { days }+ \\
\text { prednisone } 40 \mathrm{mg} \text { four times } \\
\text { daily } \times 4 \text { days and } 20 \mathrm{mg} \text { for } \\
4 \text { days }\end{array}$ & $\begin{array}{l}\text { Assessed at } 28 \text { days: } \\
\text { - Total symptom score } \\
\text { - Nasal obstruction } \\
\text { - Rhinorrhoea } \\
\text { - Sneezing } \\
\text { - Nasal itching } \\
\text { - Cough }\end{array}$ & $\begin{array}{l}\text { At } 28 \text { days: } \\
\text { - No significant reduction in } \\
\text { total symptom scores } \\
\text { - Significant decreased } \\
\text { nasal obstruction } \\
\text { - No significant decrease in } \\
\text { other symptoms }\end{array}$ \\
\hline $\begin{array}{l}\text { McCormick } \\
1996^{(275)}\end{array}$ & DBPCT & $\begin{array}{l}68 \text { children (age } 6 \text { (6-16y) } \\
\text { with acute post-viral } \\
\text { rhinosinusitis }\end{array}$ & $\begin{array}{l}\text { - Nasal oxymetazolone and } \\
\text { oral syrup containing bromp- } \\
\text { heniramine and phenylpro- } \\
\text { panolamine (age dependent } \\
\text { dosage) }(n=34) \\
\text { - Placebo }(n=34) \\
\text { All children received amoxicil- } \\
\text { lin three times daily dosage } \\
\text { depending on weight }\end{array}$ & $\begin{array}{l}\text { Assessed at day } 3 \text { and 14: } \\
\text { - Mean symptom score }\end{array}$ & $\begin{array}{l}\text { No differences between the } \\
\text { treatment groups. }\end{array}$ \\
\hline
\end{tabular}


the use of decongestants in acute post-viral rhinosinusitis.

\subsubsection{Saline}

\subsubsection{Summary of the evidence}

Saline irrigation and sprays are commonly recommended by primary health care physicians, as well as otorhinolaryngologists, as part of the treatment for ARS.

We identified three RCTs evaluating the impact of nasal saline irrigations/sprays on clinical outcomes in adult patients with ARS. Studies that included either ABRS or post-viral rhinosinusitis were included. The studies were different in design, duration and outcome measurements. Both studies reported no effect (Tables 4.6.11. and 4.6.12.).

Adam et al. ${ }^{(278)}$ examined 75 adults with ABRS in a partially blinded RCT. Hypertonic and normal saline nasal spray three times daily were compared. The study found no difference in symptoms between the groups on day 3 of their illness and no difference between the groups in the duration of recovery. Rabago et al. ${ }^{(279)}$ examined in a non-blinded RCT the use of daily, high volume (150ml per side), hypertonic saline on patients with post-viral ARS and CRS. Eighty percent of the study population had a diagnosis of post-viral ARS, so this study was included. Outcomes were measured at six weeks, three months and six months. Results at six weeks are included as this is closest to the relevant ARS symptom time frame. The intervention group had no significant improvement in their Rhinosinusitis Disability Index (RSDI), single-Item Sinus Severity Assessment (SIA) and SF12 score. Gelardi et al. ${ }^{(280)}$ evaluated the difference between the efficacy of two irrigation systems in 20 patients with acute (presumed post-viral) rhinosinusitis and who were further treated with levofloxacin $500 \mathrm{mg} /$ day for 10 days and topical naphazoline (two puffs in each nostril) twice daily for seven days. Patients were randomly assigned to either a nasal syringe $(10 \mathrm{~mL}$ saline solution, three times daily for 14 days) or a high volume $(250 \mathrm{ml})$ warm saline irrigation system twice daily for 14 days. Nasal high volume warm saline irrigation compared to $10 \mathrm{ml}$ saline resulted in significantly less patients with purulent rhinorrhoea at seven days, and significantly less post-nasal drip at seven and 14 days. There were no differences in nasal obstruction of rhinomanometry.

\subsubsection{Conclusion}

Two relatively small studies, one in $\mathrm{ABRS}^{(278)}$, one in post-viral $\mathrm{ARS}^{(279)}$ did not find a difference between saline nasal spray versus no treatment. One very small study found a larger effect of high volume versus low volume saline rinsing on purulent rhinorrhoea and post-nasal drip. Based on the very low quality of the evidence no advice can be given about the use of nasal saline irrigation in ABRS or post-viral ARS.

\subsubsection{Steam / heated air}

Steam inhalation has been proposed as an adjunct in the treatment of ARS. A systematic review identified two RCTs that examined the role of steam inhalation however both of these papers were excluded. Little et al. ${ }^{(281)}$ examined the role of steam and analgesia in patients with respiratory tract infections. This paper was excluded as the patients were grouped into lower respiratory tract infections (LRTI) and non-LRTI cohorts. Patients with specific ARS symptoms were not specifically examined. Little et al. ${ }^{(282)}$ compared the effectiveness of steam inhalation and nasal irrigation for CRS or recurrent ARS. This study was excluded because the first follow up assessment was three months after the intervention which is beyond the timeframe for assessment in patients with ARS.

In conclusion, there are no eligible studies evaluating the effect of steam / heated air in post-viral ARS and ABRS.

\subsubsection{Physical interventions}

We identified one study evaluating the effect of ultrasound treatment in comparison to amoxicillin in the treatment of 48 patients (42 analysed) with presumed ABRS ${ }^{(234)}$. The experimental group received four consecutive days of ultrasound and the control group received a 10-day course of amoxicillin. There were no differences between the treatment groups, apart from a significantly larger decrease in pain around the nose at day 4. There were no other differences between the groups in terms of satisfaction with intervention, number of side-effects, or number of relapses. Due to the lack of a placebo group no relevant conclusions can be drawn from this study.

\subsubsection{Bacterial lysates}

\subsubsection{Summary of the evidence}

Barreto et al. ${ }^{(283)}$, evaluated the efficacy of OM-85-BV (3.5mg) versus placebo for 10 days, in addition to amoxicillin/clavulanic acid (40/10mg per $\mathrm{kg}$ for 21 days) in children with acute rhinosinusitis. They evaluated children from 18 months to nine years old with at least 30 days of the following symptoms: post-nasal discharge, nasal congestion, halitosis, facial pain and pressure, nasal mucosa irritation, fevers, retro-orbital headache and radiographic evidence of sinus opacification or oedema of bilateral paranasal sinuses. Patients were assessed on days 0,3 , $15,21,30,60,90$, and 180 for improvement of current infection and recurrent respiratory infections. Patients who received OM-85-BV had significantly shorter time to improvement when compared to the placebo group $(5.56 \pm 4.98$ vs. $10 \pm 8.49 ; \mathrm{p}<0.05)$. The convalescence time was significantly shorter in the OM-85BV group when compared to the placebo group (15.38 \pm 8.91 vs. $20.28 \pm 7.17 ; p<0.05)$. There was one adverse event, a rash, that disappeared three days after drug discontinuation (Table 4.6.6.). 
Table 4.6.11. Nasal saline spray in adult ABRS.

\begin{tabular}{|c|c|c|c|c|c|}
\hline Study & Methods & Participants & Interventions & Outcomes & Results \\
\hline $\begin{array}{l}\text { Adam } \\
1998^{(278)}\end{array}$ & $\begin{array}{l}\text { RCT, partial } \\
\text { blinding }\end{array}$ & Adults with ABRS $(n=75)$ & $\begin{array}{l}\text { - HNS spray; } 2 \text { sprays per } \\
\text { nostril, } 3 \text { times per day for } \\
10 \text { days ( } n=26 \text { ) } \\
\text { - NS spray; } 2 \text { sprays, each } \\
\text { nostril, } 3 \text { times per day for } \\
10 \text { days ( } n=23 \text { ) } \\
\text { - No treatment ( } n=26 \text { ) } \\
\text { All patients received antibi- } \\
\text { otics (unclear which one) }\end{array}$ & $\begin{array}{l}\text { - Nasal symptom score on } \\
\text { day } 3 \\
\text { - Day of well-being (day of } \\
\text { symptom resolution) }\end{array}$ & $\begin{array}{l}\text { - No significant difference } \\
\text { between the groups in: } \\
\text { - Day } 3 \text { nasal symptom } \\
\text { scores } \\
\text { - Day of well-being } \\
\text { between the groups }\end{array}$ \\
\hline
\end{tabular}

ABRS, acute bacterial rhinosinusitis; RCT, randomised controlled trial; HNS: hypertonic nasal saline; NS: nasal saline

Table 4.6.12. Saline use in adult post-viral ARS.

\begin{tabular}{|c|c|c|c|c|c|}
\hline Study & Methods & Participants & Interventions & Outcomes & Results \\
\hline $\begin{array}{l}\text { Gelardi } \\
2009^{(280)}\end{array}$ & $\begin{array}{l}\text { RCT, non- } \\
\text { blinded }\end{array}$ & 20 adults with (post-viral?) ARS & $\begin{array}{l}\text { - High volume ( } 250 \mathrm{ml}) \\
\text { warm saline irrigation sys- } \\
\text { tem twice daily for } 14 \text { days } \\
\text { - Saline applied with a } 10 \mathrm{ml} \\
\text { syringe } 3 \text { times daily for } \\
14 \text { days } \\
\text { All patients received } \\
\text { levofloxacin } 500 \mathrm{mg} / \text { day for } \\
10 \text { days and topical napha- } \\
\text { zoline } 2 \text { puffs in each nostril } \\
\text { twice daily for } 7 \text { days. }\end{array}$ & $\begin{array}{l}\text { At 7,14 and } 21 \text { days: } \\
\text { - Symptoms } \\
\text { - Nasal endoscopy } \\
\text { - Rhinomanometry }\end{array}$ & $\begin{array}{l}\text { The high volume warm } \\
\text { saline irrigation compared } \\
\text { to } 10 \mathrm{ml} \text { saline resulted in: } \\
\text { - Significantly less patients } \\
\text { with purulent rhinorrhoea } \\
\text { at } 7 \text { days } \\
\text { - Significantly less post- } \\
\text { nasal drip at } 7 \text { and } 14 \text { days } \\
\text { - No difference in rhinoma- } \\
\text { nometry }\end{array}$ \\
\hline $\begin{array}{l}\text { Rabago } \\
2002^{(279)}\end{array}$ & $\begin{array}{l}\text { RCT, non- } \\
\text { blinded }\end{array}$ & $\begin{array}{l}76 \text { adults with } 2 \text { episodes of } \\
\text { ARS or } 1 \text { episode of CRS per } \\
\text { year, for } 2 \text { consecutive years } \\
63 \text { with diagnosis post-viral ARS }\end{array}$ & $\begin{array}{l}\cdot 2.0 \% \text { saline with baking } \\
\text { soda } 150 \mathrm{ml} \text { per nostril daily } \\
(\mathrm{n}=52) \\
\text { - No treatment }\end{array}$ & $\begin{array}{l}\text { Baseline, 6w, 3m, 6m: } \\
\text { - SF-12 } \\
\text { - RSDI } \\
\text { - SIA } \\
\text { - Compliance diary }\end{array}$ & $\begin{array}{l}\text { No significant difference in } \\
\text { the reported outcomes. }\end{array}$ \\
\hline
\end{tabular}

ARS, acute rhinosinusitis; CRS, chronic rhinosinusitis; m, months; RCT, randomised controlled trial; RSDI, Rhinosinusitis Disability Index; SF-12, Short Form-12 (12-item short form QOL survey); SIA, Sinus Severity Assessment; w, weeks.

\subsubsection{Conclusion}

One study has shown benefit in the use of OM-85-BV for shortening the duration of illness in post-viral rhinosinusitis. Further studies should be conducted to determine efficacy in patients with post-viral and ABRS.

\subsubsection{Homeopathy}

\subsubsection{Summary of the evidence}

Two studies ${ }^{(284,285)}$ evaluated the use of homeopathy in acute post-viral rhinosinusitis. The study of Friese et al. ${ }^{(284)}$ is excluded from this analysis because the patients had symptoms for threeeight days at the beginning of the trial and thus did not fulfil the criteria for post-viral rhinosinusitis.

There was one study ${ }^{(285)}$ evaluating the use of Sinfrontal (Cinnabaris (Trit. D4), Ferrum phosphoricum (Trit. D3), Mercurius solubilis Hahnemanni (Trit. D6)). in acute maxillary sinusitis. This study consisted of 22 days of treatment with Sinfrontal versus a placebo and patients were evaluated at four time points over the course of the study (day $0,7,14$, and 21 ). There was a postobservational phase of eight weeks where the patients were no longer on the study medication. Outcome measures included the sinusitis severity score (SSS) which consisted of the sum score of the following six symptoms: headache, maxillary pain, maxillary pain worsening on bending forward, percussion, or pressure; nasal obstruction, purulent nasal secretion, purulent nasal discharge visualized in the middle meatus of purulent post-nasal discharge. Each symptom was scored on a 0-4 scale ( 0 - not present, 4 - very severe). Each patient underwent sinus radiography which was scored on a modified version of the system used by van Buchem ${ }^{(243)}$ from not assessable (NA) to a score of 6 for an air-fluid level. A response was defined as a SSS of $<10$ point on day 7 and a reduction of the SSS $\geq 4$ points from day 0 to day 7 and a clinical cure was defined as complete remission of signs and symptoms ( $\mathrm{SSS}=0$ ) or substantial improvement defined as reduction of signs and symptoms (each symptom SSS $\leq 1$ 
point). There was a significant reduction in the SSS compared to placebo (5.8 $\pm 2.3[6.0$ ] point vs. $2.3 \pm 1.8[2.0$ ] points; $p<0.0001)$. Patients in the Sinfrontal group met criteria as responders significantly more than the placebo group $(66.7 \%$ vs. $5.4 \%$, $p<0.0001)$. Substantial radiographic improvement was noted in significantly more patients in the Sinfrontal group compared to placebo ( $77.2 \%$ vs. $21.4 \%, p<0.0001)$. By day $7,52.6 \%$ of patients in the Sinfrontal group were able to carry out daily activities compared to the $17.9 \%$ in the placebo group. The mean change for the EQ-VAS was significantly different for the Sinfrontal group compared to the placebo group (17.3 \pm 9.1 vs. $6.2 \pm 8.1$, $\mathrm{p}<0.01)$. Seven patients (6.3\%) had adverse events during the study. Six patients (10.5\%) in the active medication group and one (1.8\%) in the placebo group had adverse events and these were most commonly gastrointestinal complaints. The events were most often connected to symptoms from lactose exposure as this was one of the main constituents of Sinfrontal and the placebo. This study was also evaluated from a health economic perspective. Sinfrontal led to incremental savings of 275 euros $(95 \% \mathrm{Cl} 433,103)$ per patient compared with placebo over 22 days, essentially due to the markedly reduced absenteeism from work (7.83 vs. 12.9 workdays). Incremental utility amounted to 0.0087 QALYs (95\% Cl 0.0052, 0.0123), or 3.2 quality-adjusted life-days (QALDs) ${ }^{(286)}$ (Table 4.6.7.).

\subsubsection{Conclusion}

We found one study evaluating the effect of homeopathy (Sinfrontal) and showing a significant reduction of symptoms and radiographic improvement versus placebo. Based on the limited evidence the EPOS2020 steering cannot give clear advice on the use of homeopathy in acute post-viral rhinosinusitis.

\subsubsection{Herbal compounds}

\subsubsection{Summary of the evidence}

Herbal compounds have been extensively explored in the treatment of upper respiratory tract diseases. Numerous randomized studies, of varying quality, have been performed to assess the efficacy and safety of herbal compounds in the treatment of viral, post-viral and acute bacterial ARS. However, in many studies it is unclear which groups of ARS patients have been studied. We found six DBPC randomized studies of the efficacy of herbal compounds in the treatment of presumed post-viral ARS (Table 4.6.13.). Of these six trials, in four trials it is however unclear what the exact ARS phenotype of the patients was ${ }^{(228,287-289)}$. In these studies the duration of the symptoms after the acute onset was often not indicated and some patients were included that had signs that could point to ABRS such as high fever, or severe unilateral pain ${ }^{(228,287)}$. The only two studies that clearly include post-viral patients are the studies by Pfaar et al. evaluating the effectiveness of Cyclamen europaeum nasal spray versus placebo as an adjunct to amoxicillin ${ }^{(290)}$ and the study from Bachert evaluating pelargonium sidoides versus placebo(291). The frozen, dried, natural fluid extract of the Cyclamen europaeum plant delivered intranasally is thought to have beneficial effects in relieving congestion by facilitating nasal drainage and has an anti-inflammatory effect. Cyclamen europaeum nasal spray as an adjunct to medical therapy (amoxicillin 500mg three times daily for days) was compared to placebo in 99 patients with post-viral rhinosinusitis ${ }^{(290)}$. There was no difference between the groups for change in mean rhinosinusitis visual analogue scale scores of total symptom, nasal obstruction, facial pain or pressure, smell dysfunction, mucous secretions or sleep quality after 5-7 days. Similar trends were observed at day 12-15. Of note, a reduction in facial pain significantly favoured Cyclamen europaeum (MD: - $1.20,[-2.32,-0.08] ; p=0.04$ ) for the per protocol-population after five to seven days. Endoscopic evaluation demonstrated that mucous oedema or nasal obstruction was reduced to a significantly greater extent with Cyclamen europaeum than placebo after 5-7 days (MD: -0.76, [-1.44 to -0.08]; $\mathrm{p}<0.03$ ). There were no significant between group differences for mucopurulent secretion scores in the middle meatus at any time points evaluated. No patients needed additional treatment for rhinosinusitis during the study, and there were no medical complications associated with progression of ARS. Adverse events were reported by $67 \%$ of the Cyclamen europaeum group and $29 \%$ of placebo recipients but no serious adverse events were reported. A DBPC study of Cyclamen europaeum versus placebo in a subgroup of 29 patients with presumed (post)viral rhinosinusitis showed a comparable improvement in total symptom scores but a significant difference between treatment groups in change in percent of sinus opacification in favour of Cyclamen. More treatment-related adverse events were reported in the placebo group (37.5\%) than in the Cyclamen group (15\%). As the two studies were very different no meta-analysis could be performed.

Pelargonium sidoides (P. sidoides), is an herbal remedy thought to be effective in the treatment of upper respiratory infections ${ }^{(292)}$. Only one RCT has evaluated its efficacy in 103 patients with presumed post-viral ARS(291). Patients were randomized to receive P. sidoides 60 drops orally three times daily or a matched placebo for a maximum of 22 days. The mean decrease in the sinusitis severity score at day 7 was 5.5 points in the P. sidoides group and 2.5 points in the placebo group (between group difference of 3.0 points; $95 \% \mathrm{Cl}$ [2.0 to 3.9]; $\mathrm{p}<0.0001$ ). Significant treatment effects were also observed for SNOT-20 (0.6 vs. 0.2; $\mathrm{p}<0.0001)$, EQ-VAS $(18.1 \pm 14.1$ vs. $5.1 \pm 11.0 ; \mathrm{p}<0.0001)$ by day 7 in the P. sidoides group versus placebo. Similarly, the duration of days patients were unable to work ( $8.7 \pm 6.4$ vs. $15.9 \pm 11.8$; $\mathrm{p}=0.002)$, the number of patients able to work or engage in usual activities (32 [63\%] vs. 19 [37\%]), and the number of patients improved from sleep disorders (40 [82\%] vs. 27 [54\%]; 
Table 4.6.13. Herbal compounds versus placebo in acute post-viral rhinosinusitis.

\begin{tabular}{|c|c|c|c|c|c|}
\hline Study & Methods & Participants & Interventions & Outcomes & Results \\
\hline $\begin{array}{l}\text { Pfaar } \\
2012^{(290)}\end{array}$ & DBPCT & $\begin{array}{l}\text { Adults with acute post-viral } \\
\text { rhinosinusitis }(n=99)\end{array}$ & $\begin{array}{l}\text { - Cyclamen europaeum } \\
\text { nasal spray }(1.3 \mathrm{mg} \text { ) once } \\
\text { daily each nostril for } 15 \\
\text { days ( } \mathrm{n}=48 \text { ) } \\
\text { - Placebo for } 15 \text { days ( } \mathrm{n}=51 \text { ) } \\
\text { All patients received amoxi- } \\
\text { cillin } 500 \mathrm{mg} 3 \text { times daily } \\
\text { for the first } 8 \text { days }\end{array}$ & $\begin{array}{l}\text { - TSS on day 5-7 (VAS) } \\
\text { - Individual symptom } \\
\text { scores (nasal congestion, } \\
\text { mucus secretion, facial } \\
\text { pain, impairment of smell) } \\
\text { on day 5-7 and 12-15 (VAS) } \\
\text { - Endoscopic findings on } \\
\text { day 5-7 and 12-15 } \\
\text { - Treatment failure/need for } \\
\text { additional treatment } \\
\text { - Onset of medical compli- } \\
\text { cations of rhinosinusitis } \\
\text { - Sleep quality } \\
\text { - Overall patient- and } \\
\text { investigator-assessed treat- } \\
\text { ment satisfaction } \\
\text { - Safety assessment }\end{array}$ & $\begin{array}{l}\text { Compared to placebo, cyclamen } \\
\text { europaeum resulted in: } \\
\text { - Improvement in mean patient } \\
\text { ( } p=0.03 \text { ) and investigator-satis- } \\
\text { faction scores ( } p=0.04 \text { ) } \\
\text { - No difference for change in } \\
\text { mean TSS, nasal obstruction, } \\
\text { facial pain or pressure, sleep } \\
\text { quality (VAS), or endoscopic } \\
\text { mucopurulent secretions } \\
\text { - Improvement in endoscopic } \\
\text { scores for nasal obstruction/ } \\
\text { mucosal oedema at } 5-7 \text { days } \\
\text { ( } p=0.03 \text { ) } \\
\text { - No patients needed additional } \\
\text { treatment for rhinosinusitis } \\
\text { during the study, medical } \\
\text { complications associated with } \\
\text { progression of ARS } \\
\text { - Adverse events were reported } \\
\text { by } 67 \% \text { of the cyclamen euro- } \\
\text { paeum group and } 29 \% \text { of pla- } \\
\text { cebo recipients but no serious } \\
\text { adverse events were reported. }\end{array}$ \\
\hline $\begin{array}{l}\text { Ponikau } \\
2012^{(287)}\end{array}$ & DBPCT & $\begin{array}{l}\text { Adults with acute (post) } \\
\text { viral rhinosinusitis }(n=29)\end{array}$ & $\begin{array}{l}\text { - Cyclamen europaeum } \\
\text { nasal spray ( } 2.6 \mathrm{mg}) \text { once } \\
\text { daily each nostril for } 7 \text { days } \\
(\mathrm{n}=24) \\
\text { - Placebo spray sterile } \\
\text { water, once daily for } 7 \text { days } \\
(\mathrm{n}=24)\end{array}$ & $\begin{array}{l}\text { - Percent sinus opacifica- } \\
\text { tion on CT scans at days } 15 \text {, } \\
29 \text { or endpoint } \\
\text { - Reduction in TSS at } \\
\text { endpoint } \\
\text { - Endoscopic inflammation } \\
\text { at day } 7\end{array}$ & $\begin{array}{l}\text { - Significant improvement in } \\
\text { percent of sinus opacification in } \\
\text { favour of cyclamen ( } p=0.045) \text {. } \\
\text { - Comparable improvement in } \\
\text { total symptom scores. } \\
\text { - More treatment-related } \\
\text { adverse events in the placebo } \\
\text { group }(37.5 \%) \text { vs. cyclamen } \\
\text { group }(15 \%) \text {. }\end{array}$ \\
\hline $\begin{array}{l}\text { Bachert } \\
2009^{(291)}\end{array}$ & DBPCT & $\begin{array}{l}\text { Adults with mostly } \\
\text { symptoms of acute (post) } \\
\text { viral rhinosinusitis although } \\
\text { some of the patients could } \\
\text { have had ABRS }(n=103)\end{array}$ & $\begin{array}{l}\text { - Pelargonium sidoides (P. } \\
\text { sidoides) } 60 \text { drops orally } \\
\text { three times daily for maxi- } \\
\text { mum } 22 \text { days }(n=51) \\
\text { - Matched placebo three } \\
\text { times daily for maximum } 22 \\
\text { days }(n=52)\end{array}$ & $\begin{array}{l}\text { - SSS at day } 7 \\
\text { - Radiographic cure at day } \\
21 \\
\text { - SNOT-20 at day } 7 \\
\text { - EQ-VAS on day } 7 \\
\text { - Activity level on day } 7 \\
\text { - Ability to work or engage } \\
\text { in usual activities on day } 7 \\
\text { - General well-being on } \\
\text { day } 7 \\
\text { - Patient and investigator- } \\
\text { reported treatment } \\
\text { outcome on the IMOS } \\
\text { - Safety assessment }\end{array}$ & $\begin{array}{l}\text { Compared to placebo, P. sidoi- } \\
\text { des demonstrated: } \\
\text { - Greater reduction in sinusitis } \\
\text { severity score }(p<0.0001) \text { SNOT- } \\
20 \text { score }(p<0.0001) \\
\text { - Decrease in duration of inabi- } \\
\text { lity to work ( } p=0.002) \\
\text { - Increase in subjects able to } \\
\text { work or engage in usual activi- } \\
\text { ties ( } p=0.003) \text {, and subjects as- } \\
\text { sessed as a 'major improvement' } \\
\text { on IMOS ( } p<0.0001) \text { on day } 7 \\
\text { - Radiographic improvement in } \\
\text { the maxillary sinuses ( } p=0.002 \text { ) } \\
\text { - No significant radiographic } \\
\text { improvement in the frontal or } \\
\text { ethmoid sinuses at day } 21\end{array}$ \\
\hline $\begin{array}{l}\text { Federspil } \\
1997^{(288)}\end{array}$ & $\begin{array}{l}\text { Rando- } \\
\text { mized, } \\
\text { double- } \\
\text { blind, } \\
\text { double- } \\
\text { dummy, } \\
\text { trial }\end{array}$ & $\begin{array}{l}\text { Patients with acute (post) } \\
\text { viral rhinosinusitis }(n=331)\end{array}$ & $\begin{array}{l}\text { - Myrtol standardized } 4 \\
\text { capsules of } 300 \mathrm{mg} \text { daily, for } \\
6 \pm 2 \text { days ( } n=109) \\
\text { - Essential oil (unregistered) } \\
4 \text { capsules of } 300 \mathrm{mg} \text { daily, } \\
\text { for } 6 \pm 2 \text { days ( } n=110 \text { ) } \\
\text { - Matched placebo, } 4 \\
\text { capsules daily for } 6 \pm 2 \text { days } \\
\text { ( } n=111 \text { ) } \\
\text { All patients received xylo- } \\
\text { methazoline } 4 \text { dd } 2 \text { puffs }\end{array}$ & $\begin{array}{l}\text { - Difference in symptoms } \\
\text { score before and after treat- } \\
\text { ment at } 14 \text { days }\end{array}$ & $\begin{array}{l}\text { - Myrtol standardized and the } \\
\text { other essential oil proved to be } \\
\text { significantly superior to placebo } \\
\text { - Tolerance was slightly better } \\
\text { for myrtol standardized in com- } \\
\text { parison to the essential oil }\end{array}$ \\
\hline
\end{tabular}


Table 4.6.13. Herbal compounds versus placebo in acute post-viral rhinosinusitis (continued)..

\begin{tabular}{|c|c|c|c|c|c|}
\hline Study & Methods & Participants & Interventions & Outcomes & Results \\
\hline $\begin{array}{l}\text { Neubauer } \\
1994^{(289)}\end{array}$ & DBPCT & $\begin{array}{l}\text { Patients with acute } \\
\text { (post) viral rhinosinu- } \\
\text { sitis }(n=160)\end{array}$ & $\begin{array}{l}\text { - Sinupret } 2 \text { tablets three } \\
\text { times daily for } 14 \text { days } \\
\text { ( } \mathrm{n}=81 \text { ) } \\
\text { - Placebo (sugar-coated } \\
\text { tablets) } 2 \text { tablets three } \\
\text { times daily for } 14 \text { days } \\
\text { ( } \mathrm{n}=79 \text { ) } \\
\text { All patients received } \\
\text { doxycycline and xylome- } \\
\text { talzoline }\end{array}$ & $\begin{array}{l}\text { - Patient assessment of the } \\
\text { therapy (three categories: } \\
\text { asymptomatic/good ef- } \\
\text { fect/no effect) at } 14 \text { days } \\
\text { - Clinical symptoms of } \\
\text { rhinosinusitis at } 14 \text { days } \\
\text { - Radiographic finding } \\
\text { (completely opaque/sha- } \\
\text { dowed/nothing abnormal) }\end{array}$ & $\begin{array}{l}\text { Compared to placebo, Sinupret demon- } \\
\text { strated } \\
\text { - Greater improvement in patient-repor- } \\
\text { ted complete resolution of symptoms } \\
(\mathrm{p}=0.0002) \\
\text { - Improvement in radiographic findings } \\
\text { after } 14 \text { days of treatment ( } \mathrm{p}=0.02) \\
\text { - Effect on nasal obstruction and muco- } \\
\text { sal swelling } \\
\text { - No difference in response rates for } \\
\text { nasal patency, nasal discharge or } \\
\text { headaches. }\end{array}$ \\
\hline
\end{tabular}

ABRS, acute bacterial rhinosinusitis; ARS, acute rhinosinusitis; DBPCT, double blind placebo controlled trial; EQ-VAS: Euroqol visual analoque score; IMOS, Integrated Medicine Outcomes Scale; SNOT-20, Sino-nasal Outcome Test-20; SSS, Sinusitis Severity Score; TSS, total symptom scores.

$\mathrm{p}=0.003$ ) on day 7 favoured P. sidoides. The investigator assessed the treatment outcome as a 'major improvement' in 15 (30\%) in the P. sidoides group, compared to three (5.8\%) patients in the placebo group $(p<0.0001)$ and treatment outcome assessment by the patients yielded to a similar pattern in favour of the $P$. sidoides group. Results also indicated a statistically significant superiority of $\mathrm{P}$. sidoides for radiographic improvement in the maxillary sinuses (24 [69\%] vs. 22[44\%]; $\mathrm{p}=0.002$ ). Six patients $(11.8 \%)$ reported non-serious adverse events in the P. sidoides group compared to two patients (3.8\%) in the placebo group. Federpil et al. ${ }^{(288)}$ studied the efficacy of myrtol, an herbal extract from essential oils, as a therapeutic alternative for acute rhinosinusitis ( $n=331)$, in comparison to placebo and other essential oils. The results showed a statistically significant improvement of total rhinosinusitis symptoms score in the myrtol standardized group and the other essential oils group compared to placebo at 14 days (10.5 vs. 9.2 points) without difference between the myrtol and the other essential oils.

BNO 1016 (Sinupret), an extract of five herbal drugs (gentian root, primula flower, sorrel herb, elder flower, and verbena herb) that has demonstrated antimicrobial and antiviral activity, has been investigated in common cold (see Chapter 4.6.17.). One study from Neubauer et al. ${ }^{(289)}$ randomized 160 patients with symptoms and signs indicative of acute post-viral rhinosinusitis into Sinupret or placebo in addition to doxycycline and xylometazoline. A greater percentage of patients in the Sinupret group reported complete resolution of symptoms ( $60.3 \%$ vs. $25.0 \%$; $\mathrm{p}=0.0002$ ) and were observed to have improvement in radiographic findings after 14 days of treatment ( $84.0 \%$ vs. $68.4 \%$; $\mathrm{p}=0.02$ ) than in the placebo group. While Sinupret demonstrated a small but significant effect on nasal obstruction (difference in response rates: $0.23,[0.09,0.39])$ and mucous swelling $(0.32$ $[0.17,0.46])$, there was no difference in response rates for nasal patency $(0.10[-0.05,0.26])$, nasal discharge $(0[-0.17,0.20])$ or headaches $(0.15[0.00,0.3])$. The efficacy of Sinupret was compared in one study to fluticasone furoate (Table 4.6.14.) ${ }^{(293)}$. Sixty patients with ARS were randomized to Sinupret or intranasal fluticasone furoate for 14 days. No differences between the two groups were found, most likely due to type II error. No adverse events were reported in the Sinupret group. Among the patients that received fluticasone furoate, three patients reported minor adverse events (epistaxis and nasal itching).

\subsubsection{Vaccination}

There are no RCTs which demonstrate a direct effect of vaccination on post-viral ARS. A systematic review which examined inactivated parental influenza virus vaccination reported $59 \%$ efficacy in preventing confirmed influenza (RR $0.4195 \% \mathrm{Cl} 0.36$ $0.47)^{(294)}$. The pneumococcal vaccine also led to decrease in invasive diseases (meningitis, bacteremia) and acute otitis media ${ }^{(295)}$ but there was no data on decrease of ABRS or post-viral ARS (296). There has been a shift in the organisms involved in ARS after the Heptavalent conjugate pneumococcal vaccine (PCNV7) was introduced with decreased isolated streptococcus pneumoniae but increased in isolated Haemophilus influenza culture among adults with acute maxillary sinusitis ${ }^{(297)}$. How this will impact occurrences of ABRS is still under speculation ${ }^{(296,298)}$.

\subsubsection{Sodium hyaluronate}

One study evaluated 48 patients with ABRS following EPOS criteria that were treated with high molecular weight sodium hyaluronate (3\%) plus saline solution ( $3 \mathrm{~mL}$ sodium chloride$\mathrm{NaCl}-0.9 \%$ ) versus placebo using a nebulizer ampoule for nasal douching twice a day ${ }^{(299)}$. All patients received levofloxacin (500mg for 10 days) and prednisone (50mg for eight days, $25 \mathrm{mg}$ for four days and $12,5 \mathrm{mg}$ for four days). The hyaluronate group compared to placebo had significantly fewer symptoms and better smell threshold (Table 4.6.15.).

In conclusion, sodium hyaluronate may have an additive effect to antibiotics in patients with ABRS. 
Table 4.6.14. Herbal compounds versus corticosteroids in acute post-viral rhinosinusitis.

\begin{tabular}{|c|c|c|c|c|c|}
\hline Study & Methods & Participants & Interventions & Outcomes & Results \\
\hline $\begin{array}{l}\text { Passali } \\
2015^{(293)}\end{array}$ & $\begin{array}{l}\text { Randomi- } \\
\text { zed, pros- } \\
\text { pective, } \\
\text { open-label } \\
\text { study }\end{array}$ & $\begin{array}{l}\text { Adults with acute } \\
\text { (post)viral rhinosinu- } \\
\text { sitis }(n=60)\end{array}$ & $\begin{array}{l}\text { - Sinupret Forte } 1 \text { tablet } \\
3 \text { times a day for } 14 \text { days } \\
(\mathrm{n}=30) \\
\text { - FFNS } 55 \mu \mathrm{g} \text { in each nos- } \\
\text { tril, once a day for } 14 \text { days } \\
(\mathrm{n}=30)\end{array}$ & $\begin{array}{l}\text { - MSS according to the investiga- } \\
\text { tor at day } 3 \text {, day } 7,10 \text {, and } 14 \\
\text { - SNOT- } 20 \text { score at day } 3 \text {, day } 7 \text {, } \\
10 \text {, and } 14 \\
\text { - Concomitant medication for the } \\
\text { treatment of acute rhinosinusitis } \\
\text { - Premature termination due } \\
\text { to antibiotic therapy for acute } \\
\text { rhinosinusitis } \\
\text { - Percentage of patients with MSS } \\
\leq 1 \text { at day } 14 \\
\text { - Percentage of patients with MSS } \\
>1 \text { at day } 14 \\
\text { - Safety assessment }\end{array}$ & $\begin{array}{l}\text { - No statistical analyses } \\
\text { performed. } \\
\text { - Comparable mean major } \\
\text { symptom score, SNOT-20 } \\
\text { score, and percentage of } \\
\text { patients at } 14 \text { days. } \\
\text { - No adverse events were } \\
\text { reported in the Sinupret } \\
\text { group. } \\
\text { - Three patients in the FFNS } \\
\text { treatment group reported } \\
\text { minor adverse events (epis- } \\
\text { taxis and nasal itching). }\end{array}$ \\
\hline
\end{tabular}

FFNS, fluticasone fuorate nasal spray; MSS, major symptom scores; SNOT-20, Sino-nasal Outcome Test-20.

Table 4.6.15. Sodium hyaluronate versus placebo in ABRS.

\begin{tabular}{|c|c|c|c|c|c|}
\hline Study & Methods & Participants & Interventions & Outcomes & Results \\
\hline $\begin{array}{l}\text { Ciofalo } \\
2017^{(299)}\end{array}$ & DBPCT & $\begin{array}{l}\text { Adults with ABRS (EPOS } \\
\text { criteria, details not } \\
\text { given) }(n=48)\end{array}$ & $\begin{array}{l}\text { - Sodium hyaluronate ( } 3 \%) \\
\text { plus saline solution }(3 \mathrm{~mL} \\
\text { sodium chloride- } \mathrm{NaCl}-0.9 \\
\% \text { ) twice daily ( } \mathrm{n}=24) \\
\text { - Placebo using a nebulizer } \\
\text { ampoule for nasal douche } \\
\text { twice a day ( } \mathrm{n}=24) \\
\text { All patients received } \\
\text { levofloxacin ( } 500 \mathrm{mg} \text { for } \\
10 \text { days) and prednisone } \\
\text { ( } 50 \text { mg for } 8 \text { days, } 25 \mathrm{mg} \\
\text { for } 4 \text { days and } 12,5 \mathrm{mg} \text { for } \\
4 \text { days) }\end{array}$ & $\begin{array}{l}\text { At } 14-18 \text { days and } 30-35 \\
\text { days: } \\
\text { - Symptoms (0-3) } \\
\text { - Smell (0-3) } \\
\text { - Smell test (threshold, dis- } \\
\text { crimination, identification) } \\
\text { Mucociliary clearance time }\end{array}$ & $\begin{array}{l}\text { The hyaluronate group compared } \\
\text { to placebo had: } \\
\text { - Significantly less nasal obstruc- } \\
\text { tion at } 14 \text { days } \\
\text { - Significantly less nasal discharge } \\
\text { at both time points } \\
\text { - Better smell (0-2) at } 14 \text { days but } \\
\text { not at } 30 \text { days } \\
\text { - Significantly better median } \\
\text { threshold smell score at both time- } \\
\text { points but no other differences in } \\
\text { smell testing } \\
\text { - Significantly better mucociliary } \\
\text { clearance time at both timepoints }\end{array}$ \\
\hline
\end{tabular}

ABRS, acute bacterial rhinosinusitis; DBPCT, double blind placebo controlled trial.

Table 4.6.16. Mucolytics versus placebo in ABRS.

\begin{tabular}{|c|c|c|c|c|c|}
\hline Study & Methods & Participants & Interventions & Outcomes & Results \\
\hline $\begin{array}{l}\text { Unuvar } \\
2010^{(300)}\end{array}$ & DBPCT & $\begin{array}{l}\text { Children }(8.5 \pm 3.2 y)(81 \\
\text { evaluated) with ABRS } \\
(n=92)\end{array}$ & $\begin{array}{l}\text { - Erdosteine }(5-8 \mathrm{mg} / \mathrm{kg} / \\
\text { day) }(\mathrm{n}=49) \\
\text { - Placebo }(\mathrm{n}=43) \\
\text { All patients received antibi- } \\
\text { otics, dosage and duration } \\
\text { unclear }\end{array}$ & $\begin{array}{l}\text { - Cure at day } 14 \\
\text { - Daily general impression } \\
\text { for } 14 \text { days } \\
\text { - Daily symptoms for } 14 \\
\text { days }\end{array}$ & $\begin{array}{l}\text { - No difference between } \\
\text { the treatment groups }\end{array}$ \\
\hline
\end{tabular}

ABRS, acute bacterial rhinosinusitis; DBPCT, double blind placebo controlled trial.

\subsubsection{Mucolytics}

To evaluate whether mucolytic agents have an adjuvant role with antibiotics in the treatment of children with ABRS, Unuvar et al. evaluated the effectiveness of erdosteine ( $5-8 \mathrm{mg} / \mathrm{kg} /$ day) versus placebo as an adjunct to antibiotic in 92 children (age 8.5 \pm 3.2 years) with $\mathrm{ABRS}^{(300)}$. They found no significant difference between the groups (Table 4.6.16.).

\subsection{Complications of acute bacterial rhinosinusitis (ABRS)}

The complications of ABRS refer to periorbital, endocranial and osseous clinical conditions which are uncommon potentially life-threatening events. The periorbital complications include preseptal cellulitis, orbital cellulitis, subperiosteal and intraorbital abscess; rapid diagnosis and treatment (including 
intravenous antibiotics and / or surgical drainage) is imperative to avoid long-term morbidity and mortality. The endocranial complications include epidural empyema, subdural empyema, brain abscess, meningitis, encephalitis, superior sagittal and cavernous sinus thrombosis. These may present with nonspecific signs and symptoms and diagnosis requires high clinical suspicion from practitioners, especially in children. Osseous complications result from osteomyelitis and may present as a subperiosteal frontal bone abscess (Potts Puffy tumour) or a frontocutaneous fistula. The quantity and quality of high-quality studies is restricted, in part due to the frequency and emergency nature of many of the problems.

\section{There are still cases in which acute bacterial sinus infection is associated with complications which can be due to sporadic, untreated or treated microbial infections.}

\subsubsection{Introduction}

Despite the advent and broad use of antibiotic therapy during the last six decades, complications of rhinosinusitis still cause substantial morbidity and occasionally mortality. Due to contemporary diagnostic modalities [mainly CT and Magnetic Resonance Imaging (MRI)] and improved surgical techniques (mainly endoscopic sinus surgery) incidence and related morbidity and mortality from complications of bacterial rhinosinusitis have dramatically decreased. However, there are still cases in which acute bacterial sinus infection is associated with complications which can be due to sporadic ${ }^{(12)}$, untreated or treated microbial infections s01-303) $^{(30}$ they could be overlooked due to lack of availability of contemporary diagnostic and therapeutic modalities in certain populations ${ }^{(304,305)}$. Other cases include those patients in whom prescription of oral antimicrobial agents is contraindicated such as pregnant women ${ }^{(306-308)}$. Complications of ABRS are typically classified as orbital (approximately 60-80\%), intracranial (approximately 15-20\%) and rarely osseous (approximately $5 \%)^{(302,309-317)}$ (Table 4.7.1.) though occasionally some unusual complications can develop (see below and Table 4.7.4.) (302, 303, 318, 319). Recently a cohort study even associated acute bacterial rhinosinusitis with an increased risk for stroke ${ }^{(318)}$. Although a cohort design is adequate to document causality, more studies are necessary to classify stroke as a complication of chronic or untreated rhinosinusitis ${ }^{(318)}$.

\subsubsection{Epidemiology of complications}

Evidence with regard to the incidence and prevalence of the complications of rhinosinusitis is patchy and there is no consensus on the exact prevalence of the different types of complications. Furthermore, the causal link between the microbial sinus infection and the reported complications is rarely if ever reported in the literature. The incidence of ABRS complications has been shown to be approximately three per million of the population per year (see Table 4.7.1.) despite very different utilization of antibiotics in the various countries and this number has not been reduced by the advent of widespread antibiotic prescribing. In patients who are hospitalized with ABRS, the reported rate of complications varies from approximately $3 \%$ to $20 \%{ }^{(12,}$ 320,321), although there is selection bias and thus existing series likely overestimate the incidence of those complications. The highest number of admissions occurs from January to March ${ }^{(173,}$ 310). Seven studies (until 2019), attempting to collect nationwide or large-scale data were identified and the incidence results are briefly summarized below (Table 4.7.1.). In the majority of these studies, males are significantly more frequently affected than females and ABRS was more often the precipitating factor in children, whereas CRS with or without NP was more important in adults ${ }^{(173,301,322,323)}$. In all studies, the orbital complications are the most frequent while osseous appear to be relatively uncommon (Table 4.7.1.).

\section{The incidence of ABRS complications has been shown to be approximately three per million of the population per year despite very different utilization of antibiotics in the various countries and this number has not been reduced by the advent of widespread antibiotic prescribing.}

With regard to age predeliction, orbital complications seem to be more common in small children while intracranial complications can occur at any age, with a preponderance of young adults around their 20th birthday ${ }^{(301,324)}$. The course of ABRS complications has been reported to become more prolonged in parallel with the age of the patients ${ }^{(311,323)}$.

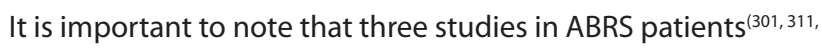
${ }^{322}$ did not record any benefit for history of oral antimicrobial drug usage prior to the development of the complications. In this regard the study by Babar-Craig ${ }^{(12)}$, which was based on returned questionnaires by members of the British Rhinology Society also showed that prescribing of antimicrobials for ABRS does not prevent the occurrence of complications. These facts, together with the risk of antibiotic resistance and possible masking of intracranial complications argue strongly against the routine use of antibiotics in $\mathrm{ABRS}^{(302)}$. These studies also emphasise the need to be constantly vigilant for complications irrespective of whether antibiotics are being taken or not and complications may occur before the patient is seen in primary care.

\section{Taking oral antibiotics did not provide any advantage in the prevention of complications.}


Table 4.7.1. Epidemiological data of complications in ABRS.

\begin{tabular}{|c|c|c|c|c|c|c|c|c|c|}
\hline $\begin{array}{l}\text { Author, } \\
\text { year, ref. }\end{array}$ & Country & Age & Disease & Patients & $\begin{array}{l}\text { (Incidence per } \\
\text { million population } \\
\text { per year) }\end{array}$ & Orbital & $\begin{array}{l}\text { Intracra- } \\
\text { nial }\end{array}$ & Bone & $\begin{array}{l}\text { Soft } \\
\text { tissue }\end{array}$ \\
\hline $\begin{array}{l}\text { Dennison } \\
2019^{(328)}\end{array}$ & Sweden & Children & ABRS & 29 & & $100 \%$ & & & \\
\hline $\begin{array}{l}\text { El Mograbi } \\
2019^{(322)}\end{array}$ & Israel & Adults & $\begin{array}{l}\text { ABRS + } \\
\text { CRS }\end{array}$ & 70 & & $\begin{array}{l}100 \% \\
(70)\end{array}$ & $\begin{array}{l}2.8 \% \\
\text { concur- } \\
\text { rent (2) }\end{array}$ & & \\
\hline $\begin{array}{l}\text { Hamill } \\
2018^{(364)}\end{array}$ & USA & Children & ABRS & 250 & & $\begin{array}{l}61.6 \% \\
(154)\end{array}$ & $\begin{array}{c}11.6 \% \\
(29)\end{array}$ & & \\
\hline $\begin{array}{l}\text { Scholin Ask } \\
2017^{(323)}\end{array}$ & Sweden & Children & $\begin{array}{l}\text { ABRS + } \\
\text { CRS }\end{array}$ & 213 & $\begin{array}{l}3.6 \text { (orbital only) } \\
\text { Hospitalization boys } \\
5.3 \text {, girls } 3.6\end{array}$ & $\begin{array}{l}80 \% \\
(171)\end{array}$ & & & \\
\hline $\begin{array}{l}\text { Nicoli } \\
2016^{(383)}\end{array}$ & Finland & $\begin{array}{l}\text { Adults / } \\
\text { children }\end{array}$ & ABRS & & $\begin{array}{l}3.2 \text { (intracranial } \\
\text { only) }\end{array}$ & & 6 & & \\
\hline $\begin{array}{l}\text { Chaiyasate } \\
2015^{(312)}\end{array}$ & China & $\begin{array}{l}\text { Adults / } \\
\text { children }\end{array}$ & ABRS & 1655 & $\begin{array}{l}85 \text { patients with } \\
\text { complications }\end{array}$ & 41 & 24 & 2 & 3 \\
\hline $\begin{array}{l}\text { Capra } \\
2015^{(320)}\end{array}$ & USA & Children & ABRS & & $\begin{array}{l}0.738 \text { children in } \\
2000 \text { and } 0.605 \text { in } \\
2009\end{array}$ & & & & \\
\hline $\begin{array}{l}\text { Miah } \\
2015^{(314)}\end{array}$ & UK & $\begin{array}{l}\text { Adults / } \\
\text { children }\end{array}$ & ABRS & $31 / 248$ & & 21 & 9 & & \\
\hline $\begin{array}{l}\text { Sedaghat } \\
2014^{(317)}\end{array}$ & USA & Children & ABRS & 696 & & $90.2 \%$ & $9.8 \%$ & & \\
\hline $\begin{array}{l}\text { Hansen } \\
2012^{(301)}\end{array}$ & $\begin{array}{l}\text { Netherlands (Natio- } \\
\text { nal Database 2004) }\end{array}$ & $\begin{array}{l}\text { Adults / } \\
\text { children }\end{array}$ & ABRS & 48 & $(48 / 16.3$ million=3 ) & $\begin{array}{l}67 \% \\
(32)\end{array}$ & $\begin{array}{l}33 \% \\
(16)\end{array}$ & & \\
\hline $\begin{array}{l}\text { Piatt } \\
2011^{(384)}\end{array}$ & $\begin{array}{l}\text { USA - National in- } \\
\text { patient database } \\
(1997,2000,2001, \\
2003,2006)\end{array}$ & Children & ABRS & 695 & $2.7-4.3$ & & & & \\
\hline $\begin{array}{l}\text { Babar-Craig } \\
2010^{(12)}\end{array}$ & $\begin{array}{l}\text { UK - national } \\
\text { ques-tionnaire }\end{array}$ & $\begin{array}{l}\text { Adults / } \\
\text { children }\end{array}$ & ABRS & 78 & $\mathrm{~N} / \mathrm{A}$ & $76 \%$ & $9 \%$ & $5 \%$ & \\
\hline Stoll $2006^{(14)}$ & France (2001-2003) & $\begin{array}{l}\text { Adults and } \\
\text { adolescents }\end{array}$ & ABRS & 43 & $(30 / 12$ million= 2.5$)$ & $\begin{array}{l}35 \% \\
(15)\end{array}$ & $\begin{array}{l}37 \% \\
(16)\end{array}$ & & $\begin{array}{l}18 \% \\
(8)\end{array}$ \\
\hline $\begin{array}{l}\text { Oxford } \\
2005^{(316)}\end{array}$ & USA & Children & ABRS/CRS & 104 & $\mathrm{~N} / \mathrm{A}$ & $\begin{array}{l}91 \% \\
(95)\end{array}$ & $\begin{array}{l}16 \% \\
(17)\end{array}$ & $\begin{array}{l}3 \% \\
(3)\end{array}$ & \\
\hline $\begin{array}{l}\text { Eufinger } \\
2001^{(313)}\end{array}$ & Germany & $\begin{array}{l}\text { Adults / } \\
\text { children }\end{array}$ & ABRS & 25 & $\mathrm{~N} / \mathrm{A}$ & $\begin{array}{l}88 \% \\
(22)\end{array}$ & $\begin{array}{c}20 \% \\
(5)\end{array}$ & $\begin{array}{l}2 \text { pat. } \\
\text { (had } \\
\text { both) }\end{array}$ & \\
\hline $\begin{array}{l}\text { Mortimore } \\
1997^{(315)}\end{array}$ & South Africa & $\begin{array}{l}\text { Adults / } \\
\text { children }\end{array}$ & ABRS/CRS & 63 & $\mathrm{~N} / \mathrm{A}$ & $\begin{array}{l}81 \% \\
(51)\end{array}$ & $\begin{array}{l}13 \% \\
(8)\end{array}$ & $\begin{array}{l}10 \% \\
(6)\end{array}$ & $\begin{array}{l}24 \% \\
(15)\end{array}$ \\
\hline
\end{tabular}

ABRS, acute bacterial rhinosinusitis; CRS, chronic rhinosinusitis; N/A, not applicable.

\subsubsection{Orbital complications of ABRS}

\subsubsection{Classification}

Orbital complications, the most commonly related to ABRS, involve (in decreasing frequency) the ethmoid, maxillary, frontal and rarely the sphenoid sinuses (Table 4.7.2.). The infection spreads directly via the thin and often dehiscent lamina papyracea or a pre-existing anatomical abnormality in cases of recurrent periorbital cellulitis or through venous return ${ }^{(302,303,}$ 309). Orbital complications commonly affect children ${ }^{(322,323,325-327)}$ a population which is known to express fewer clinical signs and symptoms so it is mandatory to have a high level of clinical suspicion for the possibility of orbital complications in children with ABRS. Hospital admission due to acute rhinosinusitis in children decreased after the introduction of pneumococcal conjugate vaccine but there was no parallel decrease in the incidence of orbital complications ${ }^{(328,329)}$.

Despite the fact that the Chandler's classification is widely used, it does present some problems. The orbital septum is the anterior limit of the orbit, therefore it has been suggested that pre-septal cellulitis should be classified as an eyelid, rather than an orbital infection ${ }^{(302)}$. In this regard it has been argued that 
pre-septal cellulitis is infrequently associated with rhinosinusitis and its clinical picture, its management and its prognosis differentiate it from all other orbital infections ${ }^{(302)}$. It has been suggested that orbital involvement (more appropriately: post-septal cellulitis) presents with swelling, exophthalmos and impaired, painful extra-ocular eye movements with diplopia. With the exception of swelling, these features are not present in pre-septal cellulitis and differentiate it from true orbital infections ${ }^{(302)}$. Additionally, cavernous sinus thrombosis, as has been suggested by Mortimore in $1997^{(315)}$ is an intracranial complication and not necessarily the end stage of orbital infection, while it is more often associated with sphenoid rather than ethmoid or frontal sinus infection ${ }^{(302)}$.

According to Chandler's classification, orbital complications may be divided into five stages based on their clinical and radiological findings $\mathrm{s}^{(309) \text { : }}$

- Stage 1: pre-septal cellulitis

- Stage 2: orbital cellulitis

- Stage 3: subperiosteal abscess

- $\quad$ Stage 4: orbital abscess

- Stage 5: cavernous sinus thrombosis

Because Chandler's classification remains the most commonly used and because we attempted to summarize the evidence from a number of papers, past and present, we still employ this classification in Table 4.7.2., although it was also debated in EPOS 2012 ${ }^{(302)}$.

Periorbital or orbital cellulitis may result from direct or vascular spread of the sinus infection ${ }^{(330-335)}$. As the spread of sinus infection through the orbit follows a well-described pattern, the initial manifestations are oedema and erythema of the medial aspects of the eyelid. Spread of infection from the maxillary or the frontal sinus results in initial presentation with swelling of the lower or upper eyelid, respectively. Consultation with an ophthalmologist, in some cases twice daily, should always be sought for clinical and medicolegal reasons and objective assessment of proptosis (exophthalmometer), orbital pressure (tonometer), visual acuity, colour vision (an important and early sign of deterioration) and eye movements should always be clearly documented ${ }^{(302,315,319)}$. Snellen charts can be easily downloaded as free-of-charge android applications and are a quick and reproducible way of assessing visual acuity and colour vision by the non-expert.

\subsubsection{Preseptal cellulitis}

Preseptal cellulitis refers to the inflammation of the eyelid and conjunctiva and involves the tissues anterior to the orbital septum, if necessary. It can be identified on the CT scan as soft tissue swelling. The MRIT2 sequence is better in demonstrating the inflammation in the soft tissues ${ }^{(336)}$. It can occur as a complication of a URTI, dacryocystitis or skin infection while rhinosinusitis is an uncommon cause ${ }^{(302,315,326)}$. Pre-septal celIulitis presents with orbital pain, eyelid oedema, erythema and fever. On clinical examination there may be no evident proptosis and no limitation of eye movement. Of note these signs of proptosis and limited eye movement can be difficult to assess in paediatric patients ${ }^{(325,326,337)}$. Most cases of pre-septal cellulitis respond to oral antibiotic therapy but if not timely and appropriately treated, they can spread beyond the orbital septum ${ }^{(334,335,}$ ${ }^{338)}$. In most cases, pre-septal cellulitis is a clinical diagnosis and due to the superficial nature of the inflammation, imaging is not necessary ${ }^{(302,336)}$.

\subsubsection{Orbital cellulitis}

Orbital cellulitis, orbital abscess and subperiosteal abscess are all complications that are more commonly associated with acute rhinosinusitis, in contrast with pre-septal cellulitis which more commonly occurs following upper respiratory tract infections (see above) ${ }^{(302)}$. As the inflammation spreads through the orbit, proptosis (a protruding eyeball) develops together with some limitation of ocular movement (diplopia can present in extreme gaze) indicating orbital cellulitis. Other typical signs of orbital cellulitis are chemosis (conjunctival oedema), ocular pain and tenderness, as well as ophthalmoplegia (restricted and painful ocular movement) of the extraocular muscles ${ }^{(302)}$. This complication requires immediate and aggressive treatment with intravenous antibiotics and should be referred for detailed CT scan with contrast of the sinuses to distinguish between orbital cellulitis, intraorbital or subperiosteal abscess ${ }^{(336)}$. However, there should be close monitoring for progression with a low threshold for surgical intervention as the few who do not respond to antibiotic therapy can progress rapidly over $24-48 \mathrm{~h}$. In cases where a concomitant intracranial complication is also suspected, MRI scan better diagnoses orbital complications ${ }^{(313,338-340)}$. All three complications (orbital cellulitis, subperiosteal and intraorbital abscess) cause eyeball proptosis and limit ocular movement. Evidence of an abscess on the CT scan, progressive orbital findings or visual deterioration after initial intravenous (i.v) antibiotic therapy should be considered as indications for orbital exploration and drainage (see below). Repeated ophthalmologic examinations of visual acuity should take place and i.v. antibiotic therapy may be converted to oral when the patient has been afebrile for 48 hours and the ophthalmological symptoms and signs are resolving $302,337,341,342)$. Indications for surgical intervention in orbital complications of ABRS:

- Evidence of subperiosteal or intraorbital abscess on CT or MRI (with potential exception for small volume abscesses).

- Reduced visual acuity/reduced colour vision/affected afferent pupillary reflex or inability to assess vision.

- Progression or no improvement in orbital signs (diplopia, 
Table 4.7.2. Orbital complications of ABRS.

\begin{tabular}{|c|c|c|c|c|c|}
\hline $\begin{array}{l}\text { Author, } \\
\text { year, ref. }\end{array}$ & $\mathbf{N}$ & Age & Disease & $\begin{array}{l}\text { Type of } \\
\text { complications }\end{array}$ & Management \\
\hline $\begin{array}{l}\text { Tachibana } \\
\text { 2019(311) }\end{array}$ & 21 & Adults / children & ABRS/CRS & $\begin{array}{l}\text { Preseptal cellulitis (4) } \\
\text { Postseptal orbital cellulitis (8) } \\
\text { Subperioteal abscess (9) }\end{array}$ & Surgical (23.8\%) \\
\hline $\begin{array}{l}\text { Trivic } \\
2019^{(346)}\end{array}$ & 61 & Children & ABRS & $\begin{array}{l}\text { Preseptal cellulitis (50.0\%) } \\
\text { Orbital cellulitis (50\%) }\end{array}$ & Medical and Surgical \\
\hline $\begin{array}{l}\text { El Mograbi } \\
2019^{(322)}\end{array}$ & 70 & Adults & ABRS/CRS & $\begin{array}{l}\text { Preseptal/orbital cellulitis (61.5\%) } \\
\text { Orbital cellulitis (23\%) } \\
\text { Subperiosteal abscess (11\%) } \\
\text { Orbital abscess (3\%) } \\
\text { Cavernous sinus thrombosis (1.5\%) }\end{array}$ & $\begin{array}{l}\text { Medical only } \\
\text { Medical only } \\
\text { Surgical, ESS (5), ESS \& EXS (2) } \\
\text { Surgical, ESS (1), ESS \& EXS (1) } \\
\text { Surgical, ESS (1) }\end{array}$ \\
\hline $\begin{array}{l}\text { Jabarin } \\
2019^{(344)}\end{array}$ & 123 & Children & ABRS & $\begin{array}{l}\text { Preseptal cellulitis (57\%) } \\
\text { Orbital cellulitis (1.5\%) } \\
\text { Subperiosteal abscess (41.5\%) }\end{array}$ & $\begin{array}{l}\text { Medical only } \\
\text { Medical only } \\
\text { Medical (29), Surgical (24) }\end{array}$ \\
\hline $\begin{array}{l}\text { Gavriel } \\
2018^{(341)}\end{array}$ & 37 & Adults & ABRS & $\begin{array}{l}\text { Preseptal cellulitis (49\%) } \\
\text { Subperiosteal abscess (51\%) }\end{array}$ & $\begin{array}{l}\text { Medical only } \\
\text { Medical (12), Surgical (7) }\end{array}$ \\
\hline $\begin{array}{l}\text { Scholin Ask } \\
2017^{(323)}\end{array}$ & 203 & Preschool children & ABRS & $\begin{array}{l}\text { Preseptal cellulitis (96.5\%) } \\
\text { Orbital cellulitis/Subperiostal abscess (2.0\%) } \\
\text { Orbital abscess (1,5\%) }\end{array}$ & $\begin{array}{l}\text { Medical only } \\
\text { Medical only } \\
\text { Medical (2), Surgical (1) }\end{array}$ \\
\hline $\begin{array}{l}\text { Chang } \\
2017^{(334)}\end{array}$ & 71 & Adults / children & $\mathrm{ABRS} / \mathrm{CRS}$ & $\begin{array}{l}\text { Preseptal cellulitis (47\%) } \\
\text { Orbital cellulitis (9.6\%) } \\
\text { Subperiosteal abscess (19.3) } \\
\text { Orbital abscess (9.6\%) }\end{array}$ & $\begin{array}{l}\text { Surgical (13) } \\
\text { Surgical (3) } \\
\text { Surgical (13) } \\
\text { Surgical (6) }\end{array}$ \\
\hline $\begin{array}{l}\mathrm{Li} \\
2017^{(385)}\end{array}$ & 28 & Children & & $\begin{array}{l}\text { Preseptal cellulitis (1) } \\
\text { Orbital cellulitis (9) } \\
\text { Subperiosteal orbital abscess (13) } \\
\text { Orbital abscess (5) }\end{array}$ & $\begin{array}{l}\text { Medical only (8) } \\
\text { Medical and Surgical (20) }\end{array}$ \\
\hline $\begin{array}{l}\text { Wan } \\
2016^{(325)}\end{array}$ & 31 & Children & ABRS & $\begin{array}{l}\text { Preseptal cellulitis (4) } \\
\text { Orbital cellulitis (14) } \\
\text { Subperiosteal abscess (13) }\end{array}$ & $\begin{array}{l}\text { Medical (16) } \\
\text { Surgical (15) }\end{array}$ \\
\hline $\begin{array}{l}\text { Miah } \\
2015^{(314)}\end{array}$ & 31 & Adults / children & ABRS & $\begin{array}{l}\text { Periorbital cellulitis (16) } \\
\text { Orbital abscess (6) } \\
\text { Intracranial abscess (9) }\end{array}$ & \\
\hline $\begin{array}{l}\text { Radovani } \\
2013^{(335)}\end{array}$ & 35 & Adults / children & ABRS & $\begin{array}{l}\text { Preseptal cellulitis (15) } \\
\text { Orbital cellulitis (10) } \\
\text { Subperiosteal abscess (6) } \\
\text { Orbital abscess (3) } \\
\text { Cavernous sinus thrombosis (1) }\end{array}$ & $\begin{array}{l}\text { Medical (12) } \\
\text { Medical and surgical (3) } \\
\text { Surgical } \\
\text { Surgical } \\
\text { Surgical } \\
\text { Surgical }\end{array}$ \\
\hline $\begin{array}{l}\text { Al-Madani } \\
2013^{(333)}\end{array}$ & 35 & Adults / children & ABRS & $\begin{array}{l}\text { Preseptal cellulitis (26) } \\
\text { Orbital cellulitis (8) } \\
\text { Orbital abscess (2) }\end{array}$ & $\begin{array}{l}\text { All medical } \\
\text { Surgical (1) }\end{array}$ \\
\hline $\begin{array}{l}\text { Huang } \\
2011^{(343)}\end{array}$ & 64 & Children & ABRS & $\begin{array}{l}\text { Subperiosteal/intraorbital abscess } 56 \% \text { (36) } \\
\text { Preseptal/orbital cellulitis } 44 \% \text { (28) }\end{array}$ & $\begin{array}{l}\text { Medical only: } 53 \%(34) \\
\text { Medical and surgical: } 47 \%(30)\end{array}$ \\
\hline $\begin{array}{l}\text { Georgakopoulos } \\
2010^{(326)}\end{array}$ & 83 & Children & ABRS/CRS & $\begin{array}{l}\text { Preseptal cellulitis 83\% (69) } \\
\text { Orbital cellulitis 12\% (10) } \\
\text { Subperiosteal abscess 5\% (4) }\end{array}$ & $\begin{array}{l}\text { Medical only: } 95 \% \text { (79) } \\
\text { Surgical and medical: } 5 \%(4)\end{array}$ \\
\hline $\begin{array}{l}\text { Siedek } \\
2008^{(345)}\end{array}$ & 127 & Adults / children & ABRS/CRS & $\begin{array}{l}\text { Preseptal cellulitis } 36 \%(46) \\
\text { Orbital cellulitis } 44 \%(56) \\
\text { Subperiosteal abscess } 6 \%(8) \\
\text { Intraorbital abscess } 14 \%(17)\end{array}$ & $\begin{array}{l}\text { Medical only: } 51 \%(65) \\
\text { Surgical: } 49 \%(62)\end{array}$ \\
\hline $\begin{array}{l}\text { Eviatar } \\
2008^{(338)}\end{array}$ & 52 & Children & ABRS & $\begin{array}{l}\text { Preseptal cellulitis } 92 \%(48) \\
\text { Subperiosteal abscess } 8 \%(4)\end{array}$ & $\begin{array}{l}\text { Medical only: } 98 \%(51) \\
\text { Surgical: } 2 \%(1)\end{array}$ \\
\hline $\begin{array}{l}\text { Mekhitarian } \\
2007^{(327)}\end{array}$ & 25 & Children & ABRS & $\begin{array}{l}\text { Preseptal cellulitis 96\% (24) } \\
\text { Subperiosteal abscess } 4 \%(1)\end{array}$ & $\begin{array}{l}\text { Medical only: } 92 \%(23) \\
\text { Surgical: } 8 \%(2)\end{array}$ \\
\hline $\begin{array}{l}\text { Oxford } \\
2006^{(348)}\end{array}$ & 43 & Children & ABRS & Subperiosteal abscess $100 \%(43)$ & Medical only: $42 \%$ (18) \\
\hline
\end{tabular}


Table 4.7.2. Orbital complications of ABRS continued

\begin{tabular}{|c|c|c|c|c|c|}
\hline $\begin{array}{l}\text { Author, } \\
\text { year, ref. }\end{array}$ & $\mathbf{N}$ & Age & Disease & $\begin{array}{l}\text { Type of } \\
\text { complications }\end{array}$ & Management \\
\hline $\begin{array}{l}\text { Mortimore } \\
1997^{(315)}\end{array}$ & 51 & Adults / children & ABRS & $\begin{array}{l}\text { Preseptal cellulitis 55\% (28) } \\
\text { Orbital cellulitis 10\% (5) } \\
\text { Subperiosteal abscess 33\% (17) } \\
\text { Intraorbital abscess } 2 \%(1)\end{array}$ & Not stated \\
\hline
\end{tabular}

ABRS, acute bacterial rhinosinusitis; CRS, chronic rhinosinusitis; ESS, endoscopic sinus surgery; EXS external sinus surgery.

ophthalmoplegia, proptosis, swelling, chemosis) after 48 hours intravenous antibiotics.

- Progression or no improvement in the general condition (fever, infection parameters) after 48 hours of intravenous antibiotics.

\subsubsection{Subperiosteal and orbital abscess}

A subperiosteal abscess forms between the periorbita and the sinuses and is located outside the ocular muscles (or extraconal). The clinical features of a subperiosteal abscess are oedema, erythema, chemosis and proptosis of the eyelid with limitation of ocular motility and as a consequence of extra-ocular muscle paralysis, the eyeball becomes fixed (ophthalmoplegia) and visual acuity diminishes. In most case series, high fever and raised leucocyte count as well as left shift (an increase in the number of immature leucocytes in the peripheral blood, particularly neutrophil band cells) have been reported as strongly associated

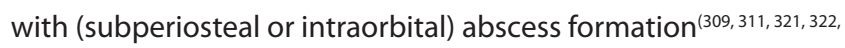
325, 326, 333-335, 339, 341, 343-346).

An orbital abscess is contained within the space defined by the ocular muscles and the eyeball ie intraconal and generally results from diagnostic delay, inappropriate antimicrobial treatment or immunosuppression of the patient ${ }^{(335,341,347)}$ with a frequency of between $1.5 \%$ and $14 \%$ (Table 4.7.2.) in paediatric studies of orbital complications. The clinical or radiological evidence of an abscess or the lack of clinical improvement after 24-48 hours of i.v. antibiotics are indications for prompt surgical exploration and drainage, preferably via endoscopic approaches.

As previously noted, a detailed CT scan of the sinuses with contrast and possibly with $3 \mathrm{D}$ reconstruction may help to distinguish between cellulitis and orbital or subperiosteal abscess. In the case of a subperiosteal abscess, the CT usually reveals oedema of the medial rectus muscle, lateralization of the periorbita and lateral downward displacement of the eyeball ${ }^{(336,}$ 337,342 ). In cases where the CT scan shows obliteration of the detail of the extraocular musculature and of the optic nerve by a confluent mass, the orbital cellulitis has progressed to an intraorbital abscess, in which case there may sometimes be air in the orbit due to the presence of anaerobic bacteria. The diagnostic accuracy of clinical examination alone to determine an orbital abscess has been reported to be approximately $80 \%$ which rises to approximately $90 \%$ with the addition of CT imaging. MRI may be useful in cases of diagnostic uncertainty after a CT scan or when intracranial complications are suspected ${ }^{(348,349)}$.

Subperiosteal abscess in children is not an absolute indication for immediate surgical intervention. Conservative measures can be safe and effective if appropriately used, depending on patient characteristics, exam findings, clinical course, and imaging ${ }^{(350)}$. An ophthalmologist should check visual acuity from the early stages of the illness. Intravenous antimicrobial therapy should cover aerobic and anaerobic pathogens. Evidence of an abscess on the CT scan or absence of clinical improvement after 24-48 hours of i.v. antibiotics are indications for orbital exploration and drainage ${ }^{(349)}$. Depending on the radiographic volume of the abscess, the decision to surgically explore may be prioritized if the volume appears to be large. In contrast, in small abscesses the most prudent decision would be to continue i.v. antibiotics and monitor the patient ${ }^{(351)}$. A current guideline mandates that pre-septal and orbital cellulitis should be treated with antibiotics while subperiosteal and intraorbital abscesses require surgical exploration which should include not just the drainage of the abscess but also of the paranasal sinuses ${ }^{(302)}$ as cellulitis can be expected to improve with proper antimicrobial therapy while drainage is the mainstay of treatment for any abscess. The guideline recommends an endoscopic approach to open the lamina papyracea and draining the abscess after complete ethmoidectomy. External approaches to lateral and medial orbital abscesses (lateral and medial orbitotomy) can also be used if necessary. However, there have been a number of recent studies showing good outcomes with i.v. antibiotics in children with subperiosteal abscesses ${ }^{(302,345,347,349)}$. In such cases, and provided there is ${ }^{(302)}$ : clear clinical improvement within 24-48 hours; no decrease in visual acuity; small (<0.5-1 ml in volume); medially located subperiosteal abscess; no significant systemic involvement; the decision might be to withhold surgical drainage and closely monitor the patient ${ }^{(341,342)}$. Importantly, it has recently been reported that the same conservative approach is also valid for adult patients with orbital abscesses. Gavriel et al. reported a similar prognosis between conservative medical and 
surgical approach in the treatment of orbital abscesses for adult patients ${ }^{(341)}$. Therefore, based on this evidence, adult patients may also be candidates for watchful waiting instead of endoscopic drainage. Blindness may result from central retinal artery occlusion, optic neuritis, corneal ulceration, or panophthalmitis. It is not infrequent for sepsis to spread intracranially as well as anteriorly into the orbit ${ }^{(302,303,319)}$. Patients with delayed admission and additional risk factors have a higher risk of irreversible blindness ${ }^{(352)}$.

\section{Subperiosteal abscess in children is not an absolute indication for immediate surgical intervention. Conservative measures can be safe and effective if appropriately used, depending on patient characteristics, exam findings, clinical course, and imaging.}

\subsubsection{Endocranial complications}

Endocranial (or intracranial) complications of ABRS are epidural or subdural empyema, brain abscess, meningitis, cerebritis and superior sagittal and cavernous sinus thrombosis (Table 4.7.3.). These complications may present with non-specific symptoms and signs of a systematic inflammatory response (high fever, headache, lethargy, reduced consciousness) or with specific central nervous system signs due to focal neurologic damage or increased intracranial pressure. Of note, it is not uncommon for any of these complications to either present with non- specific symptoms including high fever accompanied by headache, or even to be silent ${ }^{(315)}$. Although an intracranial abscess can be relatively asymptomatic, subtle affective and behavioural changes are not uncommon; these are signs of altered neurologic function, orientation and cognitive state. Gait instability and severe, progressive headache have also been reported to be common symptoms ${ }^{(302)}$. The majority of the endocranial complications of ABRS however, present with more specific signs and symptoms that suggest the intracranial inflammatory involvement, such as nausea and vomiting, neck stiffness and altered mental state ${ }^{(301,}$ $312,324,347,353-358$. The clinical expression of subdural and epidural empyema is different. Subdural empyema often presents with neurologic symptoms as an emergency case. In contrast epidural empyema is more often diagnosed on imaging studies ${ }^{(359)}$. Intracranial abscesses often become apparent through signs of increased intracranial pressure, meningeal irritation and focal neurologic deficit mainly of the third, sixth or seventh cranial nerves ${ }^{(301,305,316,330,347,349,360,361)}$.

Endocranial complications are usually associated with frontoethmoidal or sphenoid rhinosinusitis ${ }^{(312,324,354,355,357,361,362)}$. Infections can proceed from the paranasal cavities to the endocranial structures by two different routes: (a) haematologically - pathogens can pass through the diploic veins to reach the brain; (b) tissue continuity spread - pathogens can reach the intracranial structures by eroding the thin osseous walls of the sinuses ${ }^{(354-356,}$ 361). Inflammatory complications of the brain start as inflammation (ie encephalitis), which progresses to necrosis and liquefaction of brain tissue, with a reactive connective tissue capsule forming around the brain abscess.

A CT scan with contrast, as a minimum, is required for diagnosis as it allows for precise definition of osseous tissue involvement. MRI is considered to be the "gold-standard" as it is more sensitive than $\mathrm{CT}$ and should be the imaging modality of choice where available; further it has additional diagnostic value to exclude or confirm cavernous sinus thrombosis and also in cases with soft tissue involvement ${ }^{(336,357)}$. Studies show a high incidence of anaerobic organisms or mixed aerobic-anaerobic in patients with endocranial complications. In cases where meningitis is suspected and provided that any intracranial abscess has been definitely excluded by imaging, a lumbar puncture can be performed to properly determine the underlying pathogens and to customize antimicrobial therapy ${ }^{(357)}$. Pathogens most commonly isolated following lumbar puncture are Streptococcus and Staphylococcus species including methicillin-resistant (MRSA) and anaerobes ${ }^{(363-365)}$. High dose long-term i.v. antimicrobial therapy followed by neurosurgical burr hole drainage, craniotomy for evacuation of the abscess or image guided aspiration, are usually required for successful treatment ${ }^{312,349,354 \text {, }}$ 359, 361). Current treatment involves joint neurosurgical drainage procedures and drainage of the paranasal sinuses (most often the frontal sinus) can be performed endoscopically ${ }^{(359,362,366)}$. The rationale is to evacuate the intracranial collection and manage the source of infection using a rhinological approach which will provide microbiological samples. Lack of early sinus drainage has been associated with the need for repeated craniotomies ${ }^{(357,}$ ${ }^{366)}$. The prognosis of intracranial complications of acute bacterial rhinosinusitis depends on the severity of neurological signs and on the delay in diagnosis and management ${ }^{(354,366)}$. The mortality rate varies from $0 \%$ up to $19 \%{ }^{(312,361,362,367)}$, (Table 4.7.3.), and is related to cortical veins thrombosis and to cerebral vascular infarction ${ }^{(357,366)}$.

\subsubsection{Cavernous sinus thrombosis}

Cavernous sinus thrombosis is a rare complication and has been estimated at a rate of less than $10 \%$ of all the intracranial complications (Table 4.7.3.) ${ }^{(353,356)}$. The highly anastomotic venous system of the paranasal sinuses allows retrograde spread of infection to the cavernous sinus causing sepsis and multiple cranial nerve involvement ${ }^{(316,330,353,356,362,368)}$. Proptosis, ptosis, diplopia, chemosis, involvement of the eye motor nerves and impairment in the ophthalmic and maxillary branches of cranial nerve $\mathrm{V}(1)$ (ophthalmic nerve neuralgia) papilloedema and signs of meningeal irritation associated with spiking fevers and prostration establish the diagnosis ${ }^{(301,356,361)}$. Symptoms start in one eye and progress to the other. 
Table 4.7.3. Endocranial complications in ABRS.

\begin{tabular}{|c|c|c|c|c|}
\hline $\begin{array}{l}\text { Author, } \\
\text { year, ref. }\end{array}$ & $\mathbf{N}$ & Complications & $\begin{array}{l}\text { Management (all had } \\
\text { medical therapy) }\end{array}$ & Mortality / further defects \\
\hline $\begin{array}{l}\text { Mulvey } \\
2019^{(367)}\end{array}$ & 43 & $\begin{array}{l}\text { Meningitis (10) } \\
\text { Epidural empyema (28) } \\
\text { Subdural empyema (19) } \\
\text { Intracerebral abscess (2) }\end{array}$ & $\begin{array}{l}\text { ESS (43) } \\
\text { NS (23) }\end{array}$ & $\begin{array}{l}\text { No mortality. } 14 \text { patients ( } 16 \% \text { ) } \\
\text { were readmitted for worsening } \\
\text { symptoms or complications of } \\
\text { treatments. }\end{array}$ \\
\hline $\begin{array}{l}\text { Schupper } \\
2018^{(361)}\end{array}$ & 16 & $\begin{array}{l}\text { Epidural abscess (10) } \\
\text { Subdural abscess (9) } \\
\text { Intracerebral abscess (3) } \\
\text { Multiple abscesses in } 6 \text { cases } \\
\text { Cavernous sinus thrombosis (2) }\end{array}$ & $\begin{array}{l}\text { Simultaneous ESS and NS as first } \\
\text { procedure (16) }\end{array}$ & No mortality \\
\hline $\begin{array}{l}\text { Kou } \\
2018^{(362)}\end{array}$ & 22 & $\begin{array}{l}\text { Subdural empyema (10) } \\
\text { Epidural abscess (10) } \\
\text { Meningitis (5) } \\
\text { Cerebral abscess (5) } \\
\text { Cavernous sinus thrombosis (2) }\end{array}$ & $\begin{array}{l}\text { ESS (19) } \\
\text { NS (8) } \\
\text { Simultaneous ESS and NS (6) }\end{array}$ & $\begin{array}{l}\text { No mortality } \\
\text { Neurologic deficits } 4.5 \% \text { (short } \\
\text { follow up average: } 7 \text { months) }\end{array}$ \\
\hline $\begin{array}{l}\text { Patel } \\
2015^{(358)}\end{array}$ & 27 & $\begin{array}{l}\text { Epidural empyema (14) } \\
\text { Subdural empyema (9) } \\
\text { Cerebral abscess (4) }\end{array}$ & $\begin{array}{l}\text { ESS (11) } \\
\text { ESS and NS (18) } \\
\text { Only NS (3) }\end{array}$ & $\begin{array}{l}\text { No mortality } \\
\text { Neurologic deficits cognitive/per- } \\
\text { sonality changes } 33 \% \text { ( } 6 \text { months } \\
\text { follow up) }\end{array}$ \\
\hline $\begin{array}{l}\text { Garin } \\
2015^{(359)}\end{array}$ & 17 & $\begin{array}{l}\text { Subdural empyema (9) } \\
\text { Epidural empyema (8) }\end{array}$ & $\begin{array}{l}\text { ESS and NS (15) } \\
\text { Only ESS (2) }\end{array}$ & $\begin{array}{l}\text { No mortality } \\
\text { Cognitive and schooling problems: } \\
\text { subdural empyema } 67 \% \text {, epidural } \\
\text { empyema } 29 \%\end{array}$ \\
\hline $\begin{array}{l}\text { Khamassi } \\
2015^{(366)}\end{array}$ & 23 & $\begin{array}{l}\text { Subdural empyema (11) } \\
\text { Epidural empyema (7) } \\
\text { Intracerebral abscess (5) } \\
\text { Cerebral thrombophlebitis (4) }\end{array}$ & $\begin{array}{l}\text { Medical therapy only (3) } \\
\text { NS (19) } \\
\text { ESS (1) }\end{array}$ & $\begin{array}{l}\text { Mortality } 8.7 \% \\
\text { Morbidity } 34.7 \%\end{array}$ \\
\hline $\begin{array}{l}\text { Chaiyasate } \\
2015^{(312)}\end{array}$ & 24 & $\begin{array}{l}\text { Meningitis (13) } \\
\text { Brain abscess (5) } \\
\text { Cavernous thrombosis ( } 8 \text { ) } \\
\text { Transverse sinus and sigmoid sinus } \\
\text { thrombosis (2) } \\
\text { Superior sagittal sinus (1) }\end{array}$ & & $\begin{array}{l}\text { Mortality } 11.3 \% \\
\text { Morbidity } 25.3 \%\end{array}$ \\
\hline $\begin{array}{l}\text { Deutschmann } \\
2013^{(365)}\end{array}$ & 50 & $\begin{array}{l}\text { Meningitis (23) } \\
\text { Epidural abscess (10) } \\
\text { Subdural abscess (8) } \\
\text { Intracerebral abscess (3) } \\
\text { Cavernous sinus thrombosis (2) } \\
\text { Other (4) }\end{array}$ & $\begin{array}{l}\text { Medical therapy only ( } 23) \\
\text { NS (14) } \\
\text { ESS (8) } \\
\text { Other (5) }\end{array}$ & $\begin{array}{l}\text { No mortality } \\
\text { Morbidity } 6 \%\end{array}$ \\
\hline $\begin{array}{l}\text { Hansen } \\
2012^{(301)}\end{array}$ & 16 & $\begin{array}{l}\text { Subdural empyema (9) } \\
\text { Meningitis (3) } \\
\text { Epidural abscess (2) } \\
\text { Intracerebral abscess (2) } \\
\text { Encephalitis (1) } \\
\text { Superior sagittal sinus thrombosis (1) }\end{array}$ & & $\begin{array}{l}\text { Mortality } 19 \% \\
\text { Morbidity } 19 \%\end{array}$ \\
\hline $\begin{array}{l}\text { DelGaudio } \\
2010^{(355)}\end{array}$ & 23 & $\begin{array}{l}\text { Epidural empyema (8) } \\
\text { Subdural empyema (10) } \\
\text { Intracerebral abscesses (2) } \\
\text { Meningitis (3) }\end{array}$ & $\begin{array}{l}\text { Only medical therapy (3) } \\
\text { ESS (1) } \\
\text { NS (18) }\end{array}$ & $\begin{array}{l}\text { Mortality } 4 \% \\
\text { Morbidity } 12 \%\end{array}$ \\
\hline $\begin{array}{l}\text { Bayonne } \\
2009^{(354)}\end{array}$ & 25 & $\begin{array}{l}\text { Epidural empyema } \\
\text { Subdural empyema } \\
\text { Meningitis }\end{array}$ & & $\begin{array}{l}\text { Sequelae } 16 \% \\
\text { Mortality } 0 \%\end{array}$ \\
\hline $\begin{array}{l}\text { Germiller } \\
2006^{(357)}\end{array}$ & $\begin{array}{l}25 \text { (mean } \\
\text { age 13y) }\end{array}$ & $\begin{array}{l}\text { Epidural empyema (13) } \\
\text { Subdural empyema (9) } \\
\text { Meningitis (6) } \\
\text { Encephalitis (2) } \\
\text { Intracerebral abscess (2) } \\
\text { Cavernous sinus thrombosis (2) }\end{array}$ & $\begin{array}{l}\text { ESS (21) } \\
\text { EXS (7) } \\
\text { NS (13) }\end{array}$ & $\begin{array}{l}\text { Morbidity } 8 \% \\
\text { Mortality } 4 \%\end{array}$ \\
\hline $\begin{array}{l}\text { Quraishi } \\
2006^{(305)}\end{array}$ & $\begin{array}{l}12 \text { (mean } \\
\text { age } 14 \text { y) }\end{array}$ & $\begin{array}{l}\text { Frontal lobe abscess ( } 2 \text { ) } \\
\text { Subdural empyema ( } 8 \text { ) } \\
\text { Cavernous sinus thrombosis ( } 2 \text { ) }\end{array}$ & & $\begin{array}{l}\text { Mortality } 8 \% \\
\text { Morbidity } 16 \%\end{array}$ \\
\hline
\end{tabular}


Table 4.7.3. Endocranial complications in ABRS (continued).

\begin{tabular}{|c|c|c|c|c|}
\hline $\begin{array}{l}\text { Author, } \\
\text { year, ref. }\end{array}$ & $\mathbf{N}$ & Complications & $\begin{array}{l}\text { Management (all had } \\
\text { medical therapy) }\end{array}$ & Mortality / further defects \\
\hline $\begin{array}{l}\text { Oxford } \\
2005^{(316)}\end{array}$ & $\begin{array}{l}18 \text { (mean } \\
\text { age } 12 \mathrm{y})\end{array}$ & $\begin{array}{l}\text { Epidural empyema (7) } \\
\text { Subdural empyema (6) } \\
\text { Intracerebral abscess (2) } \\
\text { Meningitis (2) } \\
\text { Cavernous sinus thrombosis (1) }\end{array}$ & & $\begin{array}{l}\text { No mortality } \\
\text { Morbidity } 11 \%\end{array}$ \\
\hline $\begin{array}{l}\text { Younis } \\
2002^{(349)}\end{array}$ & 39 & $\begin{array}{l}\text { Epidural empyema (7) } \\
\text { Subdural empyema (4) } \\
\text { Meningitis ( } 21) \\
\text { Intracerebral abscess (4) } \\
\text { Superior sagittal sinus thrombosis (1) }\end{array}$ & $\begin{array}{l}\text { Medical therapy only (21) } \\
\text { NS (15) } \\
\text { EXS (4) } \\
\text { ESS (2) }\end{array}$ & $\begin{array}{l}\text { Sequelae } 10 \% \\
\text { No mortality }\end{array}$ \\
\hline $\begin{array}{l}\text { Jones } \\
2002^{(347)}\end{array}$ & 47 & $\begin{array}{l}\text { Subdural empyema } 38 \% \\
\text { Meningitis } 2 \% \\
\text { Epidural empyema } 23 \% \\
\text { Intracranial abscess 30\% }\end{array}$ & $\begin{array}{l}\text { NS ( } 47) \\
\operatorname{EXS~(17)~} \\
\operatorname{ESS}(6)\end{array}$ & $\begin{array}{l}\text { Mortality } 2 \% \\
\text { Morbidity } 19 \%\end{array}$ \\
\hline $\begin{array}{l}\text { Albu } \\
2001^{(353)}\end{array}$ & 16 & $\begin{array}{l}\text { Meningitis (6) } \\
\text { Frontal lobe abscess (6) } \\
\text { Epidural empyema (5) } \\
\text { Subdural empyema (\$) } \\
\text { Cavernous sinus thrombosis (2) }\end{array}$ & & $\begin{array}{l}\text { Mortality } 6 \% \\
\text { Morbidity } 25 \%\end{array}$ \\
\hline $\begin{array}{l}\text { Gallagher } \\
1998^{(356)}\end{array}$ & 15 & $\begin{array}{l}\text { Meningitis } 18 \% \\
\text { Cerebral abscess } 14 \% \\
\text { Epidural empyema } 23 \%\end{array}$ & & $\begin{array}{l}\text { Mortality } 7 \% \\
\text { Morbidity } 13 \%\end{array}$ \\
\hline $\begin{array}{l}\text { Clayman } \\
1991^{(324)}\end{array}$ & 24 & $\begin{array}{l}\text { Meningitis } 29 \% \\
\text { Cerebral abscess } 46 \% \\
\text { Epidural empyema } 5 \% \\
\text { Subdural empyema } 8 \% \\
\text { Cavernous sinus thrombosis } 8 \% \\
\text { Sagittal vein thrombosis } 4 \%\end{array}$ & & $\begin{array}{l}\text { Mortality 4\% } \\
\text { Morbidity 33\% }\end{array}$ \\
\hline
\end{tabular}

ABRS, acute bacterial rhinosinusitis; CRS, chronic rhinosinusitis; ESS, endoscopic sinus surgery; EXS, external sinus surgery; NS, neurosurgical procedures.

Full blood count may show increased white blood cell count with left shift, C-reactive protein (CRP), erythrocyte sedimentation rate $(E S R$, ) and $\mathrm{D}$-dimer while lumbar puncture may show elevated opening pressure and pleocytosis even in culture negative samples whilst blood cultures are frequently positive ${ }^{(353,}$ ${ }^{356)}$. Screening for thrombophilia may give false results during anticoagulation therapy and should be delayed until after treatment is completed.

The cornerstone of diagnosis is an MR venogram, demonstrating absence of venous flow in the affected cavernous sinus. Highresolution CT scan with contrast can also show filling defects ${ }^{(336,}$ 337). Previous articles described an approximate mortality rate of $30 \%$ and a morbidity rate of $60 \%$ in the adult population but more recent articles report better results ${ }^{(369)}$. Lize et al. reported seven patients who were treated for septic cavernous sinus thrombosis secondary to acute bacterial rhinosinusitis. All patients were treated with high dose i.v antibiotics, anticoagulation therapy and endoscopic sinus drainage of the infected sinuses. They described a mortality rate of $0 \%$ but one patient developed permanent unilateral visual loss and four permanent neurologic deficits ${ }^{(370)}$. Most experts recommend anticoagula- tion, in the absence of strong contraindications (only if there is no evidence of severe bleeding risk or current hemorrhage by history, exam, and imaging) but the anticoagulation remains controversial. Retrospective reviews suggest a possible decrease in mortality and reduction in neurologic morbidity when anticoagulation is combined with antibiotics for septic cavernous sinus thrombosis but without support from prospective clinical trials ${ }^{(302)}$ due to a lack of cases. Corticosteroids are often given with antibiotics but without proven efficacy. The potential benefit would be decreased inflammation and vasogenic oedema surrounding cranial nerves and orbital structures. Immediate endoscopic drainage of the affected sinus (almost always the sphenoid) is mandatory.

\subsubsection{Osseous complications}

ABRS can also affect the bony sinus walls causing osteomyelitis and subperiosteal abscess, eventually involving the brain and the nervous system. Even though the most frequent intracranial route of spread is from the frontal sinus, any sinus infection can lead to osseous complications. The most common osseous complications are osteomyelitis of the maxillary (typically in infancy) 
or the frontal bones $(330,336,365,371)$.

The frontal sinus infection can cause osteitis and osteomyelitis of the frontal sinus walls and may develop into a subperiosteal abscess with soft tissue swelling and a doughy pitting oedema on the anterior wall (Pott's puffy tumour). A sino-cutaneaous fistula is a rarer presentation. Since the inflammatory process also extends posteriorly from the frontal sinus, directly or via thrombophlebitis of the valveless diploic veins, patients may have concomitant complications, such as meningitis, epidural and subdural empyema and brain abscess ${ }^{(316,371,372)}$. Leong $^{(316,371,372)}$ reviewed 29 cases of Pott's puffy tumour (PPT) and the most common aetiology was acute frontal sinusitis (62\%). Bony complications develop in 3-10\% of complicated

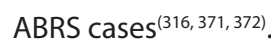

Signs and symptoms of intracranial involvement are soft tissue oedema (especially of the superior eyelid), high fever, severe headache, meningeal irritation, nausea and vomiting, diplopia, photophobia, papillary oedema, coma and focal neurological signs. Ocular signs can appear contralaterally as well. Contrastenhanced CT scan is needed to confirm the diagnosis. A lumbar puncture is not indicated if the intracranial pressure is elevated, but in certain cases it can also be of diagnostic value. Therapy originally included a combination of i.v. broad-spectrum antibiotics administration and surgical debridement of sequestered bone and drainage ${ }^{(302,321,373)}$. There is a growing body of evidence that uncomplicated PPT can be managed successfully via an endoscopic approach/minimal external drainage, combined with long-term antibiotics ${ }^{(316,371,372)}$.

There is a growing body of evidence that uncomplicated Pott's puffy tumour can be managed successfully via an endoscopic approach/ minimal external drainage, combined with longterm antibiotics.

\subsubsection{Unusual complications of rhinosinusitis}

A number of unusual complications have been reported in cases of patients with ABRS. These are presented in Table 4.7.4. It is important to note that the management of ABRS complications should always be multidisciplinary and close cooperation with other different specialties as ophthalmologists, neurologists/ neurosurgeons, paediatricians, radiologists and microbiologists is mandatory.

\subsubsection{Follow-up of complications}

ABRS complications may occasionally appear simultaneously (for example subperiosteal frontal abscess and intracranial extension, orbital and intracranial complications) and intracranial complications may have neurologic deficits. Therefore, a long term follow-up of 6-12 months is indicated for such patients in order to monitor for complete resolution of the disease as well as to exclude any disease recurrence or complication of treatment. Meticulous care of the sinuses should be the first priority.

\subsubsection{Conclusion}

Complications of bacterial rhinosinusitis are rare but potentially serious. However, a number of studies have shown that they are not prevented by routine prescribing of antibiotics so a low threshold of suspicion must always be maintained for their early diagnosis.

Table 4.7.4. Unusual complications of ABRS.

\begin{tabular}{|c|c|c|}
\hline Author, year (ref.) & Type of complication & Outcome \\
\hline Abou-Al-Ahaar 2019(386) & Clival osteomyelitis with VI, XII nerve palsy & Complete recovery after medical and surgical therapy \\
\hline Fabre $2018^{(387)}$ & Acute Stroke & Complete recovery after medical therapy and ESS \\
\hline Righini $2009^{(388)}$ & Acute ischemic stroke & Minimal neurologic sequelae after medical therapy and ESS \\
\hline Sanan $2017^{(340)}$ & Intra-optic nerve abscess with vision loss & $\begin{array}{l}\text { Stable ophthalmologic findings after medical therapy and } \\
\text { ESS }\end{array}$ \\
\hline Korkmaz $2017^{(389)}$ & Isolated unilateral upper lid ptosis & Complete recovery after medical therapy \\
\hline Huth $2015^{(390)}$ & $\begin{array}{l}\text { Thrombophlebitis temporal vein (without cavernous } \\
\text { sinus thrombosis) }\end{array}$ & Complete recovery after medical therapy and ESS \\
\hline Tien 2016(391) & Nasal septal abscess ( 5 cases) & No morbidity after medical and surgical therapy \\
\hline Zielnik-Jurkiewicz 2005(392) & Nasal septal and palatine process abscess & No morbidity after medical and surgical therapy \\
\hline Gradoni $2010^{(393)}$ & Nasal septal abscess & No morbidity after medical and surgical therapy \\
\hline Nomura 2014 ${ }^{(394)}$ & Orbital hematoma & Complete recovery after medical therapy and ESS \\
\hline
\end{tabular}


Table 4.7.4. Unusual complications of ABRS, continued.

\begin{tabular}{|c|c|c|}
\hline Author, year (ref.) & Type of complication & Outcome \\
\hline Nomura 2014(394) & Orbital hematoma & $\begin{array}{l}\text { Complete recovery after medical therapy, ESS and superior } \\
\text { orbitotomy }\end{array}$ \\
\hline Yim 2013(395) & $\begin{array}{l}\text { Orbital abscess secondary to contralateral rhinosinu- } \\
\text { sitis }\end{array}$ & $\begin{array}{l}\text { Complete recovery after medical therapy, ESS and superior } \\
\text { orbitotomy }\end{array}$ \\
\hline Chan $2009^{(396)}$ & Toxic Shock Syndrome & Complete recovery after medical therapy and ESS \\
\hline Chen MC 2019(330) & Septic shock & Complete recovery after medical therapy and ESS \\
\hline Rimal $2006^{(332)}$ & Septicaemia & Complete recovery after only medical therapy \\
\hline Suzuki $2005^{(360)}$ & $\begin{array}{l}\text { Internal carotid artery aneurysm with multiple cranial } \\
\text { nerve palsy }\end{array}$ & $\begin{array}{l}\text { Partial recovery of visual disturbance after endovascular coil } \\
\text { placement of the aneurysm }\end{array}$ \\
\hline Patel $2003^{(397)}$ & Lacrimal gland abscess & Complete recovery after medical therapy and drainage \\
\hline Mirza $2001^{(398)}$ & Lacrimal gland abscess & Complete recovery after medical therapy and ESS \\
\hline Sidwell $2001^{(399)}$ & Pyoderma gangrenosum of the eyelid & Scarring of the eyelid after medical therapy \\
\hline Sahjpaul 1999(400) & $\begin{array}{l}\text { Pituitary abscess and cavernous sinus thrombophle- } \\
\text { bitis }\end{array}$ & $\begin{array}{l}\text { Blind right eye and pituitary insufficiency after medical } \\
\text { therapy and ESS }\end{array}$ \\
\hline
\end{tabular}

ESS, endoscopic sinus surgery.

\section{References}

1. Fokkens W, Lund V, Bachert C, et al European Position Paper on Rhinosinusitis and nasal Polyps. Rhinology Suppl. 2005;18.

2. Bachert C, Hormann K, Mosges R, et al. An update on the diagnosis and treatment of sinusitis and nasal polyposis. Allergy 2003:58:176-91.

3. Hoffmans R, Wagemakers A, van Drunen C, Hellings P, Fokkens W. Acute and chronic rhinosinusitis and allergic rhinitis in relation to comorbidity, ethnicity and environment. PLoS One 2018:13:e0192330.

4. Revai K, Dobbs LA, Nair S, Patel JA, Grady $J$ J, Chonmaitree T. Incidence of acute otitis media and sinusitis complicating upper respiratory tract infection: the effect of age. Pediatrics 2007;119:e1408-12.

5. Fokkens W, Lund V, Mullol J. European position paper on rhinosinusitis and nasal polyps 2007. Rhinol Suppl 2007:20:1-136.

6. Aring AM, Chan MM. Current Concepts in Adult Acute Rhinosinusitis. Am Fam Physician 2016;94:97-105.

7. Rosenfeld RM, Piccirillo JF, Chandrasekhar SS, et al. Clinical practice guideline (update): Adult sinusitis. Otolaryngology - Head and Neck Surgery (United States) 2015;152:S1S39.

8. Smith SS, Ference EH, Evans CT, Tan BK, Kern RC, Chandra RK. The prevalence of bacterial infection in acute rhinosinusitis: a Systematic review and meta-analysis. Laryngoscope 2015;125:57-69.

9. Seresirikachorn K, Snidvongs K, Chitsuthipakorn W, et al. EPOS2012 has better specificity compared to IDSA2012 for diagnosing acute bacterial rhinosinusitis. Rhinology 2018;56:241-4

10. Jaume F, Quintó L, Alobid I, Mullol J. Overuse of diagnostic tools and medica- tions in acute rhinosinusitis in Spain: a population-based study (the PROSINUS study). BMJ open 2018;8:e018788.

11. Stjärne P, Odebäck P, Ställberg B, Lundberg J, Olsson P. High costs and burden of illness in acute rhinosinusitis: real-life treatment patterns and outcomes in Swedish primary care. Primary Care Respiratory Journal 2012;21:174-9.

12. Babar-Craig H, Gupta Y, Lund VJ. British Rhinological Society audit of the role of antibiotics in complications of acute rhinosinusitis: a national prospective audit. Rhinology 2010;48:344-7.

13. Hansen FS, Hoffmans R, Georgalas C, Fokkens WJ. Complications of acute rhinosinusitis in The Netherlands. Fam Pract 2011;29:147-53.

14. Stoll D, Klossek JM, Barbaza MO [Prospective study of 43 severe complications of acute rhinosinusitis]. Rev Laryngol Otol Rhinol (Bord) 2006;127:195-201.

15. van Gageldonk-Lafeber $A B$, Heijnen $M L$, Bartelds Al, Peters MF, van der Plas SM, Wilbrink B. A case-control study of acute respiratory tract infection in general practice patients in The Netherlands. Clin Infect Dis 2005:41:490-7.

16. Fairlie T, Shapiro DJ, Hersh AL, Hicks LA National trends in visit rates and antibiotic prescribing for adults with acute sinusitis. Arch Intern Med 2012;172:1513-4.

17. Hoffmans $R$, Schermer $T$, van der Linde $K$, et al. Rhinosinusitis in morbidity registrations in Dutch General Practice: a retrospective case-control study. BMC Fam Pract 2015;16:120.

18. Finley CR, Chan DS, Garrison S, et al. What are the most common conditions in primary care? Systematic review. Can Fam Physician 2018;64:832-40.
19. Britt H MG, Henderson J, Bayram C, Harrison C, Valenti L, Pan Y, Charles J, Pollack AJ, Wong C, Gordon J. General practice activity in Australia 2015-16. Sydney: Sydney University Press, 2016.

20. Akkina SR, Novis SJ, Keshavarzi NR, Pynnonen MA. Academic institution pilot study shows far fewer diagnoses of sinusitis than reported nationally. Laryngoscope Investig Otolaryngol 2016;1:124-9.

21. Caini S, Spreeuwenberg P, Donker G, Korevaar J, Paget J. Climatic factors and long-term trends of influenza-like illness rates in The Netherlands, 1970-2016. Environ Res 2018;167:307-13.

22. Pouwels KB, Dolk FCK, Smith DRM, Robotham JV, Smieszek T. Actual versus 'ideal' antibiotic prescribing for common conditions in English primary care. The Journal of antimicrobial chemotherapy 2018;73:19-26.

23. Dallas A, van Driel M, Morgan S, et al. Antibiotic prescribing for acute otitis media and acute sinusitis: a cross-sectional analysis of the ReCEnT study exploring the habits of early career doctors in family practice. Fam Pract 2017;34:180-7.

24. Jorgensen LC, Friis Christensen S, Cordoba Currea G, Llor C, Bjerrum L. Antibiotic prescribing in patients with acute rhinosinusitis is not in agreement with European recommendations. Scand J Prim Health Care 2013:31:101-5

25. Al-Hashel JY, Ahmed SF, Alroughani $R$, Goadsby PJ. Migraine misdiagnosis as a sinusitis, a delay that can last for many years. The Journal of headache and pain 2013;14:97.

26. Godley FA, Casiano RR, Mehle M, McGeeney B, Gottschalk C. Update on the diagnostic considerations for neurogenic nasal and 
sinus symptoms: A current review suggests adding a possible diagnosis of migraine. Am J Otolaryngol 2019;40:306-11.

27. Davies PTG, Lane RJM, Astbury T, Fontebasso M, Murphy J, Matharu M. The long and winding road: the journey taken by headache sufferers in search of help. Prim Health Care Res Dev 2019;20:e4.

28. Benninger MS, Ferguson BJ, Hadley JA, et al. Adult chronic rhinosinusitis: Definitions, diagnosis, epidemiology, and pathophysiology. Otolaryngology - Head \& Neck Surgery 2003;129:S1-32.

29. Shapiro DJ, Gonzales R, Cabana MD, Hersh AL. National trends in visit rates and antibiotic prescribing for children with acute sinusitis. Pediatrics 2011;127:28-34.

30. Leung R, Almassian S, Kern R, Conley D, Tan B, Chandra R. Patient level decision making in recurrent acute rhinosinusitis: a costbenefit threshold for surgery. Laryngoscope 2013;123:11-6.

31. Bhattacharyya N, Grebner J, Martinson NG. Recurrent Acute Rhinosinusitis: Epidemiology and Health Care Cost Burden. Otolaryngology--head and neck surgery 2012;146:307-12.

32. Lee LN, Bhattacharyya N. Regional and specialty variations in the treatment of chronic rhinosinusitis. Laryngoscope 2011;121:1092-7.

33. Costa ML, Psaltis AJ, Nayak JV, Hwang PH. Medical therapy vs surgery for recurrent acute rhinosinusitis. Int Forum Allergy Rhinol 2015;5:667-73.

34. Hirsch AG, Stewart WF, Sundaresan AS, et al. Nasal and sinus symptoms and chronic rhinosinusitis in a population-based sample. Allergy 2017;72:274-81.

35. Orlandi RR, Kingdom TT, Hwang PH, et al. International Consensus Statement on Allergy and Rhinology: Rhinosinusitis. Int Forum Allergy Rhinol 2016;6 Suppl 1:S22S209.

36. Chee L, Graham SM, Carothers DG, Ballas ZK. Immune dysfunction in refractory sinusitis in a tertiary care setting. Laryngoscope 2001;111:233-5.

37. Wise MT, Hagaman DD. An immunological approach to chronic and recurrent sinusitis. Curr Opin Otolaryngol Head Neck Surg 2007;15:10-7.

38. Cunningham-Rundles C, Bodian C. Common variable immunodeficiency: clinical and immunological features of 248 patients. Clin Immunol 1999;92:34-48.

39. Carr TF, Koterba AP, Chandra R, et al. Characterization of specific antibody deficiency in adults with medically refractory chronic rhinosinusitis. Am J Rhinol Allergy 2011;25:241-4.

40. Edwards E, Razvi S, Cunningham-Rundles C. IgA deficiency: clinical correlates and responses to pneumococcal vaccine. Clin Immunol 2004;111:93-7.

41. van Gageldonk-Lafeber AB, van der Sande MA, Heijnen ML, Peeters MF, Bartelds Al, Wilbrink B. Risk factors for acute respiratory tract infections in general practitioner patients in The Netherlands: a case-control study. BMC Infect Dis 2007;7:35.

42. Koskinen OM, Husman TM, Meklin TM, Nevalainen Al. The relationship between moisture or mould observations in houses and the state of health of their occupants. Eur Respir J 1999;14:1363-7.

43. Shaw Stewart PD. Seasonality and selective trends in viral acute respiratory tract infections q. 2016, 10.1016/j.mehy.2015.11.005.

44. De Sario M, Katsouyanni K, Michelozzi P. Climate change, extreme weather events, air pollution and respiratory health in Europe. Eur Respir J, 2013, 826-43.

45. Neumark T, Brudin L, Engstrom S, Molstad S. Trends in number of consultations and antibiotic prescriptions for respiratory tract infections between 1999 and 2005 in primary healthcare in Kalmar County, Southern Sweden. Scand J Prim Health Care 2009;27:18-24.

46. Rank MA, Wollan P, Kita H, Yawn BP. Acute exacerbations of chronic rhinosinusitis occur in a distinct seasonal pattern. J Allergy Clin Immunol 2010, 10.1016/j. jaci.2010.03.041.

47. Eccles R. An explanation for the seasonality of acute upper respiratory tract viral infections. Acta Otolaryngol 2002;122:183-91.

48. Rudmik L, Muzychuk A, Oddone Paolucci E, Mechor B. Chinook wind barosinusitis: an anatomic evaluation. Am J Rhinol Allergy 2009;23:e14-6.

49. Suonpaa J, Antila J. Increase of acute frontal sinusitis in southwestern Finland. Scand J Infect Dis 1990;22:563-8.

50. Bhattacharyya N. Air quality influences the prevalence of hay fever and sinusitis. Laryngoscope 2009;119:429-33.

51. Trevino RJ. Air pollution and its effect on the upper respiratory tract and on allergic rhinosinusitis. Otolaryngol Head Neck Surg 1996;114:239-41.

52. Zuskin E, Mustajbegovic J, Schachter EN, et al. Respiratory findings in pharmaceutical workers. Am J Ind Med 2004;46:472-9.

53. Jaakkola MS, Jaakkola JJ. Office equipment and supplies: a modern occupational health concern? Am J Epidemiol 1999;150:1223-8.

54. Duclos P, Sanderson LM, Lipsett M. The 1987 forest fire disaster in California: assessment of emergency room visits. Arch Environ Health 1990;45:53-8.

55. Kuiper JR, Hirsch AG, Bandeen-Roche K, et al. Prevalence, severity, and risk factors for acute exacerbations of nasal and sinus symptoms by chronic rhinosinusitis status. Allergy 2018;73:1244-53.

56. Dietz de Loos D, Lourijsen ES, Wildeman MAM, et al. Prevalence of chronic rhinosinusitis in the general population based on sinus radiology and symptomatology. J Allergy Clin Immunol 2019;143:1207-14.

57. Sugiura S, Yasue M, Uchida $Y$, et al. Prevalence and Risk Factors of MRI Abnormality Which Was Suspected as Sinusitis in Japanese Middle-Aged and Elderly Community Dwellers. BioMed Research International 2018;2018.
58. Alkire BC, Bhattacharyya N. An assessment of sinonasal anatomic variants potentially associated with recurrent acute rhinosinusitis. Laryngoscope 2010;120:631-4.

59. Jain R, Stow N, Douglas R. Comparison of anatomical abnormalities in patients with limited and diffuse chronic rhinosinusitis. Int Forum Allergy Rhinol 2013;3:493-6.

60. Loftus PA, Lin J, Tabaee A. Anatomic variants of the paranasal sinuses in patients with recurrent acute rhinosinusitis. International Forum of Allergy \& Rhinology 2016;6:32833.

61. Bomeli SR, Branstetter BFt, Ferguson BJ. Frequency of a dental source for acute maxillary sinusitis. Laryngoscope 2009;119:5804.

62. Mathew AL, Pai KM, Sholapurkar AA. Maxillary sinus findings in the elderly: a panoramic radiographic study. J Contemp Dent Pract 2009;10:E041-8.

63. Wuokko-Landén A, Blomgren K, Välimaa $H$. Acute rhinosinusitis-are we forgetting the possibility of a dental origin? A retrospective study of 385 patients. Acta Otolaryngol 2019, 10.1080/00016489.2019.1634837.

64. Khan A, Vandeplas G, Huynh TMT, et al. The Global Allergy and Asthma European Network (GALEN rhinosinusitis cohort: a large European cross-sectional study of chronic rhinosinusitis patients with and without nasal polyps. Rhinology 2019;57:3242.

65. Schatz M, Zeiger RS, Chen W, Yang S-J, Corrao MA, Quinn VP. The burden of rhinitis in a managed care organization. Annals of allergy, asthma \& immunology : official publication of the American College of Allergy, Asthma, \& Immunology 2008;101:240-7.

66. Scadding G, Bousquet J, Bachert C, et al. Rhinology future trends: 2017 EUFOREA debate on allergic rhinitis. Rhinology 2019;57:49-56.

67. Mortz CG, Andersen KE, Poulsen LK, Kjaer HF, Broesby-Olsen S, Bindslev-Jensen C. Atopic diseases and type I sensitization from adolescence to adulthood in an unselected population (TOACS) with focus on predictors for allergic rhinitis. Allergy 2019;74:308-17.

68. Leth-Moller KB, Skaaby T, Linneberg A. Allergic rhinitis and allergic sensitisation are still increasing among Danish adults. Allergy 2019, 10.1111/all.14046.

69. Wang $X Y, M a T T$, Wang $X Y$, et al. Prevalence of pollen-induced allergic rhinitis with high pollen exposure in grasslands of northern China. Allergy 2018;73:1232-43.

70. Reitsma S, Subramaniam S, Fokkens WWJ, Wang Y. Recent developments and highlights in rhinitis and allergen immunotherapy. Allergy 2018;73:2306-13.

71. Savolainen S. Allergy in patients with acute maxillary sinusitis. Allergy 1989;44:116-22.

72. Ulanovski D, Barenboim E, Raveh E, Grossman A, Azaria B, Shpitzer T. Sinusitis in pilots of different aircraft types: is allergic rhinitis a predisposing factor? Am J Rhinol 2008;22:122-4. 
73. Pant H, Ferguson BJ, Macardle PJ. The role of allergy in rhinosinusitis. Curr Opin Otolaryngol Head Neck Surg 2009;17:232-8.

74. Lin SW, Wang YH, Lee MY, et al. Clinical spectrum of acute rhinosinusitis among atopic and nonatopic children in Taiwan. Int J Pediatr Otorhinolaryngol 2012;76:70-5.

75. Leo G, Incorvaia C, AC-TJo, 2018 u. Could seasonal allergy be a risk factor for acute rhinosinusitis in children? J Laryngol Otol. 2018.132,2:150-153.

76. Vlastos I, Athanasopoulos I, Mastronikolis NS, et al. Impaired mucociliary clearance in allergic rhinitis patients is related to a predisposition to rhinosinusitis. Ear Nose Throat J 2009;88:E17-9.

77. Tamashiro E, Xiong G, Anselmo-Lima WT, Kreindler JL, Palmer JN, Cohen NA. Cigarette smoke exposure impairs respiratory epithelial ciliogenesis. Am J Rhinol Allergy 2009;23:117-22.

78. De S, Leong SC, Fenton JE, Carter SD, Clarke RW, Jones AS. The effect of passive smoking on the levels of matrix metalloproteinase 9 in nasal secretions of children. Am J Rhinol Allergy 2011;25:226-30.

79. Bonham GS, Wilson RW. Children's health in families with cigarette smokers. Am J Public Health 1981:71:290-3

80. Eyigor H, Basak S. [Evaluation of predisposing factors and bacteriologic agents in pediatric rhinosinusitis]. Kulak Burun Bogaz Ihtis Derg 2005;15:49-55.

81. Zuskin E, Mustajbegovic J, Schachter EN, et al. Respiratory findings in mail carriers. Int Arch Occup Environ Health 2000;73:136-43.

82. Brook I. Effects of exposure to smoking on the microbial flora of children and their parents. Int J Pediatr Otorhinolaryngol 2010;74:447-50

83. Brook I, Gober AE. Effect of smoking cessation on the microbial flora. Arch Otolaryngol Head Neck Surg 2007;133:1358.

84. Davis KS, Casey SE, Mulligan JK, Mulligan RM, Schlosser RJ, Atkinson C. Murine complement deficiency ameliorates acute cigarette smoke-induced nasal damage. Otolaryngol Head Neck Surg 2010;143:1528.

85. Pacheco-Galvan A, Hart SP, Morice AH Relationship between gastro-oesophageal reflux and airway diseases: the airway reflux paradigm. Arch Bronconeumol 2011;47:195-203.

86. Flook EP, Kumar BN. Is there evidence to link acid reflux with chronic sinusitis or any nasal symptoms? A review of the evidence. Rhinology 2011;49:11-6.

87. Tan BK, Chandra RK, Pollak J, et al. Incidence and associated premorbid diagnoses of patients with chronic rhinosinusitis. J Allergy Clin Immunol 2013;131:1350-60.

88. Adams TB, Wharton CM, Quilter L, Hirsch T. The association between mental health and acute infectious illness among a national sample of 18- to 24-year-old college students. J Am Coll Health 2008;56:657-63.

89. Taylor G, McNeill A, Girling A, Farley A,
Lindson-Hawley N, Aveyard P. Change in mental health after smoking cessation: Systematic review and meta-analysis. BMJ (Online) 2014;348.

90. Loughlin J, Poulios N, Napalkov P, Wegmuller Y, Monto AS. A study of influenza and influenza-related complications among children in a large US health insurance plan database. Pharmacoeconomics 2003;21:273-83.

91. Fokkens WJ, Lund VJ, Mullol J, et al. European Position Paper on Rhinosinusitis and Nasal Polyps 2012. Rhinol Suppl 2012;23:3 p preceding table of contents, $1-298$.

92. Eloy $\mathrm{P}$, Poirrier AL, De Dorlodot C, Van Zele T, Watelet JB, Bertrand B. Actual concepts in rhinosinusitis: a review of clinical presentations, inflammatory pathways, cytokine profiles, remodeling, and management. Curr Allergy Asthma Rep 2011;11:146-62.

93. Tan KS, Yan Y, Ong HH, Chow VTK, Shi L, Wang D-Y. Impact of Respiratory Virus Infections in Exacerbation of Acute and Chronic Rhinosinusitis. Curr Allergy Asthma Rep 2017;17:24

94. Chuang C-Y, Kao C-L, Huang L-M, et al. Human bocavirus as an important cause of respiratory tract infection in Taiwanese children. Journal of microbiology, immunology, and infection $=$ Wei mian yu gan ran za zhi 2011;44:323-7

95. Braciale TJ, Sun J, Kim TS. Regulating the adaptive immune response to respiratory virus infection. Nature Reviews Immunology 2012;12:295-305

96. Kolesnikova L, Heck S, Matrosovich T, Klenk $\mathrm{H}-\mathrm{D}$, Becker S, Matrosovich M. Influenza virus budding from the tips of cellular microvilli in differentiated human airway epithelial cells. The Journal of general virology 2013;94:971-6.

97. Tan KS, Ong HH, Yan Y, et al. In Vitro Model of Fully Differentiated Human Nasal Epithelial Cells Infected With Rhinovirus Reveals Epithelium-Initiated Immune Responses. The Journal of infectious diseases 2018;217:906-15.

98. Tan KS, Yan Y, Koh WLH, et al. Comparative Transcriptomic and Metagenomic Analyses of Influenza Virus-Infected Nasal Epithelial Cells From Multiple Individuals Reveal Specific Nasal-Initiated Signatures. Front Microbiol 2018:9:2685.

99. Greve JM, Davis G, Meyer AM, et al. The major human rhinovirus receptor is ICAM-1. Cell 1989;56:839-47.

100. Papi A, Johnston SL. Rhinovirus infection induces expression of its own receptor intercellular adhesion molecule 1 (ICAM-1) via increased NF-kappaB-mediated transcription. J Biol Chem 1999;274:9707-20.

101. Lewis TC, Henderson TA, Carpenter AR, et al. Nasal cytokine responses to natural colds in asthmatic children. Clin Exp Allergy 2012:42:1734-44

102. Medina RA, García-Sastre A. Influenza A viruses: new research developments. Nature reviews Microbiology 2011;9:590-603.
103. Masaki T, Kojima T, Okabayashi T, et al. A nuclear factor-kB signaling pathway via protein kinase $C \delta$ regulates replication of respiratory syncytial virus in polarized normal human nasal epithelial cells. Mol Biol Cell 2011;22:2144-56.

104. Lamb RA KR. Orthomyxoviridae: The viruses and their replication. In: Knipe DM HP, (ed). Fields Virology fourth edition. Philadelphia, 2001, 1487-531.

105. Sugrue RJ. Interactions between respiratory syncytial virus and the host cell: opportunities for antivirus strategies? Expert Rev Mol Med 2006:8:1-17.

106. Othumpangat S, Regier M, Piedimonte G. Nerve growth factor modulates human rhinovirus infection in airway epithelial cells by controlling ICAM-1 expression. American Journal of Physiology Lung cellular and Molecular Physiology 2012;302:L1057-66

107.van Kempen M, Bachert C, Van Cauwenberge P. An update on the pathophysiology of rhinovirus upper respiratory tract infections. Rhinology 1999;37:97-103.

108. Suptawiwat O, Tantilipikorn P, Boonarkart C, et al. Enhanced Susceptibility of Nasal Polyp Tissues to Avian and Human Influenza Viruses. PLoS One 2010;5:e12973.

109. Matrosovich MN, Matrosovich TY, Gray T, Roberts NA, Klenk H-D. Human and avian influenza viruses target different cell types in cultures of human airway epithelium. Proceedings of the National Academy of Sciences 2004;101:4620-4.

110. Matrosovich M, Zhou N, Kawaoka Y, Webster $\mathrm{R}$. The surface glycoproteins of $\mathrm{H} 5$ influenza viruses isolated from humans, chickens, and wild aquatic birds have distinguishable properties. J Virol 1999;73:1146-55.

111. Yan Y, Tan KS, Li C, et al. Human nasal epithelial cells derived from multiple subjects exhibit differential responses to H3N2 influenza virus infection in vitro. The Journal of allergy and clinical immunology 2016;138:276-81.e15.

112. Aab A, Wirz O, van de Veen W, et al. Human rhinoviruses enter and induce proliferation of B lymphocytes. Allergy 2017;72:232-43.

113. Le T, Psaltis A, Tan LW, Wormald PJ. The efficacy of topical antibiofilm agents in a sheep model of rhinosinusitis. Am J Rhinol 2008;22:560-7.

114. Wang D-Y, Li Y, Yan Y, Li C, Shi L. Upper airway stem cells: understanding the nose and role for future cell therapy. Curr Allergy Asthma Rep 2015;15:490.

115. Kast JI, McFarlane AJ, Głobińska A, et al. Respiratory syncytial virus infection influences tight junction integrity. Clin Exp Immunol 2017;190:351-9.

116. Tian T, Zi X, Peng $Y$, et al. H3N2 influenza virus infection enhances oncostatin $M$ expression in human nasal epithelium. Exp Cell Res 2018;371:322-9.

117. Yeo N-K, Jang YJ. Rhinovirus infectioninduced alteration of tight junction and adherens junction components in human nasal epithelial cells. The Laryngoscope 2009, 10.1002/lary.20764:n/a-n/a. 
118. Yan Y, Gordon WM, Wang D-Y. Nasal epithelial repair and remodeling in physical injury, infection, and inflammatory diseases. Curr Opin Otolaryngol Head Neck Surg 2013;21:263-70.

119. Watelet JB, Van Zele T, Gjomarkaj M, et al. Tissue remodelling in upper airways: where is the link with lower airway remodelling? Allergy 2006;61:1249-58.

120. Clay CC, Reader JR, Gerriets JE, Wang TT, Harrod KS, Miller LA. Enhanced viral replication and modulated innate immune responses in infant airway epithelium following H1N1 infection. J Virol 2014;88:741225.

121. Wang Z, Wan Y, Qiu C, et al. Recovery from severe H7N9 disease is associated with diverse response mechanisms dominated by CD8+ T cells. Nature Communications 2015;6:6833.

122. Andres-Terre M, McGuire HM, Pouliot Y, et al. Integrated, Multi-cohort Analysis Identifies Conserved Transcriptional Signatures across Multiple Respiratory Viruses. Immunity 2015;43:1199-211.

123. Klinkhammer J, Schnepf D, Ye L, et al. IFN- $\lambda$ prevents influenza virus spread from the upper airways to the lungs and limits virus transmission. elife 2018;7.

124. Huong TN, Yan Y, Jumat MR, et al. A sustained antiviral host response in respiratory syncytial virus infected human nasal epithelium does not prevent progeny virus production. Virology 2018;521:20-32.

125. Pedersen M, Sakakura Y, Winther B, Brofeldt S, Mygind N. Nasal mucociliary transport, number of ciliated cells, and beating pattern in naturally acquired common colds. Eur J Respir Dis Suppl 1983;128 (Pt 1):35565.

126. Kuiken T, van den Hoogen BG, van Riel DAJ, et al. Experimental human metapneumovirus infection of cynomolgus macaques (Macaca fascicularis) results in virus replication in ciliated epithelial cells and pneumocytes with associated lesions throughout the respiratory tract. Am J Pathol 2004;164:1893-900.

127. Lopez-Souza N, Favoreto S, Wong H, et al. In vitro susceptibility to rhinovirus infection is greater for bronchial than for nasal airway epithelial cells in human subjects. The Journal of allergy and clinical immunology 2009;123:1384-90.e2.

128. Smith CM, Kulkarni H, Radhakrishnan P, et al. Ciliary dyskinesia is an early feature of respiratory syncytial virus infection. The European Respiratory Journal 2014;43:48596.

129.Jumat MR, Yan Y, Ravi LI, et al. Morphogenesis of respiratory syncytial virus in human primary nasal ciliated epithelial cells occurs at surface membrane microdomains that are distinct from cilia. Virology 2015;484:395-411.

130. Raphael GD, Baraniuk JN, Kaliner MA. How and why the nose runs. The Journal of allergy and clinical immunology 1991;87:457-67.

131. Hoggard M, Wagner Mackenzie B, Jain
R, Taylor MW, Biswas K, Douglas RG. Chronic Rhinosinusitis and the Evolving Understanding of Microbial Ecology in Chronic Inflammatory Mucosal Disease. Clin Microbiol Rev 2017;30:321-48.

132. Naclerio RM, Proud D, Lichtenstein LM, et al. Kinins are generated during experimental rhinovirus colds. J Infect Dis 1988;157:13342.

133. Linden M, Greiff L, Andersson M, et al. Nasal cytokines in common cold and allergic rhinitis. Clin Exp Allergy 1995;25:166-72.

134. Yuta A, Doyle WJ, Gaumond E, et al. Rhinovirus infection induces mucus hypersecretion. Am J Physiol 1998;274:L1017-23.

135. Villenave R, Thavagnanam S, Sarlang S, et al. In vitro modeling of respiratory syncytial virus infection of pediatric bronchial epithelium, the primary target of infection in vivo. Proc Natl Acad Sci U S A 2012;109:5040-5.

136. Baños-Lara MDR, Piao B, Guerrero-Plata A. Differential mucin expression by respiratory syncytial virus and human metapneumovirus infection in human epithelial cells. Mediators Inflamm 2015;2015:347292.

137. Baos SC, Phillips DB, Wildling L, McMaster TJ, Berry M. Distribution of sialic acids on mucins and gels: a defense mechanism. Biophys J 2012;102:176-84.

138. Cohen M, Zhang X-Q, Senaati HP, et al. Influenza A penetrates host mucus by cleaving sialic acids with neuraminidase. Virol J 2013;10:321.

139. Morris DE, Cleary DW, Clarke SC. Secondary Bacterial Infections Associated with Influenza Pandemics. Front Microbiol 2017;8:1041.

140. Gipson IK, Spurr-Michaud S, Tisdale A, Menon BB. Comparison of the transmembrane mucins MUC1 and MUC16 in epithelial barrier function. PLoS One 2014;9:e100393.

141. Kim KC. Role of epithelial mucins during airway infection. Pulm Pharmacol Ther 2012;25:415-9.

142. Dhar P, McAuley J. The Role of the Cell Surface Mucin MUC1 as a Barrier to Infection and Regulator of Inflammation. Frontiers in Cellular and Infection Microbiology 2019;9.

143. McAuley JL, Corcilius L, Tan H-X, Payne RJ, McGuckin MA, Brown LE. The cell surface mucin MUC1 limits the severity of influenza A virus infection. Mucosal Immunol 2017;10:1581-93.

144. de Bentzmann S, Polette M, Zahm JM, et al. Pseudomonas aeruginosa virulence factors delay airway epithelial wound repair by altering the actin cytoskeleton and inducing overactivation of epithelial matrix metalloproteinase-2. Laboratory Investigation; Journal of Technical Methods and Pathology 2000;80:209-19.

145. Passariello C, Schippa S, Conti C, et al. Rhinoviruses promote internalisation of Staphylococcus aureus into non-fully permissive cultured pneumocytes. Microbes Infect 2006;8:758-66.

146. van Cauwenberge P, Ingels K. Effects of viral and bacterial infection on nasal and sinus mucosa. Acta Otolaryngol 1996;116:316-21.

147. Min J-Y, Shin S-H, Kwon HJ, Jang YJ. Levocetirizine inhibits rhinovirus-induced bacterial adhesion to nasal epithelial cells through down-regulation of cell adhesion molecules. Annals of Allergy, Asthma \& Immunology 2012;108:44-8.

148. Wang JH, Lee SH, Kwon HJ, Jang YJ. Clarithromycin inhibits rhinovirus-induced bacterial adhesions to nasal epithelial cells. Laryngoscope 2010;120:193-9.

149. Wang JH, Kwon HJ, Jang YJ. Rhinovirus enhances various bacterial adhesions to nasal epithelial cells simultaneously. Laryngoscope 2009;119:1406-11.

150. Jang YJ, Lee Y-H, Shin S-H. Rhinovirusinfected nasal polyp epithelial cells: effect on the activation and migration of eosinophils by airborne fungi. Annals of Allergy, Asthma \& Immunology 2010;104:434-9.

151. Mahdavinia M, Keshavarzian A, Tobin MC, Landay AL, Schleimer RP. A comprehensive review of the nasal microbiome in chronic rhinosinusitis (CRS). Clinical and experimental allergy : Journal of the British Society for Allergy and Clinical Immunology 2016;46:21-41.

152. Vareille M, Kieninger E, Edwards MR, Regamey N. The airway epithelium: soldier in the fight against respiratory viruses. Clin Microbiol Rev 2011;24:210-29.

153. Kudva A, Scheller EV, Robinson KM, et al. Influenza A inhibits Th17-mediated host defense against bacterial pneumonia in mice. Journal of immunology (Baltimore, Md : 1950) 2011;186:1666-74.

154.Diavatopoulos DA, Short KR, Price JT, et al. Influenza A virus facilitates Streptococcus pneumoniae transmission and disease. FASEB Journal 2010;24:1789-98.

155. Marks LR, Davidson BA, Knight PR, Hakansson AP. Interkingdom signaling induces Streptococcus pneumoniae biofilm dispersion and transition from asymptomatic colonization to disease. mBio 2013;4.

156. Deng Y, Yan Y, Tan KS, et al. MicroRNA-146a induction during influenza H3N2 virus infection targets and regulates TRAF6 levels in human nasal epithelial cells (hNECs). Exp Cell Res 2017;352:184-92.

157. Grainge CL, Davies DE. Epithelial injury and repair in airways diseases. Chest 2013;144:1906-12.

158. Luukkainen A, Puan KJ, Yusof N, et al. A Co-culture Model of PBMC and Stem Cell Derived Human Nasal Epithelium Reveals Rapid Activation of NK and Innate T Cells Upon Influenza A Virus Infection of the Nasal Epithelium. Front Immunol 2018;9:2514.

159. Cui L, Zheng D, Lee YH, et al. Metabolomics Investigation Reveals Metabolite Mediators Associated with Acute Lung Injury and Repair in a Murine Model of Influenza Pneumonia. Sci Rep 2016;6:26076.

160.Ivan FX, Rajapakse JC, Welsch RE, et al. Differential pulmonary transcriptomic profiles in murine lungs infected with low 
and highly virulent influenza H3N2 viruses reveal dysregulation of TREM1 signaling cytokines, and chemokines. Functional \& integrative genomics 2012;12:105-17.

161.Ivan FX, Tan KS, Phoon MC, et al. Neutrophils infected with highly virulent influenza H3N2 virus exhibit augmented early cell death and rapid induction of type I interferon signaling pathways. Genomics 2013;101:101-12.

162.Li N, Parrish M, Chan TK, et al. Influenza infection induces host DNA damage and dynamic DNA damage responses during tissue regeneration. Cell Mol Life Sci 2015;72:2973-88.

163. Raval F, Nikolajczyk BS. The Bidirectional Relationship between Metabolism and Immune Responses. Discoveries 2013;1:e6.

164. Thomas M, Yawn BP, Price D, Lund V, Mullol J, Fokkens W. EPOS Primary Care Guidelines: European Position Paper on the Primary Care Diagnosis and Management of Rhinosinusitis and Nasal Polyps 2007 - a summary. Prim Care Respir J 2008;17:79-89.

165. Steurer J, Held U, Bachmann LM, Holzmann $D$, Ott P, Miettinen OS. Clinical diagnosis of acute bacterial rhinosinusitis, typical of experts. J Eval Clin Pract 2009;15:614-9.

166. Ashworth M, Charlton J, Ballard K, Latinovic $R$, Gulliford M. Variations in antibiotic prescribing and consultation rates for acute respiratory infection in UK general practices 1995-2000. Br J Gen Pract 2005;55:603-8.

167. Hansen JG. Management of acute rhinosinusitis in Danish general practice: a survey. Clin Epidemiol 2011;3:213-6.

168. Meltzer EO, Hamilos DL. Rhinosinusitis diagnosis and management for the clinician: a synopsis of recent consensus guidelines. Mayo Clin Proc 2011;86:427-43.

169. Desrosiers M, Evans GA, Keith PK, et al. Canadian clinical practice guidelines for acute and chronic rhinosinusitis. Otolaryngol Head Neck Surg 2011;40 Suppl 2:S99-193.

170.Klossek JM, Mesbah K. Presentation and treatment of acute maxillary sinusitis in general practice: a French observational study. Rhinology 2011;49:84-9.

171. Hoffmans R, Schermer T, van Weel C, Fokkens W. Management of rhinosinusitis in Dutch general practice. Prim Care Respir J 2011;20:64-70.

172. McQuillan L, Crane LA, Kempe A. Diagnosis and management of acute sinusitis by pediatricians. Pediatrics 2009;123:e193-8.

173. Piatt JH, Jr. Intracranial suppuration complicating sinusitis among children: an epidemiological and clinical study. J Neurosurg Pediatr 2011;7:567-74

174. Kristo A, Uhari M. Timing of rhinosinusitis complications in children. Pediatr Infect Dis J 2009;28:769-71.

175. Hicks CW, Weber JG, Reid JR, Moodley $M$. Identifying and managing intracranial complications of sinusitis in children a retrospective series. Pediatr Infect Dis J 2011;30:222-6.

176. Wang DY, Wardani RS, Singh K, et al. A sur- vey on the management of acute rhinosinusitis among Asian physicians. Rhinology 2011:49:264-71

177.Dykewicz MS. 7. Rhinitis and sinusitis. J Allergy Clin Immunol 2003;111:S520-9.

178. Berg O, Carenfelt C. Analysis of symptoms and clinical signs in the maxillary sinus empyema. Acta Otolaryngol 1988;105:3439.

179. Williams Jr. JW, Simel DL, Roberts L, Samsa GP. Clinical evaluation for sinusitis. Making the diagnosis by history and physical examination. Ann Intern Med 1992;1 17:705-10.

180.Spector S. Parameters for the diagnosis and management of sinusitis J Allergy Clin Immunol 1998;dec:107-44.

181.Damm M, Quante G, Jungehuelsing M, Stennert E. Impact of functional endoscopic sinus surgery on symptoms and quality of life in chronic rhinosinusitis. Laryngoscope 2002;112:310-5.

182. Benninger MS, Senior BA. The development of the Rhinosinusitis Disability Index. Arch Otolaryngol Head Neck Surg 1997;123:1175-9.

183. Metson RB, Gliklich RE. Clinical outcomes in patients with chronic sinusitis. Laryngoscope 2000;110:24-8.

184. Ebell MH, McKay B, Dale A, Guilbault R, Ermias Y. Accuracy of Signs and Symptoms for the Diagnosis of Acute Rhinosinusitis and Acute Bacterial Rhinosinusitis. Ann Fam Med 2019;17:164-72.

185. Hansen JG, Hojbjerg T, Rosborg J. Symptoms and signs in culture-proven acute maxillary sinusitis in a general practice population. APMIS 2009;117:724-9.

186. Lindbaek M, Hjortdahl P, Johnsen UL. Use of symptoms, signs, and blood tests to diagnose acute sinus infections in primary care: comparison with computed tomography. Fam Med 1996;28:183-8.

187. Ebell MH, McKay B, Guilbault R, Ermias Y. Diagnosis of acute rhinosinusitis in primary care: a systematic review of test accuracy. $\mathrm{Br}$ J Gen Pract 2016;66:e612-e32.

188. Hansen JG, Lund E. The association between paranasal computerized tomography scans and symptoms and signs in a general practice population with acute maxillary sinusitis. APMIS 2011;119:44-8.

189. Hansen JG, Schmidt H, Rosborg J, Lund E. Predicting acute maxillary sinusitis in a general practice population. BMJ 1995;311:2336.

190.Dilger AE, Peters AT, Wunderink RG, et al. Procalcitonin as a Biomarker in Rhinosinusitis: A Systematic Review. American Journal of Rhinology \& Allergy 2018:33:103-12.

191. Gwaltney Jr. JM, Gwaltney. Rhinovirus infection of the normal human airway. Am J Respir Crit Care Med 1995;152:9.

192. Brozek JL, Bousquet J, Baena-Cagnani CE, et al. Allergic Rhinitis and its Impact on Asthma (ARIA) guidelines: 2010 revision. J Allergy Clin Immunol 2010;126:466-76.

193. Kasapoglu F, Coskun H, Ozmen OA, Akalin $\mathrm{H}$, Ener B. Acute invasive fungal rhinosinusi- tis: evaluation of 26 patients treated with endonasal or open surgical procedures. Otolaryngol Head Neck Surg 2010;143:61420.

194. Suslu AE, Ogretmenoglu O, Suslu N, Yucel OT, Onerci TM. Acute invasive fungal rhinosinusitis: our experience with 19 patients. Eur Arch Otorhinolaryngol 2009;266:77-82.

195. Marshall AH, Jones NS, Robertson IJ. CSF rhinorrhoea: the place of endoscopic sinus surgery. Br J Neurosurg 2001;15:8-12.

196. Padua FG, Bezerra TF, Voegels RL, Bento RF. The efficacy of functional endoscopic sinus surgery in the evolution of fever of unknown origin in ICU patients. Acta Otolaryngol 2011;131:166-72.

197. Jardim Vieira FM, Nunes da Silva R, Stefanini $R$, et al. Safety of sphenoid aspiration for diagnosis and treatment of intensive care unit rhinosinusitis. Am J Rhinol Allergy 2010;24:389-91.

198. Kenealy T, Arroll B. Antibiotics for the common cold and acute purulent rhinitis. Cochrane Database Syst Rev 2013:CD000247.

199. Hayward G, Thompson MJ, Perera R, Del Mar CB, Glasziou PP, Heneghan CJ. Corticosteroids for the common cold. Cochrane Database Syst Rev 2015;2016.

200.De Sutter Al, Saraswat A, van Driel ML. Antihistamines for the common cold. Cochrane Database Syst Rev 2015:CD009345.

201. Deckx L, De Sutter Al, Guo L, Mir NA, van Driel ML. Nasal decongestants in monotherapy for the common cold. Cochrane Database Syst Rev 2016;10:CD009612.

202.Li S, Yue J, Dong BR, Yang M, Lin X, Wu T. Acetaminophen (paracetamol) for the common cold in adults. Cochrane Database Syst Rev 2013:CD008800.

203.Kim SY, Chang YJ, Cho HM, Hwang YW, Moon YS. Non-steroidal anti-inflammatory drugs for the common cold. Cochrane Database Syst Rev 2009:CD006362.

204. De Sutter Al, van Driel ML, Kumar AA, Lesslar O, Skrt A. Oral antihistamine-decongestant-analgesic combinations for the common cold. Cochrane Database Syst Rev 2012:CD004976.

205. AlBalawi ZH, Othman SS, Alfaleh K. Intranasal ipratropium bromide for the common cold. Cochrane Database Syst Rev 2013;6:CD008231.

206. King D, Mitchell B, Williams CP, Spurling GK. Saline nasal irrigation for acute upper respiratory tract infections. Cochrane Database Syst Rev 2015:CD006821.

207. Singh AK, Gupta P, Verma N, et al. Fungal rhinosinusitis: Microbiological and histopathological perspective. J Clin Diagnostic Res 2017;11:DC10-DC2.

208. Quick M. Cochrane commentary: Probiotics for prevention of acute upper respiratory infection. Explore: The Journal of Science and Healing 2015;11:418-20.

209. Hemila H, Chalker E. Vitamin C for preventing and treating the common cold. Cochrane Database Syst Rev 
2013:CD000980

210. Quidel S, Gomez E, Bravo-Soto G, Ortigoza A. What are the effects of vitamin $C$ on the duration and severity of the common cold? Medwave 2018;18:e7261.

211. Gomez E, Quidel S, Bravo-Soto G, Ortigoza A. Does vitamin $C$ prevent the common cold? Medwave 2018;18:e7235.

212.Ran L, Zhao W, Wang J, et al. Extra Dose of Vitamin C Based on a Daily Supplementation Shortens the Common Cold: A Meta-Analysis of 9 Randomized Controlled Trials. BioMed Research International 2018;2018:1837634.

213. Vorilhon P, Arpajou B, Vaillant Roussel H, Merlin E, Pereira B, Cabaillot A. Efficacy of vitamin $C$ for the prevention and treatment of upper respiratory tract infection. A metaanalysis in children. Eur J Clin Pharmacol 2018;21:21

214. Simancas-Racines D, Franco JV, Guerra CV, Felix ML, Hidalgo R, Martinez-Zapata MJ. Vaccines for the common cold. Cochrane Database Syst Rev 2017;2017.

215. Lee HK, Hwang IH, Kim SY, Pyo SY. The effect of exercise on prevention of the common cold: a meta-analysis of randomized controlled trial studies. Korean Journal of Family Medicine 2014;35:119-26.

216. Karsch-Volk M, Barrett B, Kiefer D, Bauer R, Ardjomand-Woelkart K, Linde K. Echinacea for preventing and treating the common cold. Cochrane Database Syst Rev 2014:CD000530.

217.Singh M, Das RR. Zinc for the common cold. Cochrane Database Syst Rev 2011;2013:CD001364.

218. Hemila H, Petrus EJ, Fitzgerald JT, Prasad A. Zinc acetate lozenges for treating the common cold: an individual patient data metaanalysis. Br J Clin Pharmacol 2016;82:1393-8.

219. Hemila H, Fitzgerald JT, Petrus EJ, Prasad A. Zinc Acetate Lozenges May Improve the Recovery Rate of Common Cold Patients: An Individual Patient Data MetaAnalysis. Open Forum Infectious Diseases 2017;4:ofx059.

220. Koch AK, Klose $P$, Lauche $R$, et al. [A Systematic Review of Phytotherapy for Acute Rhinosinusitis]. Forschende Komplementarmedizin (2006) 2016;23:1659.

221. Jund R, Mondigler M, Steindl H, Stammer H, Stierna P, Bachert C. Clinical efficacy of a herbal drug combination in acute viral rhinosinusitis. MMW fortschritte der medizin 2015;157:6-11.

222. Jund R, Mondigler M, Steindl H, Stammer H, Stierna P, Bachert C. Clinical efficacy of a dry extract of five herbal drugs in acute viral rhinosinusitis. Rhinology 2012;50:417-26.

223. Vishnyakov VV, Sinkov DE. Herbal medicine as add-on therapy in acute Rhinosinusitis: results of an open randomized cohort study with the herbal combination Sinupret. Zeitschrift fur phytotherapie 2013;34:262-5.

224. Popovich VI, Koshel IV. Sinupret as addon therapy to saline irrigation for children with acute Post-Viral Rhinosinusitis. Clinical
Phytoscience 2017;3.

225.Kehrl W, Sonnemann U, Dethlefsen U. Therapy for acute nonpurulent rhinosinusitis with cineole: results of a doubleblind, randomized, placebo-controlled trial. Laryngoscope 2004;114:738-42.

226. Tesche S, Metternich F, Sonnemann U, Engelke JC, Dethlefsen U. The value of herbal medicines in the treatment of acute nonpurulent rhinosinusitis. Results of a doubleblind, randomised, controlled trial. Eur Arch Otorhinolaryngol 2008;265:1355-9.

227. Cáceres DD, Hancke JL, Burgos RA, S, berg F, Wikman GK. Use of visual analogue scale measurements (VAS) to asses the effectiveness of standardized Andrographis paniculata extract SHA-10 in reducing the symptoms of common cold. A randomized double blind-placebo study. Phytomedicine 1999;6:217-23.

228. Gabrielian ES, Shukarian AK, Goukasova GI, et al. A double blind, placebo-controlled study of Andrographis paniculata fixed combination Kan Jang in the treatment of acute upper respiratory tract infections including sinusitis. Phytomedicine 2002;9:589-97.

229. Hawkins J, Baker C, Cherry L, Dunne E. Black elderberry (Sambucus nigra) supplementation effectively treats upper respiratory symptoms: A meta-analysis of randomized, controlled clinical trials. Complement Ther Med 2019;42:361-5.

230.Lund VJ, Grouin JM, Eccles R, Bouter C, Chabolle F. Efficacy of fusafungine in acute rhinopharyngitis: a pooled analysis. Rhinology 2004;42:207-12.

231. Fokkens WJJ, Lund VJJ, Mullol J, et al. EPOS 2012: European position paper on rhinosinusitis and nasal polyps 2012. A summary for otorhinolaryngologists. Rhinology 2012;50:1-12.

232. Lindbaek M, Hjortdahl P, Johnsen UL. Randomised, double blind, placebo controlled trial of penicillin $V$ and amoxycillin in treatment of acute sinus infections in adults. BMJ (clinical research ed) 1996;313:325-9.

233. Hadley JA, Mösges R, Desrosiers M, Haverstock D, van Veenhuyzen D, HermanGnjidic Z. Moxifloxacin five-day therapy versus placebo in acute bacterial rhinosinusitis. Laryngoscope 2010;120:1057-62.

234. Høsøien E, Lund AB, Vasseljen O. Similar effect of therapeutic ultrasound and antibiotics for acute bacterial rhinosinusitis: a randomised trial. J Physiother 2010;56:29-32.

235. Wald ER, Nash D, Eickhoff J. Effectiveness of amoxicillin/clavulanate potassium in the treatment of acute bacterial sinusitis in children. Pediatrics 2009;124:9-15.

236. Ragab A, Farahat T, Al-Hendawy G, Samaka R, Ragab S, El-Ghobashy A. Nasal saline irrigation with or without systemic antibiotics in treatment of children with acute rhinosinusitis. Int J Pediatr Otorhinolaryngol 2015;79:2178-86.

237.Garbutt JM, Banister C, Spitznagel E, Piccirillo JF. Amoxicillin for acute rhinosi- nusitis: a randomized controlled trial. JAMA 2012;307:685-92.

238. Meltzer EO, Bachert C, Staudinger H. Treating acute rhinosinusitis: comparing efficacy and safety of mometasone furoate nasal spray, amoxicillin, and placebo. J Allergy Clin Immunol 2005;1 16:1289-95.

239. Merenstein D, Whittaker C, Chadwell T, Wegner B, D'Amico F. Are antibiotics beneficial for patients with sinusitis complaints? A randomized double-blind clinical trial. J Fam Pract 2005;54:144-51.

240. Haye R, Lingaas E, Høivik HO, Odegård T. Azithromycin versus placebo in acute infectious rhinitis with clinical symptoms but without radiological signs of maxillary sinusitis. Eur J Clin Microbiol Infect Dis 1998;17:309-12.

241.Lindbaek M, Kaastad E, Dølvik S, Johnsen U, Laerum E, Hjortdahl P. Antibiotic treatment of patients with mucosal thickening in the paranasal sinuses, and validation of cut-off points in sinus CT. Rhinology 1998:36:7-11.

242. Stalman W, van Essen GA, van der Graaf Y, de Melker RA. The end of antibiotic treatment in adults with acute sinusitis-like complaints in general practice? A placebocontrolled double-blind randomized doxycycline trial. Br J Gen Pract 1997;47:794-9.

243.van Buchem FL, Knottnerus JA, Schrijnemaekers VJ, et al. Primary-carebased randomised placebo-controlled trial of antibiotic treatment in acute maxillary sinusitis. Lancet 1997;349:683-7.

244.Garbutt JM, Goldstein M, Gellman E, Shannon W, Littenberg B. A randomized, placebo-controlled trial of antimicrobial treatment for children with clinically diagnosed acute sinusitis. Pediatrics 2001;107:619-25.

245. Kristo A, Uhari M, Luotonen J, Ilkko E, Koivunen P, Alho OP. Cefuroxime axetil versus placebo for children with acute respiratory infection and imaging evidence of sinusitis: a randomized, controlled trial. Acta Paediatr 2005;94:1208-13.

246. Khoshdel A, Panahande GR, Noorbakhsh MK, Malek Ahmadi MR, Lotfizadeh M, Parvin N. A comparison of the efficacy of amoxicillin and nasal irrigation in treatment of acute sinusitis in children. Korean J Pediatr 2014;57:479-83.

247.Bell BG, Schellevis F, Stobberingh E, Goossens H, Pringle M. A systematic review and meta-analysis of the effects of antibiotic consumption on antibiotic resistance. BMC Infect Dis 2014;14:13.

248. Goossens H, Ferech M, Vander Stichele R, Elseviers M. Outpatient antibiotic use in Europe and association with resistance: a cross-national database study. Lancet 2005;365:579-87.

249.Dolk FCK, Pouwels KB, Smith DRM, Robotham JV, Smieszek T. Antibiotics in primary care in England: which antibiotics are prescribed and for which conditions? J Antimicrob Chemother 2018;73:ii2-iii 0.

250. Antimicrobial resistance surveillance in Europe 2013 data: European Centre for 
Disease Prevention and Control, 2014.

251. Summary of the latest data on antibiotic consumption in the European Union. 2017. https://www.ecdc.europa.eu/sites/ portal/files/documents/Final_2017_EAAD_ ESAC-Net_Summary-edited $\% 20-\% 20$ FINALwith\%20erratum.pdf.

252. Costelloe C, Metcalfe C, Lovering A, Mant D, Hay AD. Effect of antibiotic prescribing in primary care on antimicrobial resistance in individual patients: systematic review and meta-analysis. Bmj 2010;340:c2096.

253. Cordoba G, Siersma V, Lopez-Valcarcel B, et al. Prescribing style and variation in antibiotic prescriptions for sore throat: crosssectional study across six countries. BMC Fam Pract 2015;16:7.

254. Lemiengre MB, Verbakel JY, Colman R, et al. Reducing inappropriate antibiotic prescribing for children in primary care: a cluster randomised controlled trial of two interventions. Br J Gen Pract 2018;68:e204-e10

255. Vervloet M, Meulepas MA, Cals JWL, Eimers M, Van Der Hoek LS, Van Dijk L. Reducing antibiotic prescriptions for respiratory tract infections in family practice: results of a cluster randomized controlled trial evaluating a multifaceted peer-group-based intervention. Npj primary care respiratory medicine 2016;26.

256.van der Velden AW, Kuyvenhoven MM, Verheij TJM. Improving antibiotic prescribing quality by an intervention embedded in the primary care practice accreditation: The ARTI4 randomized trial. J Antimicrob Chemother 2016;71:257-63.

257. Rebnord IK, S, vik H, Batman Mjelle A, Hunskaar S. Out-of-hours antibiotic prescription after screening with $C$ reactive protein: a randomised controlled study. BMJ open 2016;6.

258. Persell SD, Doctor JN, Friedberg MW, et al. Behavioral interventions to reduce inappropriate antibiotic prescribing: a randomized pilot trial. BMC Infect Dis 2016;16:373.

259. Meeker D, Linder JA, Fox CR, et al. Effect of Behavioral Interventions on Inappropriate Antibiotic Prescribing Among Primary Care Practices: a Randomized Clinical Trial. JAMA 2016;315:562-70.

260. Keith PK, Dymek A, Pfaar O, et al. Fluticasone furoate nasal spray reduces symptoms of uncomplicated acute rhinosinusitis: a randomised placebo-controlled study. Prim Care Respir J 2012;21:267-75

261. Meltzer EO, Gates D, Bachert C. Mometasone furoate nasal spray increases the number of minimal-symptom days in patients with acute rhinosinusitis. Annals of allergy, asthma and immunology 2012;108:275-9.

262. Williamson IG, Rumsby K, Benge S, et al. Antibiotics and topical nasal steroid for treatment of acute maxillary sinusitis: a randomized controlled trial. JAMA 2007;298:2487-96.

263. Bachert C, Meltzer EO. Effect of mometasone furoate nasal spray on quality of life of patients with acute rhinosinusitis.
Rhinology 2007;45:190-6.

264. Dolor RJ, Witsell DL, Hellkamp AS, Williams JW, Califf RM, Simel DL. Comparison of cefuroxime with or without intranasal fluticasone for the treatment of rhinosinusitis. The CAFFS Trial: a randomized controlled trial. JAMA 2001;286:3097-105.

265. Nayak AS, Settipane GA, Pedinoff A, et al. Effective dose range of mometasone furoate nasal spray in the treatment of acute rhinosinusitis. Annals of allergy, asthma \& immunology 2002;89:271-8.

266. Meltzer EO, Charous BL, Busse WW, Zinreich SJ, Lorber RR, Danzig MR. Added relief in the treatment of acute recurrent sinusitis with adjunctive mometasone furoate nasal spray. The Nasonex Sinusitis Group. J Allergy Clin Immunol 2000;106:630-7.

267. Svensson J, Lundberg J, Olsson P, Stjarne P, Tennvall GR. Cost-effectiveness of mometasone furoate nasal spray in the treatment of acute rhinosinusitis. Primary Care Respiratory Journal 2012;21:412-8.

268. Rahmati MB, Mohebi S, Shahmohammadi S, Rezai MS. Fluticasone nasal spray as an adjunct to Amoxicillin for acute sinusitis in children: a randomized controlled trial. Eur Rev Med Pharmacol Sci 2013;17:3068-72.

269. Barlan IB, Erkan E, Bakir M, Berrak S, Basaran MM. Intranasal budesonide spray as an adjunct to oral antibiotic therapy for acute sinusitis in children. Ann Allergy Asthma Immunol 1997;78:598-601.

270. Venekamp RP, Thompson MJ, Hayward G, et al. Systemic corticosteroids for acute sinusitis. Cochrane Database Syst Rev 2014;2014.

271. Venekamp RP, Bonten MJ, Rovers MM, Verheij TJ, Sachs AP. Systemic corticosteroid monotherapy for clinically diagnosed acute rhinosinusitis: a randomized controlled trial. CMAJ : Canadian Medical Association Journal 2012;184:E751-7.

272. Ratau NP, Snyman JR, Swanepoel C. Shortcourse, low-dose oral betamethasone as an adjunct in the treatment of acute infective sinusitis: a comparative study with placebo. Clin Drug Investig 2004;24:577-82.

273. Klossek JM, Desmonts-Gohler C, Deslandes $B$, et al. Treatment of functional signs of acute maxillary rhinosinusitis in adults: Efficacy and tolerance of administration of oral prednisone for 3 days. [French]. Presse Med 2004;33:303-9.

274. Gehanno P, Beauvillain C, Bobin S, et al. Short therapy with amoxicillin-clavulanate and corticosteroids in acute sinusitis: results of a multicentre study in adults. Scand J Infect Dis 2000;32:679-84.

275. McCormick DP, John SD, Swischuk LE, et al. A double-blind, placebo-controlled trial of decongestant-antihistamine for the treatment of sinusitis in children. Clin Pediatr (Phila) 1996;35:457-60.

276. Braun JJ, Alabert JP, Michel FB, et al. Adjunct effect of loratadine in the treatment of acute sinusitis in patients with allergic rhinitis. Allergy 1997;52:650-5.

277. Inanli S, Ozturk O, Korkmaz M, Tutkun A, Batman C. The effects of topical agents of fluticasone propionate, oxymetazoline, and $3 \%$ and $0.9 \%$ sodium chloride solutions on mucociliary clearance in the therapy of acute bacterial rhinosinusitis in vivo. Laryngoscope 2002;112:320-5.

278. Adam P, Stiffman M, Blake Jr. RL, P A, M $S$, Blake RL J. A clinical trial of hypertonic saline nasal spray in subjects with the common cold or rhinosinusitis. Arch Fam Med 1998;7:39-43.

279. Rabago D, Zgierska A, Mundt M, Barrett B, Bobula J, Maberry R. Efficacy of daily hypertonic saline nasal irrigation among patients with sinusitis: a randomized controlled trial. J Fam Pract 2002;51:1049-55.

280. Gelardi M, Mezzoli A, Fiorella ML, Carbonara M, Di Gioacchino M, Ciprandi G. Nasal irrigation with lavonase as ancillary treatment of acute rhinosinusitis: a pilot study. J Biol Regul Homeost Agents 2009;23:79-84.

281. Little P, Moore M, Kelly J, et al. Ibuprofen, paracetamol, and steam for patients with respiratory tract infections in primary care: pragmatic randomised factorial trial. BMJ : British Medical Journal 2013;347:f6041.

282. Little P, Stuart B, Mullee $M$, et al. Effectiveness of steam inhalation and nasal irrigation for chronic or recurrent sinus symptoms in primary care: a pragmatic randomized controlled trial. CMAJ 2016;188:940-9.

283. Gomez Barreto D, De la Torre C, Alvarez A, et al. Safety and efficacy of OM-85-BV plus amoxicillin/clavulanate in the treatment of subacute sinusitis and in the prevention of recurrent infections in children: Seguridad Y Eficacia De Om-85-Bv Mas Amoxicilina/ Clavulanato En El Tratamiento De La Sinusitis S. Allergol Immunopathol (Madr) 1998;26:17-22.

284. Friese KH, Zabalotnyi DI. Homeopathy in acute rhinosinusitis. A double-blind, placebo controlled study shows the effeciency and tolerability of a homeopathic combination remedy. [German]. HNO 2007;55:271-7.

285.Zabolotnyi DI, Kneis KC, Richardson A, et al. Efficacy of a complex homeopathic medication (Sinfrontal) in patients with acute maxillary sinusitis: a prospective, randomized, double-blind, placebo-controlled, multicenter clinical trial. Explore (new york, NY) 2007;3:98-109.

286. Kneis KC, Gandjour A. Economic evaluation of Sinfrontal in the treatment of acute maxillary sinusitis in adults. Applied health economics and health policy 2009;7:181-91.

287. Ponikau JU, Hamilos DL, Barreto A, et al. An exploratory trial of Cyclamen europaeum extract for acute rhinosinusitis. Laryngoscope 2012;122:1887-92.

288. Federspil P, Wulkow R, Zimmermann T. Effects of standardized Myrtol in therapy of acute sinusitis--results of a doubleblind, randomized multicenter study compared with placebo. Laryngorhinootologie 1997;76:23-7.

289. Neubauer N, MÃarz RW. Placebo-controlled, randomized double-blind clinical trial with Sinupret sugar coated tablets on the basis 
of a therapy with antibiotics and decongestant nasal drops in acute sinusitis Phytomedicine 1994;1:177-81.

290.Pfaar O, Mullol J, Anders C, Hormann K, Klimek L. Cyclamen europaeum nasal spray, a novel phytotherapeutic product for the management of acute rhinosinusitis: a randomized double-blind, placebo-controlled trial. Rhinology 2012;50:37-44.

291. Bachert C, Schapowal A, Funk P, Kieser M. Treatment of acute rhinosinusitis with the preparation from Pelargonium sidoides EPS 7630: a randomized, double-blind, placebocontrolled trial. Rhinology 2009;47:51-8.

292. Timmer A, Gunther J, Rucker G, Motschall E, Antes G, Kern WV. Pelargonium sidoides extract for acute respiratory tract infections. Cochrane Database Syst Rev 2008:CD006323.

293. Passali D, Loglisci M, Passali GC, Cassano P, Rodriguez HA, Bellussi LM. A prospective open-label study to assess the efficacy and safety of a herbal medicinal product (Sinupret) in patients with acute rhinosinusitis. ORL; Journal for Oto-rhino-laryng and its Related Specialties 2015;77:27-32.

294.Demicheli V, Jefferson T, Al-Ansary LA, Ferroni E, Rivetti A, Di Pietrantonj C. Vaccines for preventing influenza in healthy adults. Cochrane Database Syst Rev 2014;2014.

295. Myint TT, Madhava H, Balmer P, et al. The impact of 7-valent pneumococcal conjugate vaccine on invasive pneumococcal disease: a literature review. Adv Ther 2013;30:127-51.

296. Benninger MS. Acute bacterial rhinosinusitis and otitis media: changes in pathogenicity following widespread use of pneumococcal conjugate vaccine. Otolaryngol Head Neck Surg 2008;138:274-8.

297. Brook I, Gober AE. Frequency of recovery of pathogens from the nasopharynx of children with acute maxillary sinusitis before and after the introduction of vaccination with the 7-valent pneumococcal vaccine. Int J Pediatr Otorhinolaryngol 2007;71:5759.

298. Marom T, Alvarez-Fernandez PE, Jennings K, Patel JA, McCormick DP, Chonmaitree T. Acute bacterial sinusitis complicating viral upper respiratory tract infection in young children. Pediatr Infect Dis J 2014;33:803-8.

299. Ciofalo A, de Vincentiis M, Zambetti G, et al. Olfactory dysfunction in acute rhinosinusitis: intranasal sodium hyaluronate as adjuvant treatment. Eur Arch Otorhinolaryngol 2017:274:803-8

300. Unuvar E, Tamay Z, Yildiz I, et al. Effectiveness of erdosteine, a second generation mucolytic agent, in children with acute rhinosinusitis: a randomized, placebo controlled, double-blinded clinical study. Acta Paediatr 2010;99:585-9.

301. Hansen FS, Hoffmans R, Georgalas C, Fokkens WJ. Complications of acute rhinosinusitis in The Netherlands. Fam Pract 2012;29:147-53.

302. Fokkens WJ, Lund VJ, Mullol J, et al. EPOS
2012: European position paper on rhinosinusitis and nasal polyps 2012. A summary for otorhinolaryngologists. Rhinology 2012;50:1-12.

303. Wyler B, Mallon WK. Sinusitis Update. Emerg Med Clin North Am 2019;37:41-54.

304. Mehta VJ, Ling JD, Mawn LA. Socioeconomic Disparities in the Presentation of Acute Bacterial Sinusitis Complications in the Pediatric Population. Semin Ophthalmol 2016;31:405-8.

305. Quraishi H, Zevallos JP. Subdural empyema as a complication of sinusitis in the pediatric population. Int J Pediatr Otorhinolaryngol 2006;70:1581-6.

306. Domville-Lewis C, Friedland PL, Santa Maria PL. Pott's puffy tumour and intracranial complications of frontal sinusitis in pregnancy. J Laryngol Otol 2013;127 Suppl 1:S35-8.

307. Gilbey P, McGruthers L, Morency AM, Shrim A. Rhinosinusitis-related quality of life during pregnancy. Am J Rhinol Allergy 2012;26:283-6.

308. Lal D, Jategaonkar AA, Borish L, et al. Management of rhinosinusitis during pregnancy: systematic review and expert panel recommendations. Rhinology 2016;54:99104.

309. Chandler JR, Langenbrunner DJ, Stevens ER. The pathogenesis of orbital complications in acute sinusitis. Laryngoscope 1970:80:1414-28.

310. Sultesz M, Csakanyi Z, Majoros T, Farkas Z, Katona G. Acute bacterial rhinosinusitis and its complications in our pediatric otolaryngological department between 1997 and 2006. Int J Pediatr Otorhinolaryngol 2009;73:1507-12.

311. Tachibana T, Kariya S, Orita Y, et al. Factors that prolong the duration of recovery in acute rhinosinusitis with orbital complications. Acta Otolaryngol 2019;139:52-6.

312. Chaiyasate S, Fooanant S, Navacharoen N, Roongrotwattanasiri K, Tantilipikorn $\mathrm{P}_{1}$ Patumanond J. The complications of sinusitis in a tertiary care hospital: types, patient characteristics, and outcomes. Int Otolaryngol 2015;2015:709302.

313. Eufinger $\mathrm{H}$, Machtens E. Purulent pansinusitis, orbital cellulitis and rhinogenic intracranial complications. J Craniomaxillofac Surg 2001;29:111-7.

314. Miah MS, Nix P, Koukkoullis A, Sandoe J. Microbial causes of complicated acute bacterial rhinosinusitis and implications for empirical antimicrobial therapy. J Laryngol Otol 2016;130:169-75

315. Mortimore S, Wormald PJ. The Groote Schuur hospital classification of the orbital complications of sinusitis. J Laryngol Otol 1997;111:719-23.

316. Oxford LE, McClay J. Complications of acute sinusitis in children. Otolaryngology - Head \& Neck Surgery 2005;133:32-7.

317. Sedaghat AR, Wilke CO, Cunningham MJ, Ishman SL. Socioeconomic disparities in the presentation of acute bacterial sinusitis complications in children. Laryngoscope
2014:124:1700-6.

318. Lee WH, Kim JW, Lim JS, Kong IG, Choi HG. Chronic rhinosinusitis increases the risk of hemorrhagic and ischemic stroke: A longitudinal follow-up study using a national sample cohort. PLoS One 2018;13:e0193886.

319. Sharma S, Josephson GD. Orbital complications of acute sinusitis in infants: a systematic review and report of a case. JAMA Otolaryngol Head Neck Surg 2014;140:1070-3.

320. Capra G, Liming B, Boseley ME, Brigger MT. Trends in orbital complications of pediatric rhinosinusitis in the United States. JAMA Otolaryngol Head Neck Surg 2015;141:12-7.

321. Torretta S, Guastella C, Marchisio P, et al. Sinonasal-Related Orbital Infections in Children: A Clinical and Therapeutic Overview. J Clin Med 2019;8.

322. El Mograbi A, Ritter A, Najjar E, Soudry E. Orbital Complications of Rhinosinusitis in the Adult Population: Analysis of Cases Presenting to a Tertiary Medical Center Over a 13-Year Period. Ann Otol Rhinol Laryngol 2019;128:563-8.

323. Schollin Ask L, Hultman Dennison S, Stjarne $P$, et al. Most preschool children hospitalised for acute rhinosinusitis had orbital complications, more common in the youngest and among boys. Acta Paediatr 2017;106:268-73.

324. Clayman GL, Adams GL, Paugh DR, Koopmann Jr. CF. Intracranial complications of paranasal sinusitis: a combined institutional review. Laryngoscope 1991;101:2349.

325. Wan Y, Shi G, Wang H. Treatment of Orbital Complications Following Acute Rhinosinusitis in Children. Balkan Med J 2016:33:401-6.

326.Georgakopoulos CD, Eliopoulou MI, Stasinos S, Exarchou A, Pharmakakis N, Varvarigou A. Periorbital and orbital cellulitis: a 10-year review of hospitalized children. Eur J Ophthalmol 2010;20:1066-72.

327. Mekhitarian Neto L, Pignatari S, Mitsuda S, Fava AS, Stamm A. Acute sinusitis in children: a retrospective study of orbital complications. Braz J Otorhinolaryngol 2007;73:75-9.

328. Dennison SH, Ask LS, Eriksson M, et al. Serious complications due to acute rhinosinusitis in children up to five years old in Stockholm, Sweden - Still a challenge in the pneumococcal conjugate vaccine era. Int J Pediatr Otorhinolaryngol 2019;121:50-4.

329. Pena MT, Preciado D, Orestes M, Choi S. Orbital complications of acute sinusitis: changes in the post-pneumococcal vaccine era. JAMA Otolaryngol Head Neck Surg 2013;139:223-7.

330. Chen J-H, Chen M-C, Ho Y-H, Chong P-N. A rare case of septic cavernous sinus thrombosis as a complication of sphenoid sinusitis. Tzu Chi Medical Journal 2019;31:63.

331. Park HW. Orbital subperiosteal hematoma associated with sinus infection. Rhinology Journal 2010;48.

332. Rimal D. An unusual presentation of sphenoid sinusitis with septicaemia in a healthy 
young adult. Emergency Medicine Journal 2006;23:e36-e.

333. Al-Madani MV, Khatatbeh AE, Rawashdeh RZ, Al-Khtoum NF, Shawagfeh NR. The prevalence of orbital complications among children and adults with acute rhinosinusitis. Braz J Otorhinolaryngol 2013;79:716-9.

334. Chang YS, Chen PL, Hung JH, et al. Orbital complications of paranasal sinusitis in Taiwan, 1988 through 2015: Acute ophthalmological manifestations, diagnosis, and management. PLoS One 2017;12:e0184477.

335. Radovani P, Vasili D, Xhelili M, Dervishi J. Orbital complications of sinusitis. Balkan Med J 2013;30:151-4.

336. Dankbaar JW, van Bemmel AJ, Pameijer FA. Imaging findings of the orbital and intracranial complications of acute bacterial rhinosinusitis. Insights Imaging 2015;6:509-18.

337. Ho CF, Huang YC, Wang CJ, Chiu CH, Lin TY. Clinical analysis of computed tomography-staged orbital cellulitis in children. J Microbiol Immunol Infect 2007;40:518-24.

338. Eviatar E, Gavriel H, Pitaro K, Vaiman M, Goldman M, Kessler A. Conservative treatment in rhinosinusitis orbital complications in children aged 2 years and younger. Rhinology 2008;46:334-7.

339. Constantin F, Niculescu PA, Petre O, et al. Orbital cellulitis and brain abscess - rare complications of maxillo-spheno-ethmoidal rhinosinusitis. Rom J Ophthalmol 2017:61:133-6.

340. Sanan A, Shumrick C, Nyquist G, Rosen M. Intra-optic nerve abscess: A rare complication of acute sinusitis. Otolaryngology Case Reports 2017;2:13-5.

341. Gavriel H, Jabarin B, Israel O, Eviatar E. Conservative Management for Subperiosteal Orbital Abscess in Adults: A 20-Year Experience. Ann Otol Rhinol Laryngol 2018;127:162-6.

342. Hoxworth JM, Glastonbury CM. Orbital and intracranial complications of acute sinusitis. Neuroimaging Clin N Am 2010;20:511-26.

343. Huang SF, Lee TJ, Lee YS, Chen CC, Chin SC, Wang NC. Acute rhinosinusitis-related orbital infection in pediatric patients: a retrospective analysis. Ann Otol Rhinol Laryngol 2011;120:185-90.

344. Jabarin B, Marom T, Gavriel H, Eviatar E, Pitaro J. Orbital complications secondary to acute rhinosinusitis in toddlers: A unique age group. Int J Pediatr Otorhinolaryngol 2019;121:46-9.

345. Siedek V, Kremer A, Betz CS, Tschiesner U, Berghaus A, Leunig A. Management of orbital complications due to rhinosinusitis. Eur Arch Otorhinolaryngol 2010;267:1881-6.

346. Trivic A, Cevik M, Folic M, et al. Management of Orbital Complications of Acute Rhinosinusitis in Pediatric Patients: A 15-Year Single-Center Experience. Pediatr Infect Dis J 2019, 10.1097/ INF.0000000000002414.

347.Jones NS, Walker JL, Bassi S, Jones T, Punt J. The intracranial complications of rhinosinusitis: can they be prevented?
Laryngoscope 2002;112:59-63.

348. Oxford LE, McClay J. Medical and surgical management of subperiosteal orbital abscess secondary to acute sinusitis in children. Int J Pediatr Otorhinolaryngol 2006;70:1853-61.

349. Younis RT, Lazar RH, Anand VK. Intracranial complications of sinusitis: a 15-year review of 39 cases. Ear Nose Throat J 2002:81:6368,40-42,44.

350. Wong SJ, Levi J. Management of pediatric orbital cellulitis: A systematic review. Int J Pediatr Otorhinolaryngol 2018;110:123-9.

351.Todman MS, Enzer YR. Medical management versus surgical intervention of pediatric orbital cellulitis: the importance of subperiosteal abscess volume as a new criterion. Ophthal Plast Reconstr Surg 2011;27:255-9.

352.Ismi O, Vayisoğlu Y, Bal KK, Helvaci I, Görür K, Ozcan C. Surgical Treatment of Rhinosinusitis-Related Orbital Complications: Factors Affecting Irreversible Blindness. J Craniofac Surg 2018;29:1294-9.

353. Albu S, Tomescu E, Bassam S, Merca Z. Intracranial complications of sinusitis. Acta Otorhinolaryngol Belg 2001;55:265-72.

354. Bayonne E, Kania R, Tran P, Huy B, Herman P. Intracranial complications of rhinosinusitis. A review, typical imaging data and algorithm of management*. Rhinology 2009;47:59-65.

355. DelGaudio JM, Evans SH, Sobol SE, Parikh SL. Intracranial complications of sinusitis: what is the role of endoscopic sinus surgery in the acute setting. Am J Otolaryngol 2010;31:25-8

356. Gallagher RM, Gross CW, Phillips CD. Suppurative intracranial complications of sinusitis. Laryngoscope 1998;108:1635-42.

357. Germiller J, Monin D, Sparano A, Tom L. Intracranial Complications of Sinusitis in Children and Adolescents and Their Outcomes Archives of Otolaryngology -Head \& Neck Surgery 2006;132:969-76.

358. Patel AB, Hoxworth JM, Lal D. Orbital Complications Associated with the Treatment of Chronic Rhinosinusitis. Otolaryngol Clin North Am 2015;48:749-68.

359. Garin A, Thierry B, Leboulanger N, et al. Pediatric sinogenic epidural and subdural empyema: The role of endoscopic sinus surgery. Int J Pediatr Otorhinolaryngol 2015;79:1752-60.

360. Suzuki N, Suzuki M, Araki S, Sato H. A case of multiple cranial nerve palsy due to sphenoid sinusitis complicated by cerebral aneurysm. Auris Nasus Larynx 2005;32:4159.

361.Schupper AJ, Jiang W, Coulter MJ, Brigger M, Nation J. Intracranial complications of pediatric sinusitis: Identifying risk factors associated with prolonged clinical course. Int J Pediatr Otorhinolaryngol 2018;112:105.

362. Kou YF, Killeen D, Whittemore B, et al. Intracranial complications of acute sinusitis in children: The role of endoscopic sinus surgery. Int J Pediatr Otorhinolaryngol 2018;110:147-51.

363. Giannoni CM, Stewart MG, Alford EL. Intracranial complications of sinusitis. Laryngoscope 1997;107:863-7.

364. Hamill CS, Sykes KJ, Harrison CJ, Weatherly RA. Infection rates of MRSA in complicated pediatric rhinosinusitis: An up to date review. Int J Pediatr Otorhinolaryngol 2018;104:79-83.

365. Deutschmann MW, Livingstone D, Cho JJ, Vanderkooi OG, Brookes JT. The significance of Streptococcus anginosus group in intracranial complications of pediatric rhinosinusitis. JAMA Otolaryngol Head Neck Surg 2013;139:157-60.

366. Khamassi K, Mahfoudhi M, Ben Yahia A, et al. Management of Intracranial Complications of Sinusitis. Open Journal of Clinical Diagnostics 2015;5:86-95.

367. Mulvey $C L$, Kiell EP, Rizzi MD, Buzi A. The Microbiology of Complicated Acute Sinusitis among Pediatric Patients: A Case Series. Otolaryngol Head Neck Surg 2019;160:712-9.

368. Ogunleye AO, Nwaorgu OG, Lasisi AO. Complications of sinusitis in Ibadan, Nigeria. West Afr J Med 2001;20:98-101.

369. van der Poel NA, Mourits MP, de Win MML, Coutinho JM, Dikkers FG. Prognosis of septic cavernous sinus thrombosis remarkably improved: a case series of 12 patients and literature review. Eur Arch Otorhinolaryngol 2018;275:2387-95.

370. Lize F, Verillaud B, Vironneau P, et al. Septic cavernous sinus thrombosis secondary to acute bacterial sinusitis: a retrospective study of seven cases. Am J Rhinol Allergy 2015;29:e7-12.

371. Ball SL, Carrie S. Complications of rhinosinusitis. BMJ 2016;352:i795

372. Leong SC. Minimally Invasive Surgery for Pott's Puffy Tumor: Is It Time for a Paradigm Shift in Managing a 250-Year-Old Problem? Ann Otol Rhinol Laryngol 2017;126:433-7.

373. Josephson JS, Rosenberg SI. Sinusitis. Clin Symp 1994;46:1-32.

374. Uijen JH, Bindels PJ, Schellevis FG, van der Wouden JC. ENT problems in Dutch children: trends in incidence rates, antibiotic prescribing and referrals 2002-2008. Scand J Prim Health Care 2011;29:75-9.

375. Oskarsson JP, Halldorsson S. [An evaluation of diagnosis and treatment of acute sinusitis at three health care centers]. Laeknabladid 2010;96:531-5.

376. Kaliner MA, Osguthorpe JD, Fireman P, et al. Sinusitis: bench to bedside. Current findings, future directions. J Allergy Clin Immunol 1997;99:S829-48.

377. Bhattacharyya N. Contemporary assessment of the disease burden of sinusitis. Am J Rhinol Allergy 2009;23:392-5.

378. Fokkens W, Lund V, Mullol J, et al. European position paper on rhinosinusitis and nasal polyps 2007. Rhinology 2007;Suppl. 20:1136.

379. Cherry DK, Woodwell DA, Rechsteiner EA. In 
National aumbulatory medical care survey: 2005 summary., Hyattsville, 2007.

380.Louie JK, Hacker JK, Gonzales R, et al. Characterization of viral agents causing acute respiratory infection in a San Francisco University Medical Center Clinic during the influenza season. Clin Infect Dis 2005;41:822-8.

381. Varonen H, Rautakorpi UM, Huikko S, et al. Management of acute maxillary sinusitis in Finnish primary care. Results from the nationwide MIKSTRA study. Scand J Prim Health Care 2004;22:122-7.

382. Rautakorpi UM, Klaukka T, Honkanen P, et al. Antibiotic use by indication: a basis for active antibiotic policy in the community. Scand J Infect Dis 2001;33:920-6.

383. Nicoli TK, Oinas M, Niemela M, Makitie AA, Atula T. Intracranial Suppurative Complications of Sinusitis. Scand J Surg 2016;105:254-62.

384. Piatt Jr. JH. Intracranial suppuration complicating sinusitis among children: an epidemiological and clinical study. J Neurosurg Pediatr 2011;7:567-74.

385. Li N, Yu LG, Chen M, Yang L, Qin ZR, Jiang Y. [Clinical analysis of orbital complications due to rhinosinusitis in 28 cases]. Zhonghua Er Bi Yan Hou Tou Jing Wai Ke Za Zhi 2017;52:664-9.

386. Abou-Al-Shaar H, Mulvaney G, Alzhrani G, Gozal Y, Oakley G, Couldwell W. Nocardial clival osteomyelitis secondary to sphenoid sinusitis: an atypical skull base infection. Acta Neurochir 2019;161:529-34.

387. Fabre C, Atallah I, Wroblewski I, Righini CA.
Maxillary sinusitis complicated by stroke. Eur Ann Otorhinolaryngol Head Neck Dis 2018;135:449-51.

388. Righini CA, Bing F, Bessou P, Boubagra K, Reyt $E$. An acute ischemic stroke secondary to sphenoid sinusitis. Ear Nose Throat $J$ 2009;88:E23-8.

389. Korkmaz M, Güven M, Asil K. An Unusual Complication of Acute Sinusitis: Isolated Unilateral Ptosis. Annals of Otolaryngology and Rhinology 2017:4:1178.

390. Huth ME, Caversaccio MD Thrombophlebitis of the temporal vein as an extracranial complication of acute bacterial rhinosinusitis. Ear Nose Throat $J$ 2015;94:E48-51.

391.Tien DA, Krakovitz P, Anne S. Nasal septal abscess in association with pediatric acute rhinosinusitis. Int J Pediatr Otorhinolaryngol 2016;91:27-9.

392. Jurkiewicz D, Zielnik-Jurkiewicz B. Bacterial lysates in the prevention of respiratory tract infections. Otolaryngologia Polska 2018;72:1-8.

393. Gradoni P, Fois P. Nasal septal abscess complicating isolated acute sphenoiditis: case report and literature review. B-ent 2010;6:303-5.

394. Nomura K, Honkura Y, Ozawa D, et al. Endoscopic drainage of orbital subperiosteal hematoma secondary to acute rhinosinusitis in a child. Tohoku J Exp Med 2014;233:171-4.

395. Yim HB, Yoo YS, Oh SH, Lee NY. An unusual case of an orbital abscess secondary to contralateral sinusitis. Ophthalmic Plast
Reconstr Surg 2013;29:e142-3.

396. Chan KH, Kraai TL, Richter GT, Wetherall S, Todd JK. Toxic shock syndrome and rhinosinusitis in children. Arch Otolaryngol Head Neck Surg 2009;135:538-42.

397. Patel N, Khalil HM, Amirfeyz R, Kaddour HS. Lacrimal gland abscess complicating acute sinusitis. Int J Pediatr Otorhinolaryngol 2003;67:917-9.

398. Mirza S, Lobo CJ, Counter P, Farrington WT. Lacrimal gland abscess: an unusual complication of rhinosinusitis. ORL J Otorhinolaryngol Relat Spec. 2001;63(6):37981.

399. Sidwell RU, Patel NN, Francis N, Staughton RC. Pyoderma gangrenosum of the eyelid and acute rhinosinusitis. Clin Exp Dermatol 2001;26:680-2.

400. Sahjpaul RL, Lee DH. Infratentorial subdural empyema, pituitary abscess, and septic cavernous sinus thrombophlebitis secondary to paranasal sinusitis: case report. Neurosurgery 1999;44:864-6; discussion 6-8.

401. Gwaltney Jr. JM, Hendley JO, Simon G, Jordan Jr. WS. Rhinovirus infections in an industrial population. II. Characteristics of illness and antibody response. JAMA 1967;202:494-500.

402. Hawke K, van Driel ML, Buffington BJ, McGuire TM, King D. Homeopathic medicinal products for preventing and treating acute respiratory tract infections in children. Cochrane Database of Systematic Reviews. 2018;4:CD005974. 


\section{Epidemiology, predisposing factors, pathophysiology, and diagnosis of CRS}

\subsection{Epidemiology and predisposing factors of CRS}

\subsubsection{Epidemiology of CRS}

The number of papers on epidemiology of chronic rhinosinusitis (CRS) is limited compared to papers on treatment.

Surveys with questionnaires are the most common way to explore the field of CRS in order to screen the population and to determine the prevalence of CRS in the general population. It probably overestimates the prevalence of CRS due to overlap with other rhinologic diseases.

In recent years a number of population-based studies have been performed with questionnaires evaluating the prevalence of CRS based on the epidemiological definition from the EPOS2012 document ${ }^{(1)}$.

Earlier studies in the USA in 2009 based on the National Health Interview Survey found 13\% of adults to have "sinusitis"(2). The first of these studies was part of The Global Allergy and Asthma European Network (GA2LEN). It consisted of a postal questionnaire sent to a random sample of adults aged 15-75 years in 19 centres in Europe. Participants reported symptoms of CRS, and doctor diagnosed CRS, allergic rhinitis, age, gender and smoking history. Information was obtained from 57,128 responders living in 12 countries. The overall prevalence of CRS was $10.9 \%$ with a wide variation between countries ranging from 6.9 in Finland to 27.1 in Portugal. CRS was more common in smokers than in nonsmokers (odds ratio (OR) 1.7: 95\% confidence interval (CI) 1.6-1.9). The prevalence of self-reported physician-diagnosed CRS within centres was highly correlated with the prevalence of EPOS-diagnosed CRS. Subsequent studies following roughly the same method showed a prevalence of 5,5\% in Brazil(3), $8 \%$ in China ${ }^{(4)}, 11 \%$ in South Korea ${ }^{(5)}$ and $12 \%$ in the USA ${ }^{(6)}, 16 \%$ in the Netherlands ${ }^{(7)}$ and $28 \%$ in $\operatorname{Iran}^{(8)}$. In a second phase of the $G A^{2} L E N$ Survey each centre invited 120 randomly selected subjects with asthma, 120 with CRS, 40 with asthma and CRS and 120 with neither asthma or CRS for a clinical study visit with further investigations among which was a questionnaire including the same questions as for the postal survey. Of the patients having an epidemiological diagnosis of CRS in the first phase, EPOS provides two definitions of CRS: a clinical diagnosis based on symptoms, supported by signs of mucosal inflammation found on imaging or with nasal endo- scopy, and a symptom-based definition to be used in epidemiologic research, without radiologic imaging or endoscopic examination ${ }^{(1)}$. Due to the overlap of symptoms between CRS, acute rhinosinusitis and (non-)allergic rhinitis, it can be difficult or maybe impossible to discriminate between these diagnoses based on symptoms alone.

The addition of nasal endoscopy or computed tomography (CT) scan makes the diagnosis more reliable. Two studies evaluating the use of nasal endoscopy to make a clinical diagnosis of CRS in a population study showed reduction of the prevalence to $1.2 \%$ $(88 \text { of } 7,343)^{(5)}$ and $6.8 \%{ }^{(9)}$. Interestingly in the paper from Tomassen et al., $38 \%$ of symptom-negative patients had a positive endoscopy. The reason for the discrepancies between these two papers is unclear. Both papers used the EPOS criteria of clinical CRS: the presence of polyps, presence of oedema in the middle meatus or presence of thick purulent discharge in the middle meatus to define endoscopy positive CRS.

Also, two studies evaluated the use of CT scan to make a clinical diagnosis of CRS in a population study. In one study a group of subjects who underwent a computed tomographic or magnetic resonance imaging scan of the head for any non-rhinologic indication were asked to fill in the GA2LEN survey including the CRS questions ${ }^{(10)}$. The scans were evaluated according to the Lund-Mackay (LM) scoring system. Eight hundred and thirty-four subjects were included, and 107 (12.8\%) had epidemiologically based CRS according to EPOS. Of these subjects, $50 \%$ had an LM score of $0,26 \%$ had an LM score of 1 to 3 , and $23 \%$ had an LM score of 4 or greater. Twenty-five (3.0\%) subjects had clinically based CRS (based on LM score $>/=4$ ), and 53 (6.4\%) subjects had clinically based CRS (based on LM score $>0$ ). In subjects who did not report upper airway symptoms, $57 \%$ had an LM score of $0,30 \%$ had an LM score of 1 to 3, and $12 \%$ had an LM score of 4 or greater. The authors found a prevalence of $3.0 \%$ to $6.4 \%$ of clinically based CRS (depending on an LM cut-off point; i.e., $\mathrm{LM} \geq 4$ or $\mathrm{LM}>0$, respectively) in a relatively randomly selected group of subjects. The other study ${ }^{(11)}$ collected questionnaires and sinus CT scans from 646 participants selected from a source population of primary care patients. Symptom status was based on guideline criteria, and radiology was based on the LM score using cutoff points of $\geq 3,4$, or 6 . The authors found a prevalence of $11.1 \%, 9.9 \%$, and $5.7 \%$ among woman respectively, and $16.1 \%, 14.6 \%$, and $8.7 \%$ among men for the different LM cutoff 
points. When combining with symptomatology, the proportion of clinically based CRS was $1.7 \%, 1.6 \%$, and $0.45 \%$ among women and $8.8 \%, 7.5 \%$, and $3.6 \%$ among men. The figures of these two studies are very comparable, pointing to a prevalence of CRS based on symptoms and CT scan of around 3\%.

Finally, prevalence of CRS can be estimated from databases based on the diagnosis by physicians. Health care administrative databases allow for population-based cohort studies to generate large sample sizes with small financial and time expense. However, the risk of inaccurate cohort identification can lead to biased outcomes. Lui et al. showed that in 27 studies obtaining CRSspecific data from a health records database, eight different CRS case definitions were identified and 13 administrative databases were evaluated. The most commonly used CRS case definition was the ICD-9 473.x code alone ${ }^{(12)}$. The CRS case definition of >/=2 claims with a CRS ICD-9 code (471.x or 473.x) within two years of the reference case provides a balanced validity with a sensitivity of $77 \%$ and specificity of $79 \%{ }^{(13)}$. The data that can be used to evaluate prevalence and doctors' visits are limited. In the year 2000, 2405 residents of Olmsted County were given an ICD-9 diagnosis code for CRS. Among these, 1627 (67.7\%) were female and 778 (32.3\%) were male. The overall age- and sex-adjusted prevalence per 100,000 was $1955(1.96 \%)^{(14)}$. The mean age- and sex-standardized incidence of diagnosed CRS in Canada was reported to be 2.5 (range, 2.3-2.7) per 1000 population. The estimated prevalence based on age-specific incidence varied between 18.8 (95\% Cl, 18.7-18.9) and 23.3 (95\% $\mathrm{Cl}, 23.1-23.5)$ per 1000 population during 2004-2005 to 20132014 , and no obvious growing trend was found. There was high geographic variation in the diagnosed incidence and prevalence of $\mathrm{CRS}^{(15)}$.

Studies from [general practitioner (GP)] databases often suffer from suboptimal differentiation between ARS and CRS. Hof-

fmans et al. showed that GPs often do not differentiate between the two diseases ${ }^{(16)}$.

Xiao et al. ${ }^{(845)}$ retrospectively reviewed a random sample of adult patients diagnosed with CRS in 2016, based on ICD-10 codes from primary care and otorhinolaryngology departments. The percentage of patients fullfilling the criteria of the American Academy of Otolaryngology-Head and Neck Surgery (AAO-HNS) was very low from GP patients (0.97) and low from otorhinolaryngologists' patients (28.9\%). Symptom duration $<12$ weeks was higher in primary care $(81.6 \%$ vs. $53.6 \%, \mathrm{p}<0.0001)$, as was lack of evidence of inflammation ( $97.4 \%$ vs. $50.0 \%, \mathrm{p}<0.0001)$. Having $<2$ of the required symptoms was significantly higher in otorhinolaryngology $(63.8 \%$ vs. $50.8 \%, p=0.013)$. The most commonly unevaluated symptom was decreased sense of smell (97.7\% in primary care, $69.1 \%$ in otorhinolaryngology encounters). There is an obvious need for clear criteria to do research on CRS in healthcare.

\subsubsection{CRS: difference between CRSwNP and CRSsNP}

CRS can be classified into CRS with nasal polyps (CRSwNP) and CRS without nasal polyps (CRSsNP). Data on the prevalence of CRSwNP are limited. Two early Scandinavian studies ${ }^{(17,18)}$ investigated the prevalence of nasal polyps in an adult population. A random sample of 1,900 inhabitants over the age of 20 years, stratified for age and gender, was drawn from the municipal population register in Skövde, Sweden, in December 2000. The subjects were called to clinical visits that included questions about rhinitis, asthma, and aspirin intolerance and examination by nasal endoscopy. In total, 1,387 volunteers (73\% of the sample) were investigated. The prevalence of nasal polyps was $2.7 \%$ (95\% confidence interval, 1.9-3.5), and polyps were more frequent in men ( 2.2 to 1 ), the elderly ( $5 \%$ at > or $=60$ years of age), and asthmatics ${ }^{(18)}$. Similar data were found in Denmark where over a 6-year period all polyp patients diagnosed for the first time ( $n=252 ; 174$ males, 78 females) were prospectively registered at the study clinic and compared to an estimated background population. The mean estimated incidences for all age groups were 0.86 and 0.39 patients per thousand per year for males and females, respectively. With a disease duration of estimated 20 years, this would lead to a prevalence of 1.92 and 0.78 for males and females. Using a validated questionnaire/ algorithm ( $90 \%$ specificity and sensitivity) in a populationbased random sample, Klossek et al. estimated a prevalence of $2.11 \%$ (95\% Cl 1.83-2.39). CRSwNP patients ( $n=212,45 \%$ males) were aged $49.4 \pm 17.6$ years. No gender preponderance was observed, but NP tended to increase with age. Mean duration of nasal symptoms was $22.4 \pm 15.7$ years.

Tan et al. sought to determine incidence for CRSsNP and CRSwNP using electronic health records data from 446,480 Geisinger Clinic primary care patients ${ }^{(19)}$. The average incidence of CRS was $83 \pm 13$ CRSwNP cases per 100,000 person-years and $1048 \pm 78$ CRSsNP cases per 100,000 person-years. Between 2007 and 2009, 595 patients with incident CRSwNP and 7523 patients with incident CRSsNP were identified and compared with 8118 control subjects. Compared with control subjects and patients with CRSsNP, patients with CRSwNP were older and more likely to be male. Finally Won et al. found a prevalence of CRSsNP and CRSwNP of $3.5 \pm 0.2 \%$ and $2.5 \pm 0.2 \%$ (mean $\pm \mathrm{SE}$ ), respectively. When classified by age group, the prevalence of CRSwNP increased with age in adults ( $\geq 18$ years of age), which was particularly evident after 40 years of age and CRSsNP was more prevalent in subjects younger than 40 years. Those with CRSwNP were significantly more likely to be men, to have higher $\mathrm{BMI}$, to smoke cigarettes, and to have asthma than the CRSsNP or no-CRS group.

CRSwNP was significantly associated with adult-onset asthma (onset after 18 years of age) or late-onset asthma (onset after 40 years of age), whereas CRS without nasal polyps was related to childhood-onset asthma (onset before 18 years) or early-onset 
asthma (onset before 40 years) in adults. The 2 CRS subgroups showed significant associations with current asthma but not with past asthma. However, the comorbid asthma rate was lower than $10 \%$ among subjects with $\mathrm{CRS}^{(20)}$.

Soler et al. ${ }^{(21)}$ in 2012 evaluated the role of gender and race in surgical outcomes for patients with CRSwNP and asthma. In his study, African Americans with CRSwNP had less improvement after endoscopic sinus surgery than Caucasians. This difference according to ethnicity must be proven because it could be confused by other socio-economic factors such as access to healthcare in this population. This study also shows that the prevalence of CRS was lower in the Asian (7\%) and Hispanic population (8.6\%) than in the black population (13.3\%) or the Caucasian population (prevalence around 13\% too).

However, there is no significant difference regarding the gender. This is confirmed by the study of Hirsch et al. ${ }^{(6)}$ in 2017 that found that women are more likely to have acute rhinosinusitis but that there is no sex ratio for CRS. Hoffmans et al. ${ }^{(7)}$ had the same conclusions regarding ethnicity and gender in his study including 8,347 adults in the Netherlands in 2017.

Brescia et al. ${ }^{(22)}$ in 2016 examined the difference in CRS prevalence between elderly and young people. After endoscopic sinus surgery, nasal polyps recurred less often in the elderly, maybe because in CRS in the elderly, there is less eosinophilic infiltration which is known to increase the risk of recurrence. Furthermore, the proportion of patients with allergy was significantly higher in young people than in the elderly population, but there was no difference in the prevalence of asthma in both populations. Cho et al. ${ }^{(23)}$ found the same result in his study, suggesting that when CRS appears in the elderly the pathogenesis is different, less linked with allergy and eosinophilic infiltration, but more with nasal polyp formation.

\subsubsection{Predisposing factors of CRSwNP and CRSsNP}

\subsubsection{Allergy}

The relationship between atopy and CRS has been well studied and resulted in many reviews. A systematic review was performed by Wilson in 2014 ${ }^{(24)}$. A total of 24 articles were found that met the inclusion criteria; 18 articles examined the relationship between allergy and CRSwNP, with 10 articles showing an association, seven articles showing no association, and one article showing a possible association. Nine articles examined the relationship between allergy and CRSsNP, with four articles showing an association and five articles showing no association. Four studies directly compared the role of allergy in CRSWNP and CRSsNP, and, again, the results were mixed. No articles examined the outcomes of CRSsNP or CRSwNP following allergy treatment. The authors concluded that the role of allergy in CRSwNP and CRSsNP continues to be controversial, with the level of evidence poor. Based on the available data, the recom- mendation is that allergy testing and treatment are an option in CRSwNP and CRSsNP. Since 2014, a few noteworthy studies have been published. A more recent non-systematic review points to the fact that different phenotypes/endoptypes of CRS may have a variable associations with allergy. The authors point to allergic fungal rhinosinusitis (AFRS) (see 8.6) and central compartment atopic disease (CCAD) ${ }^{(25)}$ (see 8.1.2.1.) This point was also made in a recent study from the UK that analysed differences between different phenotypes of CRS and controls in secondary care ${ }^{(26)}$. The analysis included 1470 study participants: 221 controls, 553 CRSsNPs, 651 CRSwNPs and 45 AFRS. The prevalence of inhalant allergy was 13.1, 20.3, 31.0 and $33.3 \%$ respectively; house dust mite allergy was significantly higher in CRSwNPs (16\%) compared to CRSsNPs (9\%) in this study.

In conclusion, the prevalence of allergy in CRS may vary by phenotype, with CCAD and AFRS having a stronger association than CRSwNP and CRSsNP. Large studies evaluating relationships in different settings like the Philpott paper are needed.

\subsubsection{Asthma and other lower airway diseases}

Unlike for allergy and CRS, the relationship between CRS and asthma is indisputable.

The prevalence of asthma is around $25 \%$ in patients with CRS compared to $5 \%$ in the general population ${ }^{(27)}$. GA2LEN ${ }^{(28)}$ studied over 52,000 adults aged 18-75 years living in 19 centres in 12 countries and concluded that there is a strong association between asthma and CRS. The association with asthma was stronger in those reporting both CRS and allergic rhinitis. In the earlier mentioned UK study ${ }^{(26)}$, the prevalence of asthma was $9.95 \%$ in controls, $21.2 \%$ in patients with CRSsNP, $44.9 \%$ in CRSwNPs and $73.3 \%$ in patients AFRS. A subgroup of the earlier mentioned GA2LEN cohort, with and without asthma and CRS of 3337 participants were further analysed for decline in lung function. Participants with asthma had lower forced expiratory flow per second (FEV) and a steeper slope of FEV1 against age equivalent to smoking 1-2 packs of cigarettes per day. Those with atopy had a slope equivalent to controls, but neither CRS nor atopy alone were associated with such decline ${ }^{(29)}$.

Chen et al. ${ }^{(30)}$ identified patients newly diagnosed with asthma in Taiwan and analyzed the incidence of CRS in that population. After adjustment for gender, age and medical comorbidities, they showed that asthma is an independent predictor of CRS, with or without nasal polyps (OR: 2.58 for CRSsNP). The chronology of developing asthma or CRS first is variable.

A Japanese study shows that there is no significant difference in onset times between the two diseases ${ }^{(31)}$. Wheezing and respiratory discomfort are present in 31 to $42 \%$ of patients with CRSwNP, and asthma is reported by $26 \%$ of patients with CRSwNP, compared to $6 \%$ of controls ${ }^{(32)}$. On the contrary, $7 \%$ of asthmatic patients have nasal polyps with a prevalence of $13 \%$ in non-atopic asthma and 5\% in atopic asthma and they have 
more nasal symptoms than patients that have CRS without asthma ${ }^{(33)}$. In asthmatic CRS patients, a sizeable portion of CRS impact on QOL is indirectly mediated through the effect of CRS on poorer asthma control which may then drive decreased $\mathrm{QOL}^{(34)}$.

Several authors reported on the higher incidence of CRS in chronic obstructive pulmonary disease (COPD) patients ${ }^{(35)}$. The figures depend mostly on the definition of CRS applied in a study. Upper airway symptom frequency among COPD patients may be as high as $88 \%{ }^{(33)}$ but when more objective tests were applied for CRS diagnosis (such as CT scans), lower numbers were reported (53\%). CRS symptoms significantly impair COPD patients' quality of life. Smoking should be considered an important risk factor of $\mathrm{CRS}^{(35)}$.

Within the GA2LEN network a multicentre cross-sectional casecontrol study recruited 935 adults (869 eligible for analysis:

237 CRSsNP; 445 CRSwNP; 187 controls). Comorbidities such as asthma, allergy, eczema, food allergy, urticaria, and chronic obstructive pulmonary disease were significantly more frequent in CRS patients ${ }^{(36)}$. Another chronic pulmonary disease has been studied thanks to a National database analysis in Taiwan ${ }^{(37)}$. They followed 68,058 patients and found 569 patients that developed CRS during the five years of follow-up. After adjustment COPD was an independent predictor of CRS without nasal polyps. Bronchiectasis is a chronic bronchial disease with permanent and irreversible destruction and dilatation of the bronchial wall, leading to retained secretions and recurrent infections. More than $50 \%$ of bronchiectasis are idiopathic but as it is a respiratory tract disease, some researchers studied the link between it and CRS. Guilemany et al. ${ }^{(38)}$ found that $77 \%$ of bronchiectasis patients have CRS and $26 \%$ of them have nasal polyps; in both idiopathic and post-infective bronchiectasis. Some authors suggest that in bronchiectasis there is an impairment of ciliary function such as in Kartagener's syndrome, explaining these results and the sinonasal symptomatology.

\subsubsection{Non-steroidal anti-inflammatory drug (NSAID)-exa- cerbated respiratory disease (N-ERD)}

Non-steroidal anti-inflammatory drug (NSAID)-exacerbated respiratory disease (N-ERD) is a chronic eosinophilic, inflammatory disorder of the respiratory tract occurring in patients with asthma and/or CRS with nasal polyps (CRSwNP), symptoms which are exacerbated by NSAIDs, including aspirin ${ }^{(39)}$.

The pathogenesis of N-ERD is related to dysregulation of eicosanoid synthesis ${ }^{(40)}$ leading to an eosinophilic inflammation of the nasal and sinus membranes and an increased leukotriene production that is further accentuated by cyclooxygenase (COX)-1 inhibitor (aspirin or NSAIDs) ${ }^{(41)}$.

Respiratory symptoms following NSAID intake have been reported by $1.8 \%$ of the general European population and by $10 \%-20 \%$ of patients with asthma ${ }^{(42)}$. The prevalence of respiratory reactions to NSAIDs was higher in participants with CRS symptoms and asthma symptoms in last 12 months but was not associated with allergic rhinitis ${ }^{(43)}$. In the previously mentioned UK analysis ${ }^{(26)}$ the prevalence of self- reported aspirin sensitivity was 2.3 in the control group, 3.3 in CRSsNP patients, 9.6 in CRSwNP patients and 40\% in the AFRS group. In this same study the AFRS and CRSwNPs group and to a lesser extent also the CRSsNP group were significantly more likely than controls to report symptom exacerbation due to ingestion of food groups with higher potential dietary salicylate content(44). The prevalence of N-ERD among patients with CRSwNP in a tertiary referral centre was $16 \%{ }^{(45)}$. Patients with aspirin-exacerbated respiratory disease (AERD) had undergone two-fold more sinus surgeries $(p<.001)$ and were significantly younger at the time of their first surgery than were patients with CRSwNP without $\mathrm{N}$-ERD.

Upper airway disease in N-ERD patients is usually CRSwNP. On average, upper respiratory symptoms are worse, extent of opacification on CT scan and recurrence of nasal polyps after surgery are more frequent in N-ERD than in NSAIDs-tolerant CRSwNP patients $^{(39,45)}$.

Diagnosis is mainly based on patient history and aspirin provocation tests are only needed when the history is not clear (see also 5.3.4.14).

\subsubsection{Immune deficiencies}

There is good evidence that immune deficiencies are more common in patients with CRS. A meta-analysis, which included 1418 individuals with CRS from 13 studies, found that $23 \%$ of patients with difficult- to-treat CRS and $13 \%$ of individuals with recurrent CRS had immunoglobulin deficiencies ${ }^{(46)}$. A recent study shows that the prevalence of immune deficiencies could be up to $50 \%$ in "difficult to treat" cases of CRS and that immunological tests should be undertaken in these cases $^{(47)}$. Other authors suggest integrating systematically immunological tests when exploring a diagnosis of CRS, particularly when it does not respond to standard treatment or have an impact on morbidity or quality of life ${ }^{(48)}$.

The most frequent immune deficiency found in these cases is Common Variable ImmunoDeficiency (CVID) (10\% cases) and Selective immunoglobulin A (lgA) deficiency (6\% cases) $)^{(49)}$. Selective IgA deficiency is the most common immunodeficiency with a prevalence of one in 600 individuals, but usually people are asymptomati ${ }^{(47)}$. However, many of the patients diagnosed in the series included in the meta-analysis had subclass or specific antibody deficiency. The laboratory criteria for diagnosing these conditions, and the clinical implications once the diagnoses are made, are not uniformly accepted. Many of the studies of the meta-analysis were performed in tertiary referral centres and this may have biased the population of patients studied toward having underlying immune defects. These reservations aside, it 
is likely that the prevalence of hypogammaglobulinemia in CRS patients is significantly higher than in the general population.

\subsubsection{Gastro-oesophageal reflux disease (GORD)}

Gastro-oesophageal reflux disease (GORD) is a common gastrointestinal disorder that affects approximately $10 \%$ of western populations and has been hypothesized to be one of the possible factors that induces CRS and contributes to its worsening. However, the relationship between GORD and CRS remains indeterminate due to its complexity, and it is not clear that an anti-reflux treatment would be indicated for patients with recurrent CRS. A recent meta-analysis ${ }^{(50)}$ in 2016 gathered 32 publications that assessed the prevalence or incidence of CRS in a GORD population or vice-versa.

In a recent systematic review, Leason et al. included studies $(n=32)$ that consisted of studies reporting pathogenic factors $(n=20)$, epidemiological association $(n=8)$, prognostic interactions $(n=3)$, and a combination of these outcomes $(n=1)$. They concluded that the potential pathogenic roles for GORD in CRS were supported; CRS subjects had greater prevalence of intranasal Helicobacter pylori and acid reflux than subjects without CRS. A more recent study also showed that CRS patients had significantly higher incidences of gastro-oesophageal reflux measured with 24-h multichannel intraluminal impedance (MII)-pH monitoring compared with asymptomatic controls ${ }^{(51)}$. CRS is more prevalent in GORD sufferers than those without GORD. Evidence is conflicting for GORD as a factor in CRS treatment failure. A second systematic review included 12 papers and found eight cross-sectional articles that suggested a relation between CRS and GORD, especially in CRS that is refractory to clinical or surgical treatment ${ }^{(52)}$. However, the authors concluded that the groups are small and methodologies different.

The association between GORD and CRS seems to be two-way and Leason et al. suggest that direct nasopharyngeal reflux leads to gastric acid and pepsin reflux directly in the nasal cavity, mostly in a lying position, inducing nasal inflammation that worsens CRS. Another hypothesis is the intranasal presence of H. pylori, a bacteria which has in fact been found in the nasal cavity ${ }^{(53)}$; and as it is known for its aggression and its role in the pathogenicity of gastric ulcers, gastritis and gastric cancers, it is reasonable to suggest the same pathogenicity in the nasal cavity. Furthermore, as nasal pepsin, gastric acid and local eosinophilic infiltration are more prevalent in the nasal cavity in GORD patients ${ }^{(54)}$ with CRS, the induced nasal inflammation is more important and explains the results of studies ${ }^{(55)}$ finding that patients with GORD and CRS had more symptomatic CRS [higher Sino-Nasal Outcome Test (SNOT)-22 score, more CRS medication, more endoscopic sinus surgery] than patients with CRS without GORD.

Another hypothesis is that corticosteroids and antibiotics given in CRS medication could induce or worsen GORD, mostly in recurrent and aggressive CRS. This suggests that there could be a benefit of giving an anti-reflux treatment to a patient with CRS even if he has no symptoms of GORD. However, it has not yet been shown that reducing gastroesophageal reflux symptoms correlates with a reduction of CRS symptoms (see also 6.1.23.1).

\subsubsection{Nasal anatomic variations}

Anatomic variants have been studied because the obstruction of the ostiomeatal complex has been suggested as a risk factor for developing $\mathrm{CRS}^{(33)}$. A systematic review analyzing the role of septal deviations in CRS demonstrated a significant association of septal deviation and rhinosinusitis. However, the clinical effect was modest and interestingly in all studies that examined the laterality of rhinosinusitis associated with septal deviation, inflammation was found bilaterally ${ }^{(56)}$. A recent study ${ }^{(57)}$ analyzed CT and nasal endoscopies in patients with CRS and found that $88.2 \%$ of patients had a deviated nasal septum and $76.4 \%$ had concha bullosa. However, these numbers were close to those found in retrospective trials in the general population and are not significant.

Sedaghat et al. ${ }^{(58)}$ studied CT scans of patients with CRS and found that sinonasal anatomic variants such as infra-orbital cells and frontal inter-sinus cells are related to faster progression of CRS, but this prevalence has not been compared to a population without CRS. Although patients with CRS do not necessarily have higher rates of specific anatomic variations, it appears that they can affect the progression of the disease.

\subsubsection{Microbiology}

\subsubsection{Bacteria and biofilms}

The pathophysiology of CRS is multifactorial but mostly considered an inflammatory disease of the upper airways analogous to asthma in the lower airways. It is a multifactorial condition in which the microbiota may play a pathogenic role. Because the nose is not sterile, a culture of the sinus obtained via the nose will always grow microbes, and causality in CRS is not established by a positive culture. Trying to determine the role of infection based on a response to antibiotic treatment is also difficult. CRS in general also does not have a favorable reaction to treatment with either short term or long term antibiotics (see 6.1.). Studies of the microbiology of CRS have demonstrated a variety of bacteria depending on geographic location, prior surgery, and recent antibiotic use. Staphylococcus aureus $(\mathrm{SA})$ is a frequent colonizer in humans, and it is considered to be associated with chronic airway diseases including CRS and asthma. In studies of general populations, nasal SA colonization had significant relationships with asthma prevalence. In studies of patients with CRS, positive associations were also found but had a considerable heterogeneity and the results were comparable between CRS with and without nasal polyps ${ }^{(59)}$. Recent improve- 
ments in the microbiological techniques have greatly advanced our understanding of the complex nature of this interaction. The nature of the interaction between the microbiota and the local immune system is very complex and has not been fully elucidated. The role played by microbiomes in CRS is difficult to be clearly defined at the current time due to the difficulties in the laboratory techniques and small studies with limited sample size. It is likely that bacterial and fungal airway microbiomes are dynamic and experience natural shifts in diversity with time. The underlying reasons for these shifts appear to be a combination of changes in environmental climate and host factors. A small core community that persisted throughout the two year sampling period was identified: Corynebacterium, Propionibacterium and Staphylococcus, and one type of fungus, Malassezia restricta ${ }^{(60)}$.

The presence of biofilms in CRS patients was first demonstrated in 2004 via scanning electron microscopy of the nasal mucosa of CRS patients ${ }^{(61)}$. A biofilm comprises any syntrophic consortium of microorganisms in which cells stick to each other and often also to a surface. These adherent cells become embedded within a slimy extracellular matrix that is composed of extracellular polymeric substances. Because they have three-dimensional structure and represent a community lifestyle for microorganisms, they have been metaphorically described as cities for microbes. Microbes form a biofilm in response to various different factors which may include cellular recognition of specific or non-specific attachment sites on a surface, nutritional cues, or in some cases, by exposure of planktonic cells to sub-inhibitory concentrations of antibiotics. A cell that switches to the biofilm mode of growth undergoes a phenotypic shift in behaviour in which large suites of genes are differentially regulated ${ }^{(62)}$. Since the advent of biofilm detection in CRS, multiple bacterial organisms have been implicated including Staphylococus aureus, Pseudomonas aeruginosa, Haemophilus influenza and Moraxella catarrhalis. Of these, SA biofilms have the greatest association with severely recurrent and recalcitrant cases of CRS possibly because of their potential to produce antigens ${ }^{(63)}$. Estimated rates of biofilm formation in patients with CRS range from 29 to $72 \%$. CRS patients with biofilm have more severe disease preoperatively and persistence of postoperative symptoms, ongoing mucosal inflammation, and infections ${ }^{(64,65)}$.

\subsection{Virus}

The role of viral infections in inducing or exacerbating CRS has been studied and coronavirus was identified via molecular sequencing as the most common virus in patients with $\mathrm{CRS}^{(66)}$. Parainfluenza virus has been identified as a major cause of postinfectious olfactory dysfunction and could have a potential role in the pathogenesis of $\mathrm{CRS}^{(67)}$. Acute exacerbations of CRS have been increasingly recognized as an important disease entity with a significant impact on the quality of life. Current evidence supports the role of the transient viral infection as the initial inflammatory stimulus in the pathogenesis of acute exacerbations in CRS.

\subsubsection{Fungal infection}

Chronic fungal rhinosinusitis may represent a wide spectrum of disease ranging from the mild form of superficial colonization (saprophytic fungal infestation and fungal ball), allergic manifestations (AFRS) to chronic granulomatous invasive fungal rhinosinusitis (see 8.6).

\subsubsection{Ciliary impairment}

Motile ciliary disorders have been extensively investigated in primary ciliary dyskinesia ( $\mathrm{pcd}$ ), a rare and under-recognized genetic disease characterized by impaired mucociliary clearance (see 9.5). Defective mucociliary clearance, evidenced by impaired ultrastructure and/or function of respiratory cilia, have also been implicated in CRS.

In healthy individuals, mucociliary clearance (MCC) is part of the innate defense mechanism and functions to protect the airways by trapping inhaled ambient pathogens within the mucus layer of epithelial surfaces and propelling it out of the airways through coordinated cilia movement. Normal functioning of MCC consists of two equally important components: mucus production and mucus transport. When MCC is compromised, airways become vulnerable to a vicious cycle of infection and obstruction. Extrinsic factors such as pollutants and microbes can directly and indirectly impact normal cilia function. This phenomenon is evident in patients with CRS who experience relentless cycles of infection and inflammation, resulting in ciliary loss and a hyperviscous mucus blanket generating dysfunctional mucociliary coupling. In addition to direct ciliary loss, cilia surviving the microbial and/or inflammatory insults appear not to function normally ${ }^{(68)}$. Although the literature is conflicting regarding ciliary impairment and its changes in patients with CRS, recent work has suggested that a subset of patients with CRS have a blunted ciliary response to environmental stimuli that is reversible once the tissue is removed from the infected or inflamed sinonasal environment. This finding suggests that local exogenous factors can negatively modulate the ciliary dynamic response to stimuli.

Multiple environmental and exogenous factors alter the normal physiological state, and the resultant inflammatory cytokines secondarily exacerbate the impaired mucociliary clearance ${ }^{(69)}$.

\subsubsection{Smoking}

Smoking should be considered an important risk factor of $\mathrm{CRS}^{(35)}$. The GA2LEN survey showed that current smoking and ex-smoking are significantly associated with CRS(70). Tobacco is toxic to the nasal mucosa and cigarette smoking plays a significant role in diseases of the upper airway because pollutants and 
toxins in cigarette smoke are pro-inflammatory and create oxidative stress of the mucosa, leading to symptoms such as nasal obstruction, increased nasal secretion and mucosal dryness. Recent systematic reviews found a strong correlation between active and passive cigarette smoke with the prevalence of CRS. Paediatric patients exposed to secondhand smoke appear to have particularly poor outcomes ${ }^{(71,72)}$.

\subsubsection{Pollution}

The consequences of the World Trade Centre attack have clearly shown the relation between pollution and CRS. The enormous dust exposure of the firefighters resulted in a significant increase in prevalence of CRS that was correlated to the amount of inhalation and did not diminish by time since exposure ${ }^{(73)}$. Recently air pollutants were shown to correlate with CRS symptom severity that may be influenced by exposure levels, with a more pronounced impact on CRSsNP patients ${ }^{(74)}$. Geramas et al. ${ }^{(75)}$ also concluded in their 2018 review that living in poor, crowded or old housing is associated with increased prevalence of CRS. Until quite recently, the current state of the literature allowed us to make very few conclusions about the role of hazardous occupational risk in $\mathrm{CRS}^{(76)}$. However, some recent studies show associations between occupational exposure to paper dust, cleaning agents, metal dust, animals, moisture/mould/mildew, poisonous gas and physically strenuous work ${ }^{(77-79)}$. A recent Portuguese study ${ }^{(79)}$ found an important correlation between occupational dust exposure and nasal polyps' occurrence in textile workers and Mady et al. ${ }^{(74)}$ suggest that exposure to air pollutants correlates with CRS symptom severity in CRSsNP.

\subsubsection{Obstructive sleep apnoea (OSA)}

Patients with undiagnosed obstructive sleep apnoea (OSA) often present to an otorhinolaryngologist with symptoms of CRS. In a retrospective analysis of adult patients at an academic practice with a rhinologic chief complaint, the authors showed that OSA and CRS have significant overlap in symptom profiles and that the SNOT-22 can help identify those with undiagnosed OSA. OSA should be suspected in patients who report a sleeprelated item as a most important symptom and display higher psychological and sleep domain scores ${ }^{(80)}$.

A retrospective study found that obstructive sleep apnoea ${ }^{(81)}$ is an independent factor of risk for developing CRS [hazard ratio (HR) of 3.44 for males and 2.63 for females].

In a Taiwanese study evaluating patients who underwent FESS, $38 \%$ complained of daytime sleepiness, and this sleep problem was correlated with the symptom of nasal obstruction. Obstructive sleep apnoea syndrome (OSAS) was diagnosed in $64.7 \%$ of the patients, but there was no correlation with the severity of rhinosinusitis. Nasal polyps did not worsen sleep problems in the CRS patients ${ }^{(82)}$.

The exact relationship between these two overlapping diseases is not clear and further larger prospective studies are needed.

\subsubsection{Metabolic syndrome and obesity}

Using the Korean National Health and Nutrition Examination Survey (KNHANES), Lee et al. ${ }^{(83)}$ analyzed 12,015 individuals and found that the prevalence of CRS in patients with metabolic syndrome (high triglyceride level, reduced high-density lipoprotein level and elevated blood pressure) was significantly higher than in patients without metabolic syndrome (14.15 vs. $10.16 \%$; $\mathrm{p}<0.05)$. This database was also used to show that obesity(84) is an independent factor in developing CRS $(\mathrm{OR}, 1.46 ; 95 \% \mathrm{Cl}, 1.16$ $1.84 ; \mathrm{p}=0.001$ ). The hypothesis is that there is a pro-inflammatory condition secondary to excessive adipose tissue favouring the development of inflammatory diseases such as CRS. This association needs further study.

\subsubsection{Vitamin D (VD3)}

There now is substantial literature to indicate that VD3 acts as an immunomodulator of adaptive and innate immunity locally within the respiratory epithelium.

Stokes et al. included seven articles (four prospective and three retrospective studies), with a total of 539 patients in a systematic review. There were significantly lower VD3 levels in the polypoid phenotypes of CRS compared with controls. Low VD3 levels were often associated with an increased degree of inflammation ${ }^{(85)}$. The authors conclude that the available evidence indicate that there is a significant relationship between low VD3 levels and polypoid CRS phenotypes. The association between VD3 levels and disease severity and VD3 potential for drug therapy remains unclear, which warrants further research in the area.

\subsubsection{Alcohol}

An important percentage of subjects diagnosed with chronic upper airway disease report alcohol-induced worsening of their symptoms.

The highest prevalence of nasal and bronchial alcohol hyperresponsiveness is observed in patients with N-ERD, followed by patients with CRSwNP, and less frequent in CRSsNP, and healthy controls. Alcohol hyper-responsiveness is significantly more prevalent in CRSwNP patients suffering from recurrent disease and in patients with severe symptomatology ${ }^{(86)}$.

\subsubsection{Acute exacerbations of CRS (AECRS)}

\subsubsection{Epidemiology and predisposing factors}

\subsection{Epidemiology}

Acute exacerbation of chronic rhinosinusitis (AECRS) is defined as worsening of symptom intensity with return to baseline CRS symptom intensity, often after intervention with corticosteroids and or antibiotics. Exacerbation of nose and sinus symptoms, 
often considered as a bacterial infection, may in reality have a more complex background and depend on multiple factors. It could be due to a worsening of allergic rhinitis, an acute viral respiratory infection, exacerbation of asthma or other stress factors including depression.

Nasal symptoms are common in the general population and without prior sinus CT it is difficult to precisely ascertain if an exacerbation of nasal symptoms was on a background of CRS. Large scale epidemiologic studies have, therefore, previously not been available. A recent epidemiologic study has tried to overcome this by studying acute exacerbation of nasal and sinus symptoms from a general population of 200769 subjects, 7847 were selected and mailed questions on nose and sinus symptoms according to EPOS2012 every four months over 16 months. Subjects were classified into; current long-term CRS, current recent, past or never CRS. Prescription of oral antibiotics and or orals steroids were considered as a proper event of exacerbation. Prevalence peaked in the winter months and peaked among long-term current CRS subjects, where $25.9 \%$ of patients had at least one exacerbation in the last 12 months compared to $7.9 \%$ in the never CRS group ${ }^{(87)}$.

Another study has followed 209 CRS patient from a tertiary care rhinology clinic monitoring the number of CRS infections, CRS-related antibiotic prescription and CRS-related oral corticosteroids taken. Ninety patients reported zero exacerbations, whereas 119 patients had three or more episodes during the prior 12 months ${ }^{(88)}$.

In 108 asthmatic patients with CRS (69.9\% with CRSwNP) the mean number of exacerbations of CRS was in the past 3 months 0.8 episodes, it is unclear if this refer to a specific season, but if extrapolated it would suggest 2.4 episodes per year in this particular group of patients ${ }^{(89)}$. The same study showed that frequency of acute exacerbation of CRS have a negative impact on asthma control.

A study on the effect of quality of life from acute exacerbations of CRS found that in 85 patients with a mean SNOT score of 36.8 the patients had taken a mean of $1.5, \mathrm{SD}=1.9$ courses of antibiotics related to CRS in the past 12 months ${ }^{(90)}$.

Thus, the prevalence varies with the patient cohort being studied, season, and how the exacerbation was defined.

\subsection{Predisposing factors}

According to the previously mentioned epidemiologic study an increased prevalence of AECRS is more likely to be seen; in younger white, female, on medical assistance or having greater Charlson comorbidity index. Other risk factors identified in this study were CRS status, increased BMI, asthma, hay fever, migraine and history of sinus surgery ${ }^{(87)}$.

The study by Phillips et al. identified an exacerbation prone phenotype associated with high sinonasal burden (SNOT score over 24) and comorbid asthma and interestingly patients without nasal polyps, which was negatively associated with the risk of exacerbation $^{(90)}$.

\subsubsection{Pathophysiology}

\subsection{Viruses}

Over 160 serotypes of rhinoviruses have been identified and are considered important contributors or triggers of airway inflammation both in the upper and lower airway. It is believed that the rhinovirus initiates primary epithelial damage which is followed by secondary damage by the host anti-viral inflammatory response. This should ideally be timely and self-limiting, avoiding permanent inflammatory change in the sinonasal mucosa ${ }^{(91)}$. The inflammatory response starts with activation of Toll like receptors (TLR 3,7 and 9) to initiate a cellular response, which induce the expression of interferons and cytokines, which in turn leads to recruitment of innate responders such as macrophages and neutrophils. More recently evidence has emerged that eosinophils are involved in anti-viral activities and are recruited to the site of rhinovirus infections explaining among other things asthma exacerbations seen during viral respiratory tract infections ${ }^{(92)}$.

A recent comprehensive review found 147 publications studying the effect of rhinoviruses in CRS patients and concluded that; 1 ) rhinovirus infections have a higher prevalence in CRS patients than in the general population, 2) humans challenged in vivo with rhinoviruses will elicit a local inflammatory response evident by elevated cytokines (IL-6, IL-8) in nasal lavage and mucosal changes on $\mathrm{MRI}$, and 3 ) in vitro challenge of nasal epithelial cells will produce a robust cytokine response mimicking the one found in vivo supporting the causality ${ }^{(93)}$.

Rhinoviruses may also disturb the balance of the residential microbiome. A study where healthy participants were inoculated with rhinoviruses revealed a shift in the resident microbiome where the abundance of Haemophilus parainfluenzae, Neisseria subflavia and Staphylococcus aureus increased. This change in the microbiota could help explain why virus infections predisposes to secondary bacterial infections ${ }^{(94)}$.

To sum up, virus infections are likely to be the main trigger for acute CRS exacerbations, where host response initiates or accentuates an inflammatory reaction in the nose and sinus mucosa, including eosinophils, and has a potential to shift the residential microbiome towards an increased abundance of microbial airway pathogens.

\subsection{Bacteria}

The microbiology of exacerbations of CRS has been studied by Brook using traditional culture. He found that in the majority of cases the bacterial flora was similar to the one found in stable CRS patients, although Haemophilus influenzae was significantly more prevalent in the exacerbation group ( 9 positive cultures 
of 30 patients with exacerbation, compared to 2 of 32 in stable (RS) $)^{(95)}$.

A more recent study using $16 \mathrm{~S}$ ribosomal RNA for bacteria reviewed the sinus microbiotia during acute exacerbation of CRS in 134 patients (65 CRSsNP, 55 CRSwNP and 14 AFRS). Of the bacteria identified, those with the highest relative abundance were Staphylococcus spp (21.3\%), Pseudomonas (15.0\%), Streptococcus (14.4\%), and Staphylococcus aureus (12.4\%). No consistent differences among subgroups were noted and all various bacterial taxa and species were present in all clinical subgroups ${ }^{(96)}$.

In 50 patients with a history of previous sinus surgery with acute exacerbation of CRS, most were found to have Staphylococcus aureus and S. epidermidis with six patients positive for Haemophilus influenzae and 5 for Pseudomonas aeruginosa $a^{(97)}$. This once again demonstrates that common airway pathogens only explain a small number of clinical exacerbations.

In summary, virus infections are likely to be the main trigger for AECRS, where host response initiates or accentuates a symptomatic inflammatory reaction in the nose and sinus mucosa. Furthermore, rhinoviruses have the potential to shift the residential microbiome towards an increased abundance of microbial pathogens. Significant microbiological changes are, however, only identified in a small number of exacerbations suggesting other mechanisms may be responsible.

Virus infections are likely to be the main trigger for AECRS and rhinoviruses have the potential to shift the residential microbiome towards an increased abundance of microbial pathogens.

\subsection{Pathophysiology of chronic rhinosinusitis with and without nasal polyposis}

\subsubsection{Genetics and epigenetics of CRS}

\subsubsection{Genetics of CRS}

The study of DNA via the Human Genome Project in the late 1990s was supposed to revolutionise medicine genes, allowing new understandings of implicated mechanisms and identifying new drug targets, with future treatments based on personalised genetic makeup ${ }^{(98)}$. However, despite the considerable advances in technology and radical cost reductions of genotyping, early experience with use of association genetics was disappointing. Monogenic gene disorders, where transmission of variation(s) in the makeup of a single gene produce disease via well-described mechanisms (such as the Cystic Fibrosis Transmembrane Conductance Regulator (CFTR) gene responsible for cystic fibrosis), were almost never found. Instead, for complex traits (such as height, intelligence, creativity), and traits for complex diseases (such as schizophrenia, diabetes and asthma) were found to be associated with a dizzying number of variations in wide variety of genes, all offering minor contributions to the observed phe- notype ${ }^{(99)}$. Indeed, for schizophrenia, a well described disease entity with distinctive symptoms, was found to be associated with variations in almost one hundred different genes ${ }^{(100)}$. Compounding this difficulty was the genes identified are often at first glance difficult to integrate into mechanistic models - for example, one of the main associations in schizophrenia was with a gene coding for $\mathrm{C} 4$, an element of the complement casca$\mathrm{de}^{(101)}$. Additionally, identified genetic factors may not so much modify the structure of an organ or a cellular organelle, but may instead increase susceptibility to an environmental influence, such as infection or colonisation with undesirable bacteria ${ }^{(102)}$. It may then be the bacterial presence that contributes to persistence or maintenance of disease. While this complicates the direct transposition of genetic results to the clinic, the field of genomics is again progressing, and mathematical approaches being developed to make predictions based on multiple variations, rather than relying on a single one. A glimpse into the future is afforded by a recent commercial venture which analyses combinations of SNPSs from the DNA of fertilised embryos to predict the fittest ones for implantation in vitro fertilisation (https://genomicprediction.com). This nevertheless may be of limited usefulness, as a simulation exercise using real GWAS data from large families showed that simulated embryos selected to be the smartest and tallest were only around $2.5 \mathrm{IQ}$ points and 2.5 centimetres above average. Screening human embryos for polygenic traits has limited utility ${ }^{(103)}$.

CRS is nevertheless benefitting from the 'genetics revolution'. For example, in studies from the group of Desrosiers identification of candidate genes associated with epithelial and basement membrane structure and function led to exploration of barrier function in epithelial cells from CRS patients. This culminated in the recent identification of a defect in tissue repair in CRS ${ }^{(104)}$, opening up the possibility of new drug treatments, confirming that there is value in the genetics arena. Other insights still waiting to bear fruit may become clearer as we better understand the role and functions of identified putative candidate genes.

\subsubsection{Genetics: an overview}

The term 'genetics' encompasses transmissible gene variations causing or predisposing to development of disease or phenotype in question. Transmissible variations in gene function may also be induced by exposure to outside agents in a process termed epigenetic regulation, or epigenetics. In a newer challenge, previously ignored short sequences of the genome called ' $\mathrm{mi}$ croRNAs' have been found to play important roles in regulation of gene function, and transmission of de-novo genetic material via bacterial viruses termed 'bacteriophages' may also modulate genetic makeup.

The identification of a genetic basis to a disease may be difficult. Sinus physiology is a complex system, with multiple steps involved in even a single process such as pathogen recognition 
and initiation of initial defensive responses. Variations in function of a number of different genes or regulatory elements may lead to dysfunction within this system, ultimately yielding the same common disease phenotype. In addition, different genetic variations within a same gene may produce variable degrees of dysfunction.

The earliest identified genetic disorders were discovered because they showed a clear pattern of heritability, with welldefined disease phenotype or by using markers such as the sweat chloride test used in cystic fibrosis (CF). These well-characterised genetic disorders implicated a single gene with a high penetrance and strong effects, which made the search for the genetic underpinnings of the disease much simpler. In contrast, most chronic disease such as asthma and CRS are considered 'complex diseases' where multiple genes are believed to participate in disease development, with each genetic factor having weak effects and thereby making only a partial contribution. In addition, the genetic basis may not be immediately obvious. For example, while it may seem obvious that an immune deficiency may predispose to chronic infection with bacteria, a defect in a gene involved of the epithelial barrier may lead to poor epithelial regeneration following viral insult, thereby facilitating bacterial sub-epithelially and thereby yielding the same result. Despite the considerable difficulties posed by the multiplicity of factors implicated in CRS pathogenesis, strong evidence nevertheless supports that there is nonetheless a hereditary component to CRS. A classic example is CF, where homozygous mutations in the CFTR gene lead to defects in chloride transport and yield the clinical manifestations of the disease. CRS, preferentially affecting the maxillary sinuses, is a consistent feature of CF. Other examples of well-characterised genetic diseases which include CRS in their phenotype include the forms of ciliary dyskinesia which can be coded for by at least 31 different genes implicated in coding a different portion of the structural arm of the cilia ${ }^{(105)}$.

More recent work by Oakley et al. assesses the heritability of CRSwNP and CRSsNP in a more general population. In a study of 1638 patients with CRSwNP and 24,200 CRSsNP patients, they identify that first-degree family members of affected subjects are 4.1 times more likely to develop CRSwNP and 2.4 times more likely to develop CRSsNP(106). However, despite the demonstration of a heritable component, they still suggest an environmental factor as spouses of an affected patient are also 2.0 times more likely to develop CRSsNP as well. This is complemented by work from Sweden. Relatives of patients with nasal polyposis were screened for CRSwNP. 13.4\% of the relatives had nasal polyps (compared to $2.7 \%$ in a control group randomly selected from the Swedish population). Thus the relative risk of the first-degree relatives having nasal polyps when compared to the control group was $4.9^{(107)}$.

Techniques used to identify the genetic basis of disease involve comparisons between two or more groups, usually separated according to the element under study. Markers of genetic variation ('microsatellites' or single nucleotide polymorphisms (SNP)), single genes or multiple genes of a pathway are compared to identify differences in frequency of identified traits. The modern era was ushered in by the introduction of 'chips' which allowed the simultaneous analysis of 100,000+ SNPs simultaneously, interrogating the entire genome in a hypothesis-free fashion (Genome wide association study (GWAS)). More recently, whole-genome sequencing has been used, however, bioinformatic analysis of results remains a rate-limiting step. For validation, findings must be validated via replication in a second population and/or associated with genotype-specific variation in a biologic mechanism or in outcome. For a number of genetic findings, biological plausibility may not be evident as the role these genes play in normal function may not yet be described. One particular problem for CRS is difficulties with statistical methods required in genetic association studies; the size of required populations and the cost of studies. Analysing the typical one million different genetic variations simultaneously increases the risk of spurious association. Thus large, well-characterised populations (1,000-10,000 subjects) are required, with their attendant costs. Caution must be thus be used when interpreting the results from CRS genetic studies in the literature.

\subsubsection{Implications of genetic association studies of CRS} Despite these challenges, genetic assessments of CRS are suggesting links with exiting concepts of pathophysiology and extending the tantalizing promise of future results down the road. Published genetic association studies in CRS have increased in number over the past decade, increasing the number of potential gene candidates (Table 5.2.1.), and also repeatedly implicating certain genes, supporting their relevance to disease (Table 5.2.2.). Increased numbers allowed us to categorise gene candidates according to location or function. In both groups, candidate genes group loosely into regulation of immune function, barrier function, and a variety of SNPs in genes whose functions are unknown or difficult to integrate into our current vision of CRS pathophysiology. Note that the high percentages of identified genes related to immune function may reflect a selection bias of candidate genes studied rather than their actual level of implication. As an example, barrier structure and function genes were not suspected in CRS, but were identified with 'agnostic' or 'hypothesis-free' genome-wide approaches. Subsequently, dysfunction of the epithelial barrier has been confirmed in in vitro models as a novel pathway for CRS development and persistence. This improves our understanding of the disease process and opens potential new targets and approaches for therapy.

\subsubsection{Epigenetics in CRS}

Epigenetics deals with changes in organisms brought about by 
Table 5.2.1. List of genes associated with CRS in more than one study. Genes are grouped according to putative biological role: a. immune system related; b. epithelial barrier related; c. difficult to categorize.

\begin{tabular}{|c|c|}
\hline $\begin{array}{l}\text { a. Immune } \\
\text { Gene }\end{array}$ & Reference \\
\hline ALOX5AP & Al-Shemari et al. 2008 ${ }^{(804)}$; Henmyr et al. 2014(805) \\
\hline $\mathrm{AOAH}$ & Bossé et al. $2009^{(806)} ;$ Zhang et al. $2012^{(807)}$ \\
\hline IL1A & Karjalainen et al. $2003^{(846)}$; Erbek et al. $2007^{(808)}$; Mfuna Endam et al. $2010^{(809)}$ \\
\hline IL1B & Erbek et al. $2007^{(808)}$; Bernstein et al. $2009^{(810)}$ \\
\hline IL10 & Kim et al. $2009^{(811)}$; Bernstein et al. $2009^{(810)}$; Zhang $2012^{(812)}$ \\
\hline IL22RA1 & Endam $2009^{(813)} ;$ Henmyr 2014 ${ }^{(805)}$ \\
\hline IL33 & Buysschaert 2010 ${ }^{(814)}$; Kristjansson 2019(436) \\
\hline IRAK-4 & Tewfik et al. $2009^{(815)}$; Zhang et al. $2011^{(816)}$ \\
\hline NOS1 & Castano et al. 2009(817); Zhang et al. $2011^{(816)} ;$ Henmyr et al. 2014 ${ }^{(805)}$ \\
\hline NOS1AP & Zhang et al. $2011^{(816)}$; Henmyr et al. 2014 ${ }^{(805)}$ \\
\hline TAS2R38 & Adappa et al. 2014(116); Mfuna Endam et al. 2014(111); Purnell et al. 2019(117) \\
\hline TGFB1 & Henmyr et al. 2014 ${ }^{(805)}$ \\
\hline TNFA & Erbek et al. $2007^{(808)}$; Bernstein et al. 2009(810); Batikhan et al. $2010^{(818)}$ \\
\hline \multicolumn{2}{|c|}{ b. Barrier and structural } \\
\hline Gene & Reference \\
\hline None & None \\
\hline \multicolumn{2}{|c|}{ c. Not easily categorised } \\
\hline Gene & Reference \\
\hline DCBLD2 & Pasaje et al. $2012^{(819)}$; Henmyr et al. $2014^{(805)}$ \\
\hline PARS2 & Bossé et al. 2009(806); Henmyr et al. 2014(805) \\
\hline RYBP & Bossé et al. $2009^{(806)}$; Zhang et al. $2011^{(816)}$; Cormier et al. $2014^{(102)}$ \\
\hline
\end{tabular}

modifications in gene expression not resulting directly from alteration of DNA sequences ${ }^{(108)}$. This can lead to the modification of gene expression which can then be transmitted both intragenerationally and inter-generationally. "Epigenetics" denote the way that genes interact with the environment in order to produce each individual phenotype. It is of significant interest that factors associated with increased risk of severity of disease such as cigarette smoking or Staphylococcus aureus are both implicated in epigenetic modification.

Evidence of epigenetics in vivo is still limited, but nevertheless, the concepts suggested by these studies are intriguing and hold promise for the future ${ }^{(109-113)}$. Most studies assessing blood or nasal epithelia obtained from brushing or raised nasal epithelial cultures derived from patients have identified that epigenetic changes are more pronounced in epithelium than in circulating blood, supporting the importance of contact with the external environment for their development. This suggests that pathogens might be playing a role in adapting the environment for evolutionary advantage, and underlines that genetics co-exist with environment, compounding the difficulties in finding a 'single-gene' solution to the problem of CRS.

\subsubsection{Clinical uses of genetics and genotyping in CRS}

\subsection{Characterisation of unexplained immune deficien-} cies

Immune deficiencies frequently present as a clinical portrait or phenotype, where an immune defect is suspected but which may not be specifically elucidated by common blood tests ${ }^{(114)}$. However, sequencing suspect genes may identify the nature of the defect and allow for specific corrective therapy. A recent example of this has been the novel description of TLR3 receptor dysfunction first identified by sequencing patients with herpes simplex encephalitis ${ }^{(115)}$.

\subsubsection{Assessment and selection of therapy for cystic fibrosis (CF)}

CFTR genotyping is not recommended routinely in CRS patients but is instead performed only following demonstration of CFTR gene function impairment via sweat testing. In patients with positive test (high sweat chloride), this will be followed by gene testing for a panel of standard mutations then possibly gene sequencing. Type of mutation identified does not predict evo- 
Table 5.2.2. Genes reported in a single study. Genes are grouped according to putative biological role: a. immune system-related; b. epithelial barrier related; c. difficult to categorize.

\begin{tabular}{|c|c|c|c|}
\hline a. Immune system & & CACNG6 & Lee et al. $2010^{(831)}$ \\
\hline Gene & Reference & $\mathrm{CDH} 23$ & Cormier et al. 2014(102) \\
\hline ALOX15 & Kristjansson et al. $2019^{(436)}$ & K6IRS2 & Cormier et al. 2014 ${ }^{(102)}$ \\
\hline ALOX5 & Al-Shemari et al. 2008 ${ }^{(804)}$ & KCNAM1 & Purkey et al. 2014 \\
\hline BDKRB2 & Cormier et al. 2014 ${ }^{(102)}$ & KCNQ5 & Purkey et al. 2014 \\
\hline CD58 & Pasaje et al. $2011^{(819)}$ & K6IRS4 & Cormier et al. 2014 ${ }^{(102)}$ \\
\hline CD8A & Alromaih et al. $2013^{(820)}$ & LAMA2 & Bossé et al. 2009(806) \\
\hline CIITA & Bae et al. $2013^{(821)}$ & LAMB1 & Bossé et al. 2009(806) \\
\hline CNTN5 & Cormier et al. 2014(102) & LF & Zielinska-Blizniewska et al. $2012^{(827)}$ \\
\hline $\operatorname{cox} 2$ & Sitarek et al. $2012^{(822)}$ & MMP9 & Wang et al. $2010^{(832)}$ \\
\hline CYSLTR1 $(X)^{*}$ & Al-Shemari et al. 2008 ${ }^{(804)}$ & MSRA & Bossé et al. $2009^{(806)}$ \\
\hline FOXP1 & Kristjansson et al. 2019(436) & MUSK & Bossé et al. 2009(806) \\
\hline HLA-DQA1 & Kristjansson et al. 2019(436) & NARF & Cormier et al. $2014^{(102)}$ \\
\hline HLA-DQB1 & Schubert et al. 2004 ${ }^{(823)}$ & NAV3 & Bossé et al. $2009^{(806)}$ \\
\hline HLA-DRA & Bohman et al. 2017(824) & RPGR & Bukowy-Bieryłło et al. $2013^{(833)}$ \\
\hline IGFBP7 & Cormier et al. 2014(102) & c. Not easily categorised & \\
\hline IL1RL1 & Castano et al. $2009^{(817)}$ & Gene & Reference \\
\hline IL1RN & Cheng et al. 2006 & C13orf7 & Cormier et al. 2014(102) \\
\hline IL18R1 & Kristjansson et al. 2019(436) & CYP2S1 & Kristjansson et al. 2019(436) \\
\hline IL4 & Zhang et al. 2012 $2^{(807)}$ & DPP10 & Kim et al. 2015 \\
\hline MET & Sitarek et al. $2012^{(822)}$ & FAM79B & Cormier et al. $2014^{(102)}$ \\
\hline MET1 & Castano et al. $2010^{(826)}$ & GFRA1 & Cormier et al. $2014^{(102)}$ \\
\hline OSF-2 (POSTN) & Zielinska-Blizniewska et al. $2012^{(827)}$ & GNB2 & Purnell et al. $2019^{(117)}$ \\
\hline PDGFD & Cormier et al. 2014(102) & HLCS & Bohman et al. $2017^{(824)}$ \\
\hline PRKCH & Cormier et al. 2014(102) & KIAA1456 & Bossé et al. $2009^{(806)}$ \\
\hline RAC1 & Cormier et al. 2014 & MYRF & Kristjansson et al. 2019 \\
\hline SERPINA1 & Kilty et al. $2010^{(828)}$ & PHF14 & Cormier et al. 2014 \\
\hline TAS2R19 & Purnell et al. $2019^{(117)}$ & PIGT & Cormier et al. 2014 ${ }^{(102)}$ \\
\hline TNFAIP3 & Cormier et al. $2009^{(829)}$ & SLC13A3 & Cormier et al. 2014 ${ }^{(102)}$ \\
\hline TP73 & Tournas et al. $2010^{(830)}$ & SLC22A4 & Kristjansson et al. 2019 $9^{(436)}$ \\
\hline TSLP & Kristjansson et al. 2019(436) & SLC5A1 & Bohman et al. $2017^{(824)}$ \\
\hline VSIR & Bohman et al. 2017(824) & тОММ34 & Cormier et al. 2014(102) \\
\hline b. Barrier and structural & & TRHDE & Cormier et al. 2014(102) \\
\hline Gene & Reference & TRIP12 & Bossé et al. 2009(806) \\
\hline BICD2 & Bohman et al. 2017 & UBE3A & Cormier et al. $2014^{(102)}$ \\
\hline CACNA1I & Bossé et al. $2009^{(806)}$ & UBE3C & Pasaje et al. $2011^{(819)}$ \\
\hline CACNA2D1 & Cormier et al. 2014(102) & $10 p 14$ & Kristjansson et al. $2019^{(436)}$ \\
\hline
\end{tabular}

lution, as the overall clinical picture is believed to be secondary to other 'modifier' genes. However, certain genotypes may predict response to Ivacaflor, a new drug enhancing CFTR gene function.

\subsection{Predictive genetics in CRS}

Given the multiplicity of implicated factors, it is unclear that genetic polymorphisms alone will allow planning of success vs. failure following therapy. However, a number of markers are already predicting type of bacteria likely to be recovered, which offers a beginning of a classification of CRS patients. 
Table 5.2.3. Genetic polymorphisms associated with S. aureus carriage in CRSwNP patients ${ }^{(102)}$.

\begin{tabular}{l} 
a. Immune system \\
Gene \\
\hline BDKRB2 \\
\hline CNTN5 \\
\hline IGFBP7 \\
\hline PDGFD \\
\hline PRKCH \\
\hline RAC1 \\
\hline b. Barrier and structural \\
\hline Gene \\
\hline CACNA2D1 \\
\hline CDH23 \\
\hline GFRA1 \\
\hline K6IRS2 \\
\hline K6IRS4 \\
\hline TOMM34 \\
\hline C. Not easily categorised \\
\hline Gene \\
\hline C13orf7 \\
\hline FAM79B \\
\hline NARF \\
\hline PHF14 \\
\hline PIGT \\
\hline RYBP \\
\hline SLC13A3 \\
\hline TRHDE \\
\hline UBE3A \\
\hline
\end{tabular}

\subsubsection{Taste receptors: predicting Gram-negative carri-} age

TAS2R38 polymorphisms have been associated with $\mathrm{CRS}^{(116)}$. TAS2R38 codes for a type of bitter taste receptor, which is expressed in the airway and is implicated in innate immune defence. Activation of T2Rs by bitter stimuli are followed by secretion of antimicrobial peptides, production of nitric oxide, and increased ciliary beat frequency. The protective genotype codes for ability to detect phenylthiocarbamide (PTC), which can be assessed by simple taste testing. Interestingly, in CRSsNP, the non-tasting (or non-protective) TAS2R38 genotype is associated with a higher rate of gram-negative bacterial carriage and a poor outcome. To this end, many physicians now profile taste as part of patient assessment, however, this does not yet identify optimal therapy. Additionally, there is a concern that the effect may not be similar in patients with CRSwNP. Also, taste receptors may also play role or have predictive value in CRS, notably the taste receptor TAS2R19 (rs10772420)(111, 117). This remains to be validated and replicated in other populations.

\subsubsection{Staphyloccus aureus carriage in CRSwNP}

Genes associated with culture-positivity for S. aureus in CRSwNP patients has been assessed in an agnostic 'hypothesis-free' fashion using a pooling-based Genome-wide Association study ${ }^{(102)}$. Presence of S. aureus in CRSwNP patients is associated with a number of genes loosely organised along reduced engulfment of bacteria, modulation of inflammatory response, and genes of barrier elements (Table 5.2.3.). This supports that CRS patients colonised with $S$. aureus may be subject to immune impairment and dysfunction of the epithelial barrier and may thus be exquisitely sensitive to low level chronic bacterial infection with S. aureus. Attempts to predict S. aureus carriage in individual patients implicates several genes acting together to provide additive effects (Figure 5.2.1.). This supports the concept of multiple genes in a pathway interacting to yield a common final phenotype (S. aureus carriage) and aligns well with our current concepts of disease pathogenesis. Given the ubiquity of S. aureus in CRS and its association with a difficult evolution, having the capacity to identify patients at risk of $S$. aureus carriage prospectively might allow selection of patients for specific anti-S. aureus therapy.

\subsubsection{Summary and future perspectives}

The current knowledge base in genomics of CRS disease offers the tantalizing promise of identifying new mechanisms of disease development and of markers predicting optimal response to available therapies. However, for the moment, genetics do not allow prediction of disease or outcome and its uses are currently restricted to extreme cases to understand the molecular underpinnings of disease. Nevertheless, simultaneous ongoing revolutions in our understanding of CRS and the dissection of implicated mechanisms will complement increased appreciation of genetic changes. Diagnosis of particular forms of disease or identification of particular predisposing factors may help predict evolution and better tailor therapy. Identification of novel pathogenic mechanism may lead to increased scrutiny of genes in unsuspected novel pathways.

\subsubsection{Conclusion}

It is probable that over the coming years we will identify individual or complex genetic traits conferring susceptibility to CRS, evolution of disease, and response to medical or surgical treatment. Given that in other disease areas, genetic traits have already been identified which allow administration of appropriate therapy while minimizing side effects, it is certainly intriguing to explore what role genetic variations may play in CRS diagnosis and therapy.

As knowledge regarding and our appreciation of interactions of immune system / microbiome / epithelial barrier improves, we may be able to develop multi component predictive models 
Figure 5.2.1 Physiologic immune responses across mucosal boundaries are tailored to address particular classes of inciting pathogens. Type 1 responses, depicted on the left, are directed against intracellular pathogens, most commonly viruses. The canonical cytokine is IFN- $\gamma$. Type 2 responses, in the center, are directed against large, extracellular parasites. The canonical cytokines are IL-4, IL-5 and IL-13. Type 3 responses are directed against extracellular bacteria and fungi and the canonical cytokines are IL-17 and IL-22. Each type of response utilizes a rapid response mediated by an innate lymphocyte subset (ILC1, 2 and 3 respectively) that is linked to a corresponding delayed T helper subset (Th1, Th2 and Th17 respectively). In CRS, these pathways are typically coopted with chronic activation of one or more of these responses in the sinonasal tissue.

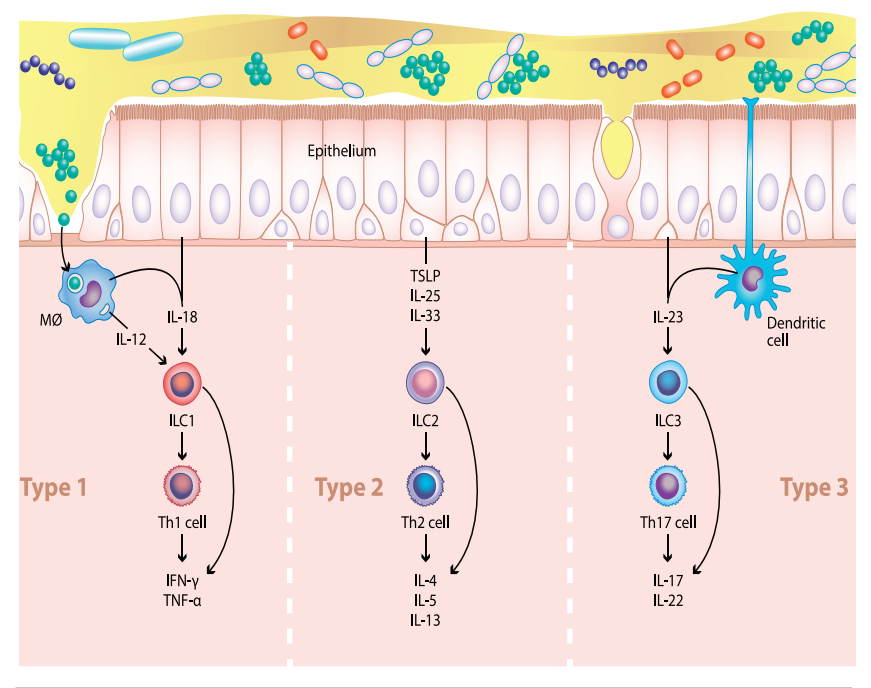

that integrate all of the interaction components and allow more rational administration of therapy and improved clinical care. It will be essential to continue to collect genetic material as a component of clinical trials to be able to verify whether identified factors and factors remaining to be discovered influence response to therapy and can be used for pharmacogenomic purposes.

\subsubsection{Inflammatory mechanisms of CRS}

The inflammatory mechanisms of chronic rhinosinusitis (CRS) are the molecular pathways leading to the establishment of the mucosal inflammation and tissue remodeling that characterizes this broad syndrome. Multiple inflammatory mechanisms are operative, interacting dynamically, and resulting in variable patterns of tissue inflammation that roughly correlate with clinical phenotype. Recent attempts have been made to subdivide CRS into inflammatory endotypes, defined as distinct pathobiological mechanisms, that may vary in time and between different sinonasal anatomic sites in individual patients. From the
Figure 5.2.2. This figure is a proposed model of the Type 1 immune response in CRS. ILC1 cells and dendritic cells set the process in motion. The Type 1 CRS has not been extensively studied and many of the molecular details have not been confirmed at the protein level. The degree of barrier damage, conceptually viewed as a form of tissue remodeling, is variable. In this figure, neutrophil activity is depicted as the primary driver of barrier damage but other factors such as Type 1 cytokines may play a role. Further study is necessary. Other forms of remodeling such as nasal polyposis are less common than seen in Type 2 CRS and the pathways less certain. Emerging evidence however, suggests that Type 1 polyps are composed of fibrin matrix, similar to Type 2 polyps.

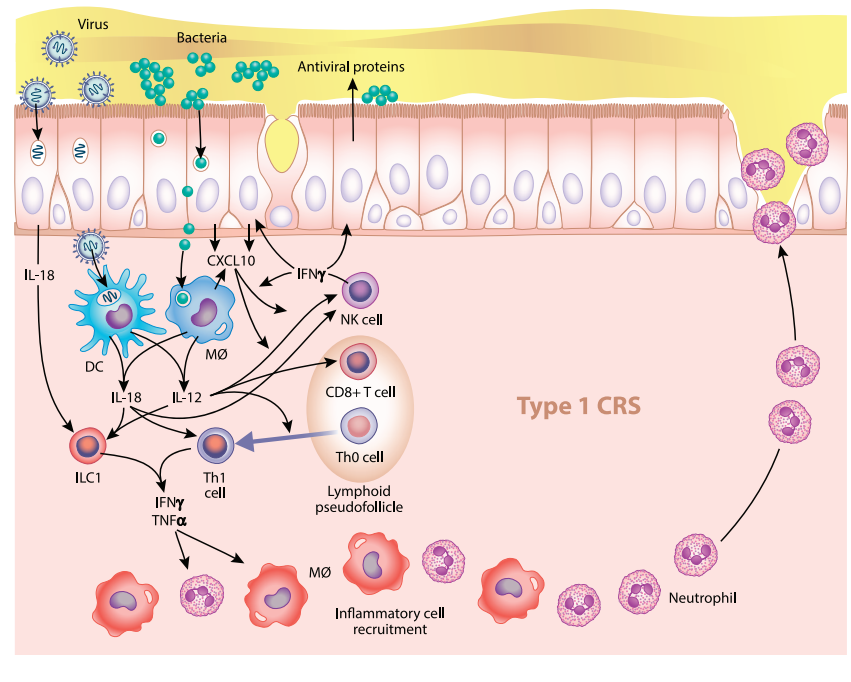

standpoint of aetiopathogenesis, the prevailing, as yet unproven, hypothesis is that these diverse inflammatory mechanisms are driven by dysfunctional interactions at the mucosal surface between the host and environmental stressors. From the host standpoint, genetic and epigenetic variation of the mucosal immune system is believed to play a key role in CRS, but multiple genes are likely involved and thus far, very few have been associated with a large effect-size. The key environmental agents also remain largely uncertain, but cigarette smoke, fungi, viruses, bacteria, pollutants and allergens have all been implicated. The most commonly discussed microbial agent is Staphylococcus aureus, but some evidence also implicates dysbiosis of the microbial community as a whole, rather than a specific dominant pathogen.

Although the host-environment interactions are complex and begin very early in life, perhaps in utero, it is important to bear in mind that CRS is predominantly an adult onset disorder. This suggests that duration, intensity and sequence of exposure, including stochastic events such as viral infection, may be key components of pathogenesis in an individual CRS patient. In 
Figure 5.2.3. This figure is a proposed model of the Type 2 immune response in CRS. ILC2 cells and dendritic cells set the process in motion. Type 2 CRS has been been the most extensively studied subset of CRS, and many of the molecular details have been confirmed at the protein level. The degree of tissue remodeling is greatest in Type 2 CRS, particularly in terms of fibrin mesh formation and barrier damage. These are shown in the figure as driven by IL-13 although other factors likely play a role as well. Inhibition of t-PA (tissue plasminogen activator) results in the deposition of a fibrin mesh that forms the tissue matrix of polyps. Fibrin mesh deposition and polyp formation is less common in Type 1 and Type 3 CRS, but also appears to involve t-PA suppression. Barrier damage in Type 2 CRS is often severe, and this may be a key driver of treatment failure. Factors driving barrier damage include autoimmunity and complement activation as well as a chronically immature state of epithelial differentiation driven by IL-13 amongst other factors. (See text for more details.)

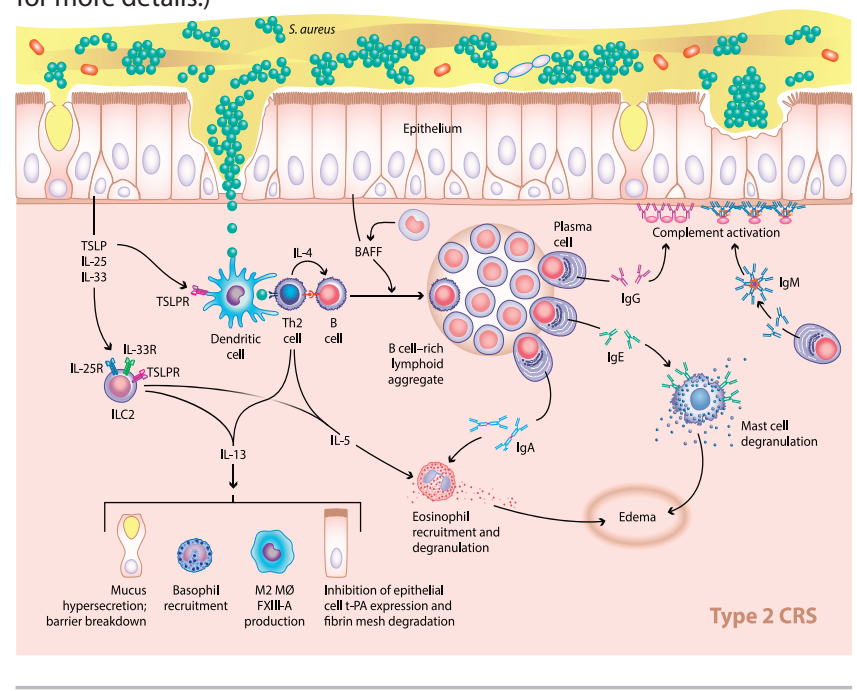

conditions of health, the sinonasal mucosa acts as a relative barrier, modulating environmental stimulation including commensal organisms with a specific, self-limited response. In cases of pathogen penetration, single or combined Type 1, 2 and 3 immunological response pathways are invoked, generating cytokine, chemokine, innate cellular and T helper (Th) responses designed to eliminate the identified class of pathogen with minimal collateral tissue damage (Figure 5.2.1.).

Type 1 canonical cytokines include IFN-gamma (IFN-g) and IL-12 with the response geared towards addressing viral pathogens (Figure 5.2.2.). Canonical Type 2 cytokines are IL-4, IL-5, and IL-13, which promote anti-helminth immunity and regulate tissue regeneration following injury (Figure 5.2.3.). Type 3 cytokines include IL-17A and IL-22 with immunologic effects directed against extracellular bacteria and fungi (Figure 5.2.4.). Each immune response pathway is orchestrated by unique innate lymphoid (ILC) and T helper (Th) subsets that secrete the majority of the key cytokines. Importantly, in vivo responses are often mixed, with significant plasticity in the ILC and Th subsets as well as a self-limited time course. In cases of CRS, mucosal
Figure 5.2.4. This figure is a proposed model of the Type 3 immune response in CRS. ILC3 cells and Th17 cells are key, as well as IL-17, IL-22 and IL-23. Type 3 CRS has not been extensively studied and many of the details have not been confirmed at the protein level. The degree of barrier damage, conceptually viewed as a form of tissue remodeling, is variable. In this figure, neutrophil activity is depicted as the primary driver of barrier damage but other factors such as Type 3 cytokines may play a role. Further study is necessary but the neutrophil response is particularly strong in Type 3 immunity. Fibrin deposition and polyp formation occurs, but with less frequency than in Type 2 CRS.

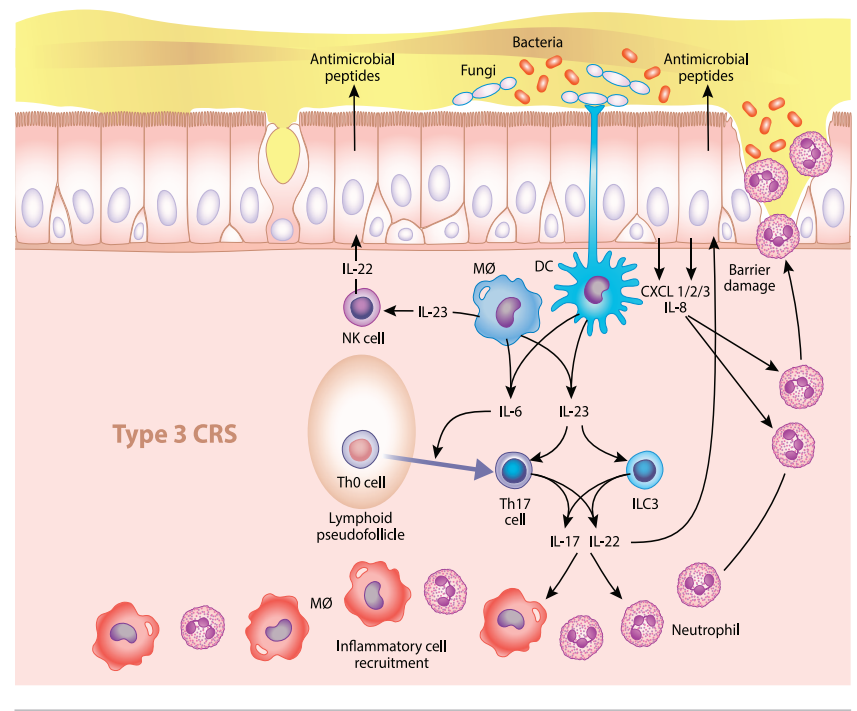

barrier penetration by environmental agents also invokes Type 1, 2 and 3 pathways; however, in contrast to the specific, selflimited, pathogen-directed response associated with physiologic host defence, the CRS response is chronic and polyclonal, directed against an array of thus far poorly characterized targets including self-antigens in some severe cases. The resulting tissue inflammation is of a mixed pattern and intensity, potentially involving multiple endotypes and exhibiting variability in clinical presentation (phenotypes), natural history, comorbidities and outcome.

CRS with Type 2 cytokines is most commonly associated with asthma and resistance to current therapies. The tissue inflammation is often associated with remodeling patterns including fibrosis, polypoid oedema, fibrin deposition and barrier failure. It is unclear if factors associated with barrier failure mediate initial barrier penetration, but failure may promote a feed forward process whereby the loss of barrier results in greater antigenic stimulation and more inflammation, further damaging the barrier and potentially fostering chronicity. Future challenges include a better understanding of: 1 ) the aetiologic factors that drive CRS 
with a goal towards prevention; 2 ) the pathophysiologic inflammatory mechanisms and relevant endotype biomarkers with a goal toward targeted therapy; 3 ) the molecular mechanisms of barrier and tissue remodeling that may play a role in persistence and recurrence.

\subsubsection{Introduction}

Chronic rhinosinusitis (CRS) is a clinical syndrome, rather than a specific disease, characterized by persistent symptomatic inflammation of the nose and paranasal sinus mucosa for more than 12 weeks. This definition is purposely broad and says nothing about aetiology, pathogenesis, clinical presentation or natural history. In a small subset of patients, CRS syndrome occurs in association with a known systemic disorder or local process. For the vast majority, aetiology is uncertain although multiple environmental and host genetic factors have been implicated. From the standpoint of pathogenesis, these host and environmental factors interact over time to trigger one or more mechanistic pathways (endotypes) of chronic tissue inflammation that lead to the clinical presentation (phenotype). This chapter will review the host and environmental factors implicated in this process and the mechanistic pathways that ensue. Overall, while this remains an area of active research, relative consensus has emerged on three points: 1 ) CRS is typically an antegrade process with the mucosal inflammation triggered by a dysfunctional interaction between exogenous agents inhaled through the nose and the host immune system; 2) specific causal factors likely vary in importance in individual patients leading to different types or patterns of tissue inflammation (endotypes); 3) the clinical characteristics (phenotypes), natural history and response to treatment will depend on 1 and 2. Since the last EPOS document, much research has been done to better characterize both the host immune response and the environmental stressors that interact in CRS. A Literature search covering January 2012 to May 2019 produced 1202 publications in English concerning pathomechanisms of human CRS. The most frequently existing search words were "eosinophil" in 401/1202 publications, "epithelium" in 370, "bacteria" in 184, "type 2" in 180, "IL-25"in 132 and "lymphocyte" in 87 publications, respectively. A systematic review of all of these publications was undertaken for EPOS 2020. This chapter will update the current understanding of CRS aetiology and pathogenesis. The environmental factors and host immune elements implicated in CRS will be reviewed first. The last section will discuss a current concept of aetiopathogenesis and endotypes resulting from host-environment interactions.

\subsubsection{Environmental factors}

Microbial agents are generally viewed as the most important environmental drivers of CRS. Molecular sequencing techniques are evolving in power and are now being applied to study the total and relative microbial abundance of the sinonasal tract and the functional activity of microbiota in health and disease. Acute viral infection has been implicated in both the initial development and subsequent exacerbations of asthma, yet the role of viruses in CRS pathogenesis remains unclear. Studies on bacteria have revealed the abundance and diversity of bacterial genera present in the nose and sinuses, further suggesting alterations in association with the presence of CRS. In particular, the presence of $S$. aureus has been hypothesized to potentiate certain inflammatory mechanisms of CRS. Fungi seem to affect CRS at least in some sporadic cases. Allergens and an array of other environmental agents also pay a role in CRS. The final part of this section will address other environmental factors such as allergens and air pollutants, which have been little studied in CRS

\subsection{Viruses}

An RNA virus has RNA as its genetic material. Usually it is singlestranded RNA (ssRNA) but it may be double-stranded RNA (dsRNA). RNA viruses that contribute to respiratory infections include rhinovirus, respiratory syncytial virus and influenza. RNA viruses have high mutation rates compared to DNA viruses because viral RNA polymerases lack the proofreading ability of DNA polymerases ${ }^{(118)}$. The common cold is caused by RNA viruses and is the most common viral disease. Ex vivo studies of mucosal tissue have shown that rhinovirus infection can be linked with exacerbations of CRS, including increased bacterial adhesion; increased susceptibility to secondary microbial infection (by bacteria, fungi, or other viruses), reduction of epithelial barrier integrity, respiratory exacerbations (as in asthma, CF and bronchiectasis subjects); epithelial damage, including necrosis; ciliary dysfunction; and impairment of mucociliary clearance and mucus overproduction ${ }^{(119,120)}$. A study group evaluated the prevalence of rhinovirus and respiratory syncytial virus by performing PCR to mucus specimens that were collected from 56 CRSwNP and 23 CRSsNP patients in Iran undergoing ESS ${ }^{(121)}$. The prevalence of at least one virus was 33\%, which is higher than that reported in the literature in healthy adults suffering from the common cold $(10 \%)^{(122)}$. Cadherin-related family member 3 gene (CDHR3) is a rhinovirus receptor and its rs6967330 risk allele (A) has been shown to be associated with childhood asthma with severe exacerbations ${ }^{(123)}$ and adult CRS, suggesting a role for aberrant rhinovirus effects in the pathogenesis of both diseases $^{(124)}$. Carlson-Jones showed virus-like particles and bacterial populations by performing flow cytometry to five sinus flush samples $^{(125)}$. Virus infection activates TLR3 and TLR7-9 signaling in human airway epithelium. Tengroth et al. showed defects in the TLR9 mediated microbial defence close to anatomical origin of polyp, which might explain virus induced polyp growth ${ }^{(126)}$. Golebski exposed epithelial cells from CRSwNP patients to various TLR-specific triggers and detected a higher TSLP- gene and protein expression in polyp compared to control epithelium, 
suggesting that this could reflect contribution of viral infections to CRSwNP pathogenesis ${ }^{(127)}$. Rowan et al. performed PCRbased sequencing on sinus samples of 21 CRS patients and 14 controls and detected positive screens in four CRSsNP patients, one CRSwNP patient and none of the controls ${ }^{(66)}$. Coronavirus was the most common virus detected. Liao et al. performed $\mathrm{PCR}$ of nine common viruses from middle meatal scrapings of 53 controls and $128 \mathrm{CRS}$ patients who were without common cold $^{(128)}$. Overall about $70 \%$ of subjects had viral infections and there were no significant differences between subject groups by virus profiles, nor were there associations of viral infection with symptoms, computed tomography or endoscopic scores ${ }^{(128)}$. A DNA virus has DNA as its genetic material and replicates using a DNA-dependent DNA polymerase. The nucleic acid is usually double-stranded DNA (dsDNA) but may also be single-stranded DNA (ssDNA), which is usually expanded to double-stranded in infected cells. Notable DNA-viruses in the head and neck region under normal conditions and during cancer pathogenesis are human herpes virus Epstein-Barr virus (EBV), and human papillomavirus (HPV), yet their role in CRS pathogenesis still needs more evidence from research. loannidis et al. detected with quantitative PCR a trend that EBV and Human Herpes Virus-6 (HHV-6) associated with nasal polyps specimens compared to controls ${ }^{(129)}$, whereas Ha et al. did not detect EBV-positive lymphoid cells in the nasal mucosa of 420 patients who had undergone surgical resection of lesions with various diagnoses such as CRS(wNP), AR, papilloma, cyst, or septal deviation ${ }^{(130)}$. Wang et al. infected cultured inferior turbinate and nasal polyp samples with either Herpes Simplex Virus-1 (HSV1) or S. aureus alone or a combination of both ${ }^{(131)}$. Epithelial damage scores were significantly higher for HSV1 and S. aureus co-infected explants compared with control explants or S. aureus only-infected explants, and significantly correlated with HSV1-invasion scores, thus suggesting that HSV-1 infection facilitates invasion of $S$. aureus $^{(131)}$. Taken together, virus infection might contribute to development and exacerbations of CRS, via damaged airway barrier, though further studies are needed.

\subsection{Bacteria}

Bacteria can be classified into five groups according to their shapes (such as cocci) or to their metabolism (such as anaerobes). This classification has been replaced by an operational taxonomic unit (OTU), in which bacteria are grouped by DNA sequence similarity of a specific taxonomic marker gene, especially when analyzing small subunit $16 \mathrm{~S}$ ribosomal RNA (16S rRNA) marker gene sequence datasets ${ }^{(132)}$. 16S rRNA is the component of the $30 \mathrm{~S}$ small and highly conserved subunit of a prokaryotic ribosome and thus 16S rRNA -genes are used for quantitation of different sinonasal bacteria. Metatranscriptomics is a functional technique profiling gene expression of complex microbial communities enhancing our understanding of the complex com- munity behavior of the microbiome ${ }^{(132)}$. In addition to expression profiling, the place and timing of sampling are important to consider when investigating host-microbial events of CRS pathogenesis. Still the greatest challenge remains in identifying causality in microbial contribution to natural homeostasis and CRS. Acute exacerbations of CRS have been more closely linked to specific bacteria, perhaps following a viral infection, but it remains unclear whether the presence or abundance of particular bacteria are a cause or a consequence of CRS development. Commensals likely play important roles in immune priming and development of healthy sinus mucosa and most microbial colonies are benign or even beneficial. The resident microbiota imbedded in the respiratory mucus effectively provide the first line of defence in that commensal organisms prevent colonization or proliferation of pathogens and also likely provide certain local metabolites that enhance mucosal health. Bacterial communities vary more between subjects than between sites within a subject, although significant regional differences also exist in a given individual(133).

Dysbiosis is a term indicating microbial imbalance and some evidence suggests that it is associated with the sinonasal microbiome of CRS patients ${ }^{(134-141)}$. Lal et al. performed 16S rRNA gene sequencing and showed that microbiome profile of CRSsNP subjects exhibited decreased diversity and anaerobic enrichment ${ }^{(142)}$. The size of an antrostomy $y^{(143)}$, as well as comorbid asthma and purulence affect resident bacterial communities and may predict surgical outcome ${ }^{(144)}$. Moreover, the sinonasal bacterial microbiomes are significantly different between healthy individuals in general ${ }^{(136)}$, and between sinonasal anatomic subsites of an individual patient. Some evidence suggests that CRS phenotypes are associated with distinct microbiomes ${ }^{(135,142,}$ ${ }^{145)}$ and the microbiomes change, at least transiently, after sinus surgery ${ }^{(146)}$. Copeland et al. detected that Escherichia was overrepresented in CRS sinuses, and that sinus samples were superior to middle meatal samples as they have less intra-patient variation $^{(137)}$. Cope et al. performed 16S rRNA gene sequencing for intraoperative sinus brushings from 59 CRS patients and 10 controls, and detected that CRS patients fall into clusters based on co-colonization pattern of Streptococcaceae, Pseudomonadaceae, Corynebacteriaceae, or Staphylococcaceae $e^{(147)}$. Each pathogenic microbiota was functionally distinct and was associated with distinct host immune responses ${ }^{(147)}$. Traditional culture techniques, as opposed to the newer molecular techniques, typically reveal only the most rapidly growing organisms. Nevertheless, large scale studies have been done over the years. Liu et al. performed bacterial culture from middle meatal swabs of 165 CRSwNP, 76 CRSsNP and 44 control subjects and detected coagulase-negative staphylococci to be closely associated with all three subject groups, whereas $S$. aureus, Streptococcus, Haemophilus, Enterobacter and Corynebacterium species were associated with CRSwNP(148). The prevalence 
of Gram-negative aerobic and facultative anaerobic bacteria was high in the non-eosinophilic CRSwNP subgroup ${ }^{(148)}$. Another study performed middle meatal cultures of 136 CRSwNP, 66 CRSsNP, and 49 control subjects and revealed that the isolates were similar in all three groups, the commonest being coagulasenegative staphylococcus, Corynebacterium and Staphylococcus epidermidis and S. aureus ${ }^{(149)}$. The CRSwNP group presented with high relative abundance of Citrobacter and the eosinophilic CRSwNP group presented with S. aureus(149). Tabet et al. performed culture of middle meatal swabs from 337 CRS patients and showed that S. aureus (33\%), Corynebacterium diphtheria (26\%), coagulase-negative staphylococci (51\%), and Pseudomonas aeruginosa (7\%) were the most frequently recovered organisms ${ }^{(150)}$. Relative abundance of the Bacteroidetes phylum in CRS has been shown to be associated with increased severity of histopathological inflammation and the presence of mucosal ulceration ${ }^{(150)}$. PCR studies have not detected DNA of Helicobacter pylori in nasal polyp tissue ${ }^{(151)}$, nor C. pneumoniae or M. pneumonia in sinus aspirates $^{(152)}$ or nasal polyp tissue ${ }^{(153)}$. Hauser et al. showed that culture of ethmoid samples from CRS patients identifies only about $50 \%$ of the taxa compared to $16 \mathrm{~S}$ rRNA gene sequencing, and $12 \%$ of isolates detected by culture were not identified by 16S rRNA gene sequencing, highlighting the need for replication by several methods ${ }^{(154)}$. In summary, traditional culture techniques are still used in the setting of acute exacerbations, but their clinical utility and pathophysiologic relevance in CRS has greatly diminished in recent years. The newer molecular microbiome data has provide support for the hypothesis that dysbiosis of the community as a whole, as opposed to individual organisms, may trigger mucosal inflammation ${ }^{(132)}$. From this perspective, the resident microbiota embedded in the respiratory mucus effectively provide the first line of defence. Commensal organisms would prevent colonization of pathogens and also likely provide certain local metabolites that enhance mucosal health. The results vary greatly, however, depending on the number of subjects, sampling methodology, techniques used, depth of sequencing, phenotype and prior treatments. As a result, any firm conclusions about the precise nature of any dysbiosis remain limited, but these newer techniques are only now being applied to large numbers of patients using standardized protocols. The largest study to date did not indicate any key role for a specific organism but did show that the depletion of two genera (Corynebacterium and Peptonophilus) was associated with CRS. It is possible that studies using narrow CRS patient groups may reveal associations with characteristic microbiomes or even the presence of specific organisms. Nevertheless, this would only be the first step towards validating the microbiome hypothesis as the association of a more specific microbial signature with a specific endotype or phenotype may not be causative.

A biofilm consists of bacterial colony embedded within an extracellular matrix of polymeric substances, which makes it resistant to environmental stress, host defences and antimicrobial treatment ${ }^{(155)}$. Biofilm layers can be detected by staining and light microscopy but characterization of bacterial species and dynamics of biofilms require advanced optical technology such as electron microscopy, confocal laser scanning microscopy, fluorescent tagging and in-situ hybridization ${ }^{(156)}$. Pathogens such as Haemophilus influenzae, Streptococcus pneumoniae, Pseudomonas aeruginosa, Moraxella catarrhalis and S. aureus can all form sinonasal biofilms in CRS patients, some of which have been associated with a worse prognosis. The bacterial composition of biofilms vary between the ethmoid bulla and middle turbinate ${ }^{(157)}$. An uncontrolled study detected bacteria in 29/30 postoperative paranasal sinus specimens and 23/62 strains were coagulase-negative Staphylococcus epidermidis and 6/62 strains were Escherichia coli(155). 58/62 strains were used to assess biofilm formation, of which about $30 \%$ were moderate to strong biofilm producers $^{(155)}$. In a Chinese study 13 out of 19 CRSwNP patients and none of the controls had biofilms, which were associated with increased mucosal inflammatory signs ${ }^{(158)}$, purulent nasal discharge, preoperative Lund-Kennedy scores, serum total lgE, and previous ESS ${ }^{(159)}$. An uncontrolled study showed that 39/84 CRS patients had both ethmoidal osteitis and bacterial biofilms, and their extent correlated ${ }^{(160)}$. In a study by Marcinkiewicz et al. biofilms were found by scanning electron microcopy in 3/10 samples from CRSwNP patients undergoing ESS; S. epidermidis was the primary isolated bacteria and, biofilm-associated neutrophil infiltration was detected with compromised antimicrobial activity ${ }^{(161)}$. Arjomandi et al., visualized sinonasal biofilms in 15 of 20 patients with CRS confirmed by microbial presence using fluorescence in-situ hybridization, and was associated with host inflammatory response involving plasma cells and eosinophil recruitment ${ }^{(162)}$. Overall, the literature indicates an association between the presence of biofilm-forming pathogens and CRS. This suggests the hypothesis that biofilms are potentially an important factor in CRS pathogenesis explaining resistance to conventional therapy. Moreover, the emergence of biofilms due to one or more stochastic events could possibly address the question of aetiology, serving as a potential initiating event. It should be kept in mind however, that the simple presence of biofilms does not indicate pathology since the sinonasal tract likely exhibits commensal and pathologic biofilms in both health and disease. Detection of the relative abundance of commensal and pathogenic biofilms would be challenging and any results very difficult to interpret. Moreover, biofilms are a survival adaptation and the presence of pathogenic biofilms may reflect a response to stress (e.g. antibiotics). Lastly, the presence of pathogenic biofilms may reflect variation in the host immune response that is unrelated to CRS causation. In summary, the role of biofilms in the aetiology and pathogenesis of CRS remains unclear.

S. aureus is the microbe most commonly associated with CRS. 
Using conventional culture techniques, this organism has been shown to colonize the nasal cavity of one-third of the general population but affects a higher percentage of patients with ${ }^{(163)}$ eosinophilic CRS, specifically CRSwNP patients with asthma ${ }^{(59,}$ ${ }^{164)}$. This organism also has the capability to reside within nasal epithelial cells, the presence of which in CRS appears to confer a poor prognosis ${ }^{(102,151,152,165,166)}$. Some molecular studies support a key role for staphylococcus as well, indicating a greater abundance of S. aureus and altered microbial composition in CRS vs. controls. The largest molecular study on the sinonasal microbiome thus far undertaken however, did not suggest a key role for S. aureus in CRS in general. At this point, aggregate evidence would suggest that $S$. aureus is most closely associated with a subpopulation of CRS patients, specifically severe CRSWNP and asthma patients exhibiting high tissue eosinophilia(160). High tissue eosinophilia is typically associated with high levels of cytokines IL-4, IL-5 and IL-13, which are also known as Type 2 cytokines ${ }^{(167)}$. (See section 5.2.2.3 for a more complete description of Type 1, 2 and 3 inflammation.)

The precise nature of the association between S. aureus and Type 2 cytokine signaling is however, unclear. The presence of Type 2 inflammation should independently favor S. aureus colonization since Type 2 inflammation suppresses aspects of the normal immune response directed against S. aureus. In addition this organism also has the capacity to secrete superantigenic toxins that can directly alter the immune response of the host, in part, triggering locally produced polyclonal lgE. This is supported by a large, multi-institutional European study, which revealed the presence of staphylococcal superantigen effects in a subpopulation of severe, Type 2 CRSwNP patients ${ }^{(153)}$. Polyp samples from Asian CRSwNP patients, which tend to have lower expression levels of Type 2 cytokines than Western polyps, show little evidence of superantigen effects ${ }^{(168,169)}$. Viewed conservatively, these findings would suggest that superantigens accentuate but do not initiate Type 2 inflammation. Challenging this perspective are recent papers suggesting that $S$. aureus is a key player actually driving Type 2 inflammation primarily via a specific pattern recognition receptor (TLR2) unrelated to, and upstream from any superantigen effects ${ }^{(170-172)}$. A recent report has demonstrated large amounts of locally expressed IgE in some Type 2 polyp patients, targeting common resident nasal bacteria including S. aureus, Streptococcus pyogenes and Hemophilus influenza ${ }^{(172)}$. While the mechanism is unclear, this study indicates that the Type2-skewing detected in CRSWNP is not solely directed against antigens derived from S. aureus. Taken together, implementation of 16s rRNA gene sequencing technology on a small scale has suggested that some degree of bacterial dysbiosis may be associated with CRS pathogenesis, although it is not yet known whether it is a primary or secondary event. The composition of microbiota varies based on age, stochastic events, prior treatment, anatomic subsite and, most importantly, individual variation. This necessitates very large, well-controlled studies, which, for the most part, have yet to be done. While a large proportion of CRS patient heterogeneity could be explained by the composition of their sinus bacterial microbiota and related host immune response, it is unclear if direct manipulation of the microbiome can be an effective treatment modality ${ }^{(147)}$. Implementation of hypothesis-free and functional (such as meta-transcriptomics) approaches may be useful in addressing both causation and therapy.

\subsection{Fungi}

Commensal fungi are present over all mucosal surfaces of the body, with potentially important roles in CRS. Initial studies suggesting that fungi were the key drivers of CRS have not been validated, but their presence, nevertheless, almost assuredly impacts mucosal health. Similar to bacteria, fungi are also capable of forming biofilms and secreting toxins but the relevance to CRS pathogenesis is uncertain. Fungi can be difficult to detect, as traditional cultures are not particularly sensitive. Molecular techniques (such as 18S rRNA gene sequencing) are now being applied however, and the knowledge gained should eventually expand our understanding of fungi in both health and CRS. Nuclear ribosomal internal transcribed spacer (ITS) region serves a universal DNA barcode marker for fungal species ${ }^{(173)}$. Hoggard et al. performed middle meatal ITS2 marker amplicon sequencing of 106 CRS and 38 control subjects and data demonstrated that there is high abundance of Malassezia spp, and while fungal communities vary seasonally, they vary little between subject $\operatorname{groups}^{(174)}$.

Zhao et al. estimated fungal biomass by extracting DNA and measuring ITS amplicon concentration using fluorometry of middle meatal swabs of 63 CRS and 27 control subjects ${ }^{(138)}$. Fungi were identified in $14 \%$ CRS patients by conventional techniques (culture and histology) ${ }^{(138)}$. This fungus-identified group had a significantly higher average ITS concentration and a significantly lower Shannon's diversity index compared to the other two groups $^{(138)}$. The most abundant organism sequenced was Aspergillus $^{(138)}$. Cleland et al. performed 18S rRNA gene sequencing of middle meatal swabs from 23 CRS and 11 control subjects, and detected ubiquitous and rich (a total of 207) fungal genera, Malassezia being the most abundant ${ }^{(175)}$. Fungal richness did not correlate with $\mathrm{QOL}$ and it decreased postoperatively ${ }^{(175)}$. Aurora et al. sequenced bacterial $16 \mathrm{~S}$ and fungal $18 \mathrm{~S}$ rRNA genes of middle meatal lavage samples from 30 CRS and 12 control subjects and detected quantitative increase in most bacterial and fungal species, and also host secreted IL-4, IL-5, IL-8, and IL-13, eosinophils and basophils in CRS relative to controls ${ }^{(176)}$. Yet the microbiomes of CRS patients were qualitatively similar to the controls ${ }^{(176)}$. Hirotsu et al. performed culture, microscopy and PCR on 35 middle meatal nasal polyps and 15 sphenoidal mucosal samples of controls ${ }^{(177)}$. No microbiological growth or 
fungal bodies were observed, whereas 16/35 samples showed amplification of fungal DNA (such as C. parapsilosis, R. mucilaginosa and Aspergillus sp), which was associated with enhanced tissue eosinophils ${ }^{(177)}$. Mohammadi et al. performed PCR and clinical examination of sinonasal samples obtained intraoperatively from various locations from 100 patients with a suspicion of noninvasive fungal rhinosinusitis, and showed that the proportion of fungal rhinosinusitis was $27 \%$ (such as Aspergillus flavus, Penicillium chrysogenum and Candida glabrata species complex), of these $41 \%$ were housekeepers by way of occupation ${ }^{(178)}$. Fungi have the capacity to stimulate significant host responses in vitro, suggesting a role in CRS pathogenesis. Using ELISA, Sproson et al. studied cytokines in nasal polyp tissue, challenged ex vivo by Alternaria alternata, Aspergillus niger, Cladosporium sphaerospermum and Penicillium notatum. Results indicated that Aspergillus niger stimulation increased pro-inflammatory cytokines TNF-a, GM-CSF and IL-6 ${ }^{(179)}$. In contrast, stimulation with Cladosporium sphaerospermum, Alternaria alternata and Penicillium notatum reduced pro-inflammatory cytokines TNF-a and IL-6, but induced a dose-dependent increase in remodeling cytokines TGF-b1 and bFGF, which suggests a possible disease-modifying role for fungi in $\mathrm{CRS}^{(179)}$. Shin et al. performed PCR to primary nasal polyp fibroblasts ${ }^{(180,181)}$, epithelium ${ }^{(182)}$ that were co-cultured with Alternaria and Aspergillus. They detected induction of IL-6, IL-8, TLR2, TLR5 and extracellular matrix in fibroblasts ${ }^{(180,181)}$ and fungi and RV-16 induced mucin gene expression in epithelial cells ${ }^{(182)}$. Rai et al. performed ELISA of serum samples for 40 CRSWNP and 20 control subjects and PCR of nasal lavages for CRSWNP group and detected that serum levels of IL-1 $\beta$, IL-17, IL-21, and TGF- $\beta$ were higher in CRSwNP group compared to controls ${ }^{(183)}$. The proportion of Aspergillus flavus positive CRSwNP patients was $78 \%$, and they had increased risk of elevated serum IL-17 ${ }^{(183)}$. Lawrence et al. co-cultured sinus epithelial cells with Aspergillus and Alternaria, from nine CRSwNP and seven control subjects and demonstrated by performing ELISA of cell supernatants that fungi increased IL-6 and IL-8 production in both CRSwNP and control groups, which was decreased by superoxide dismutase ${ }^{(184)}$. In overview, fungi are clearly present in CRS and many have the capability to stimulate an inflammatory response, at least in vitro. Any generalizable relevance to CRS pathogenesis is, however, quite uncertain as fungal directed therapies were ineffective. Much more work, using large numbers of patients and standardized techniques, will be necessary before any firm conclusions can be made about the fungal microbiome in health and CRS. Although a broad role in pathogenesis is uncertain, fungi almost certainly play key roles in two CRS subtypes: fungal balls and allergic fungal rhinosinusitis (AFRS). Aspergillus can cause a fungal ball, which is usually unilateral, and is more prevalent in middle aged to elderly females. Histologically, fungal balls are characterized by entangled masses of fungal organisms or masses of fungi embedded in fibrinous, necrotic exudate, with minimal mucosal inflammatory reaction without tissue invasion or granulomatous inflammation.

Allergic fungal rhinosinusitis was classically defined over 20 years ago as: 1) Type 1 hypersensitivity; 2) nasal polyposis; 3) characteristic CT changes; 4) eosinophilic mucus; 5) presence of non-invasive fungus in sinus contents ${ }^{(185)}$. We now know that, when using special techniques, fungi can be detected in the nose and sinuses of all patients with and without CRS, indicating that the fifth requirement needs revision. In contrast to normal patients and routine CRS, patients with fungal rhinosinusitis have large amounts of fungi that are readily detectable by routine histologic techniques. The EPOS steering group agreed that allergic fungal rhinosinusitis (AFRS) should be regarded as a subgroup of eosinophilic fungal rhinosinusitis and reserved for subjects with evidence of an allergic reaction to fungi e.g. a positive skin prick and/or specific lgE.

In comparison to typical CRSwNP patients, AFRS patients tend to be younger, express consistently high serum $\lg \mathrm{E}$, and have milder asthma in general. AFRS is also more common in humid climates with a tendency to present unilaterally ${ }^{(186)}$. Despite the phenotypic differences, distinguishing AFRS and generic CRSwNP has been problematic at the molecular level ${ }^{(187)}$. More recent work has suggested that an innate immune defect (possibly in TLR4) may account for fungal accumulation in the sinonasal cavities of AFRS patients ${ }^{(186)}$. Fungi have intrinsic protease effects that induce Type 2 cytokine signaling leading to the accumulation of eosinophilic mucin, which is histologically characterized by sloughed epithelial cells, eosinophils, Charcot-Leyden crystals, and scattered fungal hyphae. Furthermore, these same protease effects should foster a type 2 response as well, favoring local and systemic IgE responses to fungal antigens $^{(188,189)}$. The corollary of this hypothesis would be that fungi drive AFRS, with $T$ and $B$ cells targeting the fungi as a major component of the disease. Secondary barrier damage may, however, lead to the targeting of many other colonizing microbes thereby blurring the distinction between AFRS and CRSWNP as the process evolves.

\subsubsection{2 . Other environmental factors}

Immune responses against airborne allergens with or without co-existing CRS are common in Western individuals. The relationship between allergic rhinitis (AR) and chronic rhinosinusitis has been studied for decades and remains controversial and while many large studies have demonstrated an association, the significance is unclear ${ }^{(190)}$. Allergic rhinitis occurs through host sensitization to foreign proteins (allergens) across a mucosal barrier via dendritic cells and naive CD4-positive lymphocytes, with the generation of antigen-specific type 2 lymphocytes and IgE-secreting plasma cells. The sensitizations patterns for airborne allergens vary significantly by geography, genetics, living 
conditions and climate, amongst other factors ${ }^{(191-193)}$. Regardless, subsequent allergen challenge across the nasal mucosa of sensitized individuals results in cross-linking of $\operatorname{lgE}$ bound to the surface of mast cells with degranulation, mediator, chemokine and cytokine release, leading to the recruitment of other inflammatory cells. In the unoperated state, allergens should have limited access to the sinus mucosa, but they can certainly trigger inflammation of the nasal cavity reducing sinus drainage. An 'allergic phenotype of CRS' has been proposed by two separate groups of investigators, with primarily central intense nasal inflammation and milder signs of inflammation in the dependent sinuses ${ }^{(194)}$. Further study is necessary to validate whether an allergic phenotype of CRS is a significant discrete entity.

Severe CRS is, in general, not regarded as an allergic disease, but co-existing allergic rhinitis will accentuate Type 2 inflammatory mechanisms of CRS. There is limited epidemiological evidence of an association with nasal polyposis and it has been interpreted that allergy does not increase the probability of CRSWNP. A recent study in the Chinese population showed distinct mucosal immunopathologic profiles in atopic and nonatopic CRSsNP patients, suggesting that in the Chinese population the comorbid CRSsNP and AR might be more related to Type 2 mechanisms, whereas the phenotype CRSsNP without AR exhibits more non-Type 2 mechanisms ${ }^{(187)}$. Clearly, there is significant overlap between AR and CRS in terms of Type 2 cytokines. While the initiating events in CRS are unknown, allergen sensitization is, in part, related to complex interactions with the epithelial surface. Allergens typically possess intrinsic protease activity that can interact with epithelial cells through three principle pathways: direct effects on junctional proteins, reaction with cell surface protease-activated receptors and toll-like receptor 4 (TLR4)dependent epithelial activation ${ }^{(186)}$. Protease activity of allergens may elicit the release of epithelial-derived Type 2-driving cytokines and chemokines, some of which have also been implicated in CRS. In terms of potential overlap between AR and CRS pathogenesis, Kouzaki et al. showed lower host expression of two protease inhibitors (cystatin A and SPINK5) in the nasal epithelial cells extracted from patients with eosinophilic CRS compared with control and non-eosinophilic CRS groups ${ }^{(195)}$. This suggests that an imbalance of proteases and protease inhibitors within the epithelial barrier may contribute to the pathogenesis of Type 2 diseases in general ${ }^{(196)}$. As we advance our understanding of the interactions between barrier immunity and the environment as a function of time, the relationship between allergens and CRS pathogenesis should become clearer.

Exposure to tobacco smoke alters sinonasal mucosa. Xie et al. performed microscopy and ELISA on polyps obtained from 28 non-smoking and 21 smoking CRSwNP patients and detected that cigarette smoke exposure downregulates expression levels of E-prostanoid receptors and stimulates the production of pros- taglandin E2, IL-8 and TNF-a ${ }^{(197)}$. Mulligan et al. isolated polyp/ mucosal dendritic cell subsets from CRSwNP and control subjects and detected by flow cytometry that in general, exposure to tobacco smoke alters the sinonasal composition of dendritic cells ${ }^{(198)}$. Tharakan et al. grew sinonasal epithelial cells from control patients at the air-liquid interface and were able to demonstrate that cigarette smoke extract stimulation caused disruption of the epithelial junctional proteins and transepithelial electrical resistance, whereas barrier dysfunction was reversible by an antioxidant (Nrf2) activation ${ }^{(199)}$. Huang et al. performed PCR on the nasal tissues of 40 asthmatic patients with/without CRS and detected that cigarette smoke was associated with IL$17 \mathrm{~A}$ activation and lesser improvement of asthma control after sinonasal surgery ${ }^{(200)}$. Kule et al. performed polyp microscopy in 30 smoking and 47 non-smoking CRSwNP patients and showed no differences between the groups in basement membrane thickness, goblet cell hyperplasia, subepithelial oedema, submucous glands, tissue leukocyte subsets, squamous cell metaplasia, or stromal cell atypia ${ }^{(201)}$. Shin et al. stimulated nasal fibroblasts with cigarette smoke extract which had a stimulatory effect on vascular endothelial growth factor mRNA and protein expression through the TLR4, ROS, MAPK and NF-kappaB signaling pathways ${ }^{(202)}$.

Ozone is an air pollutant with known proinflammatory effects on exposed epithelia. Zhu et al. cultured polyp/mucosa from CRSWNP \pm ASA intolerance and control subjects and exposed these in vitro to ozone ${ }^{(203)}$. They showed by immunohistochemistry and ELISA that after ozone exposure, Cox $1 / 2$ expression remained unchanged in all groups, yet PGE(2) release was lowered more in ASA tolerant than in ASA intolerant patients after ozone exposure ${ }^{(203)}$. Clara cell protein 16 (CC16) is produced by non-ciliated Clara cells in the respiratory epithelium and has an anti-inflammatory role in chronic upper and lower airway eosinophilic inflammations ${ }^{(204)}$. Decreased levels of CC16 are found in the nasal secretions and plasma of patients with chronic eosinophilic inflammatory disorders, such as asthma, allergic rhinitis and CRSwNP and CRSsNP, as well as in people exposed to high levels of air pollutants ${ }^{(204)}$. Tissue metal levels are increased due to smoking, environmental and occupational exposure. Khlifi et al. studied blood concentrations of heavy metals, cadmium and nickel, in 90 CRSwNP and 171 control Tunisian subjects and higher heavy metal concentrations were associated with CRSwNP, with tobacco consumers and with CRSwNP patients with occupational exposure ${ }^{(205)}$. Among risk factors, shisha consumption, environmental exposure and occupational exposure presented the most significant association with CRSwNP(205). The same study group measured cadmium, chromium, nickel and arsenic by atomic absorption spectrometry of polyp/nasal mucosa from 55 CRSwNP subjects and they detected increased heavy metal levels in polyp tissue compared to non-polyp tissue from the same patient, suggesting a role of heavy metal exposure in 
CRSwNP pathogenesis ${ }^{(206)}$. Yamin et al. stimulated with cigarette smoke extract or dsRNA cultured polyp/mucosa from CRSsNP, CRSWNP and control subjects and detected increased TGF- $\beta 1$ and activin $A$ in CRSwNP patients, suggesting that cigarette smoke and viral infection might contribute to polyp remodeling ${ }^{(207)}$.

There is limited knowledge of the effects of nutrition on CRS pathogenesis. Vitamin D and its receptors might be involved in the regulation of the immune system and may play role in CRS. Tomaszewska et al. performed immunohistochemistry of ostiomeatal complex tissue from 52 CRSsNP, 55 CRSwNP and 59 control subjects and detected a decrease in vitamin $D$ receptor nuclear staining in CRS patients as compared to controls ${ }^{(208)}$. Vitamin D3 deficiency has been shown to cause changes in murine sinonasal immunity as measured by tissue immune cell infiltrate and nasal lavage cytokine levels ${ }^{(209)}$.

\subsubsection{Host factors}

The function of the immune system is to maintain homeostasis and to prevent or eradicate infections, but chronic activation can also be seen in a range of disorders. Host defences are grouped under innate immunity which provides immediate protection against microbial invasion, and adaptive immunity which provides a delayed but more specialized defence against infections. The innate and adaptive immune systems are presented in this section as discrete functional entities, but they largely overlap in nature. The initial portion of this section will discuss the cells and molecules that are related to innate immunity responses. Next the adaptive immune responses are discussed. The final part of this section will address other host factors, such as remodeling, which is presumably driven by the presence of the chronically activated immune response.

\subsection{Innate immunity}

Innate immunity is present in healthy individuals at baseline, prepared to block the entry of microbes and to rapidly eliminate microbes entering the host tissues. The components of innate immune system include epithelial barrier, sentinel mucosal cells (resident macrophages, dendritic cells, mast cells etc.), circulating and recruited phagocytes (monocytes and neutrophils), innate lymphoid cells, NK cells, as well as non-cellular components (e.g. complement system). The major functions of the sinonasal innate immune system include phagocytosis, recruiting immune cells to sites of infection, activation of the complement cascade to identify and clear off bacteria, antibody complexes or dead cells, identification and removal of foreign substances, and activation of the adaptive immune system through antigen presentation.

\section{Epithelial barrier}

The sinonasal tract is covered by ciliated pseudostratified columnar, olfactory, and to lesser extent layered squamous epithelium (in the nostril area). Airway epithelium plays a critical role in conducting and humidifying air, responding to trigeminal and olfactory stimuli, and host defence. Airway epithelial barrier comprises ciliated cells, olfactory cells (in olfactory epithelium) mucus-secreting goblet cells, and basal cells which are considered progenitor cells $s^{(210)}$. More recently, solitary chemosensory cells (SCCs) have also been reported in the sinonasal epithelium, playing a significant role in IL-25 production contributing to Type 2 immunity ${ }^{(211)}$.

Transcriptomics of healthy middle turbinate epithelium showed that about three quarters of protein coding genes are expressed, suggesting nasal epithelium is a very active organ ${ }^{(212)}$. Both epithelial specification and terminal differentiation are critical to epithelial homeostasis. Environmental and mucosal signals regulate epithelial stem-cell self-renewal under normal conditions $^{(213)}$ and in $\mathrm{CRS}^{(112)}$. Under physiological conditions environmental and intrinsic signals are able to alter the composition and function of epithelium rapidly. Hence investigating the role of differentially expressed epithelial genes or proteins between CRS and controls in a cross-sectional set-up is challenging. Single cell transcriptomics detect cell subtypes exhibiting distinct gene expression profiles and are able to trace cell transitions in development and disease. Ordovas-Montanes et al. performed single cell transcriptomics of polyp/scraping epithelium from 12 CRSWNP and 9 control subjects and detected differences in expression of antimicrobial genes by secretory cells, a loss of glandular cell heterogeneity, and that polyp basal progenitor cells were locked by a Type 2 memory ${ }^{(112)}$. Epithelial basal progenitor cells are able to migrate and proliferate into ciliated and goblet cells in injured regions. Epithelial cells may also de-differentiate through squamous metaplasia or epithelial to mesenchymal transition (EMT), which describes a rapid and normally reversible modulation of the epithelial phenotype toward mesenchymal cells ${ }^{(214)}$. Epithelial cells undergoing EMT lose cell-cell polarity and adhesion to become migratory and usually downregulate junctional proteins (such as E-cadherin) while acquiring mesenchymal features such as alpha-smooth muscle actin, vimentin, matrix metalloproteinases (MMPs), and the transcription factors. Studies on polyp and control tissue show that CRSwNP may be associated with elevated expression levels of MMP-1 ${ }^{(215,216)}$, MMP-2 ${ }^{(215)}$, TIMP-1 ${ }^{(217,218)}$, MMP-7 $^{(219,220)}$, MMP-9 $9^{(215,217-219,221-224)}$, and suppressed TIMP-2 ${ }^{(221)}$ and E-cadherin $^{(225-227)}$ expression. Periostin binds to several integrin molecules, including $\operatorname{aV} \beta 1, a \vee \beta 3$, and $\operatorname{aV} \beta 5$ on the epithelial cell surface to support adhesion and migration of epithelial cells, and elevated airway mucosal periostin may be useful in detecting Type-2 $\mathrm{CRSWNP}^{(228-238)}$ and asthma ${ }^{(239)}$. Kim et al. suggested that a main cellular source of periostin in eosinophilic CRSwNP are mast cells ${ }^{(240)}$. Regardless, it has been proposed that abnormalities of epithelial turnover and maturation may result in an immature, 
leaky barrier with significance in CRS pathogenesis ${ }^{(230)}$. This will be discussed further in section 5.2.2.3.3.

Epithelial physical barriers are maintained by intercellular junctions, especially tight junctions (TJs). TJs are located most apically, are linked to cytoskeleton, and inhibit solute and water movement through the paracellular space, thus establishing cell polarity ${ }^{(214)}$.TJ molecules and epithelial barrier function in CRS may be modulated by various internal and external factors such as inhaled allergens, microbial or viral infections, cytokines, hypoxia, or zinc deficiency ${ }^{(241)}$. Several genes/molecules, such as SPINK5, S100A7, S100A8/9, PCDH1, NDRG1, SPRR, and p63 are involved in modulating the physical barrier function in CRS $^{(241)}$. Epithelium membrane protein 1 (EMP1) is a TJ protein mediating cell proliferation and its gene and protein expression has been shown to be lower in nasal polyp epithelium compared to control nasal mucosa ${ }^{(242)}$. Soyka et al. detected a decreased trans-tissue resistance in biopsy specimens from patients with CRSwNP along with decreased TJ proteins ${ }^{(243)}$. Suzuki et al. measured electrical impedance in vivo using a tissue conductance meter and performed microscopy and qRT-PCR, detecting that electrical impedance did not differ between the turbinate and polyp tissue ${ }^{(244)}$. The polyp tended to show higher expression of claudin-1 but showed lower expression of tricellulin than the turbinate $^{(244)}$. Jiao et al. assessed transepithelial resistance in ALIcultured polyp/mucosal samples from 17 eosinophilic CRSWNP, 15 noneosinophilic CRSWNP and 20 control subjects and detected that integrity of epithelial TJ barrier in the nasal epithelium is compromised in Chinese patients with eosinophilic and noneosinophilic CRSwNP, and that TGF- $\beta 1$ plays an important role in inducing TJ barrier defects(245). Li et al. showed that the expression of epithelial ZO-1, claudin-1, DSG1 and DSG2 in the CRSwNP patient group and the expression of claudin-1, DSG1 and DSG2 of the CRSsNP patient group was significantly lower compared to that of the control group ${ }^{(246)}$. Overall, these data suggest that the mechanical barrier is more susceptible to penetration in patients with CRS.

Epithelial cell communication is mediated via, for example Gap junction channels, which are formed by connexin proteins and they facilitate communication between adjacent cells in homeostasis and inflammation ${ }^{(247)}$. Kim et al. performed immunofluorescence microscopy and $\mathrm{qPCR}$ to sinus mucosa biopsies from 11 CRS and 7 control subjects and detected increased gene and/ or protein expression of connexins in CRS compared to controls $^{(247)}$. The relevance to CRS pathogenesis remains unclear. Cilia are microtubule-based hair-like organelles that are fundamental to the development and physiology of many organs. Mucociliary clearance (MCC) and apical junctional complexes between epithelial cells comprise a mechanical barrier between host and environment, which provides the first line of host defence for the nose and sinuses. Coordinated ciliary beating transports debris-laden mucus from respiratory passages towards the oropharynx ${ }^{(248)}$. Genetic and acquired defects in mucociliary flow with increased mucus viscosity are associated with a high incidence of $\mathrm{CRS}^{(69,249)}$. Li et al. detected structural and functional abnormalities in the cilia obtained from CRSwNP patients ${ }^{(250,251)}$. The cytokines IFN- $\gamma$ and IL-13, but not IL-17 decrease differentiation of ciliated epithelial cells and secondary reduction in ciliary beat frequency ${ }^{(251)}$. Ma et al. cultured sinonasal epithelial cells and found that cilia loss and decreased expression of WDPCP, a ciliogenesis protein, are associated with CRScompared to controls ${ }^{(252)}$. Overall, these studies indicate that CRS is commonly associated with ciliary dysfunction.

Cilia are coated with membrane-spanning mucins and tethered mucopolysaccharides that exclude mucus from the periciliary space and promote formation of distinct mucus layers ${ }^{(214)}$. Secretory cells produce a different class of mucins, the polymeric gelforming mucins. The two major airway gel-forming mucins are MUC5AC and MUC5B. Some secretory cells, known as mucous or goblet cells, produce mucins and store them within easily visualized collections of mucin granules, whereas other cells produce and secrete mucins (especially MUC5B) but lack prominent granules ${ }^{(214)}$. Gel-forming mucins are secreted into the airway lumen and are responsible for the characteristic viscoelastic properties of the mucus gel layer. Tipirneni et al. identified that dynamic mucus strand velocities from submucosal glands, a major component of MCC, were significantly decreased in CRS patients compared to controls ${ }^{(253)}$.

Surfactants are compounds that lower the surface tension. El-Anwar et al. performed PCR and immunohistochemistry on inferior turbinate and sinus mucosal biopsies from 30 CRS, 30 primary atrophic rhinitis and 20 control subjects, and demonstrated that Surfactant protein A gene expression was increased in CRS and decreased in primary atrophic rhinitis patients compared to controls ${ }^{(254)}$.

Pattern recognition receptors (PRRs) rapidly detect microbial and other foreign molecular patterns and either maintain homoeostasis or induce immune responses. Toll-like receptors (TLR1-TLR10), NOD receptors, RIG-like receptors and dectins are among the best-known pattern recognition molecules ${ }^{(214)}$. Tengroth et al. demonstrated that nasal apical epithelium expresses abundant TLR3, TLR7, TLR9, RIG-I, and MDA-5, and that TLRagonist-stimulation leads to upregulation of several secreted cytokines $^{(255)}$. The group detected defects in the TLR9-mediated microbial defence in the turbinates of CRSwNP patients, which could in part explain virus-induced polyp growth ${ }^{(126)}$. Studies on polyp and control tissue show that CRSwNP may be related to increased epithelial expression levels of TLR $2^{(256)}$ and $4^{(256-258)}$.

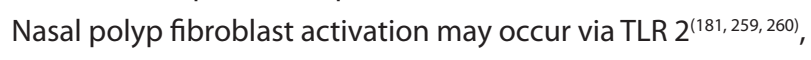
TLR $4^{(261,262)}$, TLR $5^{(181)}$, and TLR $9^{(263)}$ might be related to polyp $B$ cell activation ${ }^{(264)}$.

NOD family PRRs form a major component of the inflammasome, which is a multiprotein oligomer that maturates and 
secretes pro-inflammatory cytokines interleukin $1 \beta$ (IL-1 $\beta$ ) and interleukin 18 (IL-18). This results in pyroptosis, a form of programmed pro-inflammatory cell death distinct from apoptosis. Jardeleza et al. performed microscopy and PCR of polyp/mucosal samples of CRS patients with/without $S$. aureus biofilms, and detected involvement of inflammasome complexes and their signaling pathways in biofilm positive CRSwNP(265). Lin et al. performed microscopy and ELISA of polyp/mucosal samples from CRSwNP and control subjects and detected that NLRP3 (NACHT, LRR and PYD domains-containing protein 3 ) and caspase-1 were overexpressed in eosinophilic CRSwNP, and that the inflammasome signaling pathway was augmented by LPS but suppressed by glyburide, suggesting that inflammasome is playing a pro-inflammatory role in eosinophilic CRSwNP pathogenesis ${ }^{(266)}$.

Bitter taste receptors (T2Rs), also present on epithelial cells, function as non-classical PRRs. They are G protein-coupled receptors (GPCRs), which are widely expressed in host airway epithelial membranes. Airway T2R-mediated immune responses are activated by bacterial quinolones as well as acyl-homoserine lactones ${ }^{(267)}$ secreted by gram-negative bacteria, including Pseudomonas aeruginosa ${ }^{(268)}$. Linkage studies have demonstrated associations between taste receptor genetics and $\mathrm{CRS}^{(269)}$. Both mucosal and glandular epithelium secrete a large array of host defence molecules (such as surfactant, lactoferrin, defensin) that are known to kill or neutralize microorganisms. Disruption of this antimicrobial action might lead to the risk of colonization or infection by microorganisms, which might be associated with the pathogenesis of chronic airway inflammation. Baseline levels are secreted into the mucus and production is increased with PRR stimulation. Some evidence suggests that decreased secretion of some host defence molecules is associated with $\mathrm{CRS}^{(270)}$. Studies on polyp and control tissue show that CRSWNP may be related to decreased expression levels of PLUNC, possibly secondary to loss of glands, as well as increased surfactant-B and alpha-defensin expression ${ }^{(265,267,271,272)}$. Protease inhibitors regulate environmental proteases that might compromise barrier and they have been discussed in the earlier section on allergens (5.2.2.4).

\section{Innate lymphoid cells}

Innate lymphoid cells (ILCS) are lineage and antigen receptornegative lymphocytes including natural killer (NK) cells and at least three distinguishable cell subsets (ILC1, ILC2, ILC3) ${ }^{(167)}$ that rapidly produce cytokines (IFN- $\gamma$, IL-5, IL-13, IL-17A, IL-22) upon activation $^{(273)}$. As such, ILCs can act as first-line defenders in the airway epithelial barrier. ILC1, ILC2 and ILC3 correspond to the Th1, Th2, and Th17 subsets of CD4+ T cells, respectively ${ }^{(274)}$. The development of ILC2s, the best studied of ILC subsets, is controlled by the GATA-3 transcription factor and they produce IL-5 and IL-13 ${ }^{(275)}$. In response to tightly regulated local environmental cues, ILCs can generate characteristics of other subtypes, a process known as plasticity ${ }^{(275)}$. ILC2s can promptly shift to functional IFN- $\gamma$-producing ILC1s or IL-17-producing ILC3s, depending on the cytokines and chemokines produced by antigen presenting cells or epithelial cells. Due to as yet unknown triggers, this complex network of signals may become dysregulated $^{(275)}$. Several distinct ILC precursors (ILCP) with panILC (giving rise to all ILCs) or subset-restricted potentials have been described ${ }^{(273)}$. How and where these different ILCPs give rise to more mature tissue-resident ILCs remains unclear. Recently, environmental signals have been shown to epigenetically influence canonical ILC differentiation pathways, generating substantial functional plasticity ${ }^{(273)}$. Although there is significant intergroup plasticity, each ILC group appears to have a distinct physiologic role and cytokine profile described as Type 1,2 and 3 inflammation respectively ${ }^{(276)}$. ILC1 cells respond to viruses and intracellular bacteria, fostering a Th1 lymphocyte response and release various cytokines including IFN-g which is considered the canonical Type 1 cytokine. ILC2 cells target parasites, assist in tissue repair and also mediate allergy. They foster type 2 responses and are associated with the canonical Type 2 cytokines IL-4, IL-5 and IL-13. ILC3 cells target extracellular organisms, foster Th17 responses and release IL-17 and IL-22, canonical Type 3 cytokines $^{(277,278)}$. ILC2 cells are the best studied in CRS, playing a significant role in eosinophilic CRSwNP, and possibly all CRS that exhibits Type 2 inflammation. ILC2s are likely an important source of Type 2 cytokines that drive tissue inflammation. On the other hand, while canonical Type 1 and Type 3 cytokines are clearly present in the tissue of some CRS patients, the importance and contribution of ILC1 and ILC3 cells to this process is unclear. From the standpoint of epithelial signaling to ILCs in CRS, the key upstream epithelial cytokines thus far identified include IL-25(279-281), IL-33 (279-281), and thymic stromal lymphopoietin (TSLP) ${ }^{(127,279,280,282-284)}$. They activate ILC2 cells as well as other cell types, which is the physiologic response to parasites. A more comprehensive knowledge of the molecular mechanisms that regulate ILC development and effector functions may allow for better understanding of their role in CRS.

\section{Neutrophils}

Neutrophils, also known as polymorphonuclear leukocytes, are the most abundant leukocytes in the blood and are important in the early phagocytosis and killing of extracellular mucosal microbes ${ }^{(274)}$. Neutrophil recruitment is driven in large part by microbial stimulation (via pattern recognition receptors) or tissue damage signals, and epithelial release of IL- $8^{(285)}$. In response to bacterial or fungal infection, the production of neutrophils from bone marrow increases, rapidly leading to their increased levels in blood ${ }^{(274)}$. Neutrophils typically live for only 1-2 days, expressing receptors for products of complement activation and for antibodies that coat microbes ${ }^{(274)}$. These receptors increase 
the ability of the neutrophils to kill ingested microbes. The role of the neutrophil in CRS pathophysiology remains unclear, but they are found in all or nearly all cases, with the highest tissue levels seen in CF patients ${ }^{(286)}$. For other forms of CRS, differences appear to depend on ethnicity as well as on the presence or absence of nasal polyps. In Caucasians, neutrophilic infiltration can be demonstrated in CRS, with slightly lower levels observed in CRSsNP than in CRSwNP. Nevertheless, in general, the degree of neutrophilic infiltrate is comparable between CRSsNP and CRSwNP as opposed to the eosinophilic infiltrate, which is significantly less in CRSsNP. As a corollary, it has been suggested that CRSsNP is more distinctly a neutrophilic process, whereas CRSwNP is more eosinophilic, based on the relative degree of tissue infiltration ${ }^{(286)}$. In the subpopulation of CRSwNP patients with relatively low eosinophilic infiltration, it has been suggested that neutrophils may be the major pathologic driver of disease, analogous to 'neutrophilic' asthma ${ }^{(287)}$. In studies of polyps from Chinese patients, although both neutrophilic and eosinophilic infiltration are decreased compared with polyps in Caucasian patients, the degree of eosinophilia reduction is much more pronounced, which suggests these polyps are relatively neutrophilic ${ }^{(288,289)}$. Immunologic profiling of Asian CRSsNP demonstrated strong Type 2 and 3 inflammation with dominant neutrophil-related markers, which were associated with disease extent ${ }^{(290)}$. Neutrophils are driven most strongly by Type 3 cytokines and their infiltrates often co-exist with Type-2 cytokine-driven eosinophils, suggesting the possibility that this tissue neutrophilia may reflect a superimposed physiologic response against microbiota in CRS patients ${ }^{(286,290-292)}$. Clinically, this may explain some degree of corticosteroid resistance seen in some forms of $\mathrm{CRS}^{(293)}$.

The role of neutrophils in CRS pathogenesis is uncertain, but these cells are capable of degranulation with tissue damage including some loss of barrier integrity. In addition, similar to macrophages ${ }^{(286,291)}$, neutrophils can differentiate from the N1 phagocytic type, into the $\mathrm{N} 2$ form of neutrophils in the presence of Type 2 cytokines. Among other factors, N2 neutrophils release oncostatin M (OSM), which is important in epithelial repair and homeostasis ${ }^{(291,293,294)}$. OSM is believed to be operative in the early stages of epithelial repair but excess amounts or the diminished follow up signals have been associated with defective barrier regeneration ${ }^{(292,295)}$, helping to drive Type $2 \mathrm{CRS}^{(293)}$.

\section{Monocytes}

Monocytes are less abundant in the blood than neutrophils, and they also ingest microbes in the blood and tissues ${ }^{(274)}$. During inflammation, monocytes extravasate into the tissue and differentiate into macrophages, which may survive for a long time, even years ${ }^{(274)}$. Macrophages exist in all connective tissue and organs of the body, having diverse roles that include removal of particulates, primary response to pathogens, tissue homeostasis, coor- dination of the adaptive immune response, inflammation and tissue repair ${ }^{(296)}$. There are two types of activated macrophages, M1 and M2. M1 macrophages are the dominating phenotype observed in the early stages of inflammation, and the activation is driven by Type 1 cytokines that trigger a proinflammatory response necessary to kill intracellular pathogens. The alternative pathway is driven by Type 2 cytokines in the local milieu, which leads to activation of $\mathrm{M} 2$ macrophages; this process is important in the defence against helminths, in humoral immunity, and in tissue repair. Conversion to M2 status may be important in Type 2 CRSwNP and CCL23 may be the key chemokine for the recruitment of macrophages into the polyp, which then convert to $M 2$ in the Type 2 cytokine milieu ${ }^{(297)}$. These M2 macrophages appear to have an impaired ability to phagocytose and also secrete high levels of CCL18, a cytokine known to be chemotactic for dendritic cells, naïve T cells, and Th2 cells - all of which may contribute to the pathogenesis of CRSwNP(298). M2 macrophages are also important sources of factor XIII-A in Caucasian CRSwNP patients, which induces excessive fibrin deposition and secondary tissue oedema in nasal polyps ${ }^{(299-302)}$. In relationship to CRSsNP, macrophages have not been extensively studied, but they are often elevated, presumably play a central role in Type 1 CRS inflammation. Macrophages may also recruit both eosinophils and neutrophils to the site of inflammation $299,303,304)$.

\section{Basophils}

Basophils are granulocytes found mainly in the circulation and are known to have a role in allergic disease and immunity against parasites. They have been implicated in Type 2 responses in general, possibly serving as an early source of IL-4 driving polarization in the Th2 direction (see below) ${ }^{(167)}$. They are particularly elevated in aspirin tolerant polyps as opposed to N-ERD polyps. Their significance in CRSwNP remains unclear.

\section{Mast cells}

Mast cells are present in all connective tissues, especially under epithelia, and adjacent to blood vessels with physiologic roles in innate immunity and wound healing ${ }^{(305)}$. They can be activated by microbial products binding TLRs and by components of the complement system as part of the innate immunity or by an antibody-dependent mechanism in adaptive immunity. Mast cell granules contain vasoactive amines such as histamine that cause vasodilation and increased capillary permeability, as well as proteolytic enzymes, lipid mediators, chemokines and cytokines, which provide a defence against helminths, other pathogens and animal venoms, and which are also responsible for early onset allergic symptoms via antigen-driven lgE cross-linking ${ }^{(305)}$. Overall, the downstream effects of this process include tissue oedema, degradation of the ECM, and diminished barrier integrity. In CRS, interest has centred on a role for mast cells in nasal polyposis, in part as a result of the potential to in- 
duce, augment and maintain eosinophilic inflammation through IgE-dependent and lgE-independent processes ${ }^{(306)}$. Specifically, local IgE class switching directed against common aeroallergens may mediate mast cell activation and contribute to subsequent eosinophilic inflammation in CRSwNP, but non-IgE mediated activation mechanisms such as CD30L may also be play a role ${ }^{(307)}$. Mast cells, along with platelets (see below), may be sources of proinflammatory leukotrienes (cysLTs) and prostaglandins (PGD2) in CRSwNP and N-ERD ${ }^{(308,309)}$. Mast cells in CRSwNP are localized near the glandular tissue of polyps, but the clinical significance is unclear ${ }^{(310-312)}$. Recent clinical trials using anti-lgE for CRSwNP have also demonstrated efficacy independent of systemic allergy, which suggests the potential importance of the mast cell in at least a subtype of CRSwNP patients with local IgE production ${ }^{(313)}$. Overall, however, the relative importance of mast cells versus other cell types in the pathogenesis of CRSwNP remains unclear ${ }^{(314)}$.

\section{Eosinophils}

Eosinophils have a significant role in tissue repair and immune defence, in particular against helminths ${ }^{(274)}$. Eosinophils are also an important cell type in asthma, AR and CRS; and CRS was at one time considered by many to be a purely eosinophilic disease. Eosinophilic damage to the sinonasal mucosa was believed to be the central pathophysiologic mechanism of CRS and the hallmark of the disorder ${ }^{(315,316)}$. It was always clear, however, that non-eosinophilic forms existed, and some cases of CRS demonstrated relatively minimal eosinophilia with a predominant influx of other cell types ${ }^{(317)}$. Notably, separation of CRS tissue specimens into CRSsNP and CRSwNP demonstrated that tissue eosinophilia was much higher on average in polyps ${ }^{(318,319)}$. This close association, independent of atopy, suggested that eosinophils may be critical to polyp formation, but the relationship between CRSwNP and mucosal eosinophilia is not maintained in Asian polyps or in a demonstrable minority of Western/Caucasian polyps ${ }^{(320,321)}$ in comparison to most Caucasian polyps ${ }^{(322)}$. Taken together, these studies indicate that eosinophils are not absolutely necessary for nasal polyposis or CRS to be present. Although this might appear to diminish the importance of these cells in CRS, recent studies have demonstrated that tissue eosinophilia correlates with a relatively poor outcome independent of the presence or absence of polyps ${ }^{(323,324)}$. Consequently, whereas eosinophils are not essential for CRS to exist, they appear to be a biomarker for severe, recalcitrant disease, and may still be the cell that mediates this relatively poor prognosis ${ }^{(325,326)}$. Systemic or tissue biomarkers for eosinophilic CRS could have, therefore, a significant clinical role ${ }^{(327,328)}$.

The overall process of eosinophil recruitment, activation and survival in CRS is driven largely by epithelial cytokines, exogenous proteases, complement proteins, eicosanoids, stem cell factor and Type 2 cytokines ${ }^{(198,284,329-331)}$. The critical upstream cellular sources of the Type 2 cytokines in eosinophilic CRS include ILC2 and Th2 cells ${ }^{(332)}$. Although the pathological effects of eosinophil activity in CRS are unclear, presumably degranulation causes tissue oedema, epithelial sloughing and possbily some fibrosis, as has been reported in asthma ${ }^{(333,334)}$. Eosinophils have also been shown to exert a host defence effect against bacteria at sites of barrier damage via the formation of extracellular traps, which may have collateral negative effects on barrier integrity as well(335). The mechanism for eosinophil degranulation in CRS is unclear, but data from other tissues suggest that cross-linking of receptors for $\lg \mathrm{A}$ is an important trigger ${ }^{(336)}$. High levels of $\lg \mathrm{A}$ have been identified in nasal polyps, which suggests that this immunoglobulin may play a key role in vivo ${ }^{(337,338)}$.

The association of eosinophilia with refractory disease makes this cell a potentially important target in CRS. Eosinophils are steroid responsive, which likely explains at least some of the therapeutic effects of glucocorticoids in $\mathrm{CRS}^{(339)}$. A doubleblinded trial using oral corticosteroids demonstrated clinical efficacy and reduced IL-5 and eosinophilic cationic protein in nasal secretions ${ }^{(340)}$. Unfortunately, systemic steroids have broad tissue effects resulting in significant morbidity. A more directed approach has been suggested by recent papers which identified specific features of activated eosinophils, variations that could potentially be targeted to reduce tissue eosinophilia( ${ }^{(328)}$. This concept has been utilized in early clinical trials using anti-IL-5 and in CRSwNP with evidence for reduced polyp eosinophilia as well as clinical efficacy ${ }^{(341)}$. Although the majority of CRSwNP in Western countries demonstrate a Type 2 cytokine profile with polyp eosinophilia ${ }^{(342-344)}$, only about half demonstrated significant polyp shrinkage with anti-IL-5 mAb therapy ${ }^{(341)}$. In addition, a recent study utilizing the oral anti-eosinophil drug, dexpamipexole, demonstrated a $>90 \%$ decrease in both blood and polyp eosinophils in CRSwNP patients without significant improvement in symptoms or polyp shrinkage ${ }^{(345)}$. These results suggest that despite being a predictive marker for disease-severity, eosinophils may account for only a portion of Type 2 CRS pathophysiology.

\section{Natural killer cells}

Natural killer (NK) cells are a type of cytotoxic lymphocyte critical to the innate immune system. NK cells recognize infected and stressed cells and respond by killing these cells and by secreting the macrophage-activating cytokine IFN- $\gamma^{(274)}$. NK cells do not express immunoglobulins or T cell receptors, the antigen receptors of B and T lymphocytes. On activation by (virus-) infected cells, NK cells empty their cytoplasmic granules ${ }^{(274)}$. The granule proteins enter infected cells and induce apoptosis. Kim et al. peformed flow cytometry on peripheral blood mononuclear cells from 18 CRS and 19 control subjects, and detected that compared to the controls, impaired ability of NK cells to degranulate and to produce cytokines such as IFN- $\gamma$ and TNF- $a$ 
was associated with CRSwNP, concomitant asthma and peripheral blood eosinophilia ${ }^{(346)}$. The study group also showed that compared with healthy controls, eosinophil apoptosis mediated by NK cells was significantly decreased in CRS patients ${ }^{(347)}$. Smith et al. performed flow cytometry to peripheral blood mononuclear cells and to polyp/mucosal samples from eight CRSsNP, eight CRSWNP and eight control subjects, and detected that compared to controls, sinonasal markers of cytotoxity, granzyme B and perforin, CD8+T cells, but not the NK cells, were decreased in CRSsNP group ${ }^{(331)}$. Invariant natural killer T (iNKT) cells and mucosal associated invariant T (MAIT) cells express $T$ cell receptors of very limited diversity ${ }^{(327)}$. iNKT and MAIT cells recognise antigens presented by the $\mathrm{MHC}$ class 1-like monomorphic molecules CD1d and MR1, respectively. Both iNKT cells and MAIT cells have been identified in the skin and airways and can rapidly produce cytokines after activation ${ }^{(327)}$. Luukkainen et al. stimulated with H3N2-virus a co-culture of peripheral blood mononuclear cells and nasal epithelium, differentiated from stem/progenitor cells, and detected rapid activation of monocytes, NK-cells and innate T-cells (MAIT and $\gamma \delta$ T cells) bridging systemic models and possibly enabling the development of targeted immunomodulatory therapies ${ }^{(328)}$. Taken together, innate immunity is crucial to maintain sinonasal homeostasis, and most probably disrupted homeostasis between microbiota and host innate immune system is a key event in CRS.

\section{Platelets}

Platelets are cytoplasmic fragments that primarily function in haemostasis. In addition, since they are rapidly deployed to sites of injury, they may potentially modulate inflammatory processes by interacting with effector cells and secreting mediators ${ }^{(274)}$. Platelets are activated and a major source of proinflammatory leukotrienes (cysLTs) and prostaglandins (PGD2) in CRSwNP and, in particular N-ERD ${ }^{(348)}$. Based on released microparticles, which may indicate signs of cell injury or pathology, platelets, as well as mast cells and basophils, were more highly activated in patients with N-ERD than in patients with CRS. However, overall it is not yet clear whether platelet activation plays a direct role in the development of the aspirin-induced reactions.

\subsubsection{Mechanisms of adaptive immunity}

The separation of the innate and the adaptive immune responses into distinct compartments is artificial; in vivo, these two arms of the immune response are mutually supportive and distinctions have been blurred. Nevertheless, the separation is useful for mechanistic and temporal explanations of immune defence across mucosal barriers. Specifically, if penetration of the mechanical and innate defences is sufficiently strong, an adaptive immune response will be initiated and maintained; the overall response will be specialized to address both the specific nature and the strength of the insult. Cytokine crosstalk between epithelial cells (ECs), innate lymphocytes (ILCs), and dendritic cells (DCs) plays a key role in matching the appropriate adaptive response to foreign stimuli. In health, this maintains mucosal homeostasis with 1) tolerance of potential allergens and commensals and 2) defence against pathogens without the development of chronic inflammation. Adaptive immunity is typically divided into a T-cell response and a B-cell response, and these will be discussed as they relate to CRS.

\section{Antigen presentation, dendritic cells and $T$ cell activation}

The initiation of $T$ cell responses requires that naïve $T$ cells recognize antigens presented by dendritic cells under conditions that require multiple receptors and co-stimulatory molecules. Antigen presentation is a transition phase from the innate to the adaptive response and is mediated mainly by $D C s^{(274)}$. DCs can activate both innate and adaptive immunity via antigen capture, presentation of antigen to immature T cells, and secretion of soluble inflammatory mediators. DCs are influenced by the collective cytokine response from ECs and ILCs, and this is believed to be pivotal in shaping T-cell differentiation ${ }^{(277)}$. Plasmacytoid dendritic cells are decreased in patients with a more severe type 2 profile, suggesting an important role of the cytokines milieu in their functional response or that plasmacytoid dendritic cell could mitigate the inflamed process found in polypoid tissue ${ }^{(349,}$ ${ }^{350}$. Zheng et al. showed increased expressions of IL-17RB and ST2 on myeloid DCs associated with enhanced local type 2 inflammation in polyp ${ }^{(351)}$. Shi et al. detected distinct subsets of lesional DCs in eosinophilic and noneosinophilic CRSwNP, which could prime Th 2 cells, and Th1/Th17 cells respectively in these endotypes ${ }^{(352)}$. Lin et al. investigated the functional status of dendritic cells (DCs) in polyp/mucosa from 30 CRSwNP and 10 control subjects and detected infiltration of DCs in NP, with the majority being mature $D C s$, and that $D C s$ are able to interact with T cells via the CD40/CD40L co-stimulatory factor ${ }^{(353)}$. Antigen presentation is regulated by co-stimulatory molecules. Programmed cell death-1 (PD-1) is a negative regulator of T-cell responses ${ }^{(354)}$. Kortekaas Krohn et al. measured expression of PD-1 pathway molecules by RT-qPCR on tissue from 21 CRSwNP and 21 control subjects and detected higher PD-1 expression in CRSwNP compared to controls independently of atopic status $^{(354)}$. In accordance with this, high PD-L1 levels were associated with Asian eosinophilic CRSwNP compared to controls ${ }^{(355)}$. Overall however, at this point, only limited evidence exists to support the hypothesis that defects in antigen presentation are significant in CRS pathogenesis.

\section{$T$ cells}

T cells have multiple roles in immune defence including defence against intracellular and extracellular infection ${ }^{(274)}$. T cells activate and recruit effector cells including phagocytes, kill infected cells and provide help for B cells in immunoglobulin production. 
Memory T cells are active as a durable form of innate activity at barrier surfaces such as the sinus mucosa.

A major role of $C D 4+T$ helper cells is to send signals to other types of immune cells, including CD8+ killer cells ${ }^{(274,356)}$. Loss of T-helper cell function leaves the body vulnerable to a wide range of infections as have been detected in untreated HIV infection ${ }^{(274)}$. CD4+ T cells may differentiate into different subsets of effector cells that produce distinct sets of cytokines that assist in defence against various types of microbial infections in tissues, and a fourth subset that activates B cells in secondary lymphoid organs: Th1, Th2, Th17 and follicular helper T cell ${ }^{(274)}$. A fifth T subset are T regulatory (Treg) cells that help turn the process off. Activated CD4+ cells produce a mixture of the characteristic cytokines ${ }^{(274)}$. The Th1 subset is induced, with the help of ILC1s, by microbes that are ingested by phagocytes (primarily macrophages) ${ }^{(356)}$. Th1 cells stimulate phagocyte-mediated killing of ingested microbes via secretion of interferon- $\gamma$ (IFN- $\gamma$ ) and tumor necrosis factor $-\alpha$ and $-\beta$ (TNF- $\alpha$ and TNF- $\beta$ ), leading to macrophage activation and enhanced antigen presentation, B-cell help and class switching to production of IgG subclasses and local tissue inflammation and neutrophil activation ${ }^{(356,357)}$. Th2 cells are induced by parasitic worm infections and promote IgE-, mast cell-, and eosinophil-mediated destruction of these parasites with the help of ILC2 cells ${ }^{(274,358)}$. Th2 cells secrete the Type 2-cytokines IL-4, IL-5, and IL-13, which have important protective effects that include eosinophil effector mechanisms, immunoglobulin class switching to lgE and lgG4 and mucus production ${ }^{(359)}$. The Type2-cytokines also stimulate the alternative pathway of macrophage activation and shut down the classical pathway mentioned above. This terminates prolonged and potentially damaging reactions. Alternative M2 macrophages secrete growth factors that act on fibroblasts to increase collagen synthesis and induce fibrosis fostering tissue repair. Th17 cells develop in response to extracellular bacterial and fungal infections and induce inflammatory reactions destroying these organisms. Th17 stimulate recruitment of neutrophils and monocytes and the production of defensins via secretion of IL17A, IL-17F, and IL-22.

CD4+ T cells differentiate and acquire distinct functions to combat specific pathogens but can also adapt their functions in response to changing circumstances ${ }^{(358,360)}$. Although this phenotypic plasticity can be potentially deleterious, driving pathology, the T-cell flexibility also provides important benefits that have led to its evolutionary preservation ${ }^{(360)}$. Studies of the human $T$ cell response to microbes have advanced our understanding of CD4+ T cell functional heterogeneity, in particular with the discovery of a distinct Th1 subset involved in the response to Mycobacteria and the characterization of two types of Th17 cells specific for extracellular bacteria or fungi(356). In CRS, the protective adaptive pathways go awry, usually with an excessive and prolonged response ${ }^{(361)}$. In support of this, the numbers of regulatory T cells (Tregs) are generally reduced in CRS and this has been interpreted as diminished Treg activity leading to chronicity ${ }^{(362)}$. For the other Th subsets however, elevated numbers have been associated with $\mathrm{CRS}^{(363)}$. Elevated numbers of Th2 cells and eosinophils have been reported with Western CRSwNP, while increased Th1/Th17 and neutrophilia are associated with Asian CRSwNP and CF polyps ${ }^{(343,344,363)}$. There is emerging evidence to suggest that the prevalence of Type 2 CRS is increasing in Asia however ${ }^{(322)}$. The aetiologic factors underlying this shift are both unknown and likely complex. Nevertheless, the speed of the process suggests changes in environmental exposures with secondary epigenetic variation as a root cause. Importantly, an overall increase in $\mathrm{T} 2$ diseases was observed in Western countries beginning in the early 20th century, and the changes in Asia appear to be manifesting in those areas adopting a more Western life style. Further study of these real-time changes in Asian CRS may be useful in identifying key environmental determinants that, with avoidance, could lead to a reduction in the incidence of $C R S$.

CD4+ T cells that express Foxp3 and with immune suppressive activity are known as CD4+Tregs ${ }^{(274)}$. Tregs develop in the thymus or peripheral tissues on recognition of self-antigens and suppress the activation of potentially harmful lymphocytes specific for these self-antigens. They are also essential for maintaining tolerance and immune homeostasis ${ }^{(364-369)}$. As mentioned above, Tregs appear to be reduced in CRS and autoantibodies have been reported. Studies show that multiple Th subsets may be activated in the same CRS patient, that the cytokines produced may suppress or augment the other subsets, and this may be part of normal immunoresponse, or may affect development or clinical presentation of CRS, or may affect treatment course ${ }^{(342,}$ 370).

CD8+ cells activated by antigen and other signals differentiate into cytotoxic T cells (CTL, T-killer cell) that are able to kill infected (particularly with viruses) cells expressing the antigen ${ }^{(274)}$. CTLs also kill malignant and other damaged cells ${ }^{(274)}$. Memory $T$ cells recirculate from the tissues to the peripheral blood. As mentioned above, T cell levels are higher in CRS but the CD8+/ $\mathrm{CD} 4+\mathrm{T}$ cell ratio is also higher, indicating particular enhancement of the cytotoxic T cell population ${ }^{(371,372)}$. Any specific role for cytotoxic T cells in CRS pathogenesis, however, remains unclear $^{(372)}$.

Cell adhesion molecules located on the cell surface are involved in binding with other cells or with the extracellular matrix. Adhesion molecules on leukocytes, fibroblasts and vascular endothelium, enabling leukocyte extravasation from vessels and migration to sites of inflammation ${ }^{(373)}$. Increased expression of intercellular adhesion molecule-1 (ICAM-1) in activated eosinophils is associated with eosinophil extravasation and infiltration into NP tissue, and seems to initiate also mucosal remodeling of 
$N P^{(374)}$. Fibroblasts' expression of vascular cell adhesion molecule (VCAM) has been shown to attract eosinophils and mast cells contributing to Type 2 cytokine skewing, while ICAM attracts neutrophils and to a lesser degree, eosinophils ${ }^{(375)}$. VCAM expression was lowest in controls, higher in CRSsNP, and highest in CRSwNP, while ICAM expression was elevated in both CRS subtypes $^{(375)}$. The L-selectin ligand, peripheral lymph node addressin (PNAd), was preferentially induced in the nasal vasculature of eosinophilic CRS facilitating tissue recruitment from blood ${ }^{(376)}$. It is not clear if primary defects in cell adhesion markers in particular or effector recruitment in general, play any key role in CRS pathogenesis. Specifically, the above findings may simply be the predicted secondary effect of the tissue inflammation present in the sinus mucosa of the CRS patient. Regardless, this may lead to innovative treatments via limiting the influx of eosinophils and neutrophils into the sinus tissues.

Humoral immunity is the type of host defence mediated by antibodies, which are produced and secreted by B cells and plasma cells, functioning both in circulation and in peripheral tissues such as sinonasal mucosa ${ }^{(274)}$. Antibodies use their antigen binding regions (Fab) to bind and block harmful effects of microbes and toxins. Heavy-chain isotype (class) switching and affinity maturation enhance the protective functions of antibodies ${ }^{(274)}$. The major functions of antibodies are to block or neutralize the infectivity of microbes, coat microbes and promote their ingestion by NK-cells, to activate (IgE) mast cell and eosinophil mediated reactions against helminthic parasites, and activation of the complement system vs. Physiologically tonic secretion of secretory lgA works in concert with other innate protective factors and mucociliary flow to limit mucosal colonization without tissue-damaging inflammation ${ }^{(377)}$. This $\lg \mathrm{A}$ is of low affinity and generated by a $T$ cell independent process by extrafollicular B cells. In the case of an active breach of the respiratory mucosa, higher-affinity $\lg A$ secretion may increase and, in combination with help from IgG, a robust inflammatory response ensues ${ }^{(378)}$. These are high affinity, $T$ dependent immunoglobulins produced by follicular B cells and plasma cells. IgG is the most common circulating antibody with four subclasses (IgG1-4) and a half-life of 7-21 days. IgG has several effector functions: it neutralizes microbes and toxins; opsonizes antigens; activates the classical pathway of the complement system; is responsible for NK cell cytotoxicity events and feedback inhibition of B cells $s^{(274)}$. $\operatorname{lgD}$ is an enigmatic antibody isotype best known when co-expressed with IgM on naive $B$ cells. Class switching to $\lg E$ is regulated by Type 2 cytokines and this immunoglobulin plays several important physiologic roles that include antigen presentation, increased mast cell survival, mucosal homeostasis, and defence against viruses, bacteria, fungi and parasites ${ }^{(312,348,379-383)}$. The Fc portions of $\lg \mathrm{E}$ and $\lg \mathrm{A}$ are often mounted on mast cells and eosinophils, respectively ${ }^{(274)}$. The antigen selective portions can bind parasites, sterically inhibiting their ability to invade and also leading to degranulation, which may trigger inflammation and some degree of tissue damage.

$B$ cells and immunoglobulins have been implicated in CRS and antibody defects are the most common immunodeficiencies, typically associated with CRSsNP(384). Low immunoglobulin protection can lead to recurrent acute sinus infection and ultimately CRSsNP(385). Most cases of CRSsNP do not have an immunoglobulin deficit however, and in fact, a subset of CRSsNP patients has high tissue levels of $\operatorname{lgD}$, although any role in pathogenesis remains uncertain.

B cell activation with excessive local antibody production has more commonly been associated with CRSwNP(369-371, 386). Polyp tissue from CRSwNP patients has been found to contain high levels of $B$ cells, plasma cells, follicles that resemble germinal centres, and high levels of $\lg A, \lg M, \lg G$ and $\lg E$ that indicate local production of immunoglobulins ${ }^{(337,369-372,387,388)}$. Antibody production in CRSWNP is driven in part by elevated levels of the BAFF cytokine (B-cell activating factor) in nasal polyp tissue ${ }^{(389)}$. In addition to B-cell proliferation, BAFF has also been associated with isotype class switching to $\lg E$ and $\lg A$ as well as the development of autoimmune immunoglobulins. Tan et al. was the first to demonstrate this, with anti-dsDNA IgG and IgA autoantibodies at increased levels in nasal polyps in comparison with controls, suggesting a role for autoimmunity in the more severely affected CRSwNP patients ${ }^{(344)}$. One specfic target appears to be the self protein BP180, which is a key anchoring protein in the skin and the nasal mucosa. Anti-BP180 antibodies were elevated in CRSwNP patients, suggesting autoimmune targeting of the epithelial barrier ${ }^{(343)}$.

Local IgE has been the most commonly implicated antibody in the pathophysiology of CRSwNP. Elevated IgE levels in nasal polyps have been shown to be independent of systemic atopy, driven by BAFF with local class switching, predict poor prognosis and correlate with the presence of lgE against staphylococcal superantigenic toxins. These toxins have the capacity to act as both $B$ and $T$ cell superantigens in nasal polyps, fostering a polyclonal $\lg \mathrm{E}_{\text {response }} \mathrm{e}^{(390)}$. Studies have demonstrated that the $\lg \mathrm{E}$ in nasal polyps is polyclonal and can trigger mast cell degranulation, suggesting a significant role for IgE in the pathophysiology of this subset of CRSwNP patients ${ }^{(391)}$. The therapeutic potential of anti-lgE for nasal polyposis has recently been demonstrated in CRSWNP patients as well(313,384).

Similar to $\lg \mathrm{E}, \lg \mathrm{G} 4$ is a minor $\lg \mathrm{G}$ subtype whose production is also regulated by Type 2 (IL-4 and IL-13) cytokines. Local IgG4 expression is high in serve eosinophilic CRS, with higher ILC2, plasma cells, B cells and activated CD8+ T cells ${ }^{(362,363)}$. The complement system is a collection of circulating and cell membrane proteins that play important roles in host defence against microbes and in antibody-mediated tissue injury ${ }^{(274)}$. Complement assists the antibody-mediated lysis of microbes but can result in tissue injury, particularly in cases of autoimmu- 
nity. At this point, there is only weak evidence to support a complement defect associated with CRS pathophysiology ${ }^{(366-368)}$. The evidence for excessive complement activation in CRS is stronger, suggesting the importance of antibody-mediated events in CRS pathophysiology possibly related to autoimmunity ${ }^{(384)}$.

\subsubsection{Other inflammatory mechanisms}

Remodeling is defined as abnormal restitution of damaged tissues and these processes have been widely described in lower airway diseases. In CRS, remodeling also takes place and observed changes include fibrosis, basement membrane thickening (BMT), goblet cell hyperplasia, epithelial barrier abnormalities and polyp formation, osteitis and angiogenesis ${ }^{3392}$. ${ }^{393)}$. The remodeling patterns were thought to separate based on the presence or absence of polyps, but clear distinctions have been difficult to clearly demonstrate ${ }^{(394)}$. This finding illustrates the limitations of this classification and CRS is now viewed as a heterogeneous disorder driven by an array of inflammatory mechanisms or endotypes. The underlying working hypothesis in upper airway disease is that the inflammation leads to remodeling with cytokines, mediators, enzymes and other factors determining the remodeling pattern. Essentially, the duration and type of inflammation would determine the outcome. The alternative hypothesis, that inflammation and remodeling are independent, remains viable although currently unsubstantiated at least in the upper airway ${ }^{(395)}$.

Studies have attempted to associate cytokines and other tissue markers with remodeling changes in CRS. Periostin has been suggested as a marker for BMT and fibrosis ${ }^{(238)}$. BMT has also been related to the duration of inflammation independent of the presence of tissue eosinophilia(393, 396). TGF-b has been most closely linked to fibrosis, but IL-13 and osteopontin have also been implicated in BMT and fibrosis ${ }^{(395,397-399)}$. Fibrosis may also be secondary to surgical trauma, confounding interpretation of results.

Remodeling changes in the epithelial barrier have engendered much interest since the last EPOS publication. As discussed in 5.2.2.3.1., the epithelial barrier appears to be weakened in many forms of CRS, leading to increased permeability to foreign material suggesting this as an early factor in CRS pathogenesis. While specific areas of weakness have been identified (see section 5.2.2.43.1.), the underlying cause(s) are uncertain. Some evidence exists suggesting genetic and epigenetic factors, early stage environmental insults including an abnormal microbiome or viral injury, and possibly systemic hormone signaling defects, which could all serve as permissive factors in CRS pathogenesis ${ }^{(218,400)}$. Once established, the inflammatory cytokines of CRS also trigger increased barrier permeability, an effect most closely associated with Type 2 inflammation but which likely occurs in other types as well ${ }^{(240,243,401,402)}$. None of the above would be considered remodeling but epithelial cells undergo turnover and repair after injury through a process involving interplay with fibroblasts, termed epithelial to mesenchymal transition (EMT). Under healthy conditions, this results in maintenance of a normal barrier but in some forms of CRS, the process is abnormal, resulting in epithelial remodeling toward acanthosis, acantholysis and a very leaky barrier ${ }^{(400)}$. The EMT process is driven by an array of factors including WNT, reactive oxygen species, proteases, HIF1, IL-13 Epiregulin, Oncostatin M, and IL-1 among others ${ }^{(207,230,373,374,377,403)}$. There is some evidence that stem cells in the epithelium maintain a memory for the chronic immature EMT state in severe Type 2 CRS $^{(228)}$. This form of remodeling may lead to barrier failure with markedly increased antigen access suggesting the possibility of a feed forward mechanism, such that barrier failure leads to more inflammation accelerating barrier damage and then accelerating inflammation ${ }^{(404)}$. This concept of a remodeled, immature, very leaky barrier is most closely associated with Type 2 CRS and may account for the higher treatment failure rate using conventional therapy. Directly targeting the failed barrier may be necessary to limit recurrence ${ }^{(405)}$. Nasal polyposis is associated with marked tissue oedema, diminished collagen and extracellular matrix degradation, possibly due to imbalances in metalloproteinase activity ${ }^{(406)}$. Abnormalities of the coagulation cascade have also been associated with polyp formation including Factor $X$, tissue factor and thrombin ${ }^{(407-409)}$. Supportive studies have indicated that a crosslinked fibrin matrix is the key component of polyps. It has been proposed that this matrix draws in fluid, accounting for the polyp mass. The pathway begins with inflammation, which results in the leakage of fibrinogen, amongst other plasma proteins, out of the vasculature into the tissues, where components of the coagulation cascade catalyze formation of a fibrin matrix 'clot'. Under normal circumstances, plasminogen is activated by t-PA (tissue plasminogen activator) to form plasmin, which enzymatically degrades the fibrin matrix into dimers. Type 2 cytokines inhibit t-PA activity so in the presence of high levels of Type 2 inflammation, the matrix will be retained and grow $^{(300)}$. The intrinsic levels of t-PA vary in the tissue and they are particularly low in the ethmoid sinuses, a possible explanation for this site as the most commonly observed origin of nasal polyps. The coagulation factor XIII-A has also been shown to induce excessive fibrin depostion in nasal polyps and M2 macrophages are the probable source ${ }^{(302)}$. Eosinophils may also play a role in fibrin deposition in N-ERD patients through the expression of L-selec$\operatorname{tin}^{(410)}$. Thus far, fibrin deposition has only been demonstrated in polyps from patients with Type 2 inflammation. Very recent studies have indicated that a broad reduction in the fibrinolytic pathways via protease inhibition accounts for accumulation of the fibrin matrix in CRSwNP(411).

Tissue angiogenesis is a complex phenomenon that results in the growth of new blood vessels from the microcirculation. This process has been known to play a crucial role in tumour growth 
as well as several benign diseases ${ }^{(412)}$ and could be considered a form of remodeling in CRS. Angiogenesis is regulated by infection and immune cells VEGF, VEGFR-1, Ang-1, Ang-2, Tie-2A, Tie-2B, SDF- $1 a$ and SDF-1 $\beta$ mRNA expression to be significantly higher in CRSwNP patients compared to the control group ${ }^{(412)}$. Luukkainen et al. performed immunohistochemistry and counted lymphatic vessel density of sinus mucosa and polyp tissue of 120 subjects and detected that low, absolute and relative density of vessels and of lymphatic vessels was associated with CRSwNP and antrochoanal polyp (ACP) tissues compared to control inferior turbinate ${ }^{(413)}$. This was observed also in the inflammatory hotspot area. Higher lymphatic vessel density seems to associate with polyp recurrence ${ }^{(413)}$. In contrast, Mostafa et al. have detected a high density of lymphatic vessels in the transitional area between the sinus mucosa and the pedicle of the ACPs, compared to the control group ${ }^{(414)}$. Regarding other growth factors, differences existed in EGF pathways in CRS patients and normal subjects as well as in CRSsNP and CRSwNP(415)). GF-1 induced MUC8 and MUC5B expression is regulated by activation of the ERK1 and p38 MAPK signaling pathway in human airway epithelial cells ${ }^{(416)}$. PDGF systems play important roles in polyp pathogenesis. Fibroblast-derived PDGF may be more important than MNC-derived PDGF in the polyp developing process ${ }^{(417)}$.

Osteitis has been described in eosinophilic CRSwNP and putatively results from infection and biofilm production ${ }^{(418)}$. Downregulated pro-osteoblastic mucosal BMP signaling is strongly and significantly associated with increased osteitis in CRSwNP(419, ${ }^{420}$. There are phenotypic differences in adhesion and mineralization between osteoblasts in patients with CRS compared to controls $^{(419)}$. Different signaling pathways are involved in osteitis in CRS and are activated by the TGF- $\beta /$ Smad signaling pathway in CRSwNP versus the TGF- $\beta /$ Smad-independent signaling pathway in CRSsNP(418,420). Taken together, pathways closely related to mucosal inflammation have been much less studied than the immune system. It is possible that they have a role in CRS and would be worthy of further investigation.

Neural function is closely related to infection ${ }^{(421)}$ and the immune system. Dysfunctional innervation might contribute to the pathogenesis of CRSWNP and could in part explain olfactory loss. Wu et al. detected that axonal guidance signaling and neural growth factor pathway proteins are significantly suppressed in eosinophilic CRSwNP(422). Elevated TRPV1 levels in comorbid asthma and allergy may have a function in CRSwNP(259). Substance $P$ was released from the sensory neurons and human/ murine nasal epithelial cells within 15 minutes of local TLR7 stimulation ${ }^{(423)}$, highlighting a novel role for sensory neuropeptides as acute and local mediators of pathogen-driven inflammation, rapidly priming innate immune defences in the airway. Neurotrophin-3, a neurotrophic factor, was associated with CRSwNP compared to controls ${ }^{(424)}$. Neuronal dysfunction has been pro- posed as an endotype for chronic rhinitis, but it is also likely that these mechanistic pathways play a role in CRS as well(425).

\subsubsection{Conclusions and future needs}

A wide array of exogenous agents are inhaled through the nose and interact with the sinonasal mucosa, a process that begins at birth with rapid colonization by viruses, bacteria and fungi. In healthy individuals, the mucosa serves as a relative barrier limiting and regulating environmental interaction with the host immune system, a process that is likely beneficial to the host in a number of ways including development of tolerance, generation of important metabolites and competitive inhibition of pathogens ${ }^{(136)}$. In healthy patients, when the barrier is breached, a specific, self-limited physiologic immune response is generated, characterized by a cellular and cytokine repertoire targeting the pathogen(s). Although the in vivo response is much more complex, at the basic level Type 1 immunity targets viruses, Type 2 parasites and Type 3 extracellular bacteria and fungi, resolving with elimination of the pathogens and restoration of barrier integrity. In cases of CRS, the current working hypothesis is that barrier penetration, possibly by an alternate mechanism, results in a chronic inflammatory response that still utilizes the Type 1, 2 or 3 pathways alone, or in combinations. CRS immune responses are: a) dynamic and heterogeneous, likely exhibiting plasticity; b) not self-limiting (remaining active for months to years); c) not clearly matched to the inciting agents; and d) often associated with various types of tissue remodeling, presumably linked to the pattern of inflammation. There is no evidence at this point for a specific dominant pathogen and, based on the limited evidence currently available, the immunologic response is polyclonal, targeting antigens derived from multiple organisms, including nasal bacteria ${ }^{(168,172)}$. In some severe, recurrent CRS cases, self antigens are also targeted but the development of autoimmunity is mostly viewed as a phenomenon secondary to the chronic process ${ }^{(367)}$.

Many questions remained unanswered including the mechanism for initiation of CRS, but presumably this results from a combination of environmental stressors, genetic susceptibility and stochastic events that collectively promote barrier penetration. While the nature, intensity and sequence of the exposures are likely significant in triggering CRS, evidence supporting a role for specific environmental agents remains largely indirect, as no widely accepted animal model of CRS exists. Host genetic variation, particularly governing the sinonasal epithelium and immune response, also plays a major role but given that age of onset of CRS is typically in the fifth decade, environmental and epigenetic effects likely dominate from the standpoint of causation. Relatively few high-quality genetic studies have been performed to this point, but using adult onset asthma as a model, multiple genes are involved, each typically conferring a relatively small effect size and most having functional implications 
for the epithelial barrier and immune response ${ }^{(426)}$. CF, with mutation of the CFTR gene, is a notable exception demonstrating a strong association of the homozygous mutation with childhood onset CRS ${ }^{(427)}$. Presence of the heterozygous CFTR mutation is also associated with a CRS signal albeit less than clinical CF, and usually presenting in adulthood ${ }^{(428)}$. The impaired mucociliary flow seen with CFTR mutations is presumed to intensify microbial exposure driving the process ${ }^{(429)}$. The importance of CFTR in childhood onset CRS suggests that other barrier-related genes may play a role in CRS initiation.

The identity of the antigens triggering CRS is another area where we have little information. The limited data available suggests that common nasal bacteria, including S. aureus, are targeted by the host immune system in CRSwNP(172). In AFRS, fungi are likely targeted as well. Both nasal bacteria and fungi should induce a Type 3 immune response, yet nearly $90 \%$ of Western CRSwNP patients (and over $50 \%$ of surgical CRSsNP patients) exhibit a significant if not predominant Type 2 cytokine profile. indicating that interactions between host and bacteria are not solely driven by Type 3 mechanisms ${ }^{(153,342,400,430-434)}$. Although the reason for Type 2 skewing is very unclear, it suggests that some of the inter-related hypotheses which have been used to explain atopy including dysbiosis, may be operative in Type 2 CRS as well. Alternatively, it has been proposed that S. aureus, via multiple pathobiologic mechanisms including superantigens, is capable of shifting the immune response in a Type 2 direction ${ }^{(170)}$. Some evidence also suggests that environmental proteases and host anti-proteases interact at the mucosal surface, setting a threshold for barrier penetrance as well as skewing the immune response in the Type 2 direction ${ }^{(196,400,435)}$. One key pathway for Type 2 skewing may include the gene ALOX15, as a loss of function variation protects against nasal polyposis ${ }^{(436)}$. This gene encodes the enzyme 15LO (15 lipoxygenase), which is up-regulated by IL-13 and promotes eoxtaxin 3 expression by epithelial cells, presumably driving tissue eosinophilia in CRSWNP(437). This pathway has also been implicated in epithelial remodeling and establishment of chronically immature, leaky barrier ${ }^{(438)}$. Lastly, beyond Type 2 skewing, patients with severe Type 2 inflammation tend to be resistant to treatment with a high recurrence rate. In theory, this could relate to remodeling changes in the epithelium that persist despite treatment ${ }^{(112,400,404)}$.

The extensive pre-morbid time course, complexity of environmental exposures and lack of many strong genetic signals has diminished current enthusiasm for studies on CRS aetiology and pathogenesis. Moreover, therapies focused on addressing proposed environmental agents have not been very successful in CRS management. As a result, interest has shifted to study the mechanistic pathways (endotypes) that result within the sinus mucosal tissue itself, as opposed to searching for putative causes. This shift has been accelerated by the recent availability of biologic agents that target molecules operative in these pathways. In theory, the intensity and nature of the endotype should play a key role in determining the observed phenotype, clinical course, response to therapy, remodeling pattern in the tissue and possibly the presence of co-morbid pulmonary disease. The first large study attempting to define the endotypes of CRS with tissue data used a modified cluster analysis of preselected tissue biomarkers including Type 1, 2 and 3 canonical cytokines, neutrophilic markers, IgE to staphylococcal superantigens, albumin, TGF-beta, IL- 6 and IL-1b. Results indicated 10 endotypes including four that were characterized as IL-5 negative and six as IL-5 positive. Of these six, three were considered high IL-5 and had the strongest association with asthma and the presence of polyps ${ }^{(153,166,439)}$. There was a correlation between the Type 2 intensity and the presence of asthma and nasal polyps but data on symptoms, natural history and response therapy were unavailable. A second large scale attempt to define endotypes and tissue patterns was based on the Type 1, 2 and 3 inflammatory patterns alone ${ }^{(342)}$. The various possible combinations of the three types of inflammation yields eight endotypes. In this study, smell loss was associated with Type 2 inflammation as previously shown. The presence of Type 1 inflammation was protective against facial pain while the presence of pus was associated with Type 3 inflammation. These two classifcation schemes are the first steps towards identifying the precise mechanistic pathways potentially relevant and active in an individual patient. Type 2 inflammation is the best studied in CRS, and it is likely that subendotypes exist that vary beyond simply disease-intensity. Overall, the mechanistic pathways of Type 2 inflammation in CRS tissue are more likely to reflect an interconnected lattice network as opposed to a linear progression ${ }^{(404)}$. As a consequence, the mechanism that results in the N-ERD phenotype may be a distinct Type 2 subendotype with accentuated prostaglandin/leucotriene activity. AFRS as well as SE-lgE+ patients may be subendotypes that are more closely tied to local polyclonal IgE production. As a corollary, it is very possible that these subendotypes, if verified, would respond differently to the various Type 2 biologics currently available. Lastly, both of these CRS endotype classification schemes had one endotype (>10\%) of surgical CRS patients that had no measured inflammatory markers that were significantly greater than background control levels. The CT and symptoms scores were, however, similar to other endotypes ${ }^{(344)}$. Although the mechanism is unclear in this group, the symptoms and the mucosal oedema in the group appear to be driven by a mechansim distinct from the traditional inflammatory pathways associated with CRS. Neuroimmune dysfunction involving neuropeptides and ion channels, already proposed as an endotype of non-allergic rhinitis, could also be operative in $\mathrm{CRS}^{(425)}$.

Although much has been accomplished since the last EPOS document, many questions still remain regarding aetiology, pathogenesis and relevant inflammatory mechanisms. Future 
challenges include a better understanding of: 1) the aetiologic factors that drive CRS with a goal towards prevention; 2) the pathophysiologic inflammatory mechanisms and relevant endotype biomarkers with a goal towards targeted therapy; 3 ) the molecular mechanisms of barrier and tissue remodeling that may play a role in persistence and recurrence.

\subsection{Diagnosis and differential diagnosis}

\subsubsection{Allergic and non-allergic rhinitis}

Upper airway diseases present with a variable pattern of common symptoms such as nasal obstruction and discharge, making the epidemiological diagnosis of CRS difficult to differentiate from allergic and nonallergic rhinitis based on symptomatic grounds only. Moreover, not all patients presenting with symptoms meeting CRS criteria have evidence of disease if diagnosis is complemented with nasal endoscopy and CT.

\subsubsection{Allergic and non-allergic rhinitis}

Allergic rhinitis (AR) is clinically defined as a symptomatic disorder of the nose induced after allergen exposure by an IgEmediated inflammation of the nasal membranes. The symptoms include rhinorrhoea (anterior or posterior), nasal congestion, nasal itching, and sneezing. There is no uniform definition for epidemiological studies. Different definitions have been used in questionnaires in previous studies.

Allergic rhinitis is a very prevalent disease ${ }^{(440-443)}$. In a Dutch study using the GA2LEN questionnaire $29 \%$ of the population had allergic rhinitis, based on the question "Do you have any nasal allergies including hay fever?" and 16\% CRS based on the EPOS criteria. In this study the risk of CRS was significantly higher in respondents with a doctor's diagnosis of CRS (OR 6.83), AR (OR 2.87), asthma (OR 2.36), an adverse response after taking painkillers (OR 2.34), itchy rash (OR 1.71), or active smoking (OR 1.45).

Data on the prevalence of non-allergic rhinitis are less abundant with figures of $10-19 \%{ }^{(444,445)}$.

As far as we are aware there are no comparative studies in the literature evaluating symptom prevalence and severity in CRS, AR and NAR.

\subsubsection{CT scan in allergic rhinitis versus $C R S$}

In a group of 216 CRS patients, the patients with a positive specific in vitro allergen test result did not have higher overall rates of radiographic sinus inflammation ${ }^{(446)}$. Moreover, in patients with allergic rhinitis alone, asthma alone or both, the group with allergic rhinitis and asthma, 58\% had some abnormalities on CT scan versus $74 \%$ of the patients with only asthma, $67 \%$ with only rhinitis versus $20 \%$ in a control group. In the group with allergic rhinitis CTscan abnormalities were seen significantly more often in the "blockers" (90\%) than the "sneezers" (52\%) $)^{(447)}$.
A central radiological pattern of mucosal disease may be associated with inhalant allergen sensitization. This group may represent a CCAD subgroup of patients with mainly allergic aetiology ${ }^{(194)}$ (see also CCAD). The prevalence of allergy in CRS may vary by phenotype, with CCAD and AFRS having a stronger association than CRSwNP and CRSsNP (see 5.1 and 8.1).

\subsubsection{Differentiating AR, NAR and CRS}

Combining data from different studies in one table (Table 5.3.1.) although incomplete, leads to a picture of a significant overlap in symptomatology for prevalence and severity of symptomatology.

Koskinen et al. ${ }^{(448)}$ compared diagnostic accuracy of symptoms, endoscopy and imaging signs of CRSsNP and allergic rhinitis patients and made a prediction model for CRSsNP. They also concluded that there is a significant overlap in symptoms. The highest area under curve value (0.93) was demonstrated for a visual analogue scale score of facial pain / pressure $\geq 4 / 10$ showing $60 \%$ sensitivity and $95 \%$ specificity for detecting the CRSsNP group ( $p<.001$ ). The radiologic sign of an obstructed ostiomeatal complex showed $100 \%$ specificity and $38 \%$ sensitivity for detecting the CRSsNP group ( $\mathrm{p}<.001)$. In this study endoscopy had no added value.

\subsubsection{Conclusion}

It can be very difficult to discriminate between AR, NAR and CRS. The combination of symptoms, CT scan and maybe also nasal endoscopy can point in the right direction but significant overlap in diseases occur.

\subsubsection{Smell disorders}

\subsubsection{Symptoms}

Olfactory impairment, like facial pain is part of the four main CRS symptoms. Similar to facial pain and in contrast to nasal obstruction and rhinorrhoea, olfactory impairment may not exclusively be related to sinonasal disease and thus has a wide differential diagnosis ${ }^{(449)}$. Olfactory impairment as a symptom can be roughly subdivided into distortions (qualitative disorder), decrease or total loss (quantitative disorders) (Table 5.3.2.) Patients may present with only a qualitative, quantitative or both complaints. Identifying the exact symptom by means of inquiry and measurement ${ }^{(450)}$ (see section 5.3.5.5) is essential for establishing a correct diagnosis.

\subsubsection{Epidemiology}

The prevalence of olfactory disorders in the general population is estimated to be 3-5\% for total smell loss (anosmia) and $15-25 \%$ for partial impairment (hyposmia) ${ }^{(451,452)}$. These studies provide reliable data on how often, but not why and for what reason people have impaired olfactory function. Some of these 
Table 5.3.1 Prevalence and severity of symptoms in CRS, AR and NAR (data from different studies).

\begin{tabular}{|c|c|c|c|c|c|c|}
\hline \multirow[t]{2}{*}{ Symptom } & \multicolumn{2}{|c|}{ Chronic rhinosinusitis } & \multicolumn{2}{|c|}{ Allergic rhinitis } & \multicolumn{2}{|c|}{ Non-allergic rhinitis } \\
\hline & Prevalence (\%) & Severity & Prevalence (\%) & Severity & Prevalence & Severity \\
\hline Nasal obstruction & 92 & 6.8 & $92^{5}$ & $2.7^{\#}$ & & $5.8^{\#}$ \\
\hline Rhinorrhoea / discharge & 80 & 5.8 & $86^{5}$ & $3.6^{\#}$ & & $4.3^{\#}$ \\
\hline Post-nasal drip & 80 & 6.0 & $65^{5}$ & $5.3^{5}$ & & \\
\hline Loss of smell & 84 & 6.8 & $30^{\circledR}$ & $0^{8}$ & & \\
\hline Facial pain / pressure / headache & 67 & 5 & & $0.5^{\&}$ & & \\
\hline Nasal / palatal itch & & & $81^{5}$ & $6.8^{5}$ & & \\
\hline Itchy eyes & 45 & 3.2 & $63^{\$}$ & $2.4^{*}$ & & $2.3^{\#}$ \\
\hline Sneezing & 56 & 3.6 & $80^{5}$ & $4.2^{*}$ & & $4.0^{\#}$ \\
\hline
\end{tabular}

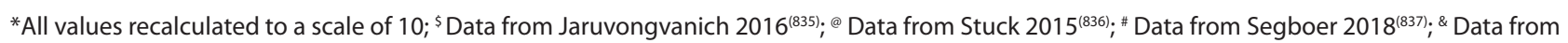
Koskinen ${ }^{(448)}$. Empty cells: no data available.

epidemiological follow-up studies suggest a link between altered olfactory function and increased mortality or development of neurodegenerative diseases ${ }^{(453,454)}$. This underlines the potential biomarker function of olfactory measurement.

\subsubsection{Causes and frequency}

In a patient with an olfactory disorder, the most important question is the cause of the smell problem. Many methods have been proposed to classify olfactory disorders such as conductive versus sensorineural or peripheral versus central. The shortcomings of these classifications are that they suggest that the place and mechanism of the disorder is known, which is not supported by the current knowledge in the field. The olfactory literature is very consistent with the suggestion that one should classify olfactory disorders currently according to the putative cause ${ }^{(449,455-462)}$. The most frequently identified causes of olfactory disorders are listed in Table 5.3.3.

The table shows that the four most frequent causes, posttraumatic, post-infectious, CRS-related and idiopathic olfactory disorders occur in very different frequencies whether they are reported from specialized smell and taste outpatient clinics or from general ENT outpatient clinics. The reports from specialized clinics underreport the frequency of CRS- related olfactory disorders, whereas this seems to be the most frequent cause encountered by the average ENT physician.

\subsubsection{Olfactory (dys-) function in CRS}

Olfactory function in CRS has a very variable degree of clinical manifestation, ranging from no impairment to total loss. The literature also shows that the extent of olfactory dysfunction depends on the underlying form of sinonasal disease. CRS with nasal polyps is more severely and more often associated with olfactory impairment than any other form of sinonasal disease ${ }^{(449,463-467)}$. The mechanism leading to olfactory impair- ment in CRS is twofold: inflammatory and purely mechanical with obstruction of the olfactory cleft ${ }^{(468,469)}$. Inflammation that characterises CRS also modifies the olfactory epithelium ${ }^{(468)}$ with consecutive functional impairment. Mechanical blockage to the olfactory cleft also decreases olfaction ${ }^{(469)}$. In most CRS patients with olfactory impairment both mechanisms are present and a mechanistic view of olfactory impairment is inadequate. It explains why not all patients per se have an olfactory benefit from surgical removal of polyps alone, but also require subsequent anti-inflammatory treatment.

CRS related olfactory dysfunction has some distinct clinical features that should be looked for during assessment since they may help to identify the olfactory impairment as sinonasal versus other causes. The first particularity mainly found in CRSrelated olfactory dysfunction is fluctuation of the olfactory complaint ${ }^{(470-473)}$. Fluctuation is hardly ever found in post-traumatic, post-infectious, and congenital or neurodegenerative causes. The second finding that can be seen more often in CRS related olfactory impairment is a gap between ortho- versus retronasal olfactory function ${ }^{(474-476)}$. Although not consistently present, certain CRS patients have well preserved retronasal olfactory function and express that as intact flavour perception whereas their orthonasal olfaction is decreased or absent. The most likely explanation is a partially preserved retronasal access to the olfactory cleft. Third, if testing is performed with tests that have thresholds and identification (see chapter 5.3.5.5) CRS- related olfactory impairment often shows a characteristic pattern of low threshold and preserved identification scores ${ }^{(464,477)}$. Finally, CRS- related olfactory impairment shows a steroid-dependent reversal ${ }^{(478)}$, which is a clear indicator of inflammatory related olfactory impairment. Olfactory dysfunction that is reversible with systemic steroids has also been found to be a reliable predictor of post-surgical olfactory outcome $\mathrm{e}^{(479-481)}$. 
Table 5.3.2. Details and characteristics of olfactory symptoms and their occurrence in CRS.

\begin{tabular}{|c|c|}
\hline Quantitative disorder & Definition / characteristics \\
\hline Parosmia / troposmia & $\begin{array}{l}\text { Distorted smell triggered by an odour source. Mostly after post-infectious / post-traumatic olfactory } \\
\text { impairment. Very rare in CRS. }\end{array}$ \\
\hline Phantosmia / olfactory hallucination & $\begin{array}{l}\text { Distorted smell occurring independently of an odour source. Wide differential diagnosis including post- } \\
\text { infectious / post-traumatic but also tumour, psychiatric or idiopathic olfactory impairment. Rare but not } \\
\text { impossible in CRS. }\end{array}$ \\
\hline Qualitative disorder & Definition / characteristics \\
\hline Normosmia & Normal olfactory function \\
\hline Hyposmia & Decreased olfactory function. Smell perceived if odours are strong / concentrated. Very frequent in CRS. \\
\hline Anosmia & $\begin{array}{l}\text { Total loss of olfactory function. No smell perception even if odours are very strong. Wide differential diag- } \\
\text { nosis but frequently encountered in severe forms of CRS. }\end{array}$ \\
\hline Hyperosmia & $\begin{array}{l}\text { Increased, above average good olfactory function. Subjectively reported in migraine, pregnancy and mul- } \\
\text { tiple chemical sensitivity but rarely confirmed by psychophysical / objective measures. Literature scarce. } \\
\text { Not reported in CRS. }\end{array}$ \\
\hline Specific anosmia & $\begin{array}{l}\text { Selective loss / inability to smell certain odours while all other odours are perceived normally. Physiologi- } \\
\text { cal state due to variable olfactory receptor expression in humans. All of us have certain specific anosmias. } \\
\text { Not related to CRS. }\end{array}$ \\
\hline
\end{tabular}

Table 5.3.3. Most frequent causes of olfactory dysfunction according to their putative caus $\mathrm{e}^{(449,455-462,473,838,839)}$ and depending on where the patient was seen (specialized outpatient clinic or in a general ENT department).

\begin{tabular}{|c|c|c|c|}
\hline Cause & $\begin{array}{l}\text { Frequency (observed in special- } \\
\text { ized smell and taste clinics) }\end{array}$ & $\begin{array}{l}\text { Frequency (in general ENT } \\
\text { consultations) }\end{array}$ & $\begin{array}{l}\text { Presence of distortions } \\
\text { (parosmia / phantosmia) }\end{array}$ \\
\hline CRS related (sinonasal) & $6-21 \%$ & $72 \%$ & rare \\
\hline Post-infectious (URTI) & $26-40 \%$ & $11 \%$ & very frequent \\
\hline Post-traumatic & $16-39 \%$ & $5 \%$ & frequent \\
\hline Idiopathic & $14-22 \%$ & $6 \%$ & variable \\
\hline Neurological-neurodegenerative & $1-2 \%$ & $\mathrm{n} / \mathrm{a}$ & variable \\
\hline Congenital & $4-10 \%$ & $1 \%$ & almost never \\
\hline Other rare causes (e.g. toxic exposure) & $9-15 \%$ & $5 \%$ & variable \\
\hline
\end{tabular}

URTI, upper respiratory tract infection.

\subsubsection{Diagnosis and assessment}

Diagnosing an olfactory deficit comprises several steps: the patient's history is crucial and one should look for all the above-mentioned elements, such as the duration, concomitant sinonasal symptoms, possible fluctuation, distortions and events preceding the olfactory deficit (e.g. trauma or URTI) in order to delimit the major causes of olfactory deficits (Table 5.3.3.). Measuring olfactory function as completely as possible (see chapter 5.3.5.5) should be done in order to assess the measurable olfactory function since purely asking is not representative of what eventually may be measured ${ }^{(482-484)}$. Finally, a thorough nasal endoscopy is mandatory. If after these steps, the putative cause remains unclear and before stating that it might be an idiopathic problem, a short systemic steroid trial and imaging (CT and $\mathrm{MRI}$ ) to rule out any hidden intranasal inflammation (especially in the olfactory cleft) and intracranial elements that explain the olfactory dysfunction should be undertaken.

\subsubsection{Treatment}

Treating CRS-related olfactory dysfunction is synonymous with treating CRS and is discussed elsewhere in the position paper. The current literature shows that olfaction mostly improves with varying degrees of reported success rates ${ }^{(463,485)}$. For post-infectious, post-traumatic, neurodegenerative and congenital olfactory disorders no real curative treatment exists. However, smell training, a regular and conscious exposure to odours has been shown to significantly improve olfactory function, especially in post-infectious and to a lesser extent post-traumatic olfactory dysfunction ${ }^{(486)}$. 
Key points | What's new since EPOS 2012

- Olfactory dysfunction is frequent in the general population

- The most frequent cause of olfactory dysfunction encountered by ENT physician is related to CRS

- CRS-related olfactory impairments have a good success rate of improvement if the underlying CRS is treated

- CRS-related olfactory impairments show some distinct clinical features that should be looked for since they help to accurately diagnose the olfactory dysfunction

\subsubsection{Facial pain}

Like all the cardinal symptoms that constitute the diagnosis of CRS, facial pain has a broad differential diagnosis. Unlike the other symptoms, however, causes for facial pain should often be sought in areas beyond the nose. Unfortunately, 'common sense', often strengthened by the judgement of primary care physicians, indicates that facial pain must derive from sinonasal disease. As such, ENT-surgeons regularly encounter patients referred for facial pain but will only diagnose CRS in the minority of them ${ }^{(487)}$. Therefore, having a basic understanding of the various causes of facial pain is pivotal to determine the right diagnosis and management. Too often patients undergo treatment, including surgery, aimed at supposed sinonasal disease, when proper history-taking would have revealed a non-sinonasal cause to be far more likely ${ }^{(488)}$.

\subsubsection{Facial pain in CRS}

Facial pain is usually a mild complaint in CRS (see section 3.1). Small studies with both CRSwNP and CRSsNP patients suggest that they report more facial pain than controls (although the controls groups were really small, $<10$ subjects per study) ${ }^{(489,}$ ${ }^{490)}$. In patients with CRSwNP, $16-18 \%$ report moderate to severe facial pain, with only a good correlation between pain and sinus disease in those with purulent discharge ${ }^{(491,492)}$. Conversely, in patients with purulent secretions from the paranasal sinuses, only $29 \%$ report facial pain ${ }^{(493)}$. As such, nasal endoscopy is advised when facial pain is the main symptom, as it has a strong negative predictive value to rule out sinogenic pain ${ }^{(494)}$. In patients with an indication for endoscopic sinus surgery, the prevalence of facial pain is higher. In a surgical cohort of patients with CRSwNP, roughly half was found to report moderate to severe facial pain pre-operatively, with marked improvement after several weeks ${ }^{(495)}$ up to seven months postoperatively ${ }^{(496)}$. Studies with mixed surgical cohorts of patients with CRSsNP or CRSwNP also showed sustained improvement of facial pain after surgery ${ }^{(497,498)}$.

Interestingly, there seems to be a difference in the prevalence or severity of headache/facial pain between patients with CRSwNP and those with CRSsNP. Studies show that both groups have comparable total symptom scores, but those without polyps have increased scores for headache and facial pain, while the other cardinal symptoms of CRS are more prevalent in patients with nasal polyps ${ }^{(499,500)}$. Moreover, the tendency for CRSsNP patients to report more severe facial pain can even be used to distinguish them from allergic rhinitis patients ${ }^{(501)}$.

There is no correlation between the location of facial pain and abnormalities (if any) of anatomically related sinuses upon imaging, both for CRSsNP and CRSwNP(502,503). Patients reporting facial pain as their main symptom have less abnormalities upon imaging than those reporting the other cardinal symptoms that

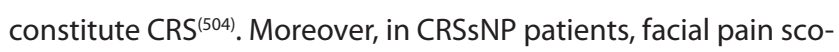
res are negatively correlated with $\mathrm{CT}$ sinus abnormalities ${ }^{(505)}$. As such, routine CT scanning in patients with facial pain is not advised, as most will have no relevant findings ${ }^{(506)}$. Proper historytaking can eliminate the need for imaging in most patients ${ }^{(507)}$. Taken together, facial pain should only be ascribed to CRS if there are other nasal symptoms and concordant abnormalities upon nasal endoscopy and/or imaging. Ideally, the pain and its intensity have a temporal relationship with the intensity of the other nasal symptoms (also indicated in the third edition of the Int Classification of Headache Disorders (ICHD), see https:// ichd-3.org). With the absence of any of these findings, a primary headache syndrome should be considered as cause of the facial pain (Table 5.3.4.).

\subsubsection{Nasal complaints in primary headache syndromes}

Unfortunately, patients with a primary headache syndrome also report nasal symptoms. In a retrospective chart review, these patients were found to report items as nasal congestion, rhinorrhea and sneezing in the majority of $\operatorname{cases}^{(508)}$. When compared to healthy controls, patients with either midfacial segment pain or CRS both have increased SNOT scores ${ }^{(508,509)}$. However, the pattern in the subdomains is different. CRS patients have higher scores for the rhinological domain, while midfacial segment pain patients score higher in the ear/facial domain (including facial pain) ${ }^{(510)}$. As such, a ratio of ear/facial domain over rhinological domain scores $>0.66$ was found to be a strong negative predictor of $\mathrm{CRS}^{(511)}$.

\subsubsection{Medication overuse headache}

Patients with a primary headache syndrome are prone to use analgesic medication in an increasing frequency. This might trigger a concomitant form of headache, which is termed medication overuse headache (MOH). According to the ICHD diagnostic criteria, it occurs 15 or more days per month, in a patient with a primary headache syndrome using medication for more than 10 
Table 5.3.4. Most frequent primary headache syndromes giving facial pain.

\begin{tabular}{|c|c|c|c|c|c|c|}
\hline & & Trigeminovas & cular headache & & Tension-type & Midfacial \\
\hline & Migraine & $\begin{array}{l}\text { Trigeminal } \\
\text { neuralgia }\end{array}$ & $\begin{array}{l}\text { Paroxysmal } \\
\text { hemicrania }\end{array}$ & Cluster headache & & \\
\hline & Paroxysmal & Paroxysmal & Paroxysmal & Paroxysmal & $\begin{array}{l}\text { Paroxysmal or } \\
\text { continuous }\end{array}$ & $\begin{array}{l}\text { Paroxysmal or } \\
\text { continuous }\end{array}$ \\
\hline Location & $\begin{array}{l}\text { Usually frontotem- } \\
\text { poral, facial }\end{array}$ & $\begin{array}{l}\text { In the distribution(s) } \\
\text { of one or more } \\
\text { divisions of the } \\
\text { trigeminal nerve }\end{array}$ & $\begin{array}{l}\text { Orbital, supraorbital } \\
\text { and/or temporal, } \\
\text { facial }\end{array}$ & $\begin{array}{l}\text { Orbital, supraorbital, } \\
\text { temporal, facial }\end{array}$ & Entire vertex & $\begin{array}{l}\text { The midface alone } \\
\text { (bridge of the nose, } \\
\text { paranasal, and/ } \\
\text { or peri-orbital) in } \\
\text { midfacial segment } \\
\text { pain. }\end{array}$ \\
\hline Side & Mostly unilateral & Unilateral & Unilateral & Unilateral & $\begin{array}{l}\text { Usually bilateral, } \\
\text { symmetrical }\end{array}$ & $\begin{array}{l}\text { Usually bilateral, } \\
\text { symmetrical }\end{array}$ \\
\hline Duration & $4-72 \mathrm{~h}$ & $\begin{array}{l}\text { Fraction of a second } \\
-2 \mathrm{~min}\end{array}$ & $2-30 \mathrm{~min}$ & $15 \mathrm{~min}-3 \mathrm{~h}$ & $\begin{array}{l}\text { Continuously pre- } \\
\text { sent to some extent } \\
\text { ( } 30 \text { min }-7 \text { days if } \\
\text { paroxysmal) }\end{array}$ & $\begin{array}{l}\text { Continuously pre- } \\
\text { sent to some extent }\end{array}$ \\
\hline Quality & $\begin{array}{l}\text { Pulsating (throb- } \\
\text { bing, piercing, } \\
\text { stabbing) }\end{array}$ & $\begin{array}{l}\text { Severe, electric } \\
\text { shock-like, shooting, } \\
\text { stabbing or sharp }\end{array}$ & $\begin{array}{l}\text { Severe pain, bur- } \\
\text { ning, piercing }\end{array}$ & $\begin{array}{l}\text { Very severe pain, } \\
\text { burning, piercing, } \\
\text { pressing }\end{array}$ & $\begin{array}{l}\text { Pressing, tightening } \\
\text { (non-pulsating). }\end{array}$ & $\begin{array}{l}\text { Pressing, tightening } \\
\text { (non-pulsating). }\end{array}$ \\
\hline $\begin{array}{l}\text { Accompa- } \\
\text { nied by }\end{array}$ & $\begin{array}{l}\text { Nausea, vomiting, } \\
\text { photophobia, } \\
\text { phonophobia }\end{array}$ & $\begin{array}{l}\text { Mild autonomic } \\
\text { symptoms such as } \\
\text { lacrimation and/ } \\
\text { or redness of the } \\
\text { ipsilateral eye may } \\
\text { be present }\end{array}$ & $\begin{array}{l}\text { Any or several of } \\
\text { ipsilateral nasal } \\
\text { congestion, rhinor- } \\
\text { rhea, lacrimation, } \\
\text { conjunctival injec- } \\
\text { tion, eyelid oedema, } \\
\text { facial sweating, } \\
\text { miosis or ptosis, } \\
\text { sense of restlessness } \\
\text { or agitation }\end{array}$ & $\begin{array}{l}\text { Any or several of } \\
\text { ipsilateral nasal } \\
\text { congestion, rhinor- } \\
\text { rhea, lacrimation, } \\
\text { conjunctival injec- } \\
\text { tion, eyelid oedema, } \\
\text { facial sweating, } \\
\text { miosis or ptosis, } \\
\text { sense of restlessness } \\
\text { or agitation }\end{array}$ & & $\begin{array}{l}\text { Hyperaesthesia } \\
\text { Increase of } \\
\text { complaints when } \\
\text { bending over } \\
\text { A sense of nasal } \\
\text { obstruction }\end{array}$ \\
\hline Others & $\begin{array}{l}\text { Prodromal state, } \\
\text { aura }\end{array}$ & $\begin{array}{l}\text { Attacks can be } \\
\text { precipitated by } \\
\text { innocuous stimuli } \\
\text { within the affected } \\
\text { trigeminal distri- } \\
\text { bution. There may } \\
\text { be concomitant } \\
\text { continuous pain of } \\
\text { moderate intensity }\end{array}$ & $\begin{array}{l}\text { Rare condition, } \\
\text { mostly males. Fre- } \\
\text { quent attacks (>5 } \\
\text { daily); a chronic } \\
\text { form exists as well; } \\
\text { absolute response } \\
\text { to indometacin }\end{array}$ & $\begin{array}{l}\text { Rare condition, } \\
\text { mostly males. Most } \\
\text { often comes in } \\
\text { bouts that last } \\
\text { weeks to months }\end{array}$ & & $\begin{array}{l}\text { Often patients } \\
\text { overuse analge- } \\
\text { sics without clear } \\
\text { benefit; if nasal } \\
\text { surgery is perfor- } \\
\text { med, it typically } \\
\text { relieves complaints } \\
\text { for several months } \\
\text { only }\end{array}$ \\
\hline
\end{tabular}

For a full overview of neuropathies and facial pain syndromes see: https://ichd-3.org; midfacial segment pain is not yet recognized in this list as a separate headache syndrome, but is established as a clinical entity.

or 15 days per month (depending on the medication group) for more than three months. More detailed information on this form of headache can be found in a recent review by Diener et al. ${ }^{(512)}$. The relevance for this chapter is that a patient referred for sinus disease because of headache or facial pain, might also be suffering from $\mathrm{MOH}$ in addition to a primary headache syndrome.

\subsubsection{Pragmatic approach}

When a patient's main complaint is facial pain:

- Invest in taking a proper history, including:

- the presence of (other) rhinosinusitis symptoms

- the correlation between the pain and other symptoms
- $\quad$ medication use (especially analgesics)

- $\quad$ other nasal conditions than CRS (e.g., allergic rhinitis)

- the response of the facial pain/headache to previous nasal treatments such as nasal and oral corticosteroids, antibiotics and/or surgery.

- Perform nasal endoscopy to exclude CRS or other rhinological causes for the facial pain

- Use CT imaging only when in doubt.

If CRS is expected as the explanation for the facial pain:

- First treat medically

- Check whether the pain improves together with the other 
signs and symptoms of CRS.

- If so, surgery might be helpful when medication alone fails to gain control of disease

- If not, reconsider the diagnosis or at least consider overlap with a primary headache syndrome or $\mathrm{MOH}$ and consider referral to a specialized neurologist

If the facial pain is not likely caused by CRS (or any other rhinological condition):

- Counsel the patient that the sinuses are not involved

- Try to further clarify the pain according to the characteristics in Table 5.3.4. Episodic/paroxysmal pain alludes to a primary headache disorder.

- It is up to the treating physician / rhinologist to what extent the treatment is carried out personally. Always consider referral to a specialized neurologist. For treatment, current literature suggests (a trial of) treatment with amitriptyline and/or triptans s $^{(513-516)}$, while others report success by offering a team-based approach ${ }^{(517)}$.

Treating a primary headache syndrome might be challenging and might need multiple medication regimes to succeed. For example, in a small retrospective cohort of patients with midfacial segment pain, success was achieved in $50 \%$ within 18 months. Most patients used a combination of drugs at that time ${ }^{(518)}$. As such, it is advised to consult a specialized neurologist dedicated to neuropathies, headache and facial pain whenever the first-line treatment (such as amitriptyline) fails.

\subsubsection{Diagnosis and differential diagnosis}

\subsubsection{Host factors}

As with all difficult to treat CRS patients, evaluation of the patient's immune system is warranted (see also 8.2). Basic testing would include a full blood count including eosinophils, specific IgE for common airway allergens and serum IgE. Immune deficiency has to be considered if the patient's history suggests frequent infections and is more common in patients with exacerbation of CRS than in the healthy population, up to $11 \%$ in recurrent acute CRS according to a recent review ${ }^{(46)}$. Revision surgery should be considered if the sinus anatomy is unfavourable.
Key points | What's new since EPOS 2012

- Many studies have explored further the relation between facial pain and CRS, showing that facial pain alone is rarely caused by CRS.

- $\quad$ Patterns in the different domains of disease-specific surveys such as the SNOT-22 help discern sinonasal facial pain from other causes.

- There is no relation between the location of facial pain and abnormalities upon imaging.

- A pragmatic approach to the patient with facial pain is provided.

- $\quad$ Facial pain without other nasal complaints or abnormalities on examination should not (primarily) be addressed surgically.

\subsubsection{Viral exacerbation}

Point of care viral assays are available and are now starting to be used clinically. The impact of these tests on antibiotic prescription and clinical outcomes in exacerbation of airway disease is currently being evaluated ${ }^{(519)}$. It is too early to form an opinion on the role of viral assays in the diagnosis of exacerbations of CRS but they may play a role in the future.

\subsubsection{Bacterial exacerbation}

A nasal swab with ensuing culture is often inconclusive. However, if positive, it will allow targeted antibiotic treatment. A repeated positive result is also informative and may suggest an antibody deficiency associated with encapsulated micro organisms such as Streptococcus Pneumonia, Haemophilus Influenzae and Moraxella Catarrhalis ${ }^{(520)}$.

\subsubsection{Biomarkers}

No unique biomarkers have been identified to diagnose an acute exacerbation of CRS. Point of care CRP or ESR have proved to be of limited value in acute rhinosinusitis ${ }^{(521)}$.

\subsubsection{Differential diagnosis}

Conditions of recurrent or cyclic nature such as migraine are more likely to be mistaken for AECRS. Many migraine sufferers have nasal symptoms with congestion, rhinorrhoea, fullness in cheek and forehead and which easily lead to the diagnosis. AECRS (522). Flare-ups of allergic rhinitis or non-allergic rhinitis are also easily confounded with AECRS and having a full understanding of the patient's airway history and immune system including allergy testing is helpful in identifying this.

Other possibilities are similar to the differential diagnosis in 
Figure 5.3.1. Delphi: Is it essential to do CT of the sinuses at initial presentation to ENT / secondary care with highly suggestive symptoms of CRS and abnormal mucosa at endoscopy?

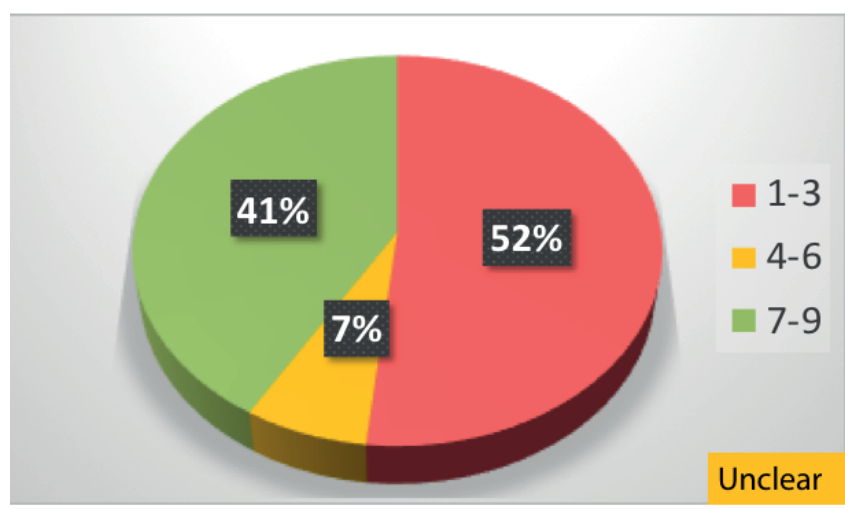

difficult-to-treat CRS including; dental infection, GORD, cystic fibrosis, primary ciliary dyskinesia, vasculitis and sarcoidosis. Conditions of recurrent or cyclic nature are more likely to be mistaken for AECRS such as migraine.

\subsubsection{Diagnostic tools}

This subchapter discusses diagnostic tools most relevant for CRS. For more extensive discussion of diagnostic tools in rhinology please refer to the European position paper on diagnostic tools in rhinology ${ }^{(450)}$.

\subsubsection{Diagnostic imaging in rhinosinusitis}

\subsection{Rationale}

Imaging is used to assess:

- Corroboration of clinical symptoms and endoscopic findings

- Anatomy and anatomical variants

- Pathology

- Diagnosis

- Extent $^{(523,524)}$

\subsection{Tests}

The different imaging modalities in diagnosing rhinosinusitis (conventional X-ray, computerized tomography (CT), cone beam $\mathrm{CT}$ and magnetic resonance imaging (MRI)) have been evaluated ${ }^{(525)}$ of which overall CT scan remains the gold standard in the radiologic evaluation of rhinologic disease, notably $\mathrm{CRS}^{(526-528)}$. The first guidelines that recommended CT sinus imaging and/or nasal endoscopy for confirmation of the symptoms-only based diagnosis of chronic rhinosinusitis were the EPOS guidelines in 2005 ${ }^{(529)}$.

However, in acute rhinosinusitis, the diagnosis is made on clinical grounds and CT is not recommended ${ }^{(1)}$ unless the condition persists despite treatment, or a complication is suspected when
Figure 5.3.2. Delphi: Is it essential to do CT of the sinuses at initial presentation to ENT / secondary care in patients with highly suggestive symptoms of CRS but normal endoscopy?

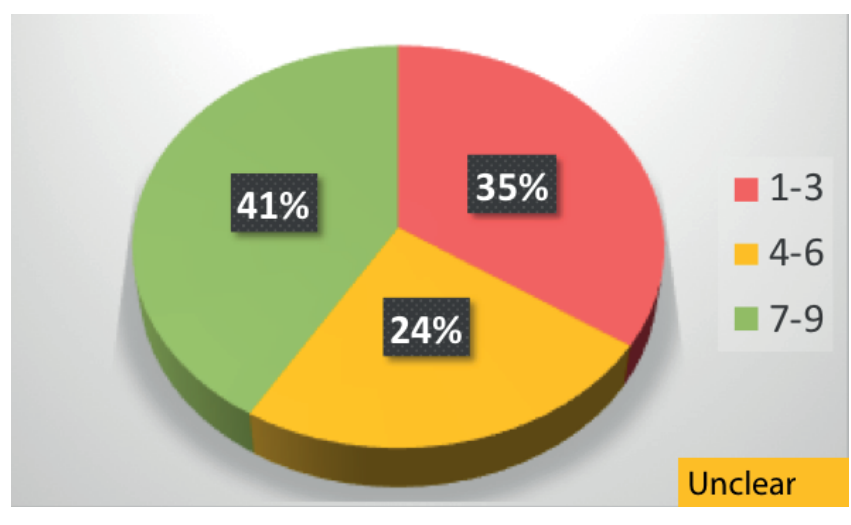

CT with contrast or MRI is used (if there is no contraindication) ${ }^{(530)}$. However, a recent study from Spain suggested that CT continues to be over-used in this context ${ }^{(531)}$. MRI and CT with contrast are also indicated if neoplasia or invasive fungal rhinosinusitis are suspected or in the further investigation of olfactory loss ${ }^{(525,526,532)}$.

It was unclear from the responses of the EPOS2020 steering group whether it was essential to do a CT of the sinuses at initial presentation to ENT / secondary care in a patient with symptoms highly suggestive of CRS irrespective of whether the mucosa was abnormal or normal at endoscopy (Figures 5.3.1., 5.3.2.).

However, the majority of the EPOS2020 steering group considered it was essential to do CT in CRS after failure of appropriate medical or surgical treatment in secondary care with continued symptoms and abnormal endoscopy (Figure 5.3.3.).

Many attempts have been made to semi-quantify the changes observed on CT scan using scoring and staging systems of varying complexity. The most commonly used of these is the Lund-Mackay system which is based on the degree of opacification for the maxillary, anterior and posterior ethmoids, frontal and sphenoid sinuses (0-none; 1-partial; 2-complete) and OMC ( 0 or 2 ) giving a maximum score of 24 or $12 /$ side $^{(533)}$. This scoring system has been validated in several studies ${ }^{(534,535)}$. Modifications of the LMS have been proposed but have not shown significant improvement in correlation with symptom severity scores or HRQOL impairment ${ }^{(536-538)}$.

In CRS after failure of appropriate medical or surgical treatment in secondary care with continued symptoms and abnormal endoscopy, the EPOS2020 steering group regarded a total LundMackay score of 0 as irrelevant, the relevance of a score of 1 or 2 due to mucosal thickening in 2 sinuses to be unclear and scores of 2 due to complete obstruction of one sinus, or a score of 3 or greater, whether uni- or bilateral to be clinically relevant (Figures 5.3.4. - 5.3.10.). 
Figure 5.3.3. Delphi: Is it essential to do CT in CRS after failure of appropriate medical or surgical treatment in secondary care with continued symptoms and abnormal endoscopy?

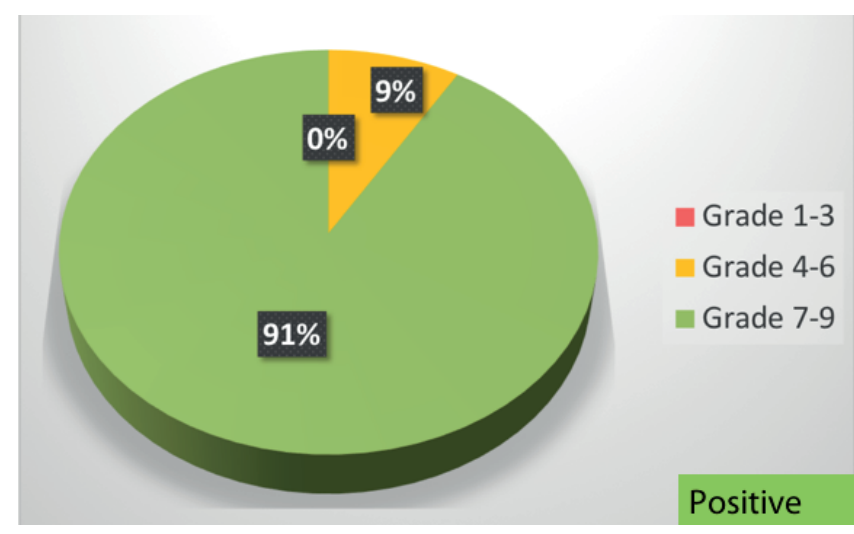

When deciding if the changes are clinically relevant, the EPOS2020 steering group felt that the importance of the following were unclear:

- Degree of mucosal thickening

- Number of sinuses affected

- Status of the ostiomeatal complex

However, the presence of complete opacification of any sinus was considered by the majority as clinically positive (Figures 5.3.11., 5.3.12., 5.3.13., 5.3.14.).

For the purposes of both medical and surgical treatment in secondary care/ENT, the EPOS020 steering group considered it was acceptable to use a previously performed CT scan of the sinuses which has been done up to six months earlier. They were unclear regarding the acceptability of a scan performed $>6$ months-3 years earlier but considered one performed $>3$ years earlier to be unacceptable for the purposes of medical treatment of CRS (Figures 5.3.15.-5.3.24.).

How and when the scan is done will influence the results ${ }^{(450)}$. Marked inflammatory changes may be observed following a viral cold which can persist for a number of weeks ${ }^{(539,540)}$ which probably accounts for the high percentage of normal asymptomatic adults who have evidence of mild mucosal thickening on CT ( 18\%) $)^{(10,541)}$.

Thus, on CT a "normal" Lund-Mackay score (LMS) for adults is $4.26(95 \% \mathrm{Cl}, 3.43 \text { to } 5.10)^{(542)}$ and for children it is 2.81 (95\% confidence interval, 2.40 to 3.22 ), with only $19.3 \%$ having a score of 0 (543). The LMS can also be applied to MRI and does not appear to overstage inflammatory disease ${ }^{(544)}$.

CRS studies comparing symptoms with CT and endoscopic findings have shown good correlation between $\mathrm{CT}$ and endoscopy but generally not between symptoms and $\mathrm{CT}$. When comparing concordance between endoscopy and CT staging in CRS, the correlation was $65 \%$ for positive and $71 \%$ for negative results ${ }^{(545)}$. However, more than $40 \%$ of patients who fulfil the symptom- based diagnosis of CRS may have normal CT and endoscopy results $^{(9,10)}$. The accuracy of CT in the diagnosis of CRS was tested, comparing CT scores with histopathologic grade of inflammation and including a control group without CRS, utilizing well designed criteria ${ }^{(543)}$. By using the ROC method, the sensitivity of CT was found to be $94 \%$ and specificity $41 \%$ using an LMS score cut-off value for CRS of greater than 2. If the value of "normal" LMS scores (i.e. >4) was applied, specificity increased to $59 \%$. Using the same method in paediatric rhinosinusitis, taking an LMS of at least 5 to indicate real disease, CT sensitivity was $86 \%$ and specificity $85 \%{ }^{(546)}$. Thus, an LMS of 2 or less has an excellent negative predictive value, and an LMS of five or more has an excellent positive predictive value, strongly indicating true disease. Weak but significant correlations were found in a small cohort of CRS patients between CT stage and scores on patient-based questionnaires in the CRSWNP subgroup but not with the CRS patient group as a whole ${ }^{(547)}$. In a similar study using 271 CRS patients comparing SNOT-22 and VAS with LMS CT score, no association was found between total SNOT-22 score and LMS overall or in CRSsNP(505). However, again some independent associations were shown with the LMS in CRwNP and both SNOT-22 and VAS $(p<0.001)$. There was no correlation between inflammation on CT and facial pain especially in CRSwNP(504, ${ }^{505)}$ and in CRSsNP the higher the SNOT-22 facial pain score, the lower the LMS $(p=0.022)$. The only symptom that significantly correlates with CT scores is olfaction ${ }^{(465,548)}$. On the other hand, CT scores were found to significantly correlate with the stage of inflammation in histopathological analysis of sinus mucosal samples in CRS, most notably in CRSsNP(549,550).

In comparison to $\mathrm{CT}$, plain sinus radiographs have shown poor sensitivity and specificity, so that even low irradiation does not justify their use. Correlation of CT scans with plain sinus radiographs for maxillary sinusitis was reasonable (78\%) but was only $52 \%$ for the ethmoids ${ }^{(551)}$ and CT out-performed ultrasonography ${ }^{(521)}$. However, CT cannot confirm acute bacterial rhinosinusitis as compared to antral puncture with positive bacteriology ${ }^{(552,553)}$. $\mathrm{CT}$ is also extremely sensitive in demonstrating 'abnormalities' in the sinonasal region. A wide range of anatomic variations are observed which are generally unrelated to the development of disease ${ }^{(541,554)}$ but are highly significant for 'safe' surgery e.g. the presence of a sphenoethmoidal cell ${ }^{(555)}$. The high resolution of modern CT and the use of detailed three planar reconstruction has significantly improved our appreciation of the complex anatomy particularly in the frontal recess and led to Int efforts to agree a classification of the area ${ }^{(523,524)}$. Thus, preoperative CT is mandatory for sinus surgery in most countries and should be available in the operating theatre ${ }^{(556,557)}$ providing an anatomical roadmap often augmented by navigation systems and other techniques such as fusion of CT and MRI.

Whilst CT is the principle modality for diagnosis in rhinosinusitis, extent of disease does not necessarily equate with prognosis ${ }^{(527,}$ 
Figure 5.3.4. Delphi: What do you regard as a clinically relevant total LundMackay score for CRS in this situation ? 0

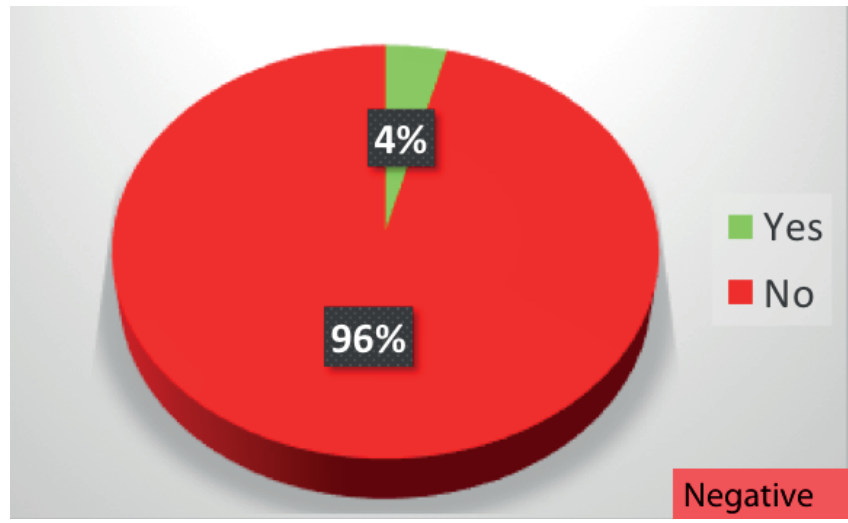

Figure 5.3.6. Delphi: What do you regard as a clinically relevant total LM score for CRS in this situation? 2 complete obstruction of one sinus

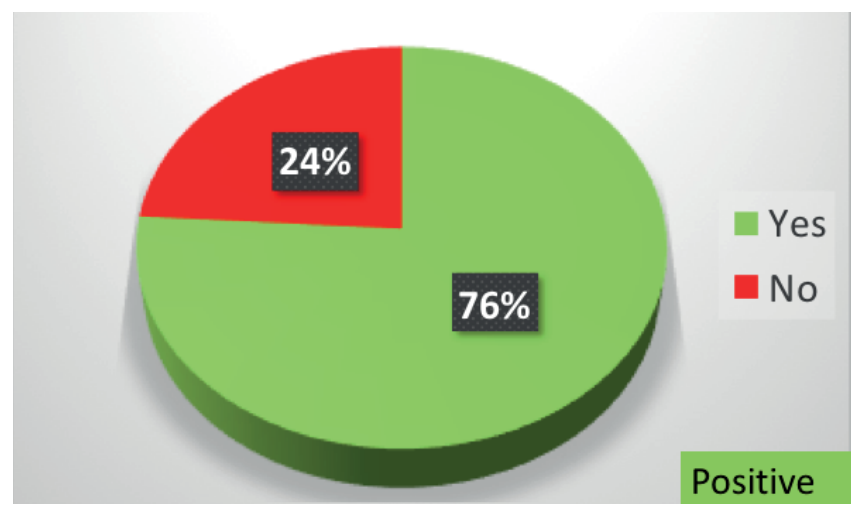

Figure 5.3.8.: Delphi What do you regard as a clinically relevant total LM score for CRS in this situation? $\mathbf{3}$ or more uni or bilateral

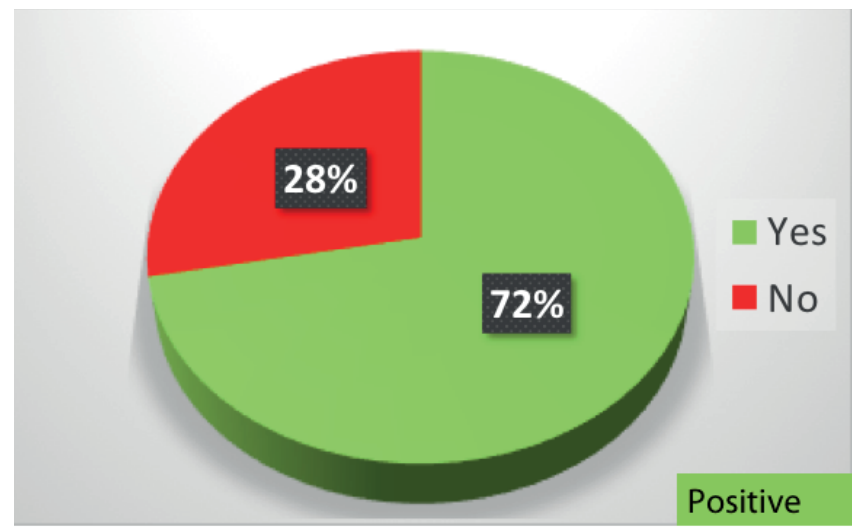

${ }^{534)}$. In a multicentre prospective study of patients undergoing surgery for CRS with nasal polyps (CRSwNP) and without (CRSSNP), the LMS was correlated with a range of parameters in 1840 CT scans ${ }^{(558)}$. This showed as expected that the higher the score, the higher the grade of polyp and the more extensive the surgery. The score also correlated with symptom reduction
Figure 5.3.5. Delphi: What do you regard as a clinically relevant total $L M$ score for CRS in this situation? 1

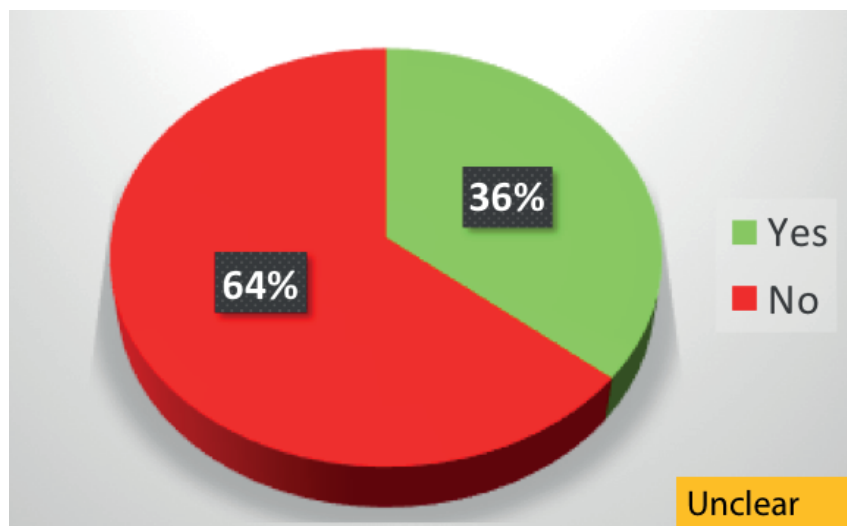

Figure 5.3.7. Delphi What do you regard as a clinically relevant total LM score for CRS in this situation? 2 mucosal thickening in 2 sinuses

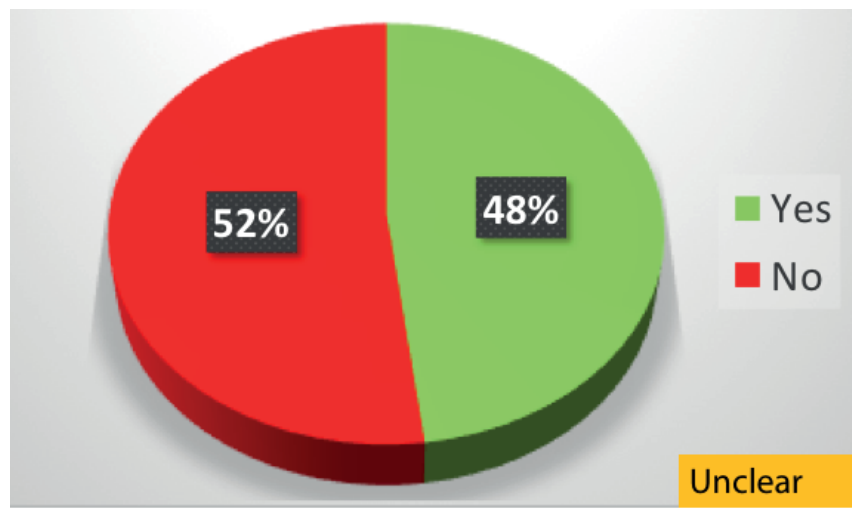

Figure 5.3.9.: Delphi What do you regard as a clinically relevant total LM score for CRS in this situation? $\mathbf{4}$ or more uni or bilateral

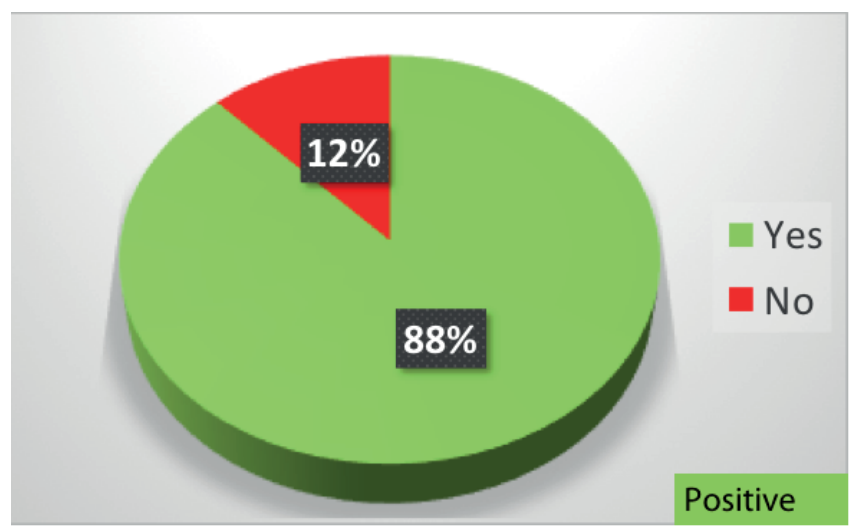

(coeff $=0.24, p=0.02)$, complication rate (odds ratio $(O R) 1.08$, 95\% confidence interval $(\mathrm{Cl})$ 1.06-1.1) and revision rates (OR $1.03,95 \% \mathrm{Cl} 1.001-1.06)$ but showed no correlation with SNOT22 scores. In another large multicentre cohort of refractory CRS patients, patients with low stage LMS are not significantly different than high stage LMS and did not show any difference 
Figure 5.3.10. Delphi: What do you regard as a clinically relevant total LM score for CRS in this situation? 5 or more uni or bilateral.

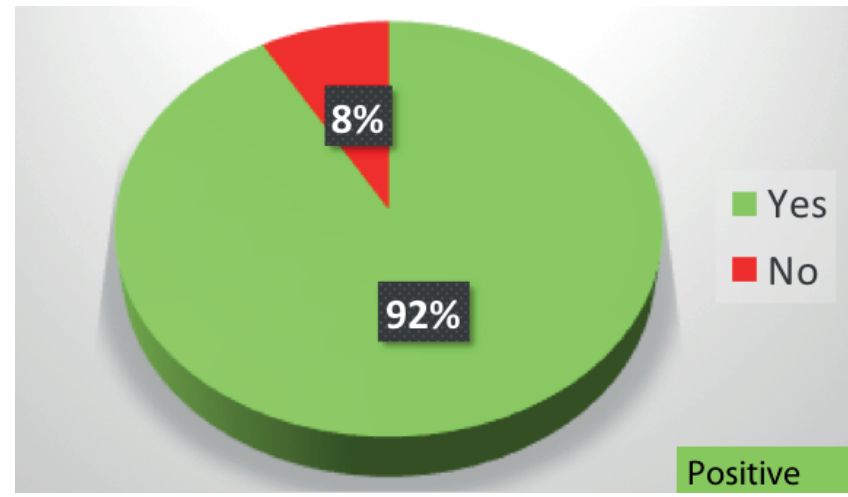

Figure 5.3.12. Delphi: When deciding if the changes are clinically relevant, how important are the following in your decision. Number of sinuses affected?

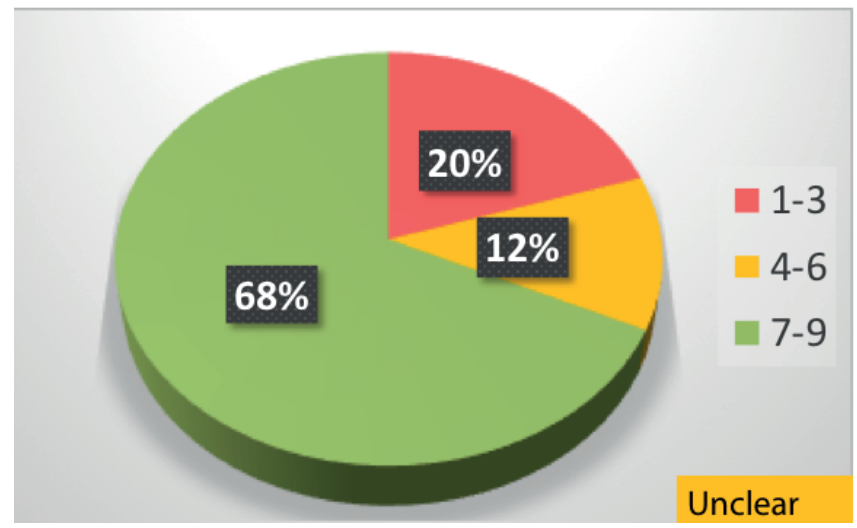

Figure 5.3.14. Delphi: When deciding if the changes are clinically relevant, how important are the following in your decision: Presence of complete opacification of any sinus?

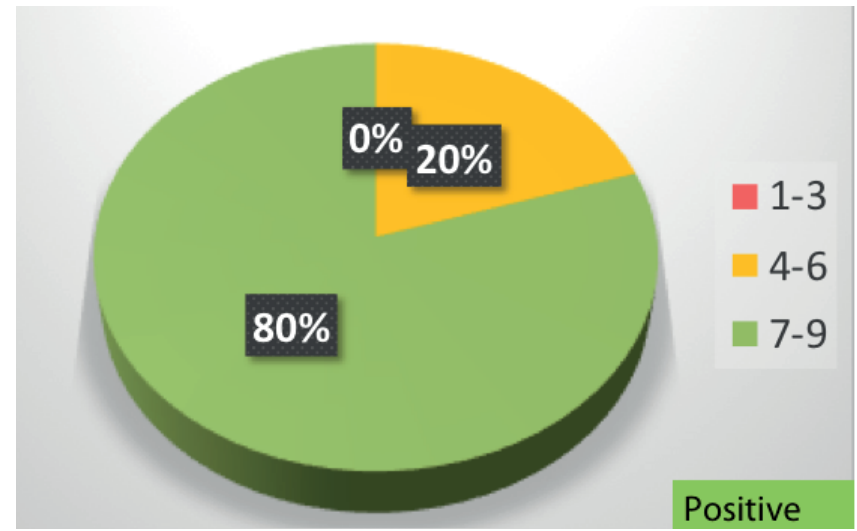

Figure 5.3.11. Delphi: When deciding if the changes are clinically relevant, how important are the following in your decision, Degree of mucosal thickening?

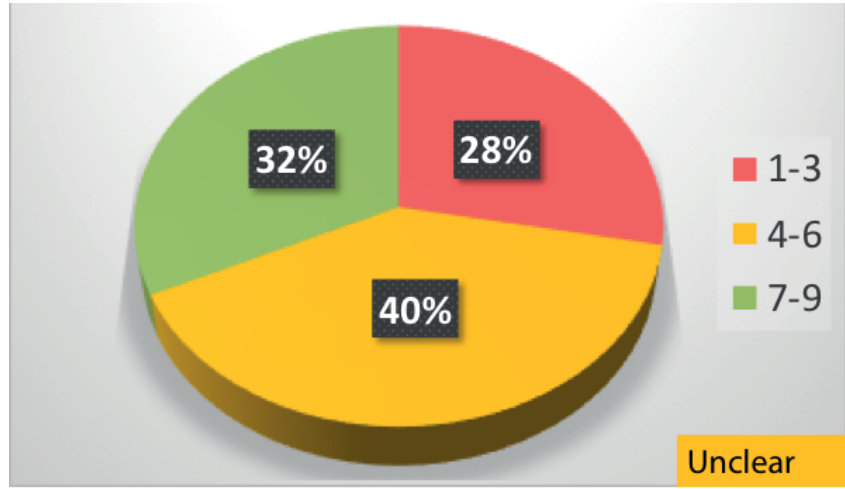

Figure 5.3.13. Delphi: When deciding if the changes are clinically relevant, how important are the following in your decision. Status of the ostiomeatal complex?

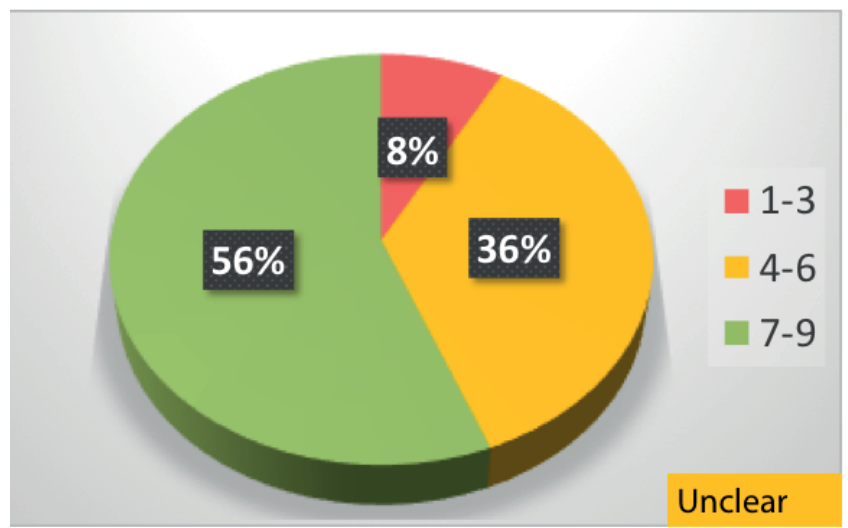

Figure 5.3.15. Delphi: For the purposes of medical treatment in secondary care/ENT is it acceptable to use the previously performed CT scan which has been done: $<3$ months ago.

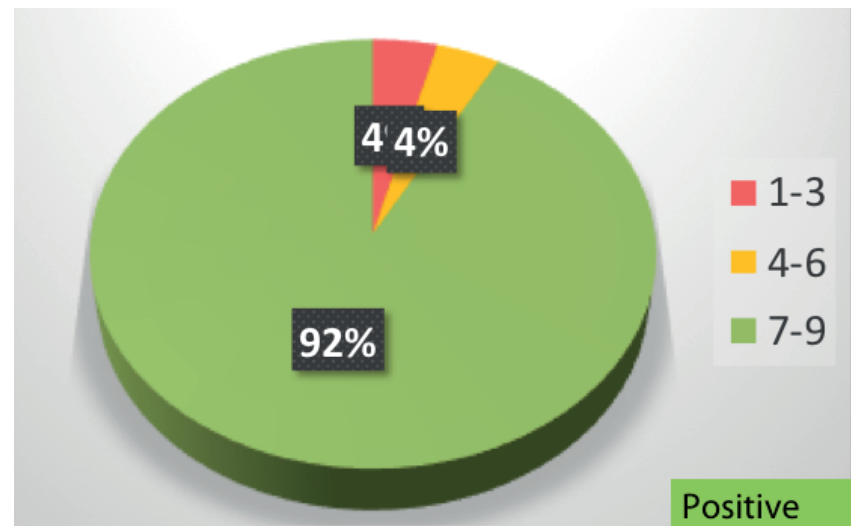


Figure 5.3.16. Delphi: For the purposes of medical treatment in secondary care/ENT is it acceptable to use the previously performed CT scan which has been done 3-6 months ago

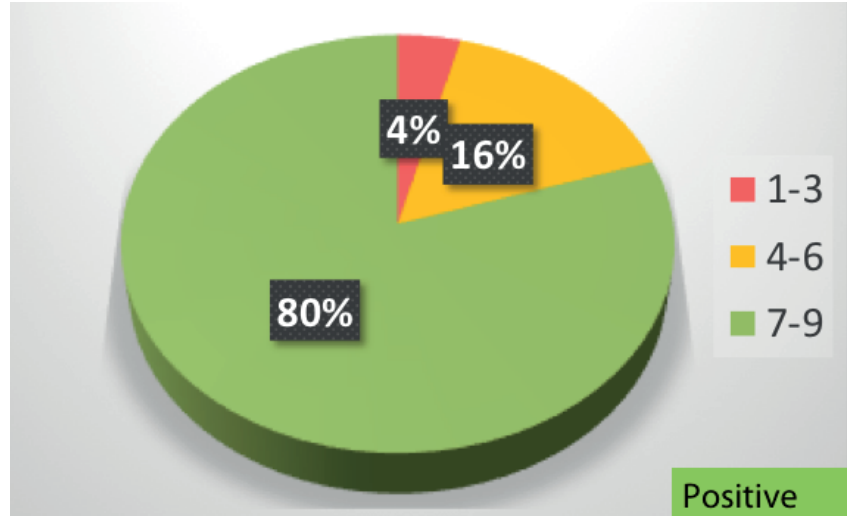

Figure 5.3.18. Delphi: For the purposes of medical treatment in secondary care/ENT is it acceptable to use the previously performed CT scan which has been done 1-3 years ago?

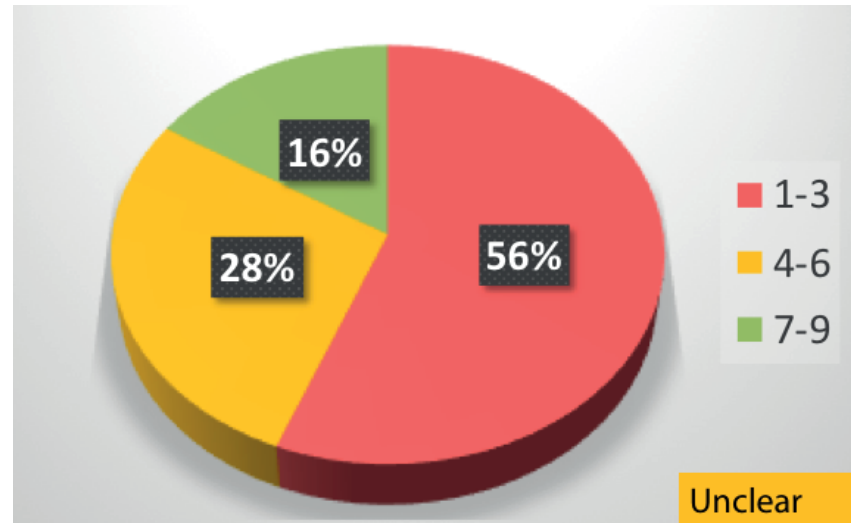

Figure 5.3.20. Delphi: For the purposes of surgical treatment in secondary care/ENT is it acceptable to use the previously performed CT scan which has been done < 3months ago?

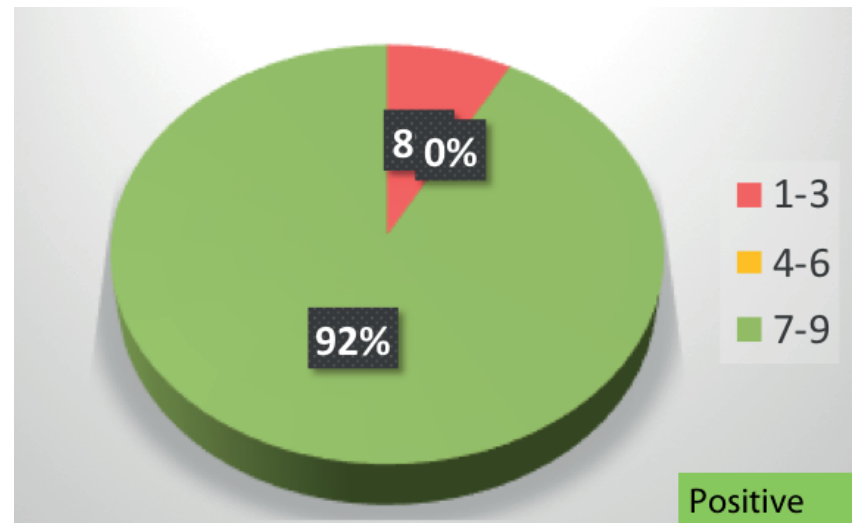

Figure 5.3.17. Delphi: For the purposes of medical treatment in secondary care/ENT is it acceptable to use the previously performed CT scan which has been done 6-12 months ago?

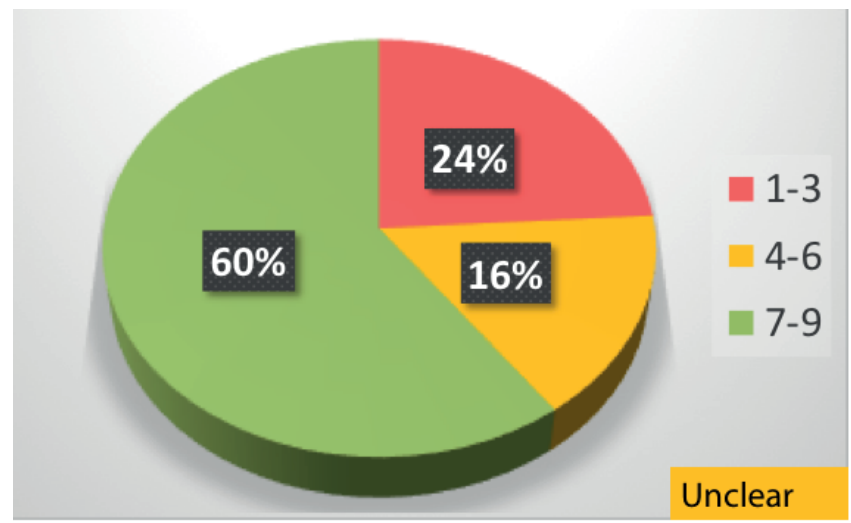

Figure 5.3.19. Delphi: For the purposes of medical treatment in secondary care/ENT is it acceptable to use the previously performed CT scan which has been done longer then 3 years ago?

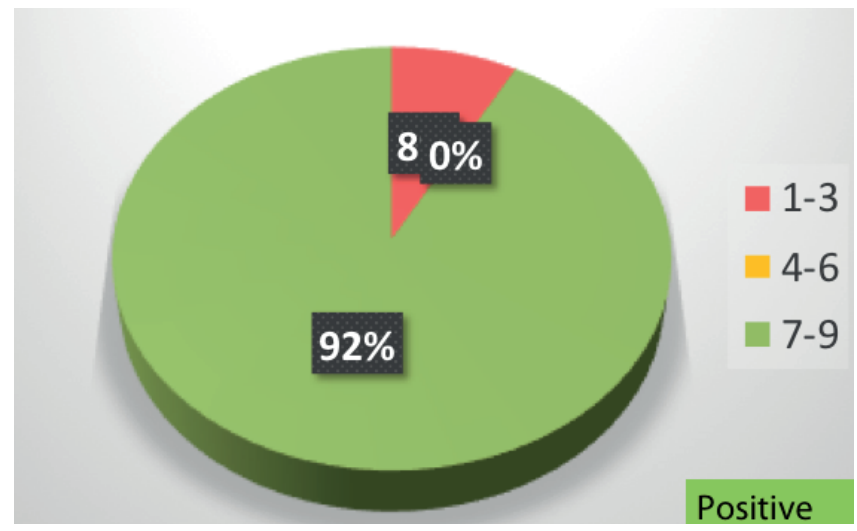

Figure 5.3.21. Delphi: For the purposes of surgical treatment in secondary care/ENT is it acceptable to use the previously performed CT scan which has been done 3-6 months ago?

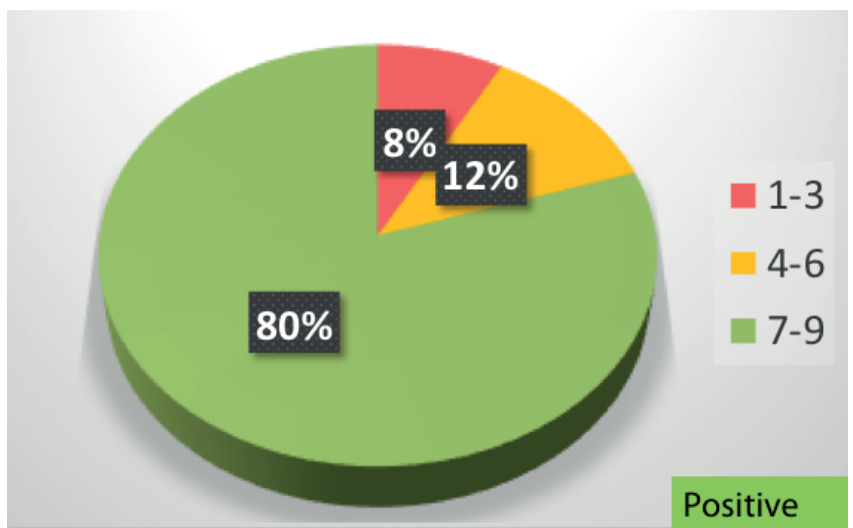


Figure 5.3.22. Delphi: For the purposes of surgical treatment in secondary care/ENT is it acceptable to use the previously performed CT scan which has been done 6-12 months ago?

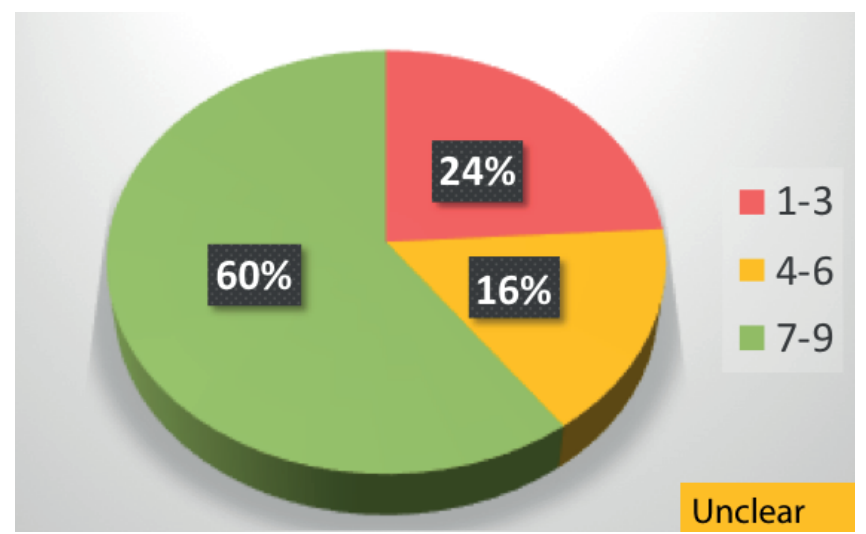

Figure 5.3.23. Delphi: For the purposes of surgical treatment in secondary care/ENT is it acceptable to use the previously performed CT scan which has been done 1 - 3 years ago?

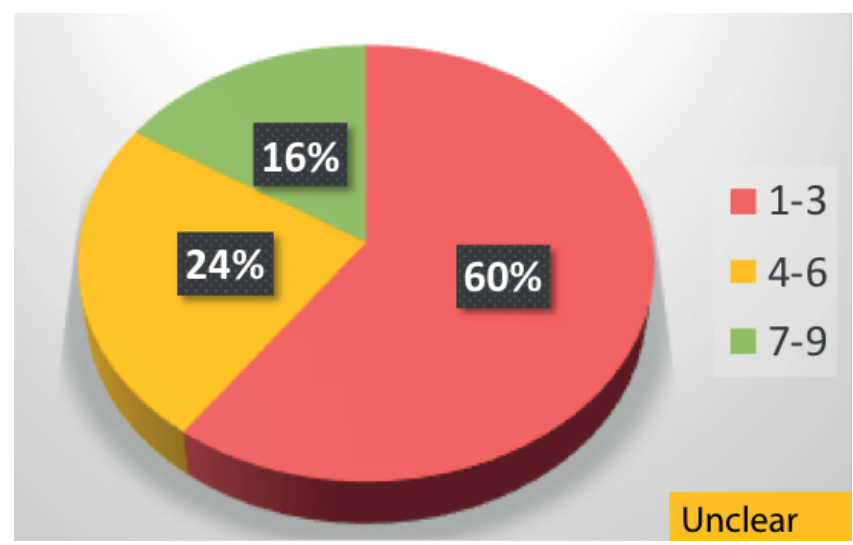

Figure 5.3.24. Delphi: For the purposes of surgical treatment in secondary care/ENT is it acceptable to use the previously performed CT scan which has been done longer then 3 years ago?

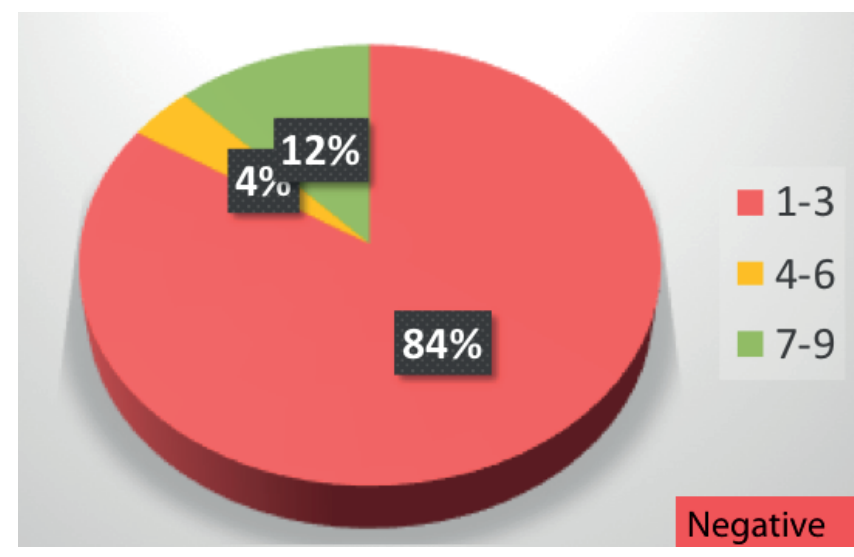

in level of improvement after endoscopic sinus surgery ${ }^{(559)}$. In contrast, a more recent study showed that the preoperative LMS in 665 patients with medically recalcitrant CRS correlated significantly with preoperative extranasal and rhinologic symptom severity on the SNOT-22 and that the LMS was an indicator of postsurgical quality of life (QOL) outcomes ${ }^{(560)}$. The LMS measures a different aspect to 'subjective' symptom scores as it correlates well with other markers of disease severity, the nature of surgery offered and its outcome. Its main value lies in diagnosis, determining extent of disease and providing inclusion criteria for studies.

The pattern of inflammation may also be important. A central radiological pattern of mucosal disease is likely associated with inhalant allergen sensitization - the so-called 'black halo' sign ${ }^{(194)}$. The radiological pattern defined by centrally limited changes in all of the paranasal sinuses was associated with allergy status and predicted atopy with $90.82 \%$ specificity, $73.53 \%$ positive predictive value, likelihood positive ratios of 2.16 and diagnostic OR of 4.59.

Other radiologic features such as the degree of neo-osteogenesis in CRS may have important prognostic implications ${ }^{(561-563)}$. Scoring systems have been developed to evaluate this on sinus CT based on bone thickness (Kennedy osteitis score: 0-3 $3^{(564)}$; Global osteitis scoring system: $0-5)^{(565)}$.

In CRS, CT imaging is the primary modality, but it is not normally recommended until after an appropriate course of medical therapy has failed ${ }^{(1,566)}$ and without an intervening acute episode. It should be noted that other studies suggest that early $\mathrm{CT}$ scanning may be more cost-effective as compared to extended courses of antibiotics given empirically and is preferred by patients ${ }^{(567-569)}$. This is especially pertinent in patients with rhinitis or atypical facial pain ${ }^{(504,570,571)}$ and may be facilitated by the availability of lower radiation protocols and/or cone beam. Hitherto, CT has not been used as an outcome measure due to ethical issues with additional radiation exposure but the technology and processing abilities of multi-detector CT (MDCT) scanners have continued to evolve and there has been a drive to reduce radiation dose whilst preserving image quality by shortening the scan time and using post-processing techniques $\left({ }^{572}\right.$, 573). Cone beam CT (CBCT), historically used for dental imaging, is also being utilised in sinonasal imaging producing high resolution images, but the longer scan time, subsequent susceptibility to motion artefact and the lack of soft tissue differentiation can be a drawback resulting in loss of some pathological detail such as the hyperdensities seen in sinonasal fungal disease ${ }^{(574)}$. However, ionizing radiation has been significantly reduced with multi-slice detector CT (MSCT) utilising low dose protocols, which reduce the dose to $0,07 \mathrm{mSv}, 10$ times lower than the standard protocol ${ }^{(575)}$. Low dose protocols have been shown to significantly shorten the duration of the procedure without compromising anatomical accuracy ${ }^{(575)}$ making them increasin- 
gly attractive as an objective outcome measure in therapeutic studies $^{(576,577) \text {. }}$

\subsubsection{Health-related quality-of-life (HRQOL) measures}

\subsection{Rationale}

Patient reporting outcome measures (PROMs) are questionnaires or instruments, developed and validated according to defined methods, that are designed to quantify patients' subjective perception of the severity of their disease in terms of the impact on quality of life. They may be disease specific or reflect global QOL. While not intended as diagnostic tools, PROMS will normally discriminate between those with and without the disease in question, and for many 'normal' population scores have been defined. However, they are best used as repeated measures to detect changes in HRQOL over time, or in response to medical or surgical intervantion.

Early reports of sinus surgery focused in clinician rated measures such as endoscopy or radiological scores, although since the mid 90 s there has been a growing focus in the use of symtoms scores and PROMS, reflecting the primary aim of treatment being the improvement in the general well-being of the patient and their quality of life. CRS related quality of life has been shown to drive patient's decision-making regarding treatment options and predicting post-operative outcomes.

\subsection{Tests}

Instruments which measure health-related quality-of-life (HR$\mathrm{QOL}$ ) are generally divided into generic HRQOL instrument and disease specific ones.

Generic questionnaires are designed to measure different domains of physical, psychological and social functioning across all health conditions. These instruments may be used to compare healthy and diseased subjects, and may also be used to estimate the impact of different diseases on general well-being of patients in comparison with other diseases patients ${ }^{(578)}$, but are also used to measure QOL in CRS patients ${ }^{(36,579-582)}$. The EQ-5D responds well to changing CRS symptomatology ${ }^{(583)}$.

Disease-specific instruments may be composed of different domains of general health affected in a given disease but also measure the severity of the specific symptoms of the disease. Such instruments are developed having identified the most common symptoms or impairments when questioning the patients about the problems they experience related to the disease. These instruments may measure both the severity and frequency of the impairment. Specific questionnaires seem to discriminate better between those with and without disease than generic PROMS, and better evaluate the impairment within the domains of a specific disease and their interactions, such that they are more relevant to clinical practice. They are of less value in comparison with other conditions.

\subsection{HRQOL measures in ARS}

Perhaps reflecting the self-limiting nature of ARS, and its relatively short duration, there are very few studies evaluating $\mathrm{HRQOL}$ in ARS and a paucity of validated measures. The SNOT- $16^{(584)}$, a derivative of the RSOM-31 questionnaire, which was initially developed for patients with CRS, has been validated for use in primary care patients with ARS. It has been shown to have high internal consistency and sensitivity to change, with a mean item score reported between 0-3 and an MCID of 0.5.

Trials of treatments in ARS report variable outcomes, but usually present 'cure' rates (described as complete resolution of symptoms, but not otherwise defined) or report scores for individual symptoms of facial pain, congestion, rhinorhoea, post-nasal drip or headache. The heteregeneity in reporting of outcomes precludes meta-analysis. There is no core outcome set for ARS.

The EPOS2020 steering group were unclear whether a QOL instrument was important for the management of acute rhinosinusitis (ARS) (Figure 5.3.25.).

For a General Health-related quality of life instrument in ARS, the EPOS2020 steering group used a wide range of possibilities. Of these the SF36 was the most popular but the SF12 was also suggested (Figure 5.3.26.).

When using a specific rhinologic health-related quality of life instrument in ARS, the majority of the EPOS2020 steering group proposed the SNOT22 (Figure 5.3.27.).

\subsection{HRQOL measures in CRS}

Chronic rhinosinusitis (CRS) has a significant impact on HRQOL and may affect different domains of HRQOL in addition to rhinological symptoms, such as fatigue, sleep quality, pain and emotional impairment (like anxiety and depression, productivity and social functioning.

A number of $\mathrm{HRQOL}$ evaluating instruments have been developed during past few decades. The key features of the most commonly used PROMs are summarised in Table 5.3.5.

The Chronic Sinusitis Survey (CSS) ${ }^{(585)}$ evaluated six item severity and duration based specific outcomes, primarily related to rhinosinusitis symptoms, pain and medication use. Rhinosinusitis Disability Index (RSDI) ${ }^{(586)}$ is a similar HRQOL instrument asking 30 questions in 3 domains, covering also some generic questions. The Rhinosinusitis Outcome Measure (RSOM-31), developed in $1995^{(587)}$ is a HRQOL questionnaire evaluating seven domains in 31 questions. RSOM-31 was reduced to SNOT-20 in 2002, and then modified into SNOT-22 in 2009. The SNOT22 is now regarded as the most appropriate instrument in the evaluation of HRQOL impairment in CRS patients, according to a recent systematic review of 15 CRS related PROMs which were analyzed using Consensus-based Standards for the selection of Health Measurement Instruments ${ }^{(588)}$. The SNOT-22 includes evaluation of the severity of major and most of the minor CRS 
Figure 5.3.25. Delphi: Is a QoL instrument important for the management of ARS ?

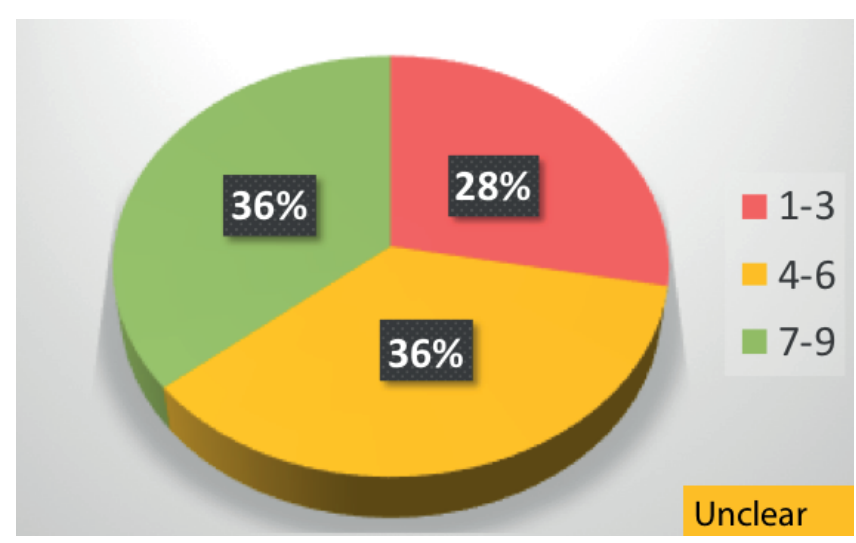

Figure 5.3.26. Delphi: If you use a general health-related quality of life instrument which would you use for ARS?

\begin{tabular}{ll}
\hline SF-36 & $9(36 \%)$ \\
\hline SF12 & $5(20 \%)$ \\
\hline I don't use one & $5(20 \%)$ \\
\hline EuroQOL & $\begin{array}{l}2(8 \%)+1(4 \%) \text { in a research setting } \\
\text { only }\end{array}$ \\
\hline A short one (e.g.NOSE) & $1(4 \%)$ \\
\hline $\begin{array}{l}\text { SF-36 or SF-12 (generic not } \\
\text { general) }\end{array}$ & $1(4 \%)$ \\
\hline I don't see ARS patients & $1(4 \%)$ \\
\hline SNOT-16 & $1(4 \%)$ \\
\hline VAS & $1(4 \%)$ \\
\hline EVA & $1(4 \%)$ \\
\hline
\end{tabular}

Multiple options given by some respondants.

Figure 5.3.27. Delphi: If you use a specific rhinologic health-related quality of life instrument, which would you use for ARS?

\begin{tabular}{|ll|}
\hline SNOT-22 & $12(48 \%)$ \\
\hline SNOT20 & $3(6 \%)$ \\
\hline I don't use & $3(6 \%)$ \\
\hline NOSE & $1(4 \%)$ \\
\hline I don't see ARS patients & $1(4 \%)$ \\
\hline EVA & $1(4 \%)$ \\
\hline SNOT unspecified & $1(4 \%)$ \\
\hline
\end{tabular}

Not all participants answered. symptoms with a nasal domain, and includes additional items in subdomains relating to sleep disturbance, emotional / psychological and aural/ facial pain domains ${ }^{(588,589)}$. Patients score each of 22 items on a Likert 6 points scale, or 0 to 5 , with a total score range of 0-110. SNOT-22 scores are influenced significantly with several demographic confounders and comorbid disorders, like gender, smoking, asthma and mental illness, including anxiety and depression ${ }^{(511,590,591)}$.

The SNOT 22 questionnaire was validated in 2009 relying on the data from a UK multicentric prospective cohort study in over 3000 patients followed pre- and postoperatively after sinus surgery ${ }^{(592,593)}$. The SNOT-22 can be stratified into 'mild' being defined on the SNOT-22 score as 8-20, 'moderate' as >20-50 and 'severe' as $>50^{(594)}$. It has been validated in several foreign languages ${ }^{(595-599)}$, for children ${ }^{(600,601)}$ and it has been used as an outcome measure in several multicentric cohort studies ${ }^{(36,590,602)}$. Patients with non-sinogenic headache have distinct SNOT-22 score patterns compared with patients with CRS. A domain 3 (Ear/facial)/domain 1 (Rhinologic) ratio greater than 0.66 is a strong negative predictor of CRS, which can be used to aid in patient counseling and potentially limit the use of unnecessary sinonasal therapeutics ${ }^{(511)}$.

With regards to changes in $\mathrm{HRQOL}$ in the setting of chronic disease, many patients may experience improvement after intervention but many may have ongoing symptoms. Repeated measures allow change in HRQOL to be monitored over time. It may also facilitate cohort studies where the effectiveness of interventions can be analysed. In order to determine if a change at individual or population level is clinicaly significant, the minimal clinically important difference (MCID) can be used. This is the smallest change in a given outcome measure that can be detected by a patient as a perceptible change. The MCID can be calculated using a number of different methods; using anchor-based methodology in a large surgical cohort the MCID has been shown to be a change in 8.9 points on the SNOT-22, while for patients undergoing medical intervention, an MCID of 12 has been proposed ${ }^{(603)}$. Scores for the SNOT-22 subdomains have also been defined ${ }^{(603,604)}$. It can be used to help define a minimum threshold for 'success' or response rate for any given intervention. A limitation of the MCID is that it is a populationderived score and the threshold for perceived benefit may vary between individuals. Individualized outcome measures, however, are complex to use and preclude comparative studies. Based on the large number of outcome measures and heterogeneity of outcomes measured in the current published literature, a Core Outcome Set (COS) for assessing outcomes in CRS has been proposed ${ }^{(605)}$. A COS is a standardized group of outcome measures that may be included in future trials to facilitate data comparisons and future meta-analyses. For the CRS COS, items in four domains were identified as imperative: patient reported symptoms and QOL, control of disease, impact 
Table 5.3.5. Characteristics of validated patient reported outcome measures for chronic rhinosinusitis.

\begin{tabular}{|c|c|c|c|c|c|c|c|c|c|}
\hline $\begin{array}{c}\text { QoL } \\
\text { Measure }\end{array}$ & $\begin{array}{c}\text { Year } \\
\text { developed }\end{array}$ & Type & $\begin{array}{l}\text { No. of } \\
\text { patients in } \\
\text { validation } \\
\text { study }\end{array}$ & $\begin{array}{l}\text { No. of } \\
\text { questions }\end{array}$ & $\begin{array}{l}\text { No. of } \\
\text { domains }\end{array}$ & $\begin{array}{l}\text { Score } \\
\text { range }\end{array}$ & Domains assessed & $\begin{array}{l}\text { Minutes } \\
\text { to } \\
\text { complete }\end{array}$ & MCID \\
\hline CSS & 1995 & HRQoL & 104 & 6 & 2 & $0-100$ & $\begin{array}{l}\text { CRS symptoms; } \\
\text { medication use }\end{array}$ & 5 & 9.75 \\
\hline RSOM-31 & 1995 & HRQoL & 142 & 31 & 7 & $0-155$ & $\begin{array}{l}\text { Nasal; eye; ear; sleep; gene- } \\
\text { ral; emotional; functional }\end{array}$ & 15 & \\
\hline RSDI & 1997 & HRQoL & 87 & 30 & 3 & $0-120$ & $\begin{array}{l}\text { Physical; functional; } \\
\text { emotional }\end{array}$ & $5-10$ & 10.35 \\
\hline SNOT-16 & 1999 & HRQoL & 47 & 16 & 0 & $0-48$ & NA & 5 & \\
\hline SNOT-20 & 2002 & HRQoL & 102 & 20 & 0 & $0-100$ & NA & 5 & 16.0 \\
\hline RSI & 2003 & $\begin{array}{l}\text { Symptom } \\
\text { score }\end{array}$ & 322 & 20 & 3 & $0-100$ & $\begin{array}{l}\text { CRS symptoms; medication } \\
\text { use; work and social }\end{array}$ & 5 & \\
\hline $\begin{array}{l}\text { Rhino- } \\
\text { QoL }\end{array}$ & 2005 & HRQoL & 49 & 17 & 3 & $0-100$ & $\begin{array}{l}\text { Symptom severity; bother- } \\
\text { someness; impact scale }\end{array}$ & 7 & $3.8-6.1$ \\
\hline RSTF & 2007 & $\begin{array}{l}\text { Symptom } \\
\text { score }\end{array}$ & 201 & 14 & 0 & $0-140$ & NA & 3 & \\
\hline SNOT-22 & 2009 & HRQoL & 2803 & 22 & 0 & $0-110$ & $\begin{array}{l}\text { Rhinologic; extranasal } \\
\text { rhinologic; ear/facial; } \\
\text { psychosocial; sleep }\end{array}$ & 7 & 8.9 \\
\hline SNQ & 2009 & $\begin{array}{l}\text { Sinusitis } \\
\text { screen }\end{array}$ & 59 & 5 & 0 & $0-35$ & NA & $<2$ & \\
\hline $\begin{array}{l}\text { DyNa- } \\
\text { Chron }\end{array}$ & 2012 & HRQoL & 759 & 78 & 6 & $0-780$ & $\begin{array}{l}\text { Nasal obstruction; anterior } \\
\text { rhinorrhea; posterior } \\
\text { rhinorrhea; sense of smell } \\
\text { difficulty; facial pain; cough }\end{array}$ & 15 & \\
\hline QOD & 2012 & HRQoL & 102 & 25 & 3 & $0-57$ & $\begin{array}{l}\text { Negative items; positive } \\
\text { items; social items }\end{array}$ & $7-10$ & $2.6-8.6$ \\
\hline DSS & 2013 & HRQoL & 48 & 6 & 2 & $0-32$ & Symptoms; HRQoL & $<2$ & \\
\hline EQ-5D & 2015 & $\begin{array}{l}\text { Generic QoL/ } \\
\text { Health state } \\
\text { utility }\end{array}$ & 350 & 15 & 5 & $0-100$ & $\begin{array}{l}\text { Mobility; self-care; usual } \\
\text { activity; pain/discomfort; } \\
\text { anxiety/depression }\end{array}$ & $<2$ & \\
\hline SCT & 2015 & $\begin{array}{l}\text { CRS-specific } \\
\text { control }\end{array}$ & 50 & 4 & 3 & $0-16$ & $\begin{array}{l}\text { Symptoms; productivity; } \\
\text { rescue medication use }\end{array}$ & 1 & \\
\hline
\end{tabular}

Data from Rudmik et al. 2015 ${ }^{(588)}$. CSS, Chronic Sinusitis Survey; CRS, chronic rhinosinusitis; DSS, Disease Severity Score; DyNaChron,

Dysfonctionnement Nasal Chronique Questionnaire; EQ-5D, EuroQoL Five-dimensional Questionnaire; HRQoL, health related quality of life; NA, not available/applicable; QOD, Questionnaire of Olfactory Disorders;QoL, quality of life; RhinoQoL,Rhinosinusitis Quality of Life questionnaire; RSDI, Rhinosinusitis Disability Index; RSI, Rhinosinusitis Severity Inventory; RSOM-31, 31-item Rhinosinusitis Outcome Measurement; RSTF, Rhinosinusitis Task Force; SNOT, Sinonasal Outcome Test; SCT, Sinonasal Control Test; SNQ, Sinonasal 5-item questionnaire.

on daily activity, and acceptability of treatment and side effects. Currently none of the established PROMS capture all the desired aspects of CRS; the SNOT-22 fails to capture disease duration or medication usage. Current recommendations include the use of SNOT-22 scores repeated over time, Lund-Kennedy (LK) endoscopic scores, and additional questions to evaluate the need for systemic medications or progression to surgery, compliance with and side effects of treatment, additional information on symptom frequency, and impact on ability to perform normal activities $^{(605)}$ (Table 5.3.6.).
The EPOS2020 steering group were unanimous that a QOL instrument was important for the management of CRS (Figure 5.3.28.).

The General Health-related quality of life instrument which was used by the majority of the EPOS2020 steering group in CRS was the SF36 but the SNOT22 and EQ-5D were also popular (Figure 5.3.29.).

When using a specific rhinologic health-related quality of life instrument in CRS, the vast majority of the EPOS2020 steering group used a SNOT22 (Figure 5.3.30.). 
Table 5.3.6. Core Outcome Set.

\begin{tabular}{lll} 
Domain & Item & Proposed measurement tool \\
\hline Patient-reported symptoms and QOL & $\begin{array}{l}\text { Overall symptom severity } \\
\text { Frequency of symptoms } \\
\text { Duration of symptoms } \\
\text { Duration of treatment effect } \\
\text { Sense of smell } \\
\text { Runny nose/nasal discharge (anterior or posterior) } \\
\text { Nasal obstruction/blockage/congestion } \\
\text { Disease-specific quality of life }\end{array}$ & $\begin{array}{l}\text { SNOT-22 repeated over time } \\
\text { Additional question required to address fre- } \\
\text { quency of symptoms }\end{array}$ \\
& $\begin{array}{l}\text { Overall control of disease } \\
\text { Need for surgery } \\
\text { Endoscopic appearances (including presence/qua- }\end{array}$ & $\begin{array}{l}\text { Need for systemic medication (steroid or } \\
\text { antibiotic) }\end{array}$ \\
& $\begin{array}{l}\text { Progression to surgery } \\
\text { lity of pus, presence and size of polyps, oedema, }\end{array}$ & Lund-Kennedy score \\
\hline crusting, inflammation) & SNOT-22 (or specific measures of productivity) \\
Impact on daily activity & $\begin{array}{l}\text { Ability to perform normal activities } \\
\text { Compliance with treatment }\end{array}$ & Measurement of compliance and side effects \\
Acceptability of treatment and side effects & $\begin{array}{l}\text { Acceptability of treatment } \\
\text { Side effects of treatment (including medical and } \\
\text { surgical) }\end{array}$ &
\end{tabular}

\subsubsection{Nasal endoscopy}

\subsection{Rationale}

Nasal endoscopy is an essential part of the rhinological examination of the nasal cavity, middle meatus, sphenoethmoidal recess and postnasal space, and is an essential part of the rhinological examination ${ }^{(450)}$. It allows identification of oedema, pus and/or polyps, assessment of sinus cavities following surgery and facilitates postoperative debridement or microbiological sampling when needed ${ }^{(606)}$. It can be used to evaluate the response to both medical and surgical treatment and allows photo / video-documentation. It is a useful educational tool for both junior staff and patients, and is generally well-tolerated.

\subsection{Test}

The standard three-pass technique for rigid nasendoscopy was

Figure 5.3.28. Delphi: Is a QoL instrument important for the management of CRS ?

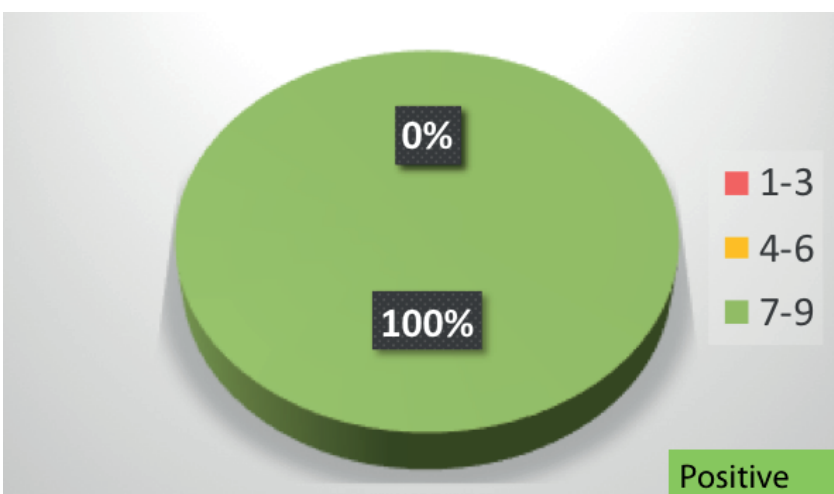

Figure 5.3.29. Delphi: If you use a general health-related quality of life instrument, which would you use for CRS *?

\begin{tabular}{|ll}
\hline SF-36 & $12(48 \%)$ \\
\hline SNOT-22 & $6(24 \%)$ \\
\hline EQ-5D & $6(24 \%)$ \\
\hline SF12 & $4(16 \%)$ \\
\hline None & $1(4 \%)$ \\
\hline VAS & $1(4 \%)$ \\
\hline
\end{tabular}

*Multiple options given by some respondants.

Figure 5.3.30. Delphi: If you use a specific rhinologic health-related quality of life instrument, which would you use for CRS *?

\begin{tabular}{|ll|}
\hline SNOT-22 & $21(84 \%)$ \\
\hline SNOT unspecified & $2(8 \%)$ \\
\hline CRS-PRO & $1(4 \%)$ \\
\hline SNOT20 & $1(4 \%)$ \\
\hline RSDI & $1(4 \%)$ \\
\hline VAS & $1(4 \%)$ \\
\hline
\end{tabular}

*Not all participants answered. 
originally described using a $4 \mathrm{~mm} 30$ degree endoscope, but it can be performed with a $2.7 \mathrm{~mm}$ endoscope and/or a zero degree endoscope instead ${ }^{(607)}$. The patient should be upright, and topical decongestant/anaesthetic spray may be used if necessary. The first pass is made along the floor of the nose to the nasopharynx, looking at the general anatomy, septum, inferior turbinates and nasal mucosa.The second pass runs above the inferior turbinate to the middle meatus then medial to the middle turbinate into the sphenoethmoidal recess.The third pass is made as the endoscope is withdrawn, when it may be possible to roll it laterally into the middle meatus. Flexible endoscopes can be used instead, and are particularly useful if the larynx and pharynx need to be assessed as well, but additional procedures cannot be performed ${ }^{(608)}$.

Endoscopy improves diagnostic accuracy compared to anterior rhinoscopy alone, up to $69.1 \%-85 \%{ }^{(609,610)}$. It is highly specific, with several studies reporting specificities of up to $95 \%(567,609$, ${ }^{611)}$. It is less sensitive, ranging from $30 \%$ to $73 \%{ }^{(609,610)}$. Various endoscopic scoring systems have been described, such as the Lund-Kennedy system and modifications thereof ${ }^{(612-614)}$. Interrater reliability is variable, with some aspects of the examination scoring highly and others less so, although overall inter-rater agreement is good ${ }^{(615)}$.

Recently, a systematic review analysed the accuracy of nasal endoscopy in diagnosing chronic rhinosinusitis (CRS) compared with paranasal sinus computed tomography (CT). Sixteen observational or retrospective studies were included resulting in a high correlation $[\mathrm{r}=0.85 ; 95 \%$ confidence interval $(\mathrm{Cl})$ (0.78-0.94), p<.0001, 1277\%) between endoscopy and CT in terms of the diagnostic accuracy for CRS. The sensitivity and specificity were $0.73[95 \% \mathrm{Cl}(0.58-0.83)]$ and 0.77 [95\% Cl (0.69-0.85)], respectively. In a subgroup analysis of studies using a Lund-Kennedy endoscopic score threshold $\geq 2$, the area under the summary curve was 0.881 , and the sensitivity and specificity were 0.84 [95\% Cl (0.78-0.93)] and 0.79 [95\% Cl (0.37-0.96)], respectively ${ }^{(616)}$

In conclusion: nasal endoscopy is is an essential part of the rhinological examination and correlates quite well with CT scan of the sinusses in patients with CRS.

\subsubsection{Allergy testing}

\subsection{Rationale}

The potential role of allergy in CRS has been discussed in 5.1.2.1.

\subsection{Test}

Sensitisation in the regular clinical practice can be measured with skin prick testing or serum specific $\lg \mathrm{E}^{(450)}$. It is important to realize the difference between sensitization to aero-allergens and allergic rhinitis. Around $60 \%$ of the total amount of sensitizations to aero-allergens are clinically relevant ${ }^{(617)}$. Depending on the allergen, from $40 \%$ (cockroach) to $87-89 \%$ (grass, mites) of the positive SPT reactions (wheal size $\geq 3 \mathrm{~mm}$ ) are associated with patient-reported clinical symptoms when exposed to the respective allergen. The risk of allergic symptoms increased significantly with larger wheal sizes for 17 of the 18 allergens tested $^{(618)}$. In exceptional circumstances in the diagnosis of CRS, mainly when occupational allergies are involved, allergy testing may be done with nasal or conjunctival allergen provocation ${ }^{(619)}$. Nasal allergen challenge tests may be performed by direct application of allergen extract solution sprayed into the nostril or by the exposure to the inhalation of allergen in the allergen provocation chamber. Unlike in skin tests, where allergen is applied uniformly, nasal challenges are based on the search of the allergen dose responsible for a verified allergic response, starting with lower dose of allergen, and increasing the dose several times until the response is reached. Several guidelines for the standardization of nasal allergen challenge tests have been published, the latest being the EAACl position paper in 2018, which are suggesting standardized application and outcome measures to prove positive response to allergen ${ }^{(620)}$. The conjunctival provocation test is also an option to prove allergic response in the target organ, as the eye is also often involved in the response to allergen ${ }^{(621)}$. The diagnostic efficacy of conjunctival provocation test was $89 \%$ in a trial where nasal allergen challenge with house dust mite was used as a standard reference, irrespective of whether the patient had ocular symptoms in natural exposure ${ }^{(622)}$.

Diagnosis of allergy in vitro is primarily based on the detection of serum specific IgE to airborne allergens, which may confirm SPT results and the presence of clinical symptoms. The other in vitro method is the basophil activation test. In vitro methods are not sensitive to patients' medication with antihistamines, have no risk of systemic reactions to allergens, and are more comfortable for the patients, as they do not result in side effects seen after SPT and after nasal or conjunctival challenges. This is very important for the diagnosis of allergy in children.

Serum specific IgE usually correlates with SPT results, and this correlation is stronger in patients with more severe symptoms and higher total IgE serum concentration. However, SPT is more sensitive than specific lgE in serum, and in most trials SPT is used as a standard reference, when testing diagnostic accuracy of slgE. Specific lgE assays which are commercially available are not completely compatible, and results using different assays may differ in sensitivity and specificity, as well as for the producer, and the allergener ${ }^{(623)}$. Different companies also produce diagnostic panels of specific lgE, to improve the speed and reduce the cost of the diagnosis if many allergens have to be tested. Such mixture panels for the most common airborne allergens have been used for years and their sensitivity and specificity may be moderate to high (70.8 and 90.7, respectively) ${ }^{(624)}$. This will probably be even more pronounced in the future, as current 
EU regulations are likely to produce a reduction in commercially available diagnostic allergens for SPT or ICT ${ }^{(625)}$. This will probably induce a switch to a broader panel of in vitro slgE diagnostics. Further development of in vivo molecular diagnostics is expected through the component resolved diagnosis (CRD). CRD allows us to identify single allergen molecular components responsible for the allergic reaction. It may help in distinguishing clinical symptoms resulting from allergen exposure from those which can be attributed to cross-reactivity. Total serum IgE is not of much value in diagnosing allergic rhinitis and but might be useful to discriminate patients that are eligible for anti-lgE treatment. In chronic urticaria and asthma total serum lgE levels and their change seems to predict the response to treatment with omalizumab ${ }^{(626,627)}$.

The basophil activation test (BAT) is a flow-cytometry based IgE functional test which detects basophil activation in the presence of allergen and specific lgE. The BAT measures the expression of activation markers on the basophil surface which are activated due to cross-linking of IgE antibodies bound to the high-affinity IgE receptor (FcERI). It imitates the conditions leading to type I hypersensitivity reaction. The use of the BAT in the diagnosis of allergy in CRS is limited.

To summarize, clinical history supported with the SPT will probably remain the golden standard of the upper airway allergy diagnosis, for some time, as it has acceptable sensitivity and specificity. The advances which are expected from the molecular in vitro diagnosis may change trend, due to improved technology which enables faster diagnosis on a broader panel of allergens. On the other hand, complications related to the availability of diagnostic allergen extracts or molecules may also shift diagnostic preference towards in vitro diagnostics. For the cases which are difficult to diagnose, nasal and conjunctival provocations test as in vivo procedures, and BAT as an in vitro procedure, may help in establishing the final diagnosis, however in the diagnosis of CRS these tests are seldom necessary.

\subsubsection{Assesment of smell}

\subsection{Rationale}

Smell loss has been reported in 60 to $80 \%$ of CRS patients ${ }^{(463,}$ ${ }^{467)}$. Olfactory impairment is significantly worse in patients with CRSwNP and smell loss is associated with higher eosinophilic inflammation in nasal polyp tissue (which was not shown in the CRSsNP phenotype) ${ }^{(628)}$. Besides nasal polyps, asthma, N-ERD, previous surgery and age have been shown to be predisposing factors for a greater smell loss ${ }^{(465,629)}$.

\subsection{Tests}

Smell impairment is a symptom which differentially affects CRS patients in terms of severity of smell disorder than is the case with rhinitis or other inflammatory sinonasal disease ${ }^{(470)}$. Incre- ased smell loss and less fluctuation is associated with the increasing severity of sinonasal disease. For that reason, it is important to evaluate sense of smell both subjectively and objectively in CRS patients. As they are commonly not fully aware of their impairment, or at least they are not able to estimate the severity of smell loss, the use of smell tests is recommended in order to objectively evaluate this disorder ${ }^{(450,630,631)}$. The tests may also be useful during the follow-up of the CRS patient, primarily to monitor the outcomes of anti-inflammatory or surgical treatment. Many olfactory tests are available but very few are well validated or used on a regular basis with a high number of observations over the last decades, especially in CRS patients ${ }^{(463)}$. The most widely used are the North American UPSIT ${ }^{(629)}$, its short version (SIT, B-SIT) and the European Sniffin'Sticks ${ }^{(632)}$. Numerous other tests exist ${ }^{(449)}$. Most tests used worldwide are pure identification tests (e.g. UPSIT) or composite tests where identification is complemented by a discrimination task and an olfactory threshold (e.g. Sniffin'Sticks extended version). They also differ significantly in time consumption (4-25 minutes) which is mainly dependent on the number of items and aspects tested ${ }^{(633)}$. For most of these tests, test-retest reliability is high and the cost of the test set more or less the same ${ }^{(634,635)}$. All identification tests have cultural biases, which is the main reason for the large number of different tests. There have been recent advances to overcome this with culturally unbiased, universally usable smell tests ${ }^{(636)}$. However, in the patient with symptoms suspicious of CRS who complains of smell impairment, the diagnostic approach to olfactory assessment should also include rigid endoscopy of the nose, which may differentiate between potential causes for inflammatory olfactory loss. Polyp presence or endonasal tumour detected at endoscopy may help explain the smell decline, or it may be only obstructive or inflammatory. However, due to interaction of sensory epithelia with inflammation in the nose, the exact aetiology and level of smell impairment may be mixed and not always entirely due to inflammation, mechanical obstruction or olfactory epithelial damage due to repeated inflammation. If the subjective and psychophysical level of smell loss does not correlate with the endoscopic finding, it may raise suspicion of a sinonasal or skull base tumour which was not seen on endoscopy. Such suspicion should lead to CT and MRI imaging of the skull base and brain and if there is an olfactory complaint, regardless of the endoscopy, there needs to be psychophysical testing. Imaging with MRI or CT may exclude olfactory and pituitary tumours and aneurysms and may detect reduced olfactory bulb volume and reduced olfactory sulcus depth in congenital smell loss ${ }^{(637-639)}$. Regarding parosmia, different studies show morpho$\operatorname{logic}^{(640,641)}$ and functional differences on MRI and functional MRI scans $^{(642)}$. However, there is no tool yet available to reliably assess the presence of olfactory distortions in a single patient apart from some questionnaires ${ }^{(643)}$.

Smell tests are classified into threshold (liminar) and supra- 
threshold (supraliminar) tests ${ }^{(644)}$. In threshold tests the subjects detect the minimum concentration of the tested odorant. Such tests most commonly use phenyl ethyl alcohol and n-butanol as the odorant substance but can be done for any substance. The test time in detection threshold test for a person with normal smell is about 15 minutes and tends to be lower for subjects with impaired smell. The test is done starting with lower concentrations with a stepwise increase until the minimally perceived dose is reached, which has to be confirmed several times. A less common type of threshold test is the recognition threshold test, where perception threshold is combined with recognition of odour. The olfactory discrimination test includes examination with three odour challenges, where two are the same, and the third is different ("find the odd one" principle). The test subject has to recognize the different odour in the set. Threshold tests can be useful in patients with CRS, where the patients with higher threshold concentration may still be performing well in the suprathreshold tests. In composite tests such as the Sniffin'Sticks, a pattern of test results with low threshold but normal suprathreshold scores have been repeatedly identified as quite typical for CRS-related olfactory impairment ${ }^{(464,645)}$.

Suprathreshold tests are used much more commonly than threshold and they are based on identification of different odours in much higher concentrations then the threshold.

These tests are usually developed as a panel of different odours, which stimulate either olfactory or trigeminal response. Panels of odours contain from 8-40 odours and in some tests they are applied to both nostrils. Time consumption depends primarily on the number of odours in the panel, and varies from 4 to 30 minutes. Tested subjects recognize the odour through a forced choice response ${ }^{(646)}$. The examiner tests the subject with a sequence of known odour stimuli which are presented to the tested subject, who has to recognize the right odour between several descriptors offered as response. The examiner usually offers 3-4 different distractors as a response to the presented odour, and the subject has to choose a single response. The smell test has to be culturally adjusted, which means that odours in the panel tested and offered responses have to be recognized and familiar to the vast majority of the population that will be tested. Only a few of the smell tests integrate liminar and supraliminar tests (Sniffin'Sticks full version, Connecticut Chemosensory Clinical Research Center Test, Barcelona Smell Test-24, T\&T olfactometer) ${ }^{(647-649)}$.

Several trials have tested the compatibility or the correlation of the results of the different smell tests in the same individuals (normal and hyposmic). These studies confirm compatibility of the results with different tests. The commonly used European tests managed to differentiate tested individuals into anosmic, hyposmic and normosmic categories, with some discrepancies within the hyposmic group, however, all the tests were able to differentiate anosmic from normosmic well ${ }^{(650)}$.
Compared to one or two decades ago, there are many well validated and especially widely used psychophysical tests available ${ }^{(651,652)}$. It is generally admitted that the value of an individual test score obtained for a single patient is more meaningful the better the test used was. It is thus important to test whenever possible with standardized psychophysical tests and use a sufficient amount of odours.

A lower number of test odours may reduce the cost and time spent. However, it introduces, based on the distribution of the normative data, a certain degree of unreliability. Such short tests should be used for screening purposes only ${ }^{(653,654)}$.

Identification tests should be exclusively used with a previously described forced choice paradigm for two reasons: hyposmic subjects perform better and it potentially gives hints about malingering or aggravation.

Retronasal testing is combining the test of smell and taste, which is close to testing flavour. Such tests apply test substances transorally, or on the tongue. Retronasal olfaction is often better preserved in CRS with polyps compared to orthonasal olfaction ${ }^{(476)}$. Using retronasal olfaction may give supplementary information for a possible inflammatory related olfactory impairment. Although many reports have been published, as yet there is no commercially available retronasal test available. However, there has been a recent European attempt for a selfbuilt retronasal test ${ }^{(655)}$.

Objective measurement of olfactory function can be done with olfactory-event related potentials. This diagnostic tool is able to confirm the absence of olfactory specific brain responses to a given olfactory stimulus and thus suspected anosmia. However, OERPs are not part of the routine work-up and rather time-consuming. Their use is largely restricted to research and medical expertise.

The EPOS2020 steering group were unclear as to whether it was essential to do a smell test at initial presentation to ENT/ secondary care with highly suggestive symptoms of CRS and abnormal mucosa on endoscopy (Figure 5.3.31.).

It was not essential to do a smell test at initial presentation to ENT / secondary care with highly suggestive symptoms of CRS and normal mucosa on endoscopy (Figure 5.3.32.).

The EPOS2020 steering group were unclear as to whether it was essential to do a smell test in CRS after failure of appropriate medical or surgical treatment in ENT / secondary care with continued symptoms and abnormal mucosa on endoscopy (Figure 5.3.33.).

\subsubsection{Objective measures of nasal airflow and patency}

\subsection{Rationale}

Chronic rhinosinusitis is characterized with chronic nasal obstruction and HRQOL impairment in CRS is also often related to 
sleep disturbance and chronic fatigue ${ }^{(656)}$, which may be related to sleep disorders. On the other hand, other upper airways disorders have symptoms which overlap with CRS, such as enlarged adenoids (nasal obstruction, smell disorders) and laryngopharyngeal reflux (postnasal drip, chronic cough). In nasal obstruction, sleep disorders and laryngopharyngeal reflux, investigation of the upper airways may help distinguish each disorder and their comorbidities.

\subsection{Evaluation of nasal patency}

Besides nasal endoscopy (see 5.3.5.2.) nasal patency may be objectively evaluated with peak nasal inspiratory flowmetry (PNIF), (active anterior) rhinomanometry (AAR), and acoustic rhinometry (AR). All of these methods have been standardized for years and their outcomes related to subjective sensation of nasal patency ${ }^{(450,657-660)}$. Newer methods are computational fluid dynamics ${ }^{(661)}$ that at the moment are mainly used for research purposes $^{(661-664)}$

The different tests are extensively discussed in the European position paper on diagnostic tools in rhinology $\mathrm{y}^{(450)}$.

The simplest objective method for nasal patency measurement is peak nasal inspiratory flowmetry ${ }^{(665-668)}$. It measures simultaneously one or both nostrils during maximal inspiration and the best of the three attempts is taken as the objective measure of nasal patency. This measure correlates best with subjective nasal patency ${ }^{(669,670)}$ and QOL after FESS ${ }^{(645)}$. PNIF and AAR have a similar and significant power to discriminate pathologic from healthy subjects ${ }^{(671,672)}$. PNIF also correlates well with $A R^{(673)}$. Besides that, this method has the advantage of being the cheapest and the most functional, in the sense that it measures real life exposure challenges, like exercise ${ }^{(674)}$, aspirin ${ }^{(675-677)}$ or allergen challenge ${ }^{(620)}$. The weakness of PNIF method is that it does not locate the narrowest point of nasal obstruction (like acoustic rhinometry). It is also influenced by collapsibility of the nasal valve, which may occur only in maximal airflow.

Objective airway testing has been used to evaluate nasal patency in patients with CRS in the diagnostic phase ${ }^{(678,679)}$ as in the evaluation of treatment ${ }^{(670,680-682)}$.

\subsubsection{Histopathology tests}

\subsection{Biopsies}

\subsection{Rationale}

To confirm diagnosis, to assist in endotyping of inflammatory disease and for research purposes.

To provide tissue uncrushed and suitable for analysis.

To explore the differential diagnosis (inflammation, respiratory epithelial adenomatoid hamartoma (REAH), infection, granuloma / vasculitis, tumour).

To confirm potential aetiologic or pathogenic mechanisms (type
Figure 5.3.31. Delphi: Is it essential to do a smell test at initial presentation to ENT/ secondary care with highly suggestive symptoms of CRS and abnormal mucosa on endoscopy?

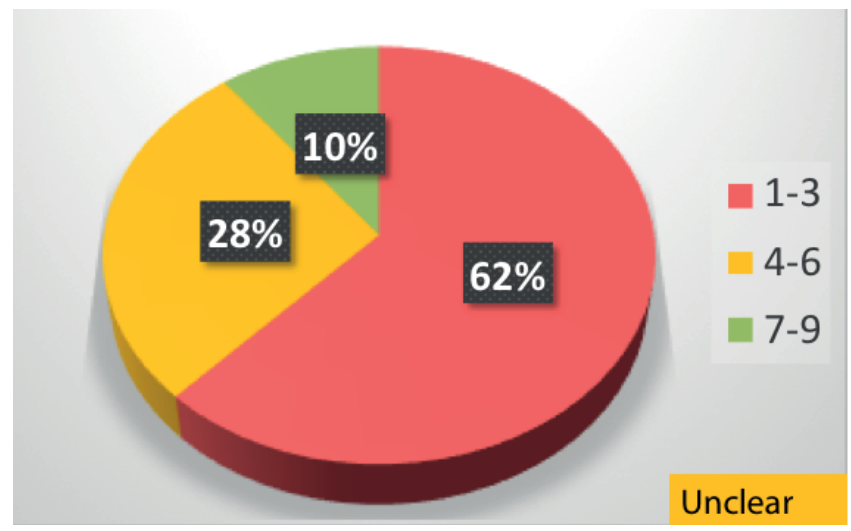

Figure 5.3.32. Delphi: Is it essential to do a smell test at initial presentation to ENT / secondary care in patients with highly suggestive symptoms of CRS but normal endoscopy?

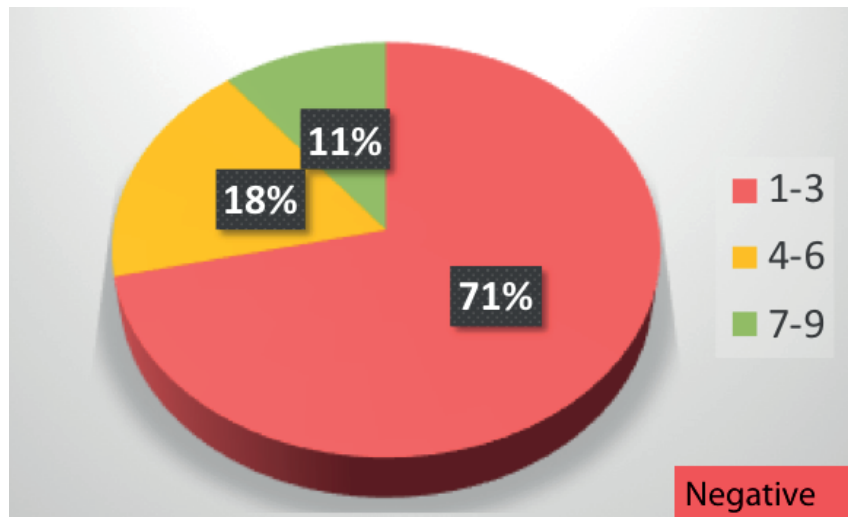

Figure 5.3.33. Delphi: Is it essential to do a smell test in CRS after failure of appropriate medical or surgical treatment in ENT / secondary care with continued symptoms and abnormal mucosa on endoscopy?

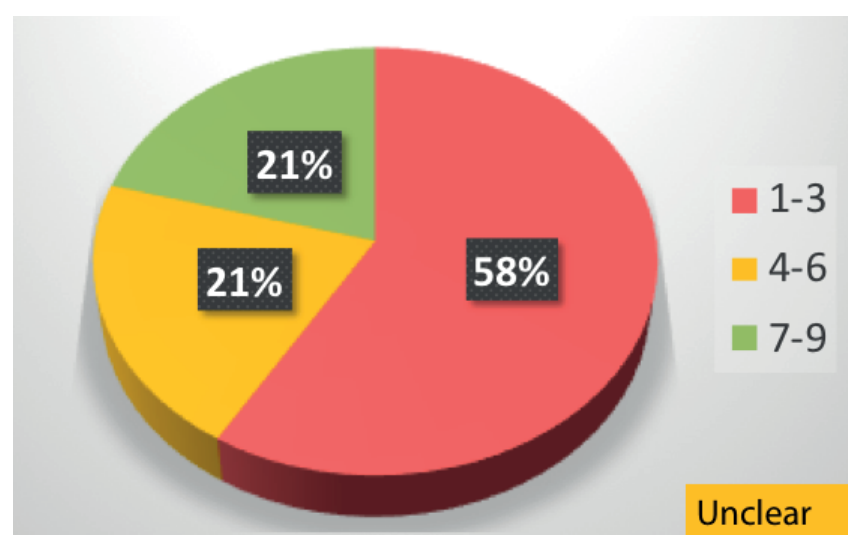


and severity of inflammation, cellular composition e.g. eosinophils, bacterial and fungal elements.

To determine nature of relationship e.g. invasive or non-invasive fungus.

To direct potential therapies e.g. biologics.

\subsection{Technique}

Nasal biopsies can be performed in an outpatient clinic under local anaesthesia or in the operating theatre ${ }^{(450)}$. The choice depends on a number of factors including the reason for performing the biopsy and the size of the biopsy required. For research purposes, the biopsy must be easily performed, painless and with a minimal complication rate. For clinical diagnosis the need for sufficient sample size has to be balanced with the burden for the patient. It is important that the biopsy is not crushed either by surgeon or scrub nurse. This can be achieved with specific Fokkens forceps ${ }^{(683)}$ or fenestrated punch forceps ${ }^{(684)}$ or in the operating theatre with scissors ${ }^{(685)}$. Small biopsies for research purposes can be taken from the inferior turbinate after local anaesthesia ${ }^{(683,684)}$. For light microscopic evaluation, the biopsy specimens can be embedded in Tissue-Tek II OCT compound in a gelatin capsule and frozen immediately. It has been shown that biopsies can safely be taken from the olfactory mucosa without affecting nasal function or the sense of smell(686). With the advent of microdebrider powered instrumentation, there were initial concerns that tissue would be unsuitable for histological analysis. However, Zweig et al. showed in a small prospective blinded study (15 cases covering a range of pathologies) that there was no significant loss of morphologic features in tissue passed through the debrider ${ }^{(687) .}$

In rhinosinusitis, where clinical diagnosis is based on well established criteria in specialist care (defined symptoms + endoscopy and/or imaging), histopathological confirmation of inflammation in surgically removed sinus mucosa is very high though there are few high quality studies. Two retrospective studies from the UK published 20 years ago evaluated histopathological findings from CRSwNP ( $\mathrm{n}=344$ and 2021 respectively) ${ }^{(688,689)}$ and confirmed the diagnosis in $95 \%$ and $98,5 \%$.

Misdiagnoses included inverted papillomas, granulomatous disease, and malignancy in $<1 \%$. Diamantopoulos et al recommended routine histology in all cases on the grounds that the medicolegal costs outweighed the service costs whilst Kale et al. concluded only first-time bilateral polyps and any unilateral polyps should undergo histology but that by implication, subsequent routine histology was not required ${ }^{(688,689)}$.

A similar retrospective cohort from the USA analyzed 380 consecutive patients (unilateral and microdebrider cases excluded), comprising 180 CRSsNP and 200 of CRSwNP(690). Two cases of apparent CRSsNP had sinonasal sarcoidosis and in the CRSwNP group, there were five inverted papillomas, two carcinomas, one invasive fungal rhinosinusitis, and one sarcoidosis. Thus diagnostic discrepancy was found in $1.1 \%$ of CRSsNP and $4.5 \%$ of CRSWNP and routine examination of nasal polyp tissue was recommended.

However, two more recent studies [from Canada (prospective) and the Netherlands (retrospective)] found only $<0.5 \%$ had an unexpected histopathological diagnosis after routine endoscopic sinus surgery for $\mathrm{CRS}^{(691,692)}$. Yeh et al. calculated that $217 \mathrm{ca}$ ses had to be screened to discover one unexpected pathology, the cost of which was $>\$ 19,000$. Van der Boer et al. considered material from 1944 operations of which only two yielded an unexpected diagnosis (inverted papilloma in each case) and questioned the need for routine histology.

A systematic review was conducted by Wong et al. to assess the usefulness of routine histopathology in CRSwNP which included six studies of which only Yeh et al. was prospective. Of the 3772 patients, 3751 had a pre-operative clinical and postoperative pathological diagnosis of inflammatory nasal polyps with an agreement proportion of $99.44 \%$. Overall there were 18 unexpected benign and three unexpected malignant diagnoses identified ( 0.48 and $0.08 \%$ respectively). The number needed to screen was 210 and 1258, respectively, and the cost to pick up one unexpected benign diagnosis was $\$ 14,557$ and $\$ 87,204$ for one unexpected malignancy. Despite this low yield, the authors concluded that there was no compelling evidence to cease routine histological examination ${ }^{(693)}$.

Therefore, should representative tissue removed at surgery in apparently uncomplicated CRS be subjected to biopsy and histopathology or only in cases that raise suspicions such as unilateral disease, friable mucosa, crusting, serosanguinous discharge?

Evidence from the literature is not strong but in cases of bilateral CRS with no other concerning clinical features, clinicians should exercise judgment in submitting polyp specimens for histopathology rather than routinely sending all polyps every time. However, there are sound medicolegal reasons to confirm the initial diagnosis in all cases and tissue may be increasingly important for endotyping with its therapeutic implications. For example, to assess eosinophils in tissue especially when considering biologics.

Subphenotyping and endotyping by degree of eosinophilic infiltration and other histological criteria is gaining traction ${ }^{\text {(694-697) }}$ and has led to the development of institutional protocols for sampling, storing and processing samples in close collaboration with histopathologists. Eosinophilic CRS (eCRS) requires quantification of the numbers of eosinophils ie number/high powered field (HPF) which varies in the literature (8-12/hpf). Sampling has been described as 'approximately the three most dense collections of eosinophils in the stroma'(694) ${ }^{\prime}$ 'the mean number of eosinophils in the mucosa counted at HPF ( $\times 400)$ in the three densest areas with cellular infiltrate beneath the epithelial surface ${ }^{\prime \prime(698)}$. Further stratification may be made between 
those with 10-100 eosinophils per HPF in two or more areas and those with $>100$ eosinophils per HPF in two or more areas( ${ }^{(694)}$. In a systematic review of 11 articles reporting high tissue eosinophilia associated with recurrence, a cut-off value of $>55$ eosinophils / HPF showed the highest sensitivity $(0.87 ; 95 \% \mathrm{Cl}, 0.82-0.91)$, specificity $(0.97 ; 95 \% \mathrm{Cl}, 0.93-0.99)$, and Diagnostic Odds Ratio $(232.7 ; 95 \% \mathrm{Cl}, 91.0-595.1)^{(699)}$. This was not affected by other factors considered in a meta-regression analysis and was, therefore, proposed as a useful tool for the diagnosis of eCRS.

A systematic review of 71 articles considering the histopathology of human sinus mucosa collected in vivo in patients with CRS was undertaken to determine if there were specific histopathological markers of inflammation that help to distinguish between the various subgroups of CRS and if such markers could serve as prognostic indicators ${ }^{(700)}$. Techniques used for the analysis included light microscopy, immunohistochemistry, in-situ hybridization, reverse-transcription polymerase chain reaction, immunoassays, Western blot, and flow cytometry. Twenty-eight considered histopathological findings that might be prognostic indicators in CRS and forty-four evaluated inflammatory biomarkers in the various subgroups of CRS. The amount of eosinophilic infiltration and the overall intensity of the inflammatory response were closely related to the prognosis and severity of disease, information that is quantifiable by light microscopy alone and does not require any specific immunohistochemistry techniques. There were discrepancies in the methodologies and results of the immunohistochemistry so a meta-analysis of the inflammatory markers was not possible, but the authors concluded that future studies were needed to determine their role as indicators of prognosis or targets for tailored therapy.

Close collaboration between surgeon, radiologist and histopathologist underpins optimal tissue sampling, especially when there is co-existent pathology in CRSwNP e.g. inverted papilloma or respiratory epithelial adenomatoid hamartomas (REAH) (701, 702).

A range of specific staining techniques, immunohistochemistry and confocal scanning laser microscopy (CSLM) coupled with a fluorescence in-situ hybridization (FISH) probe can assist in more refined analyses ${ }^{(697,703,704)}$. For research purposes also more advanced techniques like (RT)PCR an micro-arrays are be used ${ }^{(282,}$ 705, 706).

The distinction between invasive and non-invasive forms of fungal rhinosinusitis depends on the presence or absence of fungal hyphae in the mucosa (section 8.6). Special fungal stains of the secretions may be required e.g. Gomori methenamine silver combined with the presence of Charcot Leyden crystals and marked eosinophilia in AFRS ${ }^{(707)}$. In acute invasive fungal forms frozen section biopsy can assist confirmation, with a sensitivity of $87.5 \%$ and $100 \%$ specificity ${ }^{(708)}$. Granulomatous and chronic invasive fungal disease should be confirmed by histopathology but PCR may be helpful if fungal elements cannot be detected by histopathology ${ }^{(709)}$. A recent study involving 76 patients with suspected fungal rhinosinusitis, including the chronic invasive forms, showed that histopathology in these cases had a sensitivity of $<20 \%$, whereas PCR had a sensitivity of $35 \%$. However, fungal disease was proven in less than half of suspicious cases, and the result was calculated after considering all the diagnostic procedures (staining, culture, histopathology and PCR)(709). The EPOS2020 steering group did not consider it essential to do histopathology / biopsy at initial presentation to ENT / secondary care with highly suggestive symptoms of CRS irrespective of whether the mucosa was abnormal or normal on endoscopy but their responses were unclear regarding whether this was essential after failure of appropriate medical or surgical treatment in ENT / secondary care with continued symptoms and abnormal mucosa on endoscopy (Figures 5.3.34., 5.3.35., 5.3.36.).

\subsubsection{Other nasal sampling}

\subsubsection{2.1 . Rationale}

In addition to sinonasal tissue, nasal secretions and cells can also provide diagnostic and therapeutic information. The most common techniques are nasal lavage fluid, nasal suctioning, insertion of nasal packs, nasal brushing or scraping, the choice being determined by whether inflammatory markers, nasal cells for cytology or culture, or ciliary function are required.

\subsection{Techniques}

Nasal blown secretions are the easiest method to collect nasal secretions by simply blowing the nose and collecting the mucus in sterile containers directly or by letting the participants blow into tissues ${ }^{(710)}$. This method is useful in children, but the sample is often small.

In nasal lavage, fluid is introduced into the nose for a certain amount of time and then recollected, with its associated nasal secretions. It is easy to perform and well tolerated. Usually $2-10 \mathrm{~mL}$ of saline, $0.9 \%$ sodium chloride $(\mathrm{NaCl})$ at body temperature, is instilled with a syringe and the patient is asked not to swallow the fluid while the head is reclined. After approximately 10 seconds $^{(711-714)}$ the head is bent forward and the lavage fluid collected in sterile containers. Smaller volumes of iso- to mildly hypertonic saline (0.9-1.8\%) can be instilled with a nasal spray $^{(712)}$. Inflating the cuff of a paediatric tracheostomy tube into the nostril has been described to reduce loss of lavage fluid $^{(715)}$.

Sterile nasal suctioning devices with a reservoir are also available The mucus can be suctioned out and directly frozen at -80 to $-90^{\circ} \mathrm{C}$ before further processing or a known quantity of phosphate buffered saline (PBS) or similar agents can be applied which stop chemical activity such as proteolysis in the mucus ${ }^{(716)}$. The 
Figure 5.3.34. Delphi: Is it essential to do histopathology/biopsy at initial presentation in ENT/secondary care with highly suggestive symptoms of CRS and abnormal endoscopy?

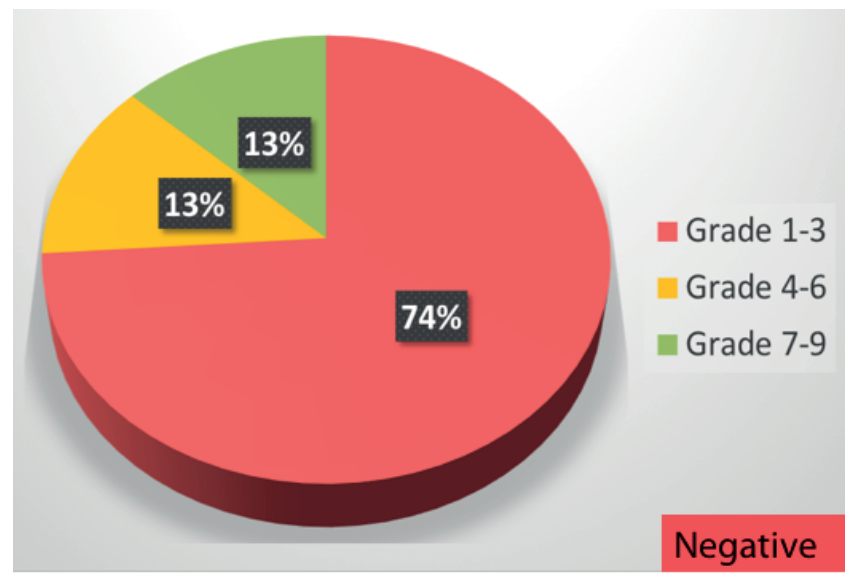

Figure 5.3.35. Delphi: Is it essential to do histopathology/biopsy at initial presentation in ENT/secondary care in patients with highly suggestive symptoms of CRS but normal endoscopy?

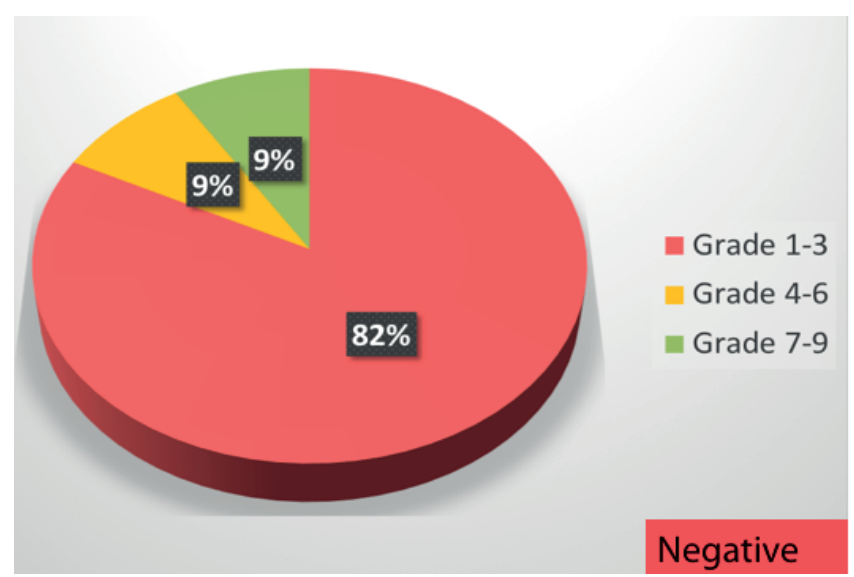

Figure 5.3.36. Delphi: Is it essential to do histopathology/biopsy in CRS after failure of appropriate medical or surgical treatment in ENT/secondary care with continued symptoms and abnormal mucosa on endoscopy?

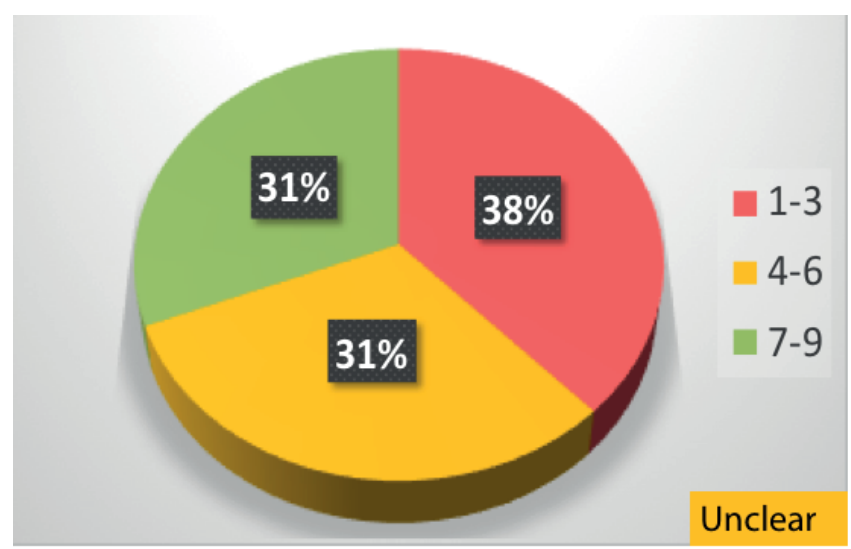

technique is well tolerated, has the advantage of minimal stimulation and/or trauma to the mucosa and can be done under endoscopic control ${ }^{(717)}$.

Nasal packing can be used to soak up nasal secretions from different areas of the nasal cavity (e.g. inferior or middle meatus) being weighed before and after insertion into the nose. The packs are left in-situ for five minutes and are then placed in a conical tube, washed in $\mathrm{NaCl}$ and mechanically squeezed out (e.g. with a syringe), then washed again and centrifuged at $1500 \mathrm{~g}$ for $10 \mathrm{~min}$ at $4^{\circ} \mathrm{C}$ to obtain all secretions $\mathrm{s}^{(717)}$. An advantage of this method is clear discrimination of sides as compared to lavage techniques but the technique is uncomfortable and irritates the mucosa. Another technique uses reticulated polyurethane foam packs (at 60 and 110 pores per inch (PPI)) that may absorb secretions better than standard packs with less stimulation of the mucosa ${ }^{(718)}$.

Nasal brushing can be performed with a soft sterile brush (bronchial brush) in the middle or inferior meatus and then placed in tubes containing an appropriate growth medium. The material is dislodged by oscillation and the contents centrifuged at $400 \mathrm{~g}$ for 10 minutes. A specimen of cells is obtained and the supernatant can be discarded or the mucus it contains may be used for further analysis ${ }^{(719)}$. Nasal scraping may also be obtained using a small disposable plastic cup (Rhinoprobe) which is more comfortable for the patient and more accurate than swabbing ${ }^{(720)}$. The material can be spread onto a slide, fixed and airdried followed by staining, usually with Wright-Giesma or Cresol-Red-O. Specimens obtained by brushing or with the Rhinoprobe can also be used to measure ciliary beat frequency or perform electron microscopy of particular importance in the diagnosis of primary ciliary dyskinesia (see 5.3.5.9). Several abnormalities may be observed in the ciliary ultrastructure including total or partial absence of dynein arms, aberrant organization of the dynein arms and/or disorientation but may also be observed in some cases of secondary ciliary disease. Therefore, EM evaluation may support diagnosis of PCD but is not $100 \%$ sensitive or specific. The EPOS2020 steering group did not consider it essential to do cytology (either brushings or lavage) at initial presentation to ENT/ secondary care with highly suggestive symptoms of CRS irrespective of whether the mucosa was abnormal or normal on endoscopy but their responses were unclear regarding whether this was essential after failure of appropriate medical or surgical treatment in ENT/secondary care with continued symptoms and abnormal mucosa on endoscopy (Figure 5.3.37., 5.3.38., 5.3.39.).

\subsubsection{Blood tests}

\subsection{Rationale}

Until recently most blood tests in patients with CRS are to diagnose immunodeficiences and vasculitic disease with sinonasal manifestations. However, recently the options to treat with 
biologicals has put more emphasis on markers of type 2 disease although until today we are not aware of biomarkers that can predict response to biologicals in CRS ${ }^{(721)}$.

Immunodeficiencies can be the cause or a contributing factor in CRS (see 5.1.2.4. Immune deficiencies and 8.2 Immunodeficiencies and their role in CRS). The testing of immune function in all patients who present with CRS is almost certainly unwarranted as it is likely to produce more false positive results than true positives. However, it is recommended that the clinical features listed below are used to identify those patients who warrant some form of immune testing for testing.

The most common immunoglobulin deficiency in the general population is IgA deficiency with a prevalence between 1:173 and 1:3024 ${ }^{(722)}$. Most patients are asymptomatic, but a deficiency of $\lg$ A would appear to predispose patients to rhinosinusitis and allergies ${ }^{(723)}$.

CRS secondary to hypogammaglobulinaemia may present to a rhinologist in a manner identical to idiopathic CRS. There are some clinical features that may elevate suspicion: association with lower respiratory tract infections (pneumonia, particularly if recurrent, or bronchiectasis) and recalcitrance to standard treatments (and particularly rapid recurrence of symptoms after stopping antibiotics).

\subsection{Tests}

\subsubsection{1 . Markers of type 2 disease}

The main biomarkers used at the moment to define type 2 disease are eosinophils, IgE levels, and in some more specialized centres periostin. Other biomarkers are currently under investigation and may provider further guidance in the future.

There is quite some evidence showing that eosinophils are a reasonable surrogate marker for type 2 disease, and that blood eosinophils are a reasonable biomarker to predict eosinophilic CRS with or without nasal polyps ${ }^{(695,724,725)}$. Blood eosinophil counts were found to be significantly correlated with the LundMackay CT and Lund-Kennedy endoscopic scores in patients with nasal polyps $(r=0.353, p=0.010$ and $r=0.444, p=0.001$, respectively) $)^{(724)}$.

Receiver operating characteristic curve analysis predicted high tissue eosinophilia at blood eosinophil levels above 0.24109/L [sensitivity $70.9 \%$, specificity $78.4 \%$, area under the curve (AUC): $0.792, \mathrm{p}<0.01]$. eCRS was predicted at eosinophil above $4.27 \%$ of total WCC (sensitivity $64.1 \%$, specificity $88.5 \%$, AUC 0.797 ; $\mathrm{p}<0.01$; positive predictive value $89.2 \%$, negative predictive value $62.4 \%$, positive likelihood ratio 5.57 , and diagnostic odds ratio 13.71)(695). A cut-off point of blood eosinophils $>0.24$ 109/L or eosinophil ratio $>4.27 \%$ of total WCC was proposed by Ho et al. ${ }^{(695)}$.

The EPOS2020 steering group were unclear as to whether it was essential to evaluate blood eosinophilia either at initial presen-
Figure 5.3.37. Delphi: Is it essential to do cytology brushings or lavage at initial presentation in ENT / secondary care with highly suggestive symptoms of CRS and abnormal endoscopy?

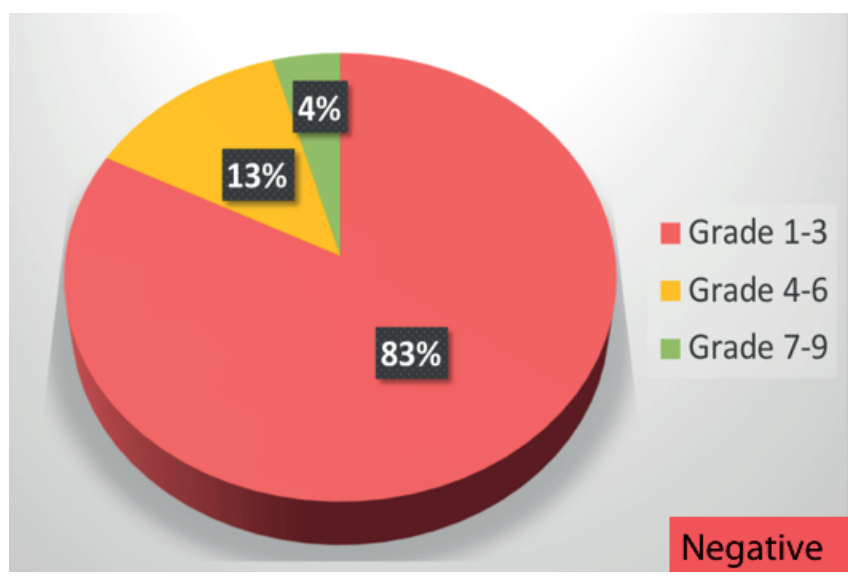

Figure 5.3.38. Delphi: Is it essential to do cytology brushings or lavage at initial presentation in ENT / secondary care in patients with highly suggestive symptoms of CRS but normal endoscopy?

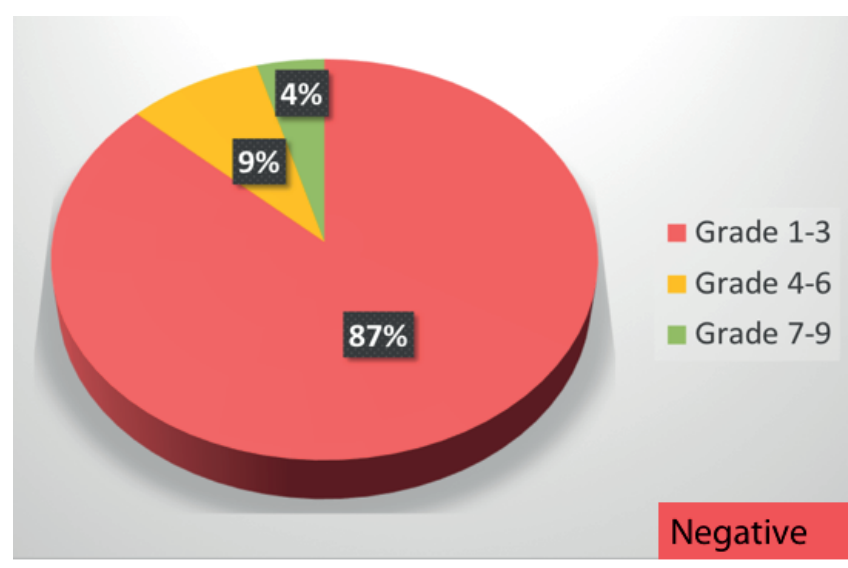

Figure 5.3.39. Delphi: Is it essential to do cytology brushings or lavage in CRS after failure of appropriate medical or surgical treatment in ENT / secondary care with continued symptoms and abnormal mucosa?

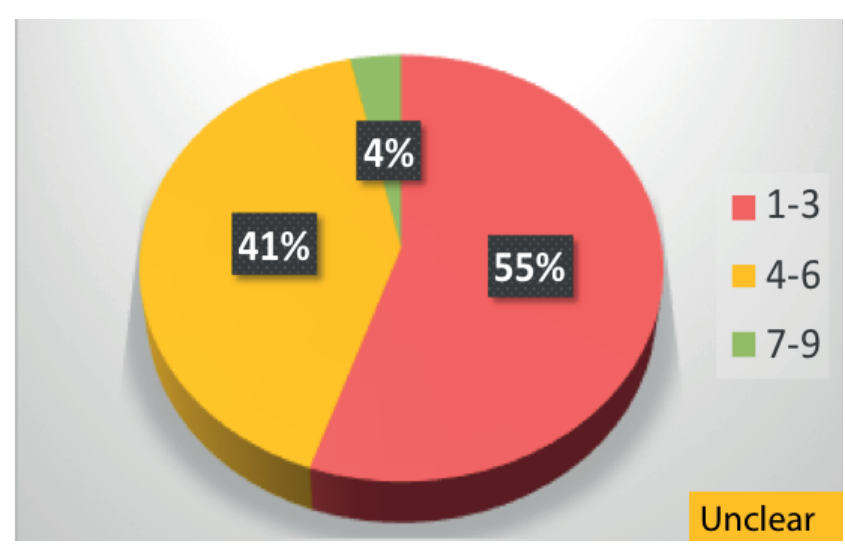


tation to ENT / secondary care with highly suggestive symptoms of CRS irrespective of whether the mucosa was abnormal or normal on endoscopy or after failure of appropriate medical or surgical treatment in ENT / secondary care with continued symptoms and abnormal mucosa on endoscopy (Figure 5.3.40., 5.3.41., 5.3.42.).

Anti-IgE treatment has been shown to be effective in the treatment of CRSwNP (see 6.1.14). There is an association between increased levels of total $\lg \mathrm{E}$, specific $\lg \mathrm{E}$, and eosinophilic inflammation in NPs, which may be of relevance in the pathophysiology of nasal polyposis ${ }^{(726)}$. Increased serum IgE has been described in a subset of patients with N-ERD ${ }^{(727)}$. A serum $\operatorname{lgE}$ cut of value of $96 \mathrm{kU} / \mathrm{L}$ was shown to be a bad predictor of CRSwNP (44\% in CRSwNP group versus 28\% in CRSsNP and 30\% in controls) $)^{(728)}$.

The EPOS2020 steering group were unclear as to whether it was essential to measure total $\lg \mathrm{E}$ either at initial presentation to ENT / secondary care with highly suggestive symptoms of CRS irrespective of whether the mucosa was abnormal or normal on endoscopy or after failure of appropriate medical or surgical treatment in ENT / secondary care with continued symptoms and abnormal mucosa on endoscopy (Figure 5.3.43., 5.3.44., 5.3.45.). Periostin is both an extracellular matrix protein and matricellular protein that is capable of activating cells by linking integrin molecules to cell receptors, promoting tissue remodeling. It may be an important biomarker for type 2 immunity and airway allergic inflammation exacerbations ${ }^{(729)}$. It has been established that periostin is a downstream molecule of interleukin (IL)-13, and that asthmatic patients with high serum periostin react more favourable to anti-IL-13 antibodies (lebrikizumab) and anti-lgE antibodies (omalizumab) ${ }^{(730)}$. Methylprednisolone and omalizumab significantly reduced serum periostin levels in CRSwNP patients ${ }^{(731)}$.

Serum periostin was found to be the sole biomarker among those tested for detecting the presence of nasal polyps and it significantly correlated with Lund-Mackay score in patients with chronic rhinosinusitis and asthma ${ }^{(732)}$. Mean serum periostin levels were markedly elevated in patients with CRS versus controls. In addition, mean periostin levels were significantly higher in CRS patients with nasal polyps as compared with those without polyps ${ }^{(728,733)}$. In the study of Maxfield et al. periostin levels did not correlate with sex, smoking history, N-ERD, oral steroid use within 1 month of surgery, use of topical steroid nasal spray or number of prior sinus operations ${ }^{(733)}$.

\subsection{Immunoglobulin}

It is not clear how much of the immunological testing should be undertaken by the rhinologist before referral to an immunologist, and this decision may reflect the individual knowledge and expertise of the rhinologist. For CRS patients suspected of having humoral immunodeficiency because of the charac-
Figure 5.3.40. Delphi: Is it essential to evaluate blood eosinophilia at initial presentation to ENT/secondary care with highly suggestive symptoms of CRS and abnormal mucosa on endoscopy?

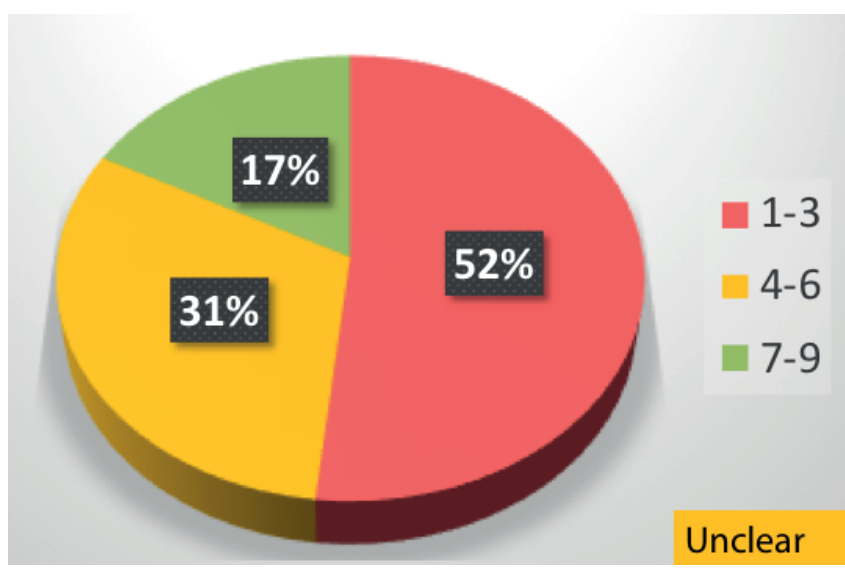

Figure 5.3.41. Delphi: Is it essential to evaluate blood eosinophilia at initial presentation to ENT/secondary care in patients with highly suggestive symptoms of CRS but normal endoscopy?

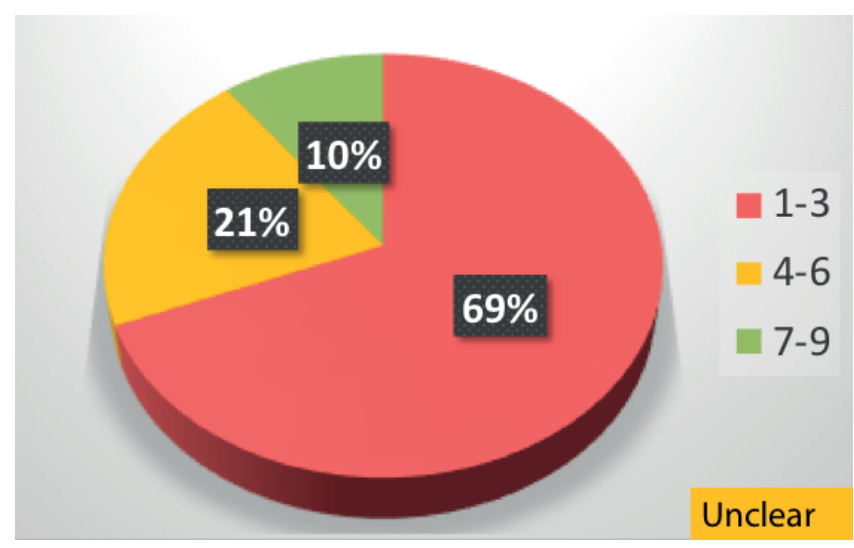

Figure 5.3.42. Delphi: Is it essential to evaluate blood eosinophilia in CRS after failure of appropriate medical or surgical treatment in ENT/secondary care with continued symptoms and abnormal mucosa on endoscopy?

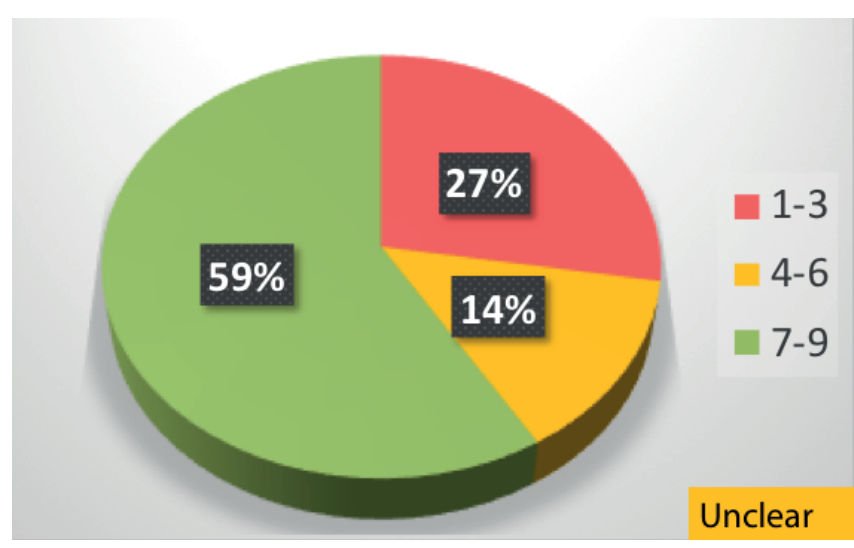


teristics of their presentation or their response to treatment, measurement of serum immunoglobulin levels ( $\lg A$ and $\lg G$ ) is the key investigation. The best approach for confirming a diagnosis of an antibody-deficiency disorder is the measurement of serum-specific antibody titers (usually lgG) in response to vaccine antigens. This approach involves immunizing a patient with protein antigens (e.g., tetanus toxoid) and polysaccharide antigens (e.g., pneumococcus) and assessing pre- and postimmunization antibody levels ${ }^{(734)}$. If the levels are normal, but the suspicion of humoral immunodeficiency is high, referral to a clinical immunologist is optimal. Further studies, such as flow cytometry can then be organized by an expert in the interpretation of these tests.

The EPOS2020 steering group did not consider it essential to do an objective test of immunodeficiency at initial presentation to ENT/ secondary care with highly suggestive symptoms of CRS irrespective of whether the mucosa was abnormal or normal on endoscopy.

Their responses were unclear regarding whether this was essential after failure of appropriate medical or surgical treatment in ENT/secondary care with continued symptoms and abnormal mucosa on endoscopy (Figure 5.3.46., 5.3.47., 5.3.48.).

\subsubsection{2 .3. Testing for vasculitis}

Vasculitic diseases with sinonasal manifestations usually but not always involve multiple organs. Rhinitis, sinusitis, septal perforation and epistaxis are the most common nasal features related to these diseases. There are two relatively common vasculitides in the sinunasal tract:

Granulomatosis with polyangiitis (GPA, formerly Wegener's granulomatosis) and Eosinophilic granulomatosis with polyangiitis (EGPA, formerly Churg-Strauss syndrome). Patients with granulomatosis with polyangiitis (GPA) affecting the sinonasal cavity usually have persistent bleeding, crusting and obstruction, and may develop a septal perforation. Sometimes the manifestations of this vasculitic condition are limited to the sinonasal cavity. GPA is strongly associated with anti-neutrophil cytoplasmic antibodies (ANCA), and the more widespread and severe the presentation, the more likely the serum ANCA is to be positive ${ }^{(735)}$. In cases limited to the sinonasal tract the ANCA can be negative, making the diagnosis challenging. The combination of clinical features, positive ANCA serology, necrotizing vasculitis and granulomatous inflammation on biopsy establish the diagnosis ${ }^{(736)}$. However, nasal biopsies often do not have enough features specific to this condition to confirm the diagnosis, and so biopsies from other involved organs may be required. Eosinophilic granulomatosis with polyangiitis (EGPA, formerly Churg-Strauss syndrome) is a necrotizing vasculitis of small and medium-sized vessels. Among a wide spectrum of non-pulmonary symptoms, nasal and sinonasal features are common and part of the diagnostic criteria for EGPA ${ }^{(737)}$. This
Figure 5.3.43. Delphi: Is it essential to measure total IgE at initial presentation to ENT/secondary care with highly suggestive symptoms of CRS and abnormal mucosa on endoscopy?

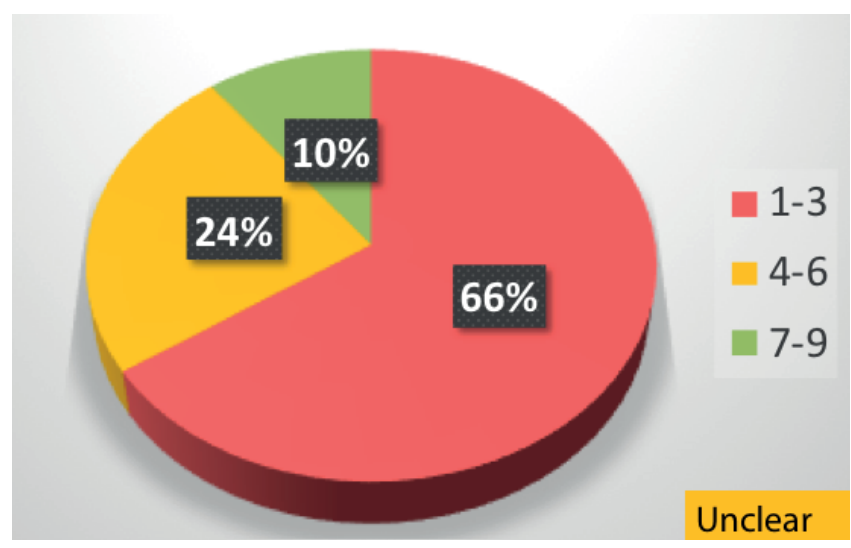

Figure 5.3.44. Delphi: Is it essential to measure total lgE at initial presentation to ENT/secondary care in patients with highly suggestive symptoms of CRS but normal endoscopy?

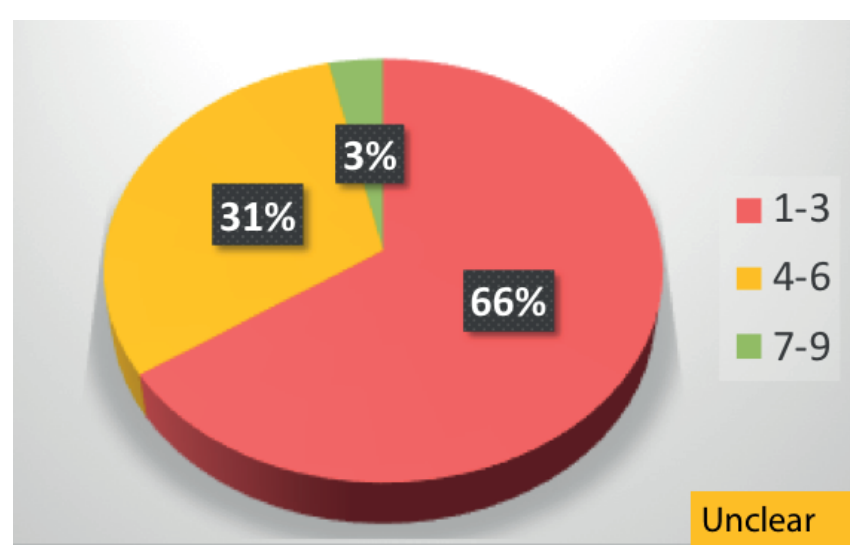

Figure 5.3.45. Delphi: Is it essential to measure total IgE in CRS after failure of appropriate medical or surgical treatment in ENT/secondary care with continued symptoms and abnormal mucosa on endoscopy?

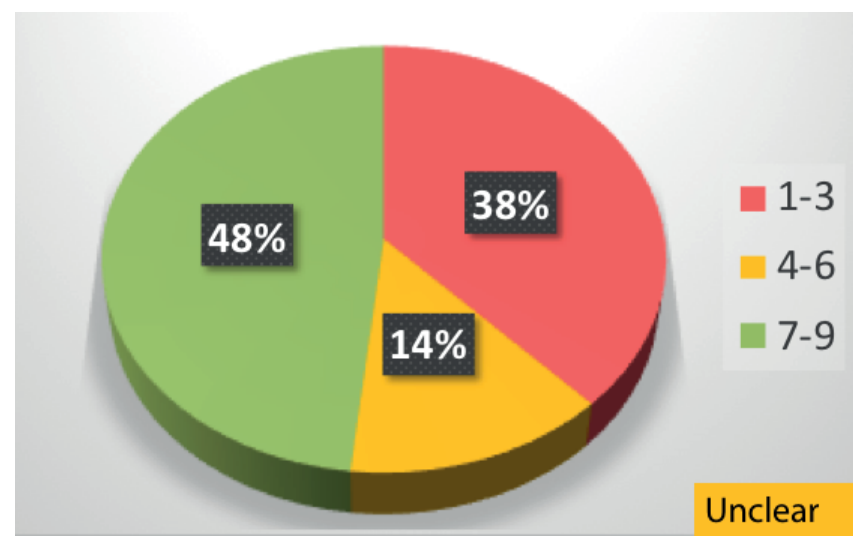


condition typically develops in patients with adult onset asthma and the sinonasal manifestation is chronic rhinosinusitis with eosinophilic nasal polyps. Many of these patients also have middle ear disease ${ }^{(738)}$.

Anti-neutrophil cytoplasmic antibodies (ANCAs) are a group of autoantibodies, mainly of the lgG type, against antigens in the cytoplasm of neutrophil granulocytes and monocytes. They are detected as a blood test in a number of autoimmune disorders, but are particularly associated with systemic vasculitis, so called ANCA-associated vasculitides. ANCAs are associated with small vessel vasculitides including granulomatosis with polyangiitis, microscopic polyangiitis, primary pauci-immune necrotizing crescentic glomerulonephritis (a type of renal-limited microscopic polyangiitis), eosinophilic granulomatosis with polyangiitis and drug induced vasculitides. PR3 directed c-ANCA is present in $80-90 \%$ of granulomatosis with polyangiitis, $20-40 \%$ of microscopic polyangiitis, $20-40 \%$ of pauci-immune crescentic glomerulonephritis and $35 \%$ of eosinophilic granulomatosis with polyangiitis. c-ANCA (atypical) is present in $80 \%$ of cystic fibrosis (with BPI as the target antigen) and also in inflammatory bowel disease, primary sclerosing cholangitis and rheumatoid arthritis (with antibodies to multiple antigenic targets). p-ANCA with MPO specificity is found $35 \%$ of eosinophilic granulomatosis with polyangiitis. p-ANCA with specificity to other antigens are associated with inflammatory bowel disease, rheumatoid arthritis, drug-induced vasculitis, autoimmune liver disease, drug induced syndromes and parasitic infections.

Cocaine and levamisole, which is a common adulterant of cocaine, can cause an ANCA positive vasculitis ${ }^{(739-741)}$.

The EPOS2020 steering group did not consider it essential to do an objective test for vasculitis at initial presentation to ENT/ secondary care with highly suggestive symptoms of CRS irrespective of whether the mucosa was abnormal or normal on endoscopy but their responses were unclear regarding whether this was essential after failure of appropriate medical or surgical treatment in ENT/secondary care with continued symptoms and abnormal mucosa on endoscopy (Figure 5.3.49., 5.3.50., 5.3.51.).

\subsection{5.}

Sarcoidosis is a complex disease with manifold clinical manifestations. A case suspicious for sarcoidosis requires clinical, radiological and histological evidence of non-caseating granulomas. Serum soluble interleukin-2 (sIL-2R), angiotensin converting enzyme (ACE) and lysozyme levels have become useful tools in the diagnosis of sarcoidosis and for evaluating disease activity, in addition to fluorodeoxyglucose-positron emission tomography (FDG-PET). Serum ACE is the most widely used laboratory test for the investigation of sarcoidosis ${ }^{(742)}$. Elevated serum levels of ACE, IL-2R, and lysozyme are usually associated with more aggressive disease and multiple-organ involvement ${ }^{(743)}$.
Figure 5.3.46. Delphi: Is it essential to do an objective test of immunodeficiency at initial presentation in ENT/secondary care with highly suggestive symptoms of CRS and abnormal endoscopy?

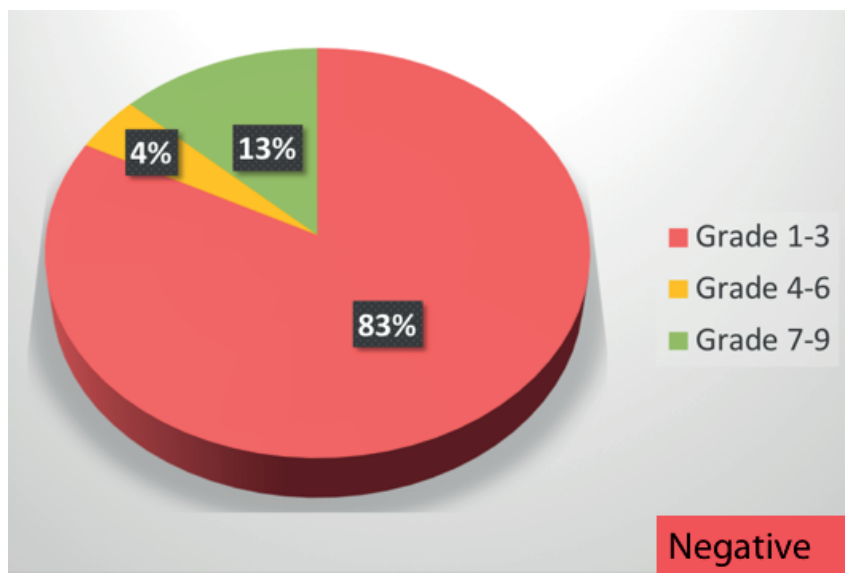

Figure 5.3.47. Delphi: Is it essential to do an objective test of immunodeficiency at initial presentation in ENT/secondary care in patients with highly suggestive symptoms of CRS but normal endoscopy?

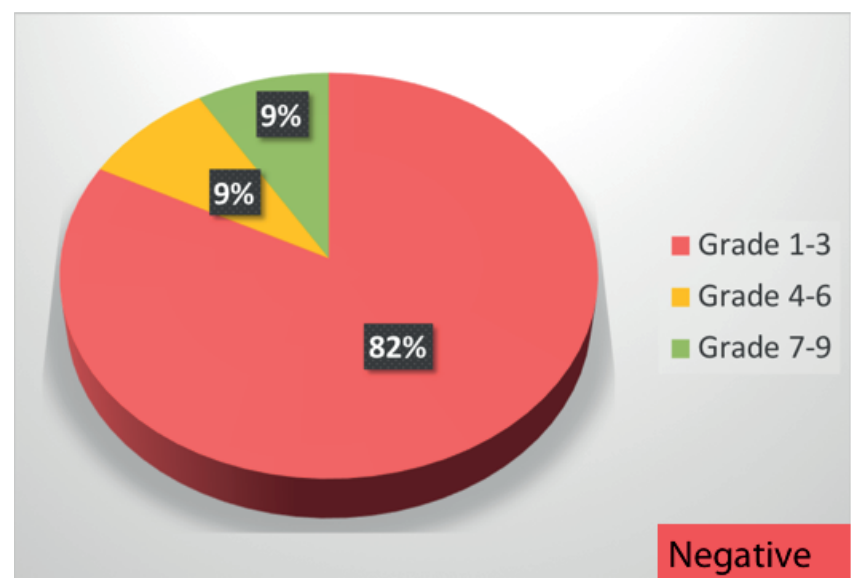

Figure 5.3.48. Delphi: Is it essential to do an objective test of immunodeficiency in CRS after failure of appropriate medical or surgical treatment in ENT/secondary care with continued symptoms and abnormal mucosa?

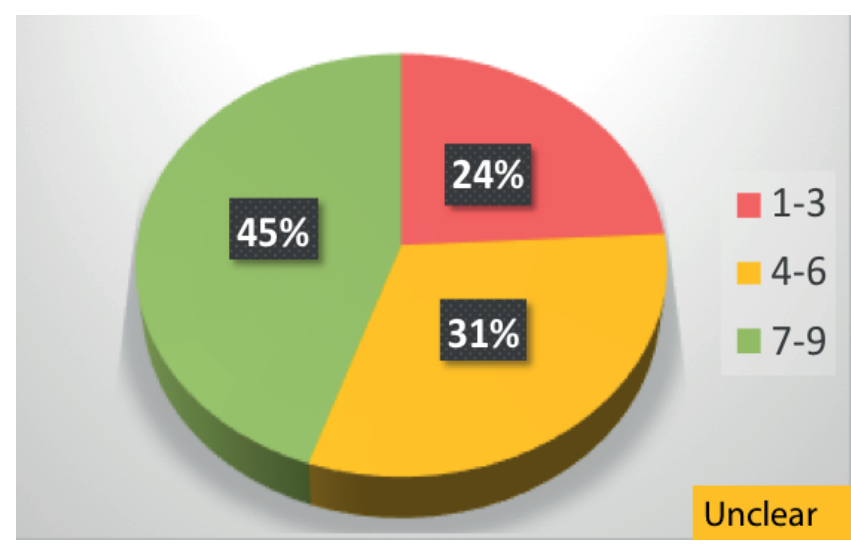




\subsection{Testing for $\mathbf{N}-E R D$}

Oral provocation test with aspirin is the most common investigation used to identify hypersensitivity reactions to aspirin (see 5.3.5.15). For details how to perform see the recently published EAACI position paper: Diagnosis and management of NSAID-Exacerbated Respiratory Disease (N-ERD) ${ }^{(39)}$. However, for those patients who have a higher risk of severe reactions, flow cytometry-assisted basophil activation testing (FAST) and functional-eicosanoid-test (FET) can be helpful tools to elucidate the diagnosis, if they are available ${ }^{(744,745)}$.

\subsubsection{Microbiology}

\subsubsection{1. Rationale}

Sinonasal microbiological sampling is important in the diagnostic work-up of patients with rhinosinusitis. The importance of microbes in the aetiology of acute bacterial rhinosinusitis (ABRS) is well recognized ${ }^{(553)}$ whereas the situation in CRS is less clear but is of increasing interest, facilitated by improved identification techniques ${ }^{(746,747)}$.

\subsection{Tests}

\subsection{Culture-dependent Techniques Sinus aspirates}

A maxillary sinus tap (MST) has historically been the gold standard technique for obtaining sinus cultures by providing a representative specimen of the antral contents with minimal nasal contamination ${ }^{(748,749)}$. It can be performed under local anaesthesia via the inferior meatus or canine fossa but is often associated with discomfort, potentially risks orbital, dental and nerve injury and only provides information on the maxillary sinus ${ }^{(748)}$.

The sphenoid sinus can also be approached directly to obtain an aspirate for culture ${ }^{(750)}$. In the past the frontal sinus has also been 'punctured' through the anterior wall to obtain a sample ${ }^{(751)}$ but this is of greater magnitude, usually requires a general anaesthetic and is generally part of a therapeutic surgical procedure rather than being purely diagnostic ${ }^{\text {(752) }}$.

\section{Nasal swabs}

Nasal swabs are the most commonly used sampling method in the nasal cavity as they are easy to use, non-invasive and generally well tolerated without the need for local anaesthesia. However, there is a poor correlation between undirected nasal/ nasopharyngeal and endoscopically directed middle meatal (EDMM) culture swabs, largely due to contamination from the nasal vestibule and cavity. EDMM swabs show a high concordance with maxillary sinus aspirates and cultures ${ }^{(753-758)}$ and thus have become the mainstay of microbial sampling in patients with rhinosinusitis (Table 5.3.7.). A meta-analysis confirmed that
Figure 5.3.49. Delphi: Is it essential to do an objective test for vasculitis at initial presentation in ENT/secondary care with highly suggestive symptoms of CRS and abnormal endoscopy?

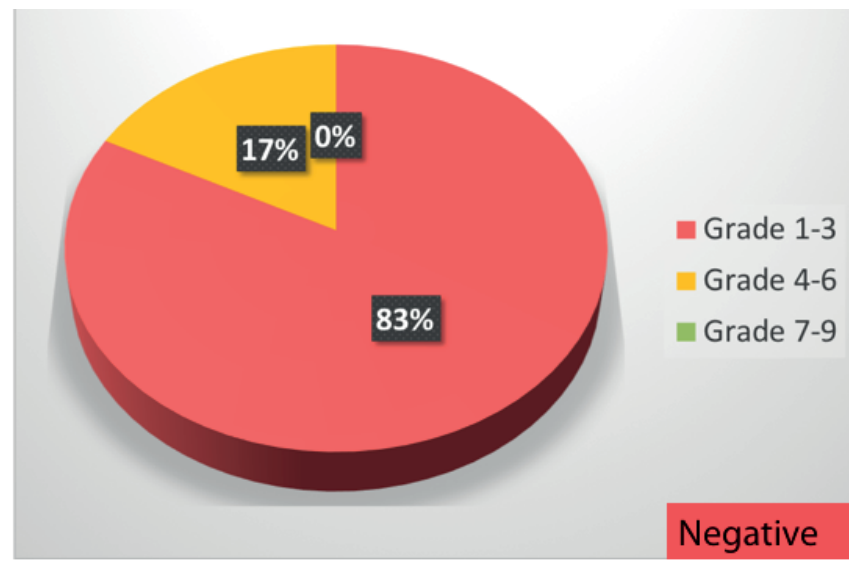

Figure 5.3.50. Delphi: Is it essential to do an objective test for vasculitis at initial presentation in ENT/secondary care in patients with highly suggestive symptoms of CRS but normal endoscopy?

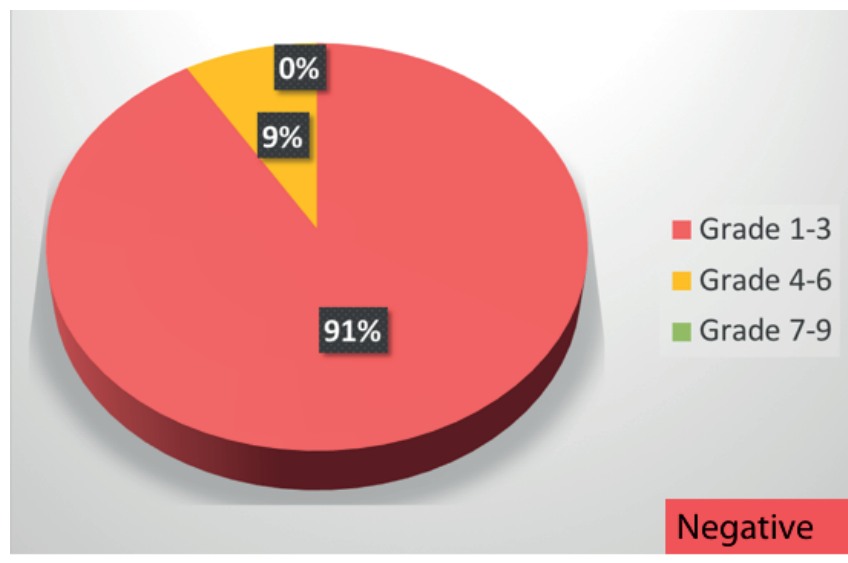

Figure 5.3.51. Delphi: Is it essential to do an objective test for vasculitis in CRS after failure of appropriate medical or surgical treatment in ENT/ secondary care with continued symptoms and abnormal mucosa on endoscopy?

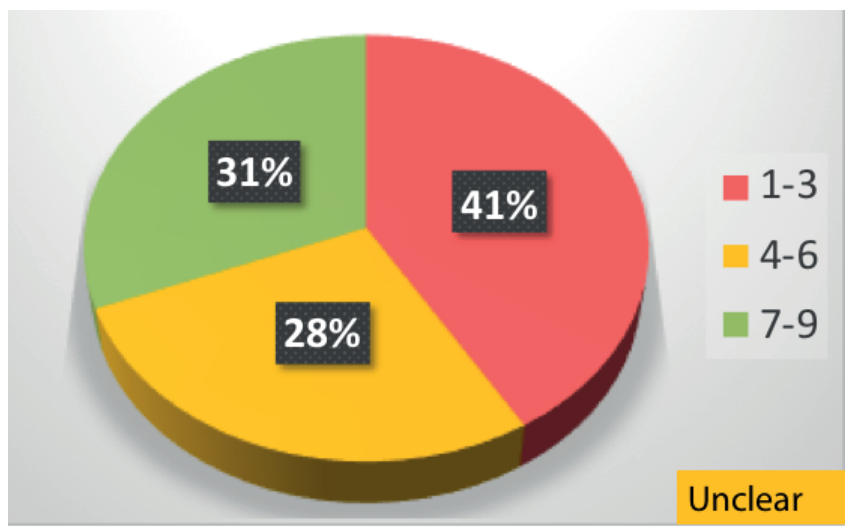


Table 5.3.7. Bacteriology of rhinosinusitis. Correlation of middle meatus versus maxillary sinus.

\begin{tabular}{|c|c|c|c|c|}
\hline Author, year, (ref) & $\begin{array}{l}\text { Number of } \\
\text { samples }\end{array}$ & $\begin{array}{l}\text { Type of rhinosi- } \\
\text { nusitis }\end{array}$ & Technique & Concordance \\
\hline Joniau $2005^{(756)}$ & 26 & ARS & Endoscopic swab (MM) vs. maxillary sinus tap & $88.5 \%$ \\
\hline Casiano $2001^{(755)}$ & 29 & ARS (intensive care) & Endoscopic tissue culture (MM) vs. maxillary sinus tap & $60.0 \%$ \\
\hline Talbot $2001^{(840)}$ & 46 & ARS & Endoscopic swab (MM) vs. maxillary sinus tap & $90.6 \%$ \\
\hline Vogan $2000^{(754)}$ & 16 & ARS & Endoscopic swab (MM) vs. maxillary sinus tap & $93.0 \%$ \\
\hline Gold and Tami $1997^{(841)}$ & 21 & CRS & Endoscopic tap (MM) vs. maxillary aspiration during ESS & $85.7 \%$ \\
\hline Klossek 1996 (757) & 65 & CRS & Endoscopic swab (MM) vs. maxillary aspiration during ESS & $73.8 \%$ \\
\hline
\end{tabular}

$A R$, acute rhinosinusitis; CRS, chronic rhinosinusitis; MM, middle meatus; ESS, endoscopic sinus surgery.

EDMM is a highly sensitive and accurate culture method for ABRS and may be more sensitive than maxillary sinus taps (MST) given the presence of pathogenic bacteria not found on antral lavage ${ }^{(759)}$. For known pathogenic bacteria for ABRS and in comparison to MST, EDMM had a sensitivity of $80.9 \%$, a specificity of $90.5 \%$, a positive predictive value of $82.6 \%$, a negative predictive value of $89.4 \%$, and an overall accuracy of $87.0 \%$ (95\% confidence interval, 81.3\%-92.8\%).

The more recent meta-analysis by Smith et al. showed that bac terial pathogens are recovered by sinus puncture or EDMM culture in $53 \%$ of patients with suspected ABRS, based on studies requiring patients to meet clinical criteria, with most studies also requiring radiographic and/or endoscopic confirmation.

There is limited research on how representative middle meatal swabs are for the remaining sinuses. Although not a direct comparative study, a recent retrospective study by Miller et al. ${ }^{(760)}$ demonstrated different pathogens in two or more of the swabs of $40 \%$ of patients undergoing multiple sinus cultures. While only $5 \%$ of the patients in this study received clinical benefit from changing the antibiotic, it does suggest that bacterial cultures from the middle meatus may not be entirely representative of all the sinuses.

EDMM culture swabs have become the mainstay of microbial sampling in patients with rhinosinusitis.

\section{Nasal blown secretions}

Nasal blown secretions may be readily obtained in primary care, particularly for paediatric patients as it avoids the discomfort of a nasal swab. The few comparative studies comparing expelled mucus to standard culture swab techniques suggest a high concordance rate (>90\%) for the detection of common upper airway pathogens when nasal secretions are present, but as might be expected, this rate reduces to less than $50 \%$ in the absence of obvious secretions ${ }^{(710,761)}$.

\section{Nasal and sinus lavage}

Nasal lavage is not an accurate technique for the culturing of sinus contents due to contamination by nasal flora and minimal sinus penetration in an unoperated patient. These issues may be circumvented by direct sinus lavage through an endoscopically placed maxillary sinus catheter. Studies comparing direct sinus lavage to EDMM swabs have demonstrated higher bacterial yields and increased recovery of anaerobic pathogens using the former technique ${ }^{(762,763)}$ and this sampling technique could be facilitated by balloon technology as many devices have inbuilt catheters that can be used for lavage drainage and antibiotic irrigation. The clinical relevance of the anaerobic organisms and the targeted culture and treatment of isolated infected sinuses remain to be studied.

\subsection{Culture-independent techniques}

Standard culture approaches offer only a limited range of defined conditions for microbial growth and, therefore, omits taxa that require alternative conditions, including slower-growing organisms that are outcompeted for a limited range of nutrient sources or those that depend upon cooperation to survive in vivo (such as cross-feeding relationships). The disparity between the identification of microbes by culture and those (viable but nonculturable or difficult to culture) identified by molecular methods has been termed "the great plate count anomaly," with estimates of the nonculturable portion of microbial communities ranging between $25 \%$ and $99 \%{ }^{(764,765)}$.

\section{Next generation sequencing}

Next generation sequencing techniques have enabled identification of micro-organisms previously unidentifiable on routine culture studies ${ }^{(450)}$. Samples can be obtained using guarded, flocculated microbial swabs or by tissue biopsy with high concordance noted between the two ${ }^{\left({ }^{(87)}\right)}$. DNA is extracted from the obtained specimens and sequenced using primers specific for conserved genetic regions within bacterial or fungal microorganisms. Sophisticated bio-informatic pipelines that reference known microbial libraries then allow identification and relative quantification of all the organisms present. Initially a research tool, such technology is now commercially available for clinical use. Studies comparing culture-directed analysis with next 
generation sequencing have shown significant discordance, with sinus culture poorly predicting resident microbiota $^{(154)}$. In direct comparisons between results of culture and sequencing approaches, the dominant bacteria identified in most patients by sequencing were identified by culture only approximately half of the time. Of those taxa present at a relative sequence abundance of $1 \%$, only about $5 \%$ were also identified by culture, revealing the extent of information on lower-abundance taxa missed by standard clinical culturing. This may in part explain poor treatment response despite culture-directed antibiotic therapy. It is possible that the incorporation of modern cultureindependent techniques into clinical practice may improve treatment outcomes, but further research is required.

Future techniques, particularly in the microbiology research on inflammatory mucosal diseases such as CRS include sequencing of all DNA (metagenomics) or all transcribed RNA (metatranscriptomics) or identification of proteins (metaproteomics) or metabolites (metabolomics) which should provide greater insights into the true diversity and structure, as well as the full genetic potential and in-situ activity, of the mucosa-associated microbiota $^{(746)}$.

\section{Outcomes}

There is no evidence that microbiological assessment of nasal or sinus samples has any impact on outcomes in rhinosinusitis. $80 \%$ of individuals with ABRS typically improve within two weeks without treatment ${ }^{(766)}$ and there is no evidence showing superiority of culture-directed antibiotic therapy over empiric treatment for this condition. Although low-level evidence suggests a possible role for culture-directed antibiotic therapy in the treatment of acute exacerbations of CRS, no high-level evidence studies exist supporting the use of antibiotics in CRS. Furthermore, there is a paucity of literature comparing outcomes of culture-directed versus empirical antibiotic treatment for this condition.

Microbial sampling for uncomplicated ABRS is not routinely recommended.

There is no evidence that microbiological assessment of nasal or sinus samples has any impact on outcomes in rhinosinusitis. Although antibiotics provide a small but significant benefit for clinical outcomes in adults and children with ABRS (see 4.6.3.1. and 4.6.3.2.) most patients suffer from post-viral ARS where antibiotics are not helpful (see 4.6.3.3. and 4.6.3.4). Furthermore, there are no studies ishowing superiority of culture-directed antibiotic therapy over empiric treatment for this condition.

\section{Recommendations}

Microbiological assessment is not routinely recommended in the diagnosis of rhinosinusitis. In ABRS non-responsive to empirical antimicrobial treatment and topical nasal steroids, referral is recommended to an ENT specialist where further diagnostic procedures, including microbiology, can be performed. The role of microbial sampling in CRS remains unclear. Prospective trials are needed to examine the relevance of routine microbiologic cultures and antibiotic treatment in CRS patients before sound recommendations can be made.

It is possible that the incorporation of modern culture-independent techniques into clinical practice may improve treatment outcomes, but further research is required. The EPOS2020 steering group did not consider it was essential to do microbiology at initial presentation to ENT/ secondary care with highly suggestive symptoms of CRS irrespective of whether the mucosa was abnormal or normal on endoscopy.

However, they were unclear as to whether it was essential to do microbiology in CRS after failure of appropriate medical or surgical treatment in ENT/secondary care with continued symptoms and abnormal mucosa on endoscopy (Figures 5.3.52., 5.3.53., 5.3.54.).

\subsubsection{Mucociliary clearance testing}

\subsection{Rationale}

By their coordinated movement, the cilia lining the respiratory epithelium transport the mucus layer with entrapped inhaled particles from the nasal cavity towards the hypopharynx. Normal mucociliary transport (MCT) is essential for the maintenance of healthy sinonasal cavities. With infection or congenital dysfunction of the cilia as in PCD, MCT is inadequate or absent. In PCD, lack of MCT may lead to CRS and bronchiectasis. Mucostasis, hypoxia, microbial products and toxic inflammatory mediators in CRS may induce secondary ciliary changes, i.e. secondary ciliary dyskinesia (SCD), with subsequent inadequate $\mathrm{MCT}^{(450)}$.

The mucociliary clearance (MCC) time is considered to be normal below 15 minutes and should be less than one hour.

The saccharine test evaluates the time taken to experience a sweet taste after placement of a $1-2 \mathrm{~mm}$ particle of saccharine on the inferior turbinate mucosa, $1 \mathrm{~cm}$ from the anterior end. The patient must sit quietly with the head bent forward and without sniffing, coughing, sneezing, drinking or eating during the investigation. Alternatively, one can monitor the time needed for a dye such as methylene blue to be transported from the mucosa of the anterior third of the nasal cavity towards the hypopharynx. Other substances including technetium- 99m-labeled iron oxide have also been used.

As the MCC time can only be measured in cooperative patients with patent nasal cavities and in the absence of severe mucosal disease, it has limited diagnostic value in certain patients. It also has low sensitivity and specificity and requires significant time investment. In the context of the clinical relevance of $\mathrm{MCT}$, it has recently been demonstrated that nasal lavages, a commonly recommended treatment for many sinonasal conditions, reduce 
Figure 5.3.52. Delphi: Is it essential to do microbiology at initial presentation to ENT/secondary care with highly suggestive symptoms of CRS and abnormal mucosa on endoscopy?

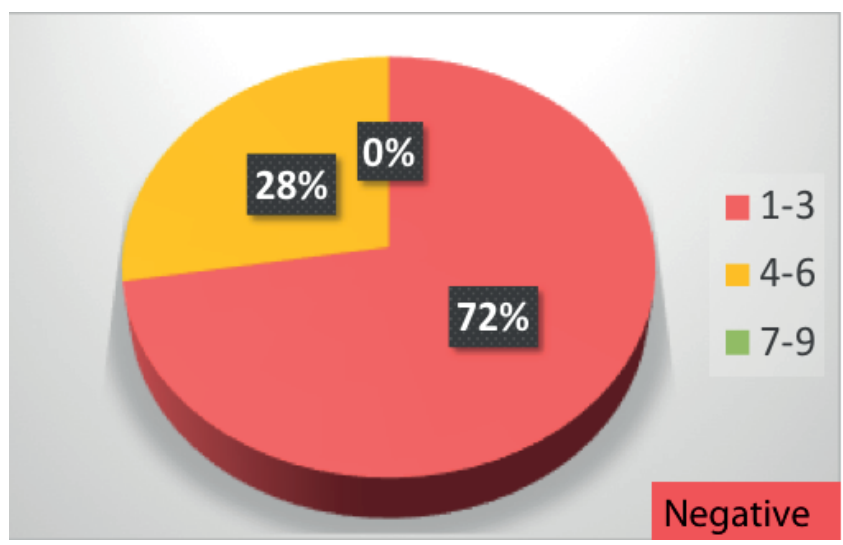

Figure 5.3.53 Delphi: Is it essential to do microbiology at initial presentation in ENT/secondary care in patients with highly suggestive symptoms of CRS but normal endoscopy?

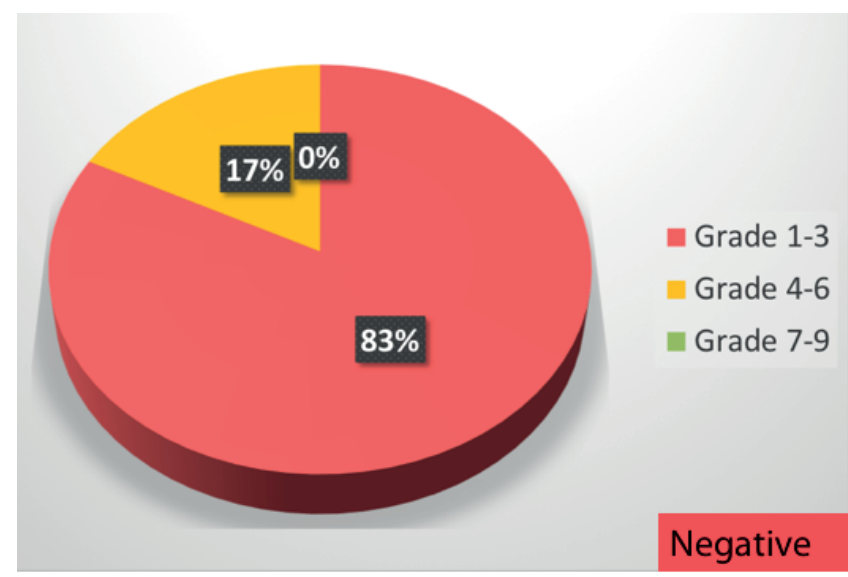

Figure 5.3.54 Delphi: Is it essential to do microbiology in CRS after failure of appropriate medical or surgical treatment in ENT/secondary care with continued symptoms and abnormal mucosa on endoscopy?

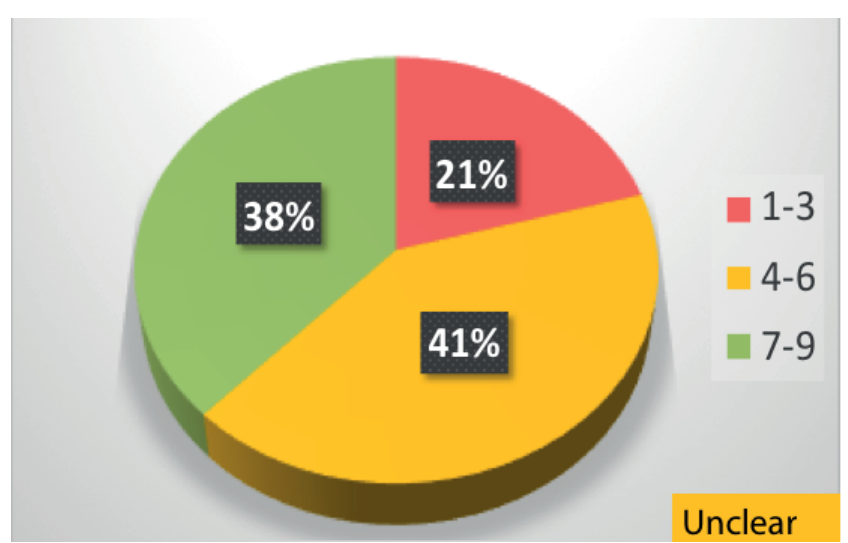

the MCC time ${ }^{(767)}$.

The EPOS2020 steering group did not consider it essential to do an objective test of mucociliary function at initial presentation to ENT / secondary care with highly suggestive symptoms of CRS irrespective of whether the mucosa was abnormal or normal on endoscopy but their responses were unclear regarding whether this was essential after failure of appropriate medical or surgical treatment in ENT/secondary care with continued symptoms and abnormal mucosa on endoscopy (Figures 5.3.55., 5.3.56., 5.3.57.).

\subsubsection{Nasal nitric oxide}

\subsection{Rationale}

Nitric oxide (NO) is a colourless, odourless gas that is present in air exhaled through the mouth or nose. NO is produced from arginine and oxygen by nitric oxide synthase (NOS). Constitutively expressed neuronal and endothelial forms exist as well as an induced form, iNOS, which appears to be upregulated within the respiratory tract in response to pro-inflammatory signals. Exhaled NO (eNO) levels are raised in eosinophilic asthma and measurement of this has become a standardised, but not yet widespread, tool in the diagnosis and management of asthma. It can potentially provide a rapid, low cost objective measure of lower airway inflammation. Most NO is produced in the sinuses, far less by the nasal mucosa and the lower respiratory tract ${ }^{(450)}$. $\mathrm{NO}$ and its metabolites are toxic to micro-organisms and likely form part of the innate defence mechanism of the respiratory tract. NO may also stimulate ciliary beat frequency within the epithelium and regulate nasal vascular tone.

\subsection{Diagnostic accuracy}

Nasal $\mathrm{NO}(\mathrm{nNO})$ can be measured in either nostril and shows no diurnal variation. The measurement is quick, reproducible, feasible and best assessed with an aspiration flow of $700 \mathrm{ml} / \mathrm{min}$ during breath-holding for 10 seconds ${ }^{(768)}$. Humming during $\mathrm{nNO}$ measurement initially increases levels considerably in healthy subjects. If low values are obtained, measuring nNO during humming can increase the specificity of the measurement ${ }^{(768)}$. Healthy controls usually have nNO levels above 300 $\mathrm{ppb}^{(769)}$. Nasal NO testing using palatal closure manoeuvres has diagnostic accuracy similar to electron microscopy and/or genetic testing for primairy ciliairy dyskinesia when cystic fibrosis has been ruled out ${ }^{(770)}$. Nasal NO values below 77 ppb are very suggestive of PCD but higher values are occasionally found. CF also has generally low values (70-300 ppb), and the presence of nasal polyps is associated with significantly lower nNO levels than in CF patients without nasal polyps ${ }^{(771)}$ due to obstruction of the sinuses. Thus, in nasal polyps, patients may paradoxically have low $\mathrm{nNO}$ and normal or high eNO from the chest.

Nasal NO is a sensitive and specific test for PCD in cooperative 
Figure 5.3.55 Delphi: Is it essential to do an objective test of mucociliary function at initial presentation in ENT/secondary care with highly suggestive symptoms of CRS and abnormal endoscopy?

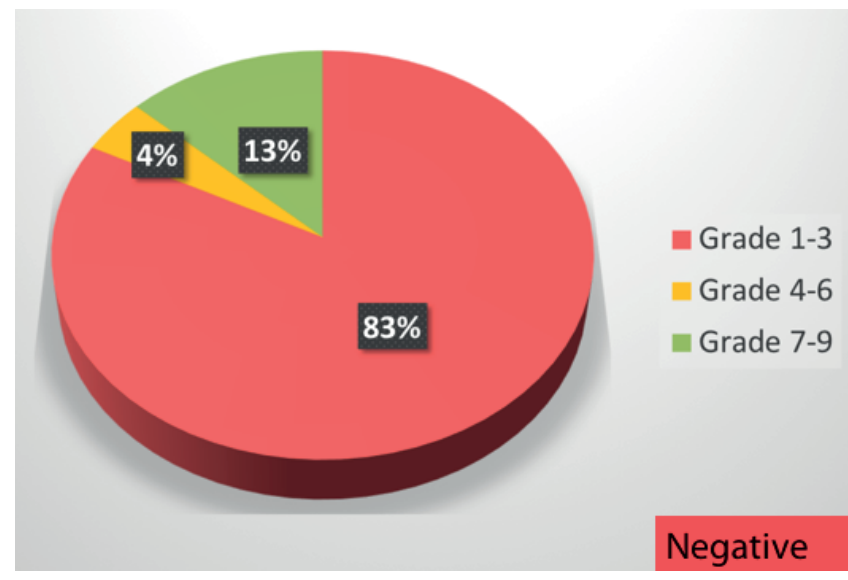

Figure 5.3.56 Delphi: Is it essential to do an objective test of mucociliary function at initial presentation in ENT/secondary care in patients with highly suggestive symptoms of CRS but normal endoscopy?

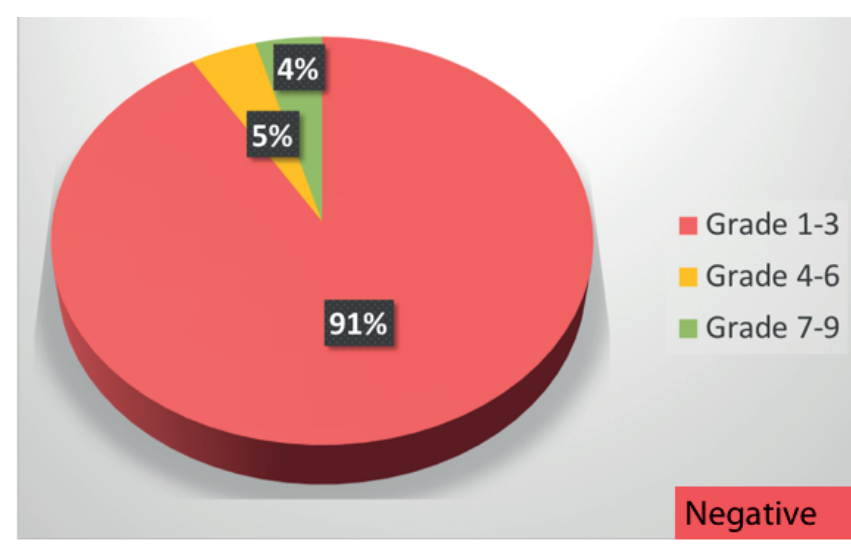

Figure 5.3.57 Delphi: Is it essential to do an objective test of mucociliary function in CRS after failure of appropriate medical or surgical treatment in ENT/secondary care with continued symptoms and abnormal mucosa?

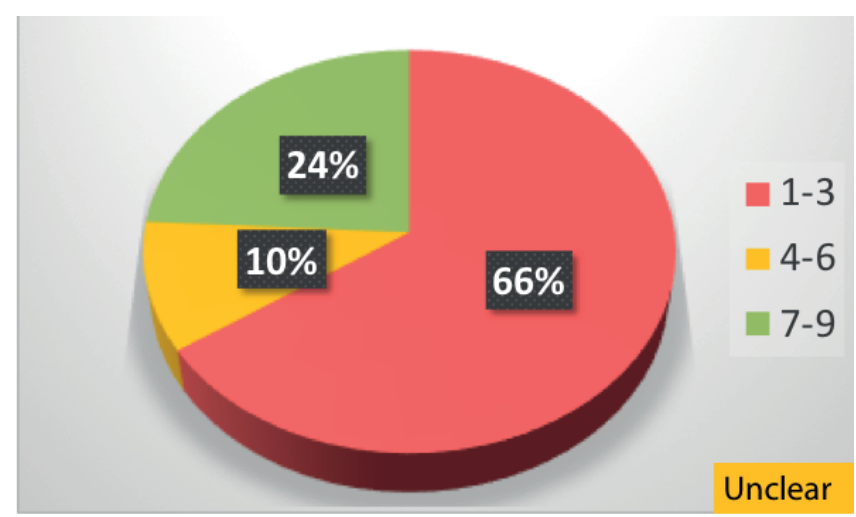

patients (generally over five years old) with a high clinical suspicion for this disease. To a lesser extent it may also be a useful adjunct in a potential diagnosis of CF. Whilst not commonly used as a specific diagnostic tool in CRS, it may be used to monitor the response to treatment in these conditions.

\subsubsection{Other tests for PCD}

\subsection{Rationale}

No ideal test is available for the diagnosis of PCD in routine practice $^{(772)}$. In cases of suspected PCD in a patient with CRS since birth, a family history of PCD and/or associated features of Kartagener syndrome (situs inversus and infertility), one should consider diagnostic tests of ciliary function by evaluation of ciliary beat frequency (CBF), EM evaluation of the dynein arms of the cilia and/or evaluation of the cilia after ciliogenesis in vitro ${ }^{(450)}$. Harvested epithelial cells can be evaluated for ciliary beat frequency (CBF) and the ciliary wave form analysed in detail by digital high-speed video imaging. The evaluation of the CBF as well as assessment of their coordinated movement can be performed by computerized programs using a Fast Fourier analysis. Normal values of CBF vary depending on the methodology used, the age of the patient and the culture conditions. The demonstration of normal CBF and beat pattern excludes the diagnosis of PCD. Recently, a novel technique for quantification of CBF has been reported using phase-contrast microscopy images, estimating ciliary motion by means of an optical flow algorithm $^{(773)}$. In a tertiary referral centre, one third of patients referred with suspected $P C D$ were eventually diagnosed with the condition ${ }^{(774)}$.

As these techniques are not available in routine ENT practice, one should rely on measuring nNO levels in cases of suspected PCD; low nNO levels have been associated with PCD and, therefore, represent an excellent screening tool (see above). In addition, screening for genetic mutations known to be associated with PCD, as well as genetic counselling, is recommended ${ }^{(775)}$.

\subsubsection{Sweat testing}

\subsection{Rationale}

Cystic fibrosis (CF), caused by mutations in the CF transmembrane conductance regulator (CFTR) gene, continues to present diagnostic challenges. It is recommended that diagnoses associated with CFTR mutations in all individuals, from newborn to adult, be established by evaluation of CFTR function with a sweat chloride test ${ }^{(776)}$. Sweat chloride testing should be performed according to approved procedural guidelines published in established, Int protocols such as the CLSI 2009 Guidelines (https://clsi.org). 
Figure 5.3.58 Delphi: Is it essential to do an objective test for cystic fibrosis at initial presentation in ENT/secondary care with highly suggestive symptoms of CRS and abnormal endoscopy?

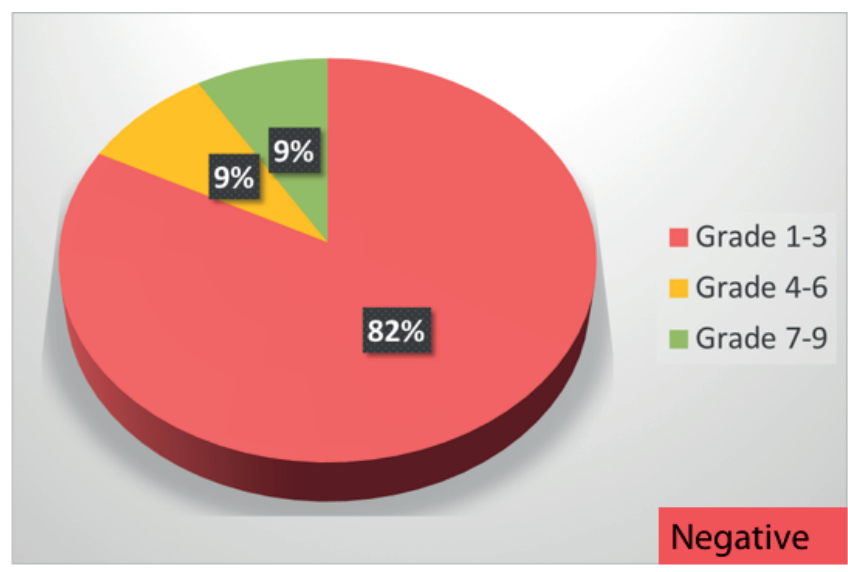

Figure 5.3.59 Delphi: Is it essential to do an objective test for cystic fibrosis at initial presentation in ENT/secondary care in patients with highly suggestive symptoms of CRS but normal endoscopy?

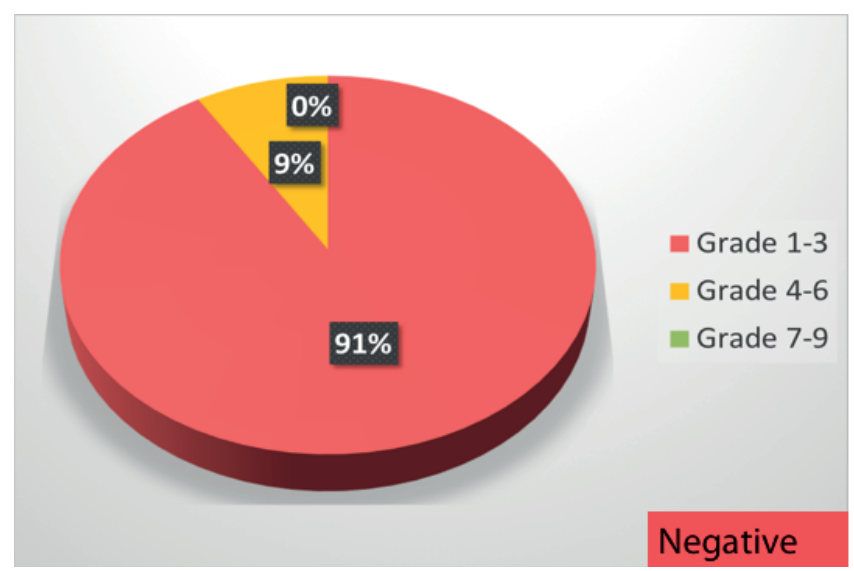

Figure 5.3.60 Delphi: Is it essential to do an objective test for cystic fibrosis in CRS after failure of appropriate medical or surgical treatment in ENT/ secondary care with continued symptoms and abnormal mucosa on endoscopy?

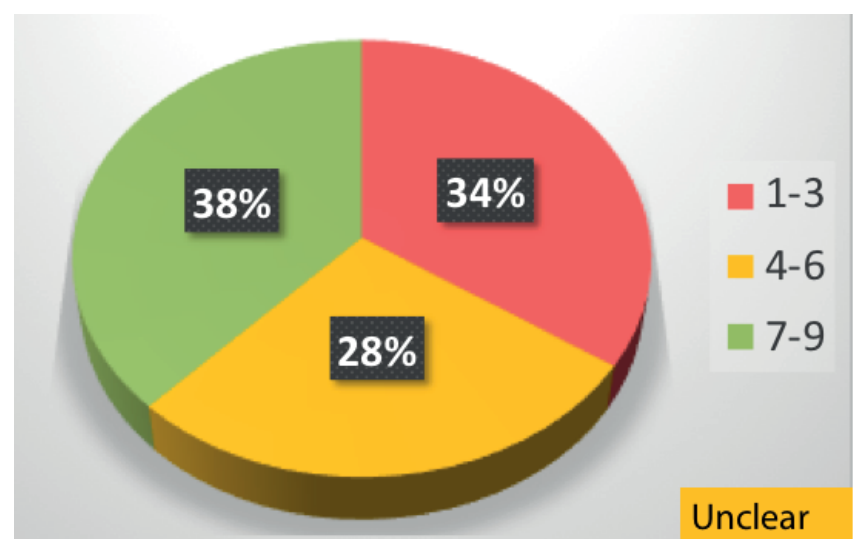

\subsection{Advice of the Consensus Guidelines from the Cystic Fibrosis Foundation}

Newborns with a positive CF newborn screen (NBS), to increase the likelihood of collecting an adequate sweat specimen, should have the test performed bilaterally and when the infant weighs $>2 \mathrm{~kg}$, and is at least 36 weeks of corrected gestational age. Newborns greater than 36 weeks gestation and $>2 \mathrm{~kg}$ body weight with a positive CF newborn screen, or positive prenatal genetic test, should have sweat chloride testing performed as soon as possible after 10 days of age, ideally by the end of the neonatal period (four weeks of age).

In infants with presumptive CF identified through NBS, CF treatment should not be delayed while efforts to establish a diagnosis of CF are initiated. Sweat chloride analysis should be performed within a few hours of sweat collection. In individuals presenting with a positive newborn screen, clinical features consistent with $\mathrm{CF}$, or a positive family history, a diagnosis of $\mathrm{CF}$ can be made if the sweat chloride value is $\geq 60 \mathrm{mmol} / \mathrm{L}$.

In individuals with a positive newborn screen, a sweat chloride $<30 \mathrm{mmol} / \mathrm{L}$ indicates that CF is unlikely.

Individuals with clinical features that may be consistent with CF who have a sweat chloride $<30 \mathrm{mmol} / \mathrm{L}$ indicates that CF is less likely. It may, however, be considered if evolving clinical criteria and/or CFTR genotyping support CF and not an alternative diagnosis. Individuals presenting with a positive newborn screen, symptoms of $\mathrm{CF}$, or a positive family history, and sweat chloride values in the intermediate range $(30-59 \mathrm{mmol} / \mathrm{L})$ on two separate occasions may have CF. They should be considered for extended CFTR gene analysis and/or CFTR functional analysis. Individuals who are screen-positive and meet sweat chloride criteria for CF diagnosis should undergo CFTR genetic testing if the CFTR genotype was not available through the screening process or is incomplete ${ }^{(776)}$.

The EPOS2020 steering group did not consider it essential to do an objective test for CF at initial presentation to ENT/ secondary care with highly suggestive symptoms of CRS irrespective of whether the mucosa was abnormal or normal on endoscopy but their responses were unclear regarding whether this was essential after failure of appropriate medical or surgical treatment in ENT / secondary care with continued symptoms and abnormal mucosa on endoscopy (Figures 5.3.58., 5.3.59. and 5.3.60.).

\subsection{Indications and possibilities of genetic testing}

Genetic testing is mainly used for CF, Primary Ciliary Dyskinesia $(P C D)$ and to a lesser extent in chronic rhinosinusitis (CRS) ${ }^{(777)}$. CF results from mutations in the cystic fibrosis transmembrane conductance regulator (CFTR) which is located on the long arm of chromosome 7. Normally a 6.5-kb mRNA encodes CFTR which is a 1490 -amino acid integral membrane protein. It is an ion channel responsible for chloride exchange in epithelial cells. To date more than 2000 CFTR variants have been described ${ }^{(778)}$. 
Newborn screenings date back to 1979 in New Zealand and are performed by serial, two-tiered, or, more recently, three-tiered/ hybrid-based methods ${ }^{(778)}$. The screenings are based on immunoreactive trypsinogen (IRT). There are three approaches: 1 ) one starts with the measurement of IRT where measurements above a defined cut-off are followed up by genetic analysis to determine specific mutations in the CFTR gene (IRT/DNA); 2) An initial IRT measurement with defined cut-offs is followed by IRT measurement on a second specimen collected two weeks later (IRT/IRT); 3) Pancreatitis-associated protein is used following an increased IRT (IRT/pancreatitis-associated protein method). Direct sandwich immunoassays with either monoclonal or polyclonal antibodies are used and may also be multiplexed. The advantage is to test multiple samples in a patient at a relatively low cost. The genetic analysis (from blood or oral mucosal scrapings) are done by Sanger sequencing and quantitative methods. Nowadays new high throughput approaches such as Next Generation Sequencing, targeted deep sequencing (TDS) and Droplet Digital PCR technologies are used ${ }^{(779)}$. Recently, Zeevi et al. ${ }^{(780)}$ proposed a new method called allele sensitive proliferation sequencing (ASP-SEQ) which is more sensitive than targeted deep sequencing or digital PCR methods.

PCD is a genetically heterogeneous autosomal-recessive motile ciliopathy associated with 32 genes. Clinical manifestations are situs inversus, neonatal respiratory distress, progressive bronchiectasis, and respiratory failure in young adulthood ${ }^{(781)}$. Tests include including $\mathrm{nNO}$, high speed videomicroscopy analysis (HSVA), transmission electron microscopy (TEM), immunofluorescent tests of ciliary proteins and genetic testing. Structural abnormalities can be confirmed by genetic testing of biallelic mutations in known PCD ${ }^{(782)}$. The most prevalent mutations occur in five genes (DNAH5; DNAH11; DNAI1; CCDC39; CCDC40) (783). Genetic tests combining Intron-exon level copy number variations (CNVs) and whole-exome sequencing (WES) seem to be the most efficient approach ${ }^{\text {(781) }}$.

For CRS genomic analysis the most studied targets are bitter taste receptors especially $\mathrm{T} 2 \mathrm{R} 38^{(784)}$ which are analysed by eg. reverse transcription polymerase chain reaction with $7900 \mathrm{HT}$ fast using allele-specific probes and primers ${ }^{(785)}$ or single nucleotide polymorphisms (SNPs) ${ }^{(117)}$. Other genetic approaches target single nucleotide polymorphism genotypes in potassium channels (eg. by Illumnia Human-Hap550 BeadChip or Illumina Human610-Quad BeadChip) ${ }^{(786)}$. A more recent genome-wide association study investigated the role of ALOX15 in CRS ${ }^{(436)}$. More targeted analyses eg. for methylated genes like FZD5 playing a role in CRSWNP can be detected using methyl-CpG-binding domain sequencing (MBD-seq) and validated using methylationspecific polymerase chain reaction ${ }^{(787)}$.

In summary, many potential genes influencing CRS have been investigated but for diagnosis or confirmation of the latter genetic testing has only been routinely established for CF and
PCD. Despite promising targets for future research bitter taste receptors are currently tested for the phenotype of patients as either supertasters, intermediate or non-tasters.

\subsubsection{Lower respiratory tract assessment}

\subsection{Rationale}

A significant number of patients with rhinosinusitis have involvement of the lower respiratory tract embodied in the concept of the 'united airways'. A patient's own assessment of their chest symptoms (wheeze, shortness of breath, chest tightness, and cough) and severity is often poor or they may even be unaware that the lower respiratory tract is affected so objective assessment is necessary ${ }^{(34)}$.

\subsection{Techniques Peak flow}

This is a simple test in which the individual stands and blows as hard as possible through a disposable filtered mouthpiece into a peak flow meter. The best of three attempts is taken and compared with charts of normative data for sex, age and height. If the result is not within the normal range, it can be repeated 15 minutes after administration of a bronchodilator such as salbutamol, to assess reversibility. A 20\% increase and absolute improvement of $60 \mathrm{l} / \mathrm{min}$ is suggestive of asthma though the diagnosis cannot be made on a single test. Alternatively, an exercise test such as running for 10-15 minutes followed by peak flow measurements every 5 minutes which show a decrease of $>10 \%$ or $>200 \mathrm{ml}$ from baseline of FEV1 for adults or a fall in PEF of $>15 \%$ in children is also indicative of asthma ${ }^{\text {(788-792) }}$.

Patients can be prescribed a peak flow meter and chart on which they can record their daily measurements, ideally performed at the same time morning and evening. They can be asked to keep the record over several weeks and to bring the chart to their next outpatient visit. Average daily diurnal variation is calculated as the day's highest PEF minus the day's lowest PEF, divided by the mean PEF for the day and then averaged over the course of a week. A variability of $>10 \%$ in adults and $>13 \%$ in children is suggestive of asthma ${ }^{(792-794)}$.

\section{Spirometry}

This technique provides additional information in the outpatient setting (Table 5.3.8.). The individual is asked to blow as hard as possible into a tube through a filtered disposable mouthpiece and expiratory effort continued as long as possible. Again, the best of three attempts is used to derive the following values:

- $\quad$ Forced expiratory volume in one second (FEV1)

- $\quad$ Forced vital capacity (FVC)

- Ratio of FEV1/FVC. This should be $>70-80 \%$ in healthy adults and $>90 \%$ in healthy children ${ }^{(305,788-791)}$.

Comparative normative data are available. Various abnormal 
Table 5.3.8. Comparison of peak expiratory flow and spirometry in the assessment of possible asthma (Scadding and Lund 2004(842))

\begin{tabular}{llll} 
& Setting & Advantages & Disadvantages \\
\hline Spirometry & Outpatients & Most reliable & Snaphot \\
& & Reproducible & Expense \\
& & Results in 15-30 mins & Trained operator \\
& & Pre- and post-bronchodilator & Patient compliance \\
Peak flow & Outpatients / Home & Sensitive & Less reliable than FEV 1 \\
& & Easy & Patient compliance \\
& & Cheap & Snapshot or wait $~$ \\
& &
\end{tabular}

patterns may be seen.

1) Obstruction: FEV1 is low, FVC normal and FEV1/FVC $<70-80 \%$ or below the lower limit of normal (lowest $5 \%$ of the reference

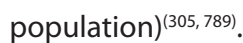

This is characteristic of asthma and likely reversible with a beta-agonist eg salbutamol, albuterol. The likelihood of a false negative result is increased if a short-acting beta-agonist was administered within 4 hours of the test, or if a long-acting beta agonist was administered within 15 hours of the test.

An increase of FEV1 by $>12 \%$ from baseline (in adults and children) or an increase of $>200 \mathrm{ml}$ after a short-acting bronchodilator (in adults) indicates significant reversibility.

Caution is advised with the use of a fixed FEV1/FVC ratio as a cut-point, as it results in false-positive diagnoses of obstruction in older adults and potentially false-negative diagnoses of obstruction in younger adults, given a natural decline in FEV1 with age ${ }^{(795,796)}$.

2) Restriction: FEV1 and FVC are low with a normal FEV1/FVC. This is characteristic of a fibrosis or granulomatous process such as sarcoid and is not reversible with bronchodilators. To confirm restriction a measurement of lung volumes (total lung capacity, residual volume) is necessary.

\section{Spirometry post-initiation of controller therapy}

In adults, if after four weeks of anti-inflammatory, controller therapy (such as an inhaled corticosteroid or combined inhaled corticosteroid and long-acting beta agonist), FEV1 has improved by over $12 \%$ and over $200 \mathrm{~mL}$ (or PEF by over 20\%), this can also be seen as supportive of a diagnosis of asthma in the appropriate clinical context ${ }^{(792)}$.

If not already involved, the presence of abnormal measurements or high clinical suspicion should prompt referal to a chest physician. They also have at their disposal other investigations including bronchial provocation testing, with or without pharmacologic agents and fractional exhaled nitric oxide as well as imaging of the chest. The availablility of expired nitric oxide measurement to rhinologists as well as pulmonologists has improved diagnosis and reduced the use of peak flow as this provides immediate confirmation of inflammation in the lower (and upper) respiratory tract (see 5.3.5.9.; Tables 5.3.8.; 5.3.9.).

\subsubsection{Diagnosis of N-ERD}

\subsection{Rationale}

NSAID-exacerbated respiratory disease (N-ERD) is a chronic eosinophilic, inflammatory disorder of the respiratory tract occurring in patients with asthma and/or chronic rhinosinusitis with nasal polyps (CRSwNP), symptoms of which are exacerbated by NSAIDs, including aspirin. As the condition is likely under-diagnosed and outcomes are worse in these patients, it is important to identify the hypersensitivity. It is also important so that affected patients can be warned about avoidance, whilst enabling the non-sensitive to use therapeutic aspirin and NSAIDs and to allow consideration of the option of aspirin desensitization in those who are sensitized ${ }^{(797)}$.

\subsection{Diagnosis}

Diagnosis of N-ERD is mainly based on a patient history of at least one documented reaction to aspirin or NSAIDs though it is recognised that history alone is not always reliable, especially as asthmatic patients are often warned to avoid these drugs ${ }^{(798)}$. Thus, aspirin provocation tests are needed when the history is not clear (Figure 5.3.61.).

\subsection{Techniques}

A provocation test may be undertaken via oral, bronchial or nasal routes (Table 5.3.10.). An oral provocation test with aspirin is the most common investigation used but is not without potential risk. Oral or bronchial challenge starts with a very low dose, usually around $30 \mathrm{mg}$ aspirin, which is then given in gradually increasing doses ${ }^{(799)}$. Although bronchial challenge can take only four hours to perform ${ }^{(800)}$, both these methods can result in severe symptoms in over $50 \%$ of subjects, especially in asthmatics, and may necessitate emergency treatment, hospital admission and close monitoring. Since the reaction can be delayed, the challenge often requires more than 1 day and and subsequent overnight admission.

Nasal provocation with lysine aspirin (LAS), a truly soluble form of aspirin, is an approach that has gained popularity as it can be performed in severe asthmatics due to the rarity of systemic reactions. It has been standardized, is reproducible ${ }^{(801)}$ and may 
Table 5.3.9. Diagnostic testing of lower respiratory tract (Brigham et al. 2015(843)).

\begin{abstract}
History of variable respiratory symptoms
Confimed variable expiratory airflow limitation
\end{abstract}

Basic measurements

Positive exercise challenge test

Assessment of a response to treatment

Bronchial provocation

Other supportive tests
Peak expiratory flow (repeated measures) Spirometry (repeated measures)

Spirometry pre-bronchodilator and post-bronchodilator Spirometry post-initiation of a controller therapy ie inhaled steroid Nonpharmacologic: exercise or eucapnic voluntary ventilation Pharmacologic: hypertonic saline, mannitol, or methacholine

Allergy testing

Exhaled nitric oxide be done in the outpatient setting but it should still be performed under medical supervision with resuscitation equipment available. The reaction does not begin until about 45 minutes after the nasal application and may persist for many hours. If nasal provocation proves negative, oral challenge should be considered. In a cohort of 150 difficult-to-treat CRSwNP patients, 100 proved positive on nasal challenge, and 31 who were negative went on to oral LAS challenge of which a further 14 gave positive results ${ }^{(802)}$.

Medication which must be stopped prior to the provocation test:

- Oral and intranasal corticosteroids (at least seven days);

- Antileukotrienes (at least seven days);

- Antihistamines (three days);

- Decongestants and cromones (one day).

Lysine-aspirin solutions at $0.1,1$ and $2 \mathrm{M}$ are instilled in the nose at 45-minute intervals and the effects assessed by clinical symptoms (VAS), objective nasal airway assessment (acoustic rhinometry, anterior rhinomanometry) and pulmonary function. A positive response is taken either as a $25 \%$ or greater decrease in the nasal airway (represented by a reduction of cross-sectional area: Amin or volume $0-12 \mathrm{~cm}$ ) as assessed by acoustic rhinometry or a $40 \%$ decrease in nasal inspiratory peak flow ${ }^{(799)}$. However, nasal inspiratory peak flow monitoring has been shown to be less sensitive to obstruction caused by aspirin than acoustic rhinometry ${ }^{(802)}$. On pulmonary function, a drop of $20 \%$ FEV1 is considered positive.

If nasal provocation proves negative, oral challenge should be considered.

However, for those patients who have a higher risk of severe reactions, flow cytometry-assisted basophil activation testing (FAST) and functional-eicosanoid-test (FET) can be helpful tools to elucidate the diagnosis, if they are available $\mathrm{e}^{(744,745)}$. Another alternative to aspirin provocation is the cellular antigen stimulation test (CAST) but a study showed that whilst the leucocytes of patients with N-ERD produced increased amounts of cysteinyl leukotrienes, the assay had a low sensitivity (25\%), a specificity of $92.3 \%$, a positive predictive value of $28.7 \%$ and a negative value of $90.7 \%$ which limit its usefulness ${ }^{(803)}$.

The EPOS2020 steering group was unclear whether one or two episodes of a respiratory reaction to aspirin or NSAIDs wasvessential for the diagnosis AERD in a patient with CRS and whether an objective test was essential to diagnose aspirin exacerbated respiratory disease for the diagnosis AERD in a patient with CRS (Figures 5.3.62., 5.3.63. and 5.3.64.).

Table 5.3.10. Different challenges to assess aspirin sensitivity (Nizankowska 2000 ${ }^{(844)}$ ).

\begin{tabular}{|lcc|}
\multicolumn{2}{c}{ History $+/-$} & \multicolumn{2}{c|}{ Challenge } \\
& Sensitivity (\%) & Specificity (\%) \\
\hline Oral & 77 & 93 \\
Bronchial & 77 & 93 \\
Nasal & 73 & 94
\end{tabular}

Figure 5.3.62. Is an objective test to diagnose aspirin exacerbated respiratory disease essential for the diagnosis N-ERD in a patient with CRS?

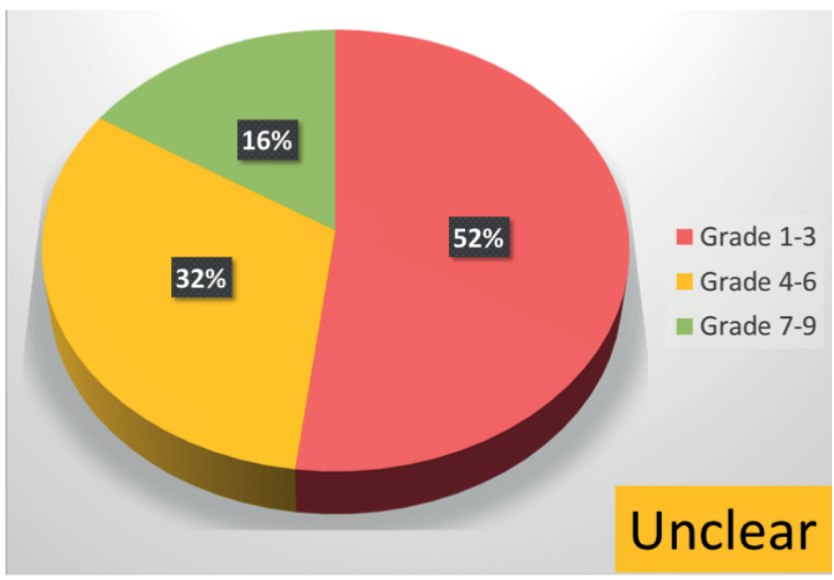




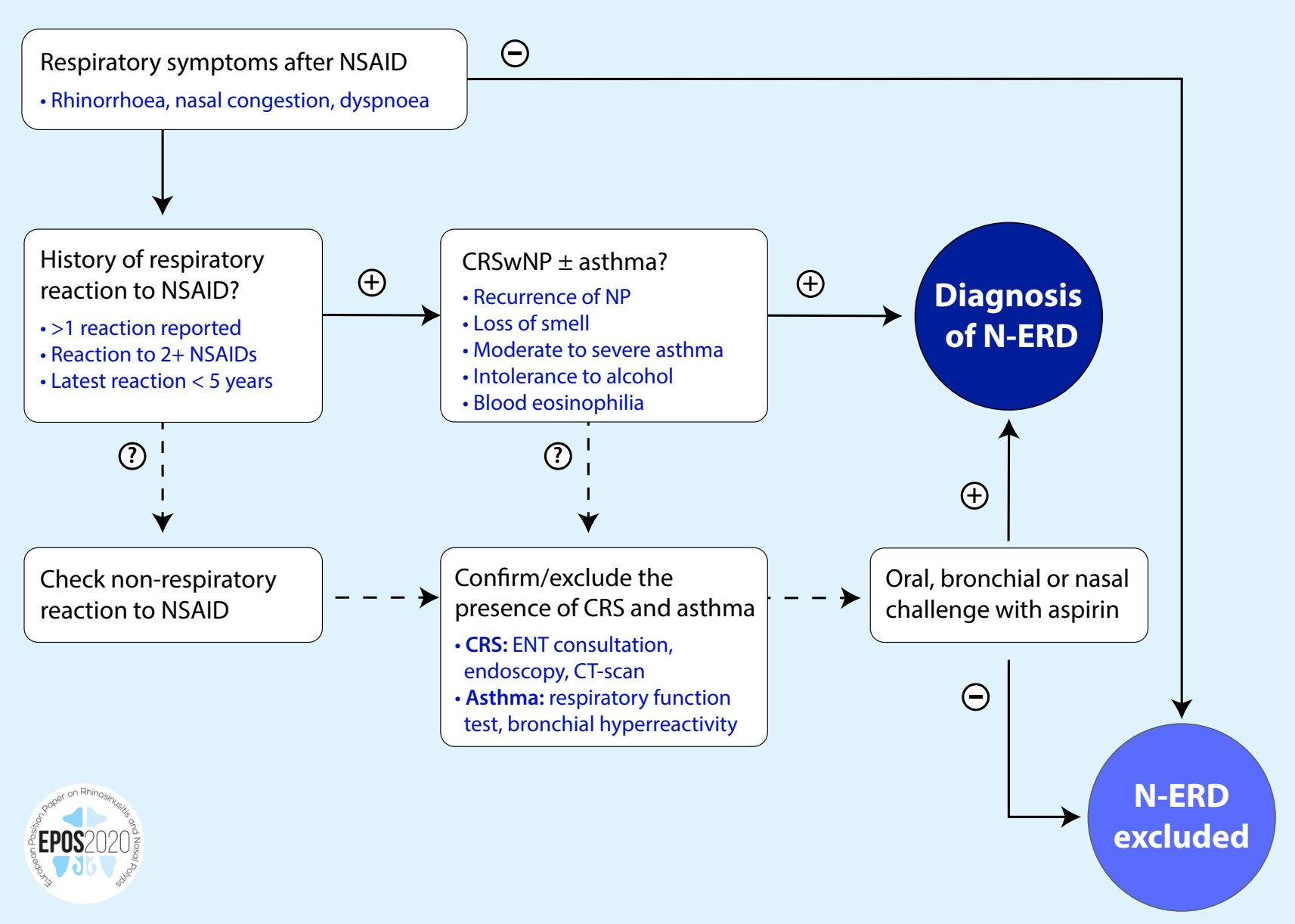

Figure 5.3.63. Is a clinical history of two episodes of respiratory reaction to aspirin or NSAIDs essential for the diagnosis N-ERD in a patient with CRS?

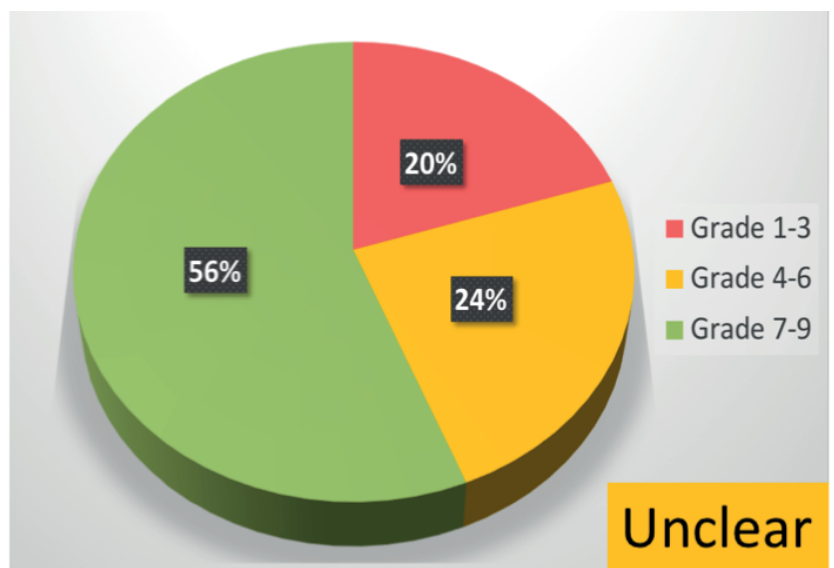

Figure 5.3.64. Is a clinical history of one episode of respiratory reaction to aspirin or NSAIDs essential for the diagnosis N-ERD in a patient with CRS?

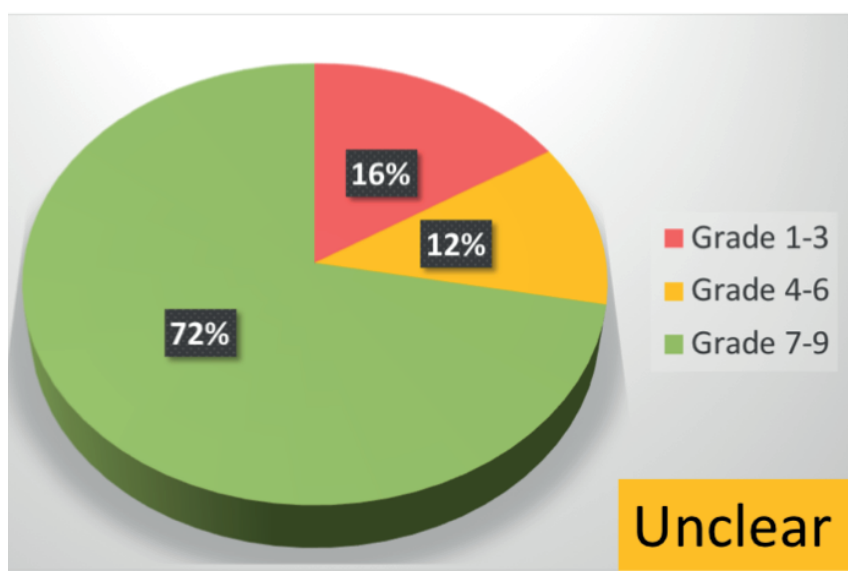


5.3.5.15.4. Recommendations

All patients with CRS should be asked about reactions to aspirin and NSAIDs.

At least one documented reaction to aspirin or NSAIDs is required to make the diagnosis of N-ERD though history alone is not always reliable. Aspirin provocation tests are needed when the history is not clear.

Nasal challenge with lysine aspirin is a good alternative to oral and bronchial methods, in terms of safety, sensitivity and specificity.

\section{References}

1. Fokkens WJ, Lund VJ, Mullol J, et al. Eur Position Paper on Rhinosinusitis and Nasal Polyps 2012. Rhinol Suppl 2012;23:3 p preceding table of contents, 1-298.

2. Pleis JR, Lucas JW, Ward BW. Summary health statistics for U.S. adults: National Health Interview Survey, 2008. Vital and health statistics Series 10, Data from the National Health Survey 2009:1-157.

3. Pilan RR, Pinna FR, Bezerra TF, et al. Prevalence of chronic rhinosinusitis in Sao Paulo. Rhinology 2012;50:129-38.

4. Shi JB, Fu QL, Zhang H, et al. Epidemiology of chronic rhinosinusitis: results from a cross-sectional survey in seven Chinese cities. Allergy 2015;70:533-9.

5. Kim JH, Cho C, Lee EJ, Suh YS, Choi BI, Kim KS. Prevalence and risk factors of chronic rhinosinusitis in South Korea according to diagnostic criteria. Rhinology 2016;54:32935.

6. Hirsch AG, Stewart WF, Sundaresan AS, et al. Nasal and sinus symptoms and chronic rhinosinusitis in a population-based sample. Allergy 2017;72:274-81.

7. Hoffmans R, Wagemakers A, van Drunen C, Hellings P, Fokkens W. Acute and chronic rhinosinusitis and allergic rhinitis in relation to comorbidity, ethnicity and environment. Plos one 2018;13:e0192330.

8. Ostovar A, Fokkens WJ, Vahdat K, Raeisi A, Mallahzadeh A, Farrokhi S. Epidemiology of chronic rhinosinusitis in Bushehr, southwestern region of Iran: a GA2LEN study. Rhinology 2019;57:43-8.

9. Tomassen P, Newson RB, Hoffmans R, et al. Reliability of EP3OS symptom criteria and nasal endoscopy in the assessment of chronic rhinosinusitis--a GA(2) LEN study. Allergy 2011;66:556-61.

10. Dietz de Loos D, Lourijsen ES, Wildeman MAM, et al. Prevalence of chronic rhinosinusitis in the general population based on sinus radiology and symptomatology. J Allergy Clin Immunol 2019;143:1207-14.

11. Hirsch AG, Nordberg C, Bandeen-Roche K, et al. Radiologic sinus inflammation and symptoms of chronic rhinosinusitis in a population-based sample. Allergy. 2019 Nov 11. doi: 10.1111/all.14106.

12. Liu CC, Lui J, Oddone Paolucci E, Rudmik L. Systematic review of the quality of economic evaluations in the otolaryngology literature. Otolaryngol Head Neck Surg 2015;152:106-15.

13. Rudmik L, Xu Y, Kukec E, Liu M, Dean S, Quan H. A validated case definition for chronic rhinosinusitis in administrative data: a Canadian perspective. Int Forum Allergy Rhinol 2016;6:1167-72.

14. Shashy RG, Moore EJ, Weaver A. Prevalence of the chronic sinusitis diagnosis in Olmsted County, Minnesota. Arch Otolaryngol Head Neck Surg 2004;130:320-3.

15. Xu Y, Quan H, Faris P, et al. Prevalence and Incidence of Diagnosed Chronic Rhinosinusitis in Alberta, Canada. JAMA Otolaryngology-Head \& Neck Surgery 2016;142:1063.

16. Hoffmans R, Schermer T, van der Linde $K$, et al. Rhinosinusitis in morbidity registrations in Dutch General Practice: a retrospective case-control study. BMC Fam Pract 2015;16:120.

17. Larsen K, Tos M. The estimated incidence of symptomatic nasal polyps. Acta Otolaryngol 2002;122:179-82.

18. Johansson L, Akerlund A, Holmberg K, Melen I, Bende M. Prevalence of nasal polyps in adults: the Skovde populationbased study. Ann Otol Rhinol Laryngol 2003;112:625-9.

19. Tan BK, Chandra RK, Pollak J, et al. Incidence and associated premorbid diagnoses of patients with chronic rhinosinusitis. J Allergy Clin Immunol 2013;131:1350-60.

20. Won HK, Kim YC, Kang MG, et al. Agerelated prevalence of chronic rhinosinusitis and nasal polyps and their relationships with asthma onset. Ann Allergy Asthma Immunol 2018;120:389-94.

21. Soler ZM, Mace JC, Litvack JR, Smith TL. Chronic Rhinosinusitis, Race, and Ethnicity. Am J Rhinol Allergy 2012;26:110-6.

22. Brescia G, Barion U, Pedruzzi B, et al. Sinonasal polyposis in the elderly. Am J Rhinol Allergy 2016;30:153-6.

23. Cho SH, Hong SJ, Han B, et al. Age-related differences in the pathogenesis of chronic rhinosinusitis. J Allergy Clin Immunol 2012, 10.1016/j.jaci.2011.12.002

24. Wilson KF, McMains KC, Orlandi RR. The association between allergy and chronic rhinosinusitis with and without nasal polyps: an evidence-based review with recommendations. Int Forum Allergy Rhinol 2014;4:93-103.

25. Marcus S, Schertzer J, Roland LT, Wise SK, Levy JM, DelGaudio JM. Central compartment atopic disease: prevalence of allergy and asthma compared with other subtypes of chronic rhinosinusitis with nasal polyps. Int Forum Allergy Rhinol 2019, 10.1002/ alr.22454.

26. Philpott CM, Erskine S, Hopkins C, et al. Prevalence of asthma, aspirin sensitivity and allergy in chronic rhinosinusitis: data from the UK National Chronic Rhinosinusitis Epidemiology Study. Respiratory research 2018;19:129.

27. Seybt MW, McMains KC, Kountakis SE. The prevalence and effect of asthma on adults with chronic rhinosinusitis. Ear Nose Throat J 2007;86:409-11.

28. Jarvis D, Newson R, Lotvall J, et al. Asthma in adults and its association with chronic rhinosinusitis: the GA2LEN survey in Europe. Allergy 2012;67:91-8.

29. Obaseki D, Potts J, Joos G, et al. The relation of airway obstruction to asthma, chronic rhinosinusitis and age: results from a population survey of adults. Allergy 2014;69:1205-14.

30. Chen YT, Chien CY, Tai SY, Huang CM, Lee CT. Asthma associated with chronic rhinosinusitis: a population-based study. Int Forum Allergy Rhinol 2016;6:1284-93.

31. Yoshimura K, Kawata R, Haruna S, et al. Clin epidemiological study of 553 patients with chronic rhinosinusitis in Japan. Allergology Int.2011;60:491-6.

32. Klossek JM, Neukirch F, Pribil C, et al. Prevalence of nasal polyposis in France: a cross-sectional, case-control study. Allergy 2005;60:233-7.

33. Tint D, Kubala S, Toskala E. Risk Factors and Comorbidities in Chronic Rhinosinusitis. Curr Allergy Asthma Rep 2016;16:16.

34. Phillips KM, Talat R, Caradonna DS, Gray ST, Sedaghat AR. Quality of life impairment due to chronic rhinosinusitis in asthmatics is mediated by asthma control. Rhinology 2019;57:430-5.

35. Milkowska-Dymanowska J, Bialas AJ, Zalewska-Janowska A, Gorski P, Piotrowski WJ. Underrecognized comorbidities of chronic obstructive pulmonary disease. Int J Chron Obstruct Pulmon Dis 2015;10:133141.

36. Khan A, Vandeplas G, Huynh TMT, et al. The Global Allergy and Asthma Eur Network (GALEN rhinosinusitis cohort: a large Eur cross-sectional study of chronic rhinosinusitis patients with and without nasal polyps. Rhinology 2019;57:32-42.

37. Chien C-Y, Tai S-Y, Wang L-F, Lee CT-C. Chronic obstructive pulmonary disease predicts chronic rhinosinusitis without nasal polyps: A population-based study. Am J Rhinol Allergy 2015;29:e75-80.

38. Guilemany JM, Angrill J, Alobid I, et al. United airways again: high prevalence of rhinosinusitis and nasal polyps in bronchiectasis. Allergy 2009;64:790-7.

39. Kowalski ML, Agache I, Bavbek S, et al. Diagnosis and management of NSAID- 
Exacerbated Respiratory Disease (N-ERD)-a EAACl position paper. Allergy 2019;74:28-39.

40. Berges-Gimeno MP, Simon RA, Stevenson DD. The natural history and Clin characteristics of aspirin-exacerbated respiratory disease. Ann Allergy Asthma Immunol 2002;89:474-8.

41. Stevens WW, Schleimer RP. AspirinExacerbated Respiratory Disease as an Endotype of Chronic Rhinosinusitis. Immunology and allergy clinics of North America 2016;36:669-80.

42. Morales DR, Guthrie B, Lipworth BJ, Jackson C, Donnan PT, Santiago VH. NSAIDexacerbated respiratory disease: a metaanalysis evaluating prevalence, mean provocative dose of aspirin and increased asthma morbidity. Allergy 2015;70:828-35.

43. Makowska JS, Burney P, Jarvis D, et al. Respiratory hypersensitivity reactions to NSAIDs in Europe: the global allergy and asthma network (GA2 LEN) survey. Allergy 2016;71:1603-11.

44. Philpott CM, Smith R, Davies-Husband CR, et al. Exploring the association between ingestion of foods with higher potential salicylate content and symptom exacerbation in chronic rhinosinusitis. Data from the National Chronic Rhinosinusitis Epidemiology Study. Rhinology 2019, 10.4193/Rhin19.027.

45. Stevens WW, Peters AT, Hirsch AG, et al. Clin Characteristics of Patients with Chronic Rhinosinusitis with Nasal Polyps, Asthma, and Aspirin-Exacerbated Respiratory Disease. J Allergy Clin Immunol Pract 2017;5:1061-70.e3.

46. Schwitzguébel AJ-P, Jandus $P$, Lacroix $J$-SS, et al. Immunoglobulin deficiency in patients with chronic rhinosinusitis: Systematic review of the literature and meta-analysis. J Allergy Clin Immunol 2015;136:1523-31.

47. Mazza JM, Lin SY. Primary immunodeficiency and recalcitrant chronic sinusitis: a systematic review. Int Forum Allergy Rhinol 2016;6:1029-33.

48. Nayan S, Alizadehfar R, Desrosiers M. Humoral Primary Immunodeficiencies in Chronic Rhinosinusitis. Curr Allergy Asthma Rep 2015;15:46.

49. Ocampo CJ, Peters AT. Antibody deficiency in chronic rhinosinusitis: epidemiology and burden of illness. Am J Rhinol Allergy 2013;27:34-8.

50. Leason SR, Barham HP, Oakley G, et al. Association of gastro-oesophageal reflux and chronic rhinosinusitis: systematic review and meta-analysis. Rhinology 2017:55:3-16.

51. Katle EJ, Hatlebakk JG, Grimstad T, Kvaloy JT, Steinsvag SK. Gastro-oesophageal reflux in patients with chronic rhino-sinusitis investigated with multichannel impedance - pH monitoring. Rhinology 2017;55:27-33.

52. Sella GCP, Tamashiro E, Anselmo-Lima WT, Valera FCP. Relation between chronic rhinosinusitis and gastroesophageal reflux in adults: systematic review. Revista Brasileira de Otorrinolaringologia 2017;83:356-63.

53. Morinaka S, Ichimiya M, Nakamura H. Detection of Helicobacter pylori in nasal and maxillary sinus specimens from patients with chronic sinusitis. Laryngoscope 2003;113:1557-63.

54. Lin YH, Chang TS, Yao YC, Li YC. Increased Risk of Chronic Sinusitis in Adults With Gastroesophgeal Reflux Disease: A Nationwide Population-Based Cohort Study. Medicine (Baltimore) 2015;94:e1642.

55. Bohnhorst I, Jawad S, Lange B, Kjeldsen J, Hansen JM, Kjeldsen AD. Prevalence of chronic rhinosinusitis in a population of patients with gastroesophageal reflux disease. Am J Rhinol Allergy 2015;29:e70-4.

56. Orlandi RR. A systematic analysis of septal deviation associated with rhinosinusitis. Laryngoscope 2010;120:1687-95.

57. Tiwari R, Goyal R. Study of Anatomical Variations on CT in Chronic Sinusitis. Indian J Otolaryngol Head Neck Surg 2015;67:1820.

58. Sedaghat AR, Gray ST, Chambers KJ, Wilke $\mathrm{CO}$, Caradonna DS. Sinonasal anatomic variants and asthma are associated with faster development of chronic rhinosinusitis in patients with allergic rhinitis. Int Forum Allergy Rhinol 2013;3:755-61.

59. Kim Y-C, Won H-K, Lee JW, et al. Staphylococcus aureus Nasal Colonization and Asthma in Adults: Systematic Review and Meta-Analysis. The J Allergy Clin Immunolln practice 2019;7:606-15.e9.

60. Wagner Mackenzie B, Chang K, Zoing M, et al. Longitudinal study of the bacterial and fungal microbiota in the human sinuses reveals seasonal and annual changes in diversity. Sci Rep 2019;9:17416.

61. Cryer J, Schipor I, Perloff JR, Palmer JN Evidence of bacterial biofilms in human chronic sinusitis. Orl 2004;66:155-8.

62. Ramakrishnan Y, Shields RC, Elbadawey MR, Wilson JA. Biofilms in chronic rhinosinusitis: what is new and where next? The J laryngology and otology 2015;129:744-51.

63. Maina IW, Patel NN, Cohen NA. Understanding the Role of Biofilms and Superantigens in Chronic Rhinosinusitis. Curr Otorhinolaryngol Rep 2018;6:253-62.

64. 64. Singhal D, Psaltis AJ, Foreman A, Wormald PJ. The impact of biofilms on outcomes after endoscopic sinus surgery. Am J Rhinol Allergy 2010;24:169-74

65. Zhang J, Li Y, Lu X, et al. Distinguishing the dominant species of pathogen in maxillary sinusitis by sequencing DNA dataset analysis. Gene 2015;561:256-60.

66. Rowan NR, Lee S, Sahu N, et al. The role of viruses in the Clin presentation of chronic rhinosinusitis. Am J Rhinol Allergy 2015;29:e197-200

67. Pawełczyk M, Kowalski ML. The Role of Human Parainfluenza Virus Infections in the Immunopathology of the Respiratory Tract. Curr Allergy Asthma Rep 2017;17:16.

68. Guan W-j, Peng Y, Zi X-X, et al. Motile Ciliary Disorders in Chronic Airway Inflammatory Diseases: Critical Target for Interventions.
Curr Allergy Asthma Rep 2018;18:48.

69. Gudis D, Zhao K-q, Cohen NA. Acquired cilia dysfunction in chronic rhinosinusitis. Am J Rhinol Allergy 2012;26:1-6.

70. Hastan D, Fokkens WJ, Bachert C, et al. Chronic rhinosinusitis in Europe--an underestimated disease. A GA(2)LEN study. Allergy 2011;66:1216-23.

71. Christensen DN, Franks ZG, McCrary HC, Saleh AA, Chang EH. A Systematic Review of the Association between Cigarette Smoke Exposure and Chronic Rhinosinusitis. Otolaryngol Head Neck Surg 2018;158:80116.

72. Hur K, Liang J, Lin SY. The role of secondhand smoke in sinusitis: a systematic review. Int Forum Allergy Rhinol 2014;4:228.

73. Weakley J, Hall CB, Liu X, et al. The effect of World Trade Center exposure on the latency of chronic rhinosinusitis diagnoses in New York City firefighters: 2001-2011. Occup Environ Med 2016;73:280-3.

74. Mady LJ, Schwarzbach HL, Moore JA, et al. Air pollutants may be environmental risk factors in chronic rhinosinusitis disease progression. Int Forum Allergy Rhinol 2018;8:377-84

75. Geramas I, Terzakis D, Hatzimanolis E, Georgalas C. Social Factors in the Development of Chronic Rhinosinusitis: a Systematic Review. Current Allergy \& Asthma Reports 2018;18:7.

76. Sundaresan AS, Hirsch AG, Storm M, et al. Occupational and environmental risk factors for chronic rhinosinusitis: a systematic review. Int Forum Allergy Rhinol 2015;5:9961003.

77. Clarhed UKE, Svendsen M, Schioler L, et al. Chronic Rhinosinusitis Related to Occupational Exposure: The Telemark Population Study. J Occup Environ Med 2018;60:656-60

78. Gao WX, Ou CQ, Fang SB, et al. Occupational and environmental risk factors for chronic rhinosinusitis in China: a multicentre crosssectional study. Respir Res 2016;17:54.

79. Veloso-Teles R, Cerejeira R, Roque-Farinha $\mathrm{R}$, von Buchwald C. Higher prevalence of nasal polyposis among textile workers: an endoscopic based and controlled study. Rhinology 2018, 10.4193/Rhin17.228.

80. Ji K, Risoli TJ, Kuchibhatla M, Chan L, Hachem RA, Jang DW. Symptom Profile of Chronic Rhinosinusitis Versus Obstructive Sleep Apnea in a Tertiary Rhinology Clinic. Ann Otol Rhinol Laryngol 2019;128:963-9.

81. Kao L-T, Hung S-H, Lin H-C, Liu C-K, Huang $\mathrm{H}-\mathrm{M}$, Wu C-S. Obstructive Sleep Apnea and the Subsequent Risk of Chronic Rhinosinusitis: A Population-Based Study. Scientific Reports 2016;6:20786.

82. Jiang RS, Liang KL, Hsin CH, Su MC. The impact of chronic rhinosinusitis on sleep-disordered breathing. Rhinology 2016;54:75-9.

83. Lee EJ, Hwang HJ, Jung CM, Kim MK, Kang MS, Kim K-S. The relationship between chronic rhinosinusitis and metabolic syn- 
drome. Am J Rhinol Allergy 2017;31:222-7.

84. Ahn J-C, Kim J-W, Lee CH, Rhee C-S Prevalence and Risk Factors of Chronic Rhinosinusitus, Allergic Rhinitis, and Nasal Septal Deviation: Results of the Korean National Health and Nutrition Survey 20082012. JAMA otolaryngology-- head \& neck surgery 2016;142:162-7.

85. Stokes PJ, Rimmer J. The relationship between serum vitamin D and chronic rhinosinusitis: A systematic review. Am J Rhinol Allergy 2016;30:23-8.

86. De Schryver E, Derycke L, Campo P, et al. Alcohol hyper-responsiveness in chronic rhinosinusitis with nasal polyps. Clin Exp Allergy 2016, 10.1111/cea.12836.

87. Kuiper JR, Hirsch AG, Bandeen-Roche K, et al. Prevalence, severity, and risk factors for acute exacerbations of nasal and sinus symptoms by chronic rhinosinusitis status. Allergy 2018;73:1244-53.

88. Phillips KM, Barbarite E, Hoehle LP, Caradonna DS, Gray ST, Sedaghat AR. Clin Traits Characterizing an ExacerbationProne Phenotype in Chronic Rhinosinusitis. Otolaryngology-Head and Neck Surgery 2019;161:890-6

89. Banoub RG, Phillips KM, Hoehle LP, Caradonna DS, Gray ST, Sedaghat AR Relationship between chronic rhinosinusitis exacerbation frequency and asthma control. Laryngoscope 2018;128:1033-8.

90. Phillips KM, Hoehle LP, Bergmark RW, et al. Chronic rhinosinusitis severity is associated with need for asthma-related systemic corticosteroids. Rhinology 2017;55:211-7.

91. Tan KS, Yan Y, Ong HH, Chow VTK, Shi L, Wang D-Y. Impact of Respiratory Virus Infections in Exacerbation of Acute and Chronic Rhinosinusitis. Curr Allergy Asthma Rep 2017;17:24.

92. Flores-Torres AS, Salinas-Carmona MC, Salinas E, Rosas-Taraco AG. Eosinophils and Respiratory Viruses. Viral Immunol 2019;32:198-207.

93. Basharat U, Aiche MM, Kim MM, Sohal M, Chang $\mathrm{EH}$. Are rhinoviruses implicated in the pathogenesis of sinusitis and chronic rhinosinusitis exacerbations? A comprehensive review. Int Forum Allergy Rhinol 2019;9:1159-88

94. Hofstra JJ, Matamoros S, van de Pol MA, et al. Changes in microbiota during experimental human Rhinovirus infection. BMC Infect Dis 2015;15:336.

95. Brook I. The role of anaerobic bacteria in sinusitis. Anaerobe 2006;12:5-12.

96. Vandelaar LJ, Hanson B, Marino M, et al. Analysis of Sinonasal Microbiota in Exacerbations of Chronic Rhinosinusitis Subgroups. OTO Open 2019:3:2473974x19875100.

97. Szaleniec J, Gibala A, Pobiega M, et al. Exacerbations of Chronic RhinosinusitisMicrobiology and Perspectives of Phage Therapy. Antibiotics (Basel) 2019;8.

98. Collins FS, Morgan M, Patrinos A. The Human Genome Project: lessons from large-scale biology. Science 2003;300:286-
90

99. Davies G, Lam M, Harris SE, et al. Study of 300,486 individuals identifies 148 independent genetic loci influencing general cognitive function. Nat Commun 2018;9:2098.

100.Bergen S, Petryshen T. Genome-wide association studies of schizophrenia: Does bigger lead to better results? Curr Oppsychiatry 2012;25:76-82.

101. Sekar A, Bialas AR, de Rivera $H$, et al. Schizophrenia risk from complex variation of complement component 4. Nature 2016:530:177-83.

102. Cormier C, Endam LM, Filali-Mouhim A, et al. A pooling-based genomewide association study identifies genetic variants associated with Staphylococcus aureus colonization in chronic rhinosinusitis patients. Int Forum Allergy Rhinol 2014;4:207-15.

103. Karavani E, Zuk O, Zeevi D, et al. Screening Human Embryos for Polygenic Traits Has Limited Utility. Cell 2019;179:1424-35.e8.

104. Valera FCP, Ruffin M, Adam D, et al. Staphylococcus aureus impairs sinonasal epithelial repair: Effects in patients with chronic rhinosinusitis with nasal polyps and control subjects. J Allergy Clin Immunol 2019;143:591-603.e3.

105.Zariwala MA, Knowles MR, Leigh MW. Primary Ciliary Dyskinesia. 2007 Jan 24 [Updated 2019 Dec 5]. In: Adam MP Ardinger $\mathrm{HH}$, Pagon RA, et al., editors. GeneReviews $^{\circledR}$ [Internet]. Seattle (WA) University of Washington, Seattle; 19932020. Available from: https://www.ncbi.nlm nih.gov/books/NBK1122/.

106. Oakley GM, Curtin K, Orb Q, Schaefer C, Orlandi RR, Alt JA. Familial risk of chronic rhinosinusitis with and without nasal polyposis: genetics or environment. Int Forum Allergy Rhinol 2015;5:276-82.

107. Bohman A, Oscarsson M, Holmberg K, et al. Heredity of nasal polyps. Rhinology 2015;53:25-8

108. Weinhold B. Epigenetics: the science of change. Environmental health perspectives 2006;114:A160-A7.

109. Kim J-Y, Kim D-K, Yu MS, Cha M-J, Yu S-L, Kang J. Role of epigenetics in the pathogenesis of chronic rhinosinusitis with nasal polyps. Molecular medicine reports 2018;17:1219-27.

110.Kidoguchi M, Noguchi E, Nakamura $T$, et al. DNA Methylation of Proximal PLAT Promoter in Chronic Rhinosinusitis With Nasal Polyps. Am J Rhinol Allergy 2018;32:374-9.

111. Endam LM, Filali-Mouhim A, Boisvert $P$ Boulet L-P, Bosse Y, Desrosiers M. Genetic variations in taste receptors are associated with chronic rhinosinusitis: a replication study. Int Forum Allergy Rhinol 2014;4:200 6.

112. Ordovas-Montanes J, Dwyer DF, Nyquist SK, et al. Allergic inflammatory memory in human respiratory epithelial progenitor cells. Nature 2018:560:649-54.

113. Seiberling KA, Church CA, Herring JL,
Sowers LC. Epigenetics of chronic rhinosinusitis and the role of the eosinophil. Int Forum Allergy Rhinol 2012;2:80-4.

114. Heimall JR, Hagin D, Hajjar J, et al. Use of Genetic Testing for Primary Immunodeficiency Patients. J Clin Immunol 2018;38:320-9.

115.Zhang SY, Jouanguy E, Ugolini S, et al. TLR3 deficiency in patients with herpes simplex encephalitis. Science 2007:317:1522-7.

116. Adappa ND, Zhang Z, Palmer JN, et al. The bitter taste receptor T2R38 is an independent risk factor for chronic rhinosinusitis requiring sinus surgery. Int Forum Allergy Rhinol 2014;4:3-7.

117. Purnell PR, Addicks BL, Zalzal HG, et al. Single Nucleotide Polymorphisms in Chemosensory Pathway Genes GNB3, TAS2R19, and TAS2R38 Are Associated with Chronic Rhinosinusitis. Int Arch Allergy Immunol 2019;180:72-8.

118. Walker AP, Fodor E. Interplay between Influenza Virus and the Host RNA Polymerase II Transcriptional Machinery. Trends in Microbiology 2019;27:398-407.

119. Meng J, Zhou P, Liu Y, et al. The development of nasal polyp disease involves early nasal mucosal inflammation and remodelling. Plos one 2013;8:e82373.

120. Hoggard M, Mackenzie BW, Jain R, Taylor MW, Biswas K, Douglas RG. Chronic Rhinosinusitis and the Evolving Understanding of Microbial Ecology in Chronic Inflammatory Mucosal Disease. Clin microbiology reviews 2017;30:321-48.

121. Abshirini $H$, Makvandi M, Ashrafi MS Hamidifard M, Saki N. Prevalence of rhinovirus and respiratory syncytial virus among patients with chronic rhinosinusitis. Jundishapur J microbiology 2015;8:e20068.

122. Chen WJ, Arnold JC, Fairchok MP, et al. Epidemiologic, Clin, and virologic characteristics of human rhinovirus infection among otherwise healthy children and adults. Rhinovirus among adults and children. J Clin Virology 2015, 10.1016/j.jcv.2015.01.007.

123. Bønnelykke K, Sleiman P, Nielsen K, et al. A genome-wide association study identifies CDHR3 as a susceptibility locus for early childhood asthma with severe exacerbations. Nature Genetics 2013;46:51.

124.Chang $\mathrm{EH}$, Willis $\mathrm{AL}$, McCrary HC, et al. Association between the CDHR3 rs6967330 risk allele and chronic rhinosinusitis. J Allergy Clin Immunol 2017;139:1990-2.e2.

125. Carlson-Jones J, Paterson JS, Newton $K$, et al. Enumerating Virus-Like Particles and Bacterial Populations in the Sinuses of Chronic Rhinosinusitis Patients Using Flow Cytometry. PloS one 2016;11:e0155003.

126. Tengroth L, Arebro J, Georen SK, Winqvist O, Cardell L-O. Deprived TLR9 expression in apparently healthy nasal mucosa might trigger polyp-growth in chronic rhinosinusitis patients. PloS one 2014;9:e105618.

127. Golebski K, van Tongeren J, van Egmond D, de Groot EJ, Fokkens WJ, van Drunen CM. Specific Induction of TSLP by the Viral RNA Analogue Poly(l:C) in Primary Epithelial 
Cells Derived from Nasal Polyps. PLoS One 2016;11:e0152808.

128. Liao B, Hu C-Y, Liu T, Liu Z. Respiratory viral infection in the chronic persistent phase of chronic rhinosinusitis. Laryngoscope 2014;124:832-7.

129. loannidis D, Lachanas VA, Florou Z, Bizakis JG, Petinaki E, Skoulakis CE. Herpes viruses and human papilloma virus in nasal polyposis and controls. Braz J Otorhinolaryngol 2015;81:658-62.

130. Ha SY, Park S. The prevalence of EpsteinBarr Virus-positive lymphoid cells in nasa mucosa: an extremely rare event. Rhinology 2014:52:403-5.

131. Wang X, Zhang N, Glorieux S, et al. Herpes simplex virus type 1 infection facilitates invasion of Staphylococcus aureus into the nasal mucosa and nasal polyp tissue. Plos one 2012;7:e39875.

132. Knight R, Vrbanac A, Taylor BC, et al. Best practices for analysing microbiomes. Nat Rev Microbiology 2018;16:410-22.

133. Earl JP, Adappa ND, Krol J, et al. Specieslevel bacterial community profiling of the healthy sinonasal microbiome using Pacific Biosciences sequencing of full-length 165 rRNA genes. Microbiome 2018;6:190.

134. Abreu NA, Nagalingam NA, Song Y, et al. Sinus microbiome diversity depletion and Corynebacterium tuberculostearicum enrichment mediates rhinosinusitis. Science translational medicine 2012;4:151 ra24

135. Gan W, Yang F, Tang Y, et al. The difference in nasal bacterial microbiome diversity between chronic rhinosinusitis patients with polyps and a control population. Int Forum Allergy Rhinol 2019, https://dx.doi. org/10.1002/alr.22297.

136. Lee K, Pletcher SD, Lynch SV, Goldberg AN, Cope EK. Heterogeneity of Microbiota Dysbiosis in Chronic Rhinosinusitis: Potential Clin Implications and Microbial Community Mechanisms Contributing to Sinonasal Inflammation. Frontiers in cellular and infection microbiology 2018;8:168.

137. Copeland E, Leonard K, Carney R, et al. Chronic Rhinosinusitis: Potential Role of Microbial Dysbiosis and Recommendations for Sampling Sites. Frontiers in cellular and infection microbiology 2018;8:57.

138.Zhao YC, Bassiouni A, Tanjararak K, Vreugde $\mathrm{S}$, Wormald P-J, Psaltis AJ. Role of fungi in chronic rhinosinusitis through ITS sequencing. Laryngoscope 2018;128:16-22.

139. Hoggard M, Biswas K, Zoing M, Mackenzie BW, Taylor MW, Douglas RG. Evidence of microbiota dysbiosis in chronic rhinosinusitis. Int Forum Allergy Rhinol 2017;7:230-9.

140. Karunasagar A, Jalastagi R, Naik A, Rai P. Detection of bacteria by 165 rRNA PCR and sequencing in culture-negative chronic rhinosinusitis. Laryngoscope 2018;128:2223-5.

141. Chalermwatanachai T, Vilchez-Vargas R, Holtappels G, et al. Chronic rhinosinusitis with nasal polyps is characterized by dysbacteriosis of the nasal microbiota. Scientific reports 2018;8:7926.

142. Lal D, Keim P, Delisle J, et al. Mapping and comparing bacterial microbiota in the sinonasal cavity of healthy, allergic rhinitis, and chronic rhinosinusitis subjects. Int Forum Allergy Rhinol 2017;7:561-9.

143. Kim AS, Willis AL, Laubitz D, et al. The effect of maxillary sinus antrostomy size on the sinus microbiome. Int Forum Allergy Rhinol 2019:9:30-8

144. Ramakrishnan VR, Hauser LJ, Feazel LM, Ir D, Robertson CE, Frank DN. Sinus microbiota varies among chronic rhinosinusitis phenotypes and predicts surgical outcome. J Allergy Clin Immunol 2015;136:334-42.e1.

145. Joss TV, Burke CM, Hudson BJ, et al. Bacterial Communities Vary between Sinuses in Chronic Rhinosinusitis Patients. Frontiers in microbiology 2015;6:1532.

146. Jain R, Hoggard M, Biswas K, Zoing M, Jiang $Y$, Douglas R. Changes in the bacterial microbiome of patients with chronic rhinosinusitis after endoscopic sinus surgery. Int Forum Allergy Rhinol 2017;7:7-15.

147. Cope EK, Goldberg AN, Pletcher SD, Lynch SV. Compositionally and functionally distinct sinus microbiota in chronic rhinosinusitis patients have immunological and Clinly divergent consequences. Microbiome 2017;5:53.

148. Liu Q, Lu X, Bo M, Qing H, Wang X, Zhang L. The microbiology of chronic rhinosinusitis with and without nasal polyps. Acta OtoLaryngologica 2014;134:1251-8.

149. Wei H-Z, Li Y-C, Wang X-D, et al. The microbiology of chronic rhinosinusitis with and without nasal polyps. Eur Arch Otorhinolaryngol. 2018;275(6):1439-1447.

150. Tabet P, Endam LM, Boisvert P, Boulet L-P, Desrosiers M. Gram-negative bacterial carriage in chronic rhinosinusitis with nasal polyposis is not associated with more severe inflammation. Int Forum Allergy Rhinol 2015:5:289-93.

151.Drilling A, Coombs GW, Tan $\mathrm{H}-\mathrm{l}$, et al. Cousins, siblings, or copies: the genomics of recurrent Staphylococcus aureus infections in chronic rhinosinusitis. Int Forum Allergy Rhinol 2014;4:953-60.

152. Ou J, Bassiouni A, Drilling A, Psaltis AJ, Vreugde S, Wormald PJ. The persistence of intracellular Staphylococcus aureus in the sinuses: a longitudinal study. Rhinology 2017;55:305-11.

153. Tomassen P, Vandeplas G, Van Zele T, et al. Inflammatory endotypes of chronic rhinosinusitis based on cluster analysis of biomarkers. J Allergy Clin Immunol 2016;137:144956.e4.

154. Hauser LJ, Feazel LM, Ir D, et al. Sinus culture poorly predicts resident microbiota. Int Forum Allergy Rhinol 2015;5:3-9.

155. Dlugaszewska J, Leszczynska M, Lenkowski M, Tatarska A, Pastusiak T, Szyfter W. The pathophysiological role of bacterial biofilms in chronic sinusitis. Eur Arch Otorhinolaryngol. 2016;273:1989-94.

156. Wilson C, Lukowicz R, Merchant S, et al. Quantitative and Qualitative Assessment Methods for Biofilm Growth: A Mini-review. Research \& reviews J engineering and tech- nology 2017;6:http://www.rroij.com/openaccess/quantitative-and-.

157. Arild Danielsen K, Eskeland $\varnothing$, Fridrich-Aas K, Cecilie Orszagh V, Bachmann-Harildstad G, Burum-Auensen E. Bacterial biofilms in chronic rhinosinusitis; distribution and prevalence. Acta Oto-Laryngologica 2016;136:109-12.

158. Wang X, Du J, Zhao C. Bacterial biofilms are associated with inflammatory cells infiltration and the innate immunity in chronic rhinosinusitis with or without nasal polyps. Inflammation 2014;37:871-9.

159. Sun Y, Zhou B, Wang C-s, et al. Clin and histopathologic features of biofilm-associated chronic rhinosinusitis with nasal polyps in Chinese patients. Chinese medical journal 2012;125:1104-9.

160.Dong D, Yulin Z, Xiao W, et al. Correlation between bacterial biofilms and osteitis in patients with chronic rhinosinusitis. Laryngoscope 2014;124:1072-8.

161. Marcinkiewicz J, Strek P, Strus M, et al. Staphylococcus epidermidis and biofilmassociated neutrophils in chronic rhinosinusitis. A pilot study. Int J experimental pathology 2015;96:378-86.

162. Arjomandi H, Gilde J, Zhu S, et al. Relationship of eosinophils and plasma cells to biofilm in chronic rhinosinusitis. Am J Rhinol Allergy 2013;27:85-90.

163. Teufelberger AR, Bröker BM, Krysko DV, Bachert C, Krysko O. <em>Staphylococcus aureus $</$ em $>$ Orchestrates Type 2 Airway Diseases. Trends in Molecular Medicine 2019, 10.1016/j.molmed.2019.05.003.

164. Feazel LM, Robertson CE, Ramakrishnan VR, Frank DN. Microbiome complexity and Staphylococcus aureus in chronic rhinosinusitis. Laryngoscope 2012;122:467-72.

165. Tan NC-W, Foreman A, Jardeleza C, Douglas R, Vreugde S, Wormald P-J. Intracellular Staphylococcus aureus: the Trojan horse of recalcitrant chronic rhinosinusitis? Int Forum Allergy Rhinol 2013;3:261-6.

166. Thunberg U, Hugosson S, Monecke S, Ehricht R, Soderquist B. Molecular characteristics of Staphylococcus aureus associated with chronic rhinosinusitis. APMIS : Acta Pathologica, Microbiologica, et Immunologica Scandinavica 2015;123:3744.

167. Voehringer D. Protective and pathological roles of mast cells and basophils. Nature Rev Immunol 2013;13:362-75.

168.Cheng K-J, Wang S-Q, Xu Y-Y. Different roles of Staphylococcus aureus enterotoxin in different subtypes of nasal polyps. Experimental and therapeutic medicine 2017;13:321-6.

169. Ba L, Zhang N, Meng J, et al. The association between bacterial colonization and inflammatory pattern in Chinese chronic rhinosinusitis patients with nasal polymps. Allergy 2011;66:1296-303

170.Lan F, Zhang N, Holtappels G, et al. Staphylococcus aureus Induces a Mucosal Type 2 Immune Response via Epithelial Cell-derived Cytokines. Am J respiratory and 
critical care medicine 2018;198:452-63.

171. Bachert C, Holtappels G, Merabishvili M, et al. Staphylococcus aureus controls interleukin-5 release in upper airway inflammation. J proteomics 2018;180:53-60.

172. Takeda K, Sakakibara S, Yamashita K, et al. Allergic conversion of protective mucosa immunity against nasal bacteria in patients with chronic rhinosinusitis with nasal polyposis. The J Allergy Clin Immuno 2019;143:1163-75.e15.

173. Zhang J, He S, Li Y, et al. Distinguishing the dominant species of pathogen in ethmoidal sinusitis by sequencing DNA dataset analysis. Experimental and therapeutic medicine 2018;16:4207-12.

174. Hoggard M, Zoing M, Biswas K, Taylor MW, Douglas RG. The sinonasal mycobiota in chronic rhinosinusitis and control patients. Rhinology 2019;57:190-9.

175. Cleland EJ, Bassiouni A, Boase S, Dowd S Vreugde S, Wormald P-J. The fungal microbiome in chronic rhinosinusitis: richness, diversity, postoperative changes and patient outcomes. Int Forum Allergy Rhinol 2014:4:259-65.

176. Aurora R, Chatterjee D, Hentzleman J, Prasad G, Sindwani R, Sanford T. Contrasting the microbiomes from healthy volunteers and patients with chronic rhinosinusitis. JAMA otolaryngology-- head \& neck surgery 2013;139:1328-38.

177. Hirotsu M, Shiozawa A, Ono N, Miwa M, Kikuchi K, Ikeda K. Fungal extracts detected in eosinophilic chronic rhinosinusitis induced cytokines from the nasal polyp cells. Laryngoscope 2014;124:E347-53.

178. Mohammadi A, Hashemi SM, Abtahi SH, Lajevardi SM, Kianipour S, Mohammadi R. An investigation on non-invasive fungal sinusitis; Molecular identification of etiologic agents. J research in medical sciences: 2017;22:67.

179. Sproson EL, Thomas KM, Lau LC, Harries PG, Howarth PH, Salib RJ. Common airborne fungi induce species-specific effects on upper airway inflammatory and remodelling responses. Rhinology 2016;54:51-5.

180. Shin S-H, Ye M-K, Choi S-Y, Kim Y-H. Effect of eosinophils activated with Alternaria on the production of extracellular matrix from nasal fibroblasts. Ann of Allergy, Asthma Immunol 2016;116:559-64.

181. Shin S-H, Ye M-K, Kim Y-H, Kim J-K. Role of TLRs in the production of chemical mediators in nasal polyp fibroblasts by fungi. Auris, Nasus, Larynx 2016;43:166-70.

182. Shin S-H, Ye M-K, Kim J-K. Effects of fungi and eosinophils on mucin gene expression in rhinovirus-infected nasal epithelial cells. Allergy, asthma \& immunology research 2014;6:149-55.

183. 183. Rai G, Ansari MA, Dar SA, et al. Serum Cytokine Profile in Patients with Chronic Rhinosinusitis with Nasal Polyposis Infected by Aspergillus flavus. Ann of laboratory medicine 2018;38:125-31.

184. Lawrence LA, Mulligan JK, Roach C, et al Superoxide dismutase reduces the inflam- matory response to Aspergillus and Alternaria in human sinonasal epithelial cells derived from patients with chronic rhinosinusitis. Am J Rhinol Allergy 2015;29:8993.

185. Ponikau JU, Sherris DA, Kern EB, et al. The diagnosis and incidence of allergic fungal sinusitis. Mayo Clin Proc 1999;74:877-84.

186. Zhang J, Chen J, Robinson C. Cellular and Molecular Events in the Airway Epithelium Defining the Interaction Between House Dust Mite Group 1 Allergens and Innate Defences. Int J molecular sciences 2018;19:3549.

187. Wang B-F, Cao P-P, Long X-B, et al. Distinct mucosal immunopathologic profiles in atopic and nonatopic chronic rhinosinusitis without nasal polyps in Central China. Int Forum Allergy Rhinol 2016;6:1013-9.

188. Kim JH, Yi JS, Gong C-H, Jang YJ. Development of Aspergillus protease with ovalbumin-induced allergic chronic rhinosinusitis model in the mouse. Am J Rhinol Allergy 2014;28:465-70.

189.Jr CSE, McKinney KA, Urrutia G, et al. Expression of protease-activated receptors in allergic fungal rhinosinusitis. Int Forum Allergy Rhinol 2014;4:266-71.

190.Wise SK, Lin SY, Toskala E, et al. Int Consensus Statement on Allergy and Rhinology: Allergic Rhinitis. Int Forum Allergy Rhinol 2018;8:108-352.

191. Andiappan AK, Puan KJ, Lee B, et al. Allergic airway diseases in a tropical urban environment are driven by dominant mono-specific sensitization against house dust mites. Allergy 2014;69:501-9.

192. Toppila-Salmi SK, Huhtala H, Karjalainen J, et al. Sensitization pattern affects the asthma risk in Finnish adult population. Allergy: Eur J Allergy Clin Immunol 2015, 10.1111/ all.12670.

193. Siroux V, Ballardini N, Soler M, et al. The asthma-rhinitis multimorbidity is associated with IgE polysensitization in adolescents and adults. Allergy 2018;73:1447-58.

194. Hamizan AW, Loftus PA, Alvarado R, et al. Allergic phenotype of chronic rhinosinusitis based on radiologic pattern of disease. Laryngoscope 2018;128:2015-21.

195. Kouzaki H, Matsumoto K, Kato T, Tojima I, Shimizu S, Shimizu T. Epithelial CellDerived Cytokines Contribute to the Pathophysiology of Eosinophilic Chronic Rhinosinusitis. J interferon \& cytokine research. 2016;36:169-79.

196. Wu D, Wei Y, Bleier BS. Emerging Role of Proteases in the Pathogenesis of Chronic Rhinosinusitis with Nasal Polyps. Frontiers in cellular and infection microbiology 2017;7:538

197. Xie L, Liu A-G, Peng L-Y, Wang S-J, Zhang Y-P, Wang X-S. Expression of E-prostanoid receptors in nasal polyp tissues of smoking and nonsmoking patients with chronic rhinosinusitis. PloS one 2018;13:e0200989.

198. Mulligan JK, P. BOC, Pasquini W, et al. Impact of tobacco smoke on upper airway dendritic cell accumulation and regulation by sinonasal epithelial cells. Int Forum Allergy Rhinol 2017;7:777-85.

199. Tharakan A, Halderman AA, Lane AP, Biswal S, Jr MR. Reversal of cigarette smoke extract-induced sinonasal epithelial cell barrier dysfunction through Nrf2 Activation. Int Forum Allergy Rhinol 2016;6:1 145-50.

200. Huang CC, Lee TJ, Huang CC, et al. Impact of cigarette smoke and IL-17A activation on asthmatic patients with chronic rhinosinusitis. Rhinology 2019;57:57-66.

201. Kule ZG, Habesoglu TE, Somay A, Deveci HS, Kule M, Gursel AO. Histopathological characteristics of nasal polyps in smokers and non-smokers. The J craniofacial surgery 2014;25:946-9.

202. Shin J-M, Park J-H, Kim H-J, Park I-H, Lee $\mathrm{H}-\mathrm{M}$. Cigarette smoke extract increases vascular endothelial growth factor production via TLR4/ROS/MAPKs/NF-kappaB pathway in nasal fibroblast. Am J Rhinol Allergy 2017;31:78-84.

203.Zhu CJ, Fruth K, Schneider A, Mann WJ, Brieger J. Impact of ozone exposure on prostaglandin release in nasal polyps. Eur archives of oto-rhino-laryngology 2012;269:1623-8.

204.Peric A, Mirkovic CS, Vojvodic D. Clara cell protein 16 release from the nasal mucosa in allergic rhinitis, chronic rhinosinusitis, and exposure to air pollutants. Arhiv za Higijenu Rada i Toksikologiju 2018;69:215-9.

205. Khlifi R, Olmedo P, Gil F, Hammami B, Hamza-Chaffai A. Cadmium and nickel in blood of Tunisian population and risk of nasosinusal polyposis disease. Environmental science and pollution research Int 2015;22:3586-93.

206. Khlifi R, Olmedo P, Gil F, Chakroun A, Hammami B, Hamza-Chaffai A. Heavy metals in normal mucosa and nasal polyp tissues from Tunisian patients. Environmental science and pollution research Int 2015;22:463-71.

207. Yamin M, Holbrook EH, Gray ST, Busaba NY, Lovett B, Hamilos DL. Profibrotic transforming growth factor beta 1 and activin A are increased in nasal polyp tissue and induced in nasal polyp epithelium by cigarette smoke and Toll-like receptor 3 ligation. Int Forum Allergy Rhinol 2015;5:573-82.

208. Tomaszewska M, Sarnowska E, Rusetska N, et al. Role of Vitamin D and Its Receptors in the Pathophysiology of Chronic Rhinosinusitis. J the Am College of Nutrition 2019;38:108-18

209. Mulligan JK, Pasquini WN, Carroll WW, et al. Dietary vitamin D3 deficiency exacerbates sinonasal inflammation and alters local 25(OH)D3 metabolism. Plos one 2017;12:e0186374.

210.Bravo DT, Soudry E, Edward JA, et al. Characterization of human upper airway epithelial progenitors. Int Forum Allergy Rhinol 2013;3:841-7

211. Kohanski MA, Workman AD, Patel NN, et al. Solitary chemosensory cells are a primary epithelial source of IL-25 in patients with chronic rhinosinusitis with nasal polyps. J 
Allergy Clin Immunol 2018;142:460-9.e7

212. Hanif T, Dhaygude K, Kankainen M, et al. Birch pollen allergen immunotherapy reprograms nasal epithelial transcriptome and recovers microbial diversity. J Allergy Clin Immunol 2019;143:2293-6.e11.

213. Yilmaz ÖH, Katajisto P, Lamming DW, et al. mTORC1 in the Paneth cell niche couples intestinal stem-cell function to calorie intake. Nature 2012;486:490.

214. Toppila-Salmi S, van Drunen CM, Fokkens WJ, et al. Molecular mechanisms of nasal epithelium in rhinitis and rhinosinusitis. Curr Allergy Asthma Rep 2015;15:495.

215. Malinsky RR, Valera FCP, Cavallari FE, et al. Matrix metalloproteinases and their impact on sinusal extension in chronic rhinosinusitis with nasal polyps. Eur archives of otorhino-laryngology 2013;270:1345-8.

216. Homma T, Kato A, Sakashita M, et al. Potential Involvement of the Epidermal Growth Factor Receptor Ligand Epiregulin and Matrix Metalloproteinase-1 in Pathogenesis of Chronic Rhinosinusitis. Am I respiratory cell and molecular biology 2017:57:334-45.

217. Muluk NB, Arikan OK, Atasoy P, Kilic R, Yalcinozan ET. The role of MMP-2, MMP-9, and TIMP-1 in the pathogenesis of nasal polyps: Immunohistochemical assessment at eight different levels in the epithelial, subepithelial, and deep layers of the mucosa. ENT J. 2015;94:E1-13.

218. Wang L-F, Chien C-Y, Chiang F-Y, Chai C-Y, Tai C-F. Corelationship between matrix metalloproteinase 2 and 9 expression and severity of chronic rhinosinusitis with nasal polyposis. Am J Rhinol Allergy 2012;26:e1-4.

219. Chen X, Chang L, Li X, et al. TC17/IL-17A Up-Regulated the Expression of MMP-9 via NF-kappaB Pathway in Nasal Epithelial Cells of Patients With Chronic Rhinosinusitis. Front Immunol 2018;9:2121.

220. Yang LY, Li X, Li WT, et al. Vgamma1+ gammadeltaT Cells Are Correlated With Increasing Expression of Eosinophil Cationic Protein and Metalloproteinase-7 in Chronic Rhinosinusitis With Nasal Polyps Inducing the Formation of Edema. Allergy, asthma \& immunology research 2017;9:142-51.

221. Li X, Tao Y, Li X. Expression of MMP-9/TIMP-2 in nasal polyps and its functional implications. Int J Clin and experimental pathology 2015;8:14556-61.

222. Suzuki M, Ramezanpour M, Cooksley C, et al. Sirtuin-1 Controls Poly (I:C)-Dependent Matrix Metalloproteinase 9 Activation in Primary Human Nasal Epithelial Cells. Am J respiratory cell and molecular biology 2018:59:500-10.

223. Xiang R, Zhang Q-P, Zhang W, et al. Different effects of allergic rhinitis on nasal mucosa remodeling in chronic rhinosinusitis with and without nasal polyps. Eur archives of oto-rhino-laryngology 2019;276:115-30.

224. Yeo N-K, Eom D-W, Oh MY, Lim HW, Song YJ. Expression of matrix metalloproteinase 2 and 9 and tissue inhibitor of metalloproteinase 1 in nonrecurrent vs recur- rent nasal polyps. Ann of Allergy, Asthma \& Immunology2013;111:205-10.

225. Hupin C, Gohy S, Bouzin C, Lecocq M, Polette M, Pilette $C$. Features of mesenchymal transition in the airway epithelium from chronic rhinosinusitis. Allergy 2014;69:15409

226. Kim B, Lee H-J, Im N-R, et al. Effect of matrix metalloproteinase inhibitor on disrupted E-cadherin after acid exposure in the human nasal epithelium. Laryngoscope 2018;128:E1-E7.

227. Deng H, Sun $Y$, Wang $W$, et al. The hippo pathway effector Yes-associated protein promotes epithelial proliferation and remodeling in chronic rhinosinusitis with nasal polyps. Allergy 2019;74:731-42.

228. Ishida A, Ohta N, Suzuki Y, et al. Expression of Pendrin Periostin in Allergic Rhinitis Chronic Rhinosinusitis. Allergology Int 2012;61:589-95

229. Lehmann AE, Scangas GA, Bergmark RW, Rassi EE, Stankovic KM, Metson R. Periostin and Inflammatory Disease: Implications for Chronic Rhinosinusitis. Otolaryngology-head and neck surgery 2019, https://dx.doi. org/10.1177/0194599819838782:1945998 19838782.

230. Shiono O, Sakuma Y, Komatsu M, et al. Differential expression of periostin in the nasal polyp may represent distinct histological features of chronic rhinosinusitis. Auris, Nasus, Larynx 2015;42:123-7.

231. Yang H-W, Park J-H, Shin J-M, Lee H-M. Glucocorticoids ameliorate periostininduced tissue remodeling in chronic rhinosinusitis with nasal polyps. Clin Experimental allergy J 2018, https://dx.doi. org/10.1111/cea.13267.

232.Milonski J, Zielinska-Blizniewska $\mathrm{H}$ Przybylowska K, et al. Significance of CYCLOOXYGENASE-2(COX-2), PERIOSTIN (POSTN) and INTERLEUKIN-4(IL-4) gene expression in the pathogenesis of chronic rhinosinusitis with nasal polyps. Eur archives of oto-rhino-laryngology Surgery 2015;272:3715-20

233. Ohta N, Ishida A, Kurakami K, et al. Expressions and roles of periostin in otolaryngological diseases. Allergology Int : official J the Japanese Society of Allergology 2014:63:171-80

234. Xu M, Chen D, Zhou H, Zhang W, Xu J, Chen $\mathrm{L}$. The Role of Periostin in the Occurrence and Progression of Eosinophilic Chronic Sinusitis with Nasal Polyps. Scientific reports 2017;7:9479.

235. Laury AM, Hilgarth R, Nusrat A, Wise SK. Periostin and receptor activator of nuclear factor kappa-B ligand expression in allergic fungal rhinosinusitis. Int Forum Allergy Rhinol 2014;4:716-24.

236. Wei Y, Ma R, Zhang J, et al. Excessive periostin expression and Th2 response in patients with nasal polyps: association with asthma. J thoracic disease 2018;10:6585-97.

237. Wang $M$, Wang $X$, Zhang $N$, et al. Association of periostin expression with eosinophilic inflammation in nasal polyps. J
Allergy Clin Immunol 2015;136:1700-9.

238. Ebenezer JA, Christensen JM, Oliver BG, et al. Periostin as a marker of mucosal remodelling in chronic rhinosinusitis. Rhinology 2017;55:234-41.

239. Carpagnano GE, Scioscia G, Lacedonia D, et al. Looking for Airways Periostin in Severe Asthma: Could It Be Useful for Clustering Type 2 Endotype? Chest 2018;154:1083-90.

240.Kim DW, Kulka M, Jo A, et al. Cross-talk between human mast cells and epithelial cells by lgE-mediated periostin production in eosinophilic nasal polyps. The J Allergy Clin Immunol 2017;139:1692-5.e6.

241. Jiao J, Wang C, Zhang L. Epithelial physical barrier defects in chronic rhinosinusitis. Expert review of Clin immunology 2019;15:679-88

242. Yu XM, Li CW, Li YY, et al. Down-regulation of EMP1 is associated with epithelial hyperplasia and metaplasia in nasal polyps. Histopathology 2013;63:686-95.

243. Soyka MB, Wawrzyniak P, Eiwegger T, et al. Defective epithelial barrier in chronic rhinosinusitis: the regulation of tight junctions by IFN-gamma and IL-4. J Allergy Clin Immunol 2012;130:1087-96.e10.

244. Suzuki H, Koizumi H, Ikezaki S, et al. Electrical Impedance and Expression of Tight Junction Components of the Nasal Turbinate and Polyp. ORL J Otorhinolaryngol Relat Spec. 2016;78:16-25.

245. Jiao J, Wang M, Duan S, et al. Transforming growth factor-beta1 decreases epithelial tight junction integrity in chronic rhinosinusitis with nasal polyps. J Allergy Clin Immunol 2018;141:1160-3.e9.

246. Li Y, Wang $X$, Wang $R$, et al. The expression of epithelial intercellular junctional proteins in the sinonasal tissue of subjects with chronic rhinosinusitis: a histopathologic study.ORL J Otorhinolaryngol Relat Spec. 2014;76:110-9.

247. Kim R, Chang G, Hu R, Phillips A, Douglas R. Connexin gap junction channels and chronic rhinosinusitis. Int Forum Allergy Rhinol 2016;6:611-7.

248.Toppila-Salmi SK, Drunen CMv, Fokkens WJ, et al. Molecular Mechanisms of Nasal Epithelium in Rhinitis and Rhinosinusitis. Curr Allergy Asthma Rep 2015, 10.1007/ s11882-014-0495-8.

249. Cutting GR. Modifier genetics: cystic fibrosis. Annu Rev Genomics Hum Genet 2005:6:237-60.

250. Li YY, Li CW, Chao SS, et al. Impairment of cilia architecture and ciliogenesis in hyperplastic nasal epithelium from nasal polyps. J Allergy Clin Immunol 2014;134:1282-92.

251. Jiao J, Duan S, Meng N, Li Y, Fan E, Zhang L. Role of IFN-gamma, IL-13, and IL-17 on mucociliary differentiation of nasal epithelial cells in chronic rhinosinusitis with nasal polyps. Clin and experimental allergy : 2016:46:449-60.

252. Ma $Y$, Sun $Y$, Jiang $L$, et al. WDPCP regulates the ciliogenesis of human sinonasal epithelial cells in chronic rhinosinusitis. Cytoskeleton (Hoboken, NJ) 2017;74:82-90.

253.Tipirneni KE, Zhang S, Cho D-Y, et al. 
Submucosal gland mucus strand velocity is decreased in chronic rhinosinusitis. Int Forum Allergy Rhinol 2018;8:509-12.

254.El-Anwar M, Hamed AA, Mohamed AES, Nofal AA-F, Mohamed MA, Abdel-Aziz $H$. Surfactant protein a expression in chronic rhinosinusitis and atrophic rhinitis. Int archives of otorhinolaryngology 2015;19:130-4.

255. Tengroth L, Millrud CR, Kvarnhammar AM, Georen SK, Latif L, Cardell L-O. Functional effects of Toll-like receptor (TLR)3, 7, 9, RIG-I and MDA-5 stimulation in nasal epithelial cells. PloS one 2014;9:e98239.

256. Sun Y, Zhou B, Wang C, et al. Biofilm formation and Toll-like receptor 2, Toll-like receptor 4, and NF-kappaB expression in sinus tissues of patients with chronic rhinosinusitis. Am J Rhinol Allergy 2012;26:104-9.

257. Hu H, Li H. Prunetin inhibits lipopolysaccharide-induced inflammatory cytokine production and MUC5AC expression by inactivating the TLR4/MyD88 pathway in human nasal epithelial cells. Biomedicine \& pharmacotherapy $=$ Biomedecine \& pharmacotherapie 2018;106:1469-77.

258. Shimizu S, Kouzaki H, Kato T, Tojima I, Shimizu T. HMGB1-TLR4 signaling contributes to the secretion of interleukin 6 and interleukin 8 by nasal epithelial cells. Am J Rhinol Allergy 2016;30:167-72.

259.Tsai Y-J, Chi JC-Y, Hao C-Y, Wu W-B. Peptidoglycan induces bradykinin receptor 1 expression through Toll-like receptor 2 and NF-kappaB signaling pathway in human nasal mucosa-derived fibroblasts of chronic rhinosinusitis patients. J cellular physiology 2018;233:7226-38.

260. Shin SH, Kim YH, Jin HS, Kang SH. Alternaria Induces Production of Thymic Stromal Lymphopoietin in Nasal Fibroblasts Through Toll-like Receptor 2. Allergy, asthma \& immunology research 2016;8:63-8.

261. Cho J-S, Kang J-H, Um J-Y, Han I-H, Park I-H, Lee H-M. Lipopolysaccharide induces pro-inflammatory cytokines and MMP production via TLR4 in nasal polyp-derived fibroblast and organ culture. PloS one 2014;9:e90683.

262. Cho J-S, Kim J-A, Park J-H, Park I-H, Han I-H, Lee H-M. Toll-like receptor 4-mediated expression of interleukin-32 via the c-Jun $\mathrm{N}$-terminal kinase/protein kinase B/cyclic adenosine monophosphate response element binding protein pathway in chronic rhinosinusitis with nasal polyps. Int Forum Allergy Rhinol 2016;6:1020-8.

263. Park SK, Jin SY, Yeon SH, et al. Role of Tolllike receptor 9 signaling on activation of nasal polyp-derived fibroblasts and its association with nasal polypogenesis. Int Forum Allergy Rhinol 2018;8:1001-12.

264.264. Xu J, Lee J-W, Park S-K, et al. Toll-like receptor 9 ligands increase type I interferon induced B-cell activating factor expression in chronic rhinosinusitis with nasal polyposis. Clin immunology (Orlando, Fla) 2018;197:19-26.

265.Jardeleza C, Miljkovic D, Baker L, et al.
Inflammasome gene expression alterations in Staphylococcus aureus biofilmassociated chronic rhinosinusitis. Rhinology 2013:51:315-22

266. Lin H, Li Z, Lin D, Zheng C, Zhang W. Role of NLRP3 Inflammasome in Eosinophilic and Non-eosinophilic Chronic Rhinosinusitis with Nasal Polyps. Inflammation 2016;39:2045-52.

267. Freund JR, Mansfield CJ, Doghramji LJ, et al. Activation of airway epithelial bitter taste receptors by Pseudomonas aeruginosa quinolones modulates calcium, cyclic-AMP and nitric oxide signaling. J Biol Chem 2018:293:9824-40.

268. Lee RJ, Cohen NA. Bitter and sweet taste receptors in the respiratory epithelium in health and disease. J Molecular Medicine 2014;92:1235-44.

269. Cohen NA. The genetics of the bitter taste receptor T2R38 in upper airway innate immunity and implications for chronic rhinosinusitis. Laryngoscope 2017;127:44-51.

270. Tieu DD, Peters AT, Carter RG, et al. Evidence for diminished levels of epithelial psoriasin and calprotectin in chronic rhinosinusitis. J Allergy Clin Immunol 2010;125:667-75.

271. Seshadri S, Lin DC, Rosati M, et al. Reduced expression of antimicrobial PLUNC proteins in nasal polyp tissues of patients with chronic rhinosinusitis. Allergy 2012;67:920-8.

272.Tsybikov NN, Egorova EV, Kuznik BI, Fefelova EV, Magen E. Biomarker assessment in chronic rhinitis and chronic rhinosinusitis: Endothelin-1, TARC/CCL17, neopterin, and alpha-defensins. Allergy and asthma proceedings 2016;37:35-42.

273. Lim Al, Verrier T, Vosshenrich CA, Di Santo JP. Developmental options and functional plasticity of innate lymphoid cells. Curr Opimmunology 2017;44:61-8.

274. Abbas AK, Lichtman AH, Pillai S. Basic Immunology: Functions and Disorders of the Immune System. 2019:1-319.

275. Krabbendam L, Bal SM, Spits H, Golebski K. New insights into the function, development, and plasticity of type 2 innate lymphoid cells. Immunological reviews 2018;286:74-85

276. Ebbo M, Crinier A, Vély F, Vivier E. Innate lymphoid cells: major players in inflammatory diseases. Nat Rev Immunology 2017;17:665.

277. Kortekaas Krohn I, Shikhagaie MM, Golebski $\mathrm{K}$, et al. Emerging roles of innate lymphoid cells in inflammatory diseases: Clin implications. Allergy 2018;73:837-50.

278. Shikhagaie MM, Germar K, Bal SM, Ros $X R$, Spits H. Innate lymphoid cells in autoimmunity: emerging regulators in rheumatic diseases. Nat Rev Rheumatology 2017;13:164.

279. Dogan M, Sahin M, Yenisey C. Increased TSLP, IL-33, IL-25, IL-19, IL 21 and amphiregulin (AREG) levels in chronic rhinosinusitis with nasal polyp. Eur archives of otorhino-laryngology 2019, https://dx.doi. org/10.1007/s00405-019-05379-8

280. Nagata Y, Maruoka S, Gon Y, et al. Expression of IL-25, IL-33, and Thymic Stromal Lymphopoietin in Nasal Polyp Gland Duct Epithelium in Patients With Chronic Rhinosinusitis. Am J Rhinol Allergy 2019, https://dx.doi.org/10.1177/1945892419835 333:1945892419835333

281. Lam M, Hull L, Imrie A, et al. Interleukin-25 and interleukin-33 as mediators of eosinophilic inflammation in chronic rhinosinusitis. Am J Rhinol Allergy 2015;29:175-81.

282.Wang WW, Lu DM, Zheng M, Zhang JG, Zhang B. TSLP regulates eotaxin-1 production by nasal epithelial cells from patients with eosinophilic CRSwNP. Rhinology 2018:56:370-7.

283. Ouyang Y, Fan E, Li Y, Wang X, Zhang L. Clin characteristics and expression of thymic stromal lymphopoetin in eosinophilic and non-eosinophilic chronic rhinosinusitis. ORL; journal for oto-rhino-laryngology and its related specialties 2013;75:37-45.

284. Nagarkar DR, Poposki JA, Tan BK, et al. Thymic stromal lymphopoietin activity is increased in nasal polyps of patients with chronic rhinosinusitis. The J Allergy Clin Immunol 2013;132:593-600.e12.

285. Rudack C, Steinhoff M, Mooren F, et al. PAR-2 activation regulates IL-8 and GROalpha synthesis by NF-kappaB, but not RANTES, IL-6, eotaxin or TARC expression in nasal epithelium. Clin Exp Allergy 2007:37:1009-22.

286. Derycke L, Zhang N, Holtappels G, Dutre T, Bachert C. IL-17A as a regulator of neutrophil survival in nasal polyp disease of patients with and without cystic fibrosis. J cystic fibrosis 2012;11:193-200.

287.van Drunen CM, Reinartz S, Wigman J, Fokkens WJ. Inflammation in chronic rhinosinusitis and nasal polyposis. Immunol Allergy Clin North Am 2009;29:621-9.

288.Zhang N, Holtappels G, Claeys C, Huang $G$, van Cauwenberge P, Bachert C. Pattern of inflammation and impact of Staphylococcus aureus enterotoxins in nasal polyps from southern China. Am J Rhinol 2006;20:445-50.

289. Zhang N, Van Zele T, Perez-Novo C, et al. Different types of T-effector cells orchestrate mucosal inflammation in chronic sinus disease. J Allergy Clin Immunol 2008;122:961-8.

290. Kim DW, Eun KM, Roh EY, Shin S, Kim D-K. Chronic Rhinosinusitis without Nasal Polyps in Asian Patients Shows Mixed Inflammatory Patterns and NeutrophilRelated Disease Severity. Mediators of inflammation 2019;2019:7138643.

291. Wang H, Li Z-Y, Jiang W-X, et al. The activation and function of IL-36gamma in neutrophilic inflammation in chronic rhinosinusitis. The J Allergy Clin Immunol 2018;141:1646-58.

292. Kim DW, Kim D-K, Jo A, et al. Age-Related Decline of Neutrophilic Inflammation Is Associated with Better Postoperative Prognosis in Non-eosinophilic Nasal Polyps. PloS one 2016;11:e0148442.

293. Pothoven KL, Norton JE, Suh LA, et al. 
Neutrophils are a major source of the epithelial barrier disrupting cytokine oncostatin $M$ in patients with mucosal airways disease. J Allergy Clin Immunol 2017;139:196678.e9.

294. Morse JC, Li P, Ely KA, et al. Chronic rhinosinusitis in elderly patients is associated with an exaggerated neutrophilic proinflammatory response to pathogenic bacteria. Allergy Clin Immunol 2019;143:990-1002.e6.

295.Wu D, Mueller SK, Nocera AL, Finn K, Libermann TA, Bleier BS. TREM-1 Neutrophil Activation Pathway Is Suppressed in Eosinophilic Nasal Polyps. Am J Rhinol Allergy 2018;32:359-68.

296. Banks CA, Schlosser RJ, Wang EW, Casey SE, Mulligan RM, Mulligan JK. Macrophage Infiltrate Is Elevated in CRSwNP Sinonasa Tissue Regardless of Atopic Status. Otolaryngology--head and neck surgery 2014;151:215-20.

297.Poposki JA, Uzzaman A, Nagarkar DR, et al. Increased expression of the chemokine CCL23 in eosinophilic chronic rhinosinusitis with nasal polyps. J Allergy Clin Immunol 2011;128:73-81 e4.

298. Krysko O, Holtappels G, Zhang N, et al. Alternatively activated macrophages and impaired phagocytosis of S. aureus in chronic rhinosinusitis. Allergy 2011;66:396403.

299. Khawar MB, Abbasi MH, Sheikh N. IL-32: A Novel Pluripotent Inflammatory Interleukin, towards Gastric Inflammation, Gastric Cancer, and Chronic Rhino Sinusitis. Mediators of inflammation 2016;2016:8413768.

300. Takabayashi T, Kato A, Peters AT, et al. Excessive fibrin deposition in nasal polyps caused by fibrinolytic impairment through reduction of tissue plasminogen activator expression. Am J respiratory and critical care medicine 2013;187:49-57.

301. Peterson S, Poposki JA, Nagarkar DR, et al. Increased expression of CC chemokine ligand 18 in patients with chronic rhinosinusitis with nasal polyps. J Allergy Clin Immunol 2012;129:119.

302. Takabayashi T, Kato A, Peters AT, et al. Increased expression of factor XIII-A in patients with chronic rhinosinusitis with nasal polyps. J Allergy Clin Immunol 2013;132:584-92.e4.

303.Peric A, Baletic N, Sotirovic J, SpadijerMirkovic C. Macrophage inflammatory protein-1 production and eosinophil infiltration in chronic rhinosinusitis with nasal polyps. The Ann of Otology, Rhinology, and Laryngology 2015;124:266-72.

304. Kaczmarek M, Banaszewski J, Leszczynska $M$, et al. High frequency of macrophages expressing elevated level of CD80, PD-Ls and TLR1 in nasal polyps of CRS patients. Immunobiology 2019;224:154-62.

305. Piliponsky AM, Romani L. The contribution of mast cells to bacterial and fungal infection immunity. Immunological Reviews 2018;282:188-97.

306. Pawankar R, Lee KH, Nonaka M, Takizawa
R. Role of mast cells and basophils in chronic rhinosinusitis. Clin Allergy Immunol 2007;20:93-101.

307. Cao PP, Zhang YN, Liao B, et al. Increased local IgE production induced by common aeroallergens and phenotypic alteration of mast cells in Chinese eosinophilic, but not non-eosinophilic, chronic rhinosinusitis with nasal polyps. Clin Exp Allergy 2014:44:690-700.

308. Cahill KN, Laidlaw TM. Pathogenesis of Aspirin-Induced Reactions in AspirinExacerbated Respiratory Disease. Immunology and allergy clinics of North America 2016;36:681-91.

309. Borish L. Aspirin-exacerbated Respiratory Disease: A Syndrome of Mast Cell-mediated PgD2 Overproduction. Am J Respiratory and Critical Care Medicine 2019;200:651-2.

310. Takabayashi T, Kato A, Peters AT, et al. Glandular mast cells with distinct phenotype are highly elevated in chronic rhinosinusitis with nasal polyps. J Allergy Clin Immunol 2012;130:410-20.e5.

311.Zhai G-T, Li J-X, Zhang X-H, Liao B, Lu X, Liu Z. Increased accumulation of CD30 ligand-positive mast cells associates with eosinophilic inflammation in nasal polyps. Laryngoscope 2019;129:E110-E7.

312.Zhai G-T, Wang H, Li J-X, et al. IgD-activated mast cells induce IgE synthesis in B cells in nasal polyps. J Allergy Clin Immunol 2018;142:1489-99.e23

313. Gevaert P, Calus L, Van Zele T, et al. Omalizumab is effective in allergic and nonallergic patients with nasal polyps and asthma. J Allergy Clin Immunol 2013;131:110-6. e1.

314. Kern RC. Biologics and the treatment of chronic rhinosinusitis. J Allergy Clin Immunol, 2013, 117-8.

315. Kaliner MA, Osguthorpe JD, Fireman P, et al. Sinusitis: bench to bedside. Current findings, future directions [published erratum appears in Otolaryngol Head Neck Surg 1997 Sep;117(3 Pt 1):187]. Otolaryngol Head Neck Surg 1997;116:S1-20

316. Harlin SL, Ansel DG, Lane SR, Myers J, Kephart GM, Gleich GJ. A Clin and pathologic study of chronic sinusitis: the role of the eosinophil. J Allergy Clin Immunol 1988;81:867-75.

317. Polzehl D, Moeller P, Riechelmann H, Perner S. Distinct features of chronic rhinosinusitis with and without nasal polyps. Allergy 2006;61:1275-9.

318. Payne SC, Borish L, Steinke JW. Genetics and phenotyping in chronic sinusitis. J Allergy Clin Immunol 2011, S0091-6749(11)008463.

319. Lou H, Zhang N, Bachert C, Zhang L. Highlights of eosinophilic chronic rhinosinusitis with nasal polyps in definition, prognosis, and advancement. Int Forum Allergy Rhinol 2018;8:1218-25.

320. Cao PP, Li HB, Wang BF, et al. Distinct immunopathologic characteristics of various types of chronic rhinosinusitis in adult Chinese. J Allergy Clin Immunol 2009,
S0091-6749(09)00793-3

321. Payne SC, Early SB, Huyett P, Han JK, Borish L, Steinke JW. Evidence for distinct histological profile of nasal polyps: with and without eosinophilia. Laryngoscope 2011;121:2262-7.

322.Zhang Y, Gevaert E, Lou H, et al. Chronic rhinosinusitis in Asia. J Allergy Clin Immunol 2017;140:1230-9.

323. Soler ZM, Sauer D, Mace J, Smith TL. Impact of mucosal eosinophilia and nasal polyposis on quality-of-life outcomes after sinus surgery. Otolaryngol Head Neck Surg 2010;142:64-71.

324. Vlaminck S, Vauterin T, Hellings PW, et al. The importance of local eosinophilia in the surgical outcome of chronic rhinosinusitis: a 3-year prospective observational study. Am J Rhinol Allergy 2014;28:260-4.

325. Tokunaga T, Sakashita M, Haruna T, et al. Novel scoring system and algorithm for classifying chronic rhinosinusitis: the JESREC Study. Allergy 2015;70:995-1003.

326. Tajudeen BA, Ganti A, Kuhar HN, et al. The presence of eosinophil aggregates correlates with increased postoperative prednisone requirement. Laryngoscope 2019;129:794-9.

327. Yip KH, Papadopoulos M, Pant H, Tumes DJ. The role of invariant $T$ cells in inflammation of the skin and airways. Seminars in immunopathology 2019;41:401-10.

328. Luukkainen A, Puan KJ, Yusof N, et al. A Co-culture Model of PBMC and Stem Cell Derived Human Nasal Epithelium Reveals Rapid Activation of NK and Innate T Cells Upon Influenza A Virus Infection of the Nasal Epithelium. Frontiers in immunology 2018;9:2514.

329. Kowalski ML, Lewandowska-Polak A Wozniak J, et al. Association of stem cell factor expression in nasal polyp epithelial cells with aspirin sensitivity and asthma. Allergy 2005:60:631-7.

330.330. Van Zele T, Coppieters F, Gevaert P, Holtappels G, Van Cauwenberge P, Bachert C. Local complement activation in nasal polyposis. Laryngoscope 2009;1 19:1753-8.

331.Smith SE, Schlosser RJ, Yawn JR, Mattos JL, Soler ZM, Mulligan JK. Sinonasal T-cell expression of cytotoxic mediators granzyme $B$ and perforin is reduced in patients with chronic rhinosinusitis. Am J Rhinol Allergy 2017;31:352-6.

332. Poposki JA, Klingler Al, Tan BK, et al. Group 2 innate lymphoid cells are elevated and activated in chronic rhinosinusitis with nasal polyps. Immunity, inflammation and disease 2017;5:233-43.

333. Flood-Page P, Menzies-Gow A, Phipps S, et al. Anti-IL-5 treatment reduces deposition of ECM proteins in the bronchial subepithelial basement membrane of mild atopic asthmatics. J Clin Invest 2003;112:1029-36.

334. Bernardes JF, Shan J, Tewfik M, Hamid Q, Frenkiel S, Eidelman DH. Protein nitration in chronic sinusitis and nasal polyposis: Role of eosinophils. Otolaryngology - Head \& Neck Surgery 2004;131:696-703. 
335. Gevaert E, Zhang N, Krysko O, et al. Extracellular eosinophilic traps in association with Staphylococcus aureus at the site of epithelial barrier defects in patients with severe airway inflammation. J Allergy Clin Immunol 2017;139:1849-60.e6.

336. Pleass RJ, Lang ML, Kerr MA, Woof JM. $\lg A$ is a more potent inducer of NADPH oxidase activation and degranulation in blood eosinophils than IgE. Mol Immunol 2007:44:1401-8.

337. Feldman S, Kasjanski R, Poposki J, et al. Chronic airway inflammation provides a unique environment for $\mathrm{B}$ cell activation and antibody production. Clin and experimental allergy : J the British Society for Allergy and Clin Immunology 2017;47:45766.

338. an Zele T, Gevaert P, Holtappels G, van Cauwenberge $\mathrm{P}$, Bachert C. Local immunoglobulin production in nasal polyposis is modulated by superantigens. Clin Exp Allergy 2007;37:1840-7.

339. Schleimer RP, Bochner BS. The effects of glucocorticoids on human eosinophils. Allergy Clin Immunol 1994;94:1202-13.

340.Van Zele T, Gevaert P, Holtappels G, et al. Oral steroids and doxycycline: Two different approaches to treat nasal polyps. J Allergy Clin Immunol 2010;125:1069-76.e4.

341. Bachert C, Sousa AR, Lund VJ, et al. Reduced need for surgery in severe nasal polyposis with mepolizumab: Randomized trial. Allergy Clin Immunol 2017;140:1024-31.e14.

342. Stevens WW, Peters AT, Tan BK, et al. Associations Between Inflammatory Endotypes and Clin Presentations in Chronic Rhinosinusitis. J Allergy Clin Immunol In practice 2019, 10.1016/j. jaip.2019.05.009.

343. Wang X, Zhang N, Bo M, et al. Diversity of TH cytokine profiles in patients with chronic rhinosinusitis: A multicenter study in Europe, Asia, and Oceania. J Allergy Clin Immunol 2016;138:1344-53.

344.Tan BK, Klingler Al, Poposki JA, et al. Heterogeneous inflammatory patterns in chronic rhinosinusitis without nasal polyps in Chicago, Illinois. J Allergy Clin Immunol 2017;139:699-703.e7

345. Laidlaw TM, Prussin C, Panettieri RA, et al. Dexpramipexole depletes blood and tissue eosinophils in nasal polyps with no change in polyp size. Laryngoscope 2019;129:E61e6.

346. Kim JH, Kim GE, Cho GS, et al. Natural killer cells from patients with chronic rhinosinusitis have impaired effector functions. Plos one 2013;8:e77177

347. Kim JH, Choi GE, Lee B-J, et al. Natural killer cells regulate eosinophilic inflammation in chronic rhinosinusitis. Scientific reports 2016;6:27615

348. Baba S, Kondo K, Suzukawa M, Ohta K, Yamasoba T. Distribution, subtype population, and IgE positivity of mast cells in chronic rhinosinusitis with nasal polyps. Ann of Allergy, Asthma \& Immunology 2017:119:120-8
349. Perez-Novo C, Pezato R. Dendritic cell subset expression in severe chronic rhinosinusitis with nasal polyps. Curr Opallergy and Clin immunology 2017;17:1-4.

350. Pezato R, Perez-Novo C, Holtappels G, et al. The expression of dendritic cell subsets in severe chronic rhinosinusitis with nasal polyps is altered. Immunobiology 2014;219:729-36.

351.Zheng R, Wang D, Wang K, et al. Elevated expression of IL-17RB and ST2 on myeloid dendritic cells is associated with a Th2 skewed eosinophilic inflammation in nasal polyps. Clin and translational allergy 2018:8:50.

352. Shi L-L, Song J, Xiong P, et al. Diseasespecific T-helper cell polarizing function of lesional dendritic cells in different types of chronic rhinosinusitis with nasal polyps. Am J respiratory and critical care medicine 2014;190:628-38.

353. Lin X, Zhuang X, Li C, Wang X. Interactions between dendritic cells and T lymphocytes in pathogenesis of nasal polyps. Experimental and therapeutic medicine 2018;15:5167-72.

354. Krohn IK, Bobic S, Dooley J, et al. Programmed cell death-1 expression correlates with disease severity and IL-5 in chronic rhinosinusitis with nasal polyps. Allergy 2017;72:985-93.

355. Liu CC, Zhang HL, Zhi LL, et al. CDK5 Regulates PD-L1 Expression and Cel Maturation in Dendritic Cells of CRSwNP. Inflammation 2019:42:135-44.

356. Sallusto F. Heterogeneity of Human CD4+ T Cells Against Microbes. Annual Review of Immunology 2016;34:317-34.

357. Commins SP, Borish L, Steinke JW. Immunologic messenger molecules: cytokines, interferons, and chemokines. J Allergy Clin Immunol 2010;125:S53-72.

358. O'Shea JJ, Paul WE. Mechanisms underlying lineage commitment and plasticity of helper CD4+ T cells. Science 2010;327:1098-102.

359. Henry EK, Inclan-Rico JM, Siracusa MC. Type 2 cytokine responses: regulating immunity to helminth parasites and allergic inflammation. Current pharmacology reports 2017:3:346-59.

360. DuPage M, Bluestone JA. Harnessing the plasticity of $C D 4+T$ cells to treat immunemediated disease. Nat Rev Immunology 2016;16:149.

361.Tan BK, Min J-Y, Hulse KE. Acquired Immunity in Chronic Rhinosinusitis. Curr Allergy Asthma Rep 2017;17:49.

362. Palmer C, Mulligan JK, Smith SE, Atkinson C. The role of regulatory $T$ cells in the regulation of upper airway inflammation. Am J Rhinol Allergy 2017;31:345-51.

363. Derycke L, Eyerich S, Crombruggen KV, et al. Mixed T helper cell signatures in chronic rhinosinusitis with and without polyps. Plos one 2014;9:e97581.

364. Pant H, Macardle P. CD8(+) T cells implicated in the pathogenesis of allergic fungal rhinosinusitis. Allergy \& rhinology 2014:5:146-56.
365. Macri GF, Greco A, Marinelli C, et al. Evidence and role of autoantibodies in chronic rhinosinusitis with nasal polyps. Int $J$ Immunopathology and Pharmacology 2014;27:155-61

366. Lefrancois $P$, Chapdelaine $H$, Cote $B$, Desrosiers M. A role for auto-immunity in chronic rhinosinusitis? Lessons learned from sub-epidermal bullous disorders of the skin. Allergy Asthma Clin Immunol 2016;12:38.

367. Tan BK, Li QZ, Suh L, et al. Evidence for intranasal antinuclear autoantibodies in patients with chronic rhinosinusitis with nasal polyps. J Allergy Clin Immunol 2011;128:1198206 e1.

368. Jeffe JS, Seshadri S, Hamill KJ, et al. A role for anti-BP180 autoantibodies in chronic rhinosinusitis. Laryngoscope 2013;123:210411.

369. Tsybikov NN, Egorova EV, Kuznik BI, Fefelova EV, Magen E. Anticytokine autoantibodies in chronic rhinosinusitis. Allergy and asthma proceedings 2015;36:473-80.

370.Wang M, Zhang N, Zheng M, et al. Crosstalk between $\mathrm{TH} 2$ and $\mathrm{TH} 17$ pathways in patients with chronic rhinosinusitis with nasal polyps. J Allergy Clin Immunol 2019, 10.1016/j.jaci.2019.06.023.

371.Ickrath $P$, Kleinsasser $N$, Ding $X$, et al. Accumulation of CD69+ tissue-resident memory $T$ cells in the nasal polyps of patients with chronic rhinosinusitis. Int $J$ molecular medicine 2018;42:1116-24.

372.Xiao L, Jia L, Bai L, et al. Phenotypic and functional characteristics of $\mid \mathrm{L}-21$ expressing CD8(+) T cells in human nasal polyps. Scientific reports 2016;6:30362.

373. Kong D-H, Kim YK, Kim MR, Jang JH, Lee S. Emerging Roles of Vascular Cell Adhesion Molecule-1 (VCAM-1) in Immunological Disorders and Cancer. Int J molecular sciences 2018;19:1057.

374. Batzakakis D, Stathas T, Mastronikolis N, Kourousis C, Aletras A, Naxakis S. Adhesion molecules as predictors of nasal polyposis recurrence. Am J Rhinol Allergy 2014;28:202.

375. Oyer SL, Nagel W, Mulligan JK. Differential expression of adhesion molecules by sinonasal fibroblasts among control and chronic rhinosinusitis patients. Am J Rhinol Allergy 2013:27:381-6.

376. Tsutsumiuchi T, Hoshino H, Fujieda S, Kobayashi M. Induction of peripheral lymph node addressin in human nasal mucosa with eosinophilic chronic rhinosinusitis. Pathology 2019;51:268-73.

377. Cerutti A, Chen K, Chorny A. Immunoglobulin responses at the mucosal interface. Annu Rev Immunol 2011:29:27393.

378. Aazami H, Seif F, Ghalehbaghi B, et al. Levels of total IgA and IgA subclasses in the serum of chronic rhinosinusitis patients. Medical J the Islamic Republic of Iran 2018;32:94.

379. Gevaert P, Nouri-Aria KT, Wu H, et al. Local receptor revision and class switching to IgE in chronic rhinosinusitis with nasal polyps. Allergy 2013;68:55-63. 
380. Baba S, Kondo K, Toma-Hirano M, et al. Loca increase in IgE and class switch recombination to lgE in nasal polyps in chronic rhinosinusitis. Clin and experimental allergy 2014;44:701-12.

381. Dilidaer, Zheng Y, Liu Z, et al. Increased BAFF expression in nasal polyps is associated with local lgE production, Th2 response and concomitant asthma. Eur archives of oto-rhino-laryngology2017;274:1883-90

382.382. Stone KD, Prussin C, Metcalfe DD. IgE, mast cells, basophils, and eosinophils. J Allergy Clin Immunol 2010;125:S73-80.

383. McCoy KD, Ronchi F, Geuking MB. Hostmicrobiota interactions and adaptive immunity. Immunological reviews 2017;279:63-9.

384. Tan BK, Peters AT, Schleimer RP, Hulse KE. Pathogenic and protective roles of $B$ cells and antibodies in patients with chronic rhinosinusitis. J Allergy Clin Immunol 2018;141:1553-60.

385. Keswani A, Dunn NM, Manzur A, et al. The Clin Significance of Specific Antibody Deficiency (SAD) Severity in Chronic Rhinosinusitis (CRS). J Allergy Clin Immunolln practice 2017:5:1105-11.

386. Hulse KE, Norton JE, Suh L, et al. Chronic rhinosinusitis with nasal polyps is characterized by B-cell inflammation and EBVinduced protein 2 expression. J Allergy Clin Immunol 2013;131:1075-83, 83.e1-7.

387. Gevaert P, Holtappels G, Johansson SG, Cuvelier C, Cauwenberge P, Bachert C. Organization of secondary lymphoid tissue and local IgE formation to Staphylococcus aureus enterotoxins in nasal polyp tissue. Allergy 2005;60:71-9.

388. Lau A, Lester S, Moraitis S, et al. Tertiary lymphoid organs in recalcitrant chronic rhinosinusitis. J Allergy Clin Immunol 2017;139:1371-3.e6

389. Kato A, Peters A, Suh L, et al. Evidence of a role for $B$ cell-activating factor of the TNF family in the pathogenesis of chronic rhinosinusitis with nasal polyps. J Allergy Clin Immunol 2008;121:1385-92, 92 e1-2.

390. Chen J-B, James LK, Davies AM, et al. Antibodies and superantibodies in patients with chronic rhinosinusitis with nasal polyps. J Allergy Clin Immunol 2017;139:1195 204.e11.

391.Patou J, Holtappels G, Affleck K, van Cauwenberge P, Bachert C. Syk-kinase inhibition prevents mast cell activation in nasal polyps. Rhinology 2011;49:100-6.

392. Stevens WW, Lee RJ, Schleimer RP, Cohen NA. Chronic rhinosinusitis pathogenesis. J Allergy Clin Immunol 2015;136:1442-53.

393. Barham HP, Osborn JL, Snidvongs K, Mrad N, Sacks R, Harvey RJ. Remodeling changes of the upper airway with chronic rhinosinusitis. Int Forum Allergy Rhinol 2015;5:565-72.

394. Kuhar HN, Tajudeen BA, Mahdavinia M, Gattuso P, Ghai R, Batra PS. Inflammatory infiltrate and mucosal remodeling in chronic rhinosinusitis with and without polyps: structured histopathologic analysis. Int
Forum Allergy Rhinol 2017;7:679-89.

395. Samitas K, Carter A, Kariyawasam HH, Xanthou G. Upper and lower airway remodelling mechanisms in asthma, allergic rhinitis and chronic rhinosinusitis: The one airway concept revisited. Allergy 2018;73:9931002.

396. Rehl RM, Balla AA, Cabay RJ, Hearp ML, Pytynia KB, Joe SA. Mucosal remodeling in chronic rhinosinusitis. Am J Rhinol 2007;21:651-7.

397. Kountakis SE, Arango P, Bradley D, Wade ZK, Borish L. Molecular and cellular staging for the severity of chronic rhinosinusitis. Laryngoscope 2004;114:1895-905.

398. Van Bruaene N, Bachert C. Tissue remodeling in chronic rhinosinusitis. Curr Opin Allergy Clin Immunol 2011;11:8-11.

399. Shi LL, Xiong P, Zhang L, et al. Features of airway remodeling in different types of Chinese chronic rhinosinusitis are associated with inflammation patterns. Allergy 2013;68:101-9.

400.Schleimer RP, Berdnikovs S. Etiology of epithelial barrier dysfunction in patients with type 2 inflammatory diseases. The J Allergy Clin Immunol 2017;139:1752-61.

401.Zhang N, Van Crombruggen K, Gevaert $E$, Bachert C. Barrier function of the nasal mucosa in health and type-2 biased airway diseases. Allergy 2016;71:295-307.

402. Ramezanpour M, Moraitis S, Smith JLP, Wormald PJ, Vreugde S. Th17 Cytokines Disrupt the Airway Mucosal Barrier in Chronic Rhinosinusitis. Mediators of inflammation 2016;2016:9798206.

403. Pothoven KL, Norton JE, Hulse KE, et al. Oncostatin M promotes mucosal epithelial barrier dysfunction, and its expression is increased in patients with eosinophilic mucosal disease. J Allergy Clin Immunol 2015:136:737-46.e4.

404. Schleimer RP. Immunopathogenesis of Chronic Rhinosinusitis and Nasal Polyposis. Annual review of pathology 2017;12:331-57.

405. Pothoven KL, Schleimer RP. The barrier hypothesis and Oncostatin M: Restoration of epithelial barrier function as a novel therapeutic strategy for the treatment of type 2 inflammatory disease. Tissue barriers 2017;5:e1341367.

406. de Borja Callejas F, Picado C, MartinezAnton A, et al. Differential expression of remodeling markers by tissue structure in nasal polyposis. Am J Rhinol Allergy 2013;27:e69-74

407. Shimizu S, Gabazza EC, Ogawa T, et al. Role of thrombin in chronic rhinosinusitis-associated tissue remodeling. Am J Rhinol Allergy 2011;25:7-11.

408. Shimizu S, Tojima I, Takezawa K, Matsumoto K, Kouzaki H, Shimizu T. Thrombin and activated coagulation factor $X$ stimulate the release of cytokines and fibronectin from nasal polyp fibroblasts via proteaseactivated receptors. Am J Rhinol Allergy 2017:31:13-8

409. Shimizu S, Ogawa T, Takezawa K, Tojima
I, Kouzaki H, Shimizu T. Tissue factor and tissue factor pathway inhibitor in nasal mucosa and nasal secretions of chronic rhinosinusitis with nasal polyp. Am J Rhinol Allergy 2015;29:235-42.

410.Takabayashi T, Tanaka Y, Susuki D, et al. Increased expression of L-plastin in nasal polyp of patients with nonsteroidal antiinflammatory drug-exacerbated respiratory disease. Allergy 2019;74:1307-16

411. Mueller SK, Nocera AL, Dillon ST, Libermann TA, Wendler O, Bleier BS. Tissue and Exosomal Serine Protease Inhibitors Are Significantly Overexpressed in Chronic Rhinosinusitis With Nasal Polyps. Am J Rhinol Allergy 2019, https://dx.doi.org/10.11 77/1945892419831108:1945892419831108.

412. Karatzanis AD, Samara KD, Antoniou KM et al. Investigation of angiogenetic pathways in nasal polyposis. Molecular medicine reports 2012;5:1158-62.

413. Luukkainen A, Seppala M, Renkonen J, et al. Low lymphatic vessel density associates with chronic rhinosinusitis with nasal polyps. Rhinology 2017;55:181-91.

414. Mostafa HS, Fawzy TO, Jabri WR, Ayad E. Lymphatic obstruction: a novel etiologic factor in the formation of antrochoanal polyps. Ann of Otology, Rhinology, and Laryngology 2014;123:381-6.

415. Li Y, Li L, Wang T, et al. Analysis of epidermal growth factor signaling in nasal mucosa epithelial cell proliferation involved in chronic rhinosinusitis. Chinese medical journal 2014;127:3449-53.

416. Bae CH, Kim JS, Song S-Y, Kim Y-W, Park S-Y, Kim Y-D. Insulin-like growth factor-1 induces MUC8 and MUC5B expression via ERK1 and p38 MAPK in human airway epithelial cells. Biochemical and biophysical research communications 2013:430:683-8.

417. Muluk NB, Arikan OK, Atasoy P, Kilic R, Yalcinozan ET. The role of platelet-derived growth factor in the pathogenesis of sinonasal polyps: immunohistochemical assessment in epithelial, subepithelial and deep layers of the mucosa. Clin and experimental otorhinolaryngology 2013;6:152-60.

418. Stevens PR, Tessema B, Brown SM, Parham K, Gronowicz G. Chronic rhinosinusitis osteoblasts differ in cellular properties from normal bone. Int Forum Allergy Rhinol 2015;5:124-31.

419. Wu D, Nocera AL, Mueller SK, Finn K, Libermann TA, Bleier BS. Osteitis is associated with dysregulated pro-osteoblastic activity in patients with nasal polyps. Laryngoscope 2019;129:E102-E9.

420. Wang $M$, Ye T, Liang $N$, et al. Differing roles for TGF-beta/Smad signaling in osteitis in chronic rhinosinusitis with and without nasal polyps. Am J Rhinol Allergy 2015;29:e152-9.

421. Hoggard M, Nocera A, Biswas K, Taylor MW Douglas RG, Bleier BS. The sinonasal microbiota, neural signaling, and depression in chronic rhinosinusitis. Int Forum Allergy Rhinol 2018:8:394-405. 
422.Wu D, Mueller SK, Nocera AL, Finn K, Libermann TA, Bleier BS. Axonal Guidance Signaling Pathway Is Suppressed in Human Nasal Polyps. Am J Rhinol Allergy 2018;32:208-16.

423. Larsson $O$, Tengroth $L, X u Y, U d d m a n R$, Kumlien Georén S, Cardell L-O. Substance P represents a novel first-line defense mechanism in the nose. J Allergy Clin Immunol 2018;141:128-36.e3.

424.Ismi O, Kara T, Polat G, et al. Is there any effect of neurotrophin-3 on the pathogenesis of non-allergic nasal polyps? The J laryngology and otology 2018;132:724-8.

425. De Greve G, Hellings PW, Fokkens WJ, Pugin B, Steelant B, Seys SF. Endotype-driven treatment in chronic upper airway diseases. Clin Transl Allergy 2017;7:22.

426. Kim KW, Ober C. Lessons Learned From GWAS of Asthma. Allergy, asthma \& immunology research 2019;11:170-87.

427. Hsu J, Avila PC, Kern RC, Hayes MG, Schleimer RP, Pinto JM. Genetics of chronic rhinosinusitis: state of the field and directions forward. J Allergy Clin Immunol 2013;131:975-7.

428. Wang X, Moylan B, Leopold DA, et al. Mutation in the gene responsible for cystic fibrosis and predisposition to chronic rhinosinusitis in the general population. Jama 2000;284:1814-9.

429. Hamilos DL. Chronic Rhinosinusitis in Patients with Cystic Fibrosis. The J Allergy Clin Immunol In practice 2016;4:605-12.

430.Lynch SV, Boushey HA. The microbiome and development of allergic disease. Curr Opallergy and Clin immunology 2016;16:165-71.

431. Liu AH. Revisiting the hygiene hypothesis for allergy and asthma. The J Allergy Clin Immunol 2015;136:860-5.

432. Burbank AJ, Sood AK, Kesic MJ, Peden DB Hernandez ML. Environmental determinants of allergy and asthma in early life. J Allergy Clin Immunol 2017;140:1-12.

433. Yang IV, Lozupone CA, Schwartz DA. The environment, epigenome, and asthma. The J Allergy Clin Immunol 2017;140:14-23.

434. Kloepfer KM, Sarsani VK, Poroyko V, et al. Community-acquired rhinovirus infection is associated with changes in the airway microbiome. J Allergy Clin Immunol, 2017, 312-5.e8.

435. Kouzaki H, Matsumoto K, Kikuoka H, et al. Endogenous Protease Inhibitors in Airway Epithelial Cells Contribute to Eosinophilic Chronic Rhinosinusitis. Am J respiratory and critical care medicine 2017;195:737-47.

436.436. Kristjansson RP, Benonisdottir S, Davidsson OB, et al. A loss-of-function variant in ALOX15 protects against nasal polyps and chronic rhinosinusitis. Nature genetics 2019;51:267-76.

437. Li Z, Zeng M, Deng Y, et al. 15-Lipoxygenase 1 in nasal polyps promotes CCL26/eotaxin 3 expression through extracellular signalregulated kinase activation. J Allergy Clin Immunol 2019, 10.1016/j.jaci.2019.06.037.
438. Yan B, Wang Y, Li Y, Wang C, Zhang L. Inhibition of arachidonate 15-lipoxygenase reduces the epithelial-mesenchymal transition in eosinophilic chronic rhinosinusitis with nasal polyps. Int Forum Allergy Rhinol 2019;9:270-80

439. Bachert C, Zhang N, Hellings PW, Bousquet J. Endotype-driven care pathways in patients with chronic rhinosinusitis. The J Allergy Clin Immunol 2018;141:1543-51.

440. Leth-Moller KB, Skaaby T, Linneberg A. Allergic rhinitis and allergic sensitisation are still increasing among Danish adults. Allergy 2019, 10.1111/all.14046.

441. Wang XY, Ma TT, Wang XY, et al. Prevalence of pollen-induced allergic rhinitis with high pollen exposure in grasslands of northern China. Allergy 2018;73:1232-43.

442. Wang XD, Zheng M, Lou HF, et al. An increased prevalence of self-reported allergic rhinitis in major Chinese cities from 2005 to 2011. Allergy 2016;71:1170-80.

443. Bauchau V, Durham SR. Prevalence and rate of diagnosis of allergic rhinitis in Europe. Eur Respir J 2004;24:758-64.

444. Cazzoletti L, Ferrari M, Olivieri M, et al. The gender, age and risk factor distribution differs in self-reported allergic and non-allergic rhinitis: a cross-sectional populationbased study. Allergy Asthma Clin Immunol 2015;11:36-

445. Olsson P, Berglind N, Bellander T, Stjarne P. Prevalence of self-reported allergic and non-allergic rhinitis symptoms in Stockholm: relation to age, gender, olfactory sense and smoking. Acta Otolaryngol 2003; 123:75-80.

446. Brook CD, Kuperstock JE, Rubin SJ, Ryan MW, Platt MP. The association of allergic sensitization with radiographic sinus opacification. Am J Rhinol Allergy 2017;31:12-5.

447. Sahay S, Gera K, Bhargava SK, Shah A. Occurrence and impact of sinusitis in patients with asthma and/or allergic rhinitis. J Asthma 2016;53:635-43.

448. Koskinen A, Numminen J, Markkola A, et al. Diagnostic Accuracy of Symptoms, Endoscopy, and Imaging Signs of Chronic Rhinosinusitis Without Nasal Polyps Compared to Allergic Rhinitis. Am J Rhino Allergy 2018, 10.1177/1945892418762891.

449. Hummel T, Whitcroft KL, Andrews $P$, et al Position paper on olfactory dysfunction. Rhinology Supplement 2017;54:1-30.

450. Rimmer J, Hellings P, Lund VJ, et al. Eur position paper on diagnostic tools in rhinology. Rhinology 2019;57:1-41

451. Stogbauer J, Wirkner K, Engel C, et al. Prevalence and risk factors of smell dysfunction - a comparison between five German population-based studies. Rhinology 2019, 10.4193/Rhin19.181.

452. Landis BN, Hummel T. New evidence for high occurrence of olfactory dysfunctions within the population. Am J Med 2006;119:91-2.

453. Liu B, Luo Z, Pinto JM, et al. Relationship Between Poor Olfaction and Mortality
Among Community-Dwelling Older Adults: A Cohort Study. Ann Intern Med 2019;170:673-81.

454. Pinto JM, Wroblewski KE, Kern DW, Schumm LP, McClintock MK. Olfactory dysfunction predicts 5-year mortality in older adults. PLoS One 2014;9:e107541.

455. Ciofalo A, Filiaci F, Romeo R, Zambetti G, Vestri AR. Epidemiological aspects of olfactory dysfunction. Rhinology 2006;44:78-82.

456. Damm M, Temmel A, Welge-Lussen A, et al. [Olfactory dysfunctions. Epidemiology and therapy in Germany, Austria and Switzerland]. HNO 2004;52:112-20.

457. Deems DA, Doty RL, Settle RG, et al. Smell and taste disorders, a study of 750 patients from the University of Pennsylvania Smell and Taste Center. Arch Otolaryngol Head Neck Surg. 1991;117:519-28.

458. Fark T, Hummel T. Olfactory disorders: distribution according to age and gender in 3,400 patients. Eur Arch Otorhinolaryngol 2013;270:777-9

459. Nordin S, Bramerson A. Complaints of olfactory disorders: epidemiology, assessment and Clin implications. Curr Opin Allergy Clin Immunol 2008:8:10-5.

460. Quint C, Temmel AF, Schickinger B, Pabinger $S$, Ramberger P, Hummel T. Patterns of nonconductive olfactory disorders in eastern Austria: a study of 120 patients from the Department of Otorhinolaryngology at the University of Vienna. Wien Klin Wochenschr 2001;113:52-7.

461.Temmel AF, Quint C, SchickingerFischer B, Klimek L, Stoller E, Hummel T. Characteristics of olfactory disorders in relation to major causes of olfactory loss. Arch Otolaryngol Head Neck Surg 2002;128:63541.

462. Forster G, Damm M, Gudziol H, et al. [Olfactory dysfunction. Epidemiology, pathophsiological classification, diagnosis and therapy]. HNO 2004;52:679-84.

463. Haxel BR. Recovery of olfaction after sinus surgery for chronic rhinosinusitis: A review. Laryngoscope 2019;129:1053-9.

464. Klimek L, Hummel T, Moll B, Kobal G, Mann WJ. Lateralized and bilateral olfactory function in patients with chronic sinusitis compared with healthy control subjects. Laryngoscope 1998;108:111-4.

465. Litvack JR, Mace JC, Smith TL. Olfactory function and disease severity in chronic rhinosinusitis. Am J Rhinol Allergy 2009;23:139-44.

466. Kohli P, Naik AN, Harruff EE, Nguyen SA, Schlosser RJ, Soler ZM. The prevalence of olfactory dysfunction in chronic rhinosinusitis. Laryngoscope 2017;127:309-20.

467.Soler ZM, Kohli P, Storck KA, Schlosser RJ. Olfactory Impairment in Chronic Rhinosinusitis Using Threshold, Discrimination, and Identification Scores. Chem Sens 2016:41:713-9.

468. Lane AP, Turner J, May L, Reed R. A genetic model of chronic rhinosinusitis-associated olfactory inflammation reveals revers- 
ible functional impairment and dramatic neuroepithelial reorganization. J Neurosci 2010;30:2324-9.

469. Pfaar O, Landis BN, Frasnelli J, Huttenbrink KB, Hummel T. Mechanical obstruction of the olfactory cleft reveals differences between orthonasal and retronasal olfactory functions. Chem Sens 2006;31:27-31.

470. Apter AJ, Gent JF, Frank ME. Fluctuating olfactory sensitivity and distorted odor perception in allergic rhinitis. Arch Otolaryngol Head Neck Surg 1999;125:1005-10.

471. Landis BN, Hsieh JW, Coppin G. Circadian anosmia: A rare Clin presentation. Laryngoscope 2018;128:1537-9.

472. Negoias S, Friedrich H, Caversaccio MD, Landis BN. Rapidly fluctuating anosmia: A Clin sign for unilateral smell impairment. Laryngoscope 2016;126:E57-9.

473. Seiden AM, Duncan HJ. The diagnosis of a conductive olfactory loss. Laryngoscope 2001;111:9-14.

474. Ganjaei KG, Soler ZM, Storck KA, Rowan NR, Othieno FA, Schlosser RJ. Variability in Retronasal Odor Identification Among Patients With Chronic Rhinosinusitis. Am J Rhinol Allergy 2018;32:424-31.

475. Othieno F, Schlosser RJ, Storck KA, Rowan NR, Smith TL, Soler ZM. Retronasal olfaction in chronic rhinosinusitis. Laryngoscope 2018;128:2437-42.

476. Landis BN, Giger R, Ricchetti A, et al. Retronasal olfactory function in nasal polyposis. Laryngoscope 2003;113:1993-7.

477. Whitcroft KL, Cuevas M, Haehner A, Hummel T. Patterns of olfactory impairment reflect underlying disease etiology. Laryngoscope 2017;127:291-5.

478. Stevens MH. Steroid-dependent anosmia. Laryngoscope 2001;111:200-3.

479. Jankowski R, Bodino C. Olfaction in patients with nasal polyposis: effects of systemic steroids and radical ethmoidectomy with middle turbinate resection (nasalization). Rhinology 2003:41:220-30.

480.Bogdanov V, Walliczek-Dworschak U, Whitcroft KL, Landis BN, Hummel T. Response to Glucocorticosteroids Predicts Olfactory Outcome After ESS in Chronic Rhinosinusitis. Laryngoscope 2019, 10.1002/lary.28233.

481. Rives P, Espitalier F, Michel G, Blanc X, Fortun C, Malard O. Prospective evaluation of oral corticosteroid as a predictor of postoperative olfactory recovery after functional endoscopic surgery for nasal polyposis. Eur Arch Otorhinolaryngol 2019;276:3359-66.

482. Haxel BR, Bertz-Duffy S, Fruth K, Letzel S, Mann WJ, Muttray A. Comparison of subjective olfaction ratings in patients with and without olfactory disorders. J Laryngology \& Otology 2012;126:692-7.

483. Landis BN, Hummel T, Hugentobler M, Giger R, Lacroix JS. Ratings of overall olfactory function. Chem Sens 2003;28:691-4.

484. Philpott C, Wolstenholme C, Goodenough P, Clark A, Murty G. Comparison of Subjective Perception with Objective Measurement of
Olfaction. Otolaryngology - Head and Neck Surgery 2006;134:488-90.

485. Kohli P, Naik AN, Farhood Z, et al. Olfactory Outcomes after Endoscopic Sinus Surgery for Chronic Rhinosinusitis: A Meta-analysis. Otolaryngology - Head \& Neck Surgery 2016;155:936-48.

486. Sorokowska A, Drechsler E, Karwowski M, Hummel T. Effects of olfactory training: a meta-analysis. Rhinology 2017;55:17-26.

487. Foroughipour M, Sharifian SM, Shoeibi A, Ebdali Barabad N, Bakhshaee M. Causes of headache in patients with a primary diagnosis of sinus headache. Eur Arch Otorhinolaryngol 2011;268:1593-6.

488. Jones NS, Cooney TR. Facial pain and sinonasal surgery. Rhinology 2003;41:193-200.

489. DeConde AS, Suh JD, Mace JC, Alt JA, Smith TL. Outcomes of complete vs targeted approaches to endoscopic sinus surgery. Int Forum Allergy Rhinol 2015;5:691-700.

490. Van Zele T, Claeys S, Gevaert P, et al. Differentiation of chronic sinus diseases by measurement of inflammatory mediators. Allergy 2006;61:1280-9.

491. Eweiss AZ, Lund VJ, Barlow J, Rose G. Do patients with chronic rhinosinusitis with nasal polyps suffer with facial pain? Rhinology 2013;51:231-5.

492. Fahy C, Jones NS. Nasal polyposis and facial pain. Clin Otolaryngol Allied Sci 2001;26:510-3.

493. Clifton NJ, Jones NS. Prevalence of facial pain in 108 consecutive patients with paranasal mucopurulent discharge at endoscopy. J Laryngol Otol 2007;121:345-8.

494. Kieff DA, Busaba NY. Negative predictive value of normal nasal endoscopy for sinus disease as a cause of isolated facial pain. J Laryngol Otol 2011;125:1038-41.

495. Nguyen DT, Felix-Ravelo M, Arous F, Nguyen-Thi PL, Jankowski R. Facial pain/ headache before and after surgery in patients with nasal polyposis. Acta Otolaryngol 2015;135:1045-50.

496. Nguyen DT, Felix-Ravelo M, Sonnet MH, et al. Assessment of facial pain and headache before and after nasal polyposis surgery with the DyNaChron questionnaire. Eur Ann Otorhinolaryngol Head Neck Dis 2016;133:301-5.

497. Moretz IW, Kountakis SE. Subjective headache before and after endoscopic sinus surgery. Am J Rhinol 2006;20:305-7.

498. Phillips JS, Vowler SL, Salam MA. Endoscopic sinus surgery for 'sinus headache'. Rhinology 2007;45:14-9.

499. Dietz de Loos DA, Hopkins C, Fokkens WJ. Symptoms in chronic rhinosinusitis with and without nasal polyps. Laryngoscope 2013;123:57-63.

500. Gregurić T, Baudoin T, Tomljenović D, Grgić M, Štefanović M, Kalogjera L. Relationship between nasal septal deformity, symptoms and disease severity in chronic rhinosinusitis. Eur archives of oto-rhino-laryngology 2016;273:671-7.

501.Koskinen A, Numminen J, Markkola A, et al. Diagnostic Accuracy of Symptoms, Endoscopy, and Imaging Signs of Chronic Rhinosinusitis Without Nasal Polyps Compared to Allergic Rhinitis. Am J Rhinol Allergy 2018.

502. Shields G, Seikaly H, LeBoeuf $M$, et al. Correlation between facial pain or headache and computed tomography in rhinosinusitis in Canadian and U.S. subjects. Laryngoscope 2003;113:943-5.

503. Falco JJ, Thomas AJ, Quin X, et al. Lack of correlation between patient reported location and severity of facial pain and radiographic burden of disease in chronic rhinosinusitis. Int Forum Allergy Rhinol 2016;6:1173-81.

504. Agius AM. Long-term follow-up of patients with facial pain in chronic rhinosinusitis-correlation with nasal endoscopy and CT. Rhinology 2010;48:65-70.

505. Greguric T, Trkulja V, Baudoin T, Grgic MV, Smigovec I, Kalogjera L. Association between computed tomography findings and Clin symptoms in chronic rhinosinusitis with and without nasal polyps. Eur Arch Otorhinolaryngol 2017;274:2165-73.

506.Valenca MM, Valenca LP, Menezes TL. Computed tomography scan of the head in patients with migraine or tension-type headache. Arq Neuropsiquiatr 2002;60:5427.

507. Amir I, Yeo JCL, Ram B. Audit of CT scanning of paranasal sinuses in patients referred with facial pain. Rhinology 2012;50:442-6.

508. Lal D, Rounds AB, Rank MA, Divekar R. Clin and 22-item Sino-Nasal Outcome Test symptom patterns in primary headache disorder patients presenting to otolaryngologists with "sinus" headaches, pain or pressure. Int Forum Allergy Rhinol 2015;5:40816.

509.Perry BF, Login IS, Kountakis SE. Nonrhinologic headache in a tertiary rhinology practice. Otolaryngology - Head \& Neck Surgery 2004;130:449-52.

510. Leong SC, Tsang HK, Wilkie MD, Banhegyi G. Characterisation of patients with endoscopy-negative, computer tomography-negative midfacial segment pain using the sinonasal outcome test. Rhinology 2014;52:7883.

511. Wu D, Gray ST, Holbrook EH, BuSaba NY, Bleier BS. SNOT-22 score patterns strongly negatively predict chronic rhinosinusitis in patients with headache. Int Forum Allergy Rhinol 2019;9:9-15.

512. Diener HC, Dodick D, Evers S, et al. Pathophysiology, prevention, and treatment of medication overuse headache. Lancet Neurol 2019;18:891-902.

513. Kari E, DelGaudio JM. Treatment of sinus headache as migraine: the diagnostic utility of triptans. Laryngoscope 2008;1 18:2235-9.

514.Ishkanian G, Blumenthal H, Webster CJ, Richardson MS, Ames M. Efficacy of sumatriptan tablets in migraineurs selfdescribed or physician-diagnosed as having sinus headache: a randomized, double- 
blind, placebo-controlled study. Clin Ther 2007;29:99-109.

515. West B, Jones NS. Endoscopy-negative, computed tomography-negative facial pain in a nasal clinic. Laryngoscope 2001;111:581-6.

516. Agius AM, Jones NS, Muscat R. Prospective three-year follow up of a cohort study of 240 patients with chronic facial pain. J Laryngol Otol 2014;128:518-26.

517.Lal D, Rounds A, Dodick DW. Comprehensive management of patients presenting to the otolaryngologist for sinus pressure, pain, or headache. Laryngoscope 2015;125:303-10.

518. Leong SC, Lazarova L, Tsang HK, Banhegyi G. Treatment outcomes of midfacial segment pain: experience from the Liverpool multi-disciplinary team facial pain clinic. Rhinology 2015;53:35-40.

519. Brendish NJ, Mills S, Ewings S, Clark TW. Impact of point-of-care testing for respiratory viruses on antibiotic use in adults with exacerbation of airways disease. J Infect 2019;79:357-62.

520. Chiarella SE, Grammer LC. Immune deficiency in chronic rhinosinusitis: screening and treatment. Expert Rev Clin Immunol 2017;13:117-23.

521. Ebell MH, McKay B, Guilbault R, Ermias Y. Diagnosis of acute rhinosinusitis in primary care: a systematic review of test accuracy. British J General Practice 2016;66:e612-e32.

522. Godley FA, Casiano RR, Mehle M, McGeeney B, Gottschalk C. Update on the diagnostic considerations for neurogenic nasal and sinus symptoms: A current review suggests adding a possible diagnosis of migraine. Am J otolaryngology 2019;40:306-11.

523. Lund VJ, Stammberger H, Fokkens WJ, et al. Eur position paper on the anatomical terminology of the internal nose and paranasal sinuses. Rhinology Supplement 2014;24:134.

524. Wormald PJ, Bassiouni A, Callejas CA, et al. The Int Classification of the radiological Complexity (ICC) of frontal recess and frontal sinus. Int Forum Allergy Rhinol 2017;7:332-7.

525. Expert Panel on Neurologic I, Kirsch CFE, Bykowski J, et al. ACR Appropriateness Criteria((R)) Sinonasal Disease. J Am Coll Radiol 2017;14:S550-S9.

526. Younis RT, Anand VK, Davidson B. The role of computed tomography and magnetic resonance imaging in patients with sinusitis with complications. Laryngoscope 2002;112:224-9.

527. Bhattacharyya N. A comparison of symptom scores and radiographic staging systems in chronic rhinosinusitis. Am J Rhinol 2005;19:175-9.

528. Lund VJ, Kennedy DW. Staging for rhinosinusitis. Otolaryngology - Head \& Neck Surgery 1997;117:S35-40.

529. Fokkens W, Lund V, Bachert C, et al. Eur Position Paper on Rhinosinusitis and nasal Polyps. Rhinology 2005;18.
530. Bayonne E, Kania R, Tran P, Huy B, Herman P. Intracranial complications of rhinosinusitis. A review, typical imaging data and algorithm of management*. Rhinology 2009;47:59-65.

531.Jaume F, Quintó L, Alobid I, Mullol J. Overuse of diagnostic tools and medications in acute rhinosinusitis in Spain: a population-based study (the PROSINUS study). BMJ open 2018;8:e018788.

532. Rombaux P, Duprez T, Hummel T. Olfactory bulb volume in the Clin assessment of olfactory dysfunction. Rhinology 2009;47:39.

533. Lund VJ, Mackay IS. Staging in rhinosinusitus. Rhinology 1993;31:183-4.

534. Metson R, Gliklich RE, Stankiewicz JA, et al. Comparison of Sinus Computed Tomography Staging Systems. Otolaryngology-Head and Neck Surgery 1997;117:372-9

535. Oluwole M, Russell N, Tan L, Gardiner Q, White P. A comparison of computerized tomographic staging systems in chronic sinusitis. Clin otolaryngology and allied sciences 1996;21:91-5.

536. Likness MM, Pallanch JF, Sherris DA, Kita $\mathrm{H}$, Mashtare TL, Ponikau JU. Computed tomography scans as an objective measure of disease severity in chronic rhinosinusitis. Otolaryngology - Head and Neck Surgery, 2014, 305-11.

537. Okushi T, Nakayama T, Morimoto S, et al. A modified Lund-Mackay system for radiological evaluation of chronic rhinosinusitis. Auris Nasus Larynx 2013;40:548-53.

538. Sedaghat AR, Bhattacharyya N. Chronic rhinosinusitis symptoms and computed tomography staging: improved correlation by incorporating radiographic density. Int Forum Allergy Rhinol 2012;2:386-91.

539. Gwaltney JM, Phillips CD, Miller RD, Riker DK. Computed Tomographic Study of the Common Cold. New England J Medicine 1994;330:25-30

540. A. Leopold D, T. Stafford C, W. Sod E, et al. Clin Course of Acute Maxillary Sinusitis Documented by Sequential MRI Scanning. Am J Rhinol 1994;8:19-28.

541. Lloyd G, Lund V, Scadding G. Computerised tomography in the pre-operative evaluation of functional endoscopic sinus surgery. J Laryngology and Otology 1991;105:181-5.

542. Ashraf N, Bhattacharyya N. Determination of the "Incidental" Lund Score for the Staging of Chronic Rhinosinusitis. Otolaryngology-Head and Neck Surgery 2001;125:483-6.

543. Bhattacharyya N, Fried MP. The accuracy of computed tomography in the diagnosis of chronic rhinosinusitis. Laryngoscope 2003;113:125-9.

544. Lin HW, Bhattacharyya N. Diagnostic and Staging Accuracy of Magnetic Resonance Imaging for the Assessment of Sinonasal Disease. Am J Rhinol Allergy 2009;23:36-9.

545. Stankiewicz JA, Chow JM. Nasal endoscopy and the definition and diagnosis of chronic rhinosinusitis. Otolaryngol Head Neck Surg 2002;126:623-7.

546. Hill M, Bhattacharyya N, Hall TR, Lufkin R, Shapiro NL. Incidental paranasal sinus imaging abnormalities and the normal Lund score in children. Otolaryngol Head Neck Surg 2004;130:171-5.

547.Zheng Y, Zhao Y, Lv D, et al. Correlation between computed tomography staging and quality of life instruments in patients with chronic rhinosinusitis. Am J Rhinol Allergy 2010;24:e41-e5.

548. Konstantinidis I, Triaridis S, Printza A, Vital $\checkmark$, Ferekidis E, Constantinidis J. Olfactory dysfunction in nasal polyposis: correlation with computed tomography findings. ORL J Otorhinolaryngol Relat Spec 2007;69:22632.

549. Soler ZM, Sauer DA, Mace J, Smith TL. Relationship between Clin measures and histopathologic findings in chronic rhinosinusitis. Otolaryngology - Head and Neck Surgery 2009;141:454-61.

550. Bhattacharyya N. Relationship between mucosal inflammation, computed tomography, and symptomatology in chronic rhinosinusitis without polyposis. Ann Otol Rhinol Laryngol 2008;117:517-22.

551. Varonen H, Savolainen S, Kunnamo I, Heikkinen R, Revonta M. Acute rhinosinusitis in primary care: a comparison of symptoms, signs, ultrasound, and radiography. Rhinology 2003;41:37-43.

552. Ebell MH, McKay B, Dale A, Guilbault R, Ermias Y. Accuracy of Signs and Symptoms for the Diagnosis of Acute Rhinosinusitis and Acute Bacterial Rhinosinusitis. Ann Fam Med 2019;17:164-72.

553. Smith SS, Ference EH, Evans CT, Tan BK, Kern RC, Chandra RK. The prevalence of bacterial infection in acute rhinosinusitis: a Systematic review and meta-analysis. Laryngoscope 2015;125:57-69.

554. Jones NS, Strobl A, Holland I. A study of the CT findings in 100 patients with rhinosinusitis and 100 controls. Clin otolaryngology and allied sciences 1997;22:47-51.

555.Lund VJ, Stammberger H, Nicolai P, et al. Eur position paper on endoscopic management of tumours of the nose, paranasal sinuses and skull base. Rhinology Supplement 2010;22:1-143.

556.Zinreich SJ, Kennedy DW, Rosenbaum AE, Gayler BW, Kumar AJ, Stammberger $H$. Paranasal sinuses: $C T$ imaging requirements for endoscopic surgery. Radiology 1987;163:769-75.

557. Lund VJ, Savy L, Lloyd G. Imaging for endoscopic sinus surgery in adults. $J$ Laryngology Otology 2000;1 14:395-7.

558. Hopkins C, Browne JP, Slack R, Lund V, Brown P. The Lund-Mackay staging system for chronic rhinosinusitis: How is it used and what does it predict? Otolaryngology-Head and Neck Surgery 2007;137:555-61.

559. Rudmik L, MacE J, Smith T. Low-stage computed tomography chronic rhinosinusitis: What is the role of endoscopic sinus sur- 
gery? Laryngoscope 2011;121:417-21.

560. Brooks SG, Trope M, Blasetti M, et al. Preoperative Lund-Mackay computed tomography score is associated with preoperative symptom severity and predicts quality-of-life outcome trajectories after sinus surgery. Int Forum Allergy Rhinol 2018;8:668-75.

561. Leung N, Mawby TAR, Turner H, Qureishi A. Osteitis and chronic rhinosinusitis: a review of the current literature. Eur Archives of Oto-Rhino-Laryngology 2016;273:2917-23.

562. Sacks PL, Snidvongs K, Rom D, Earls P, Sacks R, Harvey RJ. The impact of neo-osteogenesis on disease control in chronic rhinosinusitis after primary surgery. Int Forum Allergy Rhinol 2013;3:823-7.

563. Videler WJM, Georgalas C, Menger DJ, Freling NJM, van Drunen CM, Fokkens WJ. Osteitic bone in recalcitrant chronic rhinosinusitis. Rhinology 2011;49:139-47.

564. Lee JT, Kennedy DW, Palmer JN, Feldman M, Chiu AG. The Incidence of Concurrent Osteitis in Patients with Chronic Rhinosinusitis: A Clinicopathological Study. Am J Rhinol 2006;20:278-82.

565. Georgalas C, Videler W, Freling N, Fokkens W. Global Osteitis Scoring Scale and chronic rhinosinusitis: a marker of revision surgery. Clin Otolaryngol 2010;35:455-61.

566. Orlandi RR, Kingdom TT, Hwang PH, et al. Int Consensus Statement on Allergy and Rhinology: Rhinosinusitis. Int Forum Allergy Rhinol 2016;6 Suppl 1:S22-S209.

567. Amine $M$, Lininger $L$, Fargo KN, Welch KC. Outcomes of endoscopy and computed tomography in patients with chronic rhinosinusitis. Int Forum Allergy Rhinol 2013;3:73-9.

568. Daramola OO, Lidder AK, Ramli R, et al. Patient knowledge and perception of computed tomography scan in the management of chronic rhinosinusitis symptoms. Laryngoscope 2015;125:791-5.

569. Leung RM, Chandra RK, Kern RC, Conley DB, Tan BK. Primary care and upfront computed tomography scanning in the diagnosis of chronic rhinosinusitis: A cost-based decision analysis. Laryngoscope 2014;124:12-8.

570. Leung R, Kern R, Jordan N, et al. Upfront computed tomography scanning is more cost-beneficial than empiric medical therapy in the initial management of chronic rhinosinusitis. Int Forum Allergy Rhinol

571. Tan BK, Chandra RK, Conley DB, Tudor RS, Kern RC. A randomized trial examining the effect of pretreatment point-of-care computed tomography imaging on the management of patients with chronic rhinosinusitis symptoms. Int Forum Allergy Rhinol 2011;1:229-34.

572. Bulla S, Blanke P, Hassepass F, et al Reducing the radiation dose for low-dose $\mathrm{CT}$ of the paranasal sinuses using iterative reconstruction: Feasibility and image quality. Eur J Radiology 2012;81:2246-50.

573. Sodickson A. CT radiation risks coming into clearer focus. BMJ 2013;346:f3102-f.
574. Yamauchi T, Tani A, Yokoyama S, Ogawa $H$. Assessment of non-invasive chronic fungal rhinosinusitis by cone beam CT: comparison with multidetector CT findings. Fukushima J Med Sci 2017;63:100-5.

575. Fraczek M, Guzinski M, Morawska-Kochman $M$, Krecicki $T$. Investigation of sinonasal anatomy via low-dose multidetector CT examination in chronic rhinosinusitis patients with higher risk for perioperative complications. Eur Archives of Oto-RhinoLaryngology 2017;274:787-93.

576. Gevaert P, Van Bruaene N, Cattaert T, et al. Mepolizumab, a humanized anti-IL-5 mAb, as a treatment option for severe nasal polyposis. J Allergy Clin Immunol 2011;128:9889.

577.Pinto JM, Mehta N, DiTineo M, Wang J, Baroody FM, Naclerio RM. A randomized, double-blind, placebo-controlled trial of anti-lgE for chronic rhinosinusitis. Rhinology 2010;48:318-24

578.van Agthoven M, Fokkens WJ, van de Merwe JP, Marijke van Bolhuis E, Uyl-de Groot CA, Busschbach JJ. Quality of life of patients with refractory chronic rhinosinusitis: effects of filgrastim treatment. Am J Rhinol 2001;15:231-7.

579. van der Veen J, Seys SF, Timmermans M et al. Real-life study showing uncontrolled rhinosinusitis after sinus surgery in a tertiary referral centre. Allergy 2017;72:282-90.

580. Sahlstrand-Johnson P, Hopkins C, Ohlsson $B$, Ahlner-Elmqvist M. The effect of endoscopic sinus surgery on quality of life and absenteeism in patients with chronic rhinosinuitis - a multi-centre study. Rhinology 2017;55:251-61.

581. Erskine SE, Hopkins C, Clark A, et al. Chronic rhinosinusitis and mood disturbance. Rhinology 2017;55:113-9.

582. Khan A, Huynh TMT, Vandeplas G, et al. The GALEN rhinosinusitis cohort: chronic rhinosinusitis with nasal polyps affects healthrelated quality of life. Rhinology 2019, 10.4193/Rhin19.158.

583. Hoehle LP, Phillips KM, Speth MM, Caradonna DS, Gray ST, Sedaghat AR. Responsiveness and minimal Clinly important difference for the EQ-5D in chronic rhinosinusitis. Rhinology 2019;57:110-6.

584.Garbutt J, Spitznagel E, Piccirillo J. Use of the modified SNOT-16 in primary care patients with Clinly diagnosed acute rhinosinusitis. Arch Otolaryngol Head Neck Surg. 2011;137:792-7.

585. Gliklich RE, Metson R. Techniques for outcomes research in chronic sinusitis. Laryngoscope 1995;105:387-90.

586. Benninger MS, Senior BA. The development of the Rhinosinusitis Disability Index. Arch Otolaryngol Head Neck Surg 1997;123:1175-9.

587. Piccirillo J. Psychometric and clinimetric validity of the 31-item rhinosinusitis outcome measure. Am J Rhinol 1995:297-306.

588. Rudmik L, Hopkins C, Peters A, Smith TL, Schlosser RJ, Soler ZM. Patient-reported outcome measures for adult chronic rhinosinusitis: A systematic review and quality assessment. J Allergy Clin Immunol 2015;136:1532-40.e2.

589. DeConde AS, Mace JC, Bodner T, et al. SNOT-22 quality of life domains differentially predict treatment modality selection in chronic rhinosinusitis. Int Forum Allergy Rhinol 2014;4:972-9.

590.Sedaghat AR, Gray ST, Caradonna SD, Caradonna DS. Clustering of chronic rhinosinusitis symptomatology reveals novel associations with objective Clin and demographic characteristics. Am J Rhinol Allergy 2015;29:100-5.

591. Gray ST, Phillips KM, Hoehle LP, Caradonna DS, Sedaghat AR. The 22-item Sino-Nasal Outcome Test accurately reflects patientreported control of chronic rhinosinusitis symptomatology. Int Forum Allergy Rhinol 2017;7:945-51.

592. Hopkins C, Slack R, Lund V, Brown P, Copley $\mathrm{L}$, Browne J. Long-term outcomes from the English national comparative audit of surgery for nasal polyposis and chronic rhinosinusitis. Laryngoscope 2009;119:2459-65.

593. Hopkins C, Gillett S, Slack R, Lund VJ, Browne JP. Psychometric validity of the 22-item Sinonasal Outcome Test. Clin Otolaryngology 2009;34:447-54.

594. Toma S, Hopkins C. Stratification of SNOT-22 scores into mild, moderate or severe and relationship with other subjective instruments. Rhinology 2016;54:129-33.

595. de los Santos G, Reyes P, del Castillo R, Fragola C, Royuela A. Cross-cultural adaptation and validation of the sino-nasal outcome test (SNOT-22) for Spanish-speaking patients. Eur Archives of Oto-RhinoLaryngology 2015;272:3335-40.

596.Zuo KJ, Fang JQ, Piccirillo JF, Wang H, Xu G. [Development of the Sino-Nasal Outcome Test-20 Chinese version (SNOT-20 CV)]. Zhonghua Er Bi Yan Hou Tou Jing Wai Ke Za Zhi 2008;43:751-6.

597. Lu W, Qi F, Gao ZQ, Feng GD, Yuan XD, Jin XF. [Quality of life survey on patients with chronic rhinosinusitis by using Chinese version of the 22-item sinonasal outcome test (SNOT-22)]. Zhonghua Er Bi Yan Hou Tou Jing Wai Ke Za Zhi 2008;43:18-21.

598. Alanazy F, Al Dousary S, Albosaily A, Aldriweesh $T$, Alsaleh $S$, Aldrees $T$. Psychometric Arabic Sino-Nasal Outcome test-22: Validation and translation in chronic rhinosinusitis patients. Ann of Saudi Medicine 2018;38:502-7.

599. Cakir Cetin A, Kumus O, Keskinoglu P, Sutay S, Ecevit MC. Turkish validation of the SinoNasal Outcome Test-22. Clin Otolaryngol 2019;44:557-64.

600. Sami AS, Scadding GK. Rhinosinusitis in secondary school children-part 1: pilot study of the MSNOT-20 Young Person Questionnaire (MSYPQ). Rhinology 2014;52:215-24.

601.Sami AS, Scadding GK. Rhinosinusitis in secondary school children-part 2: main project analysis of MSNOT-20 Young 
Persons Questionnaire (MSYPQ). Rhinology 2014;52:225-30.

602. Rudmik L, Soler ZM, Hopkins C. Using postoperative SNOT-22 to help predict the probability of revision sinus surgery. Rhinology 2016;54:111-6.

603. Phillips KM, Bergmark RW, Hoehle LP, Caradonna DS, Gray ST, Sedaghat AR. Chronic rhinosinusitis exacerbations are differentially associated with lost productivity based on asthma status. Rhinology 2018;56:323-9.

604. Chowdhury NI, Mace JC, Bodner TE, et al. Does Medical Therapy Improve SinoNasal Outcomes Test-22 Domain Scores? An Analysis of Clinly Important Differences. Laryngoscope 2019;129:31-6.

605. Hopkins C, Hettige R, Soni-Jaiswal A, et al. CHronic Rhinosinusitis Outcome MEasures (CHROME), developing a core outcome set for trials of interventions in chronic rhinosinusitis. Rhinology 2018;56:22-32.

606. Autio TJ, Koskenkorva T, Narkio M, Leino TK, Koivunen P, Alho OP. Diagnostic accuracy of history and physical examination in bacterial acute rhinosinusitis. Laryngoscope 2015;125:1541-6.

607. Bolger WE, Kennedy DW. Nasal endoscopy in the outpatient clinic. Otolaryngologic Clinics of North America 1992;25:791-802.

608. Druce HM. Diagnosis of sinusitis in adults: history, physical examination, nasal cytology, echo, and rhinoscope. J Allergy Clin Immunol 1992;90:436-41.

609. Bhattacharyya N, Lee LN. Evaluating the diagnosis of chronic rhinosinusitis based on Clin guidelines and endoscopy. Otolaryngology-Head and Neck Surgery 2010;143:147-51.

610. Hughes RG, Jones NS. The role of nasal endoscopy in outpatient management. Clin otolaryngology allied sciences 1998;23:2246.

611. Shargorodsky J, Bhattacharyya N. What is the role of nasal endoscopy in the diagnosis of chronic rhinosinusitis? Laryngoscope 2013;123:4-6.

612. Lund VJ, Kennedy DW. Quantification for staging sinusitis. The Staging and Therapy Group. Ann Otol Rhinol Laryngol Suppl 1995;167:17-21.

613. Psaltis AJ, Li G, Vaezeafshar R, Cho K-S, Hwang PH. Modification of the lund-kennedy endoscopic scoring system improves its reliability and correlation with patientreported outcome measures. Laryngoscope 2014;124:2216-23.

614.Zhang L, Zhang LH. Comparison of different endoscopic scoring systems in patients with chronic rhinosinusitis: reliability, validity, responsiveness and correlation. Rhinology 2017;55:363-8.

615. Annamalai S, Davis J, Kubba H. How Subjective is Nasal Endoscopy? A Study of Interrater Agreement using the Lund and Mackay Scoring System. Am J Rhinol 2004;18:301-3.

616.Kim DH, Seo Y, Kim KM, Lee S, Hwang
SH. Usefulness of Nasal Endoscopy for Diagnosing Patients With Chronic Rhinosinusitis: A Meta-Analysis. Am J Rhinol Allergy 2019, 10.1177/1945892419892157:1 945892419892157.

617. Burbach GJ, Heinzerling LM, Edenharter G, et al. GA2LEN skin test study II: Clin relevance of inhalant allergen sensitizations in Europe. Allergy: Eur J Allergy Clin Immunol 2009:64:1507-15.

618. Haahtela T, Burbach GJ, Bachert C, et al. Clin relevance is associated with allergenspecific wheal size in skin prick testing. Clin Exp Allergy 2014;44:407-16.

619.van Kampen V, de Blay F, Folletti I, et al. EAACl position paper: skin prick testing in the diagnosis of occupational type I allergies. Allergy 2013;68:580-4.

620.Augé J, Vent J, Agache I, et al. EAACl Position paper on the standardization of nasal allergen challenges. Allergy 2018;73:1597-608.

621. Fauquert J-L, Jedrzejczak-Czechowicz M, Rondon C, et al. Conjunctival allergen provocation test : guidelines for daily practice. Allergy 2017;72:43-54.

622. Riechelmann H, Epple B, Gropper G. Comparison of Conjunctival and Nasal Provocation Test in Allergic Rhinitis to House Dust Mite. Int Arch Allergy Immunol2003;130:51-9.

623. Alimuddin S, Rengganis I, Rumende CM, Setiati S. Comparison of Specific Immunoglobulin E with the Skin Prick Test in the Diagnosis of House Dust Mites and Cockroach Sensitization in Patients with Asthma and/or Allergic Rhinitis. Acta Med Indones 2018;50:125-31.

624. Vidal C, Gude F, Boquete O, et al. Evaluation of the phadiatop test in the diagnosis of allergic sensitization in a general adult population. J Investig Allergol Clin Immunol 2005;15:124-30

625. Klimek L, Hoffmann HJ, Renz $H$, et al. Diagnostic test allergens used for $<i>$ in vivo $</ i>$ diagnosis of allergic diseases are at risk: a Eur Perspective. Allergy 2015;70:132931.

626. Ertas R, Ozyurt K, Atasoy M, Hawro T, Maurer $M$. The Clin response to omalizumab in chronic spontaneous urticaria patients is linked to and predicted by lgE levels and their change. Allergy 2018;73:705-12.

627. Tajiri T, Matsumoto H, Gon Y, et al. Utility of serum periostin and free lgE levels in evaluating responsiveness to omalizum$\mathrm{ab}$ in patients with severe asthma. Allergy 2016;71:1472-9.

628. Lavin J, Min JY, Lidder AK, et al. Superior turbinate eosinophilia correlates with olfactory deficit in chronic rhinosinusitis patients. Laryngoscope 2017, 10.1002/lary.26555.

629. Danielides V, Katotomichelakis $M$, Balatsouras D, et al. Evaluation of prognostic factors for olfaction in nasal polyposis treated by Endoscopic Sinus Surgery. Rhinology 2009;47:172-80

630.Lötsch J, Hummel T. Clin Usefulness of
Self-Rated Olfactory Performance-A Data Science-Based Assessment of 6000 Patients. Chem Sens 2019;44:357-64.

631. Wang X, Zhang C, Xia X, Yang Y, Zhou C. Effect of gender on odor identification at different life stages: a meta-analysis. Rhinology 2019;57(5):322-330..

632. Apter AJ, Gent JF, Frank ME. Fluctuating Olfactory Sensitivity and Distorted Odor Perception in Allergic Rhinitis. Arch Otolaryngol Head Neck Surg 1999;125:1005-10.

633. Hummel T, Konnerth CG, Rosenheim K, Kobal G. Screening of olfactory function with a four-minute odor identification test: reliability, normative data, and investigations in patients with olfactory loss. Ann Otol Rhinol Laryngol 2001;110:976-81.

634. Doty RL, McKeown DA, Lee WW, Shaman P. A study of the test-retest reliability of ten olfactory tests. Chem Senses 1995;20:64556.

635. Haehner A, Mayer A-M, Landis BN, et al. High Test-Retest Reliability of the Extended Version of the \&quot;Sniffin' Sticks\&quot; Test. Chem Sens 2009;34:705-11.

636. Hsieh JW, Keller A, Wong M, Jiang R-S, Vosshall LB. SMELL-S and SMELL-R: Olfactory tests not influenced by odor-specific insensitivity or prior olfactory experience. Proc Nat Acad Sci 2017;114:11275-84.

637. Soler ZM, Pallanch JF, Sansoni ER, et al. Volumetric computed tomography analysis of the olfactory cleft in patients with chronic rhinosinusitis. Int Forum Allergy Rhinol 2015;5:846-54.

638. Rombaux P, Weitz H, Mouraux A, et al. Olfactory Function Assessed With Orthonasal and Retronasal Testing, Olfactory Bulb Volume, and Chemosensory Event-Related Potentials. Arch Otolaryngol Head Neck Surg 2006;132:1346-51.

639. Huart C, Meusel T, Gerber J, Duprez T, Rombaux P, Hummel T. The Depth of the Olfactory Sulcus Is an Indicator of Congenital Anosmia. Am J Neuroradiology 2011;32:1911-4

640. Mueller A, Rodewald A, Reden J, Gerber J, von Kummer R, Hummel T. Reduced olfactory bulb volume in post-traumatic and post-infectious olfactory dysfunction. NeuroReport 2005;16:475-8.

641. Bitter T, Siegert F, Gudziol H, et al. Gray matter alterations in parosmia. Neuroscience 2011;177:177-82.

642.Iannilli E, Leopold DA, Hornung DE, Hummel T. Advances in Understanding Parosmia: An fMRI Study. ORL 2019;81:18592.

643. Landis BN,Frasnelli J, Croy I, Hummel T. Evaluating the clinical usefulness of structured questions in parosmia assessment. Laryngoscope 210;120:1707-1713.

644. Doty RL. Measurement of chemosensory function. World J otorhinolaryngology head and neck surgery 2018;4:11-28.

645. Whitcroft KL, Andrews PJ, Randhawa PS. Peak nasal inspiratory flow correlates with 
quality of life in functional endoscopic sinus surgery. Clin Otolaryngology 2017;42:118792.

646. Doty RL. Olfactory dysfunction and its measurement in the clinic. World J otorhinolaryngology - head and neck surgery 2015;1:28-33.

647. Hummel T, Sekinger B, Wolf SR, Pauli E, Kobal G. 'Sniffin' sticks': olfactory performance assessed by the combined testing of odor identification, odor discrimination and olfactory threshold. Chem Senses 1997;22:39-52.

648. Toledano Munoz A, Gonzalez E, Rodriguez $G$, Galindo N. The validity of CCCRC test in patients with nasal polyposis. Rhinology 2007:45:54-8.

649. Cardesin A, Alobid I, Benitez P, et al. Barcelona Smell Test - 24 (BAST-24): validation and smell characteristics in the healthy Spanish population. Rhinology 2006;44:839.

650. Koskinen S, Vento S, Malmberg H, Tuorila $\mathrm{H}$. Correspondence Between Three Olfactory Tests and Suprathreshold Odor Intensity Ratings. Acta Oto-Laryngologica 2004;124:1072-7.

651. Hummel T, Kobal G, Gudziol H, Mackay-Sim A. Normative data for the "Sniffin' Sticks" including tests of odor identification, odor discrimination, and olfactory thresholds: an upgrade based on a group of more than 3,000 subjects. Eur Archives of Oto-RhinoLaryngology 2007;264:237-43.

652. Thomas-Danguin T, Rouby C, Sicard G, et al. Development of the ETOC: a Eur test of olfactory capabilities. Rhinology 2003;41:142-51

653. Briner HR, Simmen D. Smell diskettes as screening test of olfaction. Rhinology 1999;37:145-8.

654. Sorokowska A, Oleszkiewicz A, Minovi A, Konnerth CG, Hummel T. Fast Screening of Olfactory Function Using the Q-Sticks Test. ORL J Otorhinolaryngol Relat Spec 2019, 10.1159/000500559:1-7.

655. Croy I, Hoffmann H, Philpott C, et al. Retronasal testing of olfactory function: an investigation and comparison in seven countries. Eur Archives of Oto-RhinoLaryngology 2014;271:1087-95.

656. Hoven KM, Aarstad HJ, Bjorvatn B, Lundemo EH, Steinsvag SK. Correlation between Excessive Daytime Sleepiness (EDS) and self-reported and objective nasal characteristics. Rhinology 2018;56:316-22.

657. Wong EHC, Eccles R. Comparison of classic and 4-phase rhinomanometry methods, is there any difference? Rhinology 2014:52:360-5.

658. Vogt K, Bachmann-Harildstad G, Lintermann A, Nechyporenko A, Peters F, Wernecke KD. The new agreement of the Int RIGA consensus conference on nasal airway function tests. Rhinology 2018;56:133-43.

659. Volstad I, Olafsson T, Steinsvik EA, Dahl FA, Skrindo I, Bachmann-Harildstad G. Minimal unilateral peak nasal inspiratory flow cor- relates with patient reported nasal obstruction. Rhinology 2019, 10.4193/Rhin19.178.

660.Wong EH, Eccles R. Comparison of classic and 4-phase rhinomanometry methods, is there any difference? Rhinology 2014;52:360-5.

661. Leite SHP, Jain R, Douglas RG. The Clin implications of computerised fluid dynamic modelling in rhinology. Rhinology 2019;57:2-9.

662. Wong E, Inthavong K, Singh N. Comment on the Eur position paper on diagnostic tools in rhinology aeuro" computational fluid dynamics. Rhinology 2019;57:477-8.

663. Rimmer J. Reply to the letter by Wong et al. Rhinology 2019;57:479.

664. Farzal Z, Del Signore AG, Zanation AM, et al. A computational fluid dynamics analysis of the effects of size and shape of anterior nasal septal perforations. Rhinology 2019;57:153-9.

665. Ozkul HM, Balikci HH, Gurdal MM, et al. Normal Range of Peak Nasal Inspiratory Flow and Its Role in Nasal Septal Surgery. J Craniofacial Surgery 2013;24:900-2.

666. Ottaviano G, Scadding GK, Scarpa B, Accordi D, Staffieri A, Lund VJ. Unilateral peak nasal inspiratory flow, normal values in adult population. Rhinology 2012:50:386-92.

667. Ottaviano G, Scadding GK, lacono V, Scarpa B, Martini A, Lund VJ. Peak nasal inspiratory flow and peak expiratory flow. Upright and sitting values in an adult population. Rhinology. 2016;54(2):160-3.

668. Ottaviano G, Fokkens WJ. Measurements of nasal airflow and patency: a critical review with emphasis on the use of peak nasal inspiratory flow in daily practice. Allergy 2016;71:162-74.

669. Sipilä J, Suonpää J, Silvoniemi P, Laippala P. Correlations between Subjective Sensation of Nasal Patency and Rhinomanometry in both Unilateral and Total Nasal Assessment. ORL 1995:57:260-3.

670.Ta NH, Hopkins C, Vennik J, Philpott C. Optimising trial outcomes and patient retention for the MACRO trial for chronic rhinosinusitis. Rhinology 2019;57:358-66.

671. Ottaviano G, Lund VJ, Nardello E, et al. Comparison between unilateral PNIF and rhinomanometry in healthy and obstructed noses. Rhinology 2014;52:25-30.

672. Pendolino AL, Nardello E, Lund VJ, et al. Comparison between unilateral PNIF and rhinomanometry in the evaluation of nasal cycle. Rhinology 2018;56:122-6.

673. Lange B, Thilsing T, Baelum J, Pedersen OF, Holst R, Kjeldsen A. Acoustic rhinometry in persons recruited from the general population and diagnosed with chronic rhinosinusitis according to EPOS. Eur Arch Otorhinolaryngol 2014;271:1961-6.

674. Marioni G, Ottaviano G, Staffieri A, et al. Nasal functional modifications after physical exercise: olfactory threshold and peak nasal inspiratory flow. Rhinology 2010;48:277-80.

675. Celikel S, Stevenson D, Erkorkmaz U, White AA. Use of nasal inspiratory flow rates in the measurement of aspirin-induced respiratory reactions. Ann of Allergy, Asthma and Immunology 2013;111:252-5.

676. Walters KM, Simon RA, Woessner KM Wineinger NE, White AA. Effect of misoprostol on patients with aspirin-exacerbated respiratory disease undergoing aspirin challenge and desensitization. Ann Allergy Asthma Immunol 2017;1 19:71-6.

677.Lee DK, Haggart K, Lipworth BJ. Reproducibility of response to nasal lysineaspirin challenge in patients with aspirininduced asthma. Ann Allergy Asthma Immunol 2004:93:185-8.

678. Munoz-Cano R, Salvador R, Valero A, et al. Accuracy of acoustic rhinometry versus computed tomography in the evaluation of nasal cavity in patients with nasal polyposis. Rhinology 2010;48:224-7.

679.Dinis PB, Haider H, Gomes A. Rhinomanometry, sinus CT-scan and allergy testing in the diagnostic assessment of chronic nasal obstruction. Rhinology 1997;35:158-60.

680. Alobid I, Benitez P, Valero A, Munoz R, Langdon C, Mullol J. Oral and intranasal steroid treatments improve nasal patency and paradoxically increase nasal nitric oxide in patients with severe nasal polyposis. Rhinology 2012;50:171-7.

681. Ecevit MC, Erdag TK, Dogan E, Sutay S. Effect of steroids for nasal polyposis surgery: a placebo-controlled, randomized, doubleblind study. Laryngoscope 2015;125:2041-5.

682. Hox V, Bobic S, Callebaux I, Jorissen M Hellings PW. Nasal obstruction and smell impairment in nasal polyp disease: correlation between objective and subjective parameters. Rhinology 2010;48:426-32.

683. Fokkens WJ, Vroom TM, Gerritsma V, Rijntjes E. A biopsy method to obtain high quality specimens of nasal mucosa. Rhinology 1988;26:293-5.

684.Prior AJ, Calderon MA, Lavelle RJ, Davies RJ. Nasal biopsy: indications, techniques and complications. Respiratory medicine 1995;89:161-9

685. Segal N, Osyntsov L, Olchowski J, Kordeluk S, Plakht Y. Nose biopsy: a comparison between two sampling techniques. Eur Archives of Oto-Rhino-Laryngology 2016;273:1445-8.

686. Andrews $P$, Poirrier A-L, Lund V, Choi D. Safety of human olfactory mucosal biopsy for the purpose of olfactory ensheathing cell harvest and nerve repair: a prospective controlled study in patients undergoing endoscopic sinus surgery. Rhinology 2016;54:183-91.

687. Zweig JL, Schaitkin BM, Fan CY, Barnes EL. Histopathology of tissue samples removed using the microdebrider technique: implications for endoscopic sinus surgery. Am J Rhinol 2000;14:27-32.

688. Kale SU, Mohite U, Rowlands D, Drake-Lee AB. Clin and histopathological correlation of nasal polyps: are there any surprises? Clin Otolaryngol Allied Sci 2001;26:321-3. 
689. Diamantopoulos, II, Jones NS, Lowe J. All nasal polyps need histological examination: an audit-based appraisal of Clin practice. J Laryngol Otol 2000;114:755-9.

690.Busaba NY, de Oliveira LV, Kieff DL. Correlation between preoperative Clin diagnosis and histopathological findings in patients with rhinosinusitis. Am J Rhinol 2005; 19:153-7.

691. Yeh DH, Wong J, Hoffbauer S, Wehrli B, Sommer D, Rotenberg BW. The utility of routine polyp histopathology after endoscopic sinus surgery. Int Forum Allergy Rhinol 2014;4:926-30.

692. van den Boer C, Brutel G, de Vries N. Is routine histopathological examination of FESS material useful? Eur Arch Otorhinolaryngol 2010;267:381-4

693. Wong JS, Hoffbauer S, Yeh DH, Rotenberg B, Gupta M, Sommer DD. The usefulness of routine histopathology of bilateral nasal polyps - a systematic review, meta-analysis, and cost evaluation. J Otolaryngology: Head and Neck Surgery 2015;44:46.

694.Snidvongs K, Lam M, Sacks R, et al. Structured histopathology profiling of chronic rhinosinusitis in routine practice. Int Forum Allergy Rhinol 2012;2:376-85

695. Ho J, Hamizan AW, Alvarado R, Rimmer J, Sewell WA, Harvey RJ. Systemic Predictors of Eosinophilic Chronic Rhinosinusitis. Am J Rhinol Allergy 2018;32:252-7.

696. Jiang WX, Cao PP, Li ZY, et al. A retrospective study of changes of histopathology of nasa polyps in adult Chinese in central China. Rhinology. 2019;57(4):261-267.

697. Jin P, Zi X, Charn TC, et al. Histopathological features of antrochoanal polyps in Chinese patients. Rhinology 2018;56:378-85.

698. Nakayama T, Sugimoto N, Okada N, et al. JESREC score and mucosal eosinophilia can predict endotypes of chronic rhinosinusitis with nasal polyps. Auris, Nasus, Larynx 2019;46:374-83

699. McHugh T, Snidvongs K, Xie M, Banglawala S, Sommer D. High tissue eosinophilia as a marker to predict recurrence for eosinophilic chronic rhinosinusitis: a systematic review and meta-analysis. Int Forum Allergy Rhinol 2018;8:1421-9.

700.Jiang N, Kern RC, Altman KW. Histopathological evaluation of chronic rhinosinusitis: a critical review. Am J Rhinol Allergy 2013;27:396-402.

701. Davison WL, Pearlman AN, Donatelli LA, Conley LM. Respiratory epithelial adenomatoid hamartomas: An increasingly common diagnosis in the setting of nasal polyps. Am J Rhinol Allergy 2016;30:139-46.

702. Nguyen DT, Gauchotte G, Arous F, Vignaud JM, Jankowski R. Respiratory epithelial adenomatoid hamartoma of the nose: an updated review. Am J Rhinol Allergy 2014;28:187-92

703. Vital D, Holzmann D, Huber GF, Moch $H$, Morand GB, Ikenberg K. PD-L1 is a positive prognostic factor in squamous cell carcinoma of the nasal vestibule. Rhinology
2018;56:255-60

704. Danielsen KA, Eskeland O, Fridrich-Aas K, Orszagh VC, Bachmann-Harildstad G, Burum-Auensen E. Bacterial biofilms in patients with chronic rhinosinusitis: confocal scanning laser microscopy study. Rhinology 2014;52:150-5.

705. Cornet ME, Kostamo K, Rinia AB, et al. Novel roles for nasal epithelium in the pathogenesis of chronic rhinosinusitis with nasal polyps. Rhinology 2019;57:169-79.

706. Biggs TC, Hayes SM, Harries PG, et al Immunological profiling of key inflammatory drivers of nasal polyp formation and growth in chronic rhinosinusitis. Rhinology. 2019;57(5):336-342

707. Gungor AA. On chronic rhinosinusitis and the prevalence of fungal sinus disease: problems of diagnostic accuracy and a proposed classification of chronic rhinosinusitis. Am J Otolaryngol 2012;33:543-8.

708. Melancon CC, Clinger JD. The Use of Frozen Section in the Early Diagnosis of Acute Invasive Fungal Sinusitis. Otolaryngol Head Neck Surg 2017;157:314-9.

709. Chakrabarti A, Denning DW, Ferguson BJ, et al. Fungal rhinosinusitis: a categorization and definitional schema address ing current controversies. Laryngoscope 2009;119:1809-18.

710. Leach AJ, Stubbs E, Hare K, Beissbarth J, Morris PS. Comparison of Nasal Swabs with Nose Blowing for Community-Based Pneumococcal Surveillance of Healthy Children. J Clin Microbiology 2008;46:20812

711. Beiersdorf N, Schien M, Hentschel J, Pfister W, Markert UR, Mainz JG. Soluble inflammation markers in nasal lavage from CF patients and healthy controls. J Cystic Fibrosis 2013;12:249-57

712. Castelli S, Arasi S, Pawankar R, Matricard PM. Collection of nasal secretions and tears and their use in allergology. Curr OpAllergy and Clin Immunology, 2018, 1-9.

713. Meng Y, Wang Y, Lou H, et al. Specific immunoglobulin $E$ in nasal secretions for the diagnosis of local allergic rhinitis. Rhinology. 2019;57(4):313-320

714. Manji J, Thamboo A, Tacey M, Garnis C Chadha NK. The presence of Interleukin-13 in nasal lavage may be a predictor of nasal polyposis in pediatric patients with cystic fibrosis. Rhinology 2018:56:261-7.

715. AlAhmari MD, Sapsford RJ, Wedzicha JA, Hurst JR. Intersession repeatability of a novel nasal lavage technique. Translational research: J Lab Clin Med 2011;158:163-8.

716. Tomazic PV, Birner-Gruenberger R, Leitner A, Obrist B, Spoerk S, Lang-Loidolt D. Nasal mucus proteomic changes reflect altered immune responses and epithelial permeability in patients with allergic rhinitis. Allergy Clin Immunol 2014;133:741-50.

717. Wolf A, Liesinger L, Spoerk S, et al. Olfactory cleft proteome does not reflect olfactory performance in patients with idiopathic and postinfectious olfactory disorder: A pilot study. Scientific Reports 2018;8:17554.

718. Lü FX, Esch RE. Novel nasal secretion collection method for the analysis of allergen specific antibodies and inflammatory biomarkers. J Immunological Methods 2010;356:6-17.

719. Mosler K, Coraux C, Fragaki K, et al. Feasibility of nasal epithelial brushing for the study of airway epithelial functions in CF infants. J Cystic Fibrosis 2008;7:44-53.

720. Pipolo C, Bianchini S, Barberi S, et al. Nasal cytology in children: scraping or swabbing? Rhinology 2017:55:242-50

721. Fokkens WJ, Lund V, Bachert $C$, et al. EUFOREA consensus on biologics for CRSwNP with or without asthma. Allergy 2019;74:2312-9

722. Singh K, Chang C, Gershwin ME. IgA deficiency and autoimmunity. Autoimmunity Reviews 2014;13:163-77.

723. Bonilla FA, Khan DA, Ballas ZK, et al. Practice parameter for the diagnosis and management of primary immunodeficiency. J Allergy Clin Immunol 2015;136:1186-205. e78.

724. Aslan F, Altun E, Paksoy S, Turan G. Could Eosinophilia predict Clin severity in nasal polyps? Multidisciplinary respiratory medicine 2017:12:21

725. Fokkens WJ, Reitsma S. Medical algorithms: Management of chronic rhinosinusitis. Allergy 2019;74:1415-6.

726. Bachert C, Gevaert P, Holtappels G, Johansson SG, van Cauwenberge P. Total and specific lgE in nasal polyps is related to local eosinophilic inflammation. J Allergy Clin Immunol 2001;107:607-14.

727. Johns CB, Laidlaw TM. Elevated total serum $\lg$ E in nonatopic patients with aspirin-exacerbated respiratory disease. Am J Rhinol Allergy 2014;28:287-9.

728. Jonstam K, Westman M, Holtappels G, Holweg CTJ, Bachert C. Serum periostin, IgE, and SE-lgE can be used as biomarkers to identify moderate to severe chronic rhinosinusitis with nasal polyps. J Allergy Clin Immunol 2017;140:1705-8.e3.

729. Izuhara K, Nunomura S, Nanri Y, Ono J, Takai M, Kawaguchi A. Periostin: An emerging biomarker for allergic diseases. Allergy 2019;74:2116-28.

730. Izuhara K, Ohta S, Ono J. Using Periostin as a Biomarker in the Treatment of Asthma. Allergy, asthma immunol Res 2016:8:491-8.

731. De Schryver E, Derycke L, Calus L, et al. The effect of systemic treatments on periostin expression reflects their interference with the eosinophilic inflammation in chronic rhinosinusitis with nasal polyps. Rhinology 2017:55:152-60

732. Asano T, Kanemitsu Y, Takemura M, et al. Serum Periostin as a Biomarker for Comorbid Chronic Rhinosinusitis in Patients with Asthma. Ann of the Am Thoracic Society 2017;14:667-75.

733. Maxfield AZ, Landegger LD, Brook CD, et al. Periostin as a Biomarker for Nasal Polyps in Chronic Rhinosinusitis. Otolaryngology-- 
head and neck surgery 2018;158:181-6.

734. McCusker C, Upton J, Warrington R. Primary immunodeficiency. Allergy, Asthma Clin Immunol 2018;14:61.

735. Lutalo PMK, D'Cruz DP. Diagnosis and classification of granulomatosis with polyangiitis (aka Wegener's granulomatosis). Autoimmunity 2014;48-49:94-8.

736. Greco A, Marinelli C, Fusconi M, et al. Clinic manifestations in granulomatosis with polyangiitis. Int J immunopathology and pharmacology 2016;29:151-9.

737. Seccia V, Baldini C, Latorre M, et al. Focus on the Involvement of the Nose and Paranasal Sinuses in Eosinophilic Granulomatosis with Polyangiitis (Churg-Strauss Syndrome) Nasal Cytology Reveals Infiltration of Eosinophils as a Very Common Feature. Int Arch Allergy Immunol2018;175:61-9.

738.Solans-Laque R, Fraile G, Rodriguez Carballeira $M$, et al. Clin characteristics and outcome of Spanish patients with ANCAassociated vasculitides: Impact of the vasculitis type, ANCA specificity, and treatment on mortality and morbidity. Medicine (Baltimore) 2017;96:e6083.

739. Trimarchi M, Bertazzoni G, Bussi M. Cocaine induced midline destructive lesions. Rhinology 2014;52:104-11.

740. Larocque A, Hoffman RS. Levamisole in cocaine: unexpected news from an old acquaintance. Clin toxicology (Philadelphia Pa) 2012;50:231-41.

741. Rowshani AT, Schot LJ, ten Berge IJ. C-ANCA as a serological pitfall. Lancet 2004;363:782.

742. Gungor S, Ozseker F, Yalcinsoy M, et al. Conventional markers in determination of activity of sarcoidosis. Int Immunopharmacology 2015;25:174-9.

743. Thi Hong Nguyen C, Kambe N, Kishimoto I, Ueda-Hayakawa I, Okamoto H. Serum soluble interleukin-2 receptor level is more sensitive than angiotensin-converting enzyme or lysozyme for diagnosis of sarcoidosis and may be a marker of multiple organ involvement. J Dermatology 2017;44:789-97.

744.Baenkler H-W. Functional-eicosanoidtest (FET) and disease. J Physiology and Pharmacology 2006;57:65-72.

745. Kim MS, Cho YJ. Flow Cytometry-Assisted Basophil Activation Test as a Safe Diagnostic Tool for Aspirin/NSAID Hypersenstivity. Allergy, asthma \& immunology research 2012:4:137-42.

746. Hoggard M, Wagner Mackenzie B, Jain R, Taylor MW, Biswas K, Douglas RG. Chronic Rhinosinusitis and the Evolving Understanding of Microbial Ecology in Chronic Inflammatory Mucosal Disease. Clin microbiology reviews 2017;30:321-48.

747. Mahdavinia M, Keshavarzian A, Tobin MC, Landay AL, Schleimer RP. A comprehensive review of the nasal microbiome in chronic rhinosinusitis (CRS). Clin and experimental allergy. 2016;46:21-41

748. Benninger MS, Appelbaum PC, Denneny JC, Osguthorpe DJ, Stankiewicz JA. Maxillary sinus puncture and culture in the diag- nosis of acute rhinosinusitis: The case for pursuing alternative culture methods. Otolaryngology - Head and Neck Surgery 2002;127:7-12.

749. Tantilipikorn P, Fritz M, Tanabodee J, Lanza DC, Kennedy DW. A Comparison of Endoscopic Culture Techniques for Chronic Rhinosinusitis. Am J Rhinol 2002;16:255-60.

750.750. Jardim Vieira FM, Nunes da Silva R, Stefanini R, et al. Safety of sphenoid aspiration for diagnosis and treatment of intensive care unit rhinosinusitis. Am J Rhinol Allergy 2010;24:389-91.

751. Hutchinson CA. External Proof-Puncture of the Frontal Sinus. J Laryngology Otology 1939;54:259-60.

752. Schlosser RJ, London SD, Gwaltney JM, Jr., Gross CW. Microbiology of chronic frontal sinusitis. Laryngoscope 2001;111:1330-2.

753. Dubin MG, Ebert CS, Coffey CS, Melroy CT, Sonnenburg RE, Senior BA. Concordance of middle meatal swab and maxillary sinus aspirate in acute and chronic sinusitis: a meta-analysis. Am J Rhinol 2005;19:462-70.

754.Vogan JC, Bolger WE, Keyes AS. Endoscopically guided sinonasal cultures: a direct comparison with maxillary sinus aspirate cultures. Otolaryngol Head Neck Surg 2000;122:370-3.

755. Casiano RR, Cohn S, Villasuso 3rd E, et al. Comparison of antral tap with endoscopically directed nasal culture. Laryngoscope 2001;111:1333-7.

756.Joniau S, Vlaminck S, Van Landuyt $H$, Kuhweide R, Dick C. Microbiology of sinus puncture versus middle meatal aspiration in acute bacterial maxillary sinusitis. Am J Rhinol 2005;19:135-40.

757. Klossek JM, Dubreuil L, Richet H, Richet B, Sedallian A, Beutter P. Bacteriology of the adult middle meatus. J Laryngol Otol 1996;110:847-9.

758. Gold SM, Tami TA. Role of middle meatus aspiration culture in the diagnosis of chronic sinusitis. Laryngoscope 1997;107:1586-9.

759. Benninger MS, Payne SC, Ferguson BJ Hadley JA, Ahmad N. Endoscopically directed middle meatal cultures versus maxillary sinus taps in acute bacterial maxillary rhinosinusitis: a meta-analysis. Otolaryngol Head Neck Surg 2006;134:3-9.

760. Miller C, Davis GE. Are multiple sinus cultures necessary during sinus surgery for chronic rhinosinusitis? Int Forum Allergy Rhinol 2018;8:504-8.

761.761. van den Bergh MR, Bogaert D, Dun $L$, et al. Alternative sampling methods for detecting bacterial pathogens in children with upper respiratory tract infections. J Clin Microbiol 2012;50:4134-7.

762. Hsin CH, Chen TH, Su MC, Jiang RS, Liu CM. Aspiration technique improves reliability of endoscopically directed middle meatal cultures in pediatric rhinosinusitis. Am J Rhinol Allergy 2010;24:205-9.

763. Ikeda K, Ono N, lizuka T, et al. Bacteriologic evaluation of sinus aspirates taken by balloon catheter devices in chronic rhi- nosinusitis: preliminary study. ORL J Otorhinolaryngol Relat Spec 2011;73:271-4.

764. Browne HP, Forster SC, Anonye BO, et al. Culturing of 'unculturable' human microbiota reveals novel taxa and extensive sporulation. Nature 2016;533:543-6.

765. Hugenholtz P. Exploring prokaryotic diversity in the genomic era. Genome Biol 2002;3:Reviews0003.

766. Ahovuo-Saloranta A, Rautakorpi UM, Borisenko OV, Liira H, Williams Jr. JW, Makela M. Antibiotics for acute maxillary sinusitis in adults. Cochrane Database Syst Rev 2014, 10.1002/14651858.CD000243. pub3:CD000243.

767. Sauvalle M, Alvo A. Effect of the temperature of nasal lavages on mucociliary clearance: a randomised controlled trial. Eur Archives of Oto-Rhino-Laryngology 2018;275:2403-6.

768. Struben VMD, Wieringa $\mathrm{MH}$, Mantingh $\mathrm{CJ}$, Jongste JC, Feenstra L. Nasal NO measurement by direct sampling from the nose during breathhold: aspiration flow, nasal resistance and reproducibility. Eur Archives of Oto-Rhino-Laryngology 2006;263:723-8.

769. Boon M, Meyts I, Proesmans M, Vermeulen $F L$, Jorissen M, De Boeck K. Diagnostic accuracy of nitric oxide measurements to detect primary ciliary dyskinesia. Eur J Clin Investigation 2014:44:477-85.

770. Shapiro AJ, Josephson M, Rosenfeld $M$, et al. Accuracy of Nasal Nitric Oxide Measurement as a Diagnostic Test for Primary Ciliary Dyskinesia: A Systematic Review and Meta-Analysis. Ann of the Am Thoracic Society 2017;14:AnnATS.201701062SR.

771. de Winter-de Groot KM, van Haren Noman S, Speleman L, Schilder AGM, van der Ent CK. Nasal Nitric Oxide Levels and Nasal Polyposis in Children and Adolescents With Cystic Fibrosis. JAMA Otolaryngology-Head \& Neck Surgery 2013;139:931.

772. Takeuchi K, Kitano M, Ishinaga H, et al. Recent advances in primary ciliary dyskinesia. Auris Nasus Larynx 2016;43:229-36.

773. Parrilla E, Armengot M, Mata M, et al. Primary ciliary dyskinesia assessment by means of optical flow analysis of phasecontrast microscopy images. Computerized Medical Imaging and Graphics 2014;38:16370.

774. Kouis P, Yiallouros PK, Middleton N, Evans JS, Kyriacou K, Papatheodorou SI. Prevalence of primary ciliary dyskinesia in consecutive referrals of suspect cases and the transmission electron microscopy detection rate: a systematic review and meta-analysis. Pediatric Research 2017:81:398-405.

775. Olbrich H, Cremers C, Loges Niki T, et al. Loss-of-Function GAS8 Mutations Cause Primary Ciliary Dyskinesia and Disrupt the Nexin-Dynein Regulatory Complex. The Am J Human Genetics 2015;97:546-54.

776. Farrell PM, White TB, Ren CL, et al. Diagnosis of Cystic Fibrosis: Consensus Guidelines from the Cystic Fibrosis Foundation. J 
Pediatr 2017;181s:S4-S15.e1.

777. Yoo F, Suh JD. What is the evidence for genetics in chronic rhinosinusitis? Curr Op Otolaryngology Head and Neck Surgery 2017;25:54-63.

778. Wiencek JR, Lo SF. Advances in the Diagnosis and Management of Cystic Fibrosis in the Genomic Era. Clin Chem 2018;64:898-908.

779. Bergougnoux A, Taulan-Cadars M, Claustres M, Raynal C. Current and future molecular approaches in the diagnosis of cystic fibrosis. Expert Rev Respir Med 2018;12:415-26.

780.Zeevi DA, Zahdeh F, Kling $Y$, et al. Noninvasive paternal exclusion testing for cystic fibrosis in the first five to eight weeks of gestation. Sci Rep 2018;8:15941.

781. Marshall CR, Scherer SW, Zariwala MA, et al. Whole-Exome Sequencing and Targeted Copy Number Analysis in Primary Ciliary Dyskinesia. G3 (Bethesda) 2015;5:1775-81.

782. Damseh N, Quercia N, Rumman N, Dell SD, Kim RH. Primary ciliary dyskinesia: mechanisms and management. Application of Clin Genetics 2017;10:67-74.

783. Knowles MR, Zariwala M, Leigh M. Primary Ciliary Dyskinesia. Clinics in Chest Medicine 2016;37:449-61.

784. Lee RJ, Cohen NA. Role of the bitter taste receptor T2R38 in upper respiratory infection and chronic rhinosinusitis. Curr Opallergy and Clin immunology 2015;15:14-20.

785. Cantone E, Negri R, Roscetto E, et al. In Vivo Biofilm Formation, Gram-Negative Infections and TAS2R38 Polymorphisms in CRSW NP Patients. Laryngoscope 2018;128:E339-E45.

786. Purkey MT, Li J, Mentch F, et al. Genetic variation in genes encoding airway epithelial potassium channels is associated with chronic rhinosinusitis in a pediatric population. PloS one 2014;9:e89329-e.

787. Kim JY, Cha MJ, Park YS, et al. Upregulation of FZD5 in Eosinophilic Chronic Rhinosinusitis with Nasal Polyps by Epigenetic Modification. Mol Cells 2019:42:345-55.

788. Scadding G, Lund VJ. Investigative Rhinology. Taylor \& Francis, 2004.

789. Pellegrino R, Viegi G, Brusasco V, et al. Interpretative strategies for lung function tests. Eur Respir J 2005;26:948-68.

790. NICE: Quality Standard for Asthma. 2013. https://www.nice.org.uk/guidance/qs25.

791. Bacharier LB, Boner A, Carlsen KH, et al. Diagnosis and treatment of asthma in childhood: a PRACTALL consensus report. Allergy 2008;63:5-34.

792. GINA Report: Global Strategy for Asthma Management and Prevention. 2018. https:// ginasthma.org/2018-gina-report-globalstrategy-for-asthmamanagement-and-prevention/.

793. Reddel HK, Taylor DR, Bateman ED, et al. An official Am Thoracic Society/Eur Respiratory Society statement: asthma control and exacerbations: standardizing endpoints for
Clin asthma trials and Clin practice. Am J Respir Crit Care Med 2009;180:59-99.

794. Brouwer AF, Brand PL. Asthma education and monitoring: what has been shown to work. Paediatr Respir Rev 2008;9:193-9; quiz 9-200.

795. Hardie JA, Buist AS, Vollmer WM, Ellingsen I, Bakke PS, Morkve O. Risk of over-diagnosis of COPD in asymptomatic elderly neversmokers. Eur Respir J 2002;20:1117-22.

796. Roberts SD, Farber MO, Knox KS, et al. FEV1/ FVC ratio of $70 \%$ misclassifies patients with obstruction at the extremes of age. Chest 2006;130:200-6.

797. Kowalski ML, Agache I, Bavbek S, et al. Diagnosis and management of NSAIDExacerbated Respiratory Disease (N-ERD)-a EAACI position paper. Allergy 2018, 10.1111/all.13599.

798. Viola M, Rumi G, Valluzzi RL, Gaeta F, Caruso C, Romano A. Assessing potential determinants of positive provocation tests in subjects with NSAID hypersensitivity. Clin Exp Allergy 2011;41:96-103.

799. Nizankowska-Mogilnicka E, Bochenek G, Mastalerz L, et al. EAACI/GA2LEN guideline: aspirin provocation tests for diagnosis of aspirin hypersensitivity. Allergy 2007:62:1111-8.

800. Barranco P, Bobolea I, Larco Jl, Prior N, Lopez-Serrano MC, Quirce S. Diagnosis of aspirin-induced asthma combining the bronchial and the oral challenge tests: a pilot study. J Investig Allergol Clin Immunol 2009:19:446-52.

801. Milewski M, Mastalerz L, Nizankowska E, Szczeklik A. Nasal provocation test with lysine-aspirin for diagnosis of aspirinsensitive asthma. J Allergy Clin Immunol 1998;101:581-6.

802. Miller B, Mirakian R, Gane S, et al. Nasal lysine aspirin challenge in the diagnosis of aspirin - exacerbated respiratory disease. Clin Experimental Allergy 2013;43:874-80.

803. Bavbek S, Dursun AB, Birben E, Kalayci O, Misirligil Z. Cellular allergen stimulation test with acetylsalicylic acid-lysine is not a useful test to discriminate between asthmatic patients with and without acetylsalicylic acid sensitivity. Int Arch Allergy Immunol 2009;149:58-64.

804. Al-Shemari H, Bosse $Y$, Hudson TJ, et al. Influence of leukotriene gene polymorphisms on chronic rhinosinusitis. BMC Med Genet 2008;9:21.

805. Henmyr V, Vandeplas G, Hallden C, et al. Replication study of genetic variants associated with chronic rhinosinusitis and nasal polyposis. J Allergy Clin Immunol 2014;133:273-5.

806. Bosse Y, Bacot F, Montpetit A, et al. Identification of susceptibility genes for complex diseases using pooling-based genome-wide association scans. Hum Genet 2009;125:305-18.

807. Zhang Y, Endam LM, Filali-Mouhim A, et al. Polymorphisms in RYBP and $\mathrm{AOAH}$ genes are associated with chronic rhinosinusitis in a Chinese population: a replication study. PLoS One 2012;7:e39247.

808. Erbek SS, Yurtcu E, Erbek S, Atac FB, Sahin FI, Cakmak O. Proinflammatory cytokine single nucleotide polymorphisms in nasal polyposis. Arch Otolaryngol Head Neck Surg 2007;133:705-9.

809. Mfuna Endam L, Cormier C, Bosse Y, FilaliMouhim A, Desrosiers M. Association of IL1A, IL1B, and TNF gene polymorphisms with chronic rhinosinusitis with and without nasal polyposis: A replication study. Arch Otolaryngol Head Neck Surg 2010;136:187-92.

810.Bernstein JM, Anon JB, Rontal M, Conroy J, Wang C, Sucheston L. Genetic polymorphisms in chronic hyperplastic sinusitis with nasal polyposis. Laryngoscope 2009;119:1258-64.

811.Kim SH, Yang EM, Lee HN, Cho BY, Ye YM, Park HS. Combined effect of IL-10 and TGFbeta1 promoter polymorphisms as a risk factor for aspirin-intolerant asthma and rhinosinusitis. Allergy 2009;64:1221-5.

812.Zhang ML, Ni PH, Cai CP, Chen NJ, Wang SL. [Association of susceptibility to chronic rhinosinusitis with genetic polymorphisms of IL-4 and IL-10]. Zhonghua Er Bi Yan Hou Tou Jing Wai Ke Za Zhi 2012:47:212-7.

813. Endam LM, Bosse Y, Filali-Mouhim A, et al. Polymorphisms in the interleukin-22 receptor alpha-1 gene are associated with severe chronic rhinosinusitis. Otolaryngol Head Neck Surg 2009;140:741-7.

814. Buysschaert ID, Grulois V, Eloy P, et al. Genetic evidence for a role of IL33 in nasal polyposis. Allergy 2010;65:616-22.

815. Tewfik MA, Bosse $Y$, Lemire $M$, et al. Polymorphisms in interleukin-1 receptorassociated kinase 4 are associated with total serum IgE. Allergy 2009;64:746-53.

816. Zhang Y, Endam LM, Filali-Mouhim A, Bosse Y, Castano R, Desrosiers M. Polymorphisms in the nitric oxide synthase 1 gene are associated with severe chronic rhinosinusitis. Am J Rhinol Allergy 2011;25:e49-54.

817. Castano R, Bosse Y, Endam LM, Desrosiers M. Evidence of association of interleukin-1 receptor-like 1 gene polymorphisms with chronic rhinosinusitis. Am J Rhinol Allergy 2009;23:377-84.

818. Batikhan H, Gokcan MK, Beder E, Akar N, Ozturk A, Gerceker M. Association of the tumor necrosis factor-alpha -308 G/A polymorphism with nasal polyposis. Eur Arch Otorhinolaryngol 2010;267:903-8.

819. Pasaje CF, Kim JH, Park BL, et al. UBE3C genetic variations as potent markers of nasal polyps in Korean asthma patients. J Hum Genet 2011;56:797-800.

820. Alromaih S, Mfuna-Endam L, Bosse Y, FilaliMouhim A, Desrosiers M. CD8A gene polymorphisms predict severity factors in chronic rhinosinusitis. Int Forum Allergy Rhinol 2013;3:605-11.

821. Bae JS, Pasaje CFA, Park BL, et al. Genetic association analysis of CIITA variations with nasal polyp pathogenesis in asth- 
matic patients. Molecular medicine reports 2013;7:927-34.

822. Sitarek P, Zielinska-Blizniewska H, Dziki L, et al. Association of the $-14 \mathrm{C} / \mathrm{G}$ MET and the -765G/C COX-2 gene polymorphisms with the risk of chronic rhinosinusitis with nasa polyps in a Polish population. DNA and cell biology 2012;31:1258-66.

823. Schubert MS, Hutcheson PS, Graff RJ, Santiago L, Slavin RG. HLA-DQB 1*03 in allergic fungal sinusitis and other chronic hypertrophic rhinosinusitis disorders. J Allergy Clin Immunol 2004;114:1376-83.

824. Bohman A, Juodakis J, Oscarsson M, Bacelis J, Bende M, Naluai AT. A family-based genome-wide association study of chronic rhinosinusitis with nasal polyps implicates several genes in the disease pathogenesis. PloS one 2017;12:e0185244.

825.Cheng YK, Lin CD, Chang WC, et al. Increased prevalence of interleukin-1 receptor antagonist gene polymorphism in patients with chronic rhinosinusitis. Archives of Otolaryngology -- Head \& Neck Surgery 2006;132:285-90.

826. Castano R, Bosse Y, Endam LM, FilaliMouhim A, Desrosiers M. c-MET pathway involvement in chronic rhinosinusitis: a genetic association analysis. Otolaryngol Head Neck Surg 2010;142:662-5.

827. Zielinska-Blizniewska H, Sitarek P, Milonski J, et al. Association of the $-33 \mathrm{C} / \mathrm{G}$ OSF-2 and the $140 \mathrm{~A} / \mathrm{G}$ LF gene polymorphisms with the risk of chronic rhinosinusitis with nasal polyps in a Polish population. Molecular biology reports 2012;39:5449-57.

828. Kilty SJ, Bosse Y, Cormier C, Endam LM, Desrosiers MY. Polymorphisms in the SERPINA1 (Alpha-1-Antitrypsin) gene are associated with severe chronic rhinosinusitis unresponsive to medical therapy. Am J Rhinol Allergy 2010;24:e4-9.

829. Cormier C, Bosse Y, Mfuna L, Hudson TJ,
Desrosiers M. Polymorphisms in the tumour necrosis factor alpha-induced protein 3 (TNFAIP3) gene are associated with chronic rhinosinusitis. J Otolaryngol Head Neck Surg 2009;38:133-41.

830. Tournas A, Mfuna L, Bosse Y, Filali-Mouhim A, Grenier JP, Desrosiers M. A pooling-based genome-wide association study implicates the p73 gene in chronic rhinosinusitis. J Otolaryngol Head Neck Surg 2010;39:18895.

831. Lee JS, Kim JH, Bae JS, et al. Association of CACNG6 polymorphisms with aspirin-intolerance asthmatics in a Korean population. BMC Med Genet 2010;11:138.

832. Wang LF, Chien CY, Tai CF, Kuo WR, Hsi E, Juo $\mathrm{SH}$. Matrix metalloproteinase-9 gene polymorphisms in nasal polyposis. BMC Med Genet 2010;11:85.

833. Bukowy-Bieryllo Z, Zietkiewicz E, Loges NT, et al. RPGR mutations might cause reduced orientation of respiratory cilia. Pediatr Pulmonol 2013;48:352-63.

834.Kim S-H, Choi H, Yoon M-G, Ye Y-M, Park $\mathrm{H}$-S. Dipeptidyl-peptidase 10 as a genetic biomarker for the aspirin-exacerbated respiratory disease phenotype. Ann of Allergy, Asthma \& Immunology 2015;114:208-13.

835. Jaruvongvanich $\vee$, Mongkolpathumrat P, Chantaphakul H, Klaewsongkram J. Extranasal symptoms of allergic rhinitis are difficult to treat and affect quality of life. Allergol Int 2016;65:199-203.

836. Stuck BA, Hummel T. Olfaction in allergic rhinitis: A systematic review. J Allergy Clin Immunol 2015;136:1460-70.

837. Segboer CL, Terreehorst I, Gevorgyan A, Hellings PW, van Drunen CM, Fokkens WJ. Quality of life is significantly impaired in nonallergic rhinitis patients. Allergy 2018;73:1094-100.

838. Miwa T, Furukawa M, Tsukatani T, Costanzo RM, DiNardo LJ, Reiter ER. Impact of olfac- tory impairment on quality of life and disability. Arch Otolaryngol Head Neck Surg 2001;127:497-503.

839. Mori J, Aiba T, Sugiura M, et al. Clin study of olfactory disturbance. Acta Otolaryngol Suppl 1998;538:197-201.

840.Talbot GH, Kennedy DW, Scheld WM Granito K. Rigid nasal endoscopy versus sinus puncture and aspiration for microbiologic documentation of acute bacterial maxillary sinusitis. Clin Infect Dis 2001;33:1668-75.

841. Gold SM, Tami TA. Role of middle meatus aspiration culture in the diagnosis of chronic sinusitis. Laryngoscope 1997;107:1586-9.

842.Scadding GK. Medical management of chronic rhinosinusitis. Immunology Allergy Clinics of North America 2004;24:103-18.

843. Brigham EP, West NE. Diagnosis of asthma: diagnostic testing. Int Forum Allergy Rhinol. 2015;5:S27-S30.

844. Nizankowska E, Bestynska-Krypel A, Cmiel A, Szczeklik A. Oral and bronchial provocation tests with aspirin for diagnosis of aspirin-induced asthma. Eur Resp J 2000;15:8639.

845.Xiao CC, Anderson M, Harless LD, Liang J. Shortcomings in the diagnosis of chronic rhinosinusitis: evaluating diagnosis by otolaryngologists and primary care physicians. Int Forum Allergy Rhinol. 2018;8(10):110713.

846. Karjalainen J, Joki-Erkkila VP, Hulkkonen J, Pessi T, Nieminen MM, Aromaa A, et al. The IL1A genotype is associated with nasal polyposis in asthmatic adults. Allergy. 2003;58:393-6.

847. Bassiouni A, Cleland EJ, Psaltis AJ, Vreugde S, Wormald PJ. Sinonasal microbiome sampling: a comparison of techniques. PLoS One. 2015;10(4):e0123216. 


\section{Management of chronic rhinosinusitis in adults}

\subsection{Medical Management}

\subsubsection{Short-term oral antibiotics for chronic rhinosinusitis (CRS) and exacerbations of CRS}

\subsubsection{Summary of the evidence}

Short-term courses of antibiotics are prescribed at high rates in patients with chronic rhinosinusitis (CRS) in primary and secondary care ${ }^{(1,2)}$. There is a need for stronger evidence on the role of antibiotics in CRS management. In this review, short-term antibiotic treatment is defined as a treatment duration of four weeks or less. To date, there have been two randomized clinical trials published that utilized a placebo control investigating the effect of short-term antibiotics in $\mathrm{CRS}^{(3,4)}$. A single-centre placebo-controlled trial ${ }^{(3)}$ of 32 patients with acute on chronic exacerbation of CRS (Table 6.1.1.1). Acute exacerbation of CRS was defined as an acute worsening of sinonasal symptoms in the last four weeks (nasal secretion, nasal obstruction/congestion, sense of smell, and/or facial pain) in patients with underlying CRS. Patients were randomized to receive amoxicillin/ clavulanate vs. placebo for two weeks. Although both groups had significant symptom improvement in nasal secretions and nasal obstruction compared to baseline, there was no significant between-group-differences on Visual Analog Scale-Severity
Scoring Assessment [nasal secretion: mean difference (MD) $-2(-16.1,12.1), p=0.44$; nasal obstruction: MD $-6.6(-10.6,24)$, $p=0.78]$. There was no difference between nasal endoscopy scores at day 14 between the groups (total endoscopy score, $\mathrm{p}=0.88$; nasal polyps, $\mathrm{p}=0.58$; oedema, $\mathrm{p}=0.36$; nasal secretion, $\mathrm{p}=0.42$ ). The quality of life (Sino-nasal Outcome Test, SNOT-22) improvement was similar between groups after three months [MD -2.7 $(-20.36,14), p=0.75]$. Bacterial eradication was observed in $29 \%$ of those treated with amoxicillin/clavulanate compared to $9 \%$ of those in the placebo group, but no significant statistical difference was observed between groups $(p=0.37)$. Although no severe adverse events were reported in either group, two patients who received amoxicillin/clavulanate complained of mild abdominal colic and diarrhoea.

Van Zele et al. ${ }^{(4)}$ conducted a double-blinded placebo-controlled randomized controlled trial (RCT) of the effect of doxycycline in treatment of CRS with nasal polyps (CRSwNP) in 28 patients. Doxycycline treatment significantly reduced postnasal drip symptom scores at week $2(p=0.044)$ and showed a trend for reduction in rhinorrhoea at week $8(p=0.058)$ (no corrections for multi-testing). Doxycycline had no significant effect on nasal congestion, or loss of sense of smell during the study period nor on postnasal drip and rhinorrhoea at the other time points. The doxycycline group was noted to have a small but significant reduction in nasal polyp size $(0,5$ on a scale of 8$)$

Table 6.1.1.1. Short-term antibiotics for the treatment of patients with CRS.

\begin{tabular}{|c|c|c|c|c|c|}
\hline Study & Methods & Participants & Interventions & Outcomes & Results \\
\hline $\begin{array}{l}\text { Sabino } \\
2017^{(3)}\end{array}$ & DBPCT & $\begin{array}{l}32 \text { patients ( }>18 \\
\text { years of age) with } \\
\text { acute on chronic } \\
\text { exacerbation of } \\
\text { CRSsNP }(n=12) \text { or } \\
\text { CRSwNP }(n=20)\end{array}$ & $\begin{array}{l}\text { - Amoxicillin-clavu- } \\
\text { lanate } 875 \text { mg/125 } \\
\text { mg, orally, twice } \\
\text { daily for } 14 \text { days } \\
\text { ( } n=21) \\
\text { - Placebo twice daily } \\
\text { for } 14 \text { days }(n=11)\end{array}$ & $\begin{array}{l}\text { - Visual Analog Scale-Se- } \\
\text { verity Scoring of clinical } \\
\text { symptoms at } 2 \text { wks } \\
\text { - Nasal endoscopy at } 2 \text { wks } \\
\text { - Middle meatus swab at } \\
2 \text { wks } \\
\text { - Lund-Kennedy scores at } \\
2 \text { wks } \\
\text { - SNOT-22 at } 2 \text { wks and } 3 \\
\text { months }\end{array}$ & $\begin{array}{l}\text { - No significant difference in symptoms scores, SNOT- } \\
\text { 22, endoscopy scores or bacteriologic eradication } \\
\text { at day } 14 \text { compared to baseline in the } 2 \text { treatment } \\
\text { groups } \\
\text { - No significant differences in SNOT-22 at } 3 \text { months }\end{array}$ \\
\hline $\begin{array}{l}\text { Van Zele } \\
2010^{(4)}\end{array}$ & DBPCT & $\begin{array}{l}47 \text { patients with } \\
\text { recurrent bilateral } \\
\text { nasal polyps } \\
\text { after surgery or } \\
\text { massive bilateral } \\
\text { nasal polyps } \\
\text { (grade } 3 \text { or } 4 \text { ) }\end{array}$ & $\begin{array}{l}\text { Oral methylprednis- } \\
\text { olone }(32 \mathrm{mg} / \mathrm{d} \text { on } \\
\text { days } 1-5 ; 16 \mathrm{mg} / \mathrm{d} \\
\text { on days } 6-10 ; \text { and } 8 \\
\text { mg/d on days } 11 \text { - } \\
20)(\mathrm{n}=19) \\
\text { - Oral doxycycline } \\
(200 \mathrm{mg} \text { on day } 1 \text {, } \\
100 \mathrm{mg} / \mathrm{d} \text { on days } \\
2-20)(\mathrm{n}=14) \\
\text { - Placebo for } 20 \text { days } \\
(\mathrm{n}=14)\end{array}$ & $\begin{array}{l}\text { - Nasal symptoms (anterior } \\
\text { rhinorrhoea, nasal obstruc- } \\
\text { tion, postnasal drip, and } \\
\text { loss of sense of smell) at } 1 \text {, } \\
\text { 2,4, 8, and } 12 \text { weeks } \\
\text { - Total nasal polyp score } \\
\text { (0-8) at 1,2,4, 8, and } 12 \\
\text { weeks } \\
\text { - Nasal peak inspiratory flow } \\
\text { at 1,2, 4, 8, and } 12 \text { weeks } \\
\text { - Serum eosinophil counts } \\
\text { and serum ECP } \\
\text { - IL-5, IgE, MMP-9, MPO and } \\
\text { ECP in nasal secretions }\end{array}$ & $\begin{array}{l}\text { Doxycycline treatment compared to placebo resulted in: } \\
\text { - Significantly reduced postnasal drip symptom scores } \\
\text { at week } 2 \text { ( } p=0.044) \\
\text { - Trend of reduction in rhinorrhoea at } 8 \text { wks ( } p=0.058 \text { ) } \\
\text { - No significant differences in all other symptoms and } \\
\text { time points. } \\
\text { - Small (0.5 on scale of } 8 \text { ) but significant reduction in } \\
\text { nasal polyp size for } 3 \text { months compared to placebo } \\
\text { - } p=0.015) \text {. } \\
\text { - No significant increase in PNIF over the entire study } \\
\text { - Significant reduction in MPO in nasal secretions for } 2 \\
\text { months and MPP-9 for } 2 \text { weeks }\end{array}$ \\
\hline
\end{tabular}

CRSwNP, chronic rhinosinusitis with nasal polyps; CRSsNP, chronic rhinosinusitis without nasal polyps; DBPCT, double-blind placebo controlled trial; ECP, eosinophil cationic protein; MPP-9, Matrix metalloproteinase; MPO, myeloperoxidase; PNIF, peak nasal inspiratory flow; SNOT, Sino-nasal Outcome Test. 
for three months compared to placebo $(p=0.015)$. There were no significant differences in peak nasal inspiratory flow (PNIF). Although a significant decrease in serum IL-5Ra levels was observed at one month in the doxycycline group compared to placebo ( $p=0.01$ ), this decrease was not sustained throughout the study period. Doxycycline had no effect on serum eosinophil counts or serum eosinophil cationic protein (ECP) levels compared to placebo. A significant decrease in ECP levels in nasal secretions was observed at one month in the doxycycline groups ( $p=0.032$ ), but the significance of this difference was likely resultant from an increase in the mediator in the placebo group. Similarly, a significantly lower level of immunoglobulin $\mathrm{E}(\mathrm{IgE})$ in nasal secretions was observed in the doxycycline treatment groups at four weeks compared to placebo ( $p=0.003)$, while an increase in this mediator was observed in the placebo group. Levels of IL-5 in nasal secretions progressively increased during the treatment period for the doxycycline treatment group (significance of change compared to placebo not reported). Doxycycline treatment demonstrated significantly reduced level of myeloperoxidase in nasal secretions compared to placebo during the study period (week $8 ; p=0.022$ ), suggestive of a reduction in neutrophilic activity. A significant decrease in levels of matrix metalloproteinase 9 (MMP-9) was observed in the doxycycline group compared to placebo at week $1(p=0.025)$ and week $2(p=0.028)$.

There were seven randomized studies identified that provided a parallel comparison of various antibiotic therapies (Table 6.1.1.2). One multi-centre, double-blind, double-placebo study ${ }^{(5)}$ evaluated the effects of short-term ciprofloxacin and amoxicillin/clavulanate in CRS. CRS was defined as unilateral or bilateral inflammation without nasal polyps of at least three months duration. Overall, both treatment groups had similar clinical cure rates (58.6\% vs. $51.2 \%$ ) and bacteriologic clearance rates (88.9\% vs. $90.5 \%$ ). However, patients with a positive culture who received ciprofloxacin were more likely to maintain bacteriologic clearance at 40 days post-treatment (83.3\% vs. 67.6\%, p=0.043). Endoscopy at the end of treatment showed purulent discharge in the middle meatus had cleared in a higher proportion of ciprofloxacin-treated patients $(p=0.05)$, while inflammatory reaction resolved in a higher proportion of amoxicillin/clavulanate-treated patients $(p=0.04)$. Ciprofloxacin was better tolerated during the study period with only $12.4 \%$ of patients reporting at least one adverse event, compared to $25 \%$ in the amoxicillin/clavulanate recipients $(p=0.012)$. Gastrointestinal-related events were the most common complaints in both treatment groups and all adverse events resolved by the end of treatment. There was no placebo arm. In a single-centre, clinical trial ${ }^{(6)}, 59$ patients with CRS were randomized to receive clarithromycin versus amoxicillin/ clavulanate. Patients that demonstrated clinical symptoms of chronic rhinosinusitis [headache, rhinorrhoea, coughing, post-nasal drip (PND), feeling of facial fullness, and nasal congestion for more than 12 weeks] with at least one CT finding (sinus opacity, air-fluid level or thickening of mucus layer) were recruited for the study. Clinical symptom improvement was found to be comparable between amoxicillin/clavulanate and clarithromycin for PND (40.7\% vs. 46.7\%; $\mathrm{p}=0.802)$, headache ( $40.7 \%$ vs. $50.0 \% ; p=0.626)$, cough $(48.1 \%$ vs. $43.3 \% ; p=0.626)$, rhinorrhoea ( $48.1 \%$ vs. $43.3 \%$; $p=0.502)$, epistaxis ( $14.8 \%$ vs. $10.0 \% ; p=0.473)$, facial pain or pressure $(55.6 \%$ vs. $56.7 \%$; $\mathrm{p}=0.995)$, and nasal congestion ( $81.5 \%$ vs. $63.4 \% ; p=0.255)$, respectively. The authors reported no significant difference in the rates of adverse events between both groups, with diarrhoea (10.3\%) observed most commonly in the amoxicillin/ clavulanate group and anorexia (10.0\%) reported in the clarithromycin group.

Fan et al..$^{(7)}$, conducted an open label study in 43 patients with chronic rhinosinusitis without nasal polyps (CRSsNP) comparing the effects of low versus high dose clarithromycin for 14 days. Patients recruited for the study demonstrated at least 12 weeks of two or more clinical symptoms (including nasal obstruction, nasal secretion, postnasal drip, facial pain or pressure, headache and reduction or loss of sense of smell), visible secretions in the middle nasal meatus and/or olfactory cleft on endoscopic examination, and no nasal polyps. Patients on low dose clarithromycin did not experience any significant changes in median visual analogue scale scores of nasal congestion (5.4 vs. 5.4), rhinorrhoea (6.8 vs. 6.8), PND (4.5 vs. 4.3) or loss of smell after four weeks compared to baseline. However, those in the high-dose group had sustained improvement in MVAS scores up to week 4 for nasal congestion (3.8 vs. 5.7; $p<0.025$ ), rhinorrhoea ( 2.0 vs. $6.5 ; p<0.025)$, PND (1.6 vs. 3.9, $p<0.025)$, and loss of smell (1.0 vs. $2.2, p<0.025)$ compared to baseline. There was also significant improvement in both SNOT 20 and endoscopic LundKennedy scoring for the high-dose group at two and four weeks compared to baseline, as well as when compared to the lowdose group $(p<0.025)$. Significant differences in nasal symptom scores were also observed between the low-dose and high dose groups at week 2 and week 4 (statistical significance not reported). Interleukin-8 (IL-8) decreased significantly following low and high dose antibiotic therapy at two and four weeks $(p<0.025)$. The high dose treatment group also significantly decreased levels of IL-5 at two and four weeks compared to baseline and levels of IL-5 and IL- 8 when compared to the low dose group $(p<0.025)$.

Namyslowski et al. ${ }^{(8)}$ investigated 14 days of amoxicillin/ clavulanate vs. cefuroxime in 115 patients with clinical and radiographic features of CRS. CRS was defined as clinical symptoms of headache, facial pain, presence of purulent or mucopurulent nasal discharge or PND, nasal obstruction and objective findings including $\mathrm{X}$-ray documentation of sinus opacification, air-fluid levels or mucosal thickening. The authors focused on unilateral or bilateral chronic maxillary rhinosinusitis, performing maxillary punctures to evaluate microbiologic status and bacteriologic eradication post-treatment. There was no significant difference in the observed rates of clinical cure in the amoxicillin/clavulanate group as compared to the cefuroxime group ( $98.2 \%$ vs. $91.1 \%, p=0.2$ ) or efficacy of bacteriologic eradication (rates for this outcome variable not specified). One patient from each of the study arms interrupted treatment due to diarrhoea. 
Table 6.1.1.2. Comparison of various short-term antibiotics for the treatment of patients with CRS.

\begin{tabular}{|c|c|c|c|c|c|}
\hline Study & Methods & Participants & Interventions & Outcomes & Results \\
\hline $\begin{array}{l}\text { Fan } \\
2014^{(7)}\end{array}$ & $\begin{array}{l}\text { Open-label, } \\
\text { parallel-group } \\
\text { randomized } \\
\text { clinical trial }\end{array}$ & $\begin{array}{l}43 \text { patients } \\
\text { (>20 years } \\
\text { of age) with } \\
\text { CRSsNP }\end{array}$ & $\begin{array}{l}\text { - Clarithromycin } \\
250 \text { mg daily for } \\
14 \text { days ( } n=20) \\
\text { - Clarithromycin } \\
500 \text { mg twice dai- } \\
\text { ly for } 7 \text { days, then } \\
250 \text { mg twice } \\
\text { daily for } 7 \text { days } \\
(n=23)\end{array}$ & $\begin{array}{l}\text { At } 2 \text { and } 4 \text { weeks: } \\
\text { - Nasal symptom assessment } \\
\text { - Endoscopic inspection } \\
\text { (Lund-Kennedy scores) } \\
\text { - SNOT-20 } \\
\text { - Interleukin-5 levels in nasal } \\
\text { - } \text { secretions } \\
\text { Interleukin-8 levels in nasal } \\
\text { secretions }\end{array}$ & $\begin{array}{l}\text { High-dose group demonstrated: } \\
\text { - Significant improvement in nasal symptom } \\
\text { scores, Lund-Kennedy and SNOT-20 at weeks } 2 \\
\text { and } 4 \text { compared with the baseline values and } \\
\text { low dose group } \\
\text { - IL-8 levels in nasal secretions were significantly } \\
\text { decreased at weeks } 2 \text { and } 4 \text { compared with the } \\
\text { baseline values and low-dose group values } \\
\text { - IL-5 levels were significantly decreased at } \\
\text { weeks } 2 \text { and } 4 \text { compared with the baseline val- } \\
\text { ues and the low-dose group values }\end{array}$ \\
\hline $\begin{array}{l}\text { Amini } \\
2009^{(6)}\end{array}$ & $\begin{array}{l}\text { Parallel-group } \\
\text { randomized } \\
\text { clinical trial }\end{array}$ & $\begin{array}{l}59 \text { CRS } \\
\text { patients }\end{array}$ & $\begin{array}{l}\text { - Clarithromycin } \\
500 \text { mg daily for } 3 \\
\text { weeks ( } n=30) \\
\text { - Amoxicillin-clavu- } \\
\text { lanate } 625 \text { mg } \\
\text { three times } \\
\text { daily for } 3 \text { weeks } \\
(n=29)\end{array}$ & $\begin{array}{l}\text { - Clinical efficacy at 7, 17, 21, } \\
28,42 \text { and } 56 \text { days } \\
\text { - } \text { Radiographic status at } 8 \\
\text { weeks } \\
\text { - Adverse effects }\end{array}$ & $\begin{array}{l}\text { - No statistically significant differences in clinical } \\
\text { improvement of symptoms or adverse events } \\
\text { between treatment groups }\end{array}$ \\
\hline $\begin{array}{l}\text { Jareoncharsri } \\
2004^{(12)}\end{array}$ & $\begin{array}{l}\text { Open, } \\
\text { parallel-group } \\
\text { randomized } \\
\text { clinical trial }\end{array}$ & $\begin{array}{l}60 \text { patients } \\
\text { ( }>16 \text { years } \\
\text { of age) } \\
\text { with acute } \\
\text { maxillary } \\
\text { sinusitis } \\
\text { ( } n=48) \\
\text { and acute } \\
\text { exacerbation } \\
\text { of CRS }(n=12)\end{array}$ & $\begin{array}{l}\text { - Levofloxacin } 300 \\
\text { mg daily for } 14 \\
\text { days ( } n=34) \\
\text { - Amoxicillin clavu- } \\
\text { lanate } 625 \text { mg } \\
\text { three times daily } \\
\text { for } 14 \text { days ( } n=26)\end{array}$ & $\begin{array}{l}\text { - Clinical response at day } 4 \\
\text { and } 21 \\
\text { - Plain film radiologic } \\
\text { evaluation at day } 14 \\
\text { - Bacteriologic efficacy at day } \\
14 \\
\text { - Adverse drug reactions at } \\
\text { - } \text { day } 14 \\
\text { - } \text { Laboratory tests at day } 14 \\
\text { - Vital signs at day } 14\end{array}$ & $\begin{array}{l}\text { - Mean total symptom score at } 21 \text { days, radio- } \\
\text { logic improvement, bacteriologic eradication, } \\
\text { laboratory tests, vital signs and adverse events } \\
\text { at } 14 \text { days were comparable between both } \\
\text { groups. } \\
\text { - Subgroup analysis of chronic sinusitis group } \\
\text { not performed }\end{array}$ \\
\hline $\begin{array}{l}\text { Namyslowski } \\
2002^{(9)}\end{array}$ & $\begin{array}{l}\text { Open, } \\
\text { parallel-group } \\
\text { randomized } \\
\text { clinical trial }\end{array}$ & $\begin{array}{l}231 \text { patients } \\
\text { ( }>18 \text { years } \\
\text { of age) with } \\
\text { CRS or acute } \\
\text { exacerbation } \\
\text { of CRS }\end{array}$ & $\begin{array}{l}\text { - Amoxicillin } \\
\text { clavulanate } \\
875 / 125 \mathrm{mg} \text { twice } \\
\text { daily for } 14 \text { days } \\
\text { ( } \mathrm{n}=115) \\
\text { - Cefuroxime } \\
\text { 500mg twice } \\
\text { daily for } 14 \text { days } \\
(\mathrm{n}=116)\end{array}$ & $\begin{array}{l}\text { - Clinical response at day } 3 \text { to } 5 \text {, } \\
15 \text { to } 18, \text { week } 2 \text { to } 4 \\
\text { - Treatment compliance at day } \\
15 \text { to } 18 \\
\text { - Bacteriologic response at day } \\
15 \text { to } 18 \\
\text { - Global severity of infection } \\
\text { - Adveek } 2 \text { to } 4 \\
\text { - Adve events }\end{array}$ & $\begin{array}{l}\text { - No significant difference in clinical cure rates } \\
\text { or bacteriologic eradication between the } \\
\text { amoxicillin-clavulanate treatment group vs. the } \\
\text { cefuroxime treatment group at day } 15-18 \text {. } \\
\text { - Significant improvement in symptoms of infec- } \\
\text { tion at day } 3 \text { to } 5 \text { in the amoxicillin clavulanate } \\
\text { group compared to cefuroxime ( } 81 \% \text { vs } 56 \% \text {; } \\
\text { p=0.0137) } \\
\text { - Persistent, purulent nasal discharge following } \\
\text { treatment was noted to be significantly higher } \\
\text { in the cefuroxime group at day } 3-5(3 \% \text { vs. } 12 \% \text {; } \\
\text { p=0.036). } \\
\text { - Clinical relapse at week } 2 \text { to } 4 \text { was significantly } \\
\text { higher in the cefuroxime group in the clinically } \\
\text { evaluable patients (0\% vs. } 8 \% \text {; } p=0.0049 \text { ) and } \\
\text { the intention-to-treat population (0.09\% vs. } 7 \% \text {; } \\
\text { p=0.03). } \\
\text { - Adverse events were comparable between the } \\
2 \text { treatment groups. Diarrhoea was the most } \\
\text { common adverse event observed in both } \\
\text { treatment groups. }\end{array}$ \\
\hline $\begin{array}{l}\text { Namyslowski } \\
1998^{(8)}\end{array}$ & $\begin{array}{l}\text { Parallel-group } \\
\text { randomized } \\
\text { clinical trial }\end{array}$ & $\begin{array}{l}115 \text { patients } \\
\text { (>18 years } \\
\text { of age) with } \\
\text { unilateral or } \\
\text { bilateral CRS }\end{array}$ & $\begin{array}{l}\text { - Amoxicillin } \\
\text { clavulanate } \\
875 / 125 \mathrm{mg} \text { twice } \\
\text { daily for } 14 \text { days } \\
(\mathrm{n}=55) \\
\text { - Cefuroxime } \\
\text { 500mg twice } \\
\text { daily for } 14 \text { days } \\
(n=56)\end{array}$ & $\begin{array}{l}\text { - Clinical efficacy evaluated at } \\
\text { week } 2 \text { to } 4 \\
\text { - Bacteriologic response } \\
\text { evaluated at week } 2 \text { to } 4 \\
\text { - Adverse events }\end{array}$ & $\begin{array}{l}\text { - No significant difference in the observed rates } \\
\text { of clinical cure or bacteriologic eradication in } \\
\text { the amoxicillin-clavulanate group as compared } \\
\text { to the cefuroxime group at week } 2 \text { to } 4 \\
\text { - Adverse events (e.g. diarrhoea) were compara- } \\
\text { ble between the } 2 \text { treatment groups. }\end{array}$ \\
\hline
\end{tabular}




\begin{tabular}{|c|c|c|c|c|c|}
\hline Study & Methods & Participants & Interventions & Outcomes & Results \\
\hline $\begin{array}{l}\text { Legent } \\
1994^{(5)}\end{array}$ & $\begin{array}{l}\text { Double-blind, } \\
\text { parallel-group, } \\
\text { double-placebo } \\
\text { randomized } \\
\text { clinical trial }\end{array}$ & $\begin{array}{l}251 \text { patients } \\
\text { ( }>18 \text { years } \\
\text { of age) with } \\
\text { unilateral } \\
\text { or bilateral } \\
\text { CRSsNP }\end{array}$ & $\begin{array}{l}\text { - Ciprofloxacin } \\
500 \mathrm{mg} \text { twice } \\
\text { daily for } 9 \text { days } \\
(\mathrm{n}=122) \\
\text { - Amoxicillin clavu- } \\
\text { lanate } 500 \mathrm{mg} \\
\text { three times daily } \\
\text { for } 9 \text { days }(\mathrm{n}=129)\end{array}$ & $\begin{array}{l}\text { - Clinical efficacy at day } 10 \\
\text { and } 40 \\
\text { - Bacteriologic eradication at } \\
\text { day } 10 \text { and } 40 \\
\text { - Clinical tolerance at day } 10 \\
\text { - Adverse events }\end{array}$ & $\begin{array}{l}\text { - Ciprofloxacin and amoxicillin clavulanate had } \\
\text { similar clinical cure rates ( } 58.6 \% \text { vs. } 51.2 \%) \text { and } \\
\text { bacteriologic clearance rates ( } 88.9 \% \text { vs. } 90.5 \%) \text {. } \\
\text { - Endoscopy at day } 10 \text { showed purulent } \\
\text { discharge in the middle meatus had cleared } \\
\text { in a higher proportion of ciprofloxacin-treated } \\
\text { patients ( } p=0.05 \text { ) } \\
\text { - Inflammatory reaction on endoscopy at day } 10 \\
\text { resolved in a higher proportion of amoxicillin } \\
\text { clavulanate-treated patients ( } p=0.04 \text { ). } \\
\text { - Patients with a positive culture who received } \\
\text { Ciprofloxacin were more likely to maintain bac- } \\
\text { teriologic clearance at } 40 \text { days post-treatment } \\
\text { (83.3\% vs. } 67.6 \%, p=0.043) \text {. } \\
\text { - Ciprofloxacin-recipients had lower adverse } \\
\text { events }(12.4 \% \text { vs. } 25 \%, p=0.012 \text { ) }\end{array}$ \\
\hline $\begin{array}{l}\text { Huck } \\
1993^{(11)}\end{array}$ & $\begin{array}{l}\text { Parallel-group } \\
\text { randomized } \\
\text { clinical trial }\end{array}$ & $\begin{array}{l}56 \text { acute } \\
\text { rhinosinusitis, } \\
25 \text { recurrent } \\
\text { rhinosinusitis, } \\
15 \text { chronic } \\
\text { maxillary } \\
\text { sinusitis }\end{array}$ & $\begin{array}{l}\text { - Cefaclor } 500 \mathrm{mg} \\
\text { twice daily for } 10 \\
\text { days ( } n=5) \\
\text { - Amoxicillin } \\
500 \mathrm{mg} 3 \text { times } \\
\text { daily for } 10 \text { days } \\
(n=10)\end{array}$ & $\begin{array}{l}\text { - Clinical evaluation at day } 2 \text {, } \\
16 \text { to } 18 \\
\text { - Sinus X-rays at day } 16 \text { to } 18 \\
\text { - Adverse events }\end{array}$ & $\begin{array}{l}\text { - No significant differences between the groups } \\
\text { in the CRS group }\end{array}$ \\
\hline
\end{tabular}

CRS, chronic rhinosinusitis; CRSsNP, chronic rhinosinusitis without nasal polyps; IL, interleukin; SNOT, Sino-nasal Outcome Test.

In a second study from Namyslowski et al. ${ }^{(9)}, 231$ participants with either chronic rhinosinusitis or an acute exacerbation of CRS were randomized to receive amoxicillin/clavulanate or cefuroxime for 14 days. CRS was defined as at least one major symptom (including post-nasal discharge, rhinorrhoea, cough) and constitutional symptoms (including headaches, facial pain, tooth pain, halitosis, sore throat, earache, increased wheeze or fever) plus an abnormal X-ray (including opacification, abnormal air-fluid level or greater than $5 \mathrm{~mm}$ swelling of mucosa) lasting for at least three months. Acute exacerbation of CRS was defined as the presence of inflamed sinuses on X-ray associated with two or more symptoms (including purulent nasal discharge, headache, molar tenderness and fever) lasting $\leq$ four weeks, and a patient history of at least two episodes of acute rhinosinusitis requiring antibiotic treatment during the 12 months prior to study inclusion. It is unclear how many patients had an acute exacerbation. All patients had a maxillary sinus puncture confirming infection. There was no significant difference in clinical cure rates between the amoxicillin/clavulanate treatment group versus the cefuroxime treatment group in the clinically evaluable patients ( $95 \%$ vs $88 \% ; \mathrm{p}=0.07 ; \mathrm{MD} 95 \% \mathrm{Cl}=0.6 \%$ to $15 \%$ ) or the intention-to-treat populations ( $92 \%$ vs $86 \% ; \mathrm{p}=0.15 ; \mathrm{MD} 95 \% \mathrm{Cl}=-2 \%$ to $14 \%$ ). At the day 3-5 assessment, the amoxicillin/clavulanate group showed improvement in symptoms of infection, compared the cefuroxime group ( $81 \%$ vs. $56 \%, p=0.0137)$. Persistent, purulent nasal discharge following treatment was noted to be significantly higher in the cefuroxime group at day 15 to 18 (3\% vs. $12 \% ; p=0.036)$.

All other signs and symptoms including inflammation of the sinuses ( $6 \%$ vs. $6 \%)$, fever ( $11 \%$ vs. $0 \%)$, headache ( $6 \%$ vs. $13 \%)$, and molar tenderness ( $5 \%$ vs. $9 \%$ ) were not reported to be significantly different between the amoxicillin/clavulanate treated and cefuroxime treated patients. Bacterial eradication rates were similar for amoxicillin/clavulanate and cefuroxime in the bacteriologically evaluable population ( $66 \%$ vs. $68 \%$; $\mathrm{p}=0.85 ; \mathrm{MD} 95 \% \mathrm{Cl}=-18 \%$ to $15 \%)$ and the intention-to-treat population ( $42 \%$ vs. $40 \%$ ). Clinical relapse was observed to be significantly higher in the cefuroxime group in the clinically evaluable patients ( $0 \%$ vs. $8 \%$; $p=0.0049$ ) and the intention-totreat population $(0.09 \%$ vs. $7 \% ; p=0.03)$. Adverse events were observed in $7 \%$ of the amoxicillin/clavulanate treated group and $9.5 \%$ in the cefuroxime-treated group. Diarrhoea was the most common adverse event observed in both treatment groups. Seven patients discontinued treatment due to amoxicillin/ clavulanate-related adverse events, compared to four patients in the cefuroxime-treated group. It was unclear if the second study from Namyslowski et al. ${ }^{(9)}$ represented longitudinal patient recruitment from the first Namyslowski et al. study ${ }^{(10)}$. Given the similarities in the two studies and the lack of clarity in their patient recruitment, a meta-analysis of the study outcomes was not attempted. Huck et al. ${ }^{(11)}$ evaluated cefaclor versus. amoxicillin over 10 days. The study included patients with: acute rhinosinusitis (ARS) $(n=56)$, defined as patients with rhinosinusitis symptoms for fewer than 14 days with no previous episodes in the previous year; recurrent rhinosinusitis, defined as more than one episode per year with improvement between episodes ( $n=25)$; and CRS defined as non-resolving sinus disease ( $n=15$ ). While $40 \%$ of the patients on amoxicillin and $20 \%$ of the patients on cefaclor were found to have improvement posttherapy, these numbers were too small for statistical analysis. Jareoncharsri et al. ${ }^{(12)}$ investigated the role of levofloxacin and amoxicillin/clavulanate in patients with acute maxillary sinusitis and acute exacerbation of CRS. Patients with acute rhinosinusitis were defined based on symptoms and signs of rhinosinusitis, including obstruction, purulent nasal discharge, postnasal drip, loss of smell, foul smell and headache, lasting less than four 
weeks and acute exacerbation of CRS was defined as acute worsening of chronic symptoms lasting less than four weeks. The authors demonstrated comparable symptom improvement (68.7\% vs. $69.8 \%)$, radiological improvement (61.8\% vs. $61.5 \%)$, bacteriologic eradication (78.5\% vs. $70.0 \%, \mathrm{p}>0.05)$ and adverse events ( $8.6 \%$ vs. $7.7 \%)$ in levofloxacin recipients as compared to amoxicillin/clavulanate amongst patients with acute maxillary sinusitis and acute exacerbation of CRS. However, the sample size in the subgroup of patients with acute exacerbation of CRS $(n=12)$ was small.

\section{It is uncertain whether or not the use of a short course of antibiotics has an impact on patient outcomes in adults with CRS compared with placebo.}

\subsubsection{Conclusion}

The EPOS2020 steering group, due to the very low quality of the evidence, is uncertain whether or not the use of a short course of antibiotics has an impact on patient outcomes in adults with acute exacerbations of CRS compared with placebo. Also, due to the very low quality of the evidence, it is uncertain whether or not the use of a short course of antibiotics has an impact on patient outcomes in adults with CRS compared with placebo. Gastrointestinal-related adverse events (diarrhoea and anorexia) are frequently reported. There is a need for larger high-quality trials, especially to evaluate the use of short courses of antibiotics in acute exacerbations of CRS.

\subsubsection{Long-term antibiotics}

\subsubsection{Long-term treatment with systemic macrolide antibiotics vs. placebo in CRS}

For this systematic review, long-term antibiotic treatment was defined as a treatment duration longer than four weeks. While treatment with antibiotics is common practice in the treatment of acute rhinosinusitis, there have been few placebo-controlled studies on the effects of this practice. There are two such studies that evaluate the outcomes after long-term treatment with macrolide antibiotics ${ }^{(13,14)}$. There have been multiple open studies evaluating the effects of long-term macrolide antibiotics which have had promising results with decreases in inflammatory markers, changes in mucus consistency, or endoscopic and radiographic scoring systems ${ }^{(15-21)}$. Macrolide antibiotics at a low-dose are thought to express an immunemodulating effect and their use has been incorporated into standard cystic fibrosis (CF) care ${ }^{(22-24)}$. However, there are studies comparing nasal steroid sprays to macrolide antibiotics without evidence of improvement in the macrolide group over the use of a nasal steroid spray ${ }^{(25,26)}$. Two meta-analysis containing studies in the Chinese literature have been published recently, however the authors indicated that none of the studies not reviewed here were double blind, placebo controlled trials $(\mathrm{DBPCT})^{(21,27)}$.

Two studies ${ }^{(13,14)}$ (Figure 6.1.2.1.) have completed placebocontrolled trials of the effects of long-term macrolide therapy on signs, symptoms, and patient reported quality of life outcomes. Both studies evaluated 12-week treatment, however Wallwork et al. evaluated roxithromycin at $150 \mathrm{mg}$ daily, while Videler et al. evaluated azithromycin (AZM) 500mg per week. Wallwork and colleagues showed significant improvement in SNOT-20 scores, saccharin transit time, and nasal endoscopy. Once the groups were sub-divided into low and high IgE levels $(<200 \mu \mathrm{g} / \mathrm{L}$ vs. $>200 \mu \mathrm{g} / \mathrm{L}$ ), the low IgE group had significant improvement in saccharin transit time, endoscopy, and nasal lavage of IL-8 ${ }^{2}$. However, Videler et al. did not show any improvement in their outcomes and IgE was not measured so no subgroup analysis was possible. At the end of the studies, Videler and colleagues had an improvement in $51 \%$ of patients on AZM and $35 \%$ of the placebo group without significance. Interestingly, when the groups were re-evaluated by phone 12 weeks after completion of the antibiotics, $50 \%$ of the AZM group reported "improvement/cure" compared to $9 \%$ in the placebo group. This was statistically significant $(p=0.017)$. Wallwork and colleagues had a response rate in the roxithromycin group of $67 \%$ compared to $22 \%$ in the placebo group.

While these studies had similar study sizes (60 vs. 64), their inclusion criteria were different. Wallwork et al. included only patients without nasal polyps, while Videler included patients both with and without nasal polyps. As stated above, the Wallwork et al. study sub-analyzed patients based upon low and high IgE levels. The high IgE group had the larger majority of non-responders. It is possible that sub-group analysis of the Videler study would have shown a high proportion of elevated IgE patients. These two studies highlight the importance of appropriate patient selection when using macrolide therapy. Furthermore, the once weekly dosing regime utilised by Videler et al may have compromised the study in comparison with low dose daily dosing by Wallwork et al. Further studies on the efficacy of macrolide therapy have evaluated different biochemical markers in those who do and do not respond to macrolide therapy, while some have identified lower levels of $\lg$ E to be significantly associated with response to macrolides ${ }^{(28)}$,

Figure 6.1.2.1. Forest plot of the effect of macrolides versus placebo on responder scores in CRS patients.

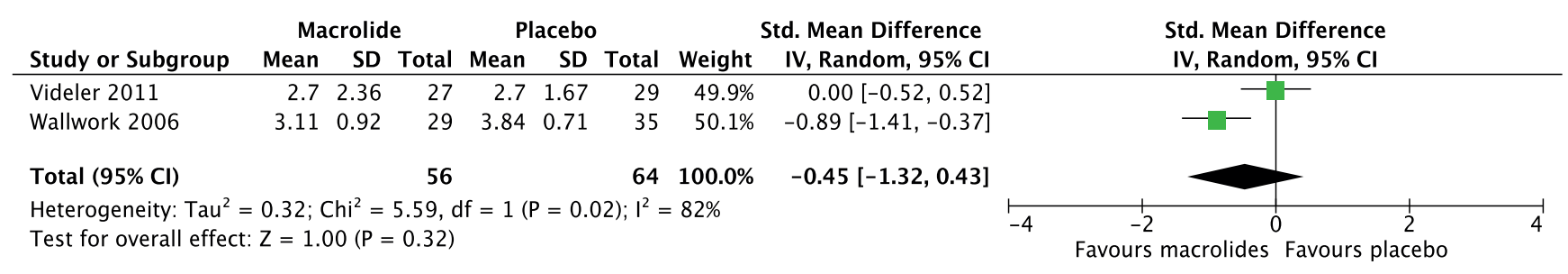


Figure 6.1.2.2. Forest plot of the effect of macrolides versus placebo on SNOT scores in CRS patients.

\begin{tabular}{|c|c|c|c|c|c|c|c|c|c|c|c|c|}
\hline Study or Subgroup & Mean & SD & Total & Mean & SD & Total & Weight & IV, Random, 95\% Cl & IV, Random & $\mathrm{m}, 95 \% \mathrm{Cl}$ & & \\
\hline Videler 2011 & 2 & 1.34 & 27 & 1.48 & 0.88 & 29 & $49.7 \%$ & $0.46[-0.08,0.99]$ & & - & & \\
\hline Wallwork 2006 & 2.34 & 1.02 & 29 & 2.88 & 0.71 & 35 & $50.3 \%$ & $-0.62[-1.12,-0.11]$ & $-1-$ & & & \\
\hline Total $(95 \% \mathrm{Cl})$ & & & 56 & & & 64 & $100.0 \%$ & $-0.08[-1.14,0.97]$ & & & & \\
\hline \multicolumn{9}{|c|}{$\begin{array}{l}\text { Heterogeneity: } \mathrm{Tau}^{2}=0.51 ; \mathrm{Chi}^{2}=8.24, \mathrm{df}=1(\mathrm{P}=0.004) ; \mathrm{I}^{2}=88 \% \\
\text { Test for overall effect: } \mathrm{Z}=0.16(\mathrm{P}=0.88)\end{array}$} & ${ }^{-1}$ Macrolide ${ }^{0}$ & Placebo & 1 & 2 \\
\hline
\end{tabular}

others have not shown this direct correlation

Pooled data analysis of the responder score (Table 6.1.2.1.; Figure 6.1.2.1.) and the SNOT scores (Figure 6.1.2.2) did not show any significant benefit in the macrolide group [responder score: $0.45(-1.32,0.43), \mathrm{p}=0.32$, two trials, 120 patients], [SNOT score: standard mean difference (SMD) 0.08(-1.14, 0.97), $\mathrm{p}=0.88$, two trials, 120 patients]. Both analyses showed significant heterogeneity.

\subsubsection{Long-term antibiotics in CRS vs. topical corticosteroids}

Two double blind studies ${ }^{(29,30)}$ evaluated long-term antibiotics in comparison with topical corticosteroids in patients with and without nasal polyps (Table 6.1.2.2.)

Haxel et al. assessed erythromycin plus fluticasone furoate in CRS patients with or without NP. All patients were treated post-surgery. There was no significant improvement in SNOT20 , olfaction, saccharin trasit time, endoscopy scores, or nasal health between the groups ${ }^{(29)}$. Subgroup analysis determined that CRSsNP patients trended towards significant improvement in the erythromycin group, however, only the endoscopy scores were significant. Amali et al. ${ }^{(30)}$ evaluated azithromycin plus fluticasone versus fluticasone in patients with and without nasal polyps. There was significant improvement in SNOT-22 scores in the azithromycin group when compared to fluticasone.

Pooled data analysis of SNOT scores showed no significant benefit in the macrolide over topical corticosteroids [SMD $0.21(-1.28,2.09), p=0.83$, two trials, 118 patients]. The $I^{2}$ was $96 \%$, suggesting a little heterogeneity $\left(X^{2}=22.94, d f=1, p<0.01\right)$. For this analysis, mean scores from SNOT-20 and SNOT-22 were taken for assessment (i.e. SNOT-22 scores were divided by 22 and SNOT-20 scores were divided by 20) (Figure 6.1.2.3).

\subsubsection{Long-term antibiotic in CRS vs. surgery}

Two studies ${ }^{(31-33)}$ evaluated treatment with long-term antibiotics versus surgical intervention for patients with and without nasal polyps. One study ${ }^{(32,33)}$ evaluated patients with and without polyps, while the other study ${ }^{(31)}$ only evaluated patients with polyps (Table 6.1.2.3.) The studies could not be combined in a meta-analysis due to the differences in design.

Ragab et al. reported on the same group of CRS patients with and without polyps ${ }^{19,20}$. The long-term antibiotic group received erythromycin for 12 weeks in addition to nasal saline irrigations and intranasal corticosteroids (INCS). The surgical group underwent surgery followed by short-term postoperative antibiotics (erythromycin), nasal saline irrigations, Dexarhinospray (DRS) for two weeks, followed by INCS. The patients were assessed at three, 6 and 12 months. There was significant improvement in visual analogue scale (VAS), SNOT20, Short Form 36 Health Survey (SF36), saccharin clearance time, and nitric oxide (NO) in each group with no significant differences between the two subgroups. Endoscopy scores increased at six and 12 months from baseline with no significant difference between groups. Subgroup analysis found no significant difference between patients with and without polyps except in total nasal volume. Patients with CRSsNP had significant improvement in nasal volume in the surgery group $(\% \Delta ; 57.3 \pm 37.6 v 41.8 \pm 42.8 ; p<0.01)$.

One study ${ }^{(31)}$ compared clarithromycin plus endoscopic sinus surgery (following eight weeks of antibiotics) to endoscopic sinus surgery alone in patients with CRSwNP. Patients were evaluated at the completion of antibiotic treatment as well as six- and 12-months post-surgery for endoscopic and nasal symptom scoring. Both groups had a significant decrease in nasal symptom score post-treatment (antibiotics vs. surgery). Both groups also had an increase in nasal symptom score from six to 12 months post-surgical intervention, but this was not significant between the two groups. Patients in the clarithromycin plus surgery group had significant improvement in endoscopy scores post-antibiotic treatment and post-surgical treatment. The surgical arm also had significant improvement of endoscopic scores. In both groups, there were patients with polyp recurrence at six and 12 months. This was significantly higher in the surgery only group at six $(27.5 \% \mathrm{v} 50 \%$; $\mathrm{p}=0.034)$ and 12 months (35\%v62.5\%; $\mathrm{p}=0.029$ ).

Figure 6.1.2.3. Forest plot of the effect of macrolides versus nasal corticosteroids on SNOT scores in CRS patients.

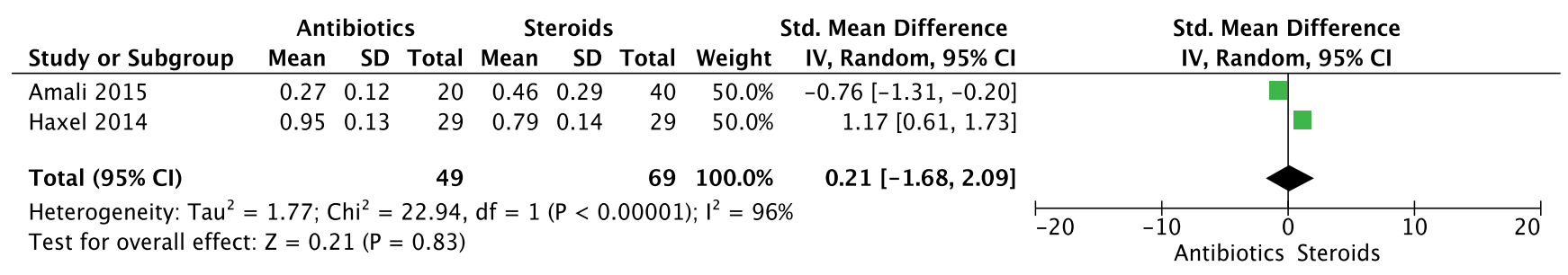


Table 6.1.2.1. Long-term antibiotic for the treatment of patients with CRS.

\begin{tabular}{|c|c|c|c|c|c|}
\hline Study & Methods & Participants & Intervention & Outcomes & Results \\
\hline $\begin{array}{l}\text { Videler } \\
2011^{(13)}\end{array}$ & DBPCT & $\begin{array}{l}60 \text { CRSWNP } \\
\text { and CRSsNP }\end{array}$ & $\begin{array}{l}\text { - Azithromycin } \\
500 \text { mg weekly for } \\
12 \text { weeks ( } n=29) \\
\text { - Placebo weekly for } \\
12 \text { weeks }(n=31)\end{array}$ & $\begin{array}{l}\text { All assessed at 6, 12, } 14 \text { weeks } \\
\text { - Patient response rating scale (1-5, } 1 \text { desperately worse, } 4 \text { cured) } \\
\text { - SNOT-22 } \\
\text { - VAS (nasal obstruction, rhinorrhea, PND, facial pain, smell reduc- } \\
\text { tion, general health, headache, toothache, tearing, coughing, } \\
\text { nasal bleeding, crusts, fatigue, nausea, vomiting/diaorrhea) } \\
\text { - SF36 } \\
\text { - Endoscopy scores } \\
\text { - PNIF } \\
\text { - Culfaction } \\
\text { Cultures }\end{array}$ & $\begin{array}{l}\text { No significant } \\
\text { effects }\end{array}$ \\
\hline $\begin{array}{l}\text { Wallwork } \\
2006^{(14)}\end{array}$ & DBPCT & 64 CRSsNP & $\begin{array}{l}\text { - Roxithromycin } \\
150 \mathrm{mg} \text { daily for } 12 \\
\text { weeks ( } n=29) \\
\text { - Placebo daily for } 12 \\
\text { weeks ( } n=35)\end{array}$ & $\begin{array}{l}\text { All at } 12 \text { weeks } \\
\text { - Patient Response rating Scale (1-6, } 1 \text { completely improved, } 6 \\
\text { much worse) } \\
\text { - SNOT-20 } \\
\text { - PNIF } \\
\text { - Saccharin transit time } \\
\text { - Olfaction } \\
\text { - Endoscopy } \\
\text { - IL8, fucose, alpha2-macroglobulin }\end{array}$ & $\begin{array}{l}\text { Significant effect } \\
\text { on SNOT-20, } \\
\text { endoscopy, } \\
\text { saccharin transit } \\
\text { time. Subgroup } \\
\text { analysis on } \\
\text { low vs high IgE } \\
\text { levels found } 93 \% \\
\text { improvement in } \\
\text { the low lgE group }\end{array}$ \\
\hline
\end{tabular}

CRSwNP, chronic rhinosinusitis with nasal polyps; CRSsNP, chronic rhinosinusitis without nasal polyps; DBPCT, Double-blind placebo-controlled trial; IL, interleukin; IgE, immunoglobulin E; PND, post-nasal drip; PNIF, peak nasal inspiratory flow; SF-36, Short-Form 36; SNOT, Sino-nasal Outcome Test; VAS, visual analogue scale.

Table 6.1.2.2. Long-term antibiotics versus topical corticosteroids for the treatment of patients with CRS.

\begin{tabular}{|c|c|c|c|c|c|}
\hline Study & Methods & Participants & Intervention & Outcomes & Results \\
\hline $\begin{array}{l}\text { Amali } \\
2015^{(30)}\end{array}$ & DBPCT & $\begin{array}{l}66 \text { CRSwNP } \\
\text { and CRSsNP (60 } \\
\text { analysed) }\end{array}$ & $\begin{array}{l}\text { - Azithromycin } 250 \text { mg once daily } \\
\text { for } 12 \text { weeks ( } n=20) \\
\text { - Placebo once daily for } 12 \text { weeks } \\
(n=40) \\
\text { All patients received fluticasone } \\
\text { proprioanate } 100 \text { mcg twice daily }\end{array}$ & - SNOT-22 at 12 weeks & $\begin{array}{l}\text { Significant improvement in SNOT } 22 \\
\text { scores in azithromycin group over } \\
\text { fluticasone }\end{array}$ \\
\hline $\begin{array}{l}\text { Haxel } \\
2015^{(29)}\end{array}$ & DBPCT & $\begin{array}{l}58 \text { CRSsNP } \\
\text { andCR SwNP } \\
\text { postoperatively }\end{array}$ & $\begin{array}{l}\text { - Erythromycin } 250 \mathrm{mg} \text { once daily } \\
\text { for } 12 \text { weeks ( } n=29 \text { ) } \\
\text { - Placebo } 250 \text { mg once daily for } 12 \\
\text { weeks ( } n=29 \\
\text { All patients received fluticasone } \\
\text { fuorate } 27.5 \text { mcg once daily }\end{array}$ & $\begin{array}{l}\text { Assessed at } 12 \text { and } 24 \text { weeks } \\
\text { - ECP } \\
\text { - MPO } \\
\text { - SNOT-20 } \\
\text { - Olfaction } \\
\text { - Saccharin transit time } \\
\text { - Nasal endoscopy score } \\
\text { - Nasal health self-rate }\end{array}$ & $\begin{array}{l}\text { No significant improvement in } \\
\text { SNOT20, olfaction, STT, endoscopy } \\
\text { scores, or nasal health. Trend towards } \\
\text { improvement in CRSsNP subgroup } \\
\text { on erythromycin, but only endoscopy } \\
\text { score was significant. }\end{array}$ \\
\hline
\end{tabular}

CRSsNP, chronic rhinosinusitis without nasal polyps; CRSwNP, chronic rhinosinusitis with nasal polyps; DBPCT, Double-blind placebocontrolled trial; ECP, eosinophil cationic protein; MPO, Myeloperoxidase ; SNOT, Sino-nasal Outcome Test; STT, Saccharin Transit Time.

\subsubsection{Long-term antibiotics versus Chinese herbal medicine}

One study ${ }^{(34)}$ compared the effect of Chinese herbal medicine (CHM), specifically Tsang-Erh-San extract granules and Houttuynia extract powder to erythromycin $250 \mathrm{mg}$ bid for eight weeks in patients with CRSsNP. The groups were assessed with the Taiwanese SNOT-20 (TWSNOT-20), endoscopy scores, saccharin transit time, and bacterial culture. In both groups, there was significant decrease in TWSNOT20, with no significant differences between groups (Table 6.1.2.4.). Both groups also had a decrease in endoscopy scores, however, this did not reach significance. Mucociliary clearance improved more significantly in the $\mathrm{CHM}$ group than erythromycin with improvement in saccharin transit time.

\subsubsection{Comparison of long-term antibiotics in CRS} One study ${ }^{(35)}$ compared one macrolide antibiotic to another in a single blinded study. The clarithromycin group was found to have improved efficacy over erythromycin (Table 6.1.2.5.)

\subsubsection{Cardiovascular risks of macrolide antibiotics} In common with a number of other drug groups, macrolides are known to prolong the QT interval, potentially increasing the 
Table 6.1.2.3. Long-term antibiotics versus surgery for the treatment of patients with CRS.

\begin{tabular}{|c|c|c|c|c|c|}
\hline Study & Methods & Participants & Intervention & Outcomes & Results \\
\hline $\begin{array}{l}\text { Peric, } \\
2014^{(31)}\end{array}$ & $\mathrm{RCT}$ & 80 CRSwNP & $\begin{array}{l}\text { - Clarithromycin } 500 \text { mg daily } \times 8 \\
\text { weeks followed by surgery }(n=40) \\
\text { - Surgery alone }(n=40)\end{array}$ & $\begin{array}{l}\text { Assessed at } 2 \text { weeks, } 6 \text { months, and } \\
12 \text { months after surgery } \\
\text { - Nasal polyp size } \\
\text { - Nasal symptom score }\end{array}$ & $\begin{array}{l}\text { Significant improvement in } \\
\text { NSS and Endoscopy score. } \\
\text { Surgical group with significantly } \\
\text { higher polyp recurrence post- } \\
\text { operatively. }\end{array}$ \\
\hline $\begin{array}{l}\text { Ragab } \\
2004 \\
2010^{(32,33)}\end{array}$ & RCT & $\begin{array}{l}90 \text { CRSwNP } \\
\text { and } \\
\text { CRSsNP ( } 89 \\
\text { evaluated) }\end{array}$ & $\begin{array}{l}\text { - Erythromycin } 500 \mathrm{mg} \text { bid } \times 2 \text { weeks } \\
\text { then } 250 \mathrm{mg} \text { bid }+ \text { alkaline douche }+ \\
\text { intranasal corticosteroid } \times 10 \text { weeks } \\
(n=45) \\
\text { - Surgery + erythromycin } 250 \mathrm{mg} \\
\text { bid } \times 2 \text { weeks + alkaline douche } \\
+ \text { intranasal corticosteroid (after } 2 \\
\text { weeks) }(n=44)\end{array}$ & $\begin{array}{l}\text { Assessed at } 6 \text { and } 12 \text { months } \\
\text { - VAS (nasal blockage, nasal } \\
\text { discharge, olfaction, facial pain, } \\
\text { headache, overall discomfort) } \\
\text { - SNOT-20 } \\
\text { - SF-36 } \\
\text { - NO } \\
\text { - STT } \\
\text { - Acoustic rhinomanometry } \\
\text { - Endoscopy }\end{array}$ & $\begin{array}{l}\text { Significant effect on SNOT-20, } \\
\text { SF36, SCT and NO in both groups. } \\
\text { No difference between groups }\end{array}$ \\
\hline
\end{tabular}

$\mathrm{RCT}$, randomised controlled trial; CRSsNP, chronic rhinosinusitis without nasal polyps; CRSwNP, chronic rhinosinusitis with nasal polyps; NO, nitric oxide; NSS, Nasal and Sinus Symptom scale ; SCT, Saccharine Clearance Time ; SF36, Short-Form 36; SNOT, Sino-nasal Outcome Test.

short-term risk of arrhythmia ${ }^{(36,37)}$. Since the CLARICOR study ${ }^{(38)}$ there have been a number of studies exploring cardiovascular events in associations between macrolides, in particular clarithromycin, some of which have found increased risks of cardiovascular events up to a year beyond the exposure to the antibiotic ${ }^{(39-41)}$ whereas this has not been demonstrated in a number of large observational studies ${ }^{(42-46)}$, though some found evidence of short-term increases in risk ${ }^{(43-46)}$. A metaanalysis published by Wong et al. ${ }^{(47)}$, demonstrated that the short-term risk of cardiovascular outcomes associated with macrolides in observational studies was estimated at 1.79 excess myocardial infarctions per 1000 patients (95\% confidence interval (Cl): 0.88 to 3.20 ). This risk was not observed in RCTs but these trials were likely underpowered to detect this. No long-term cardiovascular risk (ranging from 30 days to three years) associated with macrolides was observed. Williamson et al. ${ }^{(46)}$ in an observational cohort of 66,331 adult CRS patients receiving 320,798 prescriptions for a macrolide or penicillin, compared prescriptions for macrolide antibiotics to penicillin. Outcomes were all-cause mortality, cardiac death, myocardial infarction, stroke, diagnosis of peripheral vascular disease, and cardiac arrhythmia. A non-statistically significant trend towards increased risk of myocardial infarction during the first 30 days following macrolide prescription was observed. No statistically significant short- or long-term risks were observed for macrolide prescription nor for clarithromycin in particular, in contrast to Svanstrom et al. ${ }^{(48)}$. The apparent discrepancies between this study and others $(38,40,41,43,48)$ may be because these have predominantly investigated associations in general populations with a potentially greater prevalence of pre-existing heart conditions and other morbidities in contrast to a CRS population. Clarithromycin should not be given to patients with history of QT prolongation (congenital or documented acquired QT prolongation) or ventricular cardiac arrhythmia, including torsades de pointe or hypokalaemia (risk of prolongation of QT-time).

Concomitant administration of clarithromycin and astemizole, cisapride, pimozide and/or terfenadine is contraindicated as this may result in QT prolongation and cardiac arrhythmias, including ventricular tachycardia, ventricular fibrillation, and torsades de pointe. Also, concomitant administration of clarithromycin and ergotamine or dihydroergotamine is contraindicated, as this may result in ergot toxicity. Concomitant administration with ticagrelor or ranolazine is contraindicated. Furthermore, clarithromycin should not be used concomitantly with HMG-CoA reductase inhibitors (statins) that are extensively metabolized by CYP3A4 (lovastatin or simvastatin), due to the increased risk of myopathy, including rhabdomyolysis. As with other strong CYP3A4 inhibitors, clarithromycin should not be used in patients taking colchicine. Clarithromycin should not be used in patients who suffer from severe hepatic failure in combination with renal impairment.

\section{"As always a full clinical and drug history should be undertaken to cover these aspects and potential drug interactions".}

\subsubsection{Summary}

Two placebo-controlled studies were conducted with macrolide antibiotics. One study ${ }^{(13)}$ did not find any significant improvement in a mixed group of patients (with and without nasal polyps). The other study ${ }^{(14)}$ found improvement in SNOT-20 scores, nasal endoscopy, and saccharin transit time in CRSsNP patients treated with macrolides. Patients with low IgE were noted to have more significant improvement with macrolide treatment. The meta-analysis did not show a significant effect of macrolides over placebo for responder score and SNOT score. While both studies used macrolides, they utilized different dosages and schedules that may have affected the results. These two studies also did not look at the same subtypes of CRS. Larger placebo-controlled trials are warranted, in addition to more microbiological studies on the underlying difference in the nasal microbiome of CRSWNP and CRSsNP and these are presently on-going in several European countries ${ }^{(49)}$. Both studies evaluating the efficacy of antibiotics over topical corticosteroids used macrolide antibiotics in a 
Table 6.1.2.4. Long-term antibiotics vs. Chinese herbal medicine for the treatment of patients with CRS.

\begin{tabular}{|c|c|c|c|c|c|}
\hline Study & Methods & Participants & Intervention & Outcomes & Results \\
\hline Jiang ${ }^{(34)}$ & $\mathrm{RCT}$ & 83 (53 evaluated) CRSsNP & $\begin{array}{l}\text { - Erythromycin } \\
250 \text { mg bid x } 8 \\
\text { weeks }(n=26) \\
\text { - Chinese herbal } \\
\text { medicine }(n=27)\end{array}$ & $\begin{array}{l}\text { Assessed at } 8 \text { weeks } \\
\text { - Taiwanese SNOT-20 } \\
\text { - Nasal endoscopy } \\
\text { - Nasal swab } \\
\text { - Saccharin transit time }\end{array}$ & $\begin{array}{l}\text { - Saccharin transit time improved significantly } \\
\text { more in the Chinese herbal medicine group } \\
\text { than erythromcycin group. } \\
\text { - No significant difference between the } \\
\text { groups for all other measurements. }\end{array}$ \\
\hline
\end{tabular}

CRSsNP, chronic rhinosinusitis without nasal polyps; RCT, randomised controlled trial; SNOT, Sino-nasal Outcome Test.

Table 6.1.2.5. Long-term clarithromycin vs. erythromycin for the treatment of patients with CRS.

\begin{tabular}{|c|c|c|c|c|c|}
\hline Study & Methods & Participants & Drug & Outcomes & Results \\
\hline Hashiba $1997^{(35)}$ & $\begin{array}{l}\text { Single } \\
\text { blind }\end{array}$ & 59 CRS & $\begin{array}{l}\text { Clarithromycin } 400 \mathrm{mg} \text { twice } \\
\text { daily vs. erythromycin } 600 \mathrm{mg} \\
\text { three times daily for } 8-12 \text { weeks }\end{array}$ & $\begin{array}{l}\text { Efficacy assessed (symptoms } \\
\text { and endoscopic signs) after } 2, \\
4,8 \text { and } 12 \text { weeks. }\end{array}$ & $\begin{array}{l}\text { Clarithromycin was significantly } \\
\text { more effective when compared to } \\
\text { erythromycin }\end{array}$ \\
\hline
\end{tabular}

CRS, chronic rhinosinusitis.

population of patients with CRSwNP and CRSsNP(29, 30). In metaanalysis, macrolide antibiotics had no significant benefit over topical steroids for SNOT scores. Further studies on individual CRS subtypes would be useful to determine which patients would benefit from long-term antibiotics and more specifically macrolide antibiotics.

\section{The EPOS2020 steering group, due to the low quality of the evidence, is uncertain whether or not the use of long-term antibiotics has an impact on patient outcomes in adults with CRS, particularly in the light of potentially increased risks of cardiovascular events. There is a need for the larger high-quality trials that are presently being undertaken in Europe.}

Both studies ${ }^{(31-33)}$ comparing long-term antibiotics treatment versus surgical treatment reported similar improvement. However, Peric et al. reported more durable polyp control postsurgical intervention in patients who received pre-operative long-term antibiotics. Two reports on the same study ${ }^{(32,33)}$ found similar benefit from the use of long-term macrolide therapy when compared to surgical treatment.

Alternative medicinal treatments have been used routinely in Eastern culture and are being used more often in Western culture. There was no significant difference in a small study comparing erythromcyin versus Chinese Herbal Medicine ${ }^{(34)}$. There are indications that the use of macrolides have increased risks of cardiovascular events up to a year beyond the exposure to the antibiotic although this has not been shown in patients with $\mathrm{CRS}^{(46)}$.

\subsubsection{Conclusion}

A small number of randomized (placebo) controlled trials have evaluated the potential of long-term macrolides in the treatment of CRS. No difference has been shown compared to placebo, nor could any differences compared to other proven effective treatments like nasal corticosteroids or surgery be found. There is some indication that macrolides might be more effective in patients with low IgE. Macrolides have been shown to have some potentially serious side effects at normal full dosage and the risk of these side effects and specifically cardiac toxicity should be considered when considering the prescribing these drugs. The EPOS2020 steering group, due to the low quality of the evidence, is uncertain whether or not the use of long-term antibiotics has an impact on patient outcomes in adults with CRS, particularly in the light of potentially increased risks of cardiovascular events. There is a need for the larger highquality trials that are presently being undertaken in Europe.

\subsubsection{Topical antibiotics}

\subsubsection{Summary of the evidence}

The rationale of treating patients with recalcitrant difficultto-treat rhinosinusitis with local antibiotics is the potential role of biofilm. These patients have wide open sinuses that are perfectly accessible for local treatment but despite rinsing with saline, anti- inflammatory corticosteroid treatment and/ or systemic antibiotics, the sinus mucosa remains inflamed. Often (biofilm-forming) Staphylococcus aureus or Pseudomonas aeruginosa is cultured from these sinuses ${ }^{(50)}$, but more recent molecular microbiome studies point towards various other micro-organisms being possibly involved ${ }^{(51)}$. Culture-dependent studies need to be carefully interpreted since micro-organisms such as $\mathrm{S}$. aureus and $\mathrm{P}$. aeruginosa can easily overgrow culture plates $^{(52)}$.

In this systematic review, we identified seven randomized trials ${ }^{(53-59)}$ with topical antibiotics in difficult-to-treat CRS of which four were placebo (or saline)-controlled studies ${ }^{(53,55,56 \text {, }}$ 59) (Table 6.1.3.1.) The design and outcome parameters of the studies were too different to allow for meta-analysis. The four placebo controlled studies $(n=141)$ did not show significant improvement in symptoms by using topical antibiotics (nebulization or spray) compared to the treatment without antibiotics ${ }^{(53,55,56,59)}$. Sykes et al. compared dexamethasone, neomycin and tramazoline with dexamethasone without neomycin and a placebo group in 50 patients with CRSsNP with vehicle alone and showed significant 
Table 6.1.3.1. Topical antibiotics for the treatment of patients with difficult-to-treat CRS.

\begin{tabular}{|c|c|c|c|c|c|}
\hline Study & Methods & Participants & Interventions & Outcomes & Results \\
\hline $\begin{array}{l}\text { Bonfils } \\
2015^{(59)}\end{array}$ & $\mathrm{DBPCT}$ & $\begin{array}{l}72 \text { adults CRSwNP } \\
\text { post-surgery (55 } \\
\text { evaluated) }\end{array}$ & $\begin{array}{l}\text { - A solution of } 150 \mathrm{mg} \\
\text { tobramycin for inhalation } \\
\text { by nebulization in a single } \\
\text { dose of } 3 \mathrm{ml} \text { medium } \\
(150 \mathrm{mg} / 3 \mathrm{ml}) \text { administered } \\
\text { by the Easynose }{ }^{\circledR} \text { mesh } \\
\text { nebulizer ( } \mathrm{n}=32 \text { evaluated) } \\
\text { twice daily for } 7 \text { days } \\
\text { - Same treatment with } 3 \mathrm{ml} \\
\text { saline }(\mathrm{n}=23 \text { evaluated) }\end{array}$ & $\begin{array}{l}\text { - Bacteriological eradication } \\
\text { - Congestion, anterior or poste- } \\
\text { rior rhinorrhea, sneezing, facial } \\
\text { pain or olfactory impairment } \\
\text { (VAS) } \\
\text { - Early withdrawal } \\
\text { - Adverse effects }\end{array}$ & $\begin{array}{l}\text { - Significantly better bacterio- } \\
\text { logical eradication with active } \\
\text { treatment } \\
\text { - No significant impact on } \\
\text { symptoms }\end{array}$ \\
\hline $\begin{array}{l}\text { Shikani } \\
2013^{(58)}\end{array}$ & DBRCT & $\begin{array}{l}35 \text { adult patients } \\
\text { with refractory CRS } \\
\text { (CRSWNP and CRSsNP) } \\
\text { that had undergone } \\
\text { prior endoscopic sinus } \\
\text { surgery }\end{array}$ & $\begin{array}{l}\text { - Topical regimen group } \\
\text { (13 CRSwNP, } 12 \text { CRSsNP) } \\
\text { daily self-administered } \\
\text { topical nebulisation of } \\
\text { culture-directed antibiotics } \\
\text { (tobramycin, vancomycin, } \\
\text { mupirocin and levofloxacin) } \\
\text { and of mometasone using a } \\
\text { commercial nebuliser for } 6 \\
\text { weeks ( } n=25,13 \text { CRSwNP, } 12 \\
\text { CRSsNP) } \\
\text { - Oral regimen group } \\
\text { consisting of culture- } \\
\text { directed oral antibiotics } \\
\text { and commercially available } \\
\text { mometasone spray for } 6 \\
\text { weeks ( } n=10 \text {, (5 CRSwNP, } 5 \\
\text { CRSsNP) }\end{array}$ & $\begin{array}{l}\text { - Lund-Kennedy symptoms score } \\
\text { - Lund-Kennedy endoscopic } \\
\text { appearance scores } \\
\text { - Histological response to } \\
\text { treatment }\end{array}$ & $\begin{array}{l}\text { - No comparisons are given } \\
\text { between treatment groups } \\
\text { but in the CRSsNP there } \\
\text { appears to be no difference } \\
\text { between treatments. In the } \\
\text { CRSwNP topical treatment } \\
\text { significantly reduced both } \\
\text { LK symptom score and LK } \\
\text { endoscopic appearances and } \\
\text { oral treatment did not } \\
\text { The improvement } \\
\text { in histological score } \\
\text { (inflammation, oedema, } \\
\text { epithelial attenuation, } \\
\text { epithelial hyperplasia, } \\
\text { squamous metaplasia, fibrosis } \\
\text { and goblet cell hyperplasia) } \\
\text { was significant for the CRSwNP } \\
\text { group treated with topical } \\
\text { therapy but not for CRSsNP. } \\
\text { No comparison between } \\
\text { treatment groups was made }\end{array}$ \\
\hline $\begin{array}{l}\text { Jervis } \\
\text { Bardy } \\
2012^{(57)}\end{array}$ & DBRCT & $\begin{array}{l}25 \text { Stap. aureus- } \\
\text { positive CRSwNP } \\
\text { patients but no frank } \\
\text { polyps at moment of } \\
\text { trial with ongoing signs } \\
\text { and symptoms of CRS } \\
\text { despite complete ESS }\end{array}$ & $\begin{array}{l}125 \mathrm{mg} \text { mupirocin in a in } \\
200 \mathrm{ml} \text { proprietary buffered } \\
\text { salts blend (MUP group) + } \\
\text { 3dd placebo tablet for } 28 \\
\text { days ( } \mathrm{n}=9 \text { ) } \\
\text { - } 200 \mathrm{ml} \text { proprietary buffered } \\
\text { salts blend and } 3 \text { dd } 625 \\
\text { Augmentin }(n=13) \text { for } 28 \\
\text { days }\end{array}$ & $\begin{array}{l}\text { - Change from baseline SNOT-20 } \\
\text { (28 days) } \\
\text { - Change from baseline VAS } \\
\text { battery (summation of } 5 \\
\text { individual symptom scores plus } \\
\text { an overall symptomatology } \\
\text { score; total score range, } 0-60 \text { ) } \\
\text { (28 days) } \\
\text { - Change from baseline Lund- } \\
\text { Kennedy endoscopic score) ( } 28 \\
\text { days) } \\
\text { - Bacteriological culture at } 28 \\
\text { days }\end{array}$ & $\begin{array}{l}\text { - Change from baseline SNOT-20 } \\
\text { just not significant (0.06) } \\
\text { - Change from baseline VAS } \\
\text { battery: not significant } \\
\text { - Significantly better LK score in } \\
\text { mupirocin treated group -4.0 } \\
(-7.0 \text { to }-1.3) \text { than Augmentin } \\
3.0(-1.0 \text { to } 4.0) \text {. } \\
\text { - Significantly better bacterial } \\
\text { eradication }\end{array}$ \\
\hline $\begin{array}{l}\text { Videler } \\
2008^{(56)}\end{array}$ & $\begin{array}{l}\text { DBPC } \\
\text { cross- } \\
\text { over } \\
\text { study }\end{array}$ & $\begin{array}{l}14 \text { patients with } \\
\text { recalcitrant CRSsNP } \\
\text { [symptomatology and } \\
\text { a positive nasal culture } \\
\text { for Staph. aureus } \\
\text { returned, despite } 2 \\
\text { previous attempts to } \\
\text { treat the disease with } \\
\text { appropriate antibiotics } \\
\text { (at least } 2 \text { weeks) and } \\
\text { nasal saline irrigation] }\end{array}$ & $\begin{array}{l}\text { - } 8 \mathrm{ml} \text { solution of bacitracin/ } \\
\text { colimycin }(830 / 640 \mu \mathrm{g} / \\
\mathrm{ml}) \text { twice daily for } 8 \text { weeks } \\
(n=14) \\
\text { - } 8 \mathrm{ml} \text { placebo solution } \\
(n=14)\end{array}$ & $\begin{array}{l}\text { - Severity of symptoms (rhinor- } \\
\text { rhoea, postnasal drip, crusts, } \\
\text { headache, facial pain, smell } \\
\text { disturbance, nasal pain, nose } \\
\text { bleeds, fever, malaise, tired- } \\
\text { ness) measured using VAS } \\
\text { - Disease-Specific Symptom } \\
\text { Score } \\
\text { - SF-36 questionnaire } \\
\text { - Nasal endoscopic findings }\end{array}$ & $\begin{array}{l}\text { - No difference in severity of } \\
\text { symptoms measured by VAS, } \\
\text { disease specific symptom } \\
\text { score, SF-36 or nasal endo- } \\
\text { scopic findings }\end{array}$ \\
\hline
\end{tabular}


Table 6.1.3.1. Topical antibiotics for the treatment of patients with difficult-to-treat CRS (continued).

\begin{tabular}{|c|c|c|c|c|c|}
\hline Study & Methods & Participants & Interventions & Outcomes & Results \\
\hline $\begin{array}{l}\text { Desrosiers } \\
2001^{(55)}\end{array}$ & DBRCT & $\begin{array}{l}20 \text { patients with } \\
\text { refractory rhinosinusitis } \\
\text { who had failed } \\
\text { maximal surgical and } \\
\text { medical therapies } \\
\text { without immune } \\
\text { deficiencies }\end{array}$ & $\begin{array}{l}\text { - 3-times daily applications } \\
\text { of } 4 \mathrm{~mL} \text { of a } 20 \mathrm{mg} / \mathrm{mL} \\
\text { tobramycin-saline solution } \\
\text { as a } 4 \text {-week course of } \\
\text { large-particle nebulized } \\
\text { aerosol therapy ( } \mathrm{n}=10 \text { ?) } \\
\text { - 3-times daily applications } \\
\text { of } 4 \mathrm{~mL} \text { of saline solution } \\
\text { as a 4-week course of } \\
\text { large-particle nebulized } \\
\text { aerosol therapy }(\mathrm{n}=10 \text { ?) }\end{array}$ & $\begin{array}{l}\text { - Symptoms pain, congestion, } \\
\text { PND (VAS) 0,2,4 and } 8 \text { weeks } \\
\text { after start of treatment } \\
\text { - Quality of life (RQLQ) } \\
\text { - Mucosal oedema and secretion } \\
\text { evaluated by nasal endoscopy } \\
\text { 0,2,4 and } 8 \text { weeks after start of } \\
\text { treatment (0-3) }\end{array}$ & $\begin{array}{l}\text { - No benefit from the addition } \\
\text { of tobramycin on symptoms, } \\
\text { quality of life and/or mucosal } \\
\text { oedema or secretion }\end{array}$ \\
\hline $\begin{array}{l}\text { Sykes } \\
1986^{(53)}\end{array}$ & DBPCT & $\begin{array}{l}\text { CRSsNP defined as } \\
\text { chronic mucopurulent } \\
\text { rhinorrhoea (50), nasal } \\
\text { blockage }(28 / 50) \text { and/ } \\
\text { or facial pain }(15 / 50) \text {, } \\
\text { abnormalities at sinus } \\
\text { radiograph }(25 / 50) \text {, } \\
\text { abnormalities at } \\
\text { nasendoscopy }(28 / 50)\end{array}$ & $\begin{array}{l}\text { - Dexamethasone- } \\
\text { tramazoline-neomycin nasal } \\
\text { spray } 1 \text { application to each } \\
\text { nostril } 4 \text { times daily for } 2 \\
\text { weeks }(n=20) \\
\text { - Dexamethasone-tramazo } \\
\text { line nasal spray } 1 \text { application } \\
\text { to each nostril } 4 \text { times daily } \\
\text { for } 2 \text { weeks ( } n=20) \\
\text { - Placebo spray } 1 \text { application } \\
\text { to each nostril } 4 \text { times daily } \\
\text { for } 2 \text { weeks }(n=10)\end{array}$ & $\begin{array}{l}\text { - Daily symptom diary card } \\
\text { - Sinus radiograph } \\
\text { - Nasal mucociliary clearance } \\
\text { measured by the modified } \\
\text { saccharin method } \\
\text { - Nasal airways resistance } \\
\text { measured by active anterior } \\
\text { rhinomanometry }\end{array}$ & $\begin{array}{l}\text { - No significant impact on } \\
\text { symptoms, sinus radiograph, } \\
\text { nasal mucociliary clearance }\end{array}$ \\
\hline
\end{tabular}

CRSsNP, chronic rhinosinusitis without nasal polyps; CRSwNP, chronic rhinosinusitis with nasal polyps; DBPCT, double blind placebo controlled trial; dd, dose/day; LK, Lund-Kennedy; PND, post-nasal drip; VAS, visual analogue scale; Short-Form 36; SNOT, Sino-nasal Outcome Test.

effect of the dexamethasone/tramazoline compared to placebo but no additive effect of neomycin ${ }^{(53)}$.

Desrosiers et al. investigated 20 recalcitrant CRS patients (CRSsNP and CRSwNP) in a randomized, double-blind trial of tobramycin-saline solution or saline with quinine as control, administered thrice daily by means of a nebulizer for four weeks, followed by a four-week observation period. Both patient groups experienced improvement in signs and symptoms but the addition of tobramycin appears of no benefit ${ }^{(55)}$. The bittermasking quinine used for the placebo in this trial is now being used therapeutically to stimulate taste receptors and potentially to clear gram-negative bacteria ${ }^{(60)}$.

Videler et al. investigated the effect of nasal irrigation with bacitracin/colimycin or placebo after two weeks of levofloxacin $500 \mathrm{mg}$ twice daily for two weeks in a randomised, double blind, cross-over study in 14 patients with recalcitrant CRS in spite of surgery with positive S.aureus culture. Both groups improved and there was no difference in SF-36 and endoscopic appearance $^{(56)}$.

Bonfils et al. showed in 59 patients with CRSwNP with symptoms after surgery that inhalation by nebulization of a solution of $150 \mathrm{mg}$ tobramycin twice daily for seven days eradicated bacteria based upon post-treatment culture significantly better than placebo. Bacteriological analysis was performed on day 0 and day 10 , following Day et al. ${ }^{(61)}$ studying the presence of pathogenic strains in culture and their antibiotic susceptibility, with cytological analysis of the presence and concentration of leukocytes (D0 and D10). Pre-treatment and day 10 posttreatment bacterial isolates were compared (Table 6.1.3.1.). Presence of leukocytes and sensitivities to antimicrobials were also evaluated. However, there was no significant impact on symptoms ${ }^{(59)}$.

Of the non-placebo controlled randomised trials (RTs) Jervis-

Bardy et al. ${ }^{(57)}$ evaluated the effect of $125 \mathrm{mg}$ mupirocin in a 200 $\mathrm{ml}$ proprietary buffered salts blend (MUP group) +3 dd placebo tablet for 28 days ( $n=9)$ with Augmentin $200 \mathrm{ml}$ proprietary buffered salts blend and 3dd 625 Augmentin $(n=13)$ for 28 days in 25 CRSwNP patients but no frank polyps at the time of trial with ongoing signs and symptoms of CRS despite complete endoscopic sinus surgery (ESS). The MUP treatment eradicated $S$. aureus significantly more effectively than Augmentin at one month and five patients remained $\mathrm{S}$. aureus negative for a period of two months after the treatment, based on middle meatus swab cultures. The Lund-Kennedy (LK) endoscopic score change from baseline was significantly better in the MUP treated group -4.0 [-7.0 to -1.3] vs. Augmentin 3.0 [-1.0 to 4.0]. The change from baseline in the SNOT-20 scores approached significance (MUP -7.0 [-10.0 to -1.0] vs. Augmentin -1.0 [-4.5 to 3.0$], P \quad 0.06)$, whereas the VAS scores were not significantly different.

Finally, Shikani $2013^{(58)}$ conducted a randomized study in 35 patients with refractory chronic rhinosinusitis (CRSwNP and CRSsNP) that had undergone prior endoscopic sinus surgery. Twenty-five patients (13 CRSwNP, 12CRSsNP) daily self- 
administered topical nebulisation of culture-directed antibiotics (tobramycin, vancomycin, mupirocin and levofloxacin) and mometasone using a commercial nebuliser for six weeks. The control group of 10 patients (five CRSwNP, five CRSsNP) received culture-directed oral antibiotics and commercially available mometasone spray for six weeks. No comparisons are given between treatment groups but in the CRSsNP there appears to be no difference between treatments, in the CRSwNP topical treatment significantly reduced both LK symptom score and LK endoscopic appearances whereas oral treatment did not. The improvement in histological score (inflammation, oedema, epithelial attenuation, epithelial hyperplasia, squamous metaplasia, fibrosis and goblet cell hyperplasia) was significant for the CRSwNP group treated with topical therapy but not for CRSsNP. No comparison between treatment groups was made.

\section{Topical antibacterial therapy does not seem to be more effective than placebo in improving symptoms in patients with CRS. However, it may give a clinically non-relevant improvement in symptoms, SNOT-22 and LK endoscopic score compared to oral antibiotics.}

\subsubsection{Conclusion}

There are six RCTs evaluating the efficacy of topical antibacterial therapy in recalcitrant CRS. The four placebo controlled studies ( $n=141,3$ in CRSsNP, 1 in CRSwNP) did not show significant improvement in symptoms compared to the treatment without antibiotics ${ }^{(53,55,56,59) \text {. }}$

High-volume rinsing with mupirocin seems able to eradicate S. aureus significantly better than oral Augmentin and LK endoscopy score and SNOT-22 showed (near) significant improvement compared to Augmentin ${ }^{(57)}$. Also, topical nebulisation of culture-directed antibiotics seemed to be more effective than oral treatment especially in CRSwNP patients ${ }^{(58)}$. The latter two studies lack a placebo group and have significant other methodological issues reducing the certainty of the findings.

Topical antibacterial therapy does not seem to be more effective than placebo in improving symptoms in patients with CRS. However, it may give a clinically non-relevant improvement in symptoms, SNOT-22 and LK endoscopic score compared to oral antibiotics. The EPOS2020 steering group, due to the very low quality of the evidence, is uncertain whether or not the use of topical antibacterial therapy has an impact on patient outcomes in adults with CRS compared with placebo.

\subsubsection{Intravenous antibiotics}

There are no randomized studies evaluating the effectiveness of intravenous (IV) antibiotics in CRS.

There is one uncontrolled case series of 45 patients reporting a significant improvement in symptoms in patients receiving home IV antibiotics when previous oral antibiotic treatment and/or surgery had failed ${ }^{(62)}$.

\subsubsection{Conclusions}

Due to the very low quality of the evidence, it is uncertain whether or not the use of intravenous antibiotics therapy has an impact on patient outcomes in adults with chronic rhinosinusitis compared with placebo.

\subsubsection{Nasal corticosteroids}

\subsubsection{Summary of the evidence}

In this analysis, DBPCT trials after the year 1990, assessing the effects of nasal corticosteroids for patients with chronic rhinosinusitis, both with and without polyps are included. Diagnostic criteria for CRS should meet the current EPOS criteria for chronic rhinosinusitis ${ }^{(63)}$. Patients with allergic fungal rhinosinusitis, cystic fibrosis, immunodeficiency, primary ciliary dyskinesia, fungal balls, granulomatosis with polyangiitis are excluded. Corticosteroids delivered into the nose and the paranasal sinuses by any delivery methods generally available in clinical practice are included. Trials administering corticosteroids via catheter ${ }^{(64,65)}$ and polyp injection ${ }^{(66)}$ are excluded. Outcomes analysed in this analysis are symptoms, quality of life, endoscopy polyp size, polyp recurrence and adverse events. Of the 42 studies included in this review ( 43 articles), 40 studies were placebo-controlled trials assessing the effects of nasal corticosteroids (Table 6.1.5.1.). Four studies compared different delivery methods of nasal corticosteroids (Table 6.1.5.2.).(67-70). One article ${ }^{(71)}$ included data of a previously published article ${ }^{(72)}$ for re-analysis. Thirty-two studies evaluated patients with CRSwNP, five studied patients with CRSsNP and the other five studied a mixed population. In 24 trials most patients had previous sinus surgery and in 14 trials most had no surgery. Patients' sinus surgery status was not mentioned in four trials. A variety of nasal corticosteroids was used such as triamcinolone, mometasone furoate, hydrocortisone, budesonide, FP, and dexamethasone. Corticosteroids were delivered using nasal spray in 26 trials, nasal drop in seven trials, nebulizer in two trials, aerosol in three trials, nasal irrigation in 4 trials and turbuhaler in two trials. Four trials used a bi-directional exhalation device which generated airflow in the nose after patients blew through the device. In 13 trials postoperative nasal corticosteroids were administered. Endoscopic sinus surgery was not performed in 29 trials.

\subsubsection{Impact of nasal corticosteroids on quality of life (QOL) in patients with CRS}

There were twelve studies assessing quality of life $(\mathrm{QOL})^{(73-80)(67,}$ 81)(82, 83). Of the 12 studies, 10 assessed disease-specific quality of life ${ }^{(73-75,78-80)(67,81)(82,83)}$ and two studies assessed general health-related quality of life ${ }^{(76,77)}$. Disease-specific quality of life questionnaires used were the Sinonasal Outcome Test-22 (SNOT$22)^{(73-75)(67,81)(82,83)}$, Rhinosinusitis Outcome Measure (RSOM-31) ${ }^{(82)}$, the Chronic Sinusitis Survey (CSS) ${ }^{(79)}$, Rhinosinusitis Disability Index ${ }^{(82)}$, and a questionnaire designed by the investigators ${ }^{(80)}$.

Nasal corticostereoids have a positive impact on disease specific and general QOL in patients with CRS 
Table 6.1.5.1. Nasal corticosteroids versus placebo for the treatment of patients with CRS.

\begin{tabular}{|c|c|c|c|c|c|}
\hline Study & Methods & Participants & Interventions & Outcomes & Results \\
\hline $\begin{array}{l}\text { Leopold } \\
2019^{(73)}\end{array}$ & DBPCRCT & $\begin{array}{l}323 \text { CRSWNP, } \\
27 \% \text { previous } \\
\text { sinus surgery }\end{array}$ & $\begin{array}{l}\text { - FP using exhalation } \\
\text { delivery system } 93 \mu \mathrm{g} \\
\text { b.i.d. for } 16 \text { weeks } \\
\text { ( } n=81 \text { ) } \\
\text { - FP using exhalation } \\
\text { delivery system } 186 \mu \mathrm{g} \\
\text { b.i.d. for } 16 \text { weeks } \\
\text { ( } n=80) \\
\text { - FP using exhalation } \\
\text { delivery system } 372 \mu g \\
\text { b.i.d. for } 16 \text { weeks } \\
\text { ( } n=82 \text { ) } \\
\text { - } P \text { lacebo for } 16 \text { weeks } \\
\text { (n=80) }\end{array}$ & $\begin{array}{l}\text { - Disease specific quality } \\
\text { of life (SNOT-22) at 16, } \\
24 \text { weeks } \\
\text { - } \text { Proportion of respond- } \\
\text { ers at } 16 \text { weeks } \\
\text { - } \text { Symptom score } \\
\text { improvement }(0-3,4 \\
\text { symptoms) at } 4 \text { weeks } \\
\text { - Polyp score }(0-3) \\
\text { reduction at } 4,8,12,16 \text {, } \\
24 \text { weeks } \\
\text { - } \text { Adverse events }\end{array}$ & $\begin{array}{l}\text { - At } 16 \text { weeks, greater number of responders } \\
\text { in symptoms and polyp score reduction } \\
\text { significantly favoured FP versus placebo } \\
\text { - SNOT-22, symptoms, polyp grade were } \\
\text { significantly improved in each FP group at } \\
\text { weeks } 8,12,16 \\
\text { - There were no differences between active/ } \\
\text { placebo groups in intraocular pressure and } \\
\text { cataract } \\
\text { - Serious AEs were reported in } 1 \text { patient } \\
\text { receiving placebo (meningitis and } \\
\text { sinusitis) and } 1 \text { patient receiving } 372 \text { mg FP } \\
\text { (positional vertigo) } \\
\text { - Local adverse events were more common in } \\
\text { FP (epistaxis and nasal septal ulcer) }\end{array}$ \\
\hline $\begin{array}{l}\text { Kobayashi } \\
2018^{(74)}\end{array}$ & DBPCRCT & $\begin{array}{l}\text { CRSwNP, 35\% } \\
\text { previous sinus } \\
\text { surgery }\end{array}$ & $\begin{array}{l}\text { - Beclomethasone dipro- } \\
\text { pionate metered-dose } \\
\text { inhaler, inhalation } \\
\text { through the mouth } \\
\text { followed by exhalation } \\
\text { through the nose for } 4 \\
\text { weeks ( } n=11) \\
\text { - Placebo for } 4 \text { weeks } \\
(n=12)\end{array}$ & $\begin{array}{l}\text { - Disease specific quality } \\
\text { of life (SNOT-22) at } 4 \\
\text { weeks } \\
\text { - Proportion of respond- } \\
\text { ers at } 4 \text { weeks } \\
\text { - Polyp score }(0-4) \text { reduc- } \\
\text { tion at } 4 \text { weeks }\end{array}$ & $\begin{array}{l}\text { - At } 4 \text { weeks, greater number of responders in } \\
\text { polyp score reduction significantly favoured } \\
\text { beclomethasone dipropionate versus } \\
\text { placebo } \\
\text { - At } 4 \text { weeks, SNOT-22 improved significantly } \\
\text { in the beclomethasone dipropionate group } \\
\text { - At } 4 \text { weeks, beclomethasone dipropionate } \\
\text { reduced polyp size significantly }\end{array}$ \\
\hline Tait $2018^{(81)}$ & DBPCRCT & $\begin{array}{l}74 \text { CRS } \\
\text { (25\%CRSwNP), } \\
28 \% \text { previous } \\
\text { sinus surgery } \\
\text { (61 analyzed) }\end{array}$ & $\begin{array}{l}\text { - Budesonide irrigation } \\
1000 \mu g \text { o.d. in } 240 \mathrm{ml} \\
\text { bottle for } 4 \text { weeks } \\
(n=29) \\
\text { - Saline irrigation with } \\
\text { placebo (lactose) for } 4 \\
\text { weeks ( } n=32)\end{array}$ & $\begin{array}{l}\text { - Quality of life (SNOT-22 } \\
\text { (0-110) at } 4 \text { weeks) } \\
\text { - Symptoms (Likert scale } \\
\text { of improvement 1-7, } \\
\text { scale at } 4 \text { weeks) } \\
\text { - Endoscopy score (LKES } \\
\text { (0-20) at } 4 \text { weeks) } \\
\text { - Adverse event }\end{array}$ & $\begin{array}{l}\text { - The SNOT-22 scores at } 4 \text { weeks were not } \\
\text { different between the } 2 \text { groups } \\
\text { - Symptoms at } 4 \text { weeks were not different } \\
\text { - LKES change at } 4 \text { weeks was not different } \\
\text { There were no related adverse events. }\end{array}$ \\
\hline $\begin{array}{l}\text { Venkatesan } \\
2016^{(107)}\end{array}$ & DBPCRCT & $\begin{array}{l}22 \text { CRSwNP, } \\
\text { without sinus } \\
\text { surgery }\end{array}$ & $\begin{array}{l}\text { - Fluticasone fuorate } \\
\text { nasal spray } 110 \mu \mathrm{g} \text { o.d. } \\
\text { for } 12 \text { weeks }(n=18) \\
\text { - Placebo nasal spray for } \\
12 \text { weeks }(n=2)\end{array}$ & $\begin{array}{l}\text { - Symptom score }(0-3,5 \\
\text { symptoms) improve- } \\
\text { ment at } 12 \text { weeks } \\
\text { - Polyp score }(0-3) \text { reduc- } \\
\text { tion at } 12 \text { weeks }\end{array}$ & $\begin{array}{l}\text { - At } 12 \text { weeks, improvement by corticosteroid } \\
\text { treatment over placebo on symptom scores } \\
\text { and the polyp score were not significant }\end{array}$ \\
\hline $\begin{array}{l}\text { Zhou } \\
2016^{(106)}\end{array}$ & DBPCRCT & $\begin{array}{l}748 \text { CRSWNP, } \\
24 \% \text { previous } \\
\text { sinus surgery }\end{array}$ & $\begin{array}{l}\text { - MFNS } 200 \mu \mathrm{g} \text { b.i.d. for } \\
16 \text { weeks }(n=375) \\
\text { - Placebo spray for } 16 \\
\text { weeks }(n=373)\end{array}$ & $\begin{array}{l}\text { - Proportion of respond- } \\
\text { ers at } 16 \text { weeks } \\
\text { - Symptom score }(0-3,4 \\
\text { symptoms) improve- } \\
\text { ment at 4,8,12,16 } \\
\text { weeks } \\
\text { - Polyp score }(0-3) \\
\text { reduction at 4, 8, 12, } 16 \\
\text { weeks }\end{array}$ & $\begin{array}{l}\text { - At } 16 \text { weeks, a greater number of } \\
\text { responders was significant for MFNS } \\
\text { compared to placebo } \\
\text { - MFNS was significantly effective in } \\
\text { symptom improvement over placebo. Nasal } \\
\text { obstruction, anterior rhinorrhoea, smell } \\
\text { improvements were significant at 4, 8, 12, } 16 \\
\text { weeks. It was significant for postnasal drip at } \\
\text { 8, } 12 \text { weeks. } \\
\text { - Change from baseline in total polyp size } \\
\text { score was significantly greater for MFNS vs. } \\
\text { placebo at 4, 8, 12, } 16 \text { weeks }\end{array}$ \\
\hline $\begin{array}{l}\text { Dixon } \\
2015^{(75)}\end{array}$ & DBPCRCT & $\begin{array}{l}388 \text { CRSsNP } \\
\text { (237 adult and } \\
151 \text { children) } \\
\text { without sinus } \\
\text { surgery }\end{array}$ & $\begin{array}{l}\text { - MFNS } 200 \mu \mathrm{g} / \text { day for } \\
24 \text { weeks }(n=189) \\
\text { - Placebo for } 24 \text { weeks } \\
(n=199)\end{array}$ & $\begin{array}{l}\text { - Disease specific quality } \\
\text { of life (SNOT-22) at } 24 \\
\text { weeks } \\
\text { - Symptom score (1-60) } \\
\text { improvement at } 24 \\
\text { weeks }\end{array}$ & $\begin{array}{l}\text { - At } 24 \text { weeks, there was no difference } \\
\text { between corticosteroid and placebo in } \\
\text { SNOT-22 } \\
\text { - At } 24 \text { weeks, there was no difference } \\
\text { between groups in symptom improvement }\end{array}$ \\
\hline
\end{tabular}


Table 6.1.5.1. Nasal corticosteroids versus placebo for the treatment of patients with CRS (continued).

\begin{tabular}{|c|c|c|c|c|c|}
\hline Study & Methods & Participants & Interventions & Outcomes & Results \\
\hline $\begin{array}{l}\text { Wang } \\
2015^{(85)}\end{array}$ & DBPCRCT & $\begin{array}{l}57 \text { CRSwNP, } \\
\text { without sinus } \\
\text { surgery }\end{array}$ & $\begin{array}{l}\text { Budesonide } 1000 \mu g \\
\text { b.i.d. transnasal neb- } \\
\text { ulization for } 14 \text { days } \\
\text { ( } n=29) \\
\text { - Placebo nebulization } \\
\text { for } 14 \text { days }(n=28)\end{array}$ & $\begin{array}{l}\text { - Symptom (4 symp- } \\
\text { toms) improvement at } \\
2 \text { weeks } \\
\text { - Polyp score (0-3) reduc- } \\
\text { tion at } 2 \text { weeks } \\
\text { - Adverse events }\end{array}$ & $\begin{array}{l}\text { - At } 2 \text { weeks, budesonide transnasal } \\
\text { nebulization significantly improved total } \\
\text { nasal symptoms and } 4 \text { individual symptoms } \\
\text { - At } 2 \text { weeks, budesonide transnasal } \\
\text { nebulization significantly reduced polyp size } \\
\text { compared with placebo } \\
\text { - Morning plasma cortisol levels showed no } \\
\text { significant difference between the initial } \\
\text { treatment and the end of treatment. There } \\
\text { was no symptom of adrenal suppression in } \\
\text { any of the participants. } \\
\text { - No serious adverse events were reported }\end{array}$ \\
\hline $\begin{array}{l}\text { Rawal } \\
2015^{(82)}\end{array}$ & SBPCRCT & $\begin{array}{l}50 \text { CRSwNP } \\
\text { postoperative } \\
\text { (42 analyzed) }\end{array}$ & $\begin{array}{l}\text { - Budesonide irrigation } \\
500 \mu \mathrm{g} \text { in } 240 \mathrm{ml} \text { bottle. } \\
60 \mathrm{ml} / \mathrm{nostril} \text { b.i.d. for } \\
24 \text { weeks }(\mathrm{n}=24) \\
\text { - Saline irrigation alone } \\
\text { for } 24 \text { weeks }(n=18)\end{array}$ & $\begin{array}{l}\text { - Quality of life (SNOT-22 } \\
\text { (0-110), RSOM-31 (0- } \\
\text { 155), RSDI (0-120) at } 2 \\
\text { weeks, 3, } 6 \text { months) }\end{array}$ & $\begin{array}{l}\text { - SNOT-22, RSOM31, and RSDI scores were not } \\
\text { different between } 2 \text { arms at all } 3 \text { timepoints } \\
\text { (2 weeks, 3, } 6 \text { months). }\end{array}$ \\
\hline $\begin{array}{l}\text { Nordin } \\
2013^{(76)}\end{array}$ & DBPCRCT & $\begin{array}{l}60 \text { CRSwNP, } \\
\text { postoperative }\end{array}$ & $\begin{array}{l}\text { - Peri-operative FPND } \\
400 \mu g \text { b.i.d. for } 14 \\
\text { weeks }(n=26) \\
\text { - } \text { Peri-operative placebo } \\
\text { b.i.d. for } 14 \text { weeks } \\
(n=34)\end{array}$ & $\begin{array}{l}\text { - General quality of life, } \\
\text { using General Well } \\
\text { Being Schedule (0-110, } \\
14 \text { questions with a } \\
6 \text {-point scale and } 4 \\
\text { questions with an } \\
11 \text {-point scale) at weeks } \\
4 \text { and } 5\end{array}$ & $\begin{array}{l}\text { - At } 5 \text { weeks, both groups increased in } \\
\text { general quality of life score to a similar } \\
\text { extent without difference }\end{array}$ \\
\hline $\begin{array}{l}\text { Vento } \\
2012^{(105)}\end{array}$ & DBPCRCT & $\begin{array}{l}\text { CRSwNP, } \\
\text { postoperative }\end{array}$ & $\begin{array}{l}\text { - Postoperative triam- } \\
\text { cinolone acetonide } \\
\text { nasal aerosol } 220 \mu \mathrm{g} \\
/ \text { day as } 2 \text { actuations } \\
\text { of } 55 \mu \mathrm{g} \text {, each for } 9 \\
\text { months ( } \mathrm{n}=30 \text { ) } \\
\text { - Postoperative placebo } \\
\text { spray for } 9 \text { months } \\
(\mathrm{n}=30)\end{array}$ & $\begin{array}{l}\text { - Symptom score }(0-3,10 \\
\text { symptoms) improve- } \\
\text { ment at month 3,6,9 } \\
\text { - Polyp score }(0-3) \text { reduc- } \\
\text { tion at month 3,6,9 } \\
\text { - Polyp recurrence at } 9 \\
\text { months } \\
\text { - } \text { Adverse events }\end{array}$ & $\begin{array}{l}\text { - There was no difference in symptom } \\
\text { improvement } \\
\text { - At } 3 \text { months, triamcinolone acetonide } \\
\text { reduced polyp size and prevented regrowth } \\
\text { of nasal polyps significantly in acetylsalicylic } \\
\text { acid-tolerant patients, but it was not } \\
\text { significant at } 6 \text { and } 9 \text { months. It was not } \\
\text { significant in acetylsalicylic acid-intolerant } \\
\text { patients } \\
\text { - No serious adverse events reported. } \\
\text { There was no difference in blood in nasal } \\
\text { secretions. }\end{array}$ \\
\hline $\begin{array}{l}\text { Mosges } \\
2011^{(90)}\end{array}$ & DBPCRCT & $\begin{array}{l}59 \text { CRSsNP, } \\
\text { without sinus } \\
\text { surgery }\end{array}$ & $\begin{array}{l}\text { - MFNS } 200 \mu \mathrm{g} \text { b.i.d. for } \\
16 \text { weeks }(n=29) \\
\text { - } \text { Placebo for } 16 \text { weeks } \\
(n=30)\end{array}$ & $\begin{array}{l}\text { - Proportion of respond- } \\
\text { ers at } 16 \text { weeks } \\
\text { - Symptom score }(0-3,5 \\
\text { symptoms) improve- } \\
\text { ment at weeks } 1,4,8 \text {, } \\
12,16 \\
\text { - LK endoscopy score at } \\
\text { weeks } 1,4,8,12,16 \\
\text { - Adverse events }\end{array}$ & $\begin{array}{l}\text { - At } 16 \text { weeks, greater number of responders } \\
\text { significantly favoured MFNS over placebo } \\
\text { - At } 16 \text { weeks, symptom score was } \\
\text { significantly improved by MFNS } \\
\text { - At } 16 \text { weeks, the LK endoscopy score } \\
\text { showed a significant difference between } \\
\text { the MFNS group and the placebo group } \\
\text { - No serious adverse events reported. No } \\
\text { difference between groups in non-specific } \\
\text { complaints }\end{array}$ \\
\hline $\begin{array}{l}\text { Rotenberg } \\
2011^{(83)}\end{array}$ & DBPCRCT & $\begin{array}{l}64 \text { CRSwNP } \\
\text { (N-ERD) } \\
\text { postoperative } \\
\text { ( } 60 \text { analyzed) }\end{array}$ & $\begin{array}{l}\text { - Budesonide irrigation } \\
60 \mathrm{ml} / \text { nostril } 500 \mu \mathrm{g} \\
\text { b.i.d. (total } 1000 \mu \mathrm{g}) \text { for } \\
52 \text { weeks }(\mathrm{n}=20) \\
\text { - Saline irrigation } \\
60 \mathrm{ml} / \text { nostril and } \\
\text { budesonide spray } \\
64 \mu \mathrm{g} / \text { nostril b.i.d. } \\
\text { (total } 256 \mu \mathrm{g} \text { ) for } 52 \\
\text { weeks ( } \mathrm{n}=19 \text { ) } \\
\text { - Saline irrigation alone } \\
\text { for } 52 \text { weeks }(\mathrm{n}=21 \text { ) }\end{array}$ & $\begin{array}{l}\text { - Quality of life (SNOT-21 } \\
\text { (0-105) at 6,12 months) } \\
\text { - Endoscopy score (LKES } \\
\text { (0-12) at 6,12 months) } \\
\text { - } \text { CT score (LM score (0- } \\
\text { 24) at 6,12 months) } \\
\text { - Ocular events (IOP at } \\
\text { 6,12 months) } \\
\text { - HPA axis (ACTH (nor- } \\
\text { mal/abnormal) at 6,12 } \\
\text { months) }\end{array}$ & $\begin{array}{l}\text { - SNOT-21, LKES, and LM score results of both } \\
\text { intervention groups were not different from } \\
\text { placebo group at both } 6 \text { and } 12 \text {-month } \\
\text { timepoints (significant level was defined at } \\
\text { P }<.0167 \text { ) } \\
\text { - IOP and ACTH level were not changed at } \\
\text { both } 6 \text { and } 12 \text {-month timepoints }\end{array}$ \\
\hline
\end{tabular}


Table 6.1.5.1. Nasal corticosteroids versus placebo for the treatment of patients with CRS (continued).

\begin{tabular}{|c|c|c|c|c|c|}
\hline Study & Methods & Participants & Interventions & Outcomes & Results \\
\hline $\begin{array}{l}\text { Hansen } \\
2010^{(78)}\end{array}$ & DBPCRCT & $\begin{array}{l}20 \text { CRSsNP, all } \\
\text { previous sinus } \\
\text { surgery }\end{array}$ & $\begin{array}{l}\text { - FPNS } 400 \mu \text { g b.i.d. } \\
\text { using a bi-directional } \\
\text { exhalation delivery } \\
\text { device for } 12 \text { weeks } \\
(n=10) \\
\text { - Placebo b.i.d. using a } \\
\text { bi-directional exhala- } \\
\text { tion delivery device } \\
\text { for } 12 \text { weeks }(n=10)\end{array}$ & $\begin{array}{l}\text { - Proportion of respond- } \\
\text { ers at } 12 \text { weeks } \\
\text { - Symptom improve- } \\
\text { ment at 4, 8, } 12 \text { weeks } \\
\text { - Disease specific quality } \\
\text { of life (RSOM-31) at 4, 8, } \\
12 \text { weeks } \\
\text { - LK endoscopy score at } \\
4,8,12 \text { weeks }\end{array}$ & $\begin{array}{l}\text { - At } 12 \text { weeks, a significantly greater } \\
\text { number of responders reported symptom } \\
\text { improvement in the FP group than in the } \\
\text { placebo group. } \\
\text { - Combined symptom score was significantly } \\
\text { improved by FP compared to placebo at } \\
8,12 \text { weeks } \\
\text { - Overall RSOM-31 score and nasal subscale } \\
\text { were not different at } 12 \text { weeks but nasal } \\
\text { subscale was significantly improved with FP } \\
\text { compared to placebo at 4, } 8 \text { weeks } \\
\text { - Endoscopy score showed a significant } \\
\text { improvement of FP in oedema at } 4,8,12 \\
\text { weeks but no difference for nasal discharge. }\end{array}$ \\
\hline $\begin{array}{l}\text { Olsson } \\
2010^{(77)}\end{array}$ & DBPCRCT & $\begin{array}{l}68 \text { CRSwNP, } \\
\text { postoperative }\end{array}$ & $\begin{array}{l}\text { - Perioperative FPND } \\
400 \mu g \text { twice daily. for } \\
10 \text { weeks ( } n=30) \\
\text { - Perioperative placebo } \\
\text { b.i.d. for } 10 \text { weeks } \\
(n=38)\end{array}$ & $\begin{array}{l}\text { - Health related quality } \\
\text { of life (SF-36, } 0-100 \text {, } \\
8 \text { health domains) } \\
\text { at week } 5 \text { after sinus } \\
\text { surgery }\end{array}$ & $\begin{array}{l}\text { - At } 5 \text { weeks after sinus surgery no effect on } \\
\text { total SF36 but significant improvement in } \\
\text { the FPND group of the mental component } \\
\text { summary and } 3 \text { domains (role physical, } \\
\text { vitality, social functioning). }\end{array}$ \\
\hline $\begin{array}{l}\text { Ehnhage } \\
2009^{(95)}\end{array}$ & DBPCRCT & $\begin{array}{l}68 \text { CRSwNP, } \\
\text { postoperative }\end{array}$ & $\begin{array}{l}\text { - Perioperative FPND } \\
400 \mu \text { g b.i.d. for } 10 \\
\text { weeks ( } n=100) \\
\text { - Perioperative placebo } \\
\text { b.i.d. for } 10 \text { weeks } \\
(n=47)\end{array}$ & $\begin{array}{l}\text { - Symptom score (3 } \\
\text { symptoms, each score } \\
0-3) \text { improvement at 10, } \\
17 \text { weeks } \\
\text { - Nasal Polyp score (0-3) } \\
\text { reduction at 4, 6, 10,17 } \\
\text { weeks } \\
\text { - Adverse events }\end{array}$ & $\begin{array}{l}\text { - There was no difference between FP and } \\
\text { placebo in symptom improvement and } \\
\text { nasal polyp score reduction at } 10 \text { and } 17 \\
\text { weeks } \\
\text { - No difference in adverse events }\end{array}$ \\
\hline $\begin{array}{l}\text { Jankowski } \\
2009^{(99)}\end{array}$ & DBPCRCT & $\begin{array}{l}245 \text { CRSwNP, } \\
\text { without sinus } \\
\text { surgery }\end{array}$ & $\begin{array}{l}\text { FPNS } 200 \mu \mathrm{g} \text { bid for } 8 \\
\text { months ( } \mathrm{n}=81 \text { ) } \\
\text { - } \text { FPNS } 200 \mu \mathrm{g} \text { bid for } 1 \\
\text { month followed by FP } \\
\text { NS } 200 \mu \mathrm{g} \text { daily for } 7 \\
\text { months ( } \mathrm{n}=83 \text { ) } \\
\text { - Placebo spray for } 2 \\
\text { months followed by } \\
\text { FPNS } 200 \mu \mathrm{g} \text { bid for } 6 \\
\text { months ( } \mathrm{n}=81 \text { ) }\end{array}$ & $\begin{array}{l}\text { - Symptom score }(0-3 \text {, } \\
3 \text { symptoms), VAS im- } \\
\text { provement at } 4 \text { weeks } \\
\text { - Polyp score }(0-3) \text { reduc- } \\
\text { tion at } 4 \text { weeks }\end{array}$ & $\begin{array}{l}\text { At } 4 \text { weeks, FPNS } 400 \mu \mathrm{g} \text { / day was significantly } \\
\text { more effective than placebo } \\
\text { - in reduction of intensity of three symptoms } \\
\text { - polyp reduction }\end{array}$ \\
\hline $\begin{array}{l}\text { Jorissen } \\
2009^{(93)}\end{array}$ & DBPCRCT & $\begin{array}{l}91 \text { CRS (45\% } \\
\text { CRSwNP), } \\
\text { postoperative }\end{array}$ & $\begin{array}{l}\text { - Postoperative MFNS } \\
200 \mu \mathrm{g} \text { bid for } 6 \\
\text { months ( } \mathrm{n}=46) \\
\text { - Postoperative placebo } \\
\text { bid for } 6 \text { months } \\
(n=45)\end{array}$ & $\begin{array}{l}\text { - Symptom score (4 } \\
\text { symptoms and overall } \\
\text { discomfort, 0-10 VAS) } \\
\text { improvement at } \\
\text { months } 1,2,4,6 \\
\text { - Endoscopy score (0-2, } \\
8 \text { characteristics) at } \\
\text { days } 7,14 \text { and months } \\
1,2,4,6 \\
\text { - Adverse events }\end{array}$ & $\begin{array}{l}\text { - At } 6 \text { months, there was no difference } \\
\text { between MFNS and placebo in symptoms } \\
\text { - At } 6 \text { months, endoscopy scores were } \\
\text { reduced in both groups without difference } \\
\text { - Serious adverse events were rare without } \\
\text { difference between MFNS and placebo }\end{array}$ \\
\hline $\begin{array}{l}\text { Stjarne } \\
2009^{(114)}\end{array}$ & DBPCRCT & $\begin{array}{l}162 \text { CRSwNP } \\
\text { postoperative }\end{array}$ & $\begin{array}{l}\text { - Postoperative MFNS } \\
200 \mu \text { g o.d. for } 8 \text { weeks } \\
(n=80) \\
\text { - Postoperative placebo } \\
\text { for } 8 \text { weeks }(n=82)\end{array}$ & $\begin{array}{l}\text { - } \text { Time to relapse for } \\
\text { polyp recurrence at } 24 \\
\text { weeks } \\
\text { - Adverse events }\end{array}$ & $\begin{array}{l}\text { - At } 24 \text { weeks follow-up, postoperative use } \\
\text { of MFNS } 200 \mu \text { g once daily, provided a } \\
\text { statistically significant longer time to relapse } \\
\text { with nasal polyps than with placebo } \\
\text { - The frequency of AEs with MFNS and } \\
\text { placebo was comparable. }\end{array}$ \\
\hline
\end{tabular}


Table 6.1.5.1. Nasal corticosteroids versus placebo for the treatment of patients with CRS (continued).

\begin{tabular}{|c|c|c|c|c|c|}
\hline Study & Methods & Participants & Interventions & Outcomes & Results \\
\hline $\begin{array}{l}\text { Vlckova } \\
2009^{(72)} \\
\text { Djupesland } \\
2010^{(71)}\end{array}$ & DBPCRCT & $\begin{array}{l}109 \text { CRSwNP } \\
\text { (65\% previous } \\
\text { sinus surgery) }\end{array}$ & $\begin{array}{l}\text { - FP } 400 \mu g \text { b.i.d. using } \\
\text { a bidirectional exha- } \\
\text { lation delivery device } \\
\text { for } 12 \text { weeks }(n=54) \\
\text { - Placebo b.i.d. for } 12 \\
\text { weeks }(n=55)\end{array}$ & $\begin{array}{l}\text { - Proportion of respond- } \\
\text { ers at 4, 8, } 12 \text { weeks } \\
\text { - Polyp score (0-3) reduc- } \\
\text { tion at 4, 8, } 12 \text { weeks } \\
\text { - Adverse events }\end{array}$ & $\begin{array}{l}\text { - At 4, 8, } 12 \text { weeks, a greater number of } \\
\text { responders was significant for FP compared } \\
\text { to placebo. } \\
\text { - At 4, 8, } 12 \text { weeks, a significantly greater } \\
\text { reduction in polyp score was observed for } \\
\text { FP } \\
\text { - No serious adverse events reported. } \\
\text { Epistaxis occurred in } 11 \% \text { subjects treated } \\
\text { with FP and none in the placebo group. } \\
\text { Morning plasma cortisol concentrations } \\
\text { were unchanged. }\end{array}$ \\
\hline $\begin{array}{l}\text { Stjarne } \\
2006 a^{(104)}\end{array}$ & DBPCRCT & $\begin{array}{l}274 \text { CRSwNP, } \\
\text { sinus surgery } \\
\text { status not } \\
\text { mentioned }\end{array}$ & $\begin{array}{l}\text { - } \text { MFNS } 200 \mu \mathrm{g} \text { o.d. for } \\
16 \text { weeks }(n=94) \\
\text { - } \text { MFNS } 200 \mu \mathrm{g} \text { b.i.d. for } \\
16 \text { weeks ( } n=93) \\
\text { - } \quad \text { Placebo for } 16 \text { weeks } \\
(n=87)\end{array}$ & $\begin{array}{l}\text { - Symptom score }(0-3,4 \\
\text { symptoms) improve- } \\
\text { ment at months } 1,2 \text {, } \\
3,4 \\
\text { - Polyp score (0-3) reduc- } \\
\text { tion at months } 1,2,3,4 \\
\text { - } \text { Adverse events }\end{array}$ & $\begin{array}{l}\text { - At } 4 \text { months, both o.d. and b.i.d. doses } \\
\text { of MFNS significantly improved in nasal } \\
\text { obstruction score and anterior rhinorrhoea } \\
\text { compared to placebo. At } 4 \text { weeks, only the } \\
\text { b.i.d. dose significantly improved postnasal } \\
\text { drip. } \\
\text { - At } 4 \text { months, MFNS significantly produced } \\
\text { greater reductions in bilateral polyp grade } \\
\text { than placebo } \\
\text { - Most adverse events were mild or moderate } \\
\text { in severity without difference among } 3 \\
\text { groups. }\end{array}$ \\
\hline $\begin{array}{l}\text { Stjarne } \\
2006 b^{(80)}\end{array}$ & DBPCRCT & $\begin{array}{l}298 \text { CRSwNP } \\
\text { ( } 26 \% \text { previous } \\
\text { sinus surgery) }\end{array}$ & $\begin{array}{l}\text { - MFNS } 200 \mu \mathrm{g} \text { o.d. for } \\
16 \text { weeks ( } n=153) \\
\text { - Placebo for } 16 \text { weeks } \\
(n=145)\end{array}$ & $\begin{array}{l}\text { - Proportion of respond- } \\
\text { ers at } 16 \text { weeks } \\
\text { - Symptom score (0-3, } 3 \\
\text { symptoms) improve- } \\
\text { ment at } 12,16 \text { weeks } \\
\text { - Polyp score (0-3) reduc- } \\
\text { tion at } 12,16 \text { weeks } \\
\text { - Quality of life (4 items, } \\
1-3 \text { for } 2 \text { items and 0-3 } \\
\text { for } 2 \text { items) at } 12,16 \\
\text { weeks } \\
\text { - Adverse events }\end{array}$ & $\begin{array}{l}\text { - At } 16 \text { weeks, a greater proportion of } \\
\text { the MFNS-treated group than the } \\
\text { placebo group experienced a significant } \\
\text { improvement in } 3 \text { symptoms of nasal } \\
\text { obstruction, rhinorrhoea and sense of smell } \\
\text { - At } 16 \text { weeks, polyp size reduction } \\
\text { significantly favoured MFNS. } \\
\text { - At } 16 \text { weeks, MFNS significantly improved } \\
\text { quality of life in nose breathing, daily } \\
\text { activities, sleep disturbance. It was not } \\
\text { significant for taste and smell. } \\
\text { - Most adverse events were mild or moderate } \\
\text { in severity without difference between } \\
\text { groups. }\end{array}$ \\
\hline $\begin{array}{l}\text { Aukema } \\
2005^{(110)}\end{array}$ & DBPCRCT & $\begin{array}{l}54 \text { CRSwNP, } \\
\text { (83\% previous } \\
\text { sinus surgery) }\end{array}$ & $\begin{array}{l}\text { - FPND } 400 \mu \mathrm{g} / \text { day for } \\
12 \text { weeks }(n=27) \\
\text { - Placebo nasal drops } \\
\text { for } 12 \text { weeks }(n=27)\end{array}$ & $\begin{array}{l}\text { - Proportion of respond- } \\
\text { ers at } 12 \text { weeks } \\
\text { - Symptom score (VAS } \\
\text { 0-10) at 2, 6, } 12 \text { weeks } \\
\text { - Polyp volume estimat- } \\
\text { ed by endoscopy using } \\
\text { VAS at 2, 6, } 12 \text { weeks }\end{array}$ & $\begin{array}{l}\text { - At } 12 \text { weeks, the number of responders who } \\
\text { did not require surgery was significantly } \\
\text { greater with FPND versus placebo } \\
\text { - Nasal blockage scores were significantly } \\
\text { lower with FPND at 6, } 12 \text { weeks } \\
\text { - Polyp volume was significantly reduced in } \\
\text { the FPND group at } 12 \text { weeks }\end{array}$ \\
\hline $\begin{array}{l}\text { Rowe- } \\
\text { Jones } \\
2005^{(92)}\end{array}$ & DBPCRCT & $\begin{array}{l}109 \text { CRS (71\% } \\
\text { CRSwNP), } \\
\text { postoperative }\end{array}$ & $\begin{array}{l}\text { - Postoperative FPNS } \\
200 \mu \text { g b.i.d. for } 6 \\
\text { weeks ( } n=55) \\
\text { - Postoperative placebo } \\
\text { spray for } 6 \text { weeks } \\
(n=54)\end{array}$ & $\begin{array}{l}\text { - Symptom score (VAS, } 6 \\
\text { symptoms) improve- } \\
\text { ment at week } 6 \text { and } \\
\text { year } 1,2,3,4,5 \\
\text { - Polyp score }(0-2) \text { reduc- } \\
\text { tion at week } 6 \text { and year } \\
1,2,3,4,5 \\
\text { - LKendoscopy score at } \\
\text { week } 6 \text { and year } 1,2 \text {, } \\
3,4,5\end{array}$ & $\begin{array}{l}\text { - At } 5 \text { years, 'How do you feel overall'VAS } \\
\text { favoured FPNS significantly but total VAS } \\
\text { was not different. } \\
\text { - At } 1 \text { year, improvement in polyp score was } \\
\text { significantly better with FPNS but it was not } \\
\text { different at } 5 \text { years } \\
\text { - There was no difference in endoscopy } \\
\text { oedema and discharge score at } 1 \text { and } 5 \\
\text { years. }\end{array}$ \\
\hline $\begin{array}{l}\text { Small } \\
2005^{(103)}\end{array}$ & DBPCRCT & $\begin{array}{l}354 \text { CRSwNP, } \\
\text { sinus surgery } \\
\text { status not } \\
\text { mentioned }\end{array}$ & 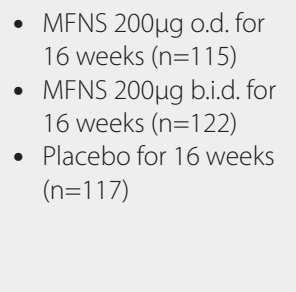 & $\begin{array}{l}\text { - Symptom score }(0-3,4 \\
\text { symptoms) improve- } \\
\text { ment at day } 8 \text { and } \\
\text { months } 1,2,3,4 \\
\text { - Polyp score }(0-3) \\
\text { reduction at day } 8 \text { and } \\
\text { months } 1,2,3,4 \\
\text { - Adverse events }\end{array}$ & $\begin{array}{l}\text { - At months 1, 2, 3, 4, MFNS significantly } \\
\text { improved nasal obstruction, loss of smell, } \\
\text { anterior rhinorrhoea, and postnasal drip } \\
\text { - At } 4 \text { months, improvement in polyp grade } \\
\text { was significantly better by MFNS } \\
\text { - Most adverse events were mild or moderate } \\
\text { in severity without difference among } 3 \\
\text { groups. 24-hour urinary free cortisol was not } \\
\text { different between groups }\end{array}$ \\
\hline
\end{tabular}


Table 6.1.5.1. Nasal corticosteroids versus placebo for the treatment of patients with CRS (continued).

\begin{tabular}{|c|c|c|c|c|c|}
\hline Study & Methods & Participants & Interventions & Outcomes & Results \\
\hline $\begin{array}{l}\text { Dijkstra } \\
2004^{(111)}\end{array}$ & DBPCRCT & $\begin{array}{l}162 \text { CRS ( } 42 \% \\
\text { CRSwNP) } \\
\text { postoperative }\end{array}$ & $\begin{array}{l}\text { - Postoperative FPNS } \\
400 \mu g \text { b.i.d. for } 1 \text { year } \\
(n=53) \\
\text { - Postoperative FPNS } \\
800 \mu g \text { b.i.d. for } 1 \text { year } \\
(n=53) \\
\text { - Postoperative placebo } \\
\text { b.i.d. for } 1 \text { year }(n=56)\end{array}$ & $\begin{array}{l}\text { - Number of patients } \\
\text { with recurrence of at } \\
1 \text { year } \\
\text { - Adverse events }\end{array}$ & $\begin{array}{l}\text { - No difference in percentage of recurrence of } \\
\text { disease between the groups } \\
\text { - The incidence of epistaxis was similar } \\
\text { between FPNS and placebo }\end{array}$ \\
\hline $\begin{array}{l}\text { Lund } \\
2004^{(112)}\end{array}$ & DBPCRCT & $\begin{array}{l}167 \text { CRSsNP, } \\
\text { without sinus } \\
\text { surgery }\end{array}$ & $\begin{array}{l}\text { - } \text { Budesonide nasal } \\
\text { spray } 128 \mu \text { g b.i.d. for } \\
20 \text { weeks ( } n=81) \\
\text { - } \begin{array}{l}\text { Placebo for } 20 \text { weeks } \\
(n=86)\end{array}\end{array}$ & $\begin{array}{l}\text { - Proportion of respond- } \\
\text { ers at } 20 \text { weeks } \\
\text { - Disease specific quality } \\
\text { of life (CSS) at } 20 \text { weeks } \\
\text { - Symptom score }(0-3,4 \\
\text { symptoms) improve- } \\
\text { ment at } 20 \text { weeks } \\
\text { - Adverse events }\end{array}$ & $\begin{array}{l}\text { - At } 20 \text { weeks, greater number of responders } \\
\text { significantly favoured budesonide over } \\
\text { placebo } \\
\text { - At } 20 \text { weeks, CSS was not different between } \\
\text { groups } \\
\text { - At } 20 \text { weeks, budesonide significantly } \\
\text { improved combined symptom score } \\
\text { compared to placebo. } \\
\text { - There were no serious adverse events } \\
\text { reported. Minor effects were not different } \\
\text { between groups. }\end{array}$ \\
\hline $\begin{array}{l}\text { Gulati } \\
2001^{(859)}\end{array}$ & DBPCRCT & $\begin{array}{l}63 \text { CRSWNP, } \\
\text { postoperative }\end{array}$ & $\begin{array}{l}\text { Postoperative } \\
\text { Budesonide nasal } \\
\text { sprays } 400 \mu \mathrm{g} / \text { day for } \\
12 \text { weeks ( } n=38 \text { ) } \\
\text { - Postoperative saline } \\
\text { nasal sprays for } 12 \\
\text { weeks ( } n=25 \text { ) }\end{array}$ & $\begin{array}{l}\text { - Proportion of respond- } \\
\text { ers at } 12 \text { weeks } \\
\text { - Symptom improve- } \\
\text { ment (4 symptoms) at } \\
12 \text { weeks } \\
\text { - Polyp recurrence at } 12 \\
\text { weeks }\end{array}$ & $\begin{array}{l}\text { - At } 12 \text { weeks, greater responder of symptom } \\
\text { improvement in the Budesonide group } \\
\text { than placebo group was significant for } \\
\text { rhinorrhoea and sneezing. } \\
\text { - At } 12 \text { weeks, budesonide treated patients } \\
\text { had significantly lower rate of polyp } \\
\text { recurrence. }\end{array}$ \\
\hline $\begin{array}{l}\text { Jankowski } \\
2001^{(98)}\end{array}$ & DBPCRCT & $\begin{array}{l}183 \text { CRSwNP, } \\
\text { all previous } \\
\text { sinus surgery }\end{array}$ & $\begin{array}{l}\text { - } \text { Budesonide nasal } \\
\text { spray } 128 \mu \text { g daily for } 8 \\
\text { weeks }(n=48) \\
\text { - } \text { Budesonide nasal } \\
\text { spray } 128 \mu \text { gid for } 8 \\
\text { weeks ( } n=48 \text { ) } \\
\text { - } \text { Budesonide nasal } \\
\text { spray } 256 \mu \text { daily for } 8 \\
\text { weeks ( } n=42 \text { ) } \\
\text { - Placebo spray for } 8 \\
\text { weeks ( } n=45)\end{array}$ & $\begin{array}{l}\text { - Symptom score }(0-4,4 \\
\text { symptoms) improve- } \\
\text { ment at 4,8 weeks } \\
\text { - Polyp score (0-3) reduc- } \\
\text { tion at 4, } 8 \text { weeks } \\
\text { - Adverse events }\end{array}$ & $\begin{array}{l}\text { - Combined symptom scores improved } \\
\text { significantly in all budesonide-treated } \\
\text { groups at 4,8 weeks. } \\
\text { - Budesonide nasal spray (all regimens) } \\
\text { significantly reduced polyp size at 4,8 } \\
\text { weeks. } \\
\text { - Budesonide nasal spray was well } \\
\text { tolerated. The adverse events reported } \\
\text { more frequently with budesonide nasal } \\
\text { spray treatment were blood-tinged nasal } \\
\text { secretions. }\end{array}$ \\
\hline $\begin{array}{l}\text { Parikh } \\
2001^{(91)}\end{array}$ & DBPCRCT & $\begin{array}{l}22 \text { CRS } \\
(18 \% \text { CRSwNP), } \\
>50 \% \\
\text { previous sinus } \\
\text { surgery }\end{array}$ & $\begin{array}{l}\text { - FPNS } 200 \mu \text { g b.i.d. for } 8 \\
\text { weeks }(n=9) \\
\text { - Placebo for } 8 \text { weeks } \\
(n=13)\end{array}$ & $\begin{array}{l}\text { - Symptom score (VAS, } 9 \\
\text { symptoms) improve- } \\
\text { ment at } 8 \text { weeks } \\
\text { - LK endoscopy score at } \\
8 \text { weeks }\end{array}$ & $\begin{array}{l}\text { - There was no difference between FPNS and } \\
\text { placebo in symptoms and endoscopy score. }\end{array}$ \\
\hline $\begin{array}{l}\text { Filiaci } \\
2000^{(96)}\end{array}$ & DBPCRCT & $\begin{array}{l}157 \text { CRSwNP, } \\
41 \% \text { previous } \\
\text { sinus surgery }\end{array}$ & 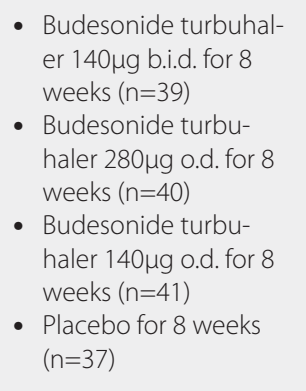 & $\begin{array}{l}\text { - Proportion of respond- } \\
\text { ers at } 8 \text { weeks } \\
\text { - Symptom score ( } 3 \\
\text { symptoms, each score } \\
\text { 0-3) improvement at } 8 \\
\text { weeks } \\
\text { - Polyp score (0-40) } \\
\text { reduction at } 8 \text { weeks } \\
\text { - Adverse events }\end{array}$ & $\begin{array}{l}\text { - At } 8 \text { weeks, substantial control of symptoms } \\
\text { was significantly superior in patients } \\
\text { receiving budesonide (70\%) than placebo } \\
\text { ( } 45 \%) \\
\text { - At } 8 \text { weeks, the mean reduction in total } \\
\text { polyp score was significantly greater in the } \\
\text { group receiving budesonide, } 140 \mu \mathrm{g} \text { twice } \\
\text { daily, than in the placebo group } \\
\text { - Budesonide was well tolerated, and the } \\
\text { proportion of patients reporting adverse } \\
\text { events were similar in the four treatment } \\
\text { groups. }\end{array}$ \\
\hline
\end{tabular}


Table 6.1.5.1. Nasal corticosteroids versus placebo for the treatment of patients with CRS (continued).

\begin{tabular}{|c|c|c|c|c|c|}
\hline Study & Methods & Participants & Interventions & Outcomes & Results \\
\hline $\begin{array}{l}\text { Keith } \\
2000^{(100)}\end{array}$ & DBPCRCT & $\begin{array}{l}104 \text { CRSwNP, } \\
69 \% \text { previous } \\
\text { sinus surgery }\end{array}$ & $\begin{array}{l}\text { - FPNS } 400 \mu \mathrm{g} \text { daily for } \\
12 \text { weeks }(n=52) \\
\text { - Placebo for } 12 \text { weeks } \\
(n=52)\end{array}$ & $\begin{array}{l}\text { - Proportion of respond- } \\
\text { - } \text { ers at } 12 \text { weeks } \\
\text { nasal blockage, overall } \\
\text { rhinitis, smell) improve- } \\
\text { ment at } 4,8,12 \text { weeks } \\
\text { - Polyp score }(0-3) \text { reduc- } \\
\text { tion at } 12 \text { weeks } \\
\text { - Adverse events }\end{array}$ & $\begin{array}{l}\text { - At } 12 \text { weeks, number of responders } \\
\text { significantly favoured FP nasal spray over } \\
\text { placebo in reduction of nasal blockage } \\
\text { - At } 12 \text { weeks, symptom score reduction } \\
\text { significantly favoured FP nasal spray in nasal } \\
\text { blockage and overall rhinitis but smelling } \\
\text { score was not significant } \\
\text { - At } 12 \text { weeks, number of responders was } \\
\text { not statistically significant in polyp size } \\
\text { reduction } \\
\text { - No serious adverse events. More patients } \\
\text { reported epistaxis in the FPNS group } \\
\text { (19\%) than in the placebo group (4\%). No } \\
\text { individual's serum cortisol level fell below } \\
\text { the lower threshold limit. }\end{array}$ \\
\hline $\begin{array}{l}\text { Pentilla } \\
2000^{(102)}\end{array}$ & DBPCRCT & $\begin{array}{l}142 \text { CRSwNP, } \\
71 \% \text { previous } \\
\text { sinus surgery }\end{array}$ & $\begin{array}{l}\text { - } \text { FPNS } 400 \mu \mathrm{g} \text { o.d. for } 12 \\
\text { weeks }(\mathrm{n}=48) \\
\text { - } \text { FPNS } 400 \mu \mathrm{g} \text { b.i.d. for } \\
12 \text { weeks ( } n=47) \\
\text { - } \quad \text { Placebo for } 12 \text { weeks } \\
(n=47)\end{array}$ & $\begin{array}{l}\text { - Proportion of respond- } \\
\text { ers at } 12 \text { weeks } \\
\text { - Symptom score } \\
(0-3, \text { nasal blockage } \\
\text { and overall rhinitis) } \\
\text { improvement at weeks } \\
4,8,12 \\
\text { - Polyp score }(0-3) \text { reduc- } \\
\text { tion at weeks } 4,8,12 \\
\text { - Adverse events }\end{array}$ & $\begin{array}{l}\text { - At } 12 \text { weeks, greater number of responders } \\
\text { significantly favoured FPNS over placebo } \\
\text { - At } 12 \text { weeks, nasal blockage showed } \\
\text { significant improvements with both doses } \\
\text { of FPNS over placebo, but overall rhinitis } \\
\text { showed significant improvement only for } \\
\text { FPNS } 400 \text { Hg o.d. } \\
\text { - At 4, } 8,12 \text { weeks, polyp size was reduced } \\
\text { significantly by FPNS } 400 \text { mg b.i.d. as } \\
\text { compared with placebo } \\
\text { - There were no serious adverse events. There } \\
\text { was no difference in side effects between } \\
\text { groups. }\end{array}$ \\
\hline $\begin{array}{l}\text { Holmstrom } \\
1999^{(97)}\end{array}$ & DBPCRCT & $\begin{array}{l}100 \text { CRSwNP } \\
\text { without sinus } \\
\text { surgery }\end{array}$ & $\begin{array}{l}\text { - FPNS } 400 \mu \mathrm{g} / \text { day for } \\
12 \text { weeks }(n=49) \\
\text { - Placebo spray for } 12 \\
\text { weeks }(n=51)\end{array}$ & $\begin{array}{l}\text { - Proportion of respond- } \\
\text { ers at } 12 \text { weeks } \\
\text { - Polyp score (0-3) reduc- } \\
\text { tion at } 26 \text { weeks } \\
\text { - Adverse events }\end{array}$ & $\begin{array}{l}\text { - At } 26 \text { weeks, a greater number of } \\
\text { responders in polyp score reduction was } \\
\text { found with FPNS versus placebo } \\
\text { - No serious adverse events reported }\end{array}$ \\
\hline $\begin{array}{l}\text { Lund } \\
1998^{(79)}\end{array}$ & DBPCRCT & $\begin{array}{l}29 \text { CRSwNP, } \\
66 \% \text { previous } \\
\text { sinus surgery }\end{array}$ & $\begin{array}{l}\text { - FPNS } 200 \mu \mathrm{g} \text { b.i.d. for } \\
12 \text { weeks }(\mathrm{n}=10) \\
\text { - Beclomethasone } \\
\text { dipropionate } 200 \mu \mathrm{g} \\
\text { b.i.d. for } 12 \text { weeks } \\
(\mathrm{n}=10) \\
\text { - Placebo for } 12 \text { weeks } \\
(\mathrm{n}=9)\end{array}$ & $\begin{array}{l}\text { - Symptom score }(0-4,4 \\
\text { symptoms) improve- } \\
\text { ment at } 12 \text { weeks } \\
\text { - Polyp score (0-3) reduc- } \\
\text { tion at } 12 \text { weeks } \\
\text { - Adverse events }\end{array}$ & $\begin{array}{l}\text { - At } 12 \text { weeks, the overall symptom score } \\
\text { was lower for both treatment groups, it } \\
\text { did not reach statistical significance when } \\
\text { compared with placebo } \\
\text { - At } 12 \text { weeks, the polyp score reduction } \\
\text { was significant in the FPNS-treated group } \\
\text { but not significant in the beclomethasone } \\
\text { group } \\
\text { - No serious adverse events. No drug related } \\
\text { adverse events. }\end{array}$ \\
\hline Tos $1998^{(69)}$ & DBPCRCT & $\begin{array}{l}138 \text { CRSWNP, } \\
\text { all previous } \\
\text { sinus surgery }\end{array}$ & $\begin{array}{l}\text { - } \text { Budesonide aqua } \\
\text { spray } 128 \mu \text { b.i.d. for } 6 \\
\text { weeks ( } n=46 \text { ) } \\
\text { - } \text { Budesonide turbuhal- } \\
\text { er } 200 \mu \text { b b.i.d. for } 6 \\
\text { weeks ( } n=45 \text { ) } \\
\text { - Placebo aqua spray for } \\
6 \text { weeks ( } n=24 \text { ) } \\
\text { - Placebo turbuhaler for } \\
6 \text { weeks }(n=23)\end{array}$ & $\begin{array}{l}\text { - Proportion of respond- } \\
\text { ers at } 6 \text { weeks } \\
\text { - Symptom score }(0-3,3 \\
\text { symptoms) improve- } \\
\text { ment at } 6 \text { weeks } \\
\text { - Polyp score }(0-3) \text { reduc- } \\
\text { tion at } 6 \text { weeks } \\
\text { - Adverse events }\end{array}$ & $\begin{array}{l}\text { - The proportion of responders after } 6 \text { weeks } \\
\text { treatment was not significantly different } \\
\text { between the aqua and turbuhaler-treated } \\
\text { groups } \\
\text { - At } 6 \text { weeks, budesonide aqua spray showed } \\
\text { a significantly superior efficacy over } \\
\text { turbuhaler for blocked nose, runny nose and } \\
\text { sneezing } \\
\text { - At } 6 \text { weeks, there was no statistical } \\
\text { difference between aqua and turbuhaler in } \\
\text { polyp size reduction } \\
\text { - No serious adverse events }\end{array}$ \\
\hline $\begin{array}{l}\text { Holmberg } \\
1997^{(109)}\end{array}$ & DBPCRCT & $\begin{array}{l}55 \text { CRSwNP, all } \\
\text { previous sinus } \\
\text { surgery }\end{array}$ & $\begin{array}{l}\text { - FPNS } 400 \mu \mathrm{g} / \text { day for } \\
26 \text { weeks }(n=15) \\
\text { - } \text { Beclomethasone } \\
\text { dipropionate aqueous } \\
\text { nasal spray for } 26 \\
\text { weeks ( } n=16) \\
\text { - Placebo nasal spray for } \\
26 \text { weeks }(n=11)\end{array}$ & $\begin{array}{l}\text { - Proportion of respond- } \\
\text { ers at } 26 \text { weeks } \\
\text { - Symptom improve- } \\
\text { ment }(0-3,5 \text { symptoms) } \\
\text { at 4,8, 26, } 38 \text { weeks } \\
\text { - Polyp score }(0-4) \\
\text { reduction at 4, 8, 26, } 28 \\
\text { - } \text { - Adverse events }\end{array}$ & $\begin{array}{l}\text { - At } 26 \text { weeks, greater response of symptom } \\
\text { improvement in the corticosteroids group } \\
\text { than in the placebo group, but it was } \\
\text { significant only for beclomethasone } \\
\text { - At } 26 \text { weeks, symptom score improvement } \\
\text { was significantly greater than placebo only } \\
\text { for beclomethasone } \\
\text { - Reduction in polyp size at } 26 \text { weeks } \\
\text { was only statistically significant for } \\
\text { beclomethasone } \\
\text { - A drug related adverse event was } \\
\text { epistaxis. No serious adverse events were } \\
\text { experienced. }\end{array}$ \\
\hline
\end{tabular}


Table 6.1.5.1. Nasal corticosteroids versus placebo for the treatment of patients with CRS (continued).

\begin{tabular}{|c|c|c|c|c|c|}
\hline Study & Methods & Participants & Interventions & Outcomes & Results \\
\hline $\begin{array}{l}\text { Lildholdt } \\
1995^{(101)}\end{array}$ & DBPCRCT & $\begin{array}{l}126 \text { CRSwNP, } \\
\text { sinus surgery } \\
\text { status not } \\
\text { mentioned }\end{array}$ & $\begin{array}{l}\text { - Budesonide turbuhal- } \\
\text { er } 200 \mu \mathrm{g} \text { b.i.d. for } 4 \\
\text { weeks } \\
\text { - Budesonide turbuhal- } \\
\text { er } 400 \mu \mathrm{g} \text { b.i.d. for } 4 \\
\text { weeks } \\
\text { - Placebo for } 4 \text { weeks }\end{array}$ & $\begin{array}{l}\text { - Proportion of respond- } \\
\text { ers at } 4 \text { weeks } \\
\text { - Symptom score (0-3,3 } \\
\text { symptoms) improve- } \\
\text { ment at } 4 \text { weeks } \\
\text { - Polyp score (0-3) reduc- } \\
\text { tion at } 4 \text { weeks } \\
\text { - Adverse events }\end{array}$ & $\begin{array}{l}\text { - At } 4 \text { weeks, greater number of responders } \\
\text { significantly favoured budesonide over } \\
\text { placebo } \\
\text { - At } 4 \text { weeks, significant improvement } \\
\text { of symptom score and polyp score in } \\
\text { budesonide group } \\
\text { - No serious adverse events reported. }\end{array}$ \\
\hline $\begin{array}{l}\text { Johansen } \\
1993^{(70)}\end{array}$ & DBPCRCT & $\begin{array}{l}86 \text { CRSwNP, } \\
\text { sinus surgery } \\
\text { status not } \\
\text { mentioned }\end{array}$ & $\begin{array}{l}\text { - Budesonide nasal } \\
\text { spray } 100 \mu \mathrm{g} \text { bid for } 3 \\
\text { months } \\
\text { - Budesonide aerosol } \\
100 \mu \mathrm{g} \text { bid for } 3 \\
\text { months } \\
\text { - Placebo for } 3 \text { months }\end{array}$ & $\begin{array}{l}\text { - Symptom score }(0-3,3 \\
\text { symptoms) improve- } \\
\text { ment weekly up to } 12 \\
\text { weeks } \\
\text { - Polyp score (0-3) reduc- } \\
\text { tion at } 4,8,12 \text { weeks } \\
\text { - Adverse events }\end{array}$ & $\begin{array}{l}\text { - After } 4 \text { weeks, both budesonide spray } \\
\text { and aerosol were significantly effective in } \\
\text { patients with small and medium sized polyp } \\
\text { symptoms } \\
\text { - At } 4,8,12 \text { weeks, budesonide reduced } \\
\text { polyp size significantly } \\
\text { - Few side effects were reported without } \\
\text { difference between groups. }\end{array}$ \\
\hline $\begin{array}{l}\text { Qvarnberg } \\
1992^{(868)}\end{array}$ & DBPCRCT & $\begin{array}{l}40 \text { CRSsNP, all } \\
\text { previous sinus } \\
\text { surgery }\end{array}$ & $\begin{array}{l}\text { - Budesonide aerosol } \\
200 \mu g \text { b.i.d. for } 12 \\
\text { weeks }(n=20) \\
\text { - Placebo for } 12 \text { weeks } \\
(n=20)\end{array}$ & $\begin{array}{l}\text { - Proportion of respond- } \\
\text { ers at } 12 \text { weeks } \\
\text { - Symptom score }(0-3,4 \\
\text { symptoms) improve- } \\
\text { ment at weeks } 1,2,12\end{array}$ & $\begin{array}{l}\text { - At } 12 \text { weeks, the number of responders was } \\
\text { not different } \\
\text { - At } 12 \text { weeks, facial pain and facial sensitivity } \\
\text { were significantly improved by budesonide } \\
\text { but not nasal blockage, nasal discharge and } \\
\text { postnasal drip. }\end{array}$ \\
\hline $\begin{array}{l}\text { Ruhno } \\
1990^{(113)}\end{array}$ & DBPCRCT & $\begin{array}{l}36 \text { CRSwNP, } \\
\text { previous } \\
\text { sinus surgery } \\
\text { unclear }\end{array}$ & $\begin{array}{l}\text { - Budesonide spray } \\
400 \mu g \text { b.i.d. for } 4 \\
\text { weeks ( } n=18 \text { ) } \\
\text { - placebo spray for } 4 \\
\text { weeks ( } n=18 \text { ) }\end{array}$ & $\begin{array}{l}\text { - Symptom score }(0-4,6 \\
\text { symptoms) improve- } \\
\text { ment at week } 4 \text {. } \\
\text { - Adverse events }\end{array}$ & $\begin{array}{l}\text { - At } 4 \text { weeks, effect on mean symptom score } \\
\text { was not significant. Only improvement in } \\
\text { blockage significantly favoured budesonide } \\
\text { - No serious adverse events reported. }\end{array}$ \\
\hline
\end{tabular}

ACTH, Adrenocorticotropic Hormone ; AE, Adverse Event ; b.i.d., twice daily; CSS, Chronic Sinusitus Survey ; CRSsNP, chronic rhinosinusitis without nasal polyps; CRSwNP, chronic rhinosinusitis with nasal polyps; DBPCRCT, double blind placebo controlled randomised controlled trial; FPND, Fluticasone Propionate Nasal drops ; FPNS, Fluticasone Propionate Nasal spray ; HPA, Hypothalamic Pituitary Adrenal ; IOP, intraocular pressure; LK, Lund-Kennedy; LKES, Lund-Kennedy endoscopy scoring ; LM, Lund-Mackay; MFNS, Mometasone fuorate nasal spray; N-ERD, NSAID-exacerbated respiratory disease ; o.d., once daily; RSOM-31, Rhinosinusitis Outcome Measure-31 ; RSDI, Rhinosinusitis Disability Index; SNOT, Sino-nasal Outcome Test.

The studies evaluating the Sinonasal Outcome Test-22 were combined in a meta-analysis although the treatment time evaluated was quite different (from four weeks ${ }^{(74)}$ to 24 weeks ${ }^{(73)}$. The meta-analysis favoured nasal corticosteroids over placebo in the improvement of disease specific quality of life (MD $--5.46,95 \% \mathrm{Cl}-8.08$ to $-2.84, \mathrm{p}<0.001 ; 6 \mathrm{RCTs}, \mathrm{n}=715$; Figure 6.1.5.1). Although statistically significant, the effect size of 5.46 with $95 \% \mathrm{Cl}$ of -8.08 to -2.84 is smaller than the MCID (minimal clinically important difference) of 8.9 and may not be clinically significant ${ }^{(84)}$. When general QOL was measured with SF-36, nasal corticosteroids significantly improved the Mental subscale but not the Physical subscale at five weeks ${ }^{(77)}$. However, when psychological well-being was measured with General Well Being Schedule, improvement in general QOL was not different between nasal corticosteroids and placebo ${ }^{(76)}$.

\subsubsection{Impact of nasal corticosteroids on symptoms in patients with CRS}

Eighteen studies evaluating symptoms could be combined in a meta-analysis (Figures 6.1.5.2-6.1.5.3). The meta-analysis favoured nasal corticosteroids over placebo in the improvement in symptoms for both symptom score [SMD - $0.63,95 \%$ confidence interval $(\mathrm{Cl})-0.89$ to $-0.37, \mathrm{p}<0.01 ; 12$ randomised controlled trials (RCTs), $n=1690$; Figure 6.1.5.2] and the proportion of responders ( $\mathrm{RR} 0.66,95 \% \mathrm{Cl} 0.59$ to $0.73, \mathrm{p}<0.01$; 12 RCTs, $n=1646$; Figure 6.1.5.3).

When subgroup analysis by CRS subtype was performed, the magnitude of beneficial effects of nasal corticosteroids in the symptoms score improvement was significantly greater $(p<0.01)$ in patients with polyps (SMD $-0.93,95 \% \mathrm{Cl}-1.43$ to $-0.44, p<0.01$; $6 \mathrm{RCTs}, \mathrm{n}=1096$ ), than patients without nasal polyps (SMD -0.30 , $95 \% \mathrm{Cl}-0.46$ to $-0.14, \mathrm{p}<0.01 ; 6 \mathrm{RCTs}, \mathrm{n}=594$; Figure 6.1.5.4) but the proportion of responders was similar (Figure 6.1.5.5). Patients who had previous sinus surgery had similar change in symptoms score and proportion of responders than patients without sinus surgery (RR $0.63,95 \% \mathrm{Cl} 0.47$ to $0.84, \mathrm{p}<0.01 ; 5$ RCTs, $\mathrm{n}=298$ versus $\mathrm{RR} 0.67,95 \% \mathrm{Cl} 0.59$ to $0.77, \mathrm{p}<0.01 ; 6 \mathrm{RCTs}$, $\mathrm{n}=1264$; Figure 6.1.5.6).

\subsubsection{Regimes varied across studies}

When the impact of type of corticosteroids on symptoms was assessed, benefits in symptom improvement were found in all types without difference. Beneficial effects on symptom scores reduction favoured fluticasone propionate (SMD -0.50; $95 \% \mathrm{Cl}[-0.76,-0.23])$, budesonide (SMD $-1.35 ; 95 \% \mathrm{Cl}[-2.60$, $-0.11])$ and mometasone furoate (SMD $-0.37 ; 95 \% \mathrm{Cl}[-0.53$, $-0.22]$ ) significantly over placebo without difference among 
corticosteroids type ( $p=0.24)$ (Figure 6.1.5.7).

Corticosteroid dosages varied across studies. In general, nasal corticosteroids were administered with dosages not higher than doubling rhinitis dosages. Nevertheless, there were studies giving higher dosages (e.g. fluticasone propionate $>400 \mu \mathrm{g}$ per day; budesonide $>512 \mu \mathrm{g}$ per day; mometasone furoate $>400 \mu$ g per day $)^{(72,73,78,85)}$. When impact of different dosages of nasal steroids on symptoms in CRS patients compared to placebo was assessed, there was a trend toward greater effects in the subgroup of studies giving higher dosages (SMD -1.20; $95 \% \mathrm{Cl}[-2.06,-0.33])$ than regular dosages (SMD $-0.35 ; 95 \% \mathrm{Cl}$ $[-0.50,-0.20])$. However, this difference did not reach statistical significance $(p=0.06)$ (Figure 6.1.5.8).

\section{Long term treatment with nasal corticosteroids is effective and safe in patients with CRS.}

Duration of treatment varied across studies from four weeks to 52 weeks. Of the 42 studies included in this review, there were 13 studies giving nasal corticosteroids for less than 12 weeks and there were three studies giving nasal corticosteroids for as long as 52 weeks. As nasal corticosteroids are aimed for controlling chronic inflammatory process of CRS so that, in general they are administered for a period of 12 weeks or greater. Nevertheless, when impact of duration of nasal steroids treatment on symptoms in CRS patients compared to placebo was assessed, there was no difference between the duration of treatment of $<12$ weeks and $\geq 12$ weeks in symptoms (SMD $-1.42 ; 95 \% \mathrm{Cl}[-2.70,-0.14]$ versus SMD $-0.39 ; 95 \% \mathrm{Cl}[-0.52,-0.26])$, $\mathrm{p}=0.12$ ) and the proportion of responders (RR $0.63 ; 95 \% \mathrm{Cl}[0.51$, $0.77]$ versus $\mathrm{RR} 0.64 ; 95 \% \mathrm{Cl}[0.55,0.75]), \mathrm{p}=0.84)$.

\subsubsection{Delivery methods}

Devices affect the beneficial effects of nasal corticosteroids over placebo in the improvement in symptoms score.

One study reported on the delivery via a nebulizer and showed the largest effect (SMD $-2.79,95 \% \mathrm{Cl}-3.53$ to $-2.05, \mathrm{p}<0.001 ; 1$ $\mathrm{RCT}, \mathrm{n}=57$ ), followed by a study reporting on turbuhaler (SMD $-1.06,95 \% \mathrm{Cl}-1.58$ to $-0.55, \mathrm{p}<0.01 ; 1 \mathrm{RCT}, \mathrm{n}=47$ ), bi-directional exhalation device (SMD $-0.68,95 \% \mathrm{Cl}-0.92$ to $-0.44, \mathrm{p}<0.01 ; 3$ $\mathrm{RCTs}, \mathrm{n}=285$ ) and nasal spray (SMD $-0.30,95 \% \mathrm{Cl}-0.41$ to -0.19 , $p<0.01 ; 7$ RCTs, $n=1281$ ) (Figure 6.1.5.9.). Data from trials using nasal drops could not be pooled. Differences are statistically significant for both three-subgroup comparison and pair comparisons ( $p<0.01$ for all comparisons). However, there was no difference between subgroups in proportion of responders. Four studies compared different delivery methods of nasal corticosteroids directly(Table 6.1.5.2). Corticosteroid nasal spray was compared to corticosteroid nasal drops ${ }^{(68)}$, corticosteroid irrigation ${ }^{(67)}$, corticosteroid aerosol ${ }^{(70)}$ and corticosteroid turbuhaler ${ }^{(69)}$. Data favoured corticosteroid drops and corticosteroid nasal irrigation over corticosteroid spray. Demirel and colleagues ${ }^{(68)}$ randomized 34 patients with CRSwNP to receive fluticasone propionate in the form of aqueous nasal spray $100 \mu \mathrm{g}$ twice daily, or nasal drop $400 \mu \mathrm{g}$ once or twice daily for 12 weeks and they found that corticosteroid drop brought the greatest effects in decreasing nasal polyp size, and improving symptoms. Harvey and colleagues performed a double-blind placebo-controlled randomized trial by giving post-operative $2 \mathrm{mg}$ mometasone delivered by either spray or irrigation to 44 patients with $\mathrm{CRS}^{(67)}$. The corticosteroid nasal irrigation group showed greater improvement than corticosteroid spray in nasal blockage, drainage, total visual analogue scale, endoscopy score and radiological score at 12 months.

Nasal corticosteroid irrigation is an alternative delivery method which aims to increase the exposure of corticosteroid onto the sinus mucosa. Harvey and colleagues investigated total paranasal sinus distribution in 10 cadaver heads after nasal irrigation and nasal spray with water-soluble iodinated radiopaque contrast medium. Blinded scan assessment showed significantly greater total sinus distribution by nasal irrigation with netipot and squeeze bottle than pressurized spray ${ }^{(86)}$. When a human study to define the fluid dynamics during nasal irrigation was conducted by the same group, the data revealed the exposure of fluid onto the sinus mucosa with retention of fluid of around $2.5 \%$ or $5.8 \mathrm{~mL}$ for the $240 \mathrm{~mL}$ irrigations ${ }^{(87)}$. In addition, these two studies also assessed the impact of endoscopic sinus surgery on fluid distribution and showed that sinus surgery greatly enhanced the delivery of nasal solutions. Apart from the general therapeutic goals of topical management in providing pharmaceutical delivery, simultaneous mechanical lavage by nasal corticosteroid irrigation enhances the mechanical removal of mucus, inflammatory products, and bacteria/ biofilm ${ }^{(87)}$.

To assess the effects of nasal corticosteroid irrigation compared

Figure 6.1.5.1. Forest plot of the effect of nasal corticosteroids versus placebo on disease specific quality of life (SNOT-22) in patients with CRS.

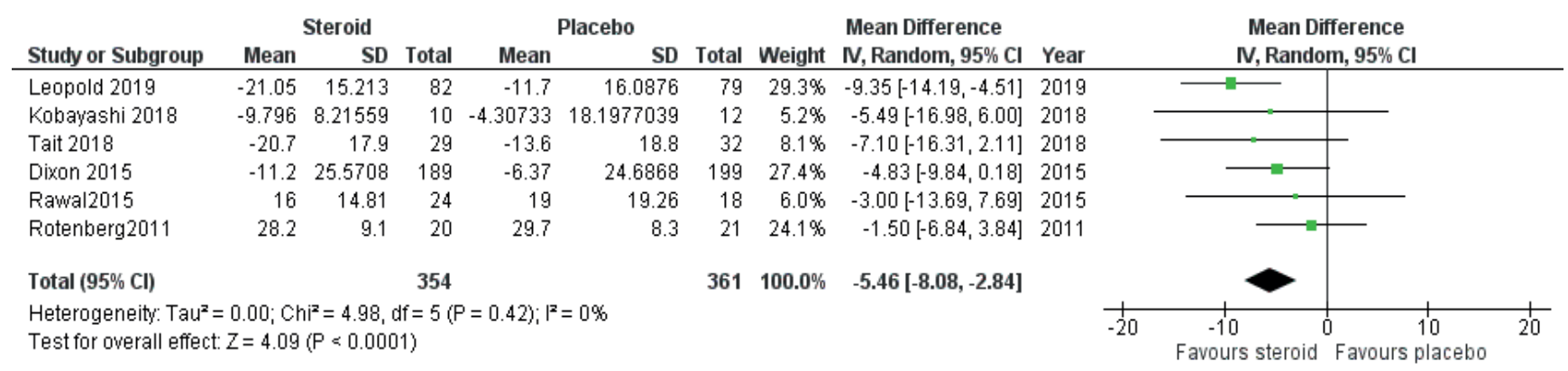


Figure 6.1.5.2. Forest plot of the effect of nasal corticosteroids versus placebo on change in symptom score in patients with CRS.

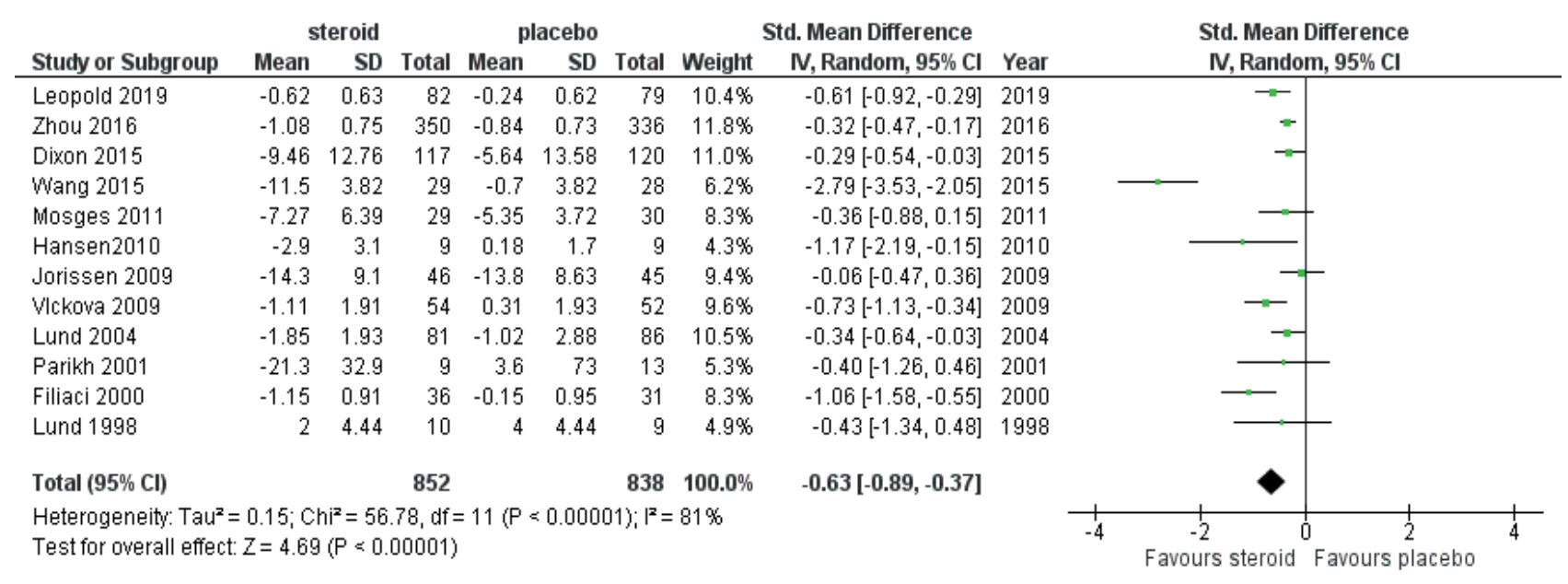

Figure 6.1.5.3. Forest plot of the effect of nasal corticosteroids versus placebo on the proportion of responders in patients with CRS.

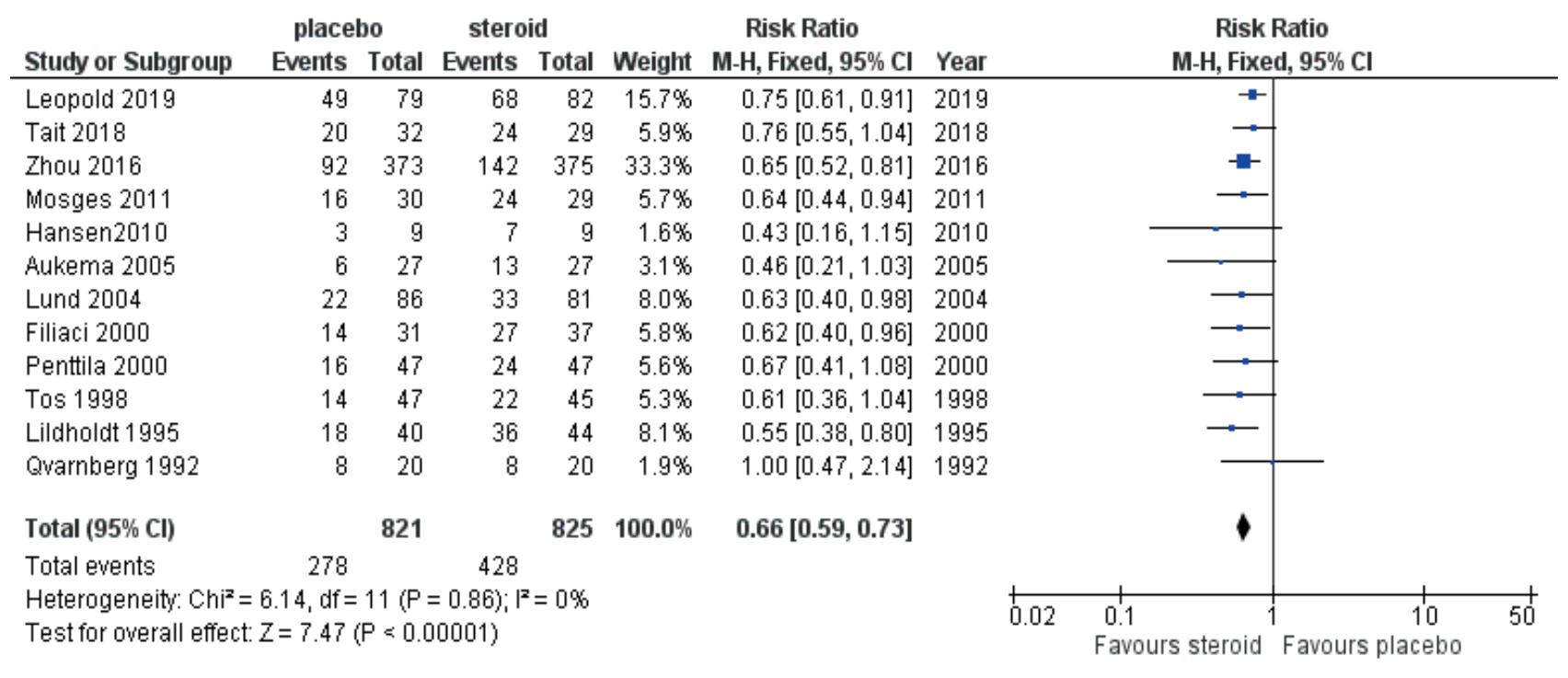

to control, four RCTs were identified (Table 6.1.5.3). There were three double-blind ${ }^{(67,81,83)}$ and one single-blind randomized controlled trial ${ }^{(82)}$. Of a total of four RCTs, one trial studied patients with CRSwNP ${ }^{(82)}$, one trial studied a mixed population of CRS with a majority of CRSsNP(81), one trial studied a mixed population of CRS with a majority of CRSWNP(67) and one trial studied patients with aspirin exacerbated respiratory disease ${ }^{(83)}$. All studies used a sinus rinse bottle kit as a device for nasal corticosteroid irrigation. However, volumes of corticosteroid irrigation varied. Two studies utilized a volume of $60 \mathrm{ml}$ on each nostril twice daily ${ }^{(82,83)}$, while the other two studies used a higher volume of $240 \mathrm{ml}$ once daily ${ }^{(67,81)}$. Three trials administered nasal corticosteroid irrigation after ESS ${ }^{(67,82,83)}$ and one trial used it without sinus surgery ${ }^{(81)}$. Regarding extension of surgery, one study explicitly stated it would perform a complete full-house ESS ${ }^{(67)}$ while one study stated that basic functional endoscopic sinus surgery (FESS) was performed ${ }^{(83)}$ and one study did not provide information ${ }^{(82)}$. The study of nasal corticosteroid irrigation without sinus surgery stated that the majority (77\%) of patients were un-operated patients ${ }^{(81)}$. Three studies followed the patients up for more than three months (12 months for two trials ${ }^{(67,83)}$ and six months for one trial( $\left.{ }^{(82)}\right)$. One study assessed the outcome at four weeks ${ }^{(81)}$.

Three RCTs compared nasal corticosteroid irrigation with placebo irrigation. There were heterogeneities across studies regarding patient population, surgical status and the volume of corticosteroid irrigation. Tait et al. performed a double blind $\mathrm{RCT}$ giving nasal corticosteroid irrigation without sinus surgery to patients with CRS with a majority of CRSsNP. At the short term of four-week irrigation, disease-specific quality of life and endoscopy scorewere not different when compared to saline irrigation with placebo ${ }^{(81)}$.

Rawal et al. performed a single blind RCT studying patients with CRSwNP. They gave nasal corticosteroid irrigation after ESS. Although a total volume of $240 \mathrm{ml}$ was rinsed per day, a volume of $60 \mathrm{ml}$ was used per nostril for each irrigation. At 24 weeks, disease-specific quality of life was not different when compared with control. Rotenberg et al. performed a double blind RCT 
Figure 6.1.5.4. Forest plot of the effect of nasal corticosteroids versus placebo on the change in symptom score in subgroups of CRS patients.

\begin{tabular}{|c|c|c|c|c|c|c|c|c|c|c|c|c|}
\hline \multirow{3}{*}{$\frac{\text { Study or Subgroup }}{1.2 .1 \text { CRSsNP }}$} & \multicolumn{3}{|c|}{ Favors steroid } & \multicolumn{3}{|c|}{ Favors placebo } & \multicolumn{3}{|c|}{ Std. Mean Difference } & \multirow{2}{*}{\multicolumn{3}{|c|}{$\begin{array}{l}\text { Std. Mean Difference } \\
\text { IV, Random, } 95 \% \mathrm{CI}\end{array}$}} \\
\hline & \multirow{2}{*}{ Mean } & \multirow[t]{2}{*}{ SD } & \multirow[t]{2}{*}{ Total } & \multirow[t]{2}{*}{ Mean } & \multirow[t]{2}{*}{ SD } & \multirow[t]{2}{*}{ Total } & \multirow{2}{*}{ Weight } & \multirow[t]{2}{*}{ IV, Random, $95 \% \mathrm{CI}$} & \multirow[t]{2}{*}{ Year } & & & \\
\hline & & & & & & & & & & & & \\
\hline Dixon 2015 & -9.46 & 12.76 & 117 & -5.64 & 13.58 & 120 & $11.0 \%$ & $-0.29[-0.54,-0.03]$ & 2015 & $-1-$ & & \\
\hline Mosges 2011 & -7.27 & 6.39 & 29 & -5.35 & 3.72 & 30 & $8.3 \%$ & $-0.36[-0.88,0.15]$ & 2011 & & & \\
\hline Hansen 2010 & -2.9 & 3.1 & 9 & 0.18 & 1.7 & 9 & $4.3 \%$ & $-1.17[-2.19,-0.15]$ & 2010 & & & \\
\hline Jorissen 2009 & -14.3 & 9.1 & 46 & -13.8 & 8.63 & 45 & $9.4 \%$ & $-0.06[-0.47,0.36]$ & 2009 & & & \\
\hline Lund 2004 & -1.85 & 1.93 & 81 & -1.02 & 2.88 & 86 & $10.5 \%$ & $-0.34[-0.64,-0.03]$ & 2004 & $\rightarrow$ & & \\
\hline $\begin{array}{l}\text { Parikh } 2001 \\
\text { Subtotal }(95 \% \mathrm{Cl})\end{array}$ & -21.3 & 32.9 & $\begin{array}{r}9 \\
291\end{array}$ & 3.6 & 73 & $\begin{array}{r}13 \\
303\end{array}$ & $\begin{array}{r}5.3 \% \\
48.8 \%\end{array}$ & $\begin{array}{r}-0.40[-1.26,0.46] \\
-0.30[-0.46,-0.14]\end{array}$ & 2001 & $\diamond$ & & \\
\hline \multicolumn{13}{|c|}{$\begin{array}{l}\text { Heterogeneity: } \operatorname{Tau}^{2}=0.00 ; \mathrm{Chi}^{2}=4.33, \mathrm{df}=5(P=0.50) ; \mathrm{I}^{2}=0 \% \\
\text { Test for overall effect: } Z=3.61(P=0.0003)\end{array}$} \\
\hline \multicolumn{13}{|l|}{ 1.2.2 CRSwNP } \\
\hline Leopold 2019 & -0.62 & 0.63 & 82 & -0.24 & 0.62 & 79 & $10.4 \%$ & $-0.61[-0.92,-0.29]$ & 2019 & - & & \\
\hline Zhou 2016 & -1.08 & 0.75 & 350 & -0.84 & 0.73 & 336 & $11.8 \%$ & $-0.32[-0.47,-0.17]$ & 2016 & - & & \\
\hline Wang 2015 & -11.5 & 3.82 & 29 & -0.7 & 3.82 & 28 & $6.2 \%$ & $-2.79[-3.53,-2.05]$ & 2015 & & & \\
\hline Vlckova 2009 & -1.11 & 1.91 & 54 & 0.31 & 1.93 & 52 & $9.6 \%$ & $-0.73[-1.13,-0.34]$ & 2009 & & & \\
\hline Filiaci 2000 & -1.15 & 0.91 & 36 & -0.15 & 0.95 & 31 & $8.3 \%$ & $-1.06[-1.58,-0.55]$ & 2000 & & & \\
\hline \multicolumn{13}{|c|}{$\begin{array}{l}\text { Heterogeneity: } \mathrm{Tau}^{2}=0.31 ; \mathrm{Chi}^{2}=48.12, \mathrm{df}=5(P<0.00001) ; \mathrm{I}^{2}=90 \% \\
\text { Test for overall effect: } \mathrm{Z}=3.70(\mathrm{P}=0.0002)\end{array}$} \\
\hline Total $(95 \% \mathrm{Cl})$ & & & 852 & & & 838 & $100.0 \%$ & $-0.63[-0.89,-0.37]$ & & & & \\
\hline \multicolumn{10}{|c|}{$\begin{array}{l}\text { Heterogeneity: } \mathrm{Tau}^{2}=0.15 ; \mathrm{Chi}^{2}=56.78, \mathrm{df}=11 \\
\text { Test for overall effect: } \mathrm{Z}=4.69(\mathrm{P}<0.00001)\end{array}$} & $\begin{array}{ccc}-4 & -2 & -1 \\
& \text { Favours corticosteroid }\end{array}$ & Favours pl & $\begin{array}{l}2 \\
\text { lacebo }\end{array}$ \\
\hline
\end{tabular}

Figure 6.1.5.5. Forest plot of the effect of nasal corticosteroids versus placebo on the proportion of responders in patients with subgroups of CRS with (CRSwNP) and without (CRSsNP) nasal polyps compared to placebo.with CRS.

Steroid Placebo Risk Ratio

Study or Subgroup Events Total Events Total Weight $\mathrm{M}-\mathrm{H}$, Fixed, $95 \% \mathrm{Cl}$ Year $\quad \mathrm{M}-\mathrm{H}, \mathrm{Fixed}, \mathbf{9 5 \%} \mathrm{Cl}$

$\begin{array}{lrrrrrrr} & & & & & \\ \text { 1.6.1 CRSsNP } & 20 & 32 & 24 & 29 & 5.9 \% & 0.76[0.55,1.04] & 2018 \\ \text { Tait 2018 } & 16 & 30 & 24 & 29 & 5.7 \% & 0.64[0.44,0.94] & 2011 \\ \text { Mosges 2011 } & 3 & 9 & 7 & 9 & 1.6 \% & 0.43[0.16,1.15] & 2010 \\ \text { Hansen2010 } & 22 & 86 & 33 & 81 & 8.0 \% & 0.63[0.40,0.98] & 2004 \\ \text { Lund 2004 } & 8 & 20 & 8 & 20 & 1.9 \% & 1.00[0.47,2.14] & 1992 \\ \text { Qvarnberg 1992 } & & \mathbf{1 7 7} & & \mathbf{1 6 8} & \mathbf{2 3 . 2 \%} & \mathbf{0 . 6 8 [ 0 . 5 5 , 0 . 8 4 ]} & \\ \text { Subtotal (95\% CI) } & 69 & & 96 & & & & \end{array}$

Heterogeneity: $\mathrm{Chi}^{2}=2.45, \mathrm{df}=4(\mathrm{P}=0.65) ; \mathrm{I}^{2}=0 \%$

Test for overall effect: $Z=3.52(P=0.0004)$

\subsubsection{CRSWNP}

Leopold 2019

Zhou 2016

Aukema 2005

Penttila 2000

Filiaci 2000

Tos 1998

Lildholdt 1995

Subtotal $(95 \% \mathrm{Cl})$

Total events

Heterogeneity: $\mathrm{Chi}^{2}=3.54, \mathrm{df}=6(\mathrm{P}=0.74) ; \mathrm{I}^{2}=0 \%$

Test for overall effect: $Z=6.58(P<0.00001)$

Total $(95 \% \mathrm{CI})$

821 278

$825 \quad 100.0 \%$

$0.66[0.59,0.73]$

Total events 428

$0.75[0.61,0.91] 2019$ $0.65[0.52,0.81] 2016$ $0.46[0.21,1.03] 2005$ $0.67[0.41,1.08] 2000$ $0.62[0.40,0.96] 2000$ $0.61[0.36,1.04] 1998$ $0.55[0.38,0.80] 1995$ $0.65[0.57,0.74]$

Heterogeneity: $\mathrm{Chi}^{2}=6.14, \mathrm{df}=11(\mathrm{P}=0.86) ; \mathrm{I}^{2}=0 \%$

Test for overall effect: $Z=7.47(P<0.00001)$

Test for subgroup differences: $\mathrm{Chi}^{2}=0.14, \mathrm{df}=1(\mathrm{P}=0.71), \mathrm{I}^{2}=0 \%$

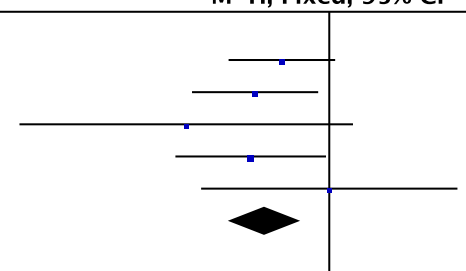

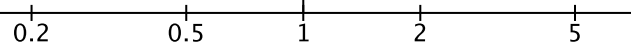

Favours corticosteroids Favours placebo 
Figure 6.1.5.6. Forest plot of the effect of nasal corticosteroids versus placebo on the proportion of responders in subgroups of CRS patients with and without sinus surgery.

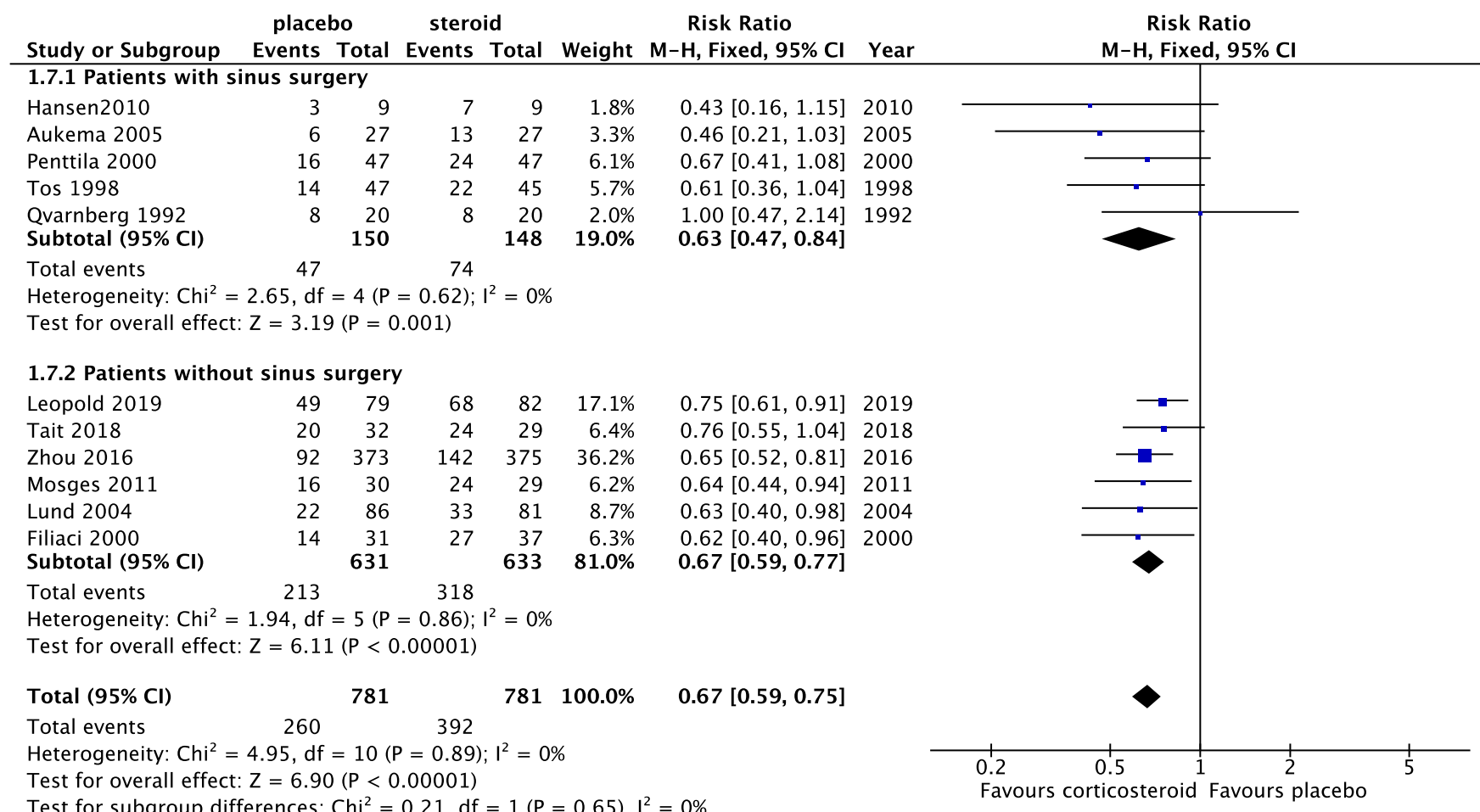

Figure 6.1.5.7. Forest plot of the effect of nasal corticosteroids versus placebo on change in symptom score in CRS.

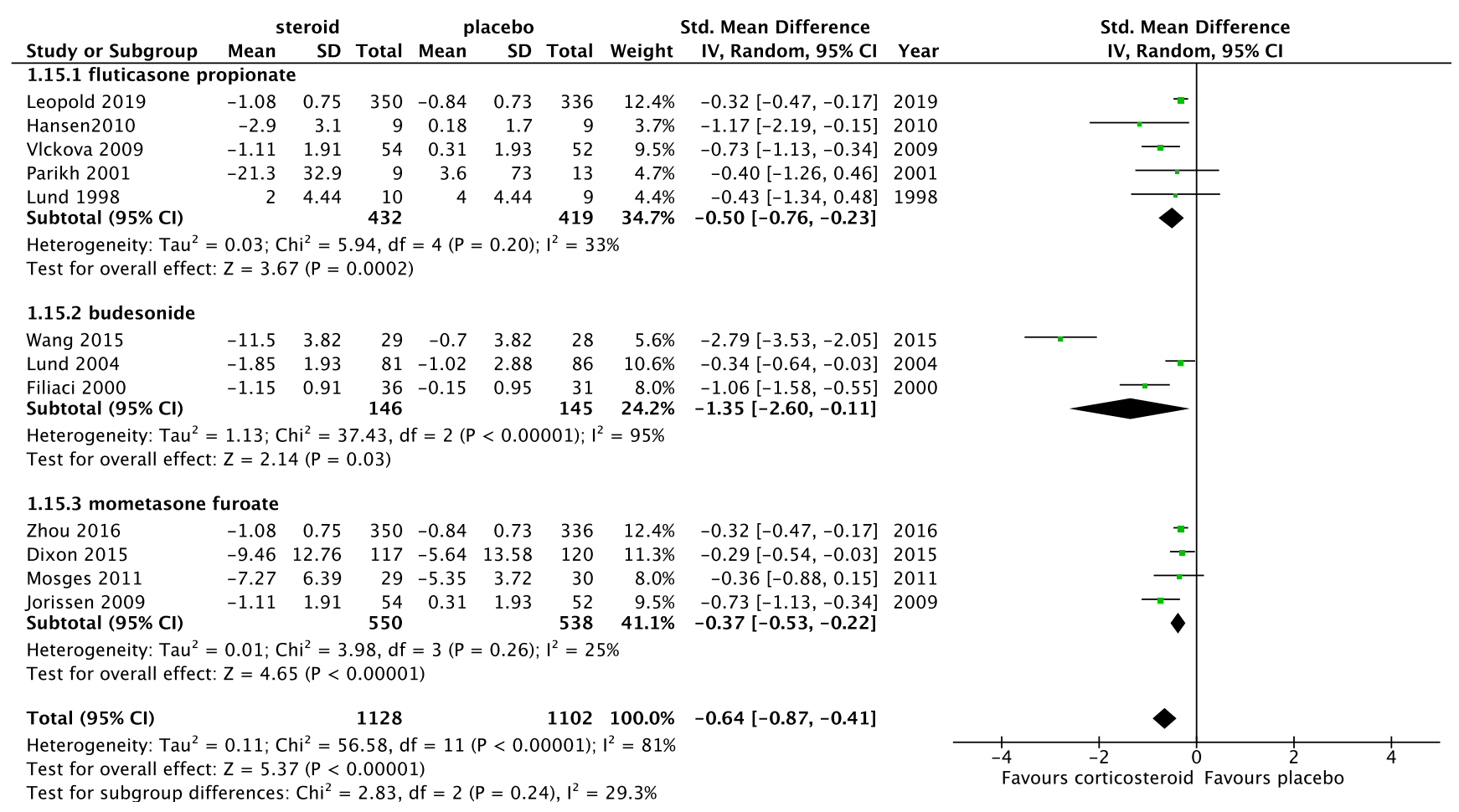


studying postoperative patients with aspirin exacerbated respiratory disease. Likewise, a volume of $60 \mathrm{ml}$ was used per nostril for each irrigation and disease-specific quality of life and endoscopic score were not different when compared with control at one-year timepoint ${ }^{(82)}$.

One trial compared nasal corticosteroid irrigation with nasal corticosteroid spray. Harvey et al. performed a double blind, double dummy, placebo-controlled RCT studying patients with CRS (77\% CRSwNP). They established a neo-sinus cavity that could be managed with topical medication by creating a complete spheno-ethmoidectomy, wide antrostomy with either a complete Draf Ila or Draf III. Nasal corticosteroid irrigation was administered using a squeeze bottle to deliver a total volume of $240 \mathrm{ml}$. When compared to corticosteroid spray at one year, the mometasone irrigation group had significantly greater improvement than spray in symptoms improvement and endoscopic score reduction.

When meta-analysis was performed to assess the impact of corticosteroid irrigation on SNOT improvement at six months, data were pooled from two RCTs ${ }^{(82,83)}$. A study by Tait et al. ${ }^{(81)}$ was not included because SNOT was assessed at four weeks. A study by Harvey et al. ${ }^{(67)}$ was not included because the control group received mometasone nasal spray. Meta-analysis showed no difference between nasal corticosteroid irrigation and placebo in SNOT improvement at six months (SMD - $0.17,95 \% \mathrm{Cl}-0.61$ to $0.26, p=0.44 ; 2 R C T s, n=83$ ). (Figure 6.1.5.10.) It is not clear why benefits of corticosteroid irrigation over saline irrigation are not shown yet by meta-analysis. Patients with high tissue eosinophilia are the most corticosteroid responsive and should be favourable cases ${ }^{(88)}$. Various factors impact corticosteroid penetration and influence the effectiveness of corticosteroids.
These factors include patients' surgical status with a wide access for topical medical therapy and delivery methods using high pressure and large volume ${ }^{(89)}$. It is notable that trials reporting no benefit used budesonide irrigation $1000 \mu \mathrm{g}$ per day in a $240 \mathrm{ml}$ bottle with a volume of $60 \mathrm{ml}$ after a basic FESS ${ }^{(82,83)}$ while a trial reporting benefits used mometasone irrigation $2000 \mu \mathrm{g}$ per day in a $240 \mathrm{ml}$ bottle with a volume of $240 \mathrm{ml}$ after a fullhouse FESS ${ }^{(67)}$.

\subsubsection{Impact of nasal corticosteroids on nasal endoscopy} Endoscopy score was assessed in six studies. Lund-Kennedy endoscopy score was assessed in six studies evaluating discharge, oedema, crusting, polyp and scars or adhesions ${ }^{(78,}$ 90-92)(81, 83). Jorissen and colleagues used their own scoring system assessing sinus ostia stenosis, polypoid change, synechia, remucosalized areas, secretions, inflammation, oedema and crusts ${ }^{(93)}$. The standardized mean difference was used for data analysis. When data were pooled for endoscopy score assessment, nasal corticosteroids were effective in decreasing endoscopy scores over placebo (SMD $-0.49,95 \% \mathrm{Cl}-0.73$ to $-0.25, \mathrm{p}<0.01 ; 6 \mathrm{RCTs}, \mathrm{n}=286$; Figure 6.1.5.11).

Nasal polyp size reduction was assessed in 25 studies. NPS on a 3-point scale (0-2) per side (0-4 for both sides), taken from endoscopic score described by Lund and Mackay ${ }^{(94)}$ was used in one study Rowe-Jones $2005^{(92)}$. NPS on a 4-point scale (0-3) per side (0-6 for both sides), described by Johansen et al. ${ }^{(70)}(0=$ no polyps; $1=$ polyps in the middle meatus, not reaching below the inferior border of middle turbinate; 2 = nasal polyps reaching below the inferior border of the middle turbinate, but not the lower border of the inferior turbinate; $3=$ nasal polyps reaching lower than the inferior border of the inferior turbinate and/

Figure 6.1.5.8. Forest plot of the effect of different dosages of nasal corticosteroids versus placebo on symptoms in CRS patients.

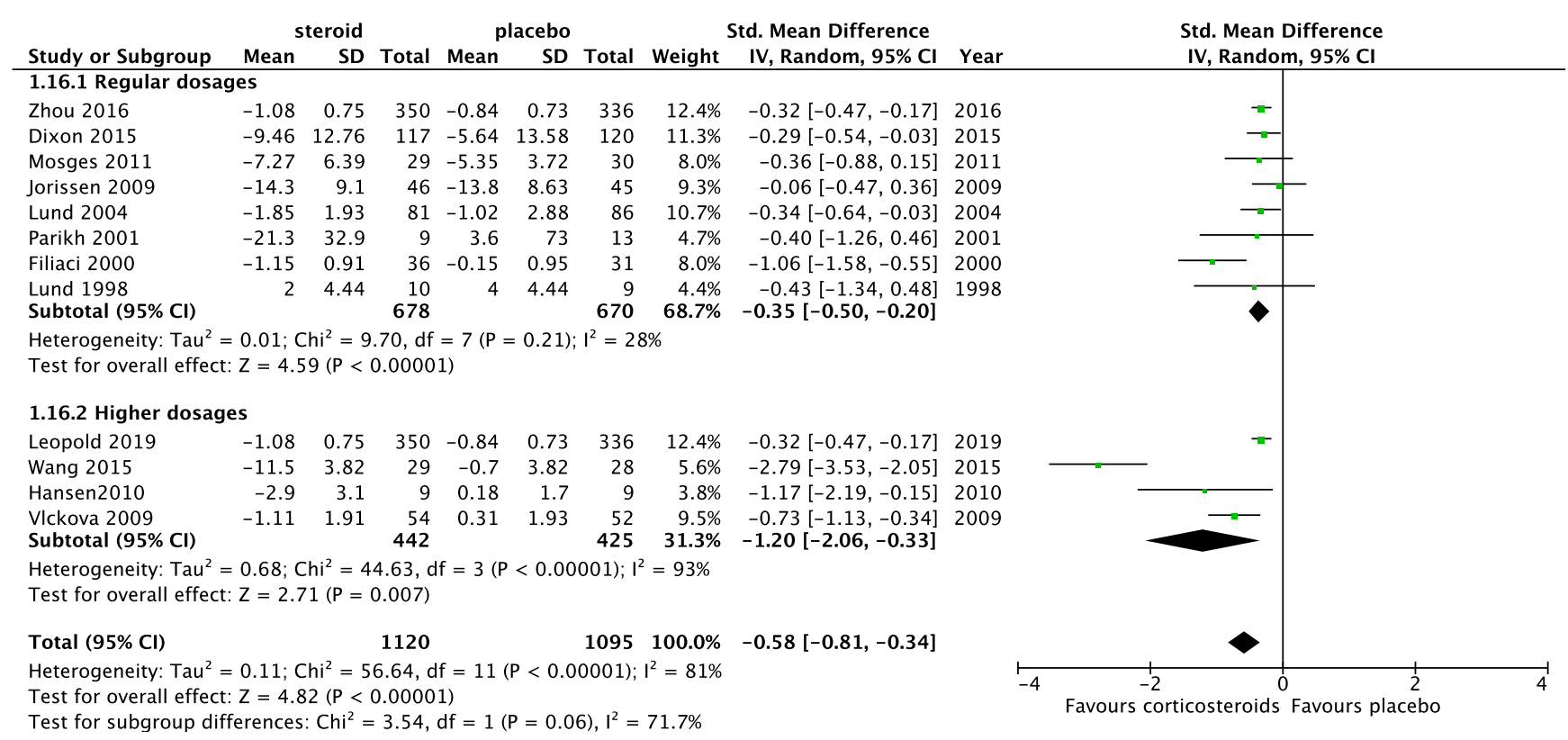


or medial to the middle turbinate) was used in 18 studies $^{(69-73,}$ ${ }^{80,95-106)}$. NPS on a 4-point scale (0-3), described by Lund et al. ${ }^{(79)}$ ( $0=$ no nasal polyps; $1=$ nasal polyps within the middle meatus; $2=$ nasal polyps outside the middle meatus; $3=$ completely obstructive nasal polyps) was used in 3 studies $^{(79,85,107)}$. NPS on a 5 -point scale (0-4), described by Meltzer et al. ${ }^{(108)}$. ( $0=$ no visible nasal polyps; $1=$ small amount of polypoid disease confined within the middle meatus; 2 = multiple nasal polyps occupying the middle meatus; 3 =nasal polyps extending beyond the middle meatus, within the sphenoethmoid recess but not totally obstructing, or both; $4=$ nasal polyps completely obstructing the nasal cavity) was used in one study by Kobayashi et al. ${ }^{(74)}$. NPS on a 5 -point scale $(0-4)(0=$ No nasal polyps; $1=$ small nasal polyps not requiring polypectomy; $2=$ medium nasal polyps not requiring polypectomy; $3=$ large nasal polyps requiring polypectomy; $4=$ completely obstructive nasal polyps requiring polypectomy) was used in one study by Holmberg et al. ${ }^{(109)}$.
Visual analogue scale was assessed in one study ${ }^{(110)}$. Nasal corticosteroids reduced polyp score (SMD -0.87, 95\% $\mathrm{Cl}-1.17$ to $-0.57, \mathrm{p}<0.01 ; 2 \mathrm{RCTs}, \mathrm{n}=184$; Figure 6.1.5.12) and brought greater proportion of responder over placebo in polyp size reduction ( $\mathrm{RR} 0.45,95 \% \mathrm{Cl} 0.36$ to $0.57, \mathrm{p}<0.01 ; 7 \mathrm{RCTs}, \mathrm{n}=$ 889; See Figure 6.1.5.13).

In addition, when nasal corticosteroids were administered after endoscopic sinus surgery, they prevented polyp recurrence significantly better than placebo (RR $0.73,95 \% \mathrm{Cl} 0.56$ to 0.94 , $\mathrm{p}<0.01$; 3 RCTs, $n=256$; Figure 6.1.5.14).

Patients who had previous sinus surgery had significantly greater change of polyp size reduction than placebo (RR 0.31 , $95 \% \mathrm{Cl} 0.20$ to $0.49, \mathrm{p}<0.01 ; 3 \mathrm{RCTs}, \mathrm{n}=307$ versus $\mathrm{RR} 0.54,95 \%$ $\mathrm{Cl} 0.41$ to $0.70, \mathrm{p}=0.04 ; 4 \mathrm{RCTs}, \mathrm{n}=582$; Figure 6.1.5.15).

\subsubsection{Adverse events of nasal corticosteroids}

Of the 42 double-blind randomized controlled studies

Table 6.1.5.2. Comparison of different delivery methods of nasal corticosteroids for the treatment of patients with CRS.

\begin{tabular}{|c|c|c|c|c|c|}
\hline Study & Methods & Participants & Interventions & Outcomes & Results \\
\hline $\begin{array}{l}\text { Harvey } \\
2018^{(67)}\end{array}$ & DBPCRCT & $\begin{array}{l}44 \text { CRS (77\% } \\
\text { CRSwNP), } \\
\text { postoperative }\end{array}$ & $\begin{array}{l}\text { - Mometasone spray and } \\
\text { placebo irrigation for } 1 \\
\text { year ( } n=23) \\
\text { - Mometasone irrigation } \\
\text { and placebo spray for } 1 \\
\text { year }(n=21)\end{array}$ & $\begin{array}{l}\text { Quality of life (SNOT-22) } \\
\text { at } 1 \text { year } \\
\text { Symptom score (VAS total } \\
\text { and } 10 \text { symptoms) at } 1 \\
\text { year } \\
\text { Endoscopy score (MLKS) } \\
0-6 \text { for inflammation, 0-2 } \\
\text { for mucus, 0-2 for pus, } \\
\text { assessment of } 10 \text { cavities) } \\
\text { at } 1 \text { year } \\
\text { Adverse events }\end{array}$ & $\begin{array}{l}\text { - At } 1 \text { year, there was no difference in SNOT- } \\
22 \text { between the mometasone irrigation and } \\
\text { mometasone spray groups } \\
\text { - At } 1 \text { year, the mometasone irrigation group } \\
\text { had significantly greater improvement than the } \\
\text { mometasone spray group in total VAS, nasal } \\
\text { blockage and nasal drainage } \\
\text { - A significantly greater improvement on } \\
\text { endoscopy score in the mometasone irrigation } \\
\text { group was revealed at } 1 \text { year. } \\
\text { - There were no medication reactions observed } \\
\text { in either group }\end{array}$ \\
\hline $\begin{array}{l}\text { Demirel } \\
2008^{(68)}\end{array}$ & DBRCT & $\begin{array}{l}34 \text { CRSwNP, } \\
\text { postoperative } \\
\text { (13/34 } \\
\text { patients had } \\
\text { polypectomy) }\end{array}$ & $\begin{array}{l}\text { - FPNS } 100 \mu \mathrm{g} \text { twice daily for } \\
12 \text { weeks }(n=11) \\
\text { - FPND } 400 \mu \mathrm{g} \text { once daily for } \\
12 \text { weeks ( } n=10) \\
\text { - FPND } 400 \mu \mathrm{g} \text { twice daily for } \\
12 \text { weeks }(n=13)\end{array}$ & $\begin{array}{l}\text { Symptom score }(0-3,4 \\
\text { symptoms) improvement } \\
\text { at } 12 \text { weeks } \\
\text { Polyp score }(0-3) \text { reduc- } \\
\text { tion at } 12 \text { weeks }\end{array}$ & $\begin{array}{l}\text { - At } 12 \text { weeks, FPNS showed significantly greater } \\
\text { efficacy than FPND in improving rhinitis } \\
\text { symptoms, smelling and nasal blockage, } \\
\text { - At } 12 \text { weeks, FPNS showed significantly greater } \\
\text { efficacy than FPND in decreasing nasal polyp } \\
\text { size and increasing total nasal volume. }\end{array}$ \\
\hline $\begin{array}{l}\text { Tos } \\
1998^{(69)}\end{array}$ & DBPCRCT & $\begin{array}{l}138 \text { CRSwNP, } \\
\text { postoperative }\end{array}$ & $\begin{array}{l}\text { - Budesonide aqua spray } \\
128 \mu \mathrm{g} \text { twice daily for } 6 \\
\text { weeks ( } n=46 \text { ) } \\
\text { - Budesonide turbuhaler } \\
200 \mu \mathrm{g} \text { twice daily for } 6 \\
\text { weeks ( } n=45 \text { ) } \\
\text { - Placebo aqua spray for } 6 \\
\text { weeks ( } n=24 \text { ) } \\
\text { - Placebo turbuhaler for } 6 \\
\text { weeks ( } n=23 \text { ) }\end{array}$ & $\begin{array}{l}\text { Proportion of responders } \\
\text { at } 6 \text { weeks } \\
\text { Symptom score }(0-3,3 \\
\text { symptoms) improvement } \\
\text { at } 6 \text { weeks } \\
\text { Polyp score }(0-3) \text { reduc- } \\
\text { tion at } 6 \text { weeks } \\
\text { Adverse events }\end{array}$ & $\begin{array}{l}\text { - The proportion of responders after } 6 \text { weeks } \\
\text { treatment was not significantly different } \\
\text { between the aqua and turbuhaler-treated } \\
\text { groups } \\
\text { - At } 6 \text { weeks, budesonide aqua spray showed a } \\
\text { significantly superior efficacy over turbuhaler } \\
\text { for blocked nose, runny nose and sneezing } \\
\text { - At } 6 \text { weeks, there was no statistical difference } \\
\text { between aqua and turbuhaler in polyp size } \\
\text { reduction } \\
\text { - No serious adverse events }\end{array}$ \\
\hline $\begin{array}{l}\text { Johansen } \\
\text { 1993(70) }\end{array}$ & DBPCRCT & $\begin{array}{l}86 \text { CRSwNP, } \\
\text { sinus surgery } \\
\text { status not } \\
\text { mentioned }\end{array}$ & 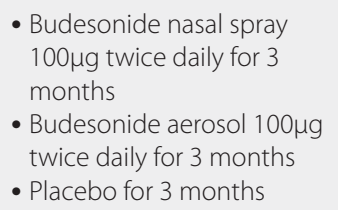 & $\begin{array}{l}\text { Symptom score }(0-3,3 \\
\text { symptoms) improvement } \\
\text { weekly up to } 12 \text { weeks } \\
\text { Polyp score }(0-3) \text { reduc- } \\
\text { tion at } 4,8,12 \text { weeks } \\
\text { Adverse events }\end{array}$ & $\begin{array}{l}\text { - budesonide spray and aerosol were not } \\
\text { different in symptom improvement and polyp } \\
\text { size reduction } \\
\text { - Few side effects of budesonide spray and } \\
\text { aerosol were reported without difference } \\
\text { between groups. }\end{array}$ \\
\hline
\end{tabular}

CRS, chronic rhinosinusitis; CRSwNP, chronic rhinosinusitis with nasal polyps; DBPCRCT, double blind placebo controlled randomised controlled trial; DBRCT, double blind randomised controlled trial; FPNS, Fluticasone Propionate Nasal spray ; MLKS, Modified Lund-Kennedy Score; SNOT, Sino-nasal Outcome Test; VAS, visual analogue scale. 
Table 6.1.5.3. Nasal corticosteroid irrigation in CRS patients.

\begin{tabular}{|c|c|c|c|c|c|}
\hline Study & Methods & Participants & Interventions & Outcomes & Results \\
\hline $\begin{array}{l}\text { Harvey } \\
2018^{(67)}\end{array}$ & DBPCRCT & $\begin{array}{l}44 \text { CRS (77\% CRSwNP), } \\
\text { postoperative ( } 35 \\
\text { analyzed) }\end{array}$ & 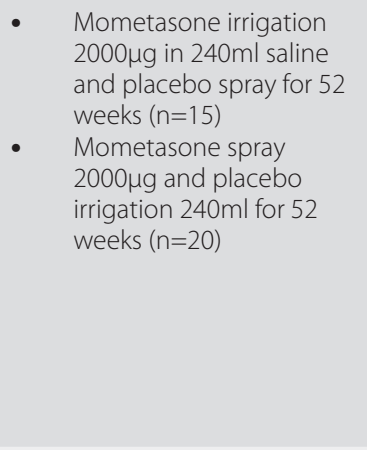 & $\begin{array}{l}\text { - Quality of life (SNOT-22 } \\
\text { (0-110) at } 52 \text { weeks) } \\
\text { - Symptoms (VAS (0-100) at } \\
52 \text { weeks) } \\
\text { - Endoscopy score (MLKS } \\
\text { (0-100) at } 52 \text { weeks) } \\
\text { - CT score (LM score (0-24) } \\
\text { at } 52 \text { weeks) } \\
\text { - Adverse event }\end{array}$ & $\begin{array}{l}\text { - SNOT-22 changes at } 12 \text { months } \\
\text { are not different between } \\
\text { mometasone irrigation and spray } \\
\text { - Total VAS scores at } 12 \text { months } \\
\text { significantly favoured } \\
\text { mometasone irrigation over spray } \\
\text { - Endoscopy scores at } 12 \\
\text { months significantly favoured } \\
\text { mometasone irrigation over spray } \\
\text { - CT scores at } 12 \text { months } \\
\text { significantly favoured } \\
\text { mometasone irrigation over spray } \\
\text { - No treatment-related adverse } \\
\text { event was observed. }\end{array}$ \\
\hline $\begin{array}{l}\text { Tait } \\
2018^{(81)}\end{array}$ & DBPCRCT & $\begin{array}{l}74 \text { CRS ( } 25 \% \text { CRSwNP), } \\
\text { with } 28 \% \text { history of } \\
\text { prior ESS ( } 61 \text { finally } \\
\text { analysed) }\end{array}$ & $\begin{array}{l}\text { - Budesonide irrigation } \\
1000 \mu \mathrm{g} \text { once daily in } 240 \mathrm{ml} \\
\text { bottle for } 4 \text { weeks ( } \mathrm{n}=29) \\
\text { - Saline irrigation with pla- } \\
\text { cebo (lactose) for } 4 \text { weeks } \\
(\mathrm{n}=32)\end{array}$ & $\begin{array}{l}\text { - Quality of life (SNOT-22 } \\
\text { (0-110) at } 4 \text { weeks) } \\
\text { - Symptoms (Likert scale of } \\
\text { improvement 1-7, scale at } \\
4 \text { weeks) } \\
\text { - Endoscopy score (LKES } \\
\text { (0-20) at } 4 \text { weeks) } \\
\text { - Adverse event }\end{array}$ & $\begin{array}{l}\text { - The SNOT- } 22 \text { scores at } 4 \text { weeks } \\
\text { were not different between } 2 \\
\text { groups } \\
\text { - Symptoms at } 4 \text { weeks were not } \\
\text { different } \\
\text { - LKES change at } 4 \text { weeks was not } \\
\text { different } \\
\text { - There were no related adverse } \\
\text { events. }\end{array}$ \\
\hline $\begin{array}{l}\text { Rawal } \\
2015^{(82)}\end{array}$ & SBPCRCT & $\begin{array}{l}50 \text { CRSwNP, with } 100 \% \\
\text { postoperative (42 finally } \\
\text { analysed) }\end{array}$ & $\begin{array}{l}\text { - Budesonide irrigation } 500 \mu \mathrm{g} \\
\text { in } 240 \mathrm{ml} \text { bottle. } 60 \mathrm{ml} / \mathrm{nostril} \\
\text { twice daily for } 24 \text { weeks } \\
(\mathrm{n}=24) \\
\text { - Saline irrigation alone for } 24 \\
\text { weeks }(n=18)\end{array}$ & $\begin{array}{l}\text { - Quality of life (SNOT-22 } \\
\text { (0-110), RSOM-31 (0-155), } \\
\text { RSDI (0-120) at } 2 \text { weeks, 3, } \\
6 \text { months) }\end{array}$ & $\begin{array}{l}\text { - SNOT-22, RSOM31, and RSDI } \\
\text { scores were not different between } \\
2 \text { arms at all } 3 \text { timepoints ( } 2 \text { weeks, } \\
3,6 \text { months). }\end{array}$ \\
\hline $\begin{array}{l}\text { Rotenberg } \\
2011^{(83)}\end{array}$ & DBPCRCT & $\begin{array}{l}64 \text { CRSwNP (Samter's } \\
\text { triad), with } 100 \% \text { post- } \\
\text { operative (basic FESS) } \\
\text { (60 finally analysed) }\end{array}$ & $\begin{array}{l}\text { - Budesonide irrigation } 60 \mathrm{ml} / \\
\text { nostril } 500 \mu \mathrm{g} \text { twice daily } \\
\text { (total } 1000 \mu \mathrm{g} \text { ) for } 52 \text { weeks } \\
\text { ( } \mathrm{n}=20 \text { ) } \\
\text { - Saline irrigation } 60 \mathrm{ml} / \mathrm{nostril} \\
\text { and budesonide spray } 64 \mu \mathrm{g} / \\
\text { nostril twice daily (total } \\
256 \mu \mathrm{g} \text { ) for } 52 \text { weeks }(\mathrm{n}=19 \text { ) } \\
\text { - Saline irrigation alone for } 52 \\
\text { weeks ( } n=21 \text { ) }\end{array}$ & $\begin{array}{l}\text { - Quality of life (SNOT-21 (0- } \\
\text { 105) at 6,12 months) } \\
\text { - Endoscopy score (LKES } \\
\text { (0-12) at 6,12 months) } \\
\text { - CT score (LM score (0-24) } \\
\text { at 6,12 months) } \\
\text { - Ocular events (IOP at 6,12 } \\
\text { months) } \\
\text { - HPA axis (ACTH (normal/ } \\
\text { abnormal) at 6,12 months) }\end{array}$ & $\begin{array}{l}\text { - SNOT-21, LKES, and LM score } \\
\text { results of both intervention groups } \\
\text { were not different from placebo } \\
\text { group at both } 6 \text { and } 12 \text {-month } \\
\text { timepoints (significant level was } \\
\text { defined at } \mathrm{p}<.0167 \text { ) } \\
\text { - IOP and ACTH level were not } \\
\text { changed at both } 6 \text { and } 12 \text {-month } \\
\text { timepoints }\end{array}$ \\
\hline
\end{tabular}

ACTH, Adrenocorticotropic Hormone ; CRS, chronic rhinosinusitis; CRSwNP, chronic rhinosinusitis with nasal polyps; DBPCRCT, double blind placebo controlled randomisedcontrolled trial; FESS, functional endoscopic sinus surgery; HPA, Hypothalamic Pituitary Adrenal ; IOP, intraocular pressure; LKES, Lund-Kennedy endoscopy scoring ; LM, Lund-Mackay; MLKS, Modified Lund-Kennedy Score; SBPCRCT, single blind placebo controlled randomised controlled trial; SNOT, Sino-nasal Outcome Test; VAS, visual analogue scale; RSDI, Rhinosinusitis Disability Index; RSOM-31, Rhinosinusitis Outcome Measure-31.

included in this review (43 articles), adverse events of nasal corticosteroids compared to placebo were assessed in 26 studies $^{(67,69,70,72,73,79-81,83,85,90,93,95,96,98,100-105,109,111-114)}$. All these studies found that nasal corticosteroids were well tolerated and safe. Drug related major adverse events were not reported. Minor adverse events (if any) had mild to moderate severity. Of the 26, 18 studies found that adverse events (including epistaxis) caused by nasal corticosteroids were not different from placebo ${ }^{(69,70,79,80,85,90,93,95,96,101-105,111-114)}$. Five studies reported that a greater number of patients in the nasal corticosteroid group had epistaxis than in the placebo group ${ }^{(72,73,98,100,109)}$. When data were pooled for meta-analysis, nasal corticosteroids resulted in increase in the risk of epistaxis compared to placebo (RR 3.49, 95\% Cl 2.42 to 5.05; 16 RCTs, $n=2021$; Figure 6.1.5.16). Nasal septal ulcer was reported more frequently in the nasal corticosteroid group in one study ${ }^{(72,73)}$. The hypothalamicpituitary-adrenal axis suppression assessed in four studies found that serum cortisol ${ }^{(72,85,100)}$ and 24-hour urinary free cortisol $^{(103)}$ were unchanged with no difference from the placebo group. No patients in both groups had clinical adrenal insufficiency. Nasal steroids did not increase intraocular pressure and did not cause cataract ${ }^{(73)}$. This is concordance with a systematic review that also concluded that nasal corticosteroids do not affect intraocular pressure or lens opacity: a systematic review of controlled trials ${ }^{(115)}$. There was no difference between nasal steroids and placebo in asthma exacerbation ${ }^{(95)}$, and acute bacterial rhinosinusitis ${ }^{(95,116)}$. although one study did describe more infections ${ }^{(117)}$.

\subsubsection{Conclusion}

There is high-quality evidence that long term use of nasal corticosteroids is effective and safe for treating patients with CRS. They have impact on nasal symptoms and quality of life improvement, although the effect on SNOT-22 is smaller the 
Figure 6.1.5.9. Forest plot of the effect of different delivery methods of nasal corticosteroids versus placebo on symptoms scores in CRS patients.

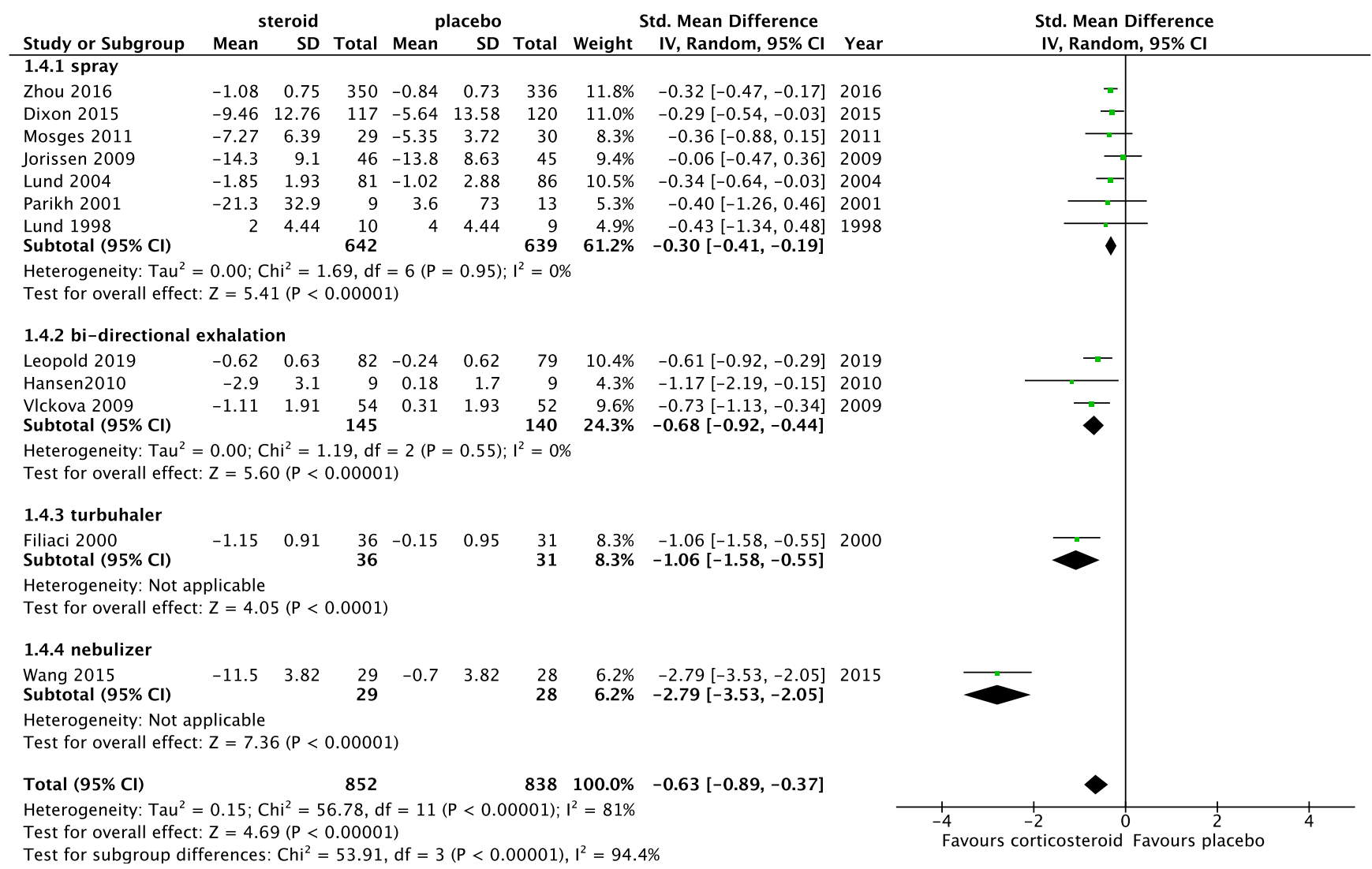

Figure 6.1.5.10. Forrest plot of the effect on SNOT score at six months after steroid irrigation versus saline irrgation in CRS patients.

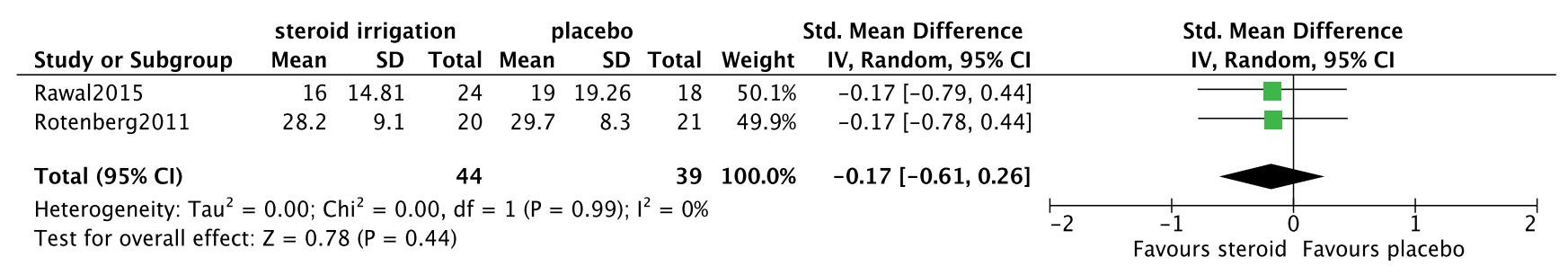

Figure 6.1.5.11. Forest plot of the effect of nasal corticosteroid versus placebo on nasal endoscopy score in patients with CRS.

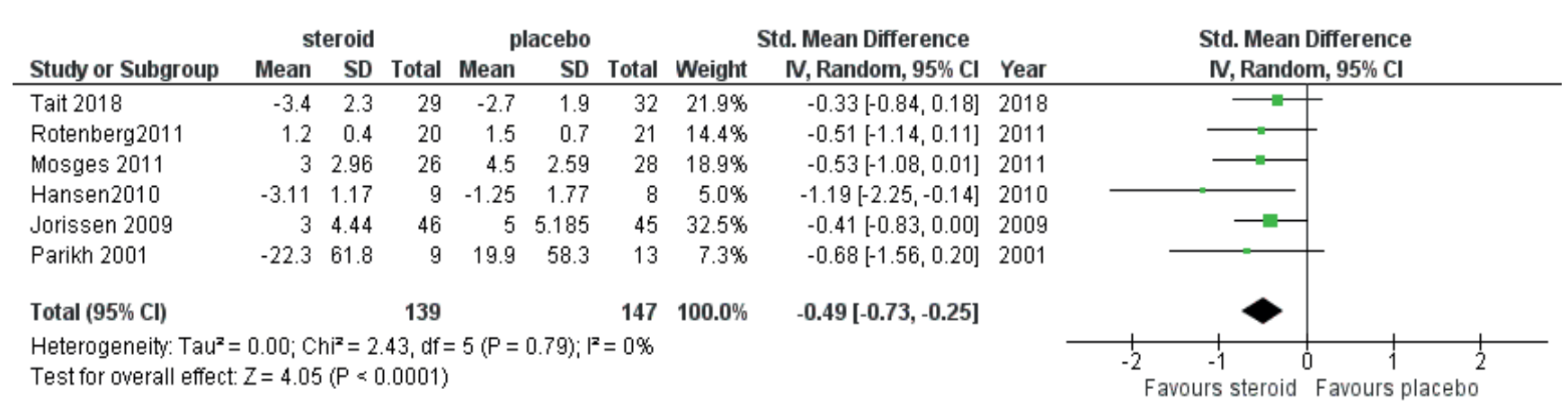


Figure 6.1.5.12. Forest plot of the effect of nasal corticosteroid versus placebo on nasal polyp score in patients with CRSwNP.

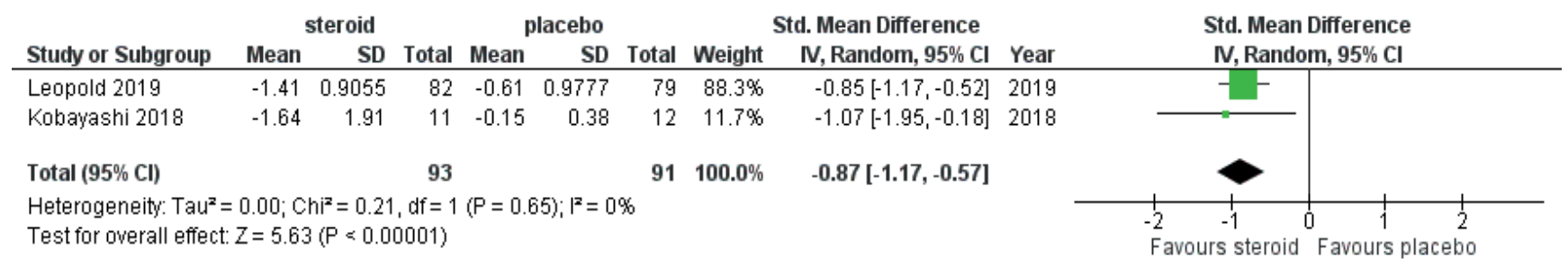

Figure 6.1.5.13. Forest plot of the effect of nasal corticosteroid versus placebo on the proportion of responders in nasal polyp score reduction.

\begin{tabular}{|c|c|c|c|c|c|c|c|c|c|c|c|}
\hline Study or Subgroup & placebo & $\begin{array}{l}\text { bo } \\
\text { Total }\end{array}$ & $\begin{array}{l}\text { stero } \\
\text { Events }\end{array}$ & $\begin{array}{l}\text { id } \\
\text { Total }\end{array}$ & \multicolumn{3}{|c|}{ Risk Ratio } & \multicolumn{4}{|c|}{$\begin{array}{c}\text { Risk Ratio } \\
\text { M-H, Fixed, 95\% Cl }\end{array}$} \\
\hline Leopold 2019 & 7 & 79 & 23 & 82 & $13.2 \%$ & $0.32[0.14,0.69]$ & 2019 & & & & \\
\hline Kobayashi 2018 & 2 & 12 & 8 & 11 & $4.9 \%$ & $0.23[0.06,0.85]$ & 2018 & & & & \\
\hline Vlckova 2009 & 5 & 55 & 31 & 54 & $18.3 \%$ & $0.16[0.07,0.38]$ & 2009 & & & & \\
\hline Stjarne 2006b & 39 & 145 & 63 & 153 & $35.8 \%$ & $0.65[0.47,0.91]$ & 2006 & & $\rightarrow-$ & & \\
\hline Penttila 2000 & 7 & 47 & 19 & 47 & $11.1 \%$ & $0.37[0.17,0.79]$ & 2000 & & & & \\
\hline Keith 2000 & 8 & 52 & 14 & 52 & $8.2 \%$ & $0.57[0.26,1.25]$ & 2000 & & & & \\
\hline Holmstrom 1999 & 8 & 49 & 15 & 51 & $8.6 \%$ & $0.56[0.26,1.19]$ & 1999 & & & & \\
\hline Total $(95 \% \mathrm{Cl})$ & & 439 & & 450 & $100.0 \%$ & $0.45[0.36,0.57]$ & & & 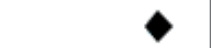 & & \\
\hline Total events & 76 & & 173 & & & & & & & & \\
\hline $\begin{array}{l}\text { Heterogeneity: } \mathrm{Chi}^{2}= \\
\text { Test for overall effect }\end{array}$ & $\begin{array}{l}13.20, d f \\
Z=6.67\end{array}$ & $\begin{array}{l}=6(P= \\
P<0.0\end{array}$ & $\begin{array}{l}=0.04) ; I^{2} \\
00001)\end{array}$ & $=55 \%$ & & & & 0.01 & $\begin{array}{c}0.1 \\
\text { Favours steroid }\end{array}$ & $\begin{array}{cc}1 & 10 \\
\text { Favours placebo }\end{array}$ & 100 \\
\hline
\end{tabular}

Figure 6.1.5.14. Forest plot of the effect of nasal corticosteroid versus placebo on the prevention of nasal polyp recurrence after sinus surgery in CRSwNP patients

\begin{tabular}{|c|c|c|c|c|c|c|c|c|c|c|c|c|}
\hline \multirow[b]{2}{*}{ Study or Subgroup } & \multicolumn{2}{|c|}{ steroid } & \multicolumn{2}{|c|}{ placebo } & \multicolumn{3}{|c|}{ Risk Ratio } & \multirow{2}{*}{\multicolumn{5}{|c|}{$\begin{array}{c}\text { Risk Ratio } \\
\text { M-H, Fixed, } 95 \% \mathrm{Cl}\end{array}$}} \\
\hline & Events & Total & Events & Total & Weight & M-H, Fixed, $95 \% \mathrm{Cl}$ & Year & & & & & \\
\hline Stjarne 2009 & 26 & 79 & 35 & 80 & $52.2 \%$ & $0.75[0.50,1.12]$ & 2009 & & & -1 & & \\
\hline Dijkstra 2004 & 4 & 13 & 7 & 21 & $8.0 \%$ & $0.92[0.33,2.55]$ & 2004 & & & & & \\
\hline Gulati 2001 & 22 & 38 & 22 & 25 & $39.8 \%$ & $0.66[0.48,0.89]$ & 2001 & & & - & & \\
\hline Total $(95 \% \mathrm{Cl})$ & & 130 & & 126 & $100.0 \%$ & $0.73[0.56,0.94]$ & & & & $\gamma$ & & \\
\hline Total events & 52 & & 64 & & & & & & & & & \\
\hline $\begin{array}{l}\text { Heterogeneity: } \mathrm{Chi}^{2} \text { = } \\
\text { Test for overall effect }\end{array}$ & $\begin{array}{l}0.66, \mathrm{df}= \\
Z=2.40\end{array}$ & $\begin{array}{l}2(P= \\
P=0.0\end{array}$ & $\begin{array}{l}0.72) ; 1^{2}= \\
\text { 2) }\end{array}$ & $=0 \%$ & & & & 0.01 & $\begin{array}{c}0.1 \\
\text { Favour }\end{array}$ & steroid & $\begin{array}{c}10 \\
\text { Favours placebo }\end{array}$ & 100 \\
\hline
\end{tabular}

minimal clinically important difference. The effect size on symptomatology is larger in CRSwNP (SMD - $0.93,95 \% \mathrm{Cl}-1.43$ to -0.44 ) than in CRSsNP (SMD - $0.30,95 \% \mathrm{Cl}-0.46$ ). The metaanalysis did not show differences between different kinds of nasal corticosteroids. Although in meta-analysis higher dosages and some different delivery methods seem to have a larger effect size on symptomatology direct comparisons are mostly missing. For chronic rhinosinusitis with nasal polyposis, nasal corticosteroids reduce nasal polyp size. When administered after endoscopic sinus surgery, nasal corticosteroids prevent polyp recurrence. Nasal corticosteroids are well tolerated. Most adverse events reported are mild to moderate in severity. Nasal corticosteroids do not affect intraocular pressure or lens opacity. The EPOS2020 steering committee advises to use nasal corticosteroids in patients with CRS. Based on the low to very low quality of the evidence for higher dosages or different delivery methods and the paucity of direct comparisons the steering committee cannot advise in favour of higher dosages or certain delivery methods.

\subsubsection{Corticosteroid-eluting implants}

\subsubsection{Corticosteroid-eluting implants following endoscopic sinus surgery, summary of the evidence} Steroid-eluting stents, spacers and dressings can improve local drug delivery immediately following endoscopic sinus surgery or be placed in office in postoperative patients. The placement immediately after surgery will be discussed in chapter 6.2.7.4.

\subsubsection{Corticosteroid-eluting implants in office, summary of the evidence}

In addition to the postoperative studies, two studies evaluated the effect of placement of a corticosteroid-eluting sinus implant delivering $1350 \mu \mathrm{g}$ of mometasone furoate (MF) in the ethmoid of patients with recurrent polyposis after sinus surgery over approximately 90 days $^{(118,119)}$, as well as the follow-up of $\mathrm{Han}^{(120)}$. Both the treated and the placebo group also used $200 \mu \mathrm{g}$ mometasone furoate nasal spray once daily (Table 6.1.6.1.) Some of the parameters could be combined for the two studies: 
Figure 6.1.5.15. Forest plot of the effect of nasal corticosteroid versus placebo on the proportion of patients with nasal polyp score reduction in subgroups of CRS patients with and without sinus surgery.

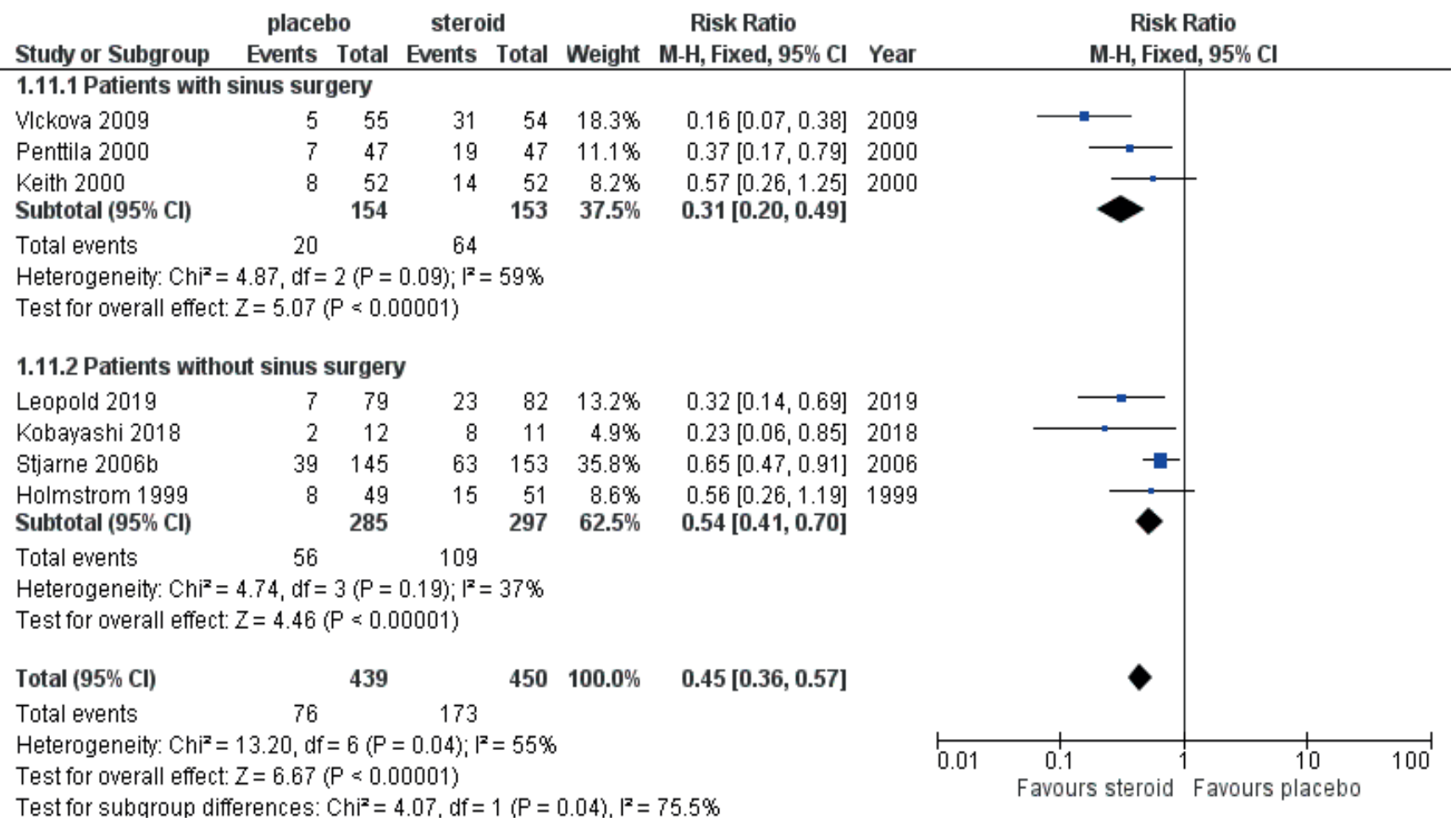

Figure 6.1.5.16. Forest plot of the effect of nasal corticosteroid versus placebo on the proportion of CRS patients with epistaxis.

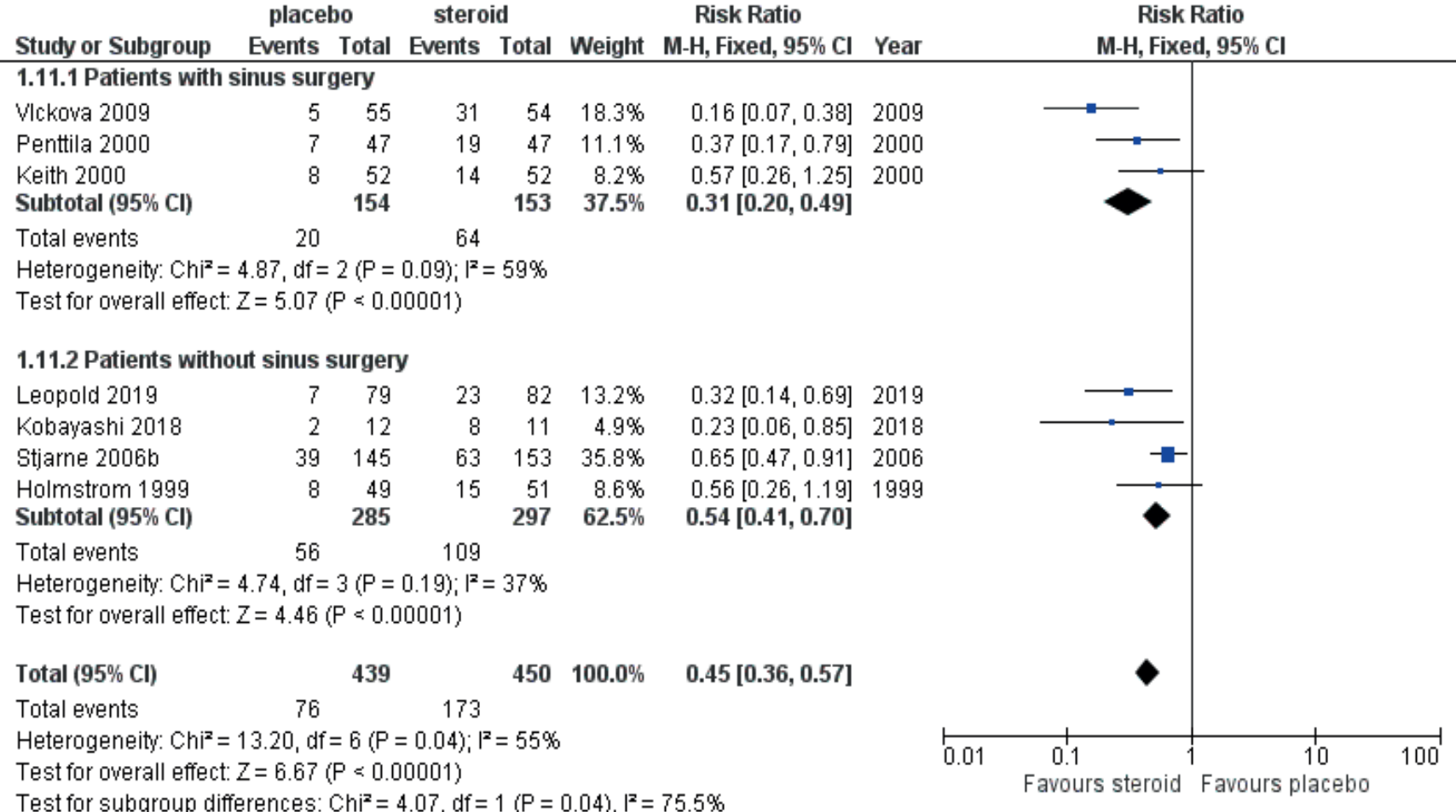


the change from baseline to day 90 in nasal obstruction/ congestion score (0-3), evaluated for seven days before clinic visits, showed MD 0.28 (95\% Cl 0.09, 0.48); 366 participants; two studies $(12=43 \%)$, lower $=$ better, indicating less symptoms of nasal obstruction/congestion in the patients that were treated with corticosteroid-eluting sinus implant than the placebo (Figure 6.1.6.1).

The change from baseline to day 90 in bilateral polyp grade (08/0-4), showed an SMD 0.40 ( $95 \%$ Cl 0.19, 0.61); 392 participants; two studies; $12=0 \%$ ), lower = better, indicating less disease in the patients that were treated with corticosteroid-eluting sinus implant than the placebo (Figure 6.1.6.2).
The patients indicated for sinus surgery at day 90 (\%) showed an OR of 0.37 (95\% Cl 0.23, 0.61); 398 participants; two studies (I2 $=0 \%$, indicating less likelihood that the patients treated with corticosteroid-eluting sinus implant than the placebo would need sinus surgery (Figure 6.1.6.3).

In one study ${ }^{(118)}$ sense of smell (scale $0-5$ ) and facial pain (scale $0-5)$ were also evaluated. There was a significant improvement in smell MD - $0.46(95 \% \mathrm{Cl}-0.85 ;-0.06)$ in the patients that were treated with corticosteroid-eluting sinus implant compared with the placebo. No differences in facial pain scores were found.

Figure 6.1.6.1. Forest plot of the effect of corticosteroid-eluting implants versus placebo on the change from baseline to day 90 in nasal obstruction/congestion score in patients with CRS.

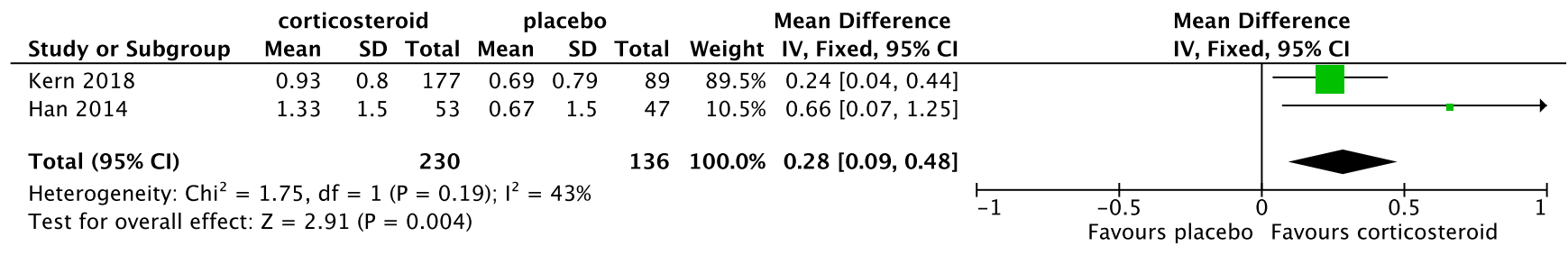

Figure 6.1.6.2. Forest plot of the effect of corticosteroid-eluting implants versus placebo on the change from baseline to day 90 in bilateral Nasal Polyp Score (NPS) in patients with CRS.

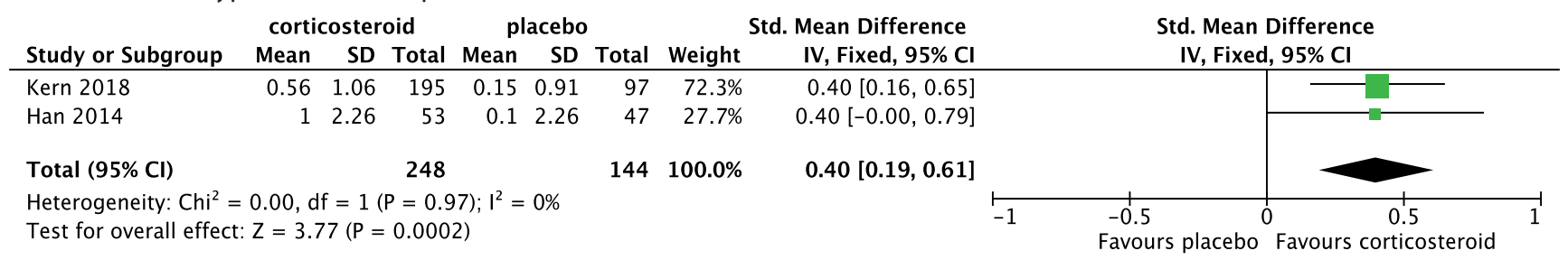

Figure 6.1.6.3. Forest plot of the effect of corticosteroid-eluting implants versus placebo on the reduction of indicated sinus surgery at day $90(\%)$.

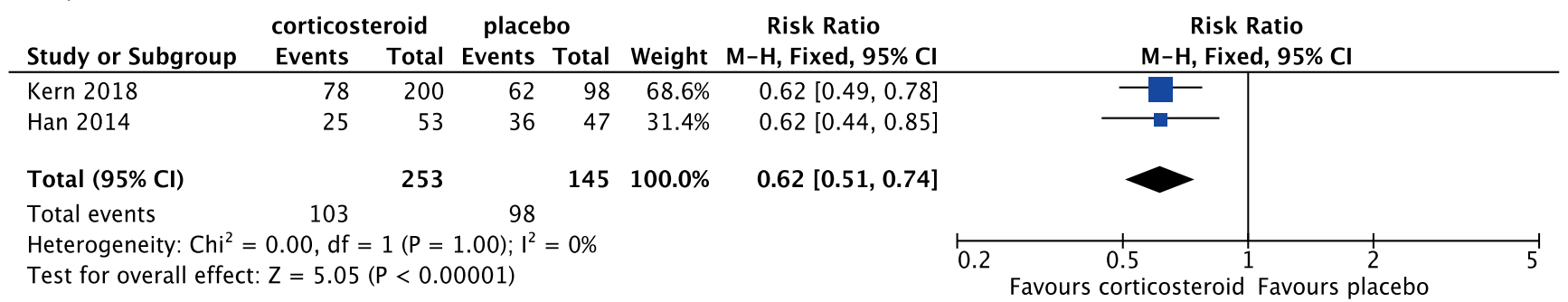

Figure 6.1.7.1. Forest plot of the effect of short course of systemic corticosteroid therapy versus placebo on total symptom score 2-3 weeks after start of the therapy in CRS patients.

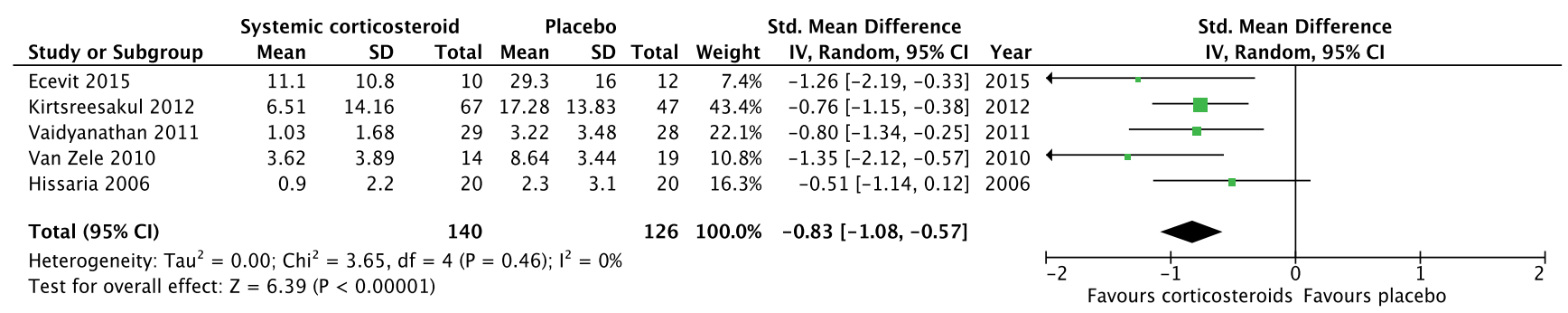


Table 6.1.6.1. Corticosteroid-eluting implants for the in the office treatment of patients with CRS.

\begin{tabular}{|c|c|c|c|c|c|}
\hline Study & Methods & Participants & Interventions & Outcomes & Results \\
\hline $\begin{array}{l}\text { Kern } \\
2018^{(118)}\end{array}$ & DBRPCT & $\begin{array}{l}301 \text { CRSwNP } \\
\text { patients }\end{array}$ & 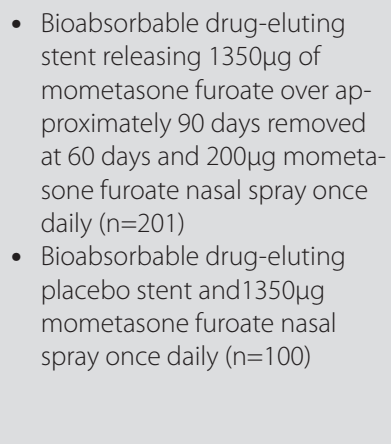 & $\begin{array}{l}\text { - Change from baseline to day } 30 \text { and day } 90 \text { in } \\
\text { nasal obstruction/congestion score (0-3) } \\
\text { - Change from baseline to day } 90 \text { in bilateral } \\
\text { polyp grade (0-8), as determined by indepen- } \\
\text { dent, blinded panel } \\
\text { - Patients indicated for sinus surgery at day } 90 \\
\text { - Change from baseline to day } 90 \text { of ethmoid } \\
\text { sinus obstruction (0-100) by independent, } \\
\text { blinded panel } \\
\text { - Change from baseline to day } 90 \text { in sense of } \\
\text { smell (0-5) and facial pain (0-5) } \\
\text { - Adverse events }\end{array}$ & $\begin{array}{l}\text { - All outcomes } \\
\text { significant better in } \\
\text { the mometasone } \\
\text { furoate stent group } \\
\text { than the placebo stent } \\
\text { group but facial pain. } \\
\text { - No difference in } \\
\text { adverse events. }\end{array}$ \\
\hline $\begin{array}{l}\text { Forwith } \\
2016^{(120)}\end{array}$ & $\begin{array}{l}\text { DBRPCT } \\
\text { (Follow- } \\
\text { up of Han } \\
2014^{(119)} \text { ) }\end{array}$ & $\begin{array}{l}100 \text { CRSwNP } \\
\text { patients }\end{array}$ & 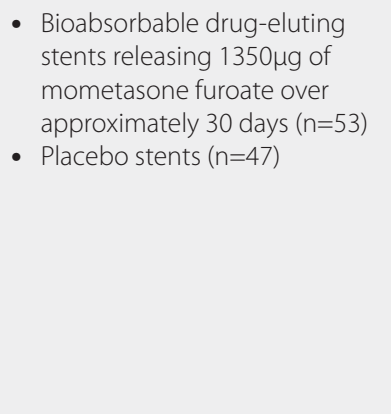 & $\begin{array}{l}\text { - Change from baseline to day obstruction/ } \\
\text { congestion score (0-3) (subset of patients) at } \\
6 \text { months } \\
\text { - Change from baseline in bilateral polyp grade } \\
\text { (0-4) at } 6 \text { months } \\
\text { - Patients indicated for sinus surgery at } 6 \\
\text { months (\%) } \\
\text { - Change from baseline of ethmoid sinus } \\
\text { obstruction (0-100) at } 6 \text { months } \\
\text { - Adverse events }\end{array}$ & $\begin{array}{l}\text { At } 6 \text { months, treated } \\
\text { patients compared to } \\
\text { placebo patients had: } \\
\text { - Significant reduction } \\
\text { in ethmoid snus } \\
\text { obstruction }(p<0.001) \\
\text { and bilateral polyp } \\
\text { grade }(p=0.018) \\
\text { - Significantly smaller } \\
\text { change of sinus } \\
\text { surgery }\end{array}$ \\
\hline $\begin{array}{l}\text { Han } \\
2014^{(119)}\end{array}$ & DBRPCT & $\begin{array}{l}100 \text { CRSWNP } \\
\text { patients }\end{array}$ & $\begin{array}{l}\text { - Bioabsorbable drug-eluting } \\
\text { stents releasing } 1350 \mu \mathrm{g} \text { of } \\
\text { mometasone furoate over ap- } \\
\text { proximately } 90 \text { days removed } \\
\text { at } 60 \text { days and } 200 \mu \mathrm{g} \text { mometa- } \\
\text { sone furoate nasal spray once } \\
\text { daily ( } \mathrm{n}=53 \text { ) } \\
\text { - Bioabsorbable drug-eluting } \\
\text { placebo stent and } 1350 \mu \mathrm{g} \\
\text { mometasone furoate nasal } \\
\text { spray once daily }(n=47)\end{array}$ & $\begin{array}{l}\text { - Change from baseline to day obstruction/ } \\
\text { congestion score (0-3) (subset of patients) at } \\
\text { day } 7,14,30,45,60 \text {, and } 90 \\
\text { - Change from baseline in bilateral polyp grade } \\
\text { ( } 0-4) \text { at day } 7,14,30,45,60 \text {, and } 90 \\
\text { - Patients indicated for sinus surgery an inter- } \\
\text { vention at day } 90(\%) \\
\text { - Change from baseline of ethmoid sinus ob- } \\
\text { - Atruction (0-100) at day } 7,14,30,45,60 \text { and } 90 \\
\text { - Adverse events } \\
\text { Ocular safety }\end{array}$ & $\begin{array}{l}\text { - Significant reduc- } \\
\text { tion in obstruction/ } \\
\text { congestion (day } \\
30 \text { and 90), polyps } \\
\text { grade, ethmoid sinus } \\
\text { obstruction } \\
\text { - No significant } \\
\text { differences in adverse } \\
\text { events or ocular safety }\end{array}$ \\
\hline
\end{tabular}

CRSwNP, chronic rhinosinusitis with nasal polyps; DBRPCT, double blind randomised placebo controlled trial.

\subsubsection{Safety of corticosteroid-eluting implants}

Most of the safety studies have been performed directly after surgery and the full safety data are discussed in chapter 6.2.7.3. None of the studies showed impact on ocular safety ${ }^{(119,121,}$ ${ }^{122)}$. Local (drug related) adverse events were limited and not reported more often in the local corticosteroid group than the placebo group.

\subsubsection{Conclusion}

The placement of corticosteroid-eluting sinus implants in the ethmoid of patients with recurrent polyposis after sinus surgery has a significant but small ( 0.3 on a $0-3$ scale) impact on nasal obstruction but is significantly reduced the need for surgery [odds ratio (OR) 0.34] and reduced nasal polyp score. Based on the moderate to high quality of the evidence the steering group considered the use of corticosteroid-eluting sinus implants in the ethmoid an option. Larger studies including long term safety data are needed.

\subsubsection{Short courses of systemic corticosteroids}

\section{A short course of systemic corticosteroid, with or without local corticosteroid treatment results in a significant reduction in total symptom score and nasal polyp score in patients with CRSwNP.}

\subsubsection{Summary of the evidence}

Short courses of systemic corticosteroids (7-21 days), usually combined with local corticosteroids, are a widely used treatment for chronic rhinosinusitis with nasal polyps. Systemic corticosteroids reduce the inflammatory response and may reduce the size of nasal polyps. Interestingly especially inflammation of the olfactory mucosa is reduced often resulting in a rapid improvement in olfaction (days) without visual change in polyp volume.

In this analysis, only studies comparing a short course of systemic corticosteroids using a DBPCRT design are included. Different forms of systemic corticosteroids were used (Table 
6.1.7.2. for comparison of corticosteroids) although most studies used prednis(ol)one. In seven DBPCRCTs a short course of systemic corticosteroids, sometimes combined with local corticosteroids, was compared to placebo ${ }^{(4,123-128)}$ (Table 6.1.7.2.). All studies were done in patients with CRSwNP. We are not aware of any RCTs with systemic corticosteroids in CRSsNP. Five studies evaluated the effect of oral (methyl)prednis(ol)one in dosages ranging from $25-60 \mathrm{mg}$ for $7-20$ days $\mathrm{s}^{(4,124,126-128)}$. Three studies only used the systemic corticosteroids $(4,124,128)$ and two studies used a form of local corticosteroids after the systemic course ${ }^{(126,}$ 127). All five studies measured some form of symptom score 2-4 weeks after the start of the treatment (for Hissaria we took the nasal domain of the Rhinosinusitis Outcome Measure-31 (RSOM-31)) and the meta-analysis showed a significant effect on total symptom score (SMD -1.51 (95\% Cl -1.08, -1.57); 266 participants; four studies; $12=0 \%)(p<0.00001)$ (Figure 6.1.7.1). However, at 10-12 weeks after the start of the treatment the difference was no longer significant SMD $-0.13(95 \% \mathrm{Cl}-0.41$, $0.15) ; 200$ participants; 3 studies; $12=0 \%),(p=0.38$ ) (Figure 6.1.7.2). Four studies could be included in a meta-analysis on nasal polyp score at 2-3 weeks after the start of the corticosteroid therapy. The meta-analysis showed a significant effect of oral corticosteroid treatment over placebo: SMD -1.51 (95\% Cl -2.12, -0.90); 237 participants; four studies; $12=70 \% ; \mathrm{p}<0.00001$ ) (Figure 6.1.7.3).

At 10-12 weeks after the start of the treatment the difference was still significant SMD $-0.51(95 \% \mathrm{Cl}-0.80,-0.21) ; 203$ participants; three studies; $12=4 \%),(p=0.0007)$ (Figure 6.1.7.4). Two studies (118 postoperative CRSwNP patients) evaluated the postoperative use of systemic corticosteroids ${ }^{(123,125)}$. Both studies reported no significant differences in SNOT-22 in the first 2-3 months after surgery (Figure 6.1.7.5) and in Shen et al. even six months after surgery. Shen et al. ${ }^{(123)}$ found a significantly lower Lund-Kennedy endoscopy score in the prednisone group for the high eosinophils group at three months and a trend at six months (no correction for multitesting).

The adverse effects of short courses of systemic corticosteroids can include insomnia, mood changes and gastrointestinal changes. Short-term courses of systemic corticosteroids are generally safe, but there have been reports of associated avascular necrosis and a few cases of fatal varicella-zoster in immunocompetent patients ${ }^{(129)}$. It is not clear from the literature whether the dose or duration of the systemic corticosteroids are

Figure 6.1.7.2. Forest plot of the effect of short course of systemic corticosteroid therapy versus placebo on total symptom score 1012 weeks after start of the therapy in CRS patients.

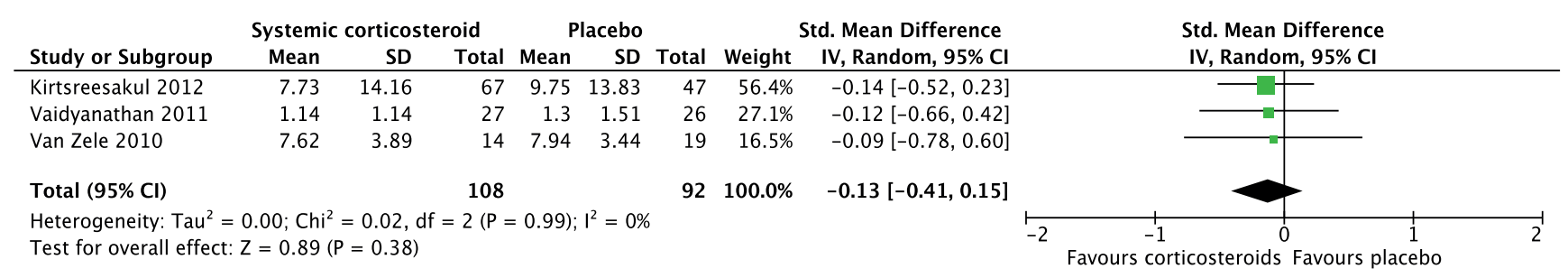

Figure 6.1.7.3. Forest plot of the effect of short course of systemic corticosteroid therapy versus placebo on polyp score 2-3 weeks after start of the therapy in CRS patients.

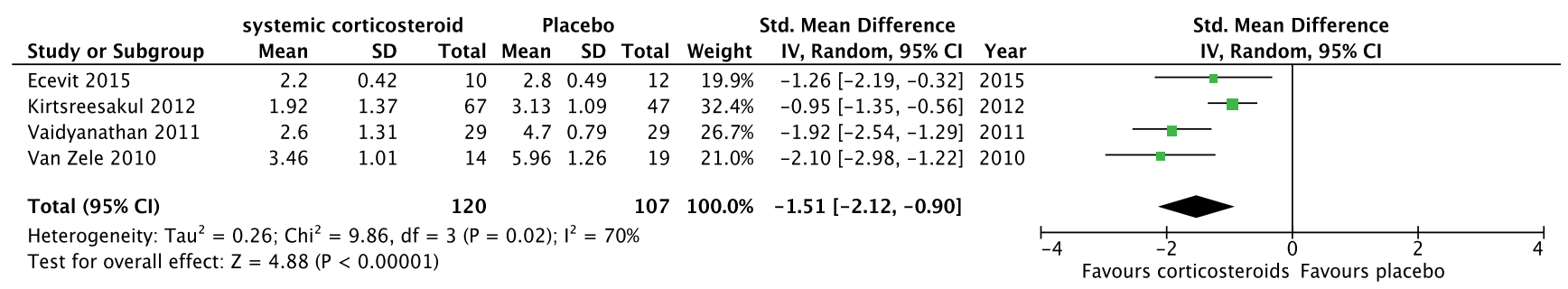

Figure 6.1.7.4. Forest plot of the effect of short course of systemic corticosteroid therapy versus placebo on polyp score 10-12 weeks after start of the therapy in CRS patients.

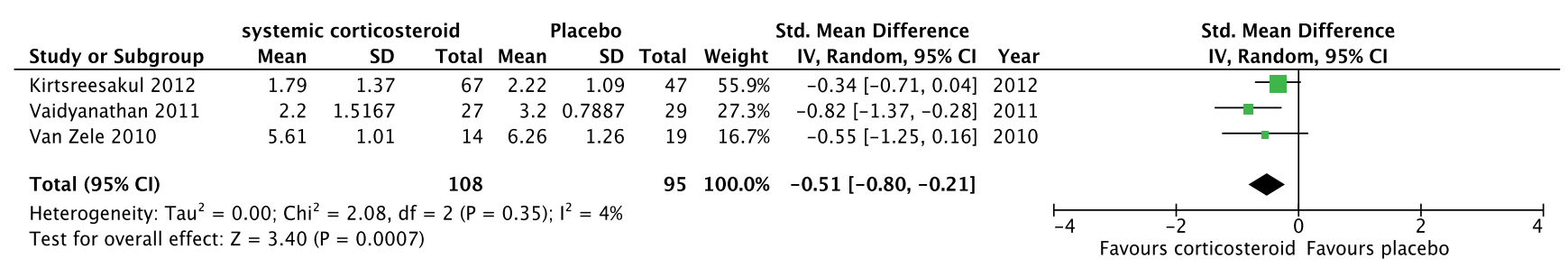


Figure 6.1.7.5. Forest plot of the effect on SNOT-22 of postoperative short course of systemic corticosteroid therapy versus placebo 2-3 months after surgery in CRS patients.

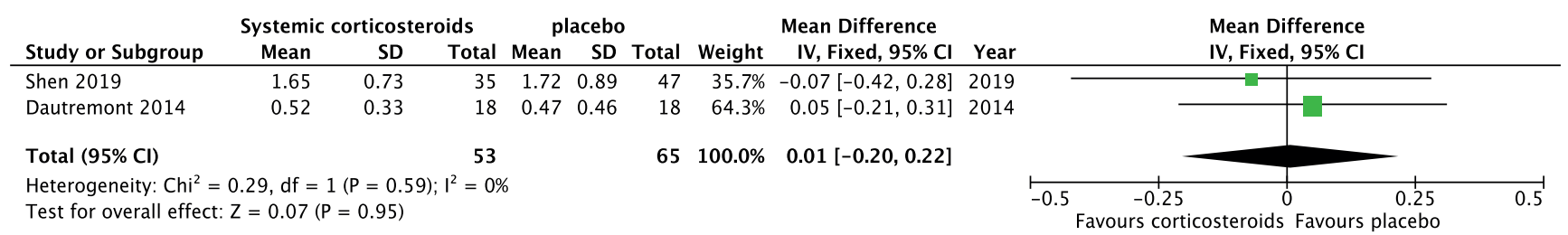

Table 6.1.7.1. Short courses of systemic corticosteroids for the treatment of patients with CRSwNP.

\begin{tabular}{|c|c|c|c|c|c|}
\hline Author & Methods & Participants & Interventions & Outcomes & Results \\
\hline $\begin{array}{l}\text { Shen } \\
2019^{(123)}\end{array}$ & $\begin{array}{l}\text { DBPCT, } \\
\text { postoperative }\end{array}$ & $\begin{array}{l}100 \text { patients with } \\
\text { bilateral CRSwNP } \\
\text { postoperatively } \\
\text { ( } 82 \text { included) } \\
\text { stratified } \\
\text { by tissue } \\
\text { eosinophils } \\
\text { (> } 10 \text { tissue } \\
\text { eosinophils/HPF) }\end{array}$ & $\begin{array}{l}\text { - Oral prednisolone } \\
15 \mathrm{mg} \text { twice a day for } 2 \\
\text { weeks combined with a } \\
\text { nasal spray of } 100 \mu \mathrm{g} \text { of } \\
\text { mometasone furoate once } \\
\text { daily ( } \mathrm{n}=35 \text { ) } \\
\text { - Placebo twice a day for } \\
2 \text { weeks combined with } \\
\text { a nasal spray of } 100 \mu \mathrm{g} \text { of } \\
\text { mometasone furoate once } \\
\text { daily }(\mathrm{n}=47)\end{array}$ & $\begin{array}{l}\text { - Symptoms: head fullness, smell, } \\
\text { cough, nasal obstruction, head- } \\
\text { ache, foul odour, facial pressure, } \\
\text { post nasal drip, and rhinorrhoea } \\
\text { (VAS) } \\
\text { - SNOT-22 at 1, } 3 \text { and } 6 \text { months } \\
\text { postoperatively } \\
\text { - LKESs } 2 \text { weeks and 2,3 and } 6 \\
\text { months postoperatively. } \\
\text { - Safety and side effects assess- } \\
\text { ment }\end{array}$ & $\begin{array}{l}\text { - No differences in symptoms and } \\
\text { SNOT-22 at } 1,3 \text { and } 6 \text { months } \\
\text { - LKES: trend }(0,05) \text { at } 6 \text { months } \\
\text { for whole group, significantly } \\
\text { lower in prednisolone group for } \\
\text { the high eosinophils group at } 3 \\
\text { months and trend at } 6 \text { months } \\
\text { (no correction for multi-testing) }\end{array}$ \\
\hline $\begin{array}{l}\text { Ecevit } \\
2015^{(124)}\end{array}$ & DBPCT & $\begin{array}{l}23 \text { patients with } \\
\text { bilateral CRSwNP }\end{array}$ & $\begin{array}{l}\text { - Prednisolone } 60 \text { mg ( } 60 \\
\text { tablets) for } 7 \text { days, then } \\
\text { reduced to } 10 \mathrm{mg} \text { taken } \\
\text { every other day, stopping } \\
\text { on day } 17 \text { ( } \mathrm{n}=11) \\
\text { - Placebo ( } 6 \text { tablets) for } 7 \\
\text { days, then reduced to } 1 \\
\text { tablet every other day, } \\
\text { stopping on day } 17(\mathrm{n}=12)\end{array}$ & $\begin{array}{l}\text { - Symptoms (VAS) at day 15-17 } \\
\text { - Sense of smell (CCCRC) (butanol } \\
\text { olfactory threshold test) day } \\
\text { 15-17 } \\
\text { - Nasal polys score (0-6) } \\
\text { PNIF day 15-17 } \\
\text { - Adverse events }\end{array}$ & $\begin{array}{l}\text { - Significantly more improvement } \\
\text { in the corticosteroid group } \\
\text { compared to placebo in disease } \\
\text { severity, smell, and PNIF } \\
\text { - No differences in adverse events }\end{array}$ \\
\hline $\begin{array}{l}\text { Dautremont } \\
2014^{(125)}\end{array}$ & DBPCT & $\begin{array}{l}36 \text { CRSwNP } \\
\text { patients } \\
\text { postoperatively }\end{array}$ & $\begin{array}{l}\text { - Prednisone } 30 \text { mg daily for } \\
7 \text { days and } 1 \text { dissolvable } \\
\text { spacer soaked with } 2 \mathrm{ml} \\
\text { triamcinolone ( } 40 \mathrm{mg} / \\
\mathrm{ml} \text { ) and high-volume } \\
\text { budesonide irrigation } \\
\text { once daily ( } 1 \mathrm{mg} \text { in } 240 \mathrm{ml} \\
\text { of saline ( } \mathrm{n}=18 \text { ) } \\
\text { - Placebo daily for } 7 \text { days } \\
\text { and } 1 \text { dissolvable spacer } \\
\text { soaked with } 2 \mathrm{ml} \text { triam- } \\
\text { cinolone ( } 40 \mathrm{mg} / \mathrm{ml} \text { ) and } \\
\text { high-volume budesonide } \\
\text { irrigation once daily ( } 1 \mathrm{mg} \\
\text { in } 240 \mathrm{ml} \text { of saline }(\mathrm{n}=18 \text { ) }\end{array}$ & $\begin{array}{l}\text { - Endoscopy (LK score) at } 1 \text { week, } \\
3 \text { weeks, and } 2 \text { months postop- } \\
\text { eratively. } \\
\text { - SNOT-22 at } 1 \text { week, } 3 \text { weeks, and } \\
2 \text { months postoperatively. }\end{array}$ & $\begin{array}{l}\text { - No significant differences in } \\
\text { mean endoscopic scores or } \\
\text { SNOT-22 at any of the timepoints. }\end{array}$ \\
\hline $\begin{array}{l}\text { Kirtsreesakul } \\
2012^{(126)}\end{array}$ & $\begin{array}{l}\text { DBPCT 3:2 } \\
\text { randomization }\end{array}$ & $\begin{array}{l}117 \text { CRSwNP } \\
\text { (114 included) }\end{array}$ & $\begin{array}{l}\text { - Oral prednisolone } 50 \text { mg } \\
\text { daily for } 14 \text { days followed } \\
\text { by mometasone furoate } \\
\text { nasal spray (MFNS) at } 200 \\
\text { micrograms twice daily for } \\
10 \text { weeks ( } n=67 \text { ) } \\
\text { - Placebo for } 14 \text { days } \\
\text { followed by MFNS at } 200 \\
\text { micrograms twice daily for } \\
10 \text { weeks ( } n=47 \text { ) }\end{array}$ & $\begin{array}{l}\text { - Disease severity (added symp- } \\
\text { tom score (0-7) for blocked } \\
\text { nose, runny nose, sneezing, na- } \\
\text { sal itching, hyposmia, postnasal } \\
\text { drip, cough and sinonasal pain } \\
\text { at } 2,7 \text { and } 12 \text { weeks } \\
\text { - Nasal polyp score (0-6) } \\
\text { - PEFR } \\
\text { - Adverse events }\end{array}$ & $\begin{array}{l}\text { The prednisolone treated group } \\
\text { showed compared to placebo: } \\
\text { - Significantly greater improve- } \\
\text { ments in all nasal symptoms, } \\
\text { nasal airflow, and polyp size at } \\
2 \text { weeks } \\
\text { - Significantly greater improve- } \\
\text { ments in most symptoms, nasal } \\
\text { airflow, and polyp size at } 7 \text { weeks } \\
\text { - Significantly greater improve- } \\
\text { ments in smell, nasal airflow, and } \\
\text { polyp size at } 12 \text { weeks }\end{array}$ \\
\hline
\end{tabular}


Table 6.1.7.1. Short courses of systemic corticosteroids for the treatment of patients with CRSwNP (continued).

\begin{tabular}{|c|c|c|c|c|c|}
\hline Author & Methods & Participants & Interventions & Outcomes & Results \\
\hline $\begin{array}{l}\text { Vaidyanathan } \\
2011^{(127)}\end{array}$ & $\mathrm{DBPCT}$ & $\begin{array}{l}60 \text { CRSwNP (51 } \\
\text { included) }\end{array}$ & 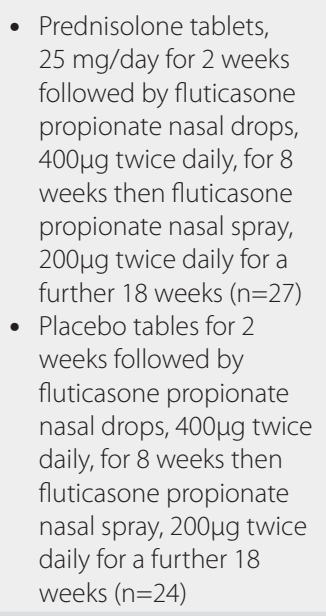 & $\begin{array}{l}\text { - RQLQ at } 2 \text { weeks and } 6 \text { months } \\
\text { - Total nasal symptoms score at } 2 \\
\text { weeks and } 6 \text { months } \\
\text { - Nasal polyps score (0-6) } \\
\text { - PNIF } \\
\text { - Responders at } 28 \text { weeks (im- } \\
\text { - } \text { provement more that MID) } \\
\text { nerum eosinophil-derived } \\
\text { - High-sensitivity C-reactive } \\
\text { - } \text { protein levels } \\
\text { - } \text { and corright urinary free cortisol for creatinine } \\
\text { - } 08: 00 \text { am serum cortisol } \\
\text { tropic hormone-simulation test } \\
\text { - Markers of bone turnover }\end{array}$ & $\begin{array}{l}\text { Oral corticosteroid therapy } \\
\text { followed by topical steroid therapy } \\
\text { compared to nasal corticosteroid } \\
\text { alone resulted in a significant larger } \\
\text { reduction in: } \\
\text { - \% responders at } 28 \text { wks } \\
\text { - RQLQ at } 2 \text { wks } \\
\text { - Total nasal symptom score at } \\
2 \text { wks } \\
\text { - Nasal polyp score at } 2 \text { and } 10 \\
\text { wks } \\
\text { - PNIF at } 2 \text { wks }\end{array}$ \\
\hline $\begin{array}{l}\text { Van Zele } \\
2010^{(4)}\end{array}$ & $\mathrm{DBPCT}$ & 33 CRSwNP & $\begin{array}{l}\text { - Oral methylprednisolone } \\
(32 \mathrm{mg} / \text { day on days } 1 \text { to } \\
5 ; 16 \mathrm{mg} / \text { day on days } 6 \text { to } \\
10 ; 8 \mathrm{mg} / \text { day on days } 11 \\
\text { to } 20)(\mathrm{n}=14) \\
\text { - Placebo, } 20 \text { days }(\mathrm{n}=19)\end{array}$ & $\begin{array}{l}\text { - Disease severity, measured by } \\
\text { patient-assessed symptoms } \\
\text { (anterior rhinorrhoea, nasal } \\
\text { obstruction, post-nasal drip } \\
\text { and loss of sense of smell) at } 20 \\
\text { days and } 12 \text { weeks. } \\
\text { - Nasal polyp score (0-8) } \\
\text { - PNIF } \\
\text { - Blood eosinophils } \\
\text { - Serum ECP, soluble IL-5ra } \\
\text { - ECP, IL-5, IgE, MMP9 and MPO in } \\
\text { nasal secretion } \\
\text { - Need for rescue surgery/ nasal } \\
\text { - steroids } \\
\text { - Adverse events }\end{array}$ & $\begin{array}{l}\text { Oral corticosteroids compared to } \\
\text { placebo for } 20 \text { days resulted in: } \\
\text { - Reduced symptoms for } 4 \text { weeks } \\
\text { - Reduced polyp score and PNIF } \\
\text { for } 55 \text { days }\end{array}$ \\
\hline $\begin{array}{l}\text { Hissaria } \\
2006^{(128)}\end{array}$ & DBPCT & 40 CRSwNP & $\begin{array}{l}\text { - 50mg of prednisolone } \\
\text { once daily for } 14 \text { days } \\
(n=20) \\
\text { - Placebo once daily for } 14 \\
\text { days }(n=20)\end{array}$ & $\begin{array}{l}\text { - Nasal symptoms } \\
\text { - RSOM-31, and nasal domain of } \\
\text { RSOM-31 at } 2 \text { weeks } \\
\text { - Polyp score } \\
\text { - } \text { MRI } \\
\text { - Responders at } 2 \text { weeks }\end{array}$ & $\begin{array}{l}\text { Significantly better results of } \\
\text { systemic corticosteroid compared } \\
\text { to placebo for: } \\
\text { - Symptoms of stuffy/blocked } \\
\text { nose, runny nose, sneezing, } \\
\text { decreased sensation of smell, } \\
\text { and postnasal drip showed } \\
\text { significant improvement after } \\
\text { treatment with corticosteroids } \\
\text { (p<.05) as opposed to placebo } \\
\text { - RSOM-31 and nasal domain of } \\
\text { RSOM-31 }\end{array}$ \\
\hline
\end{tabular}

CCCRC, The Conneticut Chemosensory Clinical Research Centre ; CRSwNP, chronic rhinosinusitis with nasal polyps; DBPCT, double blind placebo controlled trial; ECP, eosinophil cationic protein; IgE, immunoglobulin E; IL-5, interleukin-5; LK, Lund-Kennedy; LKES, Lund-Kennedy Endoscopic Scores; MFNS, Mometasone furoate nasal spray; MID, Minimal important difference ; MMP9, matrix metalloproteinase 9; MPO, Myeloperoxidase ; MRI, magnetic resonance imaging; PEFR, Peak Expiratory Flow Rate ; PNIF, peak nasal inspiratory flow; RSOM-31, Rhinosinusitis Outcome Measure-31 ; RQLQ, Rhinoconjunctivitis Quality of Life Questionnaire ; SNOT, Sino-nasal Outcome Test; VAS, visual analogue scale.

important. In the lower airways, evidence is not strong enough to reveal whether shorter or lower-dose regimens are generally less effective than longer or higher-dose regimens in asthma or indeed that the latter are associated with more adverse events ${ }^{(130)}$ although in chronic obstructive pulmonary disease (COPD) there is data that shorter courses are equally effective ${ }^{(131)}$. When given over the longer term, or through many repeated short courses, it is possible to develop osteoporosis ${ }^{(132)}$.

\subsubsection{Conclusion}

A short course of systemic corticosteroid, with or without local corticosteroid treatment results in a significant reduction in total symptom score and nasal polyp score. Although the effect on the nasal polyp score remains significant up to three months after the start of treatment by that time there is no longer an effect on the symptom score. The EPOS2020 steering group felt that 1-2 courses of systemic corticosteroids per year can be a useful addition to nasal corticosteroid treatment in patients with partially or uncontrolled disease. A short course of systemic corticosteroid postoperatively does not seem to have an effect on quality of life. 
Table 6.1.7.2. Corticosteroid dose equivalents ${ }^{(869-872)}$

\begin{tabular}{|cc}
\hline Equivalent Dose & Steroid \\
$1.2 \mathrm{mg}$ & Betamethasone (long-acting) \\
$1.5 \mathrm{mg}$ & Dexamethasone (long-acting) \\
$8 \mathrm{mg}$ & Methylprednisolone (intermediate-acting) \\
$8 \mathrm{mg}$ & Triamcinolone (intermediate-acting) \\
$10 \mathrm{mg}$ & Prednisone (intermediate-acting) \\
$10 \mathrm{mg}$ & Prednisolone (intermediate-acting) ${ }^{[4,5]}$ \\
$40 \mathrm{mg}$ & Hydrocortisone (short-acting) \\
$50 \mathrm{mg}$ & Cortisone (short-acting) \\
\end{tabular}

\subsubsection{Antihistamines (oral and topical)}

\subsubsection{Summary of the evidence}

The role of sensitisation in CRSsNP is unclear (see 5.1.2). It is tempting to speculate that allergic inflammation in the nose predisposes the atopic individual to the development of CRS. However, papers evaluating atopy as a risk factor to CRS are conflicting.

Notwithstanding the lack of hard epidemiologic evidence for a clear causal relationship between allergy and CRS, there is some indication that failure to address allergy as a contributing factor to CRS diminishes the probability of success of a surgical intervention $^{(133)}$. In a real life retrospective study among allergy patients undergoing immunotherapy, those who felt most helped by immunotherapy were the subjects with a history of recurrent rhinosinusitis, and about half of the patients who had had sinus surgery before believed that the surgery alone was not sufficient to completely resolve the recurrent episodes of infection ${ }^{(133)}$.

In a large German retrospective database, both ENT specialists (3.5\%) and GPs (2.2\%) prescribed low rates of antihistamines in patients with CRSsNP and slightly higher rates in CRSwNP $(5.4 \%$ and $3.1 \%$, respectively) ${ }^{(134)}$.

There are no studies evaluating the effect of antihistamines in patients with CRSsNP(135).

There is one study evaluating the effect of antihistamines in patients with CRSwNP(135, 136)

Haye et al. in a DBPCT randomised 45 patients with CRSwNP (of which 16 were allergic) to receive either $20 \mathrm{mg}$ of cetirizine or placebo for three months (Table 6.1.8.1). The authors reported that cetirizine reduced days with a score for nasal sneezing and rhinorrhoea lower than one from $70-80 \%$ (rhinorrhoea) and 80 -
$90 \%$ (sneezing) in the placebo group to $90-100 \%$ in the cetirizine treated group ${ }^{(136)}$ at all time points). However, data for patients with allergies and those without were not reported separately. There was no effect of the cetirizine on nasal polyp size ${ }^{(136)}$.

\subsubsection{Conclusion}

The quality of the evidence using GRADE comparing antihistamines with placebo was very low. Evidence was downgraded because of the small number of studies and because the most important measures of efficacy were not reported. There is insufficient evidence to decide on the effect of the regular use of antihistamines in the treatment of patients with CRS.

\subsubsection{Anti-leukotrienes}

\subsubsection{Summary of the evidence}

Cysteinyl leukotrienes (CysLT) are a class of inflammatory mediators synthesized by eosinophils and mast cells through the breakdown of arachidonic acid. CysLT are known to play a role in the pathophysiology of rhinitis, asthma and possibly also CRSwNP, promoting bronchoconstriction, mucus production, oedema and chemotaxis of eosinophils and neutrophils. Overproduction of CysLTs and upregulation of the receptor has been demonstrated in allergic rhinitis, asthma, and CRS with nasal polyposis (CRSwNP) (5.1.2).

There are no data on the efficacy of CysLT in CRSsNP

There are limited data on the efficacy of CysLT in CRSwNP, mainly on montelukast. There are no RCTs performed in patients with CRSwNP with other CysLT inhibitors such as zafirlukast, zileuton (-lipoxygenase inhibitor) and pranlukast.

Wentzel et al..(137) performed a systematic review in 2013

Table 6.1.8.1. Antihistamines for the treatment of patients with CRS.

\begin{tabular}{|c|c|c|c|c|c|}
\hline Study & Methods & Participants & Interventions & Outcomes & Results \\
\hline Haye $1998^{(136)}$ & DBPCT & $\begin{array}{l}45 \text { patients } \\
\text { with CRSwNP }\end{array}$ & $\begin{array}{l}\text { - } 20 \text { mg of cetirizine } \\
\text { for } 3 \text { months }(n=23) \\
\text { - } \text { Placebo for } 3 \\
\text { months }(n=22)\end{array}$ & $\begin{array}{l}\text { - Total symptom score at 4, } 8 \text { and } 12 \\
\text { weeks } \\
\text { - Days with a symptom score } \leq 1 \text { for na- } \\
\text { sal obstruction, rhinorrhoea, sneezing } \\
\text { at } 4,8 \text { and } 12 \text { weeks } \\
\text { - Polyp score at } 4,8 \text { and } 12 \text { weeks }\end{array}$ & $\begin{array}{l}\text { - } \text { - Cetirizine significantly reduced days } \\
\text { with a score for nasal sneezing and } \\
\text { rhinorrhea } \leq 1 \text { at all time points and } \\
\text { nasal obstruction at week } 8 \\
\text { - No change in polyp score }\end{array}$ \\
\hline
\end{tabular}

CRSwNP, chronic rhinosinusitis with nasal polyps; DBPCT, double blind placebo controlled trial. 
describing two placebo-controlled RCTs of which only one was double blind ${ }^{(138,139)}$ and three non-placebo controlled $\mathrm{RTs}^{(140-142)}$ which included a total of 179 CRSwNP patients. The study by Mostafa indicates that it was double blind but no explanation of how the blinding was done is given, the other two studies were not blinded. The non-placebo controlled RCTs compared montelukast to intranasal corticosteroids ${ }^{(140,}$ ${ }^{142)}$ postoperatively and the combination of montelukast and nasal corticosteroids versus nasal corticosteroids alone after a course of oral steroids ${ }^{(141)}$. They concluded that both placebocontrolled RTs showed significant improvements in symptoms and local inflammatory mediators over the four- to six-week course of treatment compared to placebo. It was not possible to combine the two studies in a meta-analysis. The two small studies comparing montelukast to nasal corticosteroids showed similar improvement compared to baseline for both treatments (Table 6.1.9.1.).

Two studies have been performed after the publication of the Wentzel review ${ }^{(137)}$ both of which were prospective RCTs, adding oral montelukast to regular treatment. Suri et al. ${ }^{(143)}$ randomized 40 consecutive adult CRSwNP patients into two groups. Subjects were treated with oral prednisolone for 14 days and budesonide nasal spray for eight weeks with or without additional oral montelukast. Subjects treated with additional oral montelukast reported a statistically significant improvement in overall symptom score, sense of smell, and sneezing after eight weeks which remained for the four weeks after stopping the treatment (Table 6.1.9.2.).

Van Gerven et al. ${ }^{(144)}$ evaluated the efficacy of montelukast as an add-on treatment to intranasal corticosteroids spray (INCS) in 72 postoperative CRSwNP patients for one year. They found no significant differences between the two treatment arms in total symptom score, nasal polyp score and LMK score. They concluded that the addition of montelukast to INCS should not be recommended in the treatment of postoperative CRSWNP patients.

\section{The EPOS2020 steering group does not advise adding montelukast to nasal corticosteroid but studies evaluating the effect of montelukast in patients that failed nasal corticosteroids are missing.}

We performed a meta-analysis of the three studies evaluating the addition of montelukast to INCS either postoperatively or after systemic corticosteroid treatment for eight weeks. There was no significant additional effect from adding montelukast to intranasal corticosteroids after either surgery ${ }^{(144)}$ or systemic corticosteroids for two weeks ${ }^{(141,143)}$ (Figure 6.1.9.1.).

Various neuropsychiatric events have been reported as adverse events of anti-leukotrienes; however, the evidence of the association is conflicting ${ }^{(145-148)}$.

\subsubsection{Conclusion}

The quality of the evidence using GRADE comparing montelukast with placebo was very low. Evidence was downgraded because of the small number of studies and patients in the studies and because only one study was double blinded. Based on the available evidence, the EPOS2020 steering group is unsure about the potential use of montelukast in CRS and does not recommend its use unless in situations where patients do not tolerate nasal corticosteroids. Also, the quality of the evidence comparing montelukast with nasal corticosteroid is low. Based on the evidence, the steering group does not advise adding montelukast to nasal corticosteroid but studies evaluating the effect of montelukast in patients that failed nasal corticosteroids are missing.

\subsubsection{Decongestants}

\subsubsection{Summary of the evidence}

The use of topical decongestants has the theoretical potential to reduce mucosal oedema by inducing vasoconstriction ${ }^{(149)}$. However, prolonged use can lead to rhinitis medicamentosa and worse symptoms of nasal obstruction upon decongestant discontinuation ${ }^{(150)}$.

Nasal decongestion per se does not have effect on nasal polyp size ${ }^{(151)}$.

Two RCTs have been performed evaluating the effect of local decongestants in the treatment of CRS (Table 6.1.10.1). Kirtsreesakul et al. evaluated in a DBPCT the additive effect of oxymetazoline administration on nasal steroid therapy and whether rebound congestion develops after oxymetazoline treatment in 68 CRSwNP patients ${ }^{(152)}$. Patients were randomly assigned in a 1:1 ratio to receive either oxymetazoline plus mometasone furoate nasal spray (MFNS) or placebo plus MFNS, two sprays per nostril twice daily, with an interval of five minutes between each medication for four weeks. All the

Figure 6.1.9.1. Forest plot of the effect of the added effect of montelukast on intranasal corticosteroids at 12 weeks in CRSwNP patients

\begin{tabular}{|c|c|c|c|c|c|c|c|c|c|c|c|}
\hline \multirow[b]{2}{*}{ Study or Subgroup } & \multicolumn{3}{|c|}{ INCS and Montelukast } & \multicolumn{3}{|c|}{ INCS } & \multicolumn{2}{|r|}{ Std. Mean Difference } & \multirow{2}{*}{\multicolumn{2}{|c|}{$\begin{array}{l}\text { Std. Mean Difference } \\
\text { IV, Random, } 95 \% \mathrm{CI}\end{array}$}} & \\
\hline & Mean & SD & Total & Mean & SD & Total & Weight & IV, Random, $95 \% \mathrm{CI}$ & & & \\
\hline van Gerven 2018 & 12.3 & 3.7 & 36 & 12 & 3.7 & 36 & $36.4 \%$ & $0.08[-0.38,0.54]$ & & -1 & \\
\hline Suri 2015 & 4.1 & 0.93 & 20 & 5.2 & 0.93 & 20 & $31.7 \%$ & $-1.16[-1.83,-0.48]$ & 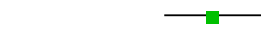 & & \\
\hline Stewart 2008 & 5.2 & 2.8 & 19 & 4.9 & 3 & 16 & $31.9 \%$ & $0.10[-0.56,0.77]$ & & & \\
\hline Total $(95 \% \mathrm{Cl})$ & & & 75 & & & 72 & $100.0 \%$ & $-0.31[-1.07,0.46]$ & & & \\
\hline $\begin{array}{l}\text { Heterogeneity: } \mathrm{Tau}^{2} \\
\text { Test for overall effec }\end{array}$ & $\begin{array}{l}0.37 ; \mathrm{Chi}^{2} \\
\mathrm{Z}=0.78\end{array}$ & $\begin{array}{l}9.95, \mathrm{c} \\
=0.44)\end{array}$ & $=2(\mathrm{P}$ & $=0.00$ & 7); $1^{2}=$ & $=80 \%$ & & & $\begin{array}{cc}-4 & 1 \\
& -2 \\
& \text { Favours monteluk }\end{array}$ & $\begin{array}{l}0 \\
\text { st Favours pla }\end{array}$ & $\begin{array}{l}1 \\
2 \\
\text { acebo }\end{array}$ \\
\hline
\end{tabular}


Table 6.1.9.1. Montelukast for the treatment of patients with CRS.

\begin{tabular}{|c|c|c|c|c|c|}
\hline Study & Methods & Participants & Interventions & Outcomes & Results \\
\hline $\begin{array}{l}\text { Schaper, } \\
2011^{(139)}\end{array}$ & $\begin{array}{l}\text { SBPCT } \\
\text { crossover }\end{array}$ & $\begin{array}{l}24 \text { CRSWNP } \\
\text { patients and } \\
\text { asthma (12 } \\
\text { with N-ERD) }\end{array}$ & $\begin{array}{l}\text { - Montelukast } 10 \\
\text { mg } 1 d d \text { for } 6 \\
\text { weeks }(n=24) \\
\text { - Placebo 1dd for } 4 \\
\text { weeks }(n=24)\end{array}$ & $\begin{array}{l}\text { - Nasal symptoms (0-12) } \\
\text { - Nasal endoscopy } \\
\text { - Anterior rhinomanometry } \\
\text { - } \text { Olfactometry } \\
\text { - } \text { Mediators in nasal secretion } \\
\text { - Lung function }\end{array}$ & $\begin{array}{l}\text { - Total symptom score improved from } 5.9 \text { to } 1.75 \text { in } \\
\text { montelukast group and not in placebo. No direct } \\
\text { comparison. No data for placebo } \\
\text { - Significant reduction in oedema, hypersecretion, } \\
\text { blockage compared to placebo at nasal endoscopy } \\
\text { - Significant improvement in nasal airflow } \\
\text { - Significant reduction in inflammatory mediators } \\
\text { and eosinophils in nasal secretion } \\
\text { - Significant improvement in lung function }\end{array}$ \\
\hline Pauli, 2007(138) & DBPCT & $\begin{array}{l}30 \text { CRSwNP } \\
\text { patients }\end{array}$ & $\begin{array}{l}\text { - Montelukast } 10 \\
\text { mg } 1 \text { dd for } 4 \\
\text { weeks }(n=20) \\
\text { - Placebo } 1 \text { dd for } 4 \\
\text { weeks }(n=10)\end{array}$ & $\begin{array}{l}\text { - } \mathrm{HRQL} \text { (health related quality } \\
\text { of life questionnaire) at } 4 \\
\text { wks } \\
\text { - Nasal endoscopy at } 4 \text { wks } \\
\text { - } \text { ECP in nasal secretion at } \\
4 \text { wks }\end{array}$ & $\begin{array}{l}\text { - Significant reduction in most domains of HRQL } \\
\text { - No significant difference in nasal endoscopy score } \\
\text { or ECP in nasal secretion }\end{array}$ \\
\hline
\end{tabular}

CRSwNP, chronic rhinosinusitis with nasal polyps; DBPCT, double-blind placebo-controlled trial; ECP, eosinophil cationic protein; $\mathrm{HRQOL}$, health related quality of life; N-ERD, NSAID-exacerbated respiratory disease; SBPCT, single-blind placebo-controlled trial.

Table 6.1.9.2. Montelukast added to intranasal corticosteroids for the treatment of patients with CRS.

\begin{tabular}{|c|c|c|c|c|c|}
\hline Study & Methods & Participants & Interventions & Outcomes & Results \\
\hline $\begin{array}{l}\text { Van Gerven } \\
2018^{(144)}\end{array}$ & RCT & $\begin{array}{l}72 \text { CRSwNP } \\
\text { postoperative }\end{array}$ & $\begin{array}{l}\text { - CRSwNP montelukast } 10 \mathrm{mg} 1 \mathrm{dd} \text { together with } \\
\text { mometasone furoate } 200 \mu \mathrm{g} 2 \text { times a day for } \\
1 \text { year ( } n=36 \text { ) } \\
\text { - CRSwNP mometasone furoate } 200 \mu \mathrm{gg} 2 \text { times a } \\
\text { day for } 1 \text { year }(n=36)\end{array}$ & $\begin{array}{l}\text { - T5SS (T5SS) at 3,6 } \\
\text { and } 12 \text { months } \\
\text { - Nasal polyp score } \\
\text { (NPS) at 3,6 and } \\
12 \text { months } \\
\text { - LMS at 3,6 and } 12 \\
\text { months } \\
\text { - BAST } 24 \text { at 3,6 and } \\
12 \text { months }\end{array}$ & $\begin{array}{l}\text { - No significant difference } \\
\text { between the treatments for any } \\
\text { outcome measured }\end{array}$ \\
\hline $\begin{array}{l}\text { Suri } \\
2015^{(143)}\end{array}$ & RCT & 40 CRSWNP & $\begin{array}{l}\text { - Montelukast } 10 \mathrm{mg} \text { for } 8 \text { weeks+ prednisolone } \\
35 \mathrm{mg} \text { reducing by } 5 \mathrm{mg} \text { every second day } \\
\text { over } 14 \text { days+ budesonide nasal spray } 2 \text { me- } \\
\text { tered doses to each nostril for } 8 \text { weeks ( } n=20 \text { ) } \\
\text { - Prednisolone } 35 \mathrm{mg} \text { reducing by } 5 \mathrm{mg} \text { every } \\
\text { second day over } 14 \text { days+ budesonide nasal } \\
\text { spray } 2 \text { metered doses to each nostril for } 8 \\
\text { weeks }(n=20)\end{array}$ & $\begin{array}{l}\text { Total symptoms } \\
\text { and nasal blockage, } \\
\text { headache, facial } \\
\text { pain, sense of smell, } \\
\text { nasal discharge and } \\
\text { sneezing }(0-10) \text { at } 8 \\
\text { and } 12 \text { weeks }\end{array}$ & $\begin{array}{l}\text { - Significant better effect of } \\
\text { montelukast group for total } \\
\text { symptoms ( } 8 \text { and } 12 \text { wks), } \\
\text { headache ( } 12 \text { wks), sense } \\
\text { of smell ( } 8 \text { and } 12 \text { wks) and } \\
\text { sneezing ( } 8 \text { wks) }\end{array}$ \\
\hline $\begin{array}{l}\text { Stewart } \\
2008^{(141)}\end{array}$ & RCT & $\begin{array}{l}38 \text { CRSwNP } \\
\text { (35 analysed) }\end{array}$ & $\begin{array}{l}\text { - Montelukast } 10 \mathrm{mg} \text { for } 8 \text { weeks+ prednisolone } \\
35 \mathrm{mg} \text { reducing by } 5 \mathrm{mg} \text { every second day over } \\
14 \text { days+ budesonide nasal spray } 2 \text { metered } \\
\text { doses to each nostril for } 8 \text { weeks ( } \mathrm{n}=20 \text { ) } \\
\text { - Prednisolone } 35 \mathrm{mg} \text { reducing by } 5 \mathrm{mg} \text { every } \\
\text { second day over } 14 \text { days+ budesonide nasal } \\
\text { spray } 2 \text { metered doses to each nostril for } 8 \\
\text { weeks }(n=20)\end{array}$ & $\begin{array}{l}\text { Total symptoms } \\
\text { and nasal block- } \\
\text { age, headache, } \\
\text { facial pain, sense } \\
\text { of smell, nasal dis- } \\
\text { charge, sneezing } \\
\text { at } 8 \text { and } 12 \text { weeks } \\
\text { - SF36 }\end{array}$ & $\begin{array}{l}\text { - Significant better effect of } \\
\text { montelukast group for facial } \\
\text { pain ( } 8 \text { wks) headache ( } 8 \text { wks), } \\
\text { sneezing ( } 8 \text { wks) } \\
\text { - No significant difference } \\
\text { between the treatments for any } \\
\text { outcome measured at } 12 \text { wks } \\
\text { - No significant difference in SF36 }\end{array}$ \\
\hline
\end{tabular}

BAST-24, Barcelona Smell Test 24 odours; CRSwNP, chronic rhinosinusitis with nasal polyps; LMS, Lund-McKay score; RCT, randomized clinical trial; SF36, short form 36; T5SS; Total 5 Symptom Score.

patients were then treated with MFNS, two sprays per nostril twice daily for two weeks. At four weeks after beginning the treatment, the oxymetazoline-MFNS group showed significantly greater improvement in blocked nose, hyposmia, peak nasal inspiratory flow (PNIF), nasal mucociliary clearance time (NMCCT) and total nasal polyps score (NPS) than the placeboMFNS group. During the two weeks in which all patients were only treated with MFNS both groups showed continuing improvement in all outcome variables but the oxymetazolineMFNS group still showed significantly greater improvement in blocked nose, hyposmia, NMCCT and total NPS, but not PNIF, than the placebo-MFNS group at the end of the study. There was no evidence of rebound congestion after four weeks of oxymetazoline treatment. This lack of rebound congestion when nasal decongestants are combined with nasal corticosteroids has also been shown in two studies in allergic rhinitis where 2-4 
Table 6.1.10.1. Nasal decongestants for the treatment of patients with CRS.

\begin{tabular}{|c|c|c|c|c|c|}
\hline Study & Methods & Participants & Interventions & Outcomes & Results \\
\hline $\begin{array}{l}\text { Kirtsreesakul, } \\
2016^{(152)}\end{array}$ & DBPCRCT & $\begin{array}{l}68 \text { CRSwNP } \\
\text { patients }\end{array}$ & $\begin{array}{l}\text { - Oxymetazoline plus MFNS } 2 \text { sprays per } \\
\text { nostril twice daily, with an interval of } 5 \\
\text { minutes between each medication for } \\
4 \text { weeks followed by } 2 \text { weeks of MFNS } \\
\text { only ( } n=34 \text { ) } \\
\text { - Placebo plus MFNS, } 2 \text { sprays per nostril } \\
\text { twice daily, with an interval of } 5 \text { minutes } \\
\text { between each medication for } 4 \text { weeks } \\
\text { followed by } 2 \text { weeks of MFNS only } \\
(n=34)\end{array}$ & $\begin{array}{l}\text { - Symptoms, } \\
\text { - Nasal patency } \\
\text { - Nasal polyp size } \\
\text { - NMCCT was evaluated at } \\
4 \text { and } 6 \text { weeks } \\
\text { - Adverse events }\end{array}$ & $\begin{array}{l}\text { - Combination of oxymetazoline } \\
\text { plus MFNS significantly more } \\
\text { - } \text { effective than MFNS alone on: } \\
\text { - Symptoms of nasal blockage } \\
\text { - } \quad \text { nas smell at } 4 \text { and } 6 \text { wks } \\
\text { - Nasal polyp size at } 4 \text { and } 6 \text { wks } \\
\text { - NMCCT at } 4 \text { and } 6 \text { wks } \\
\text { - No signs of rebound swelling }\end{array}$ \\
\hline $\begin{array}{l}\text { Humphreys, } \\
2009^{(155)}\end{array}$ & DBPCT & $\begin{array}{l}47 \text { CRS } \\
\text { patients } \\
\text { postoperative }\end{array}$ & $\begin{array}{l}\text { - Xylometazoline hydrochloride } 0.1 \% \\
\text { adult nasal spray }(n=24) \\
\text { - A physiological sterile saline aerosol } \\
(n=23)\end{array}$ & $\begin{array}{l}\text { - Symptom scores at day } \\
10 \text { postoperatively }\end{array}$ & $\begin{array}{l}\text { - No differences between the } \\
\text { groups }\end{array}$ \\
\hline
\end{tabular}

DBPCRCT, double-blind placebo-controlled randomized clinical trial; MFNS, mometasone furoate nasal spray; NMCCT, nasal mucociliary clearance time.

weeks' treatment with the combination of nasal decongestant and corticosteroid was more effective than nasal corticosteroid alone and did not show rebound swelling ${ }^{(153,154)}$.

In the second trial Humphreys at al. compared topical decongestant, xylometazoline, to a saline spray in 47 patients in the FESS early postoperative period ${ }^{(155)}$. Postoperative VAS symptom scores for nasal obstruction, rhinorrhoea, pain, loss of sense of smell and bleeding assessed at day 10 postoperatively did not show any difference between the groups.

\subsubsection{Conclusion}

There is one small study in CRSwNP patients showing a significantly better effect of oxymetazoline combined with MFNS than MFNS alone without inducing rebound swelling ${ }^{(152)}$. This is consistent with effects seen in the treatment of allergic rhinitis where the combination of nasal corticosteroid with decongestant also seemed to prevent rebound swelling ${ }^{(153,154)}$. There was no effect of xylometazoline compared to saline in the early postoperative period ${ }^{(155)}$. This review found a low level of certainty that adding a nasal decongestant to intranasal corticosteroids improves symptomatology in CRS. Although the risk of rebound swelling was not shown in this study, the EPOS2020 steering group suggests in general not to use nasal decongestants in CRS. In situations where the nose is very blocked, the temporary addition of a nasal decongestant to the nasal corticosteroid treatment can be considered.

\subsubsection{Saline}

\subsubsection{Summary}

Nasal saline irrigation is considered to be an important aspect of the management of CRS. Saline nasal irrigation may improve nasal mucosa function through several physiological effects including mechanical removal of mucus and crusts, improvement in mucus clearance, enhanced ciliary beat activity, disruption and removal of antigens, biofilms and inflammatory mediators, and increases hydration of the sol layer. Also, saline can be the carrier to supply sufficient volume to transport medication into the sinus. There is however little consensus on the best method of irrigation and the tonicity (concentration) of the saline solution, nor on the volume (low or high), pressure (low or high), and frequency, devices, and head position when rinsing.

This systematic review identified 33 RCT studies (14 in the postoperative phase after FESS) evaluating the impact of nasal saline irrigations/spray on clinical outcomes in adult CRS (Tables 6.1.11.1- 6.1.11.8). The studies were very different in design, duration and outcome measurements. Twenty studies reported a positive effect of some form of saline irrigation ${ }^{(156-175)}$.

Eight reported no effect ${ }^{(158,176-182)}$ and in five studies the effect compared to baseline was not mentioned ${ }^{(183-187)}$.

Six studies evaluated the effect of a form of saline irrigation versus a form of no irrigation of the nose (Table 6.1.11.1). The studies could not be combined in a meta-analysis. Four studies showed no or hardly any (e.g. on one symptom) effect of saline irrigation. The two most recent studies $\left({ }^{(163,165)}\right.$ did show significant positive effect of isotonic saline irrigation versus no irrigation. The differences between the positive and negative studies are not very clear but potential reasons for them could be the length of the treatment/evaluation (the positive studies had a mean duration of 7.5 months (3-12 months), the negative ones of six weeks (1-13 weeks).

In conclusion, studies evaluating a form of saline irrigation versus a form of no irrigation of the nose do not lead to a concurrent conclusion. More studies are necessary to further analyse this important question for patients and professionals.

\subsubsection{What is the best method?}

Two studies evaluated different methods of applying saline. A prospective, cross-over study evaluated the effectiveness of three methods of nasal irrigation (nasal spray, nebulisation with RinoFLow and nasal douching while kneeling with the head on the floor) on distribution of saline to the nasal cavity and paranasal sinuses ${ }^{(188)}$. The authors showed that nasal douches are more effective in distributing irrigation solution to the maxillary sinus and frontal recess, but all techniques were able 
Table 6.1.11.1. Saline nasal irrigation versus no irrigation for the treatment of patients with CRS.

\begin{tabular}{|c|c|c|c|c|c|}
\hline Study & Methods & Participants & Interventions & Outcomes & Results \\
\hline $\begin{array}{l}\text { Giotakis } \\
2016^{(163)}\end{array}$ & RCT not blinded & $\begin{array}{l}174 \text { CRSwNP } \\
\text { patients (158 } \\
\text { evaluated), } \\
\text { postoperative }\end{array}$ & $\begin{array}{l}\text { - } 250 \mathrm{ml} \text { of } 1,175 \% \text { Emser Salt }{ }^{\oplus} \text { solution } \\
\text { (EmsSalt) }(n=59) \text { twice daily for one year } \\
\text { - } 250 \mathrm{ml} \text { of isosmotic mineral salt mixture } \\
\text { (IsoMix) ( } n=58) \text { twice daily for one year } \\
\text { - No irrigation ( } n=57) \text { for one year } \\
\text { All patients were allowed to use } \\
\text { budesonide nasal spray in limited doses } \\
\text { in the first } 6 \text { months and corticoid and } \\
\text { antibiotic nasal ointment in the first } 14 \\
\text { days. }\end{array}$ & $\begin{array}{l}\text { At 3,6,9 and } 12 \\
\text { months: } \\
\text { - Nasal symptoms } \\
\text { - RQLQ } \\
\text { - Missed work days } \\
\text { - Postoperative condi- } \\
\text { tion of the mucosa }\end{array}$ & $\begin{array}{l}\text { - Significantly better results in } \\
\text { nasal symptoms and RQLQ } \\
\text { between irrigation and no } \\
\text { irrigation } \\
\text { - No significant difference } \\
\text { in missed work days and } \\
\text { mucosa } \\
\text { - No significant differences } \\
\text { between Emser Salt and } \\
\text { the isosmotic mineral salt } \\
\text { mixture }\end{array}$ \\
\hline $\begin{array}{l}\text { Nikakhlagh } \\
2016^{(165)}\end{array}$ & RCT not blinded & $\begin{array}{l}185 \mathrm{CRS} \\
\text { patients }\end{array}$ & $\begin{array}{l}\text { After } 3 \text { weeks of antibiotics patients } \\
\text { received: } \\
\text { - Hypertonic lavage (volume and } \\
\text { frequency unclear) for } 3 \text { months } \\
\text { - Isotonic lavage for } 3 \text { months } \\
\text { - Hypertonic lavage for } 3 \text { months } \\
\text { - No lavage for } 3 \text { months }\end{array}$ & $\begin{array}{l}\text { After } 3 \text { months: } \\
\text { - Percentage of } \\
\text { patients with symp- } \\
\text { toms of headache, } \\
\text { discharge from nose } \\
\text { and throat, nasal ob- } \\
\text { struction, coughing, } \\
\text { olfaction, feeling of } \\
\text { fullness in the face }\end{array}$ & $\begin{array}{l}\text { - Isotonic was more effective } \\
\text { than hypertonic, hypotonic } \\
\text { and no lavage }\end{array}$ \\
\hline $\begin{array}{l}\text { Jiang } \\
2014^{(179)}\end{array}$ & $\begin{array}{l}\mathrm{RCT} \text {, no blinding, } \\
\text { postoperative }\end{array}$ & $\begin{array}{l}185 \text { patients } \\
\text { undergoing } \\
\text { FESS ( } 110 \\
\text { evaluated) }\end{array}$ & $\begin{array}{l}\text { EAW for nasal irrigation daily for } 2 \text { months } \\
\text { starting } 1 \text { months after FESS }(n=36) \\
\text { Neutral NS daily for } 2 \text { months starting } 1 \\
\text { months after FESS ( } n=35) \\
\text { No nasal irrigation after surgery }(n=39)\end{array}$ & $\begin{array}{l}\text { Two months after FESS: } \\
\text { - SNOT-20 } \\
\text { - Mean MCA } \\
\text { - Smell threshold } \\
\text { - UPSIT-TC } \\
\text { - Saccharine transit } \\
\text { - } \text { time } \\
\text { - Endoscopic score }\end{array}$ & $\begin{array}{l}\text { - No significant differences } \\
\text { in postoperative SNOT-20 } \\
\text { scores, mean MCA } \text {, }_{2} \text { smell } \\
\text { threshold, UPSIT-TC scores, } \\
\text { saccharine transit time, and } \\
\text { endoscopic scores among } \\
\text { the three groups. }\end{array}$ \\
\hline $\begin{array}{l}\text { Freeman, } \\
2008^{(158)}\end{array}$ & $\begin{array}{l}\text { Within-subject, } \\
\text { single- } \\
\text { blinded RCT } \\
\text { postoperatively }\end{array}$ & 22 CRS patients & $\begin{array}{l}\text { - } 2 \mathrm{ml} \text { isotonic saline via a mucosal atomi- } \\
\text { sation device one side of the nose, three } \\
\text { times per day for } 6 \text { weeks } \\
\text { - No saline other side of the nose }\end{array}$ & $\begin{array}{l}\text { At } 3 \text { weeks and } 3 \\
\text { months: } \\
\text { - Adhesions, } \\
\text { discharge, polyps, } \\
\text { crusting, oedema (0- } \\
\text { 3) at endoscopy }\end{array}$ & $\begin{array}{l}\text { - Significant positive effect of } \\
\text { saline atomisation on dis- } \\
\text { charge, no other differences }\end{array}$ \\
\hline $\begin{array}{l}\text { Liang } \\
2008^{(175)}\end{array}$ & $\begin{array}{l}\mathrm{RCT} \text {, no blinding, } \\
\text { postoperative }\end{array}$ & $\begin{array}{l}77 \text { patients } \\
\text { undergoing } \\
\text { FESS }\end{array}$ & $\begin{array}{l}\text { - Pulsatile nasal irrigation for } 3 \text { months } \\
\text { - No treatment }\end{array}$ & $\begin{array}{l}\text { At } 2 \text { weeks and 1, } \\
2 \text { and } 3 \text { months } \\
\text { postoperatively: } \\
\text { - Symptom score } \\
\text { - Endoscopy score }\end{array}$ & $\begin{array}{l}\text { - No significant effect of } \\
\text { nasal irrigation for the whole } \\
\text { group } \\
\text { - Significant positive effect of } \\
\text { nasal irrigation in patients } \\
\text { with mild CRS but not with } \\
\text { severe CRS (based on CT } \\
\text { scan > LM >12) }\end{array}$ \\
\hline $\begin{array}{l}\text { Pinto } \\
2006^{(178)}\end{array}$ & $\begin{array}{l}\text { Postoperative, } \\
\text { RCT, double } \\
\text { blinded for } \\
\text { sprays }\end{array}$ & $\begin{array}{l}60 \text { patients } \\
\text { with histories } \\
\text { of frequent } \\
\text { sinusitis after } \\
\text { FESS }\end{array}$ & $\begin{array}{l}\text { - NS ( } n=20) \text { four times per day, two puffs } \\
\text { on each side, for the first } 5 \text { postoperative } \\
\text { days. } \\
\text { - Buffered HS }(n=20) 4 \text { times per day, } 2 \\
\text { puffs on each side, for the first } 5 \text { postop- } \\
\text { erative days. } \\
\text { - No spray }(n=20)\end{array}$ & $\begin{array}{l}\text { For } 5 \text { days: } \\
\text { - Symptoms (nasal } \\
\text { obstruction/conges- } \\
\text { tion, nasal discharge/ } \\
\text { postnasal drip, pain/ } \\
\text { pressure, headache, } \\
\text { and trouble sleep- } \\
\text { ing/insomnia) (0-4) } \\
\text { - Pain medication }\end{array}$ & $\begin{array}{l}\text { - Significantly more nasal } \\
\text { discharge in hypertonic } \\
\text { saline group compared to } \\
\text { the other groups } \\
\text { - No other significant differ- } \\
\text { ences }\end{array}$ \\
\hline $\begin{array}{l}\text { Heatley } \\
2001^{(177)}\end{array}$ & RCT, no blinding & $\begin{array}{l}150 \text { CRS } \\
\text { patients ( } 128 \\
\text { completed the } \\
\text { study) }\end{array}$ & $\begin{array}{l}\text { - Nasal irrigation with bulb syringe with } \\
\text { hypertonic saline for } 2 \text { weeks } \\
\text { - Nasal irrigation with nasal irrigation pot } \\
\text { with hypertonic saline for } 2 \text { weeks } \\
\text { - Reflexology massage for } 2 \text { weeks }\end{array}$ & $\begin{array}{l}\text { - RSOM-31 at } 2 \text { weeks } \\
\text { - SF-36 at } 2 \text { weeks } \\
\text { - Use of medication at } \\
2 \text { weeks }\end{array}$ & $\begin{array}{l}\text { - No significant differences } \\
\text { between the groups }\end{array}$ \\
\hline
\end{tabular}

CRS, chronic rhinosinusitis; CRSwNP, Chronic Rhinosinusitis with nasal polyps; CT, computed tomography; EAW, electrolyzed acid water ; FESS, functional endoscopic sinus surgery; HS, hypertonic saline; LM, Lund-Mackay; MCA ${ }_{2}$, second minimal crosssectional area; NS, normal saline; RCT, randomised controlled trial; RSOM-31, Rhinosinusitis Outcome Measure-31; SF-36, Short Form 36; UPSIT-TC, University of Pennsylvania Smell Identification Test - Traditional Chinese. 
to reach the anterior and posterior nasal cavity in patients postFESS and healthy subjects. The spray and the nebulizer were unable to penetrate any of the sinuses or the frontal recess in the controls. However, effect on symptoms was not measured. One study in 86 CRS patients compared a squeeze bottle with a nasal spray for one month and found no differences between the SNOT-22, Nasal and Sinus Symptom Scale (NSS) or PeriOperative Sinus Endoscopy (POSE) score between the two ways of administration ${ }^{(162)}$. Although the study showed a significant improvement in both groups there was no difference between patients treated with the squeeze bottle compared to saline nasal spray (Table 6.1.11.2). The same was shown by Salib et al. who evaluated a low-volume high-pressure nasal spray and high-volume low-pressure saline application system following functional endoscopic sinus surgery and found no differences in SNOT-22 and only one timepoint difference for the LK score in favour of high volume ${ }^{(189)}$. The studies could not be combined into a meta-analysis because of insufficient data.

To conclude, although nasal douches have been shown to be more effective in distributing irrigation solution to the sinuses, there are no studies that show that nasal douches are also more effective in reducing symptoms and signs of CRS.

\section{Nasal irrigation with isotonic saline or Ringer's lactate is an effective treatment in CRS patients.}

\subsubsection{What is the best content?}

Six studies evaluated the differences between hypertonic and normal saline ${ }^{(157,161,163,165,178,184)}$ (Table 6.1.11.3). Three studies (325 patients) found, in general, better results with isotonic than hypertonic saline ${ }^{(157,165,178)}$. Two studies (248 patients) found no difference ${ }^{(161,163)}$ and one study (60 patients) showed hypertonic nasal spray to have significantly better results on congestion and cough than isotonic saline ${ }^{(184)}$.

Two studies ${ }^{(156,159)}$ evaluated the differences between Dead Sea salt and other hypertonic saline irrigation alone ${ }^{(156)}$ or in combination with fluticasone dipropionate ${ }^{(159)}$. The studies showed that hypertonic saline alone was less effective than the other two treatments. One study in 74 CRS patients directly after FESS evaluated the differences between isotonic saline irrigation $(n=25)$ with lactated Ringer's irrigation $(n=26)$ and hypertonic saline nasal irrigation $(n=23)$ for six weeks ${ }^{(161)}$. Lactated Ringer's resulted in a significantly larger reduction in SNOT and VAS total symptom score over six weeks than the other two irrigations. In conclusion, in general, studies do not show that rinsing with hypertonic saline is more effective than with isotonic saline. There is one postoperative study showing that irrigation with lactated Ringer's solution is more effective than both isotonic and hypertonic saline irrigation ${ }^{(161)}$.

\subsubsection{What is the best temperature?}

One study compared a single saline irrigation at room temperature with irrigation with saline at 40 degrees Celsius on mucociliairy transit time and nasal obstruction and found no differences ${ }^{(164)}$ (Table 6.1.11.4).

\subsubsection{Will additions help?}

\subsection{Antiseptic/mucolytic/baby shampoo}

Three studies evaluated the addition of antiseptic/mucolytic/ baby shampoo solution and showed little or no differences between the groups but the baby-shampoo group was reported to have significantly more side effects and had more patients stop the solution $^{(160,174,183)}$ (Table 6.1.11.5). Moreover, it has been shown in healthy persons to create congestion and reversible smell loss ${ }^{(190)}$.

\subsection{5.2. Sodium hyaluronate in CRS patients (both CRSsNP and CRSwNP).}

Six Italian DBPCT or single blind (blinding often not clearly described) studies evaluated the addition of $9 \mathrm{mg}$ of sodium hyaluronate to saline ${ }^{(166-168,185,186,191)}$. Five studies evaluated nebulisation with a small volume $\mathrm{e}^{(167,168,185,186,191)}$ and one study rinsing with $250 \mathrm{ml}$. The five studies evaluating nebulization in general reported positive outcomes, the study evaluation addition of sodium hyaluronate to saline rinsing found in general no significant effect (Table 6.1.11.6).

In conclusion, sodium hyaluronate might have a positive effect on postoperative symptoms when compared to a saline spray. Adding sodium hyaluronate to saline rinsing postoperatively did not show an effect in one study.

\subsection{Honey in nasal saline irrigation}

Honey is a viscous, supersaturated sugar solution derived from nectar gathered and modified by the honeybee, Apis mellifera. Honey has been used since ancient times as a remedy in wound care. Honey appears to heal partial thickness burns more quickly than conventional treatment (which included polyurethane film, paraffin gauze, soframycin-impregnated gauze, sterile linen and leaving the burns exposed) and infected post-operative wounds more quickly than antiseptics and gauze ${ }^{(192)}$. In otolaryngology, studies have investigated the effect of honey and other bee products like propolis and royal jelly in oral infections, infections of the respiratory tract, in rhinosinusitis, tonsillectomy and head and neck surgery ${ }^{(193)}$.

Three studies ${ }^{(169,170,176)}$ evaluated the addition of honey to saline irrigation $^{(169,176)}$ or spray ${ }^{(170)}$. None of the studies did find any relevant differences between the honey treated group and the placebo group (Table 6.1.11.7). However, in all studies patients also received oral antibiotics and/or nasal corticosteroids which reduces the possibility of finding significant differences.

\section{The addition of Xylitol, sodium hyaluronate, and xyloglucan to nasal saline irrigation may have a positive effect.}

\subsection{Xylitol nasal irrigation}

Xylitol is a five-carbon sugar alcohol that occurs naturally in many fruits and vegetables and is used widely in the food industry as a sweetener. It has gained extensive attention in the past decade as a natural antibacterial agent and more recently as an anti-biofilm agent, particularly in periodontics. It decreases 
the salt concentration of human airway surface liquid that contains many antimicrobial substances, which can contribute to the improvement of the innate immune system, and thereby prevent airway infections. Moreover, through disruption of the glucose cell-wall transport and intracellular glycolysis, xylitol can inhibit bacterial growth.

Two small studies analysed the efficacy of xylitol rinsing in the treatment of patients with $\operatorname{CRS}^{(180,181)}$. Both studies reported a significant better effect of xylitol compared to saline in the reduction of SNOT and total symptoms visual analogue scale (VAS). The studies could not be combined into a meta-analysis because of missing data (Table 6.1.11.8).

\subsection{Dexpanthenol in nasal saline irrigation}

Dexpanthenol is an analog of pantothenic acid, which has been claimed to promote wound healing ${ }^{(194)}$. It has been shown to improve symptoms in patients with atrophic rhinitis ${ }^{(195)}$. Two studies evaluated the effect of dexpanthenol in saline ${ }^{(171,187)}$. Both studies showed no effect on the majority of symptoms and endoscopy but a superior efficacy over placebo on mucociliary clearance (in the Fooanant study only in patients with CRSwNP) (Table 6.1.11.9).

\subsection{Xyloglucan in nasal saline irrigation}

Xyloglucan, a natural polysaccharide derived from tamarind seeds, possesses a "mucin-like" molecular structure that confers mucoadhesive properties, allowing xyloglucan formulations to act as a barrier capable of reducing bacterial adherence and invasion and to preserve tight junctions and paracellular flux, as observed in different in vitro and in vivo studies ${ }^{(196)}$. There is one study ${ }^{(182)}$ evaluating the effect of xyloglucan nasal spray compared to saline that indicated that xyloglucan spray resulted in a significant reduction in symptoms and signs of disease (Table 6.1.11.10) and saline did not. However, no direct comparison between the groups was made. In conclusion there is limited evidence that xyloglucan nasal spray may have a positive effect on symptoms and signs of disease in patients with CRS [level $2 \mathrm{~b}$ (downgraded because of

Table 6.1.11.2. Methods of nasal irrigation for the treatment of patients with CRS.

\begin{tabular}{|c|c|c|c|c|c|}
\hline Study & Methods & Participants & Interventions & Outcomes & Results \\
\hline $\begin{array}{l}\text { Macdonald } \\
2015^{(162)}\end{array}$ & $\begin{array}{l}\text { Single blind } \mathrm{RCT} \\
\text { postoperative }\end{array}$ & $\begin{array}{l}86 \text { CRS patients } \\
\text { (55 CRSwNP) } \\
\text { directly } \\
\text { postoperatively }\end{array}$ & $\begin{array}{l}\text { - Saline squeeze bottle }(n=43) \text { for } 1 \text { month } \\
\text { - Saline nasal spray }(n=43) \text { for } 1 \text { month }\end{array}$ & $\begin{array}{l}\text { - } \text { SNOT-22 } \\
\text { - } \text { POSE } \\
\text { - } \text { Nasal and } \\
\text { sinus symp- } \\
\text { tom scale } \\
(0-15)\end{array}$ & $\begin{array}{l}\text { - Significant improvement } \\
\text { in both groups for SNOT- } \\
22, \text { POSE, NSS } \\
\text { - No difference between } \\
\text { treatment groups }\end{array}$ \\
\hline $\begin{array}{l}\text { Salib } \\
2013^{(189)}\end{array}$ & $\begin{array}{l}\text { Single blind } \mathrm{RCT} \\
\text { postoperative }\end{array}$ & $\begin{array}{l}31 \text { CRS patients } \\
\text { directly } \\
\text { postoperatively } \\
\text { ( } 24 \text { evaluated) ( } 17 \\
\text { CRSwNP) }\end{array}$ & $\begin{array}{l}\text { - Sterimar }{ }^{T M} \text { Isotonic two sprays three times } \\
\text { daily in one nostril for a period of } 12 \text { weeks } \\
\text { after surgery }(n=24) \text {. } \\
\text { - Sinus Rinse } 120 \mathrm{ml} \text { three times daily in one } \\
\text { nostril for a period of } 12 \text { weeks after surgery. } \\
\text { All patients received } 1 \text {-week course of antibiotics, } \\
\text { topical nasal steroids, and a 10-day course of } \\
\text { oral steroids in patients with nasal polyposis. }\end{array}$ & $\begin{array}{l}\text { At 2,4 and } \\
12 \text { weeks } \\
\text { following } \\
\text { surgery: } \\
\text { - SNOT-22 } \\
\text { - LK score }\end{array}$ & $\begin{array}{l}\text { At 2,4 and } 12 \text { weeks: } \\
\text { - No difference in SNOT-22 } \\
\text { at any timepoint } \\
\text { - Statistical difference for } \\
\text { LK score at } 4 \text { weeks and } \\
\text { trend at } 2 \text { weeks }\end{array}$ \\
\hline
\end{tabular}

CRS,Chronic Rhinosinusitis; Single blind RCT, Single Blind Randomised Controlled Trial; CRSwNP, Chronic Rhinosinusitis with nasal polyps;SNOT-22, Sino-Nasal Outcome Test-22; POSE, Perioperative Sinus Endoscopy score; NSS, Nasal and Sinus Symptom scale; LK score, Lund-Kennedy score;

Table 6.1.11.3. Hypertonic versus isotonic saline irrigation for the treatment of patients with CRS.

\begin{tabular}{|c|c|c|c|c|c|}
\hline Study & Methods & Participants & Interventions & Outcomes & Results \\
\hline Giotakis $2016^{(163)}$ & $\begin{array}{l}\text { RCT not } \\
\text { blinded }\end{array}$ & $\begin{array}{l}174 \text { CRSwNP } \\
\text { patients (158 } \\
\text { evaluated), } \\
\text { postoperative }\end{array}$ & $\begin{array}{l}\text { - } 250 \mathrm{ml} \text { of } 1,175 \% \text { Emser Salt }{ }^{\oplus} \text { solution } \\
\text { (EmsSalt) }(n=59) \text { twice daily for one } \\
\text { year } \\
\text { - } 250 \mathrm{ml} \text { of isosmotic mineral salt } \\
\text { mixture (IsoMix) ( } n=58 \text { ) twice daily } \\
\text { for one year } \\
\text { - No irrigation ( } n=57) \\
\text { - All patients were allowed to use } \\
\text { budesonide nasal spray in limited } \\
\text { doses in the first } 6 \text { months and corti- } \\
\text { coid and antibiotic nasal ointment in } \\
\text { the first } 14 \text { days. }\end{array}$ & $\begin{array}{l}\text { At 3,6,9 and } 12 \text { months: } \\
\text { - } \quad \text { Nasal symptoms } \\
\text { - } \quad \text { MQLQ } \\
\text { - } \quad \text { Missed work days } \\
\quad \text { Postoperative condi- } \\
\text { tion of the mucosa }\end{array}$ & $\begin{array}{l}\text { - } \quad \text { Significantly better } \\
\text { results in nasal } \\
\text { symptoms and RQLQ } \\
\text { between irrigation and } \\
\text { no irrigation } \\
\text { - No significant differ- } \\
\text { ence in missed work } \\
\text { days and mucosa } \\
\text { - } \quad \text { so significant differ- } \\
\text { ences between Emser } \\
\text { Salt and the isosmotic } \\
\text { mineral salt mixture }\end{array}$ \\
\hline
\end{tabular}


Table 6.1.11.3. Hypertonic versus isotonic saline irrigation for the treatment of patients with CRS (continued).

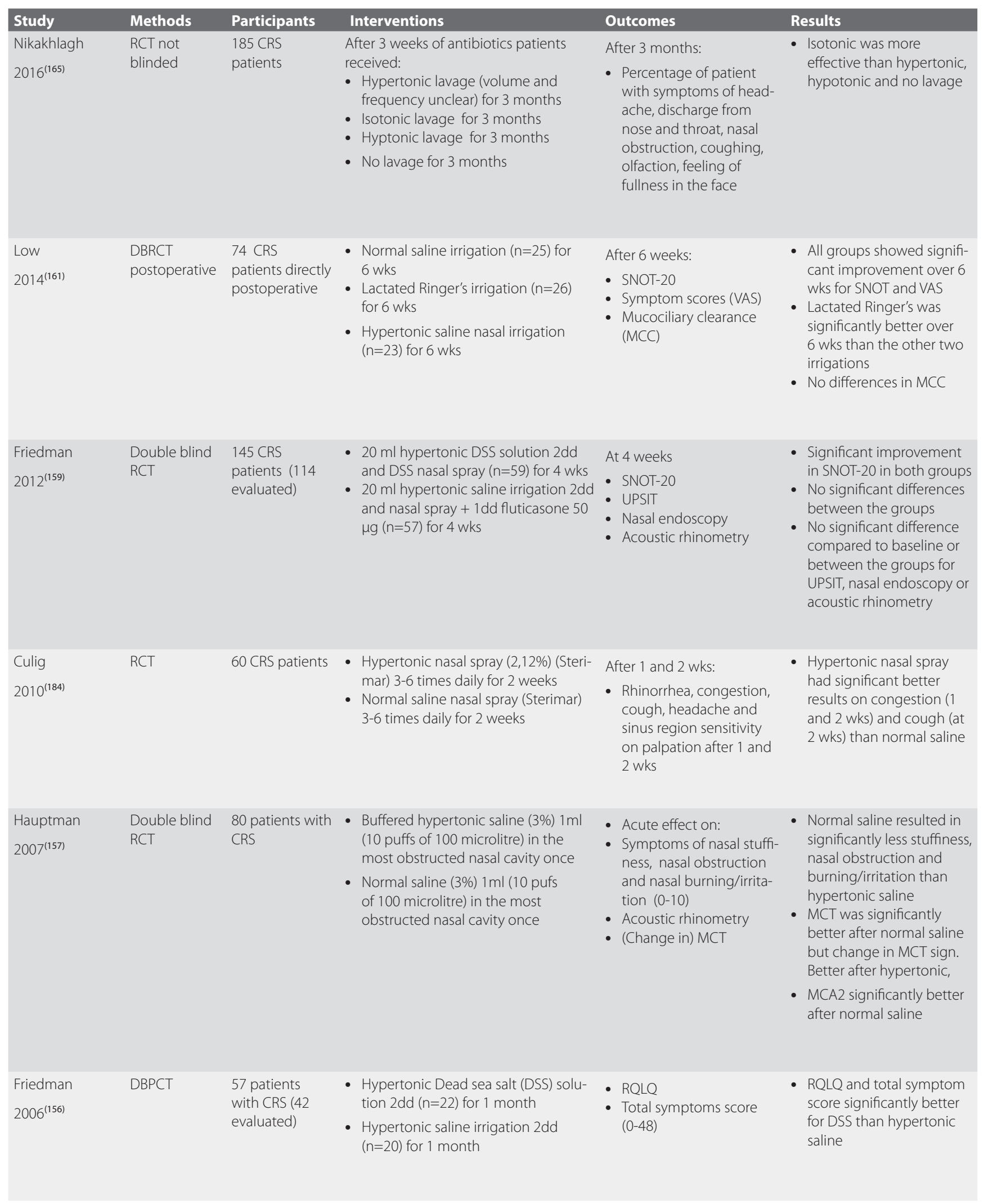


Table 6.1.11.3. Hypertonic versus isotonic saline irrigation for the treatment of patients with CRS (continued).

\begin{tabular}{|c|c|c|c|c|c|}
\hline Pinto & Postoperative, & 60 patients & - Normal saline (NS; $n=20) 4$ times & - Symptoms (nasal & - Significantly more nasal \\
\hline $2006^{(178)}$ & $\begin{array}{l}\text { RCT, double } \\
\text { blinded for } \\
\text { sprays }\end{array}$ & $\begin{array}{l}\text { with histories } \\
\text { of frequent } \\
\text { sinusitis after } \\
\text { FESS }\end{array}$ & $\begin{array}{l}\text { per day, two puffs on each side, for } \\
\text { the first } 5 \text { postoperative days. } \\
\text { - Buffered hypertonic saline (HS; } n= \\
20) 4 \text { times per day, two puffs on } \\
\text { each side, for the first } 5 \text { postoperative } \\
\text { days. } \\
\text { - No spray }(n=20)\end{array}$ & $\begin{array}{l}\text { obstruction/congestion, } \\
\text { nasal discharge/postna- } \\
\text { sal drip, pain/pressure, } \\
\text { headache, and trouble } \\
\text { sleeping/insomnia) (0-4) } \\
\text { for } 5 \text { days } \\
\text { - Pain medication for } 5 \\
\text { days }\end{array}$ & $\begin{array}{l}\text { discharge in hypertonic } \\
\text { saline group compared to } \\
\text { the other groups } \\
\text { - No other significant } \\
\text { differences }\end{array}$ \\
\hline
\end{tabular}

CRS, chronic rhinosinusitis; CRSwNP, chronic rhinosinusitis with nasal polyps; DBPCT, double blind placebo controlled trial; DBRCT, double blind randomised controlled trial; dd, daily dosage; DSS, Dead Sea Salt solution; FESS, functional endoscopic sinus surgery; HDSS, Hypertonic Dead sea salt; HS, hypertonic saline; MCA2, Minimal cross-sectional area 2; MCT, mucociliary clearance time; NS, normal saline; RCT, randomised controlled trial; RQLQ, Rhinoconjunctivitis Quality of Life Questionnaire .

Table 6.1.11.4. Temperature of nasal saline for irrigation for the treatment of patients with CRS.

\begin{tabular}{|c|c|c|c|c|c|}
\hline Study & Methods & Participants & Interventions & Outcomes & Results \\
\hline $\begin{array}{l}\text { Nimsakul } \\
2018^{(164)}\end{array}$ & $\begin{array}{l}\text { RCT not } \\
\text { blinded }\end{array}$ & 23 CRS patients & $\begin{array}{l}\text { - } 250 \mathrm{ml} \text { saline at room temperature }(\mathrm{n}=9) \\
\text { - } 250 \mathrm{ml} \text { saline at } 40^{\circ} \mathrm{C}(\mathrm{n}=12)\end{array}$ & $\begin{array}{l}\text { - Saccharin transit time (STT) } \\
\text { - PNIF } \\
\text { - Total nasal resistance measured } \\
\text { by anterior rhinomanometry } \\
\text { - Total nasal volume measured by } \\
\text { acoustic rhinometry } \\
\text { - Nasal obstruction score (0-5) } \\
\text { - Breathe-comfort score (0-5) } \\
\text { - Adverse events. }\end{array}$ & $\begin{array}{l}\text { - Significant im- } \\
\text { provement in STT } \\
\text { in both groups } \\
\text { - No differences } \\
\text { between the } \\
\text { groups } \\
\text { - No differences in } \\
\text { other outcomes }\end{array}$ \\
\hline
\end{tabular}

CRS, chronic rhinosinusitis; PNIF, peak nasal inspiratory flow; RCT, randomised controlled trial; STT, saccharin transit time.

Table 6.1.11.5. Saline irrigation with addition of antiseptic/mucolytic/baby shampoo solution for the treatment of patients with CRS.

\begin{tabular}{|c|c|c|c|c|c|}
\hline Study & Methods & Participants & Interventions & Outcomes & Results \\
\hline Yu $2016^{(174)}$ & DBPCT & $\begin{array}{l}43 \text { CRS patients } \\
\text { (39 evaluated) }\end{array}$ & $\begin{array}{l}\text { - Low-concentration } \mathrm{HOCl} \\
30 \mathrm{ml} \text { twice daily using a } \\
\text { Salicid device } \\
\text { - Saline } 30 \mathrm{ml} \text { twice daily } \\
\text { using a Sham Salicid } \\
\text { device }\end{array}$ & $\begin{array}{l}\text { At 1,2,4 and } 8 \text { weeks: } \\
\text { - SNOT-20 } \\
\text { - RSDI } \\
\text { - Endoscopy (LK score) } \\
\text { - Adverse events }\end{array}$ & $\begin{array}{l}\text { Low-concentration } \mathrm{HOCl} \text { versus saline } \\
\text { resulted in significantly reduced } \\
\text { - SNOT-20 at } 2 \text { and } 4 \text { weeks } \\
\text { - None of the other outcomes } \\
\text { - No treatment related adverse events }\end{array}$ \\
\hline Farag $2013^{(160)}$ & $\begin{array}{l}\text { Single } \\
\text { blind RCT } \\
\text { postoperative }\end{array}$ & $\begin{array}{l}40 \text { CRS patients } \\
\text { directly } \\
\text { postoperative }\end{array}$ & $\begin{array}{l}\text { - } 120 \mathrm{ml} \text { hypertonic Saline } \\
\text { solution 3dd until } 4 \\
\text { months postop } \\
\text { - } 120 \mathrm{ml} \text { of } 1 \% \text { baby sham- } \\
\text { poo solution } 3 \text { dd until } 4 \\
\text { months postop }\end{array}$ & $\begin{array}{l}\text { - } \text { RSOM-31 } \\
\text { - SNOT-22 } \\
\text { - Olfaction with phenyl } \\
\text { ethyl alcohol (PEA) } \\
\text { threshold test } \\
\text { - Adverse events }\end{array}$ & $\begin{array}{l}\text { - Significant decreases in scores for } \\
\text { both the SNOT-22and RSOM-31 } \\
\text { overtime for both treatments but no } \\
\text { difference between the treatments } \\
\text { - PEA thresholds showed improve- } \\
\text { ment but no difference between } \\
\text { the treatments } \\
\text { - The baby-shampoo group reported } \\
\text { significantly more side effects and } \\
\text { had more patients stop the solution }\end{array}$ \\
\hline Pigret $1996^{(183)}$ & $\begin{array}{l}\text { Single- } \\
\text { blind RCT } \\
\text { postoperative }\end{array}$ & $\begin{array}{l}20 \text { CRSwNP } \\
\text { patients after } \\
\text { endoscopic } \\
\text { endonasal } \\
\text { ethmoidectomy }\end{array}$ & $\begin{array}{l}\text { - Pressurized seawater (vol- } \\
\text { ume unclear) ( } n=10) 3 d d \\
\text { - } 10 \mathrm{ml} \text { of saline plus benzo- } \\
\text { dodecinium (antiseptic) } \\
\text { plus oleosorbate (mucol- } \\
\text { ytic) }(n=10) 3 d d\end{array}$ & $\begin{array}{l}\text { - Residual nasal crusts } \\
\text { weight at } 21 \pm 2 \text { days } \\
\text { secretions after } 21 \pm 2 \\
\text { days } \\
\text { - Visual analogue scale } \\
\text { for nasal obstruction, } \\
\text { rhinorrhoea, cacosmia } \\
\text { and facial pain }\end{array}$ & $\begin{array}{l}\text { No significant differences although } \\
\text { the mean residual crust weight was } \\
\text { higher in the pressurized seawater } \\
\text { group }(1,756 \pm 688 \mathrm{mg}) \text { than in the } \\
\text { antiseptic/mucolytic saline group } \\
\text { (932 } \pm 414 \mathrm{mg})\end{array}$ \\
\hline
\end{tabular}

DBPCT, double blind placebo controlled trial; LK, Lund-Kennedy; $\mathrm{HOCl}$, Hypochlorous acid; PEA, phenyl ethyl alcohol; RSDI, Rhinosinusitis Disability Index; SNOT, Sino-nasal Outcome Test; RCT, randomised controlled trial; RSOM-31, Rhinosinusitis Outcome Measure-31. 
Table 6.1.11.6. Saline irrigation with addition of sodium hyaluronate for the treatment of patients with CRS.

\begin{tabular}{|c|c|c|c|c|c|}
\hline Study & Methods & Participants & Interventions & Outcomes & Results \\
\hline $\begin{array}{l}\text { Mozzanica } \\
2019^{(166)}\end{array}$ & $\mathrm{DBPCT}$ & $\begin{array}{l}56 \text { CRSsNP } \\
\text { patients } \\
\text { postoperatively }\end{array}$ & $\begin{array}{l}\text { - } 250 \mathrm{ml} \text { saline plus } 9 \mathrm{mg} \text { sodium } \\
\text { hyaluronate twice daily for } 6 \\
\text { weeks ( } \mathrm{n}=26) \\
\text { - Saline twice daily for } 6 \text { weeks } \\
(n=30)\end{array}$ & $\begin{array}{l}\text { At } 3 \text { and } 6 \text { weeks } \\
\text { postoperatively: } \\
\text { - SNOT-22 } \\
\text { - NOSE } \\
\text { - Symptoms (VAS) } \\
\text { - LK score }\end{array}$ & $\begin{array}{l}\text { - No differences in SNOT-22, NOSE, } \\
\text { symptoms and total LK score at } 6 \\
\text { weeks } \\
\text { - Significantly better result of NOSE } \\
\text { score at } 3 \text { weeks in sodium hyaluronate } \\
\text { group } \\
\text { - Significantly better result for headache } \\
\text { and smell alteration at three weeks in } \\
\text { sodium hyaluronate group } \\
\text { - Significantly reduced scar formation (3 } \\
\text { and } 6 \text { weeks), crusting ( } 3 \text { weeks) and } \\
\text { secretion ( } 6 \text { weeks) }\end{array}$ \\
\hline $\begin{array}{l}\text { Cantone } \\
2016^{(191)}\end{array}$ & DBPCT & $\begin{array}{l}80 \text { CRS patients } \\
\text { (40 allergic and } \\
40 \text { non-allergic) }\end{array}$ & 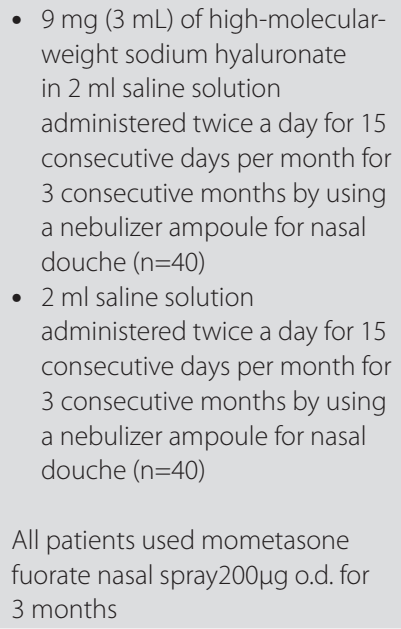 & $\begin{array}{l}\text { At } 3 \text { months: } \\
\text { - SF-36 } \\
\text { - SNOT-22 } \\
\text { - Obstruction (VAS) } \\
\text { - Nasal discharge (VAS) } \\
\text { - LK score }\end{array}$ & $\begin{array}{l}\text { Significantly better result of sodium } \\
\text { hyaluronate than saline on top of MFNS } \\
\text { for: } \\
\text { - SF36 } \\
\text { - SNOT-22 } \\
\text { - Obstruction (VAS) } \\
\text { - Nasal discharge (VAS) } \\
\text { - LK score }\end{array}$ \\
\hline $\begin{array}{l}\text { Cantone } \\
2014^{(185)}\end{array}$ & DBPCT & $\begin{array}{l}124 \text { CRSwNP } \\
\text { (122 evaluated) } \\
\text { postoperatively }\end{array}$ & $\begin{array}{l}\text { - Intranasal nebulization of } 9 \mathrm{mg} \\
\text { sodium hyaluronate }(3 \mathrm{~mL}) \text { plus } \\
\text { saline solution }(2 \mathrm{~mL}) \text { twice daily } \\
\text { for } 30 \text { days }(\mathrm{n}=62) \\
\text { - Intranasal nebulization of saline } \\
(5 \mathrm{ml}) \text { twice daily for } 30 \text { days } \\
(\mathrm{n}=60)\end{array}$ & $\begin{array}{l}\text { At } 30 \text { days postoperatively: } \\
\text { - SF-36 } \\
\text { - SNOT-22 } \\
\text { - Symptoms (VAS) } \\
\text { - Endoscopic score }\end{array}$ & $\begin{array}{l}\text { Significantly better result of sodium } \\
\text { hyaluronate than saline for: } \\
\text { - SF36 } \\
\text { - SNOT-22 } \\
\text { - Total symptoms (VAS) } \\
\text { - Endoscopic score }\end{array}$ \\
\hline $\begin{array}{l}\text { Casale, } \\
2014^{(167)}\end{array}$ & $\begin{array}{l}\text { RCT } \\
\text { (single } \\
\text { blind?) }\end{array}$ & $\begin{array}{l}33 \text { CRS patients } \\
\text { postoperatively }\end{array}$ & $\begin{array}{l}\text { - Nebulization of } 3 \mathrm{~mL} \text { containing } \\
\text { 9mg sodium hyaluronate nasal } \\
\text { washes plus saline twice daily } \\
\text { for } 1 \text { month }(n=18) \\
\text { - Nebulization of } 5 \mathrm{~mL} \text { saline } \\
\text { twice daily for } 1 \text { month }(n=15)\end{array}$ & $\begin{array}{l}\text { At } 2 \text { and } 4 \text { weeks } \\
\text { postoperatively: } \\
\text { - CRS questionnaire } \\
\text { - Visual analogic scale (VAS) } \\
\text { - Nasal endoscopy }\end{array}$ & $\begin{array}{l}\text { - VAS score of hyaluronate significantly } \\
\text { lower than saline at } 2 \text { and } 4 \text { weeks } \\
\text { - CRS score significantly lower than } \\
\text { saline at } 2 \text { and } 4 \text { weeks } \\
\text { - Significantly less crusts and oedema } \\
\text { and secretions }\end{array}$ \\
\hline $\begin{array}{l}\text { Gelardi } \\
2013^{(186)}\end{array}$ & $\begin{array}{l}\mathrm{RCT} \\
\text { (single } \\
\text { blind?) }\end{array}$ & $\begin{array}{l}36 \text { CRSwNP } \\
\text { postoperative }\end{array}$ & $\begin{array}{l}\text { - Intranasal nebulization of } \\
\text { sodium hyaluronate } 9 \text { mg in } \\
3 m L \text { twice daily for } 30 \text { days } \\
(n=19) \\
\text { - Intranasal nebulization saline } \\
5 m L \text { twice daily for } 30 \text { days } \\
(n=17)\end{array}$ & $\begin{array}{l}\text { At } 30 \text { days postoperatively: } \\
\text { - Nasal obstruction, burning, } \\
\text { dryness of the mucosa } \\
\text { (VAS) } \\
\text { - Presence or absence of } \\
\text { rhinorrhoea } \\
\text { - Mucociliary clearance time } \\
\text { - Presence of exudate at } \\
\text { endoscopy }\end{array}$ & $\begin{array}{l}\text { Sodium hyaluronate group had } \\
\text { compared to saline: } \\
\text { - Significantly faster mucociliary clear- } \\
\text { ance } \\
\text { - Lower incidence of rhinorrhoea } \\
\text { - Less nasal obstruction } \\
\text { - A lower incidence of exudate on } \\
\text { - } \text { endoscopy } \\
\text { - Better tolerability }\end{array}$ \\
\hline $\begin{array}{l}\text { Macchi } \\
2013^{(168)}\end{array}$ & DBPCT & $\begin{array}{l}46 \text { CRSwNP } \\
\text { patients } \\
\text { postoperatively }\end{array}$ & $\begin{array}{l}\text { - Nebulization of } 6 \mathrm{ml} \text { containing } \\
\text { mas sodium hyaluronate nasal } \\
\text { washes plus saline for } 15 \text { days/ } \\
\text { mo over } 3 \text { months ( } n=23 \text { ) } \\
\text { - Nebulization of } 6 \mathrm{~mL} \text { isotonic } \\
\text { saline for } 15 \text { days } / \text { mo over } 3 \\
\text { months }(n=23)\end{array}$ & $\begin{array}{l}\text { At } 3 \text { months postoperatively: } \\
\text { - Prevalence of symptoms } \\
\text { - Endoscopy } \\
\text { - Cytology } \\
\text { - Biofilm }\end{array}$ & $\begin{array}{l}\text { Significantly better result of sodium } \\
\text { hyaluronate than saline for: } \\
\text { - Nasal dyspnea (obstruction?) } \\
\text { - Normal mucosa } \\
\text { - Absence of secretion }\end{array}$ \\
\hline
\end{tabular}

CRS, chronic rhinosinusitis; CRSsNP, chronic rhinosinusitis without nasal polyps; CRSwNP, chronic rhinosinusitis with nasal polyps; DBPCT, double blind placebo controlled trial; LK, Lund-Kennedy; MFNS, Mometasone fuorate nasal spray; NOSE, Nasal Obstruction Symptom Evaluation; RCT, randomised controlled trial; SF 36, Short Form 36; SNOT, Sino-nasal Outcome Test; VAS, visual analogue scale. 
Table 6.1.11.7. Saline irrigation with addition of honey for the treatment of patients with CRS.

\begin{tabular}{|c|c|c|c|c|c|}
\hline Study & Methods & Participants & Interventions & Outcomes & Results \\
\hline $\begin{array}{l}\text { Ooi } \\
2019^{(176)}\end{array}$ & $\begin{array}{l}\text { Single blind } \\
\text { (investigator) } \\
\text { placebo } \\
\text { controlled } \\
\text { trial }\end{array}$ & $\begin{array}{l}25 \text { recalcitrant } \\
\text { CRS patients }\end{array}$ & $\begin{array}{l}\text { - } 14 \text { days of twice-daily } 16.5 \% \text { Manuka Honey }+ \\
1.3 \mathrm{mg} / \mathrm{mL} \text { augmented methylglyoxal sinonasal } \\
\text { rinses } \\
\text { - } 14 \text { days of twice-daily saline sinonasal rinses } \\
\text { Both groups received } 10 \text { days of culture-directed } \\
\text { antibiotic therapy }\end{array}$ & $\begin{array}{l}\text { At } 14 \text { days: } \\
\text { - SNOT-22 } \\
\text { - Symptom scores (VAS) } \\
\text { - UPSIT } \\
\text { - Endoscopy (LK score) } \\
\text { - Microbiology } \\
\text { - } \text { Adverse events }\end{array}$ & $\begin{array}{l}\text { - No significant } \\
\text { differences } \\
\text { in SNOT-22, } \\
\text { symptoms, } \\
\text { UPSIT, LK score, } \\
\text { microbiology or } \\
\text { adverse events }\end{array}$ \\
\hline $\begin{array}{l}\text { Lee } \\
2016^{(169)}\end{array}$ & $\begin{array}{l}\text { Single blind } \\
\text { (investigator) } \\
\text { placebo } \\
\text { controlled } \\
\text { trial }\end{array}$ & $\begin{array}{l}49 \text { CRS patients } \\
\text { (42 analysed) } \\
\text { postoperatively }\end{array}$ & $\begin{array}{l}\text { - } 120 \mathrm{ml} \text { saline containing } 12 \mathrm{mg} \text { of manuka honey } \\
\text { twice daily for } 30 \text { days } \\
\text { - } 120 \mathrm{ml} \text { saline twice daily for } 30 \text { days } \\
\text { - All patients received culture-directed oral antibi- } \\
\text { otic, and/or oral steroids for up to } 3 \text { weeks, and/ } \\
\text { or high-volume topical steroid sinus irrigations } \\
\text { (budesonide } 0.5 \mathrm{mg} / 2 \mathrm{~mL} \text { vial or } 0.6 \mathrm{mg} / 2 \mathrm{~mL} \text { cap- } \\
\text { sule, } 0.5 \text { bottle to each nasal cavity twice daily) } \\
\text { polyps or inflammation for } 30 \text { days. } \\
\text { - The dose of budesonide was determined when } \\
\text { the patient filled the prescription; if insur- } \\
\text { ance covered it, then the vial version at the } \\
0.5 \mathrm{mg} / 2 \mathrm{~mL} \text { dose was used, and if not covered, } \\
\text { then the capsule version at the } 0.6 \mathrm{mg} / 2 \mathrm{~mL} \text { dose } \\
\text { was used. }\end{array}$ & $\begin{array}{l}\text { At } 30 \text { days: } \\
\text { - } \text { SNOT-22 } \\
\text { - LK endoscopy score } \\
\text { - } \text { Bacterial culture }\end{array}$ & $\begin{array}{l}\text { - No significant } \\
\text { differences } \\
\text { between the } \\
\text { groups } \\
\text { - In the subgroup } \\
\text { not using } \\
\text { antibiotics manuka } \\
\text { honey significantly } \\
\text { reduced bacteria }\end{array}$ \\
\hline $\begin{array}{l}\text { Hashemian } \\
2015^{(170)}\end{array}$ & DBPCT & $\begin{array}{l}64 \text { CRS patients } \\
\text { postoperatively } \\
\text { (53 analysed) }\end{array}$ & $\begin{array}{l}\text { - Thyme/honey nasal spray } 2 \text { puffs per nostril } \\
\text { twice daily ( } n=27 \text { ) } \\
\text { - Placebo nasal spray } 2 \text { puffs per nostril twice daily } \\
\text { ( } n=26) \\
\text { All patients received cefixime } 400 \mathrm{mg} \text { daily for } 10 \\
\text { days postoperatively and were instructed to } \\
\text { rinse their nasal cavity initially with saline three } \\
\text { times daily, and then use fluticasone nasal spray } \\
(50 \mathrm{mcg} \text { ) twice daily. }\end{array}$ & $\begin{array}{l}\text { At } 60 \text { days: } \\
\text { - SNOT-22 } \\
\text { - CT-scan } \\
\text { - Endoscopy score }(0-8)\end{array}$ & $\begin{array}{l}\text { - No significant } \\
\text { differences } \\
\text { between the } \\
\text { groups }\end{array}$ \\
\hline
\end{tabular}

CRS, chronic rhinosinusitis; LK, Lund-Kennedy; SNOT, Sino-nasal Outcome Test; UPSIT, University of Pennsylvania Smell Identification Test; VAS, visual analogic scale.

Table 6.1.11.8. Saline irrigation with addition of xylitol for the treatment of patients with CRS.

\begin{tabular}{|c|c|c|c|c|c|}
\hline Study & Methods & Participants & Interventions & Outcomes & Results \\
\hline $\begin{array}{l}\operatorname{Lin} \\
2017^{(180)}\end{array}$ & DBPCT & $\begin{array}{l}30 \text { CRS } \\
\text { patients ( } 25 \\
\text { evaluated) }\end{array}$ & $\begin{array}{l}\text { - } 240 \mathrm{ml} \text { of water containing } 12 \mathrm{~g} \text { xylitol once } \\
\text { daily for } 30 \text { days ( } \mathrm{n}=13 \text { ) } \\
\text { - } 240 \mathrm{ml} \text { of saline once daily for } 30 \text { days } \\
\text { - } 3 \text { days washout between treatments }(n=12)\end{array}$ & $\begin{array}{l}\text { At } 30 \text { days: } \\
\text { - SNOT-22 } \\
\text { - VAS (symptoms) } \\
\text { - Nasal NO } \\
\text { - iNOS mRNA in the right max- } \\
\text { illary sinus }\end{array}$ & $\begin{array}{l}\text { At } 30 \text { the xylitol group } \\
\text { had significant better } \\
\text { result than the saline } \\
\text { group for: } \\
\text { - SNOT-22 } \\
\text { - VAS (symptoms) } \\
\text { - Nasal NO } \\
\text { - Induction of iNOS }\end{array}$ \\
\hline $\begin{array}{l}\text { Weissman } \\
2011^{(181)}\end{array}$ & $\begin{array}{l}\text { DBPC } \\
\text { crossover } \\
\text { study }\end{array}$ & $\begin{array}{l}20 \text { CRS } \\
\text { patients (15 } \\
\text { evaluated) }\end{array}$ & $\begin{array}{l}\text { - } 240 \mathrm{ml} \text { of water containing } 12 \mathrm{~g} \text { xylitol once } \\
\text { daily for } 10 \text { days }(n=20) \\
\text { - } 240 \mathrm{ml} \text { of saline once daily for } 10 \text { days } 3 \text { days } \\
\text { washout between treatments }(n=20)\end{array}$ & $\begin{array}{l}\text { - Change in SNOT-20 versus } \\
\text { baseline } \\
\text { - Change in VAS (how you think } \\
\text { your nose/sinuses are feeling } \\
\text { overall) versus baseline }\end{array}$ & $\begin{array}{l}\text { Significant larger } \\
\text { reduction in SNOT-20 in } \\
\text { the xylitol group versus } \\
\text { the saline group } \\
\text { No differences in VAS } \\
\text { score }\end{array}$ \\
\hline
\end{tabular}

CRS, chronic rhinosinusitis; DBPC, double blind placebo controlled; DBPCT, double blind placebo controlled trial; iNOS, inducible Nitic Oxide; mRNA, messenger Ribonucleic Acid; NO, nitric oxide; SNOT, Sino-nasal Outcome Test; VAS, visual analogue scale. 
Table 6.1.11.9. Saline irrigation with addition of dexpanthenol for the treatment of patients with CRS.

\begin{tabular}{|c|c|c|c|c|c|}
\hline Study & Methods & Participants & Interventions & Outcomes & Results \\
\hline $\begin{array}{l}\text { Tantilipikorn } \\
2012^{(171)}\end{array}$ & $\mathrm{DBPCT}$ & $\begin{array}{l}50 \text { CRS patients } \\
\text { postoperatively }\end{array}$ & $\begin{array}{l}\text { - Dexpanthenol nasal spray } 4 \\
\text { times a day for } 6 \text { weeks }(n=25) \\
\text { - Saline nasal spray } 4 \text { times a } \\
\text { day for } 6 \text { weeks }(n=25)\end{array}$ & $\begin{array}{l}\text { - \% of patients with symp- } \\
\text { toms at 2, } 4 \text { and } 6 \text { wks } \\
\text { - \% of patients with endo- } \\
\text { scopic abnormalities at 2,4 } \\
\text { and } 6 \text { wks } \\
\text { - Mucociliary clearance time } \\
\text { at } 6 \text { wks }\end{array}$ & $\begin{array}{l}\text { - Majority of symptoms and signs did } \\
\text { not show significant differences } \\
\text { - Dexpanthenol nasal spray has } \\
\text { superior efficacy compared } \\
\text { with normal saline nasal spray } \\
\text { on improvement of mucociliary } \\
\text { clearance and smell at } 6 \text { wks and } \\
\text { inferior effect on nasal discharge }\end{array}$ \\
\hline $\begin{array}{l}\text { Fooanant } \\
2008^{(187)}\end{array}$ & $\mathrm{RCT}$ & $\begin{array}{l}128 \text { CRS } \\
\text { patients ( } 110 \\
\text { evaluated) } \\
\text { postoperatively }\end{array}$ & $\begin{array}{l}\text { - Dexpanthenol (5\%) in sea } \\
\text { water nasal spray } 2 \text { puffs per } \\
\text { nostril, twice a day for } 4 \text { weeks } \\
\text { - Saline nasal irrigation (volume } \\
\text { unclear) twice a day for } 4 \\
\text { weeks }\end{array}$ & $\begin{array}{l}\text { - At 1, 2, 3, 4,6 and } 12 \text { weeks: } \\
\text { - Total nasal symptom score } \\
\text { - Symptoms } \\
\text { - Nasal crusts } \\
\text { - Mucociliary clearance (only } \\
\text { at } 4 \text { wks) }\end{array}$ & $\begin{array}{l}\text { - No significant differences in total } \\
\text { nasal symptom score, nasal crusts } \\
\text { (but at wk } 2 \text { less in saline group), } \\
\text { mucociliary clearance (but for } \\
\text { subgroup of CRSwNP) }\end{array}$ \\
\hline
\end{tabular}

CRS, chronic rhinosinusitis; CRSwNP, chronic rhinosinusitis with nasal polyps; DBPCT, double blind placebo controlled trial; RCT, randomized controlled trial.

Table 6.1.11.10. Saline irrigation with addition of Hyaluronate for the treatment of patients with CRS.

\begin{tabular}{|c|c|c|c|c|c|}
\hline Study & Methods & Participants & Interventions & Outcomes & Results \\
\hline $\begin{array}{l}\text { Mozzanica } \\
2019^{(166)}\end{array}$ & $\mathrm{DBPCT}$ & $\begin{array}{l}56 \text { CRSsNP } \\
\text { patients } \\
\text { postoperatively }\end{array}$ & $\begin{array}{l}\text { - } 250 \mathrm{ml} \text { saline plus } 9 \mathrm{mg} \text { sodium } \\
\text { hyaluronate twice daily for } 6 \text { weeks } \\
(n=26) \\
\text { - Saline twice daily for } 6 \text { weeks }(n=30)\end{array}$ & $\begin{array}{l}\text { At } 3 \text { and } 6 \text { weeks } \\
\text { postoperatively: } \\
\text { - SNOT-22 } \\
\text { - NOSE } \\
\text { - Symptoms (VAS) } \\
\text { - LK score }\end{array}$ & $\begin{array}{l}\text { - No differences in SNOT-22, NOSE, } \\
\text { symptoms and total LK score at } 6 \text { weeks } \\
\text { - Significantly better result of NOSE score } \\
\text { at } 3 \text { weeks in sodium hyaluronate group } \\
\text { - Significantly better result for headache } \\
\text { and smell alteration at three weeks in } \\
\text { sodium hyaluronate group } \\
\text { - Significantly reduced scar formation (3 } \\
\text { and } 6 \text { weeks), crusting ( } 3 \text { weeks) and } \\
\text { secretion (6 weeks) }\end{array}$ \\
\hline $\begin{array}{l}\text { Cantone } \\
2016^{(191)}\end{array}$ & DBPCT & $\begin{array}{l}80 \text { CRS patients } \\
\text { (40 allergic and } \\
40 \text { non-allergic) }\end{array}$ & $\begin{array}{l}\text { - } 9 \text { mg ( } 3 \mathrm{~mL} \text { ) of high-molecular- } \\
\text { weight sodium hyaluronate in } 2 \mathrm{ml} \\
\text { saline solution administered twice } \\
\text { a day for } 15 \text { consecutive days per } \\
\text { month for } 3 \text { consecutive months by } \\
\text { using a nebulizer ampoule for nasal } \\
\text { douche ( } \mathrm{n}=40 \text { ) } \\
\text { - } 2 \text { ml saline solution administered } \\
\text { twice a day for } 15 \text { consecutive days } \\
\text { per month for } 3 \text { consecutive months } \\
\text { by using a nebulizer ampoule for } \\
\text { nasal douche ( } \mathrm{n}=40 \text { ) } \\
\text { All patients used MFNS } 200 \mu \mathrm{g} \text { once } \\
\text { daily for } 3 \text { months }\end{array}$ & $\begin{array}{l}\text { At } 3 \text { months: } \\
\text { - SF-36 } \\
\text { - SNOT-22 } \\
\text { - Obstruction (VAS) } \\
\text { - Nasal discharge } \\
\text { (VAS) } \\
\text { - LK score }\end{array}$ & $\begin{array}{l}\text { Significantly better result of sodium } \\
\text { hyaluronate than saline on top of MFNS for: } \\
\text { - SF36 } \\
\text { - SNOT-22 } \\
\text { - Obstruction (VAS) } \\
\text { - Nasal discharge (VAS) } \\
\text { - LK score }\end{array}$ \\
\hline $\begin{array}{l}\text { Cantone } \\
2014^{(185)}\end{array}$ & DBPCT & $\begin{array}{l}124 \text { CRSwNP } \\
\text { (122 evaluated) } \\
\text { postoperatively }\end{array}$ & $\begin{array}{l}\text { - Intranasal nebulization of } 9 \mathrm{mg} \\
\text { sodium hyaluronate }(3 \mathrm{~mL}) \text { plus saline } \\
\text { solution }(2 \mathrm{~mL}) \text { twice daily for } 30 \text { days } \\
\text { ( } \mathrm{n}=62) \\
\text { - Intranasal nebulization of saline }(5 \mathrm{ml}) \\
\text { twice daily for } 30 \text { days }(\mathrm{n}=60)\end{array}$ & $\begin{array}{l}\text { At } 30 \text { days } \\
\text { postoperatively: } \\
\text { - SF-36 } \\
\text { - SNOT-22 } \\
\text { - Symptoms (VAS) } \\
\text { - Endoscopic score }\end{array}$ & $\begin{array}{l}\text { Significantly better result of sodium } \\
\text { hyaluronate than saline for: } \\
\text { - SF36 } \\
\text { - SNOT-22 } \\
\text { - Total symptoms (VAS) } \\
\text { - Endoscopic score }\end{array}$ \\
\hline $\begin{array}{l}\text { Casale, } \\
2014^{(167)}\end{array}$ & $\begin{array}{l}\text { RCT } \\
\text { (single } \\
\text { blind?) }\end{array}$ & $\begin{array}{l}33 \text { CRS patients } \\
\text { postoperatively }\end{array}$ & $\begin{array}{l}\text { - Nebulization of } 3 \mathrm{ml} \text { containing } 9 \mathrm{mg} \\
\text { sodium hyaluronate nasal washes } \\
\text { plus saline twice daily for } 1 \text { month } \\
\text { ( } n=18) \\
\text { - Nebulization of } 5 \mathrm{~mL} \text { saline twice daily } \\
\text { for } 1 \text { month }(n=15)\end{array}$ & $\begin{array}{l}\text { At } 2 \text { and } 4 \text { weeks } \\
\text { postoperatively: } \\
\text { - CRS questionnaire } \\
\text { - Visual analogic } \\
\text { scale (VAS) } \\
\text { - Nasal endoscopy }\end{array}$ & $\begin{array}{l}\text { - VAS score of hyaluronate significantly } \\
\text { lower than saline at } 2 \text { and } 4 \text { weeks } \\
\text { - CRS score significantly lower than saline } \\
\text { at } 2 \text { and } 4 \text { weeks } \\
\text { - Significantly less crusts and oedema and } \\
\text { secretions }\end{array}$ \\
\hline
\end{tabular}


Table 6.1.11.10. Saline irrigation with addition of Hyaluronate for the treatment of patients with CRS (continued)

\begin{tabular}{|c|c|c|c|c|c|}
\hline Study & Methods & Participants & Interventions & Outcomes & Results \\
\hline $\begin{array}{l}\text { Gelardi } \\
2013^{(186)}\end{array}$ & $\begin{array}{l}\text { RCT } \\
\text { (single } \\
\text { blind?) }\end{array}$ & $\begin{array}{l}36 \text { CRSwNP } \\
\text { postoperative }\end{array}$ & $\begin{array}{l}\text { - Intranasal nebulization of sodium } \\
\text { hyaluronate } 9 \mathrm{mg} \text { in } 3 \mathrm{ml} \text { twice daily } \\
\text { for } 30 \text { days ( } \mathrm{n}=19) \\
\text { - Intranasal nebulization saline } 5 \mathrm{ml} \\
\text { twice daily for } 30 \text { days }(\mathrm{n}=17)\end{array}$ & $\begin{array}{l}\text { At } 30 \text { days } \\
\text { postoperatively: } \\
\text { - Nasal obstruc-tion, } \\
\text { burning, dryness of } \\
\text { the mucosa (VAS) } \\
\text { - Presence or ab- } \\
\text { sence of rhinor- } \\
\text { rhoea } \\
\text { - } \quad \text { Mucociliary clear- } \\
\text { - } \text { ance time } \\
\text { Presence of exu- } \\
\text { date at endoscopy }\end{array}$ & $\begin{array}{l}\text { Sodium hyaluronate group had compared } \\
\text { to saline: } \\
\text { - Significantly faster mu-cociliary clearance } \\
\text { - Lower incidence of rhinorrhoea } \\
\text { - Less nasal obstruction } \\
\text { - A lower incidence of exudate on endos- } \\
\text { copy } \\
\text { - Better tolerability }\end{array}$ \\
\hline $\begin{array}{l}\text { Macchi } \\
2013^{(168)}\end{array}$ & DBPCT & $\begin{array}{l}46 \text { CRSwNP } \\
\text { patients } \\
\text { postoperatively }\end{array}$ & $\begin{array}{l}\text { - Nebulization of } 6 \mathrm{ml} \text { containing } 9 \mathrm{mg} \\
\text { sodium hyaluronate nasal washes } \\
\text { plus saline for } 15 \text { days/mo over } 3 \\
\text { months ( } n=23 \text { ) } \\
\text { - Nebulization of } 6 \mathrm{~mL} \text { isotonic saline } \\
\text { for } 15 \text { days/mo over } 3 \text { months ( } n=23 \text { ) }\end{array}$ & $\begin{array}{l}\text { At } 3 \text { months } \\
\text { postoperatively: } \\
\text { - Prevalence of } \\
\text { symptoms } \\
\text { - Endoscopy } \\
\text { - Cytology } \\
\text { - Biofilm }\end{array}$ & $\begin{array}{l}\text { Significantly better result of sodium } \\
\text { hyaluronate than saline for: } \\
\text { - Nasal dyspnea (obstruction?) } \\
\text { - Normal mucosa } \\
\text { - Absence of secretion }\end{array}$ \\
\hline
\end{tabular}

CRS, chronic rhinosinusitis; CRSsNP, chronic rhinosinusitis without nasal polyps; CRSwNP, chronic rhinosinusitis with nasal polyps; DBPCT, double blind placebo controlled trial; LK, Lund-Kennedy; MFNS, Mometasone fuorate nasal spray; NOSE, Nasal Obstruction Symptom Evaluation; RCT, randomized controlled trial; SF-36, Short Form-36; SNOT, Sino-nasal Outcome Test; VAS, visual analogue scale.

Table 6.1.11.11. Thermal water for the treatment of patients with CRS.

\begin{tabular}{|c|c|c|c|c|c|}
\hline Study & Methods & Participants & Interventions & Outcomes & Results \\
\hline $\begin{array}{l}\text { Ottaviano } \\
2011^{(197)}\end{array}$ & DBPCT & $\begin{array}{l}80 \text { CRS } \\
\text { patients }\end{array}$ & $\begin{array}{l}\text { - Sulphurous, salty, bromic, iodic (SSBI) } \\
\text { thermal water ( } 5 \mathrm{~mL}, 4 \text { times daily for } \\
\text { one month }(n=40) \\
\text { - Saline } 5 \mathrm{~mL}, 4 \text { times daily for one month } \\
(n=40)\end{array}$ & $\begin{array}{l}\text { At } 1 \text { month: } \\
\text { - Nasal endoscopy } \\
\text { - Rhinomanometry }\end{array}$ & $\begin{array}{l}\text { - No significant differences } \\
\text { between thermal water and } \\
\text { saline }\end{array}$ \\
\hline $\begin{array}{l}\text { Salami } \\
2010^{(873)}\end{array}$ & DBPCT & $\begin{array}{l}\text { Eighty } \\
\text { patients with } \\
\text { CRS }\end{array}$ & $\begin{array}{l}\text { - Sulphurous thermal water (Tabiano SPA } \\
\text { Mineral Water) applied as warm inhala- } \\
\text { tion }\left(38^{\circ} \mathrm{C} \text {, at a distance of } 20 \mathrm{~cm} \text { from }\right. \\
\text { the patient's face for } 10 \text { min) combined } \\
\text { with nasal irrigations with the same } \\
\text { water for } 6 \text { minutes for } 12 \text { days ( } \mathrm{n}=40 \text { ) } \\
\text { - Placebo water (saline made white and } \\
\text { with sulphur) applied as warm inhala- } \\
\text { tion }\left(38^{\circ} \mathrm{C} \text {, at a distance of } 20 \mathrm{~cm} \text { from }\right. \\
\text { the patient's face for } 10 \text { min) combined } \\
\text { with nasal irrigations with the same } \\
\text { water for } 6 \text { minutes for } 12 \text { days ( } n=40)\end{array}$ & $\begin{array}{l}\text { At } 12 \text { days and } 3 \text { months: } \\
\text { - Symptoms (VAS) } \\
\text { - Clinical parameters of } \\
\text { recurrence } \\
\text { - Nasal resistance measured } \\
\text { with anterior active rhino- } \\
\text { - Manometry } \\
\text { - Mucociliary clearance }\end{array}$ & $\begin{array}{l}\text { At } 12 \text { days and } 3 \text { months the } \\
\text { thermal water compared to placebo } \\
\text { significantly reduced } \\
\text { - Symptoms (VAS) } \\
\text { - Clinical parameters of recurrence } \\
\text { - Nasal resistance measured with } \\
\text { anterior active rhinomanometry } \\
\text { - Mucociliary clearance }\end{array}$ \\
\hline $\begin{array}{l}\text { Passali } \\
2008^{(198)}\end{array}$ & $\mathrm{RCT}$ & $\begin{array}{l}55 \text { CRS } \\
\text { patients or } \\
\text { patients with } \\
\text { recurrent RS }\end{array}$ & $\begin{array}{l}\text { Thermal (salt-bromine-iodic) water } \\
\text { nasal spray } 4 \text { times daily }(n=30) \text { for } \\
4 \text { weeks } \\
\text { - Saline nasal spray }(n=25) \text { for } 4 \text { weeks }\end{array}$ & $\begin{array}{l}\text { At } 4 \text { weeks: } \\
\text { - Symptoms of headache, } \\
\text { rhinorrhoea, hyposmia } \\
(0-3) \\
\text { - Nasal endoscopy } \\
\text { - Nasal resistance measured } \\
\text { with anterior active rhino- } \\
\text { manometry } \\
\text { - Mucociliary clearance }\end{array}$ & $\begin{array}{l}\text { At } 4 \text { weeks a significant reduction in } \\
\text { the thermal water nasal spray versus } \\
\text { saline in: } \\
\text { - Symptoms } \\
\text { - Nasal endoscopy } \\
\text { - Rhinomanometry } \\
\text { - Mucociliary clearance }\end{array}$ \\
\hline $\begin{array}{l}\text { Passali } \\
2008^{(199)}\end{array}$ & $\mathrm{RCT}$ & $\begin{array}{l}120 \text { CRS } \\
\text { patients or } \\
\text { patients with } \\
\text { recurrent RS }\end{array}$ & $\begin{array}{l}\text { - Crenotherapy treatment (vapour } \\
\text { inhalation, aerosol and nasal douching) } \\
\text { with thermal (salt-bromine-iodic water) } \\
\text { water for } 14 \text { days at Salsomaggiore } \\
\text { Thermal baths } \\
\text { - Nasal douching and aerosol with saline } \\
\text { twice a day for } 14 \text { days at the Rhino- } \\
\text { logic Centre of the ENT Clinic of Siena } \\
\text { University. }\end{array}$ & $\begin{array}{l}\text { At } 2 \text { weeks: } \\
\text { - Symptoms of nasal } \\
\text { obstruction, rhinorrhoea, } \\
\text { nightly awakenings } \\
\text { - Percent of patients with } \\
\text { hyposmia } \\
\text { - Nasal endoscopy (conges- } \\
\text { tion, secretion) } \\
\text { - Mucociliary clearance }\end{array}$ & $\begin{array}{l}\text { At } 4 \text { weeks a significant reduction in } \\
\text { the thermal water nasal spray versus } \\
\text { saline in: } \\
\text { - Symptoms } \\
\text { - Nasal endoscopy } \\
\text { - Mucociliary clearance } \\
\text { - No difference in percentage of } \\
\text { patients with hyposmia }\end{array}$ \\
\hline
\end{tabular}

CRS, chronic rhinosinusitis; DBPCT, double blind placebo controlled trial; RCT, randomised controlled trial; SPA, ; VAS, visual analogue scale. 
quality of the study.

\subsection{Thermal water}

Three Italian studies evaluated the use of thermal water, as nasal irrigation ${ }^{(197)}$, nasal spray ${ }^{(198)}$ or as a combination treatment (vapour inhalation, aerosol and nasal douching) ${ }^{(199)}$ (Table 6.1.11.11). Only one study was double blind ${ }^{(197)}$.

The double blind study of Ottaviano showed no significant differences between the treatments (calculated from data given). The other two studies showed significant improvement of symptoms, nasal endoscopy and nasal mucociliairy clearance. The studies were of very low-quality making conclusions difficult.

\subsubsection{Adverse effects}

Adverse effects of saline irrigations are rare, but include local irritation, ear pain, nosebleeds, headache, nasal burning, and nasal drainage are mentioned. The use of baby-shampoo has been shown to create congestion and reversible smell loss.

\subsubsection{Conclusions}

There are a large number of trials evaluating the efficacy of nasal irrigation. However, the quality of the studies is not always very good which makes it difficult to give a strong recommendation. However, the data show: Nasal irrigation with isotonic saline or Ringer's lactate has efficacy in CRS patients.

There is insufficient data to show that a large volume is more effective than a nasal spray.

The addition of xylitol, sodium hyaluronate, and xyloglucan to nasal saline irrigation may have a positive effect. The addition of baby shampoo, honey, or dexpanthenol as well as higher temperature and higher salt concentration do not confer additional benefit. The steering group advises the use of nasal saline irrigation with isotonic saline or Ringer's lactate potentially with the addition of xylitol, sodium hyaluronate, and xyloglucan and advises against the use of baby shampoo and hypertonic saline solutions due to side effects.

\subsubsection{Aspirin treatment after desensitization (ATAD) in N-ERD}

\subsubsection{Summary of the evidence}

Non-steroidal anti-inflammatory drug (NSAID)-exacerbated respiratory disease (N-ERD) is a chronic eosinophilic, inflammatory disorder of the respiratory tract occurring in patients with asthma and/or CRSwNP, symptoms of which are exacerbated by NSAIDs, including aspirin. Upper airway disease in N-ERD patients is usually CRSwNP. On average, upper respiratory symptoms are worse, extent of opacification on computed tomography (CT) scan and recurrence of nasal polyps after surgery are more frequent in N-ERD than in NSAIDstolerant CRSwNP patients ${ }^{(200,201)}$.

The management options are essentially based on strict avoidance of the culprit drug and cross-reactive drugs. Patient education is important, since NSAIDs respiratory symptoms are not limited to a specific drug, but may appear after the intake of other, strong cyclooxygenase (COX)-1 inhibitors. Weak COX-1 inhibitors (paracetamol) as well as preferential (meloxicam, nimesulide) and selective (celecoxib and etoricoxib) COX-2 inhibitors are usually well tolerated by most N-ERD patients ${ }^{(201)}$. In N-ERD patients, aspirin may induce a period lasting 24 to 72 hours, in which patients are refractory to repeated aspirin challenges and experience clinical improvement ${ }^{(202)}$. Based on this observation, several oral and nasal aspirin desensitization protocols were developed. Most widely used is the Scrippsclinics oral aspirin desensitization protocol, in which, following a stepwise dose increase, $625 \mathrm{mg}$ aspirin is orally administered twice daily ${ }^{(203)}$. Desensitization procedures can be performed in both outpatient and hospital settings and should be supervised by an experienced physician, but to ensure safety and effectiveness of desensitization, it is advised that one of the well-established protocols is followed with gradual dose increase, with at least 90-120 min intervals between doses $^{(203)}$.

\subsubsection{Aspirin treatment after desensitization with oral aspirin} Since EPOS2012 four DBPCTs with ATAD have been performed (Table 6.1.12.1)(204-207). Three of these studies could be metaanalysed to show that ATAD results in a significant and clinically relevant reduction of the SNOT score of 11,9 points (based on SNOT-22)(204-206). Pooled data analysis of the SNOT score showed significant benefit in the ATAD group over placebo (MD -0.54[$0,76,-0,31], p=0.00001$, three trials, 85 patients). The 12 was $0 \%$. For this analysis, mean scores from SNOT-20 and SNOT-22 were taken for assessment (i.e. SNOT-22 scores were divided by 22 and SNOT-20 scores were divided by 20) (Figure 6.1.12.1.). Also, two studies from the same group reported on a significant reduction in total symptoms.

Pooled data analysis of the total symptoms score (total score 27) showed significant benefit in the ATAD group over placebo (MD -3.51 [-5.2-, -1.81], $\mathrm{p}=0.0001$, two trials, 70 patients) (Figure 6.1.12.2.) and Forced Expiratory Volume 1 (FEV1) (MD 6.43 [3.26-, 9.61], $\mathrm{p}=0.0001$, two trials, 70 patients) (Figure 6.1.12.3.).

Aspirin treatment after desensitization (ATAD) with oral aspirin is effective in improving QOL and total nasal symptom score in patients with N-ERD.

The study by Fruth ${ }^{(207)}$ was interesting in that it had a 36 months follow-up. Unfortunately, 39 out of 70 patients, 18 of the aspirin and 21 of the placebo group, discontinued the treatment in that period, although none because of side effects of the aspirin. In the patients that completed the full follow-up period there was a trend to less recurrences of polyps in the ATAD group compared to placebo and the ATAD groups showed significantly fewer overall nasal and paranasal complaints, less quality-oflife impairment by nasal and paranasal complaints, and better general health condition.

\subsubsection{Aspirin treatment after desensitization with nasal lysine-aspirin}

Nasal administration of lysine-aspirin reduces the risk of severe 
Table 6.1.12.1. ATAD in the treatment of CRS patients with N-ERD.

\begin{tabular}{|c|c|c|c|c|c|}
\hline Study & Methods & Participants & Interventions & Outcomes & Results \\
\hline $\begin{array}{l}\text { Mortazavi } \\
2017^{(204)}\end{array}$ & DBPCT & $\begin{array}{l}41 \text { CRSwNP } \\
\text { (38 analysed) } \\
\text { with aspirin } \\
\text { intolerance }\end{array}$ & $\begin{array}{l}\text { - } 650 \text { mg of aspirin twice } \\
\text { every day for } 1 \text { month } \\
\text { and } 325 \text { mg of the same } \\
\text { twice daily for the next } 5 \\
\text { months }(n=19) \\
\text { - Placebo }(n=19)\end{array}$ & $\begin{array}{l}\text { - SNOT-22 at } 6 \text { months } \\
\text { - Symptom Score (0-27) at } 6 \text { months } \\
\text { - Medication Score at } 6 \text { months } \\
\text { - } \text { CT scan (LM score) at } 6 \text { months } \\
\text { - Serum IL-4 and IL-5 } \\
\text { - Asthma exacerbations }\end{array}$ & $\begin{array}{l}\text { In the aspirin treated group } \\
\text { compared to placebo at } 6 \\
\text { months: } \\
\text { - Decreased SNOT, symptom } \\
\text { score, medication score } \\
\text { - Higher FEV1 } \\
\text { - Lower serum IL-5 }\end{array}$ \\
\hline $\begin{array}{l}\text { Esmaeilzadeh, } \\
2015^{(205)}\end{array}$ & DBPCT & $\begin{array}{l}34 \text { CRSwNP } \\
\text { (32 analysed) } \\
\text { with aspirin } \\
\text { intolerance }\end{array}$ & $\begin{array}{l}\text { - } 650 \text { mg of aspirin twice } \\
\text { every day for } 1 \text { month } \\
\text { and } 325 \text { mg of the same } \\
\text { twice daily for the next } 5 \\
\text { months }(n=16) \\
\text { - Placebo }(n=16)\end{array}$ & $\begin{array}{l}\text { - SNOT-22 at } 1 \text { and } 6 \text { months } \\
\text { - Symptom Score at } 1 \text { and } 6 \text { months } \\
\text { - Medication Score at } 1 \text { and } 6 \text { months } \\
\text { - } \text { CT scan (LM score) at } 6 \text { months } \\
\text { - FEV1 at } 1 \text { and } 6 \text { months } \\
\text { - Serum IL-10, IFN- } \gamma \text { and TGF- } \beta \text { at } 6 \\
\text { - } \text { months } \\
\text { - Asthma exacerbations } \\
\text { Adverse events }\end{array}$ & $\begin{array}{l}\text { In the aspirin treated group } \\
\text { compared to placebo at } 6 \\
\text { months: } \\
\text { - Decreased SNOT and symptom } \\
\text { score } \\
\text { - Higher FEV1 } \\
\text { - No significant difference in } \\
\text { other parameters measured } \\
\text { - } 1 \text { patient in aspirin groups had } \\
\text { severe intestinal bleeding }\end{array}$ \\
\hline $\begin{array}{l}\text { Świerczyńska- } \\
\text { Krępa 2014(206) }\end{array}$ & DBPCT & $\begin{array}{l}34 \text { CRSwNP } \\
\text { [20 aspirin } \\
\text { intolerant } \\
\text { (AIA), } 14 \\
\text { aspirin } \\
\text { tolerant (ATA)] }\end{array}$ & $\begin{array}{l}\text { - } 624 \text { mg of aspirin } 1 \mathrm{dd} \\
\text { for } 6 \text { months [ } \mathrm{n}=18(12 \\
\text { AIA)] } \\
\text { - Placebo for } 6 \text { months } \\
{[n=16(8 \text { AIA)] }}\end{array}$ & $\begin{array}{l}\text { - Nasal symptoms monthly } \\
\text { - SNOT20 monthly } \\
\text { - PNIF monthly } \\
\text { - Asthma Control Questionnaire (ACQ) } \\
\text { monthly } \\
\text { - Spirometric parameters (and PEF) } \\
\text { - } \text { Blonthly } \\
\text { - Corticosteroid doses monthly } \\
\text { - Urinary leukotriene E4 and the (PG) } \\
\text { D2 metabolite 9a,11b-PGF2 after 1,3, } \\
5 \text { and } 6 \text { months. }\end{array}$ & $\begin{array}{l}\text { - Only the patients with AIA } \\
\text { subjected to AD reported } \\
\text { improvements in smell and } \\
\text { reductions in sneezing and } \\
\text { nasal blockade } \\
\text { - The SNOT20 and Asthma } \\
\text { Control Questionnaire scores } \\
\text { of these patients decreased, } \\
\text { and their peak nasal inspiratory } \\
\text { flows increased } \\
\text { - The dosages of inhaled } \\
\text { corticosteroids were reduced } \\
\text { - No changes in leukotriene E4 } \\
\text { or 9a,11b-PGF2 levels after AD }\end{array}$ \\
\hline $\begin{array}{l}\text { Fruth } \\
2013^{(207)}\end{array}$ & $\begin{array}{l}\text { DBPCT } 6 \\
\text { wks post- } \\
\text { operative }\end{array}$ & $\begin{array}{l}70 \text { CRSwNP } \\
\text { with N-ERD } \\
\text { ( } 30 \text { evaluated) }\end{array}$ & $\begin{array}{l}\text { - After desensitization } \\
\text { maintenance dose of } \\
100 \text { mg daily for } 36 \\
\text { months ( } n=18) \\
\text { - After desensitization } \\
\text { maintenance dose of } \\
\text { placebo daily for } 36 \\
\text { months ( } n=13)\end{array}$ & $\begin{array}{l}\text { - QOL (Rhinosinusitis Disability Index } \\
\text { questionnaire) at 6, 12, } 24 \text { and } 36 \\
\text { - Symths } \\
\text { airway obstruction, postnasal drip, } \\
\text { cephalgia, impairment of olfactory } \\
\text { function } \\
\text { - Coughing and sneezing (0-2) } \\
\text { - Total symptom score }(0-20) \\
\text { - Smell (Sniffin' Sticks) at 6, 12, } 24 \text { and } \\
36 \text { months } \\
\text { - Nasal polyp score (0-3) } \\
\text { - Overall nasal and paranasal com- } \\
\text { - plaints [1 (best)-7 (worst)] } \\
\text { - Quality-of-life impairment by nasal } \\
\text { and paranasal complaints (1-7) } \\
\text { - General health condition (1-7) } \\
\text { - Revision sinus surgery }\end{array}$ & $\begin{array}{l}\text { In aspirin treated group compared } \\
\text { to placebo at } 36 \text { months: } \\
\text { - Significantly better quality of } \\
\text { life score } \\
\text { - Significantly lower total } \\
\text { symptom score } \\
\text { - Lower quality of life } \\
\text { impairment by nasal and } \\
\text { paranasal complaints } \\
\text { - Lower overall nasal and } \\
\text { paranasal complaints, quality } \\
\text { of life impairment by nasal } \\
\text { and paranasal complaints and } \\
\text { general health condition } \\
\text { - Less revision sinus surgery }\end{array}$ \\
\hline
\end{tabular}

$A C Q$, Asthma Control Questionnaire; ; AD, Aspirin Desensitizatio ; AIA, Aspirin-induced Asthma ; ATA, Aspirin-tolerant Asthma ; CRSwNP, chronic rhinosinusitis with nasal polyps; CT, computed tomography; DBPCT, double blind placebo controlled trial; FEV1, forced expiratory volume 1; IFN, interferon; IL, interleukin; LM, Lund-Mackay; N-ERD, NSAID-exacerbated respiratory disease ; PEF, Pulmonary Expiratory Flow ; PG, Prostaglandin ; PNIF, peak nasal inspiratory flow; QOL, quality of life; SNOT, Sino-nasal Outcome Test; TGF, Transforming Growth Factor . 
Table 6.1.12.2. Nasal lysine aspirin solution for the treatment of CRS patients with N-ERD.

\begin{tabular}{|c|c|c|c|c|c|}
\hline Study & Methods & Participants & Interventions & Outcomes & Results \\
\hline Parikh $2005^{(211)}$ & $\begin{array}{l}\text { DBPC } \\
\text { cross-over } \\
\text { trial }\end{array}$ & $\begin{array}{l}22 \text { aspirin- } \\
\text { sensitive patients } \\
\text { (19 CRSwNP), } 11 \\
\text { completed the } \\
\text { full study ( } 2 \text { one } \\
\text { phase of cross- } \\
\text { over) }\end{array}$ & $\begin{array}{l}\text { - Incremental doses of lysine aspirin } \\
\text { solution (LAS) until a dose of } 16 \text { mg } \\
\text { was tolerated followed by LAS every } \\
48 \text { hours for } 6 \text { months ( } n=11 \text { ) } \\
\text { - Incremental doses of lysine aspirin } \\
\text { solution (LAS) until a dose of } 16 \text { mg } \\
\text { was tolerated followed by placebo } \\
\text { every } 48 \text { hours for } 6 \text { months ( } n=11 \text { ) }\end{array}$ & $\begin{array}{l}\text { - Nasal and pulmonary symptom } \\
\text { - Pcores } \\
\text { - Neak expiratory flow rate (PEFR) } \\
\text { - Nasal inspiratory flow (PNIF), } \\
\text { - Acoustic rhinometry }\end{array}$ & $\begin{array}{l}\text { - No significant } \\
\text { differences } \\
\text { between the } \\
\text { groups }\end{array}$ \\
\hline
\end{tabular}

DBPC, Double Blind Placebo Controlled; CRSwNP, Chronic Rhinosinusitis with nasal polyps; LAS, lysine aspirin solution; PEFR, peak expiratory flow rate; PNIF, peak nasal inspiratory flow

Table 6.1.12.3. Low salicylate diet for the treatment of CRS patients with N-ERD.

\begin{tabular}{|c|c|c|c|c|c|}
\hline Study & Methods & Participants & Interventions & Outcomes & Results \\
\hline $\begin{array}{l}\text { Sommer } \\
2015^{(874)}\end{array}$ & $\begin{array}{l}\text { Crossover } \\
\text { RCT }\end{array}$ & $\begin{array}{l}14 \text { aspirin-sensitive } \\
\text { patients ( } 10 \\
\text { evaluated) }\end{array}$ & $\begin{array}{l}6 \text { weeks low-salicylate } \\
(n=10) \\
\text { - } 6 \text { weeks normal diet } \\
(n=10)\end{array}$ & $\begin{array}{l}\text { - } \text { SNOT-22 } \\
\text { - Nasal Symptom Severity Score (NSSS) } \\
\text { - Lund-Kennedy score } \\
\text { - POSE } \\
\text { - } \text { ACQ-7 }\end{array}$ & $\begin{array}{l}\text { - No significant differences } \\
\text { apart from POSE }\end{array}$ \\
\hline $\begin{array}{l}\text { Sommer } \\
2016^{(875)}\end{array}$ & $\begin{array}{l}\text { Partially } \\
\text { single blind } \\
\text { crossover } \\
\text { RCT }\end{array}$ & $\begin{array}{l}30 \text { aspirin-sensitive } \\
\text { patients ( } 27 \\
\text { evaluated) including } \\
\text { the patients } \\
\text { from }^{(874)}\end{array}$ & $\begin{array}{l}6 \text { weeks low-salicylate } \\
(n=30) \\
\text { - } 6 \text { weeks normal diet } \\
(n=30)\end{array}$ & $\begin{array}{l}\text { - SNOT-22 } \\
\text { - Nasal Symptom Severity Score (NSSS) } \\
\text { - Lund-Kennedy score } \\
\text { - POSE } \\
\text { - } \text { ACQ-7 }\end{array}$ & $\begin{array}{l}\text { - All parameters significantly } \\
\text { better in patients on low- } \\
\text { salicylate diet }\end{array}$ \\
\hline
\end{tabular}

RCT, Randomised Controlled Trial ; SNOT, Sino-Nasal Outcome ; NSSS, Nasal Symptom Severity Score; LK, Lund-Kennedy; POSE, Perioperative Sinus Endoscopy score ; ACQ, Asthma Control Questionnaire

Table 6.1.12.4. Prasugrel for the treatment of CRS patients with N-ERD.

\begin{tabular}{|c|c|c|c|c|c|}
\hline Study & Methods & Participants & Interventions & Outcomes & Results \\
\hline $\begin{array}{l}\text { Laidlaw } \\
2018^{(876)}\end{array}$ & $\begin{array}{l}\text { DBPC } \\
\text { crossover } \\
\text { trial }\end{array}$ & $\begin{array}{l}46 \text { aspirin- } \\
\text { sensitive patients } \\
\text { ( } 40 \text { evaluated), } \\
\text { unclear how many } \\
\text { had CRS }\end{array}$ & $\begin{array}{l}\text { - Prasugrel } 5 \mathrm{mg} / \mathrm{d} \text { for patients weigh- } \\
\text { ing }<60 \mathrm{~kg} \text { or Prasugrel } 10 \mathrm{mg} / \mathrm{d} \text { for } \\
\text { patients weighing } \geq 60 \mathrm{~kg} \text { after a } 60 \text { - } \\
\text { mg loading dose) for } 4 \text { weeks ( } \mathrm{n}=40 \text { ) } \\
\text { - Placebo for } 4 \text { weeks } n=40 \text { ) }\end{array}$ & $\begin{array}{l}\text { - Change in the provocative dose of aspirin } \\
\text { at } 4 \text { weeks } \\
\text { - Changes in lung function, urinary eicosa- } \\
\text { noids, plasma tryptase, platelet-leukocyte } \\
\text { aggregates, and platelet activation at } 4 \\
\text { weeks }\end{array}$ & $\begin{array}{l}\text { - No } \\
\text { significant } \\
\text { differences }\end{array}$ \\
\hline
\end{tabular}

CRS, Chronic Rhinosinusitis; DBPC, Double Blind Placebo Controlled

hypersensitivity reactions and the frequency of gastrointestinal side effects associated with oral aspirin desensitization. Some retrospective studies reported clinical benefit from nasal lysine-aspirin treatment ${ }^{(208-210)}$. In a randomized, double-blind placebo-controlled cross over trial, N-ERD patients with positive nasal lysine-aspirin challenge received either $16 \mathrm{mg}$ nasal lysine aspirin or placebo every 48 hours for six months. Of 22 patients entering the trial, 11 were eligible for analysis. Multivariate analysis of measured parameters did not reveal a significant clinical benefit to patients receiving topical lysine-aspirin compared with placebo ${ }^{(211)}$ (Table 6.1.12.2).

\subsubsection{Diets}

Recently two crossover RCTs evaluated the effect of a low salicylate diet on sinus (SNOT-22) and asthma (ACQ-7) quality of life. The second study contained the data of the first study. The second study showed significant and clinically relevant improvement in SNOT22 (15.5 points) and ACQ-7 (5 points). Also, the Nasal Symptom Severity Score, the Lund-Kennedy score and the POSE showed significantly better results with the low salicylate diet than with the normal diet (Table 6.1.12.3). Moreover, a cohort study was performed on the effect of a high omega-3/low omega- 6 diet for the treatment of aspirin exacerbated respiratory disease (AERD) ${ }^{(212)}$. The authors concluded that a high omega-3/low omega- 6 diet may be an appropriate adjunct treatment option for patients with AERD.

\subsubsection{Prasugrel}

Finally, a DBPC crossover trial was performed with Prasugrel. Prasugrel is a platelet inhibitor and an irreversible antagonist of $P 2 Y_{12}$ ADP receptors. The study showed no significant effects on change in aspirin provocation dose or lung function (Table 6.1.12.4).

\subsubsection{Side effects of ATAD}

ATAD is associated with adverse effects (mostly gastrointestinal), and the incidence of adverse symptoms related to aspirin intake ranges from $0 \%$ to $34 \%{ }^{(201)}$. In order to reduce the prevalence of 
Figure 6.1.12.1. Forest plot of the effect of ATAD versus standard treatment alone on the SNOT score six months after start of the treatment in patients with CRSwNP.

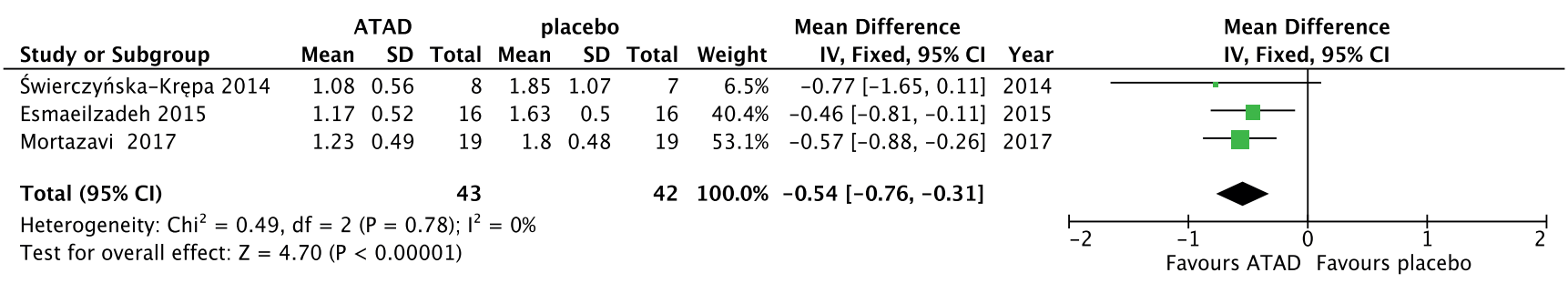

Figure 6.1.12.2. Forest plot of the effect of ATAD versus standard treatment alone on the symptom score six months after start of the treatment in patients with CRSwNP.

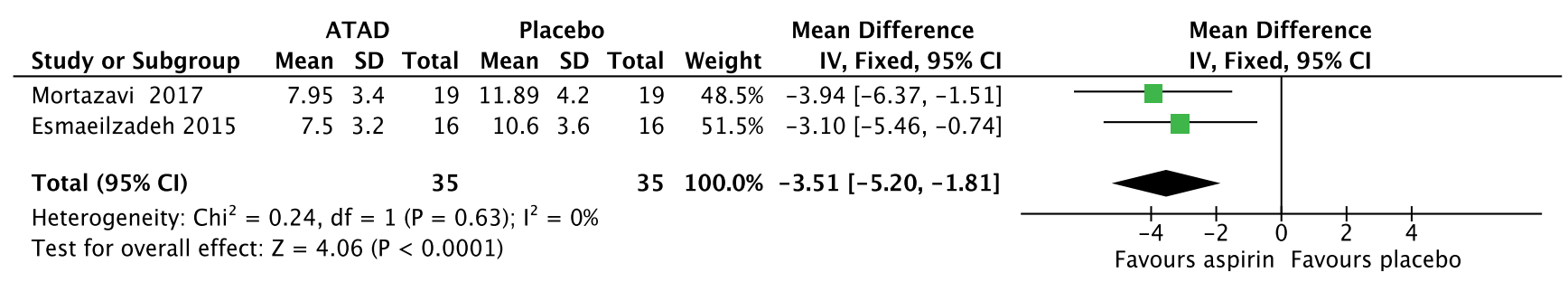

Figure 6.1.12.3. Forest plot of the effect of ATAD versus standard treatment alone on the FEV1 six months after start of the treatment in patients with CRSwNP.

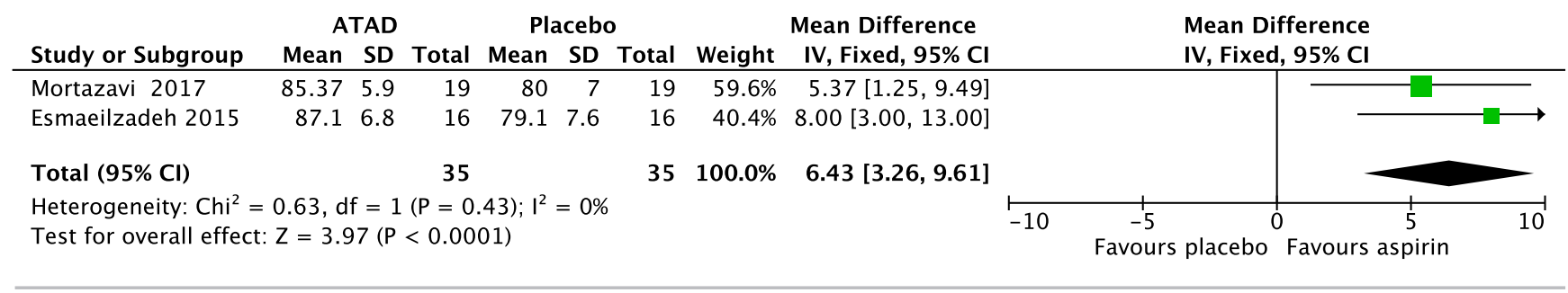

adverse effects associated with aspirin treatment, appropriate preventive measures (Helicobacter pylori eradication, PPI, and H2blockers) should be introduced and continued during the treatment ${ }^{(201)}$.

\subsubsection{Conclusions}

Oral ATAD has been shown to be significantly more effective and clinically relevant than placebo in improving QOL (measured with SNOT) and total nasal symptom score in patients with $\mathrm{N}$-ERD. However, the change in SNOT from treating with oral ATAD compared to placebo did not reach the clinically important mean difference (CIMD) (MD -0.54[-0,76, -0,31], $\mathrm{p}=0.00001$, three trials, 85 patients). ATAD reduced symptoms after six months compared to placebo (with MD -3.51 [-5.2-, -1.81 ], $p=0.0001$, two trials, 70 patients on a scale of 27), the FEV1 showed a significant reduction compared to placebo and the data were consistent. However, ATAD is associated with significant adverse effects, and the risks of not taking the medication strictly on a daily basis puts a burden on patient and caregiver. Based on these data, the EPOS2020 steering group suggests that ATAD can be a treatment for N-ERD patients with CRSwNP whenever there is confidence in the patient's compliance.

Lysine aspirin and platelet inhibitors (like Prasugrel) have not been shown to be an effective treatment in CRSwNP patients with N-ERD and are not advised. Diets, like low salicylate diet were shown to improve endoscopic scores and may improve symptoms compared to a normal diet in patients with N-ERD. However, the quality of the data at this moment is not enough to draw further conclusions.

\subsubsection{Antimycotics}

\subsubsection{Introduction}

Although the aetiology of chronic rhinosinusitis is not fully understood, it may involve abnormalities in the host response to irritants, commensal and pathogenic organisms and allergens. There is much debate regarding the role of fungus in the aetiology of chronic rhinosinusitis. Intranasal fungus can be demonstratedin nearly all diseased and normal sinuses ${ }^{(213-215)}$. Although fungi are ubiquitous in our environment, there are several forms of sinus disease that are associated with fungi as pathogens. However, rather than the fungi determining the disease process, it is usually the host immune state that 
determines the clinical presentation. The role of antifungal therapies in the setting of established fungal-related CRS is summarised in Fungal associated RS (Chapter 9.6). This chapter summarises the role of antimycotics in the setting of CRSwNP and CRSsNP.

Based on fungal detection and the presence of allergic mucus in almost all patients with chronic rhinosinusitis, Ponikau and co-authors proposed that CRS is generally caused by a dysregulated, but IgE independent immune response to fungal elements present on the mucosal surface ${ }^{(213)}$.

Consequently, reduction of fungus load should influence disease severity in all subtypes of CRS. This hypothesis led to a series of investigations, as proof of this concept of fungal-driven inflammation in CRS. In these studies, patients complying with the AAO-HNS or EPOS definitions of CRS were included ${ }^{(216,}$ 217) irrespective of the presence of eosinophilic mucus and/or fungus detection. In this systematic review studies of patients fulfilling the AAO-HNS or EPOS definitions of CRS (with or without nasal polyps) are evaluated. Subgroup analyses explore potential differences where possible.

\subsubsection{Topical amphotericin $B$}

In most trials with antifungals in CRS, amphotericin B was applied topically, either as a nasal spray or as a nasal irrigation. The majority of patients included in these trials suffered from CRSwNP. However, the presence of nasal polyps was not explicitly reported in all trials. Recently a Cochrane review has been published evaluating the effect of topical amphotericin $B$ in CRS with or without NP but not in patients that recently had surgery ${ }^{(218)}$. The authors included eight studies (490 adult participants). No studies that specifically investigated the effect of antifungals in patients with AFRS were included (Chapter 8.6.5.4). Seven studies (437 participants) were included that used amphotericin B (six studies; 383 participants). The authors concluded that due to the very low quality of the evidence, it is uncertain whether the use of topical or systemic antifungals has an impact on patient outcomes in adults with CRS compared with placebo or no treatment. In terms of adverse effects, they concluded that topical antifungals may lead to more local irritation compared with placebo.

\section{Local and systemic antifungal treatments do not have a positive effect of $Q O L$, symptoms and signs of disease in patients with CRS.}

We systematically reviewed the literature and included nine studies [627 CRS patients (373 CRSwNP; 567 CRS patients) completed the trials], local antifungal treatment, eight studies comparing amphotericin B and in one study, fluconazole nasal drops ${ }^{(219)}$ with placebo (usually yellow coloured saline) $)^{(219-227)}$ (Table 6.1.13.1.). Three studies involved patients with CRSwNP(220, ${ }^{222,224)}$, one study ${ }^{(223)}$ involved patients with CRSsNP, and the other

Figure 6.1.13.1. Forest plot of the effect of local antifungal treatment versus placebo on SNOT score in patients with CRSwNP after 2-3 months of treatment.

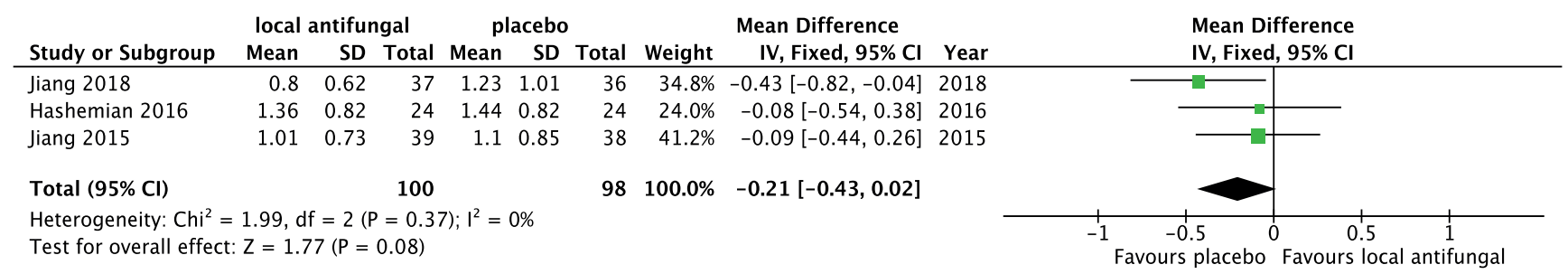

Figure 6.1.13.2. Forest plot of the effect of local antifungal treatment versus placebo on total symptom score in patients with CRSwNP after 2-3 months of treatment.

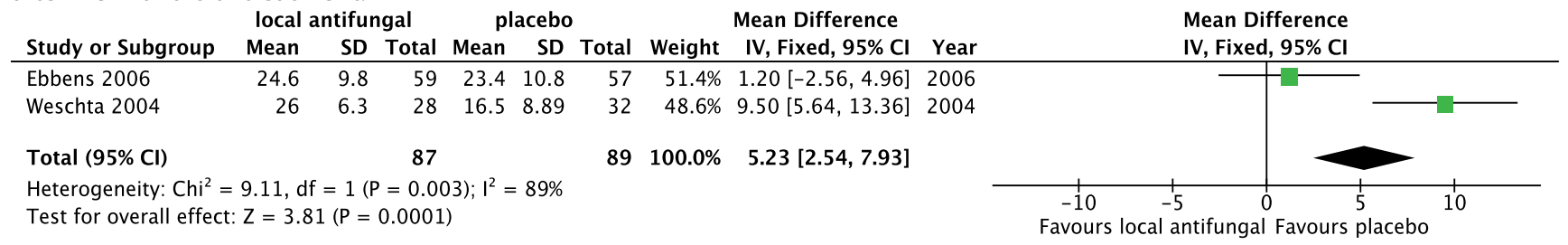

Figure 6.1.13.3. Forest plot of the effect of local antifungal treatment versus placebo on nasal endoscopy score in patients with CRSwNP after 2-3 months of treatment.

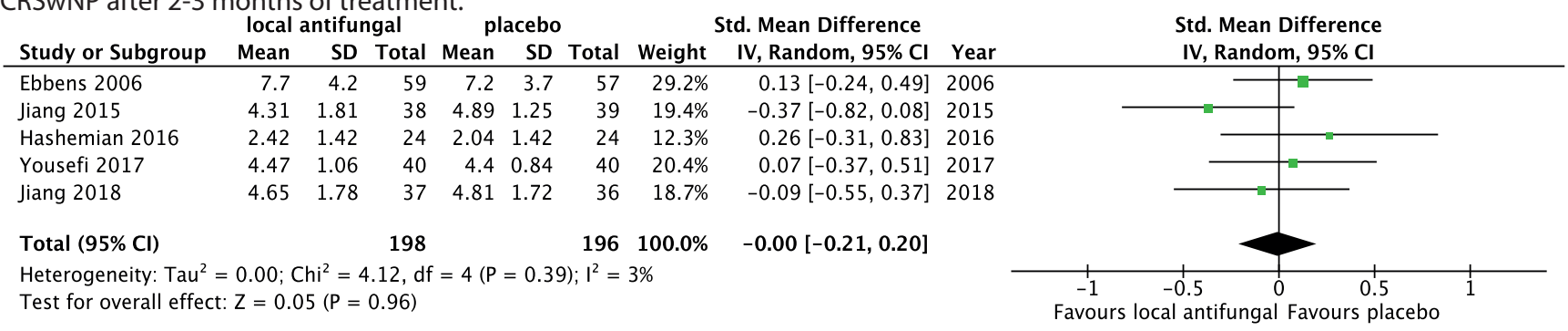


Figure 6.1.13.4. Forest plot of the effect of local antifungal treatment versus placebo on CT scan score in patients with CRSwNP after 2-3 months of treatment

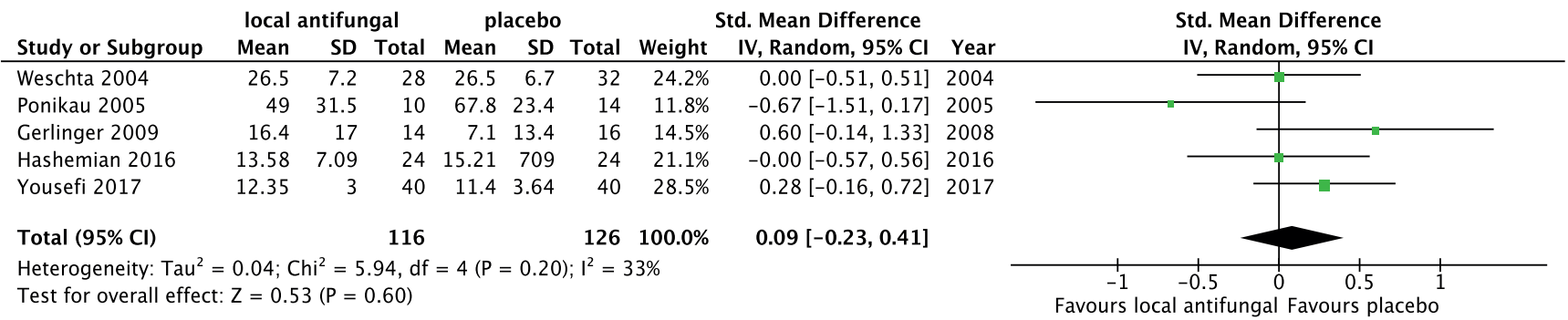

Table 6.1.13.1. Local antifungal treatment for the treatment of patients with CRS.

\begin{tabular}{|c|c|c|c|c|c|}
\hline Study & Methods & Participants & Interventions & Outcomes & Results \\
\hline $\begin{array}{l}\text { Jiang } \\
2018^{(227)}\end{array}$ & DBPCT & $\begin{array}{l}79 \text { CRS } \\
\text { patients ( } 26 \\
\text { CRSwNP) }(73 \\
\text { completed the } \\
\text { trial) starting } \\
1 \text { month } \\
\text { postoperative } \\
\text { FESS }\end{array}$ & $\begin{array}{l}50 \mathrm{~mL} \text { of } 200 \mu \mathrm{g} / \mathrm{mL} \text { of amphotericin } \\
\text { B per nostril (daily dose } 20 \mathrm{mg} \text { ) once } \\
\text { a day ( } \mathrm{n}=37 \text { ) for } 2 \text { months } \\
50 \mathrm{~mL} \text { of saline ( } \mathrm{n}=36)\end{array}$ & $\begin{array}{l}\text { - Taiwanese SNOT-22 at } 2 \\
\text { - Enonths } \\
\text { - Smell test at } 2 \text { months } \\
\text { - Saccharine transit test at } 2 \\
\text { months } \\
\text { - Bacterial culture at } 2 \text { months }\end{array}$ & $\begin{array}{l}\text { No significant difference for any } \\
\text { outcome }\end{array}$ \\
\hline $\begin{array}{l}\text { Yousefi } \\
2017^{(226)}\end{array}$ & DBPCT & $\begin{array}{l}80 \text { CRS patients } \\
\text { (40 CRSwNP) }\end{array}$ & $\begin{array}{l}\text { - } 10 \mathrm{~mL} \text { (5 mL per nostril) of } 200 \text { mg/ } \\
\mathrm{mL} \text { amphotericin B twice daily (daily } \\
\text { dose } 8 \mathrm{mg} \text { ) for } 3 \text { months } \\
\text { - } 10 \mathrm{~mL} \text { placebo ( } 5 \mathrm{~mL} \text { per nostril) } \\
\text { twice daily for } 3 \text { months }\end{array}$ & $\begin{array}{l}\text { - } \text { RSOM-31 at } 3 \text { months } \\
\text { - VAS symptom score (for nasal } \\
\text { congestion, post-nasal drip, } \\
\text { reduction in the sense of smell } \\
\text { and facial pain) at } 3 \text { months } \\
\text { - Nasal endoscopy at } 13 \text { weeks } \\
\text { - Blood levels of IgE, IL-4, IL- } \\
\text { 5,and IL-13 } \\
\text { - CT and MRI }\end{array}$ & $\begin{array}{l}\text { No significant difference for } \\
\text { any outcome apart from facial } \\
\text { pain that was better in the } \\
\text { amphotericin-treated group }\end{array}$ \\
\hline $\begin{array}{l}\text { Hashemian } \\
2016^{(219)}\end{array}$ & DBPCT & $\begin{array}{l}54 \text { CRS patients } \\
\text { ( } 21 \text { CRSwNP) } \\
\text { ( } 48 \text { completed } \\
\text { the study) }\end{array}$ & $\begin{array}{l}\text { - } 12 \text { drops per day, } 2 \text { times a day of } \\
\text { fluconazole nasal drops }(0.2 \%,) \text { (daily } \\
\text { dose } 1.2 \mathrm{mg}) \text { for } 8 \text { wks }(n=27) \\
\text { - Placebo nasal drops }(12 \text { drops per } \\
\text { day, } 2 \text { times a day) for } 8 \text { wks }(n=27)\end{array}$ & $\begin{array}{l}\text { - } \text { SNOT-20 at } 8 \text { wks } \\
\text { - Endoscopy at } 8 \text { wks } \\
\text { - CT scan at } 8 \text { wks } \\
\text { - Adverse effects }\end{array}$ & $\begin{array}{l}\text { No significant difference for any } \\
\text { outcome }\end{array}$ \\
\hline $\begin{array}{l}\text { Jiang } \\
2015^{(225)}\end{array}$ & DBPCT & $\begin{array}{l}87 \text { CRS } \\
\text { patients ( } 33 \\
\text { CRSwNP) (77 } \\
\text { completed the } \\
\text { trial) starting } \\
1 \text { month } \\
\text { postoperative } \\
\text { FESS }\end{array}$ & $\begin{array}{l}\text { - } 50 \mathrm{~mL} \text { of } 100 \mu \mathrm{g} / \mathrm{mL} \text { of amphotericin } \\
\text { B per nostril (daily dose } 20 \mathrm{mg} \text { ) once } \\
\text { a day ( } \mathrm{n}=37) \text { for } 2 \text { months } \\
\text { - } 50 \mathrm{~mL} \text { of saline }(\mathrm{n}=36)\end{array}$ & $\begin{array}{l}\text { - Taiwanese SNOT-22 at } 2 \\
\text { - Enonths } \\
\text { - Smell test at } 2 \text { months } \\
\text { - Saccharine transit test at } 2 \\
\text { months } \\
\text { - Bacterial culture at } 2 \text { months }\end{array}$ & $\begin{array}{l}\text { No significant difference for any } \\
\text { outcome }\end{array}$ \\
\hline $\begin{array}{l}\text { Gerlinger } \\
2009^{(224)}\end{array}$ & DBPCT & $\begin{array}{l}33 \text { CRSwNP } \\
\text { patients ( } 30 \\
\text { completed the } \\
\text { trial) directly } \\
\text { postoperative } \\
\text { FESS }\end{array}$ & $\begin{array}{l}\text { - Amphotericin B ( } 5 \mathrm{mg} / \mathrm{mL}) \text {, nasal } \\
\text { spray, } 2 \text { puffs per nostril }(100 \mu \mathrm{\mu l} \text { per } \\
\text { nostril), twice daily (total daily dose } \\
=4.8 \mathrm{mg}) \text { for } 8 \text { wks }(\mathrm{n}=16) \\
\text { - Placebo nasal spray }(0.2 \mu \mathrm{g} / \mathrm{mL} \\
\text { acriflavin chloride solution ) for } 8 \\
\text { wks ( } n=17)\end{array}$ & $\begin{array}{l}\text { - } \text { CT scan at } 8 \text { wks } \\
\text { - SNAQ-11 } 1^{(877)} \text { at } 8 \text { wks } \\
\text { - Nasal endoscopy at } 8 \text { wks }\end{array}$ & $\begin{array}{l}\text { No significant difference for any } \\
\text { outcome }\end{array}$ \\
\hline $\begin{array}{l}\text { Liang } \\
2008^{(223)}\end{array}$ & DBPCT & $\begin{array}{l}70 \text { CRSsNP (64 } \\
\text { completed the } \\
\text { study) }\end{array}$ & $\begin{array}{l}\text { - } 250 \mathrm{~mL} \text { of amphotericin B } \\
\text { (containing } 10 \text { mg of amphotericin } \\
\text { B) per nostril once daily (total daily } \\
\text { dose }=20 \mathrm{mg} \text { ) for } 4 \text { wks ( } n=35 \text { ) } \\
\text { - } 250 \mathrm{~mL} \text { of placebo per nostril once } \\
\text { daily (total daily dose }=20 \mathrm{mg} \text { ) for } 4 \\
\text { wks ( } \mathrm{n}=35 \text { ) }\end{array}$ & $\begin{array}{l}\text { - Chinese RSOM-31 at } 2 \text { and } \\
4 \text { wks } \\
\text { - Endoscopy } \\
\text { - Fungal and bacterial cultures }\end{array}$ & $\begin{array}{l}\text { No significant difference for any } \\
\text { outcome }\end{array}$ \\
\hline
\end{tabular}




\begin{tabular}{|c|c|c|c|c|c|}
\hline Study & Methods & Participants & Interventions & Outcomes & Results \\
\hline $\begin{array}{l}\text { Ebbens } \\
2006^{(222)}\end{array}$ & DBPCT & $\begin{array}{l}116 \text { CRSwNP } \\
\text { patients ( } 111 \\
\text { completed the } \\
\text { study) }\end{array}$ & $\begin{array}{l}\text { - } 25 \mathrm{~mL} \text { of amphotericin B }(100 \mathrm{\mu g} / \\
\mathrm{mL} \text { ) in } 2.5 \% \text { glucose applied to } \\
\text { each nostril twice daily for } 13 \text { weeks } \\
\text { ( } \mathrm{n}=59 \text { ) (daily dose } 10 \mathrm{mg}) \\
\text { - } 25 \mathrm{~mL} \text { of placebo nasal lavage ( } 3.4 \\
\mathrm{~mL} / \mathrm{L} \text { Cernevit in } 2.5 \% \text { glucose) } \\
\text { applied to each nostril twice daily for } \\
13 \text { weeks ( } \mathrm{n}=57 \text { ) }\end{array}$ & $\begin{array}{l}\text { - RSOM-31 at } 13 \text { weeks } \\
\text { - Total VAS symptom score (the } \\
\text { sum of VAS for nasal block- } \\
\text { age, rhinorrhoea, facial pain, } \\
\text { postnasal drip and anosmia) at } \\
13 \text { weeks } \\
\text { - SF36 at } 13 \text { weeks } \\
\text { - Endoscopy at } 13 \text { weeks } \\
\text { - PNIF at } 13 \text { weeks } \\
\text { - Levels of pro-inflammatory } \\
\text { cytokines, chemokines and } \\
\text { growth factors and albumin } \\
\text { - Adverse effects }\end{array}$ & $\begin{array}{l}\text { No significant differences in any } \\
\text { parameter measured }\end{array}$ \\
\hline $\begin{array}{l}\text { Ponikau } \\
2005^{(221)}\end{array}$ & DBPCT & $\begin{array}{l}30 \text { CRS patients } \\
\text { (24 completed } \\
\text { the study) }\end{array}$ & $\begin{array}{l}\text { - } 20 \mathrm{~mL} \text { amphotericin B solution ( } 250 \\
\mu \mathrm{g} / \mathrm{mL} \text { ) per nostril twice daily for } 6 \\
\text { months ( } n=15 \text { ) (daily dose } 20 \mathrm{mg} \text { ) } \\
\text { - } 20 \mathrm{~mL} \text { placebo solution per nostril } \\
\text { twice a day for } 6 \text { months }(n=15)\end{array}$ & $\begin{array}{l}\text { - SNOT-20 at } 3 \text { and } 6 \text { months } \\
\text { - Endoscopy at } 3 \text { and } 6 \text { months. } \\
\text { - CT scan at } 6 \text { months } \\
\text { - Adverse effects }\end{array}$ & $\begin{array}{l}\text { - No significant differences in } \\
\text { SNOT-20 at } 3 \text { and } 6 \text { months } \\
\text { - Significant better change } \\
\text { from baseline for endoscopy } \\
\text { at } 3 \text { and } 6 \text { months and for } \\
\text { CT scan at } 6 \text { months in } \\
\text { the amphotericin B group } \\
\text { compared to placebo } \\
\text { - } 2 \text { patients in the amphotericin } \\
\text { B group complained of } \\
\text { burning }\end{array}$ \\
\hline $\begin{array}{l}\text { Weschta } \\
2004^{(220)}\end{array}$ & DBPCT & $\begin{array}{l}78 \text { CRSwNP } \\
\text { patients ( } 60 \\
\text { completed the } \\
\text { study) }\end{array}$ & $\begin{array}{l}\text { - Amphotericin B ( } 3 \mathrm{mg} / \mathrm{mL} \text { ), nasal } \\
\text { spray, } 2 \text { puffs per nostril ( } 200 \text { } \mu \text { Lper } \\
\text { nostril), } 4 \text { times daily (total daily dose } \\
=4.8 \mathrm{mg} \text { ) for } 8 \text { wks ( } \mathrm{n}=39 \text { ) } \\
\text { - Placebo nasal spray: saline solution } \\
\text { containing tartrazine, chinin sulfate, } \\
\text { 1-(4-sulfo-1-phenylazo)-2-naphthol- } \\
\text { 6-sulfo acid, choline in } 5 \% \text { glucose } \\
\text { solution, } 2 \text { puffs per nostril, } 4 \text { times } \\
\text { daily for } 8 \text { wks ( } \mathrm{n}=39 \text { ) }\end{array}$ & $\begin{array}{l}\text { - Rhinosinusitis quality of life } \\
\text { score (RQL) (0-36) at } 8 \text { wks } \\
\text { - VAS for nasal blockage, facial } \\
\text { pain, smell, nasal discharge } \\
\text { sneezing at } 8 \text { wks } \\
\text { - Endoscopy at } 8 \text { wks } \\
\text { - CT scan at } 8 \text { wks } \\
\text { - Adverse events }\end{array}$ & $\begin{array}{l}\text { No significant differences in any } \\
\text { parameter but symptom scores } \\
\text { that were better in the placebo } \\
\text { treated group than in the } \\
\text { amphotericin B treated group }\end{array}$ \\
\hline
\end{tabular}

CRS, Chronic Rhinosinusitis; CRSsNP, Chronic Rhinosinusitis without nasal polyps ; CRSwNP, Chronic Rhinosinusitis with nasal polyps; DBPCT, Double Blind Placebo Controlled Trial ; SNOT, Sino-Nasal Outcome ; RSOM-31, Rhinosinusitis Outcome Measure-31 ; VAS, Visual Analogue Scale ;anti IgE, anti-immunoglobulin E; IL, Interleukin; CT, computed tomography; MRI, Magnetic Resonance Imaging; SNAQ, Sino-nasal Questionnaire ; SF-36, Short Form 36 ; PNIF, Peak Nasal Inspiratory Flow ; RQL, Rhinosinusitis quality of life score.

studies were either mixed populations or it was not clear what kind of CRS patients were included.

The volumes and total daily dose of local antifungal treatment varied and we also included three studies ${ }^{(224,225,227)}$ that were performed postoperatively.

None of the studies showed a significant difference for total symptoms or QOL in favour of the antifungal.

Three studies using SNOT (SNOT-22 or SNOT-20) could be combined in a meta-analysis (Figure 6.1.13.1).

The analysis showed a trend for placebo to have a better impact on SNOT score than local fungal treatment.

Two studies could be combined in a meta-analysis, showing a more favourable outcome in symptomatology for placebo than for oral antifungal treatment (Figure 6.1.13.2).

Five studies ${ }^{(219,222,25-227)}$ could be combined into a meta-analysis evaluating the effect of local antifungal on nasal endoscopy. The analysis showed no difference between local antifungal treatment and placebo (Figure 6.1.13.3).

Finally, five studies could also be meta-analysed concerning the effect of local antifungal treatment on CT scan opacification $219-221,224,226)$. Also, in this analysis no significant difference was found between the two options (Figure 6.1.13.4).

\subsubsection{Systemic antifungal treatment}

One study evaluated the effect of systemic antifungal treatment with terbinafine vs. placebo (53 participants) and reported that there was little or no difference between the groups in diseasespecific health-related quality of life or disease severity score (both low-quality evidence) ${ }^{(886)}$. Systemic antifungals may lead to more hepatic toxicity events ( $R R 3.35,95 \% \mathrm{Cl} 0.14$ to 78.60 ) but fewer gastrointestinal disturbances (RR $0.37,95 \% \mathrm{Cl} 0.04$ to 3.36), compared to placebo, although the evidence was of low quality. This study did not find a difference in CT scan score between the groups. Generic health-related quality of life and nasal endoscopic score were not measured.

\subsubsection{Conclusions}

Local and systemic antifungal treatments do not have a positive effect of QOL, symptoms and signs of disease in patients with CRS. The EPOS2020 steering group advises against the use of these treatments in patients with CRS. The EPOS2020 steering group advises against the use of anti-mycotics in CRS. 


\subsubsection{Anti-lgE}

\subsubsection{Summary of the evidence}

The pathophysiology of CRSwNP is characterized by prominent local production of IgE that may contribute to chronic inflammation by continuously activating mast cells ${ }^{(228)}$. Two randomized clinical trials ${ }^{(229,230)}$ have investigated the role of omalizumab, a recombinant humanized monoclonal antibody, in CRS management (Table 6.1.14.1). The mechanism of action of omalizumab involves its selective binding to free circulating IgE which decreases the expression of IgE receptors on mast cells, basophils and dendritic cells, and interferes with activation of these effector cells $s^{(229,231,232)}$.

In the study by Gevaert et al. ${ }^{(229)}, 24$ patients with CRSwNP and comorbid asthma for more than two years were randomized to the subcutaneous administration of four to eight doses of omalizumab or placebo. Total serum IgE levels were between 20 and $700 \mathrm{kU} / \mathrm{mL}$. The subcutaneous administration of four to eight doses of omalizumab was not found to result in significant reduction in RSOM-31 or SF-36. But the physical domain of SF-36 and the Asthma Quality of Life Questionnaire (AQLQ) demonstrated a significant improvement in the omalizumab group, whereas no significant changes were seen in the placebo group. Anti-lgE resulted in significantly lower symptoms scores for nasal congestion ( $p=0.003)$, anterior rhinorrhoea $(p=0.003)$, loss of sense of smell $(p=0.004)$, wheeze $(p=0.02)$ and dyspnoea $(p=0.02)$ compared to baseline. Cough and spirometric results did not improve with anti-lgE treatment.

A linear mixed model demonstrated a lower total nasal polyp score (NPS) in the omalizumab group compared to the placebo group throughout the study period $(p=0.2)$. Lund-MacKay scores were significantly better in the omalizumab group compared to placebo $(p=0.04)$ on radiologic imaging. The clinical and radiographic improvements following use of anti-lgE therapy were seen independent of serum IgE levels. At 16 weeks, a decrease in total NPS was seen in allergic $(-2.57 ; p=0.03)$ and non-allergic patients $(-2.75 ; p=0.06)$. An improvement in LundMackay CT scan scores was observed in the allergic patients (22.61, $\mathrm{p}=0.04)$, but this was not observed in nonallergic patients (20.66, $p=0.75$ ). By contrast, the total AQLQ score demonstrated an improvement in the nonallergic group (259.4, $p=0.03)$, but not in the allergic group (212.3, $\mathrm{p}=0.12)$. At least one adverse event was observed in 22 of 23 (95.7\%) included patients. The most commonly reported adverse event was a common cold in the omalizumab group which occurred at a higher rate in the treatment group compared to the control $(p=0.02)$. One patient developed fatal lymphoblastic lymphoma in the omalizumab group one year following the study. Of note, four of eight (50\%) of patients in the control group were excluded from the analysis. Pinto et al. ${ }^{(230)}$ conducted a randomized, double-blind, placebocontrolled trial of anti-IgE for CRS in 14 patients ( 12 of 14 with CRSwNP) refractory to standard treatment. Participants were required to have a serum total IgE between 30 - $700 \mathrm{IU} /$ $\mathrm{ml}$. All patients received omalizumab, $0.016 \mathrm{mg} / \mathrm{kg}$ per IU subcutaneously, or placebo injections every two to four weeks for six months. There was no significant net difference in SNOT20 scores across treatments (median omalizumab -5.5 , placebo
$-2.3, \mathrm{p}<0.60)$. The median change in SNOT-20 scores across the study period was consistent with a clinically significant improvement (defined as at least 0.8 ) in the omalizumab group and no clinically significant change in the placebo group $(-1.05$ vs. $-0.20, p<0.78)$. There were no significant differences between treatments for any domain except for vitality (omalizumab 9.4, placebo 12.5, p < 0.05). Similarly, there were no statistical differences in total University of Pennsylvania Smell Identification Test (UPSIT) score, nasal endoscopy scores, sinus opacity in CT scan, median change in percent eosinophils in nasal lavage, median PNIF), and median total nasal symptom scores between the omalizumab and placebo groups. Patients on omalizumab used fewer courses of steroids over the trial (median 0 vs. 1, p<0.043) and less use of antibiotics (0 vs. 1, $\mathrm{p}<0.32$ ) compared to placebo. No side effects or adverse events occurred during the study. The investigators were limited by poor subject recruitment due to perceived risk of placebo, high time commitment, high patient volume not meeting study inclusion criteria and a U.S. Food and Drug Administration (FDA)-issued warning about anaphylaxis associated with omalizumab. Recent unpublished data point to a significant impact on baseline in nasal polyp score and change from baseline in average daily nasal congestion score, SNOT-22 and sense of smell.

Concerning side effects, at this moment there is a vast experience with anti-immunoglobulin-E ( $\lg \mathrm{E})$ treatment indicating a very small chance of anaphylaxis ${ }^{(233)}$ but some indication of an association with arterial and venous thromboembolic events leading to cardiovascular and cerebrovascular accidents ${ }^{(234)}$.

\subsubsection{Conclusion}

Anti-IgE therapy has been proposed as a promising biologic therapy for CRS. Two RCTs that evaluated anti-IgE monoclonal antibody included patients with serum IgE levels between 20 $\mathrm{kU} / \mathrm{mL}$ and $700 \mathrm{kU} / \mathrm{mL}$ and compared omalizumab $(0.016 \mathrm{mg} /$ $\mathrm{kg}$ per IU total serum IgE/mL; max dose of $375 \mathrm{mg}$ ) to placebo. Both these small studies ( $<30$ patients) did not show impact on disease specific QOL but one study did show an effect on the physical domain of SF-36 and AQLQ ${ }^{(229)}$. One study ${ }^{(229)}$ demonstrated lower symptom scores (change from baseline in anti lgE group) for nasal congestion, anterior rhinorrhoea, loss of sense of smell, wheeze and dyspnoea, a significant reduction of NPS on endoscopic examination, and Lund-MacKay scores on radiologic imaging. Due to the small study population in the existing studies, further studies with larger population sizes are needed and are underway. The available data are insufficient to advise on the use of anti-IgE in CRSwNP at this moment.

\subsubsection{Anti-IL5}

\subsubsection{Summary of the evidence}

Previous treatment of CRS has included both topical and systemic corticosteroids, long-term antibiotics, and surgical intervention. However, some patients have recalcitrant disease despite utilization of best practices. Interleukin 5 (IL-5) is essential for final differentiation into an eosinophil and prolongs 
Table 6.1.14.1. Anti-lgE therapy for the treatment of patients with CRSwNP.

\begin{tabular}{|c|c|c|c|c|c|}
\hline Study & Methods & Participants & Interventions & Outcomes & Results \\
\hline \multirow[t]{3}{*}{$\begin{array}{l}\text { Gevaert et al. } \\
2013^{(229)}\end{array}$} & DBPRCT & $\begin{array}{l}24 \text { patients } \\
\text { (>18 years) } \\
\text { with CRSwNP }\end{array}$ & $\begin{array}{l}\text { - Omalizumab } \\
\text { every } 2 \text { weeks/8 } \\
\text { injections in } \\
\text { total or every } \\
4 \text { weeks/4 in- } \\
\text { jections in total } \\
\text { based on total } \\
\text { serum IgE levels } \\
\text { and body weight } \\
\text { (maximum } \\
\text { dose of } 375 \mathrm{mg}) \\
\text { ( } n=16) \\
\text { - Placebo }(n=8)\end{array}$ & $\begin{array}{l}\text { At } 16 \text { weeks: } \\
\text { - Quality of life question- } \\
\text { naires (RSOM-31, AQLQ, } \\
\text { SF-36) } \\
\text { - symptoms score } \\
\text { - Total endoscopic polyp } \\
\text { score } \\
\text { - Lund-Mackay CT score } \\
\text { - Spirometric results } \\
\text { - Adverse events }\end{array}$ & $\begin{array}{l}\text { Anti-IgE compared to placebo resulted in: } \\
\text { - No significant impact on QOL but significant im- } \\
\text { provement compared to baseline of anti-IgE treated } \\
\text { group for AQLQ, physical domain of SF-36 and some } \\
\text { subdomains of RSOM-31 } \\
\text { - Lower symptoms scores (change from baseline in anti } \\
\text { lgE group) for nasal congestion, anterior rhinorrhea, } \\
\text { loss of sense of smell, wheeze and dyspnea } \\
\text { - Reduction of polyp size on endoscopic examination } \\
\text { - Improvement in Lund-MacKay scores } \\
\text { - Increased common colds ( } p=0.02 \text { ) } \\
\text { - One event of fatal lymphoblastic lymphoma in } 1 \text { year } \\
\text { following the study }\end{array}$ \\
\hline & & & & & $\begin{array}{l}\text { Subgroup group analysis of allergic vs non-allergic } \\
\text { patients demonstrated: }\end{array}$ \\
\hline & & & & & $\begin{array}{l}\text { - A decrease in total polyps score in allergic }(-2.57 \text {; } \mathrm{p}= \\
0.03) \text { and non-allergic patients }(-2.75 ; \mathrm{p}=0.06) \text {. } \\
\text { - Improvement in Lund-Mackay } C T \text { scan scores was } \\
\text { observed in the allergic patients }(22.61, \mathrm{p}=0.04) \text {, but } \\
\text { not observed in nonallergic patients. } \\
\text { - Improvement in total AQLQ score in the nonallergic } \\
\text { group }(259.4, \mathrm{p}=0.03) \text {, but not in the allergic group } \\
(212.3, \mathrm{p}=0.12)\end{array}$ \\
\hline $\begin{array}{l}\text { Pinto et al. } \\
2010^{(230)}\end{array}$ & DBPRCT & $\begin{array}{l}14 \text { patients } \\
\text { with CRS } \\
\text { (12 CRSwNP, } \\
2 \text { CRSsNP) }\end{array}$ & $\begin{array}{l}7 \text { patients received } \\
\text { omalizumab (0.016 } \\
\mathrm{mg} / \mathrm{kg} \text { per IU total } \\
\text { serum IgE/mL } \\
\text { subcutaneously) } \\
7 \text { patients received } \\
\text { placebo injections }\end{array}$ & 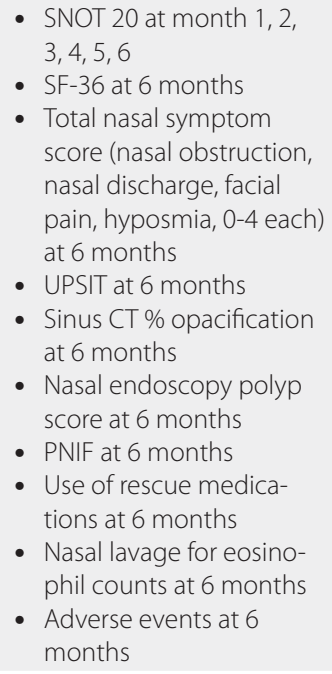 & $\begin{array}{l}\text { Omalizumab compared to placebo resulted in: } \\
\text { - No significant difference in SNOT-20 or SF-36 } \\
\text { - Increased median change in sinus opacity in coronal } \\
\text { CT scans } \\
\text { - Fewer courses of steroids over the trial (median } 0 \text { vs } 1 \text {, } \\
\text { - }<<0.043 \text { ) } \\
\text { - Less use of antibiotics ( } 0 \text { vs } 1, p<0.32 \text { ) } \\
\text { effects or adverse events in either treatment group }\end{array}$ \\
\hline
\end{tabular}

CRSwNP,Chronic Rhinosinusitis with nasal polyps; RSOM-31, Rhinosinusitis Outcome Measure-31; AQLQ, Asthma Quality of Life Questionnaire; SF-36, Short Form 36; QOL, Quality of Life; IgE, Immunoglobulin E; CRS, Chronic Rhinosinusitis; CRSsNP, Chronic Rhinosinusitis without nasal polyps; SNOT, Sino-Nasal Outcome; UPSIT, University of Pennsylvania Smell Identification Test; PNIF, Peak Nasal Inspiratory Flow.

survival of the mature cell in the tissue ${ }^{(235)}$. IL-5 has been noted to be elevated in nasal polyp tissue, and therefore has made it a potential target for the management of patients with nonallergic asthma and CRSwNP ${ }^{(236,237)}$. A few drugs have undergone testing and been available on the market, for the purpose of this review we will discuss mepolizumab and reslizumab, both blockers of free IL-5, as they have undergone randomized controlled trial testing. RCT studies on benralizumab, a blocker of IL-5Ra, are ongoing.

Three placebo-controlled studies ${ }^{(238-240)}$ evaluating anti-IL5 monoclonal antibodies were identified. Two of these studies evaluated mepolizumab ${ }^{(238,239)}$, while one study evaluated reslizumab ${ }^{(240)}$. Despite, these studies being placebo-controlled trials, the data was unable to be pooled for meta-analysis, therefore each paper will be discussed below (Table 6.1.15.1.). Bachert et al. ${ }^{(238)}$ evaluated the efficacy of mepoluzimab in patients with CRSwNP who were also on INCS (FPNS 200 $\mu \mathrm{g}$ ). All patients were given an infusion every four weeks for a total of six doses of either mepolizumab $750 \mathrm{mg}$ or placebo. Efficacy was measured as the number of patients who no longer needed revision surgery based upon VAS scores of CRS severity and NPS following the implementation of mepolizumab. Assessments of other QOL, symptoms, and lung function were also completed. One-hundred-and-five patients were initially included and 
74 ultimately completed treatment through to week 25 for assessment. More patients in the mepolizumab group no longer met the criteria for surgery when compared to those in the placebo group at week 25 (ITT, 16(30\%) vs. 5 (10\%); $=0.006$ ). There were also significant differences in VAS scores for rhinorrhoea, nasal blockage, mucus, and loss of smell (treatment difference: $-2.3(-3.4$ to -1.2$) ; p<0.001,-1.8(-2.9$ to -0.7$) ; p=0.002$, $-2.1(-3.2$ to -1.0$) ; p<0.001,-1.9(-2.9$ to -0.9$) ; p<0.001)$. SNOT-22 scores also significantly improved in the mepolizumab group at weeks 25 (treatment difference: $-13.2(-22.2$ to -4.2$) ; p=0.005$ ). There was statistical benefit in PNIF at 25 weeks (treatment difference: $26.7(3.1$ to 50.2$) ; p=0.027$, but no statistical difference in olfaction or pulmonary function testing. There were no significant differences in adverse events.

Gevaert et al. ${ }^{(239)}$ performed a double blind, placebo-controlled trial evaluating the effect of mepolizumab on nasal polyposis in 30 patients with grade 3 or 4 polyps or those who had recurrence following surgery. Patients received either $750 \mathrm{mg}$ of mepolizumab or placebo via IV injection 28 days apart from two doses. Patients were assessed for changes in symptom scores, NPS, CT scores, PNIF, and biological activity (defined as blood eosinophil counts, cytokine measurements in nasal secretions). Safety and adverse events data was also collected. The mepoluzimab group showed no significant difference in symptoms but had a greater change from baseline polyp score resulting in a significant treatment difference when compared to placebo (-1.30(1.51); $\mathrm{p}=0.028)$. There was a significant reduction in the mepolizumab treated group when compared to placebo in blood eosinophil counts, serum eosinophil cationic protein (ECP) levels, and serum IL-5Ra between the groups (raw data not available). There was no difference in the nasal ECP, IL-5, or total IgE levels.

Gevaert et al. ${ }^{(240)}$ performed a double blind, placebo-controlled, two-centre safety and pharmacokinetics study evaluating reslizumab in 24 patients with CRSwNP. Biologic activity of the monoclonal antibody was assessed via endoscopic NPS, sinonasal symptoms, peripheral eosinophils, peripheral and local eotaxin levels, ECP, and IL5 levels. Patients $(n=24)$ were divided into three groups: receiving $1 \mathrm{mg} / \mathrm{kg}$ of reslizumab, $3 \mathrm{mg} / \mathrm{kg}$ of reslizumab, or a placebo via IV for one dose. Adverse events and safety data were also obtained. Twenty-three (95.8\%) subjects reported at least one adverse event, which was most often a URI. There was no significant difference in symptom scores or peak nasal inspiratory flow in both treatment groups compared to placebo. The NPS was only significantly less at week 12 in the $1 \mathrm{mg} / \mathrm{kg}$ treatment group compared to baseline values. However, this study was not powered to determine differences in efficacy variables. Reslizumab caused a significant decrease in blood eosinophil counts in both treatment groups when compared to the placebo group. These results were evident as early as 12 hours following administration. Similar findings were also true of serum ECP and IL5Ra, however, eosinophil counts did undergo significant rebound in both treatment groups at week 24 and 32 following treatment administration.

\subsubsection{Safety}

The only data that was able to be pooled for meta-analysis was the number of patients who suffered from an adverse event. There was no statistical significance between the placebo and anti-IL-5 monoclonal antibody group (SMD 0.72(0.29,1.77), $\mathrm{p}=0.48$, two trials, 129 patients) (Figure 6.1.15.1.).

Unlike in CRS, there is a significant experience with anti-II5 in other Th2 driven diseases like asthma that do show a favourable safety profile for the time used until now ${ }^{(241-244)}$. In all these studies, patients had previously undergone conventional therapy including sinus surgery and topical corticosteroid irrigations with these medications reserved as a treatment option for patients who are considered recalcitrant despite best efforts. This is an important adjunct to our armamentarium for treatment of these difficult to control CRS patients. The relative position of the different monoclonal antibodies one to another has to be determined.

\subsubsection{Conclusion}

At the moment the only anti-II-5 treatment studied in CRS is mepolizumab. There is only one large sufficiently powered study that showed a significant reduction in patients' need for surgery and an improvement in symptoms. The EPOS2020 steering group advises use of mepolizumab in patients with CRSwNP fulfilling the criteria for treatment with monoclonal antibodies (245) (see chapter 1).

\section{Dupilumab is the only monoclonal antibody that is approved for the treatment of CRSWNP at the moment. The panel advises to use dupilumab in patients with CRSWNP fulfilling the criteria for treatment with monoclonal antibodies.}

Figure 6.1.15.1. Forest plot of the effect of anti-IL-5 biologics versus placebo on adverse events in patients with CRSwNP.

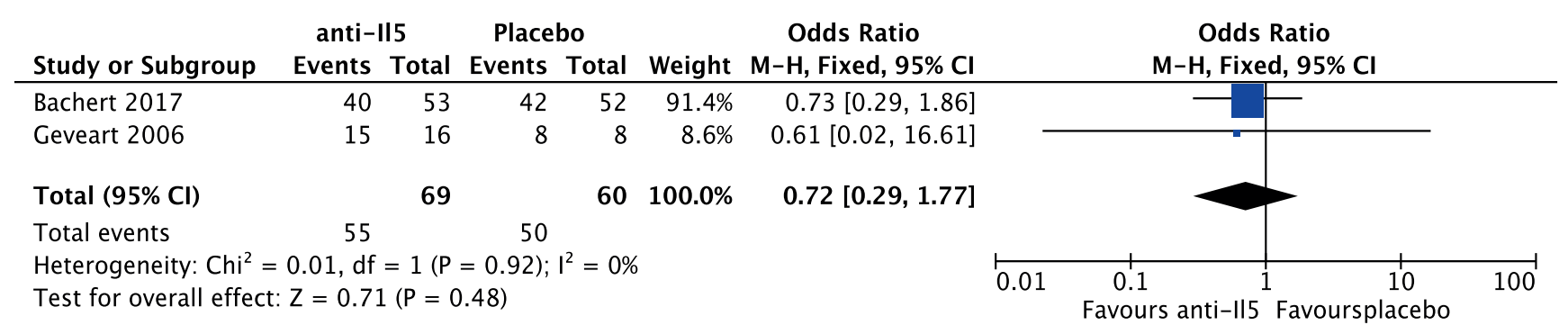


Table 6.1.15.1. Anti-IL-5 for the treatment of patients with CRSwNP.

\begin{tabular}{|c|c|c|c|c|c|}
\hline Study & Methods & Participants & Interventions & Outcomes & Results \\
\hline $\begin{array}{l}\text { Bachert } \\
2017^{(238)}\end{array}$ & DBPCT & $\begin{array}{l}105 \text { Severe } \\
\text { CRSwNP }\end{array}$ & $\begin{array}{l}\text { Mepolizumab } 750 \\
\text { mg IV } 4 \text { weeks } \times 6 \\
\text { months }(n=54) \vee \\
\text { placebo infusion q } \\
4 \text { weeks } 6 \text { months } \\
(n=53)\end{array}$ & $\begin{array}{l}\text { Assessed at } 25 \text { weeks } \\
\text { - Number of patients no longer } \\
\text { needing revision surgery } \\
\text { - VAS polyp severity (0-10), } \\
\text { - Endoscopic polyp score (0-8) } \\
\text { - Symptom scores - VAS symp- } \\
\text { toms - (rhinorrhoea, mucus, nasal } \\
\text { blockage, loss of sense of smell), } \\
\text { SNOT22, EQ-5D, PNIF, olfaction } \\
\text { (0-12) } \\
\text { - Eosinophils } \\
\text { - FEV1/FVC/PEFR }\end{array}$ & $\begin{array}{l}\text { - Patients in the mepolizumab group no longer } \\
\text { met criteria for surgery compared to placebo } \\
\text { (ITT< } 16(30 \%) \text { vs } 5(10 \%) ; p=0.006) \\
\text { - Significant improvement in the mepolizumab } \\
\text { group for VAS scores (rhinorrhoea, mucus, nasal } \\
\text { blockage, and loss of smell, in SNOT22 (28.8(22) } \\
\text { vs 38.2(24.5), and PNIF (mean difference, 26.7(3.1- } \\
50.2), p=0.027) \\
\text { - Significant improvement in the mepolizumab } \\
\text { group over placebo in nasal polyp severity VAS } \\
\text { scores (mean change; - } 1.8(-2.9-0.8), p=0.001 \\
\text { - Blood eosinophils decreased in the mepolizumab } \\
\text { group, but not placebo group. } \\
\text { - No difference in EQ-5D scores, smell scores, or } \\
\text { lung function between the groups. }\end{array}$ \\
\hline $\begin{array}{l}\text { Gevaert } \\
2011^{(239)}\end{array}$ & DBPCT & $\begin{array}{l}30 \text { Severe } \\
\text { or recurrent } \\
\text { CRSwNP }\end{array}$ & $\begin{array}{l}\text { Mepolizumab } \\
750 \mathrm{mg} \text { IV } 4 \text { weeks } \\
\times 2 \text { doses }(n=20) \text { v } \\
\text { placebo q4weeks } \times \\
2 \text { doses }(n=10)\end{array}$ & $\begin{array}{l}\text { Assessed at } 8 \text { weeks } \\
\text { - Nasal polyp score (0-8) } \\
\text { - CT scan (improved, worse, no } \\
\text { change) } \\
\text { - PNIF } \\
\text { - Symptom score - anterior rhinor- } \\
\text { rhoea, nasal obstruction, PND, loss } \\
\text { of smell (0-12) } \\
\text { - Biological activity (peripheral } \\
\text { blood eosinophil counts, serums } \\
\text { ECP, serum IL5Ra, nasal ECP, IL5, } \\
\text { lgE). }\end{array}$ & $\begin{array}{l}\text { - No significant changes in symptom scores } \\
\text { - Significant improvement in total polyp score at } \\
\text { week } 8 \text { (treatment difference of }-1.3(\mathrm{SD}, 1.51 \text { ), } \\
\mathrm{p}=0.028 \text { ) with increased improvement in the me- } \\
\text { polizumab group ( } 60 \% \text { v } 10 \% \text {, OR } 13.5, \mathrm{p}=0.018 \text { ) } \\
\text { - Improved change from baseline in PNIF in mepo- } \\
\text { lizumab group compared to placebo (raw data } \\
\text { not available) } \\
\text { - Significant reduction in blood eosinophil counts, } \\
\text { serum ECP levels, and serum IL5Ra in the mepoli- } \\
\text { zumab group, but not in nasal ECP, IL5, or IgE }\end{array}$ \\
\hline $\begin{array}{l}\text { Gevaert } \\
2006^{(240)}\end{array}$ & DBPCT & 24 CRSWNP & $\begin{array}{l}\text { Reslizumab 3mg/ } \\
\mathrm{kg}(\mathrm{n}=8) \text { or } 1 \mathrm{mg} / \\
\mathrm{kg}(\mathrm{n}=8) \times 1 \mathrm{dose} \mathrm{v} \\
\text { placebo }(\mathrm{n}=8)\end{array}$ & $\begin{array}{l}\text { Assessed at } 4 \text { weeks } \\
\text { - Endoscopy polyp size (0-8) } \\
\text { - Symptoms (anterior rhinorrhoea, } \\
\text { nasal obstruction, PND, loss } \\
\text { of sense of smell) (scoring not } \\
\text { obvious) } \\
\text { - Nasal peak inspiratory flow } \\
\text { - Eosinophil count (local and pe- } \\
\text { ripheral), ECP (local and peripher- } \\
\text { al), eotaxin (local and peripheral), } \\
\text { IL5 (local and peripheral) }\end{array}$ & $\begin{array}{l}\text { - No significant improvement in symptom scores } \\
\text { or nasal peak inspiratory flow } \\
\text { - Nasal polyp score was significantly decreased } \\
\text { at } 1 \text { time point for } 1 \mathrm{mg} / \mathrm{kg} \text { group (raw data not } \\
\text { available) } \\
\text { - Significant decrease in blood eosinophils, serum } \\
\text { ECP, and soluable IL5Ra (raw data not available) }\end{array}$ \\
\hline
\end{tabular}

CRSwNP, Chronic Rhinosinusitis with nasal polyps; PND, post nasal drip, ECP, eosinophil cationic protein; PNIF, peak nasal inspiratory flow; DBPCT, double blind placebo controlled trial; OR, Odds ratio; SD , Standard deviation; FEV1, forced expiratory volume 1; FVC, Forced Vital Capacity, PEFR, Peak Expiratory Flow Rate; SNOT, Sino-Nasal Outcome.

\subsubsection{Anti-IL4/IL13}

\subsubsection{Summary of the evidence}

IL-4 and IL-13 are potent mediators of type 2 immunity with both overlapping and distinct functions. IL-13 and IL-4 partly share the same receptor and signalling pathways and both are deeply involved in IgE synthesis, eosinophil activation, mucus secretion and airways remodelling. IL-4 is a major differentiation factor driving a $\mathrm{TH} 2$ type response by initiating $\mathrm{T}$ cell differentiation toward the TH2 subtype. IL-4 also induces the production of type 2 associated cytokine and chemokines such as IL-5, IL-9, IL-13, TARC and fotaxin. Furthermore, IL-4 and IL-13 are primarily responsible for isotype class switching of B cells to produce $\lg ^{(246)}$.

\subsection{Dupilumab}

There is recent data on the efficacy of dupilumab in CRSwNP
(Table 6.1.16.1). Dupilumab is a fully human monoclonal antibody to the IL-4 receptor $\alpha$ subunit, which inhibits signaling of IL-4 and IL-13 that is given as a subcutaneous injection. There are no other RCT's performed in patients with CRSwNP with the other anti-IL-4/IL-13 agents such as Tralokinumab and Lebrikizumab-IL-13.

Bachert et al. ${ }^{(247)}$ performed a proof of concept double blinded, placebo controlled parallel group study in 2013, which randomized 60 adult CRSwNP into two groups. After a fourweek run-in period of treatment with mometasone, patients were randomly allocated to add-on therapy with subcutaneous dupilumab (600mg loading followed by 15 weekly doses of $300 \mathrm{mg}$ ) or matched placebo for 16 weeks. Patients treated with dupilumab had significant improvement in SNOT-22, rhinosinusitis disease severity (VAS), nasal blockage, UPSIT smell score, nasal polyp score (NPS), and CT score (LMS), and asthma outcomes ( $\mathrm{FEV}_{1}$ and control) compared to placebo. 
Bachert et al. ${ }^{(248)}$ published the results of two randomised double-blind, multicentre, placebo-controlled, parallel group phase 3 trials which evaluated dupilumab added to standard-of-care in adults with severe CRSwNP. In the liberty NP SINUS-24 study, patients were randomized 1:1 to 24 weeks subcutaneous dupilumab $300 \mathrm{mg}$ or placebo every two weeks. Liberty NP SINUS-52, patients were randomized 1:1:1 to 52 weeks dupilumab $300 \mathrm{mg}$ every two weeks, 24 weeks every two weeks then 28 weeks dupilumab $300 \mathrm{mg}$ every four weeks, or 52 weeks placebo every two weeks. In both studies, treatment with dupilumab significantly improved SNOT-22, rhinosinusitis disease severity (VAS), nasal blockage, UPSIT smell score, NPS, LMS, and asthma outcomes ( $\mathrm{FEV}_{1}$ and control) compared to control.

There was one study from Wenzel et al. ${ }^{(249)}$ evaluating SNOT-22 in a group of patients with persistent, moderate-to-severe asthma and eosinophilia. Unfortunately, it was not evaluated whether these patients actually had CRS so we could not include the study. Four posthoc studies have been performed that are not taken into account in this meta-analysis ${ }^{(250-253)}$.

The Bachert studies were very much done in the same way and could be combined into a meta-analysis of the three studies evaluating dupilumab added to standard-of-care treatment to patients with CRSwNP(247, 248).

The SNOT-22 score (scale 0-110) at 4-6 months showed a significant and clinically relevant decrease of MD - 19.61 (95\% Cl -22.53 - -16.69); 784 participants; two studies; $12=0 \%$ ), (Figure 6.1.16.1).

The Rhinosinusitis disease severity (VAS) at 4-6 months showed a significant and clinically relevant decrease of MD - 2.54 (95\% Cl -2.84 - -2.23); 784 participants; two studies; $12=40 \%$ ), (Figure 6.1.16.2).

The nasal congestion/obstruction score at 4-6 months showed a significant and clinically relevant decrease of MD - 0.86 (95\% $\mathrm{Cl}-0.98-0.75)$; 784 participants; two studies; $12=0 \%$ ), (Figure 6.1.16.3).

Smell was evaluated using the UPSIT. The UPSIT score at 4-6 months showed a significant and clinically relevant decrease of MD 10.83 (95\% Cl 9.59 - 12.08); 784 participants; two studies; 12 $=0 \%$ ), (Figure 6.1.16.4).

The nasal polyp score was the primary endpoint in these studies. The mean nasal polyp score in these studies was around 6 indicating severe polyp disease. The nasal polyp score at 4-6 months showed a significant decrease of MD $-1.79(95 \% \mathrm{Cl}$ -2.01 - -1.56); 784 participants; two studies; $12=65 \%$ ), (Figure 6.1.16.5).

The Lund-Mackay score (scale 0-48) at 4-6 months showed a significant decrease of SMD -1.50 (95\% Cl-1.84 - -1.16); 784 participants; two studies; $12=71 \%$ ), (Figure 6.1.16.6).

Finally, there was a significant impact on ACQ and FEV1. Because ACQ5 and ACQ6 were used, the data could not be combined into a meta-analysis but both studies showed a significant improvement over placebo. The FEV1 could be combined into a meta-analysis and showed a significant improvement in FEV1 (I) at 4-6 months of MD 0.21 (95\% Cl $0.20-0.22)$; 488 participants; two studies; $12=0 \%$ ), (Figure 6.1.16.7).

The most common adverse events (nasopharyngitis, worsening nasal polyps and asthma, headache, epistaxis, injection-site erythema) were more frequent with placebo. When evaluating all trials with dupilumab the drug seems to induce conjunctivitis in trials in patients with atopic dermatitis but no trials with asthma and CRSwNP(254). No other adverse events have been reported in the literature up until now.

\subsubsection{Conclusion}

Dupilumab is the only monoclonal antibody that is approved for the treatment of CRSWNP at the moment. The panel advises to use dupilumab in patients with CRSwNP fulfilling the criteria for treatment with monoclonal antibodies ${ }^{(245)}$.

\subsubsection{Probiotics}

\subsubsection{Summary of the evidence}

A locally disturbed commensal microbiome might be an aetiological factor in chronic rhinosinusitis. Despite significant heterogeneity in study design, tissue sampling, processing and bioinformatics analysis, consistent findings have emerged from the recent literature. Healthy individuals and CRS patients have similar overall bacterial burden of disease and share many common phyla. CRS patients, however, routinely show reductions in markers of biodiversity. Both medical and surgical treatments appear to influence the sinonasal microbiome, with certain bacterial strains associated with better treatment outcomes. The presence of microbial dysbiosis in CRS is now supported by numerous studies. Whether this dysbiosis is a cause or rather an association of the disease process remains unclear $^{(255,256)}$.

We identified two studies on probiotic therapy, one oral(257) and one evaluating a nasal spray ${ }^{(258)}$ (Table 6.1.17.1.). Both studies evaluated SNOT and showed no significant difference between probiotic and placebo. The study of Mukerji et al. showed a significant reduction in SNOT-20 and symptoms after four weeks of treatment in the probiotic group and not the placebo group compared to baseline, but the differences between the groups were not significant and the change from baseline was not found after eight weeks.

Also, no differences in the bacteria cultured or inflammatory mediators were found.

\subsubsection{Conclusion}

Although probiotic therapies show theoretical promise, the two studies performed so far did not show any differences compared to placebo. For this reason, the EPOS2020 steering group advises against the use of probiotics for the treatment of patients with CRS.

\subsubsection{Muco-active drugs}

\subsubsection{Summary of the evidence}

Mucoactive drugs are regularly used as a therapeutic option for mucus alteration, including hypersecretion. The 
Figure 6.1.16.1. Forest plot of the effect of Dupilumab versus placebo on SNOT-22 score in CRSwNP patients at 4-6 months.

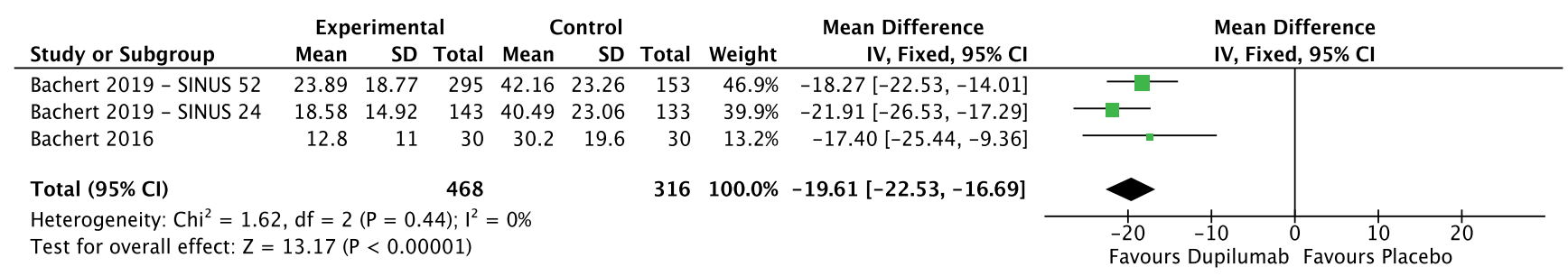

Figure 6.1.16.2. Forest plot of the effect of Dupilumab versus placebo on the rhinosinusitis disease severity (VAS) in CRSwNP patients at 4-6 months.

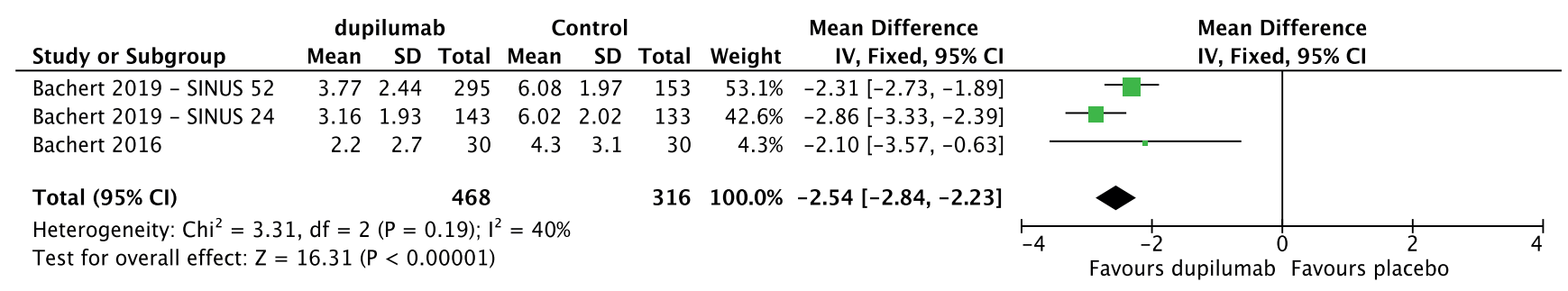

Figure 6.1.16.3. Forest plot of the effect of Dupilumab versus placebo on the change in nasal congestion/obstruction score in CRSwNP patients at 4-6 months.

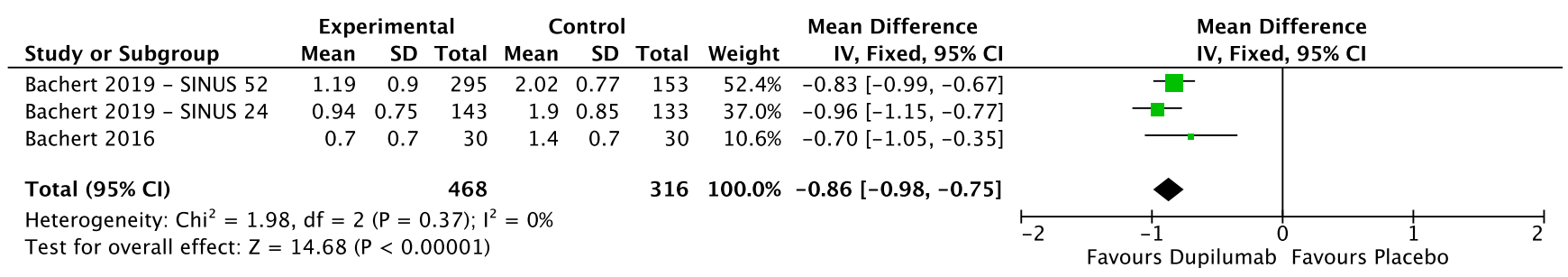

Figure 6.1.16.4. Forest plot of the effect of Dupilumab versus placebo on UPSIT score in CRSwNP patients at 4-6 months.

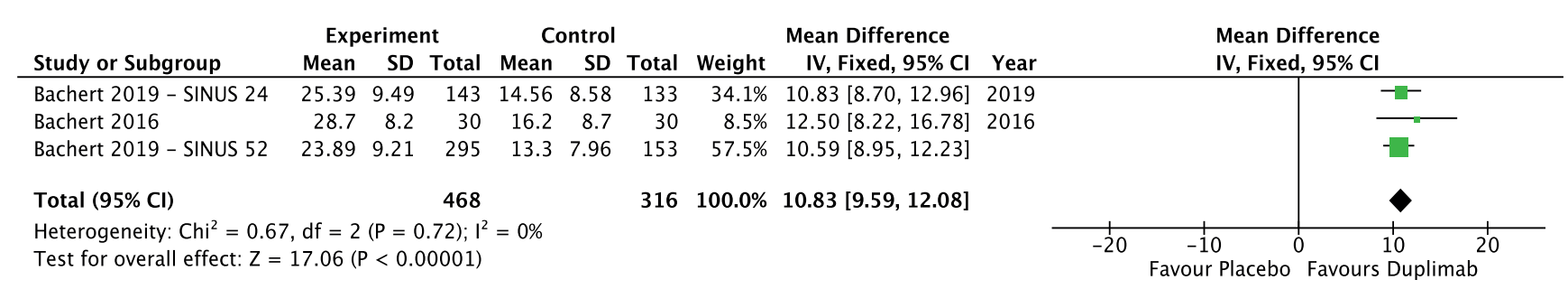

Figure 6.1.16.5. Forest plot of the effect of Dupilumab versus placebo on the change in nasal polyp score in CRSwNP patients at 4-6 months.

\begin{tabular}{|c|c|c|c|c|c|c|c|c|c|c|c|}
\hline \multirow{3}{*}{$\begin{array}{l}\text { Study or Subgroup } \\
\text { Bachert } 2019-\text { SINUS } 52\end{array}$} & \multicolumn{3}{|c|}{ Experimental } & \multicolumn{3}{|c|}{ Control } & \multicolumn{2}{|r|}{ Std. Mean Difference } & \multirow{2}{*}{\multicolumn{3}{|c|}{$\begin{array}{l}\text { Std. Mean Difference } \\
\text { IV, Random, } 95 \% \mathrm{Cl}\end{array}$}} \\
\hline & Mean & SD & Total & Mean & SD & Total & Weight & IV, Random, 95\% CI & & & \\
\hline & 4.46 & 1.89 & 295 & 6.09 & 1.19 & 153 & $46.1 \%$ & $-0.97[-1.17,-0.76]$ & & & \\
\hline Bachert 2019 - SINUS 24 & 3.75 & 1.98 & 143 & 5.94 & 1.44 & 133 & $38.3 \%$ & $-1.25[-1.51,-1.00]$ & - & & \\
\hline Bachert 2016 & 4 & 1.9 & 30 & 5.4 & 1.5 & 30 & $15.7 \%$ & $-0.81[-1.33,-0.28]$ & & & \\
\hline Total $(95 \% \mathrm{Cl})$ & & & 468 & & & 316 & $100.0 \%$ & $-1.05[-1.29,-0.82]$ & & & \\
\hline \multicolumn{9}{|c|}{$\begin{array}{l}\text { Heterogeneity: } \operatorname{Tau}^{2}=0.02 ; \mathrm{Chi}^{2}=3.85, \mathrm{df}=2(\mathrm{P}=0.15) ; \mathrm{I}^{2}=48 \% \\
\text { Test for overall effect: } \mathrm{Z}=8.71(\mathrm{P}<0.00001)\end{array}$} & $\begin{array}{c}-1 \\
\text { Favours D }\end{array}$ & 0 & 1 \\
\hline
\end{tabular}


Figure 6.1.16.6. Forest plot of the effect of dupilumab versus placebo on the Lund-Mackay score in CRSwNP patients at 4-6 months.

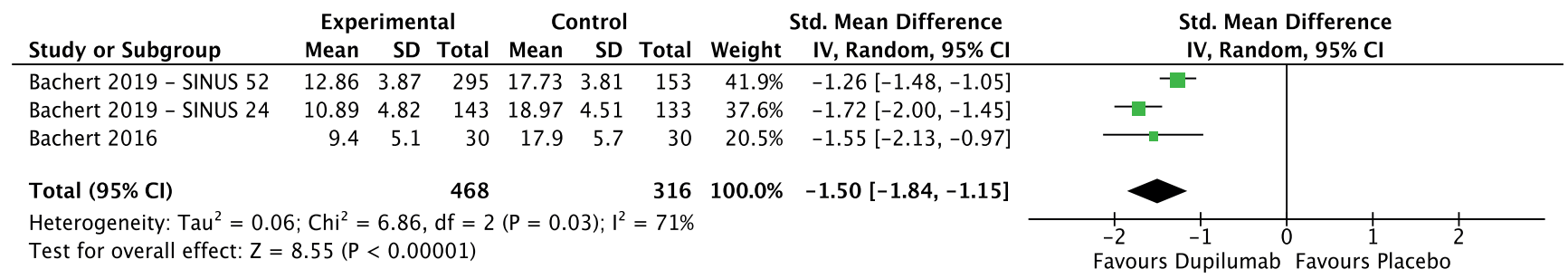

Figure 6.1.16.7. Forest plot of the effect of dupilumab versus placebo on the change in FEV1 in CRSwNP patients at 4-6 months.

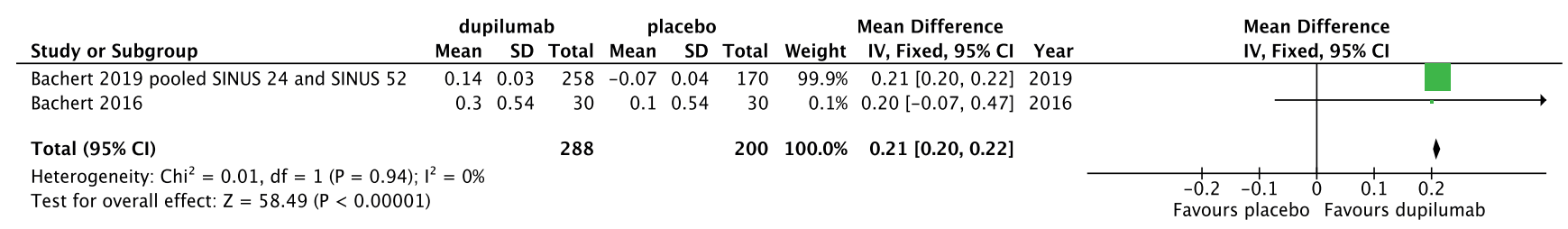

Table 6.1.16.1. Dupilumab for the treatment of patients with CRSwNP.

\begin{tabular}{|c|c|c|c|c|c|}
\hline Study & Methods & Participants & Interventions & Outcomes & Results \\
\hline $\begin{array}{l}\text { Bachert } \\
2019^{(248)}\end{array}$ & $2 \times \mathrm{DBPRCT}$ & $\begin{array}{l}\text { SINUS-24: } \\
276 \text { CRSwNP } \\
\text { patients } \\
\text { SINUS-52: } \\
448 \text { CRSwNP } \\
\text { patients } \\
\text { pts }\end{array}$ & $\begin{array}{l}\text { SINUS-24: } \\
\text { - Dupilumab ( } 300 \mathrm{mg} \text { q2w) for } 24 \text { weeks } \\
\text { ( } \mathrm{n}=143 \text { ) } \\
1 \text { placebo for } 24 \text { weeks ( } \mathrm{n}=133 \text { ) } \\
\text { SINUS-52: } \\
\text { - Dupilumab } 300 \mathrm{mg} \text { every } 2 \text { week for } 52 \\
\text { weeks ( } n=150 \text { ) } \\
\text { - Dupilumab } 300 \text { mg every } 2 \text { week for } 24 \\
\text { weeks then } 300 \text { mg every } 4 \text { weeks for } \\
28 \text { weeks ( } n=145 \text { ) } \\
\text { - Placebo every } 2 \text { week for } 52 \text { weeks } \\
\text { ( } n=153 \text { ) } \\
\text { - Both studies } 4 \text { weeks run-in and during } \\
\text { the trial mometasone nasal spray } 100 \\
\mu g \text { in each nostril twice daily }\end{array}$ & $\begin{array}{l}\text { At } 24 \text { and } 52 \text { weeks: } \\
\text { - } \text { SNOT-22 } \\
\text { - Rhinosinusitis disease } \\
\text { severity (VAS) } \\
\text { - Nasal congestion } \\
\text { - Smell (UPSIT) } \\
\text { - Nasal polyp score (NPS) } \\
\text { - CT scan (LMS) } \\
\text { - } \text { ACQ5 } \\
\text { - FEV1 }\end{array}$ & $\begin{array}{l}\text { Dupilumab resulted in significant } \\
\text { improved outcomes in both studies } \\
\text { compared to placebo at } 24 \text { and } 52 \\
\text { weeks: } \\
\text { - SNOT } 22 \\
\text { - Rhinosinusitis disease severity (VAS) } \\
\text { - Nasal congestion } \\
\text { - Smell (UPSIT) } \\
\text { - NPS } \\
\text { - LMS } \\
\text { - ACQ5 } \\
\text { - FEV1 }\end{array}$ \\
\hline $\begin{array}{l}\text { Bachert } \\
2016^{(247)}\end{array}$ & DBPRCT & $\begin{array}{l}60 \text { CRSwNP } \\
\text { patients } \\
\text { refractory to } \\
\text { INCS }\end{array}$ & $\begin{array}{l}\text { - Dupilimab (600mg loading, 300mg } \\
\text { weekly) }(n=30) \\
\text { - Placebo ( } n=30) \\
\text { - } 4 \text { weeks run-in and during the trial } \\
\text { mometasone nasal spray } 100 \mu \mathrm{g} \text { in } \\
\text { each nostril twice daily }\end{array}$ & $\begin{array}{l}\text { At } 16 \text { weeks: } \\
\text { - } \text { QOL (SNOT-22) } \\
\text { - Rhinosinusitis disease } \\
\text { severity (VAS) } \\
\text { - Nasal congestion } \\
\text { - Smell (UPSIT) } \\
\text { - Nasal polyp score (NPS) } \\
\text { - CT scan (LMS) } \\
\text { - } \text { ACQ6 } \\
\text { - FEV1 }\end{array}$ & $\begin{array}{l}\text { Dupilumab resulted in significant } \\
\text { improved outcomes in both studies } \\
\text { compared to placebo at } 16 \text { weeks: } \\
\text { - SNOT } 22 \\
\text { - Rhinosinusitis disease severity (VAS) } \\
\text { - Nasal congestion } \\
\text { - Smell (UPSIT) } \\
\text { - NPS } \\
\text { - LMS } \\
\text { - ACQ6 } \\
\text { - FEV1 }\end{array}$ \\
\hline
\end{tabular}

DBPRCT, Double Blind Placebo Controlled Trial; CRSwNP, Chronic Rhinosinusitis with nasal polyps; pts, patients; SNOT, Sino-Nasal Outcome; VAS, Visual Analogue Scale; UPSIT, University of Pennsylvania Smell Identification Test; NPS, Nasal Polyp Score; LMS, Lund-Mackay Score; ACQ5, Asthma Control Questionnaire 5 ; FEV1, Forced Expiratory Volume; INCS, Intranasal corticosteroids spray; QOL, Quality of Life; ACQ6, Asthma Control Questionnaire 6; 
Table 6.1.17.1. Probiotics for the treatment of patients with CRS.

\begin{tabular}{|c|c|c|c|c|c|}
\hline Study & Methods & Participants & Interventions & Outcomes & Results \\
\hline $\begin{array}{l}\text { Martensson } \\
2017^{(258)}\end{array}$ & $\begin{array}{l}\text { DBPC } \\
\text { crossover } \\
\text { trial with at } \\
\text { least } 4 \text { wks } \\
\text { washout }\end{array}$ & $\begin{array}{l}21 \text { CRSsNP } \\
\text { patients }\end{array}$ & $\begin{array}{l}\text { - Nasal spray containing LAB (a mixture of } 9 \\
\text { lactobacilli and } 4 \text { bifidobacteria) for } 14 \text { days } \\
\text { - Placebo nasal spray for } 14 \text { days }\end{array}$ & $\begin{array}{l}\text { - } \text { SNOT-22 } \\
\text { - } \text { Microbiology } \\
\text { - Inflammatory mediators in } \\
\text { nasal lavage fluid }\end{array}$ & $\begin{array}{l}\text { - No significant } \\
\text { differences in any } \\
\text { parameter evaluated }\end{array}$ \\
\hline $\begin{array}{l}\text { Mukerij } \\
2009^{(257)}\end{array}$ & DBPCT & $\begin{array}{l}77 \text { CRS } \\
\text { patients }\end{array}$ & $\begin{array}{l}\text { - Probiotic Lactobacillus rhamnosus R0011 } \\
\text { strain (500 million active cells/tablet) twice } \\
\text { daily for } 4 \text { weeks }(n=39) \\
\text { - Oral placebo treatment twice daily for } 4 \\
\text { weeks }(n=38)\end{array}$ & $\begin{array}{l}\text { At } 4 \text { and } 8 \text { weeks: } \\
\text { - SNOT-20 } \\
\text { - Symptoms }\end{array}$ & $\begin{array}{l}\text { - No significant } \\
\text { differences between } \\
\text { probiotic and placebo }\end{array}$ \\
\hline
\end{tabular}

CRS, Chronic Rhinosinusitis; CRSsNP, Chronic Rhinosinusitis without nasal polyps ; DBPC, Double blind placebo controlled; DBPCT, Double Blind Placebo Controlled Trial ; SNOT-22, Sino-Nasal Outcome Test-22 ; SNOT-20, Sino-Nasal Outcome Test-20.

drugs can be divided into expectorants (like hypertonic saline), mucoregulators that regulate mucous secretion (like carbocisteine and anticholinergic agents), mucolytics that decrease mucous viscosity (like $\mathrm{N}$-acetylcysteine, erdosteine and DNase) and mucokinetics: drugs that increase mucociliary clearance by acting on the cilia (like bronchodilators and surfactants).

Evaluation in the lower airways has shown that treatment with mucolytics may produce a small reduction in acute exacerbations and a small effect on overall quality of life in patients with chronic bronchitis or COPD ${ }^{(259)}$. Small benefits of mannitol, inhalation of normal and hypertonic saline has been found in patients with chronic lung diseases outside $\mathrm{CF}^{(260)}$. Adverse effects of rhDNase on lung function suggest this should not be administered in non-CF bronchiectasis ${ }^{(260)}$.

Data in CRS patients are very limited (Table 6.1.18.1.). There is one RCT evaluating the addition of S-carboxymethylcysteine $\left(1500 \mathrm{mg} /\right.$ day) to clarithromycin $(200 \mathrm{mg} / \text { day })^{(261)}$. The authors found no significant differences in effect on SNOT-20 or CT scan at 12 weeks but a significantly higher percentage of patients with a remarkably effective symptomatic response at 12 weeks, but not at four and eight weeks and also a significantly higher percentage of patients with improved characteristics of nasal discharge and reduced post-nasal discharge. There were slightly more adverse events than with clarithromycin alone, but none were serious.

A second study is a non-randomized prospective noninterventional study evaluating the effect of erdosteine ${ }^{(262)}$. Erdosteine was originally developed as a mucolytic agent. Moreover, it has also been shown to have anti-bacterial, antioxidant and most importantly anti-inflammatory effects ${ }^{(262,263)}$.

\subsubsection{Conclusion}

Data on the effect of muco-active agents in CRS are very limited. The only DBPCT showed a significantly higher percentage of patients with effective response and improved characteristics of nasal discharge at 12 weeks. The EPOS2020 steering group considered the quality of the data insufficient to advise on the use of muco-active agents in the treatment of patients with CRS.

\subsubsection{Herbal treatment}

\subsubsection{Summary of the evidence}

Herbal medicine represents a relatively frequently used complementary and alternative treatment for CRS. However, the safety and efficacy of herbal medicine for the treatment of CRS is currently uncertain. Most of the studies are not randomized. We found five RCTs: four with a local application ${ }^{(264-267)}$ and one very large study with the tablet BNO 1016 (268).

BNO 1016 has been established for use in patients with acute rhinosinusitis and is marketed under the brand name 'Sinupret extract CT'. It contains the dry extract of five herbal drugs, namely gentian root, primula flower, sorrel herb, elder flower, verbena herb, in a ratio of 1:3:3:3:3. The efficacy in acute rhinosinusitis has been demonstrated in a randomised, placebocontrolled trial(269). A DBPCT with 929 patients examined BNO 1016 (Sinupret extract) in CRS. Two different Sinupret extract doses (240mg or $480 \mathrm{mg}$ daily) were compared to placebo, with a treatment period of 12 weeks. The primary endpoint was the mean Major Symptom Score (MSS) in weeks 8 and 12 of treatment. Secondary endpoints included symptom severity by VAS, work activity and QOL (SNOT-20 score). Sinupret extract was not superior over placebo regarding the primary endpoint. However, in patients with a diagnosis of CRS for $>1$ year and a baseline MSS $>9$ (out of $\max 15$ ) a post-hoc sensitivity analysis showed a significant benefit in MSS and work activity (WPAI:GH) at 12 weeks of treatment with the use of Sinupret extract when compared to placebo ${ }^{(268)}$. In total, 1,215 adverse events were reported by 528 (57.0\%) patients during the treatment period, most of mild to moderate intensity. A relationship with the study drug was suspected in 18 patients $(5.9 \%)$ treated with the $480 \mathrm{mg}$ Sinupret extract, 14 patients (4.4\%) treated with $240 \mathrm{mg}$ and in 12 patients (3.9\%) who received placebo. The most common drug-related adverse events reported for the $480 \mathrm{mg}, 240 \mathrm{mg}$ and placebo were upper abdominal pain $(1.3 \%$, $1.3 \%, 0.7 \%)$, diarrhoea $(0.3 \%, 0.6 \%, 1.3 \%)$ and headache $(1.3 \%$, $0.3 \%, 0.7 \%)$. Adverse events led to discontinuation in 20 patients (total $2.2 \%, 1.6 \%$ in placebo group, $2.0 \%$ in $240 \mathrm{mg}$ and $3.0 \%$ in $480 \mathrm{mg}$ ). Serious adverse events were noted in 11 patients 
Table 6.1.18.1. Muco-active drugs for the treatment of patients with CRS.

\begin{tabular}{|c|c|c|c|c|c|}
\hline Study & Methods & Participants & Interventions & Outcomes & Results \\
\hline $\begin{array}{l}\text { Hoza } \\
2013^{(262)}\end{array}$ & $\begin{array}{l}\text { Non- } \\
\text { randomized } \\
\text { prospective } \\
\text { non- } \\
\text { interventional } \\
\text { post- } \\
\text { authorisation } \\
\text { study }\end{array}$ & $\begin{array}{l}60 \text { CRSwNP } \\
\text { patients }\end{array}$ & $\begin{array}{l}\text { - Erdosteine } 300 \mathrm{mg} \text { twice } \\
\text { a day for the period of } 3 \\
\text { months ( } n=33 \text { ) } \\
\text { - Erdosteine } 300 \mathrm{mg} \text { twice } \\
\text { a day combined with } \\
\text { mometasone nasal spray } \\
\text { (dosage unknown) for the } \\
\text { period of } 3 \text { months ( } n=27 \text { ) }\end{array}$ & $\begin{array}{ll}\text { - } & \text { SNOT-22 } \\
\text { - } & \text { NPS } \\
\text { - } & \text { Adverse events }\end{array}$ & $\begin{array}{l}\text { - After } 3 \text { months the erdosteine only } \\
\text { group compared to the erdosteine and } \\
\text { mometasone groups had significantly } \\
\text { better change in SNOT-22 and polyp } \\
\text { score } \\
\text { - There was no difference in adverse } \\
\text { events and adverse events were mild }\end{array}$ \\
\hline $\begin{array}{l}\text { Majima } \\
2012^{(261)}\end{array}$ & $\mathrm{RCT}$ & $\begin{array}{l}425 \text { CRS } \\
\text { (318 } \\
\text { analysed) }\end{array}$ & $\begin{array}{l}\text { - Clarithromycin ( } 200 \mathrm{mg} / \text { day) } \\
\text { for } 12 \text { weeks ( } n=213) \\
\text { - Clarithromycin ( } 200 \mathrm{mg} / \\
\text { day) and S-carboxymeth- } \\
\text { ylcysteine ( } 1500 \mathrm{mg} / \text { day; } \\
\text { combination group) for } 12 \\
\text { weeks ( } n=212)\end{array}$ & $\begin{array}{l}\text { - SNOT-20 at } 12 \text { weeks } \\
\text { - CT scan at } 12 \text { weeks } \\
\text { - } \% \text { of patients with remark- } \\
\text { ably effective or effective } \\
\text { response at } 4,8 \text { and } 12 \\
\text { weeks } \\
\text { - } \% \text { of patients with im- } \\
\text { - } \text { provement of endoscopy } \\
\text { - Adverse events }\end{array}$ & $\begin{array}{l}\text { - No significant difference in effect of } \\
\text { - SNOT-20 or CT scan at } 12 \text { weeks } \\
\text { wignificantly higher } \% \text { of patients } \\
\text { response at } 12 \text { weeks, but not at } 4 \text { and } \\
8 \text { weeks } \\
\text { - Significant higher \% of patients with im- } \\
\text { proved characteristics of nasal discharge } \\
\text { and reduced post-nasal discharge } \\
\text { - No serious adverse events }\end{array}$ \\
\hline
\end{tabular}

CRS,Chronic Rhinosinusitis; CRSwNP, Chronic Rhinosinusitis with nasal polyps; SNOT-22, Sino-Nasal Outcome Test-22; NPS, Nasal Polyp Score; SNOT-20, Sino-Nasal Outcome Test-20; RCT, Randomised Controlled Trial;

(1.2\%) during the treatment period; no causal relationship with the study medication was seen for all of these. No medically relevant differences in laboratory parameters or vital signs were observed for patients treated with Sinupret extract compared to placebo.

Of the four local studies, three measured quality of life with SNOT $^{(264-266)}$, two symptoms severity ${ }^{(265,267)}$ and two CT scan ${ }^{(264,}$ ${ }^{266)}$. The studies were too different to combine into a metaanalysis. One study found a significant improvement in the group treated with Nigella sativa nasal spray versus saline in SNOT-20, endoscopy and CT scan at four weeks' treatment. Two studies compared to treatment with FPNS. One showed no difference compared to fluticasone diproporanate nasal spray (FPNS) but in the other comparing intranasal administration of Sinupim (P. anisium) drops to FPNS, interestingly, the intranasal administration of $P$. anisium led to a significantly greater improvement $(p=0.012)$ in SNOT-22 score when compared to FPNS. However, the lack of blinding increases performance bias $^{(266)}$. Sinupim was well tolerated by patients. There were no serious adverse effects in either group.

\subsubsection{Conclusion}

Of five RCTs evaluating herbal treatment, a large DBPCT, although a post-hoc sensitivity analysis, showed a significant benefit in major symptom score at 12 weeks of treatment over placebo in patients with a diagnosis of CRS for $>1$ year and a baseline MSS $>9$ (out of max 15) showed overall no effect ${ }^{(268)}$. Of the four studies evaluating different local herbal treatment three showed a favorable effect. However, not all studies were blinded and the quality of the studies was variable.

The treatment does not show significantly more adverse events than placebo. The quality of the evidence for the local treatment is low.

Based on the available data, the EPOS2020 group cannot advise on the use of herbal medicine in CRS.

\subsubsection{Traditional Chinese medicine and acupuncture}

Complementary and alternative medicine (CAM) is commonly inquired about and often used by patients with $\mathrm{CRS}^{(270)}$. Many different CAM therapies are available including herbal medications, homeopathy and traditional Chinese medicine. We identified five RCTs for review.

\subsection{1. Traditional Chinese Medicine}

Two studies examined the use of Traditional Chinese Medicine in CRS. One study in 53 CRSsNP compared the Chinese herbal medicine (CHM) Tsang-Erh-San with Houttuynia with an erythromycin control group ${ }^{(34)}$ (Table 6.1.20.1). At eight weeks both groups had a significant improvement in their SNOT-20 score, however there was no significant difference between the two groups. Neither intervention influenced the endoscopic score or bacterial culture rate. The validity of these improvements is unclear due to the lack of placebo control. Another study in 97 postoperative CRS patients compared the same CHM (Tsang-Erh-San with Houttuynia) with amoxicillin and placebo ${ }^{(271)}$. All three groups had significant improvements in their RSOM-31 and endoscopic score at eight weeks postoperatively, however there was no significant difference between the three groups. There was no significant difference post-operatively between the three groups on their acoustic rhinometry and CT scores.

\subsubsection{Traditional Chinese acupuncture}

In some countries acupuncture is used to treat CRS although there is hardly any documentation on the effect. We found two studies from the same group evaluating health-related 
Table 6.1.20.1. Traditional Chinese Medicine for the treatment of patients with CRS.

\begin{tabular}{|c|c|c|c|c|c|}
\hline Study & Methods & Participants & Interventions & Outcomes & Results \\
\hline $\begin{array}{l}\text { Jiang } \\
2012^{(34)}\end{array}$ & DBPCT & $\begin{array}{l}53 \text { CRSsNP } \\
\text { patients }\end{array}$ & $\begin{array}{l}\text { - Chinese Herbal Medicine ( } 2 \mathrm{~g} \text { of Tsang-Erh- } \\
\text { San extract granules and } 1 \mathrm{gm} \text { of Houttuyn- } \\
\text { ia extract powder twice daily for } 8 \text { weeks) } \\
\text { with erythromycin placebo ( } \mathrm{n}=26 \text { ) } \\
\text { - Erythromycin ( } 250 \mathrm{mg} \text { twice daily for } 8 \\
\text { weeks) with CHM placebo }(n=27)\end{array}$ & $\begin{array}{l}\text { - SNOT-20 } \\
\text { - Endoscopic score } \\
\text { - Saccharin transit test } \\
\text { - Bacterial culture rate before and } \\
\text { at } 8 \text { weeks of treatment }\end{array}$ & $\begin{array}{l}\text { - No significant differ- } \\
\text { ence in any endpoints } \\
\text { between the groups }\end{array}$ \\
\hline $\begin{array}{l}\text { Liang } \\
2011^{(271)}\end{array}$ & DBPCT & $\begin{array}{l}97 \text { CRS } \\
\text { postoperative }\end{array}$ & $\begin{array}{l}\text { - Chinese Herbal Medicine ( } 2 \mathrm{~g} \text { of Tsang-Erh- } \\
\text { San extract granules and } 1 \mathrm{gm} \text { of Houttuyn- } \\
\text { ia extract powder twice daily for } 8 \text { weeks) } \\
\text { with } 4 \text { weeks of amoxicillin placebo ( } n=33 \text { ) } \\
\text { - Amoxicillin ( } 250 \text { mg three times dailly for } \\
4 \text { weeks and CHM placebo for } 8 \text { weeks } \\
(n=34) \\
\text { - Placebo }(n=30)\end{array}$ & $\begin{array}{l}\text { - } \text { RSOM-31 } \\
\text { - Endoscopic score } \\
\text { - } \text { Acoustic rhinometry } \\
\text { - } \text { Nasal swabs at } 8 \text { weeks post- } \\
\text { operative } \\
\text { - } \text { CT (LM-scoreat } 12 \text { weeks post- } \\
\text { operative }\end{array}$ & $\begin{array}{l}\text { - No significant differ- } \\
\text { ence in any endpoints } \\
\text { between the groups }\end{array}$ \\
\hline
\end{tabular}

Table 6.1.20.2. Acupuncture for the treatment of patients with CRS.

\begin{tabular}{|c|c|c|c|c|c|}
\hline Study & Methods & Participants & Interventions & Outcomes & Results \\
\hline $\begin{array}{l}\text { Stavem } \\
2008^{(272)}\end{array}$ & $\begin{array}{l}\text { RCT } \\
\text { (Investigator } \\
\text { not blinded. } \\
\text { Patient's } \\
\text { partially } \\
\text { blinded.) }\end{array}$ & $\begin{array}{l}66 \text { CRSsNP (49 } \\
\text { evaluated) }\end{array}$ & $\begin{array}{l}\text { - Traditional Chinese Acupuncture } \\
\text { (10 treatments over } 4 \text { weeks) ( } n=17) \\
\text { - Sham acupuncture ( } 10 \text { treatments } \\
\text { - Medical management (xylometazo- } \\
\text { line and normal saline irrigation } \\
\text { for } 1 \text { week, oral corticosteroids for } \\
14 \text { days, and antibiotics - either } \\
\text { cephalexin 1,500mg daily for } 10 \\
\text { days or azithromycin } 500 \text { mg for } 7 \\
\text { days) ( } n=17 \text { ) }\end{array}$ & $\begin{array}{l}\text { - CSS } \\
\text { - SF-36 at baseline } \\
\text { and } 12 \text { weeks }\end{array}$ & $\begin{array}{l}\text { - No significant difference in any endpoints } \\
\text { between the groups }\end{array}$ \\
\hline $\begin{array}{l}\text { Rössberg } \\
2005^{(274)}\end{array}$ & $\mathrm{RCT}$ & $\begin{array}{l}55 \text { CRS patients } \\
\text { (49 evaluated at } \\
4 \text { weeks, } 37 \text { at } \\
12 \text { weeks and } \\
29 \text { at } 1 \text { year) }\end{array}$ & $\begin{array}{l}\text { - Traditional Chinese acupuncture } 10 \\
\text { treatments with bilateral acupoints } \\
\text { over a period of } 4 \text { weeks ( } n=25 \text { ) } \\
\text { - Minimal acupuncture at non-acu- } \\
\text { points } 10 \text { treatments with bilateral } \\
\text { acupoints over a period of } 4 \text { weeks } \\
\text { ( } n=19) \\
\text { - Conventional medical therapy } \\
\text { consisting of xylometazoline } \\
\text { saline locally for } 1 \text { week, and oral } \\
\text { corticosteroids for } 14 \text { days ( } n=21 \text { ). } \\
\text { In addition, cefalexin } 1500 \text { mg daily } \\
\text { for } 10 \text { days ( } n=14 \text { ) or azithromycin } \\
500 \text { mg for } 7 \text { days ( } n=6 \text { ) }\end{array}$ & $\begin{array}{l}\text { - } \text { SF-36 at } 12 \\
\text { weeks } \\
\text { - } \mathrm{QOL} \text { (VAS) at } 12 \\
\text { weeks } \\
\text { - } \text { Total symptom } \\
\text { score at } 4,12 \\
\text { and } 52 \text { weeks } \\
\text { - } \text { CT scan at } 4 \\
\text { weeks }\end{array}$ & $\begin{array}{l}\text { - Conventional treatment improved CT } \\
\text { scan at } 4 \text { weeks contrary to both acu- } \\
\text { - } \text { Tuncture groups } \\
\text { improvement ( } p=0.06 \text { ) compared both } \\
\text { acupuncture groups } \\
\text { - Significant improvement in SF-36 and } \\
\text { QOL (VAS) at } 12 \text { weeks for conventional } \\
\text { treatment contrary to both acupuncture } \\
\text { groups } \\
\text { - After } 12 \text { months, } 15 \text { patients reported } \\
\text { having had surgical intervention for } \\
\text { chronic sinusitis (conventional group } 5 \text {, } \\
\text { acupuncture } 6, \text { sham 4), and there was lit- } \\
\text { tle difference in symptom score between } \\
\text { the groups ( } p=0.32 \text { ). }\end{array}$ \\
\hline
\end{tabular}

CSS, Chronic sinusitis survey ;CRS,Chronic Rhinosinusitis; CRSsNP, Chronic Rhinosinusitis without nasal polyps ; RCT, Randomised Controlled Trial ; SF-36, Short form 36; QOL, Quality of Life ;VAS, Visual Analogue Scale ; CT, computed tomography. 
quality of life comparing traditional Chinese acupuncture, sham acupuncture, and 2-4 weeks of medication with antibiotics, corticosteroids, $0.9 \%$ sodium chloride solution, and local decongestants ${ }^{(272,273)}$. Change in health-related quality of life was assessed over 4-52 weeks using the Chronic Sinusitis Survey (CSS), Short form 36 (SF-36) questionnaires, total symptom scores and CT scan. One study ${ }^{(274)}$ in general showed better effect of the conventional treatment than both traditional Chinese acupuncture and sham acupuncture, the other did not show difference in effects on CSS or SF-36 between the traditional Chinese acupuncture and the sham acupuncture groups (Table 6.1.20.2).

\subsubsection{Conclusion}

There is no evidence that traditional Chinese medicine or acupuncture is more effective than placebo in the treatment of CRS. The safety of Chinese medicine is unclear because most of the papers are not ready accessible ${ }^{(275)}$. Minor and serious adverse events can occur during the use of acupuncture and related modalities, contrary to the common impression that acupuncture is harmless ${ }^{(276)}$. For this reason, the EPOS2020 steering group advises against the use of traditional Chinese medicine or acupuncture.

\subsubsection{Topical furosemide and oral verapamil}

\subsubsection{Summary of the evidence}

While surgical intervention has often been the mainstay in treatment of nasal polyps, the propensity for some patients to relapse has led to further investigations with therapies aimed at preventing recurrence. Two of these medications are verapamil and furosemide.

Verapamil is a non-dihydropyridine Ca+-channel blocker, given orally, and an inhibitor of P-gp and can block IL-5, IL-6, and thymic stromal lymphopoietin (TSLP) secretion. One double blind placebo-controlled trial(277) evaluated the use of oral Verapamil for treatment of patients with CRSwNP. Patients were treated with either $80 \mathrm{mg}$ of Verapamil three times per day versus placebo for eight weeks.

Patients were evaluated at two, four and eight weeks for changes in quality of life (SNOT-22), symptoms (VAS), or changes in CT scan (LMS). Patients were also evaluated for side effects from the medication. There was significant improvement from baseline to week eight in SNOT-22 scores (MD -27.7 95\%CI[49.36 to -6.05$], p=0.01$ ) and VAS scores (MD $-37.9795 \% \mathrm{CI}[-$ 60.01 to -15.93$], p=0.001$ ) in the verapamil group compared to placebo. There was also improvement in Lund-Mackay score in the verapamil group with an absolute mean difference of -5.20 (95\% Cl, -9.66 to $-0.74 ; \mathrm{p}=0.02$ ). There were also significant decreases in total Lund-Kennedy score in the verapamil group at week four with an LSM difference of $-2.8(95 \% \mathrm{Cl},-4.3$ to -0.98; $p=0.003)$. This trend continued to week eight but was no longer statistically significant. Patients with higher BMls had less improvement with verapamil and this is thought to be due to higher required doses that would otherwise be supratherapeutic (Table 6.1.21.1). Studies with local verapamil are ongoing (https://ichgcp.net/clinical-trials-registry/ NCT03102190).

Furosemide, in contrast, is a loop diuretic that acts on $\mathrm{Na}+$ and $\mathrm{K}+$ channels and can increase absorption of these ions, as well as water. This dehydrates the respiratory epithelial surface. A few studies have been completed evaluating their efficacy in the prevention of nasal polyp recurrence (Table 6.1.21.2). Hashemian et al. ${ }^{(887)}$ conducted a double-blind, placebocontrolled trial assessing the efficacy of topical furosemide on nasal polyps. Patients were randomly assigned to receive either postoperative nasal spray, two puffs twice daily for two months of either $300 \mu \mathrm{g}$ of furosemide per day or placebo. Patients were assessed six months after surgery for NPS (Meltzer score), CT scoring (LMS), QOL (SNOT-22), and VAS polyp scoring. Eighty-four patients completed treatment and were able to be evaluated, with 42 patients in each group $(n=84)$. During the study time, there were no clinically significant adverse effects. After treatment, significantly more patients in the furosemide group had a polyp score of 0 , compared to the placebo group ( $79 \%$ vs $38 \% ; p<0.01$ ). There was a significant effect of furosemide on SNOT-22 scores (MD, 8.05; 95\%Cl, 3.24-12.85) and VAS scores (MD, $0.81 ; 95 \% \mathrm{Cl}, 0.22-1.39$ ) compared to placebo. However, the Lund-Mackay score did no show significant differences (MD, 2.52; 95\% $\mathrm{Cl},-0.35-5.39$ ).

Kroflic et al. ${ }^{(278)}$ evaluated the efficacy of oral methylprednisolone $(1 \mathrm{mg} / \mathrm{kg} /$ day) or inhalation of $6.6 \mathrm{mmol} / \mathrm{l}$ furosemide solution/10 min through a jet nebulizer-i.e., max. $20 \mathrm{mg} /$ day furosemide for seven days on patient reported symptoms (nasal obstruction, nasal hypersecretion, loss of smell (0-3), postnasal drip, snoring, sneezing, cough, swelling, epistaxis, headache (0-1), fatigue, temperature, previous surgery (0-1) adding up to a total symptom score of 20), NPS (Malm 0-6), and histomorphometric analysis of eosinophils, mastocytes, and edema. Both groups showed significant improvement in subjective symptom scores (pre-steroid mean 15.5 \pm 3.4 to post-steroid 9.5 $\pm 3.6, \mathrm{p}<0.001$; pre-furosemide $15.6 \pm 3.9$ to post-furosemide $9.8 \pm 3.7, \mathrm{p}<0.001$ ) and NPS (steroid: $2.4 \pm 0.7$ to $2.0 \pm 0.8, p<0.001$; furosemide: $2.2 \pm 0.9$ to $1.7 \pm 0.9 ; p<0.001$ ).

Table 6.1.21.1. Oral Verapamil for the treatment of patients with CRSwNP.

\begin{tabular}{|c|c|c|c|c|c|}
\hline Study & Methods & Participants & Interventions & Outcomes & Results \\
\hline $\begin{array}{l}\text { Miyake } \\
2017^{(277)}\end{array}$ & DBPCT & $\begin{array}{l}\text { CRSWNP } \\
(n=20)\end{array}$ & $\begin{array}{l}\text { Verapamil } 80 \text { mg four } \\
\text { times daily for } 8 \text { weeks } \\
(n=10) \text { vs placebo for } 8 \\
\text { weeks }(n=10)\end{array}$ & $\begin{array}{l}\text { At 2,4, and } 8 \text { weeks } \\
\text { - Quality of life (SNOT-22) } \\
\text { - Symptoms (VAS) } \\
\text { - } \text { CT scan (LM score) } \\
\text { - Nasal endoscopy (Lund } \\
\text { Kennedy Score) }\end{array}$ & $\begin{array}{l}\text { - Significant improvement in SNOT-22, VAS, } \\
\text { and LM score at } 8 \text { weeks } \\
\text { - Significant improvement in Lund-Kennedy } \\
\text { score at } 4 \text { weeks, but not significant at } 8 \\
\text { weeks } \\
\text { - Less improvement patients with higher BMI }\end{array}$ \\
\hline
\end{tabular}


Table 6.1.21.1. Oral Verapamil for the treatment of patients with CRSwNP.

\begin{tabular}{|c|c|c|c|c|c|}
\hline Study & Methods & Participants & Interventions & Outcomes & Results \\
\hline $\begin{array}{l}\text { Hashemian } \\
2016^{(887)}\end{array}$ & DBPCRCT & $\begin{array}{l}\text { CRSWNP } \\
(\mathrm{N}=84)\end{array}$ & $\begin{array}{l}\text { - } 2 \text { puffs twice daily for } 2 \\
\text { months of either } 300 \mu \mathrm{g} \text { of } \\
\text { furosemide per day }(\mathrm{n}=42) \\
\text { - } 2 \text { puffs twice daily for } 2 \\
\text { months placebo per day } \\
(n=42)\end{array}$ & $\begin{array}{l}\text { Assessed at } 6 \text { months: } \\
\text { - QOL (SNOT-22, 0-110) } \\
\text { - VAS polyps score (0-10) } \\
\text { - NPS (Meltzer score, 0-8) } \\
\text { - CT PNS scoring (0-30) }\end{array}$ & $\begin{array}{l}\text { At } 6 \text { months furosemide versus placebo } \\
\text { resulted in: } \\
\text { - Significantly reduced SNOT-22 scores } \\
\text { (difference, } 8.05 ; 95 \% \mathrm{Cl}, 3.24-12.85 \text { ) and } \\
\text { VAS polyp scores (difference, } 0.81 ; 95 \% \mathrm{Cl} \text {, } \\
\text { 0.22-1.39). } \\
\text { - Significantly more patients with a polyp } \\
\text { score of } 0 \text { (79\% vs 38\%; } \mathrm{P}<0.01 \text { ). } \\
\text { - No differences in CT PNS scores }\end{array}$ \\
\hline $\begin{array}{l}\text { Kroflic } \\
2006^{(278)}\end{array}$ & $\mathrm{RCT}$ & $\begin{array}{l}\text { CRSWNP } \\
(n=40)\end{array}$ & $\begin{array}{l}\text { - Inhaled 6.6mmol/L furo- } \\
\text { semide over } 10 \text { minutes } \\
\text { (max } 20 \mathrm{mg} / \text { day) }(\mathrm{n}=20) \\
\text { for } 7 \text { days } \\
\text { - Methylprednisolone } 1 \mathrm{mg} / \\
\text { kg/day }(\mathrm{n}=20)\end{array}$ & $\begin{array}{l}\text { After } 1 \text { week: } \\
\text { - Total symptom score (0-20) } \\
\text { - Nasal obstruction, nasal } \\
\text { hypersecretion, loss of smell } \\
\text { (0-3), postnasal drip, taste, } \\
\text { snoring, sneezing, cough, } \\
\text { swelling, epistaxis, headache } \\
\text { (0-1), and fatigue, tempera- } \\
\text { ture, previous surgery (0-1) } \\
\text { adding up to total symptom } \\
\text { score (0-20) } \\
\text { - NPS (Malm score, 0-6) } \\
\text { - Tissue eosinophil count } \\
\text { - Tissue mastocyte count } \\
\text { - Tissue oedema }\end{array}$ & $\begin{array}{l}\text { - No significant differences between furo- } \\
\text { semide and Methylprednisolone }=\S \text { Both } \\
\text { treatments were significantly effective in } \\
\text { reduction of nasal symptoms and polyp } \\
\text { scores } \\
\text { - Steroid group has significant reduction in } \\
\text { eosinophils, but no effect on mastocytes } \\
\text { or oedema. There was no improvement in } \\
\text { the furosemide group }\end{array}$ \\
\hline $\begin{array}{l}\text { Passali } \\
2003^{(279)}\end{array}$ & $\mathrm{RCT}$ & $\begin{array}{l}\text { CRSwNP post- } \\
\text { operatively } \\
(n=170)\end{array}$ & $\begin{array}{l}\text { - Furosemide } 2 \text { puffs }(50 \mu \mathrm{g} / \\
\text { puff) per day per nostril } \\
\text { for } 30 \text { days ( } n=97) \text { vs. } \\
\text { mometasone fuorate } \\
\text { nasal spray } 2 \text { puffs per day } \\
\text { per nostril (puff }=100 \mu \mathrm{g} \text { ) } \\
\text { for } 30 \text { days ( } n=33 \text { ) both } \\
\text { with months of no treat- } \\
\text { ment) vs. no additional } \\
\text { treatment }(n=40)\end{array}$ & $\begin{array}{l}\text { Assessed every } 6 \text { months (for } \\
\text { up to } 9 \text { years) } \\
\text { - Acoustic rhinometry } \\
\text { - Nasal endoscopy }\end{array}$ & $\begin{array}{l}\text { - Surgery restored normal patency in all } \\
\text { patients during the first year of follow-up } \\
\text { - The untreated group had significant } \\
\text { worsening of nasal volumes at the end of } \\
6 \text { years ( } 16.6 \pm 1.3 \mathrm{~cm} 3 ; \mathrm{p}=0.02 \text { ) } \\
\text { - More patients in the untreated group had } \\
\text { relapse of nasal polyps compared to furo- } \\
\text { semide or MFNS ( } 30 \% \text { vs. } 17.5 \% \text { vs. } 24.2 \% \text {; } \\
\text { no p value available) } \\
\text { - Patients without treatment had a signifi- } \\
\text { cantly higher NPS of recurrence compared } \\
\text { to furosemide and mometasone ( } 20 \% \text { vs. } \\
2 \% \text { vs. } 3 \% ; p<0.05 \text { ) }\end{array}$ \\
\hline
\end{tabular}

CRSwNP: chronic rhinosinusitis with nasal polyps; DBPCRCT: Double-blind placebo-controlled randomiced control trial; RCT: randomiced control trial; QOL: Quality of Life; SNOT-22: Sino-Nasal Outcome Test-22; VAS: Visual analogue score; NPS: Net promoter score; CT PNS; Computed Tomography para nasal sinuses.

There was no significant difference between the two groups. Given the small sample size, this may be considered to be a type 2 error. The steroid group had significant improvement in polyp eosinophil counts $(50-2.35 \pm 392.66$ to $190.65 \pm 291.41$; $\mathrm{p}<0.01)$, while the furosemide group did not $(280.95 \pm 364.58$ to $382.80 \pm 370.60 ; p=0.185)$. Neither group had significant changes in oedema or tissue mastocytes.

Passali et al. ${ }^{(279)}$ conducted a pseudo-randomized trial on patients with CRSwNP following surgery. Patients were randomized to either receive topical furosemide, no treatment, or topical mometasone furoate nasal spray (MFNS) postoperatively after either anterior or full ethmoidectomy. For six years (1991-1997) patients were randomized between topical furosemide and no treatment and after 1997 randomization was done between furesomide and MFNS. No details about the randomization process are given. Follow-up ranged from 1-9 years over the group as a whole. Patients in the furosemide group received two puffs per day per nostril (each puff $=50 \mu \mathrm{g}$ ) for 30 days, then one month of no treatment and then repetition of the furesomide treatment until a total of four months per year. The MFNS group received two puffs per day per nostril (puff $=100 \mu \mathrm{g}$ ) for 30 days then following a similar regimen to the furosemide group, and the no treatment group received no further intervention following surgery. Patients were assessed for relapse. Relapse was based on acoustic rhinometry active anterior rhinomanometry, and the presence of nasal polyp recurrence. Acoustic rhinometry and nasal endoscopy was assessed every six months in each patient. NPS was as follows: stage 0 , no nasal polyps seen; stage 1 , nasal polyps confined to the middle meatus, with acoustic rhinometry values within the normal range $\left(24.5 \pm 1.5 \mathrm{~cm}^{3}\right)$; stage 2 , nasal polyps prolapsing beyond the middle turbinate, with less than a $10 \%$ reduction of nasal volumes (per AR); and stage 3, sub-obstructive nasal polyps requiring another operation ( $>50 \%$ reduction of nasal volumes). The no treatment group had a significant worsening of nasal volumes at the end of their six-year followup $\left(16.6 \pm 1.3 \mathrm{~cm}^{3} ; p=0.02\right)$. More patients in the untreated group had relapse compared to furosemide or mometasone 
Table 6.1.22.1. Capsaicin for the treatment of patients with CRSwNP.

\begin{tabular}{|c|c|c|c|c|c|}
\hline Study & Methods & Participants & Interventions & Outcomes & Results \\
\hline $\begin{array}{l}\text { Zheng } \\
2000^{(282)}\end{array}$ & DBPCRCT & $\begin{array}{l}\text { CRSwNP post- } \\
\text { polypectomy } \\
(\mathrm{n}=51)\end{array}$ & $\begin{array}{l}\text { - Capsaicin }\left(3 \times 10^{-6} \mathrm{~mol},\right. \\
\text { dissolved in } 70 \% \text { ethanol) } \\
(n=29) \\
\text { - vehicle alone ( } 70 \% \text { etha- } \\
\text { nol) }(n=22) \text { once a week } \\
\text { times } 5 \text { weeks }\end{array}$ & $\begin{array}{l}\text { Assessed at } 9 \text { months: } \\
\text { - Nasal blockage (0-5) } \\
\text { - Rhinorrhoea (0-5) } \\
\text { - NPS (0-6) }\end{array}$ & $\begin{array}{l}\text { At } 9 \text { months there was no difference in } \\
\text { subjective rhinorrhoea in either group. } \\
\text { - The capsaicin group at } 9 \text { months group had } \\
\text { a significant decrease in NR compared to } \\
\text { the vehicle group } \\
\text { - NPS data? }\end{array}$ \\
\hline $\begin{array}{l}\text { Filiaci } \\
1996^{(281)}\end{array}$ & DBPCRCT & CRSwNP $(n=30)$ & $\begin{array}{l}\text { - Capsaicin } 0.1 \mathrm{~mL}(30 \mu \mathrm{mol}) \\
\text { in each fossa once weekly } \\
\text { for } 5 \text { weeks (after anes- } \\
\text { thetizing the nasal cavity) } \\
(\mathrm{n}=15) \\
\text { - } 0.1 \mathrm{~mL} \text { physiologic solution } \\
(\mathrm{n}=15) \text { in each fossa once } \\
\text { weekly for } 5 \text { weeks }\end{array}$ & $\begin{array}{l}\text { Assessed at } 1 \text { and } 3 \text { months: } \\
\text { - Nasal symptoms: obstruc- } \\
\text { tion, secretion, itching, } \\
\text { sneezing (0-5) } \\
\text { - NPS (0-6) } \\
\text { - Nasal resistance (active } \\
\text { anterior rhinomanometry) } \\
\text { - Specific nasal hyperreactivi- } \\
\text { ty (provocation testing with } \\
\text { cold water with rhinoma- } \\
\text { nometric assessment) } \\
\text { - Nasal tissue eosinophils }\end{array}$ & $\begin{array}{l}\text { At } 1 \text { and } 3 \text { months when comparing the } \\
\text { capsaicin group versus the controls, the } \\
\text { capsaicin group had: } \\
\text { - A significantly larger decrease in all nasal } \\
\text { - symptoms } \\
\text { A significant larger decrease difference in } \\
\text { NPS } \\
\text { - Significantly larger improvement in nasal } \\
\text { resistance } \\
\text { - Significantly improved nasal hyperreactivity } \\
\text { - Significantly increased nasal tissue eosin- } \\
\text { ophils }\end{array}$ \\
\hline
\end{tabular}

CRSwNP, Chronic Rhinosinusitis with nasal polyps; DBPCRCT, Double Blind Placebo Controlled Randomised Controlled Trial ; NPS, Nasal Polyp Score.

Figure 6.1.22.1. Forest plot of the effect of capsaicin versus placebo on nasal obstruction in CRSwNP patientsmonths.

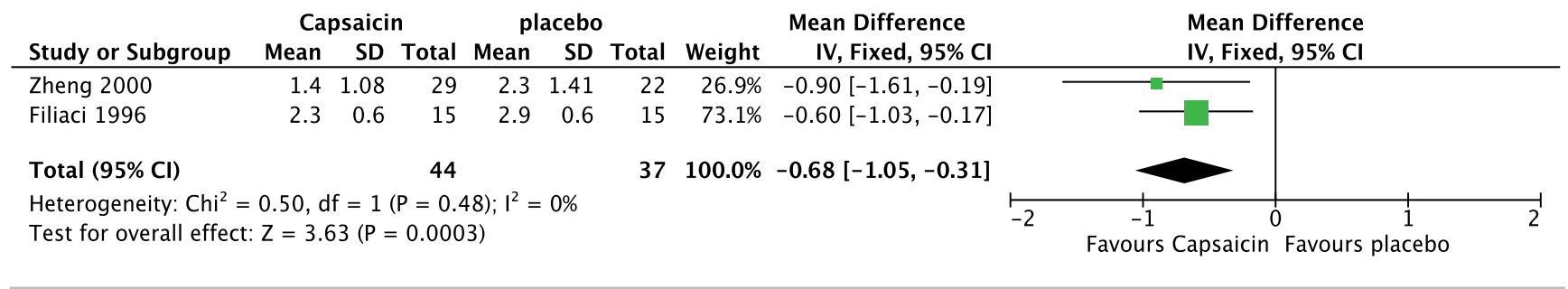

(30\%v17.5\%v24.2\%; no p value available). Patients without treatment had a significantly higher NPS of polyp recurrence compared to furosemide and mometasone fuorate nasal spray ( $20 \%$ vs $2 \%$ vs $3 \%$; $p<0.05$ ).

Another report of Passali ${ }^{(280)}$ appears to report on a subgroup of the patients of the 2003 study ${ }^{(279)}$.

\subsubsection{Conclusion}

There is some indication that verapamil might have antiinflammatory effects. A very small pilot study showed significant improvement in QOL (SNOT-22), polyp score (VAS), and CT scan (LMS) of oral verapamil over placebo. (Potential) side effects limited the dosage.

There is some indication that topical furosemide might have a beneficial effect on symptoms and signs of CRSwNP. A recent DBPCT study showed significantly reduced QOL (SNOT-22) scores and polyp score (VAS), and significantly more patients with an NPS of 0 in the furosemide nasal spray treated group versus placebo. There was no indication of a difference in adverse events between topical furosemide and placebo. The quality of the evidence for oral verapamil and topical furosemide is very low. Based on the potential side effects the EPOS2020 steering group advises against the use of oral verapamil. The group also cannot advise on local furosemide. Further placebo-controlled studies on both local furosemide and local verapamil are needed to determine efficacy and appropriate patient selection.

\subsubsection{Capsaicin}

\subsubsection{Summary of the evidence}

Neuropeptides and parasympathetic nerves in the nasal mucosa are thought to potentially play a role in the formation of nasal polyps. Therefore, capsaicin, a medication that can be applied topically has been considered for possible prevention of nasal polyp recurrence. Capsaicin, which acts upon the $C$ fibers, is thought to neuromodulate nasal hyper-reactivity and potentially prevent nasal polyp recurrence. Two studies completed double blind, placebo-controlled, randomized trials (Table 6.1.22.1).

Filiaci et al. ${ }^{(281)}$, evaluated 30 patients, with half of them receiving capsaicin $0.1 \mathrm{~mL}(30 \mu \mathrm{mol})$ in each fossa versus $0.1 \mathrm{~mL}$ physiologic solution in each fossa once weekly for five weeks. Patients were then assessed at three months for nasal symptoms, including obstruction, itching, and sneezing, endoscopic polyp scoring, nasal resistance, specific nasal hyperreactivity, and eosinophils in nasal polyp tissue. The capsaicin group had a significant decrease in all nasal symptoms compared to placebo $(p<0.01)$. There was a significant difference in the nasal polyp scores (NPS) between the capsaicin and control group at one month $(p<0.05)$ and three months $(p<0.01)$. There was significant improvement in nasal resistance in the capsaicin group compared to the control group at three months post-treatment $(p<0.01)$. There was a significant difference in nasal hyperreactivity between 
Table 6.1.23.1. Omeprazole for the treatment of patients with CRS.

\begin{tabular}{|c|c|c|c|c|c|}
\hline Study & Methods & Participants & Interventions & Outcomes & Results \\
\hline $\begin{array}{l}\text { Anzic } \\
2018^{(287)}\end{array}$ & DBPCT & $\begin{array}{l}60 \text { CRS } \\
\text { patients with } \\
\text { GORD }\end{array}$ & $\begin{array}{l}\text { - Omeprazol } 20 \mathrm{mg} \text { once } \\
\text { daily }(n=33) \text { for } 8 \text { weeks } \\
\text { - Placebo once daily }(n=27) \\
\text { for } 8 \text { weeks }\end{array}$ & $\begin{array}{l}\text { At } 8 \text { weeks: } \\
\text { - CRS symptom score (0-48) } \\
\text { - Nasal endoscopy score } \\
\text { - RSI score } \\
\text { - RFS }\end{array}$ & $\begin{array}{l}\text { No significant differences at } 8 \text { weeks } \\
\text { between Omeprazole and placebo apart } \\
\text { from reflux finding score }\end{array}$ \\
\hline
\end{tabular}

CRS,Chronic Rhinosinusitis; DBPCT, Double Blind Placebo Controlled Trial ; GORD, Gastro-oesophageal reflux disease ; RSI, Reflux symptom index; RFS, Reflux finding score.

the capsaicin and control group at three months $(p<0.01)$. Nasal tissue eosinophils were significantly increased in the patients undergoing capsaicin treatment at one and three months $(<0.01)$.

Zheng et al. ${ }^{(282)}$, also conducted a double blind, placebocontrolled trial comparing capsaicin $\left(3 \times 10^{-6} \mathrm{~mol}\right.$, dissolved in $70 \%$ ethanol) versus vehicle alone ( $70 \%$ ethanol) once a week for five weeks, following nasal polypectomy. Patients were assessed at nine months for nasal resistance (0-5), rhinorrhoea (0-5), and NPS (0-6 unilateral). The capsaicin group had a significant decrease in nasal resistance compared to the vehicle group. $(p<0.01)$. There was no difference in subjective rhinorrhea in either group. Patients treated with capsaicin had lower nasal polyp score when compared to the control group (stage 0: $41 \%$ vs $4.5 \%, \mathrm{p}<0.01$ ). Adverse events were not mentioned in both studies but are know from studies in NAR to be mostly limited to local irritation ${ }^{(283)}$.

Pooled data analysis of the nasal obstruction measurement (0-5) showed significant benefit in the capsaicin group (SMD $-0.68[-1.05,-0.31], \mathrm{p}<0.0003$, two trials, 81 patients). The 12 was $0 \%$, suggesting no heterogeneity $\left(X^{2}=0, d f=1, p=0.98\right)$ (Figure 6.1.22.1).

Pooled data analysis of nasal polyp score showed significant benefit in the capsaicin group (SMD -1.82[-2.93, -0.7], $\mathrm{p}<0.01$, two trials, 81 patients). The $\mathrm{I} 2$ was $0 \%$, suggesting no heterogeneity $\left(X^{2}=0, d f=1, p=0.98\right)$ (Figure 6.1.22.2).

\subsubsection{Conclusion}

Two small double-blind placebo-controlled studies ${ }^{(281,282)}$ have evaluated the efficacy of capsaicin. Capsaicin showed a significant decrease in nasal obstruction and nasal polyp score, however data on other symptoms like rhinorrhea and smell are either non-significant or unreported. The data were consistent over the two studies, but we downgraded for imprecision and risk of bias. It has to be mentioned that blinding of capsaicin treatment is very difficult/impossible. The quality of the evidence is low and the EPOS steering group concludes that Capsaicin may be an option in treatment of CRS in patients with CRSwNP but that larger studies are needed.

\subsubsection{Proton-pump inhibitors in patients with GORD}

\subsubsection{Summary of the evidence}

There is some of evidence demonstrating an association between gastro-oesophageal reflux disease (GORD) and CRS. GORD is defined by reflux of gastric contents, resulting in troublesome symptoms and/or complications. CRS patients have been shown to have significantly higher incidences of gastro-oesophageal reflux compared with asymptomatic controls $^{(284)}$.

CRS is more prevalent in GORD sufferers than those without GORD although the diseases have overlapping symptoms like the feeling of mucus in the throat ${ }^{(285)}$. While CRS is a multifactorial process, the evidence suggests that GORD may play a role in CRS, at least in some patients. Potential pathogenic roles for GORD in CRS could be a greater prevalence of intranasal Helicobacter pylori and acid reflux into the nose/ nasopharynx. Evidence is conflicting for GORD as a factor in CRS treatment failure ${ }^{(286)}$.

There is one DBPCRCT evaluating the role of proton-pump inhibitors in CRS ${ }^{(287)}$ (Table 6.1.23.1). Sixty patients ( 28 women, aged 19-87 years) with CRS and GORD were treated with eight weeks of omeprazole $20 \mathrm{mg}$ once daily (o.d.). Although the

Table 6.1.24.1. Bacterial lysates for the treatment of patients with CRS.

\begin{tabular}{|c|c|c|c|c|c|}
\hline Study & Methods & Participants & Interventions & Outcomes & Results \\
\hline Heintz 1989(294) & DBPCT & $\begin{array}{l}284 \text { CRS patients } \\
\text { (some patients had } \\
\text { persistent RS after } \\
\text { sinus puncture) ( } 258 \\
\text { analysed) }\end{array}$ & $\begin{array}{l}\text { - Broncho-Vaxom, } 1 \text { capsule } \\
\text { daily for a period of } 10 \text { days per } \\
\text { month during } 3 \text { consecutive } \\
\text { months ( } n=127 \text { ) } \\
\text { - Placebo } 1 \text { capsule daily for a } \\
\text { period of } 10 \text { days per month } \\
\text { during } 3 \text { consecutive months } \\
(n=121)\end{array}$ & $\begin{array}{l}\text { - Symptom score (0-4) } \\
\text { for headache, purulent } \\
\text { nasal discharge, cough } \\
\text { and expectoration } \\
\text { - X-ray sinus } \\
\text { - Adverse events }\end{array}$ & $\begin{array}{l}\text { - Significant reduction in } \\
\text { symptoms } \\
\text { - Significant reduction in } \\
\text { opacification of X-ray sinus } \\
\text { - No difference in adverse } \\
\text { events }\end{array}$ \\
\hline
\end{tabular}


Table 6.1.26.1. Phototherapy for the treatment of patients with CRS.

\begin{tabular}{|c|c|c|c|c|c|}
\hline Study & Methods & Participants & Interventions & Outcomes & Results \\
\hline $\begin{array}{l}\text { Dulguerov } \\
2017^{(296)}\end{array}$ & DBPCT & $\begin{array}{l}50 \text { CRSsNP } \\
\text { patients }\end{array}$ & $\begin{array}{l}\text { - Mixed visible and } \\
\text { ultraviolet (UVA and } \\
\text { UVB) light source ap- } \\
\text { plication for } 3 \text { weeks } \\
(n=26) \\
\text { - Low intensity visible } \\
\text { light alone for } 3 \text { weeks } \\
(n=24)\end{array}$ & $\begin{array}{l}\text { At the end of } 3 \text { wk treatment and } \\
\text { one month after treatment: } \\
\text { - RSDI } \\
\text { - symptom severity (nasal obstruc- } \\
\text { tion, sense of smell, rhinorrhoea, } \\
\text { and facial pain) (VAS) } \\
\text { - Rhinomanometry } \\
\text { - Olfactory thresholds } \\
\text { - Nasal nitric nxide } \\
\text { - Carbon monoxide }\end{array}$ & $\begin{array}{l}\text { - No significant difference in any outcome } \\
\text { measured except carbon monoxide } \\
\text { - Significant increase in carbon monoxide } \\
\text { production in the treatment group as } \\
\text { compared to controls at the end of treat- } \\
\text { ment ( } 5.24 \text { vs. } 3.88, p=0.048 \text { ), however no } \\
\text { significant difference at } 1 \text { month following } \\
\text { treatment ( } 5.25 \text { vs. } 4.07, p=0.13 \text { ) }\end{array}$ \\
\hline $\begin{array}{l}\text { Kiricsi et al. } \\
2017^{(300)}\end{array}$ & $\mathrm{RCT}$ & $\begin{array}{l}87 \text { subjects } \\
\text { (76 evaluated) } \\
\text { CRSwNP } \\
\text { patients }\end{array}$ & 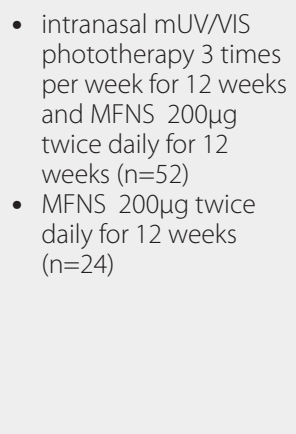 & $\begin{array}{l}\text { At week 12, week } 16 \text { and } 26 \text { weeks } \\
\text { - NOSE questionnaire } \\
\text { - Nasal symptom score (total nasal } \\
\text { symptom score and separate } \\
\text { symptom) } \\
\text { - Nasal endoscopy and polyp size } \\
\text { (0-5) } 3 \\
\text { - PNIF } \\
\text { - Acoustic rhinometry } 3 \\
\text { - Smell threshold } \\
\text { - Exhaled nasal nitric oxide }\end{array}$ & 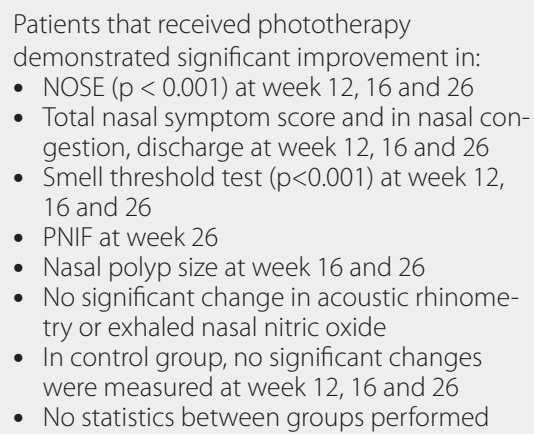 \\
\hline
\end{tabular}

CRSsNP, Chronic Rhinosinusitis without nasal polyps;CRSwNP, Chronic Rhinosinusitis with nasal polyps; DBPCT, Double Blind Placebo Controlled Trial ; RCT, Randomised Controlled Trial ; mUV/VIS, Mixed ultraviolet and visible light; UVA, Ultraviolet A; UVB, Ultraviolet B; MFNS, Mometasone Furoate Nasal spray ; RSDI, Rhinosinusitis Disability Index; VAS, Visual Analogue Scale ; NOSE, Nasal Obstruction Sympton Evaluation ; PNIF, Peak Nasal Inspiratory Flow ; NO, Nitric Oxide.

authors conclude that CRS symptom score, nasal endoscopy score, reflux symptom (index) score, and reflux finding scores were significantly reduced after eight weeks omeprazole treatment compared to placebo using ANOVA for repeated measurements or McNemar's test, only the reflux finding score showed a significant difference between the groups at the eight weeks measurement. Unfortunately, the matching of the groups at baseline was not good as the CRS and RSI were significantly different from each other at baseline. The long-term use of proton pump inhibitors is not without risk and has been associated with increased risk of cardiovascular disease and morbidity ${ }^{(288,289)}$.

\subsubsection{Conclusion}

There is one DBPCT trial evaluating the effect of omeprazole $20 \mathrm{mg}$ once daily for eight weeks in 60 patients that was illmatched at baseline making evaluating of the results difficult. At the end of the eight weeks treatment period there were no differences in CRS and nasal endoscopy scores. Moreover, long term use of proton pump inhibitors has been associated with increased risk of cardiovascular disease. The EPOS2020 steering group therefore does advise against the use of proton pump inhibitors in the treatment of CRS.

\subsubsection{Bacterial lysates}

\subsubsection{Summary of the evidence}

Bacterial lysates are constituted by a mixture of bacterial antigens derived from different respiratory bacterial species.
Different antigens are mixed and excipients are added in order to prepare lysate tablets. Bacterial lysates have both a specific and non-specific immunostimulating properties. Broncho-Vaxom ${ }^{\circledast}(\mathrm{OM}-85 \mathrm{BV})$ is a lysate of 21 strains of 8 bacteria(Staphylococcus aureus, Haemophilus influenzae, Streptococcus pyogenes, Moraxella catarrhalis, Klebsiella pneumoniae, Klebsiella ozaenae, Streptococcus viridans and, Diplococcus pneumoniae), that is used as an immunostimulant for the prevention and treatment of upper and lower airway infections and has the ability to enhance mucosal barrier function and regulate immune responses ${ }^{(290,291)}$. A recent metaanalysis showed that Broncho-Vaxom was positively correlated with a reduction in the frequency of respiratory infection $[M D=-2.33,95 \% \mathrm{Cl}(-2.75,-1.90), \mathrm{P}<0.00001]$ compared to the control group. The Broncho-Vaxom group was more effective than control groups in relation to the duration of antibiotics course, infections, fever, cough, and wheezing, increasing serum immunoglobulin levels (IgG, IgA or $\lg M)$, and T-lymphocytes subtype $\left(\mathrm{CD} 3+, \mathrm{CD} 4+\right.$, or CD8+) ${ }^{(292)}$. OM-85 BV has ciliostimulatory and immunogenic properties in an epithelial cell culture system that may be partially responsible for its observed efficacy as a respiratory therapeutic. These responses were NO-dependent and consistent with T2R activation ${ }^{(293)}$. Oral administration of Broncho-Vaxom(R) (OM-85 BV) was also shown to alleviate allergic rhinitis symptoms and to improve the overall mucosal immunity via restoring and maintaining the normal Th1/Th2 cytokine balance as an underlying cellular/ signaling mechanism ${ }^{(290)}$.

There is one DBRCT in adults with potential CRS patients 
although the population has been insufficiently described and some patients had persistent RS after sinus puncture ${ }^{(294)}$ (Table 6.1.24.1). Broncho-Vaxom or placebo (one capsule daily for a period of 10 days per month during three consecutive months) was administered to 284 patients presenting with chronic purulent rhinosinusitis. Patients were clinically examined before admittance to the study and at one, two, three and six months after treatment initiation Broncho-Vaxom therapy resulted in a significant decrease in purulent nasal discharge and headache over the full six months period compared to placebo. Also, the effect on the opacification of the sinus X-ray was significantly better in the Broncho-Vaxom treated group than the placebo group. No major side effects were reported, with comparable and good tolerability for $\mathrm{OM}-85$ and placebo.

\subsubsection{Conclusion}

There is one DBRCT from 1989 comparing the bacterial lysate Broncho-Vaxom to placebo in a large group of CRS patients resulting in a significant decrease in purulent nasal discharge and headache over the full six months period compared to placebo and reduced opacification of the sinus X-ray. Based on this limited evidence the EPOS2020 steering group cannot advise on the use of Broncho-Vaxom in the treatment of CRS. Larger studies of longer duration are needed with proper patient characterization and outcome evaluation.

\subsubsection{Homeopathy}

\subsubsection{Summary of the evidence}

Homeopathy is an alternative medicine. Its practitioners, called homeopaths, believe that a substance that causes symptoms of a disease in healthy people would cure similar symptoms in sick people. Homeopathic preparations are termed remedies and are made using a process of homeopathic dilution, in which a chosen substance is repeatedly diluted in alcohol or water, typically until nothing of the original substance remains in the product. Homeopathy in CRS involves treating both the underlying disease and its predominant symptoms with dilutions of substances that create/start the disease. There is much debate as to whether these dilutions actually contain any molecules of the original substance and hence whether any clinical effect is attributable to a placebo effect or study bias.

\subsubsection{Conclusion}

We could not retrieve RCTs on homeopathy in CRS published after 1990. Based on the available evidence, the steering group of EPOS2020 cannot advise on the use of homeopathy in the treatment of CRS.

\subsubsection{Phototherapy}

\subsubsection{Summary of the evidence}

The immunomodulative effect of phototherapy has been investigated as a potential therapeutic target for CRS patients. Ultraviolet phototherapy has been found to have a profound immunosuppressive effect with reduction of Langerhans cell number and function, induction of apoptosis in infiltrating $T$ cells, and induction of immune-modulatory cytokines such as TNF- $a$ and IL-10 ${ }^{(295)}$. Two RCT have been performed (Table 6.1.26.1.). Dulguerov et al. (2017) ${ }^{(296)}$ conducted a randomized, blinded and placebo-controlled study of mixed visible and ultraviolet light therapy (310-600 nm light spectrum) as compared to visible light alone in 50 patients with CRS without nasal polyps according to the EPOS diagnostic criteria ${ }^{(297)}$. Patients in the treatment group did not demonstrate a significant improvement in quality of life (QOL) (RSDI; 0.71 vs. $0.72 ; p=0.84$ ), nasal obstruction ( 4.87 vs. $4.56, p=0.63$ ), sense of smell ( 3.78 vs. $4.25 ; p=0.54$ ), rhinorrhoea ( 5.56 vs. $4.18 ; p=0.09$ ), facial pain ( 3.76 vs. $3.78, p=0.43)$, total nasal resistance $(0.65$ vs. $0.67, \mathrm{p}=0.88$ ), total nasal resistance after vasoconstrictor ( $0.56 \mathrm{vs}$. $0.44, p=0.12$ ), difference in total nasal resistance before and after vasoconstrictor ( 0.09 vs. $0.24, p=0.21)$, or nitric oxide production ( 571.45 vs. $656.85, p=0.21$ ) as compared to the controls at the end of treatment. Similar findings were observed at one month post-treatment. The authors observed an increase in carbon monoxide production in the treatment group as compared to controls at the end of treatment (5.24 vs. $3.88, p=0.048$ ), however this difference was not sustained at one month following treatment ( 5.25 vs. $4.07, p=0.13$ )

Studies of mixed ultraviolet/visible light have demonstrated the penetrative effect of this light therapy into polyp tissue to reach the inflammatory cells in the stroma and induce a dose-dependent increase of eosinophil and T-cell apoptosis ${ }^{(298,}$ ${ }^{299)}$. Kiricsi and colleagues conducted a randomized clinical trial comparing a combination therapy of mixed ultraviolet/visible

6.1.27. Filgastrim (r-met-HuG-CSF) for the treatment of patients with CRS.

\begin{tabular}{|c|c|c|c|c|c|}
\hline Study & Methods & Participants & Interventions & Outcomes & Results \\
\hline $\begin{array}{l}\text { Van Agthoven } \\
2001^{(301)}\end{array}$ & DBPCT & $\begin{array}{l}56 \text { CRSsNP } \\
\text { patients with } \\
\text { recalcitrant } \\
\text { CRS }\end{array}$ & $\begin{array}{l}\text { - Filgastrim } 300 \mathrm{mg} \text { subcutaneously once a day for } \\
\text { the first } 14 \text { days and every other day for another } \\
10 \text { weeks } \\
\text { - Placebo subcutaneously once a day for the first } \\
14 \text { days and every other day for another } 10 \text { weeks } \\
\text { All patients were treated at the beginning of the trial } \\
\text { with a combination of } 500-750 \mathrm{mg} \text { of ciprofloxacin } \\
\text { twice daily and } 450-600 \mathrm{mg} \text { of clindamycin three } \\
\text { times a day for } 14 \text { days. }\end{array}$ & $\begin{array}{l}\text { - At 4,8,12 and } 24 \text { weeks: } \\
\text { - SF-36 } \\
\text { - EuroQol } \\
\text { - McGill pain question- } \\
\text { naire }\end{array}$ & $\begin{array}{l}\text { - No significant } \\
\text { differences between } \\
\text { the groups }\end{array}$ \\
\hline
\end{tabular}

CRS, Chronic Rhinosinusitis ;CRSsNP, Chronic Rhinosinusitis without nasal polyps ; DBPCT, Double Blind Placebo Controlled Trial ; SF-36, Short Form 36. 
light phototherapy and intranasal corticosteroids and intranasal steroids in 87 subjects with CRSwNP(300). The authors used a mixed ultraviolet/visible light device containing 5\% ultraviolet B, $25 \%$ of ultraviolet $A$ and $70 \%$ of visible light, with the spectrum between 280 and $650 \mathrm{~nm}$. Mixed ultraviolet/visible light phototherapy led to a significant improvement in nasal congestion, nasal discharge, total nasal score, Nasal Obstruction Symptom Evaluation (NOSE) score and sense of smell $(p<0.001)$. The authors observed a significant improvement in nasal endoscopic scores in staging of polyps on the left and right side $(p<0.001$ and $p<0.008$, respectively). There was no significant improvement in these parameters in the control group. There was no significant improvement in acoustic rhinometry scores or exhaled nasal NO in the phototherapy or control groups. No comparisons were made between the two groups.

\subsubsection{Conclusion}

Two randomized clinical trials of phototherapy in CRS has shown variable results. The DBPCT trial did not show any effect. The non- blinded RCT in CRSwNP patients with phototherapy on top of nasal corticosteroid treatment showed a significant reduction in NOSE scale, symptomatology, smell and polyp score in the phototherapy group and not in the nasal corticosteroid only group. However, no comparisons between the groups were made.

The quality of the evidence for the use of phototherapy in patients with CRS is very low. Based on the evidence the EPOS2020 steering group cannot make recommendation on the use of phototherapy in patients with CRS.

\subsubsection{Filgastrim (r-met-HuG-CSF)}

The proliferation and differentiation of neutrophils are promoted by the administration of recombinant human granulocyte colony-stimulating factor ( $r$ G-CSF) (Table 6.1.27.). Clinical studies in subjects who are non-neutropenic indicate that rG-CSF may be beneficial as adjunctive therapy for treatment of serious bacterial and opportunistic fungal infections in patients who are nonneutropenic, including those with alterations in neutrophil function. Van Agthoven et al. evaluated in a double-blind placebo-controlled randomized clinical trial, the influence of filgastrim administration on QOL using the EuroQol, the Short Form (SF)-36, and the McGill pain questionnaire of 56 refractory chronic rhinosinusitis patients who did not respond to regular treatments during a 24-week trial $^{(301)}$. There was no significant difference in effect on QOL between the two groups.

\subsubsection{Topical barriers e.g. algae - carrageenans}

One alternative approach to prevention and treatment of infections of the nasal mucosa is the creation of a protective physical barrier in the nasal cavity with carrageenans, high molecular weight sulphated polysaccharides derived from red seaweed (Rhodophyceae). Several randomized clinical trials have investigated carrageenans as a potent antiviral agent in the management of upper respiratory tract infections ${ }^{(302-305)}$. To date, there have been no randomized clinical trials of topical carrageenans in CRS.

\subsubsection{Collodial Silver}

Colloidal silver (CS), a widely used naturopathic agent, has shown anti-biofilm properties both in vitro and within a rhinosinusitis animal model. A study in 22 patients evaluated the efficacy of CS as a topical nasal spray in patients with refractory CRSsNP and found no meaningful subjective or objective improvements ${ }^{(306)}$. No participants experienced negative health effects directly attributable to the administration of intranasal CS.

\subsubsection{Immunotherapy}

A systematic review evaluating the effect of immunotherapy (IT) found no RCTs. Lower level evidence resulted in seven studies of which three were prospective ${ }^{(307)}$.

The authors concluded that symptom scores improved in patients treated with IT when compared with baseline data and control patients. Objective endoscopic exam measures improved with IT treatment in short-term studies. Significant improvements were observed in radiographic assessments, and there was a decreased necessity for revision surgery, interventional office visits, and intranasal and oral steroid use. After highlighting the paucity of the data, they concluded that there is weak evidence to support the use of IT as an adjunctive treatment in CRS patients, particularly in the postoperative period. We are not aware of studies published after this systematic review.

\subsubsection{New potentials not on the market today}

\subsubsection{Introduction}

Many cytokines, alarmins, markers on effector cells, and novel options (like DNAzyme and biophages) that could potentially be used as therapeutic targets have been discussed in chapter 5.2.2. and more details can be found there. Here we focus on cytokines, alarmins, markers on effector cells, and novel options that are considered as new potential treatments at the moment.

\subsubsection{GATA-3 DNAzyme}

It is obvious that type 2 immune reactions are the most clinically relevant immune deviations in nasal polyposis as type 2 is associated with comorbid asthma and recurrent disease after sinus surgery and comorbid asthma ${ }^{(308)}$. Clinical relevance has been underscored by, current Phase 3 trials with anti-lgE, anti-IL-5 or IL-5 receptor alpha strategies, or anti-IL-4 receptor alpha (see chapter 6.1.14 - 6.1.16 for more details). Future concepts using more upstream targets, including GATA-3, focus even more on type 2 immune responses, as GATA- 3 is the transcription factor essential for Th2 and ILC2 development and function ${ }^{(309)}$. DNAzymes are single-stranded catalytic DNA molecules that bind and cleave specific sequences in a 
target mRNA molecule, such as GATA3 mRNA; this prevents the development of Th2 and ILC2 cells ${ }^{(310)}$. Their potential as novel therapeutic agents has been demonstrated in a variety of disease models, including allergic asthma ${ }^{(310,311)}$ and $\mathrm{COPD}^{(312)}$. As compared to baseline, GATA3-specific DNAzyme attenuated the late asthmatic airway response in subjects with allergic asthma and sputum eosinophilia by on average $34 \%$ and the early asthmatic response by on average $11 \%{ }^{(313)}$. The study also showed penetration of the drug into nasal polyp tissue and specifically into Th2 cells, underscoring its potential in CRSwNP. At the same time, the treatment was well tolerated. There is potential to develop a local nasal therapy for CRSwNP, as it has been demonstrated for asthma.

\subsubsection{Anti-siglec-8}

Eosinophils, mast cells, and basophils selectively express sialic acid-binding immunoglobulin-like lectin (Siglec) 8 , which is highly expressed in healthy and diseased subjects. As its activation may cause cell death, Siglec- 8 represents a potential therapeutic target ${ }^{(314)}$. Siglec-8 engagement in short-term IL-5activated eosinophils causes reactive oxygen species production and Src family kinases phosphorylation: both are essential in mediating Siglec-8-induced cell death ${ }^{(315)}$. A proof-of-concept study with the antibody AK001 however failed to show efficacy (unpublished). Presently, new antibodies against Siglec-8 are in early clinical development.

\subsubsection{Anti-OX40L}

In severe asthma, anti-OX40L may be a potential therapeutic target $^{(316)}$, as it allows the development of regulatory $\mathrm{T}$ (Treg) cells, which might suppress inflammatory T cells. However, the combination of two monoclonal antibody therapies (anti-OX40 L and anti-TSLP) on Treg frequency using a human model of allergic asthma failed to deliver the expected suppression ${ }^{(317)}$. In addition, despite anti-inflammatory efficacy, decreasing serum IgE (by 17\%) and sputum eosinophils (by 75\%), treatment with anti-OX40L monoclonal antibodies failed to attenuate the early and late airway response to inhaled allergen ${ }^{(318)}$. So far, no studies with anti-OX40L have been performed in CRSwNP.

\subsubsection{Anti-TNF alpha}

In addition to type 2 inflammatory components, also nontype2/type 2-low or type 1, bacteria or endotoxins-triggered, neutrophilic responses may comprise future targets for monoclonal antibody therapy in $\mathrm{CRS}^{(319)}$. In a placebo-controlled study in healthy volunteers comparing anti-TNF with oral corticosteroids (OCS), the endotoxin-induced neutrophilic response in sputum was significantly modulated by anti-TNF in contrast to OCS and control treatment ${ }^{(319)}$. Therefore, anti-TNF could have potential in patients with neutrophilic COPD without infectious cause ${ }^{(320)}$, but also in mixed T1/T2 immune responses in severe asthma ${ }^{(321,322)}$. Theoretically, also in CRS anti-type 1 strategies may be useful, but no trials have been performed yet. As a caveat: in several studies, anti-TNF treatment was associated with serious side effects, especially infections ${ }^{(323)}$.

\subsubsection{Anti-IL-17 alpha}

Neutrophilic inflammation in CRSsNP, as well in as in NP from patients with cystic fibrosis (CF) is predominantly driven by IL17, with ILC3s being an important source of IL-17, while its role in CRSwNP with or without asthma is questionable ${ }^{(324)}$. Findings from early clinical studies with anti-IL-17 agents, including brodalumab, secukinumab, and ixekizumab provide strong evidence for the role of IL-17 signaling in the pathophysiology of a range of inflammatory diseases ${ }^{(325)}$. However, data from a pivotal randomized, placebo-controlled double-blind trial with brodalumab in 300 patients with moderate to severe asthma, appeared rather disappointing ${ }^{(326)}$. Studies in CRSsNP and CF patients with CRSwNP should be performed to understand the potential of this approach in this disease.

\subsubsection{Anti-CXCR2 receptor}

Another therapeutic strategy in neutrophilic airway disease targets the IL-8 receptor CXCR2 to reduce airway neutrophilia ${ }^{(327)}$. Two placebo-controlled studies with CXCR2 antagonists have been conducted in patients with uncontrolled severe (neutrophilic) asthma ${ }^{(328,329)}$. Despite reductions in sputum and blood neutrophils in one study ${ }^{(328)}$, substantial clinical effectiveness was not obtained in either study. In line with the negative outcomes in clinical studies targeting IL17RA, these findings challenge a crucial role of neutrophils as potential therapeutic targets in asthma, while so far no studies in CRS targeting neutrophils have been conducted.

\subsubsection{Anti-GM-CSF/IL-3/IL-5}

Granulocyte-macrophage colony stimulating factor (GMCSF), IL-3 and IL-5 stimulate pro-inflammatory activities of haematopoietic cells, through interaction with the receptor complex incorporating the shared $\beta$ common ( $\beta c, C D 131$ ) receptor ${ }^{(330)}$. These cytokines are critical mediators in the pathogenesis of inflammatory airway disease. A novel human monoclonal antibody (CSL311) may interact with all three cytokines at the same time, specifically targeting eosinophil survival. In an ex vivo study, CSL311 inhibited the survival of inflammatory cells in induced sputum from allergic asthmatic subjects undergoing allergen bronchoprovocation ${ }^{(330)}$. Presently, a phase 1 study of CSL311 in mild asthma has commenced (https://clinicaltrials.gov/ct2/show/NCT04082754).

\subsubsection{Anti-TSLP}

Upon stimulation of the airway epithelium, several alarmins may be released, including thymic stromal lymphopoietin (TSLP), IL-33, and IL-25 which may induce downstream type 2 responses both via the innate and adaptive pathways. These cytokines may serve as targets for future treatment of type 2 disease ${ }^{(331)}$. TSLP is a cytokine that triggers dendritic cell-mediated TH2 inflammatory responses, enhances IL-1-dependent TH2 cytokine production in mast cells and together with IL-33 or IL-25 activates ILC2s. Exposure of nasal polyp-derived epithelium to a virus, in contrast to healthy control epithelium, triggers the production of TSLP ${ }^{(332)}$. Although increased levels of TSLP mRNA have been found in nasal polyps of CRSwNP patients compared 
to uncinate tissue from patients with CRS or control subjects, the TSLP protein was significantly decreased, obviously degraded by tissue proteases ${ }^{(333)}$. A relatively small double-blind, placebocontrolled trial in 31 patients with mild allergic asthma was performed using three monthly doses of AMG 157 (700 mg) or placebo intravenously ${ }^{(334)}$. AMG 157 attenuated allergen-induced early and late asthmatic responses, and decreased blood and sputum eosinophils. Whether anti-TSLP therapeutics will have clinical value in CRSwNP remains to be demonstrated

\subsubsection{Anti-IL-25}

As mentinoned above, upon stimulation of the airway epithelium, several alarmins may be released, including IL-25 which may induce downstream type 2 responses both via the innate and adaptive pathways and may thus serve as targets for future treatment of type 2 disease. IL-25 expression has also been found to be upregulated in the mucosa of patients with CRSwNP versus healthy controls and to positively correlate with the expression of several inflammatory markers, including GATA3 (see above), TGF- $\beta 1$, and TGF- $\beta 2$. Anti-IL-25 treatment reduced the number of nasal polyps and mucosal thickness due to oedema as well as the infiltration of inflammatory cells in a mouse model of CRSwNP(335). Based on these data, it has been speculated that IL-25 antagonism could be specifically promising in Asian nasal polyposis.

\subsubsection{Anti-IL33}

Interleukin 33 is a key upstream mediator of type 2 inflammation within the airways. This alarmin binds to a heterodimeric cell-surface receptor consisting of a IL-1 receptor accessory protein and ST2 on immune cells, such as TH2 cells, ILC2s, basophils, eosinophils, mast cells, dendritic cells (DCs) and others, activating intracellular signaling pathways and thus supporting the allergic airway inflammation. Direct exposure of airway epithelium to $S$. aureus increases the expression of IL-33 and TSLP, and consecutively of IL- 5 and IL-13 in nasal polyp tissue, accompanied by elevated expression levels of TSLP and IL-33 receptors ${ }^{(336)}$. In mice, repeated intratracheal exposure to SpID, a staphylococcal protein, led to IL-33 and eotaxin production, eosinophilia, bronchial hyperreactivity, and goblet cell hyperplasia in the airways. Blocking IL-33 activity with a soluble ST2 receptor significantly decreased the numbers of eosinophils in this model ${ }^{(337)}$. Furthermore, IL-33 may induce mucus overproduction in human epithelial cells ${ }^{(338)}$. In CRSWNP, ILC2s were found to be increased versus control and CRSsNP tissue, spontaneously releasing type 2 cytokines including IL-5 and IL-13 ${ }^{(339)}$. ILC2s can be found specifically in high numbers in CRSwNP patients with comorbid type 2 disease such as asthma ${ }^{(340,341)}$. The regulation of IL-33 and thus the effect of serine-protease like proteins (SPLS) in tissue are strongly dependent on the regulation of proteases and anti-proteases ${ }^{(342)}$. This regulation, as well as specific antibodies to IL-33 or its receptor, may be used for therapeutic intervention in CRSwNP in the future.

\subsubsection{Biphasic antibodies}

Novel highly potent bispecific antibodies, e.g. anti-TSLP/IL13 antibodies, that concurrently inhibit the signaling of two or more cytokines, e.g. anti-IL4Ralpha/anti-IL5, may be designed and developed for human airway disease ${ }^{(343)}$.

\subsubsection{Anti-IL7R (CD127)}

Interleukin (IL)-7 is a cytokine that is constitutively produced by stromal cells in non-haematological tissues, by lymphoid tissue (thymus and bone marrow), and by lymphatic endothelial cells. An increasing body of both pre-clinical and clinical evidence suggests that elevated levels of IL-7 and/or the IL-7 receptor (IL-7R, CD127) are associated with various disease/inflammatory states. In inflamed tissues, several cell types, including macrophages, dendritic cells, and fibroblasts produce IL-7. IL-7 primarily acts on T cells and ILCs, which are increased at the inflammatory sites that abundantly express CD127(344). Although IL-7R signalling is crucial to most T lymphocytes and ILCs, most predominantly ILC2 and ILC3s ${ }^{(345)}$, regulatory T cells (Tregs) exhibit low - to - undetectable expression of the receptor. It was hypothesized that blocking IL- 7R signalling may selectively spare Tregs function while exerting inhibitory effects on other potentially pathogenic T cell types ${ }^{(346)}$.

The substantial amelioration of inflammation and immunopathology in experimental animal models for these diseases by blocking IL-7(receptor) supports this role of IL-7 and demonstrates that IL-7 and its receptor represent novel targets for immunotherapy ${ }^{(347)}$.

CD127 is also a component of TSLPR and is required for efficient TSLP signalling ${ }^{(348)}$. Furthermore, TSLPR complex-mediated inflammation has been associated with a number of allergic inflammatory diseases ${ }^{(349)}$.

To date, one double-blind (a study of a single intravenous infusion of anti-CD127 mAb) has been performed ${ }^{(350)}$. Therapy was well tolerated and blocked IL-7 receptor signalling upon full target engagement. Although there was no discernible impact on peripheral T cell subsets in healthy subjects, anti-CD127 may effectively modulate the autoinflammatory activity of pathogenic T cells/ILCs in diseased tissues.

\subsubsection{Anti-DP2 or CRTH2}

Prostaglandin D2 (PGD2) is a metabolite of the arachidonic acid via the cyclooxygenase (COX) pathway and a key mediator in the pathophysiology of allergic rhinitis, chronic rhinosinusitis and asthma ${ }^{(351)}$. PGD2 exerts its bio-active activities through interaction with the DP1 receptor and DP2 or chemoattractant receptor-homologous molecule on Th2 cells (CRTH2), located on several structural and inflammatory cells. Through DP2 receptors located on Th2 cells, innate lymphoid type 2 cells (ILC2), and eosinophils, PGD2 interlinks the adaptive and innate immune pathways and, hence, seems an attractive target for type2 disorders ${ }^{(352,353)}$. CRTH2 antagonists represent a category of small molecules (SM) currently under development for the treatment of asthma and related syndromes ${ }^{(354,355)}$. In the initial proof of concept studies, CRTH2 antagonists showed an overall modest protection against allergen-mediated airway responses of the upper ${ }^{(356)}$ and lower respiratory tract ${ }^{(357,358)}$. When given as monotherapy or on top of standard controllers to patients with mild to moderate asthma for four to 12 weeks, a 
similarly modest effectiveness was observed on clinical indices such as symptom scores, disease control, lung function and inflammatory markers ${ }^{(359-362)}$. Until recently, in studies of allergic airway disease, the overall therapeutic effectiveness of CRTH2 antagonists compared to antihistamines ${ }^{(356)}$ and leukotriene receptor antagonists (LTRA) ${ }^{(362)}$, while some studies showed improved effectiveness in selected patient populations ${ }^{(361,363)}$. While previous data showed upregulation of the PGD2 pathway and $\mathrm{CRTH} 2$ in (more) severe uncontrolled type 2 asthma ${ }^{(364) \text {, }}$ 12 weeks of treatment with a dual DP/CRTH2 antagonist (AMG853) failed to show clinical effectiveness in patients with moderate to severe asthma uncontrolled by corticosteroids ${ }^{(365)}$. Alternatively, in a similar study population, another $\mathrm{CRTH} 2$ receptor antagonist, fevipiprant, on top of corticosteroids for 12 weeks, reduced airway eosinophils and improved clinical and physiological parameters in patients with moderate-severe asthma with sputum eosinophils ${ }^{(366)}$.

Although, no data have been published on CRTH2 antagonists in CRSwNP so far, based on their mechanism of action, it is plausible to assume that these SM may have clinical potential in patients with type2 disease despite different clinical presentations which include severe eosinophilic and/or corticosteroid-resistant asthma and CRSwNP. Future studies of CRTH2 antagonists or combinations with LTRA or biologicals on top of corticosteroids should provide the evidence ${ }^{(367)}$.

\subsubsection{Bacteriophages and anti-biofilm agents}

As antibiotic treatment may not be effective or may lead to resistance of germs, alternative therapeutic strategies against S. aureus and other frequent germs in the nose and paranasal sinuses may be useful. Theoretically, viruses targeting and killing germs (called bacteriophages) have been proposed to kill even multidrug resistant S. aureus ${ }^{(368)}$. In addition, a cocktail of bacteriophages could possibly reduce biofilm formation in vitro $^{(369,370)}$. This approach may be enforced by targeting the essential iron metabolism for bacterial growth, survival and pathogenesis ${ }^{(371)}$. In an ex-vivo experiment using nasal polyp mucosa, it was shown that a $\mathrm{S}$. aureus-bacteriophage could kill naturally occurring nasal $\mathrm{S}$. aureus and reduce the $\mathrm{S}$. aureus driven IL-5 synthesis ${ }^{(372)}$. However, no clinical studies have been performed yet in human airway disease, while potential safety issues need to be addressed first.

\section{During pregnancy it is advised to continue nasal corticosteroid sprays for CRS maintenance.}

\subsubsection{CRS treatment during pregnancy}

\subsubsection{Summary of the evidence}

A recent systematic review evaluating CRS treatment during pregnancy found no relevant level 1, 2, or 3 studies $^{(373)}$. A panel of experts formulated a number of recommendations. They advised to continue using nasal corticosteroids because modern nasal corticosteroids including budesonide, fluticasone and mometasone should be safe to use for CRS maintenance during pregnancy at recommended doses. The off-label use of budesonide irrigations or CCS nasal drops is not recommended. There is fairly good evidence for the safety of inhaled CCS in asthma during pregnancy. Budesonide is category $B$ in pregnancy and remains the agent for which the preponderance of safety data exists ${ }^{(374)}$. Newer generation sprays have negligible systemic absorption and may be safe to use, but evidence that these medications during pregnancy are not associated with any untoward risks is lacking.

The panel concluded that oral corticosteroids in short bursts may be safe after the first trimester. Use is better justified in severe CRS, especially if causing exacerbation of asthma. They advised consultation with patient's obstetrician.

Based upon the asthma literature, oral corticosteroids use would be expected to be associated with slightly increased risk of cleft lip with or without cleft palate, increased incidence of preeclampsia, and the delivery of both preterm and low birth weight infants. Oral corticosteroids cause hyperglycaemia and can lead to/worsen diabetes, causing additional maternalfoetal risks. Patients should undergo diabetes testing prior to use, especially if a longer course of corticosteroids is being considered. First trimester use has the greatest risk of potential teratogenicity. The risks of oral corticosteroids use are outweighed by risks of undertreated asthma, but this justification is less so in CRS and clinical judgment must be exercised. Oral corticosteroids are considered to be compatible with breastfeeding. The panel advised to use oral antibiotics that do not harm the foetus for ABRS or acute exacerbations of CRS. Penicillin and cephalosporin are the safest classes, and can be given when endoscopic evidence of purulence is present. Antibiotics that put the foetus at risk such as tetracyclines, aminoglycosides, trimethoprim-sulfamethaxazole and fluoroquinolones should not be used during pregnancy, nor long-term macrolide or doxycycline. The panel advises to avoid anti-leukotrienes for CRS maintenance during pregnancy because of the low-quality evidence of efficacy of

Figure 6.2.1.1. Forest plot of the effect of preoperative use of corticosteroids versus placebo on surgical blood loss.

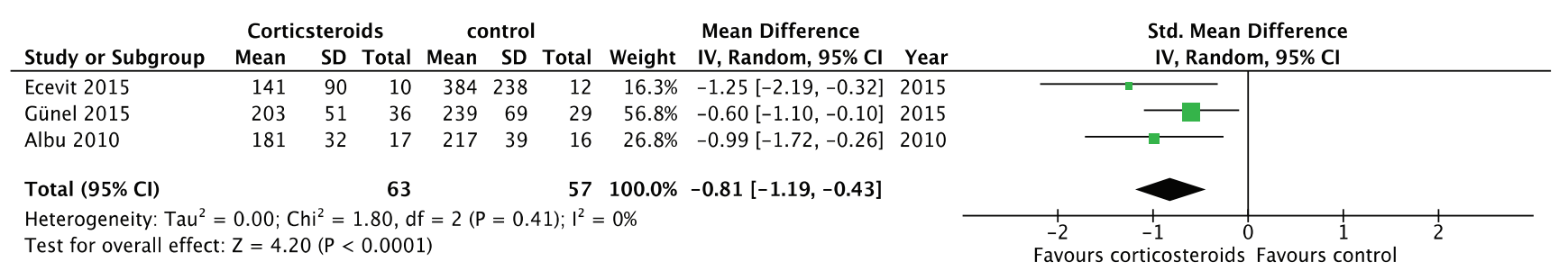


Figure 6.2.1.2. Forrest plot of the effect of perioperative use of corticosteroids versus placebo on the quality of the surgical field.

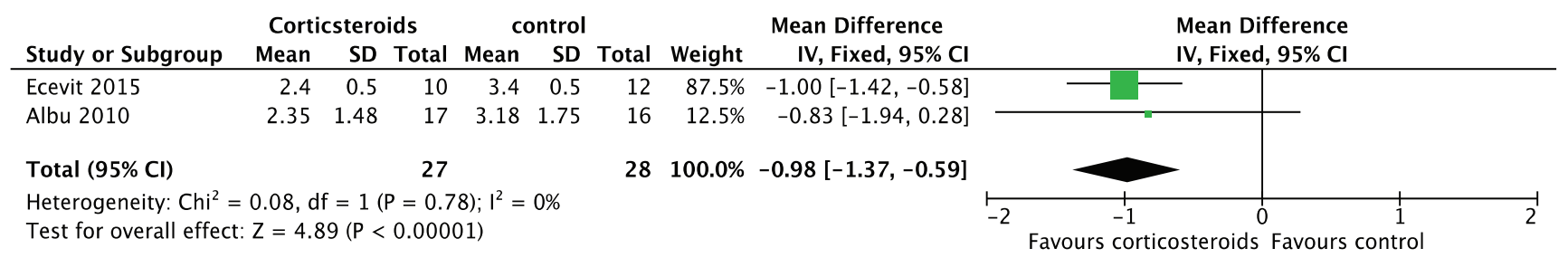

Figure 6.2.1.3. Forrest plot of the effect of preoperative use of corticosteroids versus placebo on duration of surgery.

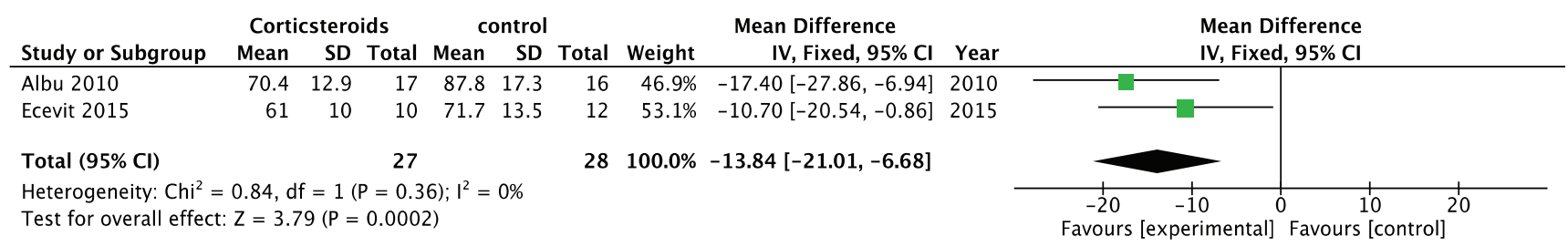

Table 6.2.1.1. Effect of preoperative corticosteroids on sinus surgery.

\begin{tabular}{|c|c|c|c|c|c|}
\hline Study & Methods & Participants & Interventions & Outcomes & Results \\
\hline $\begin{array}{l}\text { Ecevit } \\
2015^{(124)}\end{array}$ & DBPCT & $\begin{array}{l}23 \text { CRSwNP pa- } \\
\text { tients having FESS } \\
\text { ( } 22 \text { evaluated) }\end{array}$ & $\begin{array}{l}\text { - } 60 \text { mg prednisolone once daily for } 7 \\
\text { days followed by } 10 \text { mg predniso- } \\
\text { lone every other day for 8-10 days } \\
\text { preoperatively } \\
\text { - Placebo once daily for } 7 \text { days fol- } \\
\text { lowed by placebo every other day } \\
\text { for 8-10 days preoperatively }\end{array}$ & $\begin{array}{l}\text { - } \text { Blood loss (ml) } \\
\text { (Boezaart, 0-5) } \\
\text { - Operation time } \\
\text { - } \text { Hospital stay }\end{array}$ & $\begin{array}{l}\text { Treatment with topical } \\
\text { corticosteroid compared to } \\
\text { placebo resulted in significantly: } \\
\text { - Reduced blood loss } \\
\text { - Improved surgical field quality } \\
\text { - Decreased operation time } \\
\text { - Hospital stay }\end{array}$ \\
\hline $\begin{array}{l}\text { Gunel } \\
2015^{(379)}\end{array}$ & $\mathrm{DBPCT}$ & $\begin{array}{l}65 \text { CRSwNP pa- } \\
\text { tients having FESS }\end{array}$ & $\begin{array}{l}\text { - Oral prednisolone ( } 1 \mathrm{mg} / \mathrm{kg}) \text {, once } \\
\text { daily for } 2 \text { days and then tapered } \\
\text { down until day } 10(\mathrm{n}=36) \text { preoper- } \\
\text { atively } \\
\text { - Placebo once daily for } 2 \text { days and } \\
\text { then tapered down until day } 10 \\
(\mathrm{n}=29) \text { preoperatively }\end{array}$ & $\begin{array}{l}\text { - } \begin{array}{l}\text { Blood loss (ml) } \\
\text { - }\end{array} \text { Surgical field quality } \\
\text { (VAS) }\end{array}$ & $\begin{array}{l}\text { Treatment with oral corticosteroid } \\
\text { compared to placebo resulted in } \\
\text { - No difference in blood loss or } \\
\text { surgical field quality }\end{array}$ \\
\hline $\begin{array}{l}\text { Albu } \\
2010^{(380)}\end{array}$ & $\mathrm{DBPCT}$ & $\begin{array}{l}70 \text { CRS (33 CRSwNP) } \\
\text { patients having FESS }\end{array}$ & $\begin{array}{l}\text { - Mometasone nasal spray } 200 \text { mcg } \\
\text { twice daily } 4 \text { weeks preoperatively } \\
(n=35) \\
\text { - Placebo nasal spray twice daily } 4 \\
\text { weeks preoperatively }(n=35)\end{array}$ & $\begin{array}{l}\text { - } \text { Blood loss (ml) } \\
\text { - } \text { (Borgical field quality } \\
\text { - Operation time }\end{array}$ & $\begin{array}{l}\text { Treatment with topical } \\
\text { corticosteroid compared to } \\
\text { placebo resulted in significantly: } \\
\text { - Reduced blood loss } \\
\text { - Improved surgical field quality } \\
\text { - Decreased operation time }\end{array}$ \\
\hline $\begin{array}{l}\text { Wright } \\
2007 \text { (381) }\end{array}$ & $\mathrm{DBPCT}$ & $\begin{array}{l}26 \text { CRSwNP patients } \\
\text { having FESS }\end{array}$ & $\begin{array}{l}\text { - Oral prednisolone } 30 \text { mg daily for } 5 \\
\text { days preoperatively } \\
\text { and then } 30 \text { mg daily for } 9 \text { days } \\
\text { preoperatively ( } n=11 \text { ) } \\
\text { - Placebo once daily for } 5 \text { days pre- } \\
\text { operatively } \\
\text { and for } 9 \text { days preoperatively }(n=15)\end{array}$ & $\begin{array}{l}\text { - } \text { Blood loss (ml) } \\
\text { - Operation time }\end{array}$ & $\begin{array}{l}\text { Treatment with oral corticosteroid } \\
\text { compared to placebo resulted } \\
\text { in no difference in blood loss or } \\
\text { surgical field quality }\end{array}$ \\
\hline
\end{tabular}

DBPCT, Double Blind Placebo Controlled Trial; CRSwNP, Chronic Rhinosinusitis with nasal polyps; VAS, Visual Analogue Scale; FESS, Functional Endoscopic Sinus Surgery. 
anti-leukotrienes in CRS (see 6.1.9.2.). However, montelukast can be continued / initiated for recalcitrant asthma during pregnancy, especially in those with prior response. Furthermore, they advised to avoid oral decongestants and first-generation antihistamines should be avoided given their sedative and anticholinergic properties. Allergen immunotherapy is likely safe to continue during pregnancy. However, initiation or build-up of immunotherapy should be not be conducted during pregnancy. Aspirin therapy for AERD should be discontinued during pregnancy.

\subsubsection{Conclusion}

Expert panel recommendations for rhinosinusitis management during pregnancy included continuing nasal corticosteroid sprays for CRS maintenance, using pregnancy-safe antibiotics for acute rhinosinusitis and CRS exacerbations, and discontinuing aspirin desensitization for aspirin exacerbated respiratory disease.

\subsection{Surgical treatment}

\subsubsection{Primary endoscopic surgery}

\subsubsection{Preoperative CT scanning - is the timing of the scan prior to the operation important?}

To date, previous iterations of EPOS and other guidelines have not stipulated clear guidance on timing, although the current American College of Radiology (ACR) appropriateness criteria describe CT scanning without contrast as the imaging modality of choice for diagnosing $\mathrm{CRS}^{3}$. A CT scan may or may not have already been performed as part of the diagnostic work-up for CRS patients; for those where endoscopic findings confirm the diagnosis, the CT scan may be reserved until the point at which surgical intervention is being contemplated, unless concerns exist at first presentation about any unusual features such as a mucocoele ${ }^{(375)}$.

\section{A CT scan is mandatory prior to surgery, both to confirm the presence and extent of disease.}

A scan is mandatory prior to surgery, both to confirm the presence and extent of disease, and to identify any anatomical features that may predispose to the risk of complications. Multiplanar images are required to fully assess the anatomy. There are a number of systems published to facilitate systematic preoperative interpretation of the images; the CLOSE mnemonic is widely used ${ }^{(376)}$.

\footnotetext{
C Cribriform niche - depth and asymmetry should be evaluated

L Lamina papyracea - examined for dehiscence

O (Onodi) spheno-ethmoidal cells

$S$ Sphenoid sinus - examined for dehiscence of bone overlying the optic nerve and carotid artery

E Ethmoidal arteries - the position of the anterior and posterior ethmoid artery relative to the skull base should be assessed
}

\section{Provided no surgical procedures have been performed in the interim period, it is not essential to repeat CT imaging.}

Provided no surgical procedures have been performed in the interim period, it is not essential to repeat CT imaging if a scan has been performed at an earlier date. Only one study has evaluated this to date; Huang et al. identified 56 patients undergoing repeated imaging prior to ESS, with a median interval of 782 days. There was no significant difference in radiological extent of disease measured using Lund-Mackay scores and the repeat scan did not change the proposed extent of surgery.

\subsubsection{Preoperative medication - what should this consist of to optimise the surgical field and outcome?}

Excess bleeding during sinus surgery may result in an increase in operative time, difficulty of surgery and potentially serious complications. Bleeding during FESS is evaluated by measuring the amount of blood loss, although this is quite a crude method, assessment of the surgeon of the quality of the surgical field and the operating time (see also 5.3.5.1.).

Methods to reduce bleeding are the pre-operative stopping of medication that can cause bleeding like anti-coagulants and/ or aspirin/NSAIDS although we are not aware of any studies in endoscopic sinus surgery showing a difference. However, for tonsillectomy a meta-analysis on reoperation for postoperative haemorrhage reveals a 7.2-fold increased risk in the aspirin group $^{(377)}$.

\subsection{Corticosteroids}

It has been shown that increased inflammation increases bleeding during surgery ${ }^{(378)}$. The use of preoperative corticosteroids improves the quality of the surgical filed, the duration of the surgery and the operation time.

Four DBPCT studies evaluated the effect of corticosteroids during the surgery ${ }^{(124,379-381)}$ (Table 6.2.1.1.). Two of these studies used systemic corticosteroids 5-15 days before the surgery ${ }^{124,}$ ${ }^{379)}$. One study used systemic corticosteroids five days before the surgery and nine days after surgery ${ }^{(381)}$, and one a corticosteroid nasal spray for four weeks before the surgery ${ }^{(380)}$. All studies evaluated surgical blood loss. One study did not report standard deviations s $^{(381)}$ and one did not estimate blood loss. Three studies ${ }^{(124,379,380)}$ could be combined into a meta-analysis showing a significant but rather small mean reduction of blood loss of $-54 \mathrm{ml}(\mathrm{Cl}-100--7)$. There was a significant heterogeneity $\left(I^{2} 74 \%\right)$ so data are presented as SMD (SMD -0.81 (Cl-1.19-0.43), three studies, 120 patients (Figure 6.2.1.1.).

Two studies evaluated the quality of the surgical field using the Boezaart scale (0-5) $)^{(382)}$ and could be combined into a metaanalysis $^{(124,380)}$. It showed a significant impact on the quality of the surgical field of the use of preoperative corticosteroids (MD -0.98 (Cl-1.37 - -.059, two studies, 55 patients) (Figure 6.2.1.2.). Finally, data were pooled from two out of four studies which evaluated the effect of corticosteroids on the duration of the surgery ${ }^{(124,380)}$. Corticosteroids resulted in a significant reduction 
of surgery time of almost 14 minutes (MD $-13.84 \mathrm{Cl}-21.02--6.68$,

2 studies, 55 patients) (Figure 6.2.1.3.).

Interestingly there does not seem to be a difference between four weeks of local corticosteroids or 15 days of systemic corticosteroids. It is not always clear whether the systemic corticosteroids were given on top of local corticosteroids or separate. The study of Ecevit ${ }^{(124)}$ giving systemic corticosteroids after local steroids failed, but it was not disclosed whether the local corticosteroids were continued.

In conclusion: The use of perioperative corticosteroids reduces blood loss and operation time and improves the quality of the surgical field. This effect was shown to occur in one study with nasal corticosteroids and three with systemic corticosteroids. Whether there is an additive effect on systemic corticosteroids on top of nasal corticosteroids is unclear. The EPOS steering group advises to use (nasal) corticosteroids before endoscopic sinus surgery.

There are no other medications that have been shown to improve the surgical field and outcome. Antibiotics are not helpful (see also 6.1.1.).

Peroperatively a number of measures can be taken to improve the surgical field. For a review of those please see 6.2.4.

\subsubsection{Indications for surgery - what evidence do we have for when to operate?}

The principle that sinus surgery should be considered only in patients with sinus disease refractory to a trial of primary medical therapy has been upheld since endoscopic surgery was first introduced $^{(383)}$. However, there is a lack of clarity regarding what constitutes an adequate trial of medical therapy (both in terms of therapeutic classes, modes of delivery and duration), the importance of patient compliance with treatment and how to determine failure of this approach. This likely contributes to wide variations in surgical intervention rates, found in North America and Europe, both between states and between countries. In the USA, a mean adjusted rate of 0.94 was found per 1000 population in 2015 , with a four-fold difference between highest (1.8) and lowest rates $(0.5)^{(384)}$. In the UK the mean adjusted rate is nearly half; 0.53 per 1000 population, with a five-fold difference between highest and lowest regions (https://www. england.nhs.uk/rightcare/products/atlas/). Mean annual adjusted rates in Alberta, Canada are 0.33/1000 (385) and in Finland ${ }^{(386)}$ the nationwide annual rate of ESS is 0.71 per 1000 people, but variation remains very high. In Finland hospital district rates varied from $0.25 / 1000$ ( $95 \% \mathrm{Cl} 0.18$ to 0.32 ) to $1.15 / 1000$ (95\% Cl 1.09 1.21). In the Canadian study, the mean extremal quotient for health status areas was 6.9, indicating a seven-fold difference between the highest and lowest regions. Overall, there has been a progressive increase in intervention rates, despite declining rates of CRS diagnoses being recorded ${ }^{(387)}$, and increases in the number of sinus procedures being performed. Geographical variation in disease prevalence, access to healthcare and varying patient preferences may account for some of the variation, but in each study areas of high variation were found immediately adjacent to areas of low variation. A recent study shows that variation persists when separating CRS according to polyp status( ${ }^{(388)}$.

\section{There is a lack of clarity regarding what constitutes an adequate trial of medical therapy (both in terms of therapeutic classes, modes of delivery and duration), the importance of patient compliance with treatment and how to determine failure of this approach.}

There are few attempts in the literature to standardise indications for surgery. A systematic review of studies reporting outcomes from ESS identified 387 published studies in a fiveyear period, however only $21 \%$ reported medical treatment employed prior to surgical intervention ${ }^{(389)}$. Medical therapy regimens reported included topical intranasal corticosteroids in $91 \%$ studies (mean duration eight weeks), systemic corticosteroids in 61\% (18 days), oral antibiotics in 89\% (23 days), saline irrigations in $39 \%$ and oral antihistamines in $11 \%$.

\section{As the aim of surgery is to improve the severity of patient's symptoms, the decision to operate should only be made in patients with symptomatic disease, with the exception of patients with actual or impending complications.}

One study to evaluate factors (including demographics, clinical measures, social support and personality type) that influence a patient's choice to pursue surgery found that only their preoperative symptom severity, measured using the Sino-Nasal Outcome Test (SNOT)-22, predicted the choice for surgical intervention ${ }^{(390)}$. Preoperative symptom scores are predictive of postoperative improvement and the chance of achieving a clinically significant change in symptom scores ${ }^{(84,391)}$. Furthermore, postoperative changes in symptom scores can also predict need for revision surgery ${ }^{(392)}$.

As previously mentioned, a CT scan is considered mandatory prior to undertaking sinus surgery for surgical planning. However, there is evidence that a relatively high proportion of patients undergoing surgery have $\mathrm{CT}$ scores, measured using the Lund-Mackay score, within the range of the normal population; in the 2006 UK comparative audit of surgery, a prospective study enrolling 3128 patients undergoing sinus surgery in 87 different hospitals ${ }^{(393)}, 35 \%$ patients with CRSsNP had a $\mathrm{LMS}<=4$, as did $8 \%$ CRSwNP. There is a poor correlation between preoperative LMS and quality of life measures ${ }^{(394)}$, nonetheless, evidence of at least some disease on CT scan would be desirable. In a small study of patients with low-stage CRS on CT (LMS1-3) by Rudmik et al., improvements in QOL were comparable to those with more extensive disease ${ }^{(395)}$. In a 2017 publication evaluating the severity of disease in patients undergoing balloon sinus dilation, $57 \%$ had LMS $<=4$, with $19 \%$ of the total study cohort having no opacification or mucosal oedema in any sinus. In contrast to the study in low-stage disease, a randomised trial of balloon dilatation in patients with no evidence of sinus disease (LMS=0), surgery was no better than a sham procedure in terms of improving QOL or headache severity ${ }^{(396)}$.

An early paper on indications for sinus surgery summarised "if the patient has an appropriate history, appropriate physical 
findings, and appropriate $\mathrm{CT}$, endoscopic sinus surgery typically will be indicated and beneficial"(383). In the 25 years following publication there has been little to further refine that description. In an attempt to define evidence-based indications for surgery, Rudmik utilised the RAND/UCLA appropriateness methodology, with an international, multidisciplinary panel of 10 experts in CRS who completed two rounds of a modified Delphi ranking process along with a face-to-face meeting ${ }^{(397)}$. A total of 624 clinical scenarios were ranked, 312 scenarios each for CRS with and without nasal polyps. For adult patients with uncomplicated CRS with nasal polyps, it was agreed that ESS could be appropriately offered when the CT Lund-Mackay score was $>/=1$ and there had been a minimum trial of at least eight weeks' duration of a topical intranasal corticosteroid plus a short-course of systemic corticosteroid with a posttreatment total SNOT-22 score $>/=20$. For adult patients with uncomplicated CRS without nasal polyps, ESS could be appropriately offered when the CT Lund-Mackay score is $>/=$ 1 and there had been a minimum trial of at least eight weeks' duration of a topical intranasal corticosteroid plus either a short-course of a broad spectrum/culture-directed systemic antibiotic or the use of a prolonged course of systemic lowdose anti-inflammatory antibiotic with a post-treatment total SNOT-22 score $>/=20$. These criteria were considered the minimal threshold, and not all patients who meet the criteria should have surgery, but application should reduce unnecessary surgery and practice variation. A subsequent study applied the criteria retrospectively to patients recruited to a multicentre cohort study and found that patients where surgery was deemed 'inappropriate' reported significantly less improvement in their quality of life ${ }^{(398)}$.

As there are strong recommendations for use of intranasal corticosteroids and saline irrigation, based on high-quality level 1 evidence ${ }^{(399,400)}$, guidelines are consistent in recommending usage prior to offering surgery. In contrast, international guidelines are conflicted regarding whether long-term antibiotics and oral steroids should be included as part of adequate medical therapy (AMT), reflecting conflicting evidence in the current literature $(297,397,401)$, and concerns with regards to side-effects.

\subsubsection{Prediction of the success of surgery}

Brooks et al. studied whether Lund-McKay CT score (LMS) was predictive of postoperative QOL outcomes in adult patients with medically recalcitrant CRS $(n=665)$ in a prospective, observational cohort study ${ }^{(402)}$. It showed a significant association of preoperative $\mathrm{CT}$ scores with preoperative SNOT-22 scores and postoperative SNOT-22 scores, driven by extranasal and rhinologic subdomains of the QOL questionnaire. Patients in the lowest preoperative LMS quartile had the lowest mean change in SNOT-22 scores at 12 months (16.8 points; $95 \%$ confidence interval [Cl], 12.2-21.3). Patients in the second and third lowest preoperative LMS quartiles had mean changes at 12 months of 21.1 points $(95 \% \mathrm{Cl}, 16.7-25.4)$ and 23.1 points $(95 \%$ $\mathrm{Cl}$ 18.3-27.9). Patients in the highest preoperative LMS quartile had the greatest improvement in SNOT-22 scores after FESS (29.9 points; $95 \% \mathrm{Cl}, 24.9-34.8$ ). The difference in QOL change at 12 months between the highest and lowest preoperative LMS quartiles was 13.1 points $(95 \% \mathrm{Cl}, 6.0-20.2 ; \mathrm{p}<0.001)$. The key limitation of this study may be the setting, as this was conducted in a large tertiary otolaryngology setting and thus may not be transferable to wider practice.

Lal et al. tried to assess retrospectively features that might predict outcomes from endoscopic sinus surgery (ESS) with the help of clinical and endotypic features, CT, histopathology and the SNOT-22 in 146 adults that underwent ESS ${ }^{(403)}$. They were able to differentiate severity grades of CRSsNPs as clusters from middle burdened (cluster D) to severely burdened (cluster A), the latter with high scores in all four domains. Asthma burden and eosinophilia were significantly higher in cluster $C$ $(p=0.03)$ and three months after ESS, all groups had significantly improved $(p<0.0001)$. At six months, patients in cluster $C$ tended to worsen.

In a prospective, observational multi-centre study on 690 patients with CRS, Soler et al. tried to identify clusters of patients based on three common clinical variables ${ }^{(404)}$. SNOT-22 was completed at baseline and 18 months after continued medical or surgical treatment (according to patient preferences). Fortytwo percent (289/690) of patients had undergone prior sinus surgery and comorbidities, such as allergy $(25 \%, 172 / 690)$, asthma $(37 \%, 255 / 690)$, and nasal polyps (37\%, 254/690) were common. To assess factors associated with achieving a minimal clinically important difference (MCID) for each QOL measure after either medical or surgical treatments, controlling for baseline value and follow-up duration, a logistic regression was performed. Three variables were shown to allow a discrimination of patients: preoperative SNOT-22 score, age and missed productivity. Extent of the surgery was, however, left to the criterion of each treating surgeon, which does not allow one to draw conclusions about the potential effect of various extensions of the surgical procedure.

Using a similar regression to determine risk adjusted outcomes in a prospective cohort of 3128 patients undergoing sinus surgery, Hopkins et al. ${ }^{(393)}$ found the preoperative SNOT-22 score to be a strong predictor of postoperative SNOT-22, with those having higher preoperative scores achieving greater reductions in symptom scores but having persistently higher scores postoperatively. Older patients achieved greater improvement, patients with asthma less improvement. Male patients reported lower scores at all time points, but there was no difference in the overall amount of symptom improvement by gender.

More recently, a systematic review and meta-analysis with meta-regression analysis identified the variables among 3048 patients that significantly correlated with an improved SNOT-22 outcome $^{(405)}$ namely: older age, asthma, prior ESS, and a high preoperative SNOT-22 score. It seems obvious that a higher SNOT-22 at baseline is more likely to be reduced after surgery. This association applied to older patients as well, who reported a higher SNOT-22 score preoperatively. Current tobacco use status and the length of follow-up correlated with poorer SNOT22 outcomes.

In summary, the preoperative symptom score has consistently 
been shown to the best predictor of success of surgery with those with higher preoperative scores achieving greater improvement in symptoms scores.

\section{The preoperative symptom score has consistently been shown to the best predictor of success of surgery with those with higher preoperative scores achieving greater improvement in symptoms scores.}

\subsubsection{Timing of the surgery relevant to the duration of the} disease - does this affect outcome?

In order to evaluate the impact of timing of surgery on outcomes, data from both the UK prospective audit of surgery for CRS and electronic datasets were analysed by Hopkins et al. ${ }^{(406)}$. Patients were classified according to the duration of their CRS until their first surgical intervention for CRS. Three cohorts of patients were defined: early cohort - less than 12 months; mid cohort - 12-60 months; and late cohort - more than 60 months of symptoms. In the UK Audit 1493 patients having primary surgery were identified; $11.5 \%$ in the early group, $50.2 \%$ in the mid group and $38.2 \%$ in the late group. Patients in the early group had not only a greater percentage improvement in their symptoms, but the improvement was better maintained over five years. At five years there was a significantly higher proportion of patients in the early group maintaining a clinically significant improvement over baseline (71.5\%) than in either the mid (57.3\%) or late (53.0\%) groups. Using healthcare interactions as a proxy for symptomatic improvement in CPRD (assuming that higher frequency of healthcare visits and prescription medications reflect a poorer outcome from surgery), a similar pattern was found. Patients having early surgery saw their GP less frequently and received fewer prescription medications each year after surgery than the mid or late cohorts. These results were further replicated in a US based electronic dataset, MarketScan ${ }^{(407)}$.

Perhaps of even greater interest to the population as a whole, the impact of ESS on the subsequent development of asthma was also analysed. It was found, using both UK and US datasets, that ESS was associated with a reduction in the incidence of new asthma diagnoses following surgery, and that the reduction was greatest in those having early surgery ${ }^{(408)}$.

Other groups have subsequently studied the impact of timing of surgery. A prospective study in Sweden found that patients with less than 12 months of sinus disease derived greatest benefit after $\mathrm{ESS}^{(409)}$. In contrast, a multi-centred cohort study in the US found that improvements in QOL were greater in those with longer pre-operative symptom duration. The most recently published study evaluated the effect of surgical wait times and found that prolonged wait times were associated with detrimental outcomes ${ }^{(410)}$.

Although the timing of surgery has not been formerly evaluated in a randomised trial, there is a growing body of evidence suggesting that lengthy delays in intervention are detrimental to symptom improvement. The mechanism for this is not yet clear. Reduction in type 2 inflammation and prevention of irreversible remodeling of the mucosa by facilitating improved access to topical therapies are potentially disease-modifying benefits of surgery.

There is a growing body of evidence suggesting that lengthy delays in intervention are detrimental to symptom improvement.

\subsubsection{Does the type or extent of surgical procedure affect outcomes?}

Many acronyms have been applied to FESS since its inception in the 1980 s to characterise extent of the surgical procedure. First reported in 1996, minimally invasive sinus technique (MIST) is a conservative approach involving simple ventilation of the lower sinuses and has been considered to settle cases even when severe disease is present some advocating its use in current practice ${ }^{(411,412)}$; however, it is likely that selecting cases with less aggressive endotypes of CRS may be the appropriate for the use of MIST. A key advocate of MIST in the literature is Peter Catalano at Massachusetts, USA who first published a case series of 85 patients undergoing MIST in $2003^{(413)}$. The series included a diverse age range from four to 81 years old but with an equal male/female divide. Using the Chronic Sinusitis Survey, patients were followed up for two years on average. The mean CSS symptom score showed $124 \%$ improvement, with a 30\% improvement in the mean CSS medication score and a $62 \%$ improvement in the mean CSS total score (all p-values < $0.00001)$. Thirteen percent of patients were symptomatically worse and $8 \%$ were unchanged. By the end of the follow up period, $6 \%$ had had revision MIST surgery and were reported to have improved afterwards.

\subsection{Maxillary sinus}

After the anterior ethmoid sinuses, the maxillary sinus is most frequently targeted in all forms of sinus surgery, regardless of extent ${ }^{(393)}$ with a historic principle of the value of addressing this sinus to improve OMC functionality or vice versa ${ }^{(414)}$. Wadwongtham and Aeumjaturapat studied differences in the patency rate between a large middle meatal antrostomy and an undisturbed maxillary ostium in endoscopic sinus surgery for CRSwNP in 60 cases with a similar extent of polyposis in a randomized setting ${ }^{(415)}$. The patency rate of a large middle meatal antrostomy was $71.7-85 \%$ compared to $61.7-65 \%$ of the undisturbed maxillary ostium cases. There was a statistically significant difference only in early phase evaluation between the two surgical techniques ( $p$-value $=0.002$ ). Thirty-six of 60 cases $(60 \%)$ had good results with adequate drainage openings, no infection and no recurrent polyps at the final evaluation. Early and small nasal polyps (grade I polyp) was the main correlation factor to the success of endoscopic sinus surgery for CRSwNP $(p=0.017)$. Patency was strongly dependent on the recurrence rate of the polyposis causing blockage.

Albu and Tomescu conducted a prospective randomised controlled study on 133 patients to assess whether the size of a small middle meatal antrostomy $(<6 \mathrm{~mm})$ or a large one $(>16 \mathrm{~mm})$ had an influence in relieving the symptoms of rhinogenic chronic maxillary rhinosinusitis (obstruction, 
headache, and nasal discharge). The postoperative size of the ostia did not correlate with the clinical outcome. However, persistent accessory maxillary ostia and scarring within the ethmoid cavity/sinuses were statistically significant predictors of poor surgical outcome(416).

Similarly, Kim et al. studied 98 patients with CRSwNPs undergoing a maxillary antrostomy where 32 patients (group A), had the inflamed/polypoid mucosa radically (but not completely) removed with a powered microdebrider, 28 patients (group B), where only part of the mucosa was removed (mucosa in the inferior half of the sinus was preserved), and 38 patients (group C), where only enlargement of the maxillary ostium was performed without any removal of mucosa ${ }^{(417)}$. Pre- and postoperative changes in symptoms, endoscopic polyp grade, and Lund-Mackay CT score were compared between the three groups. After twelve months follow up, the study found no significant differences in symptoms (on VAS), endoscopic 4-point grading of the sinus mucosa or CT scores (LMS) between the three groups.

In a prospective, randomized, single-blinded study Myller et al. compared sides of the nose for either preservation or enlargement of the maxillary sinus ostium ${ }^{(418)}$. In 30 patients with CRSsNP they performed ESS with uncinectomy on one side and an additional maxillary antrostomy on the other side. On the enlarged side, the natural ostium was widened posteriorly to double its size; of note 25 of the 30 patients also had a bilateral ethmoid bullectomy. LMS and the ostial diameters were analysed with $1 \mathrm{~mm}$ slice thickness CT scans taken preoperatively and nine months post-operatively and were used for comparison of the two operative techniques. Also, the correlation between CT findings and subjective outcomes was studied with simple questionnaires using a total symptoms score out of 16 points; intervals for the latter were one month pre-operatively and one, 9 and 36 months postoperatively. Comparison of the preoperative and postoperative CT scans revealed a significant reduction of LM score was achieved on both sides, regardless of the type of procedure performed. The postoperative area of the ostium remained significantly larger on the antrostomy side compared to the uncinectomy side. A large maxillary sinus ostium size was associated with a lower postoperative LMS, however, the postoperative symptoms scores did not correlate to any of the postoperative CT-values ( $p$ $>0.05$ ) suggesting that it does not provide superior symptom relief.

Byun and Lee compared partial vs total resection of the uncinate process by assigning 25 patients with unilateral maxillary sinusitis (chronic maxillary sinusitis, fungal sinusitis, antrochoanal polyp, and odontogenic sinusitis) randomly to either surgical procedure ${ }^{(419)}$. CRSwNP cases were excluded. The lower half of the uncinate process was resected in the partial group. Time required for the uncinectomy, healing period for the uncinectomy site and complications such as synechiae formation in the middle meatus were compared between the groups. Partial uncinectomy had a lower complication rate, a shorter surgical procedure and more rapid healing. However, outcomes with respect to symptom benefit, or the status of the maxillary sinus were not assessed either radiologically or endoscopically.

It is notable that some of these studies fail to address the normal physiology of the maxillary sinus. The critical factor is whether the natural ostium is patent or not, as the mucociliary pathway always beats towards the natural ostium, regardless of the size of the surgical antrostomy. (See Chapter 6.2.1.6.4. for more radical maxillary sinus procedures.)

A randomised prospective study comparing a limited approach at one centre with a more extensive approach at another centre was conducted by Kuehnemund et al. in 2002 on 65 CRS patients (polyp status unknown) ${ }^{(420)}$. The limited approach involved infundibulotomy, ethmoid bullectomy and maxillary antrostomy; the extensive approach involved infundibulotomy, ethmoidectomy, sphenoidotomy, opening of the frontal recess, maxillary antrostomy and reduction of the middle turbinate. With the caveat that a non-validated symptom score was used, the authors concluded that a more limited surgical approach may avoid perioperative complications in less experienced hands while still achieving the goal of improved ventilation and drainage; these findings obviously require corroboration two decades on with the use of a validated PROM to compare the groups and better patient characterisation.

Antral washout is now mainly of historic interest and a study by Pang et al. in 1996 failed to show any benefit for the addition of antral washout to medical treatment of CRS in an RCT on 114 patients ${ }^{(421)}$. However, a study in 2013 from South Korea compared canine fossa puncture (CFP) with middle meatal antrostomy (MMA) in 25 patients with severely diseased unilateral maxillary sinuses ${ }^{(422)}$. Subjective outcomes were evaluated preoperatively and at three, 6 , and 12 months postoperatively using the SNOT-20 and a visual analogue scale (VAS). SNOT-20 and VAS scores improved significantly at three, 6 , and 12 months post-procedure in both groups. However, significant improvement of SNOT-20 at 12 months and VAS scores for purulent discharge, foul odour, and postnasal drip at six and 12 months were observed in the CFP group compared with the MMA group which was attributed to the removal of severe maxillary mucosal disease that cannot be reached through MMA.

\subsection{Ethmoid sinuses}

A small prospective study published in 2017 explored the role of ethmoid punch sinusotomy using the Relieva Circa ${ }^{\mathrm{TM}}$ device in a group of 10 CRSsNP patients who failed medical management ${ }^{(423)}$.management. At follow up, $90 \%$ of puncture sites in anterior and posterior ethmoids remained patent with stenosis present in $28 \%$. Comparative LM scores showed a reduction of a score of 1 or 2 down to 0 . Comparative pre and post SNOT-22 scores showed a significant mean reduction of $33.1(p<.0001)$, but clearly this device requires further evaluation in an appropriately powered RCT setting to determine its place as part of MIST surgical strategy.

\subsection{Olfactory niche}

A single RCT has looked at the role of clearing of olfactory cleft 
polyps with a microdebrider during ESS for CRSwNPs to assess olfactory outcomes ${ }^{(424)}$. The University of Pennsylvania Smell Identification Test (UPSIT), along with VAS and SNOT-20 were used to assess patients at baseline and six months. Seventeen patients were randomised to receive either cleft polyp removal or to have the cleft left undisturbed. All parameters improved in those having olfactory cleft surgery but only the VAS in those without $(p<0.05)$. There was a statistically significant difference in UPSIT scores between the groups at six months postoperatively $(p=0.00512)$, although long-term results showed regression to baseline. Whilst 17 patients is a small sample size, this gives some indication that careful debridement in the olfactory cleft may be effective in managing olfactory dysfunction in patients undergoing ESS for CRSwNPs, with the caveat that decline may be observed over a longer period.

\subsection{Radical approaches}

A cohort study published in 2016 reported that extended ESS (EESS )was taken to include resection of the middle and superior turbinates along with a total ethmoidectomy ${ }^{(425)}$. Forty-seven patients with CRSwNP and asthma underwent either EESS or less radical "FESS". Outcomes measured at one year suggested better outcomes for olfaction (as measured by VAS only) in the EESS group; endoscopic changes were improved in this group too but otherwise there were no differences between the groups. This study has a small sample size and without randomisation and psychophysical olfactory testing, limit its applicability. A randomised, controlled trial with parallel design was conducted between July 2000 and December 2004, including 119 patients with CRSwNP (grade III or higher)(426). Patients were randomly allocated to two treatment groups: classical endoscopic middle meatal antrostomy and endoscopic radical antrectomy. The description of the latter corresponds to a medial maxillectomy with complete removal of the maxillary sinus mucosa, a mega-antrostomy through the middle meatus and an adjuvant canine fossa puncture. The antrostomy was inferiorly extended to the level of the inferior meatus (with resection of the middle part of the inferior turbinate) and superiorly to the orbital floor. There was a significant difference between the groups: $32 \%$ of patients in the endoscopic middle meatal antrostomy group were considered surgical failures, compared with $14.5 \%$ of the endoscopic radical antrectomy group ( $p=0.023$ ). Unhealthy maxillary sinuses were significantly more prevalent in the endoscopic middle meatal antrostomy group $(p=0.029)$.

In a RCT, Jakob et al. compared outcomes of 40 cases with chronic maxillary sinusitis treated with a Caldwell-Luc against 40 cases with a middle meatal antrostomy ${ }^{(427)}$. Over $77.5 \%$ of participants had CRSwNP. After 12 months, $44 \%$ of the Caldwell-Luc patients and $89 \%$ of the FESS patients reported improvement of their symptoms, showing that endoscopic middle meatal antrostomy is superior to Caldwell-Luc in managing patients with CRSwNP surgically and further evidence that the Caldwell-Luc approach should no longer be part of practice for routine $\mathrm{ESS}^{(428)}$. An older RCT of 150 patients with chronic maxillary rhinosinusitis found that while both the Caldwell Luc and ESS approaches improved post-operative symptoms, there was stenosis of the maxillary ostium in $18.6 \%$ of the operated sinuses in the ESS group and stenosis of $31.4 \%$ of the sinuses in the Caldwell-Luc group but with large polyps found more frequently after ESS. In addition $41 \%$ of the Caldwell-Luc patients had problems with changes in sensation of the cheek and 23\% experienced severe pain. However, it is worth noting that 16 surgeons undertook the Caldwell-Luc procedure whereas only one surgeon performed ESS ${ }^{(102)}$. A follow-up study was published in 1997 on 128 of the original cohort 5-9 years after their operation. One year after surgery $51 \%$ of the C-L patients and $77 \%$ of the FES patients reported no symptoms or distinct improvement in their global symptoms, whereas $5-9$ years postoperatively $82 \%$ of the C-L and $76 \%$ of the ESS patients reported this outcome, respectively. Thirteen C-L operated patients (18\%) and 14 FES patients (20\%) have been reoperated during 7-9 years follow-up ${ }^{(429)}$. Medial maxillectomy for CRS was considered by Woodworth et al, but only in patients having failed primary surgery ${ }^{(430)}$.

\subsection{Extended frontal sinus approaches}

Alsharif et al. conducted a retrospective cohort study of 50 consecutive CRSwNPs patients ${ }^{(431)}$. In those patients $(n=12)$ in whom a full resection of the mucosa ("reboot technique) in all sinuses, including the frontal sinuses via a Draf type III was performed the recurrence of nasal polyposis was significantly reduced for 30 months postoperatively compared to the current mucosa-sparing ESS. This is a novel approach but needs further studies, and it is not clear whether the improved outcome was related to mucosal removal or the more extensive approach itself required to achieve complete clearance.

In 2009, Silvermann et al. performed a systematic review on endoscopic frontal sinus surgery including retrospective case series without control (16 articles), prospective case series without control (three articles), and retrospective case series with internal control (four articles); mostly level 3 evidence ${ }^{(432)}$. The surgical technique was described as frontal sinusotomy $(n=4)$, frontal sinusotomy or frontal sinus drillout $(n=1)$, frontal sinus drillout alone $(n=1)$, endoscopic modified Lothrop procedure (EMLP) $(n=9)$, Draf types I, II, or III $(n=6)$; trans-septal frontal sinusotomy $(n=1)$; and frontal sinus rescue procedure $(n=1)$. The mean number of subjects was 44 , and the average follow-up period was 23.6 months. Surgical success rate ranged from 50 to $100 \%$. Unfortunately, due to the limitations of the literature base at the time of this particular review, there is little to conclude.

Abuzei et al. provided a thorough meta-analysis of the Draf III after failure of primary FESS ${ }^{(433)}$. Although this evidence would support the use of the Draf III approach in recalcitrant frontal sinus disease following failure of primary ESS, it is not relevant here in considering primary surgery. A prior review performed in the same year as Silverman et al, also only included revision cases $^{(434)}$; other studies are also irrelevant to the considerations of primary surgery for the same reason ${ }^{(435-440)}$.

\subsubsection{Recommendations for when more extensive or radical surgery is required}

Regarding whether all sinuses should be opened regardless 
of disease extent or whether surgery should be targeted to affected sinuses, DeConde considered this dilemma in their observational study ${ }^{(441)}$. Their results allowed them to compare 147 subjects undergoing complete "full house" surgery with 164 subjects where the surgery was targeted to less than all of the sinuses. The "complete" group had a significantly higher prevalence of asthma, acetylsalicylic acid (ASA) sensitivity, CRSwNPs and revision cases $(p \leq 0.002)$. They also had a higher mean improvement in SNOT-22 and smell test scores (B-SIT) $(p=0.011$ and 0.005 respectively). The key limitation of the study was composition of the comparator groups, as inevitably in a non-RCT setting the patients with a higher burden of global airway disease received the complete surgical option. Masterson et al. conducted a retrospective review of 149 patients who underwent "extensive" ESS (EESS) in a district general hospital ${ }^{(442)}$. SNOT-22 scores were collected pre- and post-op as well as surgical revision rates and perioperative complications and this data was compared with the UK National Comparative Audit, the majority of whom underwent polypectomy alone or limited sinus surgery extending to the anterior ethmoid cavity. Revision rate at 36 months was significantly lower at $4 \%$ as compared to $12.3 \%$ in the national audit. No differences were seen in complication rates and significant improvements in SNOT-22 scores were seen. Although described as "extensive", this cohort can probably be considered as "complete" as per the DeConde study above. For primary surgery in CRSsNPs, more extensive surgery, such as a Draf III or endoscopic medial maxillectomy is traditionally less likely to be warranted given the nature of the pathology. The question for the surgeon counselling the patient will be as to what the goal of the surgery is in the individual patient. Even in CRSwNPs, the weight of evidence for extended approaches lies in revision cases and not for primary surgery, especially with respect to the frontal sinus where a more conservative approach is usually deemed appropriate ${ }^{(443,444)}$.

\subsubsection{What is the value of adjunctive surgery?}

\subsection{Septoplasty}

An underpowered prospective trial including 26 patients, designed to determine the role of addressing a deviated nasal symptom in ESS, claimed that septoplasty alone could be more effective than septoplasty and ESS in treating $\mathrm{CRS}^{(445)}$. This used a 14-item symptom questionnaire and CT scores to compare the two groups and suggested that $93 \%$ felt symptomatically better from septoplasty alone compared to $81 \%$ from septoplasty and ESS ( $n=9)$. Another study considered this with specific reference to maxillary rhinosinusitis and again had a small sample size $(n=40)$, but showed the opposite, that ESS with septoplasty was superior for overall symptom control when CRSwNPs was present $^{(446)}$.

There are three questions the sinus surgeon should consider about a deviated nasal septum (DNS) when deciding whether to address it during ESS:

- Does the DNS contribute to significant nasal obstruction?

- Does the DNS prevent access to the sinuses during ESS?

- Will correction of the DNS facilitate better postoperative care?
A study by Smith et al. looking at surgical outcomes across three academic North American centres found not only that septal deviation prevalence varied significantly across the sites (from $16 \%$ to $54 \%$ ), as did subsequent rates of septoplasty, but also that septoplasty significantly improved postoperative SNOT-22 scores ${ }^{(447)}$. The reasons for the difference in rate of septoplasty in the centres was unclear and the authors concluded that further study was needed to determine clear indications for septal surgery within the scope of ESS. However, given that it was not just the nasal domain of the SNOT-22 that showed improvements, there appear to be wider benefits to this adjunctive procedure that may well be due to improved delivery of postoperative topical medications to the sinuses.

\subsection{Turbinate surgery}

As per adjunctive septal surgery, evidence for turbinate surgery is limited. One study considered two methods of turbinate surgery during ESS where 54 patients with CRS were randomised to receive either an out-fracture and radiofrequency ablation of the ITs or just out-fracture alone. SNOT-20 and Lund-Kennedy scores were significantly better in the coblation group ${ }^{(448)}$. Another small-scale study of only 16 patients showed no discernible benefit of middle turbinate resection for CRSwNPs cases within six months postoperatively but as an underpowered study is of little value ${ }^{(449)}$.

\subsection{Septoplasty and turbinate surgery together} Data from Hospital Episode Statistics in the UK shows that in 2017-8 in England, there were 11,177 cases of ESS for CRSwNP in which $6 \%$ also underwent septoplasty and turbinate surgery, $13 \%$ also underwent septoplasty and $11 \%$ underwent turbinate surgery, equating to approximately 1 in 3 of all cases of ESS for CRSwNP. The same data for CRSsNP shows that of 3876 cases of ESS, $12 \%$ underwent septoplasty and turbinate surgery, $16 \%$ underwent septoplasty and $14 \%$ underwent turbinate surgery; thus approximately $40 \%$ of all cases of CRSsNPs. Overall for all cases of CRS, 33\% of ESS cases underwent adjunctive septoplasty and/or turbinate surgery. Whilst this data does not tell us anything about outcomes, it does indicate surgeon choice in these cases. It is not clear whether "turbinate surgery" involved the middle or inferior turbinates.

\subsubsection{Powered instruments}

Although powered instrumentation is now the standard of care in many hospitals, only four studies have looked at this specifically. In 2013, Saafan et al. conducted a prospective RCT to compare the efficacy and safety of powered versus conventional endoscopic sinus surgery instruments in patients undergoing ESS for CRSwNPs with a sample size of 200 patients ${ }^{(450)}$. The study addressed both intra-operative conditions and postoperative outcomes including complications across the peri-operative period. The only key notable statistically significant difference in symptom improvement on VAS at 12 months between the two groups was for olfaction in the powered group $(p<0.001)$. The operative time and surgical conditions, as well as the incidence of post-operative synechiae were significantly better in the powered group with a trend towards improvement in 
time to mucosalisation of the sinus cavities. In a separate study of 60 patients randomised by side of nose to have powered instrumentation on only one side of the nose, also supported reduced operative times in the powered sides ${ }^{(451)}$. However, a more recent trial found that there was no significant difference at 13 months between 48 patients managed with conventional instruments and 49 patients where the microdebrider was used, when using Kupferberg nasal endoscopy grades, 20-item SNOT scores, Lund-Mackay scores and mucociliary clearance time as outcome measures ${ }^{(452)}$. This corroborated the findings of an older study from 2003 looking at similar outcomes ${ }^{(453)}$. Indeed Tirelli et al. in 2013 analyzed 311 cases of bilateral CRSwNP in a prospective randomized single-blind study using eachpatient as their own control, in that one side was operated on using the Blakesley forceps and the opposite side using the microdebrider. After a follow-up mean of $13.3 \pm 1.2$ months the Blakesley forceps caused a significantly lower NP recurrence rate than the microdebrider $(p<0.001)$, though the microdebrider was more effective in preventing synechia formation $(p<0.05)^{(454)}$. Interestingly a study comparing conventional cutting and non-cutting instruments also showed less synechiae in those patients where cutting instruments were used at a mean of 12 years follow-up ${ }^{(455)}$.

Initially concerns that tissue taken with powered instruments would not be suitable for histological diagnosis were dismissed by a study from McGarry et al. which showed that tissue could be collected in an in-line specimen trap and was comparable to conventionally obtained tissue for histology ${ }^{(456)}$.

A more recent development by Medtronic is the "hydrodebrider" using a pressurised saline irrigation to clean the sinus cavities during ESS. However, a study looking at the sinus "jet wash" showed no subjective or objective treatment benefits, although was an underpowered study with only 12 patients and compared sides of the nose ${ }^{(457)}$.

\subsubsection{Balloon sinuplasty}

A RCT in 2012, the REMODEL trial, in 2012 tested the hypotheses that symptom improvement after balloon dilation was noninferior to ESS and that balloon dilation was superior to ESS for post-operative debridements. The study recruited CRS patients with disease limited to the maxillary sinuses with or without anterior ethmoid disease. Ninetytwo patients (50 balloon dilation; $42 \mathrm{ESS}$ ) were treated and the results showed a mean SNOT-20 improvement of $1.67 \pm 1.10$ and $1.60 \pm 0.96$ in the balloon and FESS arms, respectively. Both groups showed clinically meaningful and statistically significant $(p<0.0001)$ improvement and the balloon arm was non-inferior to FESS $(p<0.001)$. The balloon arm showed superiority for reduction in post-operative debridements $(p<0.0001)$. Postoperative complications such as epistaxis, analgesic use and recovery time were all significantly lower in the balloon arm $(p<0.01)$. No other complications were reported but one revision surgery was reported in each arm. This study serves to underline that in a healthcare model where patients present early, the balloon sinuplasty may have a role to play in milder cases of CRS ${ }^{(458)}$. In the UK, the National Institute for Health and Care Excellence has sanctioned use of the XprESS multi-sinus dilation system for treating uncomplicated chronic rhinosinusitis after medical treatment has failed. The specific criteria they recommend for the application of balloon sinuplasty include selecting CRS patients who do not have severe nasal polyposis or complicated cases and recommend for the procedure to be performed under local anaesthesia, however the economic modelling for this does not reflect UK practice ${ }^{(459)}$.

These recommendations were supported by a systematic review $^{(460)}$ of 9 studies including the REMODEL RCT, which included the original 92 patients, but as an expanded cohort of 135 patients with medically refractory CRS, treated with ESS or in-office balloon dilation. One hundred thirty patients had 12-month data, 66 had 18-month data, and 25 had 24-month data. Outcomes were comparable for FESS and balloon dilation, with significant reductions in symptom scores, absenteeism, health care visits and antibiotic usage in both groups. Recovery was faster in the balloon dilation group. It should be noted that all included patients had very limited sinus disease radiologically.

Bizhaki et al. whose 2014 study was included by Jenks subsequently published three papers considering quality of life, mucociliary clearance and nasal airway resistance at six month follow-up in their cohort ${ }^{(461-463)}$. They showed improvement in QoL (as measured by SNOT-22), no change in mucociliary clearance (as measured by saccharine test, methylene-blue dye and albumin labelled with Tc99m) and a decrease in nasal airway resistance (as measured by acoustic rhinometry and rhinomanometry) in both groups, but no statistical difference between balloon sinuplasty and ESS.

A more recent study by Minni et al. ${ }^{(464)}$ randomised 102 patients into mild and moderate/severe frontal CRS based on the LM score and by balloon catheter dilation (BCD) or ESS. The primary outcomes were radiology score and SNOT-20. No significant differences were shown between the groups except in those patients undergoing BCD for moderate/severe CRS who had a significant improvement in SNOT-20.

\subsubsection{Drug-eluting stents (DESs)}

\subsection{Nasal packing/spacers soaked with medications (See also section below on nasal packing)}

Drug eluting stents are expensive; a cheaper alternative utilizing absorbable dressings soaked in triamcinolone has been evaluated in a number of trials; however, the rate and amount of drug-elution is unknown. Cote and Wright randomized 19 patients to receive either a steroid or saline soaked absorbable dressing, with significant improvements in post-operative endoscopy scores being seen up to six months postoperatively ${ }^{(465)}$. Xu et al. replicated the study in 80 patients, with similar outcomes of improved endoscopy scores with triamcinaolone ${ }^{(466)}$. Hong et al. ${ }^{(467)}$ found that serum cortisol levels were suppressed on day 2 after surgery in patients receiving triamcinolone soaked dressings, but that levels had normalized by day 10 , suggesting that the triamcinolone is absorbed in a short pace of time. Unlike the drug-eluting stents that achieve controlled release over longer periods. Dautremont et al. took this a step further and in patients 
receiving a Nasopore dressing soaked in $2 \mathrm{ml}(80 \mathrm{mg})$ of triamcinolone during ESS, randomised 36 patients to receive either prednisolone $30 \mathrm{mg}$ for seven days or placebo tablet equivalent ${ }^{(125)}$. There was no significant difference between the two groups in terms of SNOT-22 or endoscopic scores up to two months, but the small sample size and short follow up period probably preclude too much being derived from this in terms of abandoning the use of perioperative OCS.

Triamcinolone versus saline soaked polyvinyl alcohol packs were considered in a recent study. The pack was removed on the second postoperative day and the cavities evaluated endoscopically in the third postoperative week, showing a significant reduction in the average Lund-Kennedy score in the treatment side compared to the control site and a greater reduction in crusting, oedema and scarring though only the reduction in oedema was statistically significant ${ }^{(468)}$.

In a pilot study of only 10 CRS patients undergoing ESS, a doxycycline releasing stent significantly lowered MMP-9 concentrations and bacterial colonization on the treated side compared with a placebo stent on the opposite side, and improved postoperative healing quality at 3 months after ESS ${ }^{(469)}$.

\subsection{DESs placed during ESS}

A growing number of DESs are emerging in the market for which the PROPEL system has the most data ${ }^{(470)}$. The Propel bioabsorbable steroid-releasing implants contain mometasone furoate $370 \mu \mathrm{g}$ and have been specifically assessed for improving postsurgical outcomes in the frontal sinus ostia ${ }^{(471)}$. This publication reported data from the pooled analysis of two RCTs considered 160 patients randomised by side of nose to receive an implant in one frontal sinus ostium with the contralateral side as a control and patients were followed up for three months. A key outcome assessed was the need for postoperative surgical interventions (debridement of obstructive adhesions or scar tissue formation in the frontal recess), which were reduced on the treated side by $47 \%$ ( $95 \%$ confidence interval -60.7 to -27.9) at one month; this was significantly reduced at three months ( $p<0.05$, relative reduction $30.2 \%$ ). The need for oral corticosteroids was also reduced by $37 \%$. There was also an increase in the estimated frontal sinus ostium diameter (absolute difference, $1 \mathrm{~mm}$ ), favoring the treated side. Other subgroup analyses did not show any significant findings for the influence of the LMS and the use of the Draf IIB procedure. However, the studies are undermined by the failure to include an arm with an implant that did not release steroid.

However, another product, Sinuband FP, a bioabsorbable, fluticasone propionate (FP)-eluting implant, was used in a small randomized, partially double-blind, single-tertiaryreferral-centre, controlled trial $(n=30)$ for patients with CRSwNP, undergoing ESS that included an ethmoidectomy. Sinuband with and without steroid was compared with Merocel as a control. Postoperative endoscopic appearances were better in the Sinuband FP arm ${ }^{(121)}$. A systematic review of two RCTs covering 143 patients found that DESs led to some significant post-operative changes including a $40 \%$ reduction in the use of OCS and $46 \%$ reduction in frank polyposis with improvements in synechiae formation compared to the Merocel group ${ }^{(472)}$.
A systematic review in 2015 considered seven studies (five prospective randomized controlled trials and two prospective single-cohort studies) involving 394 sinuses receiving steroid-eluting bioabsorbable intranasal devices. Patients were followed up for two to six months. Six studies showed efficacy with statistical significance $(p<0.05)$ for their use in reducing adhesion formation, polyp formation, inflammation, LundKennedy scores, and perioperative sinus endoscopy scores $^{(473)}$.

Economic modelling has been undertaken to support the use of a steroid stent after ESS based on prevention of post-operative division of adhesions within 60 days of ESS ${ }^{(474)}$, however this is clearly a very short period for the ongoing management of CRS patients, and adhesions may not be of clinical significance. A more recent economic analysis by the same in a US setting, also considering a reduction in polyp recurrence, gave similar support to DESs being cost-effective to use ${ }^{(475)}$.

\subsection{DESs placed in outpatients}

Other stents have been designed with the outpatient clinic setting in mind. One of these is the SINUVA implant, which is designed to deliver $1350 \mu \mathrm{g}$ of mometasone furoate to the ethmoid sinus mucosa over a three-month period. As a follow on to the RESOLVE I trial ${ }^{(120)}$, month period. As a follow on to the RESOLVE I trial, the RESOLVE II RCT by Kern et al, recruited 301 adult patients with CRSwNPs who had undergone a previous ethmoidectomy, had failed further medical therapy including INCS and $\geq 1$ course of OCS and were being considered for revision ESS ${ }^{(118)}$. Eligible patients had bilateral placement of the implants into the ethmoids under local anaesthesia. The control arm was a sham procedure to insert the implants and then remove them immediately. Patients in both arms received mometasone furoate $200 \mu \mathrm{g}$ nasal spray once daily. Nasal obstruction/congestion symptom scores and endoscopic polyp scores were used as outcome measures. There was a 2:1 randomisation resulting in 201 stent recipients and 99 sham procedure recipients (allowing for 1 excluded for extensive adhesions). In the treatment arm there were significant reductions in symptom score $(\mathrm{p}=0.0074)$ and polyp grade $(p=0.0073)$ compared to controls. After threemonths significant reductions were also seen in the secondary outcomes: $39 \%$ of patients went on to revision ESS compared to $63 \%$ of controls $(p=0.0004)$, symptom scores for nasal obstruction and smell loss were significantly better in the implant group $(p=0.0248$ and 0.0470). Facial pain/pressure was not better in the treatment group ( $p=0.9130$ ) but there was only one serious adverse event for one patient who suffered epistaxis. The SINUVA implant requires removal at the end of the three-month period and thus far has only been trialed in patients who have already undergone surgery.

By contrast, an alternative delivery vehicle for mometasone furoate is the LYR-210 (Lyra) which delivers corticosteroid to the sinonasal mucosa over six months and can be used for patients who have not had ESS following failure of conventional medical therapy. The LYR-210 system is placed into the middle meatus under local anaesthesia and slowly expands. The device has been evaluated in a small prospective, multicentre, open-label 
study including 20 patients with CRS (12 CRSsNPs and eight CRSwNPs) destined for ESS ${ }^{(476)}$.ESS. As an early phase trial, the study was set up to assess adverse events within four weeks as the primary outcome measure and found none. There were no safety concerns regarding plasma drug concentration, morning serum cortisol levels and intraocular pressures (IOPs). Although designed to be bioabsorbable, $86 \%$ of the devices were still retained in the middle meatus at six months. There were significant reductions in their SNOT-22 scores through to the end of the study period $(p<0.01)$. This device clearly requires further trial evaluation and may need removal after the treatment period, but does hold additional promise for a clinic based alternative treatment to ESS, which may be useful for those patients with other comorbidities less suitable for ESS under general anaesthesia.

\subsubsection{Packing and spacers}

At the present time the use of packing and spacers remains largely a matter of surgeon preference. They can be divided into spacers and non-absorbable materials and absorbable material including gels and other haemostats that may be instilled into nasal and/or sinus cavities.

\subsection{Gels, haemostats and absorbable dressings}

A meta-analysis by Coey et al. looking at fibrin tissue adhesive (FTA) compared to nasal packing was limited to only four studies with significant heterogeneity and bias, so mainly concluded that although there was a trend towards packing reducing bleeding and FTA reducing obstruction and granulation, the decision to use FTA has to be a cost-based one currently as there were no significant differences ${ }^{(477)}$. Carboxymethylcellulose (CMC) (mesh or gel) was evaluated in one study randomising by side of nose and showed no improvement in patient comfort on the treated side as compared to the unpacked opposite side ${ }^{(478)}$. Similar findings for improved comfort were found with a study using Nasopore in one side of the nose and gauze packing in the other ${ }^{(479)}$.

A gelatin dressing called Cutanplast was studied by Cho et al, randomizing $100 \mathrm{ESS}$ patients by side of the nose $\mathrm{e}^{(480)}$. Both the Merocel control and Cutanplast packs were effective at avoiding post-operative bleeding until the point of removal of the Merocel where bleeding did occur. There was no significant difference between the two in terms of cost or wound healing but the Cutanplast was associated with less patient discomfort. A study comparing polyurethane packing (Nasopore) versus no packing showed benefit for this packing with significant improvement in the patency of the OMC on the side with resorbable material at 4 weeks after surgery and at week 12 , a significant improvement in synechiae formation and nasal discharge ${ }^{(481)}$.

Impregnation of dissolvable dressings with corticosteroids has been studied by Zhao et al. who randomised 64 CRSwNP patients undergoing bilateral ESS by side of the nose, to receive $4 \mathrm{~mL}$ or $8 \mathrm{~mL}$ of mometasone furoate soaked Nasopore on one side and the same amount of normal saline-soaked Nasopore on the contralateral side ${ }^{(482)}$. The Nasopore was subsequently removed at seven or 14 days post-operatively, thus giving four comparators of treatment. Using the POSE and Lund-Kennedy scores to assess outcomes, they found that leaving $8 \mathrm{ml}$ of steroid soaked Nasopore for two weeks resulted in the best outcome with significant differences sustained at three months postoperatively. Similarly, Xu et al. used triamcinolone soaked Nasopore in 80 CRSwNPs undergoing bilateral ESS ${ }^{(466)}$. In this trial, patients were randomised by treatment, not by side of nose but were followed up for the same interval postoperatively. They utilised the same endoscopic scores but also included the SNOT-20 and the Sniffin' Sticks as outcome measures and found significant improvements in all except the SNOT-20 scores when comparing the triamcinolone dressing group to the saline control group. Another trial to compare triamcinolone soaked Nasopore also found significant differences in endoscopic scores at six months compared to the saline soaked dressing, albeit with a sample of only 19 patients ${ }^{(465)}$. The most recent study from Brazil used Nasopore soaked with either betamethasone or ciprofloxacin and compared with saline soaked Nasopore; the steroid group had the best 90-day appearance with respect to reduction of mucosal oedema $(p=0.007)^{(483)}$. These studies give credence to the use of steroid impregnation of nasal dressings at the end of ESS. In a recent study by Bing et al., 31 patients with CRS were randomised to receive gelatin sponge (control) or the topical application of acellular dermal matrix held with gelatin sponge in one or other surgical cavity. Lund-Kennedy scores in the acellular dermal matrix group were significantly decreased compared with the control group at 8 vs. $2(p<0.05)$ weeks and epithelialization time at eight weeks in the acellular dermal matrix groups was significantly decreased compared to the control group ${ }^{(484)}$.

Calcium alginate absorbable dressings were compared with chitin-coated gauze in a cohort of 40 CRS patients undergoing ESS, finding that the absorbable dressing was associated with lower postoperative symptoms, improved QoL and reduced pain though there was no control $\mathrm{arm}^{(485)}$. Calcium alginate was compared with CMC in 27 CRS patients undergoing ESS, After surgery, one nostril was packed with calcium alginate and the other with carboxymethyl cellulose. In contrast to Okushi, no significant differences in VAS scores for postoperative pain, discomfort from nasal discharge or pain during packing removal was found between the two sides. However, adhesion severity and oedema scores at four weeks were significantly lower with calcium alginate packing(486).

\subsection{Spacers and non-absorbable dressings}

A range of commercially available materials exist, although spacers can be created at low cost using simple nasal tampons and surgical gloves; a study looking at the impact on healing comparing a tampon with and a tampon without a glove finger covering (randomised by side of nose) showed only a benefit for the former in terms of patient comfort ${ }^{(487)}$. Evidence for the use of spacers is limited by the quality of the existing studies; a meta-analysis looking at post-operative outcomes showed only a trend towards reduced synechiae formation, but again, despite 18 included studies suffered from significant heteroegeneity ${ }^{(488)}$. 
Another RCT by Baguley et al. included 42 patients undergoing ESS for CRS who were randomized to have a silastic splint placed into the middle meatus on one side of the nose but not the other at the completion of surgery. Splints were removed two weeks postoperatively and symptom scores were recorded for each side of the nose up to 12 weeks after surgery and ethmoid cavities were graded at the six- and 12-week visits along with assessment of adhesions. The middle meatal silastic splints reduced adhesions but increased early nasal obstruction and discomfort. Their use did not significantly change symptom or ethmoid cavity scores at 12 weeks $^{(489)}$.

A similar study by Chan in 36 patients also investigated the effect of a unilateral middle meatal silastic stent up to 24 weeks. Middle turbinate lateralization was observed in 13 sides without a stent vs. one side with a stent. There was a significant reduction in adhesions at weeks 2 and $8(p<0.001)$ and crusting $(p<0.01)$ in the stent side compared to control but little difference at six months, nor for symptoms and in this study, the stent was well-tolerated ${ }^{(490)}$.

A meta-analysis comparing absorbable and non-absorbable dressings found no major differences in terms of symptoms after surgery ${ }^{(491)}$. A subsequent meta-analysis did not favour one over the other, again due to the issue of heterogeneity, although there were trends to reduced synechiae in patients receiving absorbable dressings ${ }^{(492)}$. A single RCT did however favour improvement in adhesions in those with absorbable dressings compared to saline irrigations only ${ }^{(493)}$. The most recent metaanalysis available gave greater conviction in its findings (grade of recommendation in brackets) ${ }^{(494)}$.

Reduction in postoperative bleeding: no difference when comparing absorbable packing to no packing (A) but slightly more effective than non-absorbable (C).

Reduction in postoperative synechiae: Absorbable packing was more effective than non-absorbable packing (B) and no packing (C); non-absorbable packing was also more effective than none (A). Reduction in postoperative comfort: Absorbable packing is more comfortable compared to non-absorbable (A) or no packing (B).

An interesting take on the packing debate was published by Stern-Shavit et al. who conducted a systematic review of all randomized control trials comparing packing to no packing in ESS, and generated a decision-analysis model which indicated that middle meatal packing is not advantageous for patients undergoing ESS though absorbable packing materials have a less adverse effect on quality of life than non-absorbable materials $^{(495)}$.

Nonetheless, all the meta-analyses concluded that better designed studies are needed to derive more conclusive findings in the future. Further discussion on the role of packs and spacers in prevention of synechiae can be found below in section 6.2.1.22.

\subsubsection{Role of image guidance and robotics}

\subsection{Image guidance, computer navigation and augmented reality}

The use of image guidance for ESS has been gradually increasing and in the USA has been supported by the AAONHS guidelines for ESS and the International Consensus Statement on Allergy. and Rhinology. for Rhinosinusitis in 2016(496)(401, 497).

The examples they list as appropriate for IGS include:

- Revision sinus surgery.

- Distorted sinus anatomy of development, postoperative, or traumatic origin.

- $\quad$ Extensive sino-nasal polyposis.

- Pathology involving the frontal, posterior ethmoid and sphenoid sinuses.

- Disease abutting the skull base, orbit, optic nerve or carotid artery.

- CSF rhinorrhea or conditions where there is a skull base defect.

- Benign and malignant sino-nasal neoplasms.

In terms of evaluating the role of image-guided surgery (IGS) or computer-assisted sinus surgery (CASS), key issues to address are:

- Its role in reducing complication rates

- Its role in improving clinical outcomes

- Its value in training

- Its cost-effectiveness

\subsection{Complication rates in IGS surgery}

A systematic review by Smith et al. in 2007 identified five studies for inclusion but made limited conclusions suggesting merely that the studies that needed to be done to determine safety were not ethically possible ${ }^{(498)}$. Tabee et al. compared complication rates from a single centre before and after the introduction of IGS ${ }^{(499)}$. The series included 179 non-IGS cases and 60 IGS cases. No statistically significant difference in the incidence of major intraoperative or postoperative complications was seen (all 5-6\%). The key notable difference was of a higher incidence of intraoperative cerebrospinal fluid leak in the non-IGS group ( $0 \%$ vs. $2.2 \%$ ). A retrospective cohort study from the USA in 2005-8 looked at 78,944 primary FESS cases and 4151 revision cases from insurance databases for Florida and California and found that in those who had surgery involving the frontal sinus or IGS were at higher risk for major complications $^{(500)}$. Their findings are probably likely to reflect the case selection including factors such as disease extent, anatomical factors and possibly surgical overconfidence. Masterson et al. reported on an eight-year case series of ESS using IGS and included 110 CRS patients ${ }^{(501)}$. Their results showed that four patients had a major complication (3\%) and three cases were abandoned due to excessive bleeding during surgery. Masterson et al. used IGS in a case series of $250 \mathrm{CRS/}$ AFRS patients undergoing ESS and reported a $1.2 \%$ major complication rate with only two cases requiring revision during the study period of four years ${ }^{(502)}$.

Considering the measures of operative time and blood loss, Fried et al compared cases with $(n=97)$ and without $(n=63)$ IGS during ESS ${ }^{(503)}$. The disadvantage of this study was that the groups differed in composition with the IGS group having 74\% of patients with CRSwNPs as compared to $40 \%$ in the non-IGS group. The IGS group had an average estimated blood loss 
(EBL) of $134 \mathrm{ml}$ and an average procedure time of 154 minutes as compared to $94 \mathrm{ml}$ and 133 minutes in the non-IGS group. However, the non-IGS group had seven major complications and one minor complication compared to one and three respectively in the IGS group and three revision operations in three months as compared to one. So from this study, the higher disease burden most likely contributed to longer operation times and higher blood loss in the IGS group but nonetheless they had lower complication rates.

Dalgorf et al. undertook a more recent systematic review and meta-analysis in $2013^{(504)}$. Of the 55 included studies, 14 were comparative cohorts of IGS and non-IGS cases used for the meta-analysis. In contrast to some of the above studies and previous meta-analyses ${ }^{(505)}$, they found that major and total complications were more common in the non-IGS group (major $\mathrm{RR}=0.48 ; 95 \%$ confidence interval $[\mathrm{Cl}]$, 0.28$0.82 ; \mathrm{P}=0.007$ ), (total $\mathrm{RR}=0.66 ; 95 \% \mathrm{Cl}, 0.47-0.94 ; \mathrm{P}=0.02$ ). Looking specifically at orbital, intracranial and haemorrhage complications separately, the forest plots also favoured IGS but the RRs were not statistically significant. Finally, they considered completion of the operation but again there was no significant advantage shown by IGS in this respect. Conversely, another systematic review was published that same year with opposite findings ${ }^{(506)}$, finding that the use of IGS did not decrease surgical complications or improve surgical outcomes. However, their recommendations were based on only six studies with limitations in the studies' methodology. Interestingly, despite this, within the paper the authors state a preponderance of benefit over harm and suggest that IGS helps reduce complications.

\subsection{Clinical outcomes of IGS surgery}

With respect to quality of life outcomes using IGS, Javer et al. used the RSOM-31 to compare IGS and non-IGS cases $^{(507)}$. Statistically significant improvements between preoperative and postoperative were seen for both groups considered as a whole but when the two groups were compared, the IGS group demonstrated an overall greater improvement in quality of life $(p<0.001)$. Tabee et al. found no difference with IGS in SNOT-22 scores in their single centre study with mean postoperative scores of 23.6 and 23.4 in the IGS and non-IGS groups respectively ${ }^{(499)}$.

Tabee et al. also compared and revision rates from a single centre before and after the introduction of IGS and found no significant differences in revision rates $(7 \%)^{(499)}$. A previous study from the same centre in 2003 reported an $8 \%$ revision rate for ESS when using IGS but with no comparator group this simply underlines the $7 \%$ rate reported in the more recent study ${ }^{(508)}$. Masterson et al. reported that 11 patients needed revision surgery $(10 \%)$ in their aforementioned series ${ }^{(501)}$ and Farhadi et al. reported revision rates of $11.11 \%$, and $7.1 \%$ for CRSwNPs and CRSsNPs cases after ESS with IGS in a case series of 62 patients over five years, but again with no comparator group ${ }^{(509)}$.

\section{One of the key advantages of image guided surgery is the enhancement of the learning curve in training of ESS.}

\subsection{Training value of IGS}

A small number of studies have addressed the value of IGS with respect to training. Theodoraki et al. reported on eight trainee surgeons undertaking 32 ESS cases and the influence of IGS on stress levels ${ }^{(510)}$. The cases were randomised by side of nose such that one side of each patient was operated using IGS and the other without. The trainee surgeons were monitored throughout for various parameters including heart rate variability, respiratory rate and masticator EMG. Stress was identified as an increase of heart rate frequency and a decrease of heart rate variability. They showed that stress levels were higher compared to before and after ESS but that the use of IGS did not significantly alter this except in those trainees who had performed more than 30 FESS procedures already who had a slightly decreased mental workload when using the navigation (unquantified statistically). In a separate publication, based on the same set of trainees and procedures, the authors reported how often the trainee used the navigation pointer and then changed their technique during the IGS side of the ESS ${ }^{(511)}$. They showed that procedures lasted on average 16 minutes longer with the navigation, but that in only $10-13 \%$ of cases, did it change the surgical strategy after the use of the pointer. The questionnaire completed by participants revealed a significantly increased willingness to take risks when using the image guidance system. No trainee complained of diminished surgical skills when using the navigation system. On the contrary, almost all surgeons reported a better anatomical understanding with the additional information from image guidance.

Augmented reality is starting to become available to rhinologists in the field, with preliminary work suggesting the accuracy of this approach to be submillimetric ${ }^{(512,513)}$. Initial systems available include features such as targets planned by the surgeon pre-operatively and "anti-targets" such as key structures to avoid and help prevent complications ${ }^{(514)}$. Future systems are likely to enable overlays of critical anatomy on to the live endoscopic images ${ }^{(515)}$. Certainly, one of the key advantages to these new developments will be for training to enhance the learning curve in $\mathrm{ESS}^{(513)}$.

\subsection{Cost effectiveness of IGS}

Masterson et al. considered the cost of providing an IGS service and estimated that during their study period of eight years it was approximately $£ 110,000-120,000^{(501)}$. However, their suggestion that IGS would reduce overall costs by approximately $£ 70,000$ when compared to non-IGS was based on 19 CRSsNPs patients in a military cost model, so the likely generalisability is low. The ICOR guidelines assessment was that the benefits of IGS outweigh the risk and potentially outweigh the costs ${ }^{(401)}$. Further studies to determine any specific cost benefit from the use of IGS during ESS need to be undertaken. 


\subsection{Robot-assisted ESS}

Robotic surgery in ESS has yet to find its place mainly due to the limitations on the size of instrumentation, but development is underway ${ }^{(516-523)}$. It is certainly not at the stage of trans-oral robotic surgery now being used increasingly for oropharyngeal cancers $^{(524)}$. One application considered is for a robot to hold the endoscope freeing the surgeon to undertake the ESS with both hands ${ }^{(525,526)}$. Beyond this there are yet to be trials reported on the use of robot-assisted ESS for CRS management.

\subsubsection{Office procedures}

Given the relative costs of an operating theatre setting compared to an ambulatory outpatient setting, there has been an increasing vogue to perform more procedures out of the conventional theatre setting including polypectomy, stent placement and balloon sinuplasty. Balloon technology lends itself well to this setting, as it avoids cutting tissue and thus reduces likely associated bleeding. The role of balloon sinuplasty is discussed in detail above, but with specific respect to the outpatient setting, this section considers key advantages and disadvantages to techniques that can be utilized. The aforementioned RESOLVE trial proposed steroid-eluting sinus implants for in-office treatment of recurrent sinonasal polyposis ${ }^{(119,120)}$, however, the selected patients had already failed a primary procedure. The LYR-210 device placement is also discussed above in section 6.2.1.11.3.

Levy et al. reported on two studies that looked at patients undergoing balloon sinuplasty as part of ESS in the clinic compared to the operating theatre showed that the outcomes were better when conducted in theatre ${ }^{(527)}$. It should be noted that these patients appeared to have mild to moderate disease based on a mean LMS of 8 in the Achar study. Kilty et al. recently published their data from the EPIC study showing that with good patient selection, a local anaesthetic polypectomy using the POLYPVAC could achieve good quality of life outcomes ${ }^{(528)}$.

\subsubsection{Postoperative interventions, e.g. debridement}

\subsubsection{1. Post-operative debridement}

A recent Cochrane review has considered the role of postoperative debridement in managing post-operative patients after ESS ${ }^{(529)}$. Four studies incorporating 152 participants and a duration of follow up between three and 12 months were included. Two studies were split-nose studies comparing differing interventions by side of the nose in the same patients. As patients were unblinded, the risk of bias was high and with only one study considering HRQoL scores, there is limited inference from the analysis. However, just one study did show lower, but not statistically significant lower, SNOT-22 scores at six months in the debridement group. Other studies using VAS scores showed no significant differences in total symptom score. All four studies assessed the postoperative endoscopic appearance of the sinonasal cavities using the Lund-Kennedy score. The 'split-nose' studies revealed better, but not statistically significant endoscopic scores in the debridement group but a closer focus on the adhesion formation score found this was significantly lower in the debrided patients and the number needed to treat was five patients for at least one patient to avoid an adhesion. Overall, it is not possible to comment on what impact this has in the longer term without further studies. A prior systematic review had similar conclusions with the addition that patients undergoing debridement appeared to suffer more postoperative pain ${ }^{(530)}$ which was also confirmed by Varsak in 2016 (531).

\subsubsection{2. Saline irrigations}

Rudmik et al. ${ }^{(532)}$ undertook a systematic review of the literature and identified six RCTs which evaluated the effects of postoperative saline irrigation; studies suggest improved symptom-based and endoscopic outcomes with low risk of harm and therefore that recommend use, commencing 24-48 hours after surgery. This was also supported by a more recent study concentrating on the maxillary sinus in which those using $1000 \mathrm{ml}$ of saline with an irrigation system, twice a week for the first two weeks, once a week for the next two weeks, and twice in the last month had significantly better healing than those who used no irrigation ${ }^{(533)}$. A more recent meta-analysis found only three trials deemed eligible for inclusion but nonetheless reported that nasal irrigation using normal saline and various solutions was found to be effective in reducing symptom scores and endoscopic scores for CRS after FESS ${ }^{(534)}$.

\subsection{Intranasal medications}

Given the nature of CRS as a chronic condition, maintenance treatment is more often than not an essential feature of ongoing treatment. However, the evidence supporting any specific regimen is variable. A study looking at 60 patients with $\mathrm{N}$-ERD, randomised to receive either saline, budesonide and saline separately or budesonide and saline together showed no obvious benefit in any one arm, however the sample size of this study would appear too small to show sufficient benefit ${ }^{(83)}$. Fandino et al. published a systematic review and meta-analysis to look at the role of INCS in CRSwNPs ${ }^{(535)}$. Eleven studies including $10 \mathrm{RCTs}$ and one prospective cohort study demonstrated a significant benefit in symptom scores and polyp scores up to one year postoperatively, with a significant reduction in the odds of polyp recurrence. Three studies in the analysis also collected data adrenocorticotropic hormone levels postintervention finding no elevations postoperatively. Budesonide irrigations have become a popular choice for rhinologists managing post-operative CRSwNPs, but there is now a growing body of evidence to support their use $\mathrm{e}^{(81,88,536-538)}$. Snidvongs et al. ${ }^{(88)}$ studied the use of either budesonide $1 \mathrm{mg}$ or betamethasone $1 \mathrm{mg}$ delivered in a $240 \mathrm{~mL}$ sinus squeeze bottle daily in a cohort of 111 patients. After a mean follow up period of 56 weeks, baseline and posttreatment SNOT-22 and endoscopy scores revealed significant improvements (all, $\mathrm{p}<0.001$ ). Those with high tissue eosinophilia had significantly more improvement on symptom and endoscopic scores than those without. These findings were confirmed by a more recent study by the same team, showing that the corticosteroid nasal irrigation group (using mometasone) had greater improvement in nasal blockage $(p=0.029)$, a greater improvement on LMS $(p=0.031)$ and less inflammation 
endoscopically at 12 months $(p=0.018)^{(67)}$. One-year on from surgery, symptom scores were all lower in the corticosteroid irrigation group (Table 6.1.11.9.).

One study from Thailand considered the use of dexpanthenol topically ${ }^{(171)}$. Dexpanthenol is an alcoholic analogue of D-pantothenic acid and acts as a precursor of coenzyme A necessary for acetylation and thus may enhance the effect of acetylcholine; it is used typically as a dermatological agent. Fifty patients enrolled were randomised to receive either dexpanthenol or normal saline nasal spray intranasally four times a day for six weeks postoperatively. Overall there were no significant differences in symptoms between the two groups with nasal discharge reportedly better but olfactory loss worse in the dexpanthenol group. The main claim was improvement in mucociliary clearance time, however this test is increasingly acknowledged to have little bearing on patient symptoms.

\subsection{Postoperative antibiotics}

There is no evidence to date for the use of postoperative antibiotics. In a study randomising post-ESS patients to coamoxiclav or no treatment, no differences were observed in the symptom and endoscopic scores, between the two groups after ESS ${ }^{(539)}$. Furthermore, there were no differences seen in rates of bacterial culture and drug sensitivity to co-amoxiclav. A prospective single-blind comparative effectiveness trial including 187 Chinese patients with CRS randomised subjects to receive fluticasone propionate nasal spray (200 microgram) or clarithromycin (250mg once daily) for three months after ESS ${ }^{(540)}$. Patients were followed up to one year after randomisation and outcomes included symptom severity (VAS) and endoscopic findings. Both groups saw reductions in the total and individual symptom scores, and total and individual endoscopic scores. There was no significant difference between the groups even when stratified to CRSsNP and eosinophilic and noneosinophilic CRSwNP. Neither group showed a preponderance to rescue medications either. The study design is unusual in comparing an INCS against an antibiotic rather than an INCS being included in both arms to see the additive effect of the long-term macrolide therapy after ESS.

Whilst a meta-analysis in 2012 had been unable to demonstrate a statistically significant reduction in infection, symptom scores, or endoscopic scores to support the routine use of postoperative prophylactic antibiotics following endoscopic sinus surgery, there have been concerns about the possibility of toxic shock when packs have been left in place postoperatively ${ }^{(541)}$. However, a meta-analysis was performed in 2017 to examine this issue ${ }^{(542)}$. Six studies, with a total of 990 patients undergoing ESS or septoplasty, met the inclusion criteria and there were no reports of toxic shock syndrome. The application of topical antibiotics was considered in a prospective randomized multicentre trial included 321 CRS patients undergoing ESS who received either one week of oral antibiotics and a saline-soaked bioabsorbable middle meatal (MM) sponge (control group) or no oral antibiotics and the placement of a bacitracin-soaked bioabsorbable sponge in the MM (study group). The three-week postoperative infection rate was not significantly different between the study and control groups: $5.4 \%$ versus $3.8 \%$ and there was no significant difference in MM granulations, synechia, middle turbinate lateralization, or sponge retention. However, no control group of no antibiotics was included and the use of antibiotics in any form is questionnable ${ }^{(543)}$.

\subsection{Postoperative systemic steroids}

One randomised trial has shown no impact of postoperative systemic steroids in symptom scores but did find improved endoscopic appearances at two weeks postoperatively ${ }^{(381)}$. The risks of systemic steroids must be considered against this shortterm benefit.

\subsubsection{How do we measure success versus failure?}

Early retrospective reports of endoscopic sinus surgery, whilst in its infancy, simply report 'success', as deemed by the operating surgeon without any attempt to define how this was measured. Need for revision surgery was often used to define failure. As outcome assessment evolved, focus moved to reporting symptom improvement ${ }^{(544)}$ and then to utilizing validated patient-rated outcome measures ${ }^{(545)}$ (See also chapter 5.3.5.2). The UK Audit of Sinonasal surgery was one of the first large multi-centered prospective cohort studies to use PROMS to measure the effectiveness of surgery. This study reported a statistically significant, large improvement in disease specific quality of life, maintained over a five-year period ${ }^{(546)}$. A recent publication has shown this improvement to be durable for more than 10 years in patients available for long term followup $^{(547)}$. A systematic review of outcomes in 40 unique cohorts has shown a mean improvement of 24.4 points ${ }^{(548)}$. Few studies have specifically compared outcomes of CRSwNP and CRSsNP with different reported effects. However, the metaanalysis performed by Soler et al. found no impact; only preoperative symptom scores and asthma status were found to be significantly associated with improvement in HRQOL (with studies with higher pre-operative SNOT-22 scores and higher asthma prevalence reporting greater improvement after ESS). However, looking at population means overlooks chance of success at an individual patient level. To better understand a 'successful' outcome, the concept of a'minimal clinically important difference' is usually applied, that is the smallest change in patient rated outcome scores that a patient can perceive as a real change. This has been calculated for a number of different PROMS; for the SNOT-22 it is 8.9 points ${ }^{(549)}$. Applying this to cohort series suggests that only $65-75 \%$ patients achieve a successful outcome from surgery. Baseline preoperative SNOT-22 score is the best predictor of achieving improvement in $\mathrm{HRQOL}$ greater that the MCID ${ }^{(84,391)}$; a baseline threshold of 20 points in the SNOT-22 is required to have a greater than $50 \%$ chance of achieving the MCID.

A limitation of the MCID is that it is usually calculated from population-defined statistics (using Cohen's effect size or comparing global ratings of improved versus no change to mean changes in symptom scores), while individual patients may hold different values. Asking patents simply about postoperative satisfaction or improvement does not allow for baseline measures to be used to mitigate against a placebo 
effect of intervention. This likely explains in part why patient satisfaction after surgery is higher than reported rates of achieving the $\mathrm{MCID}^{(550)}$. In addition, patients may also prioritize improvement in individual symptoms above overall quality of life. Improvement in nasal obstruction was rated as the most important symptom by $93 \%$ CRS patients, change in sense of smell second, with lower rates of importance placed on all other symptoms in a study evaluating the relationship between patient satisfaction and changes in overall symptom scores alongside individual symptoms. It found that satisfaction was better correlated with the magnitude of change in symptom scores and achieving improvement in the most important symptoms than achieving the $\mathrm{MCID}^{(550)}$. An independent study also found that in patients with sub MCID improvement in SNOT-22 scores, improvement in the nasal subdomain was also associated with reporting overall improvement in CRS symptoms ${ }^{(551)}$.

A systematic review by Chester et al. considered symptom specific outcomes after ESS ${ }^{(552)}$. A total of 2070 patients with CRS were studied a mean of 13.9 months after ESS. All symptoms demonstrated improvement compared with their respective preoperative severity scores by an overall effect size (ES) of 1.19 (95\% confidence interval, 0.96 to $1.41 ; I(2)=81.7 \%$ ) using the random-effects model. Nasal obstruction (ES, 1.73) improved the most, with facial pain (ES, 1.13) and postnasal discharge (ES, 1.19) demonstrating moderate improvements. Hyposmia (ES, 0.97) and headache (ES, 0.98) improved the least. A recent systematic review looking specifically at olfactory outcomes included thirty-one studies and demonstrated significant improvement in mixed CRS patients (those with and without polyps) through visual analog scales $(-0.83, p=.001)$, altered taste/smell item on Sinonasal Outcome Test $(-1.32, p<.00001)$, 40-item Smell Identification Test (3.49, p=.0010), and Sniffin' Sticks identification $(0.34, \mathrm{p}=.03)$. When separated, polyp patients and dysosmic patients experienced the highest levels of olfactory improvement.

The need for revision surgery should not necessarily be considered a treatment failure in the setting of chronic disease. However, it is important that patients are made aware of the chance of subsequent revision surgery. Prospective cohort studies report revision surgery rates of $11 \%$ at three years ${ }^{(393)}$, $19 \%$ at five years ${ }^{(546)}$, and $17 \%$ at 10 years ${ }^{(547)}$. Patients with CRSwNP report higher rates of revision surgery $(11 \%, 21 \%$ and $25 \%$ at three, five and 10 years respectively) than CRSsNP (10\%, 15\% and $\%$ at three, five and 10 years respectively). A population-based study using electronic healthcare records found long-term revision rates of $15.9 \%$ with a mean followup of 9.7 years, with female gender, older age at first surgery, presence of nasal polyps, comorbid asthma and allergy and a family history of CRS all being associated with a higher risk of requiring revision ${ }^{(553)}$. Revision rates in CRSwNP were $29.9 \%$ compared with $9.8 \%$ for CRSsNP. Loftus et al. have performed a systematic review looking at revision surgery rates in 45 studies and 34220 patients with CRSwNP(554). There was a mean revision rate of $16.2 \%$ with a weighted mean follow-up of 89 months. Increased rates were reported in allergic fungal rhinosinusitis (28.7\%), N-ERD (27.2\%), asthma (22.6\%) and prior polypectomy
(26\%). A recently published study with endoscopic follow-up at over 12 years after surgery, found $78.9 \%$ of CRSwNP patients had endoscopic recurrence of disease, and $36.8 \%$ had undergone revision surgery ${ }^{(555)}$.

\subsubsection{What are the outcomes of endoscopic sinus surgery?}

A large body of evidence from non-randomised cohort studies demonstrates clinically significant QoL improvements for patients after ESS. In 2009, long-term outcomes were reported on 3128 patients who underwent surgery for CRS from the National Comparative Audit of Surgery for Nasal Polyposis and Chronic Rhinosinusitis in England and Wales ${ }^{(393)}$. Mean SNOT22 score was found to significantly and clinically improve from a preoperative baseline value of 40.9 to 28.2 postoperatively, with greater improvement found in patients with CRSwNP compared with CRSsNP, although in both subgroups the effect was large. Improvements in $\mathrm{QOL}$ were maintained up to five years following surgery ${ }^{(546)}$. In 2010, Smith and colleagues ${ }^{(556)}$ published a multi-institutional cohort study of 302 patients with CRS from three medical centres followed for an average of 17.4 months after ESS. Subsequent prospective, multiinstitutional studies demonstrated that patients who elected to pursue ESS experienced clinically significant improvements in QoL, decreased utilization of oral antibiotics and systemic corticosteroids, fewer missed days of work/school(557), and a reduction in the consumption of rhinosinusitis-related health care and imaging studies ${ }^{(558,559)}$. A systematic review performed in $2005^{(560)}$ identified forty-five studies that reported either symptoms or QoL changes following ESS, with 44 of 45 studies being level 4 or 5 evidence. Although heterogeneity in study design and outcome measures utilized precluded meta-analysis, all studies were found to report significant improvement in at least one outcome measure. A systematic review and meta-analysis of cohort studies using the SNOT-22 to evaluate outcomes after sinus surgery included 40 unique patient cohorts published from 2008 to $2016^{(548)}$. All studies showed a statistically significant change in mean SNOT-22 scores between baseline and postoperative time points $(p<.001)$, ranging from 12.7 to 44.8 , at an average follow-up of 10.6 months. The summary change in mean SNOT-22 across all studies was 24.4 (95\% Cl: 22.0-26.8 The magnitude of change is quite variable across the cohorts and appeared to be influenced by a number of factors including baseline SNOT-22 score, asthma prevalence, and length of follow-up.

There are few comparative studies that allow direct comparison between medical and surgical treatment. Ragab et al. ${ }^{(32)}$

randomised ninety patients with CRS, both with and without NP were equally randomized to either medical or surgical therapy. Both the medical and surgical treatment of CRSsNP significantly improved almost all the subjective and objective parameters of CRS measured $(p<0.01)$, with no significant difference being found between the medical and surgical groups at 12 months after randomisation ( $p>0.05$ ), except for the total nasal volume in CRS $(p<.01)$ and CRS without polyposis $(p<0.01)$ groups, in which the surgical treatment demonstrated greater changes. All patients received six weeks of Dexarhinaspray prior to 
recruitment and patients who were no longer symptomatic were excluded, although there was no threshold for ongoing symptoms. A limitation of this study is that patients had only received a relatively short course of intranasal corticosteroids for six weeks prior to surgery, which does not reflect current practice of surgery being utilised after 'maximum' medical therapy has failed.

Blomquist et al. also compared medical and medical-surgical treatment of CRSwNP in 32 patients but using sinus CT. They were randomized to unilateral endoscopic sinus surgery after pretreatment with oral prednisolone for 10 days and nasal budesonide bilaterally for one month. Postoperatively, they were given nasal steroids (budesonide) bilaterally for one year. They were assessed with nasal endoscopy, symptom scores, and olfactory thresholds and followed for 12 months. CT of the sinuses was performed before and one year after operation. This showed a significant improvement on the operated side in the CT total LM scores, and scores of the OMC and maxillary sinus, but no significant differences on the unoperated side. Similarly olfaction was less improved on the unoperated side ${ }^{(561)}$. Several studies have compared outcomes in patients with refractory CRS who chose to undergo surgery, compared with those who elected not to pursue surgery and instead continued with medical therapy ${ }^{(557,562)}$. Patients who elected surgical management reported more improvement than patients who were medically managed on both the RSDI and CSS QoL instruments; in addition, surgical patients reported less use of oral antibiotics and oral steroids and fewer missed days of work and/or school, maintained through to 12 months follow-up. A subset of patients who had initially elected medical therapy crossed over to the surgical cohort, and outcomes in this crossover group mirrored those of the surgical cohort overall. A subsequent prospective study evaluated patients with refractory CRS who received ongoing medical therapy while awaiting surgery ${ }^{(563)}$, during which time SNOT-22 scores deteriorated from mean 57.6 to 66.1 , while after ESS SNOT-22 scores improved from mean 66.1 to 16.0 with an average follow up of 15 months. Patients also experienced improvements in endoscopy scores and a reduction in lost workdays and medication utilization following ESS. Patel et al. undertook a meta-analysis in 2017 that demonstrated that ESS, resulted in greater QoL scores and nasal endoscopy scores than medical therapy, and that patients undergoing surgery experienced greater improvement in health utility, olfaction, and cost-effectiveness following $\mathrm{ESS}^{(564)}$. A systematic review concentrating solely on olfaction by Kohli et al considered 31 studies and concluded that ESS improves nearly all subjective and objective measures of olfaction in CRS. Patients with CRSwNP or preoperative olfactory dysfunction improve the most ${ }^{(565)}$. This can be anticipated up to one year postoperatively ${ }^{(566)}$.

A study designed to look specifically at changes in pain following ESS enrolled 252 patients ${ }^{(567)}$. Amongst those included, $42 \%$ had no preoperative pain. Patients were followed up at six and 12 months. In the patients with no preoperative pain, none developed any new pain postoperatively. Among the 146 patients with preoperative pain, $56 \%$ had no pain, residual symptoms, or further sequelae, $29 \%$ reported a marked improvement of pain or discomfort, $6 \%$ had the same degree of pain or discomfort as before, $2 \%$ reported worse pain or discomfort and $7 \%$ reported new pain or discomfort. A meta-analysis looking at symptom response to ESS, found that depression is associated with poorer preoperative and postoperative quality-of-life scores but that the majority of major CRS symptoms improve to a similar degree ${ }^{(568)}$. The overall effect size was 1.19 (95\% confidence interval, 0.96-1.41; I (2) = 82\%), with specific symptoms effect sizes as follows: headache 0.98 , hyposmia 0.97 and nasal obstruction 1.73 . Fatigue and bodily pain were found to be more severe than general population normative values but were also found to improve following ESS (effect size 0.5 SD). Chester et al. performed a meta-analysis of 28 studies specifically considering fatigue finding substantial improvement after ESS based on pre- and postoperative comparisons. A subgroup analysis of 11 studies reporting results by the SF-36 vitality domain scores demonstrated a moderate-sized combined effect ${ }^{(569)}$. As up to $75 \%$ of CRS patients suffer from sleep disturbance, Sukato et al. undertook a meta-analysis of seven papers considering sleep improvement after ESS ${ }^{(570)}$. There was high heterogeneity in the studies (12 = 95\%-99\%) but sleep quality, as measured by the Epworth Sleepiness Scale and Pittsburgh Sleep Quality Index surveys, shows substantial improvement after surgery for CRS, with smaller improvement seen for ApnoeaHypopnoea Index. The future conduct of better studies was recommended.

The effect of ESS on the lower respiratory tract has been considered in a number of studies. A meta-analysis in 2013 identified 22 studies involving 891 patients. Patients reported improved overall asthma control in $76.1 \%$ (95\% Cl,71.9\%$80.3 \%)$ of cases. The frequency of asthma attacks decreased in $84.8 \%$ (95\% Cl, 76.6\%-93.0\%) of patients and the number of hospitalizations decreased in $64.4 \%$ (95\% Cl, 53.3\%-75.6\%). Decreased use of oral corticosteroids was seen in $72.8 \%$ (95\% $\mathrm{Cl}, 67.5 \%-78.1 \%)$; inhaled corticosteroid use decreased in $28.5 \%$ (95\% Cl, 22.6\%-34.5\%) and bronchodilator use decreased in $36.3 \%$ ( $95 \% \mathrm{Cl}, 28.9 \%$ to $43.7 \%$ ) of patients. Mean improvement in predicted forced expiratory volume at one second (FEV1) was $1.62 \%$,but was not statistically significant $(p=0.877)$. Overall ESS in patients with concomitant asthma can anticipate clinical improvement ${ }^{(571)}$.

\subsubsection{How do we manage expectation of patients? (see also chapter 9)}

Expectations of patients need to be considered with respect to outcomes of surgery, risk of complications, perioperative recovery and return to normal activities, and likely benefits of surgery and need for ongoing treatment.

A survey of patient concerns prior to ESS found that overall patients had a low-level concern before surgery, with the risk of revision surgery and waiting time being the most important drivers ${ }^{(572)}$. Revision rates are discussed in 6.2.2.

Patients should be informed of the likelihood of success based on population studies above, their preoperative symptom severity and most important symptoms. Many different factors 
have been proposed to predict outcome; Some factors have been associated with a greater mean improvement following ESS, such as CRSwNP subtype, while others have been associated with decreased mean improvement postoperatively, such as comorbid obesity and anxiety ${ }^{(573)}$.

The majority of patients are still likely to achieve clinically significant improvements regardless of associated demographic factors. The best predictor of surgical outcomes with respect to improvements in HRQOL is the patient's preoperative disease burden. A prospective cohort study of 2,263 patients found that improvement in SNOT-22 scores after ESS, and the proportion of patients achieving an improvement of at least one MCID increased with greater baseline disease severity ${ }^{(84)}$. Only those patients with a baseline SNOT-22 score of more than 21 to 30 had a mean change greater than or equivalent to the MCID, while patients with a score of 31 or more had a greater than $70 \%$ chance of achieving a MCID. However, patients with more severe baseline scores remained more symptomatic following surgery despite greater SNOT-22 improvement.

It is likely that in time, biomarkers and better endotyping will allow more accurate prediction of risk of recurrent disease. Cluster and multivariate analyses have shown that patients with eosinophil dominant CRSwNP have the highest level of polyp recurrence ${ }^{(574,575)}$. One study found that a cutoff value of $27 \%$ tissue eosinophils predicted recurrence with $96.7 \%$ sensitivity and $92.5 \%{ }^{(575)}$; and an absolute count of 55 eosinophils per high power field predicted recurrence with $87.4 \%$ sensitivity and $97.1 \%$ specificity ${ }^{(576)}$.

It should be made clear to patients that the aim of surgery is to improve disease control, and postoperative medication will be required. Studies have shown lower revision rates with ongoing use of intranasal corticosteroid sprays ${ }^{(92)}$, and increased benefits of corticosteroid irrigation over sprays alone with respect to endoscopy scores ${ }^{(67)}$. A cure is often an unrealistic aim except in the setting of isolated sinus obstruction. Patients should be counselled that their symptom load will likely be reduced, but many will not achieve post-operative scores comparable to the normal population.

\subsubsection{Postoperative recovery - pain, return to normal activity}

It seems obvious that the less extensive the surgery, the faster the recovery. Byun and Lee found a healing duration for partial uncinectomy to be of $1.77 \pm 0.59$ weeks, while total uncinectomy took $2.42 \pm 0.79$ weeks $(p=0.034)^{(419)}$. Sinus surgery usually involved more than just an uncinectomy, and the difference between these two groups is perhaps unexpected. In a randomized trial Wu et al. studied a protocol of perioperative care for enhanced recovery after (ESS) surgery (ERAS) in 102 patients with CRSwNP(577), including giving NSAIDs prior to and after surgery, and short acting opiods and topical local anaesthesia to the enhanced recovery group, who reported significantly better post-operative scores measured with the Medical Outcomes Study Sleep Scale and Kolcaba
Comfort Scale Questionnaire. The incidence rates of nausea/ emesis, haemorrhage, aspiration and falls, were not increased in the ERAS group compared with those in the control group. Kemppainen et al. studied the effect of acetaminophen / paracetamol intake on the recovery of 78 patients after FESS in a randomized study ${ }^{(578)}$. The "scheduled" group $(n=38)$ was asked to take two paracetamol/acetaminophen $665 \mathrm{mg}$ modified-release tablets three times a day during the first five postoperative days, whereas the control group $(n=40)$ took them only as needed. Most patients underwent a limited FESS procedure. Return to normal daily activities occured after 8.8 (SD 4.8) days in the "scheduled" group versus in 10.3 (SD 7.0) days in the "as needed" group (mean difference 1.5; $95 \% \mathrm{Cl}$ of the difference -1.3 to $4.2 ; p=0.29$ ). The mean of worst pain was 3.4 (2.9) in the scheduled group compared with 5.2 (3.0) in the "as needed" group on an 11-point scale (mean difference 1.7; 95\% $\mathrm{Cl}$ of the difference $0.4-5.2 ; \mathrm{p}=0.019$ ).

Tyler et al. compared the efficacy of perioperative intravenous acetaminophen with that of placebo in improving early postoperative pain after ESS in a RCT on 62 patients. Although in the first postoperative hour the VAS scale favoured the active group, at 12 and $24 \mathrm{~h}$ average pain scores were lower in the placebo group. The authors concluded that considering the high costs and inconclusive results the administration of intravenous acetaminophen (ivAPAP) could not be recommended for the control of perioperative pain reduction after ESS ${ }^{(579)}$.

Given the growing concerns with opioid over-use and addiction, a number of recent studies have assessed the need for postoperative opioid analgesia. In a single-institution retrospective study, Raikundalia et al. the opioid usage was assessed at the first postoperative visit in 136 patients who underwent FESS. Multinomial logistic regression with backward stepwise variable selection method revealed that concurrent septoplasty and young age were found to increase the odds of opioid usage. The number of sinuses opened and the presence of extended frontal and maxillary sinusotomies were not associated with increased opioid use. Patients undergoing bilateral FESS had higher opioid consumption than those who underwent unilateral FESS ${ }^{(580)}$. Locketz et al. surveyed 219 patients undergoing ESS surgery across five different centres ${ }^{(581)}$. Opiod consumption was positively correlated with postoperative, but no association was found with the procedure performed. The majority of patients took less than five opioid based tablets in the first three postoperative days; $23 \%$ did not require any. Certainly, if opioids are prescribed on discharge, only a small number should be given.

Szczygielski and co-workers compared Stammberger Sinus Dressing (SSD) with dissolvable sinu-knit (DSK) ( $\mathrm{n}=26$ sides) to routine nasal packing in latex gloves finger (RNP) $(n=23$ sides) for pain, among other issues ${ }^{(582)}$. Mean pain level for SSD was 0.85 (range from 0 to 3 ) and for DSK 2.1 (range 0 to 5) whereas for RNP was 5.6 (range from 2 to 9). Akbari et al. compared sides of the nose where 35 patients undergoing ESS for CRS were randomly allocated to receive a covered Merocel spacer on one side of the nose; the other side then received an uncovered spacer ${ }^{(487)}$. There was no significant difference in histopathological evaluation of mucosal inflammation but a 
significant difference in discomfort experienced by patients on removal of the spacers at one week, favoured the rubber glove covered spacers.

\section{Studies suggest that use of absorbable packing will reduce pain and discomfort associated with sinus surgery.}

Comparison of Merocel on one side and Nasopore on the other in 30 patients with moderate to severe CRS found comparable outcomes with regards to ease of nasal packing and control of postoperative bleeding ${ }^{(583)}$. Sequential postoperative nasal endoscopy revealed that Nasopore is kinder to the mucosa with a lower incidence of synechiae, infection and oedema, with better biocompatibility and safety. Patients' symptom questionnaires showed a significant difference in favour of Nasopore as no pack removal was needed. Also, in a separate study, Nasopore was able to significantly reduce nasal obstruction within the first 10 days after surgery $(p<0.005)$, with significant improvement of the headache and pressure in the forehead on the first follow-up visit as well as the pain in the nose on the first and second visit $(p<0.005)^{(487,582-585)}$. Shinkwin et al. compared Surgicel Nu-knit (a haemostatic material) with Vasolene ribbon gauze and Merocel packs, in 60 patients undergoing bilateral nasal surgery (ESS or inferior turbinaectomy). Surgicel Nu-knit caused significantly less discomfort and less bleeding following removal than Vasolene gauze or Merocel ( $p<0.01$, respectively). Compared to Merocel sponges, Surgicel Nu-knit caused significantly less discomfort on removal $(p<0.01)$. Bleeding following removal was also significantly less compared to the other packs ${ }^{(586)}$.

Lignocaine-soaked polyurethane foam packs as compared to saline soaked foam significantly reduced postoperative pain without serious changes on vital signs during the early postoperative period after ESS in an RCT on 63 patients ${ }^{(587)}$. Haytoglu et al. reported on the use of packing soaked in different anaesthetic agents in the management of pain after FESS in 150 patients with CRSw/sNP, using $2 \%$ lignocaine, 0.25 $\%$ bupivacaine, $0.2 \%$ ropivacaine, $2 \%$ prilocaine and $0.9 \% \mathrm{NaCl}$ as control. All received acetaminophen $(250 \mathrm{mg} / 5 \mathrm{ml})$ was used in $10-15 \mathrm{mg} / \mathrm{kg}$ per dose (four times a day). In the saline group, $93 \%$ of the patients needed additional painkiller whereas, in the bupivacaine group, additional painkiller use was $20 \%$ less than the other groups ${ }^{(588)}$. Similarly levobupivacaine-soaked PVA sponge sinus packs after FESS has been shown to be an effective, easy, and quick method to control postoperative pain $^{(589)}$.

Fentanyl-soaked absorbable packing (Nasopore or Merocel) has also been shown to significantly reduced acute postoperative pain after FESS and septoplasty without serious adverse effects ${ }^{(590)}$. Fentanyl-soaked absorbable packing (Nasopore or Merocel) has also been shown to significantly reduced acute postoperative pain after FESS and septoplasty without serious adverse effects ${ }^{(590)}$.

A systematic review of the use of sphenopalatine ganglion block (SPGB) with a local anaesthetic to decrease postoperative pain after endoscopic sinus surgery (ESS) is controversial, especially given that the procedure generally is associated with only mild pain post-operatively ${ }^{(591)}$. Eight articles were included for analysis and showed that intraoperative bleeding, postoperative pain, nausea and vomiting and recovery from sedation in the treatment group were significantly reduced compared with the control group with no notable adverse effects $(p<0.02)$. The transnasal approach also had better outcomes than the transoral route.

\subsubsection{Complications of surgery - acute and chronic}

\section{Acute / perioperative complications}

The advent of powered instrumentation does bring with it the increased potential for microdebrider related injuries ${ }^{(592,}$ 593). However, the microdebrider is not the sole source of intraoperative complications and as mentioned above, close inspection of and familiarisation with patients' preoperative CT scans is key to avoiding complications ${ }^{(594)}$. Key areas for potential pitfalls include:

Anterior ethmoid arteries - in a minority of cases the anterior ethmoid artery may lie within a bony "mesentery" beneath the skull base where it is at greater risk of injury from through-cutting instruments, particularly in the presence of a supra-orbital ethmoid cell.

Aerated posterior spheno-ethmoidal (Onodi) cells greater risk of optic nerve injury during ethmoidectomy/ sphenoidotomy; these anatomical variants are more common in some ethnic groups e.g. South East Asians ${ }^{(595,}$ 596).

Dehiscence of the lamina papryacea can be identified on coronal and axial CT sequences - this may be due to expansion by polyps in the ethmoids or to preceding facial trauma but in primary surgery will not be due to any prior intervention.

Hypoplasia of the maxillary sinus - results in the possibility of the lamina lying more medial in the nose than the maxillary wall; thus, a surgeon may breach the lamina believing they are entering the anterior ethmoids

Infra-orbital ethmoid (Haller) cells - seen best on coronal CT planes; may affect entry to the maxillary sinus and ethmoid infundibulum

Skull base defects - again in primary surgery, this would only be a consideration if there had been previous trauma or neurosurgery,in cases where massive expansion by uncontrolled polyp disease had eroded bony margins or if there was a congenital dehiscence eg meningocoele

Dehiscence of the internal carotid artery - this can be found in the sphenoid sinuses (and in some cases sphenoethmoidal cells) and renders the artery vulnerable to injury during surgery.

In 2014 a retrospective cohort analysis of two American state 
Table 6.2.1.1. Grading system for complications of ESS (599)

\begin{tabular}{|c|c|c|}
\hline Grade & Complication & Frequency \\
\hline $\begin{array}{l}\text { Grade-l: minor complication } \\
\text { (intraoperative management, } \\
\text { no persistent harm) }\end{array}$ & $\begin{array}{l}\text { Diffuse or arterial bleeding }<1,000 \mathrm{ml} \\
\text { Injury of the lamina papyracea } \\
\text { Emphysema and periorbital ecchymosis } \\
\text { Intranasal and soft tissue infection }\end{array}$ & $3.1 \%$ \\
\hline $\begin{array}{l}\text { Grade-II: major complication } \\
\text { (intraoperative management or } \\
\text { revision, no persistent harm) }\end{array}$ & $\begin{array}{l}\text { Diffuse or arterial bleeding }>1,000 \mathrm{ml} \\
\text { Bleeding requiring selective cauterising/clipping of sphenopalatine artery or anterior } \\
\text { ethmoidal artery } \\
\text { Bleeding requiring revision } \\
\text { CSF leak } \\
\text { Injury of the lacrimal duct }\end{array}$ & $0.9 \%$ \\
\hline $\begin{array}{l}\text { Grade-III: serious complication } \\
\text { (high risk of persistent deficit) }\end{array}$ & $\begin{array}{l}\text { Meningitis with or without proven leak } \\
\text { Intracerebral haemorrhage } \\
\text { Intracerebral abscess } \\
\text { Temporary or persistent neurologic deficit } \\
\text { Retro-orbital haemorrhage } \\
\text { Injury of optic nerve } \\
\text { Injury of orbital muscles with diplopia } \\
\text { Any reduction of vision and blindness } \\
\text { Injury of the internal carotid artery } \\
\text { Toxic shock syndrome } \\
\text { Sepsis } \\
\text { Death }\end{array}$ & $0.04 \%$ \\
\hline
\end{tabular}

insurance databases was undertaken from 2005 to $2008^{(500)}$. This yielded data from 78,944 primary ESS cases where 288 major complications were identified $(0.36 \%, 95 \% \mathrm{Cl} 0.32 \%-0.40 \%)$. The rate of major complications was $0.36 \%$ in primary cases $(n=288)$. Risk factors for major complications identified in their multivariate analysis include age $>40$ years, frontal sinus surgery and use of image guidance.

The rate of major complications following revision FESS $(0.46 \%)$ was found to be similar to primary cases. Skull base complications were defined as a CSF leak or dural injury within 180 days, or bacterial meningitis within 30 days, after ESS. Orbital complications were defined as diplopia, paralytic strabismus, optic nerve injury, epiphora, orbital haemorrhage, or blindness/visual disturbance within 30 days after ESS.

Additionally, a patient was considered to have an orbital complication if a person had canthotomy/canthoplasty, strabismus surgery, or orbital decompression in the same time frame. Haemorrhagic complications were counted if a diagnosis of internal carotid artery injury, epistaxis requiring transfusion, or a procedure to control haemorrhage occurred within 30 days after surgery. Some caution should be applied in interpreting these results, as in the time period studied, use of image guidance would have been relatively uncommon and these results may represent a learning curve with respect to the technique. Another study based on an insurance database from the USA was also published the same year by Benninger et al ${ }^{(597)}$. This reported adverse events in 388 patients of 9105 patients as follows: haemorrhage $0.24 \%$, cerebrospinal fluid leak $0.14 \%$ and orbital complications $0.58 \%) ; 6.28 \%$ of patients had revision surgery.

A retrospective study of one surgeon's practice over a 25 year period looked at 3,402 CRS patients and found a total of 105 patients who had suffered complications for an overall patient complication rate of $3 \%$ : haemorrhage $1.2 \%$, orbital complications $0.9 \%$, and CSF leak $0.6 \%{ }^{(598)}$. In this series, risk factor identified were age, revision surgery, CRSwNPs, anatomic variation, extensive disease, overall health, medications and powered instrumentation. The use of image guidance or surgical experience did not eliminate complications from occurring.

Another large study from one department looked at data from 2,596 patients with ARS or CRS undergoing ESS between 2000 and 2005. They reported minor complications (minor bleeding, perforation of the lamina papyracea) in 3.1\%, and major complications such as severe haemorrhage and CSF leaks in $0.9 \%$ with only one serious complication of meningitis ( $0.04 \%)$ ${ }^{(599)}$. In the UK the Sinonasal Audit performed in 2001 collected data on 3128 patients undergoing ESS for CRS and reported the following complication rates: excessive bleeding in $5 \%$ of patients during the operation and in $1 \%$ of patients after the operation, intra-orbital complications in $0.2 \%$ and CSF leak in $0.06 \%{ }^{(600)}$. A smaller series over a four year period included $250 \mathrm{CRS} /$ AFRS patients undergoing ESS and five complications were reported: two patients suffered significant epistaxis, one patient who underwent concurrent microlaryngoscopy suffered a pneumocephalus, there were no orbital complications and two general complications (transient ischaemic attack and pulmonary embolus) ${ }^{(502)}$. 
The aforementioned study by Siedek et al. proposed a grading system for complications of ESS as follows ${ }^{(599)}$ (Table6.2.1.1.):

\section{Grade I complications \\ Breach of the lamina papryacea and orbital fat injury}

Fortunately, serious ophthalmic injuries from ESS are rare but the initial recognition and management of them is crucial to avoiding any permanent sequelae ${ }^{(601-603)}$. The most common site of orbital injury during ESS is the medial orbital wall(604) during ethmoidectomy or even (aberrant) maxillary antrostomy or uncinectomy. Proponents of the retrograde approach to uncinectomy would support the notion that this avoids penetrating the orbit as the target for removal is pulled away from the orbit.

\section{Epistaxis}

Postoperative haemorrhage is typically reported as occurring as frequently as $2-4 \%{ }^{(605)}$, with one case series reporting 22 of 290 patients with post-operative epistaxis being admitted to hospital as a consequence ${ }^{(597)}$; admission with or without packing appears to be a criterion for defining significant postoperative epistaxis although most of the relevant publications do not specify this. One aforementioned study that did specify this defined the significance of bleeding within two weeks postoperatively as follows: $0=$ no bleeding, $1=$ bleeding from the nose that ceased with a gauze soaked in a mixture of 1:1000 epinephrine and 1\% lignocaine applied intra-nasally for five minutes, 2 =continuous bleeding that could not be controlled with a temporary gauze, and requiring repacking ${ }^{(606)}$. This trial found a mean score of 1.11 on the side of the Merocel dressing and 0.13 on the side of the Merogel dressing which was statistically significant $(p=0.001)$.

Hot saline irrigation during surgery is a potential therapeutic modifier of the surgical field and may modify blood loss during surgery. Gan et al. randomised 62 patients with CRS patients to receive either hot saline irrigation $(\mathrm{HSI})$ at $49^{\circ} \mathrm{C}$ or saline at room temperature $\left(18^{\circ} \mathrm{C}\right), 20 \mathrm{~mL}$ every 10 minutes, for the duration of $\mathrm{ESS}^{(607)}$. The Boezaart scores were only significantly better for HSI if the surgery lasted more than two hours but did significantly reduce the blood loss per minute for all cases in which HSI was used compared to the saline at room temperature. So, the inference for practice is that HSI may have a role for complex cases lasting over two hours and may be another therapeutic option for sinus surgeons to consider, especially if other treatment adjuncts are contraindicated.

Two almost identical RCTs have considered injection of the pterygopalatine canal with local anaesthetic (xylocaine 1 or $2 \%)$ and adrenaline $(1: 100,000 \text { or } 1: 80,000)^{(608,609)}$. The weaker solution (Valdes) produced no decrease in intraoperative surgical field bleeding, blood loss or duration of surgery whereas in the other, there was a significant reduction in blood loss.

An RCT to look at the use of a fibrin sealant in 494 patients undergoing endonasal surgery showed a significant lower postoperative bleeding rate when used ${ }^{(610,611)}$. The trial randomised patients to receive fibrin sealant or nasal packing and they were further defined as receiving one of three interventions: septoplasty, ESS or both ESS and septoplasty; all groups received turbinate surgery. Postoperative haemorrhage occurred in $22.9-25 \%$ of patients with nasal packing whereas $3.12-4.65 \%$ in the fibrin sealant groups experienced late haemorrhage only. No allergic reactions to the sealant were reported.

\section{Grade II complications Nasolacrimal injury}

It is thought that lacrimal duct injury is relatively common in ESS but that it rarely causes clinical sequelae ${ }^{(612)}$. The natural ostium of the maxillary sinus usually lies directly posterior to the nasolacrimal duct and the relationship can be seen on axial slices of preoperative $\mathrm{CT}$ imaging. Use of angled endoscopes will help the surgeon to avoid unnecessary injury after removal of the uncinate process.

\section{Cerebrospinal fluid leak}

In addition to the aforementioned reports of CSF leaks from ESS in large cohorts, recent evidence from an interesting study has suggested that occult CSF leaks may be as numerous as $13 \%$ of cases $^{(613)}$. This particular study collected samples from 57 patients after ESS to perform a hydrogel 6 beta2-transferrin assay and where CSF positive samples were detected with continuing rhinorrhoea, reanalysis after more than one year was conducted but they found that the clinical course was more often uneventful.

\section{Grade III complications \\ Medial rectus injury}

These complications typically occur when the lamina is breached and the microdebrider is being used ${ }^{(593,614)}$. If the appearance of orbital fat is not quickly recognised, damage of the medial rectus can quickly follow ${ }^{(615)}$. Injuries range from simple contusion to complete transection of the medial rectus, with and without entrapment ${ }^{(603)}$. Unless seen promptly by ophthalmology, these injuries can be very difficult to rectify and lead to disabling diplopia and strabismus ${ }^{(602,616)}$. Botox A injections have been proposed in one study to treat strabismus due to medial rectus injury ${ }^{(617)}$.

\section{Retrobulbar haemorrhage}

Orbital bleeding typically occurs due to either arterial damage such as to the anterior ethmoid artery, or venous bleeding that may occur with pulling of peri-orbital fat ${ }^{(601)}$. The consequence of such of bleeding into the orbit is a rapid increase in intraocular pressure leading to ischaemia of the optic nerve and if unresolved, irreversible damage causing blindness ${ }^{(593)}$.

\section{Direct optic nerve injury}

Injury to the optic nerve is most likely to occur in the presence of a spheno-ethmoidal cell where the optic nerve can lie dehiscent, however, the nerve can also lie close to the lamina at the back of the posterior ethmoid ${ }^{(602)}$ 


\section{Damage to the internal carotid artery}

Internal carotid artery (ICA) injury is a recognised and feared complication of ESS which can result in stroke, cranial nerve palsies, and death. A systematic review by Chin et al. in 2016 found 25 articles reporting 50 cases. The majority occurred during skull base procedures ( 34 cases) with 16 associated with CRS. The most commonly injured ICA segment was the cavernous (34 cases), followed by the ophthalmic (three cases). Injuries occurred more commonly on the left (1.3:1). Stereotactic image guidance was reported in two cases. Initial hemostasis was achieved with packing in 35 cases, endoscopic clip sacrifice in four cases, bipolar coagulation with the intent to seal defect in three cases, and bipolar coagulation with the intent to sacrifice the ICA in one case. Intraoperative or immediate postoperative interventional angiography was reported in 27 cases $^{(618)}$.

One of most successful strategies to deal with the immediate aftermath of ICA damage based on a sheep model is the application of a crushed muscle patch ${ }^{(619)}$. Subsequent imaging to exclude a pseudoaneurysm is strongly recommended.

\section{Chronic complication \\ Synechiae}

Synechiae formation after ESS is common with reports in frequency of between 4 and $35 \%(450,620)$. A recent retrospective case series to consider this specifically found that 38 of 200 ESS cases (19\%) had synechiae formation with concurrent septoplasty found to be the key risk factor for this ${ }^{(621)}$. Some trials have looked at the role of absorbable and non-absorbable packs in preventing synechiae. Verim et al. in 2013 compared Nasopore with standard Merocel in 56 ESS patients comparing sides of the nose and found no significant differences, although a coin toss was used for randomisation of side of the nose ${ }^{(606)}$. In a separate trial of 66 ESS patients comparing the hyaluronic acid pad Merogel versus the standard Merocel dressing, the percentage of adhesions seen at 12 weeks was only $5 \%$ but $30 \%$ in the Merocel group $(p<0.001)^{(622)}$. A systematic review in 2013 concluded that data from six RCTs showed no significant difference in adhesion rates if non-absorbable spacers are used for at least 48 hours postoperatively and whilst steroidal spacers may reduce adhesions, more consistent data reporting is required for future meta-analyses ${ }^{(623)}$.

The anti-adhesive properties of a thermosensitive poloxamer was compared to Merogel in ESS and showed similar antiadhesion rates of 92 vs. $89 \%$. Adhesions, oedema, and infection in the middle meatus were not significantly different between the thermosensitive poloxamer group and the Merogel group at all postoperative periods ${ }^{(624)}$.

A systematic review and meta-analysis of hyaluronan nasal dressings in 2017 included 352 patients but concluded that compared to controls the hyaluronan had no benefit in reducing synechiae formation ${ }^{(625)}$. Whilst the hyaluronan significantly promoted reepithelization (OR 3.18, 95\% Cl 1.33-7.59; $\mathrm{p}=0.009$ ) and reduced oedema (OR $0.45,95 \% \mathrm{Cl}, 0.23-0.89 ; \mathrm{p}=0.02$ ) after ESS, the ORs for synechiae, crusting and infection were $0.45,1.00$, and 0.84 respectively. Fong et al. also conducted a meta-analysis published in 2017 considering 13 (501 patients) and also demonstrated a lower risk ratio of adhesions in the hyaluronic acid intervention group (42 out of 283 cases) compared to the control group (81 out of 282) of 0.52 (95\% confidence interval $\left.=0.37-0.72^{(626)}\right)$.

A meta-analysis of whether chitosan dressings improved healing after ESS was conducted by Zhou et al. in 2017 $7^{(627)}$. Four RCTs involving 268 patients were included in the meta-analysis. Overall following ESS, compared with control intervention, chitosan dressing significantly reduced synechia $(R R=0.25$; $95 \% \mathrm{Cl} 0.13-0.49 ; \mathrm{p}<0.0001)$ and promoted hemostasis ( $R R=$ $1.70 ; 95 \% \mathrm{Cl} 1.37-2.11 ; \mathrm{p}<0.00001)$, but showed no impact on granulations, mucosal oedema, crusting and infection (RRs = $1.18,0.88,0.85$ and 0.88 respectively). Compared to control intervention, chitosan dressing could significantly decrease synechiae and improve hemostasis, but had no effect on granulations, mucosal oedema, crusting and infection. Access to the middle meatus for postoperative care and inspection can be compromised by middle turbinate lateralisation and synechiae formation in this area. Bolger ${ }^{(628)}$ recommended scarifying the medial side of the middle turbinate and adjacent septum to achieve stabilization of the middle turbinate and maintain access to the middle meatus back in 1999. A more recent RCT on 120 patients using a turbinate-septal suture showed this to be effective in preventing lateralization ${ }^{(629)}$. Maharaj et al. proposed the basal lamella relaxing incision as a means to achieve this but only found a non-significant difference in olfaction as a potential $\operatorname{advantage}^{(630)}$.

\section{Mucocoele formation}

The time of mucocele formation after initial ESS in one series was $<22$ months as compared to $<10$ years after external sinonasal surgery or trauma ${ }^{(631)}$; however, another series reported mucocoele development 15-24 years after initial surgery ${ }^{(632)}$. Of course, not all mucocoeles are caused by ESS with case series reporting $25 \%$ to $35 \%$ having a prior history of ESS ${ }^{(633)}$; another reported on 36 cases of CRSwNP where $92 \%$ had a prior history of ESS and the remaining three patients had previously unoperated disease ${ }^{(634)}$; certainly the presence of CRSwNP regardless of prior ESS appears to be a potential predisposing factor ${ }^{(633)}$. The mucocoele location is typically frontal or ethmoidal in origin ${ }^{(635)}$.

\section{Key points | What's new since EPOS 2012}

- This is a new chapter, expanding on the brief section on surgery and associated outcomes in 2012. There has been a large number of prospective studies and large datasets exploring outcomes in surgical series. As a result, we are better able to predict outcome, both at population level but also for the individual patient.

- As yet we cannot fully account for variation in intervention rates or define what the optimum extent of surgery should be. There remains a need for randomised trials comparing outcomes with medical treatment. 


\section{There are many reasons why patients require revision surgery Phenotyping and endotyping, where possible, may facilitate counselling the patient regarding prognosis and expected out- come of the surgery itself and aid identification of the postoperative medical management that has the greatest likelihood of controlling the patient's symptoms.}

\subsubsection{Revision endoscopic surgery}

The surgeon's goals in revision surgery can vary greatly. Revision surgery may imply more comprehensive dissection or exteriorization of the existing sinuses. It may be as simple as removal of adhesions, scarring or correcting lateralized turbinates. Alternatively, it may be a decision to apply more extended techniques such as Draf III frontal surgery, medial maxillectomy or bone removal of the sphenoid face. Future studies should clarify what is the goal of any revision surgery.

\subsubsection{Consider the type of rhinosinusitis / phenotype which benefits or is likely to fail and the reasons which increase the chance of revision}

There are many reasons why patients require revision surgery. Phenotyping and endotyping, where possible, may facilitate counselling the patient regarding prognosis and expected outcome of the surgery itself and aid identification of the postoperative medical management that has the greatest likelihood of controlling the patient's symptoms. They may also assist in identifying those at risk of poor outcome though failure of primary sinus surgery is often multifactorial and inadequate surgery is only one aspect, with host immunity and adherence to medical management equally important to consider. From a practical clinical perspective, in the CRSsNP phenotype group, patients with previous multiple surgeries or frank pus and / or purulent crusting in the nasal cavity on endoscopy or at the time of surgery are likely to do worse. Several prospective blinded studies have shown a correlation between in vitro biofilm-producing Pseudomonas aeruginosa and Staphylococcus aureus found in post-operative cavities and poor outcomes with increased rates of revision surgery ${ }^{(636,637)}$. Tomassen at al. investigated cytokines in CRSsNP and found that four IL-5 negative clusters made up the majority of CRSsNP patients. However, there was also an important subgroup of CRSsNP patients with a type 2 profile ${ }^{(638)}$.

A recent study in CRSsNP patients, using preoperative SNOT scores, revealed four clusters. Highest disease burden was found in patients with tissue eosinophilia and asthma. Other subgroups included those with a high score in the rhinologic domain, and those with a high score in the psychologicalsleep domain in both the moderately burdened and a mildly burdened group. All groups experienced improvement six months after surgery but those with asthma and a high tissue eosinophil count had higher SNOT-22 scores postoperatively ${ }^{(403)}$. Whilst revision surgery may be related to the pathomechanisms of the mucosal inflammation, indicated by clinical signs or biomarkers, another reason may be iatrogenic due to inadequate initial surgery for the disease endotype leading to suboptimal postoperative results and continuing symptoms. The most common findings in patients undergoing revision surgery due to iatrogenic reasons are middle turbinate lateralization, adhesions and scar formation in the middle meatus,

incompletely resected uncinate process and retained ethmoid cells (see below) ${ }^{(639)}$.

Another important factor that contributes to the need for revision ESS is surgeon-specific performance studied by Rudmik et al. ${ }^{(640)}$. This may explain why the frontal sinus, which is the hardest to approach, is the sinus with the highest revision rates. They found that $16 \%$ of surgeons followed for five years had lower-than-expected performance, indicating a potential area for improvement in quality of care. The presence of nasal polyps, repeated systemic corticosteroid courses, allergy, asthma, female gender, family history of CRS, older age at initial surgery and occupational dust exposure were associated with increased risk of undergoing ESS revision, while concurrent septoplasty reduced the rate of revision ESS. It has also been shown that the time between revision surgeries decreases with each additional revision surgery performed in a large mixed CRS population ${ }^{(553)}$. In CRSwNP, rates of revision ESS tend to be higher. In several studies, recurrence of nasal polyps has been found to occur earlier in time and more frequently ${ }^{(641-644)} A$ retrospective study included 23,542 CRS patients followed for one year showed that the frequency of revision ESS for CRSwNP was 3.5\% vs. $1.6 \%$ for CRSsNP ${ }^{(645)}$. Miglani et al. ${ }^{(646)}$ reported a similar result in a retrospective review of 424 CRS patients with and without nasal polyps, finding an overall frequency of $4 \%$ for revision ESS, $88 \%$ of which were necessary in the CRSwNP patients. According to Chen et al. ${ }^{(647)}$ CRSwNP associated with asthma also has a higher rate of revision ESS (25\%). The authors recommend extensive endoscopic sinus surgery (EESS) or radical sinus surgery for refractory CRS, since it is associated with better outcomes compared to FESS, specifically when CRSwNP is present. EESS improved olfaction and did not increase frontal ostium stenosis compared to FESS ${ }^{(425)}$.

Other co-morbidities such as N-ERD and cystic fibrosis (CF) also increase the chances of repeated surgery. In a study comprising $106 \mathrm{CF}$ patients, the necessity for revision surgery was $28 \%$ within three years of surgery ${ }^{(648)}$.A review of risk factors for recurrent sinus surgery in 81 patients with CF found that the most relevant was a high Lund-Mackay scores at their initial surgery ${ }^{(649)}$. Similarly, another retrospective study on 49 CF patients found the grade of polyp (0-3) correlated with revision ${ }^{(650)}$.

In a systematic review by Adelman et al. on the management of N-ERD (Samter's Triad) looked at 18 studies (686 patients), of whom $59.4 \%$ had undergone previous surgery ${ }^{(651)}$. This ranged from a mean of 1.9-5.4 times and was mainly ESS, but $14 \%$ had undergone a Caldwell-Luc procedure.

Immunodeficiency and GPA patients also required more revision surgery. Another study with 61,339 patients (mixed population of (RS) followed for five years, demonstrated that 4,078 patients $(6.65 \%)$ required revision ESS. The mean time to revision surgery 
was 20 months, with $43 \%$ of the patients having revision surgery during the first year. CRSwNP patients accounted for $9.9 \%$ of all revision cases vs. $7.9 \%$ for CRSsNP, concluding that patients with nasal polyps are approximately twice more likely than those without NP to require revision ESS ${ }^{(652)}$.

Modifiable exposures to risk factors associated with recurrence must be identified and patients must be encouraged to discontinue their exposure if possible. Occupational dust exposure has been associated with higher rates of recurrence of polyps i.e. $48 \%$, of which $13 \%$ needed revision surgery, compared with recurrence rates of $3 \%$ in the patients with no exposure, and for which none required re-intervention ${ }^{(4,6)}$. Smoking is another modifiable risk factor, described by $\mathrm{Wu}^{(653)}$ in a retrospective analysis of 490 patients with CRSwNP followed over a 25 -year period who underwent revision ESS by a single surgeon. They reported a mean time to revision of 4.8 years, shorter by almost half in patients who smoked ( 2.82 years). One of the first studies that showed a difference of inflammatory patterns in recurrent and non-recurrent CRSwNPs was performed by Van Zele et al. ${ }^{(654)}$ CRSwNP patients who needed revision surgery showed a predominant Th2-type inflammation and higher concentrations of IL-5, ECP, IgE, and Staphylococcus enterotoxin $\lg \mathrm{E}$ (SE-IgE). IL-5 protein within the polyp and SE-IgE were linked to recurrence after the first surgery, in contrast to IFN- $\gamma$ which was linked to non-recurring disease. Thus, the type of inflammation, specifically Th- 2 inflammation, can predict whether a patient is likely to have recurrence and revision ESS. Identifying inflammatory patterns in CRSwNP by endotyping ${ }^{(638)}$ therefore, may be helpful to identify patients who need more extensive surgery, or may be suitable for specific treatments with biologics ${ }^{(655)}$.

\subsubsection{What are the goals of revision?}

In both CRSsNP and wNP, the surgeon's goal is often to create a common cavity accessible to local therapy. It may be necessary to address anatomical variations which obstruct access, removal of residual cells, scarring or neo-osteogenesis, widening of ostia and removal of partitions, mainly in the ethmoids. Addressing the middle turbinate by partial trimming has shown improved results in some studies without seeming to cause any negative effects, although the latter has mainly been investigated in CRSwNP(656, 657).

The patient's goal is to experience symptom reduction, reduced need for medication and improved quality of life and these are generally the primary outcomes evaluated by surgeons in patients with CRS. In CRSWNP, the prolongation of recurrencefree time or avoidance of recurrence are further goals. There is a well-defined consensus that optimization of patientreported outcome measures (PROMs) is the primary goal of treatment ${ }^{(654)}$. Several tools are used for the evaluation of medical and surgical interventions, including the Rhinosinusitis Disability Index (RSDI), 20 and 22-item Sino-Nasal Outcome Test (SNOT-20, SNOT-22), Chronic Sinusitis Survey (CSS), Adelaide Disease Severity Score (ADSS), Patient Response Score (PRS), Rhino-sinusitis Symptom Inventory (RSI) and Rhinosinusitis
Outcome Measure (RSOM)(see section 5.3.4.2). For medically recalcitrant cases, surgery is the most effective way to improve QoL. In a prospective cohort study of 668 CRS (mixed population) patients undergoing primary FESS with a 60-month follow-up by Rudmik et al. ${ }^{(640)}$ failing to achieve an improvement of more than one minimal clinically important difference (MCID; 9 points in SNOT-22) at three months after primary ESS and a deterioration of greater than one MCID (> 9 points) from the three- to 12-month follow-up periods was associated with an increased risk of revision ESS.

\section{The goal of revision surgery can be different for the surgeon and the patient. These discrepancies have to be discussed}

A multi-institutional observational study demonstrated a tendency of improvement in postoperative PROMs and olfactory measures in complete surgery (Draf Ila, Ilb, or III) compared to a targeted approach in $\mathrm{CRS}^{(441,655)}$.

A systematic review included 12 studies containing data from 1308 CRS patients (mixed population) to evaluate the impact of PROMs in revision ESS. The authors found improvements in five main symptoms of nasal obstruction, rhinorrhoea, post-nasal drip, facial pain and anosmia. The benefits persisted for a mean of 21.7 months follow-up after revision surgery. The review described the importance of using PROMs to report primary and revision sinus surgery outcomes, with the SNOT-22 test being the most commonly used ${ }^{(658)}$.

\subsubsection{When should revision surgery be performed?}

Revision surgery is often seen as the last resort when medical therapy has failed. It should be considered when there is persistence of post-treatment symptoms together with objective CT and endoscopic evidence of mucosal thickening or mucopurulent discharge, nasal polyps or mucosal oedema after at least a two-month course of culture-directed antibiotics, topical and oral steroids, and nasal douching ${ }^{(431,}$ ${ }^{659)}$. It is important to ascertain that the patient has adhered to this medical therapy prior to revision surgery. Whereas the recurrence of symptomatic nasal polyps in CRSwNP prompts surgery, the indication for revision surgery in CRSsNP is not so easily determined, especially in the absence of any obvious obstruction. It is not clear from the literature whether surgery in the absence of mucocoele formation, drainage issues or residual cells is of benefit in CRSsNP. Studies investigating the timing of revision surgery in CRSsNP are lacking. However, in a mixed CRS population, delayed surgery does not seem to negatively affect postoperative improvement ${ }^{(660)}$.

\subsubsection{Preoperative assessment}

When symptoms persist after ESS, and optimal clinical treatment with antibiotics and steroids fail, a nasal endoscopy and a CT is indicated in an attempt to confirm the site and extent of the inflammation and to help the planning of surgery.

In revision surgery a number of features on the CT scan are of interest ${ }^{(639,661)}$. 


\section{Sinus anatomy and post-operative findings}

i) Anterior-posterior diameter in the frontal recess, and overall size of the sinuses (pneumatisation)

ii) Remaining cells that may obstruct drainage or block access to local treatment

(incomplete anterior or posterior ethmoidectomy)

iii) Lateralisation of the middle turbinate

iv) Residual uncinate process

iv) Synechiae or stenosis of previously opened sinuses

It is evident from the literature that frontal sinus clearance is the most challenging part of endoscopic sinus surgery. The most common findings are retained agger nasi cells, neoosteogenesis within the frontal recess, lateral scarring of the middle turbinate, residual anterior ethmoid air cells, and residual frontal cells ${ }^{(662)}$.

\section{Remaining/recurring mucosal disease despite patent drainage pathways}

This finding would indicate ongoing inflammation and/or infection in spite of patent drainage pathways. Although this is not an unusual situation in a clinical setting, there is no literature available to advise on a treatment algorithm.

\section{Neo-osteogenesis}

Neo-osteogenesis is positively correlated to disease severity and as such is a poor prognostic factor and should be part of the evaluation of the preoperative $\mathrm{CT}$ in preparation for revision surgery. A recent study concluded that the presence of $P$. aeruginosa in the sinuses is an independent predictor of neoosteogenesis, whereas $\mathrm{S}$. aureus is not. The number of previous surgeries and the Lund-Mackay score are also independently associated with the severity of neo-osteogenesis ${ }^{(663)}$.

\subsubsection{What should be the extent of a revision operation and} what are the outcomes?

It is difficult to draw any firm conclusion from the literature regarding the ideal extent of surgery as many studies are retrospective, lack a control group and do not make a distinction between CRSwNP and CRSsNP. Furthermore, the patientreported outcome is not dependent on surgery alone, but a combination of surgery, post-operative medical management and patient phenotype / endotype.

Nonetheless, recent review publications have confirmed that revision surgery in both CRSWNP and CRSsNP provides tangible benefits in the majority of patients though extent of the surgery is rarely addressed. Revision surgery is equally effective as primary surgery for CRSwNP and CRSsNP on QoL outcome instruments, in a cohort of $48^{(664)}$. In contrast, a prospective multi-institutional study in 302 patients showed that primary surgery was approximately 2 times more likely to improve than revision cases. This may reflect the grade of disease severity in the revision group which was composed of a mixed CRS population ${ }^{(556)}$. Bhattacharrya addressed the issue of outcomes in a small prospective cohort of 21 adult patients undergoing revision $\mathrm{ESS}^{(665)}$. Data were analyzed for symptom score changes and effect sizes, changes in medication, and economic variables and compared with those of a contemporaneous control group of patients undergoing primary ESS and matched for age, sex, and Lund-Mackay score at a mean follow-up of 12.4 months. Large effect sizes indicating significant symptom improvements were noted for nasal obstruction (effect size,-1.9), hyposmia $(-0.9)$, and headache $(-0.6)$, as well as nasal $(-1.1)$ and total symptom domains $(-0.9 ; \mathrm{p}<.05$ in all cases). These improvements in clinical symptoms were statistically similar to corresponding improvements in the matched cohort of patients undergoing primary ESS.

A systematic review was undertaken by Prasad et al. in 2017 to evaluate the impact of revision ESS(RESS) on PROMs ${ }^{(658)}$. This produced 12 studies (1308 patients), three used the Rhinosinusitis Disability Index and Chronic Sinusitis Survey in tandem; four used the 20-item Sino-Nasal Outcome Test (SNOT20) ${ }^{(403)}$ and the 22 -item Sino-Nasal Outcome Test (SNOT-22)(403); the remaining five used either the Adelaide Disease Severity Score, Patient Response Score (PRS), Rhinosinusitis Symptom Inventory, Rhinosinusitis Outcome Measure 31, or the Chinese version of the University of Pennsylvania Smell Identification Test. Studies consistently reported improvement in the five key symptoms of nasal obstruction, rhinorrhea, postnasal drip, facial pain, and anosmia, although improvement in halitosis, fever, dental pain, and cough were modest.

A study in CRSsNP patients comparing different sizes of the maxillary ostial antrostomy could not detect any influence of this on the main rhinosinusitis symptoms (obstruction, headache, and nasal discharge) ${ }^{(416)}$. Another retrospective review in CRSsNP patients suggests that endoscopic maxillary megaantrostomy is beneficial in recalcitrant maxillary rhinosinusitis in which medical therapy and previous endoscopic antrostomy have been unsuccessful. In a cohort of 122 patients (163 sides) who underwent endoscopic maxillary mega-antrostomy, outcomes were $72.4 \%$ reporting complete or significant improvement, $27.6 \%$ reporting partial improvement and $0 \%$ reporting worsening ${ }^{(666)}$.

There is still conflicting evidence regarding middle turbinate resection (MTR), but recent cadaveric studies confirmed better distributions of topical drugs after partial and complete middle turbinate resection ${ }^{(667,668)}$. The resulting postponement of revision surgery ${ }^{(647)}$ can justify MTR in primary surgery, especially in diffuse sinus pathology andevidence in a mixed CRS population is emerging that it may also improve patientreported outcomes in revision surgery ${ }^{(657)}$. However, if one subscribes to the 'reboot' concept, the middle turbinate should be maintained as it rarely develops polyps and provides the epithelium for re-epithelialization of the sinuses after 'reboot' surgery ${ }^{(655)}$.

The extent of the surgery depends on the severity of the disease and the type of inflammation (endotype). Schalek et al. ${ }^{(667)}$ suggest that in cases of severe, diffuse or recurrent polypoid disease, better outcomes can be achieved by more extensive surgical approaches. The concept of completely removing diseased sinus mucosa in the ethmoids to reduce disease burden and allow a healthier mucosa to develop was proposed by Jankowski in $2006^{(669)}$. It was considered controversial at the time but in recent years it has been relaunched as 'rebooting' 
of the mucosa. Not only the extent of inflammation seen on the $\mathrm{CT}$ but intraoperative judgment will be the main factors to determine the scope of the surgery ${ }^{(425,431)}$. Thus severe Th2 CRSwNP disease, often accompanied by asthma, which is associated with an increased risk of recurrence, might better be approached by 'reboot' surgical techniques ${ }^{(647)}$. To date the role of complete diseased mucosa removal in CRSsNP remains unclear. Jankowski et al. ${ }^{(699)}$ compared radical with conservative surgery for CRSwNP and showed reduced recurrence after the radical approach, albeit with different surgeons responsible for the two approaches. A recent retrospective case-control study in patients with Th-2 pattern CRSwNP done by Alsharif et al. ${ }^{(431)}$ reported an important reduction in polyp recurrence and prolongation of recurrence-free time after complete removal of the diseased sinus mucosa. This allowed healthy re-epithelialization from the preserved nasal mucosa, using the partial and full 'reboot' techniques as compared to the classical ESS approach.

This paper defined 'reboot surgery' as complete removal of all inflamed sinus mucosa to allow the regrowth of functional mucosa and divided it into 'partial' and 'full'. Partial involves the clearance of all sinonasal polyps and mucosa down to the periosteum whereas a full reboot procedure includes the mucosa of the frontal recess and the frontal sinuses with Draf III access and removal of all frontal sinus mucosa and a sufficiently large frontal opening to avoid stenosis(655). The full 'reboot' approach showed a better result as compared to a partial 'reboot'ESS though this was not statistically significant. Further research is needed to support the 'reboot' vs. EESS approaches to identify the role of complete removal of the sinus mucosa. Postoperative obstruction of the frontal sinus drainage as a cause of chronic frontal sinusitis and mucocoeles are a major concern after CRSwNP surgery ${ }^{(670,671)}$. In these cases, an endoscopic modified Draf III can be offered. This procedure is generally reserved for revision surgery after primary FESS. If the patient's primary FESS was not performed adequately by the previous surgeon, a revision Draf lla can be offered before a Draf III is undertaken. If the previous frontal surgery has been properly performed and failed, then a Draf III is indicated ${ }^{(436)}$. The Draf III is generally undertaken in severe cases, showing statistically significant improvement in recurrence rates in CRSwNP, especially in patients with asthma and aspirinexacerbated respiratory disease ${ }^{(672)}$. As might be expected, revision Draf III has a higher failure rate compared to primary Draf III ${ }^{(673)}$. With advancement in technology, instrumentation, and techniques, the Draf III has evolved into an efficient and safe procedure, and likely will be more commonly used as a primary procedure in high-risk patients If the risk factors for all patients undergoing Draf III are assessed, these factors (asthma, CRSwP, $\mathrm{LM}>16$, narrow frontal ostium $<4 \mathrm{~mm}$ ) provide a reasonable basis for considering a Draf III in patients who otherwise would undergo revision ESS. This particular subset may well benefit from a primary Draf III ${ }^{(672)}$.

The revision rate reported by Bassiouni et al. after ESS including a Draf Ila was 37\% compared to $7 \%$ for patients that underwent a Draf III, suggesting the superiority of Draf III surgery in patients with CRSWNP(674). After long-term follow-up for a minimum of seven years of 153 CRSwNP patients, Benkhatar et al. found that $6.5 \%$ required at least one revision surgery for chronic frontal sinusitis, with a mean delay from initial procedure to diagnosis of 46 months (four months to 11 years). Circular mucosal damage in combination with a narrow frontal opening can lead to closure of the frontal sinus drainage pathway and hence a higher risk of frontal sinus revision ESS ${ }^{(670)}$.

A meta-analysis of the Draf III or endoscopic modified Lothrop procedure (EMLP), as it is often called in the United States was conducted from 1990 to 2016 by Shih et al. ${ }^{(438)}$ reporting clinical outcomes of Draf III. A total of 1,205 patients were abstracted from 29 articles with a mean follow-up of $29.1+/-10.3$ months. The overall rate of significant or complete symptom improvement was $86.5 \%$ (95\% confidence interval [CI]: $84.2 \%$ 88.7\%). The overall patency rate was $90.7 \%$ (95\% Cl: $89.1 \%$ $92.3 \%)$, with a revision rate of $12.6 \%$ (95\% Cl: $10.6 \%-14.3 \%)$.

Table 6.2.3.1. Potential indications for external approaches to the maxillary and frontal sinuses in CRSsNP and CRSwNP.

\begin{tabular}{|c|c|}
\hline Maxillary sinus & Frontal sinus \\
\hline $\begin{array}{l}\text { Anterolateral/-inferior pathology requiring access but inaccessible } \\
\text { endonasally }\end{array}$ & $\begin{array}{l}\text { Narrow frontal recess that cannot be kept open. } \\
\text { Far lateral pathology or pathology on the orbital roof requiring access but } \\
\text { inaccessible endonasally. }\end{array}$ \\
\hline Osteoneogenesis that cannot be treated endoscopically & $\begin{array}{l}\text { Some forms of frontal osteomyelitis. } \\
\text { Previous trauma and severe frontal recess stenosis. } \\
\text { Complicated acute frontal sinusitis e.g. sub-periosteal frontal abscess that does not } \\
\text { resolve with antibiotics. } \\
\text { Extensive neo-osteogenesis at frontal recess area. }\end{array}$ \\
\hline Failure of endoscopic approach & $\begin{array}{l}\text { Failure of endoscopic approach. } \\
\text { Need for fat obliteration or cranialisation. }\end{array}$ \\
\hline
\end{tabular}

CRSsNP, Chronic Rhinosinusitis without nasal polyps; CRSwNP,Chronic Rhinosinusitis with nasal polyps. 
Compared to the early cohort, patients in the contemporary cohort underwent Draf III more often for tumours $(p<.001)$, had higher rates of complete or significant symptom improvement (90.0\% vs. $82.6 \%, \mathrm{p}<.001)$; and trended toward greater patency rates $(92.1 \%$ vs. $88.6 \%, p=5.052)$. Overall $79 \%$ were performed for recalcitrant CRS but when the cohort was divided into 19902008 vs. 2009-2016, whilst there was no difference in symptom improvement or patency, the revision rate was higher in the more recent group ( $14.5 \%$ vs. $9.2 \%, p=5.016)$ when Draf III has been performed more frequently for tumors. Among 114 failure cases in 16 studies, $82.5 \%$ patients were revised with Draf III, $11.4 \%$ salvaged with an osteoplastic flap and $6.1 \%$ with standard revision ESS. The revision rate increased significantly when follow-up exceeded two years which may also have reflected the indications for surgery.

Abuzeid et al. also published a meta-analysis in 2018 but only considered studies from 2000-2016, including 11 studies and 778 patients, $86.5 \%$ of whom had CRS $^{(433)}$. Mean follow-up duration was 28.4 months and the mean number of surgeries prior to Draf III was 3.5. In a subgroup analysis of seven studies in which all 357 patients had CRS, symptom improvement was $75.9 \%$ of cases and $23.1 \%$ experienced polyp recurrence. The cerebrospinal fluid leak rate was $2.5 \%$ and restenosis of the neoostium occurred in $17.1 \%$ with complete closure occurring in $3.9 \%$ of cases. The reoperation rate for Draf III was 9.0\%. Aspirin sensitivity was associated with an increased risk of cerebrospinal fluid (CSF) leak ( $p=0.0339$ ) and a reduced incidence of neoostium closure $(p=0.0001)$. Aspirin sensitivity and asthma were associated with a reduced incidence of reoperation $(p=0.001)$ and increased symptom improvement $(p<0.005)$. Restenosis or closure of the frontal neo-ostium was associated with less symptom improvement $(p<0.04)$ but not with reoperation, as above.

\subsubsection{Is there any difference in postoperative management for revision vs. primary?}

Current postoperative management of ESS patients varies greatly between individual surgeons. This variation in current practice indicates the lack of consensus on the topic. Generally postoperative management is aimed at maintaining a clean surgical cavity, in which topical steroids can act and to maintain patency of the sinus cavities, especially the frontal drainage pathway. The long-term follow-up will depend on the underlying pathophysiology, sinus patency, and control of sinonasal inflammation with medical therapy.

\section{Topical steroids and saline washes}

Escalation of treatment beyond nasal steroid sprays and saline washes is called for in a revision case. Steroid nasal lavage was shown to have superior distribution in the postoperative sinuses compared to steroid nasal sprays. Harvey et al.'s DBRCT in a mixed CRS population ( $\mathrm{n}=35$ analysable) showed benefit for mometasone irrigation as compared to spray delivery up to one year post-surgery, though both groups showed improvement ${ }^{(67)}$. No trial of steroid washes has exclusively studied the CRSsNP population. An undefined CRS population, $(n=61)$, participated in a double-blind, placebo-controlled, randomized clinical trial comparing saline/lactose with saline/budesonide large volume, low pressure washes. The average improvement in SNOT-22 scores was 20.7 points for those in the budesonide group and 13.6 points for those in the saline/lactose group, with a mean difference of 7 points in favour of the budesonide group ${ }^{(81)}$. A recent meta-analysis pooled three studies (mixed CRS population) and concluded that nasal irrigation was beneficial, but found no clinical benefit from adding corticosteroid in nasal lavage compared to saline alone as post-operative treatment ${ }^{(534)}$.

\section{Long term antibiotic treatment}

Long-term treatment, four weeks or more, with macrolide antibiotics has support in the literature in CRSsNP patients where the eosinophilic inflammation is low to moderate and serum IgE within the normal range $\mathrm{e}^{(14,27,675)}$. So far doxycycline has only been investigated in CRSwNP(4). Trimethoprim / sulfamethoxazole has been shown to improve patient reported outcomes in 2 uncontrolled clinical observational trials of 'purulent $\mathrm{CRS}^{\prime(676)}$ and 'recalcitrant $\mathrm{CRS}^{\prime(677)}$. None of these studies have focused specifically on patients undergoing revision surgery. The use of culture-directed antibiotic therapy and topical and/or systemic steroid administration depends on the underlying pathophysiology and postoperative wound healing ${ }^{(678)}$.

\section{Antimicrobial photodynamic therapy (aPDT)}

Antimicrobial photodynamic therapy (aPDT) has demonstrated in vitro efficacy for the eradication of bacterial biofilms associated with chronic rhinosinusitis (CRS) as well as antiinflammatory properties ${ }^{(679-682)}$. Previous case series have suggested effectiveness in patients with CRS unresponsive to medication and surgery ${ }^{(683)}$. More recently an RCT was undertaken to evaluate the clinical effects of aPDT for refractory CRS and included 47 patients at two centres ${ }^{(684)}$. Twenty-three patients with CRSsNPs and 24 with CRSwNPs unresponsive to medical and surgical therapy underwent one of three treatments: a single treatment with aPDT, two treatments with aPDT separated by a four-week interval, or endoscopic irrigation with saline (control). Outcome measures included SNOT-22, endoscopic scores, UPSIT, microbiology results and endoscopic scores. Significant improvements were seen following aPDT treatment in both symptomatic and endoscopic scoring, with the greatest effect occurring in the CRSwNPs group receiving two treatments $(p=0.007)$. The aPDT treatments were well tolerated, with temporary mild pressure in the treated sinus(es) being the most common adverse event. The formal trial results have yet to be reported but these findings do show early promise

\section{More active debridement?}

Debridement improves postoperative endoscopic appearances, minimizing postoperative infection, granulation, and potential restenosis especially in patients who had significant mucosa and bone removal (Draf IIB and III), but it is unclear if it has any longterm positive effects on disease-specific health-related quality of life or disease severity as reported by two recent reviews in an mixed CRS population ${ }^{(529,530)}$. 
Generally, as in primary ESS, the first postoperative debridement is seven to 10 days after surgery and thereafter the visits are individualized depending on the specific needs of the patients. Revision surgery may be an indication for the parallel use of biologics in the future ${ }^{(685)}$. The surgery should be performed after several months of biologic treatment to establish its efficacy, and the need for surgery should then be determined depending on presence of polyps, symptoms and QoL of the patients.

\subsubsection{General conclusions}

Clinical experience and evidence in the literature suggest that revision surgery in CRSWNP and to a lesser extent CRSsNP is beneficial. However, the quality of the scientific evidence is generally low.

\subsubsection{Indications for external surgery}

The need for external approaches in CRS is radically reduced with the introduction of endoscopic sinus surgery (ESS) led by a philosophical move away from radical removal of diseased mucosa to minimally invasive functional restoration of drainage pathways and enabling postoperative topical medication delivery to the sinonasal cavities. With the exception of a small number of indications in the maxillary and frontal sinuses, few areas of the sinuses cannot now be reached endoscopically, facilitated by advanced techniques using angled endoscopes and powered instrumentation. Thus, the indications for external approaches are now limited areas that cannot be reached/kept open endoscopically. Nevertheless, in the absence of adequate instrumentation or experience, external approaches still provide an option and may be combined with endoscopic techniques to provide a postoperative transnasal route for topical medical treatment delivery. The current potential indications for an external approach are listed in Table 6.2.3.1.

There are only three papers on two prospective randomised trials on this topic ${ }^{(426,429,686)}$ the rest of the publications being non-comparative cohorts which are largely retrospective.

\subsubsection{Maxillary sinus}

The Caldwell-Luc operation, or radical antrostomy is done through a sublabial anterior maxillotomy which has proven its value in several large published series ${ }^{(687-689)}$. This approach is now rarely used for inflammatory diseases, supplanted by the many variants of ESS ${ }^{(690)}$ but in spite of high success rates obtained with endoscopic approaches, maxillary antrostomy failures still occur ${ }^{(414)}$ and among revision endoscopic surgery patients, one study reported $39 \%$ stenosis of the middle meatal antrostomy ${ }^{(691)}$.

The major concerns in recalcitrant maxillary rhinosinusitis include impaired mucociliary clearance either primary such as occurs in cystic fibrosis or primary ciliary dyskinesia, or associated with biofilm formation or secondary to previous surgery ${ }^{(692)}$. Today even in the presence of persistent disease requiring surgical management, extended endoscopic approaches such as endoscopic modified medial maxillectomy or inferior meatal antrostomy are generally utilized as the next option in preference to Caldwell-Luc (C-L $)^{(690)}$. Nevertheless, in spite of these efforts, if the disease is difficult to reach endonasally, involves a large foreign body or infected bone, a classical C-L procedure ${ }^{(693)}$, an external endoscopic-assisted sublabial anterior maxillotomy ${ }^{(690)}$ or a Denker procedure still remain viable options ${ }^{(694)}$. Canine fossa trephination has also been reported to be of use in patients with N-ERD and in removal of debris and polyps in allergic fungal rhinosinusitis ${ }^{(695)}$. Pentilla et al. ${ }^{(686)}$ performed one of the few prospective randomised studies comparing standard C-L to FESS in 150 consecutive adult patients with chronic maxillary rhinosinusitis after failure of medical treatment. The endoscopic surgery was performed by one surgeon whereas 16 surgeons undertook the C-L. There were 143 patients available for the follow-up at a median of 12 months. The patients' global evaluation showed marked improvement in $51 \%$ of the C-L group and in $77 \%$ of the FESS group. Overall subjective symptoms deteriorated in $5.5 \%$ of C-L operated patients, but not at all in the FESS group. At the time, these results seemed to favour FESS over C-L. However, a contemporaneous study compared pre- and post-operative histopathologic mucosal changes in chronic maxillary rhinosinusitis after FESS (60 sinuses) and C-L (55 sinuses) ${ }^{(696)}$. The C-L operation reduced almost all parameters, whereas only oedema and inflammatory cells were significantly reduced after FESS. No valid correlations were found when comparing histology with patient symptoms. Furthermore, in 1997 Pentilla et al. ${ }^{(429)}$ published a reassessment of their cohort with questionnaires obtained from 128 patients (85\%) 5-9 years after operation. At this point $82 \%$ of the C-L and $76 \%$ of the FESS patients respectively reported significant improvement. Thirteen C-L operated patients (18\%) and 14 FESS patients (20\%) had been re-operated during the 7-9 years follow-up.

A second randomised, controlled trial with parallel design, including 119 patients who fulfilled the clinical, radiological and histopathological criteria for chronic, hyperplastic, eosinophilic rhinosinusitis was performed by Abd el-Fattah ${ }^{(426)}$. Patients were randomly allocated to two treatment groups: classical endoscopic middle meatal antrostomy as part of FESS and endoscopic radical antrectomy through a combination of canine fossa puncture and an endonasal endoscopic procedure, with complete removal of maxillary sinus mucosa. The radical group showed significantly better impact on symptoms of nasal obstruction and rhinorrhoea. Thirty-two per cent of patients in the endoscopic middle meatal antrostomy group were considered surgical failures, compared with $14.5 \%$ of the endoscopic radical antrectomy group $(p=0.023)$. Unhealthy maxillary sinuses were significantly more prevalent in the endoscopic middle meatal antrostomy group $(p=0.029)$. The authors concluded that in patients fulfilling the criteria suggestive of chronic, irreversible sinonasal pathology, primary endoscopic radical antrectomy was significantly better than endoscopic middle meatal antrostomy, based on subjective and objective findings.

In a study of Denker's procedure, Videler et al. conducted a prospective questionnaire-based study on 23 patients with refractory CRS. They had undergone a median of six previous sinonasal operations (range, 3-11) prior to undergoing the 
Denker's procedure, which combines all sinuses, except the frontal sinus, into one large cavity, via a sublabial approach to the anterior angle between the bony nose and antral walls. Preoperative and postoperative symptoms at 12 and 24 months were compared. Patients reported a significant improvement in rhinorrhoea ( $p=0.001)$, feelings of congestion $(p=0.02)$, and nasal obstruction $(p=0.03)$. Reduced olfactory perception and asthma did not improve.

Interestingly in a review of 670 Caldwell-Luc procedures, the most frequent complication was recurrent nasal obstruction, which occurred in $28 \%$ of patients ${ }^{(687)}$. Informed consent should also include other possible complications such as paraesthesia, facial pain, oroantral fistula, dacrocystitis, facial asymmetry and devitalised teeth ${ }^{(686,687,689,697)}$. Techniques that may help reduce complications include gentle tissue retraction, protection of the infraorbital nerve, limitation of the size of the anterior antrostomy, and avoidance of cracks in the bony wall of the antrum ${ }^{(698)}$. The best area in which to perform a canine fossa puncture without injuring the infraorbital nerve or anterior superior alveolar nerve has been shown to be at the intersection of the mid-pupillary line and a horizontal line through the floor of the nasal vestibule ${ }^{(699)}$.

\subsubsection{Ethmoid and sphenoid sinuses}

Endonasal ESS, due to its minimally invasive nature, has largely supplanted external approaches to the ethmoids and sphenoid in $\mathrm{CRS}^{(690)}$. An external ethmoidectomy through a modified Lynch-Howarth incision made along the inferior border of the medial eyebrow and carried inferiorly in a curvilinear fashion allows access to the ethmoid sinuses, medial orbit, anterior skull base, frontal recess, supero-medial orbit, sphenoid sinus and orbital apex. However, in addition to the usual risks engendered by proximity to the orbit and skull base, it carries the additional risk of complications such as facial scars, forehead paraesthesia, and telecanthus. The removal of the medial orbital bone frequently led to medialisation of the orbital contents which combined with failure to preserve mucosa produced stenosis of frontal sinus outflow with secondary inflammatory problems, contributing to its loss of popularity ${ }^{(689,690,700-702)}$.

In the era of ESS, external approaches to the frontal sinuses still have value used as an adjunct or alone but their indications are diminishing. However, external approaches should be kept in our rhinosurgical armamentarium and taught to the younger generations.

\subsubsection{Frontal sinus}

The frontal sinuses remain a challenge for the sinus surgeons even in the endonasal endoscopic era, due to their complex anatomy. Traditionally, all frontal sinus pathologies including CRS were addressed via external approaches such as frontal sinus trephination, external frontoethmoidectomy, and frontal sinus osteoplastic flap with or without obliteration or cranialization $^{(688-690,700,703-708)}$. These have largely been replaced by endoscopic techniques culminating in median drainage (Draf III or an endoscopic modified Lothrop procedure (EMLP)).
However, the classical external procedures may still be used alone or in combination with endoscopic approaches for lesions that cannot be reached endoscopically, very narrow entrance to the frontal sinus that cannot be kept open e.g. due to neoosteogenesis in the frontal recess and very rarely recalcitrant inflammation, particularly involving the far lateral region of the frontal sinus, disease in a type III/IV frontal cell, osteomyelitis, and/or previous trauma (Table 6.2.3.1.).

For the inflammatory diseases of the frontal sinus, a frontal sinus trephine as an adjunct to endonasal procedures can be performed ${ }^{(709)}$. Frontal sinus trephination is a safe procedure where complications are relatively rare. Nowadays, this technique is mainly performed as a mini-trephination, enhanced by the use of an endoscope, which improves visualization and facilitates instrumentation through the trephine. Indications include: complicated chronic frontal rhinosinusitis or frontal osteomyelitis; frontal sinusitis with difficult anatomic variants, such as type III and IV or intrafrontal cells ${ }^{(595)}$; and far lateral frontal sinus diseases as well as complicated acute frontal sinusitis ${ }^{(688-690)}$. Seiberling et al. ${ }^{(710)}$ reported a $92 \%$ frontal sinus patency rate after 188 mini-trephination procedures, with an average follow-up of 25.5 months and with 12 complications, the most common being infection of the trephine site. The indications for trephination were difficulty in finding the frontal recess, severe oedema / polyps, frontal cells (type III or IV or intrafrontal cells and intersinus septum cell), as an adjunct during a Draf III and when there was a need for postoperative irrigation. In a systematic review, Patel et al. ${ }^{(711)}$ concluded that in the era of ESS, frontal sinus trephination has remained the most routinely utilized of all external procedures either as a supplement to the endonasal endoscopic approach or used alone to provide adequate access for both endoscopic visualization and instrumentation into the far lateral and superior areas of the frontal sinus.

External frontoethmoidectomy through a Lynch-Howarth incision have been used historically in CRSsNP, usually as a transorbital approach to the frontal sinus by resection of the ethmoid sinuses and the creation of a contiguous space into the nasal cavity. Nevertheless, it had high failure rate due to orbital medialisation as mentioned above ${ }^{(702)}$. Although this procedure is rarely used nowadays, it may still have some limited utility in the presence of complex anatomy and in the presence of significant neo-osteogenesis of the frontal recess ${ }^{(688,689)}$. Use of the frontal sinus osteoplastic flap (OPF) with or without obliteration (FSO) for recalcitrant frontal sinus disease has also decreased significantly with the advancement of endoscopic techniques. This procedure is extensive and requires a coronal, mid-forehead or brow incision, as well as osteotomies to create the OPF. With this approach, the entire frontal sinus pathology can be exposed, removed and all the frontal sinus mucosa drilled out, followed by abdominal fat obliteration of the sinus if required ${ }^{(688-690,704,705,712-717)}$. Extensive neo-osteogenesis of the frontal recess secondary to previously failed endoscopic approaches is the most common indication for this technique in inflammatory disease, followed by some cases of laterally placed frontal sinus mucocoeles with very narrow diameter of the Draf III opening, some cases of osteomyelitis that cannot 
be cured with antibiotics, some cases of 'allergic' fungal frontal rhinosinusitis and other laterally located pathologies.

Despite success rates up to $85-90 \%$, long term studies have shown that the OPF with FSO can have significant morbidity with cosmetic deformity, cerebrospinal fluid leak, forehead numbness and postoperative headaches ${ }^{(703-705)}$. Weber et al. ${ }^{(705)}$ described a $10 \%$ incidence of mucocoele formation after FSO detected five years after surgery by MRI. Hansen et al. ${ }^{(706)}$ demonstrated that the prevalence of mucocoeles and revision rate was $7,5 \%$ in 40 consecutive obliterations with an average follow-up of 80 months. Even more problematic is infection of the frontal bone flap which in the 1970s was reported at $18 \%{ }^{(704)}$. If this occurred, removal of the affected frontal bone as in a Reidel procedure could be considered with a view to reconstruction at a later date ${ }^{(718)}$.

In the most severe cases of refractory frontal rhinosinusitis that have failed traditional endoscopic and open approaches, or in cases of osteomyelitis of the posterior table of the frontal sinus, cranialization of the sinus may be the final surgical resort ${ }^{(707,708)}$. In 2011, van Dijk et al. ${ }^{(708)}$ reported 15 patients with refractory chronic frontal rhinosinusitis treated by cranialization of the frontal sinus, from 1989 to 2008 . All of the patients had better quality of life after a mean follow-up of 6.5 years.

In a retrospective review of 717 frontal sinus procedures for inflammatory disease in 683 patients at a tertiary academic centre over a three-year period, Hahn et al. ${ }^{(715)}$ reported that most cases of chronic frontal rhinosinusitis can be treated by endoscopic sinus surgery though this may be unsuccessful in some situations such as distortion of critical landmarks, neo-osteogenesis of the frontal recess, and lateral location of diseased mucosa. Whilst, $>94 \%$ of these cases were successfully treated endoscopically, 38 external procedures were performed in 32 patients (5.3\%). Fourteen external procedures (2\%) were performed in isolation and 24 procedures (3.3\%) in combination with ESS, 16 of whom had concurrent obliteration. The osteoplastic flap was used in 24 cases (3.3\%), the remaining external procedures were frontal sinus trephines. In this series, the most common indication for the external approach was the neo-osteogenesis of the frontal recess (63.2\%). More significantly, $79.1 \%$ of the cases of neo-osteogenesis were associated with previous ESS with a mean of 2.5 procedures, indicating that repeated surgical manipulation in the frontal recess leads to scarring and osteoneogenesis that limit the success of subsequent endoscopic approaches. In these cases, the osteoplastic flap may provide a viable alternative. Although there were no major complications with the external operations, but $9 / 38$ (23.7\%) patients required revision surgery for persistent/recurrent symptoms.

In the era of ESS, external approaches to the frontal sinuses still have value used as an adjunct or alone but their indications are diminishing. However, external approaches should be kept in our rhinosurgical armamentarium and taught to the younger generations.

Figure 6.2.4.1. Forest plot of the effect of reverse Trendelenburg position compared to the horizontal position on surgical field quality.

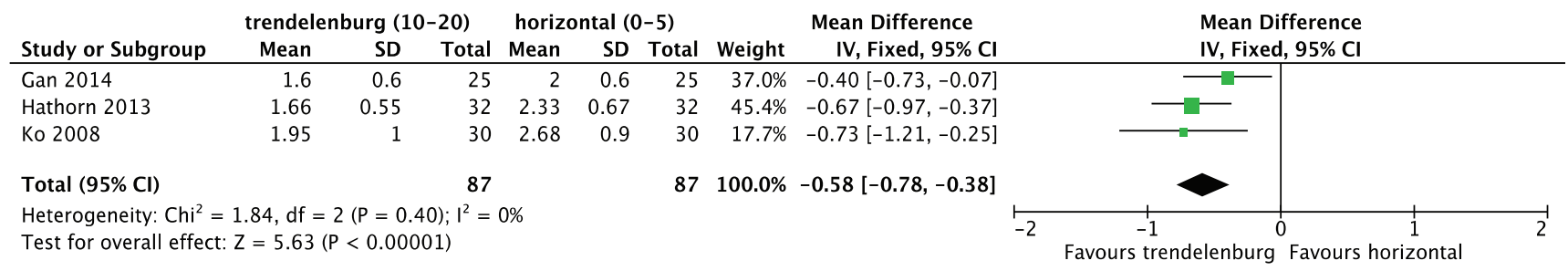

Figure 6.2.4.2. Forest plot of the effect of reverse Trendelenburg position compared to the horizontal position on blood loss.

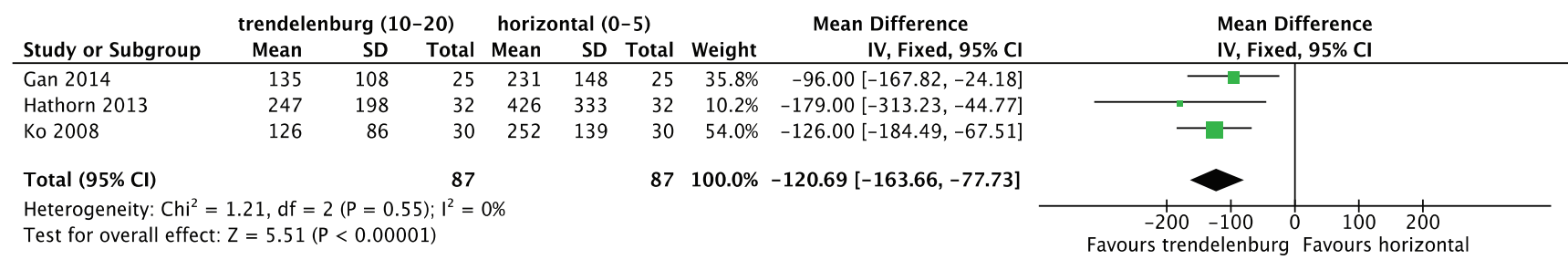

Figure 6.2.4.3. Forest plot of the effect of reverse Trendelenburg position compared to the horizontal position on operation time.

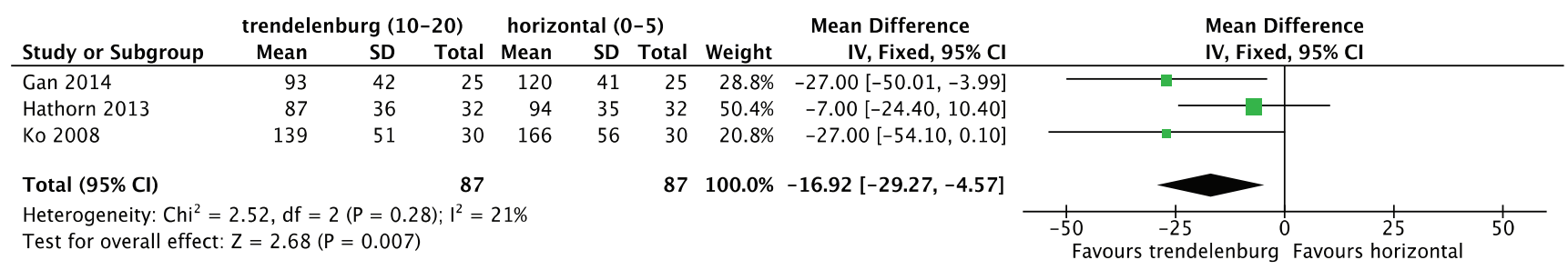


Table 6.2.4.1. Effect of reverse Trendelenburg position on endoscopic sinus surgery.

\begin{tabular}{|c|c|c|c|c|c|}
\hline Study & Methods & Participants & Interventions & Outcomes & Results \\
\hline $\begin{array}{l}\text { Gan } \\
2014^{(878)}\end{array}$ & RCT & $\begin{array}{l}\text { CRS patients } \\
\text { having FESS } \\
\text { ( } 22 \text { evaluated) } \\
(n=75)\end{array}$ & $\begin{array}{l}\text { Reverse Trendelenburg } 20 \text { degree } \\
\text { Reverse Trendelenburg } 10 \text { degree } \\
\text { Reverse Trendelenburg } 5 \text { degree }\end{array}$ & $\begin{array}{ll}\cdot & \text { Blood loss }(\mathrm{ml}) \\
\cdot & \text { Surgical field quality } \\
\text { (Boezaart, 0-5) } \\
\text {. } & \text { Operation time }\end{array}$ & $\begin{array}{l}\text { - Larger degrees of reverse Trendelenburg } \\
\text { improved surgical field quality but did } \\
\text { not improve blood loss or operation } \\
\text { time }\end{array}$ \\
\hline $\begin{array}{l}\text { Hathorn } \\
2013^{(879)}\end{array}$ & RCT & $\begin{array}{l}\text { CRS patients } \\
\text { having FESS } \\
(\mathrm{n}=64)\end{array}$ & $\begin{array}{l}\text { Reverse Trendelenburg } 15 \text { degree } \\
\text { Horizontal position }\end{array}$ & 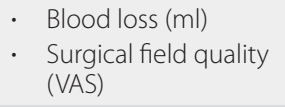 & $\begin{array}{l}\text { Reverse Trendelenburg position } \\
\text { improved surgical field quality and } \\
\text { reduced blood loss and operation time }\end{array}$ \\
\hline $\begin{array}{l}\text { Ko } \\
2008^{(880)}\end{array}$ & RCT & $\begin{array}{l}\text { CRS ( } 33 \text { CRSwNP) } \\
\text { patients having } \\
\text { FESS ( } n=70)\end{array}$ & $\begin{array}{l}\text { Mometasone nasal spray } 200 \\
\text { mcg twice daily } 4 \text { weeks preoper- } \\
\text { atively }(n=35) \\
\text { Placebo nasal spray twice daily } 4 \\
\text { weeks preoperatively ( } n=35)\end{array}$ & $\begin{array}{l}\text { - } \text { Blood loss (ml) } \\
\text { Surgical field quality } \\
\text { (Boezaart, 0-5) } \\
\text { - Operation time }\end{array}$ & $\begin{array}{l}\text { Treatment with topical corticoid compared } \\
\text { to placebo resulted in significantly: } \\
\text { - reduced blood loss } \\
\text { - improved surgical field quality } \\
\text { - decreased operation time }\end{array}$ \\
\hline
\end{tabular}

RCT,Randomised Controlled Trial; CRS,Chronic Rhinosinusitis; FESS, Functional Endoscopic Sinus Surg; CRSwNP, Chronic Rhinosinusitis with nasal polyps; VAS, Visual Analogue Scale.

\subsubsection{Peroperative measures to improve surgical field and outcomes}

Successful ESS highly depends on accurate identification of anatomic landmarks within a circumscribed surgical field. Bleeding from the sinonasal mucosa impairs endoscopic visualization and may lead to complications due to the close proximity to the skull base, orbit and vital neurovascular structures. Actions should be taken to minimise peroperative bleeding and optimize surgical visualization, whilst maintaining the physiological homeostasis of the patient. These include patient position, local anaesthetics and vasoconstrictors, type of general anaesthetic agent and controlled hypotension.

\subsubsection{Patient positioning}

A reverse Trendelenberg position of 5-15' (head elevation) has been shown to improve the surgical field in other surgical disciplines by reducing the central venous pressure from 9.2$1.7 \mathrm{~mm} \mathrm{Hg}^{(719)}$.

Three studies have been performed evaluating the effect of reverse Trendelenburg position on surgical field quality, blood loss and operation time (Table 6.2.4.1). The studies could be combined into a meta-analysis where reverse Trendelenburg position (10-20 degrees) was compared to horizontal position (0-5 degrees).

The positioning in reverse Trendelenburg position compared to the horizontal position resulted in a significant improvement of the surgical field quality measured with the Boezaart scale ${ }^{(382)}$ with a MD of $-0.58(\mathrm{Cl}-0.78--0.38)$, three trials, 194 patients, $\mathrm{p}<0.00001$ (Figure 6.2.4.1.). The positioning in reverse Trendelenburg position compared to the horizontal position also resulted in a significant mean reduction of the peroperative blood loss of $120 \mathrm{ml}(\mathrm{Cl}-78 .-164 \mathrm{ml}), 3$ trials, 194 patients, $p<0.00001$ (Figure 6.2.4.2.).

Finally, the positioning in reverse Trendelenburg position compared to the horizontal position also resulted in a significant reduction of operation time with a $\mathrm{MD}$ of $-17 \mathrm{~min}(\mathrm{Cl}-30--5$ $\mathrm{min}$ ), three trials, 194 patients, $\mathrm{p}<0.0007$ (Figure 6.2.4.3.).

\subsubsection{Local anaesthesia and vasoconstrictors}

Since its inception, it has been possible to conduct ESS under local anaesthesia. There are a number of potential advantages and disadvantages though there are few scientific studies on these topics. Gittelman et al. ${ }^{(720)}$ considered 232 patients undergoing ethmoidectomy, 149 under local (LA) and 83 under general anaesthesia (GA) in a retrospective study without statistical analysis. There was less blood loss in the LA group $(23 \mathrm{ml}$ vs. $58 \mathrm{ml}$ ) but the complication rate was higher under LA ( $8.7 \%$ vs. $2.4 \%$ ) which included four cases of severe bleeding. In contrast Lee et al. ${ }^{(721)}$ reported $1.4 \%$ complication rate (only $0.5 \%$ major) in a non-comparative series of 554 patients having ESS under LA with sedation.

A range of sedatives may accompany $L A$, given by mouth, intramuscular or intravenous routes. These include benzodiazepines such as midazolam ${ }^{(721)}$, opiates such as morphine ${ }^{(721)}$ or pethidine ${ }^{(722)}$. Local anaesthetic agents (and vasoconstrictors) may be applied topically to the mucosa or as regional blocks related to the sinonasal neurovascular anatomy as described by Rontal et al. ${ }^{(723)}$. Common combinations of anaesthetic agents and vasoconstrictors include cocaine or lignocaine (lidocaine) with adrenaline $+/$ - xylometazoline in varying concentrations ${ }^{(721,722,724-728)}$. Few high-quality studies have been performed to investigate these regimes. However, in an RCT Sarmento et al. ${ }^{(729)}$ divided 49 patients undergoing ESS into three groups using only topical solutions of adrenaline in different concentrations (1:2,000, 1:10,000 and 1:50,000). Surgery time was shorter in the group using adrenaline 1:2,000, which also showed less bleeding in all evaluations (objective and subjective $-p<0.0001$ ).

\subsection{Regional blocks}

A systematic review of the literature up to 2018 was performed on preoperative injections of local anaesthetic and vasoconstrictor into the greater palatine canal (GPC) to decrease surgical bleeding during endoscopic sinus surgery (ESS) ${ }^{(730)}$. Five articles comparing preoperative GPC injection (treatment group) with a placebo or no treatment (control group) were included ${ }^{(609,731-734)}$. The endoscopic grade of nasal bleeding in the treatment group was significantly reduced when compared with the control group and no significant adverse effects were reported in the studies. A subgroup analysis showed 
adrenalin 1:80,000 was significantly more effective in reducing intraoperative bleeding than adrenalin 1:100,000 (Level of evidence la).

\subsection{Topical agents}

Cocaine is an excellent anaesthetic and vasoconstrictor but is potentially cardiotoxic. In three national studies on the use of cocaine in the Netherlands, UK and USA, the majority of ENT respondents (84-90\%) had used cocaine as an anaesthetic and between $11-26 \%$ had observed adverse effects, mainly tachycardia ${ }^{(735-737)}$. To avoid these, it is recommended that serum levels should not exceed $3-4 \mathrm{mg} / \mathrm{kg}$ body weight which generally equates to a maximum of $200 \mathrm{mg}$ in a healthy adult ${ }^{(722,726)}$. In theory the cardiotoxicity might be enhanced by the combination of cocaine with adrenaline though this was not supported by Pfleiderer and Brockbank's study ${ }^{(738)}$. In 30 patients, they showed peak serum cocaine concentrations were lower in those receiving the combined agents. In a series of 554 patients undergoing ESS Lee et al. ${ }^{(721)}$ used $25 \%$ cocaine paste and $0.01 \%$ adrenaline and reported $<10 \%$ tachycardia and no arrhythmias or other adverse reactions.

A combination of topical lignocaine and oxymetazoline has been shown to be as effective as cocaine in terms of anaesthesia and vasoconstriction ${ }^{(739,740)}$. In a study on children undergoing FESS, oxymetazoline alone was shown to be as effective as phenylephrine or cocaine in terms of vasoconstriction which improved bleeding and visualisation ${ }^{(741)}$. Tetracaine combined with oxymetazoline was even more effective than both lignocaine with oxymetazoline or cocaine in terms of analgesia ${ }^{(742,743)}$. However, this latter study measured pain thresholds associated with stimulation of the septum in healthy subjects which may not be relevant to the surgical situation. Of greater clinical relevance, cocaine was shown to be superior when compared directly with lignocaine for intranasal antroscopy ${ }^{(744)}$.

Javer et al. ${ }^{(745)}$ showed that infiltration with $2-3 \mathrm{mls}$ of $0.25 \%$ bupivacaine plus 1:200,000 adrenaline (one side) versus saline (one side) gave a no significant reduction in intraoperative blood loss during FESS ( $n=46)$. An RCT comparing 1:200,000 and 1:100,000 adrenaline also failed to show any difference in blood loss ${ }^{(746)}$, indicating that neither concentration is effective as a vasoconstrictor when used as infiltration. Furthermore, Lee et al. ${ }^{(74)}$ could show no difference in blood loss when they compared infiltration versus topical application of $4-5 \mathrm{mls}$ of 1:100,000 adrenalin. However, when four concentrations of topical adrenaline $(20 \mathrm{mls})$ were compared, Sarmento et al. ${ }^{(729)}$ showed haemostatic superiority for 1:2000 adrenalin over lower concentrations.

Adrenaline may also produce potential cardiovascular side effects including effects on the blood pressure, tachycardia and arrhythmias. These have been investigated using infiltration with concentrations of 1:100,000-1:400,000 during ESS though effects have been variable with both increases ${ }^{(746,748,749)}$ and reductions ${ }^{(746,750)}$ in heart rate and blood pressure. This is attributed to the biphasic effect of adrenalin, which produces vasoconstriction at low concentrations and vasodilation at high concentrations.

Whilst injection of lignocaine $(4 \mathrm{ml}$ of $1 \%)$ produced no circulatory change ${ }^{(748,749)}$, the addition of 10 -20ug of adrenalin dropped the blood pressure by $25-35 \%$ and increased the heart rate slightly during the first two minutes post-infiltration but the effect was transitory. It is noteworthy that although tachycardia occurred in up to $21 \%$ of the 1022 individuals studied, no serious side effects were experienced.

\section{The EPOS steering group advises to use TIVA, and to put the patient in a reverse Trendelenberg position during endoscopic sinus surgery.}

\subsection{Anaesthetic agent}

Total intravenous anaesthesia (TIVA) has been suggested as an alternative option to traditional inhalational agents, due to the physiologic decrease in cardiac output but without the peripheral vasodilation associated with inhalational anaesthesia $(\mathrm{IA})^{(751)}$. This potentially produces a better visualisation of the surgical field but there were significant limitations in all the studies included in this systematic review, leading to the inevitable conclusion that better high-quality studies are required ${ }^{(752)}$ (Table 6.2.4.2.). A randomized controlled trial performed by Brunner et al. ${ }^{(753)}$ compared the effect of TIVA versus IA on intraoperative visualization of patients undergoing ESS in high-grade inflammatory sinus disease defined as either sinonasal polyposis or a preoperative Lund-Mackay score of $>12$. Visibility was measured using the 10-point Wormald Surgical Field Grading Scale. Operative blood loss, complications, and change in quality of life evaluated by SNOT-22 were also measured. TIVA appeared to significantly improve endoscopic visualization and total blood loss during ESS but no difference in operative time, total time of hospital stay, or postoperative antiemetic requirement. No complications or serious adverse events were reported in either study arm.

Little et al. ${ }^{(754)}$ conducted an RCT on 30 fit patients undergoing bilateral ESS for CRS randomised to maintenance anaesthesia with i.v. propofol or inhaled desflurane and showed that TIVA was associated with a statistically significant reduction in mean Wormald score of the surgical field compared to desflurane (4.21 vs. 5.53, $\mathrm{p}=0.024)$. Mean Boezaart score was also lower in the TIVA arm ( 2.18 vs. $2.76, p=0.034$ ). Secondary outcomes including surgical duration, time to extubation, and estimated blood loss were not found to be statistically significant between the two groups.

These two studies ${ }^{(753,754)}$ confirm the earlier findings of Wormald et al. who compared the effects of TIVA using propofol and remifentanil with IA agents, sevoflurane and fentanyl on blood pressure, heart rate and surgical field (Boezaart scale). TIVA improved the surgical field and there was a reduction in both blood pressure and heart rate ${ }^{(726)}$.

The majority of studies included in a subsequent review of the literature on TIVA versus IA also showed improvement in the surgical field and reduced blood loss ${ }^{(755)}$. Another more recent meta-analysis done by Lu et al. ${ }^{(756)}$ analysed 157 articles and identified 15 RCTs (828 ESS cases) that satisfied their selection 
Table 6.2.4.2. Studies describing anaesthetic techniques in endoscopic sinus surgery.

\begin{tabular}{|c|c|c|c|c|}
\hline Study & Comparison & Outcome measure & $\mathbf{n}$ & Findings \\
\hline Beule $2007^{(757)}$ & $\begin{array}{l}S / F \vee P / F \\
L M>12\end{array}$ & $\begin{array}{l}\text { HR, blood loss, blood loss/min, } \\
\text { platelet function, surgical field } \\
\text { (VAS) }\end{array}$ & 52 & $\begin{array}{l}\text { HR:NSD } \\
\text { Blood loss: NSD } \\
\text { Blood loss/min: NSD } \\
\text { Surgical field: NSD } \\
\text { Platelet function: reduced both gps, worse P/F gp }\end{array}$ \\
\hline Eberhart 2003 ${ }^{(764)}$ & TIVA (P/R) V I/A & $\begin{array}{l}\text { BP, HR, surgical field (VAS, } \\
\text { Boezaart), dryness (VAS), blood } \\
\text { loss }\end{array}$ & 90 & $\begin{array}{l}\text { BP:NSD } \\
\text { TIVA: HR lower } \\
\text { Surgical field improved } \\
\text { Blood loss: NSD }\end{array}$ \\
\hline Nekhendzy $2007^{(758)}$ & $\begin{array}{l}\text { Hyper v } \\
\text { hypo v normocapnia }\end{array}$ & $\begin{array}{l}\text { Blood loss } \\
\text { Surgical field }\end{array}$ & 180 & $\begin{array}{l}\text { Blood loss: NSD } \\
\text { Surgical field: NSD } \\
\text { Hypocapnia gp: } \\
\text { Higher need for antihypertensives } \\
\text { Increased blood loss with increased CT score, duration of } \\
\text { surgery }\end{array}$ \\
\hline Tirelli 2004 ${ }^{(765)}$ & $\begin{array}{l}\text { TIVA (P/R) } \\
V I / F\end{array}$ & $B P$, HR, surgical field (Boezaart) & 64 & $\begin{array}{l}\text { Surgical field: better with TIVA } \\
\text { HR, BP: NSD (trend to be lower in TIVA gp) }\end{array}$ \\
\hline Wormald $2005^{(726)}$ & $\begin{array}{l}\text { TIVA (P/R) } \\
\vee S / F\end{array}$ & BP, HR, surgical field (Boezaart) & 56 & $\begin{array}{l}\text { TIVA: } \\
\text { Surgical field: better } \\
\text { Improvement correlated independently with lower BP and HR }\end{array}$ \\
\hline Ahn 2008 $8^{(881)}$ & $S / R \vee P / R$ & $\begin{array}{l}\mathrm{Hb} \text {, blood loss } \\
\text { Surgical field } \\
\text { L-M score }\end{array}$ & 40 & High L-M score P/R gave less blood loss, better surgical field \\
\hline Kaygusuz 2008(882) & $\mathrm{D} / \mathrm{R} \vee \mathrm{I} / \mathrm{R}$ & $\begin{array}{l}\text { BP, HR, } \\
\text { Blood loss } \\
\text { Surgical field } \\
\text { Time to extubation }\end{array}$ & 63 & $\begin{array}{l}\text { Blood loss NSD } \\
\text { Surgical field NSD } \\
\text { Time to extubation quicker with D/R }\end{array}$ \\
\hline Yoo 2010(883) & $\begin{array}{l}\text { TIVA (P/R) } \\
\vee S / R \vee D / R\end{array}$ & $\mathrm{BP}, \mathrm{HR}$, surgical field & 60 & $B P, H R$, surgical field NSD \\
\hline Brunner 2018 & $\begin{array}{l}\text { TIVA (P/R) } \\
\text { VIA (S/F) } \\
\text { LM }>12\end{array}$ & $\begin{array}{l}\text { Surgical field (Wormald) } \\
\text { Blood loss } \\
\text { Complications } \\
\text { SNOT } 22\end{array}$ & 72 & $\begin{array}{l}\text { TIVA: } \\
\text { Surgical field ((Wormald): } \\
\text { better } \\
\text { Blood loss:better } \\
\text { Complications and SNOT22: NSD }\end{array}$ \\
\hline Little $2018^{(754)}$ & TIVA (P/R) v IA (D) & $\begin{array}{l}\text { Surgical field (Wormald and } \\
\text { Boezaart) }\end{array}$ & 30 & $\begin{array}{l}\text { TIVA: } \\
\text { Surgical field (Wormald and Boezaart): better }\end{array}$ \\
\hline
\end{tabular}

Intravenous agents: F:fentanyl; P:propofol;R:remifentanil

Inhalational agents: D:Desflurane; l:isoflurane; S:sevoflurane, BP, blood pressure; CT, computed tomography; HR, heart rate; LM, Lund-Mackay; NSD, no significant difference; TIVA, total intravenous anaesthetic; VAS, visual analogue scale, Adapted from Timperley $2010^{(755)}$.

criteria. the pooled analysis demonstrated significantly superior surgical visibility scores for TIVA compared to IA based on 10 -point grading scores $(p=0.049$, visual analog scale; $p=0.009$, Wormald scale) and 5 -point grading scores ( $p=0.002$, Boezaart scale). Blood loss was significantly less following TIVA ( $p=0.003$ ) with no significant difference in heart rate, mean arterial pressure, duration of surgery and anaesthesia.

However, not all studies show superiority for TIVA over IA such as that by Beule et al. ${ }^{(757)}$ who showed no advantage in respect to heart rate, blood loss and surgical field. These authors also considered platelet function which was impaired in both groups but was worse in the propofol and fentanyl group.

Raised CO2 potentially has an adverse effect on bleeding in the surgical field as it is known to cause smooth muscle relaxation and vasodilation. However, Nekhendzy et al. ${ }^{(758)}$ could not show a difference in either the surgical field or amount of blood loss during ESS when patients with hyper-, hypo- or normocapnia were compared.

Controlled hypotension is an important tool for better visualisation of the surgical site ${ }^{(759)}$ and several additional methods such as beta blocker premedication or intraoperative infusions, either with nitroglycerin or beta blockers have been used to achieve controlled hypotension during ESS. Other agents such as a 2 agonists are also being used, either replacing or as adjuncts to classical TIVA agents (remifentanil and propofol) for their enhanced hypotensive effects ${ }^{(760,761)}$. A meta-analysis performed by Khosla et al. ${ }^{(762)}$ of 1148 patients in 2015 indicated that total intravenous anaesthesia (TIVA) was statistically more beneficial than conventional balanced anaesthesia, providing an average difference in blood loss of $75.3 \mathrm{~mL}$ and the use of preoperative steroids is statistically more beneficial than placebo, with an improved difference in blood loss of $28 \mathrm{~mL}$.

In 2016 Boonmark et al. ${ }^{(763)}$ conducted a Cochrane review of deliberate hypotension with propofol under anaesthesia for functional endoscopic sinus surgery (FESS). Only four studies with 278 participants fulfilled the inclusion criteria of an RCT which compared propofol with other hypotensive techniques during FESS with regard to blood loss and operative conditions in both adults and children ${ }^{(726,764-766)}$. The primary outcome was total blood loss (TBL). Other outcomes included surgical field quality, operation time, mortality within 24 hours, complications, and failure to reach target blood pressure. Deliberate hypotension with propofol did not decrease TBL (millilitres) 
when compared with inhalation anaesthetics in either children (one study; 70 participants; very low-quality evidence), or adults (one study; 88 participants; moderate-quality evidence). Propofol improved the surgical field by less than one category on a scale from 0 (no bleeding) to 5 (severe bleeding) (mean difference $-0.64,95 \% \mathrm{Cl}-0.91$ to -0.37 ; four studies; 277 participants; low-quality evidence), but there was no difference in operation time (three studies; 214 participants; low quality evidence). Failure to lower blood pressure to target was less common in the propofol group (risk ratio of failure with propofol $0.24,95 \% \mathrm{Cl} 0.09$ to 0.66 ; one study; 88 participants; moderatequality evidence). The authors concluded that using propofol to achieve deliberate hypotension probably improves the surgical field, but the effect is small and deliberate hypotension with propofol did not decrease TBL and the operation time. However, as most studies included few participants and provided lowquality evidence, results should be interpreted with caution and better powered RCTs are required.

Dexmedetomidine, one of these recent agents, is a selective a2 agonist, used in combination with general anaesthesia for its sedative, analgesic, and haemodynamic stability. Gupta et al. ${ }^{(767)}$ compared two groups of 25 patients. Patients from group $D$ received dexmedetomidine $1 \mu \mathrm{g} / \mathrm{kg}$ for $10 \mathrm{~min}$, followed by an infusion at $0.4-0.7 \mu \mathrm{g} / \mathrm{kg} / \mathrm{h}$ and patients in group $C$ received the same amount of saline. Surgeons experienced an ideal surgical site with minimum bleeding and only sporadic suction in 21 (84\%) patients in group D, compared to no patients in group $\mathrm{C}$ despite a low systemic blood pressure. Also group D patients needed less isoflurane to maintain the systolic blood. The authors concluded that dexmedetomidine is effective and safe in providing an 'dry' surgical field together with its many inherent advantages of analgesia, sedation, and anaesthetic-sparing effect. It was also associated with a longer but smoother recovery time from anaesthesia. However, Kim and colleagues ${ }^{(768)}$ did not find any significant improvement in surgical field with an infusion of dexmedetomidine in comparison with remifentanil though the extubation time was significantly shorter. Two earlier RCTs ${ }^{(769,770)}$ showed an infusion of dexmedetomidine of benefit when FESS was performed under local anaesthesia or conscious sedation. Qiao et al. ${ }^{(771)}$ also showed the drug is also effective in an intranasal atomised form at significantly decreasing blood loss by a median of $75 \mathrm{ml}$ compared to $100 \mathrm{ml}$ in the control group, thus optimising surgical field visualisation.

In contrast, Karabayirli et al. ${ }^{(772)}$ compared dexmedetomidine with remifentanil in two groups of 25 patients. No significant differences were found between the groups in intraoperative bleeding, surgical field, requirement of sevoflurane, rates of nausea, vomiting, or demands for additional analgesia. These authors concluded that for controlled hypotension during FESS, dexmedetomidine had no additional benefits compared to remifentanil and was associated with higher recovery time and first-hour postoperative sedation scores.

Clonidine, another a2 agonist, may also be considered. A randomised comparison of a clonidine-based vs remifentanilbased hypotensive anaesthetic regimen was conducted during ESS in patients with CRSwNP and CRSsNP $(n=47)^{(773)}$. Assessment of video recordings were blindly assessed by a third surgeon not involved in patient care. A significantly lower proportion of patients in the clonidine arm had Boezaart scores higher than 2, with significantly lower mean Boezaart scores both at 60 and 120 minutes.

A systematic review of the use of a2-adrenergic agonists in 13 RCTs ( $n=896$ patients) assessed the efficacy of clonidine, dexmedetomidine, or both ${ }^{(774)}$. The RCTs consistently showed that a2-adrenergic agonists reduced bleeding and improved surgical field quality during ESS. However, adverse event reporting was often omitted.

In another RCT, a single preoperative single dose of bisoprolol fumarate $2.5 \mathrm{mg}$, (a cardioselective $\beta 1$-adrenergic blocking agent used to treat high blood pressure) has also been shown to significantly reduce the blood loss during surgery and improved the visualization of the operating field during FESS $(n=50)^{(775)}$. Esmolol infusion, another $\beta 1$-adrenergic blocking agent, has also been shown to improve surgical fields during sevoflurane anaesthesia for FESS ${ }^{(761)}$.

Another method for achieving controlled hypotension is magnesium sulphate infusions. An RCT comparing magnesium sulphate, diltiazem or saline each given as a bolus $(n=45)$ showed significant reduction in blood loss in both active arms ${ }^{(776)}$. However, when magnesium sulphate was compared with remifentanil in an RCT of 104 patients undergoing ESS, remifentanil was superior in reducing operative bleeding, better visibility, shorter operative time, and earlier recovery with minimum postoperative sedation, allowing ESS to be performed as a day case $\mathrm{e}^{\text {(777). }}$.

Although controlled hypotension is an important tool for creating an optimal surgical field, its systemic effects should be closely monitored and adjusted accordingly throughout the operation. It should also be acknowledged that intraoperative hypotension can be a major risk factor for several complications due to end organ injury such as perioperative stroke, cognitive dysfunction and renal failure. Thus, its level should be set according to the risks stratified for each individual patient. The POISE study ${ }^{(778)}$ revealed the impact of perioperative haemodynamics on postoperative events. A higher incidence of postoperative stroke in patients on $\beta$-blockers was emphasized in this study, potentially due to the hypotension they induce. Bijker et al. ${ }^{(779)}$ considered the relationship of intraoperative hypotension to postoperative stoke and found that a decrease in mean arterial blood pressure of more than $30 \%$ from baseline and the cumulative duration of these episodes were associated with an increased risk of postoperative stoke. In another study, Futier et al. ${ }^{(780)}$ showed that intraoperative blood pressure management targeting an individualized systolic blood pressure, according to patients' resting blood pressure, decreased postoperative organ dysfunctions and neurological complications.

Di Mauro et al. ${ }^{(781)}$ considered the role of intraoperative stroke volume variation on bleeding during FESS in 55 patients, ASA I to III. Intraoperative bleeding was assessed using the Boezaart score and from a wide range of haemodynamic parameters, only a negative correlation between surgical bleeding and stroke 
Figure 6.2.4.4. Forest plot of the effect of mitomycin versus control on adhesion formation after sinus surgery.

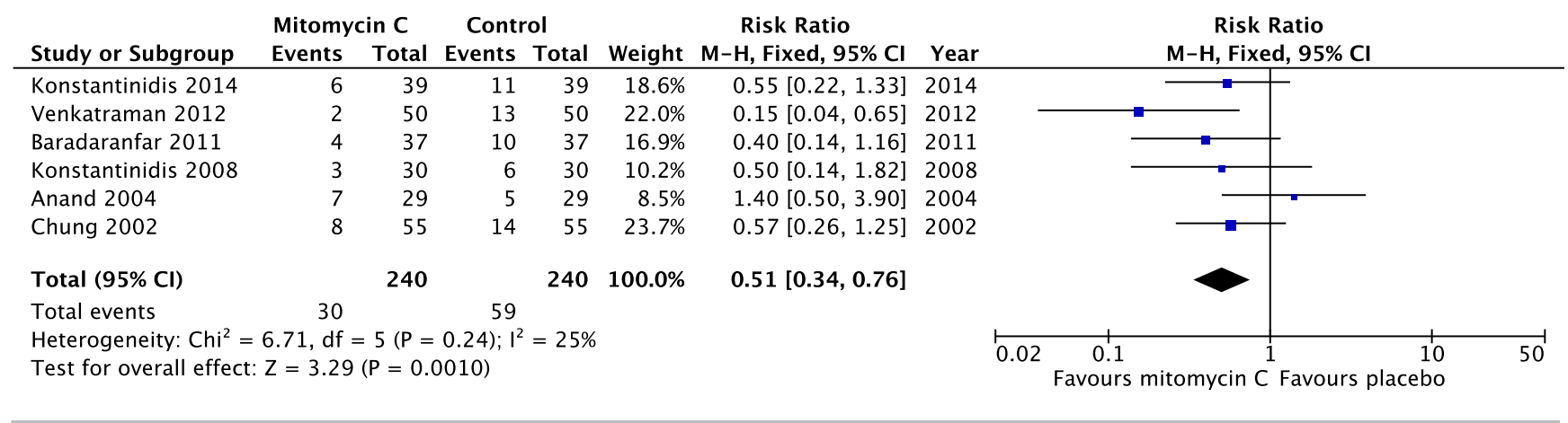

volume variation (SVV) was found. Furthermore, a cut-off of $12.5 \%$ in SVV is optimal to distinguish the group with the better surgical visibility from the group with the worst one.

Overall, patient preoperative status, the specific surgical needs and the vulnerability of end organs should guide perioperative blood pressure management. Individualised planning of the perioperative haemodynamic target, based on close cooperation between surgeon and anaesthetist is crucial, combined with accurate and continuous physiological monitoring.

Finally, there are some other aspects, such as the mode of anaesthesia which can impact on surgical field. Gilbey et al. ${ }^{(782)}$ considered a small cohort of 22 patients undergoing FESS who were randomly assigned to be ventilated during surgery under general anaesthesia by either high frequency jet ventilation (HFJV) or intermittent positive pressure ventilation (IPPV). When the quality of the surgical field was assessed (Boezaart score) and the total blood loss was measured, HFJV significantly reduced the amount of intraoperative bleeding and thus significantly improved the quality of the surgical field possibly due to increased venous return due to lower intrathoracic pressures resulted in less bleeding and improved operating conditions. The use of pharyngeal packs during FESS have not been shown to increase postoperative pain, nausea or vomiting $^{(783)}$ as compared to no packing whereas one RCT in 47 patients showed superiority for packing the nasopharynx rather than hypopharynx ${ }^{(784)}$ but the need for packs of any sort has largely been superseded by the use of the laryngeal mask ${ }^{(785)}$.

\subsubsection{Desmopressin}

The benefits of intranasal desmopressin in achieving the same benefits as steroids during ESS, have been demonstrated in a

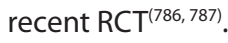

\subsubsection{Tranexmic acid}

Perioperative tranexamic acid has been evaluated in seven placebo controlled RCTS, including 562 participants, reviewed in a 2019 systematic review ${ }^{(788)}$. Operative time (standardized mean difference $(S M D)=-0.60)$ and intraoperative blood loss $(\mathrm{SMD}=-0.66)$ were statistically lower in the tranexamic group than placebo group. This achieved over $100 \mathrm{ml}$ reduction in blood loss in the active treatment groups. The quality of the surgical field and surgeon satisfaction were statistically higher in the treatment group than the placebo group. By contrast, there were no significant differences in the hemodynamic and coagulation profiles of the two groups, and tranexamic acid had no significant effect on thrombotic events compared to placebo. The relatively small number of patients included in RCTs may miss important differences in rare adverse events. A large population-based cohort study, including nearly 45,000 patients undergoing hip arthroplasty, which has a high risk of thromboembolic events, found no difference in the risk of thrombo-embolic or cardiovascular events or death ${ }^{(789)}$. Although there are no similar studies specifically in sinus surgery, the absence of increased risk should not differ from that found for arthroplasty.

In a study looking at both intravenous tranexamic acid (TXA) and epsilon aminocaproic acid (EACA) on bleeding and surgical field quality during FESS similar improvements in duration of surgery and volume of blood loss were observed in the TXA and EACA groups compared to control. The postoperative haemoglobin was significantly lower in the control group compared to TXA group and EACA group and both TXA and EACA groups had comparable improved quality of the surgical field with most of patients classified as grade 1 and 2 according to Boezaart scale while the control group had most of the patients in grade $3^{(790)}$.

\subsubsection{Mitomycin}

Mitomycin $\mathrm{C}$ is an antibiotic isolated from the broth of Streptomyces caespitosus, which acts as an alkylating agent able to inhibit DNA synthesis. It has also been shown to inhibit fibroblast proliferation and activity, which can reduce scar formation. Topical MMC has been used at diverse places in the otorhinolaryngological surgery like ear, larynx and in endoscopic sinus surgery. A significant number of randomized controlled trials have been performed to evaluate the effect of mitomycin c on adhesions formation during sinus surgery ${ }^{(791-799)}$. Most studies used 0.4 or $0.5 \mathrm{mg} / \mathrm{ml}$ mitomycin on a carrier like cotton pledgets, neuropatties, or otowick placed in the relevant sinus for 4-5 minutes. Patients were evaluated between one week and mean 15 months. Six studies could be included in a meta-analysis: there was a significant positive effect of mitomycin versus control on adhesion formation after sinus surgery RR $0.51 \mathrm{Cl} 0.3400 .76$ ), 480 patients six studies, $\mathrm{p}=0.001$ ) (Figure 6.2.4.4.). However, in some studies the difference becomes smaller over time ${ }^{(798)}$. 
Two studies evaluated whether mitomycin could prevent stenosis $^{(793,795)}$. Both concluded that mitocycin was not able to improve the relative size over an extended period of time. No adverse events were observed.

In conclusion: The application of mitomycin for 4-5 minutes at the end of surgery prevents adhesions compared to control but is not able to improve the relative size of ostia over time. No adverse events were observed. Based on the quality of the evidence the EPOS2020 steering groups advises that mitomycin can be used to prevent adhesion formation after sinus surgery.

\subsubsection{Conclusion}

There is Level 1 evidence that preoperative injections of local anaesthetic and vasoconstrictor into the greater palatine canal reduces intraoperative bleeding in ESS and a concentration of $1: 80,000$ adrenaline is the most effective. When used topically, 1:2000 adrenaline showed haemostatic superiority over lower concentrations.

The evidence is less clear as to whether cocaine, lignocaine + oxymetazoline or tetracaine + oxymetazoline is superior for local anaesthesia and vasoconstriction.There is level I evidence that simple strategies like patient positioning in the reverse-Trendelenberg position and more complex controlled hypotension on an individualised basis can be successful in reducing intraoperative bleeding. Use of propofol to achieve deliberate hypotension probably improves the surgical field, but the effect is small whereas there is level 1 evidence that a2adrenergic agonists reduce bleeding and improve surgical field quality during ESS. However, overall the literature shows TIVA is superior to IA in improving the surgical field and reduced blood loss.

The EPOS steering group advises based on the evidence above to use TIVA, and to put the patient in a reverse Trendelenberg position during endoscopic sinus surgery. The quality of the evidence does not allow for an advice on the best local anaesthesia and vasoconstriction.

\subsubsection{Informed consent/patient information in CRS}

\subsubsection{Informed consent in clinical practice}

Informed consent is "a process for getting permission before conducting a healthcare intervention on a person (or for disclosing personal information about them)".

For consent to be valid, it must be voluntary and informed, and the person consenting must have the capacity to make the decision. This means that consent must not be influenced by pressure from others and the individual must understand the information given to them. They must receive information regarding the benefits and risks of the treatment, whether there are alternative treatments and what will happen if treatment does not go ahead.

The principle of consent is an important part of medical ethics and international human rights law with significant medicolegal implications. This is increasingly pertinent with the expanding field of genomics ${ }^{(800)}$. Lynn-Macrae et al. ${ }^{(801)}$ found informed consent issues in $37 \%$ of 41 medicolegal cases involving ESS and this issue remained at $27 \%$ in the following 10 years $^{(802)}$. The advent of precision or personalised medicine has emphasised the patient-physician interaction, which has proved particularly pertinent to chronic rhinosinusitis ${ }^{(803)}$. In the UK, since 2015 there has been a statutory Duty of Candour on all health professionals to be "open and honest with patients and their families when something goes wrong that appears to have caused or could lead to significant harm in the future" even if it has not yet done $\mathrm{so}^{(804)}$. This has also had a major impact on informed consent. Informed consent applies to all treatments, both medical and surgical but surgery features more prominently in the literature where the balance of benefit versus risk may be more immediately evident ${ }^{(805)}$.

The level of detail given for any particular procedure varies considerably from country to country and institution to institution ${ }^{(806,807)}$. Traditionally, the "prudent patient standard" in which the physician disclosed all risks that it was deemed a reasonably prudent patient would consider material to their decision to undergo or refuse a particular procedure" has often been adopted ${ }^{(807,808)}$. When discussing endoscopic surgery for an essentially non-fatal condition such as CRS, complications range from mild to catastrophic which has previously made the consent process difficult. An American survey nearly 20 years ago found that $60 \%$ of responding surgeons would discuss with patients pre-operatively risks likely to occur in $1 \%$ or greater of cases $^{(806)}$. However, specifically the majority mentioned CSF leaks (99\%), bleeding (97\%), orbital injury (97\%) and infection (85\%) whereas $40 \%$ discussed smell impairment, cerebrovascular accident (18\%), myocardial infarct (8\%) and $28 \%$ death. In the last decade a more 'patient-centred' approach has evolved as studies have shown that the majority of patients undergoing ENT procedures expect to be told about all complications ${ }^{(808,809)}$ (see section 6.2.1.20.). Interestingly when Wolf et al. repeated their survey in patients, $69 \%$ wanted to be informed about any risk occurring at a frequency of $1 \%$ or greater but for the specific complications, $83 \%$ wanted to know about orbital damage and CSF leaks, 76\% revision surgery, $74 \%$ smell impairment, $73 \%$ bleeding and myocardial infarct and $72 \%$ cerebrovascular accident, highlighting a discrepancy in priorities ${ }^{(807)}$.

In the UK, the case of Montgomery vs. Lanarkshire Health Board $2015^{(810)}$ established that patients must have all information available pre-operatively, not what the doctor thinks they should know. In some respects, this legal imperative to explain all complications makes the situation easier, however distressing it may be to the patient. It is also incumbent on the surgeon to discuss their own results, both outcomes and complications in the context of their peers.

Studies have shown that age and educational status affect consent, with higher education and younger age improving understanding and retention of information ${ }^{(809,811-814)}$. Similarly, these factors are also associated with an increased wish to know about complications associated with the lowest incidence, irrespective of their severity ${ }^{(812)}$. 
Whatever the process of informed consent, after a relatively short time, many patients have poor recall of the information given, which is important when there can be a significant interval between consent and an elective procedure ${ }^{(808,809,815)}$. Aremu et al. ${ }^{(816)}$ showed that only $51 \%$ recalled complications of common ENT procedures, including for CRS, after a mean of 21 days. This was significantly improved to $62 \%$ if a handout was also given. The length of time spent on surgical consent is also an important predictor of comprehension and is maximised when it takes between 15 and 30 minutes, albeit in non-ENT procedures $^{(817)}$.

Those physicians perceived by their patients as concerned, accessible and wiling to communicate, at least in obstetrics, are least likely to be sued ${ }^{(818)}$. In the USA and elsewhere many physicians will bring the patient in for a visit shortly before surgery and obtain consent to improve recall of risks. Others obtain consent on the day of surgery though in some countries such as the UK, doctors are encouraged or mandated to obtain consent more than 24 hours in advance of surgery and definitely before a premedication has been given on the day of surgery. A variable proportion of patients (8-66\%), will also seek further information elsewhere which is now greatly facilitated by the internet ${ }^{(808,809)}$. While sometimes contributing misinformation, some issues around consent might be improved by the use of modern technology. A significant early improvement in overall risk recall was demonstrated in patients who completed a multimedia consent educational module compared to a control group although this difference was not found 3-4 weeks later ${ }^{(819)}$. Whatever the environment, the consent process is greatly strengthened by careful documentation and individual checklist forms are recommended by many authors ${ }^{(820)}$. Bagnall and colleagues $^{(821)}$ conducted a systematic review of the literature on taking consent combined with semi-structured interviews to identify key components of the surgical consent procedure. Thirty-three components were identified, the most frequently cited of which were explaining the diagnosis and procedure, general and specific risks, assessing the patient's mental capacity to give consent and offering alternative options. Least common was disclosure of outcomes for the same procedure by the individual surgeon and others. The need for better training in this area was highlighted. The EPOS panel members provided comment on informed consent in their respective countries. The following had similar systems and legislation to the UK in place : Australia, Canada, China, New Zealand, Singapore and USA. In Sweden and Denmark written consent is not required prior to a surgical procedure. This is also the case in the Netherlands where the Dutch Medical Treatment Agreement Act (WGBO) regulates the rights of the patient. This law states that patients are entitled to information and that they must give permission for treatment. The WGBO also regulates the privacy of the patient, the right to a second opinion, the right of patients to view their own medical records and the representation of patients if they cannot decide for themselves. In addition, the WGBO requires healthcare providers to keep a medical record but the law does not dictate exactly what must be discussed nor are percentages of complications given. Similarly, in Greece and Denmark, there is no uniform nationwide documentation and only complications occurring more often than $1 \%$ are usually discussed. As in the UK, consent must be given at least 24 hours in advance of the surgery. In several countries, for example Brazil and Spain, the national ENT society, often with legal input, provides templates for consent to its members. These usually include explanations of the operation (indications, technique, consequences of surgery, potential complications and postoperative care). The form is signed by both patient and surgeon. Many countries have now adopted the WHO checklist [www. WHO.int] which requires that informed consent has been given and signed by both parties, without which the operation cannot proceed. In Spain having a signed informed consent is not always deemed sufficient and in some trial cases, evidence of physical interaction between patient and doctor has been deemed necessary. As a consequence, doctors are strongly encouraged to make handwritten comments on the discussion that took place.

Most consent forms include sections for minors/parents/ guardians and those with language problems. In the case of children, some countries such as Greece require the signature of both parents. For linguistic issues, the name and signature of the translator is also required to signify that the patient has understood the procedure.

Several respondents commented that since the European Union's GDPR (General Data Protection Regulations) were implemented in May 2018, these had led to increased complexity of the consent documentation, particularly with respect to any research implications ${ }^{(822,823)}$.

\subsubsection{Informed consent in research}

Following the declaration of Helsinki in $1975^{(824)}$, the next major milestone in protecting patients who volunteer for medical research was the introduction of the International Clinical Harmonisation of Good Clinical Practice guidelines in $1996^{(825)}$. With regards to the consent process within this framework, the guidelines stipulate the following parameters:

"In any research on human beings, each potential subject must be adequately informed of the aims, methods, anticipated benefits and potential hazards of the study and the discomfort it may entail. He or she should be informed that he or she is at liberty to abstain from participation in the study and that he or she is free to withdraw his or her consent to participation at any time. The physician should then obtain the subject's freely-given informed consent, preferably in writing".

"When obtaining informed consent for the research project, the physician should be particularly cautious if the subject is in a dependent relationship to him or her or may consent under duress. In that case the informed consent should be obtained by a physician who is not engaged in the investigation and who is completely independent of this official relationship".

\section{"In case of legal incompetence, informed consent should be}


obtained from the legal guardian in accordance with national legislation. Where physical or mental incapacity makes it impossible to obtain informed consent, or when the subject is a minor, permission from the responsible relative replaces that of the subject in accordance with national legislation. Whenever the minor child is in fact able to give consent, the minor's consent must be obtained in addition to the consent of the minor's legal guardian".

In some institutions in the USA child consent is referred to as "assent" and is usually obtained from children above seven years old.

In both future diagnosis and research, especially in the fields of genetics and genomics, where big data can be shared or published, data protection is mandatory and has been the subject of a number of publications ${ }^{(826-830)}$

\section{Excerpt from ICH E6 Guideline for Good Clinical Practice: Informed Consent Explanation}

Both the informed consent discussion and the written informed consent form and any other written information to be provided to subjects should include explanations of the following:

a) That the trial involves research.

b) The purpose of the trial.

c) The trial treatment(s) and the probability for random assignment to each treatment.

d) The trial procedures to be followed, including all invasive procedures.

e) The subject's responsibilities.

f) Those aspects of the trial that are experimental.

g) The reasonably foreseeable risks or inconveniences to the subject and, when applicable, to an embryo, fetus, or nursing infant.

h) The reasonably expected benefits. When there is no intended clinical benefit to the subject, the subject should be made aware of this.

i) The alternative procedure(s) or course(s) of treatment that may be available to the subject, and their important potential benefits and risks.

j) The compensation and/or treatment available to the subject in the event of trial-related injury.

k) The anticipated prorated payment*, if any, to the subject for participating in the trial.

I) The anticipated expenses, if any, to the subject for participating in the trial.

m) That the subject's participation in the trial is voluntary and that the subject may refuse to participate or withdraw from the trial, at any time, without penalty or loss of benefits to which the subject is otherwise entitled.

n) That the monitor(s), the auditor(s), the IRB/IEC**, and the regulatory authority(ies) will be granted direct access to the subject's original medical records for verification of clinical trial procedures and/or data, without violating the confidentiality of the subject, to the extent permitted by the applicable laws and regulations and that, by signing a written informed consent form, the subject or the subject's legally acceptable representative is authorising such access. o) That records identifying the subject will be kept confidential and, to the extent permitted by the applicable laws and/or regulations, will not be made publicly available. If the results of the trial are published, the subject's identity will remain confidential.

p) That the subject or the subject's legally acceptable representative will be informed in a timely manner if information becomes available that may be relevant to the subject's willingness to continue participation in the trial.

q) The person(s) to contact for further information regarding the trial and the rights of trial subjects, and whom to contact in the event of trial-related injury.

r) The foreseeable circumstances and/or reasons under which the subject's participation in the trial may be terminated.

s) The expected duration of the subject's participation in the trial.

t) The approximate number of subjects involved in the trial. * NB. Some countries e.g. Spain, do not allow payments to participants in clinical studies.

* Similar documents are produced in other countries and refer to the relevant regulatory authorities such as the FDA (Federal Drugs Administration in the USA.

\subsubsection{Training in surgery}

As in other surgical disciplines, training for FESS involves personal studies using a variety of materials from textbooks to online resources (anatomy, pathophysiology etc.), lectures, clinical rounds, clinical fellowships and physical models ${ }^{(831)}$. The use of audiovisual techniques during FESS allows trainees to directly observe their teachers on screens that compensates for the impracticability of assisting as occurred in open surgeries ${ }^{(832)}$. In addition, teachers can supervise every surgical step of their trainees on screen and give focused commands and instructions. Sandhaus et al. proposed a standardized terminology of commands to facilitate the trainees' understanding of what they are supposed to do so as to clearly execute single surgical steps ${ }^{(833)}$.

An additional benefit to audio-visually enhanced training is the use of intraoperative navigation to integrate the knowledge of endoscopic and CT anatomy. This is particularly important in CRSWNP where the sinonasal anatomy may be distorted by the pathology as well as previous surgery. It was shown that the sense of direction and orientation during surgery was enhanced, the latter being a critical factor in training ${ }^{(834)}$. However, especially in inexperienced trainees, over-confidence and dependence on the system and risk overestimation must be considered $^{(835)}$.

Functional endoscopic sinus surgery techniques can furthermore be practiced through cadaveric dissections ${ }^{(836)}$. An understanding of the endoscopic view of sinonasal anatomy is essential and, in particular fresh frozen, cadavers offer an optimal simulation of real surgery though polyp tissue itself cannot be simulated in cadavers. However, the handling of powered instruments and potential complications can be 
practised in cadavers, and the dissections can be evaluated in a variety of ways (e.g. pre- and post-dissection CTs) to improve training outcomes ${ }^{(837)}$. The shortcomings of cadaveric dissections are the costs and limited availability in certain centres and countries. Despite this a large number of dissection courses are held around the globe.

A more accessible and cheaper alternative to human cadaveric dissection are animal models like sheep or lamb heads, synthetic human models and simulator training dissection ${ }^{(838)}$. Despite the fact that sheep sinus anatomy differs from human anatomy, its similarities are appropriate to train surgical skills, handling instruments, visualisation and orientation with the endoscope and dissection steps can be practiced especially when standardized training steps are applied ${ }^{(839-841)}$.

Apart from cadaveric dissection, synthetic models of human heads and the sinonasal tract like the S.I.M.O.N.T. (Sinus Model Otorhino Neuro Trainer) have been introduced. Here silicone/ plastic anatomic models based on CT anatomy have been created to train endoscopic sinus surgery ${ }^{(842)}$. The advantage here lies in the availability and reproducibility as well as the assessment of different tasks accomplished by the trainees ${ }^{(843)}$. A disadvantage of these models is that the haptic feedback of tissue composition such as mucosa cannot be simulated. However, these models can be combined with animal specimens to simulate surgical complications such as carotid artery injury and their management. The carotid arteries of anaesthetised sheep dissected and attached to the S.IM.O.N.T. model can be injured to create live bleeding scenarios ${ }^{(619)}$. As with cadavers, the costs of production of these models need to be considered. To overcome these shortcomings 3D printing could be a future alternative where the sinonasal system is printed according to $\mathrm{CT}$ anatomy and various materials are utilised to simulate the difference between e.g. bone and mucosa ${ }^{(844)}$ offering cheap and reproducible training prior to a specific surgical case $\mathrm{e}^{(844,845)}$.

Virtual Reality (VR) simulators are another tool to teach and learn endoscopic sinus surgery and do not rely on cadavers and models allowing almost unlimited access. The first simulators appeared in the late 1990s and were combined with head models to simulate working through the nose and could be combined with mechanical arms to the endoscope and instruments for haptic feedback. The surgical steps could be followed in a VR programmed environment on the monitor ${ }^{1846,}$ ${ }^{847)}$. Advances in computer technology have led to more detailed graphical 3D anatomy representation and implementation of various training programs ${ }^{(831,848)}$. Also, it is possible to simulate powered instrumentation like shavers, irrigation and other tools that can be activated by foot pedals similar to real life surgery ${ }^{(849)}$. Examples of sinonasal VR simulators are Madigan Endoscopic SinusSurgery Simulator: ES3, Nasal Endoscopy Simulator: NES, Voxel-Man SinuSurgery and the Dextroscope ${ }^{(850,}$ ${ }^{851)}$. Validity of these simulators was studied by comparing the surgical performance of two groups after simulator training vs conventional material training ${ }^{(852)}$ or sinonasal anatomic knowledge after training with VR simulator versus textbooks ${ }^{(853)}$.
Despite further technological advances, VR simulators still need more improvement to approach real-life or cadaver conditions, but are considered as a useful additional tool in surgical training ${ }^{(849)}$. Training of spatial orientation and mechanical manipulation of instruments and endoscopes are considered more important than haptic feedback in simulators ${ }^{(834)}$. To date the quality of evidence in validation studies is still low. Moreover, no studies focus on outcomes after surgery correlated to previous VR training ${ }^{(854,855)}$.

\subsubsection{Peri-operative medication}

\subsubsection{Introduction}

Medication can be given before, during or after the surgery. An overview of medication for CRS in general is given in chapter 6.1. Medication given before surgery to enhance the surgical field are discussed in 6.2.1.2., peri-operative medication and measures to enhance the surgical field during surgery are discussed in 6.2.4. This chapter discusses the effect of perioperative treatment on the period directly after surgery.

\subsubsection{Antibiotics}

\subsection{Summary of data}

There have been four randomized clinical trials investigating short-term postoperative antibiotics following ESS, including three placebo-controlled studies (Table 6.2.7.1). Short-term antibiotics was defined as less than 30 days of treatment. To date, there have been no studies of short-term preoperative antibiotics. None of the studies ${ }^{(539,856-858)}$ showed differences between antibiotics versus placebo/control apart from a shortterm reduction in symptoms of nasal obstruction and nasal discharge on day 5 postoperatively and significant lower POSE endoscopy scores on day 5 and 12 in the study of Albu ${ }^{(857)}$. After stopping the antibiotics no differences were seen. It was not possible to include the trials in a meta-analysis.

Jiang ${ }^{(539)}$ compared amoxicillin/clavulanic acid to no treatment following ESS and showed that the bacterial culture rate became higher after FESS in the antibiotic group but that the rate of antibiotic sensitivity to amoxicillin/ clavulanic acid did not change. No changes were seen in the control group. One study from Huvenne et al. evaluated the effect of placement of a doxycycline-releasing stent in the ethmoid in 10 postoperative CRS patients and showed that the doxycyclinereleasing stents significantly improved postoperative healing quality after functional endoscopic sinus surgery and lowered MMP-9 concentrations and bacterial colonization locally ${ }^{(469)}$.

\subsection{Conclusions}

The use of postoperative antibiotics in four (placebo) controlled trials in 371 patients did not show significant difference in objective or subjective clinical outcomes compared to controls but these studies may have been underpowered to detect a benefit. Based on the low quality of the current evidence the EPOS2020 steering group is not able to advise on the use of postoperative short-term antibiotics after endoscopic sinus surgery. There is a need for large-scale RCTs to answer this for daily practice very relevant question. Studies are also needed to evaluate the effect of short-term preoperative antibiotics. 
Table 6.2.7.1. Short-term postoperative antibiotics in CRS.

\begin{tabular}{|c|c|c|c|c|c|}
\hline Study & Methods & Participants & Interventions & Outcomes & Results \\
\hline $\begin{array}{l}\text { Albu } \\
2010^{(857)}\end{array}$ & DBPCT & $\begin{array}{l}75 \text { CRS } \\
\text { patients ( } 40 \\
\text { CRSwNP) }\end{array}$ & $\begin{array}{l}\text { - Amoxicillin + clavulanate } 625 \\
\text { mg twice daily for } 14 \text { days } \\
(n=40) \\
\text { - Placebo twice daily for } 14 \\
\text { days }(n=35)\end{array}$ & $\begin{array}{l}\text { - Symptom questionnaire day } \\
5,12,21 \text { and } 30 \\
\text { - Perioperative sinus endosco- } \\
\text { py (POSE) score at day 5, 12, } \\
21 \text { and } 30 \\
\text { - Endoscopic examination at } \\
\text { day } 5 \text { until all blood crusts } \\
\text { resolved }\end{array}$ & $\begin{array}{l}\text { Amoxicillin + clavulanate versus placebo } \\
\text { resulted in: } \\
\text { - Significant lower scores for nasal } \\
\text { obstruction and nasal discharge on } \\
\text { postoperative day } 5 \\
\text { - Significant lower POSE scores on day } 5 \\
\text { and } 12 \\
\text { - No statistical difference between groups } \\
\text { in overall symptom scores or POSE } \\
\text { scores at } 21 \text { or } 30 \text { days } \\
\text { - Patients displaying blood crusts within } \\
12 \text { days post-surgery were lower in the } \\
\text { antibiotic treated group as compared to } \\
\text { the placebo group ( } \mathrm{p}=0.02 \text { ) }\end{array}$ \\
\hline $\begin{array}{l}\text { Schalek } \\
2009^{(856)}\end{array}$ & DBPCT & $\begin{array}{l}23 \text { CRS } \\
\text { patients }\end{array}$ & $\begin{array}{l}\text { - Amoxicillin + clavulanate, } \\
\text { quinolone or co-trimoxazole } \\
\text { for } 3 \text { weeks ( } n=13) \\
\text { - Placebo for } 3 \text { weeks }(n=10)\end{array}$ & $\begin{array}{l}\text { - SNOT-22 (Czech translation) } \\
\text { at } 3 \text { and } 6 \text { months } \\
\text { - Clinical symptom-specific } \\
\text { scores at } 3 \text { and } 6 \text { months } \\
\text { - Endoscopic score at } 3 \text { and } 6 \\
\text { months }\end{array}$ & $\begin{array}{l}\text { - No statistically significant difference in } \\
\text { SNOT-22 quality of life scores, average } \\
\text { symptom score, or endoscopic scores } \\
\text { compared to placebo at } 3 \text { and } 6 \text { months } \\
\text { - Mean endoscopic scores after } 3 \text { months } \\
\text { approached significance ( } \mathrm{p}=0.056 \text { ) } \\
\text { - There was no statistical difference with } \\
\text { regard to which particular antibiotic was } \\
\text { used }\end{array}$ \\
\hline $\begin{array}{l}\text { Jiang } \\
2008^{(539)}\end{array}$ & $\mathrm{RCT}$ & $\begin{array}{l}71 \mathrm{CRS} \\
\text { patients }\end{array}$ & $\begin{array}{l}\text { - Amoxicillin + clavulanate } 375 \\
\text { mg three times daily for } 3 \\
\text { weeks }(n=31) \\
\text { - No treatment }(n=40)\end{array}$ & $\begin{array}{l}\text { - Rhinosinusitis symptom } \\
\text { scores at week } 3 \\
\text { - Antibiotic sensitivity rate at } \\
\text { week } 3 \\
\text { - Culture rate at week } 3 \\
\text { - Endoscopic scores at week } 3\end{array}$ & $\begin{array}{l}\text { - No significant difference in the short- } \\
\text { term subjective or objective outcomes } \\
\text { of CRS } 3 \text { weeks after endoscopic sinus } \\
\text { surgery } \\
\text { - Bacterial culture rates increased in the } \\
\text { study group after FESS ( } 38.7 \% \text { vs. } 61.3 \% \text {, } \\
p=0.014) \text { but no significant difference } \\
\text { in antibiotic sensitivity to amoxicillin/ } \\
\text { clavulanate }\end{array}$ \\
\hline $\begin{array}{l}\text { Annys } \\
2000^{(858)}\end{array}$ & DBPCT & $\begin{array}{l}202 \text { CRS } \\
\text { patients }\end{array}$ & $\begin{array}{l}\text { - Cefuroxime axetil } 250 \text { mg } \\
\text { twice daily }(n=101) \\
\text { - Placebo twice daily } \\
\text { all patients received } \\
\text { nasal saline and nasal } \\
\text { corticosteroids }(n=101)\end{array}$ & $\begin{array}{l}\text { - Symptoms } \\
\text { - Nasal endoscopy }\end{array}$ & $\begin{array}{l}\text { - No significant differences between the } \\
\text { groups }\end{array}$ \\
\hline
\end{tabular}

DBPCT, Double Blind Placebo Controlled Trial; CRS,Chronic Rhinosinusitis; CRSwNP, Chronic Rhinosinusitis with nasal polyps; RCT, Randomised Controlled Trial; SNOT-22, Sino-Nasal Outcome Test-22; POSE, Perioperative Sinus Endoscopy score;

\section{The use of peri-operative corticosteroids reduces blood loss and operation time and improves the quality of the surgical field.}

\subsubsection{Peri-operative corticosteroids}

Studies evaluating the effect of corticosteroids peri-operatively can be divided in studies evaluating the effect of the treatment during the surgery and studies evaluating the postoperative period. The studies evaluating the effect of corticosteroids during surgery are discussed in chapter 6.2.1.2.

\subsection{Summary of data of effect of peri-operative} corticosteroids on postoperative state of disease in CRS Next to the direct effect of peri-operative corticosteroids during surgery one might wonder whether it is useful to give (intranasal) corticosteroids after the surgery to prevent recurrence of disease/reduce symptoms after surgery.
Eleven papers on 10 studies evaluated the effect of corticosteroids starting peri-operatively on the signs and symptoms of disease in the weeks-years after the surgery (Table 6.2.7.2).

Three studies ${ }^{(76,77,83)}$ evaluated the effect of peri-operative corticosteroids on postoperative QOL using different outcome measurements. Two studies ${ }^{(76,77)}$ evaluated general quality of life five weeks after surgery and found no impact of postoperative FNDP on general quality of life although one study ${ }^{(76,77)}$ found an impact on the mental component summary and three domains of the SF-36.

Three studies evaluated the proportion of patients that was relapse free at nine months, although relapse was differently defined. When combining the three studies into a meta-analysis no significant impact was seen on the proportion of patients that was relapse free at nine months RR 1.33 ( $\mathrm{Cl} 0.59-2.98)$, three studies, 284 patients, $\mathrm{p}=0.49$ ) (Figure 6.2.7.1). 
Table 6.2.7.2. Effect of corticosteroid treatment versus placebo postoperatively.

\begin{tabular}{|c|c|c|c|c|c|}
\hline Study & Methods & Participants & Interventions & Outcomes & Results \\
\hline $\begin{array}{l}\text { Nordin } \\
2013^{(76)}\end{array}$ & DBPCRCT & $\begin{array}{l}60 \text { CRSwNP, } \\
\text { postoperative }\end{array}$ & 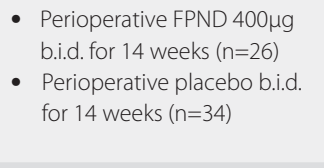 & $\begin{array}{l}\text { - General quality of life, using Gen- } \\
\text { eral Well Being Schedule (0-110, } 14 \\
\text { questions with a 6-point scale and } 4 \\
\text { questions with an 11-point scale) at } \\
\text { weeks } 4 \text { and } 5\end{array}$ & $\begin{array}{l}\text { At } 5 \text { weeks, both groups increased in general } \\
\text { quality of life score to a similar extent without } \\
\text { difference }\end{array}$ \\
\hline $\begin{array}{l}\text { Vento } \\
2012^{(105)}\end{array}$ & DBPCRCT & $\begin{array}{l}\text { CRSwNP, } \\
\text { postoperative }\end{array}$ & $\begin{array}{l}\text { - Postoperative triamcinolone } \\
\text { acetonide nasal aerosol } \\
220 \mu \mathrm{g} / \text { day as } 2 \text { actuations } \\
\text { of } 55 \mu \mathrm{g} \text {, each for } 9 \text { months } \\
(\mathrm{n}=30) \\
\text { - Postoperative placebo spray } \\
\text { for } 9 \text { months ( } n=30)\end{array}$ & $\begin{array}{l}\text { - Symptom score (0-3, } 10 \text { symptoms) } \\
\text { improvement at month 3,6,9 } \\
\text { - Nasal polyp score (0-3) reduction at } \\
\text { - } \text { month } 3,6,9 \\
\text { - Polyp recurrence at } 9 \text { months } \\
\text { - Adverse events }\end{array}$ & $\begin{array}{l}\text { Postoperative triamcinolone versus placebo } \\
\text { resulted in: } \\
\text { - No difference in symptom improvement } \\
\text { - No difference in nasal polyp score reduction } \\
\text { - Reduction in polyp size/regrowth in N-ERD } \\
\text { patients at } 3 \text { months, but not } 6 \text { and } 9 \text { months } \\
\text { - } \quad \text { No serid-intolerant patients } \\
\text { - Nous adverse events reported. }\end{array}$ \\
\hline $\begin{array}{l}\text { Rotenberg } \\
2011^{(83)}\end{array}$ & DBPCRCT & $\begin{array}{l}64 \text { CRSwNP (N-ERD) } \\
\text { postoperative ( } 60 \\
\text { analyzed) }\end{array}$ & $\begin{array}{l}\text { - Budesonide irrigation } \\
60 \mathrm{ml} / \mathrm{nostril} 500 \mu \mathrm{g} \text { b.i.d. } \\
\text { (total } 1000 \mu \mathrm{g} \text { ) for } 52 \text { weeks } \\
\text { ( } \mathrm{n}=20 \text { ) } \\
\text { - Saline irrigation } 60 \mathrm{ml} / \\
\text { nostril and budesonide } \\
\text { spray } 64 \mu \mathrm{g} / \text { nostril b.i.d. } \\
\text { (total } 256 \mu \mathrm{g} \text { ) for } 52 \text { weeks } \\
\text { ( } \mathrm{n}=19) \\
\text { - Saline irrigation alone for } 52 \\
\text { weeks }(\mathrm{n}=21)\end{array}$ & $\begin{array}{l}\text { - } \text { Quality of life (SNOT-21 (0-105) at 6,12 } \\
\text { - Enonths) } \\
\text { months) } \\
\text { - } \text { CT score (LM score (0-24) at 6,12 } \\
\text { months) } \\
\text { - Ocular events (IOP at 6,12 months) } \\
\text { - HPA axis (ACTH(normal/abnormal) at } \\
\text { 6,12 months) }\end{array}$ & $\begin{array}{l}\text { - SNOT-21, LKES, and LM score results of both } \\
\text { intervention groups were not different from } \\
\text { placebo group at both } 6 \text { and } 12 \text { month } \\
\text { timepoints (significance level was defined } \\
\text { at } p<.0167 \text { ) } \\
\text { - IOP and ACTH level were not changed at } \\
\text { both } 6 \text { and } 12 \text { month timepoints }\end{array}$ \\
\hline $\begin{array}{l}\text { Olsson } \\
2010^{(77)}\end{array}$ & DBPCRCT & $\begin{array}{l}68 \text { CRSwNP, } \\
\text { postoperative }\end{array}$ & 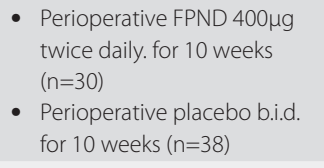 & $\begin{array}{l}\text { - Health related quality of life (SF-36, } \\
0-100,8 \text { health domains) at week } 5 \\
\text { after sinus surgery }\end{array}$ & $\begin{array}{l}\text { - At } 5 \text { weeks after sinus surgery no effect on } \\
\text { total SF36 but significant improvement in } \\
\text { the FPND group of the mental component } \\
\text { summary and } 3 \text { domains (role physical, } \\
\text { vitality, social functioning) }\end{array}$ \\
\hline $\begin{array}{l}\text { Ehnhage } \\
2009^{(95)}\end{array}$ & DBPCRCT & $\begin{array}{l}68 \text { CRSwNP } \\
(52 \text { evalauted) } \\
\text { postoperative }\end{array}$ & $\begin{array}{l}\text { - Perioperative FPND } 400 \mu \mathrm{g} \\
\text { b.i.d. for } 10 \text { weeks }(n=23) \\
\text { - Perioperative placebo b.i.d. } \\
\text { for } 10 \text { weeks }(n=29)\end{array}$ & $\begin{array}{l}\text { - Symptom score (3 symptoms, each } \\
\text { score 0-3) improvement at 10,17 } \\
\text { weeks } \\
\text { - Nasal Polyp score (0-3) reduction at 4, } \\
6,10,17 \text { weeks } \\
\text { - Adverse events }\end{array}$ & $\begin{array}{l}\text { - There was no difference between FPND and } \\
\text { placebo in symptom improvement and nasal } \\
\text { polyp score reduction at } 10 \text { and } 17 \text { weeks } \\
\text { - No difference in adverse events }\end{array}$ \\
\hline $\begin{array}{l}\text { Jorissen } \\
\text { 2009(93) }\end{array}$ & $\mathrm{DBPCT}$ & $\begin{array}{l}99 \text { CRSwNP patients } \\
\text { having FESS ( } 67 \\
\text { evaluated) }\end{array}$ & 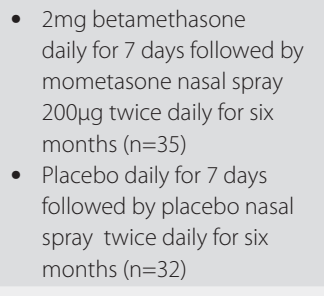 & $\begin{array}{l}\text { At } 1 \text { and } 2 \text { weeks and 1,2,4 and } 6 \\
\text { months postoperatively } \\
\text { - Total symptom score (VAS) } \\
\text { - Nasal endoscopy score (0-14) } \\
\text { - Endoscopic combination score (for } \\
\text { inflammation, oedema, and polyps) } \\
\text { - \% subjects requiring rescue medi- } \\
\text { cation }\end{array}$ & $\begin{array}{l}\text { At } 1 \text { and } 2 \text { weeks and 1,2,4 and } 6 \text { months: } \\
\text { - No significant difference in total symptom } \\
\text { score, nasal endoscopy or \% subjects } \\
\text { requiring rescue medication } \\
\text { - Significant reduction in endoscopic } \\
\text { combination score at 1,2 weeks and } 1 \text { and } \\
2 \text { months }\end{array}$ \\
\hline $\begin{array}{l}\text { Stjarne } \\
2009^{(114)}\end{array}$ & DBPCRCT & $\begin{array}{l}162 \text { CRSwNP } \\
\text { postoperative }\end{array}$ & $\begin{array}{l}\text { Postoperative MFNS } 200 \mu \mathrm{g} \\
\text { o.d. for } 8 \text { weeks }(n=80) \\
\text { - Postoperative placebo for } 8 \\
\text { weeks }(n=82)\end{array}$ & $\begin{array}{l}\text { - Time to relapse for polyp recurrence } \\
\text { - at } 24 \text { weeks } \\
\text { - Adverse events }\end{array}$ & $\begin{array}{l}\text { - At } 24 \text { weeks follow-up, postoperative use } \\
\text { of MFNS, } 200 \mu \mathrm{g} \text { once daily, provided a } \\
\text { statistically significant longer time to relapse } \\
\text { with nasal polyps than with placebo } \\
\text { - The frequency of AEs with MFNS and placebo } \\
\text { was comparable. }\end{array}$ \\
\hline $\begin{array}{l}\text { Wright } \\
2007^{(381)}\end{array}$ & $\mathrm{DBPCT}$ & $\begin{array}{l}26 \text { CRSwNP patients } \\
\text { having FESS }\end{array}$ & $\begin{array}{l}\text { - Prednisone } 30 \text { mg daily for } \\
5 \text { days preoperative and } 9 \\
\text { days postoperative }(n=11) \\
\text { - } \text { Placebo daily for } 5 \text { days } \\
\text { preoperative and } 9 \text { days } \\
\text { postoperative }(n=15)\end{array}$ & $\begin{array}{l}\text { Measured at 2,4,12 and } 26 \text { weeks: } \\
\text { - Overall symptomatology } \\
\text { - } \quad \text { Separate symptoms } \\
\text { - POSE score }\end{array}$ & $\begin{array}{l}\text { Treatment with oral corticoid compared to } \\
\text { placebo resulted in } \\
\text { - No difference in total and/or separate } \\
\text { - Improms } \\
\text { - Improved LK score at } 2,4 \text {, and } 26 \text { weeks } \\
\text { - Improved POSE score at } 2 \text { weeks }\end{array}$ \\
\hline $\begin{array}{l}\text { Rowe-Jones } \\
2005^{(92)}\end{array}$ & $\mathrm{DBPCT}$ & $\begin{array}{l}109 \text { CRS patients } \\
\text { ( } 77 \text { CRSwNP) } \\
\text { having FESS ( } 72 \\
\text { evaluated after } 5 \\
\text { years) }\end{array}$ & $\begin{array}{l}\text { - } \begin{array}{l}\text { FPANS } 200 \text { mcg twice daily } \\
(n=40)\end{array} \\
\text { - Placebo twice daily }(n=32)\end{array}$ & $\begin{array}{l}\text { Yearly evaluation of: } \\
\text { How do you feel today (VAS) } \\
\text { - Total symptoms (VAS) } \\
\text { - Nasal polyp score } \\
\text { - Oedema score } \\
\text { - Discharge score } \\
\text { - Olfaction } \\
\text { - Nasal volume (acoustic rhinometry) }\end{array}$ & $\begin{array}{l}\text { Treatment with FPANS resulted in: } \\
\text { - No significant differences in total symptoms } \\
\text { at } 1 \text { and } 5 \text { years follow-up } \\
\text { - Significant difference at } 1 \text { and } 5 \text { years in nasal } \\
\text { endoscopy score and total nasal volume }\end{array}$ \\
\hline
\end{tabular}


Table 6.2.7.2. Effect of corticosteroid treatment versus placebo postoperatively (continued).

\begin{tabular}{|c|c|c|c|c|c|}
\hline Study & Methods & Participants & Interventions & Outcomes & Results \\
\hline $\begin{array}{l}\text { Dijkstra } \\
2004^{(111)}\end{array}$ & DBPCT & $\begin{array}{l}162 \text { CRS patients } \\
(55 \text { CRSWNP) } \\
\text { having FESS }\end{array}$ & $\begin{array}{l}\text { - FPANS } 800 \text { mg twice daily } \\
\text { for } 1 \text { year ( } n=54 \text { ) } \\
\text { - FPANS } 400 \text { mg twice daily } \\
\text { daily for } 1 \text { year ( } n=54) \\
\text { - placebo twice daily for } 1 \\
\text { year daily }(n=54)\end{array}$ & $\begin{array}{l}\text { During one year: } \\
\text { - Recurrence or persistent disease }\end{array}$ & $\begin{array}{l}\text { No significant difference in the recurrence rate } \\
\text { during one year between the groups }\end{array}$ \\
\hline $\begin{array}{l}\text { Gulati } \\
2001^{1859)}\end{array}$ & DBPCRCT & $\begin{array}{l}\text { - } 63 \text { CRSWNP, } \\
\text { postoperative }\end{array}$ & $\begin{array}{l}\text { - Postoperative budesonide } \\
\text { nasal spray } 400 \mu \mathrm{g} / \text { day for } \\
12 \text { weeks ( } n=38) \\
\text { - Postoperative saline nasal } \\
\text { sprays for } 12 \text { weeks }(n=25)\end{array}$ & $\begin{array}{l}\text { - Proportion of responders at } 12 \text { weeks } \\
\text { - Symptom improvement ( } 4 \text { symptoms) } \\
\text { at } 12 \text { weeks } \\
\text { - Polyp recurrence at } 12 \text { weeks }\end{array}$ & $\begin{array}{l}\text { - At } 12 \text { weeks, greater responder of symptom } \\
\text { improvement in the budesonide group than } \\
\text { placebo group was significant for rhinorrhoea } \\
\text { and sneezing. } \\
\text { - At } 12 \text { weeks, budesonide treated patients } \\
\text { had significantly lower rate of polyp } \\
\text { recurrence. }\end{array}$ \\
\hline
\end{tabular}

DBPCRCT, Double Blind Placebo Controlled Randomised Controlled Trial; DBPCT, Double Blind Placebo Controlled Trial; CRSwNP, Chronic Rhinosinusitis with nasal polyps; N-ERD, NSAID-exacerbated respiratory disease; FESS, Functional Endoscopic Sinus Surg; FPND, Fluticasone Propionate Nasal drops; SNOT-21,; LKES, Lund-Kennedy endoscopy scoring; LM, Lund-Mackay; IOP, Intraocular Pressure; ACTH, Adrenocorticotropic Hormone; HPA, Hypothalamic-Pituitary-Adrenal; CT, computed tomography; VAS, Visual Analogue Scale; FPANS, Fluticasone propionate aqueous nasal spray; MFNS, Mometasone Furoate Nasal spray; POSE, Perioperative Sinus Endoscopy score; LK, Lund-Kennedy; CRS, Chronic Rhinosinusitis.

Table 6.2.7.3. Corticosteroid-eluting implants as postoperative treatment in patients with CRS.

\begin{tabular}{|c|c|c|c|c|c|}
\hline Study & Methods & Participants & Interventions & Outcomes & Efficacy \\
\hline $\begin{array}{l}\text { Gyawali, } \\
2019^{(468)}\end{array}$ & DBPCT & $\begin{array}{l}58 \text { CRSwNP } \\
\text { postoperative }\end{array}$ & $\begin{array}{l}\text { - Non-absorbable nasal pack soaked } \\
\text { in triamcinolone ( } 2 \mathrm{ml} \text { of } 40 \mathrm{mg} / \mathrm{ml} \\
\text { solution) to one side (removed on } \\
\text { second postop day) } \\
\text { - Non-absorbable nasal pack soaked } \\
\text { in saline on the contralateral side } \\
\text { removed on second postop day) }\end{array}$ & $\begin{array}{l}\text { - POSE and LK score in third postopera- } \\
\text { tive week }\end{array}$ & $\begin{array}{l}\text { Significantly better results with } \\
\text { corticosteroid pack than placebo pack for } \\
\text { both scores }\end{array}$ \\
\hline $\begin{array}{l}\text { Hwang } \\
2018^{(860)}\end{array}$ & SBPCT & $\begin{array}{l}22 \text { CRSwNP } \\
\text { postoperative }\end{array}$ & $\begin{array}{l}\text { - Calcium alginate soaked with } 2 \mathrm{~mL} \text { of } \\
\text { triamcinolone }(40 \mathrm{mg} / \mathrm{mL})(\mathrm{n}=22) \\
\text { - Calcium alginate soaked with } 2 \mathrm{~mL} \\
\text { saline }(\mathrm{n}=22)\end{array}$ & $\begin{array}{l}\text { - POSE score at 1,4 and } 8 \text { weeks post- } \\
\text { operatively }\end{array}$ & $\begin{array}{l}\text { Significant difference in POSE score at } \\
\text { week } 8\end{array}$ \\
\hline Luong 2018 & $\mathrm{DBPCT}$ & $\begin{array}{l}80 \text { CRS patients } \\
\text { having frontal } \\
\text { sinus surgery }\end{array}$ & $\begin{array}{l}\text { - Bioabsorbable drug-eluting stents } \\
\text { releasing } 370 \mu \text { of mometasone } \\
\text { furoate over approximately } 30 \text { days } \\
\text { unilateral frontal sinus ostium ( } n=79) \\
\text { - Stent without corticosteroid unilater- } \\
\text { al frontal sinus ostium }(n=79)\end{array}$ & $\begin{array}{l}\text { - Postoperative interventions at } 30 \\
\text { days post-ESS (oral steroid or surgery) } \\
\text { (independent observer) } \\
\text { - Postoperative interventions (cortico- } \\
\text { steroid or surgery) at } 30 \text { and } 90 \text { days } \\
\text { post-ESS (clinical investigator) } \\
\text { - Endoscopic evaluation of frontal sinus } \\
\text { ostium region (inflammation, steno- } \\
\text { sis,patency) }\end{array}$ & $\begin{array}{l}\text { Implant coated with MF compared to non- } \\
\text { coated implant } \\
\text { - Significant less need for postoperative } \\
\text { intervention at } 30 \text { days (independent } \\
\text { observer) } \\
\text { - Significant less need for postoperative } \\
\text { intervention at } 30 \text { and } 90 \text { days (clinical } \\
\text { investigator) } \\
\text { - Reduction in inflammation and reste- } \\
\text { nosis and greater diameter at } 30 \text { days }\end{array}$ \\
\hline $\begin{array}{l}\text { Zhao } \\
2018^{(482)}\end{array}$ & DBPCT & $\begin{array}{l}64 \text { CRSwNP } \\
\text { postoperative }\end{array}$ & $\begin{array}{l}\text { - } 64 \text { sides: } 2 \text { pieces of } 8 \mathrm{~cm} \text { biodegrad- } \\
\text { able nasal dressing impregnated } \\
\text { (BND) with } 4 \mathrm{~mL} \text { or } 8 \mathrm{~mL} \text { of mometa- } \\
\text { sone furoate } 0.35 \mathrm{mg} / \mathrm{ml} \text { taken out } \\
\text { after } 1 \text { week or after } 2 \text { weeks } \\
64 \text { contralateral cavities received an } \\
\text { identical BND soaked in the same } \\
\text { amount of normal saline taken out } \\
\text { after } 1 \text { week or after } 2 \text { weeks }\end{array}$ & $\begin{array}{l}\text { - POSE and LK score at } 7 \text { th or } 14 \text { th } \\
\text { postoperative days and at } 1,2 \text { and } 3 \\
\text { postoperative months. }\end{array}$ & $\begin{array}{l}\text { - The POSE and Lund-Kennedy scores } \\
\text { showed that in the } 4 \mathrm{~mL} \text {, 1-week group, } \\
\text { no significant differences between the } \\
\text { sides treated with MF-soaked BNDs and } \\
\text { the normal saline-soaked control were } \\
\text { observed at any postoperative visits. } \\
\text { In the } 4 \mathrm{~mL}, 2 \text {-week group, significant } \\
\text { differences were found at the 2-week } \\
\text { and 1-month postoperative visits but } \\
\text { not at the 2-month and 3-month visits. } \\
\text { In the 8-mL, 1-week group, significant } \\
\text { differences were found at the 1-week, } \\
\text { 1-month, and 2-month postoperative } \\
\text { visits but not at the 3-month visit. In } \\
\text { the 8mL, 2-week group, significant } \\
\text { differences were found at all } \\
\text { postoperative visits. }\end{array}$ \\
\hline $\begin{array}{l}\text { Sow } \\
2018^{(861)}\end{array}$ & $\mathrm{DBPCT}$ & $\begin{array}{l}8 \text { patients with } \\
\text { CRS (CRSwNP } \\
\text { unclear) } \\
\text { postoperative }\end{array}$ & $\begin{array}{l}\text { - Hydrocortisone-impregnated } \\
\text { bioabsorbable dressing in one nasal } \\
\text { cavity and normal saline-impreg- } \\
\text { nated bioabsorbable dressing in the } \\
\text { contralateral nasal cavity }\end{array}$ & $\begin{array}{l}\text { - SNOT and POSE at 1, } 3 \text { wks and } 3 \\
\text { months post-operative }\end{array}$ & $\begin{array}{l}\text { - No statistical significance between } \\
\text { impregnated and saline dressing at } 1 \\
\text { and } 3 \text { wks and } 3 \text { months postop. }\end{array}$ \\
\hline
\end{tabular}


Table 6.2.7.3. Corticosteroid-eluting implants as postoperative treatment in patients with CRS (continued).

\begin{tabular}{|c|c|c|c|c|c|}
\hline Study & Methods & Participants & Interventions & Outcomes & Efficacy \\
\hline Ha $2018^{(862)}$ & SBPCT & $\begin{array}{l}12 \text { CRS (7 } \\
\text { CRSwNP) } \\
\text { patients } \\
\text { postoperative } \\
\text { Part of } 36 \\
\text { patients in } \\
\text { non-blinded } \\
\text { RCT }\end{array}$ & $\begin{array}{l}\text { - Chitosan-dextran gelwith } 1 \mathrm{mg} / 2 \mathrm{~mL} \\
\text { budesonide }(\mathrm{n}=5) \\
\text { - Chitosan-dextran gel }(\mathrm{n}=7)\end{array}$ & $\begin{array}{l}\text { - Percentage of baseline intraoperative } \\
\text { areas for sinus ostia at } 2 \text { weeks, } 3 \\
\text { months, and } 12 \text { months postoperative } \\
\text { - Adhesion presence (\%) and severity (0- } \\
3 \text {, mucosal oedema (0-3), granulation } \\
\text { tissue formation (0-3), evidence of pus } \\
\text { - } 2 \text { ) and crusting (0-2) at } 2 \text { weeks, } 3 \\
\text { months and } 12 \text { months postoperative }\end{array}$ & $\begin{array}{l}\text { - All sinus ostia significantly larger at } 2 \\
\text { weeks and frontal and sphenoid ostia } \\
\text { significantly larger at } 12 \text { months. } \\
\text { - Other data not analysed }\end{array}$ \\
\hline $\begin{array}{l}\text { Sabarinath, } \\
2017^{(863)}\end{array}$ & DBPCT & $\begin{array}{l}75 \text { CRS patients } \\
\text { postoperative }\end{array}$ & $\begin{array}{l}\text { - Non-absorbable nasal pack soaked } \\
\text { in triamcinolone ( } 2 \mathrm{ml} \text { of } 40 \mathrm{mg} / \mathrm{ml} \\
\text { solution) to one side (removed on } \\
\text { second postop day) } \\
\text { - Non-absorbable nasal pack soaked } \\
\text { in saline on the contralateral side } \\
\text { removed on second postop day) }\end{array}$ & $\begin{array}{l}\text { - Crusting, oedema, polypoidal change } \\
\text { (Absent; Mild; Severe) at } 1 \text { and } 2 \text { weeks, } \\
1 \text { and } 3 \text { months } \\
\text { - Discharge (Absent; thin/clear; thick/ } \\
\text { purulent) at } 1 \text { and } 2 \text { weeks, } 1 \text { and } 3 \\
\text { months }\end{array}$ & \\
\hline $\begin{array}{l}\text { Adriaensen } \\
2017^{(121)}\end{array}$ & $\mathrm{DBPCT}$ & $\begin{array}{l}30 \text { patients } \\
\text { with CRSWNP } \\
\text { randomized } \\
\text { to } 2 \text { out of } 3 \\
\text { treatments } \\
\text { on two sides } \\
\text { postoperative }\end{array}$ & 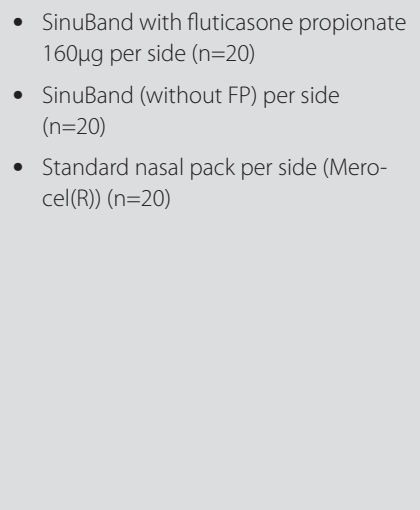 & $\begin{array}{l}\text { - Intraocular pressure } \\
\text { - Lens opacity } \\
\text { - } 24 \text {-hour urine cortisol } \\
\text { - Ethmoid inflammation (VAS) at } \\
5 / 15 / 30 / 60 \text { days } \\
\text { - } \% \text { of sinuses developing polypoid } \\
\text { - } \text { - Adhanges through first } 15 / 30 / 60 \text { days } \\
\text { - Lund-Kennedy score at } 5 / 15 / 30 / 60 \\
\text { - } \text { days } \\
\text { - } \text { Postoperative pain at } 5 / 15 / 30 / 60 \text { days } \\
\text { - Nasal discharge at } 5 / 15 / 30 / 60 \text { days }\end{array}$ & $\begin{array}{l}\text { - No significant change in 24-hour urine } \\
\text { cortisol } \\
\text { - No differences in ethmoid } \\
\text { inflammation, adhesions or LK score } \\
\text { - Significantly lower polypoid changes in } \\
\text { sides with Sinuband FP than Sinuband } \\
\text { without FP and Merocel at } 60 \text { days } \\
\text { - Significantly less pain and congestion } \\
\text { at } 60 \text { days for Sinuband FP than } \\
\text { Sinuband without FP }\end{array}$ \\
\hline $\begin{array}{l}\text { Smith } \\
2016^{(885)}\end{array}$ & DBPCT & $\begin{array}{l}80 \text { CRS patients } \\
\text { having frontal } \\
\text { sinus surgery }\end{array}$ & $\begin{array}{l}\text { - Bioabsorbable drug-eluting stents } \\
\text { releasing } 370 \mu \text { of mometasone } \\
\text { furoate over approximately } 30 \text { days } \\
\text { unilateral frontal sinus ostium ( } n=79) \\
\text { - Stent without corticosteroid unilater- } \\
\text { al frontal sinus ostium ( } n=79)\end{array}$ & $\begin{array}{l}\text { - Postoperative interventions at } 30 \\
\text { days post-ESS (oral steroid or surgery) } \\
\text { (independent observer) } \\
\text { - Postoperative interventions (cortico- } \\
\text { steroid or surgery) at } 30 \text { and } 90 \text { days } \\
\text { post-ESS (clinical investigator) } \\
\text { - Endoscopic evaluation of frontal sinus } \\
\text { ostium region (inflammation, steno- } \\
\text { sis,patency) }\end{array}$ & $\begin{array}{l}\text { Implant coated with MF compared to non- } \\
\text { coated implant } \\
\text { - Significant less need for postoperative } \\
\text { intervention at } 30 \text { days (independent } \\
\text { observer) } \\
\text { - Significant less need for postoperative } \\
\text { intervention at } 30 \text { and } 90 \text { days (clinical } \\
\text { investigator) } \\
\text { - Reduction in inflammation and } \\
\text { restenosis and greater diameter at } 30 \\
\text { days }\end{array}$ \\
\hline Xu $2016^{(466)}$ & DBPCT & $\begin{array}{l}80 \text { CRSwNP } \\
\text { postoperative } \\
\text { (58 evaluated) }\end{array}$ & $\begin{array}{l}\text { - } 28 \times \text { two pieces of } 4 \mathrm{~cm} \text { biodegrad- } \\
\text { able dressing impregnated with } 2 \mathrm{ml} \\
\text { of a } 10 \mathrm{mg} / \mathrm{ml} \text { of triamcinolone } \\
\text { - } 32 \text { pieces of } 4 \mathrm{~cm} \text { biodegradable } \\
\text { dressing impregnated with } 2 \mathrm{ml} \text { saline }\end{array}$ & $\begin{array}{l}\text { - SNOT-20 and the Sniffin' Stick test } 1 \text { and } \\
3 \text { months } \\
\text { - Lund-Kennedy (L-K) and POSE score at } \\
\text { 1,2 and } 3 \text { months. }\end{array}$ & $\begin{array}{l}\text { - No significant difference in SNOT } \\
\text { - Significant lower POSE and LK score in } \\
\text { the triamcinolone group compared to } \\
\text { the place group at } 1 \text { and } 2 \text { months }\end{array}$ \\
\hline $\begin{array}{l}\text { Bardaranfar } \\
2014^{(864)}\end{array}$ & DBPCT & $\begin{array}{l}60 \text { CRSWNP } \\
\text { postoperative }\end{array}$ & $\begin{array}{l}\text { - A sterile piece of compressed } \\
\text { Gelfoam combined with } 10 \mathrm{mg} / \\
\mathrm{mL} \text { of triamcinolone at the olfactory } \\
\text { cleft between the nasal septum and } \\
\text { middle turbinate, above anterior } \\
\text { attachment. } \\
\text { - A sterile piece of compressed } \\
\text { Gelfoam combined with saline at } \\
\text { the olfactory cleft between the nasal } \\
\text { septum and middle turbinate, above } \\
\text { anterior attachment. }\end{array}$ & $\begin{array}{l}\text { - Butanol threshold test } 8 \text { weeks post- } \\
\text { operative }\end{array}$ & $\begin{array}{l}\text { - The triamcinolone resulted in a signif- } \\
\text { cant better butanol threshold test at } 8 \\
\text { weeks than saline }\end{array}$ \\
\hline
\end{tabular}


Table 6.2.7.3. Corticosteroid-eluting implants as postoperative treatment in patients with CRS (continued).

\begin{tabular}{|c|c|c|c|c|c|}
\hline Study & Methods & Participants & Interventions & Outcomes & Efficacy \\
\hline $\begin{array}{l}\text { Hong } \\
2013^{(467)}\end{array}$ & DBPCT & $\begin{array}{l}20 \text { CRS patients } \\
\text { postoperative }\end{array}$ & $\begin{array}{l}\text { - Triamcinolone }(20 \mathrm{mg}) \text { impregnated } \\
\text { bioresorbable dressing }(n=10) \\
\text { - Saline impregnated bioresorbable } \\
\text { dressing }(n=10)\end{array}$ & $\begin{array}{l}\text { - Serum cortisol levels at day 1,2 and } 10 \\
\text { - } 12 \text {-hour urine cortisol level at day } 10 \\
\text { - Osteocalcin, blood eosinophil count, } \\
\text { and adreno-corticotropic hormone } \\
\text { (ACTH) levels at day } 2 \text { and } 10\end{array}$ & $\begin{array}{l}\text { - Serum cortisol levels significantly } \\
\text { depressed at day } 1,2 \\
\text { - } 12 \text {-hour urine cortisol level significantly } \\
\text { depressed compared to placebo at } \\
\text { day } 10^{*} \\
\text { - Significant difference in osteocalcin } \\
\text { at day } 2^{*}\end{array}$ \\
\hline $\begin{array}{l}\text { Rudmik } \\
2012^{(865)}\end{array}$ & DBPCT & $\begin{array}{l}36 \text { CRSsNP } \\
\text { patients } \\
\text { postoperative }\end{array}$ & $\begin{array}{l}\text { - Dexamethasone Sinu-Foam }{ }^{\mathrm{TM}}(16 \mathrm{mg} \\
\text { dexamethasone }(\mathrm{n}=18) \\
\text { - Sinu-Foam }{ }^{\mathrm{TM}}(\mathrm{n}=18)\end{array}$ & $\begin{array}{l}\text { - LK score at } 1 \text { week, } 4 \text { weeks and } 3 \\
\text { months } \\
\text { - Adverse events }\end{array}$ & $\begin{array}{l}\text { - No significant differences between } \\
\text { groups }\end{array}$ \\
\hline $\begin{array}{l}\text { Marple } \\
2012^{(122)}\end{array}$ & DBPCT L & $\begin{array}{l}105 \text { CRS (62 } \\
\text { CRSwNP) } \\
\text { patients } \\
\text { postoperative }\end{array}$ & $\begin{array}{l}\text { - Bioabsorbable drug-eluting stents } \\
\text { releasing } 370 \mu \mathrm{g} \text { of mometasone } \\
\text { furoate over approximately } 30 \text { days } \\
(n=105) \\
\text { - Placebo stents }(n=105)\end{array}$ & $\begin{array}{l}\text { - Frank polyposis (yes/no) at day } 30 \text { by } \\
\text { - Adhesion formation (0-5) at day } 30 \\
\text { - Need of intervention (oral corticoste- } \\
\text { roids or adhesion lysis) at day } 30 \\
\text { - All data analysed by independent panel } \\
\text { and investigators } \\
\text { - Ocular safety } \\
\text { - Drug related adverse events }\end{array}$ & $\begin{array}{l}\text { - Significant difference in mometasone } \\
\text { furoate group in need for intervention } \\
\text { and frank polyposis (independent } \\
\text { panel) } \\
\text { - No significant differences difference in } \\
\text { mometasone furoate group in need } \\
\text { of intervention and frank polyposis } \\
\text { (clinical investigators) } \\
\text { - Significant reduction in adhesions } \\
\text { (clinical investigators) }\end{array}$ \\
\hline $\begin{array}{l}\text { Murr } \\
2011^{(866)}\end{array}$ & DBPCT & $\begin{array}{l}38 \text { CRS ( } 31 \\
\text { CRSWNP) } \\
\text { patients } \\
\text { postoperative }\end{array}$ & $\begin{array}{l}\text { - Bioabsorbable drug-eluting stents } \\
\text { releasing } 370 \mu g \text { of mometasone } \\
\text { furoate over approximately } 30 \text { days } \\
(n=38) \\
\text { - Placebo stents }(n=38)\end{array}$ & $\begin{array}{l}\text { - Ethmoid sinus inflammation (VAS) at 7, } \\
14,21,30,45 \text { and } 60 \text { days } \\
\text { - } \% \text { of sinus with polypoid change }> \\
1(0-5) \text { at day } 30 \\
\text { - } \% \text { of sinus with adhesion formation } \\
\text { (0-5) and middle turbinate lateralisation } \\
\text { up to day } 30 \\
\text { - Plasma mometasone fuorate and } \\
\text { cortisol concentrations at 7, } 14,21 \text { and } \\
30 \text { days (in } 5 \text { patients with bilateral } \\
\text { mometasone furoate releasing stent) }\end{array}$ & $\begin{array}{l}\text { - Significant reduction in ethmoid } \\
\text { inflammation in mometasone furoate } \\
\text { group at } 21,28 \text { and } 45 \text { days } \\
\text { - Significant reduction in \% of sinus with } \\
\text { polypoid change at day } 30 \\
\text { - Significant reduction in \% with sinus } \\
\text { adhesion formation up to day } 30 \\
\text { - No differences in plasma } \\
\text { concentrations }\end{array}$ \\
\hline $\begin{array}{l}\text { Coté } \\
2010^{(465)}\end{array}$ & DBPCT & $\begin{array}{l}19 \text { CRSwNP } \\
\text { patients } \\
\text { postoperative }\end{array}$ & $\begin{array}{l}\text { - Triamcinolone }(80 \mathrm{mg}) \text { impregnated } \\
\text { bioresorbable dressing on one side } \\
(\mathrm{n}=19) \\
\text { - Saline-impregnated bioresorbable } \\
\text { dressing on the other side }\end{array}$ & $\begin{array}{l}\text { - LK and POSE scores at postoperative } \\
\text { days 7, 14, } 28 \text { and at } 3 \text { and } 6 \text { months } \\
\text { - Adverse events }\end{array}$ & $\begin{array}{l}\text { - Significantly better LK and POSE scores } \\
\text { at days } 7 \text { and } 14 \text { and } 3 \text { and } 6 \text { months in } \\
\text { triamcinolone-impregnated group }\end{array}$ \\
\hline
\end{tabular}

DBPCT, Double Blind Placebo Controlled Trial; SBPCT, Single Blind Placebo Controlled Trial; CRS, Chronic Rhinosinusitis; CRSwNP, Chronic Rhinosinusitis with nasal polyps; POSE, Perioperative Sinus Endoscopy score; LK, Lund-Kennedy; MF, Mometasone Furoate; post-ESS, Post Endoscopic Sinus Surg; BND, Biodegradable Nasal Dressing; SNOT, Sino-Nasal Outcome; RCT, Randomised Controlled Trial; ACTH, Adrenocorticotropic Hormone; VAS, Visual Analogue Scale; *Significance between the groups calculated based on data given in paper.

Table 6.2.7.4. Postoperative saline nasal irrigation versus no irrigation in CRS patients.

\begin{tabular}{|c|c|c|c|c|c|}
\hline Study & Methods & Participants & Interventions & Outcomes & Results \\
\hline $\begin{array}{l}\text { Liang } \\
2008^{(175)}\end{array}$ & $\begin{array}{l}\mathrm{RCT} \text {, no blinding, } \\
\text { postoperative }\end{array}$ & $\begin{array}{l}77 \text { patients } \\
\text { undergoing FESS }\end{array}$ & $\begin{array}{l}\text { - Pulsatile nasal irrigation for } 3 \\
\text { months } \\
\text { - No treatment }\end{array}$ & $\begin{array}{l}\text { At } 2 \text { weeks and 1, 2, and } 3 \\
\text { months postoperatively: } \\
\text { - Symptom score } \\
\text { - Endoscopy score }\end{array}$ & $\begin{array}{l}\text { - Significant positive effect } \\
\text { of nasal irrigation in pa- } \\
\text { tients with mild CRS but } \\
\text { not with severe CRS }\end{array}$ \\
\hline $\begin{array}{l}\text { Freeman } \\
2008^{(158)}\end{array}$ & $\begin{array}{l}\text { Within-subject, } \\
\text { single- } \\
\text { blinded RCT } \\
\text { postoperatively }\end{array}$ & 22 CRS patients & $\begin{array}{l}\text { - } 2 \mathrm{ml} \text { isotonic saline via a mucosal } \\
\text { atomisation device one side of } \\
\text { the nose, three times per day for } \\
6 \text { weeks } \\
\text { - No saline other side of the nose }\end{array}$ & $\begin{array}{l}\text { At } 3 \text { wks and } 3 \text { months: } \\
\text { - Adhesions, discharge, } \\
\text { polyps, crusting, oedema } \\
\text { (0-3) at endoscopy }\end{array}$ & $\begin{array}{l}\text { - Significant positive effect } \\
\text { of saline atomisation } \\
\text { on discharge, no other } \\
\text { differences }\end{array}$ \\
\hline $\begin{array}{l}\text { Pinto } \\
2006^{(178)}\end{array}$ & $\begin{array}{l}\text { Postoperative, } \\
\text { RCT, double } \\
\text { blinded for sprays }\end{array}$ & $\begin{array}{l}60 \text { patients } \\
\text { with histories of } \\
\text { frequent sinusitis } \\
\text { after FESS }\end{array}$ & $\begin{array}{l}\text { - Normal saline (NS; } n=20 \text { ) four } \\
\text { times per day, two puffs on each } \\
\text { side, for the first } 5 \text { postoperative } \\
\text { days. } \\
\text { - Buffered hypertonic saline (HS; } n \\
=20) 4 \text { times per day, } 2 \text { puffs on } \\
\text { each side, for the first } 5 \text { postoper- } \\
\text { ative days. } \\
\text { - Or no spray }(n=20)\end{array}$ & $\begin{array}{l}\text { For } 5 \text { days: } \\
\text { - Symptoms (nasal obstruc- } \\
\text { tion/congestion, nasal } \\
\text { discharge/postnasal drip, } \\
\text { pain/pressure, headache, } \\
\text { and trouble sleeping/in- } \\
\text { somnia) (0-4) } \\
\text { - Pain medication }\end{array}$ & $\begin{array}{l}\text { - Significantly more nasal } \\
\text { discharge in hypertonic } \\
\text { saline group compared to } \\
\text { the other groups } \\
\text { - No other significant } \\
\text { differences }\end{array}$ \\
\hline
\end{tabular}

RCT, Randomised Controlled Trial; FESS, Functional Endoscopic Sinus Surg; CRS, Chronic Rhinosinusitis. 
Figure 6.2.7.1. Forest plot of the effect of peri-operative corticosteroids versus placebo on relapse at nine months.

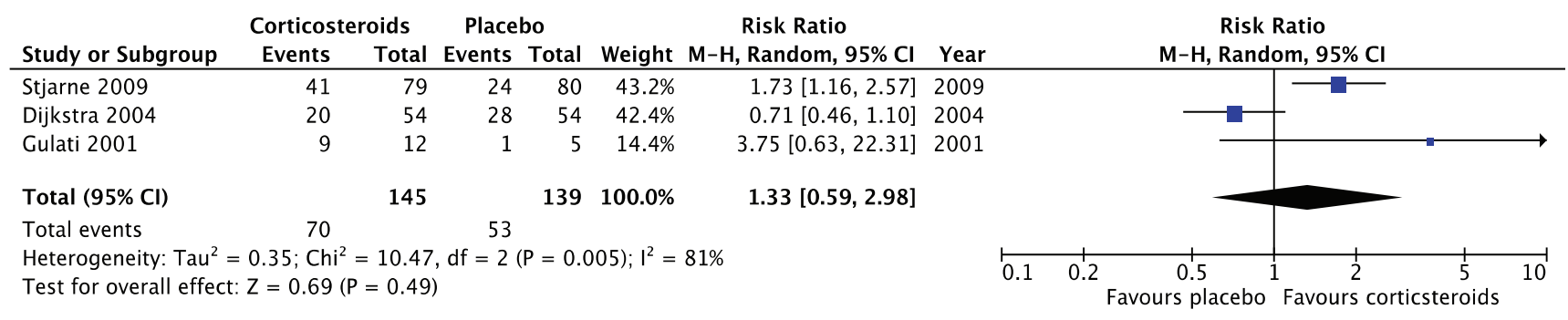

Figure 6.2.7.2. Forest plot of the effect peri-operative corticosteroids versus placebo on 6-12 months postoperative total symptoms.

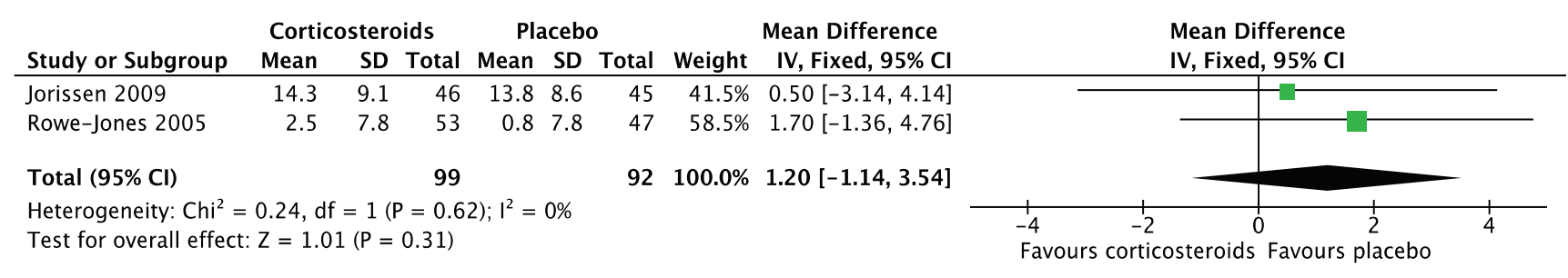

Figure 6.2.7.3. Forest plot of the effect of peri-operative corticosteroids versus placebo on six months postoperative nasal endoscopy score.

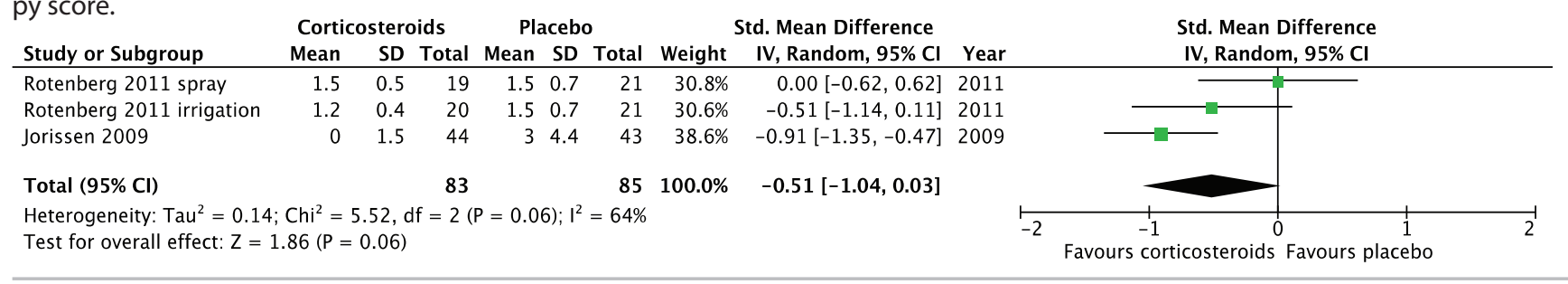

Six studies evaluated symptoms $s^{(92,93,95,105,859)}$. Five studies found no differences in symptom scores ${ }^{(92,93,95,105)}$. One study only evaluating nasal blockage and sneezing, found a difference in sneezing ${ }^{(859)}$. Two studies could be combined into a meta-analysis and showed no significant reduction in symptomatology in the corticosteroid treated group versus the placebo group MD 1.2 (Cl-1.1.4 -3.54), two studies, 201 patients, $\mathrm{p}=0.31$ ) (Figure 6.2.7.2).

Four studies evaluated a nasal polyp score. Three studies showed no difference between corticosteroid treatment and

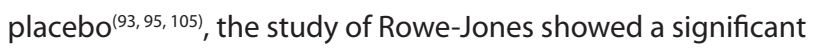
reduction in nasal polyp score after one and five years of nasal corticosteroids use versus placebo. The studies could not be combined into a meta-analysis.

Three studies evaluated a nasal endoscopy score ${ }^{(83,93,381)}$. None found a significant difference of corticosteroid treatment versus placebo. Two studies could be combined into a meta-analysis (Rotenberg had two arms) and showed a trend in reduction of endoscopy score at six months in the corticosteroid treated group versus the placebo group SMD - 0.51 ( $\mathrm{Cl}-1.04-0.03)$, two studies (one with two arms) 168 patients, $\mathrm{p}=0.06$ ) (Figure 6.2.7.3).

\subsection{Conclusion}

The quality of the evidence concerning the effect of perioperative corticosteroids to prevent recurrence of disease/ reduce postoperative was (very) low. The studies were in general small with significant heterogeneity.

There is no indication that use of peri-operative corticosteroids is more effective than placebo on the postoperative QOL, symptoms and of nasal endoscopy. Based on the quality of the available evidence the EPOS steering group cannot advise on the use of peri-operative/postoperative (nasal) corticosteroids.

\subsubsection{Corticosteroid-eluting implants}

\subsection{Summary of the evidence}

Steroid-eluting stents, spacers and dressings can improve local drug delivery immediately following endoscopic sinus surgery and reduce the recurrence of inflammation warranting systemic corticosteroids. For chronic rhinosinusitis with nasal polyposis (CRSwNP), the need for systemic corticosteroids immediately following endoscopic sinus surgery when using a steroid-eluting spacer has not been studied.

In this analysis only studies comparing a local corticosteroid containing device versus placebo are included: in 14 DBPCRCTs local corticosteroids containing steroid-eluting stents, spacers and dressings were placed in the ethmoid after surgery and compared to placebo ${ }^{(121,122,465-468,482,860-866)}$ (Table 6.2.7.3.). Twelve studies used impregnated biodegradable and two a non-absorbable packing ${ }^{(468,863)}$ impregnated with a variety of local corticosteroids including triamcinolone ${ }^{(465-468,860 \text {, }}$ ${ }^{863,864)}$, mometasone furoate ${ }^{(122,482,866)}$, hydrocortisone ${ }^{(861)}$, budesonide ${ }^{(862)}$, fluticasone proprionate ${ }^{(121)}$, and dexamethasone ${ }^{(865)}$.

Of these, seven studies evaluated patients with CRSwNP, one study ${ }^{(865)}$ patients with CRSsNP and the other six either a mixed population or the phenotype is not indicated. 
Figure 6.2.7.4. Forest plot of the effect of corticosteroid-eluting stents, spacers or dressings versus placebo on the POSE score after 3 weeks - 1 month postoperatively

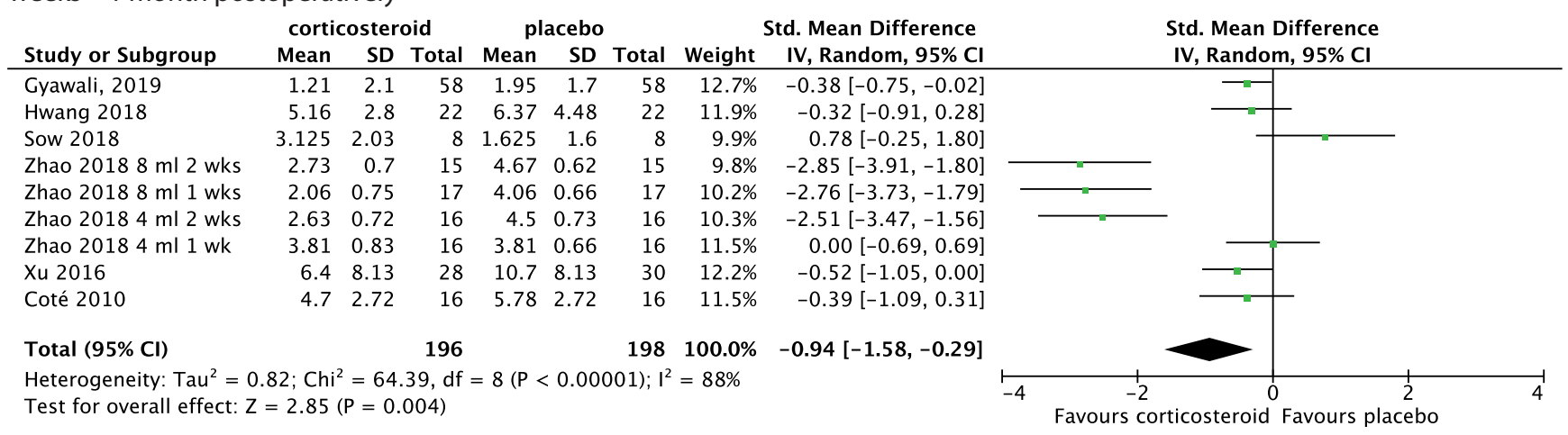

Figure 6.2.7.5. Forest plot of the effect of corticosteroid-eluting stents, spacers or dressings versus placebo on the POSE score after 3 month postoperatively

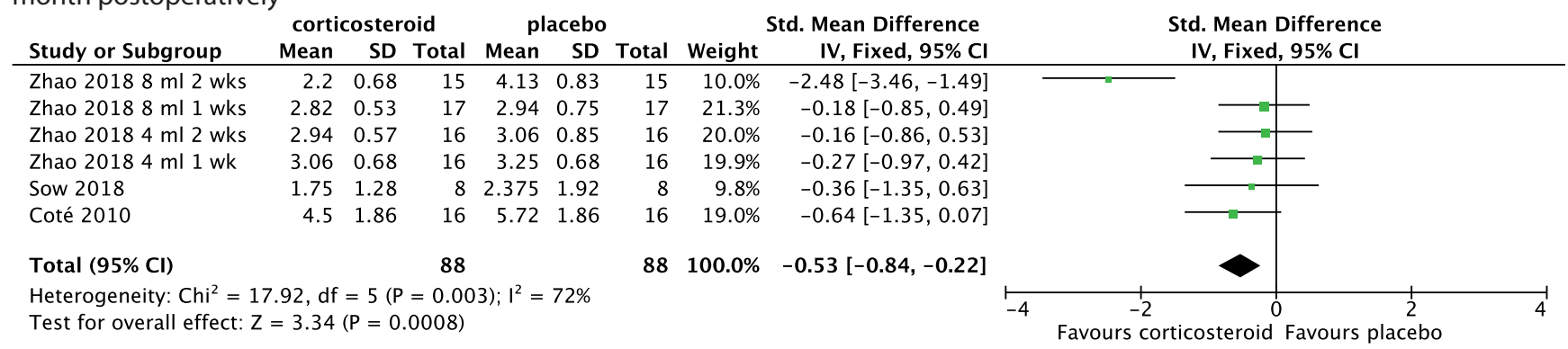

Figure 6.2.7.6. Forest plot of the effect of corticosteroid-eluting stents, spacers or dressings versus placebo on the Lund-Kennedy score after 3 month postoperatively

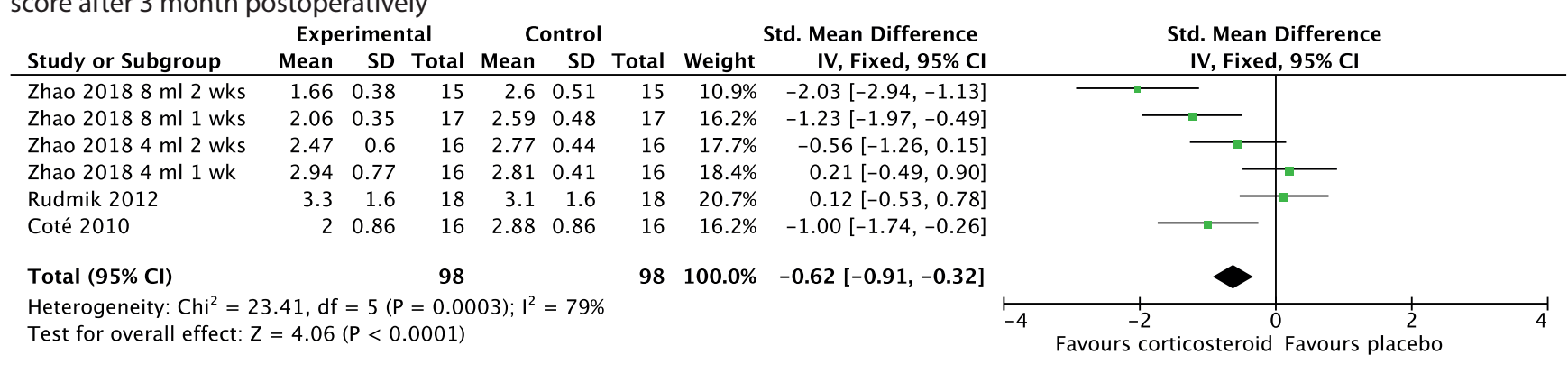

Dosages were varied and depended on the corticosteroid used. One study compared four different scenarios with two dosages and removal one and two weeks postoperatively ${ }^{(482)}$. Also, the duration of corticosteroid release varied significantly from short (1-2 weeks max) for the dressings soaked in local corticosteroids, to 30 days for the drug eluting stents ${ }^{(122,866)}$.

Two studies evaluated the effect of steroid-eluting stents, spacers and dressing placement postoperatively on QOL (SNOT) $(466,861)$. Both studies found no difference in SNOT score in the steroid treated patients/sides compared to placebo.

Symptoms were not evaluated apart from smell in two studies ${ }^{(466,864)}$ and congestion, pain and discharge in the study by Adriaensen ${ }^{(121)}$. In the study by Xu there were no significant differences between the triamcinolone treated group and the placebo group in the postoperative results of the Korean version of the Sniffin' Stick II (KVSS II) test nor the TDI (threshold, discrimination, and identification). Also, when only the anosmic/ hyposmic patients were evaluated (TDI score < below 27) no significant differences between the groups were found. Bardaranfar on the other hand found significant impact of the placement of a sterile piece of compressed Gelfoam combined with $10 \mathrm{mg} / \mathrm{mL}$ of triamcinolone at the olfactory cleft between the nasal septum and middle turbinate, above anterior attachment on the butanol threshold test ${ }^{(864)}$. Adriaensen reported significantly less nasal congestion and pain reported by the patients on the side treated with local corticosteroids than on the placebo side at day $60(p=0.036)$. All the other timepoints/measurements were not significant ${ }^{(121)}$.

A number of studies evaluated endoscopic scores (POSE) (381) (Figure 6.2.7.4.) and/or Lund-Kennedy score ${ }^{(867)}$ (Figure 6.2.7.6.) at comparable timepoints and could be combined into a metaanalysis. The POSE score at three weeks -1 mnd showed score: SMD - 1.03 (95\% Cl-1.83, -0.23); 138 participants; eight studies; $12=89 \%$ ), scale range: 0 to 16 , lower = better, indicating less disease in the patients that were treated with corticosteroideluting stents, spacers or dressings than the placebo (Figure 6.2.7 4.) . The result at three months still showed a significant effect: SMD -0,63 (95\% Cl -1.23, -0.03); 88 participants; six studies; $12=88 \%)$, $(p=0.06)$ (Figure 6.2.7.5.).

Lund-Kennedy score at three months showed: SMD -0.72 (95\% 
Table 6.2.7.5. Methods of nasal irrigation in postoperative CRS patients.

\begin{tabular}{|c|c|c|c|c|c|}
\hline Study & Methods & Participants & Interventions & Outcomes & Results \\
\hline $\begin{array}{l}\text { Macdonald } \\
2015^{(162)}\end{array}$ & $\begin{array}{l}\text { Single } \\
\text { blind RCT } \\
\text { postoperative }\end{array}$ & $\begin{array}{l}86 \text { CRS patients } \\
\text { (55 CRSwNP) } \\
\text { directly } \\
\text { postoperatively }\end{array}$ & $\begin{array}{l}\text { - Saline squeeze bottle } \\
(n=43) \text { for } 1 \text { month } \\
\text { - Saline nasal spray } \\
(n=43) \text { for } 1 \text { month }\end{array}$ & $\begin{array}{l}\text { - } \text { SNOT-22 } \\
\text { - POSE } \\
\text { - Nasal and sinus } \\
\text { symptom scale } \\
\text { (NSS) (0-15) }\end{array}$ & $\begin{array}{l}\text { - Significant improvement in both groups for SNOT- } \\
\text { 22, POSE, NSS } \\
\text { - No difference between treatment groups }\end{array}$ \\
\hline
\end{tabular}

RCT, Randomised Controlled Trial; CRS, Chronic Rhinosinusitis; CRSwNP, Chronic Rhinosinusitis with nasal polyps; SNOT-22, Sino-Nasal Outcome Test22; POSE, Perioperative Sinus Endoscopy score; NSS, Nasal and Sinus Symptom scale.

Table 6.2.7.6. Postoperative saline irrigation with antiseptic/mucolytic/babyshampoo solution in CRS patients.

\begin{tabular}{|c|c|c|c|c|c|}
\hline Study & Methods & Participants & Interventions & Outcomes & Results \\
\hline $\begin{array}{l}\text { Farag } \\
2013^{(160)}\end{array}$ & $\begin{array}{l}\text { Single } \\
\text { blind RCT } \\
\text { postoperative }\end{array}$ & $\begin{array}{l}40 \text { CRS patients } \\
\text { directly } \\
\text { postoperative }\end{array}$ & $\begin{array}{l}\text { - } 120 \mathrm{ml} \text { Hypertonic } \\
\text { Saline solution } 3 \mathrm{dd} \text { until } \\
4 \text { months postop } \\
\text { - } 120 \mathrm{ml} \text { of } 1 \% \text { baby } \\
\text { shampoo solution3dd } \\
\text { until } 4 \text { months postop }\end{array}$ & $\begin{array}{l}\text { - } \text { RSOM-31 } \\
\text { - SNOT-22 } \\
\text { - Olfaction with phenyl } \\
\text { ethyl alcohol (PEA) } \\
\text { threshold test } \\
\text { - Adverse events }\end{array}$ & $\begin{array}{l}\text { - Significant decreases in scores for both the } \\
\text { SNOT-22and RSOM-31 overtime for both } \\
\text { treatments but no difference between the } \\
\text { treatments } \\
\text { - PEA thresholds showed improvement but no } \\
\text { difference between the treatments } \\
\text { - The baby-shampoo group reported } \\
\text { significantly more side effects and had more } \\
\text { patients stop the solution }\end{array}$ \\
\hline $\begin{array}{l}\text { Pigret } \\
1996^{(183)}\end{array}$ & $\begin{array}{l}\text { Single- } \\
\text { blind RCT } \\
\text { postoperative }\end{array}$ & $\begin{array}{l}20 \text { CRSwNP } \\
\text { patients after } \\
\text { endoscopic } \\
\text { endonasal } \\
\text { ethmoidectomy }\end{array}$ & $\begin{array}{l}\text { - Pressurized seawater } \\
\text { (volume unclear) }(n=10) \\
3 d d \\
\text { - } 10 \mathrm{ml} \text { of saline plus } \\
\text { benzododecinium (anti- } \\
\text { septic) plus oleosorbate } \\
\text { (mucolytic) }(n=10) 3 d d\end{array}$ & $\begin{array}{l}\text { - Residual nasal crusts } \\
\text { weight at } 21 \pm 2 \text { days } \\
\text { secretions after } 21 \pm 2 \\
\text { days } \\
\text { - Visual analogue scale } \\
\text { for nasal obstruction, } \\
\text { rhinorrhoea, cacosmia } \\
\text { and facial pain }\end{array}$ & $\begin{array}{l}\text { - No significant differences although the mean } \\
\text { residual crust weight was higher in the } \\
\text { pressurized seawater group }(1,756 \pm 688 \mathrm{mg}) \\
\text { than in the antiseptic/mucolytic saline group } \\
(932 \pm 414 \mathrm{mg})\end{array}$ \\
\hline
\end{tabular}

CRS, Chronic Rhinosinusitis; RCT, Randomised Controlled Trial; CRSwNP, Chronic Rhinosinusitis with nasal polyps; dd, dose/day; RSOM-31, Rhinosinusitis Outcome Measure-31; SNOT-22, Sino-Nasal Outcome Test-22; PEA, Phenyl Ethyl Alcohol.

$\mathrm{Cl}$-1.36, -0.07); 98 participants; seven studies; $12=79 \%)$, scale range: 0 to 10 , lower $=$ better, indicating less disease in the patients that were treated with corticosteroid-eluting stents, spacers or dressings than the placebo (Figure 6.2.7.6.). Only one very small study evaluating off-label drug-eluting middle-meatal spacer of dexamethasone and Sinu-Foam ${ }^{\mathrm{TM}}$ in patients with CRSwNP did not show an improvement in endoscopic outcomes in the early postoperative period following $\operatorname{ESS}^{(865)}$.

For both POSE and Lund-Kennedy score there was a significant heterogeneity most likely caused by variations in the dosage of local corticosteroids: the studies reporting no effect used low potency corticosteroids like hydrocortisone ${ }^{(861)}$ and triamcinolone ${ }^{(466,860)}$, and the study by Zhao ${ }^{(482)}$ using mometasone furoate showed a dose response.

It can be concluded that postoperative placement of steroideluting stents, spacers and dressings compared to placebo does not have an influence on QOL and most likely also not on symptomatology but has a significant and most likely dose dependent effect on POSE and Lund-Kennedy scores. Whether the effect on POSE and Lund-Kennedy score (around 1 point of the mean around 5 (POSE) or 3 (LK) in the placebo group) is of imporatnce clinically remains unclear.

\subsection{Safety of corticosteroid-eluting implants}

Finally, the safety/adverse events associated with treatments with steroid-eluting stents, spacers and dressings were evaluated ${ }^{(121)}$. In this evaluation also spacers placed in the office are taken into account (see chapter 6.1.6.3; Table 6.2.7.3.) Three studies evaluated ocular safety ${ }^{(119,121,122)}$ and none showed clinically significant change in IOP and or modifications in lens opacity. Three studies evaluated systemic corticosteroid levels ${ }^{(121,467,866)}$. Hong found the serum cortisol levels to be significantly suppressed at postoperative days 1 and 2 and the 12-hour urine cortisol level at day 10 significantly lower in the local corticosteroid group compared to placebo ${ }^{(467)}$. All but one patient (taking inhaled asthma medication) had 24hour urine cortisol levels within the normal range at baseline and postoperative day 15 in the study of Adriaensen and the study of Murr showed no abnormalities in serum cortisol and mometasone furoate concentrations in five patients at 7, 14, 21 and 30 days postoperatively ${ }^{(121,467,866)}$.

Local (drug related) adverse events (seven studies) were limited and not reported more often in the local corticosteroid group than the placebo group.

\subsection{Conclusion}

It can be concluded that postoperative placement of steroid- 
Table 6.2.7.7. Postoperative saline irrigation with sodium hyaluronate in CRS patients.

\begin{tabular}{|c|c|c|c|c|c|}
\hline Study & Methods & Participants & Interventions & Outcomes & Results \\
\hline $\begin{array}{l}\text { Mozzanica } \\
2019^{(166)}\end{array}$ & DBPCT & $\begin{array}{l}56 \text { CRSsNP } \\
\text { patients } \\
\text { postoperatively }\end{array}$ & $\begin{array}{l}\text { - } 250 \mathrm{ml} \text { saline plus } 9 \mathrm{mg} \text { sodi- } \\
\text { um hyaluronate twice daily } \\
\text { for } 6 \text { weeks }(n=26) \\
\text { - Saline twice daily for } 6 \text { weeks } \\
(n=30)\end{array}$ & $\begin{array}{l}\text { At } 3 \text { and } 6 \text { weeks } \\
\text { postoperatively: } \\
\text { - SNOT-22 } \\
\text { - NOSE } \\
\text { - Symptoms (VAS) } \\
\text { - LK score }\end{array}$ & $\begin{array}{l}\text { - No differences in SNOT-22, NOSE, symptoms } \\
\text { and total LK score at } 6 \text { weeks } \\
\text { - Significantly better result of NOSE score at } 3 \\
\text { weeks in sodium hyaluronate group } \\
\text { - Significantly better result for headache and } \\
\text { smell alteration at three weeks in sodium } \\
\text { hyaluronate group } \\
\text { - Significantly reduced scar formation ( } 3 \text { and } \\
6 \text { weeks), crusting ( } 3 \text { weeks) and secretion (6 } \\
\text { weeks) }\end{array}$ \\
\hline $\begin{array}{l}\text { Cantone } \\
\text { 2014(185) }\end{array}$ & DBPCT & $\begin{array}{l}124 \text { CRSwNP } \\
\text { (122 evaluated) } \\
\text { postoperatively }\end{array}$ & $\begin{array}{l}\text { - Intranasal nebulization of } \\
9 \mathrm{mg} \text { sodium hyaluronate } \\
(3 \mathrm{~mL}) \text { plus saline solution } \\
(2 \mathrm{~mL}) \text { twice daily for } 30 \text { days } \\
(\mathrm{n}=62) \\
\text { - Intranasal nebulization of } \\
\text { saline }(5 \mathrm{~mL}) \text { twice daily for } \\
30 \text { days }(\mathrm{n}=60)\end{array}$ & $\begin{array}{l}\text { At } 30 \text { days } \\
\text { postoperatively: } \\
\text { - SF-36 } \\
\text { - SNOT-22 } \\
\text { - Symptoms (VAS) } \\
\text { - Endoscopic score }\end{array}$ & $\begin{array}{l}\text { Significantly better result of sodium hyaluronate } \\
\text { than saline for: } \\
\text { - SF36 } \\
\text { - SNOT-22 } \\
\text { - Total symptoms (VAS) } \\
\text { - Endoscopic score }\end{array}$ \\
\hline $\begin{array}{l}\text { Casale, } \\
2014^{(167)}\end{array}$ & $\begin{array}{l}\text { RCT } \\
\text { (single } \\
\text { blind?) }\end{array}$ & $\begin{array}{l}33 \text { CRS patients } \\
\text { postoperatively }\end{array}$ & $\begin{array}{l}\text { - Nebulization of } 3 \mathrm{ml} \text { contain- } \\
\text { ing } 9 \mathrm{mg} \text { sodium hyaluronate } \\
\text { nasal washes plus saline } \\
\text { twice daily for } 1 \text { month } \\
\text { ( } \mathrm{n}=18 \text { ) } \\
\text { - Nebulization of } 5 \mathrm{~mL} \text { saline } \\
\text { twice daily for } 1 \mathrm{month} \\
(\mathrm{n}=15)\end{array}$ & $\begin{array}{l}\text { At } 2 \text { and } 4 \text { weeks } \\
\text { postoperatively: } \\
\text { - CRS questionnaire } \\
\text { - Visual analogic scale } \\
\text { (VAS) } \\
\text { - Nasal endoscopy }\end{array}$ & $\begin{array}{l}\text { - VAS score of hyaluronate significantly lower } \\
\text { than saline at } 2 \text { and } 4 \text { weeks } \\
\text { - CRS score significantly lower than saline at } 2 \\
\text { and } 4 \text { weeks } \\
\text { - Significantly less crusts and oedema and } \\
\text { secretions }\end{array}$ \\
\hline $\begin{array}{l}\text { Gelardi } \\
2013^{(186)}\end{array}$ & $\begin{array}{l}\text { RCT } \\
\text { (single } \\
\text { blind?) }\end{array}$ & $\begin{array}{l}36 \text { CRSwNP } \\
\text { postoperative }\end{array}$ & $\begin{array}{l}\text { - Intranasal nebulization of } \\
\text { sodium hyaluronate } 9 \mathrm{mg} \text { in } \\
3 \mathrm{~mL} \text { twice daily for } 30 \text { days } \\
(\mathrm{n}=19) \\
\text { - Intranasal nebulization saline } \\
5 \mathrm{~mL} \text { twice daily for } 30 \text { days } \\
(\mathrm{n}=17)\end{array}$ & $\begin{array}{l}\text { At } 30 \text { days } \\
\text { postoperatively: } \\
\text { - Nasal obstruc-tion, } \\
\text { burning, dryness of the } \\
\text { mucosa (VAS) } \\
\text { - Presence or absence of } \\
\text { rhinorrhoea } \\
\text { - Mucociliary clearance } \\
\text { time } \\
\text { - Presence of exudate at } \\
\text { endoscopy }\end{array}$ & $\begin{array}{l}\text { Sodium hyaluronate group had compared to } \\
\text { saline: } \\
\text { - Significantly faster mucociliary clearance } \\
\text { - Lower incidence of rhinorrhoea } \\
\text { - Less nasal obstruction } \\
\text { - A lower incidence of exudate on endoscopy } \\
\text { - Better tolerability }\end{array}$ \\
\hline $\begin{array}{l}\text { Macchi } \\
2013^{(168)}\end{array}$ & DBPCT & $\begin{array}{l}46 \text { CRSwNP } \\
\text { patients } \\
\text { postoperatively }\end{array}$ & $\begin{array}{l}\text { - Nebulization of } 6 \mathrm{~mL} \text { contain- } \\
\text { ing } 9 \mathrm{mg} \text { sodium hyaluronate } \\
\text { nasal washes plus saline for } \\
15 \text { days/mo over } 3 \text { months } \\
\text { ( } \mathrm{n}=23 \text { ) } \\
\text { - Nebulization of } 6 \mathrm{~mL} \text { isotonic } \\
\text { saline for } 15 \text { days } / \mathrm{mo} \text { over } 3 \\
\text { months ( } \mathrm{n}=23 \text { ) }\end{array}$ & $\begin{array}{l}\text { At } 3 \text { months } \\
\text { postoperatively: } \\
\text { - Prevalence of symp- } \\
\text { toms } \\
\text { - Endoscopy } \\
\text { - Cytology } \\
\text { - Biofilm }\end{array}$ & $\begin{array}{l}\text { Significantly better result of sodium hyaluronate } \\
\text { than saline for: } \\
\text { - Nasal dyspnea (obstruction?) } \\
\text { - Normal mucosa } \\
\text { - Absence of secretion }\end{array}$ \\
\hline
\end{tabular}

CRS, Chronic Rhinosinusitis; DBPCT, Double Blind Placebo Controlled Trial; CRSsNP, Chronic Rhinosinusitis without nasal polyps; CRSwNP, Chronic Rhinosinusitis with nasal polyps; SNOT-22, Sino-Nasal Outcome Test-22; NOSE, Nasal Obstruction Sympton Evaluation; VAS, Visual Analogue Scale; LK, Lund-Kennedy; RCT, Randomised Controlled Trial; SF-36, Short Form 36.

Table 6.2.7.8.Postoperative saline irrigation with addition of honey in CRS patients.

\begin{tabular}{|c|c|c|c|c|c|}
\hline Study & Methods & Participants & Interventions & Outcomes & Results \\
\hline $\begin{array}{l}\text { Lee } \\
2016^{(169)}\end{array}$ & $\begin{array}{l}\text { Single blind } \\
\text { (investigator) } \\
\text { placebo } \\
\text { controlled } \\
\text { trial }\end{array}$ & $\begin{array}{l}49 \text { CRS patients } \\
\text { (42 analysed) } \\
\text { postoperatively }\end{array}$ & $\begin{array}{l}\text { - } 120 \mathrm{ml} \text { saline containing } 12 \mathrm{mg} \text { of manuka honey twice daily } \\
\text { for } 30 \text { days } \\
\text { - } 120 \mathrm{ml} \text { saline twice daily for } 30 \text { days } \\
\text { - All patients received culture-directed oral antibiotic, and/or } \\
\text { oral steroids for up to } 3 \text { weeks, and/or high-volume topical } \\
\text { steroid sinus irrigations (budesonide } 0.5 \mathrm{mg} / 2 \mathrm{~mL} \text { vial or } \\
0.6 \mathrm{mg} / 2 \mathrm{~mL} \text { capsule, } 0.5 \text { bottle to each nasal cavity twice daily) } \\
\text { polyps or inflammation for } 30 \text { days } \\
\text { - The dose of budesonide was determined when the patient } \\
\text { filled the prescription; if insurance covered it, then the vial ver- } \\
\text { sion at the } 0.5 \mathrm{mg} / 2 \mathrm{~mL} \text { dose was used, and if not covered, then } \\
\text { the capsule version at the } 0.6 \mathrm{mg} / 2 \mathrm{~mL} \text { dose was used }\end{array}$ & $\begin{array}{l}\text { At } 30 \text { days: } \\
\text { - SNOT-22 } \\
\text { - LK endoscopy } \\
\text { score } \\
\text { - Bacterial } \\
\text { culture }\end{array}$ & $\begin{array}{l}\text { - No significant } \\
\text { differences between } \\
\text { the groups } \\
\text { - In the subgroup not } \\
\text { using antibiotics } \\
\text { manuka honey } \\
\text { significantly } \\
\text { reduced bacteria }\end{array}$ \\
\hline
\end{tabular}


eluting stents, spacers and dressings compared to placebo does not have an influence on QOL and most likely also not on symptomatology but has a significant and most likely dose dependent effect on POSE and Lund-Kennedy scores. The EPOS2020 steering group felt the quality of the evidence was low and that based on the evidence they could not advise about the use of corticosteroid-eluting implants following endoscopic sinus surgery. For now, the use of relatively expensive readymade implants does not seem to be cost effective in the average CRSwNP patient. Larger studies including long term safety data are needed.

\subsubsection{Anti-leukotrienes}

Cysteinyl leukotrienes (CysLT) are a class of inflammatory mediators synthesized by eosinophils and mast cells through the breakdown of arachidonic acid. CysLT are known to play a role in the pathophysiology of rhinitis, asthma and possibly also CRSWNP, promoting bronchoconstriction, mucus production, oedema and chemotaxis of eosinophils and neutrophils.

Overproduction of CysLTs and upregulation of the receptor has been demonstrated in allergic rhinitis, asthma, and CRS with nasal polyposis (CRSwNP) (see chapter 5.1.2).

The quality of the evidence comparing montelukast with placebo is very low (see chapter 6.1.9.).

The only study evaluating the use of montelukast postoperatively is done by Van Gerven et al. ${ }^{(144)}$. They evaluated the efficacy of montelukast as an add-on treatment to INCS in 72 postoperative CRSwNP patients for one year and found no significant differences between the two treatment arms in total symptom score, nasal polyp score and LM score. They concluded that the addition of montelukast to INCS should not be recommended in the treatment of postoperative CRSwNP patients.

\subsubsection{Decongestants}

The use of topical decongestants has the theoretical potential to reduce mucosal oedema by inducing vasoconstriction ${ }^{(149)}$.
However, prolonged use can lead to rhinitis medicamentosa and worse symptoms of nasal obstruction upon decongestant discontinuation ${ }^{(150)}$. Nasal decongestion per se does not have effect on nasal polyp size ${ }^{(151)}$. There is one small study in CRSwNP patients showing a significantly better effect of oxymetazoline combined with MFNS than MFNS alone without inducing rebound swelling ${ }^{(152)}$. This is consistent with effects seen in the treatment of allergic rhinitis where the combination of nasal corticosteroid with decongestant also seemed to prevent rebound swelling ${ }^{(153,154)}$ (see chapter 6.1.10). There is one by Humphreys et al. who compared topical decongestant, xylometazoline, to a saline spray in 47 patients in the FESS early postoperative period ${ }^{(155)}$. Postoperative VAS symptom scores for nasal obstruction, rhinorrhoea, pain, loss of sense of smell and bleeding assessed at day 10 postoperatively did not show any difference between the groups. The EPOS2020 steering group suggests not to use nasal decongestants in the postoperative period although the quality of the evidence is low.

\subsubsection{Saline}

\subsection{Summary of the evidence}

Nasal saline irrigation is considered to be an important aspect of the management of CRS, especially in the postoperative phase. For details on saline irrigation in general please refer to chapter 6.1.11.

This systematic review identified 13 randomized controlled (RCT) studies evaluating nasal saline irrigation on clinical outcomes in adult CRS in the month after FESS (Tables 6.2.7.4.6.2.7.9). The studies were very different in design, duration and outcome measurements.

Three studies evaluated the effect of a form of saline irrigation versus a form of no irrigation of the nose (Table 6.2.7.4.). The studies could not be combined in a meta-analysis. One showed a significant positive effect of saline atomisation on discharge, but no other differences, one showed mainly positive effect in patients with mild CRS and not in patients with severe effect and

Table 6.2.7.9. Postoperative saline irrigation with addition of dexpanthenol in the treatment of CRS.

\begin{tabular}{|c|c|c|c|c|c|}
\hline Study & Methods & Participants & Interventions & Outcomes & Results \\
\hline $\begin{array}{l}\text { Tantilipikorn } \\
2012^{(171)}\end{array}$ & DBPCT & $\begin{array}{l}50 \text { CRS patients } \\
\text { postoperatively }\end{array}$ & $\begin{array}{l}\text { - Dexpanthenol nasal } \\
\text { spray } 4 \text { times a day for } 6 \\
\text { weeks ( } n=25) \\
\text { - Saline nasal spray } 4 \\
\text { times a day for } 6 \text { weeks } \\
(n=25)\end{array}$ & $\begin{array}{l}\text { - } \% \text { of patients with symp- } \\
\text { toms at 2, } 4 \text { and } 6 \text { wks } \\
\text { - } \% \text { of patients with endo- } \\
\text { scopic abnormalities at 2, } \\
4 \text { and } 6 \text { wks } \\
\text { - Mucociliary clearance time } \\
\text { at } 6 \text { wks }\end{array}$ & $\begin{array}{l}\text { - Majority of symptoms and signs did not } \\
\text { show significant differences } \\
\text { - Dexpanthenol nasal spray has superior } \\
\text { efficacy compared with normal saline } \\
\text { nasal spray on improvement of mucociliary } \\
\text { clearance and smell at } 6 \text { wks and inferior } \\
\text { effect on nasal discharge }\end{array}$ \\
\hline $\begin{array}{l}\text { Fooanant } \\
2008^{(187)}\end{array}$ & $\mathrm{RCT}$ & $\begin{array}{l}128 \text { CRS patients } \\
\text { (110 evaluated) } \\
\text { postoperatively }\end{array}$ & $\begin{array}{l}\text { Dexpanthenol (5\%) in } \\
\text { sea water nasal spray } 2 \\
\text { puffs per nostril, twice a } \\
\text { day for } 4 \text { weeks } \\
\text { - Saline nasal irrigation } \\
\text { (volume unclear) twice a } \\
\text { day for } 4 \text { weeks }\end{array}$ & $\begin{array}{l}\text { At 1,2,3,4,6 and } 12 \text { weeks: } \\
\text { - Total nasal symptom score } \\
\text { - Symptoms } \\
\text { - Nasal crusts } \\
\text { - Mucociliary clearance } \\
\text { (only at } 4 \text { wks) }\end{array}$ & $\begin{array}{l}\text { - No significant differences in total nasal } \\
\text { symptom score, nasal crusts (but at wk } 2 \\
\text { less in saline group), mucociliary clearance } \\
\text { (but for subgroup of CRSwNP) }\end{array}$ \\
\hline
\end{tabular}

DBPCT, Double Blind Placebo Controlled Trial; RCT, Randomised Controlled Trial; CRS, Chronic Rhinosinusitis; CRSwNP, Chronic Rhinosinusitis with nasal polyps. 
one did not show any differences between saline rinsing or no rinsing $(158,175,178)$.

One study in 86 CRS patients compared a squeeze bottle with a nasal spray for one month and found no differences between the SNOT-22, Nasal and Sinus Symptom Scale (NSS) or POSE between the two ways of administration ${ }^{(162)}$.

Two small studies evaluated the addition of antiseptic / mucolytic / baby shampoo solution and showed no differences between the groups but the baby-shampoo group was reported to have significantly more side effects and had more patients stop the solution ${ }^{(160,183)}$ (Table 6.2.7.6.). Moreover, it has been shown in healthy persons to create congestion and reversible smell loss ${ }^{(190)}$.

Five Italian DBPCT or single blind (blinding often not clearly described) postoperative studies evaluated the addition of $9 \mathrm{mg}$ of sodium hyaluronate to saline $\mathrm{e}^{(166-168,185,186)}$. Four studies evaluated nebulisation with a small volume ${ }^{(167,168,185,186)}$ and one study rinsing with $250 \mathrm{ml}$. The four studies evaluating nebulization in general reported positive outcomes, the study evaluation addition of sodium hyaluronate to saline rinsing found in general no significant effect (Table 6.2.7.7.).

In conclusion, sodium hyaluronate might have a positive effect on postoperative symptoms when compared to a saline spray. Adding sodium hyaluronate to saline rinsing postoperatively did not show an effect in one study. One study ${ }^{(169)}$ evaluated the addition of honey to saline irrigation. The study did find any relevant differences between the honey treated group and the placebo group (Table 6.2.7.8.).

Dexpanthenol is an analogue of pantothenic acid, which has been claimed to promote wound healing ${ }^{(194)}$. It has been shown to improve symptoms in patients with atrophic rhinitis ${ }^{(195)}$. Two studies evaluated the effect of dexpanthenol in saline ${ }^{(171,187)}$. Both studies showed no effect on the majority of symptoms and endoscopy but a superior efficacy over placebo on mucociliary clearance (in the Fooanant study only in patients with CRSwNP) (Table 6.2.7.9.).

Adverse effects of saline irrigations are rare, but include local irritation, ear pain, nosebleeds, headache, nasal burning, and nasal drainage are mentioned. The use of baby shampoo has been shown to create congestion and reversible smell loss.

\subsection{Conclusions}

The quality of the evidence evaluating the use of nasal irrigation in the postoperative phase after FESS is low. Nasal irrigation with isotonic saline or Ringer's lactate seems to be effective in CRS patients. There is insufficient data to show that large volume is more effective than nasal spray. Addition of sodium hyaluronate to nasal saline rinsing may have a positive effect in the postoperative period. Addition of baby shampoo, honey, or dexpanthenol as well as higher temperature and higher salt concentration do not show additional benefit. The steering group advises the use of nasal saline irrigation with isotonic saline or Ringer's lactate potentially with the addition sodium hyaluronate and against the use of baby shampoo and hypertonic saline solutions due to side effects in the postoperative period after FESS. However, larger well designed studies are necessary to improve the quality of our understanding.

\subsubsection{Antimycotics}

Local and systemic antifungal treatments do not have a positive effect of QOL, symptoms and signs of disease in patients with CRS. There is one study evaluating direct postoperative use of local amphotericin B nasal spray versus placebo in 33 CRSwNP patients. Eight weeks postoperatively they found no differences in the QOL questionnaire SNAQ-11, nasal endoscopy and/or CT $\operatorname{scan}^{(224)}$. The EPOS2020 steering group advises against the use of postoperative anti-mycotics in CRS.

\subsubsection{Capsaicin}

The quality of the evidence about the effect of capsaicin versus placebo is low (see chapter 6.1.22). Zheng et al. ${ }^{(282)}$, conducted a DBPCT comparing capsaicin $\left(3 \times 10^{-6} \mathrm{~mol}\right.$, dissolved in $70 \%$ ethanol) versus vehicle alone ( $70 \%$ ethanol) once a week in 51 patients for five weeks, following nasal polypectomy. Patients were assessed at nine months for nasal resistance, rhinorrhoea and nasal polyp score. The capsaicin group had a significant decrease in nasal resistance compared to the vehicle group $(p<0.01)$ and had lower nasal polyp score when compared to the control group (stage $0: 41 \%$ vs $4.5 \%, \mathrm{p}<0.01$ ). There was no difference in subjective rhinorrhoea.

A small study showed a significantly decrease in nasal resistance and nasal polyp score after five weeks of postoperative capsaicin treatment versus placebo. The quality of the evidence is low and the EPOS2020 steering group concludes that capsaicin may be an option in postoperative treatment of CRS in patients with CRSwNP but that larger studies are needed.

\subsubsection{Conclusion}

The quality of the evidence for the use of commonly administered peri-operative medication such as (nasal) corticosteroids and antibiotics is low, mainly due to insufficient and small studies. The use of peri-operative corticosteroids reduces blood loss and operation time and improves the quality of the surgical field. There is also high-quality evidence that long term use of nasal corticosteroids is effective and safe for treating patients with CRS with significant impact on nasal symptoms and quality of life improvement. Nasal corticosteroids reduce nasal polyp size and when administered after endoscopic sinus surgery, nasal corticosteroids prevent polyp recurrence. Although the quality of direct evidence postoperatively is missing, the EPOS2020 steering committee recommends using nasal corticosteroids postoperatively. Based on the low positive evidence and the excellent safety profile the group also recommends using nasal saline irrigation with isotonic saline or Ringer's lactate, potentially with the addition of sodium hyaluronate, and advise against the use of baby shampoo and hypertonic saline solutions due to side effects in the postoperative period after FESS. Otherwise, based on the low-quality evidence, the steering group cannot recommend the use of peri-operative antibiotics. The group also advises against the use of anti-mycotics. 


\section{References}

1. Lee LN, Bhattacharyya N. Regional and specialty variations in the treatment of chronic rhinosinusitis. Laryngoscope. 2011;121:1092-7.

2. Hopkins C, Williamson E, Morris S, et al. Antibiotic usage in chronic rhinosinusitis: analysis of national primary care electronic health records. Rhinology. 2019;57:420-9.

3. Sabino HA, Valera FC, Aragon DC, et al. Amoxicillinclavulanate for patients with acute exacerbation of chronic rhinosinusitis: a prospective, doubleblinded, placebo-controlled trial. Int Forum Allergy. \& Rhinol.2017;7:135-42.

4. Van Zele T, Gevaert P, Holtappels G, et al. Oral steroids and doxycycline: Two different approaches to treat nasal polyps. J Allergy Clin Immunol. 2010;125:1069-76.e4.

5. Legent F, Bordure P, Beauvillain C, Berche P. A double-blind comparison of ciprofloxacin and amoxycillin/clavulanic acid in the treatment of chronic sinusitis. Chemotherapy. 1994;40:8-15.

6. Amini M, Yarmohammadi M, Izadi P. A comparing study of clarithromycin XL with co-amoxiclav for treatment of chronic sinusitis; A clinical trial. Iranian J of Clinical Infectious Diseases. 2009;4:197201.

7. Fan $\mathrm{Y}, \mathrm{Xu} \mathrm{R}$, Hong $\mathrm{H}$, et al. High and low doses of clarithromycin treatment are associated with different clinical efficacies and immunomodulatory properties in chronic rhinosinusitis. J Laryngol. Otol. 2014;128:236-41.

8. Namysłowski G, Misiołek M, Małafiej E, et al. Randomized clinical trial comparing the efficacy and safety of Augmentin versus cefuroxime in the treatment of chronic sinusitis in adult patients. Med Sci Monit .1998;4:1551-554.

9. Namyslowski G, Misiolek M, Czecior E, et al. Comparison of the efficacy and tolerability of amoxycillin/clavulanic acid $875 \mathrm{mg}$ b.i.d. with cefuroxime $500 \mathrm{mg}$ b.i.d. in the treatment of chronic and acute exacerbation of chronic sinusitis in adults. J of chemotherapy (florence, italy) 2002;14:508-17.

10. Namysłowski G, Misiołek M, Małafiej E, Czecior E, Orecka B, Woch G. Randomized clinical trial comparing the efficacy and safety of Augmentin versus cefuroxime in the treatment of chronic sinusitis in adult patients. Med Sci Monit.1998;4:551-4.

11. Huck W, Reed BD, Nielsen RW, et al. Cefaclor vs amoxicillin in the treatment of acute, recurrent, and chronic sinusitis. Arch Fam Med. 1993;2:497503.

12. Jareoncharsri P, Bunnag C, Fooanant S, et al. An open label, randomized comparative study of levofloxacin and amoxicillin/clavulanic acid in the treatment of purulent sinusitis in adult Thai patients. Rhinology. 2004;42:23-9.

13. Videler WJ, Badia L, Harvey RJ, et al. Lack of efficacy of long-term, low-dose azithromycin in chronic rhinosinusitis: a randomized controlled trial. Allergy. 2011;66:1457-68.

14. Wallwork B, Coman W, Mackay-Sim A, Greiff L, Cervin A. A double-blind, randomized, placebocontrolled trial of macrolide in the treatment of chronic rhinosinusitis. Laryngoscope. 2006;116:189-93.
15. Ichimura K, Shimazaki Y, Ishibashi T, Higo R. Effect of new macrolide roxithromycin upon nasal polyps associated with chronic sinusitis. Auris Nasus Larynx. 1996;23:48-56.

16. Scadding GK, Lund VJ, Darby YC. The effect of long-term antibiotic therapy upon ciliary beat frequency in chronic rhinosinusitis. J Laryngol. Otol. 1995;109:24-6.

17. Suzuki H, Shimomura A, Ikeda K, Oshima T, Takasaka T. Effects of long-term low-dose macrolide administration on neutrophil recruitment and IL-8 in the nasal discharge of chronic sinusitis patients. Tohoku J Exp Med. 1997;182:115-24.

18. Hashiba M, Baba S. Efficacy of long-term administration of clarithromycin in the treatment of intractable chronic sinusitis. Acta Otolaryngol. Suppl (Stockh) 1996;525:73-8.

19. Rubin BK, Druce $H$, Ramirez OE, Palmer R. Effect of clarithromycin on nasal mucus properties in healthy subjects and in patients with purulent rhinitis. Am J Respir Crit Care Med. 1997;155:201823.

20. Cervin A, Kalm O, Sandkull P, Lindberg S. One-year low-dose erythromycin treatment of persistent chronic sinusitis after sinus surgery: clinical outcome and effects on mucociliary parameters and nasal nitric oxide. Otolaryngol. Head Neck Surg. 2002;126:481-9.

21. Shen S, Lou H, Wang C, Zhang L. Macrolide antibiotics in the treatment of chronic rhinosinusitis: evidence from a meta-analysis. J Thorac Dis. 2018;10:5913-23.

22. Wolter J, Seeney S, Bell S, Bowler S, Masel P, McCormack J. Effect of long term treatment with azithromycin on disease parameters in cystic fibrosis: a randomised trial. Thorax. 2002;57:212-6.

23. Equi AC, Davies JC, Painter $H$, et al. Exploring the mechanisms of macrolides in cystic fibrosis. Respir Med. 2006;100:687-97.

24. Saiman L, Marshall BC, Mayer-Hamblett N, et al. Azithromycin in patients with cystic fibrosis chronically infected with Pseudomonas aeruginosa: a randomized controlled trial. JAMA. 2003;290:1749-56.

25. Zeng $M$, Long XB, Cui YH, Liu Z. Comparison of efficacy of mometasone furoate versus clarithromycin in the treatment of chronic rhinosinusitis without nasal polyps in Chinese adults. American J Rhinol \& Allergy. 2011;25:e2037.

26. Zeng $\mathrm{M}$, Wang $\mathrm{H}$, Liao $\mathrm{B}$, et al. Comparison of efficacy of fluticasone propionate versus clarithromycin for postoperative treatment of different phenotypic chronic rhinosinusitis: a randomized controlled trial. Rhinology. 2019;57:101-9.

27. Huang Z, Zhou B. Clarithromycin for the treatment of adult chronic rhinosinusitis: a systematic review and meta-analysis. Int Forum Allergy. Rhinol. 2019;9:545-55.

28. Suzuki H, Ikeda K, Honma R, et al. Prognostic factors of chronic rhinosinusitis under longterm low-dose macrolide therapy. ORL J Otorhinolaryngol. Relat Spec. 2000;62:121-7.

29. Haxel BR, Clemens M, Karaiskaki N, Dippold U, Kettern L, Mann WJ. Controlled trial for long-term low-dose erythromycin after sinus surgery for chronic rhinosinusitis. Laryngoscope. 2015;125:1048-55.

30. Amali A, Saedi B, Rahavi-Ezabadi S, Ghazavi $\mathrm{H}$, Hassanpoor N. Long-term postoperative azithromycin in patients with chronic rhinosinusitis: A randomized clinical trial. Am J Rhinol.Allergy. 2015;29:421-4.

31. Peric AV, Baletic N, Milojevic M, et al. Effects of preoperative clarithromycin administration in patients with nasal polyposis. West Indian Med J 2014;63:721-7.

32. Ragab SM, Lund VJ, Scadding G. Evaluation of the medical and surgical treatment of chronic rhinosinusitis: a prospective, randomised, controlled trial. Laryngoscope. 2004;114:923-30.

33. Ragab SM, Lund VJ, Scadding G, Saleh HA, Khalifa MA. Impact of chronic rhinosinusitis therapy on quality of life: a prospective randomized controlled trial. Rhinology. 2010;48:305-11.

34. Jiang RS, Wu SH, Tsai CC, Li YH, Liang KL. Efficacy of Chinese herbal medicine compared with a macrolide in the treatment of chronic rhinosinusitis without nasal polyps. American J Rhinol \& Allergy.. 2012;26:293-7.

35. Hashiba M. Clinical efficacy of long-term macrolides therapy for chronic sinusitis Comparison between erythromycin and clarithromycin. Practica otologica 1997;90:717-27.

36. Cornett E, Novitch MB, Kaye AD, et al. Macrolide and fluoroquinolone mediated cardiac arrhythmias: clinical considerations and comprehensive review. Postgrad Med 2017;129:715-24.

37. Abo-Salem E, Fowler JC, Attari M, et al. Antibioticinduced cardiac arrhythmias. Cardiovasc Ther 2014;32:19-25.

38. Jespersen CM, Als-Nielsen B, Damgaard M, et al. Randomised placebo controlled multicentre trial to assess short term clarithromycin for patients with stable coronary heart disease: CLARICOR trial. BMJ 2006;332:22-7.

39. Mosholder AD, Lee JY, Zhou EH, et al. Long-Term Risk of Acute Myocardial Infarction, Stroke, and Death With Outpatient Use of Clarithromycin: A Retrospective Cohort Study. Am J Epidemiol 2018;187:786-92.

40. Schembri S, Williamson PA, Short PM, et al. Cardiovascular events after clarithromycin use in lower respiratory tract infections: analysis of two prospective cohort studies. BMJ 2013;346:f1235.

41. Winkel P, Hilden J, Hansen JF, et al. Clarithromycin for stable coronary heart disease increases all-cause and cardiovascular mortality and cerebrovascular morbidity over 10years in the CLARICOR randomised, blinded clinical trial. Int J Cardiol 2015;182:459-65.

42. Andersen SS, Hansen ML, Norgaard ML, et al. Clarithromycin use and risk of death in patients with ischemic heart disease. Cardiology 2010;116:89-97.

43. Inghammar M, Nibell O, Pasternak B, Melbye M, Svanstrom H, Hviid A. Long-Term Risk of Cardiovascular Death With Use of Clarithromycin and Roxithromycin: A Nationwide Cohort Study. Am J Epidemiol 2018;187:777-85.

44. Root AA, Wong AY, Ghebremichael-Weldeselassie 
$\mathrm{Y}$, et al. Evaluation of the risk of cardiovascular events with clarithromycin using both propensity score and self-controlled study designs. $\mathrm{Br} J \mathrm{Clin}$ Pharmacol 2016;82:512-21.

45. Wong AY, Root A, Douglas IJ, et al. Cardiovascular outcomes associated with use of clarithromycin: population based study. BMJ 2016;352:h6926.

46. Williamson E, Denaxas S, Morris S, et al. Risk of mortality and cardiovascular events following macrolide prescription in chronic rhinosinusitis patients: a cohort study using linked primary care electronic health records. Rhinology. 2019, 10.4193/Rhin18.237.

47. Wong AYS, Chan EW, Anand S, Worsley AJ, Wong ICK. Managing Cardiovascular Risk of Macrolides: Systematic Review and Meta-Analysis. Drug Saf 2017;40:663-77.

48. Svanstrom $H$, Pasternak B, Hviid A. Use of clarithromycin and roxithromycin and risk of cardiac death: cohort study. BMJ 2014;349:g4930.

49. Philpott C, le Conte S, Beard D, et al. Clarithromycin and endoscopic sinus surgery for adults with chronic rhinosinusitis with and without nasal polyps: study protocol for the MACRO randomised controlled trial. Trials 2019;20:246.

50. Woodhouse BM, Cleveland KW. Nebulized antibiotics for the treatment of refractory bacterial chronic rhinosinusitis. Ann Pharmacother 2011;45:798-802.

51. Biswas K, Cavubati R, Gunaratna S, et al. Comparison of Subtyping Approaches and the Underlying Drivers of Microbial Signatures for Chronic Rhinosinusitis. mSphere 2019;4.

52. Vaughn A, Shaver C, Clark D. Association Between Culture and Culture-Independent Microtyping in Recalcitrant Chronic Rhinosinusitis. Ear Nose Throat J 2019;98:94-7.

53. Sykes DA, Wilson R, Chan KL, Mackay IS, Cole PJ. Relative importance of antibiotic and improved clearance in topical treatment of chronic mucopurulent rhinosinusitis. A controlled study. Lancet 1986;2:359-60.

54. Kobayashi T, Baba S. Topical use of antibiotics for paranasal sinusitis. Rhinol Suppl 1992;14:77-81.

55. Desrosiers MY, Salas-Prato M. Treatment of chronic rhinosinusitis refractory to other treatments with topical antibiotic therapy delivered by means of a large-particle nebulizer: results of a controlled trial. Otolaryngology--Head \& Neck Surg.2001;125:2659.

56. Videler WJM, van Drunen CM, Reitsma JB, Fokkens WJ. Nebulized bacitracin/colimycin: a treatment option in recalcitrant chronic rhinosinusitis with Staphylococcus aureus? A double-blind, randomized, placebo-controlled, cross-over pilot study. Rhinology. 2008;46:92-8.

57. Jervis-Bardy J, Boase S, Psaltis A, Foreman A, Wormald PJ. A randomized trial of mupirocin sinonasal rinses versus saline in surgically recalcitrant staphylococcal chronic rhinosinusitis. Laryngoscope. 2012;122:2148-53.

58. Shikani AH, Kourelis K, Alqudah MA, et al. Multimodality topical therapy for refractory chronic rhinosinusitis: Our experience in thirteen patients with and twelve patients without nasal polyps. Clin Otolaryngol. 2013;38:254-8.
59. Bonfils P, Escabasse V, Coste A, et al. Efficacy of tobramycin aerosol in nasal polyposis. Eur Ann Otorhinolaryngol. Head Neck Dis 2015;132:119-23.

60. Workman AD, Maina IW, Brooks SG, et al. The Role of Quinine-Responsive Taste Receptor Family 2 in Airway Immune Defense and Chronic Rhinosinusitis. Front Immunol.2018;9:624.

61. Day N, Mainardi JL, Malinvaud D, Bonfils P. [Bacteriological study of ethmoid specimens from patients with nasal polyposis after ethmoidal surgery]. Ann Otolaryngol. Chir Cervicofac 2009;126:196-202.

62. Anand V, Levine $H$, Friedman $M$, et al. Intravenous antibiotics for refractory rhinosinusitis in nonsurgical patients: Preliminary findings of a prospective study. Am J Rhinol.2003;17:363-8.

63. Fokkens WJ, Lund VJ, Mullol J, et al. European Position Paper on Rhinosinusitis and Nasal Polyps 2012. Rhinol Suppl 2012;23:3 p preceding table of contents, 1-298.

64. Furukido K, Takeno S, Ueda T, Yajin K. Cytokine profile in paranasal effusions in patients with chronic sinusitis using the YAMIK sinus catheter with and without betamethasone. Eur Arch Otorhinolaryngol. 2005;262:50-4.

65. Lavigne F, Cameron L, Renzi PM, et al. Intrasinus administration of topical budesonide to allergic patients with chronic rhinosinusitis following surgery. Laryngoscope. 2002;112:858-64.

66. Kiris M, Muderris T, Yalciner G, Bercin S, Sevil E, Gul F. Intrapolyp steroid injection for nasal polyposis: randomized trial of safety and efficacy. Laryngoscope. 2016:1730-5.

67. Harvey RJ, Snidvongs K, Kalish LH, Oakley GM, Sacks R. Corticosteroid nasal irrigations are more effective than simple sprays in a randomized double-blinded placebo-controlled trial for chronic rhinosinusitis after sinus surgery. Int Forum Allergy. \& Rhinol.2018;8:461-70.

68. Demirel T, Orhan KS, Keleş N, Değer K. Comparison of the efficacy of nasal drop and nasal spray applications of fluticasone propionate in nasal polyps. Kulak burun bogaz ihtisas dergisi : KBB [J of ear, nose, and throat] 2008;18:1-6.

69. Tos M, Svendstrup F, Arndal H, et al. Efficacy of an aqueous and a powder formulation of nasal budesonide compared in patients with nasal polyps. Am J Rhinol.1998;12:183-9.

70. Johansen L, Illum P, Kristensen S, Winther L, Petersen S, Synnerstad B. The effect of budesonide (Rhinocort $\neg \mathbb{E}$ ) in the treatment of small and medium-sized nasal polyps. Clin Otolaryngol. Allied Sci 1993;18:524-7.

71. Djupesland PG, Vlckova I, Hewson G. Impact of baseline nasal polyp size and previous surgery on efficacy of fluticasone delivered with a novel device: a subgroup analysis. American J of Rhinology. \& Allergy. 2010;24:291-5.

72. Vlckova I, Navratil P, Kana R, Pavlicek P, Chrbolka P, Djupesland PG. Effective treatment of mildto-moderate nasal polyposis with fluticasone delivered by a novel device. Rhinology. 2009;47:419-26

73. Leopold DA, Elkayam D, Messina JC, et al. NAVIGATE II: randomized, double-blind trial of the exhalation delivery system with fluticasone for nasal polyposis. J Allergy Clin Immunol.2019;143:126-34.e5.

74. Kobayashi Y, Yasuba H, Asako M, et al. HFA-BDP Metered-Dose Inhaler Exhaled Through the Nose Improves Eosinophilic Chronic Rhinosinusitis With Bronchial Asthma: a Blinded, Placebo-Controlled Study. Front Immunol.2018;9:2192.

75. Dixon $\mathrm{AE}$, Castro M, Cohen Rl, et al. Efficacy of nasal mometasone for the treatment of chronic sinonasal disease in patients with inadequately controlled asthma. J Allergy Clin Immunol.2015;135:701-9.e5.

76. Nordin S, Olsson P, Hedén Blomqvist E, Stjärne $P$, Ehnhage A. Effects of FESS and additional fluticasone propionate nasal drops on psychological well-being in nasal polyposis with asthma. Acta Otolaryngol. 2013;133:939-43.

77. Olsson P, Ehnhage A, Nordin S, Stjarne P. Quality of life is improved by endoscopic surgery and fluticasone in nasal polyposis with asthma. Rhinology. 2010;48:325-30.

78. Hansen FS, Djupesland PG, Fokkens WJ. Preliminary efficacy of fluticasone delivered by a novel device in recalcitrant chronic rhinosinusitis. Rhinology. 2010;48:292-9.

79. Lund VJ, Flood J, Sykes AP, Richards DH. Effect of fluticasone in severe polyposis. Arch Otolaryngol. Head \& Neck Surg. 1998;124:513-8.

80. Stjärne $P$, Blomgren $K$, Cayé-Thomasen $P$, et al. The efficacy and safety of once-daily mometasone furoate nasal spray in nasal polyposis: A randomized, double-blind, placebo-controlled study. Acta Otolaryngol. 2006;126:606-12.

81. Tait S, Kallogjeri D, Suko J, Kukuljan S, Schneider J, Piccirillo JF. Effect of Budesonide Added to LargeVolume, Low-pressure Saline Sinus Irrigation for Chronic Rhinosinusitis: A Randomized Clinical Trial. JAMA Otolaryngol. Head Neck Surg. 2018;144:60512.

82. Rawal RB, Deal AM, Ebert C. S. J, et al. Postoperative budesonide irrigations for patients with polyposis: a blinded, randomized controlled trial. Rhinology. 2015;53:227-34.

83. Rotenberg BW, Zhang I, Arra I, Payton KB. Postoperative care for Samter's triad patients undergoing endoscopic sinus surgery: a double-blinded, randomized controlled trial. Laryngoscope. 2011;121:2702-5.

84. Hopkins C, Rudmik L, Lund VJ. The predictive value of the preoperative Sinonasal Outcome Test-22 score in patients undergoing endoscopic sinus surgery for chronic rhinosinusitis. Laryngoscope. 2015;125:1779-84.

85. Wang $C$, Lou $H$, Wang $X$, et al. Effect of budesonide transnasal nebulization in patients with eosinophilic chronic rhinosinusitis with nasal polyps. J Allergy Clin Immunol.2015;135:922-9.e6.

86. Harvey RJ, Goddard JC, Wise SK, Schlosser RJ. Effects of endoscopic sinus surgery and delivery device on cadaver sinus irrigation. Otolaryngol. Head Neck Surg. 2008;139:137-42.

87. Harvey RJ, Wallwork BD, Lund VJ. Antiinflammatory effects of macrolides: applications in chronic rhinosinusitis. Immunol.Allergy. Clin North Am 2009;29:689-703.

88. Snidvongs K, Pratt E, Chin D, Sacks R, Earls P, 
Harvey RJ. Corticosteroid nasal irrigations after endoscopic sinus surgery in the management of chronic rhinosinusitis. Int Forum Allergy. Rhinol. 2012;2:415-21.

89. Snidvongs K, Kalish L, Sacks R, Sivasubramaniam R, Cope D, Harvey RJ. Sinus surgery and delivery method influence the effectiveness of topical corticosteroids for chronic rhinosinusitis: systematic review and meta-analysis. American J of Rhinology. \& Allergy. 2013;27:221-33.

90. Mösges R, Bachert C, Rudack C, et al. Efficacy and safety of mometasone furoate nasal spray in the treatment of chronic rhinosinusitis. Adv Ther 2011;28:238-49.

91. Parikh A, Scadding GK, Darby Y, Baker RC. Topical corticosteroids in chronic rhinosinusitis: a randomized, double-blind, placebo-controlled trial using fluticasone propionate aqueous nasal spray. Rhinology. 2001;39:75-9.

92. Rowe-Jones JM, Medcalf M, Durham SR, Richards DH, Mackay IS. Functional endoscopic sinus surgery: 5 year follow up and results of a prospective, randomised, stratified, doubleblind, placebo controlled study of postoperative fluticasone propionate aqueous nasal spray. Rhinology. 2005;43:2-10.

93. Jorissen M, Bachert C. Effect of corticosteroids on wound healing after endoscopic sinus surgery. Rhinology. 2009;47:280-6.

94. Lund VJ, Mackay IS. Staging in rhinosinusitus. Rhinology. 1993;31:183-4.

95. Ehnhage A, Olsson P, Kolbeck KG, et al. Functional endoscopic sinus surgery improved asthma symptoms as well as PEFR and olfaction in patients with nasal polyposis. Allergy. 2009;64:762-9.

96. Filiaci F, Passali D, Puxeddu R, Schrewelius C. A randomized controlled trial showing efficacy of once daily intranasal budesonide in nasal polyposis. Rhinology. 2000;38:185-90.

97. Holmstrom M, Holmström M. Clinical performance of fluticasone propionate nasal drops. Allergy. 1999;54:21-5.

98. Jankowski R, Schrewelius C, Bonfils P, et al. Efficacy and tolerability of budesonide aqueous nasal spray treatment in patients with nasal polyps. Arch Otolaryngol. Head \& Neck Surg. 2001;127:447-52.

99. Jankowski R, Klossek JM, Attali V, Coste A, Serrano E. Long-term study of fluticasone propionate aqueous nasal spray in acute and maintenance therapy of nasal polyposis. Allergy. 2009;64:944-50.

100. Keith P, Nieminen J, Hollingworth K, Dolovich J. Efficacy and tolerability of fluticasone propionate nasal drops 400 microgram once daily compared with placebo for the treatment of bilateral polyposis in adults. Clin Exp Allergy. 2000;30:14608.

101. Lildholdt T, Rundcrantz H, Lindqvist N. Efficacy of topical corticosteroid powder for nasal polyps: a double-blind, placebo-controlled study of budesonide. Clin Otolaryngol. Allied Sci 1995;20:26-30.

102. Pentilla MA. Endoscopic findings after functional and radical sinus surgery: a prospective randomized study. Am J Rhinol.1994;8:71-6.

103. Small CB, Hern, Ez J, et al. Efficacy and safety of mometasone furoate nasal spray in nasal polyposis. J Allergy Clin Immunol.2005;116:1275-81.

104. Stjärne $P, M o ̈ s g e s ~ R$, Jorissen $M$, et al. A Randomized Controlled Trial of Mometasone Furoate Nasal Spray for the Treatment of Nasal Polyposis. Arch Otolaryngol. Head Neck Surg. 2006;132:179-85.

105. Vento SI, Vento K, Blomgren K, Hytönen M, Simola $M$, Malmberg H. Prevention of relapses of nasal polyposis with intranasal triamcinolone acetonide after polyp surgery: a prospective double-blind, placebo-controlled, randomised study with a 9-month follow-up. Clin Otolaryngol. 2012;37:11723.

106. Zhou B, He G, Liang J, et al. Mometasone furoate nasal spray in the treatment of nasal polyposis in Chinese patients: a double-blind, randomized, placebo-controlled trial. Int Forum Allergy. Rhinol. 2016;6:88-94

107. Venkatesan N, Lavigne P, Lavigne F, Hamid Q. Effects of fluticasone furoate on clinical and immunological outcomes (IL-17) for patients with nasal polyposis naive to steroid treatment. Annals of Otol, Rhin, and Laryngol.2016;125:213-8.

108. Meltzer EO, Hamilos DL, Hadley JA, et al. Rhinosinusitis: Developing guidance for clinical trials. Otolaryngol. Head Neck Surg. 2006;135:S3180.

109. Holmberg K, Juliusson S, Balder B, Smith DL, Richards DH, Karlsson G. Fluticasone propionate aqueous nasal spray in the treatment of nasal polyposis. Annals of allergy, asthma \& immunology 1997;78:270-6.

110. Aukema AA, Mulder PG, Fokkens WJ, et al. Treatment of nasal polyposis and chronic rhinosinusitis with fluticasone propionate nasal drops reduces need for sinus surgery. J Allergy Clin Immunol.2005;115:1017-23.

111. Dijkstra MD, Ebbens FA, Poublon RM, Fokkens WJ. Fluticasone propionate aqueous nasal spray does not influence the recurrence rate of chronic rhinosinusitis and nasal polyps 1 year after functional endoscopic sinus surgery. Clin Exp Allergy. 2004;34:1395-400.

112. Lund VJ, Black JH, Szabo LZ, Schrewelius C, Akerlund A. Efficacy and tolerability of budesonide aqueous nasal spray in chronic rhinosinusitis patients. Rhinology. 2004;42:57-62.

113. Ruhno J, Andersson B, Denburg J, et al. A doubleblind comparison of intranasal budesonide with placebo for nasal polyposis. J Allergy Clin Immunol.1990;86:946-53.

114. Stjärne P, Olsson P, Ålenius M. Use of Mometasone Furoate to Prevent Polyp Relapse After Endoscopic Sinus Surg. Arch. Otolaryng Head \& Neck Surg.2009;135:296-302.

115. Ahmadi N, Snidvongs K, Kalish L, et al. Intranasal corticosteroids do not affect intraocular pressure or lens opacity: a systematic review of controlled trials. Rhinology. 2015;53:290-302.

116. Bross-Soriano D, Arrieta-Gómez JR, Prado-Calleros H. Infections after endoscopic polypectomy using nasal steroids. Otolaryngology--Head \& Neck Surg. 2004;130:319-22.

117. Mostafa BE. Fluticasone propionate is associated with severe infection after endoscopic polypectomy. Arch Otolaryngol. Head \& Neck Surg. 1996;122:729-31.

118. Kern RC, Stolovitzky JP, Silvers SL, et al. A phase 3 trial of mometasone furoate sinus implants for chronic sinusitis with recurrent nasal polyps. Int Forum Allergy. \& Rhinol.2018;8:471-81.

119. Han JK, Forwith KD, Smith TL, et al. RESOLVE: a randomized, controlled, blinded study of bioabsorbable steroid-eluting sinus implants for in-office treatment of recurrent sinonasal polyposis. Int Forum Allergy. \& Rhinol.2014;4:861-70.

120. Forwith KD, Han JK, Stolovitzky JP, et al. RESOLVE: bioabsorbable steroid-eluting sinus implants for in-office treatment of recurrent sinonasal polyposis after sinus surgery: 6-month outcomes from a randomized, controlled, blinded study. Int Forum Allergy. \& Rhinol.2016;6:573-81.

121. Adriaensen G, Lim KH, Fokkens WJ. Safety and efficacy of a bioabsorbable fluticasone propionate-eluting sinus dressing in postoperative management of endoscopic sinus surgery: a randomized clinical trial. Int Forum Allergy. Rhinol. 2017;7:813-20.

122. Marple BF, Smith TL, Han JK, et al. Advance II: a prospective, randomized study assessing safety and efficacy of bioabsorbable steroid-releasing sinus implants. Otolaryngol--Head \& Neck Surg.2012;146:1004-11.

123. Shen K-H, Wang Y-HY-P, Hsu T-W, Hsieh L-C, Sun F-J, Wang Y-HY-P. Differential effects of postoperative oral corticosteroid on eosinophilic vs. noneosinophilic CRSwNP subtypes. Am J Otolaryngol. 2019;40:22-9.

124. Ecevit MC, Erdag TK, Dogan E, Sutay S. Effect of steroids for nasal polyposis surgery: a placebocontrolled, randomized, double-blind study. Laryngoscope. 2015;125:2041-5.

125. Dautremont JF, Mechor B, Rudmik L. The role of immediate postoperative systemic corticosteroids when utilizing a steroid-eluting spacer following sinus surgery. Otolaryngology--Head \& Neck Surg.2014;150:689-95.

126. Kirtsreesakul V, Wongsritrang K, Ruttanaphol S. Does oral prednisolone increase the efficacy of subsequent nasal steroids in treating nasal polyposis? American J Rhinol \& Allergy.. 2012;26:455-62.

127. Vaidyanathan $S$, Barnes $M$, Williamson $P$, Hopkinson P, Donnan PT, Lipworth B. Treatment of chronic rhinosinusitis with nasal polyposis with oral steroids followed by topical steroids: a randomized trial. Ann Intern Med 2011;154:293302.

128. Hissaria P, Smith W, Wormald PJ, et al. Short course of systemic corticosteroids in sinonasal polyposis: a double-blind, randomized, placebo-controlled trial with evaluation of outcome measures. J Allergy Clin Immunol.2006;118:128-33.

129. Richards RN. Side effects of short-term oral corticosteroids. J Cutan Med Surg. 2008;12:77-81.

130. Normansell R, Kew KM, Mansour G. Different oral corticosteroid regimens for acute asthma. Cochrane Database Syst Rev. 2016, 10.1002/14651858.CD011801.pub2:Cd011801.

131. Walters JA, Tan DJ, White CJ, Wood-Baker R. 
Different durations of corticosteroid therapy for exacerbations of chronic obstructive pulmonary disease. The Cochrane database of systematic reviews 2018;3:CD006897-CD.

132. Hox V, Lourijsen E, Jordens A, et al. Benefits and harm of systemic steroids for short- and long-term use in rhinitis and rhinosinusitis: an EAACl position paper. Clinical and Translational Allergy. 2020;10:1.

133. Lane AP, Pine HS, Pillsbury 3rd HC. Allergy. testing and immunotherapy in an academic otolaryngology practice: a 20-year review. Otolaryngol. Head Neck Surg. 2001;124:9-15.

134. Park JJH, Seidel DU, Bachert C, Dazert S, Kostev K. Medication use in patients with chronic rhinosinusitis in Germany - a large retrospective patient-based study. Rhinology. 2019;57:94-100.

135. Seresirikachorn K, Khattiyawittayakun L, Chitsuthipakorn W, Snidvongs K. Antihistamines for treating rhinosinusitis: systematic review and meta-analysis of randomised controlled studies. J Laryngol. Otol. 2018;132:105-10.

136. Haye R, Aanesen JP, Burtin B, Donnelly F, Duby C. The effect of cetirizine on symptoms and signs of nasal polyposis. J Laryngol. Otol. 1998;112:1042-6.

137. Wentzel JL, Soler ZM, DeYoung K, Nguyen SA, Lohia S, Schlosser RJ. Leukotriene antagonists in nasal polyposis: a meta-analysis and systematic review. American J of Rhinology. \& Allergy. 2013;27:482-9.

138. Pauli C, Fintelmann R, Klemens C, et al. Polyposis nasi--improvement in quality of life by the influence of leukotrien receptor antagonists. Laryngorhinootologie 2007;86:282-6.

139. Schaper C, Noga O, Koch B, et al. Antiinflammatory properties of montelukast, a leukotriene receptor antagonist in patients with asthma and nasal polyposis. J Investig Allergol Clin Immunol.2011;21:51-8.

140. Mostafa BE, Abdel Hay H, Mohammed HE, Yamani M. Role of leukotriene inhibitors in the postoperative management of nasal polyps. ORL; J for oto-rhino-laryngology and its related specialties 2005;67:148-53.

141. Stewart RA, Ram B, Hamilton G, Weiner J, Kane KJ. Montelukast as an adjunct to oral and inhaled steroid therapy in chronic nasal polyposis. Otolaryngology--Head \& Neck Surg.2008;139:6827.

142. Vuralkan E, Saka C, Akin I, et al. Comparison of montelukast and mometasone furoate in the prevention of recurrent nasal polyps. Ther Adv Respir Dis 2012;6:5-10.

143. Suri A, Gupta R, Gupta N, Kotwal S. Montelukast as an adjunct to treatment of chronic rhinosinusitis with polyposis: A prospective randomized controlled trial. JK Science 2015;17:92-5.

144. Van Gerven L, Langdon C, Cordero A, Cardelús S, Mullol J, Alobid I. Lack of long-term add-on effect by montelukast in postoperative chronic rhinosinusitis patients with nasal polyps. The Laryngoscope. 2018;128:1743-51.

145. Philip G, Hustad CM, Malice MP, et al. Analysis of behavior-related adverse experiences in clinical trials of montelukast. J Allergy Clin Immunol.2009;124:699-706.e8.

146. Philip G, Hustad C, Noonan G, et al. Reports of suicidality in clinical trials of montelukast. J Allergy Clin Immunol.2009;124:691-6.e6.

147. Law SWY, Wong AYS, Anand S, Wong ICK, Chan EW. Neuropsychiatric Events Associated with Leukotriene-Modifying Agents: A Systematic Review. Drug Saf 2018;41:253-65.

148. Haarman MG, van Hunsel F, de Vries TW. Adverse drug reactions of montelukast in children and adults. Pharmacol Res Perspect 2017;5.

149. Barnes ML, Biallosterski BT, Gray RD, Fardon TC, Lipworth BJ. Decongestant effects of nasal xylometazoline and mometasone furoate in persistent allergic rhinitis. Rhinology. 2005;43:2915.

150. Graf $\mathrm{P}$, Hallén $\mathrm{H}$, Juto JE. Four-week use of oxymetazoline nasal spray (Nezeril) once daily at night induces rebound swelling and nasal hyperreactivity. Acta Otolaryngol. 1995;115:71-5.

151. Johansson L, Oberg D, Melén I, Bende M. Do topical nasal decongestants affect polyps? Acta Otolaryngol. 2006;126:288-90.

152. Kirtsreesakul V, Khanuengkitkong T, Ruttanaphol S. Does oxymetazoline increase the efficacy of nasal steroids in treating nasal polyposis? American J Rhinol \& Allergy.. 2016;30:195-200.

153. Baroody FM, Brown D, Gavanescu L, DeTineo M, Naclerio RM. Oxymetazoline adds to the effectiveness of fluticasone furoate in the treatment of perennial allergic rhinitis. J Allergy Clin Immunol.2011;127:927-34.

154. Meltzer EO, Bernstein DI, Prenner BM, Berger WE, Shekar T, Teper AA. Mometasone furoate nasal spray plus oxymetazoline nasal spray: short-term efficacy and safety in seasonal allergic rhinitis. Am J Rhinol.Allergy.. 2013;27:102-8.

155. Humphreys MR, Grant D, McKean SA, Eng CY, Townend J, Evans AS. Xylometazoline hydrochloride 0.1 per cent versus physiological saline in nasal surgical aftercare: a randomised, single-blinded, comparative clinical trial. J Laryngol. Otol. 2009;123:85-90.

156. Friedman M, Vidyasagar R, Joseph N. A randomized, prospective, double-blind study on the efficacy of dead sea salt nasal irrigations. Laryngoscope. 2006;116:878-82.

157. Hauptman G, Ryan MW. The effect of saline solutions on nasal patency and mucociliary clearance in rhinosinusitis patients. Otolaryngology--Head \& Neck Surg.2007;137:81521.

158. Freeman SR, Sivayoham ES, Jepson K, de Carpentier J. A preliminary randomised controlled trial evaluating the efficacy of saline douching following endoscopic sinus surgery. Clin Otolaryngol. 2008;33:462-5.

159. Friedman M, Hamilton C, Samuelson CG, et al. Dead Sea salt irrigations vs saline irrigations with nasal steroids for symptomatic treatment of chronic rhinosinusitis: a randomized, prospective double-blind study. Int Forum Allergy. \& Rhinol.2012;2:252-7.

160. Farag AA, Deal AM, McKinney KA, et al. Singleblind randomized controlled trial of surfactant vs hypertonic saline irrigation following endoscopic endonasal surgery. Int Forum Allergy. \& Rhinol.2013;3:276-80.
161. Low TH, Woods CM, Ullah S, Carney AS. A doubleblind randomized controlled trial of normal saline, lactated Ringer's, and hypertonic saline nasal irrigation solution after endoscopic sinus surgery. American J Rhinol \& Allergy.. 2014;28:225-31.

162. Macdonald KI, Wright ED, Sowerby LJ, et al. Squeeze bottle versus saline spray after endoscopic sinus surgery for chronic rhinosinusitis: a pilot multicentre trial. American J Rhinol \& Allergy.. 2015;29:e13-7.

163. Giotakis Al, Karow EM, Scheithauer MO, Weber R, Riechelmann H. Saline irrigations following sinus surgery - a controlled, single blinded, randomized trial. Rhinology. 2016;54:302-10.

164. Nimsakul S, Ruxrungtham S, Chusakul S, Kanjanaumporn J, Aeumjaturapat S, Snidvongs K. Does Heating up Saline for Nasal Irrigation Improve Mucociliary Function in Chronic Rhinosinusitis? American J Rhinol \& Allergy. 2018;32:106-11.

165. Nikakhlagh S, Abshirini H, Lotfi M, Mohammad SM, Saki N. A comparison between the effects of nasal lavage with hypertonic, isotonic and hypotonic saline solutions for the treatment of chronic sinusitis. J of global pharma technology 2016;8:68-73.

166. Mozzanica F, Preti A, Gera R, et al. Double-blind, randomised controlled trial on the efficacy of saline nasal irrigation with sodium hyaluronate after endoscopic sinus surgery. J Laryngol. Otol. 2019;133:300-8.

167. Casale M, Sabatino L, Frari V, et al. The potential role of hyaluronan in minimizing symptoms and preventing exacerbations of chronic rhinosinusitis. American J Rhinol \& Allergy.. 2014;28:345-8.

168. Macchi A, Terranova P, Digilio E, Castelnuovo $P$. Hyaluronan plus saline nasal washes in the treatment of rhino-sinusal symptoms in patients undergoing functional endoscopic sinus surgery for rhino-sinusal remodeling. Int J Immunopathol Pharmacol 2013;26:137-45.

169. Lee VS, Humphreys IM, Purcell PL, Davis GE. Manuka honey sinus irrigation for the treatment of chronic rhinosinusitis: a randomized controlled trial. Int Forum Allergy. \& Rhinol. 2017 Apr;7(4):365372.

170. Hashemian F, Baghbanian N, Majd Z, Rouini MR, Jahanshahi J, Hashemian F. The effect of thyme honey nasal spray on chronic rhinosinusitis: a double-blind randomized controlled clinical trial. Eur Arch Otorhinolaryngol. 2015;272:1429-35.

171. Tantilipikorn $P$, Tunsuriyawong $P$, Jareoncharsri $P$, et al. A randomized, prospective, double-blind study of the efficacy of dexpanthenol nasal spray on the postoperative treatment of patients with chronic rhinosinusitis after endoscopic sinus surgery. Chotmaihet thangphaet [J of the Medical Association of Thailand] 2012;95:58-63.

172. Bachmann G, Hommel G, Michel O. Effect of irrigation of the nose with isotonic salt solution on adult patients with chronic paranasal sinus disease. Eur Arch Otorhinolaryngol.. 2000;257:53741.

173. Taccariello M, Parikh A, Darby Y, Scadding G. Nasal douching as a valuable adjunct in the management of chronic rhinosinusitis. Rhinology. 
1999;37:29-32.

174. Yu MS, Kim BH, Kang SH, Lim DJ. Lowconcentration hypochlorous acid nasal irrigation for chronic sinonasal symptoms: a prospective randomized placebo-controlled study. Eur Arch Otorhinolaryngol. 2016;274:1527-33.

175. Liang KL, Su MC, Tseng HC, Jiang RS. Impact of pulsatile nasal irrigation on the prognosis of functional endoscopic sinus surgery. J Otolaryngol. Head Neck Surg. 2008;37:148-53.

176. Ooi ML, Jothin A, Bennett C, et al. Manuka honey sinus irrigations in recalcitrant chronic rhinosinusitis: phase 1 randomized, single-blinded, placebo-controlled trial. Int Forum Allergy. Rhinol. 2019, 10.1002/alr.22423.

177. Heatley DG, McConnell KE, Kille TL, Leverson GE. Nasal irrigation for the alleviation of sinonasal symptoms. Otolaryngology--Head \& Neck Surg.2001;125:44-8.

178. Pinto JM, Elwany S, Baroody FM, Naclerio RM. Effects of saline sprays on symptoms after endoscopic sinus surgery. Am J Rhinol.2006;20:191-6.

179. Jiang RS, Liang KL, Wu SH, Su MC, Chen WK, Lu FJ. Electrolyzed acid water nasal irrigation after functional endoscopic sinus surgery. American J Rhinol \& Allergy.. 2014;28:176-81.

180. Lin L, Tang X, Wei J, Dai F, Sun G. Xylitol nasal irrigation in the treatment of chronic rhinosinusitis. Am J Otolaryngol. 2017;38:383-9.

181. Weissman JD, Fernandez F, Hwang PH. Xylitol nasal irrigation in the management of chronic rhinosinusitis: a pilot study. The Laryngoscope. 2011;121:2468-72.

182. Passali D, Fiorella R, Camaioni A, et al. Glucan solution nasal spray vs saline in the treatment of chronic rhinosinusitis: a multi-centric double blind randomised clinical trial. Clin Ter 2007;158:139-45.

183. Pigret D, Jankowski R. Management of postethmoidectomy crust formation: randomized single-blind clinical trial comparing pressurized seawater versus antiseptic/mucolytic saline. Rhinology. 1996;34:38-40.

184. Culig J, Leppée M, Vceva A, Djanic D. Efficiency of hypertonic and isotonic seawater solutions in chronic rhinosinusitis. Medicinski glasnik : official publication of the medical association of zenicadoboj canton, bosnia and herzegovina 2010;7:11623.

185. Cantone E, Castagna G, Sicignano S, et al. Impact of intranasal sodium hyaluronate on the short-term quality of life of patients undergoing functional endoscopic sinus surgery for chronic rhinosinusitis. Int Forum Allergy. \& Rhinol.2014;4:484-7.

186. Gelardi M, Passalacqua G, Fiorella ML, Quaranta N. Assessment of biofilm by nasal cytology in different forms of rhinitis and its functional correlations. Eur Ann Allergy Clin Immunol.2013;45:25-9.

187. Fooanant S, Chaiyasate S, Roongrotwattanasiri K. Comparison on the efficacy of dexpanthenol in sea water and saline in postoperative endoscopic sinus surgery. J Med Assoc Thai 2008;91:1558-63.

188. Wormald PJ, Cain T, Oates L, Hawke L, Wong I. A comparative study of three methods of nasal irrigation. Laryngoscope. 2004;114:2224-7.
189. Salib RJ, Talpallikar S, Uppal S, Nair SB. A prospective randomised single-blinded clinical trial comparing the efficacy and tolerability of the nasal douching products Sterimar ${ }^{\mathrm{TM}}$ and Sinus Rinse $^{T M}$ following functional endoscopic sinus surgery. Clin Otolaryngol. 2013;38:297-305.

190. Turner JH, Wu J, Dorminy CA, Chandra RK. Safety and tolerability of surfactant nasal irrigation. Int Forum Allergy. Rhinol. 2017;7:809-12.

191. Cantone $E$, lengo M. Effect of sodium hyluronate added to topical corticosteroids in chronic rhinosinusitis with nasal polyposis. American J Rhinol Allergy. 2016.30(5):340-343.

192. Jull AB, Cullum N, Dumville JC, Westby MJ, Deshpande S, Walker N. Honey as a topical treatment for wounds. Cochrane Database Syst Rev. 2015, 10.1002/14651858.CD005083. pub4:Cd005083.

193. Henatsch D, Wesseling F, Kross KW, Stokroos RJ. Honey and beehive products in otorhinolaryngology: a narrative review. Clin Otolaryngol. 2016;41:519-31.

194. Mosges R, Shah-Hosseini K, Hucke HP, Joisten MJ. Dexpanthenol: an Overview of its Contribution to Symptom Relief in Acute Rhinitis Treated with Decongestant Nasal Sprays. Adv Ther 2017:1-9.

195. Kehrl W, Sonnemann U. [Dexpanthenol nasal spray as an effective therapeutic principle for treatment of rhinitis sicca anterior]. Laryngorhinootologie 1998;77:506-12.

196. Pique N, Gomez-Guillen MDC, Montero MP. Xyloglucan, a Plant Polymer with Barrier Protective Properties over the Mucous Membranes: An Overview. Int J Mol Sci 2018;19.

197. Ottaviano G, Marioni G, Staffieri C, et al. Effects of sulfurous, salty, bromic, iodic thermal water nasal irrigations in nonallergic chronic rhinosinusitis: a prospective, randomized, double-blind, clinical, and cytological study. Am J Otolaryngol. 2011;32:235-9.

198. Passali D, Lauriello M, Passali GC, et al. Clinical evaluation of the efficacy of Salsomaggiore (Italy) thermal water in the treatment of rhinosinusal pathologies. Clin Ter 2008;159:181-8.

199. Passali FM, Crisanti A, Passali GC, et al. Efficacy of inhalation therapy with water of Salsomaggiore (Italy) in chronic and recurrent nasosinusal inflammation treatment. Clin Ter 2008;159:175-80.

200. Stevens WW, Peters AT, Hirsch AG, et al. Clinical Characteristics of Patients with Chronic Rhinosinusitis with Nasal Polyps, Asthma, and Aspirin-Exacerbated Respiratory Disease. J Allergy Clin Immunol.Pract 2017;5:1061-70.e3.

201. Kowalski ML, Agache I, Bavbek S, et al. Diagnosis and management of NSAID-Exacerbated Respiratory Disease (N-ERD)-a EAACI position paper. Allergy. 2019;74:28-39.

202. Pleskow WW, Stevenson DD, Mathison DA, Simon RA, Schatz M, Zeiger RS. Aspirin desensitization in aspirin-sensitive asthmatic patients: clinical manifestations and characterization of the refractory period. J Allergy Clin Immunol.1982;69:11-9.

203. White AA, Stevenson DD. Aspirin desensitization in aspirin-exacerbated respiratory disease. Immunol. Allergy. Clin North Am 2013;33:211-22.
204. Mortazavi N, Esmaeilzadeh $\mathrm{H}$, Abbasinazari M, et al. Clinical and immunological efficacy of aspirin desensitization in nasal polyp patients with aspirin-exacerbated respiratory disease. Iranian J of Pharmaceutical Research. 2017;16:1639-47.

205. Esmaeilzadeh H, Nabavi M, Aryan Z, et al. Aspirin desensitization for patients with aspirinexacerbated respiratory disease: $\mathrm{A}$ randomized double-blind placebo-controlled trial. Clin Immunol.2015;160:349-57.

206. Świerczyńska-Krępa M, Sanak M, Bochenek $G$, et al. Aspirin desensitization in patients with aspirin-induced and aspirin-tolerant asthma: a double-blind study. J Allergy Clin Immunol.2014;134:883-90.

207. Fruth K, Pogorzelski B, Schmidtmann I, et al. Lowdose aspirin desensitization in individuals with aspirin-exacerbated respiratory disease. Allergy. 2013;68:659-65.

208. Patriarca G, Bellioni P, Nucera E, et al. Intranasal treatment with lysine acetylsalicylate in patients with nasal polyposis. Ann Allergy. 1991;67:588-92.

209. Scadding GK, Hassab M, Darby YC, Lund VJ, Freedman A. Intranasal lysine aspirin in recurrent nasal polyposis. Clin Otolaryngol. Allied Sci 1995;20:561-3.

210. Ogata N, Darby Y, Scadding G. Intranasal lysineaspirin administration decreases polyp volume in patients with aspirin-intolerant asthma. J Laryngol. Otol. .2007;121:1156-60.

211. Parikh AA, Scadding GK. Intranasal lysine-aspirin in aspirin-sensitive nasal polyposis: a controlled trial. Laryngoscope. 2005;115:1385-90.

212. Schneider TR, Johns CB, Palumbo ML, Murphy KC, Cahill KN, Laidlaw TM. Dietary Fatty Acid Modification for the Treatment of AspirinExacerbated Respiratory Disease: A Prospective Pilot Trial. J Allergy Clin Immunol. Pract. 2018;6:825-31.

213. Ponikau JU, Sherris DA, Kern EB, et al. The diagnosis and incidence of allergic fungal sinusitis. Mayo Clin Proc 1999;74:877-84.

214. Lackner A, Stammberger H, Buzina W, et al. Fungi: a normal content of human nasal mucus. Am J Rhinol.2005;19:125-9.

215. Hoggard M, Zoing M, Biswas K, Taylor MW, Douglas RG. The sinonasal mycobiota in chronic rhinosinusitis and control patients. Rhinology. 2019;57:190-9.

216. Fokkens W, Lund V, Bachert C, et al. EAACI position paper on rhinosinusitis and nasal polyps executive summary. Allergy. 2005;60:583-601.

217. Meltzer EO, Hamilos DL, Hadley JA, et al. Rhinosinusitis: establishing definitions for clinical research and patient care. J Allergy Clin Immunol.2004;114:155-212.

218. Head K, Sharp S, Chong LY, Hopkins C, Philpott C. Topical and systemic antifungal therapy for chronic rhinosinusitis. Cochrane Database Syst Rev. 2018;9:CD012453.

219. Hashemian F, Hashemian F, Molaali N, Rouini M, Roohi E, Torabian S. Clinical effects of topical antifungal therapy in chronic rhinosinusitis: a randomized, double-blind, placebo-controlled trial of intranasal fluconazole. EXCLI J 2016;15:95-102.

220. Weschta M, Rimek D, Formanek M, Polzehl D, 
Podbielski A, Riechelmann H. Topical antifungal treatment of chronic rhinosinusitis with nasal polyps: a randomized, double-blind clinical trial. J Allergy Clin Immunol. 2004;113:1122-8.

221. Ponikau JU, Sherris DA, Weaver A, Kita H. Treatment of chronic rhinosinusitis with intranasal amphotericin B: a randomized, placebocontrolled, double-blind pilot trial. J Allergy Clin Immunol.2005;115:125-31.

222. Ebbens FA, Scadding GK, Badia L, et al. Amphotericin B nasal lavages: not a solution for patients with chronic rhinosinusitis. J Allergy Clin Immunol.2006;118:1149-56.

223. Liang KL, Su MC, Shiao JY, et al. Amphotericin $B$ irrigation for the treatment of chronic rhinosinusitis without nasal polyps: a randomized, placebo-controlled, double-blind study. Am J Rhinol. 2008;22:52-8.

224. Gerlinger I, Fittler A, Fonai F, Patzko A, Mayer A, Botz L. Postoperative application of amphotericin $B$ nasal spray in chronic rhinosinusitis with nasal polyposis, with a review of the antifungal therapy. Eur Arch Otorhinolaryngol. 2009;266:847-55.

225. Jiang RS, Hsu SH, Liang KL. Amphotericin B nasal irrigation as an adjuvant therapy after functional endoscopic sinus surgery. American J Rhinol \& Allergy. 2015;29:435-40.

226. Yousefi J, Akhavan A, Hoseini-Motlagh R, BanaeiBoroujeni S, Panahi Y, Khosravi MH. Effect of Amphotericin B on Treatment of Chronic Rhinosinusitis: A Double-blind Randomized Clinical Trial Razavi International J of Medicine 2017;5:e64550.

227. Jiang RS, Twu CW, Liang KL. Efficacy of nasal irrigation with $200 \mathrm{mug} / \mathrm{mL}$ amphotericin $B$ after functional endoscopic sinus surgery: a randomized, placebo-controlled, double-blind study. Int Forum Allergy. Rhinol. 2018;8:41-8.

228. Zhang N, Holtappels G, Gevaert P, et al. Mucosal tissue polyclonal $\lg E$ is functional in response to allergen and SEB. Allergy. 2011;66:141-8.

229. Gevaert P, Calus L, Van Zele T, et al. Omalizumab is effective in allergic and nonallergic patients with nasal polyps and asthma. The J Allergy Clin Immunol.2013;131:110-6.e1.

230. Pinto JM, Mehta N, DiTineo M, Wang J, Baroody FM, Naclerio RM. A randomized, double-blind, placebo-controlled trial of anti-lgE for chronic rhinosinusitis. Rhinology. 2010;48:318-24.

231. MacGlashan DW, Jr., Bochner BS, Adelman DC, et al. Down-regulation of $\mathrm{Fc}$ (epsilon)RI expression on human basophils during in vivo treatment of atopic patients with anti-IgE antibody. J Immunol.1997;158:1438-45.

232. Presta LG, Lahr SJ, Shields RL, et al. Humanization of an antibody directed against lgE. J Immunol.1993;151:2623-32.

233. Di Bona D, Fiorino I, Taurino M, et al. Long-term "real-life" safety of omalizumab in patients with severe uncontrolled asthma: A nine-year study. Respir Med 2017;130:55-60.

234. Oblitas CM, Galeano-Valle F, Vela-De La Cruz L, Del Toro-Cervera J, Demelo-Rodriguez P. Omalizumab as a Provoking Factor for Venous Thromboembolism. Drug Target Insights 2019;13:1177392819861987.
235. Denburg JA, Sehmi R, Upham J. Regulation of IL-5 receptor on eosinophil progenitors in allergic inflammation: role of retinoic acid. Int Arch Allergy. Immunol.2001;124:246-8.

236. Sahlstrand-Johnson P, Ohlsson B, Von Buchwald $C$, Jannert M, Ahlner-Elmqvist M. A multi-centre study on quality of life and absenteeism in patients with CRS referred for endoscopic surgery. Rhinology. 2011;49:420-8.

237. Bachert C, Wagenmann M, Hauser U, Rudack C. IL-5 synthesis is upregulated in human nasal polyp tissue. J Allergy Clin Immunol.1997;99:837-42.

238. Bachert C, Sousa AR, Lund VJ, et al. Reduced need for surgery in severe nasal polyposis with mepolizumab: Randomized trial. J Allergy Clin Immunol.2017;140:1024-31.e14.

239. Gevaert P, Van Bruaene N, Cattaert T, et al. Mepolizumab, a humanized anti-IL-5 mAb, as a treatment option for severe nasal polyposis. The J Allergy Clin Immunol.2011;128:988-9.

240. Gevaert P, Lang-Loidolt D, Lackner A, et al. Nasal IL-5 levels determine the response to anti-IL-5 treatment in patients with nasal polyps. J Allergy Clin Immunol.2006;118:1133-41.

241. Leung E, Al Efraij K, FitzGerald J. The safety of mepolizumab for the treatment of asthma. Expert Opin Drug Saf 2017;16:397-404.

242. Mitchell P, Leigh R. A drug safety review of treating eosinophilic asthma with monoclonal antibodies. Expert Opin Drug Saf. 2019, 10.1080/14740338.2019.1675634:1-10.

243. Tsetsos N, Goudakos JK, Daskalakis D, Konstantinidis I, Markou K. Monoclonal antibodies for the treatment of chronic rhinosinusitis with nasal polyposis: a systematic review. Rhinology. 2018;56:11-21.

244. Kang EG, Narayana PK, Pouliquen IJ, Lopez MC, Ferreira-Cornwell MC, Getsy JA. Efficacy and safety of mepolizumab administered subcutaneously for moderate to severe atopic dermatitis. Allergy. 2019, 10.1111/all.14050.

245. Fokkens WJ, Lund V, Bachert C, et al. EUFOREA consensus on biologics for CRSWNP with or without asthma. Allergy. 2019;74:2312-9.

246. Bagnasco D, Ferrando M, Varricchi G, Passalacqua G, Canonica GW. A Critical Evaluation of Anti-IL-13 and Anti-IL-4 Strategies in Severe Asthma. Int Arch Allergy. Immunol.2016;170:122-31.

247. Bachert C, Mannent L, Naclerio RM, et al. Effect of Subcutaneous Dupilumab on Nasal Polyp Burden in Patients With Chronic Sinusitis and Nasal Polyposis: A Randomized Clinical Trial. JAMA 2016;315:469-79.

248. Bachert C, Han JK, Desrosiers M, et al. Efficacy and safety of dupilumab in patients with severe chronic rhinosinusitis with nasal polyps (LIBERTY NP SINUS-24 and LIBERTY NP SINUS-52): results from two multicentre, randomised, double-blind, placebo-controlled, parallel-group phase 3 trials. Lancet 2019;394:1638-50.

249. Wenzel S, Ford L, Pearlman D, et al. Dupilumab in persistent asthma with elevated eosinophil levels. N Engl J Med 2013;368:2455-66.

250. Laidlaw TM, Mullol J, Fan C, et al. Dupilumab improves nasal polyp burden and asthma control in patients with CRSWNP and AERD. J Allergy Clin
Immunol.Pract 2019;7:2462-5.e1.

251. Bachert C, Zinreich SJ, Hellings PW, et al. Dupilumab reduces opacification across all sinuses and related symptoms in patients with CRSwNP. Rhinology. 2019, 10.4193/Rhin18.282.

252. Bachert C, Hellings PW, Mullol J, et al. Dupilumab improves health-related quality of life in patients with chronic rhinosinusitis with nasal polyposis. Allergy. 2019, 10.1111/all.13984.

253. Bachert C, Hellings PW, Mullol J, et al. Dupilumab improves patient-reported outcomes in patients with chronic rhinosinusitis with nasal polyps and comorbid asthma. J Allergy Clin Immunol.Pract 2019;7:2447-9.e2

254. Akinlade B, Guttman-Yassky E, de Bruin-Weller M, et al. Conjunctivitis in dupilumab clinical trials. $\mathrm{Br} J$ Dermatol 2019;181:459-73.

255. Psaltis AJ, Wormald PJ. Therapy of Sinonasal Microbiome in CRS: A Critical Approach. Curr Allergy. Asthma Rep 2017;17:59.

256. Cervin AU. The Potential for Topical Probiotic Treatment of Chronic Rhinosinusitis, a Personal Perspective. Front Cell Infect Microbiol 2017;7:530.

257. Mukerji SS, Pynnonen MA, Kim HM, Singer A, Tabor $M$, Terrell JE. Probiotics as adjunctive treatment for chronic rhinosinusitis: a randomized controlled trial. Otolaryngol. Head Neck Surg. 2009;140:202-8.

258. Martensson A, Abolhalaj M, Lindstedt M, et al. Clinical efficacy of a topical lactic acid bacterial microbiome in chronic rhinosinusitis: a randomized controlled trial. Laryngoscope. investigative otolaryngology 2017;2:410-6.

259. Poole P, Chong J, Cates CJ. Mucolytic agents versus placebo for chronic bronchitis or chronic obstructive pulmonary disease. Cochrane Database Syst Rev. 2015, 10.1002/14651858. CD001287.pub5:Cd001287.

260. Tarrant BJ, Le Maitre C, Romero L, et al. Mucoactive agents for chronic, non-cystic fibrosis lung disease: A systematic review and meta-analysis. Respirology. 2017;22:1084-92.

261. Majima Y, Kurono Y, Hirakawa K, et al. Efficacy of combined treatment with S-carboxymethylcysteine (carbocisteine) and clarithromycin in chronic rhinosinusitis patients without nasal polyp or with small nasal polyp. Auris Nasus Larynx. 2012;39:38-47.

262. Hoza J, Salzman R, Starek I, Schalek P, Kellnerova R. Efficacy and safety of erdosteine in the treatment of chronic rhinosinusitis with nasal polyposis - a pilot study. Rhinology. 2013;51:323-7.

263. Dal Negro RW. Erdosteine: antitussive and antiinflammatory effects. Lung. 2008;186 Suppl 1:S703.

264. Rezaeian A, Amoushahi Khouzani S. Effect of Nigella sativa Nasal Spray on the Treatment of Chronic Rhinosinusitis Without a Nasal Polyp. Allergy and Rhinology. 2018;9.

265. Anushiravani M, Bakhshaee M, Taghipour A, Mehri MR. Comparison of the therapeutic effect of the Persian Medicine Protocol with the common treatment of chronic rhinosinusitis: a randomized clinical trial. Electronic Physician [Electronic Resource] 2018;10:7017-27.

266. Vazifehkah S, Shams-Ardekani MR, Kamalinejad $M$, et al. Evaluation of a novel natural drop for 
treatment of chronic rhinosinusitis without nasal polyps: a single blind randomized trial. Int Forum Allergy. \& Rhinol.2016;6:943-9.

267. Madani AA, Azadbakht M, Kosarian M, Rabie P, Khalilian AR. Origanum vulgare inhaler in the treatment of chronic rhinosinusitis, a double blind placebo controlled randomized clinical trial. International J of biology and biotechnology 2006;3:547-50.

268. Palm J, Steiner I, Abramov-Sommariva D, et al. Assessment of efficacy and safety of the herbal medicinal product BNO 1016 in chronic rhinosinusitis. Rhinology. 2017;55:142-51.

269. Jund R, Mondigler M, Steindl H, Stammer $\mathrm{H}$, Stierna P, Bachert C. Clinical efficacy of a dry extract of five herbal drugs in acute viral rhinosinusitis. Rhinology. 2012;50:417-26.

270. Witt C, Keil T, Selim D, et al. Outcome and costs of homoeopathic and conventional treatment strategies: a comparative cohort study in patients with chronic disorders. Complement Ther Med 2005;13:79-86.

271. Liang KL, Su YC, Tsai CC, Lin JS, Jiang RS, Su MC. Postoperative care with Chinese herbal medicine or amoxicillin after functional endoscopic sinus surgery: a randomized, double-blind, placebocontrolled study. American J Rhinol \& Allergy. 2011;25:170-5.

272. Stavem K, Røssberg E, Larsson PG. Health-related quality of life in a trial of acupuncture, sham acupuncture and conventional treatment for chronic sinusitis. BMC Res Notes 2008;1:37-.

273. Rossberg E, Larsson PG, Birkeflet O, Soholt LE, Stavem K. Comparison of traditional Chinese acupuncture, minimal acupuncture at nonacupoints and conventional treatment for chronic sinusitis. Complement Ther Med. 2005;13:4-10.

274. Rössberg E, Larsson PG, Birkeflet O, Söholt LE, Stavem K. Comparison of traditional Chinese acupuncture, minimal acupuncture at nonacupoints and conventional treatment for chronic sinusitis. Complement Ther Med.2005;13:4-10.

275. Liu SH, Chuang WC, Lam W, Jiang Z, Cheng YC. Safety surveillance of traditional Chinese medicine: current and future. Drug Saf .2015;38:117-28.

276. Chan MWC, Wu XY, Wu JCY, Wong SYS, Chung VCH. Safety of Acupuncture: Overview of Systematic Reviews. Sci Rep 2017;7:3369.

277. Miyake MM, Nocera A, Levesque P, et al. Doubleblind placebo-controlled randomized clinical trial of verapamil for chronic rhinosinusitis with nasal polyps. J Allergy Clin Immunol.2017;140:271-3.

278. Kroflic B, Coer A, Baudoin T, Kalogjera L. Topical furosemide versus oral steroid in preoperative management of nasal polyposis. Eur Arch Otorhinolaryngol. 2006;263:767-71.

279. Passàli D, Bernstein JM, Passali FM, Damiani V, Passàli GC, Bellussi L. Treatment of recurrent chronic hyperplastic sinusitis with nasal polyposis. Arch Otolaryngol. Head \& Neck Surg. 2003;129:656-9.

280. Passàli D, Mezzedimi C, Passàli GC, et al. Efficacy of inhalation form of furosemide to prevent postsurgical relapses of rhinosinusal polyposis. ORL J Otorhinolaryngol. Relat Spec. 2000;62:30710 .
281. Filiaci F, Zambetti G, Luce M, Ciofalo A. Local treatment of nasal polyposis with capsaicin: preliminary findings. Allergol Immunopathol. (Madr) 1996;24:13-8.

282. Zheng C, Wang Z, Lacroix JS. Effect of intranasal treatment with capsaicin on the recurrence of polyps after polypectomy and ethmoidectomy. Acta Otolaryngol. 2000;120:62-6.

283. Gevorgyan A, Segboer C, Gorissen R, van Drunen $\mathrm{CM}$, Fokkens W. Capsaicin for non-allergic rhinitis. Cochrane Database Syst Rev. 2015, 10.1002/14651858.CD010591.pub2:Cd010591.

284. Katle EJ, Hatlebakk JG, Grimstad T, Kvaloy JT, Steinsvag SK. Gastro-oesophageal reflux in patients with chronic rhino-sinusitis investigated with multichannel impedance - $\mathrm{pH}$ monitoring. Rhinology. 2017;55:27-33.

285. Leason SR, Barham HP, Oakley G, et al. Association of gastro-oesophageal reflux and chronic rhinosinusitis: systematic review and metaanalysis. Rhinology. 2017;55:3-16.

286. Sella GCP, Tamashiro E, Anselmo-Lima WT, Valera FCP. Relation between chronic rhinosinusitis and gastroesophageal reflux in adults: systematic review. Revista Brasileira de Otorrinolaringologia 2017;83:356-63.

287. Anzic SA, Turkalj M, Zupan A, Labor M, Plavec D, Baudoin T. Eight weeks of omeprazole $20 \mathrm{mg}$ significantly reduces both laryngopharyngeal reflux and comorbid chronic rhinosinusitis signs and symptoms: Randomised, doubleblind, placebo-controlled trial. Clin Otolaryngol. 2018;43:496-501.

288. Xie Y, Bowe B, Li T, Xian H, Yan Y, Al-Aly Z. Risk of death among users of Proton Pump Inhibitors: a longitudinal observational cohort study of United States veterans. BMJ Open. 2017;7:e015735.

289. Shiraev TP, Bullen A. Proton Pump Inhibitors and Cardiovascular Events: A Systematic Review. Heart Lung Circ. 2018;27:443-50.

290. Meng Q, Li P, Li Y, et al. Broncho-vaxom alleviates persistent allergic rhinitis in patients by improving Th1/Th2 cytokine balance of nasal mucosa. Rhinology. 2019, 10.4193/Rhin 19.161.

291. Kearney SC, Dziekiewicz M, Feleszko W. Immunoregulatory and immunostimulatory responses of bacterial lysates in respiratory infections and asthma. Ann Allergy. Asthma Immunol.2015;114:364-9.

292. Yin J, Xu B, Zeng X, Shen K. Broncho-Vaxom in pediatric recurrent respiratory tract infections: A systematic review and meta-analysis. Int Immunopharmacol 2018;54:198-209.

293. Triantafillou V, Workman AD, Patel NN, et al. Broncho-Vaxom (OM-85 BV) soluble components stimulate sinonasal innate immunity. Int Forum Allergy. \& Rhinol.2019;9:370-7.

294. Heintz B, Schlenter WW, Kirsten R, Nelson K. Clinical efficacy of Broncho-Vaxom in adult patients with chronic purulent sinusitis--a multicentric, placebo-controlled, double-blind study. Int J Clin Pharmacol Ther Toxicol 1989;27:530-4.

295. Matsumura Y, Ananthaswamy HN. Toxic effects of ultraviolet radiation on the skin. Toxicol Appl Pharmacol 2004;195:298-308.

296. Dulguerov N, Guin, N., et al. Rhinophototherapy in chronic rhinosinusitis: a double blind randomized placebo-controlled trial. Rhinology. 2017;55:10612.

297. Fokkens WJ, Lund VJ, Mullol J, et al. EPOS 2012: European position paper on rhinosinusitis and nasal polyps 2012. A summary for otorhinolaryngologists. Rhinology. 2012;50:1-12.

298. Koreck Al, Csoma Z, Bodai L, et al. Rhinophototherapy: a new therapeutic tool for the management of allergic rhinitis. J Allergy Clin Immunol.2005;115:541-7.

299. Kemeny L, Koreck A. Ultraviolet light phototherapy for allergic rhinitis. J Photochem Photobiol B 2007;87:58-65.

300. Kiricsi Á, Tiszlavicz L, Rázga Z, et al. Prospective, multicenter, randomized clinical study to evaluate the clinical efficacy and tolerability of long term mixed ultraviolet and visible light phototherapy in eosinophil nasal polyps. J of photochemistry and photobiology B, biology 2017;176:118-23.

301. van Agthoven M, Fokkens WJ, van de Merwe JP, Marijke van Bolhuis E, Uyl-de Groot CA, Busschbach JJ. Quality of life of patients with refractory chronic rhinosinusitis: effects of filgrastim treatment. Am J Rhinol.2001;15:231-7.

302. Ludwig M, Enzenhofer E, Schneider S, et al. Efficacy of a carrageenan nasal spray in patients with common cold: a randomized controlled trial. Respir Res. 2013;14:124.

303. Fazekas T, Eickhoff P, Pruckner N, et al. Lessons learned from a double-blind randomised placebocontrolled study with a iota-carrageenan nasal spray as medical device in children with acute symptoms of common cold. BMC Complement Altern Med 2012;12:147.

304. Eccles R, Winther B, Johnston SL, Robinson P, Trampisch M, Koelsch S. Efficacy and safety of iotacarrageenan nasal spray versus placebo in early treatment of the common cold in adults: the ICICC trial. Respir Res.. 2015;16:121.

305. Eccles R, Meier C, Jawad M, Weinmüllner R, Grassauer A, Prieschl-Grassauer E. Efficacy and safety of an antiviral lota-Carrageenan nasal spray: a randomized, double-blind, placebocontrolled exploratory study in volunteers with early symptoms of the common cold. Respir Res. 2010;11:108.

306. Scott JR, Krishnan R, Rotenberg BW, Sowerby $\mathrm{LJ}$. The effectiveness of topical colloidal silver in recalcitrant chronic rhinosinusitis: a randomized crossover control trial. Le J d'oto-rhinolaryngologie et de chirurgie cervico-faciale [J of otolaryngology - head \& neck surgery] 2017;46:64.

307. DeYoung K, Wentzel JL, Schlosser RJ, Nguyen SA, Soler ZM. Systematic review of immunotherapy for chronic rhinosinusitis. American J of Rhinology. \& Allergy. 2014;28:145-50.

308. Bachert C, Zhang L, Gevaert P. Current and future treatment options for adult chronic rhinosinusitis: Focus on nasal polyposis. The J Allergy Clin Immunol.2015;136:1431-40.

309. Sel S, Wegmann M, Dicke T, et al. Effective prevention and therapy of experimental allergic asthma using a GATA-3-specific DNAzyme. J Allergy Clin Immunol.2008;121:910-6.e5.

310. Krug N, Hohlfeld JM, Kirsten AM, et al. Allergen- 
induced asthmatic responses modified by a GATA3-specific DNAzyme. N Engl J Med 2015;372:1987-95.

311. Garn H, Renz H. GATA-3-specific DNAzyme - A novel approach for stratified asthma therapy. Eur J Immunol.2017;47:22-30.

312. Greulich T, Hohlfeld JM, Neuser P, et al. A GATA3specific DNAzyme attenuates sputum eosinophilia in eosinophilic COPD patients: a feasibility randomized clinical trial. Respir Res. 2018;19:55.

313. Fuhst R, Runge F, Buschmann J, et al. Toxicity profile of the GATA-3-specific DNAzyme hgd40 after inhalation exposure. Pulm Pharmacol Ther 2013;26:281-9.

314. Legrand F, Cao Y, Wechsler JB, et al. Sialic acid-binding immunoglobulin-like lectin (Siglec) 8 in patients with eosinophilic disorders: Receptor expression and targeting using chimeric antibodies. J Allergy Clin Immunol.2019;143:2227-37.e10.

315. Kano G, Bochner BS, Zimmermann N. Regulation of Siglec-8-induced intracellular reactive oxygen species production and eosinophil cell death by Src family kinases. Immunobiology 2017;222:3439.

316. Catley MC, Coote J, Bari M, Tomlinson KL. Monoclonal antibodies for the treatment of asthma. Pharmacol Ther 2011;132:333-51.

317. Baatjes AJ, Smith SG, Dua B, Watson R, Gauvreau GM, O'Byrne PM. Treatment with anti-OX40L or anti-TSLP does not alter the frequency of $T$ regulatory cells in allergic asthmatics. Allergy. 2015;70:1505-8.

318. Gauvreau GM, Boulet LP, Cockcroft DW, et al. OX40L blockade and allergen-induced airway responses in subjects with mild asthma. Clinical and experimental allergy : J of the British Society for Allergy. and Clinical Immunology 2014;44:2937.

319. Michel O, Dinh PH, Doyen V, Corazza F. Anti-TNF inhibits the airways neutrophilic inflammation induced by inhaled endotoxin in human. BMC Pharmacol Toxicol 2014;15:60.

320. Antoniu SA, Mihaltan F, Ulmeanu R. Anti-TNFalpha therapies in chronic obstructive pulmonary diseases. Expert Opin Investig Drugs 2008;17:120311.

321. Cazzola M, Polosa R. Anti-TNF-alpha and Th1 cytokine-directed therapies for the treatment of asthma. Curr Opin Allergy Clin Immunol.2006;6:43-50.

322. Antoniu SA. Infliximab for chronic obstructive pulmonary disease: towards a more specific inflammation targeting? Expert Opin Investig Drugs 2006;15:181-4.

323. Winthrop KL. Risk and prevention of tuberculosis and other serious opportunistic infections associated with the inhibition of tumor necrosis factor. Nat Clin Pract Rheumatol 2006;2:602-10.

324. Golebski K, Ros XR, Nagasawa M, et al. IL-1 beta, IL-23, and TGF-beta drive plasticity of human ILC2s towards IL-17-producing ILCs in nasal inflammation. Nat Commun. 2019;10:2162.

325. Nirula A, Nilsen J, Klekotka P, et al. Effect of IL17 receptor $A$ blockade with brodalumab in inflammatory diseases. Rheumatology (Oxford) 2016;55:ii43-ii55.

326. Busse WW, Holgate S, Kerwin E, et al. Randomized, double-blind, placebo-controlled study of brodalumab, a human anti-IL-17 receptor monoclonal antibody, in moderate to severe asthma. Am J Respir Crit Care Med 2013;188:1294302.

327. Nair P, Aziz-Ur-Rehman A, Radford K. Therapeutic implications of 'neutrophilic asthma'. Curr Opin Pulm Med. 2015;21:33-8.

328. Nair P, Gaga M, Zervas E, et al. Safety and efficacy of a CXCR2 antagonist in patients with severe asthma and sputum neutrophils: a randomized, placebo-controlled clinical trial. Clin Exp Allergy. 2012;42:1097-103.

329. O'Byrne PM, Metev H, Puu M, et al. Efficacy and safety of a CXCR2 antagonist, AZD5069, in patients with uncontrolled persistent asthma: a randomised, double-blind, placebo-controlled trial. Lancet Respir Med 2016;4:797-806.

330. Panousis C, Dhagat U, Edwards KM, et al. CSL311, a novel, potent, therapeutic monoclonal antibody for the treatment of diseases mediated by the common beta chain of the IL-3, GM-CSF and IL-5 receptors. MAbs 2016;8:436-53.

331. Mitchell PD, El-Gammal Al, O'Byrne PM. Emerging monoclonal antibodies as targeted innovative therapeutic approaches to asthma. Clin Pharmacol Ther 2016;99:38-48.

332. Golebski K, van Tongeren J, van Egmond D, de Groot EJ, Fokkens WJ, van Drunen CM. Specific Induction of TSLP by the Viral RNA Analogue Poly $(\mathrm{l}: C)$ in Primary Epithelial Cells Derived from Nasal Polyps. PLoS One 2016;11:e0152808.

333. Nagarkar DR, Poposki JA, Tan BK, et al. Thymic stromal lymphopoietin activity is increased in nasal polyps of patients with chronic rhinosinusitis. The J Allergy Clin Immunol.2013;132:593-600.e12.

334. Gauvreau GM, O'Byrne PM, Boulet LP, et al. Effects of an anti-TSLP antibody on allergen-induced asthmatic responses. N Engl J Med 2014;370:210210.

335. Shin HW, Kim DK, Park MH, et al. IL-25 as a nove therapeutic target in nasal polyps of patients with chronic rhinosinusitis. J Allergy Clin Immunol.2015;135:1476-85.e7.

336. Lan F, Zhang N, Holtappels G, et al. Staphylococcus aureus Induces a Mucosal Type 2 Immune Response via Epithelial Cell-derived Cytokines. Am J Respir Crit Care Med 2018;198:452-63.

337. Teufelberger AR, Nordengrun $M, B r a u n ~ H$, et al. The IL-33/ST2 axis is crucial in type 2 airway responses induced by Staphylococcus aureus-derived serine protease-like protein D. The J Allergy Clin Immunol.2018;141:549-59.e7.

338. Ishinaga $H$, Kitano $M$, Toda $M$, et al. Interleukin-33 induces mucin gene expression and goblet cell hyperplasia in human nasal epithelial cells. Cytokine. 2017;90:60-5

339. Poposki JA, Klingler Al, Tan BK, et al. Group 2 innate lymphoid cells are elevated and activated in chronic rhinosinusitis with nasal polyps. Immunity, inflammation and disease. 2017;5:233-43.

340. Mjosberg J, Bernink J, Golebski K, et al. The transcription factor GATA3 is essential for the function of human type 2 innate lymphoid cells.
Immunity 2012;37:649-59.

341. Van Crombruggen K, Taveirne S, Holtappels G, Leclercq G, Bachert C. Innate lymphoid cells in the upper airways: importance of CD117 and IL-1RI expression. Eur Respir J. 2018;52.

342. Krysko O, Teufelberger A, Nevel SV, Krysko DV, Bachert C. Protease/antiprotease network in allergy: The role of Staphylococcus aureus protease-like proteins. Allergy. 2019, https://dx.doi. org/10.1111/all.13783.

343. Venkataramani S, Low S, Weigle B, et al. Design and characterization of Zweimab and Doppelmab, high affinity dual antagonistic anti-TSLP/IL13 bispecific antibodies. Biochem Biophys Res Commun 2018;504:19-24.

344. Bikker A, Hack CE, Lafeber FP, van Roon JA. Interleukin-7: a key mediator in T cell-driven autoimmunity, inflammation, and tissue destruction. Curr Pharm Des 2012;18:2347-56.

345. Nagasawa M, Heesters BA, Kradolfer CMA, et al. KLRG1 and NKp46 discriminate subpopulations of human CD117+CRTH2- ILCs biased toward ILC2 or ILC3. The J of Experimental Medicine. 2019;216:1762-76.

346. Liu W, Putnam AL, Xu-Yu Z, et al. CD127 expression inversely correlates with FoxP3 and suppressive function of human CD4+ T reg cells. J Exp Med. 2006;203:1701-11.

347. Belarif L, Mary C, Jacquemont L, et al. IL-7 receptor blockade blunts antigen-specific memory $\mathrm{T}$ cell responses and chronic inflammation in primates. Nat Commun. 2018;9:4483.

348. He R, Geha RS. Thymic stromal lymphopoietin. Ann NY Acad Sci. 2010;1183:13-24.

349. Krabbendam L, Bal SM, Spits H, Golebski K. New insights into the function, development, and plasticity of type 2 innate lymphoid cells. Immunol. Rev. 2018;286:74-85.

350. Ellis J, van Maurik A, Fortunato L, et al. Anti-IL-7 receptor a monoclonal antibody (GSK2618960) in healthy subjects - a randomized, double-blind, placebo-controlled study. Br J Clin Pharmacol. 2019;85:304-15.

351. Ricciotti E, FitzGerald GA. Prostaglandins and inflammation. Arterioscler Thromb Vasc Biol 2011;31:986-1000.

352. Barnes PJ. Cellular and molecular mechanisms of asthma and COPD. Clin Sci (Lond) 2017;131:1541 58.

353. Mjosberg JM, Trifari S, Crellin NK, et al. Human IL25- and IL-33-responsive type 2 innate lymphoid cells are defined by expression of CRTH2 and CD161. Nat Immunol. 2011;12:1055-62.

354. Diamant Z, Aalders W, Parulekar A, Bjermer L, Hanania NA. Targeting lipid mediators in asthma: time for reappraisal. Curr Opin Pulm Med. 2019;25:121-7.

355. Roth-Walter F, Adcock IM, Benito-Villalvilla C, et al. Comparing biologicals and small molecule drug therapies for chronic respiratory diseases: An EAACI Taskforce on Immunopharmacology position paper. Allergy. 2019;74:432-48.

356. Ratner P, Andrews CP, Hampel FC, et al. Efficacy and safety of setipiprant in seasonal allergic rhinitis: results from Phase 2 and Phase 3 randomized, 
double-blind, placebo- and active-referenced studies. Allergy., asthma, and clinical immunology : official J of the Canadian Society of Allergy. and Clinical Immunology 2017;13:18-.

357. Diamant Z, Sidharta PN, Singh D, et al. Setipiprant, a selective $\mathrm{CRTH} 2$ antagonist, reduces allergeninduced airway responses in allergic asthmatics. Clin Exp Allergy. 2014;44:1044-52.

358. Singh D, Cadden $P$, Hunter M, et al. Inhibition of the asthmatic allergen challenge response by the CRTH2 antagonist OC000459. Eur Respir J. 2013;41:46-52.

359. Barnes N, Pavord I, Chuchalin A, et al. A randomized, double-blind, placebo-controlled study of the CRTH2 antagonist OC000459 in moderate persistent asthma. Clin Exp Allergy. 2012;42:38-48.

360. Hall IP, Fowler AV, Gupta A, et al. Efficacy of BI 671800 , an oral CRTH2 antagonist, in poorly controlled asthma as sole controller and in the presence of inhaled corticosteroid treatment. Pulm Pharmacol Ther 2015;32:37-44.

361. Erpenbeck VJ, Popov TA, Miller D, et al. The oral CRTh2 antagonist QAW039 (fevipiprant): A phase II study in uncontrolled allergic asthma. Pulm Pharmacol Ther 2016;39:54-63.

362. Bateman ED, Guerreros AG, Brockhaus F, et al. Fevipiprant, an oral prostaglandin DP2 receptor (CRTh2) antagonist, in allergic asthma uncontrolled on low-dose inhaled corticosteroids. Eur Respir J. 2017;50.

363. Pettipher R, Hunter MG, Perkins CM, et al. Heightened response of eosinophilic asthmatic patients to the $\mathrm{CRTH} 2$ antagonist OC000459. Allergy. 2014;69:1223-32.

364. Fajt ML, Gelhaus SL, Freeman B, et al. Prostaglandin $\mathrm{D}(2)$ pathway upregulation: relation to asthma severity, control, and TH2 inflammation. J Allergy Clin Immunol.2013;131:1504-12.

365. Busse WW, Wenzel SE, Meltzer EO, et al. Safety and efficacy of the prostaglandin D2 receptor antagonist AMG 853 in asthmatic patients. J Allergy Clin Immunol.2013;131:339-45.

366. Gonem S, Berair R, Singapuri A, et al. Fevipiprant, a prostaglandin $\mathrm{D} 2$ receptor 2 antagonist, in patients with persistent eosinophilic asthma: a single-centre, randomised, double-blind, parallelgroup, placebo-controlled trial. Lancet Respir Med. 2016;4:699-707.

367. Xue L, Fergusson J, Salimi M, et al. Prostaglandin D2 and leukotriene E4 synergize to stimulate diverse $\mathrm{TH} 2$ functions and $\mathrm{TH} 2$ cell/neutrophil crosstalk. J Allergy Clin Immunol.2015;135:1358-66. e1-11.

368. Zhang G, Zhao Y, Paramasivan S, et al. Bacteriophage effectively kills multidrug resistant Staphylococcus aureus clinical isolates from chronic rhinosinusitis patients. Int Forum Allergy. Rhinol. 2018;8:406-14.

369. Drilling A, Morales S, Jardeleza C, Vreugde S, Speck $\mathrm{P}$, Wormald PJ. Bacteriophage reduces biofilm of Staphylococcus aureus ex vivo isolates from chronic rhinosinusitis patients. Am J Rhinol.Allergy. 2014;28:3-11.

370. Fong SA, Drilling A, Morales S, et al. Activity of Bacteriophages in Removing Biofilms of
Pseudomonas aeruginosa Isolates from Chronic Rhinosinusitis Patients. Front Cell Infect Microbiol 2017;7:418.

371. Richter K, Ramezanpour M, Thomas N, Prestidge CA, Wormald PJ, Vreugde S. Mind "De GaPP": in vitro efficacy of deferiprone and galliumprotoporphyrin against Staphylococcus aureus biofilms. Int Forum Allergy. Rhinol. 2016;6:737-43.

372. Bachert C, Holtappels G, Merabishvili M, et al. Staphylococcus aureus controls interleukin-5 release in upper airway inflammation. J Proteomics 2018;180:53-60.

373. Lal D, Jategaonkar AA, Borish L, et al. Management of rhinosinusitis during pregnancy: systematic review and expert panel recommendations. Rhinology. 2016;54:99-104.

374. Norjavaara E, de Verdier MG. Normal pregnancy outcomes in a population-based study including 2,968 pregnant women exposed to budesonide. J Allergy Clin Immunol.2003;111:736-42.

375. Dua K, Chopra H, Khurana A, Munjal M. CT scan variations in chronic sinusitis. . Indian J Radiol Imaging 2005;15:315-20.

376. Error M, Ashby S, Orlandi RR, Alt JA. Single-Blinded Prospective Implementation of a Preoperative Imaging Checklist for Endoscopic Sinus Surg. Otolaryngol. Head Neck Surg. 2018;158:177-80.

377. Burger W, Chemnitius JM, KneissI GD, Rucker G. Low-dose aspirin for secondary cardiovascular prevention - cardiovascular risks after its perioperative withdrawal versus bleeding risks with its continuation - review and meta-analysis. J Intern Med 2005;257:399-414.

378. Grzegorzek T, Kolebacz B, Stryjewska-Makuch G, Kasperska-Zajac A, Misiolek M. The influence of selected preoperative factors on the course of endoscopic surgery in patients with chronic rhinosinusitis. Adv Clin Exp Med 2014;23:69-78.

379. Gunel C, Basak HS, Bleier BS. Oral steroids and intraoperative bleeding during endoscopic sinus surgery. B-ENT 2015;11:123-8.

380. Albu S, Gocea A, Mitre I. Preoperative treatment with topical corticoids and bleeding during primary endoscopic sinus surgery. Otolaryngol. Head Neck Surg. 2010;143:573-8.

381. Wright ED, Agrawal S. Impact of perioperative systemic steroids on surgical outcomes in patients with chronic rhinosinusitis with polyposis: evaluation with the novel Perioperative Sinus Endoscopy (POSE) scoring system. Laryngoscope. 2007;117:1-28.

382. Boezaart AP, van der Merwe J, Coetzee A. Comparison of sodium nitroprusside- and esmolol-induced controlled hypotension for functional endoscopic sinus surgery. Can J Anaesth 1995;42:373-6.

383. Neel HB, Harner SG, Rice DH. Endoscopic Sinus Surg. Otolaryngology-Head and Neck Surg. 1994;111:100-10.

384. Rudmik L, Holy CE, Smith TL. Geographic variation of endoscopic sinus surgery in the united states. The Laryngoscope. 2015;125:1772-8.

385. Rudmik L, Bird C, Dean S, Dort JC, Schorn R, Kukec E. Geographic Variation of Endoscopic Sinus Surgery in Canada: An Alberta-Based Small Area Variation Analysis. Otolaryngol. Head Neck Surg.
2015;153:865-74.

386. Toppila-Salmi SK, Rihkanen H, Arffman M, Manderbacka K, Keskimäki I, Hytönen M. Regional differences in endoscopic sinus surgery in Finland. BMJ Open 2018, 10.1136/bmjopen-2018-022173.

387. Venkatraman G, Likosky DS, Zhou W, Finlayson SR, Goodman DC. Trends in endoscopic sinus surgery rates in the Medicare population. Arch Otolaryngol. Head Neck Surg. 2010;136:426-30.

388. Ference EH, Suh JD, Tan BK, Smith SS. How often is sinus surgery performed for chronic rhinosinusitis with versus without nasal polyps? Am J Rhinol. Allergy. 2018;32:34-9.

389. Dautremont JF, Rudmik L. When are we operating for chronic rhinosinusitis? A systematic review of maximal medical therapy protocols prior to endoscopic sinus surgery. International Forum of Allergy. \& Rhinology. 2015;5:1095-103.

390. Soler ZM, Rudmik L, Hwang PH, Mace JC, Schlosser RJ, Smith TL. Patient-centered decision making in the treatment of chronic rhinosinusitis. Laryngoscope. 2013;123:2341-6.

391. Rudmik L, Soler ZM, Mace JC, DeConde AS, Schlosser RJ, Smith TL. Using preoperative SNOT22 score to inform patient decision for Endoscopic sinus surgery. Laryngoscope. 2015;125:1517-22.

392. Rudmik L, Soler ZM, Hopkins C. Using postoperative SNOT-22 to help predict the probability of revision sinus surgery. Rhinology. 2016;54:111-6.

393. Hopkins C, Browne JP, Slack R, et al. The national comparative audit of surgery for nasal polyposis and chronic rhinosinusitis. Clin Otolaryngol. 2006;31:390-8.

394. Hopkins C, Browne JP, Slack R, Lund V, Brown P. The Lund-Mackay staging system for chronic rhinosinusitis: How is it used and what does it predict? Otolaryngology-Head and Neck Surg. 2007;137:555-61.

395. Rudmik L, MacE J, Smith T. Low-stage computed tomography chronic rhinosinusitis: What is the role of endoscopic sinus surgery? Laryngoscope. 2011;121:417-21.

396. Laury AM, Chen PG, McMains KC. Randomized Controlled Trial Examining the Effects of Balloon Catheter Dilation on "Sinus Pressure" / Barometric Headaches. Otolaryngol. Head Neck Surg. 2018;159:178-84.

397. Rudmik L, Soler ZM, Hopkins C, et al. Defining appropriateness criteria for endoscopic sinus surgery during management of uncomplicated adult chronic rhinosinusitis: a RAND/UCLA appropriateness study. Int Forum Allergy. Rhinol. 2016;6:557-67.

398. Beswick DM, Mace JC, Soler ZM, et al. Appropriateness criteria predict outcomes for sinus surgery and may aid in future patient selection. Laryngoscope. 2018;128:2448-54.

399. Chong LY, Head K, Hopkins C, et al. Saline irrigation for chronic rhinosinusitis. Cochrane Database Syst Rev. 2016;4:CD011995.

400. Chong LY, Head K, Hopkins C, Philpott C, Schilder AG, Burton MJ. Intranasal steroids versus placebo or no intervention for chronic rhinosinusitis. Cochrane Database Syst Rev. 2016;4:CD011996.

401. Orlandi RR, Kingdom TT, Hwang PH, et al. 
International Consensus Statement on Allergy. and Rhinology.: Rhinosinusitis. Int Forum Allergy Rhinol. 2016;6 Suppl 1:S22-S209.

402. Brooks SG, Trope M, Blasetti M, et al. Preoperative Lund-Mackay computed tomography score is associated with preoperative symptom severity and predicts quality-of-life outcome trajectories after sinus surgery. International Forum of Allergy. and Rhinology. 2018;8:668-75.

403. Lal D, Hopkins C, Divekar RD. SNOT-22-based clusters in chronic rhinosinusitis without nasal polyposis exhibit distinct endotypic and prognostic differences. Int Forum Allergy. Rhinol. 2018;8:797-805.

404. Soler ZM, Hyer JM, Rudmik L, Ramakrishnan V, Smith TL, Schlosser RJ. Cluster analysis and prediction of treatment outcomes for chronic rhinosinusitis. J Allergy Clin Immunol.2016;137:1054-62.

405. Le PT, Soler ZM, Jones R, Mattos JL, Nguyen SA, Schlosser RJ. Systematic Review and Metaanalysis of SNOT-22 Outcomes after Surgery for Chronic Rhinosinusitis with Nasal Polyposis. Otolaryngology - Head \& Neck Surg. 2018;159:41423.

406. Hopkins C, Rimmer J, Lund VJ. Does time to endoscopic sinus surgery impact outcomes in Chronic Rhinosinusitis? Prospective findings from the National Comparative Audit of Surgery for Nasal Polyposis and Chronic Rhinosinusitis. Rhinology. J 2015;53:10-7.

407. Benninger MS, Sindwani R, Holy CE, Hopkins C. Early versus delayed endoscopic sinus surgery in patients with chronic rhinosinusitis: impact on health care utilization. Otolaryngol. Head Neck Surg. 2015;152:546-52.

408. Benninger MS, Sindwani R, Holy CE, Hopkins C. Impact of medically recalcitrant chronic rhinosinusitis on incidence of asthma. Int Forum Allergy. Rhinol. 2016;6:124-9.

409. Sahlstrand-Johnson P, Hopkins C, Ohlsson B, Ahlner-Elmqvist M. The effect of endoscopic sinus surgery on quality of life and absenteeism in patients with chronic rhinosinuitis - a multi-centre study. Rhinology. 2017;55:251-61.

410. Yip J, Hao W, Eskander A, Lee JM. Wait times for endoscopic sinus surgery influence patientreported outcome measures in patients with chronic rhinosinusitis who fulfill appropriateness criteria. Int Forum Allergy. Rhinol. 2019;9:396-401.

411. Gosepath J, Mann WJ. Endonasal sinus surgery in patients with chronic rhinosinusitis. Otorinolaringologia 2003;53:109-21.

412. Catalano PJ. Minimally invasive sinus technique: what is it? Should we consider it? Curr Opin Otolaryngol. Head Neck Surg. 2004;12:34-7.

413. Catalano P, Roffman E. Outcome in patients with chronic sinusitis after the minimally invasive sinus technique. Am J Rhinol.2003;17:17-22.

414. Kennedy DW, Zinreich SJ, Shaalan H, Kuhn F, Naclerio R, Loch E. Endoscopic middle meatal antrostomy: theory, technique, and patency. Laryngoscope. 1987;97:1-9.

415. Wadwongtham W, Aeumjaturapat S. Large middle meatal antrostomy vs undisturbed maxillary ostium in the endoscopic sinus surgery of nasal polyposis. Chotmaihet thangphaet [J of the Medical Association of Thailand] 2003;86:S373-8.

416. Albu S, Tomescu E. Small and large middle meatus antrostomies in the treatment of chronic maxillary sinusitis. Otolaryngology - Head and Neck Surg. 2004;131:542-7.

417. Kim YH, Jung G, Lee SJ, Ko BY, Jang TY. Outcomes of middle meatal antrostomy after different surgical strategies. Acta Otolaryngol. 2011;131:1002-7.

418. Myller J, Dastidar P, Torkkeli T, Rautiainen M, Toppila-Salmi S. Computed tomography findings after endoscopic sinus surgery with preserving or enlarging maxillary sinus ostium surgery. Rhinology. 2011;49:438-44.

419. Byun JY, Lee JY. Usefulness of partial uncinectomy in patients with localized maxillary sinus pathology. Curr Opin Pulm Med.2014;35:594-7.

420. Kuehnemund M, Lopatin A, Amedee RG, Mann WJ. Endonasal sinus surgery: extended versus limited approach. Am J Rhinol.2002;16:187-92.

421. Pang YT, Willatt DJ. Do antral washouts have a place in the current management of chronic sinusitis? J Laryngol. Otol. 1996;110:926-8.

422. Byun JY, Lee JY. Canine fossa puncture for severe maxillary disease in unilateral chronic sinusitis with nasal polyp. Laryngoscope. 2013;123:E79-E84

423. Velasquez N, Thamboo A, Abuzeid WM, Nayak JV. Safe treatment of ethmoid sinusitis utilizing minimally invasive ethmoid punch sinusotomy in chronic rhinosinusitis without polyposis patients. Laryngoscope. 2017;127:1268-75.

424. Kuperan AB, Lieberman SM, Jourdy DN, Al-Bar MH Goldstein BJ, Casiano RR. The effect of endoscopic olfactory cleft polyp removal on olfaction. American J Rhinol \& Allergy.. 2015;29:309-13.

425. Chen FH, Deng J, Hong HY, et al. Extensive versus functional endoscopic sinus surgery for chronic rhinosinusitis with nasal polyps and asthma: A 1-year study. Am J Rhinol.Allergy. 2016;30:143-8.

426. Abd El-Fattah H, Nour YA, El-Daly A. Endoscopic radical antrectomy: A permanent replacement for the Caldwell-Luc operation. J Laryngol. Otol. 2008;122:268-76.

427. Jacob KJ, George S, Preethi S, Arunraj VS. A comparative study between endoscopic middle meatal antrostomy and caldwell-luc surgery in the treatment of chronic maxillary sinusitis. Indian J Otolaryngol. Head Neck Surg.. 2011;63:214-9.

428. Jonnalagadda S. In reference to the article a comparative study between endoscopic middle meatal antrostomy and caldwell-luc surgery in the treatment of chronic maxillary sinusitis: by k. Joe jacob . shibu george . s. Preethi .v. S. Arunraj. Indian J Otolaryngol. Head Neck Surg.. 2011;63:412.

429. Penttilä M, Rautiainen M, Puk, er J, Kataja M Functional vs. radical maxillary surgery. Failures after functional endoscopic sinus surgery. Acta Otolaryngol. Suppl 1997;529:173-6.

430. Woodworth BA, Parker RO, Schlosser RJ. Modified endoscopic medial maxillectomy for chronic maxillary sinusitis. Am J Rhinol.2006;20:317-9.

431. Alsharif $S$, Jonstam $K$, van Zele $T$, Gevaert $P$, Holtappels G, Bachert C. Endoscopic Sinus
Surgery for Type-2 CRS wNP: An EndotypeBased Retrospective Study. Laryngoscope. 2019;129:1286-92.

432. Silverman JB, Prasittivatechakool K, Busaba NY. An evidence-based review of endoscopic frontal sinus surgery. American J of Rhinology. \& Allergy. 2009;23:e59-e62.

433. Abuzeid WM, Vakil M, Lin J, et al. Endoscopic modified Lothrop procedure after failure of primary endoscopic sinus surgery: a meta-analysis. International Forum of Allergy. \& Rhinology. 2018;8:605-13.

434. Anderson P, Sindwani R. Safety and efficacy of the endoscopic modified lothrop procedure: A Systematic review and meta-analysis. Laryngoscope. 2009;119:132.

435. Hajbeygi M, Nadjafi A, Amali A, Saedi B, Sadrehosseini SM. Frontal Sinus Patency after Extended Frontal Sinusotomy Type III. Iran J Otorhinolaryngol. 2016;28:337-43.

436. Naidoo Y, Bassiouni A, Keen M, Wormald PJ. Long term outcomes for the endoscopic modified Lothrop/Draf III procedure: a 10-year review. Laryngoscope. 2014;124:43-9.

437. Ye T, Hwang PH, Huang $Z$, et al. Frontal ostium neoosteogenesis and patency after Draf III procedure: a computer-assisted study. Int Forum Allergy. Rhinol. 2014;4:739-44

438. Shih LC, Patel VS, Choby GW, Nakayama T, Hwang $\mathrm{PH}$. Evolution of the endoscopic modified Lothrop procedure: A systematic review and meta-analysis. Laryngoscope. 2018;128:317-26.

439. Wormald PJ. Salvage frontal sinus surgery: the endoscopic modified Lothrop procedure. Laryngoscope. 2003;113:276-83.

440. Samaha M, Cosenza MJ, Metson R. Endoscopic frontal sinus drillout in 100 patients. Arch Otolaryngol. Head Neck Surg. 2003;129:854-8.

441. DeConde AS, Suh JD, Mace JC, Alt JA, Smith TL. Outcomes of complete vs targeted approaches to endoscopic sinus surgery. Int Forum Allergy. Rhinol. 2015;5:691-700.

442. Masterson L, Tanweer F, Bueser T, Leong P. Extensive endoscopic sinus surgery: does this reduce the revision rate for nasal polyposis? Eur Arch Otorhinolaryngol. 2010;267:1557-61.

443. Philpott CM, McKiernan DC, Javer AR. Selecting the best approach to the frontal sinus. Indian J Otolaryngol. Head Neck Surg. 2011;63:79-84.

444. Philpott CM, Thamboo A, Lai L, Park J, Javer AR. Endoscopic frontal sinusotomy-preventing recurrence or a route to revision? Laryngoscope. 2010;120:1682-6.

445. Bayis U, Dursun E, Islam A, et al. Is septoplasty alone adequate for the treatment of chronic rhinosinusitis with septal deviation? Am J Rhinol.2005;19:612-6.

446. Goel AK, Yadav SPS, Ranga R, Gulia JS, Goel R. Comparative study of septoplasty alone and with FESS in maxillary sinusitis with septal deviation. Clinical Rhinology. 2012;5:19-24.

447. Smith TL, Mace JC, Rudmik L, et al. Comparing surgeon outcomes in endoscopic sinus surgery for chronic rhinosinusitis. Laryngoscope. 2017;127:1421. 
448. Li Y, Li P, Jin X. Clinical observation of functional endoscopic sinus surgery associated with radiofrequency ablation of inferior turbinate for chronic rhinosinusitis. Lin chuang er bi yan hou tou jing wai ke za zhi [J of clinical otorhinolaryngology, head, and neck surgery] 2015;29:788-91.

449. Hudon MA, Wright ED, Fortin-Pellerin E, Bussieres $M$. Resection versus preservation of the middle turbinate in surgery for chronic rhinosinusitis with nasal polyposis: a randomized controlled trial. J Otolaryngol Head Neck Surg. 2018;47(1):67.

450. Saafan ME, Ragab SM, Albirmawy OA, Elsherif HS. Powered versus conventional endoscopic sinus surgery instruments in management of sinonasal polyposis. Eur Arch Otorhinolaryngol. 2013;270:149-55

451. Cornet ME, Reinartz SM, Georgalas C, van Spronsen E, Fokkens WJ. The microdebrider, a step forward or an expensive gadget? Rhinology. 2012;50:191-8.

452. Behera S, Mohindra S, Patro SK, Gupta AK. Comparison by objective parameters in patients with chronic rhinosinusitis managed medically and surgically (with and without powered instruments). Allergy. \& rhinology 2016;7:121-6.

453. Selivanova O, Kuehnemund M, Mann WJ, Amedee RG. Comparison of conventional instruments and mechanical debriders for surgery of patients with chronic sinusitis. Am J Rhinol.2003;17:197-202.

454. Tirelli G, Gatto A, Spinato G, Tofanelli M. Surgical treatment of nasal polyposis: a comparison between cutting forceps and microdebrider. American J Rhinol \& Allergy.. 2013;27:e202-6.

455. Mus L, Hermans R, Jorissen M. Long-term effects of cutting versus non-cutting instruments in FESS. Rhinology. 2012;50:56-66.

456. McGarry P, Gana B, Adamson. The effect of microdebriders on tissue for histological diagnosis. Clin Otolaryngol. Allied Sci 1997;22:375-6.

457. Banglawala SM, Mulligan JK, Psaltis AJ, et al. Impact of intraoperative hydrodebrider treatment on postoperative sinonasal inflammation. American J Rhinol \& Allergy.. 2014;28:438-42.

458. Cutler J, Bikhazi N, Light J, et al. Standalone balloon dilation versus sinus surgery for chronic rhinosinusitis: a prospective, multicenter, randomized, controlled trial. American J Rhinol \& Allergy.. 2013;27:416-22.

459. NICE. XprESS multi sinus dilation system for XprESS multi sinus dilation system for treating chronic sinusitis treating chronic sinusitis. 2016. https:// www.nice.org.uk/guidance/mtg30/resources/ xpress-multi-sinus-dilation-system-for-treatingchronic-sinusitis-pdf-64371988593349.

460. Jenks M, Willits I, Turner EE, et al. The XprESS MultiSinus Dilation System for the Treatment of Chronic Sinusitis: A NICE Medical Technology Guidance. Applied Health Economics \& Health Policy 2017:15:567-82.

461. Bizaki AJ, Numminen J, Taulu R, Rautiainen M. A Controlled, Randomized Clinical Study on the Impact of Treatment on Antral Mucociliary Clearance: uncinectomy Versus Balloon Sinuplasty. Annals of Otol, Rhin, and Laryngol.2016;125:408-14.

462. Bizaki AJ, Numminen J, Taulu R, Rautiainen $M$. Decrease of nasal airway resistance and alleviations of symptoms after balloon sinuplasty in patients with isolated chronic rhinosinusitis: a prospective, randomised clinical study. Clin Otolaryngol. 2016;41:673-80.

463. Bizaki AJ, Taulu R, Numminen J, Rautiainen M. Quality of life after endoscopic sinus surgery or balloon sinuplasty: a randomized clinical study. Rhinology. 2014;52:300-5.

464. Minni A, Dragonettp A, Sciuto A, et al. Use of balloon catheter dilation vs. traditional endoscopic sinus surgery in management of light and severe chronic rhinosinusitis of the frontal sinus: a multicenter prospective randomized study. Eur Rev Med Pharmacol Sci 2018;22:285-93.

465. Côté DW, Wright ED. Triamcinolone-impregnated nasal dressing following endoscopic sinus surgery: a randomized, double-blind, placebo-controlled study. Laryngoscope. 2010;120:1269-73.

466. Xu J, Park SJ, Park HS, Han R, Rha KS, Kim YM. Effects of triamcinolone-impregnated nasal dressing on subjective and objective outcomes following endoscopic sinus surgery. Eur Arch Otorhinolaryngol. 2016;273:4351-7.

467. Hong SD, Kim JH, Dhong HJ, et al. Systemic effects and safety of triamcinolone-impregnated nasal packing after endoscopic sinus surgery: a randomized, double-blinded, placebo-controlled study. American J Rhinol \& Allergy.. 2013;27:40710.

468. Gyawali BR, Pradhan B, Thapa N. Comparison of outcomes of triamcinolone versus normal saline soaked polyvinyl alcohol pack following bilateral endoscopic sinus surgery. Rhinology. 2019;57:28792.

469. Huvenne W, Zhang N, Tijsma E, et al. Pilot study using doxycycline-releasing stents to ameliorate postoperative healing quality after sinus surgery. Wound Repair Regen 2008;16:757-67.

470. Han JK, Kern RC. Topical therapies for management of chronic rhinosinusitis: steroid implants. Int Forum Allergy. Rhinol. 2019;9:S22-s6.

471. Singh A, Luong AU, Fong KJ, et al. Bioabsorbable steroid-releasing implants in the frontal sinus ostia: a pooled analysis. International Forum of Allergy. and Rhinology. 2018;9:131-9.

472. Han JK, Marple BF, Smith TL, et al. Effect of steroid-releasing sinus implants on postoperative medical and surgical interventions: an efficacy meta-analysis. International Forum of Allergy. \& Rhinology. 2012;2:271-9.

473. Rizan C, Elhassan HA. Post-sinus surgery insertion of steroid-eluting bioabsorbable intranasal devices: A systematic review. Laryngoscope. 2016;126:86-92

474. Rudmik L, Smith TL. Economic Evaluation of a Steroid-Eluting Sinus Implant following Endoscopic Sinus Surgery for Chronic Rhinosinusitis. Otolaryngology - Head \& Neck Surg. 2014;151:359-66.

475. Rizzo JA, Rudmik L, Mallow PJ, Palli SR. Budget impact analysis of bioabsorbable drug-eluting sinus implants following endoscopic sinus surgery. J Med Econ 2016;19:829-35.

476. Douglas RG, Psaltis AJ, Rimmer J, Kuruvilla T, Cervin A, Kuang Y. Phase 1 clinical study to assess the safety of a novel drug delivery system providing long-term topical steroid therapy for chronic rhinosinusitis. Int Forum Allergy. Rhinol. 2019;9:378-87.

477. Coey JG, Whittaker PJ, Williams G, Ikram UH, Page OJR. Fibrin tissue adhesive versus nasal packing in endoscopic nasal surgery: a systematic review and meta-analysis. Rhinology. 2018;57:21-31.

478. Leunig A, Betz CS, Siedek V, Kastl KG. CMC packing in functional endoscopic sinus surgery: does it affect patient comfort? Rhinology. 2009;47:36-40.

479. Burduk PK, Wierzchowska M, Grześkowiak B, Kaźmierczak W, Wawrzyniak K. Clinical outcome and patient satisfaction using biodegradable (NasoPore) and non-biodegradable packing, a double-blind, prospective, randomized study. Braz J Otorhinolaryngol. 2017;83:23-8.

480. Cho KS, Shin SK, Lee JH, et al. The efficacy of Cutanplast nasal packing after endoscopic sinus surgery: a prospective, randomized, controlled trial. Laryngoscope. 2013;123:564-8.

481. Piski Z, Gerlinger I, Nepp N, et al. Clinical benefits of polyurethane nasal packing in endoscopic sinus surgery. Eur Arch Otorhinolaryngol. 2017;274:1449-54.

482. Zhao KQ, Yu YQ, Yu HM. Effects of mometasone furoate-impregnated biodegradable nasal dressing on endoscopic appearance in healing process following endoscopic sinus surgery: a randomized, double-blind, placebo-controlled study. Int Forum Allergy. \& Rhinol. 2018;8(11):12331241.

483. Grzeskowiak B, Wierzchowska M, Walorek R, Seredyka-Burduk M, Wawrzyniak K, Burduk PK. Steroid vs. antibiotic impregnated absorbable nasal packing for wound healing after endoscopic sinus surgery: a randomized, double blind, placebo-controlled study. Braz J Otorhinolaryngol. 2019;85:473-80.

484. Bing Z, Feng L, Wu CS, Du JT, Liu YF, Liu SX. Acellular dermal matrix contributes to epithelialization in patients with chronic sinusitis. J Biomater Appl 2019:885328218822636.

485. Okushi T, Yoshikawa M, Otori N, et al. Evaluation of symptoms and $\mathrm{QOL}$ with calcium alginate versus chitin-coated gauze for middle meatus packing after endoscopic sinus surgery. Auris Nasus Larynx. 2012;39:31-7.

486. Park DY, Chung HJ, Sim NS, et al. Comparison of calcium alginate and carboxymethyl cellulose for nasal packing after endoscopic sinus surgery: a prospective, randomised, controlled singleblinded trial. Clin Otolaryngol. 2016;41:234-40.

487. Akbari E, Philpott CM, Ostry AJ, Clark A, Javer AR. A double-blind randomised controlled trial of gloved versus ungloved merocel middle meatal spacers for endoscopic sinus surgery. Rhinology. 2012;50:306-10.

488. Hobson CE, Choby GW, Wang EW, Morton SC, Lee S. Systematic review and metaanalysis of middle meatal packing after endoscopic sinus surgery. American J of Rhinology. \& Allergy. 2015;29:13540.

489. Baguley CJ, Stow NW, Weitzel EK, Douglas RG. Silastic splints reduce middle meatal adhesions after endoscopic sinus surgery. American J Rhinol \& Allergy.. 2012;26:414-7. 
490. Chan CL, Elmiyeh B, Woods C, et al. A randomized controlled trial of a middle meatal silastic stent for reducing adhesions and middle turbinate lateralization following endoscopic sinus surgery. Int Forum Allergy. \& Rhinol.2015;5:517-23.

491. Yan M, Zheng D, Li Y, Zheng Q, Chen J, Yang B. Biodegradable nasal packings for endoscopic sinonasal surgery: A systematic review and metaanalysis. PLoS One. 2014;9(12):e115458.

492. Wang TC, Tai CJ, Tsou YA, Tsai LT, Li YF, Tsai MH. Absorbable and nonabsorbable packing after functional endoscopic sinus surgery: systematic review and meta-analysis of outcomes. Eur Arch Otorhinolaryngol. 2015;272:1825-31.

493. Bugten V, Nordgård S, Skogvoll E, Steinsvåg S. Effects of nonabsorbable packing in middle meatus after sinus surgery. Laryngoscope. 2006;116:83-8.

494. Vlastarakos PV, lacovou E, Fetta M, Tapis M, Nikolopoulos TP. How effective is postoperative packing in FESS patients? A critical analysis of published interventional studies. Eur Arch Otorhinolaryngol. 2016;273:4061-71.

495. Stern-Shavit S, Nachalon Y, Leshno M, Soudry E. Middle meatal packing in endoscopic sinus surgery-to pack or not to pack?-a decision-analysis model. Laryngoscope. 2017;127:1506-12.

496. American Academy of Otolaryngology-Head and Neck Surg. Position Statement: Intra-Operative Use of Computer Aided Surg. 2014. https://www. entnet.org/content/intra-operative-use-computeraided-surgery.

497. Miller RS, Steward DL, Tami TA, et al. The clinical effects of hyaluronic acid ester nasal dressing (Merogel) on intranasal wound healing after functional endoscopic sinus surgery. Otolaryngol. Head Neck Surg. 2003;128:862-9.

498. Smith TL, Stewart MG, Orlandi RR, Setzen M, Lanza DC. Indications for image-guided sinus surgery: The current evidence. Am J Rhinol.2007;21:80-3.

499. Tabaee A, Hsu AK, Shrime MG, Rickert S, Close LG. Quality of life and complications following imageguided endoscopic sinus surgery. Otolaryngology - Head \& Neck Surg. 2006;135:76-80.

500. Krings JG, Kallogjeri D, Wineland A, Nepple KG, Piccirillo JF, Getz AE. Complications of primary and revision functional endoscopic sinus surgery for chronic rhinosinusitis. Laryngoscope. 2014;124:838-45

501. Masterson L, Agalato E, Pearson C. Image-guided sinus surgery: practical and financial experiences from a UK centre 2001-2009. J Laryngol. Otol. 2012;126:1224-30.

502. Masterson L, Egro FM, Bewick J, et al. Qualityof-life outcomes after sinus surgery in allergic fungal rhinosinusitis versus nonfungal chronic rhinosinusitis. Am J Rhinol.Allergy. 2016;30:e30-5.

503. Fried MP, Moharir VM, Shin J, Taylor-Becker M, Morrison P. Comparison of endoscopic sinus surgery with and without image guidance. Am J Rhinol.2002;16:193-7.

504. Dalgorf DM, Sacks R, Wormald PJ, et al. Imageguided surgery influences perioperative morbidity from endoscopic sinus surgery: A systematic review and meta-analysis. Otolaryngology - Head and Neck Surg. (United States) 2013;149:17-29.
505. Sunkaraneni VS, Yeh D, Qian H, Javer AR. Computer or not? Use of image guidance during endoscopic sinus surgery for chronic rhinosinusitis at St Paul's Hospital, Vancouver, and meta-analysis. J Laryngol. Otol. 2013;127:368-77.

506. Ramakrishnan VR, Orlandi RR, Citardi MJ, Smith TL, Fried MP, Kingdom TT. The use of imageguided surgery in endoscopic sinus surgery: An evidence-based review with recommendations. International Forum of Allergy. and Rhinology. 2013;3:236-41.

507. Javer AR, Genoway KA. Patient quality of life improvements with and without computer assistance in sinus surgery: outcomes study. The J of Otolaryngology 2006;35:373-9.

508. Tabaee A, Kacker A, Kassenoff TL, Anand V. Outcome of Computer-Assisted Sinus Surg: A 5-Year Study. Am J Rhinol.2003;17:291-7.

509. Farhadi M, Jalessi M, Sharifi G, et al. Use of image guidance in endoscopic endonasal surgeries: A 5-year experience. B-ENT 2011;7:277-82.

510. Theodoraki MN, Ledderose GJ, Becker S, et al. Mental distress and effort to engage an image-guided navigation system in the surgical training of endoscopic sinus surgery: a prospective, randomised clinical trial. Eur Arch Otorhinolaryngol. 2015;272:905-13.

511. Stelter K, Ertl-Wagner B, Luz M, et al. Evaluation of an image-guided navigation system in the training of functional endoscopic sinus surgeons. A prospective, randomised clinical study. Rhinology. 2011;49:8.

512. Lapeer RJ, Jeffrey SJ, Dao JT, et al. Using a passive coordinate measurement arm for motion tracking of a rigid endoscope for augmented-reality imageguided surgery. International J of Medical Robotics and Computer Assisted Surg. 2014;10:65-77.

513. Li L, Yang J, Chu Y, et al. A Novel Augmented Reality Navigation System for Endoscopic Sinus and Skull Base Surg: A Feasibility Study. PLoS One 2016;11:e0146996.

514. Citardi MJ, Agbetoba A, Bigcas JL, Luong A. Augmented reality for endoscopic sinus surgery with surgical navigation: a cadaver study. Int Forum Allergy. Rhinol. 2016;6:523-8.

515. Citardi MJ, Yao W, Luong A. Next-Generation Surgical Navigation Systems in Sinus and Skull Base Surg. Otolaryngol. Clin North Am 2017;50:617-32.

516. Eichhorn KW, Bootz F. Clinical requirements and possible applications of robot assisted endoscopy in skull base and sinus surgery. Acta Neurochir Suppl 2011;109:237-40.

517. Eichhorn KW, Westphal R, Last C, et al. Workspace and pivot point for robot-assisted endoscope guidance in functional endonasal sinus surgery (FESS). Int J Med Robot 2015;11:30-7.

518. Eichhorn KW, Westphal R, Rilk M, et al. Robotassisted endoscope guidance versus manual endoscope guidance in functional endonasal sinus surgery (FESS). Acta Otolaryngol. 2017;137:1090-5.

519. Rilk M, Kubus D, Wahl FM, Eichhorn KWG, Wagner I, Bootz F. Demonstration of a prototype for robot assisted Endoscopic Sinus Surg. IEEE International Conference on Robotics and Automation 2010:1090-1.
520. Trevillot V, Garrel R, Dombre E, Poignet P, Sobral R, Crampette L. Robotic endoscopic sinus and skull base surgery: review of the literature and future prospects. Eur Ann Otorhinolaryngol. Head Neck Dis 2013;130:201-7.

521. Wurm J, Bumm K, Steinhart H, et al. Entwicklung eines aktiven Robotersystems für die multimodale Chirurgie der Nasennebenhöhlen. HNO. 2005;53:446-54.

522. Wurm J, Dannenmann T, Bohr C, Iro H, Bumm K. Increased safety in robotic paranasal sinus and skull base surgery with redundant navigation and automated registration. Int J Med Robot 2005;1:428.

523. Yoon $\mathrm{H}$, Oh SM, Jeong JH, et al. In Active bending endoscope robot system for navigation through sinus area. 2011 IEEE/RSJ International Conference on Intelligent Robots and Systems, 2011.

524. Paleri V, Fox H, Winter S. Transoral Robotic Surgery for Oropharyngeal Cancer. ORL J Otorhinolaryngol. Relat Spec 2018;80:156-70.

525. He Y, Hu Y, Zhang P, Zhao B, Qi X, Zhang J. HumanRobot Cooperative Control Based on Virtual Fixture in Robot-Assisted Endoscopic Sinus Surg. Applied Sciences 2019;9:1659.

526. Zhong F, Li P, Shi J, et al. Foot-controlled RobotEnabled EnDOscope Manipulator (FREEDOM) For Sinus Surg: Design, Control and Evaluation. IEEE Trans Biomed Eng 2019, 10.1109/ tbme.2019.2939557.

527. Levy JM, Marino MJ, McCoul ED. Paranasal Sinus Balloon Catheter Dilation for Treatment of Chronic Rhinosinusitis: A Systematic Review and Metaanalysis. Otolaryngology - Head \& Neck Surg. 2016;154:33-40.

528. Kilty SJ, Lasso A, Mfuna-Endam L, Desrosiers MY. Case-control study of endoscopic polypectomy in clinic (EPIC) versus endoscopic sinus surgery for chronic rhinosinusitis with polyps. Rhinology. 2018;56(2):155-7.

529. Tzelnick S, Alkan U, Leshno M, Hwang P, Soudry E. Sinonasal debridement versus no debridement for the postoperative care of patients undergoing endoscopic sinus surgery. Cochrane Database Syst Rev. 2018;11:CD011988.

530. Green R, Banigo A, Hathorn I. Postoperative nasal debridement following functional endoscopic sinus surgery, a systematic review of the literature. Clin Otolaryngol. 2015;40:2-8.

531. Varsak YK, Yuca K, Eryilmaz MA, Arbag H. Single seventh day debridement compared to frequent debridement after endoscopic sinus surgery: a randomized controlled trial. Eur Arch Otorhinolaryngol. 2016;273:689-95.

532. Rudmik L, Soler ZM, Orlandi RR, et al. Early postoperative care following endoscopic sinus surgery: an evidence-based review with recommendations. International Forum of Allergy. \& Rhinology. 2011;1:417-30.

533. Kim ST, Sung UH, Jung JH, et al. The effect of maxillary sinus irrigation on early prognostic factors after endoscopic sinus surgery: a preliminary study. American J of Rhinology. \& Allergy. 2013;27:e158-61.

534. Chen XZ, Feng SY, Chang LH, et al. The effects of nasal irrigation with various solutions after 
endoscopic sinus surgery: systematic review and meta-analysis. J Laryngol. Otol. 2018;132:673-9.

535. Fandino M, Macdonald KI, Lee J, Witterick IJ. The use of postoperative topical corticosteroids in chronic rhinosinusitis with nasal polyps: a systematic review and meta-analysis. American J of Rhinology. \& Allergy. 2013;27:e146-e57.

536. Huang ZZ, Chen XZ, Huang JC, et al. Budesonide nasal irrigation improved Lund-Kennedy endoscopic score of chronic rhinosinusitis patients after endoscopic sinus surgery. Eur Arch Otorhinolaryngol. 2019;276:1397-403.

537. Kang TW, Chung JH, Cho SH, Lee SH, Kim KR, Jeong JH. The Effectiveness of Budesonide Nasal Irrigation After Endoscopic Sinus Surgery in Chronic Rhinosinusitis With Asthma. Clin Exp Otorhinolaryngol. 2017;10:91-6.

538. Chen X, Wang H, Shi Z, et al. Long-term outcome of budesonide middle meatus treatment for chronic rhinosinusitis patients following endoscopic sinus surgery. Lin chuang er bi yan hou tou jing wai ke za zhi [J of clinical otorhinolaryngology, head, and neck surgery] 2016;30:203-5, 8.

539. Jiang RS, Liang KL, Yang KY, et al. Postoperative antibiotic care after functional endoscopic sinus surgery. Am J Rhinol.2008;22:608-12.

540. Zeng M, Wang H, Liao B, et al. Comparison of efficacy of fluticasone propionate versus clarithromycin for postoperative treatment of different phenotypic chronic rhinosinusitis: a randomized controlled trial. Rhinology. 2018;57:101-9.

541. Saleh AM, Torres KM, Murad MH, Erwin PJ, Driscoll CLW. Prophylactic perioperative antibiotic use in endoscopic sinus surgery: A systematic review and meta-analysis. Otolaryngology - Head and Neck Surg. (United States) 2012;146:533-8.

542. Lange JL, Peeden EH, Stringer SP. Are prophylactic systemic antibiotics necessary with nasal packing? A systematic review. American J of Rhinology. \& Allergy. 2017;31:240-7.

543. Wijewickrama RC, Catalano PJ, Gupta R, et al. Efficacy of targeted middle meatal antibiotics and endoscopic sinus surgery. American J Rhinol \& Allergy.. 2013;27:329-32.

544. Kennedy DW. Prognostic factors, outcomes and staging in ethmoid sinus surgery. Laryngoscope. 1992;102:1-18.

545. Gliklich RE, Metson R. Techniques for outcomes research in chronic sinusitis. Laryngoscope. 1995; 105:387-90.

546. Hopkins C, Slack R, Lund V, Brown P, Copley L, Browne J. Long-term outcomes from the English national comparative audit of surgery for nasal polyposis and chronic rhinosinusitis. Laryngoscope. 2009;119:2459-65.

547. Smith TL, Schlosser RJ, Mace JC, et al. Long-term outcomes of endoscopic sinus surgery in the management of adult chronic rhinosinusitis. Int Forum Allergy. Rhinol. 2019;9:831-41.

548. Soler ZM, Jones R, Le P, et al. Sino-Nasal outcome test- 22 outcomes after sinus surgery: A systematic review and meta-analysis. Laryngoscope. 2018;128:581-92.

549. Hopkins C, Gillett S, Slack R, Lund VJ, Browne JP. Psychometric validity of the 22-item Sinonasal
Outcome Test. Clin Otolaryngol. 2009;34:447-54

550. Mattos JL, Rudmik L, Schlosser RJ, et al. Symptom importance, patient expectations, and satisfaction in chronic rhinosinusitis. Int Forum Allergy. Rhinol. 2019;9:593-600.

551. Phillips KM, Hoehle LP, Caradonna DS, Gray ST, Sedaghat AR. Determinants of noticeable symptom improvement despite sub-MCID change in SNOT-22 score after treatment for chronic rhinosinusitis. Int Forum Allergy. Rhinol. 2019;9:508-13.

552. Chester AC, Antisdel JL, Sindwani R. Symptomspecific outcomes of endoscopic sinus surgery: a systematic review. Otolaryngology - Head \& Neck Surg. 2009;140:633-9.

553. Smith KA, Orlandi RR, Oakley G, Meeks H, Curtin $\mathrm{K}$, Alt JA. Long-term revision rates for endoscopic sinus surgery. Int Forum Allergy. Rhinol. 2019;9:402-8.

554. Loftus CA, Soler ZM, Koochakzadeh S, et al. Revision surgery rates in chronic rhinosinusitis with nasal polyps: meta-analysis of risk factors. Int Forum Allergy. Rhinol. 2019, 10.1002/alr.22487.

555. Calus L, Van Bruaene N, Bosteels C, et al. Twelveyear follow-up study after endoscopic sinus surgery in patients with chronic rhinosinusitis with nasal polyposis. Clinical and Translational Allergy. 2019;9:30.

556. Smith TL, Litvack JR, Hwang $\mathrm{PH}$, et al. Determinants of outcomes of sinus surgery: a multi-institutional prospective cohort study. Otolaryngol. Head Neck Surg.. 2010;142:55-63.

557. Smith TL, Kern R, Palmer JN, et al. Medical therapy vs surgery for chronic rhinosinusitis: a prospective, multi-institutional study with 1-year follow-up. Int Forum Allergy. Rhinol. 2013;3:4-9.

558. Purcell PL, Beck S, Davis GE. The impact of endoscopic sinus surgery on total direct healthcare costs among patients with chronic rhinosinusitis. Int Forum Allergy. Rhinol. 2015;5:498-505.

559. Bhattacharyya N. The economic burden and symptom manifestations of chronic rhinosinusitis. Am J Rhinol.2003;17:27-32.

560. Smith TL, Batra PS, Seiden AM, Hannley M. Evidence supporting endoscopic sinus surgery in the management of adult chronic rhinosinusitis: a systematic review. Am J Rhinol.2005;19:537-43.

561. Blomqvist EH, Lundblad L, Bergstedt H, Stjärne P. A randomized prospective study comparing medical and medical-surgical treatment of nasal polyposis by CT. Acta Otolaryngol. 2009;129:545-9.

562. Smith KA, Smith TL, Mace JC, Rudmik L. Endoscopic sinus surgery compared to continued medical therapy for patients with refractory chronic rhinosinusitis. Int Forum Allergy. Rhinol. 2014;4:823-7.

563. Smith KA, Rudmik L. Impact of continued medical therapy in patients with refractory chronic rhinosinusitis. International Forum of Allergy. \& Rhinology. 2014;4:34-8.

564. Patel ZM, Thamboo A, Rudmik L, Nayak JV, Smith TL, Hwang PH. Surgical therapy vs continued medical therapy for medically refractory chronic rhinosinusitis: a systematic review and metaanalysis. International Forum of Allergy. \&
Rhinology. 2017;7:119-27.

565. Kohli P, Naik AN, Farhood Z, et al. Olfactory Outcomes after Endoscopic Sinus Surgery for Chronic Rhinosinusitis: A Meta-analysis. Otolaryngology - Head \& Neck Surg. 2016;155:93648.

566. Ehnhage A, Olsson P, Kolbeck KG, Skedinger M, Stjarne $P$. One year after endoscopic sinus surgery in polyposis: asthma, olfaction, and quality-oflife outcomes. Otolaryngol. Head Neck Surg. 2012;146:834-41.

567. Acquadro MA, Salman SD, Joseph MP. Analysis of pain and endoscopic sinus surgery for sinusitis. Ann Otol. Rhinol Laryngol. 1997;106:305-9.

568. Chester AC. Symptom outcomes following endoscopic sinus surgery. Curr Opin Otolaryngol. Head Neck Surg. 2009;17:50-8.

569. Chester AC, Sindwani R, Smith TL, Bhattacharyya N. Fatigue improvement following endoscopic sinus surgery: a systematic review and meta-analysis. Laryngoscope. 2008;118:730-9.

570. Sukato DC, Abramowitz JM, Boruk M, Goldstein NA, Rosenfeld RM. Endoscopic Sinus Surgery Improves Sleep Quality in Chronic Rhinosinusitis: A Systematic Review and Meta-analysis. Otolaryngology - Head \& Neck Surg. 2018;158:24956.

571. Vashishta R, Soler ZM, Nguyen SA, Schlosser RJ. A systematic review and meta-analysis of asthma outcomes following endoscopic sinus surgery for chronic rhinosinusitis. International Forum of Allergy. \& Rhinology. 2013;3:788-94.

572. Yeung JC, Brandt MG, Franklin JH, Doyle PC, Rotenberg BW, Kilty SJ. Preoperative concerns of patients undergoing endoscopic sinus surgery. Int Forum Allergy. Rhinol. 2014;4:658-62.

573. Smith TL, Mendolia-Loffredo S, Loehrl TA, Sparapani R, Laud PW, Nattinger AB. Predictive factors and outcomes in endoscopic sinus surgery for chronic rhinosinusitis. Laryngoscope. 2005;115:2199-205.

574. Wei B, Liu F, Zhang J, et al. Multivariate analysis of inflammatory endotypes in recurrent nasal polyposis in a Chinese population. Rhinology. 2018;56:216-26.

575. Brescia G, Marioni G, Franchella S, et al. A prospective investigation of predictive parameters for post-surgical recurrences in sinonasal polyposis. Eur Arch Otorhinolaryngol. 2016;273:655-60.

576. Lou H, Meng Y, Piao Y, Wang C, Zhang L, Bachert C. Predictive significance of tissue eosinophilia for nasal polyp recurrence in the Chinese population. Am J Rhinol.Allergy. 2015;29:350-6.

577. Wu XF, Kong WF, Wang WH, et al. Enhanced recovery after surgery protocols in functional endoscopic sinus surgery for patients with chronic rhinosinusitis with nasal polyps: a randomized clinical trial. Chin Med J 2019;132:253-8.

578. Kemppainen TP, Tuomilehto H, Kokki H, Seppä $\mathrm{J}$, Nuutinen J. Pain treatment and recovery after endoscopic sinus surgery. Laryngoscope. 2007;117:1434-8.

579. Tyler MA, Lam K, Ashoori F, et al. Analgesic Effects of Intravenous Acetaminophen vs Placebo for Endoscopic Sinus Surgery and Postoperative Pain: 
a Randomized Clinical Trial. JAMA otolaryngology-head \& neck surgery 2017;143:788-94.

580. Raikundalia MD, Cheng TZ, Truong T, et al. Factors associated with opioid use after endoscopic sinus surgery. Laryngoscope. 2019;129:1751-5.

581. Locketz GD, Brant JD, Adappa ND, et al. Postoperative Opioid Use in Sinonasal Surg. Otolaryngol. Head Neck Surg. 2019;160:402-8.

582. Szczygielski K, Rapiejko P, Wojdas A, Jadczak M, Jurkiewicz D. Comparison of dissolvable sinus dressings in functional endoscopic sinus surgery. Otolaryngologia polska $=$ the polish otolaryngology 2007;61:852-6.

583. Raghunandhan S, Kameswaran M, Thomas JK. A prospective double-blinded randomized controlled study comparing the efficacy of a novel biodegradable synthetic polyurethane foam (Nasopore) vs standard polyvinyl acetate sponge (Merocel) as packing material after functional endoscopic sinus surgery: $t$. Clinical rhinology 2014;7:105-11.

584. Jurkiewicz D, Kazmierczak H, Rogowski M, et al. Evaluation of fully biodegradable nasal packings in functional endoscopic sinus surgery - a multicentre study. Otolaryngol. Pol 2015;69:11-5.

585. Jurkiewicz D, Kaźmierczak H, Rogowski M, et al. Evaluation of fully biodegradable nasal packings in functional endoscopic sinus surgery - a multicentre study. Otolaryngologia polska = the polish otolaryngology 2015;69:11-5.

586. Shinkwin CA, Beasley N, Simo R, Rushton L, Jones NS. Evaluation of Surgicel Nu-knit, Merocel and Vasolene gauze nasal packs: a randomized trial. Rhinology. 1996;34:41-3.

587. Mo JH, Park YM, Chung YJ. Effect of lidocainesoaked nasal packing on pain relief after endoscopic sinus surgery. American J Rhinol \& Allergy. 2013;27:e174-e7.

588. Haytoğlu S, Kuran G, Muluk NB, Arıkan OK. Different anesthetic agents-soaked sinus packings on pain management after functional endoscopic sinus surgery: which is the most effective? Eur Arch Otorhinolaryngol. 2016;273:1769-77.

589. Yilmaz S, Yildizbas S, Guclu E, Yaman H, Yalcin Sezen G. Topical levobupivacaine efficacy in pain control after functional endoscopic sinus surgery. Otolaryngology - Head and Neck Surg. (United States) 2013;149:777-81.

590. Kim KS, Yeo NK, Kim SS, et al. Effect of Fentanyl Nasal Packing Treatment on Patients With Acute Postoperative Pain After Nasal Operation: a Randomized Double-Blind Controlled Trial. Annals of Otol, Rhin, and Laryngol.2018;127:297-305.

591. Kim DH, Kang H, Hwang SH. The Effect of Sphenopalatine Block on the Postoperative Pain of Endoscopic Sinus Surg: A Metaanalysis. Otolaryngology - Head \& Neck Surg.2018:194599818805673.

592. Ecevit MC, Sutay S, Erdag TK. The microdebrider and its complications in endoscopic surgery for nasal polyposis. J Otolaryngol. Head Neck Surg. 2008;37:160-4.

593. Graham SM, Nerad JA. Orbital complications in endoscopic sinus surgery using powered instrumentation. Laryngoscope. 2003;113:874-8.

594. Hosemann W, Draf C. Danger points, complications and medico-legal aspects in endoscopic sinus surgery. GMS Curr Top Otorhinolaryngol. Head Neck Surg. 2013;12:Doc06.

595. Lund VJ, Stammberger H, Fokkens WJ, et al. European position paper on the anatomical terminology of the internal nose and paranasal sinuses. Rhinol Suppl 2014;24:1-34.

596. Badia L, Lund VJ, Wei W, Ho WK. Ethnic variation in sinonasal anatomy on CT-scanning. Rhinology. 2005;43:210-4.

597. Benninger MS, Holy CE. Endoscopic sinus surgery provides effective relief as observed by health care use pre- and postoperatively. Otolaryngology-Head \& Neck Surg.: official J of American Academy of Otolaryngology-Head and Neck Surg. 2014;150:893-900.

598. Stankiewicz JA, Lal D, Connor M, Welch K. Complications in endoscopic sinus surgery for chronic rhinosinusitis: a 25-year experience. Laryngoscope. 2011;121:2684-701.

599. Siedek V, Pilzweger E, Betz C, Berghaus A, Leunig A. Complications in endonasal sinus surgery: a 5-year retrospective study of 2,596 patients. Eur Arch Otorhinolaryngol. 2013;270:141-8.

600. Hopkins C, Browne JP, Slack R, et al. Complications of surgery for nasal polyposis and chronic rhinosinusitis: the results of a national audit in England and Wales. Laryngoscope. 2006;116:14949.

601. Han JK, Higgins TS. Management of orbital complications in endoscopic sinus surgery. Curr Opin Otolaryngol. Head Neck Surg. 2010;18:32-6.

602. Rene C, Rose GE, Lenthall R, Moseley I. Major orbital complications of endoscopic sinus surgery. Br J Ophthalmol 2001;85:598-603.

603. Huang CM, Meyer DR, Patrinely JR, et al. Medial rectus muscle injuries associated with functional endoscopic sinus surgery: characterization and management. Ophthal Plast Reconstr Surg. 2003;19:25-37.

604. Bhatti MT, Schmalfuss IM, Mancuso AA. Orbital complications of functional endoscopic sinus surgery: MR and CT findings. Clin Radiol 2005;60:894-904.

605. Rimmer J, Fokkens W, Chong LY, Hopkins C. Surgical versus medical interventions for chronic rhinosinusitis with nasal polyps. The Cochrane database of systematic reviews 2014;12:CD006991.

606. Verim A, Seneldir L, Naiboğlu B, et al. Role of nasal packing in surgical outcome for chronic rhinosinusitis with polyposis. Laryngoscope. 2014;124:1529-35.

607. Gan EC, Alsaleh S, Manji J, Habib AR, Amanian A, Javer AR. Hemostatic effect of hot saline irrigation during functional endoscopic sinus surgery: a randomized controlled trial. Int Forum Allergy. \& Rhinol.2014;4:877-84.

608. Mathew R, Srinivasa C, Sathyanarayana V, Suryanarayana S, Harsha P. Role of pterygopalatine fossa block in achieving relatively bloodless field during endoscopic sinus surgery. Clinical Rhinology. 2017;10:62-5.

609. Valdes CJ, Al Badaai Y, Bogado M, Samaha M. Does pterygopalatine canal injection with local anaesthetic and adrenaline decrease bleeding during functional endoscopic sinus surgery? J
Laryngol. Otol. 2014;128:814-7.

610. Vaiman M, Sarfaty S, Shlamkovich N, Segal S, Eviatar E. Fibrin sealant: alternative to nasal packing in endonasal operations. A prospective randomized study. Isr Med Assoc J 2005;7:571-4.

611. Vaiman M, Eviatar E, Shlamkovich N, Segal S. Use of fibrin glue as a hemostatic in endoscopic sinus surgery. Annals of Otol, Rhin, and Laryngol.2005;114:237-41.

612. Cohen NA, Antunes MB, Morgenstern KE. Prevention and management of lacrimal duct injury. Otolaryngol. Clin North Am 2010;43:781-8.

613. Bucher S, Kugler A, Probst E, et al. Occurrence of occult CSF leaks during standard FESS procedures. Rhinology. 2018, 10.4193/Rhin17.117.

614. Sohn JH, Hong SD, Kim JH, et al. Extraocular muscle injury during endoscopic sinus surgery: a series of 10 cases at a single center. Rhinology. 2014;52:238-45.

615. Bleier BS, Schlosser RJ. Prevention and management of medial rectus injury. Otolaryngol. Clin North Am 2010;43:801-7.

616. Thacker NM, Velez FG, Demer JL, Wang MB, Rosenbaum AL. Extraocular muscle damage associated with endoscopic sinus surgery: an ophthalmology perspective. Am J Rhinol.2005;19:400-5.

617. Hong JE, Goldberg AN, Cockerham KP. Botulinum toxin $A$ therapy for medial rectus injury during endoscopic sinus surgery. Am J Rhinol.2008;22:95-7.

618. Chin OY, Ghosh R, Fang CH, Baredes S, Liu JK, Eloy JA. Internal carotid artery injury in endoscopic endonasal surgery: A systematic review. Laryngoscope. 2016;126:582-90.

619. Valentine R, Boase S, Jervis-Bardy J, Dones Cabral JD, Robinson S, Wormald PJ. The efficacy of hemostatic techniques in the sheep model of carotid artery injury. International Forum of Allergy. and Rhinology. 2011;1:118-22.

620. Catalano PJ, Roffman EJ. Evaluation of middle meatal stenting after minimally invasive sinus techniques (MIST). Otolaryngol. Head Neck Surg. 2003;128:875-81.

621. Manji J, Habib AR, Amanian AA, Alsaleh S, Thamboo A, Javer AR. Potential risk factors associated with the development of synechiae following functional endoscopic sinus surgery. Eur Arch Otorhinolaryngol. 2018;275:1175-81.

622. Berlucchi M, Castelnuovo P, Vincenzi A, Morra B, Pasquini E. Endoscopic outcomes of resorbable nasal packing after functional endoscopic sinus surgery: a multicenter prospective randomized controlled study. Eur Arch Otorhinolaryngol. 2009;266:839-45.

623. Zhao X, Grewal A, Briel M, Lee JM. A systematic review of nonabsorbable, absorbable, and steroidimpregnated spacers following endoscopic sinus surgery. International Forum of Allergy. \& Rhinology. 2013;3:896-904.

624. Song KJ, Lee HM, Lee EJ, Kwon JH, Jo KH, Kim KS. Anti-adhesive effect of a thermosensitive poloxamer applied after the removal of nasal packing in endoscopic sinus surgery: a randomised multicentre clinical trial. Clin Otolaryngol. 2013;38:225-30. 
625. Chen J, Wang X, Chen L, Liu J. Influence of hyaluronan nasal dressing on clinical outcome after endoscopic sinus surgery: A systematic review and meta-analysis. Am J Rhinol.Allergy. 2017;31:256-9.

626. Fong E, Garcia M, Woods CM, Ooi E. Hyaluronic acid for post sinus surgery care: systematic review and meta-analysis. J Laryngol. Otol. 2017;131:S2S11.

627. Zhou JC, Zhang JJ, Zhang W, Ke ZY, Zhang B. Efficacy of chitosan dressing on endoscopic sinus surgery: a systematic review and meta-analysis. Eur Arch Otorhinolaryngol. 2017;274:3269-74.

628. Bolger WE, Kuhn FA, Kennedy DW. Middle turbinate stabilization after functional endoscopic sinus surgery: the controlled synechiae technique. Laryngoscope. 1999;109:1852-3.

629. Chen W, Wang Y, Bi Y, Chen W. Turbinate-septal suture for middle turbinate medialization: a prospective randomized trial. Laryngoscope. 2015;125:33-5.

630. Maharaj DD, Virk RS, Bansal S, Gupta AK. Comparison of basal lamella relaxing incision and combined conventional medialisation and controlled synechiae in functional endoscopic sinus surgery: a randomised prospective study. J Laryngol. Otol. 2018;132:605-10.

631. Raynal M, Peynegre R, Beautru R, Coste A. [Sinus mucoceles and surgery in iatrogenic diseases]. Ann Otolaryngol. Chir Cervicofac 1999;116:85-91.

632. Moriyama H, Nakajima T, Honda Y. Studies on mucocoeles of the ethmoid and sphenoid sinuses: analysis of 47 cases. J Laryngol. Otol. 1992;106:237.

633. Obeso S, Luis Llorente J, Pablo Rodrigo J, Sánchez R, Mancebo G, Suárez C. Paranasal sinuses mucoceles. Our experience in 72 patients. Acta Otorrinolaringologica (English Edition) 2009;60:332-9.

634. Jimenez Chobillon MA, Jankowski R. Relationship between mucoceles, nasal polyposis and nasalisation. Rhinology. 2004;42:219-24.

635. Danielsen A, Olofsson J. Endoscopic endonasal sinus surgery. A long-term follow-up study. Acta Otolaryngol. 1996;116:611-9.

636. Singhal D, Psaltis AJ, Foreman A, Wormald PJ. The impact of biofilms on outcomes after endoscopic sinus surgery. American J of Rhinology. and Allergy. 2010;24:169-74.

637. Bendouah Z, Hamad BJ, Desrosiers M, Barbeau J, Hamad WA, Desrosiers M. Biofilm formation by Staphylococcus aureus and Pseudomonas aeruginosa is associated with an unfavorable evolution after surgery for chronic sinusitis and nasal polyposis. Otolaryngology - Head \& Neck Surg. 2006;134:991-6.

638. Tomassen P, Vandeplas G, Van Zele T, et al. Inflammatory endotypes of chronic rhinosinusitis based on cluster analysis of biomarkers. J Allergy Clin Immunol.2016;137:1449-56.e4.

639. Ramadan HH. Surgical causes of failure in endoscopic sinus surgery. Laryngoscope. 1999;109:27-9.

640. Rudmik L, Xu Y, Alt JA, et al. Evaluating SurgeonSpecific Performance for Endoscopic Sinus Surg. JAMA Otolaryngol. Head Neck Surg. 2017;143:891-
8.

641. Veloso-Teles R, Cerejeira R. Endoscopic sinus surgery for chronic rhinosinusitis with nasal polyps: Clinical outcome and predictive factors of recurrence. Am J Rhinol.Allergy. 2017;31:56-62.

642. Rosenfeld RM, Piccirillo JF, Chandrasekhar SS, et al. Clinical practice guideline (update): Adult sinusitis. Otolaryngology - Head and Neck Surg.(United States) 2015;152:S1-S39.

643. Philpott C, Hopkins C, Erskine S, et al. The burden of revision sinonasal surgery in the UK-data from the Chronic Rhinosinusitis Epidemiology Study (CRES): a cross-sectional study. BMJ Open 2015;5:e006680.

644. Koskinen A, Salo R, Huhtala H, et al. Factors affecting revision rate of chronic rhinosinusitis. Laryngoscope. Investig Otolaryngol. 2016;1:96105.

645. Hunter TD, DeConde AS, Manes RP. Diseaserelated expenditures and revision rates in chronic rhinosinusitis patients after endoscopic sinus surgery. J Med Econ 2018;21:610-5.

646. Miglani A, Divekar RD, Azar A, Rank MA, Lal D. Revision endoscopic sinus surgery rates by chronic rhinosinusitis subtype. Int Forum Allergy. Rhinol. 2018;8:1047-51.

647. Chen FH, Zuo KJ, Guo YB, et al. Long-term results of endoscopic sinus surgery-oriented treatment for chronic rhinosinusitis with asthma. Laryngoscope. 2014;124:24-8.

648. Alanin MC, Aanaes K, Hoiby N, et al. Sinus surgery postpones chronic Gram-negative lung infection: cohort study of 106 patients with cystic fibrosis. Rhinology. 2016;54:206-13.

649. Becker SS, de Alarcon A, Bomeli SR, Han JK, Gross CW. Risk factors for recurrent sinus surgery in cystic fibrosis: review of a decade of experience. Am J Rhinol.2007;21:478-82.

650. Rickert S, Banuchi VE, Germana JD, Stewart MG, April MM. Cystic fibrosis and endoscopic sinus surgery: Relationship between nasal polyposis and likelihood of revision endoscopic sinus surgery in patients with cystic fibrosis. Arch Otolaryngol. Head Neck Surg. 2010;136:988-92.

651. Adelman J, McLean C, Shaigany K, Krouse JH. The Role of Surgeryin Management of Samter's Triad: A Systematic Review. Otolaryngology - Head \& Neck Surg. 2016;155:220-37.

652. Stein NR, Jafari A, DeConde AS. Revision rates and time to revision following endoscopic sinus surgery: A large database analysis. Laryngoscope. 2018;128:31-6.

653. Wu AW, Ting JY, Platt MP, Tierney HT, Metson R. Factors affecting time to revision sinus surgery for nasal polyps: a 25-year experience. Laryngoscope. 2014;124:29-33.

654. Zele TV, Holtappels G, Gevaert P, Bachert C. Differences in initial immunoprofiles between recurrent and nonrecurrent chronic rhinosinusitis with nasal polyps. American J Rhinol \& Allergy.. 2014;28:192-8.

655. Bachert C, Zhang N, Hellings PW, Bousquet J. Endotype-driven care pathways in patients with chronic rhinosinusitis. The J Allergy Clin Immunol.2018;141:1543-51.

656. Pinther S, Deeb R, Peterson EL, Standring RT, Craig
JR. Complications Are Rare From Middle Turbinate Resection: A Prospective Case Series. Am J Rhinol. Allergy. 2019, 10.1177/1945892419860299:194589 2419860299.

657. Scangas GA, Remenschneider AK, Bleier BS, Holbrook EH, Gray ST, Metson RB. Does the Timing of Middle Turbinate Resection Influence Quality-of-Life Outcomes for Patients with Chronic Rhinosinusitis? Otolaryngol. Head Neck Surg. 2017;157:874-9.

658. Prasad S, Fong E, Ooi EH. Systematic review of patient-reported outcomes after revision endoscopic sinus surgery. American J of Rhinology. \& Allergy. 2017;31:248-55.

659. Jafari A, DeConde AS. Outcomes in Medical and Surgical Treatment of Nasal Polyps. Adv Otorhinolaryngol. 2016;79:158-67.

660. Alt JA, Orlandi RR, Mace JC, Soler ZM, Smith TL. Does Delaying Endoscopic Sinus SurgeryAdversely Impact Quality-of-Life Outcomes? Laryngoscope. 2019;129:303-11.

661. Socher JA, Mello J, Baltha BB. Tomographical Findings in Adult Patients Undergoing Endoscopic Sinus Surgery Revision. Int Arch Otorhinolaryngol. 2018;22:73-80.

662. Valdes CJ, Bogado M, Samaha M. Causes of failure in endoscopic frontal sinus surgery in chronic rhinosinusitis patients. Int Forum Allergy. Rhinol. 2014;4:502-6.

663. Huang Z, Hajjij A, Li G, Nayak JV, Zhou B, Hwang $\mathrm{PH}$. Clinical predictors of neo-osteogenesis in patients with chronic rhinosinusitis. International Forum of Allergy. and Rhinology. 2015;5:303-9.

664. Litvack JR, Griest S, James KE, Smith TL. Endoscopic and quality-of-life outcomes after revision endoscopic sinus surgery. Laryngoscope. 2007; 117:2233-8.

665. Bhattacharyya N. Clinical outcomes after revision endoscopic sinus surgery. Archives of Otolaryngology -- Head \& Neck Surg.2004;130:9758.

666. Costa ML, Psaltis AJ, Nayak JV, Hwang PH. Long-term outcomes of endoscopic maxillary mega-antrostomy for refractory chronic maxillary sinusitis. Int Forum Allergy. Rhinol. 2015;5:60-5.

667. Schalek P, Otruba L, Guha A. Extent of surgery in chronic rhinosinusitis: primarily focused on nasal polyposis. Archives of Otolaryngology and Rhinology. 2017;3:109-14.

668. Halderman AA, Stokken J, Sindwani R. The effect of middle turbinate resection on topical drug distribution into the paranasal sinuses. Int Forum Allergy. Rhinol. 2016;6:1056-61.

669. Jankowski R, Pigret D, Decroocq F, Blum A, Gillet P. Comparison of radical (nasalisation) and functional ethmoidectomy in patients with severe sinonasal polyposis. A retrospective study. Rev Laryngol. Otol. Rhinol (Bord) 2006;127:131-40.

670. Benkhatar H, Khettab I, Sultanik P, Laccourreye $\mathrm{O}$, Bonfils $\mathrm{P}$. Frontal sinus revision rate after nasal polyposis surgery including frontal recess clearance and middle turbinectomy: A long-term analysis. Auris Nasus Larynx 2018;45:740-6.

671. Sama A, McClelland L, Constable J. Frontal sinus mucocoeles: new algorithm for surgical management. Rhinology. 2014;52:267-75. 
672. Naidoo Y, Bassiouni A, Keen M, Wormald PJ. Risk factors and outcomes for primary, revision, and modified Lothrop (Draf III) frontal sinus surgery. Int Forum Allergy. Rhinol. 2013;3:412-7.

673. Orgain CA, Harvey RJ. The role of frontal sinus drillouts in nasal polyposis. Curr Opin Otolaryngol. Head Neck Surg. 2018;26:34-40.

674. Bassiouni A, Wormald PJ. Role of frontal sinus surgery in nasal polyp recurrence. Laryngoscope. 2013;123:36-41.

675. Oakley GM, Christensen JM, Sacks R, Earls P, Harvey RJ. Characteristics of macrolide responders in persistent post-surgical rhinosinusitis. Rhinology. 2018;56:111-7.

676. de Bonnecaze G, Chaput B, Dupret-Bories A, Vergez $S$, Serrano E. Functional outcome after longterm low-dose trimethoprim/sulfamethoxazole in chronic rhinosinusitis with purulence: a prospective study. J Laryngol. Otol. 2018;132:6004.

677. Videler WJ, van Hee K, Reinartz SM, et al. Longterm low-dose antibiotics in recalcitrant chronic rhinosinusitis: a retrospective analysis. Rhinology. 2011;50:45-55

678. Korban ZR, Casiano RR. Standard Endoscopic Approaches in Frontal Sinus Surg: Technical Pearls and Approach Selection. Otolaryngol. Clin North Am 2016;49:989-1006.

679. Hu X, Huang YY, Wang Y, Wang X, Hamblin MR. Antimicrobial Photodynamic Therapy to Control Clinically Relevant Biofilm Infections. Front Microbiol 2018;9:1299.

680. Mahmoudi H, Bahador A, Pourhajibagher M, Alikhani MY. Antimicrobial Photodynamic Therapy: An Effective Alternative Approach to Control Bacterial Infections. J Lasers Med Sci 2018;9:15460.

681. Desrosiers M, Endam LM, Lasso A, Kilty S. Evaluation of the safety of antimicrobial photodynamic therapy (aPDT) for refractory chronic rhinosinusitis. Allergy. 2016;71:120-

682. Biel MA, Sievert C, Usacheva M, Teichert M, Balcom J. Antimicrobial photodynamic therapy treatment of chronic recurrent sinusitis biofilms. Int Forum Allergy. Rhinol. 2011;1:329-34.

683. Desrosiers M, Endam LM, Lasso A, et al. Antimicrobial photodynamic therapy for chronic rhinosinusitis. Otolaryngology - Head and Neck Surg. (United States) 2016;155:145-6.

684. Rhinology./Allergy.. Otolaryngology-Head and Neck Surg. 2016;155:144-56.

685. Bachert C, Nan Z. Medical Algorithm: Diagnosis and Treatment of Chronic Rhinosinusitis. Allergy. 2019, 10.1111/all.13823.

686. Penttilä MA, Rautiainen ME, Pukander JS, et al. Endoscopic versus Caldwell-Luc approach in chronic maxillary sinusitis: comparison of symptoms at one-year follow-up. Rhinology. 1994;32:161-5.

687. DeFreitas J, Lucente FE. The Caldwell-Luc procedure: institutional review of 670 cases: $1975-$ 1985. The Laryngoscope. 1988;98:1297-300.

688. Murr AH. Contemporary indications for external approaches to the paranasal sinuses. Otolaryngol. Clin North Am 2004;37:423-34.
689. Schneider JS, Day A, Clavenna M, Russell PT, Duncavage J. Early Practice: External Sinus Surgery and Procedures and Complications. Otolaryngol. Clin North Am 2015;48:839-50.

690. Eloy JA, Marchiano E, Vázquez A. Extended Endoscopic and Open Sinus Surgery for Refractory Chronic Rhinosinusitis Modified Lothrop procedure. 2017;50:165-82.

691. Musy PY, Kountakis SE. Anatomic findings in patients undergoing revision endoscopic sinus surgery. American J of Otolaryngology - Head \& Neck Medicine \& Surg. 2004;25:418-22.

692. Konstantinidis I, Constantinidis J. Medial maxillectomy in recalcitrant sinusitis. Curr Opin Otolaryngol. Head Neck Surg. 2014;22:68-74.

693. Barzilai G, Greenberg E, Uri N. Indications for the Caldwell-Luc approach in the endoscopic era. Otolaryngology - Head \& Neck Surg. 2005;132:21920.

694. Videler WJ, van Drunen CM, van der Meulen FW, Fokkens WJ. Radical surgery: effect on quality of life and pain in chronic rhinosinusitis. Otolaryngol. Head Neck Surg. 2007;136:261-7.

695. Seiberling KA, Church CA, Tewfik M, et al. Canine fossa trephine is a beneficial procedure in patients with Samter's triad. Rhinology. 2012;50:104-8.

696. Forsgren K, Fukami M, Kumlien J, Penttilä M, Sterna P. Endoscopic and caldwell-luc approaches in chronic maxillary sinusitis: A comparative histopathologic study on preoperative and postoperative mucosal morphology. Ann Otol. Rhinol Laryngol. .1995;104:350-7.

697. Murray JP. Complications after treatment of chronic maxillary sinus disease with caldwell-luc procedure. Laryngoscope. 1983;93:282-4.

698. Low WK. Complications of the Caldwell-Luc operation and how to avoid them. Aust N Z J Surg. 1995;65:582-4.

699. Robinson S, Wormald PJ. Patterns of innervation of the anterior maxilla: a cadaver study with relevance to canine fossa puncture of the maxillary sinus. Laryngoscope. 2005;115:1785-8.

700. Lynch RC. the Technique of a Radical Frontal Sinus Operation Which Has Given Me the Best Results. The Laryngoscope. 1921, 1-5.

701. Rubin J, Lund V, Salmon B. Fronto-Ethmoidectomy in the Treatment Of Mucocoeles -A Neglected Operation. Arch Otolaryngol. 1985;112:434-6.

702. Williams HL, Holman CB. The causes and avoidance of failure in surgery for chronic suppuration of the frontoethmo-sphenoid complex of sinuses: with a previously unreported anomaly which produces chronicity and recurrence, and the description of a surgical technique usually producing a cure of the disease. Laryngoscope. 1962;72:1179-227.

703. Tato JM, Sibbald DW, Bergaglio OE. Surgcal Treatment of the Frontal Sinus by the external route. The Laryngoscope. 1954;64:504-22.

704. Hardy JM, Montgomery WW. Osteoplastic Frontal Sinusotomy. Ann Otol. Rhinol Laryngol. 1976;85:523-32

705. Weber R, Draf W, Keerl R, et al. Osteoplastic frontal sinus surgery with fat obliteration: technique and long-term results using magnetic resonance imaging in 82 operations. The Laryngoscope.
2000;110:1037-44.

706. Hansen FS, van der Poel NA, Freling NJM, Fokkens WJ. Mucocele formation after frontal sinus obliteration. Rhinology. 2018;56:106-10.

707. Donald P. Compound frontal sinus injuries with intracranial penetration. Laryngoscope. 1978;88:225-32.

708. van Dijk JMC, Wagemakers M, Korsten-Meijer AGW, Kees Buiter CT, van der Laan BFAM, Mooij JJA. Cranialization of the frontal sinus-the final remedy for refractory chronic frontal sinusitis. J Neurosurg. 2011;116:531-5.

709. Klossek JM, Peloquin L, Friedman WH, Ferrier JC, Fontanel JP. Diffuse nasal polyposis: postoperative long-term results after endoscopic sinus surgery and frontal irrigation. Otolaryngol. Head Neck Surg. 1997;117:355-61.

710. Seiberling K, Jardeleza C, Wormald P-J. Minitrephination of the Frontal Sinus: Indications and uses in Today's Era of Sinus Surg. American J of Rhinology. \& Allergy. 2009;23:229-31.

711. Patel AB, Cain RB, Lal D. Contemporary applications of frontal sinus trephination: A systematic review of the literature. The Laryngoscope. 2015;125:2046-53.

712. Tato JM, Bergaglio OE. Cirurgia del frontal injerto de grosa. Nueva tecnica (surgery of frontal sinus. Fat grafts: new technique). Otolaryngologica 1949;3.

713. Ulualp SO, Carlson TK, Toohill RJ. In Osteoplastic Flap Versus Modified Endoscopic Lothrop Procedure in Patients with Frontal Sinus Disease. Am J Rhinol. 2000;14(1):21-6

714. Anand VK, Hiltzik DH, Kacker A, Honrado C. Osteoplastic flap for frontal sinus obliteration in the era of image-guided endoscopic sinus surgery. Am J Rhinol.2005;19:406-10.

715. Hahn S, Palmer JN, Purkey MT, Kennedy DW, Chiu AG. Indications for external frontal sinus procedures for inflammatory sinus disease. American J of Rhinology. and Allergy. 2009;23:3427.

716. Isa AY, Mennie J, McGarry GW. The frontal osteoplastic flap: does it still have a place in rhinological surgery? The J of Laryngology \& Otology 2011;125:162-8.

717. Lee JM, Palmer JN. Indications for the osteoplastic flap in the endoscopic era. Current Opinion in Otolaryngology and Head and Neck Surg. 2011;19:11-5.

718. Raghavan U, Jones NS. The place of Riedel's procedure in contemporary sinus surgery. J Laryngol. Otol. 2004;118:700-5.

719. Soonawalla ZF, Stratopoulos C, Stoneham M Wilkinson D, Britton BJ, Friend PJ. Role of the reverse-Trendelenberg patient position in maintaining low-CVP anaesthesia during liver resections. Langenbecks Arch Surg. 2008;393:1958.

720. Gittelman PD, Jacobs JB, Skorina J. Comparison of functional endoscopic sinus surgery under local and general anesthesia. Ann Otol. Rhinol Laryngol. 1993;102:289-93.

721. Lee WC, KapurTR, Ramsden WN. Local and regional anesthesia for functional endoscopic sinus surgery. Ann Otol. Rhinol Laryngol. 
1997; 106:767-9.

722. Jorissen M, Heulens H, Peters M, Feenstra L. Functional endoscopic sinus surgery under local anaesthesia: possibilities and limitations. Acta Otorhinolaryngol. Belg 1996;50:1-12.

723. Rontal M, Rontal E, Anon JB. An anatomic approach to local anesthesia for surgery of the nose and paranasal sinuses. Otolaryngol. Clin North Am 1997;30:403-20.

724. Latorre F, Klimek L. Does cocaine still have a role in nasal surgery? Drug Saf 1999;20:9-13.

725. Howard DJ, Lund VJ. The midfacial degloving approach to sinonasal disease. The $J$ of Laryngology \& Otology 1992;106:1059-62.

726. Wormald PJ, van Renen G, Perks J, Jones $J A$, Langton-Hewer CD. The effect of the total intravenous anesthesia compared with inhalational anesthesia on the surgical field during endoscopic sinus surgery. Am J Rhinol.2005;19:514-20.

727. Danielsen A. Functional endoscopic sinus surgery on a day case out-patient basis. Clin Otolaryngol. Allied Sci 1992;17:473-7.

728. Stammberger $\mathrm{H}$. Nasal and paranasal sinus endoscopy. A diagnostic and surgical approach to recurrent sinusitis. Endoscopy 1986;18:213-8.

729. Sarmento Junior KM, Tomita S, Kós AO. Topical use of adrenaline in different concentrations for endoscopic sinus surgery. Braz J Otorhinolaryngol. 2009;75:280-9.

730. Hwang SH, Kim SW, Kim SW, Kim BG, Cho JH, Kang JM. Greater palatine canal injections reduce operative bleeding during endoscopic sinus surgery: a systematic review and meta-analysis. Eur Arch Otorhinolaryngol. 2019;276:3-10.

731. Wormald PJ, Athanasiadis T, Rees G, Robinson S. An evaluation of effect of pterygopalatine fossa injection with local anesthetic and adrenalin in the control of nasal bleeding during endoscopic sinus surgery. Am J Rhinol.2005;19:288-92.

732. Mathew R, Srinivasa C, Sathyanarayana V, Suryanarayana S, Harsha P. Role of pterygopalatine fossa block in achieving relatively bloodless field during endoscopic sinus surgery. . Clinical Rhinology:: An International J 2015;125:1010-4.

733. Shankar MN, Selvan VS, Sreedharan N. An observational study comparing the effect of sphenopalatine artery block on bleeding in endoscopic sinus surgery. 2017 2017;3:5.

734. Shenoy VS, Prakash N, Kamath PM, et al. Is Pterygopalatine Fossa Injection with Adrenaline an Effective Technique for Better Surgical Field in Fess? Indian J Otolaryngol. Head Neck Surg. 2017;69:464-73.

735. De R, Uppal HS, Shehab ZP, Hilger AW, Wilson PS, Courteney-Harris R. Current practices of cocaine administration by UK otorhinolaryngologists. The J of Laryngology \& Otology 2003;117:109-12.

736. Long H, Greller H, Mercurio-Zappala M, Nelson LS, Hoffman RS. Medicinal use of cocaine: a shifting paradigm over 25 years. Laryngoscope. 2004;114:1625-9.

737. Eicehlsheim D, Friebel M, Glazenburg B. Use of cocaine as a local anaesthetic by Dutch ENT surgeons. . Ned Tijdschr v KNO-Heelokunde 2008;14:129.
738. Pfleiderer AG, Brockbank M. Cocaine and adrenaline: a safe or necessary combination in the nose? A study to determine the effect of adrenaline on the absorption and adverse side effects of cocaine. Clin Otolaryngol. Allied Sci 1988;13:421-6.

739. Tarver CP, Noorily AD, Sakai CS. A comparison of cocaine vs. lidocaine with oxymetazoline for use in nasal procedures. Otolaryngol. Head Neck Surg. 1993;109:653-9.

740. Wight RG, Cochrane T. A comparison of the effects of two commonly used vasoconstrictors on nasal mucosal blood flow and nasal airflow. Acta Otolaryngol. 1990;109:137-41.

741. Riegle EV, Gunter JB, Lusk RP, Muntz HR, Weiss KL. Comparison of vasoconstrictors for functional endoscopic sinus surgery in children. Laryngoscope. 1992;102:820-3.

742. Noorily AD, Noorily SH, Otto RA. Cocaine, lidocaine, tetracaine: which is best for topical nasal anesthesia? Anesth Analg 1995;81:724-7.

743. Noorily AD, Otto RA, Noorily SH. Intranasal anesthetic effects of lidocaine and tetracaine compared. Otolaryngol. Head Neck Surg. 1995;113:370-4.

744. Jonathan DA, Violaris NS. Comparison of cocaine and lignocaine as intranasal local anaesthetics. J Laryngol. Otol. 1988;102:628-9.

745. Javer AR, Gheriani H, Mechor B, Flamer D, Genoway $K$, Yunker WK. Effect of intraoperative injection of $0.25 \%$ bupivacaine with 1:200,000 epinephrine on intraoperative blood loss in FESS. Am J Rhinol. Allergy. 2009;23:437-41.

746. Moshaver A, Lin D, Pinto R, Witterick IJ. The hemostatic and hemodynamic effects of epinephrine during endoscopic sinus surgery: a randomized clinical trial. Arch Otolaryngol. Head Neck Surg. 2009;135:1005-9.

747. Lee TJ, Huang CC, Chang PH, Chang CJ, Chen YW. Hemostasis during functional endoscopic sinus surgery: the effect of local infiltration with adrenaline. Otolaryngol. Head Neck Surg. 2009;140:209-14.

748. Yang JJ, Li WY, Jil Q, et al. Local anesthesia for functional endoscopic sinus surgery employing small volumes of epinephrine-containing solutions of lidocaine produces profound hypotension. Acta Anaesthesiol Scand 2005;49:1471-6.

749. Zhao F, Wang Z, Yang J, Sun J, Wang Q, Xu J. Low-dosage adrenaline induces transient marked decrease of blood pressure during functional endoscopic sinus surgery. Am J Rhinol.2006;20:182-5.

750. Cohen-Kerem R, Brown S, Villaseñor LV, Witterick I. Epinephrine/Lidocaine injection vs. saline during endoscopic sinus surgery. Laryngoscope. 2008;118:1275-81.

751. DeConde AS, Thompson CF, Wu EC, Suh JD. Systematic review and meta-analysis of total intravenous anesthesia and endoscopic sinus surgery. International Forum of Allergy. \& Rhinology. 2013;3:848-54.

752. Kelly EA, Gollapudy S, Riess ML, Woehlck HJ, Loehrl TA, Poetker DM. Quality of surgical field during endoscopic sinus surgery: A systematic literature review of the effect of total intravenous compared to inhalational anesthesia. International Forum of Allergy. and Rhinology. 2013;3:474-81.

753. Brunner JP, Levy JM, Ada ML, et al. Total intravenous anesthesia improves intraoperative visualization during surgery for high-grade chronic rhinosinusitis: a double-blind randomized controlled trial. Int Forum Allergy. \& Rhinol.2018;8:1114-22.

754. Little M, Tran V, Chiarella A, Wright ED. Total intravenous anesthesia vs inhaled anesthetic for intraoperative visualization during endoscopic sinus surgery: a double blind randomized controlled trial. International Forum of Allergy. \& Rhinology. 2018;8:1123-6.

755. Timperley D, Sacks R, Parkinson RJ, Harvey RJ. Perioperative and Intraoperative Maneuvers to Optimize Surgical Outcomes in Skull Base Surg. Otolaryngol. Clin North Am 2010;43:699-730.

756. Lu VM, Phan K, Oh LJ. Total intravenous versus inhalational anesthesia in endoscopic sinus surgery: A meta-analysis. Laryngoscope. 2020;130(3):575-583.

757. Beule AG, Wilhelmi F, Kühnel TS, Hansen E, Lackner KJ, Hosemann W. Propofol versus sevoflurane: bleeding in endoscopic sinus surgery. Otolaryngology-Head Neck Surg. 2007;136:45-50.

758. Nekhendzy V, Lemmens HJ, Vaughan WC, et al. The effect of deliberate hypercapnia and hypocapnia on intraoperative blood loss and quality of surgical field during functional endoscopic sinus surgery. Anesth Analg 2007;105:1404-9, table of contents.

759. Ha TN, van Renen RG, Ludbrook GL, Wormald PJ. The effect of blood pressure and cardiac output on the quality of the surgical field and middle cerebral artery blood flow during endoscopic sinus surgery. Int Forum Allergy. \& Rhinol.2016;6:701-9.

760. El-Shmaa NS, Ezz HAA, Younes A. The efficacy of Labetalol versus Nitroglycerin for induction of controlled hypotension during sinus endoscopic surgery. A prospective, double-blind and randomized study. J Clin Anesth 2017;39:154-8.

761. Shen PH, Weitzel EK, Lai JT, Wormald PJ, Ho CS. Intravenous esmolol infusion improves surgical fields during sevoflurane-anesthetized endoscopic sinus surgery: a double-blind, randomized, placebo-controlled trial. American J Rhinol \& Allergy.. 2011;25:e208-11.

762. Khosla AJ, Pernas FG, Maeso PA. Meta-analysis and literature review of techniques to achieve hemostasis in endoscopic sinus surgery. International Forum of Allergy. and Rhinology. 2013;3:482-7.

763. Boonmak P, Boonmak S, Laopaiboon M. Deliberate hypotension with propofol under anaesthesia for functional endoscopic sinus surgery (FESS). Cochrane Database Syst Rev. 2016;2016:Cd006623.

764. Eberhart LH, Folz BJ, Wulf H, Geldner G. Intravenous anesthesia provides optimal surgical conditions during microscopic and endoscopic sinus surgery. Laryngoscope. 2003;113:1369-73.

765. Tirelli G, Bigarini S, Russolo M, Lucangelo U, Gullo A. Total intravenous anaesthesia in endoscopic sinus-nasal surgery. Acta Otorhinolaryngol. Ital 2004;24:137-44.

766. Ragab SM, Hassanin MZ. Optimizing the surgical field in pediatric functional endoscopic sinus 
surgery: a new evidence-based approach Otolaryngology--Head \& Neck Surg.2010;142:4854.

767. Gupta K, Gupta P, Bhatia K, Rastogi B, Pandey M, Agarwal $S$. Efficacy of dexmedetomidine as an anesthetic adjuvant for functional endoscopic sinus surgery under general anesthesia: A randomized-controlled study. Ain-Shams J of Anaesthesiology 2016;9:207.

768. Kim H, Ha SH, Kim CH, Lee SH, Choi SH. Efficacy of intraoperative dexmedetomidine infusion on visualization of the surgical field in endoscopic sinus surgery. Korean J Anesthesiol 2015;68:44954.

769. Goksu S, Arik H, Demiryurek S, Mumbuc S, Oner $U$, Demiryurek AT. Effects of dexmedetomidine infusion in patients undergoing functional endoscopic sinus surgery under local anaesthesia. Eur J Anaesthesiol 2008;25:22-8.

770. Guven DG, Demiraran Y, Sezen G, Kepek O, Iskender A. Evaluation of outcomes in patients given dexmedetomidine in functional endoscopic sinus surgery. Annals of Otol, Rhin, and Laryngol.2011;120:586-92.

771. Qiao H, Chen J, Li W, Shen X. Intranasal atomised dexmedetomidine optimises surgical field visualisation with decreased blood loss during endoscopic sinus surgery: a randomized study. Rhinology. 2016;54:38-44.

772. Karabayirli S, Ugur KS, Demircioglu RI, et al. Surgical conditions during FESS; comparison of dexmedetomidine and remifentanil. Eur Arch Otorhinolaryngol. 2017;274:239-45.

773. Cardesin A, Pontes C, Rosell R, et al. A randomised double blind clinical trial to compare surgical field bleeding during endoscopic sinus surgery with clonidine-based or remifentanil-based hypotensive anaesthesia. Rhinology. 2015;53:10715.

774. Quijada-Manuitt MA, Escamilla Y, Vallano A, Cardesín A, Bernal-Sprekelsen M, Pontes C. Use of a2-Adrenergic Agonists to Improve Surgical Field Visibility in Endoscopy Sinus Surg: A Systematic Review of Randomised Controlled Trials. 2018;40:136-49.e19.

775. Sophia P, Lakshmi BS, Prasad PK, Ch, ramouli KV. Pre-operative oral bisoprolol improves the surgical field during functional endoscopic sinus surgery: a randomized, controlled, prospective and doubleblinded study. Int J of scientific study 2015:47-51.

776. Aravindan A, Subramanium R, Chhabra A, et al. Magnesium sulfate or diltiazem as adjuvants to total intravenous anesthesia to reduce blood loss in functional endoscopic sinus surgery. J Clin Anesth. 2016;34:179-85.

777. Zaky, Saleh AKE. Hypotensive anesthesia during functional endoscopic sinus surgery: a comparative study of remifentanil versus magnesium sulfate infusion. Ain-Shams J of Anaesthesiology 2017;10:124.

778. Devereaux PJ, Yang H, Yusuf S, et al. Effects of extended-release metoprolol succinate in patients undergoing non-cardiac surgery (POISE trial): a randomised controlled trial. Lancet 2008;371:1839-47.

779. Bijker JB, Persoon S, Peelen LM, et al.
Intraoperative Hypotension and Perioperative Ischemic Stroke after General Surg. Anesthesiology. 2012;116:658-64.

780. Futier E, Lefrant J-Y, Guinot P-G, et al. Effect of Individualized vs Standard Blood Pressure Management Strategies on Postoperative Organ Dysfunction Among High-Risk Patients Undergoing Major Surg. JAMA 2017;318:1346.

781. Dimauro R, Lucci F, Martino F, et al. The role of intraoperative stroke volume variation on bleeding during functional endoscopic sinus surgery. Minerva Anestesiol. 2018;84:1246-53.

782. Gilbey P, Kukuev Y, Samet A, Talmon Y, Ivry S. The quality of the surgical field during functional endoscopic sinus surgery-the effect of the mode of ventilation: A randomized, prospective, doubleblind Study. Laryngoscope. 2009;119:2449-53.

783. Green R, Konuthula N, Sobrero M, et al. Use of pharyngeal packs in functional endoscopic sinus surgery: a randomized controlled trial. Laryngoscope. 2017;127:2460-5.

784. Alfiky MG, Margalani OA, Rajeh AF, et al. Nasopharyngeal versus hypopharyngeal packing during sino-nasal surgeries: Randomised controlled trial. Clin Otolaryngol. 2018;43:1235-41.

785. Atef A, Fawaz A. Comparison of laryngeal mask with endotracheal tube for anesthesia in endoscopic sinus surgery. Am J Rhinol. 2008;22:653-7.

786. Jahanshahi J, Tayebi E, Hashemian F, Bakhshaei MH, Ahmadi MS, Seif Rabiei MA. Effect of local desmopressin administration on intraoperative blood loss and quality of the surgical field during functional endoscopic sinus surgery in patients with chronic rhinosinusitis: a triple-blinded clinical trial. Eur Arch Otorhinolaryngol. 2019;276:1995-9.

787. Shao H, Kuang LT, Hou WJ, Zhang T. Effect of desmopressin administration on intraoperative blood loss and quality of the surgical field during functional endoscopic sinus surgery: a randomized, clinical trial. BMC Anesthesiol.2015;15:53.

788. Kim DH, Kim S, Kang H, Jin HJ, Hwang SH. Efficacy of tranexamic acid on operative bleeding in endoscopic sinus surgery: A meta-analysis and systematic review. Laryngoscope. 2019;129:800-7.

789. Dastrup A, Pottegard A, Hallas J, Overgaard S. Perioperative Tranexamic Acid Treatment and Risk of Cardiovascular Events or Death After Total Hip Arthroplasty: A Population-Based Cohort Study from National Danish Databases. J Bone Joint Surg. Am. 2018;100:1742-9.

790. El Shal SM, Hasanein R. Effect of intravenous tranexamic acid and epsilon aminocaproic acid on bleeding and surgical field quality during functional endoscopic sinus surgery (FESS). Egyptian J of anaesthesia. 2015;31:1-7.

791. Chung JH, Cosenza MJ, Rahbar R, Metson RB. Mitomycin $\mathrm{C}$ for the prevention of adhesion formation after endoscopic sinus surgery: a randomized, controlled study. Otolaryngology-Head \& Neck Surg. 2002;126:468-74.

792. Anand VK, Tabaee A, Kacker A, Newman JG, Huang C. The role of mitomycin $C$ in preventing synechia and stenosis after endoscopic sinus surgery. Am J Rhinol. 2004;18:311-4.
793. Chan KO, Gervais M, Tsaparas Y, Genoway KA, Manarey C, Javer AR. Effectiveness of intraoperative mitomycin $\mathrm{C}$ in maintaining the patency of a frontal sinusotomy: a preliminary report of a double-blind randomized placebocontrolled trial. Am J Rhinol. 2006;20:295-9.

794. Gupta M, Motwani G. Role of mitomycin C in reducing adhesion formation following endoscopic sinus surgery. J Laryngol. Otol. 2006;120:921-3.

795. Kim ST, Gang IG, Cha HE, Ha JS, Chung YS. Effect of mitomycin $\mathrm{C}$ on the size of antrostomy after endoscopic sinus surgery. Ann Otol. Rhinol Laryngol. 2006;115:673-8.

796. Konstantinidis I, Tsakiropoulou E, Vital I, Triaridis S, Vital V, Constantinidis J. Intra- and postoperative application of Mitomycin C in the middle meatus reduces adhesions and antrostomy stenosis after FESS. Rhinology. 2008;46:107-11.

797. Baradaranfar MH, Khadem J, Taghipoor Zahir S, Kouhi A, Dadgarnia MH, Baradarnfar A. Prevention of adhesion after endoscopic sinus surgery: role of mitomycin C. Acta Med Iran. 2011;49:131-5.

798. Venkatraman V, Balasubramanian D, Gopalakrishnan S, Saxena SK, Shanmugasundaram N. Topical Mitomycin C in functional endoscopic sinus surgery. Eur Arch Otorhinolaryngol. 2012;269:1791-4.

799. Konstantinidis I, Chatziavramidis A, Constantinidis J. A novel technique for mitomycin-c application in frontal sinus surgery. Rhinology. 2014;52:27680.

800. Rotz SJ, Kodish E. Ethical conundrums in pediatric genomics. Hematology Am Soc Hematol Educ Program 2018;2018:301-6.

801. Lynn-Macrae AG, Lynn-Macrae RA, Emani J, Kern RC, Conley DB. Medicolegal analysis of injury during endoscopic sinus surgery. The Laryngoscope. 2004;114:1492-5.

802. Winford TW, Wallin JL, Clinger JD, Graham AM. Malpractice in Treatment of Sinonasal Disease by Otolaryngologists. Otolaryngology-Head and Neck Surg. 2015;152:536-40.

803. Seys SF, Bousquet J, Bachert C, et al. mySinusitisCoach: patient empowerment in chronic rhinosinusitis using mobile technology. Rhinology. 2018;56:209-15.

804. Care Quality Commission. Regulation 20: Duty of candour. 2015. https://www.cqc.org.uk/sites/ default/files/20150327_duty_of_candour_ guidance_final.pdf. 27 August 2019.

805. Falagas ME, Karageorgopoulos DE, Grammatikos AP, Matthaiou DK. Effectiveness and safety of short vs. long duration of antibiotic therapy for acute bacterial sinusitis: a meta-analysis of randomized trials. Br J Clin Pharmacol 2009;67:161-71.

806. Wolf JS, Malekzadeh S, Berry JA, O'Malley BW. Informed consent in functional endoscopic sinus surgery. The Laryngoscope. 2002;112:774-8.

807. Wolf JS, Chiu AG, Palmer JN, O'Malley BW, Schofield K, Taylor RJ. Informed consent in endoscopic sinus surgery: the patient perspective. The Laryngoscope. 2005;115:492-4.

808. Burns P, Keogh I, Timon C. Informed consent: a patients' perspective. The $\mathrm{J}$ of laryngology and otology 2005;119:19-22. 
809. Adhikari P, Pradhananga RB. Patients' Expectations on Informed Consent before ENT Surg. International Archives of Otorhinolaryngol.ogy 2007;11:51-3.

810. Judgment: Montgomery (Appellant) $v$ Lanarkshire Health Board (Respondent) (Scotland). 2015. https://www.supremecourt.uk/cases/docs/uksc2013-0136-judgment.pdf.

811. Taub HA, Baker MT, Kline GE, Sturr JF. Comprehension of informed consent information by young-old through old-old volunteers. Exp Aging Res. 1987;13:173-8.

812. Taylor RJ, Chiu AG, Palmer JN, et al. Informed consent in sinus surgery: Link between demographics and patient desires. Laryngoscope. 2005;115:826-31.

813. Ankuda CK, Block SD, Cooper Z, et al. Measuring critical deficits in shared decision making before elective surgery. Patient Educ Couns 2014;94:32833.

814. Hekkenberg RJ, Irish JC, Rotstein LE, Brown $\mathrm{DH}$, Gullane PJ. Informed consent in head and neck surgery: how much do patients actually remember? J Otolaryngol. 1997;26:155-9.

815. Robinson G, Merav A. Informed consent: recall by patients tested postoperatively. The Annals of Thoracic Surg. 1976;22:209-12.

816. Aremu SK, Alabi BS, Segun-Busari S. The role of informed consent in risks recall in otorhinolaryngology surgeries: verbal (nonintervention) vs written (intervention) summaries of risks. Am J Otolaryngol. 2011;32:4859.

817. Fink AS, Prochazka AV, Henderson WG, et al. Predictors of Comprehension during Surgical Informed Consent. J Am Coll Surg. 2010;210:91926.

818. Hickson GB, Jenkins AD. Identifying and addressing communication failures as a means of reducing unnecessary malpractice claims. N C Med J. 68:362-4.

819. Siu JM, Rotenberg BW, Franklin JH, Sowerby LJ. Multimedia in the informed consent process for endoscopic sinus surgery: a randomized control trial. Laryngoscope. 2016;126:1273-8.

820. Dawson DE, Kraus EM. Medical malpractice and rhinology. Am J Rhinol.21:584-90.

821. Bagnall NM, Pucher PH, Johnston MJ, et al. Informing the process of consent for surgery: identification of key constructs and quality factors. J Surg Res. 2017;209:86-92.

822. Townend D. EU Laws on Privacy in Genomic Databases and Biobanking. J Law Med Ethics 2016;44:128-42.

823. de Lecuona I, Villalobos-Quesada M. European perspectives on big data applied to health: The case of biobanks and human databases. Dev World Bioeth. 2018;18:291-8.

824. Declaration of Helsinki. 1975. https://www.wma. net/wp-content/uploads/2018/07/DoH-Oct1975 pdf.

825. International Clinical Harmonisation of Good Clinical Practice guidelines. 1996. https://www.ich. org/products/guidelines.html.

826. Critselis E. Impact of the General Data Protection
Regulation on Clinical Proteomics Research. Proteomics Clin Appl 2019;13:e1800199.

827. Kaufman R. Genetic Testing Gone Wild. Manag Care. 2019;28:39.

828. Korngiebel DM, Zech JM, Chappelle A, et al. Practice Implications of Expanded Genetic Testing in Oncology. Cancer Invest. 2019;37:39-45.

829. Nicol D, Eckstein L, Bentzen HB, et al. Consent insufficient for data release. Science. 2019;364:445-

830. Stoekle HC, Turrini M, Charlier P, Deleuze JF, Herve C, Vogt G. Genetic Data, Two-Sided Markets and Dynamic Consent: United States Versus France. Sci Eng Ethics. 2019, 10.1007/s11948-019-00085-4.

831. Abou-Elhamd KEA, Al-Sultan Al, Rashad UM. Simulation in ENT medical education. J Laryngol. Otol. 2010;124:237-41.

832. Yanagisawa E. The use of video in ENT endoscopy: its value in teaching. Ear Nose Throat J. 1994;73:754-63.

833. Sandhaus H, Chen PG. Intraoperative Functional Endoscopic Sinus Surgery Training: Efficient Teaching Techniques-A New Method. Clinical Medicine Insights: Ear, Nose and Throat. 2018;11:117955061875864.

834. Bakker NH, Fokkens WJ, Grimbergen CA. Investigation of training needs for functional endoscopic sinus surgery (FESS). Rhinology. 2005;43:104-8.

835. Braun T, Betz CS, Ledderose GJ, et al. Endoscopic sinus surgery training courses: benefit and problems - a multicentre evaluation to systematically improve surgical training. Rhinology. 2012;50:246-54.

836. Rivron RP, Maran AG. The Edinburgh FESS Trainer: a cadaver-based bench-top practice system for endoscopic ethmoidal surgery. Clin Otolaryngol. Allied Sci 1991;16:426-9.

837. Zuckerman JD, Wise SK, Rogers GA, Senior BA, Schlosser RJ, DelGaudio JM. The utility of cadaver dissection in endoscopic sinus surgery training courses. Am J Rhinol. Allergy. 2009;23:218-24.

838. Chan M, Carrie S. Training and assessment in functional endoscopic sinus surgery. The $J$ of Laryngol and Otol. 2018;132:133-7.

839. Delgado-Vargas B, Romero-Salazar AL, Reyes Burneo PM, et al. Evaluation of resident's training for endoscopic sinus surgery using a sheep's head. Eur Arch Otorhinolaryngol. 2016;273:2085-9.

840. Acar B, Gunbey E, Babademez MA, Karabulut $\mathrm{H}$, Gunbey HP, Karasen RM. Utilization and Dissection for Endoscopic Sinus Surgery Training in the Residency Program. J Craniofac Surg. 2010;21:1715-8.

841. de Oliveira HF, Bollela VR, Anselmo-Lima WT, Costa CAPdO, Nakanishi M. A feasible, lowcost, reproducible lamb's head model for endoscopic sinus surgery training. PLoS One 2017;12:e0180273-e.

842. Nogueira JF Lyra M BF, Leão FS, Stamm A C Building a real endoscopic sinus and skull-base surgery simulator. 2008;3:727-8.

843. Burge SD, Bunegin L, Weitzel EK, McMains KC. The validation of an endoscopic sinus surgery skills training model: a pilot study. American J Rhinol \&
Allergy.. 2012;26:409-13.

844. Alrasheed AS, Nguyen LHP, Mongeau L, Funnell WRJ, Tewfik MA. Development and validation of a 3D-printed model of the ostiomeatal complex and frontal sinus for endoscopic sinus surgery training. International Forum of Allergy. and Rhinology. 2017;7:837-41.

845. Zhuo C, Lei L, Yulin Z, et al. Creation and validation of three-dimensional printed models for basic nasal endoscopic training. Int Forum Allergy. \& Rhinol.2019;9:695-701.

846. Rudman Don; Sessanna DY, Roni; Crawfis,Roger; Heskamp, David; Edmond, Charles; Wiet, Gregory, David; Stredney. Functional Endoscopic Sinus Surgery Training Simulator. 1998;108:1643-7.

847. Ecke U, Klimek L, Müller W, Ziegler R, Mann W. Virtual reality: preparation and execution of sinus surgery. Computer aided surg.1998;3:45-50.

848. Tolsdorff B, Pommert A, Höhne KH, et al. Virtual reality: A new paranasal sinus surgery simulator. Laryngoscope. 2010;120:420-6.

849. Varshney R, Frenkiel S, Nguyen LHP, et al. Development of the McGill simulator for endoscopic sinus surgery: a new high-fidelity virtual reality simulator for endoscopic sinus surgery. American J Rhinol \& Allergy.. 2014;28:3304.

850. Javia L, Deutsch ES. A Systematic Review of Simulators in Otolaryngology. Otolaryngology-Head \& Neck Surg.: official J of American Academy of Otolaryngology-Head and Neck Surg. 2012;147:999-1011

851. Arora A, Lau LYM, Awad Z, Darzi A, Singh A, Tolley N. Virtual reality simulation training in Otolaryngology. Int J Surg. 2014;12:87-94.

852. Fried MP, Sadoughi B, Gibber MJ, et al. From virtual reality to the operating room: the endoscopic sinus surgery simulator experiment. Otolaryngology--Head \& Neck Surg.: official J of American Academy of Otolaryngology-Head and Neck Surg. 2010;142:202-7.

853. Solyar A, Cuellar H, Sadoughi B, Olson TR, Fried MP. Endoscopic Sinus Surgery Simulator as a teaching tool for anatomy education. Am J Surg. 2008;196:120-4.

854. Piromchai P, Avery A, Laopaiboon M, Kennedy G, O'Leary $S$. Virtual reality training for improving the skills needed for performing surgery of the ear, nose or throat. Reviews 1996;112:1148.

855. Dharmawardana N, Ruthenbeck G, Woods C, et al. Validation of virtual-reality-based simulations for endoscopic sinus surgery. Clin Otolaryngol. 2015;40:569-79.

856. Schalek P, Petras P, Klement V, Hahn A. Short-term antibiotics treatment in patients with nasal polyps and enterotoxins producing Staphylococcus aureus strains. Eur Arch Otorhinolaryngol. 2009;266:1909-13.

857. Albu S, Lucaciu R. Prophylactic antibiotics in endoscopic sinus surgery: a short follow-up study. Am J Rhinol.Allergy. 2010;24:306-9.

858. Annys $E$, Jorissen M. Short term effects of antibiotics (Zinnat) after endoscopic sinus surgery. Acta Otorhinolaryngol. Belg 2000;54:23-8.

859. Gulati SK, Sharma K, Shergill GK, Kumar R. Prophylactic budesonide nasal spray after 
polypectomy. Indian J of otolaryngology and Head \& Neck Surg.2001;53:207-9.

860. Hwang CS, Al Sharhan SS, Kim BR, et al. Randomized controlled trial of steroid-soaked absorbable calcium alginate nasal packing following endoscopic sinus surgery. Laryngoscope. 2018;128:311-6.

861. Sow YL, Tang IP, Kho J, Narayanan P. Pilot study comparing steroid-impregnated and non-steroidimpregnated absorbable nasal dressing following endoscopic sinus surgery. Med J Malaysia 2018;73:244-8

862. Ha T, Valentine R, Moratti S, Hanton L, Robinson S, Wormald PJ. The efficacy of a novel budesonide chitosan gel on wound healing following endoscopic sinus surgery. International Forum of Allergy. \& Rhinology. 2018;8:435-43.

863. Sabarinath V, Harish MR, Divakaran S. Triamcinolone Impregnated Nasal Pack in Endoscopic Sinus Surg: Our Experience. Indian J of Otolaryngol \& Head \& Neck Surg. 2017;69:88-92.

864. Bardaranfar MH, Ranjbar Z, Dadgarnia MH, et al. The effect of an absorbable gelatin dressing impregnated with triamcinolone within the olfactory cleft on polypoid rhinosinusitis smell disorders. American J Rhinol \& Allergy.. 2014;28:172-5.

865. Rudmik L, Mace J, Mechor B. Effect of a dexamethasone Sinu-Foam ${ }^{\mathrm{TM}}$ middle meatal spacer on endoscopic sinus surgery outcomes: a randomized, double-blind, placebo-controlled trial. Int Forum Allergy. \& Rhinol.2012;2:248-51.

866. Murr AH, Smith TL, Hwang PH, et al. Safety and efficacy of a novel bioabsorbable, steroideluting sinus stent. Int Forum Allergy. \& Rhinol.2011;1:23-32.

867. Lund VJ, Kennedy DW. Quantification for staging sinusitis. The Staging and Therapy Group. Ann Otol. Rhinol Laryngol. Suppl 1995;167:17-21.

868. Qvarnberg Y, Kantola O, Salo J, Toivanen M, Valtonen $\mathrm{H}$, Vuori E. Influence of topical steroid treatment on maxillary sinusitis. Rhinology. 1992;30:103-12.

869. Peveling-Oberhag A, Reimann H, Weyer V, Goloborodko E, Staubach P. High-Concentration Liquid Prednisolone Formula: Filling a Therapeutic Niche in Severe Acute Attacks of Urticaria and Angioedema. Skin Pharmacol Physiol 2016;29:9-12.
870. Pirunsarn A, Kijrattanakul P, Chamnanchanunt S, Polprasert C, Rojnuckarin P. A Randomized Multicenter Trial Comparing Low-Dose Prednisolone Versus Observation for Prevention of Recurrences in Adult Immune Thrombocytopenia. Clin Appl Thromb Hemost 2018;24:867-73.

871. Sicras-Mainar A, Traseira-Lugilde S, FernandezSanchez T, Navarro-Artieda R. [Persistence to treatment and resources use with inhaled fixeddose combinations of corticosteroids and longacting beta-adrenergic agonists for the treatment of asthma: A population-based retrospective study]. Semergen 2018;44:472-84.

872. Mager DE, Lin SX, Blum RA, Lates CD, Jusko WJ. Dose equivalency evaluation of major corticosteroids: pharmacokinetics and cell trafficking and cortisol dynamics. J Clin Pharmacol 2003;43:1216-27.

873. Salami A, Dellepiane M, Strinati F, Guastini L, Mora R. Sulphurous thermal water inhalations in the treatment of chronic rhinosinusitis. Rhinology. 2010;48:71-6.

874. Sommer DD, Hoffbauer S, Au M, Sowerby LJ, Gupta MK, Nayan S. Treatment of aspirin exacerbated respiratory disease with a low salicylate diet: a pilot crossover study. Otolaryngology - Head \& Neck Surg.(united states) 2015;152:42-7.

875. Sommer DD, Rotenberg BW, Sowerby LJ, et al. A novel treatment adjunct for aspirin exacerbated respiratory disease: the low-salicylate diet: a multicenter randomized control crossover trial. Int Forum Allergy Rhinol.2016;6:385-91.

876. Laidlaw TM, Cahill KN, Cardet JC, et al. A trial of type 12 purinergic ( $\mathrm{P} 2 \mathrm{Y} 12)$ receptor inhibition with prasugrel identifies a potentially distinct endotype of patients with aspirin-exacerbated respiratory disease. J Allergy Clin Immunol. 2019. 143(1):316324.

877. Fahmy FF, McCombe A, McKiernan DC. Sino nasal assessment questionnaire, a patient focused, rhinosinusitis specific outcome measure. Rhinology. 2002;40:195-7.

878. Gan EC, Habib AR, Rajwani A, Javer AR. Fivedegree, 10-degree, and 20-degree reverse Trendelenburg position during functional endoscopic sinus surgery: a double-blind randomized controlled trial. Int Forum Allergy. \& Rhinol.2014;4:61-8.
879. Hathorn IF, Habib AR, Manji J, Javer AR. Comparing the reverse Trendelenburg and horizontal position for endoscopic sinus surgery: a randomized controlled trial. Otolaryngology--Head \& Neck Surg.2013;148:308-13.

880. Ko MT, Chuang KC, Su CY. Multiple analyses of factors related to intraoperative blood loss and the role of reverse Trendelenburg position in endoscopic sinus surgery. Laryngoscope. 2008;118:1687-91.

881. Ahn HJ, Chung SK, Dhong HJ, et al. Comparison of surgical conditions during propofol or sevoflurane anaesthesia for endoscopic sinus surgery. $\mathrm{Br} \mathrm{J}$ Anaesth 2008;100:50-4.

882. Kaygusuz K, Yildirim A, Kol IO, Gursoy S, Mimaroglu C. Hypotensive anaesthesia with remifentanil combined with desflurane or isoflurane in tympanoplasty or endoscopic sinus surgery: a randomised, controlled trial. J Laryngol. Otol. 2008;122:691-5.

883. Yoo HS, Han JH, Park SW, Kim KS. Comparison of surgical condition in endoscopic sinus surgery using remifentanil combined with propofol, sevoflurane, or desflurane. Korean J Anesthesiol 2010;59:377-82.

884. Luong A, Ow RA, Singh A, et al. Safety and effectiveness of a bioabsorbable steroid-releasing implant for the paranasal sinus ostia: a randomized clinical trial. JAMA otolaryngology - Head \& Neck Surg.2018;144:28-35.

885. Smith TL, Singh A, Luong A, et al. Randomized controlled trial of a bioabsorbable steroidreleasing implant in the frontal sinus opening. Laryngoscope. 2016;126:2659-64.

886. Kennedy DW, Kuhn FA, Hamilos DL, et al. Treatment of chronic rhinosinusitis with high-dose oral terbinafine: a double blind, placebo-controlled study. Laryngoscope. 2005;115:1793-9.

887. Hashemian F, Ghorbanian MA, Hashemian F, Mortazavi SA, Sheikhi M, Jahanshahi J, et al. Effect of Topical Furosemide on Rhinosinusal Polyposis Relapse After Endoscopic Sinus Surgery: A Randomized Clinical Trial. JAMA Otolaryngol Head Neck Surg. 2016;142(11):1045-9. 


\section{Paediatric chronic rhinosinusitis}

\subsection{Epidemiology, predisposing factors, and comorbidities}

\subsubsection{Epidemiology}

Estimates from the National Health Interview Survey in the United States in 1996 suggest that young people under the age of 18 years are affected by chronic rhinosinusitis (CRS) at a rate of 63.9 per 1000 individuals $^{(1)}$. Overall health care expenditures attributable to rhinosinusitis (acute and chronic) in 1996 were estimated at $\$ 5.8$ billion, of which $\$ 1.8$ billion $(30.6 \%)$ was for children 12 years or younger ${ }^{(2)}$. Despite growing knowledge related to the diagnosis and treatment of this disease in children, its true epidemiology in the world is not yet well described. Factors thought to contribute to the difficulty in accurate diagnosis include false positive diagnoses due to difficulty in differentiating CRS from adenoid hypertrophy, adenoiditis, and (allergic) rhinitis, incomplete evaluation (nasal endoscopy and/or imaging are not performed in many children), neglect of the disease by both doctors and care-takers, and incomplete follow-up evaluation in some children. Furthermore, race and socioeconomic status have recently been reported to influence the prevalence of the disease. Smith et al. assessed 174 children aged between six months and 18 years who presented to a single academic tertiary paediatric otolaryngology centre with a primary diagnosis of CRS (classified through the Current Procedural Terminology (CPT) codes) from March 2008 to July $2011^{(3)}$. A control group consisted of 430 children, seen in the same department over a three-month period, with other otorhinolaryngologic diagnoses. There was a higher percentage of white children and children with private health insurance among the patients with a primary diagnosis of CRS. These findings suggest that racial/ ethnic disparities and socioeconomic variables may influence the diagnosis, and this is likely related to differences in access to tertiary care, parental perception of disease severity, or different natural course of the disease process in different ethnic populations.

Despite these limitations, several studies have tried to assess the prevalence of CRS in the paediatric population. Using data obtained from a US national survey acquired over a 2 year time frame which sampled 42.1 million school age patients, Sidell et al. identified approximately $4 \%$ of the studied population (corresponding to 1.7 million individuals) with a diagnosis of CRS, based on physician diagnoses and procedure codes for medical visits/encounters ${ }^{(4)}$. Sami and Scadding used the $M S Y P Q$, a disease specific quality of life questionnaire, in 213 secondary school children (11-16 years) in East London to identify the prevalence of rhinosinusitis symptoms and impact on quality of life ${ }^{(5)}$. They show that $31.5 \%$ suffered from a combination of symptoms suggestive of rhinitis, and between $13-15 \%$ of the respondents reported facial pain or anterior/ posterior nasal drainage, symptoms suggestive of a sinus origin. More than $20 \%$ of students suffered from effects on quality of life related to these symptoms. In total, out of those with nasal and sinus symptoms, $22 \%$ had their symptoms for more than six weeks but for less than a year.

Gilani and colleagues analysed national survey databases between 2005 and 2012 and showed that CRS accounted for 5.6 million visits per year among patients $0-20$ years of age ${ }^{(6)}$. CRS was diagnosed in $2.1 \%$ of all visits, acute rhinosinusitis in $0.6 \%$ and as comparators, allergic rhinitis in $2.6 \%$, upper respiratory tract infections in $8 \%$ and otitis media in $6.7 \%$. The $5-10$ year-old and 10-15 year-old age groups were the most affected. While most of the available data is based on diagnosis codes and subjective, questionnaire-derived, information, Westman and colleagues estimated the 12-month prevalence of CRS based on questionnaire to be $1.5 \%$ in a Swedish populationbased study of 3112 adolescents ${ }^{(7)}$. They went ahead and attempted to follow-up with objective confirmation of the diagnosis leading to a reduction of the estimate of prevalence to between $0.3 \%$ to $0.8 \%$.

Together, these studies suggest that the prevalence of CRS in the paediatric population is lower than in adults. As in adults, paediatric CRS is a relevant disease in terms of quality of life. The impact of CRS on the quality of life of children is greater than that of diseases such as asthma, attention deficit hyperactivity disorder, juvenile rheumatoid arthritis, and epilepsy ${ }^{(8)}$. All these studies reflect an important negative impact on the lives of children, particularly in terms of missed classes and concentration in school, sleep quality, and physical and emotional health ${ }^{(5)}$.

Although studies of CRS in children are less common, the prevalence is lower than in adults (2-4\%), but the negative impact on quality of life seems to be similar to that observed in adults.

\subsubsection{Predisposing factors and comorbidities}

Although the understanding of the role of predisposing/ comorbid factors has evolved considerably, it is not yet clear whether these host factors (systemic and local) and the environmental conditions that affect CRS in a child induce the disease or if they share a common pathway of pathogenesis.

\subsubsection{Anatomic variations in the sinuses}

To date, there is no evidence that anatomic abnormalities contribute, in any way, to the development and maintenance of CRS in children. Studies from 1997 to 2008 suggested that, 
despite the common occurrence of these anatomical variations (concha bullosa, concha hypertrophy, septal deviation, etc.), they did not seem to correlate with the degree and existence of CRS in children ${ }^{(9-11)}$. A more recent study examining anatomical abnormalities in an observational cohort of children showed the common occurrence of anatomic abnormalities in healthy sinuses $^{(12)}$. Whereas gender did not influence the prevalence of these abnormalities, some (deviated septum and concha bullosa) seemed to have a higher point prevalence in older children. Haruna et al. investigated whether the presence of paediatric middle turbinate pneumatisation causes a narrowing of the OMC and is associated with the development of rhinosinusitis by analysing CT scans obtained from 95 children 1-15 years old ${ }^{(9)}$. Middle turbinate pneumatisation was detected in $4.6 \%$ of the nasal cavities, and the middle turbinate pneumatisation itself did not obstruct the ostiomeatal complex. Thus, a direct causal relationship between middle turbinate pneumatisation and the development of rhinosinusitis was not observed. Equally, Al-Qudah performed a prospective study of 65 cases of paediatric chronic rhinosinusitis (average age of 11.4 years) that persisted after appropriate medical therapy and examined their CT scans ${ }^{(11)}$. Agger nasi cell was the most common anatomical variation, followed by concha bullosa of the middle turbinate. No significant correlation was found between these anatomical variants and the extent of chronic rhinosinusitis, again suggesting that these abnormalities are unlikely to predict the extent and severity of chronic rhinosinusitis in the paediatric population.

\section{Anatomic variations are more frequent in older children, but do not seem to be related to the presence of CRS.}

\subsubsection{Environmental factors}

\subsection{Viral infection}

It is speculated that upper respiratory tract infections (URTIs) play a role in the development of CRS, by leading to mucosal oedema, mucus production and retention, impaired mucociliary clearance, secretion stasis, and decreased aeration of the sinus mucosa ${ }^{(13)}$. Wood et al. used the polymerase chain reaction to look for respiratory viruses in the sinus mucosa from 13 adult patients with CRS and two patients with normal sinuses and were not able to detect a signal for any of several common respiratory viruses ${ }^{(14)}$. Therefore, to date, the contribution of viral infection to paediatric CRS is poorly studied with no evidence to support the importance of viral infections to CRS in children.

\section{Thus far, there is no credible evidence that viral infections contribute to paediatric CRS.}

\subsection{Exposure to tobacco smoke}

Environmental exposure to tobacco smoke inhibits mucociliary clearance and epithelial regeneration. Both active and passive smoking have been identified as significant risk factors for the development of $\mathrm{CRS}^{(15,16)}$. Reh et al.conducted an excellent review of the adult and paediatric literature examining the effect of cigarette smoke on CRS ${ }^{(15)}$. Several of the included articles had mixed paediatric and adult populations and only Kakish et al.included data specifically related to children ${ }^{(17)}$. They reported a $68 \%$ prevalence of acute rhinosinusitis among children exposed to passive smoking, compared to a prevalence of $1.2 \%$ among children without exposure ${ }^{(17)}$. While valuable, this study only evaluated acute rhinosinusitis. Christensen et al. performed a systematic review of large population studies to determine if there was a correlation between cigarette smoke exposure and the prevalence of $\mathrm{CRS}^{(16)}$. They reported on studies that evaluated outcomes of CRS surgery in the presence of passive and/or active smoke in the paediatric population (age $<18$ years).All the studies demonstrated worse outcomes in children exposed to cigarette smoke. These included an increase in surgical revision rates ${ }^{(18,19)}$, worse SNOT scores ${ }^{(19)}$, or lack of symptom improvement after surgery ${ }^{(18-20)}$.Thus, there is some evidence that cigarette smoke worsens CRS in children, however, longitudinal and mechanistic studies are required to determine the causative effect in children.

\section{Children with CRS who are exposed to passive} smoking present with more severe disease, worse clinical scores and higher rates of revision surgery.

\subsubsection{Allergic rhinitis}

Allergic inflammation can alter the sinonasal physiology with regards to mucociliary clearance and patency of the ostia and a late-phase allergic inflammation may contribute to CRS development ${ }^{(21)}$. However, evidence tying allergic rhinitis to CRS in children is at best equivocal.

A large study in paediatric patients with CRS reported that $29.9 \%$ had both a positive skin test and an elevated serum IgE level ${ }^{(22)}$. This prevalence of atopy in children with CRS was similar to that of the general paediatric population in Italy. Importantly, only $7.4 \%$ of the children under the age of three had positive testing compared to $31.4 \%$ of the children between 3-6 years and $33.3 \%$ of children older than six suggesting a low yield for allergy testing in patients under three years of age.

Sedaghat et al. evaluated a series of 4044 consecutive patients (age $\leq 18 \mathrm{yrs}$ ) with a diagnosis of chronic rhinosinusitis in the otorhinolaryngology or allergy and immunology clinics of Boston Children's Hospital between 2002 and 2012 ${ }^{(23)}$. Twenty seven percent of the children with CRS were also diagnosed with AR which is equivalent to the typical prevalence of the disease in the paediatric age group and does not support a higher prevalence of allergic rhinitis in children with CRS. Not surprisingly, there was a significantly higher prevalence of asthma in the population of children with CRS and AR (40.7\%) than in the children without AR (9.8\%). In fact, a strong positive association was detected between the diagnosis of asthma and AR in children with CRS. Other studies have shown a higher percentage of atopy in patients with CRS than in the general population. In a study in Thailand of 100 children with a clinical diagnosis of rhinosinusitis and abnormal plain radiographs, a positive skin test to common aeroallergens was reported in 53\% of the patients, which suggests a correlation between allergic 
rhinitis and $\mathrm{CRS}^{(24)}$. Georgalas et al. studied the Bradford-Hill criteria for causation applied to the evaluation of these studies and concluded that a clear and definitive causal relationship cannot be established, especially in children ${ }^{(25)}$.

Most recently, Anamika et al. studied 110 patients, between seven and 18 years of age, diagnosed with CRS based on history and clinical examination including nasal endoscopy ${ }^{(26)}$.Patients underwent skin prick tests against 65 common aeroallergens for evidence of atopy. A positive skin prick test to at least one of the common aeroallergens was present in $52.7 \%$ of patients. Patients with CRS and atopy had significantly higher mean Lund Mackay endoscopic score and SN-5 score (denoting worse quality of life) than nonatopic patients. In contrast, an older study evaluated CT scans from allergic and nonallergic children and noted the presence of sinus opacification in $61 \%$ and $64 \%$ of allergic and nonallergic children, respectively, which argues against an important role for allergy in sinus CT abnormalities ${ }^{(27)}$. In summary, the available studies evaluating the importance of allergic disease in CRS in children do not clearly elucidate the interaction of the two diseases. They suffer from many potential pitfalls: 1) biased populations as many of the studies are from allergy and otolaryngology clinics which are more likely to be seeing patients with allergic rhinitis, 2) lack of objective confirmation of the diagnosis of CRS, 3) lack of differentiation between atopy (positive skin test) and relevant clinical allergic rhinitis (positive skin tests and relevant correlating clinical symptoms). Despite these limitations, allergy testing should be considered in the older child with CRS.

\section{According to the studies above PCRS patients are more likely to also present with AR and/or asthma. However, research on the prevalence of CRS within the population with or without AR is needed to support this correlation.}

\subsubsection{Asthma}

Both CRS and asthma are inflammatory diseases of the airways. It has been hypothesised that rhinosinusitis impacts bronchial asthma in all age groups as part of the unified airway theory ${ }^{(28)}$. In their review of Boston Children's data, about coexistent diseases, Sedaghat and colleagues reported that $18.1 \%$ of 4044 children with CRS had concomitant asthma ${ }^{(23)}$. As mentioned above, there was a higher chance of asthma if the child also had allergic rhinitis. Anfuso and colleagues evaluated cytokine expression in sinus tissues of children with CRS and normal controls and found that children with CRS and asthma had significantly higher sinus levels of multiple inflammatory cytokines supporting the contribution of upper airways disease to lower airway inflammation in patients with $\mathrm{CRS}^{(29)}$. Older, non-controlled studies have shown that pharmacologic or surgical intervention for CRS resulted in discontinuing asthma medications in $80 \%$ of a cohort of 48 children ${ }^{(30)}$. Furthermore, asthma recurred when rhinosinusitis subsequently exacerbated. These and other studies support the concept that clinical control of CRS may be important in optimizing the control of difficultto-treat asthma. However, the limitations of most available studies include the lack of good controls or randomization to different treatment modalities; therefore, the relationship

Table 7.1.1. Adenoidectomy for paediatric CRS.

\begin{tabular}{|c|c|c|c|c|}
\hline Author & Type & Intervention & Outcome measure & Result \\
\hline $\begin{array}{l}\text { Vandenberg } \\
1997^{(140)}\end{array}$ & $\begin{array}{l}\text { Retrospective case series, } \\
n=48\end{array}$ & $\begin{array}{l}\text { Adenoidectomy or } \\
\text { adenotonsillectomy }\end{array}$ & Symptoms & $\begin{array}{l}\text { Complete or near symptom resolution } \\
\text { in } 58 \% \text { of patients }\end{array}$ \\
\hline $\begin{array}{l}\text { Brietzke } \\
2008^{(33)}\end{array}$ & $\begin{array}{l}\text { Systematic literature } \\
\text { review Average sample } \\
\text { size }=46 \text { (range } \\
10-121)\end{array}$ & $\begin{array}{l}\text { Adenoidectomy ( } 8 \text { studies } \\
\text { adequate for systematic } \\
\text { literature review) }\end{array}$ & $\begin{array}{l}\text { Proportion of patients who } \\
\text { improved after surgery }\end{array}$ & $\begin{array}{l}69 \% \text { of patients improved after } \\
\text { surgery }\end{array}$ \\
\hline $\begin{array}{l}\text { Ramadan } \\
2007^{(142)}\end{array}$ & $\begin{array}{l}\text { Retrospective review of } \\
\text { prospectively collected } \\
\text { data, } n=121\end{array}$ & $\begin{array}{l}\text { Adenoidectomy } \\
55 \text { patients underwent ESS } \\
\text { after adenoidectomy and had } \\
\text { data available }\end{array}$ & $\begin{array}{l}\text { Failure of adenoidectomy, } \\
\text { Factors that contributed to } \\
\text { early failure }\end{array}$ & $\begin{array}{l}61 / 121(50 \%) \text { children failed } \\
\text { adenoidectomy. } \\
\text { Asthma and age }<7 \text { yrs. predicted } \\
\text { earlier failure and faster ESS }\end{array}$ \\
\hline $\begin{array}{l}\text { Ramadan } \\
2014^{(169)}\end{array}$ & $\begin{array}{l}\text { Retrospective } 10 \text { yr. review, } \\
n=233\end{array}$ & $\begin{array}{l}\text { Adenoidectomy preceded } \\
\text { by a CT }\end{array}$ & $\begin{array}{l}\text { Success of adenoidectomy } \\
\text { based on CT score }\end{array}$ & $\begin{array}{l}\text { Success rate }= \\
43 \% \text { in } C R S \text { group (CT score } \geq 5), \\
65 \% \text { in chronic adenoiditis group (CT } \\
\text { score }<5 \text { ) }\end{array}$ \\
\hline $\begin{array}{l}\text { Bettadahalli } \\
2017^{(143)}\end{array}$ & Prospective, $\mathrm{n}=60$ & Adenoidectomy & $\begin{array}{l}\text { Sinus symptoms and quality } \\
\text { of life scores } 6 \text { months post- } \\
\text { intervention }\end{array}$ & $\begin{array}{l}88 \% \text { of children had a significant } \\
\text { improvement in outcome measures }\end{array}$ \\
\hline $\begin{array}{l}\text { Ramadan } \\
2008^{(144)}\end{array}$ & $\begin{array}{l}\text { Retrospective review of } \\
\text { prospectively collected } \\
\text { data, } n=60\end{array}$ & $\begin{array}{l}\text { Adenoidectomy or } \\
\text { adenoidectomy with a } \\
\text { maxillary sinus wash }\end{array}$ & $\begin{array}{l}\text { Improvement of symptoms at } \\
12 \text { months }\end{array}$ & $\begin{array}{l}88 \% \text { of adenoidectomy with sinus } \\
\text { wash showed improvement, } 61 \% \text { of } \\
\text { adenoidectomy showed improvement }\end{array}$ \\
\hline
\end{tabular}

CT, computed tomography; ESS, endoscopic sinus surgery. 
between CRS and asthma in children remains largely descriptive.

\section{We observe a strong correlation between asthma and PCRS. More research is required to elucidate the nature of the pathophysiologic events underlying this observation.}

\subsubsection{Adenoids}

Recent literature reviews have supported the hypothesis that adenoids have a significant impact on the development of CRS in younger children ${ }^{(31,32)}$ (Table 7.1.1.). Supporting evidence of the importance of the adenoids is data that shows improvement in CRS in children after adenoidectomy which will be discussed in the surgical management portion of this chapter ${ }^{(33)}$. In addition to improvement of CRS symptoms after adenoidectomy in a series of 35 children, one study has shown improved mucociliary transport in these children after surgery ${ }^{(34)}$. Despite this, no studies have shown a correlation between the size of the adenoid pad and the presence of sinonasal symptoms in paediatric CRS, neither when analysing adenoid size by $\mathrm{X}$-ray ${ }^{(35)}$, by volume ${ }^{(36)}$, or by weight of the tissue removed at surgery ${ }^{(37)}$. These findings suggest that the adenoids can contribute to the development of CRS not just by mechanical obstruction but also by serving as a bacterial reservoir. In support of this hypothesis, a study by Coticchia and colleagues observed that the surface area of adenoids covered by biofilm was $94.9 \%$ in patients with CRS compared to $1.9 \%$ in patients with obstructive sleep apnoea without $\mathrm{CRS}^{(38)}$. Shin and colleagues examined adenoid size and bacteriology in 410 children under 14 years of age who underwent an adenoidectomy due to adenoid hypertrophy ${ }^{(35)}$. Common upper respiratory pathogens (Haemophilus influenzae, Streptococcus pneumoniae, and Streptococcus pyogenes) were isolated from $79.3 \%$ of the adenoids, and the rate of bacterial isolation from the adenoids correlated with the severity of sinus disease on Water's radiographs again supporting the bacterial reservoir hypothesis. Therefore, available evidence suggests that the function of adenoids as a bacterial reservoir is more important than their size in the pathophysiology of paediatric CRS. There is also some older evidence that supports a contribution of the adenoids as an immunological organ in children with CRS. One study showed a significantly lower expression of IgA in the adenoids of children with CRS compared to those with obstructive adenoids ${ }^{(39)}$. This study could not elucidate, however, whether this was primary or secondary to chronic infection, as the design was cross-sectional. Shin and colleagues showed higher levels of tissue-remodelling cytokines in adenoids obtained from patients with CRS compared to tissue obtained from patients without CRS suggesting a relationship between the adenoids and the sinuses in children with $\operatorname{CRS}^{(40)}$. In summary, the adenoids seem important in the pathophysiology of CRS in children. Indeed, the first line of surgical management of CRS in younger children is an adenoidectomy and the data supporting efficacy will be discussed in the management section.

\subsubsection{Gastro-oesophageal reflux disease}

Gastro-oesophageal reflux disease (GORD) might be related to CRS in some paediatric patients; however, its association with the respiratory disease and its incidence remain doubtful. Data are conflicting and scarce. Reflux of gastric acid into the pharynx and nasopharynx is thought to cause sinus ostium inflammation, impaired mucociliary clearance, and consequently, rhinosinusitis.

GORD has been reported at higher rates in paediatric patients with rhinopharyngitis symptoms than in a control group ${ }^{(41)}$. In a large case-control study that included 1980 children aged two to 18 years with GORD and 7920 controls, a diagnosis of rhinosinusitis was significantly more likely in the children with GORD (4.19\%) compared with the control group (1.35\%) ${ }^{(42)}$. A prospective study by Phipps and colleagues in 30 paediatric patients with CRS who were evaluated with a 24-h pH probe showed that $63 \%$ of the children with CRS had gastroesophageal reflux ${ }^{(43)}$. This study also showed that 79\% of the children with CRS experienced improvement in rhinosinusitis symptoms after medical treatment for GORD. Similar results were reported in a retrospective study showing that the rate of surgery could be reduced by $89 \%$ in children with severe CRS treated with a proton pump inhibitor for GORD ${ }^{(44)}$. In 2014, Nation and colleagues performed a retrospective chart review of 63 children, aged six months to 10 years, with rhinorrhoea, nasal congestion, and chronic cough ${ }^{(45)}$. The patients underwent maxillary cultures, adenoidectomy, and distal third oesophageal biopsies. Children with oesophageal biopsies showing oesophagitis were classified as positive for gastro-oesophageal reflux disease, and maxillary antral swabs growing a high density of bacteria were classified as positive for chronic rhinosinusitis. Younger children aged six months to five years tended to have either chronic rhinosinusitis or gastrooesophageal reflux disease, whereas older children, aged six to 10 , tended to have a more complicated aetiology of CRS and GORD. In all of these patients, GORD played an important role in many of these patients, as over $40 \%$ had gastro-oesophageal positive biopsies. Another potential causative agent that has been investigated for adenoiditis and/or CRS is Helicobacter pylori. Cedeno and colleagues failed to detect the presence of H. pylori in adenoid tissue or maxillary sinuses in patients with $\mathrm{CRS}^{(46)}$.

In summary, there is some evidence to support a potential role of GORD in CRS in children. However, the studies suffer from significant limitations including retrospective design, lack of placebo group in treatment studies, and poor characterization of the presence and extent of CRS. Therefore, routine treatment for GORD is not currently justified in children with CRS. Indeed, this conclusion is supported by the consensus statement on paediatric rhinosinusitis published by the American Academy of Otolaryngology Head and Neck Surg ${ }^{(47)}$.

The relationship between GORD and CRS seems to be strong in children. However, the evidence is weak and does not support the routine treatment of GORD in children with CRS. 


\subsubsection{Immaturity or deficiency of the paediatric immune system}

Studies in children with recurrent rhinosinusitis or CRS have demonstrated a variable percentage of immune dysfunction, including a decrease in the levels of immunoglobulins $A$, $G$, and subclasses, with a poor response to pneumococcal vaccine and low levels of immunoglobulin in response to normal vaccines. Shapiro et al. evaluated immunologic competence in 61 children with CRS who were referred for an allergy evaluation ${ }^{(48)}$. Roughly $50 \%$ of the children had evidence of environmental allergies and at least 23 of the 61 patients with refractory rhinosinusitis had impaired humoral immunity manifested by low immunoglobulin levels or vaccine hyporesponsiveness. This is clearly a much higher rate than in adults. Hidalgo and colleagues report that $6 \%$ of 78 children with CRS failed to mount an immune response to pneumococcal vaccine ${ }^{(49)}$. In 27 children with chronic or recurrent rhinosinusitis, Costa Carvalho and colleagues detected one patient with $\lg A$ andlgG2 deficiency, and another with $\lg$ I 3 deficiency ${ }^{(50)}$. Eight and 12 of 27 patients had $\lg G 2$ and IgG3 serum levels below $2.5^{\text {th }}$ percentile, respectively and no patient had an abnormalresponse to vaccination. In a large study of 307 subjects with refractory rhinosinusitis (261 adults and 46 children), Vanlerberghe et al. found laboratory evidence of humoral immunodeficiency, including IgA deficiency or IgG subclasses deficiencies, in $21.8 \%$ of the cases with no difference in prevalence between children and adults ${ }^{(51)}$. Baek and colleagues evaluated 16 children with recurrent acute or CRS and found that $50 \%$ had defects in the humoral immunity including poor responsiveness to a pneumococcal vaccine ${ }^{(52)}$. In the study by Sedaghat et al., mentioned above, 496 (12.3\%) of the 4044 children diagnosed with CRS had a diagnosis consistent with an immune disorder ${ }^{(23)}$.

On the other hand, in 2006, Bernatowska et al. evaluated the incidence of CRS in 425 children with different types of primary humoral deficiency ${ }^{(53)}$. CRS was observed most frequently in the group of patients with agammaglobulinemia (85.96\%) and less often in patients with dysgammaglobulinemia (81.81\%) and other antibody deficiencies (72.72\%). As to immunoglobulin replacement, a pilot study in six children with CRS refractory to medical management treated with intravenous immunoglobulin (IVIG) for one year showed a decrease in antibiotic intake, rhinosinusitis episodes, and an improvement in sinus imaging studies ${ }^{(54)}$.

Clearly, the reports of humoral immune deficiency in children with recurrent or chronic rhinosinusitis vary widely. This is likely influenced by the types of populations studied, measures of immune function, and accuracy in the diagnosis of rhinosinusitis. It therefore seems prudent to evaluate immune function in the child with chronic / recurrent rhinosinusitis with an immunoglobulin quantitation and titers to tetanus and diphtheria in addition to pneumococcal titers. If responses are abnormal, a repeat set of titers after pneumococcal vaccination is appropriate and if the response is not adequate, then referral to an allergist/immunologist is warranted.

\section{Children with CRS refractory to appropriate medical treatment should be evaluated for humoral immune deficiency.}

\subsubsection{Cystic fibrosis (CF) (see also chapter 8.4.)}

$\mathrm{CF}$ is an autosomal recessive disease caused by mutations in the CFTR gene that leads to the formation of thick viscous secretions, favouring infections in the respiratory tract. It is diagnosed by abnormally elevated sweat chloride levels or by genetic testing. Because the sweat chloride test is not $100 \%$ specific, the diagnosis of $C F$ is based on the presence of one or more clinical features of $C F$, in addition to evidence of an abnormality in the CFTR gene or protein. P. aeruginosa and S. aureus are the microorganisms most frequently cultured when the infection affects the sinuses ${ }^{(55)}$. It is the most common disorder encountered in children with CRSwNP. In fact, the prevalence of CRS in the population with CF is nearly $100 \%{ }^{(55-57)}$. Despite newborn screening, otorhinolaryngologists continue to receive children without a confirmed diagnosis of CF. In children with polyps and sinus disease, physicians should have a high index of suspicion for CF, particularly in the context of poor weight gain, respiratory disease and gastrointestinal abnormalities. The finding of expansion of the paranasal sinuses (pseudomucocele of the maxillary sinuses) and hypodevelopment of the frontal and sphenoidal sinuses, without evidence of bone erosion on CT scan, are strong

\section{Key points | What's new since EPOS 2012}

- The prevalence of CRS in paediatric patients has been shown to be between 2.1 to $4 \%$

- Children of 10 to 15 years are most often affected with CRS. When comparing the frequency of the disease with that of other common conditions, CRS was more prevalent than ARS in all age groups and more numerous than otitis media in the group between 15 to 20 years (0.9\%).

- Both passive and active cigarette smoking are associated with chronic rhinitis and rhinosinusitis in children.

- A clear and definitive causal relationship between AR and CRS cannot be established, especially in children.

- The adenoid may act as a reservoir for pathogenic bacteria, rather than a source of obstruction.

- The relationship between GORD and CRS in children is controversial.

- The most common immunodeficiencies related to refractory CRS in children are immunoglobulin deficiency (including IgG subclasses) and poor response to vaccines.

- Physicians treating children with nasal polyps and sinus disease should have a high index of suspicion for CF, particularly in the context of poor weight gain, respiratory disease, and gastrointestinal abnormalities. 
radiographic indicators of $\mathrm{CF}^{(58-60)}$. Wentzel et al. evaluated a group of 50 consecutive patients with CF aged 2-12 years who presented to a rhinology practice ${ }^{(60)}$. The quality-of-life visual analogue scale was strongly negatively correlated with $\mathrm{SN}-5$ scores. Additionally, worse sinus-specific QOL as measured by the $\mathrm{SN}-5$ score was associated with increased incidence in the diagnosis of rhinosinusitis, prescription of antibiotics, and missed days of school or recreational activities due to sinonasal symptoms. The authors concluded that the $\mathrm{SN}-5$ was a quick and qualitative method for monitoring CRS in this patient population. Chan et al. evaluated a group of 102 paediatric patients with CF with an age range of 2-20 (average 12.9) years by a screening questionnaire for the diagnosis of CRS and the SN-5 during a routine clinic visit ${ }^{(61)}$. Of the 102 participants, 47 completed the surveys. Depending on the diagnostic criteria used, $11-38 \%$ of the patient population had CRS. Mean domain and overall visual-analogue scale scores on the $\mathrm{SN}-5$ were consistent with a minimal effect on QOL. The authors concluded that there was a high prevalence of symptomatic CRS in this patient population, but that it had a low impact on quality of life.

\section{In cases of nasal polyps among paediatric patients, investigations for CF should be performed. Sweat chloride test remains important to confirm the disease, although newborn screening has significantly decreased the age of diagnosis of this disease.}

\subsubsection{Primary ciliary dyskinesia (see also chapter 8.5.)}

PCD is a rare autosomal recessive disorder with an estimated incidence of one in 15,000 to 20,000 births. It consists of defects in ciliary beating, resulting in decreased mucociliary clearance ${ }^{(62)}$. It is associated with frequent sinus and ear infections, situs inversus totalis (in $50 \%$ of patients), heterotaxy or situs ambiguous (in $12 \%$ of patients), and infertility (in $50 \%$ of male patients) ${ }^{(63,64)}$. The symptoms of CRS in children with complex mucociliary disorders such as PCD may be debilitating and result in respiratory compromise with decreased pulmonary function. Purulent nasal secretions, typically transported by gravity or airflow due to the decreased ciliary clearance, are observed in children with PCD during active sinus infections ${ }^{(65)}$. Indeed, in a series of 84 patients with PCD from Australia, recurrent cough and rhinosinusitis were the most common symptoms occurring in $71 \%$ and $81 \%$ of the children respectively ${ }^{(66)}$.

Screening tests for PCD include nasal nitric oxide (lower levels than controls) (see 9.5.3.3) and in vivo tests, such as the saccharin test, which documents slower mucociliary transit times. Specific diagnosis requires examination of the cilia by light and transmission electron microscopy, which is usually available only in specialised centres ${ }^{(67)}$. The most commonly described structural abnormality involves lack of the outer dynein arms, or a combined lack of both inner and outer dynein arms. Additionally, genetic testing in specialized centres is now available for roughly 20 different genes that underlie the pathophysiology of PCD (see 9.5.3.2). Nasal polyps occur in approximately $18 \%$ to $33 \%$ of patients with $P C D$, most often starting in adolescence.Kartagener's syndrome, a triad of rhinosinusitis, chronic bronchitis with bronchiectasis, and situs inversus, occurs in approximately $50 \%$ of patients with $\operatorname{PCD}^{(63,65}$, 68).

The management of CRS in children with PCD is challenging due to the rare incidence of $P C D$ and the paucity of high-quality primary literature. Control of the CRS symptoms is especially important in these children to maintain an adequate quality of life and pulmonary function. Given the rare prevalence of $P C D$ and the insufficient evidence concerning the treatment effectiveness for CRS, Mener et al. support future development of an international, multicentre database for prospective data collection $^{(63)}$. This database should include the diagnosis, treatment and outcomes of CRS for children with PCD and would encourage implementation of validated and standardised outcome measures in these children.

$P C D$ should be suspected in children with refractory CRS and pulmonary diseases, especially in those with concomitant bronchiectasis, situs inversus totalis or spermatozoid abnormalities.

\subsection{Pathophysiology}

\subsubsection{Genetics}

The information available about the genetics of CRS in children is very limited and the studies suffer from some limitations but show some interesting trends that deserve further investigation. Orb and colleagues explored the familial risk of CRS in children by mining the Utah population database which consists of computerized data records for $>7.3$ million people ${ }^{(69)}$. They identified 496 children with CRS as follows: 12 years or younger with an index ICD-9 diagnosis of CRS appearing in the medical record between 1996 and 2011, and a coupled procedural code related to CRS (nasal endoscopy, adenoidectomy and/or sinus surgery). The authors randomly selected 4959 ethnically matched controls with no history of CRS from the Utah population and individually matched 10:1 on sex and birth year to corresponding paediatric CRS cases. Siblings of patients with CRS demonstrated a 57.5 -fold increased risk of having paediatric CRS compared to controls. First cousins had a 9.0-fold increased risk and second cousins a 2.9-fold increased risk of paediatric CRS, all significant associations. Parents, first and second-degree relatives, and first cousins of paediatric cases demonstrated significant increased risks of having adult-onset CRS. Although these results suggest a significant familial risk associated with CRS, the study is limited by the accuracy of the diagnosis of CRS as it is based on review of diagnostic codes without confirmation by clinical evidence of disease.

Purkey and colleagues studied a case control sample of 828 children (average age 11.9 years) with CRS and 5,083 healthy controls from the Children's Hospital of Philadelphia ${ }^{(70)}$. Index patients had a history of CRS diagnosed by a paediatric otolaryngologist using symptom criteria, flexible endoscopy and CT evidence in almost half the cases. Children with cystic fibrosis were excluded. Single nucleotide polymorphism (SNP) 
genotyping of potassium channel genes was undertaken and showed that the locus yielding the strongest signal was at the KCNMA1 gene in Caucasian children with CRS $(p=0.022)$. There was borderline evidence for association in the KCNQ5 gene with CRS in African American children. This report suggests a genetic association between potassium channel epithelial physiology and the development of CRS in children.

In an earlier study in 58 Caucasian children with CRS (diagnosed by persistent symptoms and objective evidence of disease) and no known cystic fibrosis, $12.1 \%$ were found to carry heterozygous mutations in the cystic fibrosis transmembrane regulator (CFTR) gene, higher than the expected rate of 3-4\% in the same ethnic group ${ }^{(71)}$. These results suggest a predisposition to CRS in children who are carriers for CFTR mutations. Sedaghat and colleagues investigated the presence of mutations in genes encoding connexins, that maintain epithelial integrity via gap junctions, in patients with chronic and recurrent acute rhinosinusitis diagnosed by persistence of symptoms and objective evidence of disease by either nasal endoscopy or CT scan ${ }^{(72)}$. Of the eight paediatric patients (age between six and 16 years), only one child had a mutation in connexin 32 and of their 11 adult patients, one had a mutation in connexin 43. A previous study by the same group had shown no mutations in connexins 26 and 30 in the same group of patients ${ }^{(73)}$. Although these results suggest that mutations in the connexin gene family might not be important in the pathophysiology of CRS, the number of observations are too small to make definitive conclusions.

In summary, it is likely that the pathophysiology of paediatric CRS involves both genetic and environmental influences. Indeed, studies of monozygotic twins have not shown that both siblings always develop polyps, indicating that environmental factors are as likely as genetic ones to influence the occurrence of nasal polyps ${ }^{(74,75)}$.

Based on existing slim research, it is likely that the pathophysiology of paediatric CRS involves both genetic and environmental influences.

\section{Key points | What's new since EPOS 2012}

- A large database study suggests a significant familial risk associated with paediatric CRS.

- Studies on gene mutations in paediatric CRS show some preliminary promising results.

\subsubsection{Inflammatory mechanisms}

\subsubsection{Sinus and adenoid tissue studies}

Early studies of the cellular response in paediatric CRS in older children indicate that eosinophils and CD4-positive lymphocytes play a significant role in tissue inflammation ${ }^{(76,77)}$. In later similar studies performed in younger children, Chan and colleagues $^{(78)}$ showed that paediatric maxillary sinus mucosa had more neutrophils and significantly more lymphocytes than adult mucosa but had fewer eosinophils and major basic proteinpositive cells, with less epithelial disruption and less basement membrane thickening. These authors also showed higher numbers of CD8-positive cells, neutrophils, macrophages, B lymphocytes, and plasma cells in younger children with CRS compared with adults ${ }^{(79)}$. In another comparative study of sinus tissues between adults and children, paediatric tissue had scant eosinophils and abundant lamina propria fibrosis ${ }^{(80)}$. The differences in eosinophil versus neutrophil predominance in the specimens studied above probably relate to the age group of the children studied (eosinophil predominance in the older children and neutrophil predominance in the younger ones). In more recent studies, Wu and colleagues investigated inflammatory gene expression in tissues obtained from the sinuses of children with CRS and normal controls ${ }^{(81)}$. Using microarray analyses and validation by gene mRNA expression levels by real-time PCR, they showed that five inflammatory/ immune response gene products were significantly upregulated in tissues from CRS compared to control. Two of the products were the cytokines CXCL5 (neutrophil chemoattractant), and CXCL13 (B lymphocyte chemoattractant), contributors to the adaptive immune response. The other three were serum amyloid A1/A2 (SAA1/SAA2), serpin peptidase inhibitor member 4 (SERPINB4), and $\beta$-defensin 1 (DEFB1). The latter are proteins involved in the innate immune system. In a follow up study, the investigators evaluated the tissue distribution and cellular localization of these inflammatory markers using immuno-histochemical staining and immunofluorescence ${ }^{(82)}$. They showed that all five mediators were strongly expressed in submucosal glands and in ciliated and basal cells within the epithelium. Further, CXCL13 was expressed in macrophages, $T$ and $B$ cells but not in neutrophils and CXCL5 was detected only in T cells.

Anfuso and colleagues evaluated cytokine expression in sinus and adenoid tissues of children with CRS and normal controls and found more abundant cytokines in the tissues of the children with CRS compared to controls supporting ongoing inflammation in the disease group ${ }^{(29)}$. Importantly, compared with children with CRS and without asthma, children with CRS and asthma had significantly higher sinus levels of tumor necrosis factor- $a$ and adenoid levels of epidermal growth factor, eotaxin, fibroblast growth factor-2, growthrelated oncogene, and platelet-derived growth factor-AA. This supports the contribution of upper airways disease to lower airway inflammation in patients with CRS. Shin and colleagues evaluated inflammatory markers in adenoid homogenates of children with and without CRS undergoing adenoidectomy ${ }^{(40)}$. The adenoids from children with CRS had significantly higher levels of the T cell activation marker soluble interleukin-2 receptor (sIL-2R), as well as the levels of cytokines associated with tissue remodelling, such as transforming growth factor $\beta-1$ (TGF $\beta-1$ ), matrix metalloproteinases (MMP) 2 and 9, and tissue inhibitor of metalloproteinase (TIMP-1). Whereas there was no difference between the two groups in levels of the eosinophil activation marker, eosinophilic cationic protein (ECP), its levels were significantly higher in patients with severe CRS compared to those with mild and moderate disease. 


\subsubsection{Nasal lavage}

Passariello and colleagues evaluated levels of the cytokine (TNF-a) the antimicrobial peptide human $\beta$-defensin 2 and neutrophil-released calprotectin in nasal lavages of children (average age 3.4 years) with CRS and healthy control(83). This was done in the context of a study evaluating the benefit of crenotherapy (sulfate sodium-chloride thermal water with different mineral components by nasal aerosol administered for 15 minutes daily) in childhood CRS. They show significantly higher levels of all these proinflammatory substances in the lavage of patients with CRS compared to the control subjects.

\subsubsection{Serum}

Shin and colleagues performed allergy testing and obtained serum eosinophil counts and serum ECP and IgE levels in a group of children with CRS treated with antibiotics and controls ${ }^{(84)}$. They further divided the CRS group into responders and non-responders to a prolonged course of antibiotics. The prevalence of atopy and a family history of allergic diseases were significantly higher in the non-responder group than in the responder and control groups. Further serum eosinophil counts and levels of ECP and total IgE were significantly higher in the non-responder group than in the responder and control groups. This data suggests that eosinophilic inflammation in the context of allergy was an important factor in children with CRS who do not respond to antibiotics.

\section{Although more evidence is emerging to support upregulation of inflammatory markers in paranasal sinus tissues and nasal lavages of children with CRS, the data is relatively limited and heterogeneous and does not yet lend itself to endotyping.}

\subsubsection{Mucus}

Mucus production is an important component of the clinical picture in children with CRS. Many mucin glycoproteins have been identified and the most frequently studied are the secretory mucins MUC5AC and MUC5B. In healthy lower airways, MUC5AC is typically expressed in the goblet cells, while MUC5B is restricted to the submucosal glands ${ }^{(85)}$. In paediatric CRS, submucosal glandular hyperplasia is the characteristic phenotype and MUC5B is the predominant glandular mucin ${ }^{(86)}$. Saieg and colleagues collected secretions from patients with CRS and normal controls ${ }^{(87)}$. Many mucin glycoproteins were identified, but the relative abundance of MUC5B was found to be significantly higher in patients with CRS suggesting a parallel between the tissue and secretion glycoprotein production. In summary, multiple studies suggest upregulation of different inflammatory substances important in adaptive and innate immunity as well as tissue remodelling in sinus tissues, adenoids, nasal lavage, mucus and serum in children with CRS. Although the evidence is still scarce, these studies suggest a role for inflammatory mechanisms in paediatric CRS. Although many of the markers parallel those seen in adults, the data is very heterogeneous and does not yet lend itself to endotyping.

\section{Key points | What's new since EPOS 2012}

- Evidence of the presence of adaptive and innate immunity markers in paranasal sinus tissues and nasal lavages have been obtained from children with CRS.

- Inflammatory cytokines are present in sinus tissues of children with CRS and are more abundant when concomitant asthma is present.

- Submucosal glandular hyperplasia is the characteristic phenotype in paediatric CRS, and MUC5B is the predominant glandular mucin.

\subsection{Diagnosis and differential diagnosis}

\subsubsection{Allergic and non-allergic rhinitis}

Generally speaking, the differentiation between allergic rhinitis (AR), non-allergic rhinitis (NAR) and CRS can be challenging, although in adults careful history-taking, nasal endoscopy and allergy tests usually suffice to make a distinction (see chapter 5.3.1). In children, however, this distinction can be more complicated. Just as in CRS, both AR and NAR can present with rhinorrhoea and nasal obstruction. Furthermore, patients with AR can show increased sinus opacification on imaging ${ }^{(88)}$, although this has not been studied for children specifically. To complicate matters even further, CRS and AR often coincide, with the incidence of AR already reaching a prevalence as high as $40 \%{ }^{(89)}$.

The role of NAR in children is less well studied ${ }^{(90)}$. Most NAR subtypes are unlikely to be present in (young) children, such as drug-induced rhinitis, occupational rhinitis, hormonal rhinitis, or senile rhinitis, leaving idiopathic rhinitis as the main diagnosis of NAR in children. Several studies have investigated the prevalence of NAR in the paediatric population. In Europe, large studies indicate a prevalence varying between $6.3 \%$ (Sweden, eight-year-olds), 8.1\% (Sweden, four-year-olds) and 9.6\% (Belgium, 15 years or older) ${ }^{(91,92)}$. In an American study of 619 children with asthma, the prevalence of NAR was $11.3 \%{ }^{(93)}$.A study from Singapore with 6600 children found a prevalence of $24.9 \%$ (mean age: 7.8 years), decreasing to $10-15 \%$ in older children ${ }^{(94)}$. A Chinese study including 56 children below 15 years of age, reports a prevalence of $34 \%$. In the age group between 15 and 30 years of age, the prevalence of NAR was still $20 \%{ }^{(95)}$. Taken together, the prevalence of NAR in the paediatric population is between $6.3-34 \%$, with considerable regional differences.

In (very) young children, one could debate whether the group having complaints, but no allergic sensitization represents 'true' NAR patients. Possibly, those suffering from adenoid hypertrophy, CRS, or other sinonasal disease are erroneously included, thus inflating the numbers. Therefore, a safe estimate of the prevalence of NAR in the paediatric population would be between $10-15 \%$. These numbers are at least equal to the prevalence of paediatric CRS, making NAR an important differential diagnosis.

In order to determine the right diagnosis, a full history, physical 
Table 7.3.1. Differentiating CRS, AR and NAR.

\begin{tabular}{|c|c|c|c|}
\hline & CRS & AR & NAR \\
\hline Complaints & $\begin{array}{l}\text { Nasal obstruction } \\
\text { Rhinorrhoea } \\
\text { Cough } \\
\text { Facial pain/pressure }\end{array}$ & $\begin{array}{l}\text { Nasal itching } \\
\text { Sneezing } \\
\text { Nasal obstruction } \\
\text { Rhinorrhoea } \\
\text { Seasonal variation }\end{array}$ & $\begin{array}{l}\text { Nasal itching } \\
\text { Sneezing } \\
\text { Nasal obstruction } \\
\text { Rhinorrhoea } \\
\text { Specific triggers (smoke, mist, cold } \\
\text { dry air etc.) }\end{array}$ \\
\hline Nasal endoscopy & $\begin{array}{l}\text { Relevant pathology in the middle } \\
\text { meatus (thick secretions, pus, polyps, } \\
\text { oedema etc.) }\end{array}$ & $\begin{array}{l}\text { Generalized mucosal involvement, watery } \\
\text { rhinorrhoea, nasal hyperreactivity upon } \\
\text { endoscopy }\end{array}$ & $\begin{array}{l}\text { Generalized mucosal involvement, } \\
\text { watery rhinorrhoea, nasal } \\
\text { hyperreactivity upon endoscopy }\end{array}$ \\
\hline $\begin{array}{l}\text { Skin prick test or } \\
\text { serum specific IgE }\end{array}$ & $\begin{array}{l}\text { Negative or not clinically relevant for } \\
\text { aeroallergens". } \\
\text { Positive if AR coincides }\end{array}$ & Positive for aeroallergens" & $\begin{array}{l}\text { Negative or not clinically relevant for } \\
\text { aeroallergens" }\end{array}$ \\
\hline $\begin{array}{l}\text { Imaging } \\
\text { (CT^ or MRI) }\end{array}$ & Opacification of sinuses (if developed) & $\begin{array}{l}\text { Ideally: none, however opacification might } \\
\text { be present }\end{array}$ & $\begin{array}{l}\text { Ideally: none, however opacification } \\
\text { might be present }\end{array}$ \\
\hline
\end{tabular}

$\mathrm{CT}$, computed tomography; MRI, multi-resonance imaging; ^routinely using CTs in children is not advised because of exposure to radiation \#only clinically relevant when complaints are concordant

examination and several diagnostic tools should be used (Table 7.3.1.). Additional attention should be paid to the child's environment, such as the presence of parental smoking, pets etc.

\subsubsection{Adenoid hypertrophy/adenoiditis}

Adenoid hypertrophy/adenoiditis and CRS can be hard to distinguish, especially in the very young ${ }^{(96)}$. This has been elaborately described in chapter 7.1 and will be alluded to in the treatment section (7.4).

\subsubsection{Chronic cough}

In contrast to adult CRS, paediatric CRS can encompass chronic cough (rather than loss of smell). The important differential diagnoses to consider here are: adenoid hypertrophy/ adenoiditis, asthma, or gastro-oesophageal reflux disease. These are all addressed in chapter 7.1.

\subsubsection{Smell disorders}

In general, loss of smell can be an important sign of CRS. However, for the paediatric population, this statement does not apply consistently. Children (or their care givers) hardly ever complain of olfactory dysfunction, the very reason why "loss of smell" has been replaced by "cough" in the diagnostic construct of paediatric CRS ${ }^{(96)}$. Indeed, in a large cohort of self-declared healthy participants, up to $3.4 \%$ were found to be anosmic (and unaware). In the age group between 5-10 years, the number was as high as 7\%; and in the group between 11-20 years of age, it was still $1.4 \%{ }^{\left({ }^{(97)} \text {. }\right.}$

The real extent of CRS related olfactory impairment in children is not yet known, however it is estimated that the most frequent cause of olfactory disorders in children is also CRS related ${ }^{(98)}$. This lack of data is largely due to the inaccuracy of the testing tools available to detect olfaction problems, especially in young children, and the fact that olfactory symptoms reach the level of social consciousness only after a child reaches a certain age. The latter is currently observed in congenital anosmics whereas both the patient and their environment become aware of the absence of olfaction only in late childhood on average ${ }^{(99)}$. The way most clinical olfactory identification tests work is probably unsuited and unreliable for children below the age of 6 years (see chapter 7.3.5.5).

Acquired olfactory dysfunction due to diseases other than CRS is rare in children ${ }^{(98,100)}$. Primary disorders such as isolated congenital anosmia or syndromic forms such as Kallmann syndrome are even more uncommon. When a child presents with loss of smell, careful history-taking combined with nasal endoscopy and olfaction tests (see chapter 8.3.5.5) usually suffices to discern CRS from other causes (congenital, traumatic, oncology, psychiatric disorders etc.). In the (very) young, identification of the right diagnosis can be hard, but again, olfactory dysfunction will hardly ever be noticed or sought help for in the first place.

\subsubsection{Facial pain}

Facial pain or headache as a main (or only) complaint is usually unrelated to sinus disease. Unfortunately, patients often undergo a myriad of nasal treatments including surgery before a primary headache syndrome is suspected or diagnosed. For the paediatric population, this is true as well, although the percentage of misdiagnoses tends to be lower ${ }^{(101)}$. The fact that the paranasal sinuses are not fully developed in childhood might limit the suspicion of sinonasal disease as a cause of headaches. Of the children presenting with headache, most are diagnosed with migraine. Smith and co-workers have provided a thorough systematic review on rhinogenic headache in the paediatric population which is recommended for further reading ${ }^{(102)}$. The recommendations for children presenting with headache are the same as those for adults (see chapter 5.3.3.).

\subsubsection{Diagnostic tools in children}

In 2019, the European Position Paper on Diagnostic Tools in Rhinology. (EPPDRT) was published, providing an excellent, state-of-the-art overview of the diagnostic tools available to physicians treating sinonasal disease ${ }^{(103)}$. Below, several of these diagnostic tools will be discussed briefly, with emphasis on applicability and considerations in paediatric CRS. For more detailed information, the reader is advised to read the European 
Position Paper (which will be referred to as "the EPPDTR" below).

\subsubsection{CT scan / MRI}

The (upfront) use of imaging in children with CRS is debatable. A sinus CT-scan is mandatory when complications are suspected, or when surgery is considered/planned. In every other case, a balanced consideration must be made on the harm of the study (in case of CT: radiation; in case of MRI: long scanning times) and the possible gain in information. Current guidelines recommend "imaging gently" due to the reported increased chance of leukemia and brain tumours in children who receive CT scans, with higher risk associated with higher radiation exposure ${ }^{(104)}$. Therefore, most paediatric facilities adopt paediatric protocols that allow acquisition of the appropriate information with the least amount of radiation. In children, higher Lund-Mackay scores are generally found than in adults, which does not necessarily reflect CRS. In adults, a cut-off score of $>3$ is often used, but it has been suggested to use a higher cut-off of $>5$ in the paediatric population ${ }^{(105)}$. It seems that sinus opacification alone in the paediatric population should be interpreted with caution, even if multiple sinuses are involved. Indeed, in a cohort of 192 children (mean age: 9.0 years) with CT scans of the orbits or brain without a history of sinonasal disease, only $19.3 \%$ had completely normal sinuses ${ }^{(106)}$.

\subsubsection{QoL / SNOT}

Most of the quality-of-life (QoL) instruments described in the EPPDTR are not suitable for the paediatric population. The general QoL questionnaires depend on items as self-care, social function, etc. which are not applicable for a child. Furthermore, most disease-specific questionnaires for CRS are not validated for lower age groups. The often-used SNOT-22 was originally validated in an adult cohort (1.6) ${ }^{(107)}$. Its use in children is incidentally reported (for example in a CF-cohort ${ }^{(108)}$. The only validated disease-specific QoL questionnaire for paediatric CRS is the Sinus and Nasal Quality of Life Survey (SN5). It is validated for children aged 2-12 and filled in by their parents/care givers ${ }^{(109)}$. However, there are only a few studies using this questionnaire to generate data on QoL outcomes in paediatric $\mathrm{CRS}^{(110)}$.

\subsubsection{Endoscopy}

Performing nasal endoscopy is a pivotal step in the diagnosis of CRS. Usually it is well tolerated, even in children. Use of topical decongestants and/or anaesthetics is at the discretion of the physician. Endoscopy in children is regularly used and published upon, for example in allergic rhinitis with patients as young as two years of age $\mathrm{e}^{(111)}$.

\subsubsection{Allergy test}

As in adults, allergy tests can be performed by a skin prick test or blood tests (see 5.3.5.4.). According to the guidelines published by the European Academy of Allergy and Clinical Immunology (EAACl) Section on Pediatrics, there is no lower age limit for skin prick testing ${ }^{(112)}$. However, in younger children (roughly younger than seven years of age) regular aeroallergens are best tested using blood samples for specific lgE for reasons of patient comfort. In very young children, specific lgE can be elevated without clinical relevance and return to normal levels during their development, which could be a reason to repeat the measurement if an allergic diagnosis is doubtful despite previously elevated specific lgE levels.

\subsubsection{Assessment of smell}

As described in the EPPDRT, it is feasible to use odour identification tests in children; the Sniffin' Sticks have been validated in a cohort of 201 Dutch children ${ }^{(113)}$. Moreover, specific tests for young children (age five and above) have been developed ${ }^{(114,115)}$. One should be aware, however, that these cannot be simply applied to every country/language, as cultural differences can influence the performance of subjects on the test. Therefore, these tests need to be validated before they can be reliably applied in clinical practice or research. Below a cutoff age between five and six years and, depending on the child's cognitive maturation, currently used identification tests turn out to be unreliable and even suggest that children have lowered olfactory function. This is just a reflection of the fact that smelling and picking the right odour from a list of descriptors requires familiarity, associative and verbal capacities, and sufficient concentration which might be difficult to achieve for the younger child. It is possible to test even new-born and infant olfactory ability but that requires a different way of testing ${ }^{(116)}$. The scarce literature on olfaction in human new-borns, babies, and toddlers shows that they have an extremely developed olfactory function ${ }^{(117,118)}$.

\subsubsection{Upper airway tests}

The EPPDRT elaborates on three upper airway tests, which all can be applied in (older) children: peak nasal inspiratory flow (PNIF), acoustic rhinometry, and rhinomanometry. Of these, the PNIF is easiest to use and has been validated in children six years and older ${ }^{(119)}$. Still, in children these investigations are not very clinically relevant and are predominantly used in the research setting.

\subsubsection{Pathology test including ciliary morphology and function}

Taking mucosal biopsies in children is rarely needed. Unilateral processes warrant suspicion of malignancy or inverted papilloma, even in children. Some authors advise to routinely send antrochoanal polyps for histopathological examination as they might represent inverted papilloma ${ }^{(120)}$.For bilateral disease, biopsies are only needed if one suspects primary ciliary dyskinesia. Several tests for ciliary function are available (see EPPDTR).

\subsubsection{Blood tests}

As explained in detail in chapter 5.3.5., routine lab testing in (paediatric) CRS is not advised. As mentioned above, one should consider evaluation of the humoral immune response in children with CRS unresponsive to therapy and/or with lower airway manifestations such as recurrent pneumonia. 


\subsubsection{Nitric oxide}

Measuring nasal nitric oxide is indicated if PCD (and to some extent (F) is suspected. This non-invasive test can be performed in cooperative children, usually from the age of five onwards. It is described in detail in the EPPDTR and in chapter 5.3.5.

\subsubsection{Genetic testing}

Although there might be a genetic susceptibility for developing CRS as discussed in chapter 7.2.1, routing genetic testing is not advised. Whenever there is a suspicion of CF or PCD, genetic testing might be used. For CF, it is possible to test for mutations in the CFTR gene through a blood test or cells taken from a cheek swab. Also, sweat testing is advised. For specific details, see chapter 8.4. For PCD, genetic testing is also recommended and has greatly improved the ability to diagnose the disease (see chapter 5.3.5. for more details). This genetic test is part of a panel of diagnostic tests, which also includes microscopic evaluation of the ciliary function (see chapter 7.3.5.7.) and exhaled nitric oxide levels (see chapter 7.3.5.9.).

\section{Key points | What's new since EPOS 2012}

- In children, differentiating between CRS, AR and NAR, and adenoid hypertrophy/adenoiditis can be challenging.

- In children, olfactory dysfunction is hardly reported, even in the presence of CRS.

- As in adults, facial pain or headache as main complaints point towards a primary headache syndrome.

- Most diagnostic tools that are available for adults, can be used in children as well. Additionally, tests fo congenital diseases (genetic testing, nitric oxide and ciliary morphology) should be considered in selected cases.

\subsection{Management of paediatric CRS and co- morbidities}

Medical therapy is the mainstay of management of paediatric chronic rhinosinusitis. The most commonly used therapies include antibiotics, intranasal steroids, and saline nasal irrigation (Table 7.4.1.). There is less scientific support for other ancillary therapies.

\subsubsection{Antibiotics}

There is no good evidence in the literature to support the use of antibiotics for CRS in children. Two clinical trials conducted by the same group do not show significant differences between treatment with placebo and systemic antibiotics in children with clinical criteria commensurate with $\mathrm{CRS}^{(121,122)}$. The studies have significant limitations as the initial study did not randomize or blind the patients to the treatments and the placebo group actually received saline drops which might have been helpful in and of themselves ${ }^{(121)}$. Furthermore, one of the arms included drainage and irrigation of the maxillary sinus before initiating antibiotic therapy. The second, later study, was a randomized, double-blind comparison of cefaclor to placebo in 79 healthy children between the ages of two and 12 years with chronic rhinosinusitis ${ }^{(122)}$. All patients had a puncture and washout and were then randomized to cefaclor or placebo PO for one week and were followed at six weeks. There was no significant difference in resolution rate between the children on cefaclor (64.8\%) and those on placebo (52.5\%). The initial maxillary puncture and washout could have helped the whole group even before enrolment and influenced the outcome, making the antibiotic irrelevant, and plain radiographs were used to evaluate the sinuses. Shin and colleagues performed a case control study of patients with CRS to evaluate predictors of response to therapy ${ }^{(84)}$. They treated them with antibiotics for 12 weeks and identified responders $(n=22)$ and non-responders $(n=36)$ at the end of therapy by evaluating persistent symptoms and abnormal Waters views. The prevalence of allergic diseases and levels of serum IgE and eosinophil cationic protein as well as the number of serum eosinophils were significantly higher in the non-responder group.

lino and colleagues investigated the effect of a low dose clarithromycin regimen administered for 8-15 weeks in a group of children (1-14 years) with chronic rhinosinusitis or otitis media with effusion ${ }^{(123)}$. They also followed another group of children with the same disease with no therapy as control. Sixty-three percent of the children with CRS were free of disease at the end of therapy and there was no correlation between success of therapy and carriage in the nasopharynx of erythromycin-resistant S. pneumonia. The authors suggest that the efficacy is related to the potential anti-inflammatory effects of clarithromycin. The study is limited by lack of placebo controls and poor reporting of the improvement in disease in the control group, thus definitive conclusions about the efficacy of longterm macrolides in children is not justified.

Despite the lack of supportive evidence, it is common but unsubstantiated practice to include a course of antibiotics as part of maximal medical therapy in children with CRS. It is likely that, in many of these instances, treatment targets acute exacerbations on top of pre-existing chronic disease. Careful review of existing data suggests that the role for short term antibiotics in CRS is close to nil (see also data in adults). Furthermore, double blind, placebo-controlled trials with long term antibiotics are also negative in adults with a possibility that certain endotypes might benefit. As seen from the above, there are no supportive data for either short or long-term antibiotic regimens in the treatment of children with CRS. Intravenous antibiotic therapy for resistant CRS has been advocated as an alternative to surgical intervention. In a retrospective analysis of 70 children aged 10 months to 15 years with CRS, Don et al. found that $89 \%$ had complete resolution of symptoms after maxillary sinus irrigation and selective adenoidectomy followed by one to four weeks of culturedirected intravenous antibiotics ${ }^{(124)}$.Only eight patients required subsequent functional endoscopic sinus surgery (FESS). Cefuroxime IV was most frequently used, followed by ampicillinsulbactam, ticarcillin, clavulanate, and vancomycin. As expected, there were complications of intravenous therapy related both to line placement and the side effects of the antibiotics administered. More importantly, all the patients had irrigation of 
the maxillary sinuses, and some underwent an adenoidectomy, which, as will be detailed later, are in themselves therapeutic. It is therefore not clear from these studies whether the intravenous antibiotics, per se, were solely responsible for the success rate. Because of these issues, intravenous antibiotics are not advocated for the treatment of CRS in children and are essentially reserved to treat the complications of ARS.

\section{There is currently no evidence to support} treatment of children with CRS with either oral or intravenous antibiotics. There is also no evidence to support the utilization of prolonged macrolide therapy in children with CRS.

Proper randomized, controlled trials to evaluate the use of oral antibiotics in children with CRS are needed.

\subsubsection{Intranasal steroids}

Intranasal steroids have become an important part of the treatment algorithm in light of increasing recognition of inflammation in the aetiology of CRS. To date, there is no evidence from randomized controlled trials to support the efficacy of intranasal steroids in paediatric CRS. A multinational, randomized, double blind study evaluated the safety of mometasone furoate 100 or $200 \mathrm{mcg}$ administered either once or twice daily in children 6-17 years of age with nasal polyposis ${ }^{(125)}$. The change in 24-hour urinary free cortisol was the primary endpoint and did not differ between the mometasone and placebo treated subjects supporting the safety of treatment. Unfortunately, the study was not powered to evaluate efficacy but available data showed that mometasone administered twice daily was associated with the greatest reduction from baseline compared to placebo in polyp size, congestion and nasal drainage (anterior and posterior). Although evidence is not available to support the use of intranasal steroids in children with CRS, their efficacy in CRS with/without polyps in adults (as detailed elsewhere), as well as their favourable safety and efficacy profile in children with allergic rhinitis support the recommendation that they be part of first line therapy in children with $\mathrm{CRS}^{(47,126-128)}$.

\subsubsection{Systemic steroids}

Systemic steroids have also been used in children with CRS because of their potent anti-inflammatory properties. Ozturk and colleagues treated children with CRS with amoxicillin clavulanate for 30 days and with either a prednisone taper course for 15 days or placebo ${ }^{(129)}$. The steroid taper was given at the beginning of therapy. Compared to placebo, treatment with steroids resulted in significant improvements in CT scan score as well as symptoms of cough, nasal obstruction, postnasal discharge and total symptom score. Even though systemic steroids are effective, use for CRS in children is limited because of safety concerns.

Intranasal steroids are recommended for use in children with CRS despite the absence of good level evidence. This is based on safety in children and favorable efficacy data in adults with CRSsNP.

\section{There is one study that supports systemic steroid} use in children with CRS.

\section{A randomized, placebo controlled trial evaluating the efficacy of intranasal steroids in children with CRS is needed.}

\subsubsection{Intranasal saline}

A Cochrane review analysed randomized controlled trials in which saline was evaluated in comparison with either no treatment, a placebo, as an adjunct to other treatments, or against other treatments ${ }^{(130)}$. A total of eight trials satisfied inclusion criteria of which three were conducted in children. The studies included a broad range of delivery techniques, tonicity of saline used, and comparator treatments. Overall there was evidence that saline is beneficial in the treatment of the symptoms of CRS when used as the sole modality of treatment. Evidence also exists in favour of saline as a treatment adjunct. In one of these studies, 34 children with chronic maxillary rhinosinusitis from 3-16 years were randomized to two groups receiving 10 drops $(1 \mathrm{ml})$ of either $3.5 \%$ or isotonic saline three times a day for four weeks ${ }^{(131)}$. Four patients withdrew (three hypertonic, one isotonic) due to nasal burning sensations. Primary outcomes were symptom and radiological scores. Both hypertonic and isotonic saline led to significant improvements from baseline in postnasal drip score but only hypertonic saline therapy significantly reduced cough score and maxillary sinus opacification as assessed by Waters view. Group comparison favoured the hypertonic saline group for cough score and radiologic scores.

Wei and colleagues enrolled 40 children with CRS in a randomized, prospective, double-blind study comparing once daily irrigation with saline or saline/gentamicin for six weeks ${ }^{(132)}$ There were statistically significant improvements in quality of life scores at three and six weeks and a reduction of CT scores after six weeks in both groups with no significant difference between the groups. This suggests that nasal saline irrigation is an effective therapy in children with CRS and that addition of gentamicin to saline provided no additional benefit. A smaller prospective study enrolled 10 children with CRS refractory to antibiotics and intranasal steroids and treated them with high volume nasal irrigation once daily for 30 days $^{(133)}$. Compared to baseline the children had a significant improvement in $\mathrm{SN}-5$ and nasal quality of life scores after saline irrigation. Cho and colleagues performed a randomized, prospective study to investigate the efficacy of four weeks of nasal irrigation with low-concentration hypochlorous irrigation ( $\mathrm{HOCL}$, demonstrated to have bactericidal and fungicidal activity) or normal saline in children with $\mathrm{CRS}^{(134)}$. Total symptom scores significantly improved with both $\mathrm{HOCl}$ and normal saline nasal irrigation, with no significant difference between the two groups. Plain radiographs, obtained at baseline and after treatment, showed improvement in both groups, which was significantly greater in the group on $\mathrm{HOCl}$. Hong and colleagues retrospectively reviewed the records of children with CRS who had received nasal saline irrigation for a month and showed that $64 \%$ of the children had good compliance and carried out 
the irrigations during the follow up period ${ }^{(135)}$. Improvement of symptoms and nasal endoscopy findings were seen in the $73 \%$ of the compliant patients and the rate of surgery was significantly lower (16.3\%) for these children compared to the ones who did not show good compliance with therapy (42.9\%). In another retrospective review evaluating compliance with saline irrigation therapy, irrigations were well tolerated by more than $80 \%$ of children and adolescents and when questioned, over $70 \%$ of patients / parents thought there was an improvement in nasal symptoms with irrigation ${ }^{(136)}$. Based on the above data and the safety of this therapy, saline nasal irrigation has become mainstay of treatment of CRS in the paediatric age group. Indeed, in a survey of paediatric otolaryngologists and rhinologists in the US, $93 \%$ and $97 \%$ of the respondents respectively reported using nasal saline irrigations as part of appropriate medical therapy in paediatric $\mathrm{CRS}^{(124,137,138)}$.

\section{Saline nasal irrigation is recommended for the treatment of CRS in children. Addition of antibiotics to saline irrigations is not recommended.}

\subsubsection{Ancillary therapies}

There is no good evidence to support ancillary therapies such as antihistamines (intranasal or oral), leukotriene modifiers, decongestants (intranasal or oral), or mucus thinners in CRS in children. The use of these agents is reserved for treatment of concomitant symptomatic allergic rhinitis. Chen and colleagues performed a prospective study that evaluated the efficacy of bacterial lysate (of 21 strains of eight bacteria) administered 10 days per month for three months on recurrence of rhinosinusitis symptoms in children who had entered a remission phase after treatment for $\mathrm{CRS}^{(139)}$. At one year of follow up, the visual analogue score, nasal discharge and obstruction scores, number of days with rhinitis attacks per month and number of days with antibiotic use per month were significantly decreased in the prevention group versus the control group (nasal saline). Treatment of children with CRS for concomitant gastrooesophageal reflux disease (GORD) has been suggested by some weak evidence. Phipps and colleagues conducted a prospective study of paediatric patients with CRS and found that $63 \%$ of children with CRS had GORD documented by pH probe ${ }^{(43)}$. In addition, 79\% experienced improvement in rhinosinusitis symptoms after medical treatment of GORD. A retrospective study that lacked a placebo control showed that treatment for GORD in patients with CRS allowed many patients to improve and avoid surgery ${ }^{(44)}$. These studies were not blinded and did not include a placebo group. They also do not account for spontaneous improvement in CRS symptoms in many children over time. Therefore, routine anti-reflux treatment of children with CRS is not warranted. This conclusion is supported by the consensus statement on paediatric rhinosinusitis ${ }^{(47)}$.

\section{We do not recommend the addition of any specific ancillary therapy to the treatment regimen of children with CRS. Exceptions are using ancillary therapies when indicated for concomitant disease such as allergic rhinitis or GORD.}

\subsubsection{Surgical treatments}

Surgical intervention is considered for patients with CRS who have failed appropriate medical therapy (and, less commonly, in complicated acute rhinosinusitis). Adenoidectomy with or without antral irrigation and functional endoscopic sinus surgery (FESS) are the most commonly used modalities.

Table 7.4.1. Evidence supporting therapy of CRS in children.

\begin{tabular}{|c|c|c|}
\hline Therapy & $\begin{array}{l}\text { Level of } \\
\text { evidence }\end{array}$ & GRADE recommendation \\
\hline Antibiotics & $1 \mathrm{~b}(-)$ & $\begin{array}{l}\text { There is no high level evidence to support the efficacy of either short or long term antibiotics } \\
\text { for CRS in children. }\end{array}$ \\
\hline Nasal corticosteroids & 5 & $\begin{array}{l}\text { There is no evidence regarding the efficacy of intranasal steroids in the treatment of CRS in } \\
\text { children. Nevertheless the EPOS steering group is supportive of their use in light of their anti- } \\
\text { inflammatory effects and excellent safety record in children. }\end{array}$ \\
\hline Systemic Steroids & $1 \mathrm{~b}(+)$ & $\begin{array}{l}\text { Adding a taper course of systemic steroids to an antibiotic (not effective on its own) is more } \\
\text { effective than placebo in the treatment of paediatric CRS. Judicious use of this regimen is } \\
\text { advised considering systemic side effects. }\end{array}$ \\
\hline Saline Irrigation & $\mathrm{Ib}(+)$ & $\begin{array}{l}\text { There are a few clinical trials demonstrating the efficacy of saline irrigations in paediatric } \\
\text { patients with CRS. The EPOS steering group is supportive of the use of saline in light of the } \\
\text { excellent safety record in children. }\end{array}$ \\
\hline Adenoidectomy & 4 & $\begin{array}{l}\text { Adenoidectomy is effective in younger children with symptoms of CRS. The EPOS steering } \\
\text { group supports adenoidectomy in young children refractory to appropriate medical therapy. }\end{array}$ \\
\hline FESS & 4 & $\begin{array}{l}\text { FESS is safe and effective for the treatment of older children with CRS refractory to medical } \\
\text { therapy or previous adenoidectomy. }\end{array}$ \\
\hline
\end{tabular}

CRS, chronic rhinosinusitis; FESS, functional endoscopic sinus surgery; AMT, appropriate medical therapy. 


\subsubsection{Adenoidectomy}

Adenoidectomy is often the first-line surgical option in children with CRS, with success rates ranging between 47 and $58 \%$ of cases ${ }^{(140)}$.Anatomic obstruction and/or biofilm formation and the notion of the adenoids as a bacterial reservoir are the primary underlying rationale for adenoidectomy. In general, the revision rate for adenoidectomy is very low $(1.9 \%)^{(141)}$. In a metaanalysis of the results of adenoidectomy for symptoms of CRS in children, Brietzke and colleagues included eigth studies with a cumulative success rate of $69 \%{ }^{(33)}$. Ramadan and Tiu reported on the failures of adenoidectomy over a 10-year period and found that children younger than seven years of age and those with asthma were more likely to fail after an adenoidectomy and go on to require $\mathrm{FESS}^{(142)}$.

Clearly, adenoidectomy seems to help irrespective of whether the cause of the symptoms is adenoiditis or CRS, which are difficult to distinguish on clinical grounds. In a study that attempted to address this issue, Bhattacharyya and colleagues evaluated CT scans of two paediatric populations: 66 children with CRS about to undergo surgery and 192 children undergoing a CT scan for non-sinusitis related complaints ${ }^{(105)}$. Lund-Mackay scores of 2 or less had an excellent negative predictive value, whereas scores of 5 or more had an excellent positive predictive value for CRS, with sensitivity and specificity of $86 \%$ and $85 \%$, respectively.A score of 3-4 was considered equivocal and more weight needed to be placed on the clinical picture and endoscopic exam in these cases. It, therefore, stands to reason that a child with the typical symptoms, and a CT-LM score $<5$ probably has adenoiditis. Ramadan and colleagues hypothesized that adenoidectomy would be more successful therapy for children with adenoiditis (low CT score) as opposed to CRS (high CT score) ${ }^{(143)}$. To this end, they reviewed their patients with symptoms of CRS who underwent adenoidectomy and divided them into two groups based on their Lund Mackay CT score: the CRS group (Lund-Mackay score $\geq 5$ ) and the adenoiditis group (Lund-Mackay score $<5$ ). When the success rate of adenoidectomy at one year was evaluated, it was $65 \%$ for the adenoiditis group and $43 \%$ for the CRS proper group (with high CT scores). This supports the notion that adenoidectomy is more effective when sinus disease is not severe and the symptoms are related to adenoiditis.

Bettadahalli and colleagues report significant improvements in symptom severity scores before and six months after adenoidectomy in children with $\mathrm{CRS}^{(143)}$. Using the $\mathrm{SN}-5$ instrument, a total of 53 out of 60 children (88\%) had improved quality of life scores at the end of follow up with a significant improvement in QOL scores in all domains compared to baseline. The consensus statement of the American Academy of Otolaryngology-Head and Neck Surgery supported adenoidectomy as an effective first-line surgical procedure for children up to 12 years of age and was unable to reach consensus on the utility of adenoidectomy in patients age 13 years and older due to the absenceof supporting data for that age group ${ }^{(47)}$.

Maxillary antral irrigation is frequently performed in conjunction with an adenoidectomy. To evaluate the efficacy of this added intervention, Ramadan and colleagues analysed 60 children who underwent an adenoidectomy for CRS, 32 of which also had a sinus wash and culture via the middle meatus ${ }^{(144)}$. All children received postoperative antibiotics for two weeks and outcomes were assessed at least 12 months postoperatively. Patients who underwent an adenoidectomy alone had a $61 \%$ success rate at 12 months compared with children who underwent an adenoidectomy with a sinus wash, who had a higher success rate of $88 \%$. Children with a high Lund-Mackay CT score and asthma had better success with an adenoidectomy with a wash compared with an adenoidectomy alone. These data suggest that antral irrigation should be considered in addition to an adenoidectomy in children with asthma who have more severe disease on preoperative CT scans.

\section{Adenoidectomy with/without antral irrigation is the simplest and safest first procedure to consider in younger children with symptoms of CRS.}

\subsubsection{Balloon sinuplasty}

Balloon sinuplasty was approved by the U.S. Food and Drug Administration for use in children in the United States in 2006, and a preliminary study in children has shown the procedure to be safe and feasible ${ }^{(145)}$. Since EPOS 2012, multiple studies have been performed evaluating the efficacy of balloon sinuplasty in paediatric CRS ${ }^{(146-151)}$. Many of the studies included other procedures (adenoidectomy, sinus irrigation, anterior ethmoidectomy, turbinate reduction, ESS) that were performed simultaneously and might have contributed to success. Furthermore, none of the studies included appropriate control groups with no intervention to account for spontaneous resolution of symptoms in children.

To address whether balloon dilation provides additional benefit to maxillary sinus irrigation, Gerber and colleagues prospectively randomized children (ages 2-12 years.) with CRS to undergo either adenoidectomy with maxillary sinus irrigation (with 18 gauge needle) or adenoidectomy with maxillary sinus balloon dilation and irrigation ${ }^{(152)}$. While both groups showed significant improvement in SN-5 scores at both postoperative time points, there were no significant differences between the groups suggesting lack of additional value of maxillary sinuplasty. Ference and colleagues analysed data from ambulatory surgery databases in four states to study the utilization of balloon catheter dilation $(n=316)$ compared to traditional FESS $(n=2346)$ in patients under 18 years of age. Balloon dilation was used in $11.9 \%$ of paediatric sinus surgery and had higher average charges with no decrease in operating room time compared to procedures that only utilized FESS ${ }^{(153)}$. In a cost-effectiveness study based on decision tree analysis of different surgical scenarios in children with CRS, adenoidectomy as the sole first procedure was found to be more cost effective than adenoidectomy with balloon sinus dilation ${ }^{(154)}$. From the above, it is clear that balloon dilation in children is safe but efficacy and cost effectiveness has no supporting evidence. Therefore, this modality was not endorsed by the consensus panel of the American Academy of ORL ${ }^{(47)}$, and similarly, is not recommended by the EPOS2020 steering group. 
There is no solid evidence to support the use of balloon sinuplasty in the management of CRS in children. This modality is not recommended as part of the surgical armamentarium.

\subsubsection{Functional endoscopic sinus surgery}

There is some indication that FESS is superior to medical management (Table 7.4.2.). In a 10-year follow-up of patients who underwent FESS versus medical management alone, surgery resulted in a greater relief of nasal obstruction and purulent discharge, as well as higher parent satisfaction ${ }^{(155)}$. A prospective study by Ramadan et al. examined the outcome differences between FESS and adenoidectomies, and found that FESS resulted in improved symptom status six months post intervention ${ }^{(156)}$.Furthermore, retrospective questionnaires to the parents of children having undergone FESS for the treatment of CRS indicated a success rate that varied between 70 and $80 \%{ }^{(157)}$.A systematic review of FESS case series results has shown that surgery is effective at reducing symptoms, with an $88 \%$ success rate ${ }^{(158)}$. A more recent systematic review, confirms these positive results showing a positive outcome in between $71-100 \%$ of children after FESS as well as a significant improvement in quality of life after surgery which was evaluated in a subset of the studies ${ }^{(159)}$. In that review, the rate of major complications following paediatric FESS was $0.6 \%$, and the rate of minor complications $2 \%$. Other studies support these conclusions with improvement in symptom scores and quality of life measures after FESS in children with CRS with and without polyposis $^{(160,161)}$. However, there are no RCT and the possibility of spontaneous improvement cannot be excluded. On average, the postoperative follow up periods ranged from 1-8 years.

\section{FESS is a safe and possibly effective surgical modality children with CRS and can be used as after failure of adenoidectomy in older children refractory to medical therapy. Decisions on use depends on severity of disease, age and existing co-morbidities.}

As to predictors of success of FESS in children, the data is scant. El Sharkawy and colleagues followed 87 patients prospectively after FESS: 45 patients had allergy, 36 had no allergy, and six had nasal polyposis ${ }^{(162)}$. After a mean postoperative follow up of 28 months, the success rate (determined by improvement in CT score, nasal examination and postoperative symptoms) was not different between the groups and ranged from $85.7 \%$ to $93 \%$. The presence of asthma and higher severity of disease on CT decreased the subjective postoperative improvement and performing an adenoidectomy with FESS increased it. In another study, Lee and colleagues retrospectively reviewed the charts of children who received FESS for CRS and designated 21 patients with protracted disease and 32 patients as resolved based on the presence or lack thereof of mucopurulent discharge for more than three months after FESS ${ }^{(163)}$. Sinonasal polyposis, history of

Table 7.4.2. Functional endoscopic sinus surgery (FESS) for paediatric CRS.

\begin{tabular}{|c|c|c|c|c|}
\hline & Type & Intervention & Outcome measure & Result \\
\hline Lusk $2006^{(155)}$ & $\begin{array}{l}\text { Retrospective, age- } \\
\text { matched, cohort } \\
\text { outcome study } n=67\end{array}$ & $\begin{array}{l}\text { Endoscopic sinus } \\
\text { surgery or medical } \\
\text { management }\end{array}$ & $\begin{array}{l}\text { Post intervention symptom } \\
\text { scores ( } 10 \text { years after therapy) }\end{array}$ & $\begin{array}{l}\text { ESS resulted in significant improvements in } \\
\text { nasal obstruction and rhinorrhoea. Parental } \\
\text { assessment of improvement and satisfaction } \\
\text { were higher in the ESS group }\end{array}$ \\
\hline Ramadan 1999(156) & $\begin{array}{l}\text { Prospective, } \\
\text { nonrandomized } \\
\text { study, } \mathrm{n}=66 \text { enrolled, } \\
\mathrm{n}=61 \text { had follow up }\end{array}$ & ESS or adenoidectomy & $\begin{array}{l}\text { Symptoms } 6 \text { months postop } \\
\text { and need for revision surgery }\end{array}$ & $\begin{array}{l}\text { Significantly more children had improved } \\
\text { symptoms with ESS (77\%) compared to } \\
\text { adenoidectomy (47\%). }\end{array}$ \\
\hline Hebert $1998^{(158)}$ & $\begin{array}{l}\text { Systematic literature } \\
\text { review of studies on } \\
\text { outcomes of ESS }\end{array}$ & $\begin{array}{l}\text { ESS } \\
8 \text { articles ( } n=832 \\
\text { patients) and one set } \\
\text { of unpublished data } \\
\text { were evaluated }\end{array}$ & Positive outcome after ESS & $\begin{array}{l}\text { Positive outcome for published, unpublished } \\
\text { and combined data was } 88.4 \%, 92 \% \text {, and } \\
88.7 \% \text { respectively. }\end{array}$ \\
\hline $\begin{array}{l}\text { Vlastarakos } \\
2013^{(159)}\end{array}$ & $\begin{array}{l}\text { Systematic literature } \\
\text { review of ESS }\end{array}$ & $\begin{array}{l}15 \text { studies with a } \\
\text { total number of } 1301 \\
\text { treated patients }\end{array}$ & Positive outcome of ESS & $\begin{array}{l}\text { Positive outcome between } 71 \text { and } 100 \% \text { of } \\
\text { operated children }\end{array}$ \\
\hline Jiang $2012^{(160)}$ & $\begin{array}{l}\text { Prospective study } \\
\text { including } 270 \\
\text { patients with ESS } \\
\text { and } 273 \text { healthy } \\
\text { controls }\end{array}$ & ESS & $\begin{array}{l}\text { SNOT- } 20 \text { before and } 3-8 \text { years } \\
\text { postop }\end{array}$ & $\begin{array}{l}\text { Significant improvement in quality of life after } \\
\text { surgery }\end{array}$ \\
\hline Cornet $2013^{(161)}$ & $\begin{array}{l}\text { Combined } \\
\text { prospective and } \\
\text { retrospective study }\end{array}$ & $\begin{array}{l}\text { ESS, } n=44(18 / 44 \text { had } \\
\text { both pre and post op } \\
\text { data) }\end{array}$ & $\begin{array}{l}\text { R-SOM quality of life } \\
\text { questionnaire obtained } \\
\text { an average of } 4 \text { years post } \\
\text { operatively }\end{array}$ & $\begin{array}{l}\text { Significant improvement in R-SOM } \\
\text { postoperatively }\end{array}$ \\
\hline
\end{tabular}

ESS, endoscopic sinus surgery; SNOT-20, sino-nasal outcome test 20; R-SOM, rhinosinusitis outcome measure. 
allergic rhinitis, and male gender were more frequently observed in the protracted group than the resolved group.

Many advocate a limited approach to FESS in children, consisting of the removal of any obvious obstruction (such as polyps and concha bullosa), as well as an anterior bulla ethmoidectomy, and a maxillary antrostomy. This approach typically yields significant improvements in nasal obstruction (91\%), rhinorrhoea (90\%), PND (90\%), headache (97\%), hyposmia (89\%), and chronic cough $(96 \%)^{(164)}$. Whereas second look procedures were common after FESS to clean the cavities, the advent of absorbable packing has made it possible to avoid a second look procedure. Walner et al. found comparable rates of revision sinus surgery in children with and without a second look procedure, suggesting that it may not be necessary ${ }^{(165)}$. Ramadan and colleagues observed that the use of corticosteroids during an initial FESS might obviate a second look procedure ${ }^{(166)}$.Younis, in a review of available data, suggested that a second look is not necessary in most children after FESS ${ }^{(167)}$. Initial concerns about possible adverse effects of FESS on facial growth have been allayed by a long-term followup study by Bothwell and colleagues that showed no impact of FESS on qualitative and quantitative parameters of paediatric facial growth, as evaluated up to 10 years postoperatively ${ }^{(168)}$. Current literature supports the use of FESS in children with CRS who have failed appropriate medical treatment and possibly an earlier adenoidectomy. Other indications include orbital and intracranial complications of ARS, obstructing nasal polyposis and severe disease in cases of $\mathrm{CF}$, and patients with allergic fungal rhinosinusitis.

As seen from this section, much remains to be done relating to investigating best surgical practices for CRS in children. It is clear that initial adenoidectomy with/without irrigation is effective followed by FESS (Table 7.4.2.). Ideally, prospective, randomized, multicentre trials should be conducted where the severity of disease on CT scans and the symptom questionnaire would be matched preoperatively and the following interventions would be compared: adenoidectomy alone, adenoidectomy with a wash, and endoscopic sinus surgery.

\section{Key points | What's new since EPOS 2012}

- $\quad$ There is no (new) evidence to support the use of either short or long-term antibiotics in the treatment of CRS in children.

- Saline irrigation and intranasal corticosteroids remain acceptable modalities for the medical therapy of children with CRS

- $\quad$ Adenoidectomy is useful in the surgical therapy of CRS in young children, especially if there is limited pathology on paranasal sinus CT scans.

\section{References}

1. Adams PF, Hendershot GE, Marano MA. Current estimates from the National Health Interview Survey, 1996. Vital Health Stat. 10 1999:1-203.

2. Ray NF. Healthcare expenditures for sinusitis in 1996: contributions of asthma, rhinitis, and other airway disorders. J Allergy Clin Immunol.1999;103:408-14.

3. Smith DF, Ishman SL, Tunkel DE, Boss EF. Chronic rhinosinusitis in children: race and socioeconomic status. Otolaryngology--head and neck surgery : official journal of American Academy of Otolaryngology-Head and Neck Surg. 2013;149:63944.

4. Sidell D, Shapiro NL, Bhattacharyya N. Obesity and the risk of chronic rhinosinusitis, allergic rhinitis, and acute otitis media in school-age children. Laryngoscope. 2013;123:2360-3.

5. Sami AS, Scadding GK. Rhinosinusitis in secondary school children-part 2: main project analysis of MSNOT-20 Young Persons Questionnaire (MSYPQ). Rhinology. 2014;52:225-30.

6. Gilani S, Shin JJ. The Burden and Visit Prevalence of Pediatric Chronic Rhinosinusitis. Otolaryngology-head and neck surgery : official journal of American Academy of Otolaryngology-Head and Neck Surg. 2017;157:1048-52

7. Westman M, Stjarne P, Bergstrom A, et al. Chronic rhinosinusitis is rare but bothersome in adolescents from a Swedish population-based cohort. J Allergy Clin Immunol 2015;136:512-4.e6.

8. Cunningham JM, Chiu EJ, Landgraf JM, Gliklich RE. The health impact of chronic recurrent rhinosinusitis in children. Arch Otolaryngol. Head Neck Surg. 2000;126:1363-8.
9. Haruna S, Sawada K, Nakajima T, Moriyama H. Relationship between pediatric sinusitis and middle turbinate pneumatization - Ethmoidal sinus pyocele thought to be caused by middle turbinate pneumatization. International J of Pediatric Otorhinolaryngology 2005;69:375-9.

10. Kim HJ, Cho MJ, Lee JW, et al. The relationship between anatomic variations of paranasal sinuses and chronic sinusitis in children. Acta Otolaryngol. 2006;126:1067-72.

11. Al-Qudah M. The relationship between anatomical variations of the sino-nasal region and chronic sinusitis extension in children. Int J Pediatr Otorhinolaryngol 2008;72:817-21

12. Cohen O, Adi M, Shapira-Galitz Y, Halperin D, Warman M. Anatomic variations of the paranasal sinuses in the general pediatric population. Rhinology. 2019;57:206-12.

13. Heath J, Hartzell L, Putt C, Kennedy J. Chronic Rhinosinusitis in Children: Pathophysiology, Evaluation, and Medical Management. Current Allergy and Asthma Reports 2018;18.

14. Wood AJ, Antoszewska H, Fraser J, Douglas RG. Is chronic rhinosinusitis caused by persistent respiratory virus infection? Int Forum Allergy Rhinol 2011;1:95-100

15. Reh DD, Higgins TS, Smith TL. Impact of tobacco smoke on chronic rhinosinusitis: A review of the literature. International Forum of Allergy and Rhinology, 2012, 362-9.

16. Christensen DN, Franks ZG, McCrary HC, Saleh AA, Chang EH. A Systematic Review of the Association between Cigarette Smoke Exposure and Chronic Rhinosinusitis. Otolaryngology--head and neck surgery : official journal of American Academy of Otolaryngology-Head and Neck Surg. 2018;158:80116.

17. Kakish KS, Mahafza T, Batieha A, Ekteish F, Daoud A Clinical sinusitis in children attending primary care centers. Pediatric Infectious Disease J 2000;19:10714.

18. Ramadan HH. Surgical management of chronic sinusitis in children. Laryngoscope. 2004;114:2103-9.

19. Siedek V, Stelter K, Betz CS, Berghaus A, Leunig A. Functional endoscopic sinus surgery--a retrospective analysis of 115 children and adolescents with chronic rhinosinusitis. Int J Pediatr Otorhinolaryngol 2009;73:741-5.

20. Younis RT, Lazar RH. Criteria for Success in Pediatric Functional Endonasal Sinus Surg. Laryngoscope. 1996;106:869-73.

21. Silviu-Dan F. Pediatric chronic rhinosinusitis. Pediatric annals 2014;43:e201-9.

22. Leo G, Piacentini E, Incorvaia C, Consonni D, Frati F. Chronic rhinosinusitis and allergy. Pediatr Allergy Immunol 2007;18 Suppl 1:19-21.

23. Sedaghat AR, Phipatanakul W, Cunningham MJ. Prevalence of and associations with allergic rhinitis in children with chronic rhinosinusitis. International journal of pediatric otorhinolaryngology 2014;78:343-7.

24. Tantimongkolsuk C, Pornrattanarungsee S, Chiewvit P, Visitsunthorn N, Ungkanont K, Vichyanond P. Pediatric sinusitis: Symptom profiles with associated atopic conditions. J of the Medical Association of Thailand 2005;88:S149-S55.

25. Georgalas C, Vlastos I, Picavet V, van Drunen C, Garas G, Prokopakis E. Is chronic rhinosinusitis related to 
allergic rhinitis in adults and children? Applying epidemiological guidelines for causation. Allergy 2014;69:828-33.

26. Anamika A, Chakravarti A, Kumar R. Atopy and Quality of Life in Pediatric Chronic Rhinosinusitis. American J of Rhinology. \& Allergy 2019;33:194589241985426.

27. Iwens P, Clement PA. Sinusitis in allergic patients. Rhinology. 1994;32:65-7.

28. Mahdavinia M, 3rd LCG. Chronic rhinosinusitis and age: is the pathogenesis different? Expert review of anti-infective therapy 2013;11:1029-40.

29. Anfuso A, Ramadan H, Terrell A, et al. Sinus and adenoid inflammation in children with chronic rhinosinusitis and asthma. Ann Allergy Asthma Immunol 2015;114:103-10.

30. Rachelefsky GS, Katz RM, Siegel SC. Chronic sinus disease with associated reactive airway disease in children. Pediatrics 1984;73:526-9.

31. Neff $L$, Adil EA. What is the role of the adenoid in pediatric chronic rhinosinusitis? Laryngoscope. 2015;125:1282-3.

32. Belcher R, Virgin F. The Role of the Adenoids in Pediatric Chronic Rhinosinusitis. Medical sciences (Basel, Switzerland) 2019;7:35

33. Brietzke SE, Brigger MT. Adenoidectomy outcomes in pediatric rhinosinusitis: a meta-analysis. International J of Pediatric Otorhinolaryngology 2008;72:1541-5.

34. Arnaoutakis D, Collins WO. Correlation of mucociliary clearance and symptomatology before and after adenoidectomy in children. International J of Pediatric Otorhinolaryngology 2011;75:1318-21.

35. Shin KS, Cho SH, Kim KR, et al. The role of adenoids in pediatric rhinosinusitis. Int J Pediatr Otorhinolaryngol 2008;72:1643-50

36. Bercin AS, Ural A, Kutluhan A, Yurttas V. Relationship between sinusitis and adenoid size in pediatric age group. Ann Otol. Rhinol Laryngol. 2007;1 16:550-3.

37. Lee D, Rosenfeld RM. Adenoid bacteriology and sinonasal symptoms in children. Otolaryngol. Head Neck Surg. 1997;116:301-7.

38. Coticchia J, Zuliani G, Coleman C, et al. Biofilm surface area in the pediatric nasopharynx: Chronic rhinosinusitis vs obstructive sleep apnea. Arch Otolaryngol. Head Neck Surg. 2007;133:110-4.

39. Eun YG, Park DC, Kim SG, Kim MG, Yeo SG. Immunoglobulins and transcription factors in adenoids of children with otitis media with effusion and chronic rhinosinusitis. Int J Pediatr Otorhinolaryngol 2009;73:1412-6.

40. Shin SY, Choi GS, Park HS, Lee KH, Kim SW, Cho JS. Immunological investigation in the adenoid tissues from children with chronic rhinosinusitis. Otolaryngol. Head Neck Surg. 2009;141:91-6.

41. Contencin P, Narcy P. Nasopharyngeal pH monitoring in infants and children with chronic rhinopharyngitis. International J of Pediatric Otorhinolaryngology 1991;22:249-56.

42. El-Serag HB, Gilger M, Kuebeler M, Rabeneck L. Extraesophageal associations of gastroesophageal reflux disease in children without neurologic defects. Gastroenterology 2001;121:1294-9.

43. Phipps CD, Wood WE, Gibson WS, Cochran WJ. Gastroesophageal reflux contributing to chronic sinus disease in children: a prospective analysis. Arch
Otolaryngol. Head Neck Surg. 2000;126:831-6.

44. Bothwell MR, Parsons DS, Talbot A, Barbero GJ, Wilder B. Outcome of reflux therapy on pediatric chronic sinusitis. Otolaryngol. Head Neck Surg. 1999;121:255-62

45. Nation J, Kaufman M, Allen M, Sheyn A, Coticchia J. Incidence of gastroesophageal reflux disease and positive maxillary antral cultures in children with symptoms of chronic rhinosinusitis. International J of Pediatric Otorhinolaryngology 2014;78:218-22.

46. Cedeño EEG, Ortiz-Princz D, Figueredo SAC, Porro MEC. Adenoid hypertrophy and chronic rhinosinusitis: Helicobacter pylori on antral lavages, adenoid tissue and salival inmunoglobuline A on paediatric patients. International J of Pediatric Otorhinolaryngology 2016;80:82-7.

47. Brietzke SE, Shin JJ, Choi S, et al. Clinical consensus statement: pediatric chronic rhinosinusitis. Otolaryngology - Head \& Neck Surg. 2014;151:54253.

48. Shapiro GG, Virant FS, Furukawa CT, Pierson WE, Bierman CW. Immunologic defects in patients with refractory sinusitis. Pediatrics 1991;87:311-6.

49. Hidalgo H, Moore C, Leiva LE, Sorensen RU. Preimmunization and postimmunization pneumococcal antibody titers in children with recurrent infections. Ann Allergy Asthma Immunol 1996;76:341-6.

50. Costa Carvalho BT, Nagao AT, Arslanian C, et al. Immunological evaluation of allergic respiratory children with recurrent sinusitis. Pediatr Allergy Immunol 2005;16:534-8.

51. Vanlerberghe L, Joniau S, Jorissen M. The prevalence of humoral immunodeficiency in refractory rhinosinusitis: a retrospective analysis. B-ent 2006;2:161-6.

52. Baek JH, Seo HK, Jee HM, et al. Antibody response to pneumococcal vaccination in children with chronic or recurrent rhinosinusitis. Korean J Pediatr 2013;56:286-90.

53. Bernatowska E, Mikoluc B, Krzeski A, Piatosa B, Gromek I. Chronic rhinosinusitis in primary antibody immunodeficient patients. International J of Pediatric Otorhinolaryngology 2006;70:1587-92

54. Ramesh S, Brodsky L, Afshani E, et al. Open trial of intravenous immune serum globulin for chronic sinusitis in children. Ann Allergy Asthma Immunol 1997;79:119-24

55. Oomen KPQ, April MM. Sinonasal manifestations in cystic fibrosis. International journal of otolaryngology 2012;2012:789572-.

56. Steinke JW, Payne SC, Chen PG, Negri J, Stelow EB, Borish L. Etiology of nasal polyps in cystic fibrosis: not a unimodal disease. Ann Otol. Rhinol Laryngol. 2012;121:579-86

57. Virgin FW. Clinical chronic rhinosinusitis outcomes in pediatric patients with cystic fibrosis. Laryngoscope. Investigative Otolaryngology 2017;2:276-80.

58. Farrell PM, Rosenstein BJ, White TB, et al. Guidelines for diagnosis of cystic fibrosis in newborns through older adults: Cystic Fibrosis Foundation consensus report. The J of pediatrics 2008;153:S4-S14.

59. Pedersen MG, Højte C, Olesen HV, Pressler T, Skov M. Late diagnosis and poor nutrition in cystic fibrosis diagnosed before implementation of newborn screening. Acta Paediatrica n/a.
60. Wentzel JL, Virella-Lowell I, Schlosser RJ, Soler ZM. Quantitative sinonasal symptom assessment in an unselected pediatric population with cystic fibrosis. American J of Rhinology. \& Allergy 2015;29:357-61.

61. Chan DK, McNamara S, Park JS, Vajda J, Gibson RL, Parikh SR. Sinonasal Quality of Life in Children With Cystic Fibrosis. JAMA Otolaryngology-Head \& Neck Surg. 2016;142:743-9.

62. Sleigh MA. Primary ciliary dyskinesia. Lancet 1981;2:476.

63. Mener DJ, Lin SY, Ishman SL, Boss EF. Treatment and outcomes of chronic rhinosinusitis in children with primary ciliary dyskinesia: where is the evidence? A qualitative systematic review. International Forum of Allergy \& Rhinology. 2013;3:986-91.

64. Fretzayas A, Moustaki M. Clinical spectrum of primary ciliary dyskinesia in childhood. World J Clin Pediatr 2016;5:57-62.

65. Rollin M, Seymour K, Hariri M, Harcourt J. Rhinosinusitis, symptomatology \& absence of polyposis in children with primary ciliary dyskinesia. Rhinology. 2009;47:75-8.

66. Hosie PH, Fitzgerald DA, Jaffe A, Birman CS, Rutland J, Morgan LC. Presentation of primary ciliary dyskinesia in children: 30 years' experience. J of Paediatrics \& Child Health 2015;51:722-6.

67. Lobo J, Zariwala M, Noone P. Primary Ciliary Dyskinesia. Seminars in Respiratory and Critical Care Medicine 2015;36:169-79.

68. Goutaki M, Maurer E, Halbeisen FS, et al. The international primary ciliary dyskinesia cohort (iPCD cohort): Methods and first results. European Respiratory J 2017:49.

69. Orb Q, Curtin K, Oakley GM, et al. Familial risk of pediatric chronic rhinosinusitis. Laryngoscope. 2016;126:739-45.

70. Purkey MT, Li J, Mentch F, et al. Genetic variation in genes encoding airway epithelial potassium channels is associated with chronic rhinosinusitis in a pediatric population. PloS one 2014;9:e89329-e.

71. Raman V, Clary R, Siegrist KL, Zehnbauer B, Chatila TA. Increased prevalence of mutations in the cystic fibrosis transmembrane conductance regulator in children with chronic rhinosinusitis. Pediatrics 2002;109:E13.

72. Sedaghat AR, Cunningham MJ, Busaba NY. Connexin 32 and 43 mutations: do they play a role in chronic rhinosinusitis? Am J Otolaryngol. 2014;35:33-6.

73. BuSaba NY, Cunningham MJ. Connexin 26 and 30 genes mutations in patients with chronic rhinosinusitis. Laryngoscope. 2008;118:310-3.

74. Lockey RF, Rucknagel DL, Vanselow NA. Familial occurrence of asthma, nasal polyps and aspirin intolerance. Ann Intern Med 1973;78:57-63.

75. Settipane G. Benefit/risk ratio of aspirin. NES Allergy Proceedings 1981:96-102.

76. Baroody F. Eosinophilia in chronic childhood sinusitis. Arch Otolaryngol. Head Neck Surg. 1991:179-81.

77. Driscoll PV, Naclerio RM, Baroody FM. CD4+ lymphocytes are increased in the sinus mucosa of children with chronic sinusitis. Arch Otolaryngol. Head Neck Surg. 1996;122:1071-6.

78. Chan KH, Abzug MJ, Coffinet L, Simoes EAF, Cool C, Liu AH. Chronic rhinosinusitis in young children differs from adults: A histopathology study. J of 
Pediatrics 2004;144:206-12.

79. Coffinet L, Chan KH, Abzug MJ, Simoes EA, Cool C, Liu AH. Immunopathology of chronic rhinosinusitis in young children. J Pediatr 2009;154:754-8.

80. Berger G, Kogan T, Paker M, Berger-Achituv S, Ebner Y. Pediatric chronic rhinosinusitis histopathology: differences and similarities with the adult form. Otolaryngol. Head Neck Surg. 2011;144:85-90.

81. Wu X, Ghimbovschi S, Aujla PK, Rose MC, Pena MT Expression profiling of inflammatory mediators in pediatric sinus mucosa. Arch Otolaryngol. Head Neck Surg. 2009;135:65-72.

82. Wu X, Mimms R, Lima R, Peters-Hall J, Rose MC, Pena MT. Localization of inflammatory mediators in pediatric sinus mucosa. Arch Otolaryngol. Head Neck Surg. 2012;138:389-97.

83. Passariello A, Di Costanzo M, Terrin G, et al. Crenotherapy Modulates the Expression of Proinflammatory Cytokines and Immunoregulatory Peptides in Nasal Secretions of Children with Chronic Rhinosinusitis. American J of Rhinology. \& Allergy 2012;26:e15-e9.

84. Shin YH, Kim HS, Lee EK, et al. Eosinophil-related markers and total immunoglobulin $\mathrm{E}$ as a predictive marker for antibiotic response in chronic rhinosinusitis. Ann Saudi Med 2015;35:312-7.

85. Rose MC, Voynow JA. Respiratory tract mucin genes and mucin glycoproteins in health and disease. Physiol Rev 2006;86:245-78.

86. Penia MT, Aujla PK, Zudaire E, et al. Localization and expression of MUC5B and MUC7 mucins in pediatric sinus mucosa. Ann Otol. Rhinol Laryngol. 2007;116:389-97.

87. Saieg A, Brown KJ, Pena MT, Rose MC, Preciado D. Proteomic analysis of pediatric sinonasal secretions shows increased MUC5B mucin in CRS. Pediatr Res 2015;77:356-62

88. Ramadan HH, Fornelli R, Ortiz AO, Rodman S. Correlation of allergy and severity of sinus disease. American J of Rhinology. 1999;13:345-7.

89. Brozek JL, Bousquet J, Agache I, et al. Allergic Rhinitis and its Impact on Asthma (ARIA) guidelines-2016 revision. J Allergy Clin Immunol 2017;140:950-8.

90. Hellings PW, Klimek L, Cingi C, et al. Non-allergic rhinitis: Position paper of the European Academy of Allergy and Clinical Immunology. Allergy 2017;72:1657-65.

91. Westman M, Stjarne P, Asarnoj A, et al. Natural course and comorbidities of allergic and nonallergic rhinitis in children. J Allergy Clin Immunol 2012;129:403-8.

92. Bachert C, van Cauwenberge P, Olbrecht J, van Schoor J. Prevalence, classification and perception of allergic and nonallergic rhinitis in Belgium. Allergy 2006;61:693-8

93. Togias A, Gergen PJ, Hu JW, et al. Rhinitis in children and adolescents with asthma: Ubiquitous, difficult to control, and associated with asthma outcomes. J Allergy Clin Immunol 2019;143:1003-11.e10.

94. Chiang WC, Chen YM, Tan HK, et al. Allergic rhinitis and non-allergic rhinitis in children in the tropics: prevalence and risk associations. Pediatr Pulmonol 2012:47:1026-33.

95. Huang $Y$, Zhang $Y$, Zhang L. Prevalence of allergic and nonallergic rhinitis in a rural area of northern China based on sensitization to specific aeroallergens. Allergy Asthma Clin Immunol
2018;14:77.

96. Fokkens WJ, Lund VJ, Mullol J, et al. EPOS 2012: European position paper on rhinosinusitis and nasal polyps 2012. A summary for otorhinolaryngologists. Rhinology. 2012;50:1-12.

97. Oleszkiewicz A, Hummel T. Whose nose does not know? Demographical characterization of people unaware of anosmia. European archives of otorhino-laryngology : official journal of the European Federation of Oto-Rhino-Laryngological Societies (EUFOS) : affiliated with the German Society for Oto-Rhino-Laryngology - Head and Neck Surg. 2019;276:1849-52

98. Hauser LJ, Jensen EL, Mirsky DM, Chan KH. Pediatric anosmia: A case series. Int J Pediatr Otorhinolaryngol 2018;110:135-9.

99. Tafalla M. A world without the olfactory dimension. Anat Rec (Hoboken) 2013;296:1287-96.

100. Schriever VA, Gellrich J, von der Hagen M, Hummel T. Acquired Olfactory Dysfunction in Children and Adolescents: A Systematic Review of the Literature. Chem Senses 2018;43:571-81.

101. Senbil N, Gurer YK, Uner C, Barut Y. Sinusitis in children and adolescents with chronic or recurrent headache: a case-control study. J Headache Pain 2008;9:33-6

102. Smith BC, George LC, Svider PF, et al. Rhinogenic headache in pediatric and adolescent patients: an evidence-based review. Int Forum Allergy Rhinol 2019;9:443-51.

103. Rimmer J, Hellings P, Lund VJ, et al. European position paper on diagnostic tools in rhinology. Rhinology. 2019;57:1-41.

104. Pearce MS, Salotti JA, Little MP, et al. Radiation exposure from CT scans in childhood and subsequent risk of leukaemia and brain tumours: a retrospective cohort study. Lancet (London, England) 2012;380:499-505.

105. Bhattacharyya N, Jones DT, Hill M, Shapiro NL. The diagnostic accuracy of computed tomography in pediatric chronic rhinosinusitis. Archives of Otolaryngology -- Head \& Neck Surg. 2004;130:102932.

106. Hill M, Bhattacharyya N, Hall TR, Lufkin R, Shapiro NL. Incidental paranasal sinus imaging abnormalities and the normal Lund score in children. Otolaryngol. Head Neck Surg. 2004;130:171-5.

107. Hopkins C, Gillett S, Slack R, Lund VJ, Browne JP. Psychometric validity of the 22-item Sinonasal Outcome Test. Clinical Otolaryngology 2009;34:44754.

108. Thamboo A, Santos RC, Naidoo L, Rahmanian R, Chilvers MA, Chadha NK. Use of the SNOT-22 and UPSIT to appropriately select pediatric patients with cystic fibrosis who should be referred to an otolaryngologist: cross-sectional study. JAMA Otolaryngol. Head Neck Surg. 2014;140:934-9.

109. Kay DJ, Rosenfeld RM. Quality of life for children with persistent sinonasal symptoms. Otolaryngol. Head Neck Surg. 2003;128:17-26.

110. Ni JS, Kompelli AR, Nguyen SA, Schlosser RJ, Clemmens C, Soler ZM. The Sinus and Nasal Quality of Life Survey (SN-5) in the Management of Pediatric Chronic Rhinosinusitis: A systematic review and meta-analysis. International J of Pediatric Otorhinolaryngology 2018;111:162-9.
111. Ameli F, Brocchetti F, Tosca MA, Signori A, Ciprandi G. Nasal endoscopy in children with suspected allergic rhinitis. Laryngoscope. 2011;121:2055-9.

112. Eigenmann PA, Atanaskovic-Markovic $M, J \mathrm{OBH}$, et al. Testing children for allergies: why, how, who and when: an updated statement of the European Academy of Allergy and Clinical Immunology (EAACI) Section on Pediatrics and the EAACIClemens von Pirquet Foundation. Pediatr Allergy Immunol 2013;24:195-209.

113. van Spronsen E, Ebbens FA, Fokkens WJ. Olfactory Function in Healthy Children: Normative Data for Odor Identification. American J of Rhinology. \& Allergy 2013;27:197-201.

114. Schriever VA, Mori E, Petters W, Boerner C, Smitka M, Hummel T. The "Sniffin'Kids"Test - A 14-Item Odor Identification Test for Children. PLoS ONE 2014;9.

115. Schriever VA, Agosin E, Altundag A, et al. Development of an International Odor Identification Test for Children: The Universal Sniff Test. The J of Pediatrics 2018;198:265-72.e3.

116. Marlier L, Schaal B, Soussignan R. Neonatal responsiveness to the odor of amniotic and lacteal fluids: a test of perinatal chemosensory continuity. Child Dev 1998;69:611-23.

117. Hummel T, Bensafi M, Nikolaus J, Knecht M, Laing DG, Schaal B. Olfactory function in children assessed with psychophysical and electrophysiological techniques. Behav Brain Res 2007;180:133-8.

118. Loos HM, Reger D, Schaal B. The odour of human milk: Its chemical variability and detection by newborns. Physiol Behav 2019;199:88-99.

119. van Spronsen E, Ebbens FA, Fokkens WJ. Normal peak nasal inspiratory flow rate values in healthy children aged 6 to 11 years in the Netherlands. Rhinology. 2012;50 (1):22-5.

120. Kizil Y, Aydil U, Ceylan A, Uslu S, Basturk V, lleri F. Analysis of choanal polyps. J Craniofac Surg. 2014;25:1082-4

121. Otten FW, Grote JJ. Treatment of chronic maxillary sinusitis in children. Int J Pediatr Otorhinolaryngol 1988;15:269-78.

122. Otten HW, Antvelink JB, Ruyter de Wildt H, Rietema SJ, Siemelink RJ, Hordijk GJ. Is antibiotic treatment of chronic sinusitis effective in children? Clinical otolaryngology and allied sciences 1994;19:215-7.

123. lino Y, Sasaki Y, Miyazawa T, Kodera K. Nasopharyngeal flora and drug susceptibility in children with macrolide therapy. Laryngoscope. 2003;113:1780-5.

124. Don DM, Yellon RF, Casselbrant ML, Bluestone CD. Efficacy of a stepwise protocol that includes intravenous antibiotic therapy for the management of chronic sinusitis in children and adolescents. Arch Otolaryngol. Head Neck Surg. 2001;127:1093-8.

125. Chur V, Small CB, Stryszak P, Teper A. Safety of mometasone furoate nasal spray in the treatment of nasal polyps in children. Pediatric Allergy and Immunology 2013;24:33-8.

126. Seidman MD, Gurgel RK, Lin SY, et al. Clinical practice guideline: allergic rhinitis executive summary. Otolaryngol. Head Neck Surg. 2015;152:197-206.

127. Allen DB, Meltzer EO, Lemanske RF, Jr., et al. No growth suppression in children treated with the maximum recommended dose of fluticasone propionate aqueous nasal spray for one year. Allergy 
Asthma Proc 2002;23:407-13.

128. Schenkel EJ, Skoner DP, Bronsky EA, et al. Absence of growth retardation in children with perennial allergic rhinitis after one year of treatment with mometasone furoate aqueous nasal spray. Pediatrics 2000;105:E22.

129. Ozturk F, Bakirtas A, Ileri F, Turktas I. Efficacy and tolerability of systemic methylprednisolone in children and adolescents with chronic rhinosinusitis: a double-blind, placebo-controlled randomized trial. J of allergy and clinical immunology 2011;128:34852.

130. Harvey R, Hannan SA, Badia L, Scadding G. Nasal saline irrigations for the symptoms of chronic rhinosinusitis. Cochrane Database Syst Rev 2007:CD006394.

131. Shoseyov D, Bibi H, Shai P, Shoseyov N, Shazberg G, Hurvitz H. Treatment with hypertonic saline versus normal saline nasal wash of pediatric chronic sinusitis. J Allergy Clin Immunol 1998;101:602-5.

132. Wei JL, Sykes KJ, Johnson P, He J, Mayo MS. Safety and efficacy of once-daily nasal irrigation for the treatment of pediatric chronic rhinosinusitis. Laryngoscope. 2011;121:1989-2000.

133. Lin SY, Baugher KM, Brown DJ, Ishman SL. Effects of nasal saline lavage on pediatric sinusitis symptoms and disease-specific quality of life: a case series of 10 patients. Ear Nose Throat J 2015;94:E13-8.

134. Cho HJ, Min HJ, Chung HJ, et al. Improved outcomes after low-concentration hypochlorous acid nasal irrigation in pediatric chronic sinusitis. Laryngoscope. 2016;126:791-5.

135. Hong SD, Kim JH, Kim HY, Jang MS, Dhong HJ, Chung SK. Compliance and efficacy of saline irrigation in pediatric chronic rhinosinusitis. Auris Nasus Larynx 2014;41:46-9.

136. Jeffe JS, Bhushan B, Schroeder JW, Jr. Nasal saline irrigation in children: a study of compliance and tolerance. Int J Pediatr Otorhinolaryngol 2012;76:409-13.

137. Beswick DM, Messner AH, Hwang PH. Pediatric Chronic Rhinosinusitis Management in Rhinologists and Pediatric Otolaryngologists. Ann Otol. Rhinol Laryngol. 2017;126:634-9.

138. Beswick DM, Ramadan H, Baroody FM, Hwang PH. Practice patterns in pediatric chronic rhinosinusitis: A survey of the American Rhinologic Society. Am J Rhinol Allergy 2016;30:418-23

139. Chen J, Zhou Y, Nie J, et al. Bacterial lysate for the prevention of chronic rhinosinusitis recurrence in children. J of laryngology and otology 2017;131:5238.

140. Vandenberg SJ, Heatley DG. Efficacy of adenoidectomy in relieving symptoms of chronic sinusitis in children. Arch Otolaryngol. Head Neck Surg. 1997;123:675-8.

141. Lee CH, Hsu WC, Ko JY, Yeh TH, Lin MT, Kang KT. Revision adenoidectomy in children: a meta-analysis. Rhinology. 2019;57:411-9.
142. Ramadan HH, Tiu J. Failures of adenoidectomy for chronic rhinosinusitis in children: for whom and when do they fail? Laryngoscope. 2007;117:1080-3.

143. Bettadahalli V, Chakravarti A. Post-adenoidectomy quality of life in children with refractory chronic rhinosinusitis. The J Laryngol Otol 2017;131:1-6.

144. Ramadan HH, Cost JL. Outcome of adenoidectomy versus adenoidectomy with maxillary sinus wash for chronic rhinosinusitis in children. Laryngoscope. 2008;118:871-3.

145. Ramadan HH. Safety and feasibility of balloon sinuplasty for treatment of chronic rhinosinusitis in children. Ann Otol. Rhinol Laryngol. 2009;1 18:161-5.

146. Ramadan HH, Terrell AM. Balloon catheter sinuplasty and adenoidectomy in children with chronic rhinosinusitis. Ann Otol. Rhinol Laryngol. 2010;119:578-82.

147. Ramadan HH, Bueller H, Hester ST, Terrell AM. Sinus Balloon Catheter Dilation After Adenoidectomy Failure for Children With Chronic Rhinosinusitis. Archives of Otolaryngology-Head \& Neck Surg. 2012;138:635-7.

148. Soler ZM, Rosenbloom JS, Skarada D, Gutman M, Hoy MJ, Nguyen SA. Prospective, multicenter evaluation of balloon sinus dilation for treatment of pediatric chronic rhinosinusitis. Int Forum Allergy Rhinol 2017;7:221-9.

149. Liu J, Zhao Z, Chen Y, Xu B, Dai J, Fu Y. Clinical curative effect and safety of balloon sinuplasty in children with chronic rhinosinusitis. Int J Pediatr Otorhinolaryngol 2017;100:204-10.

150. Wang F, Song Y, Zhang X, Tan G. Sinus Balloon Catheter Dilation in Pediatric Chronic Rhinosinusitis Resistant to Medical Therapy. JAMA Otolaryngology-Head \& Neck Surg. 2015;141:52631.

151. Thottam PJ, Haupert M, Saraiya S, Dworkin J, Sirigiri R, Belenky WM. Functional endoscopic sinus surgery (FESS) alone versus balloon catheter sinuplasty (BCS) and ethmoidectomy: a comparative outcome analysis in pediatric chronic rhinosinusitis. Int J Pediatr Otorhinolaryngol 2012;76:1355-60.

152. Gerber ME, Kennedy AA. Adenoidectomy With Balloon Catheter Sinuplasty: a Randomized Trial for Pediatric Rhinosinusitis. Laryngoscope. 2018;128:2893-7.

153. Ference EH, Schroeder JW, Jr., Qureshi H, et al. Current utilization of balloon dilation versus endoscopic techniques in pediatric sinus surgery. Otolaryngol. Head Neck Surg. 2014;151:852-60.

154. House LK, Lewis AF, Ashmead MG. A costeffectiveness analysis of the up-front use of balloon catheter dilation in the treatment of pediatric chronic rhinosinusitis. Am J Otolaryngol. 2018;39:418-22.

155. Lusk RP, Bothwell MR, Piccirillo J. Long-term followup for children treated with surgical intervention for chronic rhinosinusitis. Laryngoscope. 2006;116:2099107.
156. Ramadan HH. Adenoidectomy vs endoscopic sinus surgery for the treatment of pediatric sinusitis. Arch Otolaryngol. Head Neck Surg. 1999;125:1208-11.

157. Lusk RP, Muntz HR. Endoscopic sinus surgery in children with chronic sinusitis: A pilot study. Laryngoscope. 1990;100:654-8.

158. Hebert RL, 2nd, Bent JP, 3rd. Meta-analysis of outcomes of pediatric functional endoscopic sinus surgery. 1998;108:796-9.

159. Vlastarakos PV, Fetta M, Segas JV, Maragoudakis P, Nikolopoulos TP. Functional endoscopic sinus surgery improves sinus-related symptoms and quality of life in children with chronic rhinosinusitis: a systematic analysis and meta-analysis of published interventional studies. Clinical Pediatrics 2013;52:1091-7.

160. Jiang XJ, Guo XY, Yuan W, et al. Long-term improvements in quality of life after functional endoscopic sinus surgery for adolescents with chronic rhinosinusitis. Acta Otolaryngol. 2012;132:798-802.

161. Cornet ME, Georgalas C, Reinartz SM, Fokkens WJ. Long-term results of functional endoscopic sinus surgery in children with chronic rhinosinusitis with nasal polyps. Rhinology. 2013;51:328-34.

162. El Sharkawy AA, Elmorsy SM, Eladl HM. Functional endoscopic sinus surgery in children: predictive factors of outcome. Eur Arch Otorhinolaryngol 2012;269:107-11.

163. Lee TJ, Liang CW, Chang PH, Huang CC. Risk factors for protracted sinusitis in pediatrics after endoscopic sinus surgery. Auris Nasus Larynx 2009;36:655-60.

164. Chang PH, Lee LA, Huang CC, Lai CH, Lee TJ. Functional endoscopic sinus surgery in children using a limited approach. Archives of Otolaryngology -- Head \& Neck Surg. 2004;130:10336.

165. Walner DL, Falciglia M, Willging JP, Myer 3rd CM The role of second-look nasal endoscopy after pediatric functional endoscopic sinus surgery. Arch Otolaryngol. Head Neck Surg. 1998;124:425-8.

166. Ramadan HH. Corticosteroid therapy during endoscopic sinus surgery in children: is there a need for a second look? Archives of otolaryngology--Head Neck Surg 2001;127:188-92.

167. Younis RT. The pros and cons of second-look sinonasal endoscopy after endoscopic sinus surgery in children. Archives of Otolaryngology -- Head \& Neck Surg. 2005;131:267-9.

168. Bothwell MR, Piccirillo JF, Lusk RP, Ridenour BD Long-term outcome of facial growth after functional endoscopic sinus surgery. Otolaryngol. Head Neck Surg. 2002;126:628-34

169. Ramadan H, Makary C. Can computed tomography score predict outcome of adenoidectomy for chronic rhinosinusitis in children. Am J rhinol allergy 2014:28:80-2. 


\section{Concomitant diseases in patients with chronic rhinosinusitis}

\subsection{Role of allergy in chronic rhinosinusitis}

\subsubsection{Epidemiology and predisposing factors}

The contribution of allergic inflammation to chronic rhinosinusitis (CRS) development, severity and therapeutic response are still not fully understood. It seems logical that allergic inflammation of the nasal mucosa with mucosal swelling and delayed mucociliary clearance might contribute to the development and/or severity and/or therapeutic response of $\mathrm{CRS}^{(1-3)}$. Literature is however not conclusive on the contribution of allergic inflammation to CRS, both in chronic rhinosinusitis without nasal polyps (CRSsNP) as well as chronic rhinosinusitis with nasal polyps (CRSwNP) ${ }^{(4)}$. In a systematic review Wilson et al. found nine articles that examined the relationship between allergy and CRSsNP, with four articles showing an association and five articles showing no association.

In CRSwNP they identified 18 articles, with ten showing an association, seven none and one a possible link / relationship Four studies directly compared the role of allergy in CRSWNP and CRSsNP and, again, the results were mixed. No articles examined the outcomes of CRSSNP or CRSWNP following allergy treatment. The authors concluded that the role of allergy in CRSwNP and CRSsNP continues to be controversial, with the very low level of evidence.

Since 2014 a few noteworthy studies have been published. A more recent non-systematic review points to the fact that different phenotypes / endoptypes of CRS, such as allergic fungal rhinosinusitis (AFRS) or central compartment atopic disease ${ }^{(5)}$, may have variable associations with allergy ${ }^{(6)}$. This point was also made in a recent study from the UK that analysed differences between different phenotypes of CRS and controls in secondary care ${ }^{(7)}$ and found the prevalence of self-reported inhalant allergy to be $20 \%$ in CRSwNP versus $31 \%$ in CRSwNP with the main difference in house dust mite (HDM) sensitization (9\% versus $16 \%$ ).

Earlier studies also showed that perennial allergy seems to be much more common than seasonal allergy in patients with $\mathrm{CRS}^{(1,8)}$ and positivity for perennial allergy was a risk factor of $\mathrm{CRS}^{(9,10)}$.

The effect of atopy on CRS symptom severity is unclear. Studies show no difference in symptom severity between patients with or without atopy in CRSwNP patients ${ }^{(2,11-15)}$. But in patients with CRSsNP the outcomes are more diverse with two recent studies showing a difference ${ }^{(11,16)}$ and two others no difference ${ }^{(2,13)}$.

Table 8.1.1. summarizes recent studies after EPOS2012 reporting on the association between atopic sensitisation and CRS.

\subsubsection{Pathophysiology}

Allergic inflammation of the nasal mucosa causes swelling of the mucosa with reduced mucociliary clearance ${ }^{(17,18)}$, that may contribute to the reduced clearance of pro-inflammatory agents. In addition, patients with CRSwNP usually present with type 2 helper T-cell (Th2) cytokine-mediated inflammation in the nasal mucosa, which is similar to allergic inflammation, particularly in individuals with predominant eosinophil infiltration in tissue ${ }^{(19-21)}$.

Recently, CRS has been further classified into different endotypes based on the association with type 1 or 2 inflammatory patterns ${ }^{(12,22,23)}$. Likewise, allergy is also characterized by type 2 inflammation ${ }^{(23)}$, indicating the inflammatory similarity between allergy and CRS. However, the underlying mechanisms of how allergy influences CRS are very different (see also chapter 5.2.2.).

Allergic rhinitis occurs through host sensitization to foreign proteins (allergens) across a mucosal barrier via dendritic cells and naive CD4-positive lymphocytes, with the generation of antigen-specific Th2 lymphocytes and immunoglobulin E (lgE)secreting plasma cells. Subsequent allergen challenge across the nasal mucosa of sensitized individuals may result in cross-linking of IgE bound to the surface of mast cells with degranulation, mediator, chemokine and cytokine release, leading to the recruitment of other inflammatory cells. In the unoperated state, allergens should have limited access to the sinus mucosa, but they can certainly trigger inflammation of the nasal cavity reducing sinus drainage.

Severe CRS is, in general, not regarded as an allergic disease, but co-existing allergic rhinitis may accentuate Type 2 inflammatory mechanisms of CRS. A recent study in the Chinese population showed distinct mucosal immunopathologic profiles in atopic and non-atopic CRSsNP patients, suggesting that in the Chinese population the comorbid CRSsNP and allergic rhinitis (AR) might be more related to Type 2 mechanisms, whereas the phenotype CRSsNP without AR exhibits more non-Type 2 mechanisms ${ }^{(24)}$. Allergens typically possess intrinsic protease activity that can interact with epithelial cells through three principle pathways: direct effects on junctional proteins, reaction with cell surface protease-activated receptors and toll-like receptor 4 (TLR4)dependent epithelial activation ${ }^{(25)}$. Protease activity of allergens may elicit the release of epithelial-derived Type 2-driving cytokines and chemokines, some of which have also been implicated in CRS. In terms of potential overlap between AR and CRS pathogenesis, Kouzaki et al. showed lower host expression of two protease inhibitors (cystatin A and SPINK5) in the nasal epithelial cells extracted from patients with eosinophilic CRS compared with control and non-eosinophilic CRS groups ${ }^{(25)}$. 
Table 8.1.1. The association of sensitisation with CRS. Recent studies after EPOS2012.

\begin{tabular}{|c|c|c|c|}
\hline Author & Patients & Effect & Department \\
\hline $\begin{array}{l}\text { Benjamin et al. } \\
2019^{(11)}\end{array}$ & $\begin{array}{l}\text { CRSsNP } \\
\text { CRSWNP }\end{array}$ & $\begin{array}{l}\text { The prevalence of atopy was } 52 \% \text { in CRSsNP and } 76 \% \text { in CRSwNP. } \\
\text { The atopic patients had more severe radiographic disease compared with non- } \\
\text { atopic patients in CRSsNP. }\end{array}$ & $\begin{array}{l}\text { Tertiary Allergology } \\
\text { Department, ENT }\end{array}$ \\
\hline $\begin{array}{l}\text { Shen et al. } \\
2019^{(2)}\end{array}$ & CRS & $\begin{array}{l}\text { The ImmunoCAP test was } 51 \% \text { positive in CRS patients. } \\
\text { The peripheral eosinophil count in the allergic group was higher than the non- } \\
\text { allergic group. }\end{array}$ & $\begin{array}{l}\text { Department of } \\
\text { Otolaryngology }\end{array}$ \\
\hline $\begin{array}{l}\text { Ho et al. } \\
2019^{(16)}\end{array}$ & CRS & $\begin{array}{l}\text { Positive allergen sensitization was } 53 \% \text { in CRS patients. } \\
\text { Atopy was associated with younger age at the time of surgery, CRSwNP, asthma, } \\
\text { and eosinophilic CRS. } \\
\text { Atopy was also associated with increased severity in nasal symptom score and } \\
\text { worse scores in the loss of smell/taste and need to blow nose questions in the CRS } \\
\text { population. }\end{array}$ & $\begin{array}{l}\text { Rhinology. and Skull Base } \\
\text { Research Group }\end{array}$ \\
\hline $\begin{array}{l}\text { Philpott et al. } \\
2018^{(7)}\end{array}$ & $\begin{array}{l}\text { CRSWNP } \\
\text { CRSsNP } \\
\text { Allergic fungal } \\
\text { rhinosinusitis }\end{array}$ & $\begin{array}{l}\text { The prevalence of self-reported confirmed inhalant allergy was } 13.1 \% \text { in control, } \\
20.3 \text { in CRSsNP, } 31.0 \% \text { in CRSwNP and } 33.3 \% \text { in AFRS respectively. } \\
\text { The self-reported house dust mite allergy was significantly higher in CRSwNPs } \\
\text { (16\%) compared to CRSsNPs (9\%). } \\
\text { The prevalence of self- reported aspirin sensitivity was } 2.26 \% \text { in control, } 3.25 \% \text { in } \\
\text { CRSsNP, } 9.61 \% \text { in CRSwNP and } 40 \% \text { in AFRS respectively. }\end{array}$ & Population study \\
\hline $\begin{array}{l}\text { Hamizan et al. } \\
2017^{(482)}\end{array}$ & $\begin{array}{l}\text { Patients had } \\
\text { undergone } \\
\text { nasal } \\
\text { endoscopy }\end{array}$ & $\begin{array}{l}\text { Diffuse oedema and polypoid oedema demonstrated the strongest association } \\
\text { with inhalant allergy. }\end{array}$ & $\begin{array}{l}\text { Department of } \\
\text { Otolaryngology-Head and } \\
\text { Neck Surg }\end{array}$ \\
\hline $\begin{array}{l}\text { Li et al. } \\
2016^{(12)}\end{array}$ & CRSWNP & $\begin{array}{l}\text { Atopic patients were younger than non-atopic patients. } \\
\text { There was no association between atopy status and either disease severity or } \\
\text { recurrence in patients with chronic rhinosinusitis with nasal polyps. }\end{array}$ & $\begin{array}{l}\text { Department of } \\
\text { Otorhinolaryngology - Head } \\
\text { and Neck Surg }\end{array}$ \\
\hline $\begin{array}{l}\text { Yacoub } \\
2015^{(483)}\end{array}$ & CRSWNP & $60 \%$ of patients were atopic. Patients with atopy had higher recurrence rate. & \\
\hline $\begin{array}{l}\text { Green et al. } \\
2014^{(8)}\end{array}$ & CRS & $\begin{array}{l}\text { In CRS patients, } 73 \% \text { had at least one of the postive allergen extracts in the skin } \\
\text { prick test compared with } 32 \% \text { of the control patients with chronic idiopathic } \\
\text { urticarial. } \\
\text { The perennial allergy was more common than seasonal allergy in CRS }\end{array}$ & $\begin{array}{l}\text { Allergy and Clinical } \\
\text { Immunology Branch }\end{array}$ \\
\hline
\end{tabular}

CRS, chronic rhinosinusitis; CRSsNP, chronic rhinosinusitis without nasal polyps; CRSwNP, chronic rhinosinusitis with nasal polyps.

This suggests that an imbalance of proteases and protease inhibitors within the epithelial barrier may contribute to the pathogenesis of Type 2 diseases in general(26). As we advance our understanding of the interactions between barrier immunity and the environment as a function of time, the relationship between allergens and CRS pathogenesis should become clearer.

In patients with CRSwNP, local polyclonal IgE is present in the polyp tissue ${ }^{(27-29)}$. In atopic nasal polyp patients, local IgE production can be the effect of stimulation with allergens ${ }^{(30)}$. However, local hyperimmunoglobulinemia is also present in non-atopic patients, meaning that elevated IgE levels result from other pathways as well. The cytokines interleukin (IL) 25 and IL33 can induce IgE-mediated inflammation by stimulating a non-T cell source to produce IL4. Alternatively, $\mathrm{IgE}$ is produced by stimulating innate lymphoid cells to release IL4, IL5, and IL13. IL5, and IL13, namely innate lymphoid cells (ILC). A role of mast cells in enhancing eosinophilic inflammation in chronic rhinosinusitis is suggested ${ }^{(31)}$.

\subsubsection{Central compartment atopic disease and allergy in CRS}

Central compartment atopic disease (CCAD) is a recently described variant of CRS that is significantly associated with allergy. In 2014, White et al. ${ }^{(32)}$ first presented polypoid and oedematous changes in the middle turbinate in 25 patients who tested positive for inhalant allergens. The proposed aetiology was that the anterior aspect of the middle turbinate is exposed to inhalant allergens via nasal airflow. Brunner et al. ${ }^{(33)}$ have similarly shown a higher association of allergen sensitization in patients with isolated middle turbinate changes than in those with diffuse polyposis. DelGaudio et al. ${ }^{(18)}$ revealed that other central structures, including the posterior-superior nasal septum, middle turbinates, and superior turbinates, are involved. Hamizan et al. ${ }^{(5)}$ reported that a central pattern of mucosal disease is significantly associated with allergy. Overall, this central pattern of inflammatory changes is highly associated with allergy. Further studies must be conducted to validate the aetiology and clinical course of this CRS subtype. 


\subsubsection{Diagnosis and differential diagnosis}

A suggestive history supplemented by confirmation of sensitization by demonstration of allergen-specific lgE using skin prick testing or blood analysis is as important in CRS patients as in allergic rhinitis patients. However, there is a significant overlap in symptomatology between CRS and AR. $A R$ is a very prevalent disease (see also 5.3.1.). It is not always easy to evaluate the role of sensitization to allergens in patients with CRS especially in perennial sensitisations (Table 8.1.1.). If in doubt, optimal treatment of the AR seems advisable.

\subsubsection{Treatment}

Reports on the efficacy of allergy therapy in improving the symptoms and quality of life of patients with CRS are limited. In general, the therapeutic principles for concomitant allergic diseases, such as allergic rhinitis, including allergen avoidance, pharmacotherapy and allergen-specific immunotherapy, are relevant in patients with CRS. The use of nasal corticosteroids is the mainstay of treatment for both patients with chronic rhinosinusitis and allergic rhinitis. One study observed less improvements in symptom scores, polyp score, nasal peak flow, and overall response to therapy in the skin prick test positive group than in the negative skin test group treated with budesonide nasal spray ${ }^{(34)}$. However, the presence of allergy showed no influence on the cumulative consumption of steroids between baseline and after one year in patients with CRSwNP, which indicated that the specific treatment of allergy in patients with nasal polyps may not be beneficial ${ }^{(14)}$. Current studies yield insufficient evidence to recommend oral or intranasal $\mathrm{H} 1$-antihistamines in non-allergic CRS patients (see 6.1.8. ). A systematic review evaluating the effect of immunotherapy found no RCTs and concluded after highlighting the paucity of the data that there is weak evidence to support the use of immunotherapy(IT) as an adjunctive treatment in CRS patients, particularly in the postoperative period ${ }^{(35)}$.

In conclusion: allergic rhinitis and CRS both benefit from nasal corticosteroids. There is insufficient quality evidence to advise other treatments, such as antihistamines or immunotherapy, in allergic patients with CRS. More studies are needed.

\subsection{Immunodeficiencies and their role in CRS}

Conditions that are associated with immunodeficiency are of clinical importance to rhinologists because some patients who present with CRS were predisposed to their condition by an underlying immunodeficient state. Immunodeficiency conditions may cause CRS patients to respond less favourably to standard therapies, and some patients require specific treatment for their immunodeficiencies in order for their CRS to be optimally treated.

Immunodeficiency states can be primary or secondary to other diagnoses or to immunosuppressive medication. Primary immunodeficiency conditions may be categorized according to whether the deficiency affects B cells (humoral immunity), $T$ cells (cellular immunity), phagocytes or the complement system (both innate immunity) ${ }^{(36)}$. In some cases, there is a combination of defects.

CRS is primarily associated with conditions that cause humoral deficiency, and in this section the discussion of primary immunodeficiency will be largely confined to hypogammaglobulinaemia(37).

\subsubsection{Epidemiology}

There is good evidence that immune deficiencies are more common in patients with CRS. A meta-analysis, which included 1418 individuals with CRS from 13 studies, found that $23 \%$ of patients with difficult- to-treat CRS and $13 \%$ of individuals with recurrent $\mathrm{CRS}$ had immunoglobulin deficiencies ${ }^{(38)}$. However, many of the patients diagnosed in the series included in the meta-analysis had subclass or specific antibody deficiency. The laboratory criteria for diagnosing these conditions, and the clinical implications once the diagnoses are made, are not uniformly accepted. Many of the studies of the meta-analysis were performed in tertiary referral centres and this may have biased the population of patients studied towards having underlying immune defects. These reservations aside, it is likely that the prevalence of hypogammaglobulinemia in CRS patients is very significantly higher than in the general population.

\subsubsection{Pathophysiology}

Most cases of hypogammaglobulinaemia are caused by genetic mutations. The majority of cases are sporadic, although a family history of hypogammaglobulinaemia would raise diagnostic suspicions. Immunoglobulins primarily act by opsonizing encapsulated bacteria, and consistent with that action patients with hypogammaglobulinaemia tend to be susceptible to infections with Streptococcal species, Haemophilus influenzae and Moraxella catarrhalis ${ }^{(39)}$. Patients with hypogammaglobulinaemia are predisposed to developing rhinosinusitis, pneumonia, bronchiectasis and otitis media.

\subsubsection{Diagnosis and differential diagnosis}

The causes of primary hypogammaglobulinaemia are:

1. X-linked agammaglobulinaemia

2. Common variable immunodeficiency (CVID)

3. Selective immunoglobulin $A(\lg A)$ deficiency

4. Immunoglobulin $\mathrm{G}$ (IgG) subclass deficiency

5. Selective antibody deficiency

There are other very rare causes such as Good's syndrome, which is CVID associated with thymoma and Hyper-IgE syndrome in which patients have eczema and Staphylococcal furuncles. The number of causes of primary hypogammagloblinaemia keeps expanding as the genotypes of these conditions are determined $^{(40)}$.

X-linked agammaglobulinaemia presents with recurrent respiratory tract infections in infant boys. Symptoms develop after age six months when passive protection from maternal immunoglobulins has been lost.

Common variable immunodeficiency (CVID) is more likely than $\mathrm{X}$-linked agammaglobulinaemia to present to rhinologists 
because its age of onset is often in adulthood. It is diagnosed by low immunoglobulin levels and a poor response to vaccinations. In 2015, the International Consensus Document on CVID was published proposing six diagnostic criteria for this condition, which have clarified the clinical and laboratory aspects of its diagnosis ${ }^{(41)}$. Patients with CVID are also predisposed to some autoimmune conditions as well as certain malignancies such as gastric lymphoma.

$\lg$ A deficiency is the most common immunoglobulin deficiency in the general population. The prevalence has been reported variably between 1:173 and 1:3024 $4^{(42)}$. Most patients are asymptomatic, but a deficiency of IgA would appear to predispose patients to rhinosinusitis and allergies ${ }^{(36)}$. IgG has four variants, or subclasses, each of which has slightly different functions. Subclass deficiencies are diagnosed when the serum IgG level is normal, but one or more of the subclasses are found to be deficient. Laboratory determination (both analysis and normal values) may differ between laboratories. IgG subclass deficiency is a controversial diagnosis and experts are increasingly disagreeing about the importance of this finding as a cause of repeated infections ${ }^{(37)}$. The overdiagnosis of lgG subclass deficiency as a cause of presumed immunodeficiency is not uncommon, and may lead to unnecessary long-term treatment.

Selective antibody deficiency (SAD) is diagnosed when patients have a normal serum immunoglobulin levels but attenuated responses to polysaccharide antigens ${ }^{(43)}$. The antibody response to polysaccharide vaccines such as Pneumovax is determined, however the diagnostic criteria are not universally accepted, again leading to the possibility of overdiagnosis.

CRS secondary to hypogammaglobulinaemia may present in a manner identical to idiopathic CRS. This explains why there is often a long delay between initial presentation and diagnosis of an underlying immunodeficiency. There are some clinical features that may elevate suspicion: recalcitrance to standard treatments (and particularly rapid recurrence of symptoms after stopping antibiotics) and association with lower respiratory tract infections (pneumonia, particularly if recurrent, or bronchiectasis).

The testing of immune function in all patients who present with CRS is almost certainly unwarranted as it is likely to produce more false positive results than true positives. However, it is recommended that the above clinical features are used to identify those patients who warrant some form of immune testing for testing.

\subsubsection{A work-up by ENTs before referring to immunologists} It is not clear how much of the immunological testing should be undertaken by the rhinologist before referral to an immunologist, and this decision may reflect the individual knowledge and expertise of the rhinologist.

Further studies, such as antibody responses to vaccines and flow cytometry can then be organized by an expert in the interpretation of these tests. The best approach for confirming a diagnosis of an antibody-deficiency disorder is the measurement of serum-specific antibody titers (usually $\mathrm{lgG}$ ) in response to vaccine antigens. This approach involves immunizing a patient with protein antigens (e.g. tetanus toxoid) and polysaccharide antigens (e.g., pneumococcus) and assessing pre- and post-immunization antibody levels $s^{(44)}$.

\section{For CRS patients suspected of having humoral immunodeficiency because of the characteristics of their presentation or their response to treatment, measurement of serum immunoglobulin levels is the key investigation. If the levels are normal, but the suspicion of humoral immunodeficiency is high, referral to a clinical immunologist is optimal.}

\subsubsection{Treatment}

For many patients with CRS who have subtle humoral immundeficiencies on laboratory testing, specific treatment options are not available (such as IgA deficiency) or may not be indicated (such as in lgG subclass deficiencies). It is of paramount importance that the diagnosis and its implications are established in collaboration with a clinical immunologist. It is possible for some results to be overinterpreted, leading to unnecessary treatment with IgG replacement or excessive antibiotic prescribing.

\subsubsection{Immunoglobulin replacement therapy}

The mainstay of treatment of hypogammaglobulinaemia is immunoglobulin replacement therapy. The immunoglobulin fraction is extracted from the plasma of a large number of donors so that passive immunity to an enormous number of antigens can be achieved. The optimal route of administration (intravenous or subcutaneous) has not been established ${ }^{(45)}$. However, the decision about whether to start a patient on intravenous immunoglobulin therapy and ongoing oversight of this treatment would in almost all circumstances involve a clinical immunologist.

A series of 31 patients from a single institution, most of whom had CVID, were followed closely. They had fewer sinus and chest infections, and a significant reduction in Lund-Mackay scores while receiving immunoglobulin replacement therapy ${ }^{(46)}$. However, an earlier European study did not find such a favourable impact of therapy ${ }^{(47)}$.

\section{Various antibiotic agents and regimens have been used, and often at half the usual dose. Periodically changing antibiotics monthly to every 6 months is sometimes practiced in order to reduce the development of resistance, but there are no studies that evaluated the efficacy of this practice ${ }^{(48)}$.}

\subsubsection{Prophylactic antibiotics}

Although long-term antibiotic therapy has transformed the outcomes in some types of primary immunodeficiency syndromes, controlled trials are few in the context of hypogammaglobulinaemia ${ }^{(48)}$. One long-term observational 
study of CVID patients treated with prophylactic antibiotics as they continued to have infections despite immunoglobulin replacement therapy, failed to find a reduction in the frequency of infections ${ }^{(49)}$.

\subsubsection{Vaccinations}

It has been found that some patients with low antibody levels to pneumococcal serotypes will respond favourably to conjugated pneumococcal vaccinations, reducing their requirement for antibiotics $^{(50)}$.

\subsubsection{Surgery}

The relative efficacy of surgery in the context of hypogammaglobulinaemia compared to idiopathic CRS has not been extensively reported. A nested case control study comparing the outcome of functional endoscopic sinus surgery (FESS) in patients with immunodeficiency (mostly secondary) and idiopathic CRS patients found that the immunodeficient patients responded as well as their controls ${ }^{(51)}$.

\subsubsection{CRS and secondary immune deficiencies}

In a recently reported study in patients who received rituximab for management of systemic autoimmune disorders, moderateto-severe hypogammaglobulinaemia was seen in $26 \%$ of patients, but approximately $50 \%$ of these cases improved and resolved spontaneously ${ }^{(53)}$. In addition, immunoglobulin replacement was initiated in $4.2 \%$ of patient due to recurrent infections.

A recent review on the HIV-associated manifestations in otolaryngology underscores the high prevalence of CRS in HIV patients ${ }^{(54)}$. These authors also mention that physicians need to have a high level of suspicion for atypical pathogens in this clinical context. Also iron overload, protein-energy malnutrition, or transplant patients on azole prophylaxis are also at risk ${ }^{(237)}$. Diabetes mellitus, particularly when poorly controlled, impairs both immune function and wound healing. There is however no evidence to suggest that it is a risk factor for the development of CRS. Whether having diabetes mellitus affects the outcome after FESS has been determined in a nested control study, and it was found that the diabetic patients fared as well postoperatively as the non-diabetic patients ${ }^{(55)}$.

The prevalence of secondary immune deficiency is rising due to the increased use of immunosuppressive agents such as rituximab, corticosteroids and other drugs ${ }^{(52)}$. Rituximab is a monoclonal antibody directed against CD20 that causes B-cell depletion. As the indications for rituximab are growing so is the incidence of rituximab-induced hypogammaglobulinaemia.

\subsubsection{Summary}

CRS may be associated with primary humoral immunodeficiency conditions. Most of the patients with significant humoral immunodeficiency conditions also have associated lower respiratory tract problems such as recurrent pneumonia.
The best screening tests for ORL surgeons to request are serum immunoglobulin levels. More sophisticated tests of immune function should generally be undertaken by clinical immunologists, as their interpretation requires specific expertise. The decision to treat with intravenous immunoglobulin replacement and the supervision of that treatment should ideally be made by a clinical immunologist. The potential influence of secondary causes of immunodeficincies (resulting either from co-morbidities or immunosuppressive therapy) should be considered in the management of CRS patients.

\subsection{Lower airway disease including asthma in relation to CRS}

\subsubsection{Introduction}

Health care providers dealing with CRS proactively ask for the presence of lower airway symptoms such as cough, wheezing, shortness of breath, and are ideally aware of the diagnostic and therapeutic guidelines like EPOS and GINA to optimally treat both CRS and asthma ${ }^{(56-58)}$.

Lower airway inflammation often co-exists in CRS, with up to two thirds of patients with CRS affected by comorbid asthma ${ }^{(7,59,60)}$, chronic obstructive pulmonary disease (COPD) or bronchiectasis ${ }^{(61,62)}$ (figure 8.3.1.). Pulmonary function in CRS patients is significantly reduced compared to non-CRS individuals, even in patients that do not report bronchial symptoms ${ }^{(63)}$. Rhinitis / rhinosinusitis is demonstrated to be the most prevalent co-morbidity of asthma ${ }^{(64)}$. Asthma, COPD and bronchiectasis patients have a reported prevalence of CRS in up to $57 \%{ }^{(59,65)}, 40 \%{ }^{(62)}$, and $45 \%{ }^{(66,67)}$ respectively. The HELIUS study showed CRS to be associated with adult-onset asthma ${ }^{(68)}$ and the Asthma Clinical Research Network demonstrated that CRS is associated with increased risk of asthma exacerbation ${ }^{(69)}$. In asthma patients with CRS, acute exacerbations of CRS lead to poor asthma control ${ }^{(70)}$. GINA 2019 recommends the assessment of comorbidities including CRS in every step of the therapeutic approach for asthma ${ }^{(71)}$. The presence of chronic rhinitis was associated with 30-day asthma and COPD-related hospital readmissions, in both allergic and non-allergic patients ${ }^{(72)}$. In COPD, the presence of CRS negatively impacts the severity of bronchial inflammation and COPD severity ${ }^{(73)}$.

Several immune mechanisms have been reported to be involved in the naso-bronchial interaction in patients with global airway disease. Both asthma and COPD patients show the typical sinonasal inflammatory pattern with the classic Th2 and Th2 biomarkers in the nasal secretions, as seen in rhinitis / CRS patients ${ }^{(26,74)}$.Therefore, it is not a surprise that novel biological treatments targeting inflammatory molecules such as IL4, IL-5 and IgE in both upper and lower airway inflammation are effective on both asthma and CRSwNP(75, 76)(77).

In the field of respiratory allergy and asthma, the naso-bronchial interaction has been studied by Braunstahl and coworkers ${ }^{(78,79)}$ with involvement of a systemic and/or neurogenic inflammatory component. The systemic pathway leading to bronchial disease via nasal inflammation is most likely facilitated by transmucosal 


\section{Global Airway Disease Concept}

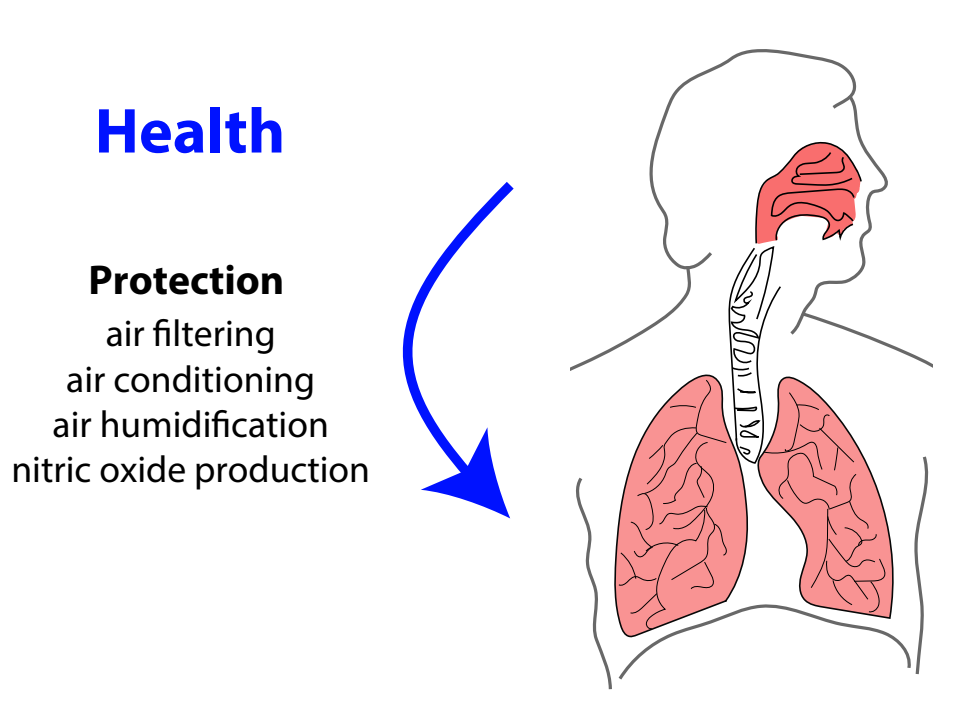

\section{Rhinitis Rhinosinusitis}

passage of exogenous particles among which are allergens ${ }^{(80)}$, and by nasal epithelial barrier dysfunction found in both rhinitis and in $\mathrm{CRS}^{(81)}$. A neurogenic pathway linking upper and lower inflammation has been demonstrated using animal models highlighting the bronchial release of neural mediator substance $P$ in the bronchi upon activation of a nasal inflammation ${ }^{(82)}$. In addition to the neural and systemic parts of the nasobronchial interaction, it seems that the microbiome in the sino-nasal cavity also plays a role in lower airway inflammation, as nasal colonization with Staphylococcus aureus showed significant relationships with asthma prevalence ${ }^{(83)}$ for both CRSwNP and CRSsNP. Animal studies have indeed demonstrated the aggravation of bronchial eosinophilia by nasal presence of Staphylococcus aureus enterotoxin $\mathrm{B}^{(84)}$. The contribution of the microbiome to asthma development in CRS patients is still under investigation ${ }^{(85)}$.

Given the epidemiologic and pathophysiologic connection between CRS and lower respiratory airway disease ${ }^{(86)}$, the concept of global airway disease has gained more interest, leading to better diagnosis and therapeutic approaches in patients with global airway disease ${ }^{(87)}$. Recently, a simple Visual Analogue Scale (VAS) for total sino-nasal symptoms has shown to correlate well with the more extended SNOT-22 questionnaire, and could be used for screening of CRS in asthma / COPD patients ${ }^{(88)}$.

The impact of lower airway disease on CRS can be arbitrarily divided into impact on disease severity and control, and outcomes of medical and surgical treatment.

8.3.2. Impact of endoscopic sinus surgery on asthma / COPD Endoscopic sinus surgery in asthma has been reported to improve multiple clinical asthma parameters ${ }^{(89)}$. In this metaanalysis, mean follow-up across all studies was 26.4 months. Patients reported improved overall asthma control in $76.1 \%$
[95\% confidence interval $(\mathrm{Cl}), 71.9 \%$ to $80.3 \%$ ] of cases. The frequency of asthma attacks decreased in $84.8 \%(95 \% \mathrm{Cl}$, $76.6 \%$ to $93.0 \%$ ) of patients and the number of hospitalizations decreased in $64.4 \%$ (95\% Cl, 53.3\% to $75.6 \%$ ). Decreased use of oral corticosteroids was seen in $72.8 \%$ ( $95 \% \mathrm{Cl}, 67.5 \%$ to $78.1 \%$ ) of patients; inhaled corticosteroid use decreased in $28.5 \%$ (95\% $\mathrm{Cl}, 22.6 \%$ to $34.5 \%$ ) and bronchodilator use decreased in $36.3 \%$ ( $95 \% \mathrm{Cl}, 28.9 \%$ to $43.7 \%$ ) of patients. Mean improvement in predicted forced expiratory volume at 1 second (FEV1) was $1.62 \%$ but was not statistically significant $(p=0.877)$. Surgical treatment for CRS has shown to be beneficial in severe asthma, with better asthma control in the most severe asthma group ${ }^{(90)}$. Contrary to asthma, at present, there is limited evidence on the impact of sinus surgery in COPD patients with CRS.

\subsubsection{Impact of asthma / COPD on CRS severity}

Patients with CRS often present with uncontrolled asthma, and endoscopic sinus surgery (ESS) improves both mini-Allergen Quality of Life Questionnaire (AQLQ) and asthma control test $(A C T)^{(90)}$. In a large-scale study on outcomes after sinus surgery in a tertiary referral centre ${ }^{(91)}$, asthma did not have an impact on the degree of control after ESS, whereas NSAIDexacerbated respiratory disease (N-ERD) was significantly associated with higher prevalence of uncontrolled CRS. In another study evaluation success of the endoscopic modified Lothrop procedure, failure rates of endoscopic modified Lothrop procedure were $8.9 \%$ for primary procedures and $21 \%$ for revision surgery. Also in this study, one of the risk factors for failure of primary endoscopic modified Lothrop procedure was $\mathrm{N}-\mathrm{ERD}^{(92)}$.

In COPD, there is a higher occurrence of nasal symptoms and pathological findings on nasal endoscopy compared with the control group, with overall reduced nasal airflow and increased nasal resistance. Pathological opacity of one or more sinuses was 
confirmed in $38 \%$ of COPD patients ${ }^{(62)}$. In bronchiectasis, CRS is associated with a greater degree of bronchiectasis severity, poorer health-related quality of life (HRQOL), reduction in smell detection, elevated levels of inflammatory markers, and reduced time to first exacerbation ${ }^{(67)}$.

\subsection{Cystic fibrosis}

Cystic fibrosis (CF) is a life-shortening genetic condition caused by a mutation in the cystic fibrosis transmembrane conductance regulator (CFTR) gene leading to defective chloride channels. $\mathrm{CF}$ is the most frequent lethal autosomal recessive disorder in the Caucasian population, but it affects all races. Ireland and the Faroe Islands have the highest prevalence of $\mathrm{CF}$ in the world with prevalence of respectively 68 per 100,000 (1:1461) in Ireland ${ }^{(93)}$ and 56 per 100,000 (1:1775) in the Faroe Islands ${ }^{(94)}$. The CFTR gene encodes the CAMP-dependent chloride channel, and a defect in the gene results in abnormaltransport of chloride and sodium across the cell. As a consequence, airway secretions contain a lower concentration of salt, causing the secretions to have more than double the viscosity of secretions of a non-CF individual. The mucus is thus dehydrated and sticky, which reduces mucociliary clearance and promotes infections; most severely leading to increased susceptibility of bacterial infections in the lower airways with the CF-pathogenic gram-negative bacteria, particularly Pseudomonas aeruginosa, Achromobacter xylosoxidans, and Burkholderia cepacia, causing progressive decline in lung function and ultimately death. Staphylococcus aureus encountered early in childhood, is generally considered to be related to poor clinical outcomes. Methicillin-resistant strains are also encountered and have been associated with worsening progression of lung disease. Often, gram-negative bacteria become the dominant pathogens in adulthood. If this is not the case, the Staph. aureus bacteria are often present as a pathogenic chronic infection of the upper and lower airways. A major goal in the treatment of patients with CF is thus to prevent or delay chronic lung infections. Preventing chronic lung infection is, however, difficult to achieve and the vast majority of patients with CF has been colonized or infected when reaching adulthood ${ }^{(95)}$.

\section{There is a high concordance of bacteria cultured from the paranasal sinuses (based on irrigations, swabs, or mucosal biopsies) and from the lungs ${ }^{(96)}$.}

Over time, P. aeruginosa develop common phenotypic traits, including conversion into alginate-overproducing mucoid variants, reduced growth rates, and loss of virulence factor production, quorum sensing, and motility. Infections often originate in the paranasal sinuses and can be responsible for the initial and temporary lung colonization or for the chronic lung infections in CF patients ${ }^{(97-99)}$. The latter is presumably due to an advantageous environment in CF sinus secretions of a higher immunoglobulin(lg) A:lgG ratio, reduced inflammation ${ }^{(100)}$, low oxygen concentration ${ }^{(101)}$, bacterial biofilm formation ${ }^{(102)}$, and lower bioavailability efficacy of IV-antibiotic treatment compared to the lungs ${ }^{(103)}$. In addition, the presence of intramucosal bacterial colonies are difficult to eradicate ${ }^{(104)}$.

$$
\begin{gathered}
\text { Bilateral nasal polyposis in children are often } \\
\text { a clinical indication of } C F^{(111)} \text {; nasal polyposis } \\
\text { in CF patients becomes more common as the } \\
\text { children age with a prevalence of up to } 50 \% \text { in } \\
\text { adolescents }{ }^{(112)} \text {. }
\end{gathered}
$$

Anatomically, CF patients often have hypoplastic sinuses, independent of previous surgery. A study on adult CF patients demonstrated $66 \%$ of the frontal sinuses were either aplastic or hypoplastic ${ }^{(105)}$. Mucoceles often leading to pyoceles and bone sclerosis are common, as well as typical inflammatory patterns, e.g. CRSwNP, mucosal swelling and bulging, or even missing the lateral nasal wall ${ }^{(105)}$. The majority of CF patients have characteristic CT findings from early childhood ${ }^{(106)}$, thus the Lund-Mackay scoring system may not be of optimal use. Alternative CT sinus scoring systems addressing CF as proposed by Eggesboe ${ }^{(107)}$, Sheikh ${ }^{(108)}$ or Rasmussen ${ }^{(109)}$ may be more useful.

Only $7 \%$ of CF patients are free from inflammatory changes in their sinonasal histology ${ }^{(110)}$.

Nasal polyps in CF tend to be more neutrophilic and less eosinophilic compared to the non-CF population ${ }^{(113)}$. CF patients are likely to under-report their symptoms of $\mathrm{CRS}^{(114,115)}$. Several patient-reported outcome measures (PROM) conclude that CF patients have symptoms of CRS two-third of whom fulfil the EPOS criteria ${ }^{(116,117)}$. Individuals who are carriers for one defective CFTR gene may be more predisposed to developing CRS. Symptoms include thick nasal discharge, postnasal discharge, nasal blockage, facial pressure, and decreased sense of smell ${ }^{(118)}$. There is a strong association between nutritional status and pulmonary function in CF and a decreased sense of smell is known to influence nutritional status in non-CF patients. Inexplicably and in contrast to patients with primary ciliary dyskinesia, patients with CF have a lower incidence of otitis media compared with the general population ${ }^{(119)}$. Several studies have investigated a correlation between CF genotype and severity of sinonasal disease, however, conflicting results were found $f^{(120,121)}$. Patients who are heterozygous for mutations in the CFTR gene may be at increased risk of CRS. However, the evidence regarding an association between heterozygote patients and CRS is sparse. Calton et al. argued in their study treating CRS in a university setting that CFTR heterozygotes have significantly higher risk for CRS and who also have smaller paranasal sinuses ${ }^{(120,121)}$.

\subsubsection{Diagnosis and differential diagnosis}

The diagnosis of $\mathrm{CF}$ is a multistep process, which involves a sweat chloride test, genetic analysis, and a clinical evaluation ${ }^{(122)}$. The diagnosis of CF is made when a patient has both clinical presentations of the disease as well as evidence of a defective CFTR channel.

Early detection accomplished by screening can prevent malnutrition and delay the imminent lung infections, and several studies have proven that the national screening program 
Table 8.4.1. CFTR mutation classes.

\begin{tabular}{lll}
\hline CFTR mutation classes & Description & Mutation example \\
\hline Class I & No functional CFTR being made & G542X \\
Class II & Incorrect trafficking of the CFTR to the cell surface & F508del \\
Class III & "Gating mutations" - the channels opening probability is affected & G551D \\
Class IV & The channels conductance is decreased & R117H \\
Class V & The synthesis of the channel is reduced & A455E \\
Class VI & The stability of the CFTR channel is decreased & r-delta-F508
\end{tabular}

improves survival and reduces morbidity in patients with $\mathrm{CF}^{(123-}$ 125).

\section{In the western part of the world national screening programs on specific genetic disorders including CF have been implemented for newborns.}

\section{Diagnostic testing/possible tests:}

Neonatal screening: a heel prick is performed in the first couple of days after birth and is tested for raised levels of immunoreactive trypsinogen, a pancreatic enzyme. Elevated level of immunoreactive trypsinogen is suggestive of CF. However, immunoreactive trypsinogen can also be elevated due to other aetiologies e.g. prematurity, stressful delivery, or if a person is a carrier of the cystic fibrosis gene. Due to this, the screening test must be followed by a diagnostic test before the CF diagnosis can be made.

Genetic testing: it is possible to test for mutations in the CFTR gene through a blood test or cells taken from a cheek swab. Identification of two disease-causing mutations in the CFTR gene confirms the diagnosis. The specificity and sensitivity are estimated as high where two disease-causing mutations are identified. However, a positive gene test is accompanied by a sweat chloride test.

\begin{abstract}
Sweat chloride test: measures the amount of chloride in the sweat. A higher amount of chloride in the sweat ( $>60 \mathrm{mmol} / \mathrm{l}$ ) compared to normal values indicates a diagnosis of CF. The test is done by placing a solution on the forearm or thigh and stimulating with a mild electric current to produce sweating. The test is tolerated well with only minor discomfort reported.
\end{abstract}

\subsubsection{Pathophysiology}

CFTR mutations can be grouped into six classes (see Table 8.4.1.), all of which alter the function of the CFTR channels and cause CF. Class I mutations result in no functional CFTR proteins being made. Class II are the most common form of mutations and result in incorrect trafficking of the CFTR to the cell surface. Class III are gating mutations and affect the channels' opening probability, and class IV mutations reduce the function of the CFTR channel by decreasing channel conductance. Class $\checkmark$ mutations reduce the synthesis of the channels and class VI decrease CFTR stability ${ }^{(126)}$. The most frequently observed mutation is the $\Delta \mathrm{F} 508$, a class II mutation, but approximately 2000 different mutations have been identified.

The presence of two mutations from either class I, II, or III are categorized as high-risk genotypes whereas at least one class IV, or V mutation are categorized as low-risk genotype. High-risk genotypes have been associated with significantly worse survival and lower lung function compared to low-risk genotype ${ }^{(127)}$.

As mentioned above, the effect of genotypes on sinonasal disease is controversial. Ferril et al. ${ }^{(128)}$ retrospectively reviewed sinonasal disease in CF patients and found a significant difference in severity of sinonasal disease when comparing patients with high-risk genotypes to low-risk genotypes. They describe how high-risk genotypes were associated with increased incidence of hypoplasia/aplasia and bony sclerosis evaluated by CT scans. Abuzeid et al. ${ }^{(129)}$ on the other hand did not demonstrate worse sinonasal disease with highrisk genotypes compared to low-risk genotypes when they retrospectively reviewed CF patients, evaluating SNOT-22 scores, nasal endoscopy scores, and CT staging scores.

The taste receptor, $\mathrm{T} 2 \mathrm{R} 38$ has been shown to play a role in detection and clearance of gram-negative organisms ${ }^{(130)}$.

\section{Non-functional polymorphisms in the T2R38 gene correlate with sinus disease severity in patients with deltaF508 homozygous mutation ${ }^{(131)}$.}

Genetic markers as a prognostic indicator for the severity of CRS in CF patients as well as a diagnostic tool that can aid in tailored treatment for patients with CF could potentially improve sinonasal QOL in patients with CF while also minimizing unnecessary healthcare treatments. Further studies on the use of genetic markers to assess sinus disease in CF patients are needed and may eventually aid in future treatment modalities.

\subsubsection{Treatment}

\subsubsection{Gene therapy}

The treatment of CF is currently symptomatic and treatment of the underlying gene defect, and thus curing the disease, has not yet been possible.

Restoration of CFTR and its function by transferring correct versions of the CFTR gene to the airways may be a first step in gene therapy. A CFTR gene replacement would be an effective treatment of $C F$ regardless of the underlying gene mutation class.

Delivery of the CFTR gene via adeno-associated virus has so far 
solely been shown in a pig model ${ }^{(132)}$. Further studies with other gene vectors and in vivo models are needed.

\section{To date there is no FDA-approved gene therapy.}

\subsubsection{TgAAV-CF}

TgAAC-CF, an adeno-associated cystic fibrosis transmembrane conductance regulator (CFTR) viral vector/gene construct, has been suggested as a therapeutic agent for the treatment of sinus disease in patients with cystic fibrosis. However, no significant effect of TgAAC-CF has been found so far, and the latest study of the effect of TgAAC-CF is more than 17 years old $^{(133)}$. The scientific evidence of the use of TgAAC-CF in the treatment of sinus disease is thus not currently available.

\subsubsection{Ivacaftor}

Ivacaftor is a gene-based therapeutic agent approved, by the US Food and Drug Administration and the European Medicines Agency for the treatment of patients with specific CF mutations. Ivacaftor is a CFTR potentiator, which increases the opening probability of the CFTR channels at the cell surface, thus increasing the flow of ions through the channel ${ }^{(134)}$. Ivacaftor is approved for the treatment of various class III mutations (G551D, G1244E, G1349D, G178R, G551S, S1251N, S1255P, S549N andS549R), and for the treatment of one class IV mutation $(\mathrm{R} 117 \mathrm{H})^{(135,136)}$.

Recently it has been shown in CF patients that the intraflagellar transport protein, IFT88, abnormally accumulates in the basal body region in ciliated respiratory epithelial cells. The latter demonstrates a novel potential pathophysiologic intracellular mechanism; and of interest in CF patients receiving ivacaftor a normalization of IFT88 localization was obtained ${ }^{(137)}$.

Ivacaftor has been shown to improve lung function in patients with $\mathrm{CF}^{(138,139)}$ and has recently demonstrated several other beneficial off-target effects, including a beneficial impact on chronic rhinosinusitis. Ivacaftor has been proven to improve rhinologic QOL in patients with CF evaluated by SNOT-20 $0^{(140)}$. Furthermore, several case reports document the reversal of sinus disease in CF patients after Ivacaftor treatment with normalized CT findings of CF sinus disease and resolved sinus symptoms $s^{(141,142)}$. Ivacaftor has also been shown to enhance the effect of some antibiotics, including ciprofloxacin ${ }^{(143)}$ and even exhibits antibacterial properties itself $f^{(144)}$.

Further studies on combination therapy and the specific effect of Ivacaftor on chronic rhinosinusitis in vivo are warranted.

\subsubsection{Tezacaftor/Ivacaftor}

Tezacaftor is a selective CFTR corrector that facilitates the cellular transport of CFTR and restores the CFTR protein in the correct position on the cell surface.Tezacaftor in combination with Ivacaftor has been approved for the treatment of patients with F508del mutations, a type II mutation ${ }^{(145)}$.

\subsubsection{Mucolytic}

As in non-CF CRS patients, treating sinonasal symptoms starts with medical therapy prior to surgical intervention ${ }^{(146)}$.

\subsection{Dornase alfa (rhDNAse)}

\section{Nasal irrigation combined with dornase alfa inhaled as a vibrating aerosol has been shown to be even more efficient than nasal irrigation solely ${ }^{(147)}$. In contrast, the use of hypertonic saline does not add significant benefit ${ }^{(148)}$.}

\subsection{Topical antimicrobials}

The use of topical antibiotics correlates with improvement in symptom and endoscopic scoring in uncontrolled studies and is safe $^{(149,150)}$. With regards to post-surgical treatment, most studies use topical and/or systemic antibiotic treatment. However, recurrent infections are often seen. Installation of resorbable antibiotics/bacteriophages/CFTR-modulating drugs/Poloxamer/ etc may help eradicate bacteria after surgery ${ }^{(151-153)}$.

\subsection{Other treatment}

There is high level of evidence that high volume nasal irrigations with saline relieve symptoms in CF patients, and the procedure is well-tolerated.

The effects of topical steroids as a medical treatment for CRS in $\mathrm{CF}$ patients are controversial.

\section{A Cochrane review (including 1 placebo controlled study) concluded that topical steroids for nasal polyposis have no significant effect on subjective nasal symptom scores but are effective in reducing nasal polyps size $^{(154)}$.}

A systematic review addressing the effect of topical steroids on CRS in CF patients did, however, including non-placebocontrolled-trials demonstrated significant benefits in CRS treatment ${ }^{(155)}$. There are no studies addressing an impact on sinonasal symptoms in CF patients with systemic steroids.

\subsubsection{Surgery}

CRS among CF patients is often refractory to medical therapy and thus sinus surgery is often recommended. Endoscopic sinus surgery in CF children and adults is a safe procedure despite anatomical variations in patients ${ }^{(156)}$. Several reports have shown that sinus surgery reduces sinonasal symptoms in CF patients although this is often only a temporary improvement with the need for revision surgery. In a study comprising 106 CF patients, the necessity for revision surgery was $28 \%$ within three years of surgery ${ }^{(157)}$. When describing the surgical procedures, several studies reported it was necessary to perform more extensive surgery, e.g. total ethmoidectomy, medial maxillectomy ${ }^{(158)}$ and various Draf procedures. Sinus surgery is also recommended to be performed in CF patients without chronic lung infection or with a transplanted lung in order to attempt to eradicate gramnegative bacteria in the paranasal sinuses, thereby avoiding or preventing re-colonisation of the lungs $\mathrm{s}^{(157,159)}$. In these cases it is important to address all the paranasal sinuses independent of the preoperative CT findings with the goal of extending the time to lung infection ${ }^{(109)}$ although even after sinus surgery the 
sinuses are not always easy to reach with local medication ${ }^{(160)}$. Children with CF undergoing sinus surgery may experience some improvement of lung function parameters, although this change may be temporary and not uniform ${ }^{(120)}$.

To conclude, several studies have evaluated the effect of sinus surgery on pulmonary function with divergent conclusions. When evaluating the reports, it is important to take into account the pre-operative lung infection status, the extent of sinus surgery, the post-operative treatment, and to keep in mind that the natural course of $C F$ is declining lung function.

\section{Detecting gram-negative sinus bacteria at an early stage is an important step towards eradicating the bacteria and avoiding a chronic bacterial sinus infection.}

Cultures from the middle meatus, cultures of nasal lavages, rising antibodies against $P$. aeruginos $a$, and declining lung function all indicate sinus infections. Nevertheless, gramnegative bacteria are also detected in the paranasal sinuses during surgery in CF patients, despite preoperative negative cultures obtained from the middle meatus or from nasal lavages $^{(161,162)}$. The importance of the microbial diversity is still unknown.

\subsection{Primary ciliary dyskinesia}

\subsubsection{Epidemiology and predisposing factors}

Primary ciliary dyskinesia (PCD), a collection of rare inherited disorders that affects motile cilia was initially termed 'immotile cilia syndrome' ${ }^{\prime(163)}$. Later, it was discovered that the cilia were not completely immotile and the name was changed to primary ciliary dyskinesia to distinguish it from secondary acquired causes $^{(164)}$.In the late 1990s, several genotype variants for PCD were identified. Despite the diversity of mutations that can cause PCD, clinical presentations tend to be consistent for all patients - productive cough, rhinitis, and recurrent upper and lower respiratory infections ${ }^{(165)}$. Patients with PCD have impaired mucociliary clearance, which leads to an elevated risk of developing recurrent respiratory infections from the time of birth ${ }^{(166)}$. Precise incidence and prevalence of PCD are difficult to estimate as there are no clinical and paraclinical criteria specific to the disease ${ }^{(167)}$. Thus, current epidemiological records for PCD might be largely underestimated due to the absence of a single, 'gold standard" diagnostic test ${ }^{(168,169)}$.

\section{Current estimated prevalence for PCD is 1:15- 30,000 live births ${ }^{(170)}$.}

In 2010, Kuehni et al. conducted a large international survey of 1,192 pediatric patients from 26 European countries and reported an incidence for PCD of 1 per 10,000-20,000 births ${ }^{(171)}$. With consanguinity as a risk factor for $P C D$, high prevalence exists in the Pakistani community in the United Kingdom $(1: 2265)^{(172)}$ and in Amish and Mennonite Communities in the United States ${ }^{(173)}$. While large discrepancies exist in epidemiological data for PCD, the challenge of confirming PCD is appreciated in all studies, with diagnostic practices possibly contributing to inaccurate estimates. Mirra et al. support this by demonstrating that many patients have an extraordinary number of physician visits (50-100) before their PCD is diagnosed. With this accounted for, they estimated the median age in Europe for diagnosis to be 5.3 years ${ }^{(174)}$.

\section{Key points | What's new since EPOS 2012}

- There is a high concordance of bacteria cultured from the paranasal sinuses (based on irrigations, swabs, or mucosal biopsies) and from the lungs ${ }^{(96)}$.

- In the western part of the world national screening programs on specific genetic disorders including CF have been implemented for newborns.

- Non-functional polymorphisms in the T2R38 gene correlate with sinus disease severity in patients with $\Delta \mathrm{F} 508$ homozygous mutation.

- Ivacaftor is a gene-based therapeutic agent approved, by the US Food and Drug Administration and the European Medicines Agency for the treatment of patients with specific CF mutations. Ivacaftor is a CFTR potentiator, which increases the opening probability of the CFTR channels at the cell surface, thus increasing the flow of ions through the channel.

- Ivacaftor has been shown to improve rhinologic QOL in patients with CF evaluated by SNOT-20.

- Tezacaftor in combination with Ivacaftor has been approved for the treatment of patients with F508del mutations, a type II mutation.

- The use of topical antibiotics correlates with improvement in symptom and endoscopic scoring in uncontrolled studies and is safe.

- Some studies recommend that sinus surgery is performed in CF patients without chronic lung infection or with a transplanted lung in order to attempt to eradicate gram-negative bacteria in the paranasal sinuses, thereby avoiding or preventing re-colonisation of the lungs. 


\subsubsection{Pathophysiology}

PCD is primarily caused by mutations in genes that affect motile cilia ${ }^{(175)}$ and is primarily inherited in an autosomal recessive manner, making family history of PCD and consanguinity among ascendants strong risk factors. There are more than 35 causal genes identified ${ }^{(176)}$, adding to the challenge of effectively detecting PCD at an early stage ${ }^{(177)}$.

The five genes most commonly mutated are DNAH5; DNAH11; DNAI1; CCDC39; CCDC40, but more than 20 other genes are associated with specific ethnic groups and geographic regions.

Knowles et al. estimates that of the 35 published PCD genes, $65-70 \%$ of patients with PCD have two mutations in one of these genes ${ }^{(178)}$. Given that cilia have many important functions within the body, defects in these structures manifest themselves in a broad spectrum of diseases. Associated conditions of PCD include hydrocephalus ${ }^{(169)}$, polycystic kidney disease ${ }^{(168,169)}$, biliary atresia ${ }^{(169)}$, scoliosis $(5 \%-10 \%)^{(178)}$, humoral immunodeficiency (6.5\%), congenital heart disease (5\%) and retinitis pigmentosa ${ }^{(169)}$. Situs inversus (i.e. Kartagener syndrome) exists in approximately half of all PCD cases. Both men and women diagnosed with PCD commonly present with fertility disorders due to the reproductive process largely dependent on ciliary function ${ }^{(169,176)}$.

\section{PCD has a strong association with history of chronic rhinosinusitis (CRS).}

PCD has a strong association with history of chronic rhinosinusitis (CRS) as it is part of the initial triad descriptor. In a critical evaluation of sinonasal disease in 64 adults with PCD, all patients reported a history of CRS ${ }^{(179)}$. PCD is associated with CRSWNP in $15 \%{ }^{(168)}$ to $30 \%$ of patients ${ }^{(180)}$, and is commonly seen in children with CRS. PCD also predisposes to bacterial infections commonly including Staphylococcus aureus, Moraxella catarrhalis, Haemophilus influenzae (181), Streptococcus pneumoniae ${ }^{(182)}$, and Pseudomonas aeruginos ${ }^{(183,}$ 184). Confirming these findings, a 2017 meta-analysis of 46 studies (1823 patients) with PCD or CF concluded that the most common organisms found in the sinuses of PCD patients were $H$. influenza followed by S. pneumoniae and P. aeruginosa ${ }^{(185)}$.

\section{The upper airway bacteriology mirrored the organisms recovered in the lower airways of $P C D$ patients ${ }^{(186,187)}$.}

\subsubsection{Diagnosis and differential diagnosis}

In the absence of hard clinical and paraclinical criteria for diagnosing PCD, confirming the diagnosis of PCD with clinical exam is a challenge because characteristic symptoms such as rhinitis, secretory otitis media, cough, and recurrent bronchitis are frequently seen in PCD- negative patients ${ }^{(188)}$. They frequently have a clinical history of lower airway disease and almost always chronic rhinitis. These symptoms can manifest as nasal discharge, episodic facial pain, and anosmia ${ }^{(188)}$.

Whenever nasal polyps are evident on nasal endoscopy of a child the diagnosis of PCD or CF must be considered.

Lucas et al. ${ }^{(189)}$ provide a decent set of conditions that support PCD diagnostic testing:

1. Situs inversus plus respiratory or nasal symptoms

2. Neonatal respiratory distress of unknown cause

3. Sibling with primary ciliary dyskinesia (PCD), particularly if symptomatic

4. Daily lifelong wet cough (note: maybe suppressed by child and under-recognized by parents)

5. If considering testing for cystic fibrosis, also consider testing for PCD particularly if rhinitis, rhinosinusitis or glue ear are present

6. Unexplained bronchiectasis

7. Serous otitis media in association with lower and upper airway symptoms

8. Cardiac disease associated with heterotaxy if there is suspicion of respiratory, nasal or ear problems

\subsubsection{Microscopy}

\section{It should be noted that cilia may appear normal in patients that present with symptoms strongly suggestive of $P C D$ due to mutations that can result in normal structure.}

Recent advances in technology have significantly improved diagnostic approaches to PCD. An electron microscropic analysis of cilia can yield valuable information about ciliary ultrastructure and function ${ }^{(188,190)}$. However, it should be noted that cilia may appear normal in patients that present with symptoms strongly suggestive of PCD due to mutations that can result in normal structure. In a respiratory epithelial biopsy with electron microscopy (EM), ciliary axonemes can be examined for defects in outer and inner dynein arms, radial spokes, and/or central microtubules ${ }^{(189,191)}$. This technique is sensitive to $70 \%$ of all PCD cases ${ }^{(192)}$. To assess cilia waveform, high speed videomicroscopy can be utilized to confirm PCD. While the technology requires experienced personnel to operate, high speed videomicroscopy is recommended as part of a panel of PCD diagnostic tests (168) - although the number of centers that can perform this worldwide is limited. Ciliary beat frequency calculations and visual assessment of ciliary motion without high speed recording devices is discouraged due to high potential for false positive and false negative results ${ }^{(168)}$. Several highly specialized centres are capable of growing primary respiratory cultures at an air liquid interface (ALI) with subsequent analysis of ciliary motion as an alternative approach to assess cilia dysfunction $^{(193)}$. 


\subsubsection{Genetics}

To test a deficiency in specific ciliary proteins such as DNAH5, DNAI2, DNALI1, and RSPH4A/RSPH1/RSPH9, immunofluorescence testing can be performed. Staining of these proteins can yield quantitative and qualitative results in determining if ciliary protein deficiencies explain the phenotype of a patient suspected of PCD. Genetic testing is also recommended as part of a panel of diagnostic PCD tests and has greatly improved the ability to diagnose PCD. With currently 35 genes known to underlie the pathophysiology of PCD, a multi-gene panel of 19 of these genes can be performed, and this is becoming more affordable, as several Clinical Laboratory Improvement Amendments (CLIA) approved labs and companies have recently begun to offer such services ${ }^{(178)}$. Genetic testing costs traditionally ranged from $\$ 1,500$ to $\$ 4,500$ ${ }^{(168)}$, which may have discouraged many individuals from being referred. Genetic panel tests can include full cystic fibrosis transmembrane regulator protein analysis ${ }^{(168)}$.

\subsubsection{Exhaled nasal nitric oxide}

A number of studies have shown that exhaled nitric oxide (NO), particularly nasal NO ( $\mathrm{nNO}$ ) production levels are low in PCD patients $(176,190)$. Nasal NO may then serve as a good diagnostic marker in assessing patients with symptoms suggestive of PCD. While a few patients with PCD have been shown to have normal NO levels, a high or normal nasal NO can assist physicians in excluding PCD as a differential, especially in patients that have a clinical history weakly suggestive of PCD ${ }^{(194)}$. Noone et al. showed in an experimental design involving 110 patients that there was a significant difference in the NO levels observed between PCD patients $(n=78)$ and the normal control group. NO measures for the PCD group had a clear separation from measures of the healthy control subjects, with a number of recordings below $100 \mathrm{nl} /$ minute $^{(190)}$. While the mechanism of low nNO in PCD is not well understood ${ }^{(195)}$, measurements of $\mathrm{nNO}$ is recommended as part of a panel of diagnostic tests for PCD in adults and children $\geq 5$ years old ${ }^{(196)}$.

\section{An nasal NO cutoff value of $<77 \mathrm{nl} / \mathrm{min}$ can allow detection of $P C D$ with a sensitivity and specificity of $98 \%$ and $>99 \%$, respectively, after excluding cystic fibrosis and acute viral respiratory infections (168).}

\subsubsection{Radiology}

Radiographic evaluation of PCD patients demonstrates that opacified paranasal sinuses on CT scans are very common and hypoplasia or aplasia of the frontal or sphenoid sinuses is found in $>50 \%$ of adult patients with PCD ${ }^{(197)}$.

\subsubsection{Treatment}

High-level evidence in managing patients with PCD is poor, with current treatment protocols mostly adopted from studies in CF or derived from small observational studies with limited follow-up period. Once a diagnosis is made, establishing a longterm relationship with an otorhinolaryngologist is encouraged. Paediatric patients with PCD are advised to visit a paediatric otorhinolaryngologist every six months ${ }^{(168,188)}$ while adult patients should visit their otorhinolaryngologists when needed (168).

Prolonged macrolide therapy has been shown to produce marked improvement in symptomatology of PCD due to the anti-inflammatory and immune-mediating properties of the antibiotic ${ }^{(180)}$.

Conservative therapy of CRS in PCD focuses on relieving symptoms. Therapeutic options include sinonasal irrigation with saline, topical steroids and long-term antibiotics. However, no prospective studies have so far evaluated the medical treatment of CRS in PCD. Presumably, sinonasal irrigation with saline is a safe method for clearing mucostasis in the upper airways as a result of impaired mucociliary clearance. Intranasal corticosteroids have a positive effect on CRS in general, and may also reduce inflammation in PCD. However, inhalation therapy with steroids is not recommended for the treatment of lower airway inflammation in PCD except in patients with concomitant asthma.

Surgical intervention (ESS) may be required when medical therapy has failed. A few minor case series and retrospective case series ${ }^{(198,199)}$ and a single prospective study ${ }^{(200)}$ have evaluated the potential benefits of CRS treatment with ESS in PCD and all these studies consistently show subjective benefit. However, further randomized controlled studies are needed to delineate the effects of surgery and adjunctive therapy.

\section{Key points | What's new since EPOS 2012}

- The number of genetic loci contributing to PCD has expanded to more than 35 .

- Diagnostic criteria now include nasal nitric oxide (nNO).

\subsection{Fungal rhinosinusitis}

Fungi are ubiquitous in our environment and with dedicated assessments they can be found in nasal mucus from almost all healthy and diseased sinuses ${ }^{(201)}$. However, there are several forms of sinus disease that are associated with fungi as pathogens. In these situations, rather than the fungi determining the disease process, it is usually the host immune state that determines the clinical presentation (Figure 8.6.1.). The definition and categorization of fungal rhinosinusitis is still controversial but the most commonly accepted system divides patients with normal immunity and immunocompromised patients ${ }^{(202)}$. For a patient with an immunocompetent state, fungi become a pathogen when they cannot be cleared by the mechanical action of mucus. In contrast to inert and non-viable material that settles on the mucus layer, fungi can multiply and create a ball or concretion of fungal hyphae. When this occurs, it becomes a fungal concretion and is widely referred to as a fungal ball. Fungal balls of the sinuses were previously referred 
to as 'mycetoma'. The fungal hyphae are not invasive, and the immune response is similar to other foreign body reactions in the immunocompetent patient. In an immunologically suppressed patient, the fungi can turn from ubiquitous material in our environment to a true invasive infective disease. It is likely that the type of fungi and the severity of immunosuppression bring about the variety of acute/chronic and granulomatous descriptions of invasive fungal rhinosinusitis. Aspergillus is common and a similar distribution of fungal species across simple fungal ball and invasive disease have been reported ${ }^{(203)}$, highlighting the importance of the host factors in the disease process. Finally, in a setting of an exuberant or hypersensitivity immune response, the fungi, if allowed to contact the mucosa for any length of time, induce a strong Th2 immune response and in turn leads to a polypoid inflammatory phenotype, referred to as Allergic Fungal Rhinosinusitis (AFRS). This can be so exuberant that distortion of the local anatomy, including the eye, cranial cavity and face is common.

Fungi are ubiquitous in our environment and with dedicated assessments they can be found in nasal mucus from almost all healthy and diseased sinuses.

There was much prior debate regarding the role of fungi in CRSwNP. Some authors had proposed that a response to fungi might be the basis for most Th2 dominated polypoid forms of CRS. However, subsequent research has not supported this ${ }^{(204)}$. Thus, this chapter will discuss these three phenotypes of 'fungal' related CRS. An intentional focus is made on AFRS as a unique phenotype, and its treatment, within the broader definition of a CRS.

\subsubsection{Fungal ball}

\subsubsection{Terminology}

Fungal ball Is a concretion or collection of fungal debris usually within a single sinus. The maxillary and sphenoid sinuses are most commonly affected. By definition, there is no invasive component to the fungal colonization. There may be an associated mixed inflammatory reaction in the involved sinus and occasionally this inflammatory reaction will involve sinuses within the same function unit, such as frontal and anterior ethmoid in the case of maxillary sinus fungal ball. To describe this entity, confusing or misleading terms such as mycetoma, aspergilloma or aspergillosis are best avoided ${ }^{(205)}$.

\subsubsection{Epidemiology and predisposing factors}

There is a reported female pre-disposition has high as 2:1(206) and others have suggested this but not to the same strength of association ${ }^{(207)}$. Fungal ball is a condition that affects most in the fifth and sixth decade ${ }^{(206)}$ with reported means of 55 years ${ }^{(208)}$ and $56 \pm 14$ years ${ }^{(209)}$. In a systematic review of isolated maxillary and sphenoid sinus opacifications, the reported age range was 38-59 years for the maxillary sinus and 38-57 years for the sphenoid sinus(210).

Fungal ball can be present for long periods with documented cases up to 17 years ${ }^{(211)}$. For newly diagnosed or formed fungal balls, enlargement is seen in $58 \%$ over time ${ }^{(211)}$. Although, fungal ball is often an incidental finding, most produce symptoms.

In 70 radiologically observed fungal balls, only $22-26.3 \%$ were asymptomatic $^{(203,211)}$.

Prior endodontic treatment is a risk factor for maxillary fungal ball formation. Case control studies with patients with fungal ball versus CRS controls demonstrated endodontic treatment was more common in both prevalence ( $89 \%$ vs. $37 \%, \mathrm{p}<0$. $01)$ and in the number of prior procedures $(1.39 \pm 0.86 \mathrm{vs}$. $0.53 \pm 0.81, \mathrm{p}<0.01)^{(212)}$. Such findings have been repeated with similar associations, demonstrating an increased prevalence of endodontic care ( $36 \%$ vs. $16 \%, p=0.001$ ) and number of teeth endodontically treated $(0.63 \pm 1.04$ vs. $0.27 \pm 0.61, p=0.001)$ and on a multivariate regression, endodontic treatment was strongly associated $(\operatorname{Exp}(B) 2.15[\mathrm{Cl} 1.26-3.67], \mathrm{p}=0.005)$ and asthma was negatively associated ${ }^{(213)}$.

Most local anatomical variants have been assessed as possible risk factors for fungal ball formation. Septal deviation amongst

Figure 8.6.1. An overview of the interaction of fungi and the human immune response.

\section{${ }_{E P O S} 2020^{\circ}$ Fungi and the human immune response}

\begin{abstract}
Allergic fungal
rhinosinusitis
\end{abstract}

Fungal ball

1
Invasive fungal rhinosinusitis 
other variants, including concha bullosa, has been implicated along with infundibulum length compared to normal controls $\left(9.71 \pm 1.43\right.$ vs. 8.6.23 $\pm 172, p<0.01^{(209)}$ but a repeat study could not demonstrate this septal association and even suggested an association with the deviation away from the involved side $\mathrm{e}^{(214)}$. The largest cross-sectional study, using the contra-lateral side as a control, of 538 patients could find no relationship with septal deviation, infraorbital (Haller) cells nor concha bullosa ${ }^{(206)}$. It is likely that anatomic variants are not a major contributor to fungal ball formation.

\section{It is likely that anatomic variants are not a major contributor to fungal ball formation.}

\subsubsection{Pathophysiology}

It is presumed that the fungal ball forms during a period of impaired mucociliary function. The relationship with endodontic treatment may simply be either that the sinus mucosa was inflamed either from periapical dental disease or from the procedure itself and this provided a period of impaired mucociliary clearance from the maxillary sinus. The potential of extrusion of root canal filling material into the sinus has been postulated as copper and zinc elements are found in both fungal ball and in endodontic materials and thought to be a cofactor for fungal growth ${ }^{(215)}$.

The role of primary ostiomeatal complex (OMC) obstruction has been assessed. Firstly, fungal ball was less likely to have OMC occlusion than CRS controls with similar maxillary sinus involvement ${ }^{(216)}$. Fungal ball patients were less likely to have frontal and anterior ethmoid radiologic changes. Sinus hypoventilation from simple OMC obstruction and thus providing an environment for growth seems unlikely and there are cases of fungal ball formation in the setting of a patent $\mathrm{OMC}^{(217)}$.

Neo-osteogenesis of maxillary sinus wall is common in fungal ball compared to normal patients and is independent of bacterial coinfection ${ }^{(218)}$. This bony change produces a smaller maxillary sinus, but a smaller maxillary sinus volume has been reported in patients with fungal ball of the maxillary sinus compared to the normal contralateral side. This finding was independent of hyperostosis on sub analysis ${ }^{(219)}$.

\subsubsection{Diagnosis and differential diagnosis}

DeShazo's initial criteria and description is still accurate for fungal ball(220).

1. Radiologic evidence of sinus opacification with or without associated flocculent calcifications

2. Mucopurulent, cheesy, or clay-like material within a sinus

3. A matted, dense conglomeration of hyphae separate from but adjacent to sinus respiratory mucosa

4. A chronic inflammatory response of variable intensity in the mucosa adjacent to fungal elements. This response includes lymphocytes, plasma cells, mast cells, and eosinophils without an eosinophil predominance or a granulomatous response. Allergic mucin is absent on hematoxylin-eosin-stained material.
5. No histologic evidence of fungal invasion of mucosa, associated blood vessels, or underlying bone visualized microscopically on Gomori methenamine silver or other special stains for fungus.

On radiology assessment, "calcifications" and erosion of the inner wall of the sinus are the two features most diagnostic for fungal ball(221). Although the term "calcifications' is used, they are probably more accurately referred to do as hyperdensities as they represent trace metal elements in the fungus. The hyperdensities are seen in the majority and reported to affect $52-77 \%(206,222,223)$. On cone beam tomography, the hyperdensities are less commonly seen ${ }^{(224)}$. On magnetic resonance imaging, fungal ball appears hypointense on T1-weighted images (64.7\%) and hyperintense on T2-weighted images (88.6.2\%) even in patients without X-ray hyderdensities ${ }^{(223)}$.

Single sinus AFRS might be similar, and some historical publications have used the term mycetoma to describe classic $\mathrm{AFRS}^{(225)}$ and thus the absence of a dominant eosinophil response, eosinophil mucin and a widely expanded sinus is still important, as per the above criteria, to separate fungal ball from isolated AFRS.

Isolated maxillary or sphenoid sinus opacification is a marker of neoplasia in $18 \%$ and malignancy in $7-10 \%$ of patients presenting with these radiologic findings. Clinicians should be wary of conservative management given the high incidence of neoplasia and consider a lower threshold for early surgical intervention ${ }^{(210)}$. Odontogenic rhinosinusitis can present with concomitant fungal ball or isolated maxillary sinus disease. However, in studies on single sinus opacification, $10-45 \%$ of isolated sphenoid sinus cases and $0-55 \%$ of isolated maxillary sinus cases are fungal balls (unpublished data)(210).

\section{Isolated maxillary or sphenoid sinus opacification is a marker of neoplasia in $18 \%$ and malignancy in $7-10 \%$ of patients presenting with these radiologic findings so clinicians should be wary of conservative management.}

\subsubsection{Treatment}

Surgery is the primary treatment for fungal balls. Although surgery was once thought to be 'curative' for fungal ball(205), there is evidence that persistent inflammation and recurrence can occur. Asymptomatic disease does not mean that surgery should be avoided ${ }^{(226)}$. In some studies, $16 \%$ of sphenoid fungal balls were associated with orbital infective complications and the loss of bone wall architecture was associated with this complication ${ }^{(208)}$. Additionally, intracranial infective complications can also be seen ${ }^{(227,228)}$. Although, fungal ball may follow an indolent course with disease present for many years ${ }^{(223)}$, the natural history of the condition is that low grade inflammation occurs, expansile changes are seen in the majority and infective complications can occur ${ }^{(229)}$. 


\subsubsection{Oral corticosteroids}

No data or studies have suggested a role for oral corticosteroids but they are often given as part of the initial perioperative care in the initial surgical removal.

\subsection{Topical corticosteroids}

No data or studies have suggested a role for topical corticosteroids but it is acknowledged that INCS are often given as part of the perioperative care after the initial surgical removal.

\subsection{Allergen immunotherapy (fungal or other)}

Immunotherapy does not play a role in the management of fungal ball and no studies have reviewed their use. Nor is inhalant allergy considered to play a role in the etiology of sinus fungal balls.

\subsection{Anti-fungals - topical or systemic}

Although it is recognized that fungal balls will develop in patients who might eventually be diagnosed with invasive fungal rhinosinusitis, it is their host immune factors that appear to predict this ${ }^{(203)}$. There are no controlled studies to provide data to support the use of antifungals in the absence of invasion.

\subsection{Other medical interventions}

Saline irrigations, concomitant use of antibiotics and intranasal corticosteroids are all reported in the perioperative care of sinus fungal ball, but no published data supports their use.

\subsection{Surgery}

Surgery is the mainstay of management of fungal ball. Most authors promote a simple antrostomy, and many reports have short-term follow up. However, a recurrence of the fungal ball itself has been reported in $4 \%$ and $3.2 \%$ in two studies with longer follow up ${ }^{(207,230)}$. Additionally, some authors have reported $50 \%$ of post treated fungal ball patients had inflammation and $14 \%$ residual debris with a simple antrostomy, whereas when an antrostomy was combined with an inferior meatal window, none had residual or inflammation ${ }^{(231)}$. Persistent dysfunction of the sinus cavity with mucostasis was reported to be as high as $18 \%{ }^{(217)}$ and some authors have proposed a medial maxillectomy for some maxillary fungal ball patients, especially in a smaller contracted sinus and when there are concerns of poor function ${ }^{(232)}$. Some studies suggest allergy and polyps are poor prognostic factors post-surgery ${ }^{(233)}$ but there is some uncertainty of the true conditions being treated in this study.

\subsubsection{Invasive fungal rhinosinusitis}

\subsubsection{Terminology}

Invasive fungal rhinosinusitis is a disease almost exclusively of immunocompromised patients, and defined as any state in which fungal hyphae can be seen 'within' the mucosal tissue, demonstrating classic angio-invasion or other infiltrative patterns ${ }^{(234)}$. Although originally several forms of invasive disease were described, granulomatous, chronic and fulminant, they all potentially represent an immunocompromised host reaction to the fungus ${ }^{(220)}$. Due to the variability in chronicity of the disease, some have divided it into acute (less than four weeks) or chronic (greater than four weeks) ${ }^{(235)}$. The type of fungus and the degree of host immune suppression is likely to create these differences and thus for the purpose of classification they are all forms of 'invasive fungal rhinosinusitis'.

\section{Invasive fungal rhinosinusitis is a disease almost exclusively of immunocompromised patients, and defined as any state in which fungal hyphae can be seen 'within' the mucosal tissue.}

\subsubsection{Epidemiology and predisposing factors}

Overall, diabetes (50\%) and haematologic malignancy (40\%) account for $90 \%$ of the immunosuppression reported in over 800 invasive rhinosinusitis patients ${ }^{(236)}$. Although neutropenia is often considered the most significant risk factor, patients with HIV/AIDS, iron overload, protein-energy malnutrition, or transplant patients on azole prophylaxis are also at risk ${ }^{(237)}$. Although invasive disease has been reported in patients without immunosuppression $^{(238,239)}$ these studies were either historical or had too few survivors to evaluate whether immunosuppression was truly excluded. A subtype of chronic invasive granulomatous rhinosinusitis has been described in otherwise healthy individuals. There are reports of such cases, often in older immunocompetent patients ${ }^{(240,241)}$ but are more prevalent from India ${ }^{(242-244)}$. Although invasive disease may occur in the absence of a well-defined immune suppression, it is likely to be uncommon.

\subsubsection{Pathophysiology}

Invasive fungal rhinosinusitis is any state in which fungal hyphae can be seen 'within' the mucosal tissue, demonstrating classic angio-invasion or other infiltrative patterns. The most common causative pathogens are the Zygomycetes (Rhizopus, Mucor, Rhizomucor) and the Aspergillus species. The phylum Zygomycota are part of the class Zygomycetes and order Mucorales and thus the term 'mucormycosis' is occasionally used. The disease process is characterized by angioinvasion of fungal hyphae (fungi within vessel walls and vascular lumen) producing thrombosis and mucosal infarction and subsequent tissue necrosis While a low grade sub-mucosal granulomatous inflammation with diffuse fibrosis and with limited fungal elements, has been described in immunocompetent hosts, this is mainly limited to India ${ }^{(243)}$. Aspergillus sp. have a uniform width, acute-branching septate (dichotomous) hyphae, often described as branching at 45 degrees, whereas Zygomycetes have non-septate hyphae with wide angle branching.

\subsubsection{Diagnosis and differential diagnosis}

The two most important diagnostic criteria for any form of 'invasive' fungal disease are ${ }^{(220)}$ :

1. Rhinosinusitis confirmed by radiological imaging (although this can be minimal) or show tissue destruction ${ }^{(245,246)}$ and

2. Histopathological evidence of hyphal forms within sinus mucosa, submucosa, blood vessels, or bone. 
There is no pathognomonic symptom for invasive fungal disease, but facial pain, facial swelling, nasal obstruction and fever, are most common and present in $50-65 \%$ of patients ${ }^{(236,}$ ${ }^{247)}$. Numbness of the face or palate, ophthalmoplegia (51\%) and/or cerebral involvement can follow quickly thereafter. The endoscopic appearance of necrosis is a hallmark sign. The middle turbinate is classically involved, as this is where aeroallergen deposition is thought to occur, but palatal, inferior turbinate and lateral wall necrosis is described ${ }^{(248)}$. Endoscopy is essential in the setting where radiologic changes are minimal.

The endoscopic appearance of necrosis is a hallmark sign Unilateral disease on radiology is typical ${ }^{(247,248)}$.Loss of contrast enhancement on magnetic resonance imaging is more sensitive $(86 \%)$ than computerized tomography $(69 \%)$ in detecting invasive fungal disease ${ }^{(249)}$. Frozen section analysis of tissue during surgery ${ }^{(250,251)}$ and in situ hybridization have both been used to define invasive disease ${ }^{(252)}$.

Serum analysis via PCR (serum or whole blood) and/or galactomannan for invasive aspergillosis can be useful. When both PCR and galactomannan for aspergillus were negative, the negative predictive value was $100 \%$ and, when both tests were positive, the positive predictive value was $88 \%{ }^{(253)}$. Galactomannan and (1,3)-beta-D-glucan are components of the Aspergillus cell wall and are therefore helpful in confirming a diagnosis of invasive Aspergillosis. Galactomannan and (1,3)-beta-D-glucan each have a sensitivity and specificity of around $80 \%$ in the detection of invasive aspergillosis from all body sites ${ }^{(253)}$.

\subsubsection{Treatment}

The three principals consistently reported in studies included:

1. Systemic antifungals therapy should be started

2. Patients should undergo, at least endoscopic surgical debridement of necrotic sinonasal tissue

3. The patient's immune suppression should be reduced when feasible

A large systematic review has been performed, 52 studies and 807 patients, 398 of whom were used for prognostic factor analysis. They reported an overall survival rate of about $50 \%$, with the following factors predicting worsened survival: advanced age, aplastic anaemia (20\%), renal / hepatic failure (24\%), intracranial and cavernous sinus extension (25\%), and neutropenia (29\%). They reported improved survival in patients who were diabetic (50\%), received liposomal amphotericin $\mathrm{B}(60 \%)$ or underwent open (54\%) or endoscopic sinus surgery (ESS) $(64 \%)^{(236)}$. However, survival and outcomes from invasive fungal rhinosinusitis remained relatively stable, over the past 20 years, with survival at $50-60 \% \%^{(236,254)}$.

\section{Patients should undergo, at least endoscopic surgical debridement of necrotic sinonasal tissue.}

Prognostic factors associated with worse survival included: haematologic malignancy (hazard ratio $[\mathrm{HR}]=3.7$ ); recent chemotherapy $(\mathrm{HR}=2.3)$; recent bone marrow transplant (BMT) $(\mathrm{HR}=2.5 ;)$; and infection with atypical fungi $(\mathrm{HR}=3.1)$. Improved survival in univariate analysis included increasing $A 1 c \%(H R=0.7)$ and surgical debridement $(\mathrm{HR}=0.1)$. One third of patients with a haematologic malignancy and invasive fungal rhinosinusitis had an absolute neutrophil count (ANC) $>1000$ at the time of diagnosis ${ }^{(247)}$. The role of an absolute neutrophil count as a prognostic factor is still debated ${ }^{(247,255)}$.

\subsection{Oral corticosteroids}

Oral corticosteroids are generally avoided as part of the treatment in invasive fungal disease due the potential additive immunosuppressive effects. However, their use may be balanced by the need to reduce oedema associated with orbital or cerebral involvement.

\subsection{Topical corticosteroids}

Topical corticosteroids do not play a role in the management of invasive fungal rhinosinusitis and no studies have review their use.

\subsection{Allergen immunotherapy (fungal or other)} Immunotherapy does not play a role in the management of invasive fungal rhinosinusitis and no studies have reviewed their use.

\subsection{Anti-fungals - topical or systemic}

Although patients with invasive fungal rhinosinusitis need surgical debridement of involved tissue, medical therapy is critical for treatment of invasive fungal rhinosinusitis. Amphotericin and the liposomal version have been shown to have positive predictive value to survival and time to commencement associated with survival ${ }^{(236)}$. In the setting of Aspergillus species, voriconazole maybe superior over amphotericin for aspergillosis ${ }^{(256)}$ and voriconazole is recommended ${ }^{(257)}$. The newer Azoles may have better bioavailability and less toxicity from studies on invasive aspergillosis that includes invasive fungal rhinosinusitis ${ }^{(258)}$. Transcutaneous retrobulbar injection of amphotericin B has been reported. Kalin et al. summarized the six cases documenting its use ${ }^{(259)}$. The amount of amphotericin injected ranged from 1-1.5 $\mathrm{ml}$ at concentrations ranging from 1-3.5 mg/ $\mathrm{ml}$ on a daily to weekly basis. Two patients died for reasons not directly related to fungal infection, but visual acuity stabilized or improved in all cases.

Conclusions
$\begin{aligned} & \text { Systemic anti-fungal therapies are an essential part } \\ & \text { of managing invasive fungal disease }\end{aligned}$
Transcutaneous retrobulbar injection of
amphotericin B may be beneficial in orbital
involvement in invasive fungal rhinosinusitis
8.6.3.5.5. Other medical interventions
Immune stimulating interventions have variably been reported
and in multivariate analyses were associated with a 70\%
reduction in mortality at one month
been used with some evidence for the diabetic patient ${ }^{(260,261)}$


and there is some evidence for iron chelating agents (desfirozx) ${ }^{(262)}$. These studies include invasive fungal rhinosinusitis within a population of patients with invasive soft tissue fungal disease.

\subsection{Surgery}

Multiple studies have shown that ESS is an independent positive prognostic factor for survival $(236,247,248,263)$.

The extent of surgical resection is largely guided by preoperative imaging and the tissue appearance during surgery. Intraoperative frozen section has been employed to guide surgical debridement. Although frozen section has an excellent PPV (100\%) for invasive disease, the NPV is $70 \%$, so a negative result does not guarantee a clear margin ${ }^{(250,264)}$.

Timing of surgery is less important than the initiation of antifungal therapy ${ }^{(265)}$ but delay of surgery $>30$ days of diagnosis predicted a poorer outcome.

\section{Managing the orbit}

At the time of diagnosis, $50-60 \%$ of patients will have orbital involvement ${ }^{(234,236)}$. In 15 patients with orbital involvement, survival was similar to patients with disease limited to the sinuses, and only one of those patients had an exenteration ${ }^{(263)}$. A meta-analysis of 224 patients with orbital mucormycosis, reported no survival benefit from orbital exenteration except in patients with fever over $38,6^{\circ} \mathrm{C}(101.58 \mathrm{~F})^{(266)}$. Similarly, Turner et al. found a similar outcome with orbital removal and survival(236) but authors from both studies acknowledge the bias that exists in assessing these outcomes as those patients with significant orbital disease were likely to have been offered surgical removal whereas limited disease was conservatively managed. Transcutaneous injection of amphotericin remains an option as described previously. Of note, in a study on patients only with orbital involvement, 2/14 patients survived and both had orbital removal ${ }^{(238)}$. Persistent enhancing tissue is a marker of infected tissue and thus many suggest conservative treatment when enhancement can still be seen on imaging.

\section{Conclusion Level}

Surgical debridement is an essential part of invasive $3 a$ fungal rhinosinusitis management

\subsubsection{Allergic fungal rhinosinusitis (AFRS)}

\subsubsection{Terminology}

Allergic fungal rhinosinusitis (AFRS) is a subset of polypoid chronic rhinosinusitis that is characterized by the presence of eosinophilic mucin with non-invasive fungal hyphae within the sinuses and a Type I hypersensitivity to fungi. It was originally described by Safirstein ${ }^{(267)}$ and Katzenstein ${ }^{(268)}$, but some clinicians who work in parts of the world where AFRS is not so common, have questioned whether the condition really exists as a separate clinical entity or is part of eosinophilic CRS or CRSwNP. Although there is some controversy about the definition of AFRS, and even whether it is a distinct clinical phenotype of CRSwNP, most terminology groups define it as a unique and distinct phenotype ${ }^{(269,270)}$. Bent and Kuhn included lgE sensitization as an inclusion criteria in their original description ${ }^{(271)}$. AFRS is, for the most part, considered an IgE mediated mucosal hypersensitivity directed against fungal antigens deposited on sinus mucosa ${ }^{(272)}$. The EPOS steering group discussed whether the term 'eosinophilic fungal rhinosinusitis' would be a better umbrella term for fungal rhinosinusitis but it was agreed that allergic fungal rhinosinusitis' should be retained as the principle term due to common usage, recognising that not all cases have evidence of an allergic reaction to fungi.

\section{Allergic fungal rhinosinusitis (AFRS) is a subset of polypoid chronic rhinosinusitis that is characterized by the presence of eosinophilic mucin with non-invasive fungal hyphae within the sinuses and a Type I hypersensitivity to fungi.}

\subsubsection{Epidemiology and predisposing factors}

AFRS accounts for about $5 \%$ to $10 \%$ of CRS cases $^{(273,274)}$. Patients presenting with AFRS do so at a younger age than other forms of CRS and with a greater proportion of females ${ }^{(275)}$. AFRS also has an association with a lower socioeconomic demographic, African Americans (in North America) and those living in warmer climates near the coast ${ }^{(276,277)}$. Atopy is a pre-defining feature of patients with AFRS and concomitant allergic disease, such as allergic rhinitis and childhood onset asthma, are common in this group.

\subsubsection{Pathophysiology}

The defining pathophysiology in AFRS is sensitization to the causative fungus as a primary and requisite feature along with mucin colonized with non-invasive fungus ${ }^{(278)}$. Although fungal sensitization may exist in CRSwNP, typically lgE levels are higher in AFRS ${ }^{(273,274)}$ studies that define AFRS as a unique phenotype in CRSwNP (Table 8.6.1.).

\subsubsection{Diagnosis and differential diagnosis} Ideally all five of the major criteria in the original Bent-Kuhn diagnostic criteria should be met to make the diagnosis as three of the five are common in most CRSwNP. These major criteria consist of the following ${ }^{(271)}$ :

1. Nasal polyposis;

2. Fungi on staining;

3. Eosinophilic mucin without fungal invasion into sinus tissue;

4. Type I hypersensitivity to fungi and;

5. Characteristic radiological findings with soft tissue differential densities on CT scanning and unilaterality or anatomically discrete sinus involvement.

The minor criteria include bone erosion, Charcot Leyden Crystals, unilateral disease, peripheral eosinophilia, positive fungal culture along with prior criteria from DeShazo ${ }^{(279)}$ :

1. the demonstration of the characteristic eosinophil-rich allergic mucin visually or histopathologically ${ }^{(280)}$;

2. a positive fungal stain or culture from the sinus at surgery;

3. the absence of immunodeficiency or diabetes; 
Table 8.6.1. Studies that define AFRS as a unique phenotype in CRSwNP.

\begin{tabular}{|c|c|c|c|}
\hline & AFRS & CRSwNP & Ref \\
\hline Demographics & $\begin{array}{l}\text { Warmer coastal climates } \\
\text { Younger } \\
\text { Female preponderance }\end{array}$ & $\begin{array}{l}\text { Older age at presentation } \\
\text { unless CCAD }\end{array}$ & $\begin{array}{l}\text { Lu-Myers et al. } 2015^{(275)} \\
\text { Miller et al. 2014(277) } \\
\text { Wise et al. } 2008^{(276)} \\
\text { Ferguson et al. } 2000^{(484)}\end{array}$ \\
\hline Comorbidities & $\begin{array}{l}\text { AR } \\
\text { Asthma (less) }\end{array}$ & $\begin{array}{l}\text { Adult onset asthma more } \\
\text { severe }\end{array}$ & Manning et al. $1998^{(485)}$ \\
\hline Associated ABPA & Uncommon & Rare & Shah et al. $2001^{(486)}$ \\
\hline Secretions & "Peanut butter" mucin & "Chewing gum" mucin & \\
\hline Lund Mackay scores & Very high & High & \\
\hline CT changes & $\begin{array}{l}\text { Hyper attenuation } \\
\text { Unilateral }\end{array}$ & Less likely to have bone erosion & Ghegan et al. $2006^{(487)}$ \\
\hline Fungal colonization & $\begin{array}{l}\text { Easily identified } \\
\text { Aspergillus species most common (others } \\
\text { include: Bipolaris, Curvularia) }\end{array}$ & $\begin{array}{l}\text { Alternaria and Cladosporium } \\
\text { species }\end{array}$ & $\begin{array}{l}\text { Granville et al. } 2004^{(488)} \\
\text { Ferguson et al. } 2000^{(484)}\end{array}$ \\
\hline Immune status & Atopic & $\begin{array}{l}\text { Allergen sensitization } \\
\text { approaches background rate }\end{array}$ & \\
\hline Fungal sensitization & $\begin{array}{l}\text { Multiple fungi } \\
\text { IgG to fungi more common } \\
\text { Greater fungal specific lgE }\end{array}$ & Less common & $\begin{array}{l}\text { Hutcheson et al. } 2010^{(489)} \\
\text { Stewart et al. 2002 }\end{array}$ \\
\hline Immunohistochemistry & Fungal specific IgE present in tissues & Poorly selected lgE in mucosa & $\begin{array}{l}\text { Collins et al. 2004(491) } \\
\text { Pratt et al. } 2010^{(492)}\end{array}$ \\
\hline Serum IgE & Higher (500 IU/mL) & Variable & $\begin{array}{l}\text { Hutcheson et al. } 2010^{(489)} \\
\text { Bakhshaee et al. } .^{(274)} \\
\text { Telmesani et al. } 2009^{(493)}\end{array}$ \\
\hline Staph. aureus co-infection & High & Less common & $\begin{array}{l}\text { Clark et al. 2013 } \\
\text { Dutre et al. } 2013^{(494)}\end{array}$ \\
\hline T cell responses & CD4+ only & Both CD4+ and Cd8+ & Pant et al. $2014^{(496)}$ \\
\hline Gene expression & $\begin{array}{l}\text { Lacking Cathepsin B, } \\
\text { sialyltransferase 1, GM2 ganglioside activator } \\
\text { protein, and S100 } \\
\text { calcium-binding protein }\end{array}$ & Broad activation & Orlandi et al. $2007^{(497)}$ \\
\hline Serum proteomics & $\begin{array}{l}\text { Spectrometry able to identify patients with } \\
\text { AFRS with } 84 \% \text { sensitivity and } 90 \% \text { specificity } \\
\text { to CRSwNP }\end{array}$ & Broad & Das et al. $2008^{(498)}$ \\
\hline HLA-MHC class II associations & HLA-DQB $1 * 03$ common & Less common & Schubert et al. 2004(499) \\
\hline
\end{tabular}

Table 8.6.1. compares AFRS with the broader group of eCRS or CRSwNP patient features.

Clinically, the younger allergic patient with unilateral disease and the typical allergic "peanut-butter-like" mucin is classic and this mucin is different from the 'chewing gum' mucin commonly associated with eosinophilic CRS ${ }^{(281,282)}$.

Imaging with computed tomography shows densely packed hyperdensities in the sinuses with expansion and erosion of the bony walls. These are a combination of the various metals (e.g. iron, magnesium, and manganese) concentrated by the fungal organisms as well as the low water and high protein content of the mucin ${ }^{(283)}$. On magnetic resonance imaging, signal voids occur on both $\mathrm{T} 1$ and $\mathrm{T} 2$ sequences. The high protein and low free water content in allergic mucin along with the presence of calcium, air, and paramagnetic metals, such as iron, magnesium, and manganese creates this classic pattern ${ }^{(283)}$ and is not from ferromagnetic elements, as previously speculated ${ }^{(284)}$.

Imaging with computed tomography shows densely packed hyperdensities in the sinuses with expansion and erosion of the bony walls.

AFRS has these defining features: 1) the fungi never invade the sinus tissue, and 2) the mucin of AFRS is the product of an allergic hypersensitivity reaction to the fungi as opposed to a simple overgrowth of mycelial elements as is seen in fungus balls.

\subsubsection{Treatment}

Unlike the management of classical CRS, the foundation of AFRS treatment is surgery. The vast majority of clinical studies in the AFRS literature indicate that medical therapy alone is usually ineffective in alleviating symptoms and that surgical intervention, alone or in combination with medical therapy, leads to improved clinical outcomes. Treatment of AFRS almost always requires surgical debridement of the involved sinuses, thus removing the antigenic stimulation for AFRS patients, but also provides wider access for surveillance, clinical debridement, and application of topical medication. Oral corticosteroids decrease recurrence after surgery, but other adjunctive pharmacologic agents, including topical and oral antifungal agents, have less evidence base for effectiveness.

Most reports on treatment options for AFRS are combined into larger series addressing CRSwNP patients and this issue is 
Table 8.6.2. Systemic corticosteroids for the treatment of patients with AFRS.

\begin{tabular}{|c|c|c|c|c|c|}
\hline Study & Methods & Participants & Interventions & Outcomes & Results \\
\hline $\begin{array}{l}\text { Prasad et al. } \\
2017^{(286)}\end{array}$ & $\mathrm{RCT}$ & $\begin{array}{l}60 \text { AFRS post- } \\
\text { surgery }\end{array}$ & $\begin{array}{l}6 \text { month postoperative corticosteroid use } \\
\text { (prednisolone, } 1 \mathrm{mg} / \mathrm{kg} / \mathrm{day} \text {, for } 1 \text { week } \\
\text { preoperatively and } 0.5 \mathrm{mg} / \mathrm{kg} / \text { day for } 4 \\
\text { weeks, oral prednisolone } 0.4 \mathrm{mg} / \mathrm{kg} / \text { day for } \\
\text { the next } 4 \text { weeks, tapered to } 0.2 \mathrm{mg} / \mathrm{kg} / \text { day } \\
\text { for the next } 2 \text { months and to } 0.1 \mathrm{mg} / \mathrm{kg} / \text { day } \\
\text { for the last } 2 \text { months } \\
2 \text { month taper (prednisolone, } 1 \mathrm{mg} / \mathrm{kg} / \mathrm{day} \text {, } \\
\text { for } 1 \text { week preoperatively and } 0.5 \mathrm{mg} / \mathrm{kg} / \\
\text { day for } 4 \text { weeks postoperatively) }\end{array}$ & Recurrence & $\begin{array}{l}6 \text { month } \\
\text { corticosteroid use } \\
\text { associated with less } \\
\text { recurrence }\end{array}$ \\
\hline $\begin{array}{l}\text { Rupa et al. } \\
2010^{(285)}\end{array}$ & RCT & $\begin{array}{l}24 \text { AFRS post- } \\
\text { surgery }\end{array}$ & $\begin{array}{l}\text { Oral prednisolone ( } 50 \mathrm{mg} \text { qd } \mathrm{x} 6 \text { weeks, then } \\
\text { additional } 6 \text { week taper) } \\
\text { - Placebo } \\
\text { - Both groups used itraconazole orally and } \\
\text { INCS }\end{array}$ & $\begin{array}{l}\text { Symptom scores } \\
\text { at } 6 \text { weeks and } 12 \\
\text { weeks } \\
\text {. Endoscopic scores } \\
\text {. Adverse effects }\end{array}$ & $\begin{array}{l}\text { Corticosteroid } \\
\text { group significantly } \\
\text { improved over } \\
\text { placebo }\end{array}$ \\
\hline $\begin{array}{l}\text { Ikram et al. } \\
2007^{(287)}\end{array}$ & $\begin{array}{l}\text { Retrospective } \\
\text { cohort }\end{array}$ & $\begin{array}{l}63 \text { AFRS post- } \\
\text { surgery }\end{array}$ & $\begin{array}{l}\text { Postoperative corticosteroids }(n=30) \\
\text { No post-operative corticosteroids }(n=33 \text { ) }\end{array}$ & $\begin{array}{l}\text { Recurrence (at } \\
2 \text { yrs post-surgery) }\end{array}$ & $\begin{array}{l}\text { Favours } \\
\text { corticosteroid use } \\
\text { postoperative } \\
\text { with lower } \\
\text { recurrence } 15.2 \% \text { vs. } \\
50 \%\end{array}$ \\
\hline
\end{tabular}

covered elsewhere in this document. It is, therefore, difficult to discern if there are varying effects in the AFRS population as opposed to the entire CRSwNP population. In general, medical therapies have been divided into oral and topical steroids, oral and topical anti-fungals, immunotherapy and others. In all but the mildest cases of AFRS, it is felt that medical therapy alone without surgical intervention, is not effective in the long term, thus most efficacy studies examining medical treatments have been performed postoperatively.

Medical therapy alone is usually ineffective in alleviating symptoms and that surgical intervention, alone or in combination with medical therapy, leads to improved clinical outcomes

\subsection{Oral corticosteroids}

Oral corticosteroid studies specific to AFRS patients have generally been conducted in the postoperative setting where benefit has been demonstrated. There are several controlled studies (Table 8.6.2.). In a randomized double blinded, placebo-controlled trial of $n=24$ AFRS patients examining the effectiveness of postoperative oral steroids, as well as the side effects, patients received oral prednisolone $(50 \mathrm{mg} \mathrm{qd} \times 6$ weeks, then additional six week taper) or placebo for two weeks after surgery ${ }^{(285)}$. All patients received fluticasone nasal spray and oral itraconazole for 12 weeks. At 12-week follow up, all 12 patients in the corticosteroid group were asymptomatic and only one in the placebo arm. All 12 patients in the corticosteroid group suffered some weight gain, five developed Cushingoid features and one developed steroid induced diabetes mellitus. In what is best described as nonblinded randomized trial, Prasad et al. describe the management of 30 patients with AFRS with six month postoperative corticosteroid use (prednisolone, 1 $\mathrm{mg}$ / $\mathrm{kg} /$ day, for one week preoperatively and $0.5 \mathrm{mg} / \mathrm{kg} /$ day for four weeks, oral prednisolone $0.4 \mathrm{mg} / \mathrm{kg} /$ day for the next four weeks, tapered to $0.2 \mathrm{mg} / \mathrm{kg} / \mathrm{day}$ for the next two months and to $0.1 \mathrm{mg} /$ $\mathrm{kg} /$ day for the last two months) compared to a group of 30 AFRS patients tapering within two months (prednisolone, $1 \mathrm{mg} / \mathrm{kg} /$ day, for one week preoperatively and $0.5 \mathrm{mg} / \mathrm{kg} /$ day for four weeks postoperatively). This study concluded that recurrence and further surgery was less likely in the six month group $(10 \%$ vs. $30 \%)^{(286)}$. See Figure 8.6.2. for the combined odds ratio of prevention of recurrence.

In a retrospective cohort by Ikram et al. a two year followup of AFRS patients treated with and without postoperative

Figure 8.6.2. Forrest plot of the effect of oral corticosteroids for the prevention of recurrence in AFRS.

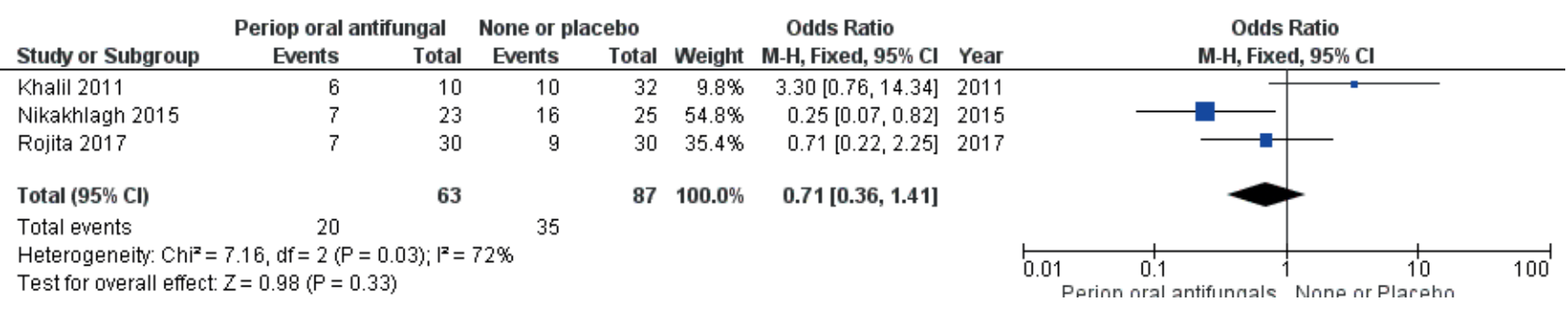


corticosteroid demonstrated a $15.2 \%$ vs. $50 \%$ recurrence along with a standardized topical corticosteroid regime ${ }^{(287)}$. Kinsella et al. reported a similar finding in 25 patients treated and none of the eight patients with recurrent disease were treated with postoperative corticosteroid ${ }^{(288)}$. Schubert et al. utilized a minimum two-month postoperative corticosteroid regime in a case series of 67 AFRS patients and demonstrated benefit in those utilizing therapy up to 12 months. Those on oral corticosteroid had the best outcomes with reduced recurrence and prolonged the time to revision surgery ${ }^{(289)}$.

Landsberg et al. demonstrated that preoperative corticosteroids given at $1 \mathrm{mg} / \mathrm{kg}$ for 10 days prior to surgery, had a more dramatic effect in AFRS compared to CRSwNP patients, with near normalization of the mucosa in the AFRS group at the time of surgery and a similar response on computed tomography ${ }^{(290)}$. Similarly, Woodworth et al. demonstrated a significant reduction in AFRS patients with postoperative corticosteroid over three weeks compared to eCRS and control patients ${ }^{(291)}$. A number of other non-placebo controlled case series have been reported with highly variable dosing protocols and durations, but generally reporting a positive effect when using postoperative oral corticosteroids ${ }^{(272,292)}$.

\section{Conclusions}

Level

Preoperative systemic corticosteroids are likely to reduces AFRS mucosa inflammation and radiologic scores

Postoperative systemic corticosteroids improve short-term outcomes in AFRS

Postoperative systemic corticosteroid are likely to reduce long term recurrence in AFRS

\subsection{Topical corticosteroids}

AFRS patients have been included in the "eCRS or CRSwNP" subgroups of other studies on intranasal corticosteroids with a general consensus that they are beneficial, safe and well tolerated $^{(293)}$. There are several studies that assess INCS in a dedicated AFRS population. A case-controlled study of surgery alone vs. surgery plus the combination of postoperative oral and topical steroid spray in AFRS patients demonstrated benefits for the combined therapy at a minimum of two year follow up, as $50 \%$ of the no steroid group recurred, while only $15 \%$ of the combined steroid group recurred ${ }^{(287)}$. Another study of low quality but an RCT, compared 34 postoperative AFRS patients which were randomized into three arms: (1) itraconazole + nasal douche $(n=11)$; (2) topical nasal steroid + nasal douche $(n=12)$; and (3) nasal douche alone $(n=11)$. Normal mucosa was observed similarly between INCS and saline only groups (16 vs. $14 \%, p=0.22$ ). The type of topical steroid used was not defined and the study was both underpowered and incompletely reported or analyzed ${ }^{(294)}$.

Dai et al. describes a study of 30 AFRS patients, randomized in a unblinded controlled study, to nebulized budesonide or topical nasal sprays. Nebulized budesonide was more effective in preventing recurrence compared to intranasal corticosteroid spray $(26.7 \% \text { vs. } 0 \%, p=0.03)^{(295)}$.

Several studies confirm that non-compliance with INCS is associated with recurrence in AFRS (Table 8.6.3.)(286, 287).

$\begin{array}{ll}\text { Conclusions } & \text { Level } \\ \begin{array}{l}\text { Non-standard INCS (nebulization) is better than } \\ \text { simple INCS sprays }\end{array} & 1 \mathrm{~b} \\ \text { INCS of any type may be useful in controlling AFRS } & 3 \mathrm{~b}\end{array}$

Table 8.6.3. Nasal corticosteroids for the treatment of patients with AFRS.

\begin{tabular}{|c|c|c|c|c|c|}
\hline Study & Methods & Participants & Interventions & Outcomes & Results \\
\hline $\begin{array}{l}\text { Dai et al. } \\
2017^{(295)}\end{array}$ & $\mathrm{RCT}$ & $\begin{array}{l}30 \text { AFRS } \\
\text { post-surgery }\end{array}$ & $\begin{array}{l}\text { - Budesonide transnasal nebulization }(n=15) \\
\text { - Topical nasal steroids }(n=15)\end{array}$ & $\begin{array}{ll}\cdot & \text { Symptoms } \\
\cdot & \text { Lund Mackay scores } \\
\cdot & \text { Kupferberg grades } \\
\cdot & \text { Adverse events }\end{array}$ & $\begin{array}{l}\text { Nebulised budesonide } \\
\text { superior to INCS spray in } \\
\text { reduction of recurrence } \\
\text { (27\% vs. 0\%) }\end{array}$ \\
\hline $\begin{array}{l}\text { Gupta et al. } \\
2007^{(294)}\end{array}$ & $\mathrm{RCT}$ & $\begin{array}{l}34 \text { AFRS } \\
\text { post-surgery }\end{array}$ & $\begin{array}{l}\text { - Oral itraconazole (200mg BD) and nasal douche } \\
(n=11) \\
\text {. Topical steroids (budesonide } 100 \mathrm{mg} B D) \text { and } \\
\text { nasal douche }(n=12) \\
\text {. Nasal douches only }(n=11)\end{array}$ & Kupferberg grades & $\begin{array}{l}\text { No differences between } \\
\text { the groups }\end{array}$ \\
\hline
\end{tabular}

Table 8.6.4. Immunotherapy for the treatment of patients with AFRS.

\begin{tabular}{|c|c|c|c|c|c|}
\hline Study & Methods & Participants & Interventions & Outcomes & Results \\
\hline $\begin{array}{l}\text { Bassichis et } \\
\text { al. } 2001^{(298)}\end{array}$ & $\begin{array}{l}\text { Retrospective } \\
\text { cohort }\end{array}$ & $\begin{array}{l}60 \text { AFRS post- } \\
\text { surgery }\end{array}$ & $\begin{array}{l}\text { Immunotherapy with standard care } \\
(\mathrm{n}=36) \\
\text {. Standard care alone ( } \mathrm{n}=24) \\
\text { - Immunotherapy to both fungal and non- } \\
\text { fungal antigens (minimum } 12 \mathrm{mths} \text { ) }\end{array}$ & $\begin{array}{l}\text { Revision surgery } \\
\text {. Clinic intervention }\end{array}$ & $\begin{array}{l}\text { Less revision surgery } \\
\text { ( } 11 \% \text { vs. } 33 \%) \text { and less } \\
\text { clinic visits in the IT } \\
\text { treated arm ( } 4.79 \text { vs. } \\
\text { 3.17) }\end{array}$ \\
\hline $\begin{array}{l}\text { Folker et al. } \\
1998^{(300)}\end{array}$ & $\begin{array}{l}\text { Retrospective } \\
\text { cohort }\end{array}$ & $\begin{array}{l}22 \text { AFRS post- } \\
\text { surgery }\end{array}$ & $\begin{array}{l}\text { Immunotherapy with standard care } \\
(n=11) \\
\text {. Standard care alone ( } n=11) \\
\text { - Immunotherapy to both fungal and non- } \\
\text { fungal antigens (mean } 33 \text { months) }\end{array}$ & $\begin{array}{l}\text { Chronic Sinusitis } \\
\text { Survey } \\
\text {. Kupferberg grades } \\
\text {. Corticosteroid use } \\
\text { (oral and topical) }\end{array}$ & $\begin{array}{l}\text { All outcomes better in } \\
\text { the IT treated group }\end{array}$ \\
\hline
\end{tabular}


Table 8.6.5. Oral antifungals for the treatment of patients with AFRS.

\begin{tabular}{|c|c|c|c|c|c|}
\hline Study & Methods & Participants & Interventions & Outcomes & Results \\
\hline $\begin{array}{l}\text { Verma et al. } \\
2017^{(307)}\end{array}$ & RCT & $\begin{array}{l}100 \text { AFRS } \\
\text { assessed post- } \\
\text { surgery }\end{array}$ & $\begin{array}{l}\text { - Itraconazole ( } 4 \text { weeks) preoperative }(n=25) \\
\text {. Itraconazole ( } 4 \text { weeks) post-operative }(n=25) \\
\text {. Surgery with antifungal therapy ( } n=50) \\
\text { douching to all groups } \\
\text { traconazole } 200 \mathrm{mg} \mathrm{(} 5 \mathrm{mg} / \mathrm{kg} \text { in children) } \\
\text { twice daily for } 2 \text { days followed by } 100 \mathrm{mg} \mathrm{(3} \\
\mathrm{mg} / \mathrm{kg} \text { in children) twice daily for remaining } 4 \\
\text { weeks therapy }\end{array}$ & $\begin{array}{l}\text { Symptoms } \\
\text { Kupferberg } \\
\text { grades } \\
\text { Radiology } \\
\text { (LM scores) } \\
\text { All assessed } \\
\text { at } 24 \text { weeks }\end{array}$ & $\begin{array}{l}\text { Although both preoperative } \\
\text { and postoperative itraconazole } \\
\text { showed significant improvement } \\
\text { in the SNOT, LM, and Kupferberg's } \\
\text { grades in the follow-up period, } \\
\text { the difference was only seen } \\
\text { between no antifungal and the } \\
\text { preoperative antifungal group } \\
\text { but included endoscopy and } \\
\text { radiology but SNOT scores were } \\
\text { similar. }\end{array}$ \\
\hline $\begin{array}{l}\text { Rojita et al. } \\
2017^{(308)}\end{array}$ & RCT & $\begin{array}{l}60 \text { AFRS post- } \\
\text { surgery }\end{array}$ & $\begin{array}{l}\text { Oral corticosteroid (prednisone } 30 \mathrm{mg} / \text { daily } \\
\text { for } 1 \mathrm{mth} \text { ) then INCS to } 6 \text { months } \\
\text {. Itraconazole } 100 \mathrm{mg} \text { bd for } 6 \text { months }\end{array}$ & $\begin{array}{l}\text { Symptoms } \\
\text { Kupferberg } \\
\text { grades } \\
\text { - Serum IgE } \\
\text {. Recurrence }\end{array}$ & $\begin{array}{l}\text { No difference between groups } \\
\text { Only } 23 \text { and } 26 \% \text { of patients had } \\
\text { normal mucosa at } 6 \text { mths }\end{array}$ \\
\hline $\begin{array}{l}\text { Nikakhlagh } \\
\text { et al. } \\
2015^{(500)}\end{array}$ & RCT & $\begin{array}{l}50 \text { AFRS post- } \\
\text { surgery }\end{array}$ & 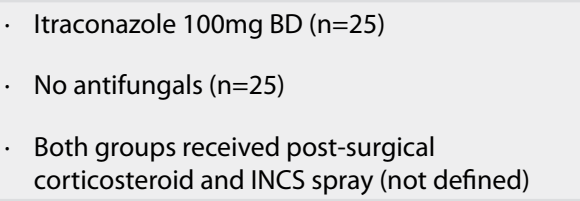 & - Recurrence & $\begin{array}{l}\text { No evidence of recurrence higher } \\
\text { in anti-fungal group ( } 44 \% \text { vs. } \\
24 \% \text { ) but no statistical analysis } \\
\text { performed }\end{array}$ \\
\hline $\begin{array}{l}\text { Khalil et al. } \\
2011^{(309)}\end{array}$ & RCT & $\begin{array}{l}50 \text { AFRS post } \\
\text { surgery }\end{array}$ & $\begin{array}{l}\cdot \text { Oral itraconazole }(n=10) \\
\text {. Fluconazole nasal spray }(n=10) \\
\text { - Oral itraconazole and Fluconazole nasal spray } \\
(n=10) \\
\text { - Fluconazole nasal irrigation }(n=10) \\
\text { - No antifungal therapy }(n=10)\end{array}$ & Recurrence & $\begin{array}{l}\text { Oral itraconazole did not show } \\
\text { benefit over other groups and } \\
\text { similar to controls ( } 66.7 \% \text { vs. } 75 \%)\end{array}$ \\
\hline $\begin{array}{l}\text { Gupta et al. } \\
2007^{(294)}\end{array}$ & RCT & $\begin{array}{l}34 \text { AFRS post- } \\
\text { surgery }\end{array}$ & $\begin{array}{l}\text { Oral itraconazole (200mg BD) and nasal } \\
\text { douche }(n=11) \\
\text {. Topical steroids (budesonide 100mg BD) and } \\
\text { nasal douche }(n=12) \\
\text {. Nasal douches only }(n=11)\end{array}$ & $\begin{array}{l}\text { Kupferberg } \\
\text { grades }\end{array}$ & $\begin{array}{l}\text { No differences between the } \\
\text { groups }\end{array}$ \\
\hline $\begin{array}{l}\text { Kupferberg } \\
\text { et al. } \\
1997^{(292)}\end{array}$ & $\begin{array}{l}\text { Retro- } \\
\text { spective } \\
\text { cohort }\end{array}$ & $\begin{array}{l}26 \text { AFRS Post } \\
\text { surgery }\end{array}$ & $\begin{array}{l}\text { No treatment }(n=11) \text {; } \\
\text { Oral corticosteroids alone }(n=10) ;(40 \mathrm{mg} \times 4 \\
\text { days, then } 30 \mathrm{mg} \times 4 \text { days, followed by } 20 \mathrm{mg} \\
\text { per day until first month) } \\
\text { Oral corticosteroids and oral antifungals (not } \\
\text { described) }(n=2) \\
\text { Oral antifungals alone }(n=3)\end{array}$ & $\begin{array}{l}\text { Symptoms } \\
\text { Kupferberg } \\
\text { grades }\end{array}$ & $\begin{array}{l}33 \% \text { of antifungal alone patients } \\
\text { had benefit but corticosteroid } \\
\text { groups did best. }\end{array}$ \\
\hline $\begin{array}{l}\text { Chan et al. } \\
2008^{(501)}\end{array}$ & $\begin{array}{l}\text { Case } \\
\text { series }\end{array}$ & $\begin{array}{l}32 \text { AFRS post- } \\
\text { surgery and } \\
\text { refractory }\end{array}$ & . Itraconazole $300 \mathrm{mg}$ daily tapered over $3 \mathrm{mths}$ & $\begin{array}{l}\text { Symptoms } \\
\text { Kupferberg } \\
\text { grades }\end{array}$ & $\begin{array}{l}\text { - Symptoms improved } \\
\text {. Endoscopic scores similar }\end{array}$ \\
\hline $\begin{array}{l}\text { Seiberling } \\
\text { et al. } \\
2009^{(304)}\end{array}$ & $\begin{array}{l}\text { Case } \\
\text { series }\end{array}$ & $\begin{array}{l}10 \text { AFRS and } \\
13 \text { AFRS } \\
\text { (without } \\
\text { atopy) post } \\
\text { surgery }\end{array}$ & . Itraconazole $100 \mathrm{mg}$ twice daily for 6 months & $\begin{array}{l}\text { Symptoms } \\
\text { Endoscopy } \\
\text { scores }\end{array}$ & $\begin{array}{l}83 \% \text { improvement in symptoms } \\
\text { and associated endoscopic } \\
\text { improvement }\end{array}$ \\
\hline
\end{tabular}


Table 8.6.5. Oral antifungals for the treatment of patients with AFRS continued.

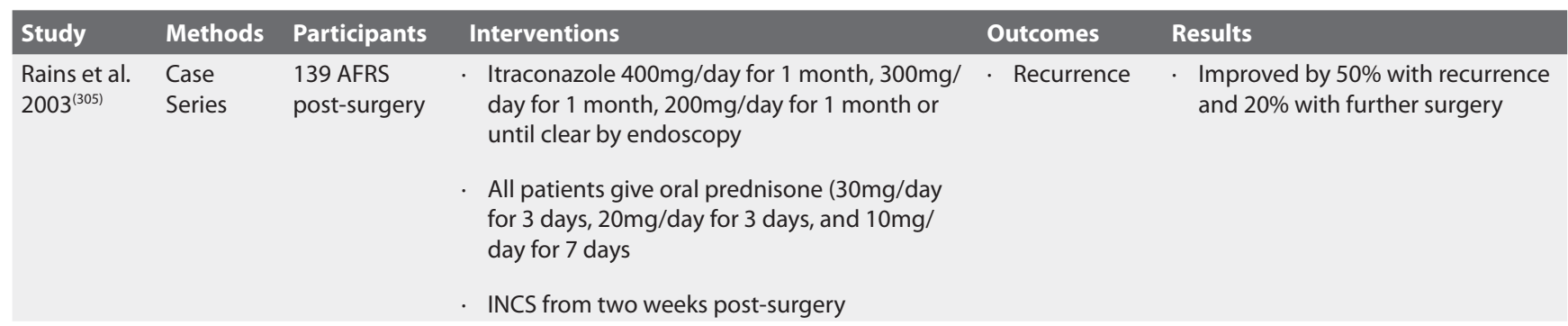

Table 8.6.6. Topical antifungals for the treatment of patients with AFRS.

\begin{tabular}{|c|c|c|c|c|c|}
\hline Study & Methods & Participants & Interventions & Outcomes & Results \\
\hline $\begin{array}{l}\text { Khalil et al. } \\
2011^{(309)}\end{array}$ & $\mathrm{RCT}$ & 50 AFRS post surgery & $\begin{array}{l}\text { Oral itraconazole }(n=10) \text { daily } 3 \text { months } \\
\text { months } \\
\text { - Oral itraconazole and fluconazole nasal } \\
\text { spray }(n=10) \text { both daily for } 3 \text { months } \\
\text { - Fluconazole nasal irrigation once/week for }(n=10) \text { bd for } 3 \\
6 \text { weeks }(n=10) \\
\text { - No antifungal therapy }(n=10)\end{array}$ & - Recurrence & $\begin{array}{l}\text { Fluconazole spray } \\
\text { (10\% recurrence). } \\
\text { Combined (14.3\%) } \\
\text { compared to } \\
\text { oral and control } \\
\text { (66.7\% vs. } 75 \%) \\
\text { - Not statistically } \\
\text { significant }\end{array}$ \\
\hline $\begin{array}{l}\text { Jen et al. } \\
2004^{(315)}\end{array}$ & Case series & 16 AFRS post surgery & $\begin{array}{l}\text { Fluconzole nasal spray (100mg/500ml, } 5 \\
\text { sprays twice daily) (3mths) } \\
\text {. Oral corticosteroid } \\
\text {. Oral itraconzole }\end{array}$ & . Symptoms & $\begin{array}{l}\text { Stable or } \\
\text { improvement in } \\
75 \% \\
\text {. Reduction in } \\
\text { oedema seen in } \\
75 \%\end{array}$ \\
\hline
\end{tabular}

\subsection{Allergen immunotherapy (fungal or other)}

Allergen immunotherapy (AIT) lends itself to placebo controlled randomized trials. However, there is a lack of such trials in AFRS. Several reviews have been undertaken, SCIT may have efficacy in the short-term(3-4 years), however, its long-term efficacy is unclear ${ }^{(296,297)}$. In a large retrospective series with immunotherapy for all fungal and non-fungal antigens, a 3-4 year course of subcutaneous immunotherapy (SCIT) demonstrated benefit 12-26 months after discontinuation by less recurrence $\mathrm{e}^{(298,299)}$ and decreased revision surgery in the immunotherapy group ${ }^{(298,300)}$. However, a subsequent study by the same group on a smaller subset of patients with longer term follow-up ranging from 46-138 months failed to demonstrate any benefit of SCIT with $60 \%$ of SCIT patients having normal mucosa or only mild oedema on endoscopy, while $100 \%$ of nonSCIT patients had normal mucosa or mild oedema ${ }^{(301)}$. This study was not randomized and obviously has the potential for bias in selecting treatment arms. Uncontrolled studies on sublingual immunotherapy have shown a benefit in IgE levels along with objective parameters ${ }^{(302)}$. Unfortunately, the atopic nature of AFRS and the inclusion of Type 1 hypersensitivity makes the concomitant treatment of AR and asthma in AFRS patients a significant confounder and clouds the results ${ }^{(303)}$.

The systematic review by Gan et al. identified two retrospective cohort studies, although misclassified as case-controlled, these studies were assessing patients with and without immunotherapy in a common population of post-surgical AFRS patients ${ }^{(293)}$ and thus more accurately had a retrospective cohort design. Both studies conclude a benefit but the confounding factors mentioned above act as potential bias (Table 8.6.4.).

$\begin{array}{ll}\text { Conclusion } & \text { Level } \\ \text { Immunotherapy to both fungal and non-fungal } & 2 \mathrm{~b} \\ \text { antigens in atopic individuals with AFRS is likely to } & \\ \text { improve symptoms and reduces revision surgery } & \end{array}$

\subsection{Anti-fungals - topical or systemic}

Limited data exists to support the use of oral antifungal agents in AFRS. Several case series have reported the benefits of systemic anti-fungal therapies in patients with $\operatorname{AFRS}^{(292,304,305)}$. It is recognized that itraconzole has anti-inflammatory effects and thus a controlled study is required ${ }^{(306)}$.

Two recent RCTs are noteworthy. The first, Verma et al., has a'no antifungal treatment' control group ${ }^{(307)}$. Although the authors found some evidence of reduction of inflammation on endoscopy and radiology, it was only between the preoperative treated and controls and symptom outcomes were similar. The other study by Rojita et al. found no difference between oral corticosteroid and itraconazole and declared them similar but a very low overall response rate was seen $(<25 \%)$ and no nasal irrigation was used ${ }^{(308)}$. The combine effect on symptoms after surgery did not favour antifungal therapy (Figure 8.6.3.) and the prevention of recurrence, including adjustment for those lost to follow-up, also did not support anti-fungal use (Figure 8.6.4.). Three very low quality RCTs have been reported. The first 
Figure 8.6.3. Forrest plot of the effect of oral antifungals for post-operative treatment of symptoms in AFRS.

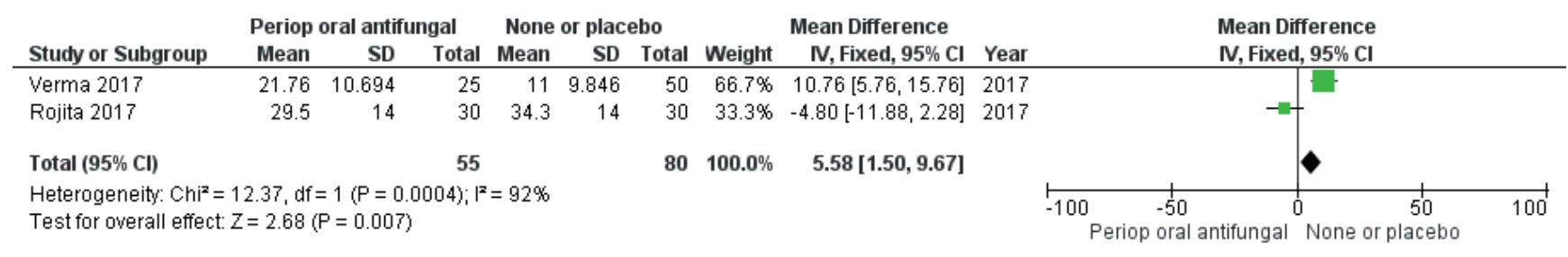

Figure 8.6.4. Forrest plot of the effect of oral antifungals for the prevention of recurrence in AFRS.

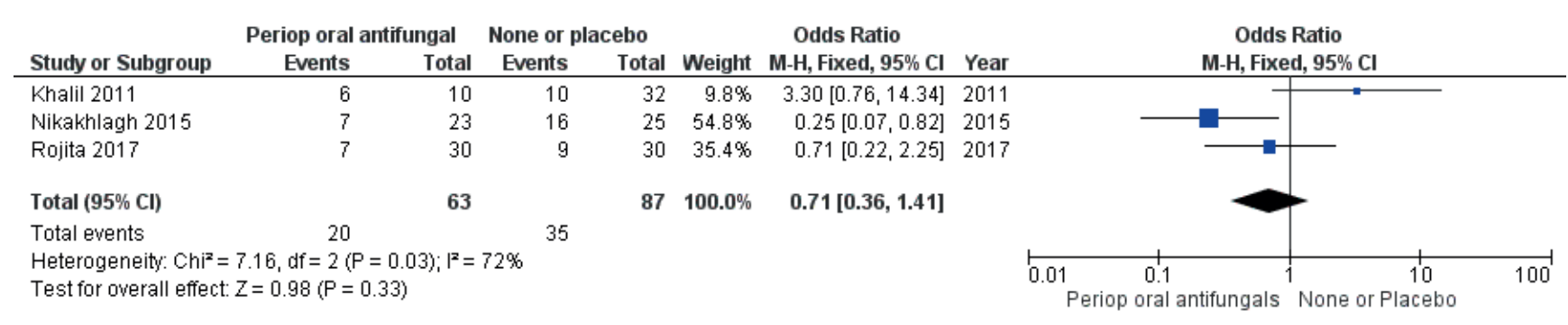

assessing a two-month treatment of oral itraconazole (100mg/ twice a day) compared to no antifungal therapy and both groups received systemic and topical corticosteroids. This study demonstrated little benefit from the addition of antifungal therapy. However, although the authors suggested there 'may be a benefit', incomplete data reporting and a lack of statistical analysis does not support this and the outcomes appear similar between groups. A similar low quality RCT by Gupta et al. did not find any benefit in a study on 34 postoperative AFRS patients which were randomized into three arms: 1 ) itraconazole + nasal douche $(n=11) ; 2)$ topical nasal steroid + nasal douche $(n=12)$; and 3) nasal douche alone $(n=11)$. The authors acknowledge that the study may be underpowered ${ }^{(294)}$. Kahil et al. reported an RCT of low quality in 50 patients in multiple treatment arms but included a 'no-antifungal treatment' group ${ }^{(309)}$. This study lacked a statistical analysis and the control group and oral antifungal group were similar and had the highest recurrences between all groups.

The prior systematic reviews on antifungal treatment in CRS included a study assessing oral antifungal (terbinafine $625 \mathrm{mg} /$ daily for six weeks). In this study AFRS patients were included within a broader CRS population ${ }^{(310)}$. This study found no benefit and along with the data from the three RCTs of low quality it is difficult to make a judgement beyond the summary metaanalysis and prior Cochrane review of topical and systemic antifungal therapies in all CRS patients, which failed to demonstrate any benefit ${ }^{(311)}$ (Table 8.6.5.).

\section{Topical antifungals}

There are very few studies that assess topical anti-fungal therapy in a dedicated AFRS population. A systematic review of the topic by Mistry and Kumar ${ }^{(312)}$ identified two RCTs that included AFRS within a broader CRS population but neither of them found convincing evidence of benefit ${ }^{(313,314)}$. Of the two studies with dedicated treatment groups in AFRS, one is a case series ${ }^{(315)}$ and the other an underpowered RCT with incomplete reporting and analysis ${ }^{(309)}$ (Table 8.6.6.).

\begin{tabular}{ll}
\hline Conclusions & Level \\
\hline $\begin{array}{l}\text { Oral antifungals may reduce inflammation and } \\
\text { recurrence in AFRS }\end{array}$ & $1 \mathrm{~b}$ \\
$\begin{array}{l}\text { Oral antifungals may reduce recurrence in AFRS, } \\
\text { although meta-analysis is negative }\end{array}$ & 4 \\
\hline $\begin{array}{l}\text { Oral antifungals do not improve symptoms in AFRS } \\
\text { Topical antifungal may be beneficial in AFRS }\end{array}$ & 4 \\
\hline
\end{tabular}

\subsection{Other medical interventions}

One single case study of leukotriene antagonist therapy in AFRS has been reported with benefit ${ }^{(316)}$.

A randomized, single-blind, prospective study of AFRS patients, who failed surgery and maximal postoperative medical management, used Manuka honey in one nostril. Overall, the group failed to demonstrate improvement ${ }^{(317)}$.

There are 8 patients from a case report and a small case series on the successful use of omalizumab to treat AFRS refractory to surgery and oral corticosteroids $s^{(318)}$. The seven $(n=7)$ patients with AFRS and asthma on omalizumab had a seven month mean treatment regime with significant reduction in SNOT22 and a $60 \%$ reduction in burden of inflammation on endoscopic scores ${ }^{(319)}$. Both studies show a reduction in corticosteroid use and other therapies.

\section{Conclusions}

Level

Antileukotrienes may reduce symptoms in AFRS 5

Manuka honey topically does not improve outcomes $1 \mathrm{~b}(-)$

Omalizumab (anti-IgE) may improve outcomes in AFRS 


\subsection{Surgery}

There is a near unanimous recommendation that surgery is required as a first stage intervention for $\operatorname{AFRS}^{(278,293,320,321)}$. The goal of surgery requires surgical debridement of the involved sinuses, thus removing the antigenic stimulation. A retrospective review reported that incomplete removal of all fungal and eosinophilic mucin contributed to disease recurrence and the need for revision surgery ${ }^{(322)}$. The recurrence rate in the surgery arm of the Ikram study ${ }^{(287)}$ was $50 \%$ at two years. Overall recurrence rates after surgery have been reported from $10 \%$ to $100 \%{ }^{(320)}$.

When skull base erosions occur with cranial neuropathies, surgery is the only successful intervention and spontaneous resolution has not been reported ${ }^{(323-326)}$. Similarly, longterm correction of proptosis is not reported without surgery. Sinus surgery alone, to address the AFRS, is usually all that is required and orbital reconstructions are not necessary ${ }^{(327)}$.

Treatment of the uninvolved contralateral side is controversial. A recent study reported that up to $30 \%$ of 'normal' contralateral sides become involved in the disease process. A recent study showed that up to $30 \%$ of "normal" contralateral sides become affected. However, the authors reported that the use of corticosteroid post-surgically dramatically reduced contralateral recurrence (OR $0.11,95 \% \mathrm{Cl} 0.01-0.87$, p value 0.01 ). If the contralateral side has early pre-operative symptoms and/or signs of inflammation radiologically then it was more likely to become involved (OR 3.49, 95\% Cl 1.19-10.22, p value 0.02$)^{(328)}$.

\section{Conclusions} Level

Surgery is required to resolve and treat AFRS including its complications $1 c$ Orbital reconstruction is not required in resolving proptosis

The contralateral sinus should be treated if there are radiologic changes of inflammation

\subsection{Vasculitis, granulomatous diseases and their role in CRS}

\subsubsection{Vasculitis}

\subsubsection{Classification}

The classification of vasculitis is challenging due to the heterogenous nature of the conditions. In 2012 the Chapel Hill Consensus on vasculitis nomenclature was revised ${ }^{(329)}$ (Table 8.7.1.) but remains mainly based on pathology, specifically affected blood vessel size (large, medium and small) which has limited clinical applicability ${ }^{(330-332)}$. The classification recognises primary and secondary vasculitis as well as single-organ disease. The latter category should not be confused with localised forms of vasculitis which may become systemic such as granulomatosis with polyangiitis (GPA) and eosinophilic granulomatosis with polyangiitis (EGPA). It may also be difficult to determine in GPA, EGPA, Behcet's and Kawasaki disease which size of vessels are involved. Similarly, the use of the antineutrophil circulating antibodies (ANCA) as a classification trait is not applicable to a subset of ANCA associated vasculitis with no detectable ANCAs. There are numerous other classification systems notably from the American College of Rheumatology, European Medicines Agency and the ACR/EULAR endorsed study to develop diagnostic and classification criteria for vasculitis (DCVAS) ${ }^{(333)}$. ANCA-associated vasculitis includes GPA, EGPA and microscopic polyangiitis (MPA) and frequently affect the upper respiratory tract and specifically the sinonasal region where they may be mistaken for more common forms of chronic rhinosinusitis.

\subsubsection{Granulomatosis with polyangitis (GPA)}

Granulomatosis with polyangitis (GPA) (previously Wegener's Granulomatosis) is defined as an idiopathic chronic inflammatory disease characterised by necrotizing granulomatous lesions and systemic vasculitis strongly associated with antineutrophil cytoplasmic antibodies (C-ANCA) (334).

\subsubsection{Epidemiology}

The true incidence of GPA has been underestimated in the past. Even with the advent of the ANCA test, many localised forms of the disease may go unrecognised. In Europe a prevalence of 23.7 per million has been reported and in the USA 30 per million ${ }^{(335)}$. The overall incidence ranges from 2.9-12/10\%/year depending on the geographic region.

\subsubsection{Pathophysiology}

The pathophysiology of GPA is most likely autoimmune. A number of in vitro and in vivo studies have indicated that ANCA induces the systemic vasculitis ${ }^{(334)}$ by binding to and activating neutrophils which causes the release of oxygen radicals, lytic enzymes and inflammatory cytokines. ANCA may also induce immune complex formation and may directly adhere to and kill endothelial cells thereby causing vasculitis ${ }^{(336)}$. Whilst PR3-ANCA (proteinase-3) is highly specific for GPA, the initial trigger may be infection or other environmental factors possibly combined with a genetic susceptibility. Staphylococcus aureus colonization of the nose is found more frequently in GPA as compared to CRS and controls (72\% v 28\% v 25\%) $)^{(337)}$ and has been strongly implicated as a causative agent, especially in relapses ${ }^{(338,339)}$ which are reduced by the use of an anti-staph agent such as trimethoprim/sulfamethoxazole ${ }^{(340)}$. A large genome wide study on 1233 patients showed a strong association with HLA-DP and genes encoding alpha ${ }_{1}$ antitrypsin (SERPINA1) and proteinase 3 (PRTN3) ${ }^{(341)}$. A review by Relle et al. ${ }^{(342)}$ also identified some susceptibility gene-CTLA4, PTPN22, COL11A2, SERPINA1 and MHCC class II gene cluster.

\subsubsection{Diagnosis and differential diagnosis}

\section{Clinical (Table 8.7.2.)}

The peak incidence is in the fourth to fifth decades, mean 50 years but GPA can occur at any age (range 9-97 years) ${ }^{(343-345)}$. Patients $>65$ years at onset have a different pattern of organ involvement, less upper respiratory tract involvement and higher morbidity and mortality ${ }^{(346,347)}$. Men and women seem equally affected and the vast majority are Caucasian $(93 \%)^{(348)}$. 
However, there is some evidence that males are more likely to have 'severe' disease and females the more 'limited' phenotype. Classically GPA affects the nose, lungs and kidneys but can present in any system and limited forms of the disease are recognised ${ }^{(348)}$. The European Vasculitis Study Group (EUVAS) distinguishes 'localized' (i.e GPA restricted to the respiratory tract) and 'early systemic' GPA (i.e., non-imminent GPA without renal organ involvement) from 'generalized' GPA ${ }^{(349)}$.

Two thirds of patients initially present with an ENT related symptom, of which the majority are rhinological ( 40\%), then otologic $(\sim 15 \%)$ and laryngopharyngeal $(\sim 5 \%)^{(350-352)}$. Nasal symptoms were commoner in those diagnosed $<40$ years (55\%) as compared to those $>60$ years $(27 \%)$. During the course of the disease, the majority experience nasal symptoms with patients experiencing crusting (75\%), discharge (70\%), nasal stuffiness (65\%), bleeding (59\%), reduced sense of smell (52\%) and facial pain $(33 \%)^{(351)}$. Hyposmia may be due to mechanical obstruction of the olfactory region, specific cranial nerve involvement ${ }^{(353)}$, secondary to immunosuppressive treatment ${ }^{(354)}$ or due to any systemic autoimmune process ${ }^{(355,356)}$. Taste is also reduced but less than smell(353). Deep seated facial pain may be secondary to sinus involvement or due to an osteitis of midfacial bone, indicative of disease activity. A characteristic supratip collapse occurs in $18-25 \%$ which may or may not be associated with septal perforation in up to $30 \%$. On endoscopic examination the nasal mucosa is usually very friable, granular and covered with old blood and crust, together with adhesions. There may be thickening of bone and/or erosion which can result in meningitis if the skull base is involved and fibrosis of the nasolacrimal duct producing epiphora. The entire internal structure of the nose may disappears creating a large featureless cavity ${ }^{(344,369)}$.

Elsewhere in the upper respiratory tract, patients may experience hearing loss (conductive, sensorineural or mixed), vertigo, tinnitus and facial nerve palsy.

In the mouth there may be gingivitis, ulceration and oro-antral fistulas though less extensively than is seen with cocaine abuse. In addition, subglottic stenosis (16\%) and vocal fold paralysis may occur, the symptoms of which may be wrongly attributed to the lower respiratory tract. Subglottic stenosis should be considered in any patients with dyspnoea, hoarseness or inspiratory stridor and is estimated to occur in about $16 \%$ of GPA patients $^{(357)}$.

Although GPA can present in many ways, often the development of relatively minor upper respiratory symptoms is associated with disproportionate unwellness, fatigue, weight loss and night sweats heralding generalised disease, the systemic symptoms and signs of which are legion. Quality of life is significantly affected in GPA as shown by the general Short Form-36 $6^{(344,}$ ${ }^{358)}$ which improves with remission. The Sinonasal Outcome Test- SNOT-22 demonstrated the significant effect of sinonasal involvement on the general health-related $\mathrm{QOL}^{(344)}$.

Disease activity can be measured using the Birmingham Vasculitis Activity Score (BVAS) ${ }^{(359)}$, a Vasculitis Damage Index $(\mathrm{VDI})^{(360)}$ and an ELK-classification of organ involvement (ENT, lung, kidney) $)^{(361)}$.
Diagnosis can be delayed by 6 months or longer ${ }^{(343,351)}$, rendering the condition potentially life threatening. The delay was greatest when patients presented with eye symptoms (>14 months) but ENT symptoms were associated with the second longest delay of $>8$ months). This is particularly disappointing as over half the patients (56\%) saw an ENT surgeon prior to their diagnosis.

In a retrospective non-controlled cohort of $89 \mathrm{GA}$ patients diagnosed between 1996 and 2016, those who presented with ENT involvement had a higher survival rate at 5 years than those who presented with other systematic disease (98\% v 78\%) and ENT involvement was an independent predictor of a better

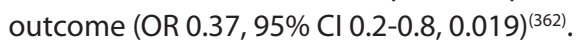

\section{Investigations (Table 8.7.3.)}

ANCA tests have become the mainstay of diagnosis in vasculitis. Indirect immunofluorescence (IIF) testing for neutrophil cytoplasmic antibodies is commonly ordered first, followed by enzyme-linked immunosorbent assays (ELISAs) for antibodies directed against proteinase 3 (PR3) and myeloperoxidase (MPO). Having been divided into c-ANCA and p-ANCA (cytoplasmic versus perinuclear) by IIF, the ELISA links them with the presence of PR3 and MPO respectively. The CANCA is $99 \%$ specific with a sensitivity of $73 \%$ using combined IIF and ELISA techniques but the increasing availability of reliable antigen-specific immunoassays (ELISA) is replacing the need for indirect immunofluorescence ${ }^{(363)}$. A positive c-ANCA test and proteinase-3 (PR3) will confirm the clinical diagnosis of GPA in up to $95 \%$ of patients with active systemic disease. However, the c-ANCA lacks sensitivity in the limited forms of the disease dropping to $50 \%$ and/or following therapy with corticosteroids so a negative ANCA does not exclude GPA.

Whilst a positive c-ANCA is usually associated with GPA and a p-ANCA with MPO and EGPA, it is recognised that 5\% of GPA patients have a positive $\mathrm{P}-\mathrm{ANCA} / \mathrm{MPO}$.

Sequential monitoring of the c-ANCA can be useful in predicting relapse ${ }^{(364)}$ but not all patients show the classical pattern of fluctuation with disease activity.

The ANCA testing is complemented by a raised ESR (erythrocyte sedimentation rate) and CRP (c-reactive protein), altered renal function on blood and urine testing (proteinuria, microscopic haematuria and red cell casts) and chest imaging (showing granulations, infiltration, necrotic cavitation, fibrosis).

\section{An ANCA test should be considered in any patient with suspicious clinical manifestations, in particular nasal crusting and bleeding, especially if they feel disproportionally unwell.}

\section{Cocaine abuse}

Cocaine abuse in the form of 'snorting' can induce significant midline destruction of the nose and palate ${ }^{(365)}$ often preceded by progressive nasal obstruction, epistaxis and crusting which closely resembles the sinonasal symptoms of GPA. The degree and distribution of destruction is greater in cocaineinduced vasculitis than GPA and was thought to arise from the 
abnormal boosting of apoptosis and cellular damage caused by cocaine $^{(366)}$. It is now thought that this is more likely caused by the illicit addition of levamisole to cocaine ${ }^{(367)}$. The c-ANCA and PR-3 can also be positive, making differentiation between the conditions difficult ${ }^{(365,368)}$ but massive apoptosis with abundant caspase- 3 and 9 expression is found in the cocaine users but not in GPA patients and ANCA reacts with human neutrophil elastase (HNE) in the cocaine group but not in autoimmune vasculitis so this may assist the differential diagnosis, supported by tissue cocaine levels.

\section{Imaging}

In a patient without a history of previous sinonasal surgery, a combination of bone destruction (60-75\%), septal loss (59\%) and new bone formation (50-78\%) on CT is highly suspicious of GPA (both localised and systemic) ${ }^{(352,369-374)}$ especially when accompanied on MRI by a fat signal from the sclerotic sinus wall ('tramlines') giving a high-signal on T1-weighted sequences ${ }^{(369)}$. However, early in the disease the most frequent finding is of non-specific mucosal thickening in the nose or paranasal sinuses (87\%) which may not raise suspicions of a vasculitis ${ }^{(369,373)}$. In contrast, progressive loss of midfacial structures can mimic an NK/T cell lymphoma.

Imaging of the lungs should be undertaken to reveal diffuse infiltration, multiple pulmonary nodules $(2-4 \mathrm{~cm}$ in diameter) or large necrotic cavitating granulomatous masses $(10 \mathrm{~cm}$ diameter or larger) some with fluid levels. Imaging of the orbit and larynx should be considered depending on clinical symptoms.

\section{Histopathology}

GPA is characterised by three key findings: granulomatous inflammation, necrosis and vasculitis affecting small to medium vessels (capillaries, venules, arterioles and arteries). The granulomas are composed of $C D 4^{+}$and $C D 8^{+} T$ cells, CD28- T cells, histiocytes, CD20+ B lymphocyte, neutrophil granulocytes, macrophages and multinucleated giant cells surrounding an area of central necrosis ${ }^{(334)}$. Occasional eosinophils may confuse the diagnosis with EGPA.

Tissue biopsy from the kidneys or lung can confirm the diagnosis but nasal biopsy, though easier to obtain, is less reliable and may be reported as 'consistent with' rather than definitively GPA as all three criteria of necrosis, granulomatous inflammation and vasculitis have to present ${ }^{(375,376)}$. Abnormal tissue from the sinuses is likely to provide a better yield ${ }^{(377)}$ as can careful endoscopically directed biopsy after cleaning and decongesting the nose ${ }^{(378)}$. Mucosal biopsy is most helpful in c-ANCA negative, localised GPA patients. A study by Borner et al. ${ }^{(379)}$ showed that although the biopsy had lower sensitivity $(47 \%)$ than the ANCA test generally, in these circumstances it had a specificity of $96 \%$ and a positive predictive value of $78 \% \mathrm{v}$ $73 \%$ with c-ANCA.

\section{Differential diagnosis}

The differential diagnosis includes any of the other granulomatous conditions such as sarcoid, NK/T cell lymphoma, a range of infectious conditions such as tuberculosis, rhinoscleroma or fungal diseases, particularly rhinosporidiosis and other autoimmune conditions e.g. SLE or polyarteritis nodosa.

\subsubsection{Treatment}

Without treatment the mean survival of systemic GPA is five months. Modern immunosuppressive treatment following a strategy of combined remission induction and maintenance has markedly improved this to a mean survival of 21.7 years from diagnosis ${ }^{(380)}$ with a significant improvement over the last four decades assisted by higher awareness and diagnosis ${ }^{(381)}$. However, $10 \%$ never achieve remission, relapses occur in up to $50 \%$ of individuals and mortality rates of $5 \%$ or higher are still reported $^{(382)}$.

\section{Systemic}

In generalised and/or severe disease regimes of high dose systemic steroids and pulsed cyclophosphamide are aimed at rapidly inducing remission to limit organ damage and to maintain this with the lowest dose to minimise the side effects of the medications ${ }^{(383)}$. Methotrexate is sometimes used to obtain remission in non-organ threatening disease and as maintenance ${ }^{(384)}$.

Monoclonal antibodies, in particular the anti-CD20 antibody, rituximab, are now widely used ${ }^{(385,386)}$. The RAVE (Rituximab for ANCA-associated Vasculitis) study ${ }^{(387)}$ on 197 patients showed intermittent infusion of rituximab to be equivalent to daily cyclophosphamide for induction of remission and superior in relapsing disease but may not improve their chronic ENT problems ${ }^{(388)}$.

For longer-term maintenance, in addition to low dose prednisolone and rituximab, azathioprine and mycophenolate mofetil have been used. These act as 'steroid-sparing', thus reducing the requisite dose of prednisolone or are used as monotherapy as they are less often associated with significant side-effects ${ }^{(383)}$. TNF-alpha inhibitors such as infliximab and etanercept have been less effective and other monoclonals such as belimumab (IgG1 antibody against B-Lys) are under investigation $^{(386)}$.

Because relapses can be triggered by many events from an episode of influenza to pregnancy, treatment needs constant surveillance. Titration of treatment against the activity of the condition and the side-effects of the drugs are generally monitored with regular ANCA, full blood counts, ESR, CRP, renal and pulmonary function tests, ideally in a multi-disciplinary setting, although the methodological range in the literature make it difficult to show the best protocol ${ }^{(389)}$. A CD8+-T cell signature may be used to identify relapse ${ }^{(383)}$. Those on longterm steroids need bone densitometry and prophylaxis against osteoporosis should be considered.

As it is not known if all localised forms spread systemically nor whether treatment of limited disease prevents dissemination, the decision to start immunosuppression can be difficult. It is unclear for how long patients need to be treated but maintenance is recommended for 18 months. In many individuals the disease eventually burns-out but they may still require a small replacement dose of steroid. 


\section{Nasal}

Nasal irrigation, a topical intranasal corticosteroid sprays or creams e.g. triamcinolone and/or a nasal lubricant such as $25 \%$ glucose and glycerine drops, honey ointment or an aqueous gel are usually recommended together with regular debridement of the crusts ${ }^{(377)}$ (Level IV evidence).

The possible aetiological role of Staphylococcus aureus has led to the use of long-term oral co-trimoxazole (trimethoprimsulfamethoxazole) and topical anti-staphylococcal creams in the nose $^{(390)}$ (Level I evidence).

Endoscopic surgery has a very limited role and is associated with poor outcomes, increased scarring and adhesions so should be a last resort. Occasionally endoscopic exploration of an opaque sinus may be done to confirm that opacification is granulation/ fibrosis rather than infection, rarely a dacrocystorhinostomy may be undertaken or repair of the skull base secondary to bone erosion in cases of meningitis ${ }^{(345,377)}$.

Repair of the septal perforation is unlikely to be successful in the long-term so septal buttons are sometimes offered though can increase the crusting. Cosmetic improvement of the external nasal deformity has been successfully undertaken once the disease has been quiescent for a reasonable period of time (e.g. one year) ${ }^{(391)}$.

\subsubsection{Eosinophilic granulomatosis with polyangiitis}

Eosinophilic granulomatosis with polyangiitis (EGPA) (previously Churg Strauss Syndrome) ${ }^{(329,392)}$, is a rare form of vasculitis characterised by adult onset asthma, severe rhinitis, nasal polyps and other systemic manifestations as a result of widespread eosinophilic granulomatous infiltration of tissues.

The Chapel Hill consensus defined it as 'eosinophil-rich granulomatous inflammation of the respiratory tract and necrotizing vasculitis of small to medium-sized vessels, associated with eosinophilia'. However, the nomenclature has been challenged as only $59 \%$ of patients with this diagnosis have evidence of polyangiitis ${ }^{(393)}$.

\subsubsection{Epidemiology}

The overall prevalence of the condition ranges from 10.7-13 cases/million inhabitants with an annual incidence of 0.5-6.8 new cases/million inhabitants ${ }^{(394)}$. However, among asthma patients the incidence is as high as 67 per million ${ }^{(395)}$.

\subsubsection{Pathophysiology}

The aetiology is unknown though it is probably an idiopathic autoimmune Th2 mediated disease affecting eosinophils, endothelial cells and lymphocytes. It has been suggested that exposure to certain allergens, drugs, vaccinations, corticosteroid withdrawal, and pulmonary infection may initiate the inflammatory cascade ${ }^{(396-398)}$. It has been suggested that antileukotriene antagonists such as zafirlukast and montelukast ${ }^{(396)}$ when used for asthma in some patients, could unmask EGPA. However, the consensus is that these drugs may be used if needed in EGPA, as the onset was either coincidental or resulted when symptoms of EGPA were unmasked after oral glucocorticosteroids were tapered and/or withdrawn ${ }^{(399,}$ ${ }^{400)}$. Other drugs implicated in triggering EGPA include antibiotics (macrolides), oestrogen replacement therapy, and carbamazepine suggesting a hypersensitivity reaction. There is also some evidence for a genetic predisposition in respect of the HLA-DRB ${ }^{*} 04$ and ${ }^{*} 07$ alleles and the related HLADRB4 ${ }^{(401,402)}$. In EGPA the anti-myeloperoxidase ANCA may

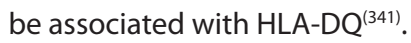

Eosinophilic infiltration and endothelial damage are likely the most important pathophysiological mechanisms. Peripheral T-cell lines from EGPA patients can produce Th2 associated cytokines such as IL4 and IL13 and IL5 is upregulated in active disease, offering therapeutic possibilities ${ }^{(403)}$. However, an exaggerated Th2 response cannot account for all the manifestations of EGPA and there is evidence of involvement of Th1 and Th17 cells secreting large amounts of IL-17a in the late stages of the disease. Eoxtaxin-3 produced by endothelial and epithelial cells may contribute to eosinophilic tissue infiltration and further tissue damage may be caused by eosinophilic cationic protein from activated eosinophils. They also secrete large amounts of IL25 which elicits Th2 responses, maintaining a vicious circle ${ }^{(403)}$.

Increased levels of IgG4 and IgG4/lgG ratios in EGPA have led to the suggestion that EGPA may be part of the $\lg G 4$ disease spectrum $^{(404)}$.

As with GPA, it is thought that localised early forms of EGPA may exist, particularly in the upper respiratory tract ${ }^{(403)}$.

\subsubsection{Diagnosis and differential diagnosis}

According to the American College of Rheumatology criteria ${ }^{(405)}$, at least four out of six criteria must be present:

1) A medical history of asthma

2) Eosinophilia $>10 \%$

3) Mono- or polyneuropathy

4) Nonfixed pulmonary infiltrates

5) Paranasal sinus abnormalities

6) Biopsy including an artery, arteriole or venule showing accumulated eosinophils in the extravascular tissue.

\section{Clinical (Table 8.7.2.)}

The male to female ratio in the literature ranges from $58: 43$ to 39:54 with mean age of between 49-52 years in the 28 studies included in a systematic review by Goldfarb ${ }^{(406)}$. It can manifest between 7-74 years with a mean age of onset of 38-54 years ${ }^{(403)}$. No ethnic predisposition has been determined ${ }^{(407)}$.

The condition often follows three clinical stages. The prodromal stage is characterised by allergic rhinitis, CRS with or without polyps and asthma ${ }^{(408,409)}$. This stage may predate the overt vasculitis by months to many years (mean eight years) ${ }^{(396)}$. The second 'eosinophilic' stage is characterised by tissue and peripheral eosinophilia.

The final 'vasculitic' phase involves extrapulmonary disease with vasculitis, associated with severe and fatal manifestations including polyneuropathies, myocarditis, cerebral haemorrhage, ischaemic strokes and bowel perforations.

In a systematic review ${ }^{(406)}$ of 1175 patients with EGPA, 48-96\% presented with head and neck manifestations, which are often sinonasal ${ }^{(410)}$. These include nasal obstruction (95\%), crusting $(75 \%)$, bleeding $(60 \%)$, rhinorrhoea $(95 \%)$, facial pain, loss of 
smell (90\%) and sneezing (80\%) associated with allergic rhinitis (43\%) and chronic rhinosinusitis with (54\%) and without nasal polyposis (70\%). In addition to nasal crusting, hypertrophy of the inferior turbinates, mucosal oedema, synechia and nasal polyps may be seen on endoscopy ${ }^{(411,412)}$. Olfactory testing using Sniffin' sticks in 62 subjects showed normosmia in 79\%, hyposmia in $11 \%$, anosmia in $29 \%$ and dysosmia in $40 \%{ }^{(412)}$. Anterior rhinomanometry in 86 subjects showed severe nasal obstruction in a third. A mean SNOT-22 of 41.5 has been found, comparable to patients with CRS without vasculitis ${ }^{(410)}$. As a consequence, patients are frequently seen by the ENT community who may not appreciate the potential for a more serious systemic disease but are in a unique position to make an early diagnosis and prevent late complications of the condition $^{(413)}$.

\section{EGPA should be considered in any patient with severe nasal polyposis who is not responding to conventional therapy}

ENT and systemic manifestations are shown in Table 8.7.2. In one series the mean delay between diagnosis and presenting to the physician with worsening symptoms was 18.5 months (range 1-71 months, SD 21.6) ${ }^{(410)}$ whereas a mean delay from diagnosis of asthma to diagnosis of EGPA of $11.8 \pm 18.2$ years has been reported ${ }^{(414)}$.

\section{Investigations (Table 8.7.3.)}

Active EGPA is characterised by marked peripheral eosinophilia (usually $>1500$ cells/ul or $>10 \%)^{(403)}$. There is usually a nonspecific increase in $\lg E$ if the patients are not taking systemic steroids for their asthma.

EGPA is one of the antineutrophil cytoplasmic antibody (ANCA)associated vasculitides. Immunofluorescent patterns with ELISA detecting antibodies to myeloperoxidase (MPO) is the most common EGPA ANCA-positivity finding but figures for p-ANCA positivity vary. Between $26-48 \%$ have been reported to have a positive $p$-ANCA but this increases to $75 \%$ with renal involvement ${ }^{(415)}$ and other studies quote $35-77 \%$ so a negative ANCA does not rule out EGPA and a positive ANCA may not be essential for the diagnosis in the head and neck phenotype ${ }^{(329,}$ $395,416-419$ ). Overall pANCA-positive patients are more likely to have peripheral neuropathy, glomerulonephritis and purpura (due to small vessel vasculitis) as compared to those with myocardial, respiratory and gastrointestinal involvement who are more often pANCA negative ${ }^{(416,420)}$.

Other biomarkers have been investigated including serum TARC/CCL17, eotaxin-3, IgG4 and IgG4/lgG ratio but none were useful in discriminating between active disease and remission ${ }^{(421)}$.

\section{Imaging}

Sinus CT does not show anything other than what would be expected in diffuse polypoid rhinosinusitis i.e. panopacification ${ }^{(422)}$. Mean Lund-Mackay scores of 9-17 have been reported in large cohorts of EGPA ${ }^{(412,422)}$. Expansion and bone erosion may be seen in the ethmoid complex with widening of the intercanthal distance. In addition, there may be evidence of mucocele formation, notably in the fronto-ethmoidal region. High resolution $\mathrm{CT}$ of the chest is recommended to demonstrate atypical 'fluffy' or nodular migratory pulmonary infiltrates which would only be seen in $64 \%$ of plain chest $x$-rays ${ }^{(423)}$. Peripheral nodules $(25 \%)$, ground glass opacities $(86 \%)$ and bronchial wall thickening and bronchiectasis (66\%) are also found on CT.

\section{Histopathology}

Histopathologically the condition is characterised by eosinophilic inflammation and infiltration, extravascular granulomas and necrotizing vasculitis in the presence of asthma. However, in studies where sinonasal tissue was examined, eosinophilic infiltration was seen in $35-100 \%$, whilst necrotising vasculitis and eosinophilic granulomas were not seen $^{(408,424) \text {. }}$

Although the histology is important, this combined with clinical criteria are required to make the diagnosis and a low threshold of suspicion is required in any patient with eosinophilic diseases i.e. significant adult-onset asthma, recurrent CRSwNP and allergic rhinitis. Once EGPA is diagnosed, investigation for lung, kidney, heart, gastrointestinal and peripheral nerve involvement is recommended as these are associated with poor prognosis ${ }^{(394)}$. This can include imaging, biopsy, bronchoalveolar lavage, renal and cardiac function tests.

\section{Differential diagnosis}

The differential diagnosis is from other eosinophilic pneumonias, idiopathic hypereosinophilic syndrome, c-ANCA positive granulomatosis and microscopic polyangiitis, sarcoid, allergic bronchopulmonary aspergillosis and parasitic infections. Thus in addition to ANCA, the EGPA Consensus Task Force recommended as a minimum initial differential diagnostic workup the following: serologic testing for toxocariasis, HIV, specific IgE and IgG for Aspergillus spp, a search for Aspergillus spp in sputum and/or bronchoalveolar lavage fluid, tryptase and vitamin B12, full blood count and CT of the chest ${ }^{(394)}$. However, EGPA can usually be distinguished from other primary systemic vasculitides by the presence of asthma and a marked blood eosinophilia.

\subsubsection{Treatment}

In 2009, the European League Against Rheumatism (EULAR) published treatment recommendations for small to medium sized vessel vasculitides. Without treatment, the mortality rate is high once the vasculitis phase is reached ${ }^{(425,426)}$. A multidisciplinary management is widely recommended in the literature ${ }^{(394)}$ and with appropriate treatment, survival rates are comparable to GPA ${ }^{(426)}$.

Long -term studies have shown that overall remission rates are good, ranging from $81-92 \%$ but over a quarter do relapse either within the first year of treatment or much later ${ }^{(427)}$. This is higher in MPO /pANCA positive patients. Overall mortality in treated patients who relapsed was only $3.1 \%$. Factors predicting poor outcome are: cardiomyopathy (an 
independent risk factor) or two features of the 'five-factor' score (FFS) (creatininemia >140umoles/litre; proteinuria >1g/day; involvement of central nervous system, gastrointestinal or heart) $(426,428)$. If one of these factors is present at onset, the five year mortality is $25.9 \%$, when two or more are present, this increases to $46 \%$ at five years ${ }^{(428)}$. Survival at one and five years was $100 \%$ if the score was 0 . The FFS was revisited in 2011 by the French Vasculitis Study group as follows: 1 ) age $>65 y$ rs; 2) cardiac symptoms; 3) gastrointestinal involvement; 4) renal insufficiency characterised by a serum creatinine $>150$ umol/l; 5) absence of ENT manifestations. With each factor accorded one point, a revised FFS of 0,1 and 2 is associated with a five-year mortality rate of $9 \%, 21 \%$ and $40 \%$ respectively ${ }^{(429)}$. A subsequent study showed that age $>65$ years was the only factor associated with higher risk of death during follow-up ${ }^{(427)}$.

\section{Systemic}

In most patients, disease control is achieved with immunosuppressant therapy, usually oral prednisolone +/cytotoxic drugs such as pulsed cyclophosphamide, azathioprine, mycophenolate mofetil and methotrexate dependent on the severity of the disease at presentation. Patients without poor prognostic factors can often be managed with glucocorticoids alone. Additional systemic treatments include intravenous immunoglobulin, leflunomide, anti-TNFa, interferon-a and plasma exchange ${ }^{(430)}$.

Rituximab is now frequently being used in selected ANCApositive patients with renal involvement or refractory disease though the evidence is less robust that in $\mathrm{GPA}^{(394,431-434)}$ and other new biologic therapies may be helpful e.g. anti-IL 5 (mepolizumab) ${ }^{(435-437)}$ or anti-lgE (omalizumab) monoclonal antibodies $^{(438,439)}$.

\section{Nasal}

Alkaline/saline nasal irrigations, topical intranasal corticosteroids and nasal lubricants are the mainstay of treatment (Level IV). Endoscopic surgery can be successfully undertaken in those whose CRS with nasal polyps do not respond to systemic and local steroids. The numbers of reported EGPA surgical cases in the literature is small( ${ }^{(440)}$ and surgery is often associated with a high recurrence rate though patients may still obtain benefit ${ }^{(422)}$. A number of patients develop paranasal sinus mucoceles which may also be dealt with endoscopically. In a series of 25 patients, $48 \%$ had undergone endoscopic sinus surgery, often multiple times ${ }^{(410)}$.

\subsubsection{Sarcoidosis}

\subsubsection{Definition}

Sarcoidosis is a chronic multi-system inflammatory disease of unknown aetiology characterised by non-caseating granuloma. The first description is sometimes ascribed to Besnier in 1889 who coined the term 'lupus pernio' for the cutaneous lesions ${ }^{(441)}$. It is usually associated with bilateral hilar lymphadenopathy, pulmonary, ocular and skin lesions ${ }^{(411,442)}$.

\subsubsection{Epidemiology}

The age-adjusted incidence is 11 cases/100,000 population in Caucasians and it is commoner in northern Europe (Sweden, Iceland) affecting an estimated 20/100,000 population versus $1.3 / 100,000$ in Japan. In the United States it is more prevalent in African Americans than whites (35.5/100,000 versus $10.9 / 100,000$ respectively). It is twice as common in women than men, who also have higher morbidity, mortality and extrapulmonary involvement. Incidence peaks in the third fourth decades, with a second peak in women between 45-65 years though any age can be affected ${ }^{(443,444)}$.

\subsubsection{Pathophysiology}

The aetiology of this condition remains unknown. It appears to be an exaggerated immune response to an unknown antigen for which a wide range of agents have been suggested. This includes infective agents such as mycobacteria or propionibacteria, chemicals (beryllium and zirconium), pine pollen and peanut dust ${ }^{(445)}$.

It is one of the few pulmonary diseases commoner in nonsmokers. Working on the World Trade Centre debris after 9.11.2001 was associated with development of sarcoidosis ${ }^{(446)}$. It is associated with cell-mediated and humoral immune abnormalities. T cells play a central role with an accumulation of $C D 4^{+} \mathrm{T}$ cells, accompanied by release of IL2 followed by a non-caseating granuloma. There may be an inverted $\mathrm{CD}^{+} /$ $\mathrm{CD}^{+}$ratio. $\mathrm{TH} 1$ cytokines such as interferon are increased as is tumour necrosis factor (TNF). There is B cell hyperreactivity and immunoglobulin production. Glycoprotein KL-6 and surfactant protein $\mathrm{D}$ are increased in pulmonary sarcoid ${ }^{(447)}$ and Th 17 cells are also thought to play a role ${ }^{(448)}$.

\subsubsection{Diagnosis and differential diagnosis}

\section{Clinical (Table 8.7.2.)}

Sarcoidosis is classically a multisystem disease and can therefore present in protean ways, the distribution of which reflects the origin of the individual series. The chances of possible organ involvement can be assessed with the WASOG sarcoidosis organ assessment instrument ${ }^{(449)}$ or more recently through the Genotype-Phenotype Relationship in Sarcoidosis study ${ }^{(450)}$. However, the disease can also be asymptomatic, found incidentally e.g. on chest imaging (5\%).

It has a propensity for the lower respiratory tract to be affected in up to $95 \%$ in some studies ${ }^{(451)}$. The upper respiratory tract is involved infrequently ${ }^{(452)}$, in approximately $3-4 \%$ of patients with generalised disease. Notably the nose is affected with blockage, crusting and bleeding ${ }^{(445,453-455)}$. Nasal polyps and septal perforations leading to saddle nose deformity have also been reported ${ }^{(411,456-458)}$. Lupus pernio of the external nose, face and ears often accompanies sinonasal sarcoidosis ${ }^{(451)}$. The ears, mouth and larynx may also be affected ${ }^{(459-461)}$.

In a series of 148 patients with proven sarcoid and referred to a specialist rhinological practice, nearly $90 \%$ suffered nasal congestion or blockage and two thirds had crusting. Bleeding or spotting of blood affects $40 \%$ and nearly a quarter had facial 
Table 8.7.1. Classification of vasculitides adopted by the 2012 International Chapel Hill Consensus Conference on the Nomenclature of Vasculitides ${ }^{(329)}$.

\section{Large vessel vasculitis (LVV)}

- Takayasu arteritis (TAK)

- Giant cell arteritis (GCA)

\section{Medium vessel vasculitis (MVV)}

- Polyarteritis nodosa (PAN)

- Kawasaki disease (KD)

\section{Small vessel vasculitis (SVV)}

Antineutrophil cytoplasmic antibody (ANCA)-associated vasculitis (AAV)

Microscopic polyangiitis (MPA)

Granulomatosis with polyangiitis (Wegener's) (GPA)

Eosinophilic granulomatosis with polyangiitis (Churg-Strauss) (EGPA)

Immune complex SVV

Anti-glomerular basement membrane (anti-GBM) disease

Cryoglobulinemic vasculitis (CV)

IgA vasculitis (Henoch-Schonlein) (IgAV)

Hypocomplementemic urticarial vasculitis (HUV) (anti-C1q vasculitis)

\section{Variable vessel vasculitis (VVV)}

Behcet's disease (BD)

- Cogan's syndrome (CS)

\section{Single-organ vasculitis (SOV)}

- Cutaneous leukocytoclastic angiitis

- Cutaneous arteritis Primary central nervous system vasculitis

Isolated aortitis

- Others

\section{Vasculitis associated with systemic disease}

Lupus vasculitis

Rheumatoid vasculitis

Sarcoid vasculitis

Others

\section{Vasculitis associated with probable aetiology}

Hepatitis C virus-associated cryoglobulinemic vasculitis

Hepatitis B virus-associated vasculitis

Syphilis-associated aortitis

Drug-associated immune complex vasculitis

Drug-associated ANCA-associated vasculitis

Cancer-associated vasculitis

Others

Table 8.7.2. Symptoms associated with different system involvement in granulomatosis with polyangiitis (GPA), eosinophilic granulomatosis with polyangiitis (EGPA) and sarcoidosis.

\begin{tabular}{|c|c|c|c|}
\hline & GPA & EGPA & Sarcoid \\
\hline Ear & $\begin{array}{l}\text { - Otalgia, otorrhoea, conductive and } \\
\text { sensorineural deafness } \\
\text {. Dizziness } \\
\text {. Tinnitus } \\
\text { - Chronic otitis media } \\
\text { - Otitis externa }\end{array}$ & $\begin{array}{l}\text { Otalgia, otorrhoea, conductive } \\
\text { and sensorineural deafness } \\
\text { - Serous otitis media, } \\
\text { mastoiditis, facial nerve palsy }\end{array}$ & $\begin{array}{l}\text { - Sensorineural and conductive deafness } \\
\text { - Vestibular dysfunction } \\
\text { - Facial nerve paralysis }\end{array}$ \\
\hline Nose and throat & $\begin{array}{l}\text { - } \text { Nasal obstruction } \\
\text {. } \text { Crusting } \\
\text {. } \text { Bleeding } \\
\text {. Rhinorrhea } \\
\text {. Facial pain, loss of smell } \\
\text {. Rhinosinusitis } \\
\text {. Septal perforation } \\
\text {. External 'saddle' deformity } \\
\text {. }\end{array}$ & 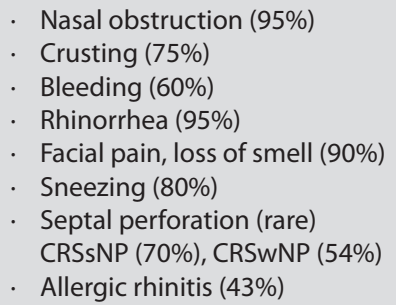 & $\begin{array}{l}\text { - Nasal obstruction }(90 \%) \\
\text {. } \text { Crusting }(66 \%) \\
\text {. } \text { Blood stained discharge }(40 \%) \\
\text {. Facial pain }(25 \%) \\
\text {. Anosmia }(70 \%) \\
\text {. Septal perforation } \\
\text {. 'Strawberry skin' nasal mucosal nodules } \\
\text { - Nasal bones - rarefaction and soft } \\
\text { tissue infiltration, adhesions }\end{array}$ \\
\hline Mouth & $\begin{array}{l}\text { - Oral ulceration } \\
\text { - Gingivitis } \\
\text { - Fistula } \\
\text {. Cranial nerve palsies }\end{array}$ & & C Cranial nerve palsies \\
\hline
\end{tabular}




\begin{tabular}{|c|c|c|c|}
\hline & GPA & EGPA & Sarcoid \\
\hline Larynx & $\begin{array}{l}\text { Hoarseness, stridor } \\
\text {. Vocal fold palsy } \\
\text {. Subglottic and tracheal stenosis }\end{array}$ & & $\begin{array}{l}\text { Hoarseness, stridor } \\
\text { - Supraglottic granulomas and stenosis } \\
\text {. Vocal fold palsies }\end{array}$ \\
\hline Lower respiratory tract & $\begin{array}{l}\text { Dyspnoea, pleuritic pain, hemoptysis } \\
\text { Pulmonary infiltrates, nodules, } \\
\text { abscesses or haemorrhage }\end{array}$ & $\begin{array}{l}\text { Asthma }(\sim 100 \%) \\
\text { Pulmonary infiltrates, nodules, } \\
\text { bronchiectasis, bronchial wall } \\
\text { thickening }\end{array}$ & $\begin{array}{l}\text { Dyspnoea, cough, chest pain, wheeze } \\
(50-95 \%) \\
\text {. Hemoptysis (rare) } \\
\text {. Pulmonary infiltrates } \\
\text {. Hilar lymphadenopathy (+ fever, } \\
\text { polyarthralgias = Lofgren syndrome, } \\
\text { mainly Scandinavians) }\end{array}$ \\
\hline Ophthalmic & $\begin{array}{l}\text { Proptosis, pain, visual loss, epiphora } \\
\text {. Scleritis, episcleritis, retinitis, retro- } \\
\text { orbital granuloma }\end{array}$ & Rare & $\begin{array}{l}\text { Epiphora, red, painful eye, } \\
\text { photophobia, dry eye, visual loss } \\
\text { Anterior and posterior uveitis, } \\
\text { iridocyclitis, keratoconjunctivitis, scleral } \\
\text { plaques } \\
\text {. Lacrimal enlargement }\end{array}$ \\
\hline Cardiac & $\begin{array}{l}\text { Arrhythmias } \\
\text { - } \text { Pericardial effusion } \\
\text { Infarction, myocarditis }\end{array}$ & $\begin{array}{l}\text { Vasculitic myocarditis (23- } \\
45 \%) \\
\text { Pericarditis } \\
\text { Myocardial infarction }\end{array}$ & $\begin{array}{l}\text { Cardiac failure and ventricular } \\
\text { arrhythmia - heart block, sudden death }\end{array}$ \\
\hline Renal & $\begin{array}{l}\text { - Glomerulonephritis } \\
\text {. } \\
\text {. Renal impairment failure }\end{array}$ & $\begin{array}{l}\text { Glomerulonephritis } \\
\text { Renal impairment } \\
\text { Renal failure }\end{array}$ & \\
\hline Gastrointestinal & $\begin{array}{l}\text { Diarrhoea } \\
\text { - Bleeding }\end{array}$ & $\begin{array}{l}\text { Abdominal pain, nausea, } \\
\text { vomiting, diarrhoea, } \\
\text { haemetemesis, melaena } \\
\text { Small bowel perforation }\end{array}$ & \\
\hline $\begin{array}{l}\text { Peripheral nervous } \\
\text { system }\end{array}$ & $\begin{array}{l}\text { Sensory or motor polyneuropathy } \\
\text {. Mononeuritis (10-50\%) }\end{array}$ & $\begin{array}{l}\text { Sensory or motor peripheral } \\
\text { neuropathy }(50-70 \%) \\
\text { Mononeuritis esp. peroneal } \\
\text { nerve }\end{array}$ & $\begin{array}{l}\text { Polyneuritis } \\
\text { - Peripheral mononeuritis } \\
\text { - Myelopathy }\end{array}$ \\
\hline Central nervous system & $\begin{array}{ll} & \text { CNS lesions } \\
\cdot & \text { Meningitis } \\
\cdot & \text { Meningeal vasculitis }\end{array}$ & $\begin{array}{l}\text { Intracranial abscess } \\
\text { Cranial neuropathies }\end{array}$ & $\begin{array}{l}\text { - Lymphocytic meningitis } \\
\text { - Cranial neuropathies } \\
\text { - Hypothalamic / pituitary dysfunction } \\
\text { (rare) }\end{array}$ \\
\hline Skin & $\begin{array}{l}\text { Purpura } \\
\text { Subcutaneous nodules } \\
\text { - Non-healing ulceration (40-50\%) }\end{array}$ & $\begin{array}{l}\text { Purpura } \\
\text { Subcutaneous nodules } \\
\text { Non-healing ulceration ( } 40- \\
50 \%)\end{array}$ & $\begin{array}{l}\text { Violaceous rash } \\
\text { - Erythema nodosum } \\
\text {. Lupus pernio } \\
\text {. } \text { Maculopapular plaques (rare) }\end{array}$ \\
\hline Musculoskeletal & $\begin{array}{l}\text { - Arthralgia } \\
\text { - Myalgia }\end{array}$ & $\begin{array}{l}\text { Arthralgia } \\
\text { Myalgia }\end{array}$ & $\begin{array}{l}\text { - Arthralgia } \\
\text { - Myalgia } \\
\text {. Dactylitis }\end{array}$ \\
\hline General / Other & $\begin{array}{l}\text { Fever } \\
\text { - Weight loss } \\
\text { - Fatigue } \\
\text {. } \\
\text { Nocturnal sweats }\end{array}$ & $\begin{array}{l}\text { Fever } \\
\text { Weight loss } \\
\text { Fatigue }\end{array}$ & $\begin{array}{l}\text { Fever } \\
\text { - } \text { Weight loss } \\
\text { - Fatigue }(45 \%) \\
\text { - Peripheral lymphadenopathy } \\
\text { - Parotitis (Heerfordt's syndrome) } \\
\text {. Hepatosplenomegaly }\end{array}$ \\
\hline
\end{tabular}

Table 8.7.3. Investigations in granulomatosis with polyangiitis (GPA), eosinophilic granulomatosis with polyangiitis (EGPA) and sarcoidosis.

\begin{tabular}{|c|c|c|c|}
\hline & GPA & EGPA & Sarcoid \\
\hline Blood & $\begin{array}{l}\text { c-ANCA/PR3 (95\% +ve with systemic } \\
\text { disease; } 50 \%+\text { ve with limited } \\
\text { disease) } \\
\text {. } \\
\text { p-ANCA ( } 5 \%+\text { ve) } \\
\text { Raised erythrocyte sedimentation } \\
\text { rate (ESR) } \\
\text {. } \text { Raised c-reactive protein }\end{array}$ & $\begin{array}{l}\text { Blood eosinophilia (>10\%) } \\
\text {. IgE - non-specific increase } \\
\text {. } \mathrm{p}-\mathrm{ANCA} \text { MPO + ve (in } 26-48 \% \text {, increasing } \\
\text { to } 75 \% \text { with renal involvement) }\end{array}$ & $\begin{array}{l}\text {. } \text { Hypercalcaemia (in } 10-13 \% \text { ) } \\
\text {. } \text { Hypercalciuria (in } ~ 30 \% \text { ) } \\
\text {. } \text { Raised alkaline phosphatase } \\
\text {. } \text { Raised angiotensin-converting } \\
\text { enzyme (in } 60-83 \% \text { ) }\end{array}$ \\
\hline Imaging & $\begin{array}{l}\text {. HRCT chest } \\
\text {. CT sinuses }\end{array}$ & $\begin{array}{l}\text {. } \mathrm{HRCT} \text { chest } \\
\text {. } \mathrm{CT} \text { sinuses }\end{array}$ & $\begin{array}{l}\text { - } \text { CXR / CT chest } \\
\text { - CT sinuses }\end{array}$ \\
\hline
\end{tabular}




\begin{tabular}{|c|c|c|c|}
\hline $\begin{array}{l}\text { Lower respiratory } \\
\text { tract }\end{array}$ & $\begin{array}{l}\text { Pulmonary function } \\
\text { Flow volume loop spirometry to } \\
\text { exclude subglottic stenosis }\end{array}$ & - Pulmonary function & $\begin{array}{l}\text { Pulmonary function test } \\
\text { Gallium }{ }^{67} \text { perfusion test } \\
\text { Broncho-alveolar lavage } \\
\text { Bronchoscopy - biopsy }\end{array}$ \\
\hline Cardiac & & - Myocardial assessment & $\begin{array}{l}\text { Cardiopulmonary exercise } \\
\text { testing } \\
\text { ECG }\end{array}$ \\
\hline Renal & $\begin{array}{l}\text { Urinalysis } \\
\text { Renal function tests } \\
\text { Tissue biopsy }\end{array}$ & $\begin{array}{l}\text { - Urinalysis } \\
\text {. Renal function tests }\end{array}$ & \\
\hline $\begin{array}{l}\text { Peripheral nervous } \\
\text { system }\end{array}$ & & - Biopsy if neuropathy & \\
\hline $\begin{array}{l}\text { Central nervous } \\
\text { system }\end{array}$ & & & MRI brain \\
\hline Skin & & & $\begin{array}{l}\text { Negative tuberculin skin test in } \\
\text { a BCG vaccinated patient } \\
\text { Biopsy of lupus pernio }\end{array}$ \\
\hline General / Other & & & FDG-PET CT \\
\hline
\end{tabular}

pain. Anosmia can result from mechanical obstruction of the olfactory region and/or sensorineural ${ }^{(453)}$. The co-existence of chronic rhinosinusitis and two of the following: nasal crusting, anosmia and epistaxis were shown to be highly specific for sarcoid rhinosinusitis ${ }^{(454)}$. This means that many of these patients may present to the ENT community but as with other granulomatous diseases, they may go unrecognised. To put this in context, the diagnosis of nasal sarcoid was made in 23 patients who presented with 'chronic rhinosinusitis' in a cohort of 5000 patients presenting to the aforementioned rhinology clinic and this has been the experience of others ${ }^{(459,460,462,463)}$. On endoscopic examination, the mucosa has been described as a 'strawberry skin' with small pale granulomas dotted over an erythematous and granular mucosa ${ }^{(411,460)}$. These appearances may be obscured by extensive crusting and eventually may become atrophic. Within the nasal cavity, the inferior turbinate and adjacent nasal septum are particularly affected with a nodular hypertrophy. However, any of the paranasal sinuses, nasopharynx, soft palate, hypopharynx and supraglottis can be involved.

\section{Investigations (Table 8.7.3.)}

There is no definitive test for sarcoid other than a positive biopsy, usually endobronchial ${ }^{(444)}$ or from skin, minor salivary glands or lymph nodes ${ }^{(411)}$. The classic appearance is of a noncaseating granuloma composed of a central area of tightly packed epithelioid, giant cells, CD4+ T cells surrounded by CD8+ $T$ and $B$ lymphocytes and fibroblasts at the periphery. Special stains should be negative for mycobacteria and fungi. Biopsying the nasal mucosa if it appears macroscopically abnormal is worthwhile as the majority will be positive (91\%) whereas if it appears normal, the biopsy will usually be negative $(92 \%)^{(453)}$. Blood tests may include raised serum and urinary calcium levels, raised alkaline phosphatase and raised serum angiotensinconverting enzyme (SACE) but none are diagnostic (sensitivity $60 \%$; specificity $70 \%$ ).

Other tests may include pulmonary and cardiopulmonary function, perfusion studies, broncho-alveolar lavage and ECG.

\section{Imaging}

CT scanning of the nose and sinuses often shows a nonspecific generalised opacification similar to that seen in chronic rhinosinusitis. This may be due to an active granulomatous process, or fibrosis after the condition has regressed and/or non-specific inflammation or infection ${ }^{(461)}$. In addition turbinate or septal nodularity (21\%), osteoneogenesis (15\%) and bone erosion (8\%) may be seen ${ }^{(464)}$. The bone erosion in the sinuses may even suggest malignancy.

The nasal bones may show rarefaction or punctuate osteolysis similar to that seen in the metacarpals or metatarsals. In CT scans of the sinuses of 39 biopsy-proven sarcoid patients, the mean Lund-Mackay score was a modest 6.2 but the nodularity of the mucosal thickening should suggest the diagnosis ${ }^{(464)}$. MRI of the sinuses may also show abnormalities consistent with the $\mathrm{CT}$ changes as will gallium ${ }^{(159)}$ scanning and positron emission tomography ${ }^{(465)}$. The lacrimal glands may be enlarged bilaterally.

Chest radiography and $\mathrm{CT}$ of the lungs may show multiple nodules or nodular infiltration but occasional these may be solitary or unilateral between $1-5 \mathrm{~cm}$ in diameter ${ }^{(466)}$.

Gallium $^{(159)}$ perfusion scans can demonstrate increased uptake in the sarcoid granuloma but have low sensitivity and specificity. MRI of the brain may show granulomatous involvement of the basal meninges.

FDG-PET is being used to monitor response in severe cases.
Sarcoid is staged according to pulmonary involvement ${ }^{(466)}$ :
0 : Normal chest imaging
I: Bilateral hilar lymphadenopathy, normal lung fields
II: Bilateral hilar lymphadenopathy and infiltrates
III: Infiltrates alone
IV: Fibrosis

\section{Differential diagnosis}

The differential diagnosis is between other granulomatous diseases such as syphilis, tuberculosis, rhinoscleroma, GPA and EGPA as well as berylliosis, leprosy, fungal disease, cat scratch fever, a range of lymphomas and diffuse granulomatous disease 
in common variable immune deficiency ${ }^{(369,371,462,467)}$. An elevated serum angiotensin-converting enzyme is suggestive of sarcoid but can be raised in other conditions such as TB, lymphoma, leprosy and Gaucher's disease.

\subsubsection{Treatment}

Sarcoidosis can present to a wide range of disciplines including ENT so as with other vasculitic/granulomatous diseases, it requires a low threshold of suspicion to make the diagnosis and is best managed in a multi-disciplinary environment ${ }^{(451)}$. In many cases, particularly Stage 1 disease, the disease undergoes spontaneous remission within two years without specific treatment. Around $75 \%$ can be managed symptomatically with NSAIDS. In those with Stage 2, 65\% spontaneously regress, Stage 3 patients with just lung disease have a $30 \%$ of remission chance and an $18 \%$ mortality rate whereas $85 \%$ of those with erythema nodosum and acute arthritis remit spontaneously. Those with more advanced disease as evidenced by raised SACE or extrapulmonary involvement will usually require treatment and about 50\% may experience a relapse, with 1 in 10 developing serious disability ${ }^{(468)}$. Severe cardiac or pulmonary involvement may lead to death in some cases and lung transplantation has been undertaken though up to two thirds develop recurrence in the allograft ${ }^{(469,470)}$. Lung function should be monitored every 6-12 months..

\section{Systemic}

The mainstay of treatment, when it is required, remains oral corticosteroids. They are used for Stage II and III pulmonary disease and for extrapulmonary involvement of critical organs $^{(468,471)}$.

Hydroxychloroquine / chloroquine are used for cutaneous and bone lesions and neurologic sarcoid as well as acute and maintenance treatment of the chest. However, they can cause retinal toxicity.

Steroid-sparing cytotoxic agents such as methotrexate (weekly), cyclophosphamide and azathioprine may be used as alternatives or in addition to systemic steroids ${ }^{(468)}$. TNF-alpha antagonists such as infliximab ${ }^{(468,472-474)}$ and thalidomide ${ }^{(475)}$ have been used in refractory disease, in particular for cutaneous, ophthalmic, hepatic and neurosarcoidosis.

\section{Nasal}

Nasal symptoms may be improved by the systemic treatment ${ }^{(461,}$
476). In addition standard topical treatments with saline or alkaline irrigations, corticosteroid sprays or drops and lubricants such as $25 \%$ glucose and glycerine drops, honey ointment or an aqueous gel are usually recommended together with regular debridement of the crusts can be used ${ }^{(451)}$ (Level IV).

Surgery has a very limited role, confined to biopsy, occasionally debulking of granulomatous masses and associated inferior turbinate hypertrophy ${ }^{(477)}$ and endoscopic sinus surgery to elucidate CT changes and possibly reduce the need for systemic steroids ${ }^{(442,457,461,462,478)}$. Surgery on the septum is not recommended, especially if the disease is active as perforation may result from the procedure ${ }^{(461)}$. Nasal reconstruction and resurfacing have also been anecdotally described for persistent lupus pernio ${ }^{(479-481)}$.

Key points | What's new since EPOS 2012
- A low threshold of suspicion should be maintained for ANCA-associated vasculitis (granulomatosis with polyangitis (GPA), eosinophilic granulomatosis with polyangitis (EGPA)) and sarcoidosis, all of which can affect the upper respiratory tract and present with apparent chronic rhinosinusitis.

- The ANCA test has become the mainstay of diagnosis in vasculitis but lacks sensitivity in limited forms of GPA (c-ANCA) and EGPA (p-ANCA).

- Cocaine abuse can produce a midline destructive process which mimics GPA.

- In GPA and EGPA systemic treatment with immunosuppression is being replaced in many cases by rituximab and other monoclonal antibodies.

- Sarcoidosis is a chronic multi-system inflammatory disease of unknown aetiology characterised by noncaseating granuloma.

- There is no definitive test for sarcoid other than a positive biopsy.

- $\quad$ Systemic steroids remain the mainstay of treatment in sarcoidosis, though hydroxychloroquine, steroid-sparing cytotoxic agents such as methotrexate andTNF-alpha antagonists such as infliximab are being used.

- In all these conditions, local treatment includes nasal rinsing, topical steroids and lubricants.

\section{References}

1. Gutman M, Torres A, Keen KJ, Houser SM. Prevalence of allergy in patients with chronic rhinosinusitis. Otolaryngology--head and neck surgery : official journal of American Academy of Otolaryngology-Head and Neck Surg. 2004;130:545-52.

2. Shen $\mathrm{K}-\mathrm{C}$, Lin $\mathrm{Y}-\mathrm{T}$, Lin C-F, Chang C-H, Yeh T-H. Allergy accelerates the disease progression of chronic rhinosinusitis. Acta oto-laryngologica 2019;139:75-9.

3. Huang S-WW. The risk of sinusitis in children with allergic rhinitis. Allergy Asthma Proc 2000;21:85-8.

4. Wilson KF, McMains KC, Orlandi RR. The association between allergy and chronic rhinosinusitis with and without nasal polyps: an evidence-based review with recommendations. International forum of allergy \& rhinology 2014;4:93-103.

5. Hamizan AW, Loftus PA, Alvarado R, et al. Allergic phenotype of chronic rhinosinusitis based on radiologic pattern of disease. Laryngoscope. 2018;128:2015-21.
6. Marcus S, Schertzer J, Roland LT, Wise SK, Levy JM, DelGaudio JM. Central compartment atopic disease: prevalence of allergy and asthma compared with other subtypes of chronic rhinosinusitis with nasal polyps. Int Forum Allergy Rhinol. 2019, 10.1002/alr.22454.

7. Philpott CM, Erskine S, Hopkins C, et al. Prevalence of asthma, aspirin sensitivity and allergy in chronic rhinosinusitis: data from the UK National Chronic Rhinosinusitis Epidemiology Study. Respiratory research 2018;19:129.

8. Green BJ, Beezhold DH, Gallinger Z, et al. Allergic 
sensitization in Canadian chronic rhinosinusitis patients. Allergy, Asthma \& Clinical Immunology 2014;10:15.

9. Houser SM, Keen KJ. The role of allergy and smoking in chronic rhinosinusitis and polyposis. Laryngoscope. 2008;118:1521-7.

10. Asero R, Bottazzi G. Nasal polyposis: a study of its association with airborne allergen hypersensitivity. Ann Allergy Asthma Immunol 2001;86:283-5.

11. Benjamin MR, Stevens WW, Li N, et al. Clinical Characteristics of Patients with Chronic Rhinosinusitis without Nasal Polyps in an Academic Setting. The journal of allergy and clinical immunology In practice 2019;7:1010-6.

12. Li QC, Cheng KJ, Wang F, Zhou SH. Role of atopy in chronic rhinosinusitis with nasal polyps: does an atopic condition affect the severity and recurrence of disease? The J of laryngology and otology 2016;130:640-4.

13. Robinson S, Douglas R, Wormald P-JJ. The relationship between atopy and chronic rhinosinusitis. American J of Rhinology. 2006;20:625-8.

14. Bonfils $P, A v a n ~ P$, Malinvaud D. Influence of allergy on the symptoms and treatment of nasal polyposis. Acta Otolaryngol. 2006;126:839-44.

15. Erbek SS, Erbek S, Topal O, Cakmak O. The Role of Allergy in the Severity of Nasal Polyposis. American J of Rhinology. 2007;21:686-90.

16. Ho J, Alvarado R, Rimmer J, Sewell WA, Harvey RJ. Atopy in chronic rhinosinusitis: impact on quality of life outcomes. International Forum of Allergy \& Rhinology. 2019;9:501-7.

17. Stammberger $\mathrm{H}$. Functional endoscopic sinus surgery. 1991.

18. DelGaudio JM, Loftus PA, Hamizan AW, Harvey RJ, Wise SK. Central Compartment Atopic Disease. American J of Rhinology. \& Allergy 2017:31:228-34.

19. Zhang $Y$, Gevaert E, Lou H, et al. Chronic rhinosinusitis in Asia. J of Allergy and Clinical Immunology 2017;140:1230-9.

20. Orlandi RR, Kingdom TT, Hwang PH, et al. International Consensus Statement on Allergy and Rhinology: Rhinosinusitis. Int Forum Allergy Rhinol. 2016;6 Suppl 1:S22-S209.

21. Hirose K, Iwata A, Tamachi T, Nakajima H. Allergic airway inflammation: key players beyond the Th2 cell pathway. Immunological Reviews 2017;278:145-61

22. Lou H, Meng Y, Piao Y, Wang C, Zhang L, Bachert C. Predictive significance of tissue eosinophilia for nasal polyp recurrence in the Chinese population. Am J Rhinol Allergy 2015;29:350-6.

23. Kato A. Group 2 Innate Lymphoid Cells in Airway Diseases. Chest 2019, 10.1016/j. chest.2019.04.101.

24. Wang B-F, Cao P-P, Long X-B, et al. Distinct mucosal immunopathologic profiles in atopic and nonatopic chronic rhinosinusitis without nasal polyps in Central China. International forum of allergy \& rhinology 2016;6:1013-9.

25. Zhang J, Chen J, Robinson C. Cellular and Molecular Events in the Airway Epithelium Defining the Interaction Between House Dust Mite Group 1 Allergens and Innate Defences. International journal of molecular sciences 2018;19:3549.
26. De Greve G, Hellings PW, Fokkens WJ, Pugin B, Steelant B, Seys SF. Endotype-driven treatment in chronic upper airway diseases. Clin Transl Allergy 2017;7:22.

27. Smurthwaite L, Durham SR. Local IgE synthesis in allergic rhinitis and asthma. Curr Allergy Asthma Rep 2002;2:231-8.

28. Wise SK, Ahn CN, Schlosser RJ. Localized immu noglobulin E expression in allergic rhinitis and nasal polyposis. Curr Opin Otolaryngol. Head Neck Surg. 2009;17:216-22.

29. Gevaert P, Holtappels G, Johansson SGO, Cuvelier $\mathrm{C}$, Cauwenberge $\mathrm{P}$, Bachert $\mathrm{C}$. Organization of secondary lymphoid tissue and local lgE formation to Staphylococcus aureus enterotoxins in nasal polyp tissue. Allergy 2005;60:71-9.

30. Schryver ED, Devuyst L, Derycke L, et al. Local immunoglobulin e in the nasal mucosa: clinical implications. Allergy, asthma \& immunology research 2015;7:321-31

31. Kortekaas Krohn I, Shikhagaie MM, Golebski K, et al. Emerging roles of innate lymphoid cells in inflammatory diseases: Clinical implications. Allergy 2018;73:837-50.

32. White LJ, Rotella MR, DelGaudio JM. Polypoid changes of the middle turbinate as an indicator of atopic disease. International forum of allergy \& rhinology 2014;4:376-80

33. Brunner JP, Jawad BA, McCoul ED. Polypoid Change of the Middle Turbinate and Paranasal Sinus Polyposis Are Distinct Entities. Otolaryngology-Head and Neck Surg. 2017;157:519-23.

34. Kirtsreesakul V. Role of allergy in the therapeutic response of nasal polyps. Asian Pacific journal of allergy and immunology 2002;20:141-6.

35. DeYoung K, Wentzel JL, Schlosser RJ, Nguyen SA Soler ZM. Systematic review of immunotherapy for chronic rhinosinusitis. American J of Rhinology. \& Allergy 2014;28:145-50

36. Bonilla FA, Khan DA, Ballas ZK, et al. Practice parameter for the diagnosis and management of primary immunodeficiency. J of Allergy and Clinical Immunology 2015;136:1186-205.e78.

37. Nayan S, Alizadehfar R, Desrosiers M. Humoral Primary Immunodeficiencies in Chronic Rhinosinusitis. Current Allergy and Asthma Reports 2015;15:46.

38. Schwitzguébel AJ-P, Jandus P, Lacroix J-SS, et al. Immunoglobulin deficiency in patients with chronic rhinosinusitis: Systematic review of the literature and meta-analysis. J of Allergy \& Clinical Immunology 2015;136:1523-31.

39. Resnick ES, Moshier EL, Godbold JH, Cunningham-Rundles C. Morbidity and mortality in common variable immune deficiency over 4 decades. Blood 2012;119:1650-7.

40. Picard C, Bobby Gaspar H, Al-Herz W, et al. International Union of Immunological Societies: 2017 Primary Immunodeficiency Diseases Committee Report on Inborn Errors of Immunity. J of clinical immunology 2018;38:96-128.

41. Bonilla FA, Barlan I, Chapel $\mathrm{H}$, et al. International Consensus Document (ICON): Common Variable Immunodeficiency Disorders. The journal of allergy and clinical immunology In practice 2016;4:38-59.
42. Singh $\mathrm{K}$, Chang C, Gershwin ME. IgA deficiency and autoimmunity. Autoimmunity Reviews 2014;13:163-77.

43. Frieri M. Good's syndrome, CVID, and selective antibody deficiency in patients with chronic rhinosinusitis. Current allergy and asthma reports 2014;14:438.

44. McCusker C, Upton J, Warrington R. Primary immunodeficiency. Allergy, Asthma \& Clinical Immunology 2018;14:61.

45. Gill PK, Betschel SD. Timing of infections in patients with primary immunodeficiencies treated with intravenous immunoglobulin (IVIg). Allergy Asthma Clin Immunol 2018;14:35.

46. Walsh JE, Gurrola JG, Graham SM, Mott SL, Ballas ZK. Immunoglobulin replacement therapy reduces chronic rhinosinusitis in patients with antibody deficiency. International forum of allergy \& rhinology 2017;7:30-6.

47. Quinti I, Soresina A, Spadaro G, et al. Long-term follow-up and outcome of a large cohort of patients with common variable immunodeficiency. J Clin Immunol 2007;27:308-16.

48. Kuruvilla M, de la Morena MT. Antibiotic Prophylaxis in Primary Immune Deficiency Disorders. J Allergy and Clin Immunol. : In Practice 2013;1:573-82.

49. Bayrakci B, Ersoy F, Sanal O, Kiliç S, Metin A, Tezcan I. The efficacy of immunoglobulin replacement therapy in the long-term follow-up of the B-cell deficiencies (XLA, HIM, CVID). Turk J Pediatr. 2005;47(3):239-46

50. Kashani S, Carr TF, Grammer LC, et al. Clinical Characteristics of Adults With Chronic Rhinosinusitis and Specific Antibody Deficiency. J Allergy Clin Immunol. 2015;3:236-42.

51. Khalid AN, Mace JC, Smith TL. Outcomes of sinus surgery in ambulatory patients with immune dysfunction. Am J Rhinol Allergy 2010;24:230-3.

52. Duraisingham SS, Buckland M, Dempster J, Lorenzo L, Grigoriadou S, Longhurst HJ. Primary vs. secondary antibody deficiency: clinical features and infection outcomes of immunoglobulin replacement. PloS one 2014;9:e100324.

53. Roberts DM, Jones RB, Smith RM, et al. Rituximab-associated hypogammaglobulinemia: incidence, predictors and outcomes in patients with multi-system autoimmune disease. J of autoimmunity 2015;57:60-5.

54. lacovou E, Vlastarakos PV, Papacharalampous G, Kampessis G, Nikolopoulos TP. Diagnosis and treatment of HIV-associated manifestations in otolaryngology. Infectious disease reports 2012;4:e9.

55. Hajjij A, Mace JC, Soler ZM, Smith TL, Hwang PH. The impact of diabetes mellitus on outcomes of endoscopic sinus surgery: a nested case-control study. International forum of allergy \& rhinology 2015;5:533-40.

56. Khan A, Vandeplas G, Huynh TMT, et al. The Global Allergy and Asthma European Network (GALEN rhinosinusitis cohort: a large European cross-sectional study of chronic rhinosinusitis patients with and without nasal polyps. Rhinology. 2019;57:32-42.

57. Hellings PW, Fokkens WJ, Bachert $C_{\text {, et al }}$ 
Positioning the principles of precision medicine in care pathways for allergic rhinitis and chronic rhinosinusitis - A EUFOREA-ARIA-EPOS-AIRWAYS ICP statement. Allergy 2017;72:1297-305.

58. Valiulis A, Bousquet J, Veryga A, et al. Vilnius Declaration on chronic respiratory diseases: multisectoral care pathways embedding guided self-management, $\mathrm{mHealth}$ and air pollution in chronic respiratory diseases. Clin Transl Allergy 2019;9:7.

59. Ostovar A, Fokkens WJ, Vahdat K, Raeisi A, Mallahzadeh A, Farrokhi S. Epidemiology of chronic rhinosinusitis in Bushehr, southwestern region of Iran: a GA2LEN study. Rhinology. 2019;57:43-8.

60. Massoth L, Anderson C, McKinney KA. Asthma and Chronic Rhinosinusitis: Diagnosis and Medical Management. Med Sci (Basel) 2019;7.

61. Yang $\mathrm{M}, \mathrm{Chen} \mathrm{H}$, Zhang $\mathrm{Y}$, et al. Long-term use of inhaled corticosteroids and risk of upper respiratory tract infection in chronic obstructive pulmonary disease: a meta-analysis. Inhalation Toxicology 2017;29:219-26.

62. Hakansson K, Konge L, Thomsen SF, Backer $\mathrm{V}$, von Buchwald C. Sinonasal inflammation in COPD: a systematic review. Eur Respir J 2013;42:1402-11

63. Kariya S, Okano M, Oto T, et al. Pulmonary function in patients with chronic rhinosinusitis and allergic rhinitis. J Laryngol. Otol. 2014;128:25562.

64. Tomisa G, Horvath A, Szalai Z, Muller V, Tamasi L. Prevalence and impact of risk factors for poor asthma outcomes in a large, specialist-managed patient cohort: a real-life study. J Asthma Allergy 2019;12:297-307.

65. Maspero JF, Katelaris $\mathrm{CH}$, Busse WW, et al. Dupilumab Efficacy in Uncontrolled, Moderate-to-Severe Asthma with Self-Reported Chronic Rhinosinusitis. J Allergy Clin Immunol Pract 2019, 10.1016/j.jaip.2019.07.016.

66. Somani SN, Kwah JH, Yeh C, et al. Prevalence and characterization of chronic rhinosinusitis in patients with non-cystic fibrosis bronchiectasis at a tertiary care center in the United States. Int Forum Allergy Rhinol. 2019;9:1424-9.

67. Handley E, Nicolson CH, Hew M, Lee AL. Prevalence and Clinical Implications of Chronic Rhinosinusitis in People with Bronchiectasis: A Systematic Review. J Allergy and Clin Immunol. : In Practice 2019;7:2004-12.e1.

68. Aarab R, Vijverberg SJH, Prins M, et al. Prevalence of and factors associated with adult-onset asthma in different ethnic groups: The HELIUS study. Respir Med 2019;150:113-9.

69. Grossman NL, Ortega VE, King TS, et al. Exacerbation-prone asthma in the context of race and ancestry in Asthma Clinical Research Network trials. J Allergy Clin Immunol 2019;144:1524-33.

70. Banoub RG, Phillips KM, Hoehle LP, Caradonna DS, Gray ST, Sedaghat AR. Relationship between chronic rhinosinusitis exacerbation frequency and asthma control. Laryngoscope. 2018;128:1033-8

71. Reddel HK, FitzGerald JM, Bateman ED, et al. GINA 2019: a fundamental change in asthma management: Treatment of asthma with short-acting bronchodilators alone is no longer recommended for adults and adolescents. Eur Respir J 2019;53.

72. Singh U, Wangia-Anderson V, Bernstein JA. Chronic Rhinitis Is a High-Risk Comorbidity for 30-Day Hospital Readmission of Patients with Asthma and Chronic Obstructive Pulmonary Disease. J Allergy and Clin Immunol. : In Practice 2019;7:279-85.e6.

73. Yang X, Xu Y, Jin J, Li R, Liu X, Sun Y. Chronic rhinosinusitis is associated with higher prevalence and severity of bronchiectasis in patients with COPD. Int J Chron Obstruct Pulmon Dis 2017;12:655-62.

74. Hens G, Vanaudenaerde BM, Bullens DM, et al. Sinonasal pathology in nonallergic asthma and COPD: 'united airway disease' beyond the scope of allergy. Allergy 2008;63:261-7.

75. Bachert C, Han JK, Desrosiers M, et al. Efficacy and safety of dupilumab in patients with severe chronic rhinosinusitis with nasal polyps (LIBERTY NP SINUS-24 and LIBERTY NP SINUS-52): results from two multicentre, randomised, double-blind, placebo-controlled, parallel-group phase 3 trials. Lancet 2019;394:1638-50.

76. Weinstein SF, Katial RK, Bardin P, et al. Effects of Reslizumab on Asthma Outcomes in a Subgroup of Eosinophilic Asthma Patients with Self-Reported Chronic Rhinosinusitis with Nasal Polyps. J Allergy Clin Immunol Pract 2019;7:589-96.e3.

77. Tiotiu A, Oster JP, Roux P, et al. Omalizumab's effectiveness in severe allergic asthma and nasal polyps: a real-life study. J Investig Allergol Clin Immunol 2019, 10.18176/jiaci.0391:0.

78. Braunstahl GJ, Overbeek SE, Fokkens WJ, et al. Segmental bronchoprovocation in allergic rhinitis patients affects mast cell and basophil numbers in nasal and bronchial mucosa. Am J Respir Crit Care Med 2001;164:858-65.

79. Braunstahl GJ, Kleinjan A, Overbeek SE, Prins JB, Hoogsteden HC, Fokkens WJ. Segmental bronchial provocation induces nasal inflammation in allergic rhinitis patients. Am J Respir Crit Care Med 2000;161:2051-7.

80. Hens G, Bobic S, Reekmans K, Ceuppens JL, Hellings PW. Rapid systemic uptake of allergens through the respiratory mucosa. J Allergy Clin Immunol 2007;120:472-4.

81. Steelant B, Seys SF, Boeckxstaens G, Akdis CA, Ceuppens JL, Hellings PW. Restoring airway epithelial barrier dysfunction: a new therapeutic challenge in allergic airway disease. Rhinology. 2016;54:195-205.

82. Hens G, Raap U, Vanoirbeek J, et al. Selective nasal allergen provocation induces substance P-mediated bronchial hyperresponsiveness. Am J Respir Cell Mol Biol 2011;44:517-23.

83. Kim Y-C, Won H-K, Lee JW, et al. Staphylococcus aureus Nasal Colonization and Asthma in Adults: Systematic Review and Meta-Analysis. The journal of allergy and clinical immunologyln practice 2019;7:606-15.e9.

84. Hellings PW, Hens G, Meyts I, et al. Aggravation of bronchial eosinophilia in mice by nasal and bronchial exposure to Staphylococcus aureus enterotoxin B. Clin Exp Allergy 2006;36:1063-71.

85. De Boeck I, Wittouck S, Martens K, et al. Anterior Nares Diversity and Pathobionts Represent Sinus
Microbiome in Chronic Rhinosinusitis. mSphere 2019;4:e00532-19.

86. Yoo KH, Ahn HR, Park JK, et al. Burden of Respiratory Disease in Korea: An Observational Study on Allergic Rhinitis, Asthma, COPD, and Rhinosinusitis. Allergy Asthma Immunol Res 2016;8:527-34.

87. Hellings PW, Prokopakis EP. Global airway disease beyond allergy. Curr Allergy Asthma Rep 2010;10:143-9.

88. Doulaptsi M, Prokopakis E, Seys S, Pugin B, Steelant B, Hellings P. Visual analogue scale for sino-nasal symptoms severity correlates with sino-nasal outcome test 22: paving the way for a simple outcome tool of CRS burden. Clin Transl Allergy 2018;8:32.

89. Vashishta R, Soler ZM, Nguyen SA, Schlosser RJ. A systematic review and meta-analysis of asthma outcomes following endoscopic sinus surgery for chronic rhinosinusitis. International Forum of Allergy \& Rhinology. 2013;3:788-94.

90. Schlosser RJ, Smith TL, Mace J, Soler ZM. Asthma quality of life and control after sinus surgery in patients with chronic rhinosinusitis. Allergy 2017;72:483-91.

91. van der Veen J, Seys SF, Timmermans M, et al. Real-life study showing uncontrolled rhinosinusitis after sinus surgery in a tertiary referral centre. Allergy 2017;72:282-90.

92. Morrissey DK, Bassiouni A, Psaltis AJ, Naidoo Y, Wormald PJ. Outcomes of revision endoscopic modified Lothrop procedure. Int Forum Allergy Rhinol. 2016;6:518-22.

93. Cashman SM, Patino A, Delgado MG, Byrne L, Denham B, De Arce M. The Irish cystic fibrosis database. J of medical genetics 1995;32:972-5.

94. Schwartz M, Sørensen N, Brandt NJ, Høgdall E, Holm T. High incidence of cystic fibrosis on The Faroe Islands: a molecular and genealogical study. Human Genetics 1995;95:703-6.

95. Lyczak JB, Cannon CL, Pier GB. Lung infections associated with cystic fibrosis. Clinical microbiology reviews 2002;15:194-222.

96. Aanaes K, Johansen HK, Skov M, et al. Clinical effects of sinus surgery and adjuvant therapy in cystic fibrosis patients - can chronic lung infections be postponed? Rhinology. 2013;51:222-30.

97. Markussen T, Marvig RL, Gómez-Lozano M, et al. Environmental Heterogeneity Drives Within-Host Diversification and Evolution of $<$ span class="named-content genus-species" id="named-content-1" $>$ Pseudomonas aeruginosa</span>. mBio 2014;5:e01592-14.

98. Mainz JG, Naehrlich L, Schien M, et al. Concordant genotype of upper and lower airways $P$ aeruginosa and $S$ aureus isolates in cystic fibrosis. Thorax 2009;64:535-40.

99. Pletcher SD, Goldberg AN, Cope EK. Loss of Microbial Niche Specificity Between the Upper and Lower Airways in Patients With Cystic Fibrosis. Laryngoscope. 2019;129:544-50.

100. Johansen HK, Aanaes K, Pressler T, et al. Colonisation and infection of the paranasal sinuses in cystic fibrosis patients is accompanied by a reduced PMN response. J Cyst Fibros 2012;11:52531.

101. Aanaes K, Rickelt LF, Johansen HK, et al. De- 
creased mucosal oxygen tension in the maxillary sinuses in patients with cystic fibrosis. J Cyst Fibros 2011;10:114-20.

102. Aanaes K, Eickhardt S, Johansen HK, et al. Sinus biofilms in patients with cystic fibrosis: is adjusted eradication therapy needed? Eur Arch Otorhinolaryngol 2015;272:2291-7.

103. Doht F, Hentschel J, Fischer N, et al. Reduced effect of intravenous antibiotic treatment on sinonasal markers in pulmonary inflammation. Rhinology. 2015;53:249-59.

104. Kim RJT, Park L, Wood AJ, Yin T, Jain R, Douglas RG. Chronic rhinosinusitis and cystic fibrosis: the interaction between sinus bacteria and mucosal immunity. International Forum of Allergy \& Rhinology. 2015;5:380-5.

105. Orlandi RR, Wiggins RH. Radiological Sinonasal Findings in Adults with Cystic Fibrosis. American J of Rhinology. \& Allergy 2009;23:307-11.

106. Berkhout MC, Klerx-Melis F, Fokkens WJ, Nuijsink $M$, van Aalderen WM, Heijerman HG. CT-abnormalities, bacteriology and symptoms of sinonasal disease in children with Cystic Fibrosis. J Cyst Fibros 2016;15:816-24.

107. Eggesbo HB, Sovik S, Dolvik S, Eiklid K, Kolmannskog F. Proposal of a CT scoring system of the paranasal sinuses in diagnosing cystic fibrosis. Eur Radiol 2003;13:1451-60.

108. Sheikh SI, Handly B, Ryan-Wenger NA, et al. Novel Computed Tomography Scoring System for Sinus Disease in Adults With Cystic Fibrosis. Ann Otol. Rhinol Laryngol. 2016;125:838-43.

109. Rasmussen J, Aanaes K, Norling R, Nielsen KG, Johansen HK, von Buchwald C. CT of the paranasal sinuses is not a valid indicator for sinus surgery in CF patients. J Cyst Fibros 2012;11:93-9.

110. Babinski D, Trawinska-Bartnicka M. Rhinosinusitis in cystic fibrosis: not a simple story. Int J Pediatr Otorhinolaryngol 2008;72:619-24.

111. Marshak T, Rivlin Y, Bentur L, Ronen O, Uri N. Prevalence of rhinosinusitis among atypical cystic fibrosis patients. Eur Arch Otorhinolaryngol 2011;268:519-24.

112. Schraven SP, Wehrmann M, Wagner W, Blumenstock G, Koitschev A. Prevalence and histopathology of chronic polypoid sinusitis in pediatric patients with cystic fibrosis. J Cyst Fibros 2011;10:181-6.

113. Steinke JW, Payne SC, Chen PG, Negri J, Stelow EB, Borish L. Etiology of nasal polyps in cystic fibrosis: not a unimodal disease. Ann Otol. Rhinol Laryngol. 2012;121:579-86.

114. Taylor RJ, Miller JD, Rose AS, et al. Comprehensive quality of life outcomes for pediatric patients undergoing endoscopic sinus surgery. Rhinology. 2014;52:327-33.

115. King VvB. Upper Respiratory Disease, Sinusitis, and Polyposis. Clinical reviews in allergy 1991;9:143-57.

116. Berkhout MC, van Rooden CJ, Rijntjes E, Fokkens WJ, el Bouazzaoui LH, Heijerman HG. Sinonasal manifestations of cystic fibrosis: a correlation between genotype and phenotype? J Cyst Fibros 2014;13:442-8.

117. Morlacchi LC, Greer M, Tudorache I, et al. The burden of sinus disease in cystic fibrosis lung transplant recipients. Transpl Infect Dis 2018;20:e12924.

118. Bock JM, Schien M, Fischer C, et al. Importance to question sinonasal symptoms and to perform rhinoscopy and rhinomanometry in cystic fibrosis patients. Pediatr Pulmonol 2017;52:167-74.

119. Kreicher KL, Bauschard MJ, Clemmens CS, Riva CM, Meyer TA. Audiometric assessment of pediatric patients with cystic fibrosis. J Cyst Fibros 2018;17:383-90.

120. Kovell LC, Wang J, Ishman SL, Zeitlin PL, Boss EF. Cystic Fibrosis and Sinusitis in Children:Outcomes and Socioeconomic Status. Otolaryngology-Head and Neck Surg. 2011;145:146-53.

121. Calton JB, Koripella PC, Willis AL, Le CH, Chiu AG, Chang EH. Paranasal sinus size is decreased in CFTR heterozygotes with chronic rhinosinusitis. Int Forum Allergy Rhinol. 2017;7:256-60.

122. Farrell PM, White TB, Ren CL, et al. Diagnosis of Cystic Fibrosis: Consensus Guidelines from the Cystic Fibrosis Foundation. J Pediatr 2017;181s:S4-S15.e1.

123. Mastella G, Zanolla L, Castellani C, et al. Neonatal Screening for Cystic Fibrosis: Long-Term Clinical Balance. Pancreatology 2001;1:531-7.

124. Lai HJ, Cheng Y, Farrell PM. The survival advantage of patients with cystic fibrosis diagnosed through neonatal screening: evidence from the United States Cystic Fibrosis Foundation registry data. J Pediatr 2005;147:S57-63.

125. Manji J, Thamboo A, Tacey M, Garnis C, Chadha NK. The presence of Interleukin-13 in nasal lavage may be a predictor of nasal polyposis in pediatric patients with cystic fibrosis. Rhinology. 2018;56:261-7.

126. Boyle MP, De Boeck K. A new era in the treatment of cystic fibrosis: correction of the underlying CFTR defect. Lancet Respir Med 2013;1:158-63.

127. McKone EF, Goss CH, Aitken ML. CFTR genotype as a predictor of prognosis in cystic fibrosis. Chest 2006;130:1441-7.

128. Ferril GR, Nick JA, Getz AE, et al. Comparison of radiographic and clinical characteristics of lowrisk and high-risk cystic fibrosis genotypes. Int Forum Allergy Rhinol. 2014;4:915-20.

129. Abuzeid WM, Song C, Fastenberg JH, et al. Correlations between cystic fibrosis genotype and sinus disease severity in chronic rhinosinusitis. Laryngoscope. 2018;128:1752-8.

130. Lee RJ, Xiong G, Kofonow JM, et al. T2R38 taste receptor polymorphisms underlie susceptibility to upper respiratory infection. J Clin Invest 2012;122:4145-59.

131. Adappa ND, Workman AD, Hadjiliadis D, et al T2R38 genotype is correlated with sinonasal quality of life in homozygous DeltaF508 cystic fibrosis patients. Int Forum Allergy Rhinol. 2016;6:356-61.

132. Potash AE, Wallen TJ, Karp PH, et al. Adenoviral gene transfer corrects the ion transport defect in the sinus epithelia of a porcine CF model. Mol Ther 2013;21:947-53.

133. Wagner JA, Nepomuceno IB, Messner AH, et al. A phase II, double-blind, randomized, placebo-controlled clinical trial of tgAAVCF using maxillary sinus delivery in patients with cystic fibrosis with antrostomies. Human gene therapy 2002;13:1349-59.

134. Van Goor F, Hadida S, Grootenhuis PD, et al. Rescue of CF airway epithelial cell function in vitro by a CFTR potentiator, VX-770. Proc Natl Acad Sci U S A 2009;106:18825-30.

135. US Food and Drug Administration. FDA expands approved use of Kalydeco to treat additional mutations of cystic fibrosis. 2017. https://www. fda.gov/news-events/press-announcements/ fda-expands-approved-use-kalydeco-treat-additional-mutations-cystic-fibrosis. 29 August 2019.

136. European Medicines Agency. Kalydeco. 2012. https://www.ema.europa.eu/en/medicines/human/EPAR/kalydeco. 29 August 2019.

137. Stevens EM, Vladar EK, Alanin MC, Christensen ST, von BuchwaldC, Milla C. Ciliary Localization of the Intraflagellar Transport Protein IFT88 is Disrupted in Cystic Fibrosis. Am J Respir Cell Mol Biol. 2020;62(1):120-123.

138. Accurso FJ, Rowe SM, Clancy JP, et al. Effect of VX-770 in persons with cystic fibrosis and the G551D-CFTR mutation. N Engl J Med 2010;363:1991-2003.

139. Sawicki GS, McKone EF, Pasta DJ, et al. Sustained Benefit from ivacaftor demonstrated by combining clinical trial and cystic fibrosis patient registry data. Am J Respir Crit Care Med 2015;192:836-42

140. McCormick J, Cho DY, Lampkin B, et al. Ivacaftor improves rhinologic, psychologic, and sleep-related quality of life in G551D cystic fibrosis patients. Int Forum Allergy Rhinol. 2019;9:292-7.

141. Chang EH, Tang XX, Shah VS, et al. Medical reversal of chronic sinusitis in a cystic fibrosis patient with ivacaftor. International forum of allergy \& rhinology 2015;5:178-81.

142. Vreede CL, Berkhout MC, Sprij AJ, Fokkens WJ, Heijerman HGM. Ivacaftor and sinonasal pathology in a cystic fibrosis patient with genotype deltaF508/S1215N. J Cyst Fibros 2014;14:412-3.

143. Cho DY, Lim DJ, Mackey C, et al. Ivacaftor, a Cystic Fibrosis Transmembrane Conductance Regulator Potentiator, Enhances Ciprofloxacin Activity Against Pseudomonas aeruginosa. Am J Rhinol Allergy 2019;33:129-36.

144. Reznikov LR, Abou Alaiwa MH, Dohrn CL, et al. Antibacterial properties of the CFTR potentiator ivacaftor. J Cyst Fibros 2014;13:515-9.

145. Philpott CM, Smith R, Davies-Husband CR, et al. Exploring the association between ingestion of foods with higher potential salicylate content and symptom exacerbation in chronic rhinosinusitis. Data from the National Chronic Rhinosinusitis Epidemiology Study. Rhinology. 2019, 10.4193/Rhin 19.027.

146. Ayoub N, Thamboo A, Habib A-R, Nayak JV, Hwang PH. Determinants and outcomes of upfront surgery versus medical therapy for chronic rhinosinusitis in cystic fibrosis. International Forum of Allergy \& Rhinology. 2017;7:450-8.

147. Cimmino $M$, Nardone $M$, Cavaliere $M$, et al. Dornase alfa as postoperative therapy in cystic fibrosis sinonasal disease. Archives of otolaryngology--head \& neck surgery 2005;131:1097101 
148. Mainz JG, Schumacher U, Schadlich K, et al. Sino nasal inhalation of isotonic versus hypertonic saline (6.0\%) in CF patients with chronic rhinosinusitis - Results of a multicenter, prospective, randomized, double-blind, controlled trial. J Cyst Fibros 2016;15:e57-e66.

149. Lee VS, Davis GE. Culture-directed topical antibiotic treatment for chronic rhinosinusitis. American journal of rhinology \& allergy 2016;30:414-7.

150. Berkhout MC, van Velzen AJ, Touw DJ, de Kok BM, Fokkens WJ, Heijerman HGM. Systemic absorption of nasally administered tobramycin and colistin in patients with cystic fibrosis. The J of antimicrobial chemotherapy 2014;69:3112-5.

151. Cho DY, Lim DJ, Mackey C, et al. In-vitro evaluation of a ciprofloxacin- and ivacaftor-coated sinus stent against Pseudomonas aeruginosa biofilms. Int Forum Allergy Rhinol. 2019;9:48692.

152. Fong SA, Drilling A, Morales S, et al. Activity of Bacteriophages in Removing Biofilms of Pseudomonas aeruginosa Isolates from Chronic Rhinosinusitis Patients. Front Cell Infect Microbiol 2017;7:418.

153. Hashemi MM, Holden BS, Taylor MF, et al. Antibacterial and Antifungal Activities of Poloxamer Micelles Containing Ceragenin CSA-131 on Ciliated Tissues. Molecules (Basel, Switzerland) 2018;23:596.

154. Beer H, Southern KW, Swift AC. Topical nasal steroids for treating nasal polyposis in people with cystic fibrosis. Cochrane Database Syst Rev 2015, 10.1002/14651858.CD008253.pub4:Cd008253.

155. Liang J, Higgins T, Ishman SL, Boss EF, Benke JR, Lin SY. Medical management of chronic rhinosinusitis in cystic fibrosis: a systematic review. Laryngoscope. 2014;124:1308-13.

156. Tumin D, Hayes D, Kirkby SE, Tobias JD, McKee C. Safety of endoscopic sinus surgery in children with cystic fibrosis. International J of Pediatric Otorhinolaryngology 2017;98:25-8.

157. Alanin MC, Aanaes K, Hoiby N, et al. Sinus surgery postpones chronic Gram-negative lung infection: cohort study of 106 patients with cystic fibrosis. Rhinology. 2016;54:206-13.

158. Virgin FW, Rowe SM, Wade MB, et al. Extensive surgical and comprehensive postoperative medical management for cystic fibrosis chronic rhinosinusitis. Am J Rhinol Allergy 2012;26:70-5.

159. Vital D, Hofer M, Benden C, Holzmann D, Boehler A. Impact of Sinus Surgery on Pseudomonal Airway Colonization, Bronchiolitis Obliterans Syndrome and Survival in Cystic Fibrosis Lung Transplant Recipients. Respiration 2013;86:2531.

160. Aanaes K, Alanin MC, Nielsen KG, et al. The accessibility of topical treatment in the paranasal sinuses on operated cystic fibrosis patients assessed by scintigraphy. Rhinology. 2018;56:26873.

161. Rudkjobing VB, Aanaes K, Wolff TY, von Buchwald C, Johansen HK, Thomsen TR. An exploratory study of microbial diversity in sinus infections of cystic fibrosis patients by molecular methods. J Cyst Fibros 2014;13:645-52.

162. Hoggard M, Biswas K, Zoing M, Wagner Mackenzie B, Taylor MW, Douglas RG. Evidence of microbiota dysbiosis in chronic rhinosinusitis. Int Forum Allergy Rhinol. 2017;7:230-9.

163. Afzelius BA. A human syndrome caused by immotile cilia. Science 1976;193:317-9.

164. Sleigh MA. Primary ciliary dyskinesia. Lancet 1981;2:476.

165. Goutaki M, Meier AB, Halbeisen FS, et al. Clinical manifestations in primary ciliary dyskinesia: systematic review and meta-analysis. The European respiratory journal 2016;48:1081-95.

166. Armengot Carceller M, Mata Roig M, Milara Payá X, Cortijo Gimeno J. Discinesia ciliar primaria. Ciliopatías. Acta Otorrinolaringológica Española 2010;61:149-59.

167. Rubbo B, Lucas JS. Clinical care for primary ciliary dyskinesia: current challenges and future directions. European Respiratory Review 2017;26:170023.

168. Shapiro AJ, Zariwala MA, Ferkol T, et al. Diagnosis, monitoring, and treatment of primary ciliary dyskinesia: PCD foundation consensus recommendations based on state of the art review. Pediatric Pulmonology 2016;51:115-32.

169. Honoré I, Burgel P-R. Primary ciliary dyskinesia in adults. Revue des Maladies Respiratoires 2016;33:165-89.

170. Bush A, Chodhari R, Collins N, et al. Primary ciliary dyskinesia: current state of the art. Arch Dis Child 2007;92:1136-40.

171. Kuehni CE, Frischer T, Strippoli M-PF, et al. Factors influencing age at diagnosis of primary ciliary dyskinesia in European children. The European respiratory journal 2010;36:1248-58.

172. O'Callaghan C, Chetcuti P, Moya E. High prevalence of primary ciliary dyskinesia in a British Asian population. Archives of Disease in Childhood 2010;95:51-2.

173. Ferkol TW, Puffenberger EG, Lie H, et al. Primary Ciliary Dyskinesia-Causing Mutations in Amish and Mennonite Communities. The J of Pediatrics 2013;163:383-7.

174. Mirra V, Werner C, Santamaria F. Primary Ciliary Dyskinesia: An Update on Clinical Aspects, Genetics, Diagnosis, and Future Treatment Strategies. Frontiers in Pediatrics 2017;5:135.

175. Bush A, Hogg C. Primary ciliary dyskinesia: recent advances in epidemiology, diagnosis, management and relationship with the expanding spectrum of ciliopathy. Expert Review of Respiratory Medicine 2012;6:663-82.

176. Frija-Masson J, Bassinet L, Honoré I, et al. Clinical characteristics, functional respiratory decline and follow-up in adult patients with primary ciliary dyskinesia. Thorax 2017;72:154-60.

177. Praveen K, Davis EE, Katsanis N. Unique among ciliopathies: primary ciliary dyskinesia, a motile cilia disorder. F1000Prime Reports 2015;7:36.

178. Knowles MR, Zariwala M, Leigh M. Primary Ciliary Dyskinesia. Clinics in Chest Medicine 2016;37:449-61.

179. Bequignon E, Dupuy L, Zerah-Lancner F, et al. Critical Evaluation of Sinonasal Disease in 64 Adults with Primary Ciliary Dyskinesia. J of Clinical Medicine 2019;8:619.

180. Campbell R. Managing upper respiratory tract complications of primary ciliary dyskinesia in children. Current Opinion in Allergy \& Clinical Immunology 2012;12:32-8.

181. Wijers CD, Chmiel JF, Gaston BM. Bacterial infections in patients with primary ciliary dyskinesia: Comparison with cystic fibrosis. Chronic respiratory disease 2017;14:392-406.

182. Rogers GB, Carroll MP, Zain NMM, et al. Complexity, Temporal Stability, and Clinical Correlates of Airway Bacterial Community Composition in Primary Ciliary Dyskinesia. J of Clinical Microbiology 2013;51:4029-35.

183. Mall MA, Danahay H, Boucher RC. Emerging Concepts and Therapies for Mucoobstructive Lung Disease. Annals of the American Thoracic Society 2018;15:S216-S26.

184. Ellerman A, Bisgaard H. Longitudinal study of lung function in a cohort of primary ciliary dyskinesia. The European respiratory journal 1997; 10:2376-9.

185. Moller ME, Alanin MC, Gronhoj C, Aanaes K, Hoiby N, von Buchwald C. Sinus bacteriology in patients with cystic fibrosis or primary ciliary dyskinesia: A systematic review. American J of Rhinology. \& Allergy 2017;31:293-8.

186. Alanin MC, Johansen HK, Aanaes K, et al. Simultaneous sinus and lung infections in patients with primary ciliary dyskinesia. Acta Otolaryngol. 2015;135:58-63.

187. Arndal E, Johansen HK, Haagensen JAJ, et al. Primary ciliary dyskinesia patients have the same $<\mathrm{em}>\mathrm{P}$. aeruginosa $</ \mathrm{em}>$ clone in sinuses and lungs. European Respiratory J 2019, 10.1183/13993003.01472-2019:1901472.

188. Barbato A, Frischer T, Kuehni CE, et al. Primary ciliary dyskinesia: a consensus statement on diagnostic and treatment approaches in children. Eur Respir J 2009;34:1264-76.

189. Lucas JS, Burgess A, Mitchison HM, et al. Diagnosis and management of primary ciliary dyskinesia. Archives of Disease in Childhood 2014;99:850-6.

190. Noone PG, Leigh MW, Sannuti A, et al. Primary Ciliary Dyskinesia. American J of Respiratory and Critical Care Medicine 2004;169:459-67.

191. O'Callaghan C, Rutman A, Williams GM, Hirst RA. Inner dynein arm defects causing primary ciliary dyskinesia: repeat testing required. European Respiratory J 2011;38:603-7.

192. Knowles MR, Daniels LA, Davis SD, Zariwala MA, Leigh MW. Primary ciliary dyskinesia. Recent advances in diagnostics, genetics, and characterization of clinical disease. American J of Respiratory \& Critical Care Medicine 2013;188:913-22.

193. Hirst RA, Jackson CL, Coles JL, et al. Culture of primary ciliary dyskinesia epithelial cells at air-liquid interface can alter ciliary phenotype but remains a robust and informative diagnostic aid. PLoS One 2014;9:e89675.

194. Karadag B, James AJ, Gültekin E, Wilson NM, Bush A. Nasal and lower airway level of nitric oxide in children with primary ciliary dyskinesia. The European respiratory journal 1999;13:14025.

195. Meng Q-H, Springall DR, Bishop AE, et al. Lack of inducible nitric oxide synthase in bronchial epithelium: a possible mechanism of susceptibility 
to infection in cystic fibrosis. The J of Pathology 1998;184:323-31.

196. Leigh MW, Hazucha MJ, Chawla KK, et al. Standardizing nasal nitric oxide measurement as a test for primary ciliary dyskinesia. Annals of the American Thoracic Society 2013;10:574-81.

197. Pifferi M, Bush A, Caramella D, et al. Agenesis of paranasal sinuses and nasal nitric oxide in primary ciliary dyskinesia. Eur Respir J 2011;37:566-71.

198. Parsons DS, Greene BA. A treatment for primary ciliary dyskinesia: efficacy of functional endoscopic sinus surgery. Laryngoscope. 1993;103:1269-72.

199. Mygind N, Pedersen M. Nose-, sinus- and ear-symptoms in 27 patients with primary ciliary dyskinesia. Eur J Respir Dis Suppl 1983;127:96101.

200. Alanin MC, Aanaes K, Høiby N, et al. Sinus surgery can improve quality of life, lung infections, and lung function in patients with primary ciliary dyskinesia. International Forum of Allergy \& Rhinology. 2017;7:240-7

201. Buzina W, Braun H, Freudenschuss K, Lackner A, Habermann W, Stammberger H. Fungal biodiversity--as found in nasal mucus. Med Mycol 2003;41:149-61.

202. Chakrabarti A, Denning DW, Ferguson BJ, et al. Fungal rhinosinusitis: a categorization and definitional schema addressing current controversies. Laryngoscope. 2009;119:1809-18.

203. Toussain G, Botterel F, Alsamad IA, et al. Sinus fungal balls: characteristics and management in patients with host factors for invasive infection. Rhinology. 2012;50:269-76.

204. Orlandi RR, Marple BF. Fungus and chronic rhinosinusitis: weighing the evidence. Otolaryngol. Head Neck Surg. 2010;143:611-3.

205. Grosjean P, Weber R. Fungus balls of the paranasal sinuses: a review. Eur Arch Otorhinolaryngol 2007;264:461-70.

206. Yoon YH, Xu J, Park SK, Heo JH, Kim YM, Rha KS. A retrospective analysis of 538 sinonasal fungus ball cases treated at a single tertiary medical center in Korea (1996-2015). Int Forum Allergy Rhinol. 2017;7:1070-5.

207. Nomura K, Asaka D, Nakayama T, et al. Sinus fungus ball in the Japanese population: clinical and imaging characteristics of 104 cases. Int J Otolaryngol. 2013;2013:731640.

208. Jung JH, Cho GS, Chung YS, Lee BJ. Clinical characteristics and outcome in patients with isolated sphenoid sinus aspergilloma. Auris Nasus Larynx 2013;40:189-93.

209. Shin JM, Baek BJ, Byun JY, Jun YJ, Lee JY. Analysis of sinonasal anatomical variations associated with maxillary sinus fungal balls. Auris Nasus Larynx 2016;43:524-8.

210. Knisely A, Holmes T, Barham H, Sacks R, Harvey R. Isolated sphenoid sinus opacification: A systematic review. Am J Otolaryngol. 2017;38:237-43.

211. Seo MY, Lee SH, Ryu G, et al. Clinical pattern of fungal balls in the paranasal sinuses: our experience with 70 patients. Eur Arch Otorhinolaryngol 2019;276:1035-8.

212. Mensi M, Piccioni M, Marsili F, Nicolai P, Sapelli $\mathrm{PL}$, Latronico N. Risk of maxillary fungus ball in patients with endodontic treatment on maxillary teeth: a case-control study. Oral Surg Oral Med Oral Pathol Oral Radiol Endod. 2007;103:433-6.

213. Park GY, Kim HY, Min JY, Dhong HJ, Chung SK. Endodontic treatment: a significant risk factor for the development of maxillary fungal ball. Clin Exp Otorhinolaryngol 2010;3:136-40.

214. Oshima H, Nomura K, Sugawara M, Arakawa K, Oshima T, Katori Y. Septal deviation is associated with maxillary sinus fungus ball in male patients. The Tohoku journal of experimental medicine 2014;232:201-6.

215. Nicolai P, Mensi M, Marsili F, et al. Maxillary fungus ball: zinc-oxide endodontic materials as a risk factor. Acta Otorhinolaryngol Ital 2015;35:93-6.

216. Tsai TL, Guo YC, Ho CY, Lin CZ. The role of ostiomeatal complex obstruction in maxillary fungus ball. Otolaryngology - Head \& Neck Surg. 2006;134:494-8.

217. Cavada M, Wong E, Orgain CA, et al. Fungal ball of the maxillary sinus and persistent sinus dysfunction after antrostomy. Am J Otolaryngol. 2020;41(4):102541.

218. Jun YJ, Shin JM, Lee JY, Baek BJ. Bony Changes in a Unilateral Maxillary Sinus Fungal Ball. J Craniofac Surg. 2018;29:e44-e7.

219. Michel J, Radulesco T, Mancini J, et al. Maxillary sinus volume: new physiopathological data in fungal ball genesis? A retrospective study. Clin Otolaryngol. 2017;42:831-6.

220. deShazo RD, O'Brien M, Chapin K, Soto-Aguilar M, Gardner L, Swain R. A new classification and diagnostic criteria for invasive fungal sinusitis. Archives of Otolaryngology -- Head \& Neck Surg. 1997;123:1181-8.

221. Chen JC, Ho CY. The significance of computed tomographic findings in the diagnosis of fungus ball in the paranasal sinuses. Am J Rhinol Allergy 2012;26:117-9.

222. Lee JS, Shin SY, Lee KH, Kim SW, Cho JS. Change of prevalence and clinical aspects of fungal ball according to temporal difference. Eur Arch Otorhinolaryngol 2013;270:1673-7.

223. Seo YJ, Kim J, Kim K, Lee JG, Kim CH, Yoon JH. Radiologic characteristics of sinonasal fungus ball: an analysis of 119 cases. Acta Radiol 2011;52:790-5.

224. Yamauchi T, Tani A, Yokoyama S, Ogawa H. Assessment of non-invasive chronic fungal rhinosinusitis by cone beam CT: comparison with multidetector CT findings. Fukushima J Med Sci 2017;63:100-5.

225. Palacios E, Jones W, Alvernia J. Sinonasal mycetoma. Ear Nose Throat J 2008;87:606-8.

226. Bowman J, Panizza B, Gandhi M. Sphenoid sinus fungal balls. Ann Otol. Rhinol Laryngol. 2007;116:514-9.

227. Botturi A, Salmaggi A, Pollo B, Lamperti E, Erbetta A, Boiardi A. Meningitis following relapsing painful ophthalmoplegia in aspergillus sphenoidal sinusitis: a case report. Neurol Sci 2006;27:284-7.

228. Hendriks T, Leedman S, Quick M, Acharya A. Non-invasive fungal sinusitis resulting in multiple cranial nerve neuropathies. BMJ Case Rep
2019;12.

229. Eloy P, Grenier J, Pirlet A, Poirrier AL, Stephens JS, Rombaux P. Sphenoid sinus fungall ball: a retrospective study over a 10-year period. Rhinology. 2013;51:181-8

230. Klossek JM, Peloquin L, Friedman WH, Ferrier JC, Fontanel JP. Diffuse nasal polyposis: postoperative long-term results after endoscopic sinus surgery and frontal irrigation. Otolaryngol. Head Neck Surg. 1997;117:355-61.

231. Sawatsubashi M, Murakami D, Umezaki T, Komune S. Endonasal endoscopic surgery with combined middle and inferior meatal antrostomies for fungal maxillary sinusitis. J Laryngol. Otol. 2015;129 Suppl 2:S52-5.

232. Nomura K, Ikushima H, Ozawa D, et al. Endoscopic Modified Medial Maxillectomy for Fungal Ball of the Hypoplastic Maxillary Sinus With Bony Hypertrophy. J Craniofac Surg. 2018;29:e304-e7.

233. Xu R, Ma L, Shi J, et al. Endoscopic sinus surgery for fungal ball rhinosinusitis in South China: long-term results and analysis of prognostic factors. Acta Otolaryngol. 2012;132:519-24.

234. Trief D, Gray ST, Jakobiec FA, et al. Invasive fungal disease of the sinus and orbit: a comparison between mucormycosis and Aspergillus. $\mathrm{Br} \mathrm{J}$ Ophthalmol 2016;100:184-8.

235. Deutsch PG, Whittaker J, Prasad S. Invasive and Non-Invasive Fungal Rhinosinusitis-A Review and Update of the Evidence. Medicina (Kaunas) 2019;55.

236. Turner JH, Soudry E, Nayak JV, Hwang PH. Survival outcomes in acute invasive fungal sinusitis: a systematic review and quantitative synthesis of published evidence. Laryngoscope. 2013;123:1112-8.

237. Marty FM, Cosimi LA, Baden LR. Breakthrough zygomycosis after voriconazole treatment in recipients of hematopoietic stem-cell transplants. N Engl J Med 2004;350:950-2.

238. Thurtell MJ, Chiu AL, Goold LA, et al. Neuro-ophthalmology of invasive fungal sinusitis: 14 consecutive patients and a review of the literature. Clin Exp Ophthalmol 2013;41:567-76.

239. Washburn RG, Kennedy DW, Begley MG, Henderson DK, Bennett JE. Chronic fungal sinusitis in apparently normal hosts. Medicine (Baltimore) 1988;67:231-47

240. Scharf JL, Soliman AMS. Chronic rhizopus invasive fungal rhinosinusitis in an immunocompetent host. Laryngoscope. 2004;114:1533-5.

241. Halderman AA, Stokken J, Sindwani R. The effect of middle turbinate resection on topical drug distribution into the paranasal sinuses. Int Forum Allergy Rhinol. 2016;6:1056-61.

242. Baeesa SS, Bakhaidar M, Ahamed NAB, Madani TA. Invasive Orbital Apex Aspergillosis with Mycotic Aneurysm Formation and Subarachnoid Hemorrhage in Immunocompetent Patients. World Neurosurg 2017;102:42-8.

243. Aggarwal E, Mulay K, Menon V, Sundar G, Honavar SG, Sharma M. Isolated Orbital Aspergillosis in Immunocompetent Patients: A Multicenter Study. Am J Ophthalmol 2016;165:125-32.

244. Mody KH, Ali MJ, Vemuganti GK, Nalamada S, Naik MN, Honavar SG. Orbital aspergillosis in 
immunocompetent patients. Br J Ophthalmol 2014;98:1379-84

245. Slonimsky G, Slonimsky E, Yakirevitch A, et al. The significance of Computed Tomography in invasive paranasal mucormycosis. Rhinology. 2018;56:54-8.

246. Lund VJ, Lloyd G, Savy L, Howard D. Fungal rhinosinusitis. J Laryngol. Otol. 2000;114:76-80.

247. Wandell GM, Miller C, Rathor A, et al. A multi-institutional review of outcomes in biopsy-proven acute invasive fungal sinusitis. Int Forum Allergy Rhinol. 2018;8:1459-68.

248. Payne SJ, Mitzner R, Kunchala S, Roland L, McGinn JD. Acute Invasive Fungal Rhinosinusitis: A 15-Year Experience with 41 Patients. Otolaryngol. Head Neck Surg. 2016;154:759-64.

249. Groppo ER, El-Sayed IH, Aiken AH, Glastonbury $\mathrm{CM}$. Computed tomography and magnetic resonance imaging characteristics of acute invasive fungal sinusitis. Arch Otolaryngol. Head Neck Surg. 2011;137:1005-10.

250. Hennessy M, McGinn J, White B, Payne S, Warrick Jl, Crist H. Frozen Section as a Rapid and Accurate Method for Diagnosing Acute Invasive Fungal Rhinosinusitis. Otolaryngology - Head and Neck Surg. (United States) 2018;159:576-80.

251. Melancon CC, Clinger JD. The Use of Frozen Section in the Early Diagnosis of Acute Invasive Fungal Sinusitis. Otolaryngol. Head Neck Surg. 2017; 157:314-9.

252. Montone KT, LiVolsi VA, Lanza DC, et al. In situ hybridization for specific fungal organisms in acute invasive fungal rhinosinusitis. Am J Clin Pathol 2011;135:190-9.

253. Arvanitis M, Anagnostou T, Mylonakis E. Galactomannan and Polymerase Chain Reaction-Based Screening for Invasive Aspergillosis Among High-Risk Hematology Patients: A Diagnostic Meta-analysis. Clin Infect Dis 2015;61:1263-72.

254. Vaughan C, Bartolo A, Vallabh N, Leong SC. A meta-analysis of survival factors in rhino-orbital-cerebral mucormycosis-has anything changed in the past 20 years? Clin Otolaryngol. 2018;43:1454-64.

255. Zappasodi P, Rossi M, Castagnola C, et al. Resolution of invasive fungal sinusitis in immunocompromised patients: neutrophil count is crucial beside a combined medical and surgical approach. Ann Hematol 2010;89:737-9.

256. Herbrecht R, Denning DW, Patterson TF, et al. Voriconazole versus amphotericin B for primary therapy of invasive aspergillosis. N Engl J Med 2002;347:408-15.

257. Walsh TJ, Anaissie EJ, Denning DW, et al. Treatment of aspergillosis: clinical practice guidelines of the Infectious Diseases Society of America. Clin Infect Dis 2008;46:327-60.

258. Maertens JA, Raad, II, Marr KA, et al. Isavuconazole versus voriconazole for primary treatment of invasive mould disease caused by Aspergillus and other filamentous fungi (SECURE): a phase 3, randomised-controlled non-inferiority trial. Lancet 2016;387:760-9.

259. Kalin-Hajdu E, Hirabayashi KE, Vagefi MR, Kersten RC. Invasive fungal sinusitis: treatment of the orbit. Curr Opin Ophthalmol 2017;28:522-33.
260. Tragiannidis A, Groll AH. Hyperbaric oxygen therapy and other adjunctive treatments for zygomycosis. Clin Microbiol Infect 2009;15 Suppl 5:82-6.

261. John BV, Chamilos G, Kontoyiannis DP. Hyperbaric oxygen as an adjunctive treatment for zygomycosis. Clin Microbiol Infect 2005;11:5157.

262. Spellberg B, Ibrahim AS, Chin-Hong PV, et al. The Deferasirox-AmBisome Therapy for Mucormycosis (DEFEAT Mucor) study: a randomized, double-blinded, placebo-controlled trial. J Antimicrob Chemother 2012;67:715-22.

263. Roxbury CR, Smith DF, Higgins TS, et al. Complete surgical resection and short-term survival in acute invasive fungal rhinosinusitis. Am J Rhinol. Allergy 2017;31:109-16.

264. Papagiannopoulos P, Lin DM, Al-Khudari S, et al. Utility of intraoperative frozen sections in surgical decision making for acute invasive fungal rhinosinusitis. International Forum of Allergy \& Rhinology. 2017;7:502-7.

265. Yohai RA, Bullock JD, Aziz AA, Markert RJ. Survival factors in rhino-orbital-cerebral mucormycosis. Surv Ophthalmol 1994;39:3-22.

266. Hargrove RN, Wesley RE, Klippenstein KA, Fleming JC, Haik BG. Indications for orbital exenteration in mucormycosis. Ophthalmic Plast Reconstr Surg. 2006;22:286-91.

267. Safirstein BH. Allergic bronchopulmonary aspergillosis with obstruction of the upper respiratory tract. Chest 1976;70:788-90.

268. Katzenstein AL, Sale SR, Greenberger PA. Allergic Aspergillus sinusitis: a newly recognized form of sinusitis. J Allergy Clin Immunol 1983;72:89-93.

269. Dykewicz MS, Rodrigues JM, Slavin RG. Allergic fungal rhinosinusitis. The $J$ of allergy and clinical immunology 2018;142:341-51.

270. Peters AT, Spector S, Hsu J, et al. Diagnosis and management of rhinosinusitis: a practice parameter update. Ann Allergy Asthma Immunol 2014; 113:347-85.

271. Bent 3rd JP, Kuhn FA, Bent JP, Kuhn FA. Diagnosis of allergic fungal sinusitis. Otolaryngol. Head Neck Surg. 1994;111:580-8.

272. Kuhn FA, Javer AR. Allergic fungal rhinosinusitis: perioperative management, prevention of recurrence, and role of steroids and antifungal agents. Otolaryngol. Clin North Am 2000;33:419-33.

273. Bakhshaee M, Fereidouni M, Mohajer MN, Majidi MR, Azad FJ, Moghiman T. The prevalence of allergic fungal rhinosinusitis in sinonasal polyposis. Eur Arch Otorhinolaryngol 2013;270:3095-8.

274. Bakhshaee M, Fereidouni M, Nourollahian M, Movahed R. The presence of fungal-specific $\lg \mathrm{E}$ in serum and sinonasal tissue among patients with sinonasal polyposis. European archives of oto-rhino-laryngology : official journal of the European Federation of Oto-Rhino-Laryngological Societies (EUFOS) : affiliated with the German Society for Oto-Rhino-Laryngology - Head and Neck Surg. 2014;271:2871-5.

275. Lu-Myers Y, Deal AM, Miller JD, et al. Comparison of Socioeconomic and Demographic Factors in Patients with Chronic Rhinosinusitis and Allergic Fungal Rhinosinusitis. Otolaryngol. Head Neck
Surg. 2015;153:137-43

276. Wise SK, Ghegan MD, Gorham E, Schlosser RJ. Socioeconomic factors in the diagnosis of allergic fungal rhinosinusitis. Otolaryngol. Head Neck Surg. 2008;138:38-42.

277. Miller JD, Deal AM, McKinney KA, et al. Markers of disease severity and socioeconomic factors in allergic fungal rhinosinusitis. Int Forum Allergy Rhinol. 2014;4:272-9.

278. Hoyt AEW, Borish L, Gurrola J, Payne S. Allergic Fungal Rhinosinusitis. The journal of allergy and clinical immunology In practice 2016;4:599-604.

279. deShazo RD, Swain RE. Diagnostic criteria for allergic fungal sinusitis. J of Allergy \& Clinical Immunology 1995;96:24-35.

280. Thaitrakool W, Sukswai N, Keelawat S, et al. Histopathology of ethmoid mucosa versus polyp tissue in diagnosing eosinophilic mucin rhinosinusitis. Rhinology. 2019;57:67-72.

281. Luong A, Marple BF. Allergic fungal rhinosinusitis. Current Allergy \& Asthma Reports 2004:4:465-70.

282. Marple BF. Allergic fungal rhinosinusitis: current theories and management strategies. Laryngoscope. 2001;111:1006-19.

283. Aribandi M, McCoy VA, Bazan C, 3rd. Imaging features of invasive and noninvasive fungal sinusitis: a review. Radiographics 2007;27:128396.

284. Manning SC, Merkel M, Kriesel K, Vuitch F, Marple B. Computed tomography and magnetic resonance diagnosis of allergic fungal sinusitis. Laryngoscope. 1997;107:170-6.

285. Rupa V, Jacob M, Mathews MS, Seshadri MS. A prospective, randomised, placebo-controlled trial of postoperative oral steroid in allergic fungal sinusitis. Eur Arch Otorhinolaryngol 2010;267:233-8

286. Prasad BK. Study of factors responsible for recidivism in allergic fungal rhinosinusitis. Ear, nose, \& throat journal 2017;96:E18-E21.

287. Ikram M, Abbas A, Suhail A, Onali MA, Akhtar S, lqbal M. Management of allergic fungal sinusitis with postoperative oral and nasal steroids: a controlled study. Ear Nose Throat J 2009;88:E811.

288. Kinsella JB, Bradfield JJ, Gourley WK, Calhoun $\mathrm{KH}$, Rassekh CH. Allergic fungal sinusitis. Clin Otolaryngol. Allied Sci 1996;21:389-92.

289. Schubert MS, Goetz DW. Evaluation and treatment of allergic fungal sinusitis. I. Demographics and diagnosis. The $J$ of allergy and clinical immunology 1998;102:387-94.

290. Landsberg R, Segev Y, DeRowe A, Landau T, Khafif A, Fliss DM. Systemic corticosteroids for allergic fungal rhinosinusitis and chronic rhinosinusitis with nasal polyposis: a comparative study. Otolaryngol. Head Neck Surg. 2007; 136:252-7

291. Woodworth BA, Joseph K, Kaplan AP, Schlosser RJ. Alterations in eotaxin, monocyte chemoattractant protein-4, interleukin-5, and interleukin-13 after systemic steroid treatment for nasal polyps. Otolaryngology - Head \& Neck Surg. 2004;131:585-9.

292. Kupferberg SB, Bent JP, 3rd, Kuhn FA. Prognosis 
for allergic fungal sinusitis. Otolaryngol. Head Neck Surg. 1997;117:35-41.

293. Gan EC, Thamboo A, Rudmik L, Hwang PH, Ferguson BJ, Javer AR. Medical management of allergic fungal rhinosinusitis following endoscopic sinus surgery: an evidence-based review and recommendations. International Forum of Allergy \& Rhinology. 2014;4:702-15.

294. Gupta RP, Bahadur S, Thakar A, Handa KK, Sarkaar C. Management protocols of Allergic Fungal Sinusitis. Indian J Otolaryngol. Head Neck Surg. 2007;59:35-40.

295. Dai Q, Duan C, Liu Q, Yu H. Effect of nebulized budesonide on decreasing the recurrence of allergic fungal rhinosinusitis. American journal of otolaryngology 2017;38:321-4.

296. Patadia MO, Welch KC. Role of immunotherapy in allergic fungal rhinosinusitis. Curr Opin Otolaryngol. Head Neck Surg. 2015;23:21-8.

297. Hall AG, deShazo RD. Immunotherapy for allergic fungal sinusitis. Curr Opin Allergy Clin Immunol 2012;12:629-34.

298. Bassichis BA, Marple BF, Mabry RL, Newcomer MT, Schwade ND. Use of immunotherapy in previously treated patients with allergic fungal sinusitis. Otolaryngol. Head Neck Surg. 2001;125:487-90

299. Mabry RL, Marple BF, Folker RJ, Mabry CS. Immunotherapy for allergic fungal sinusitis: three years' experience. Otolaryngol. Head Neck Surg. 1998;119:648-51

300. Folker RJ, Marple BF, Mabry RL, Mabry CS. Treatment of allergic fungal sinusitis: a comparison trial of postoperative immunotherapy with specific fungal antigens. Laryngoscope. 1998;108:1623-7.

301. Marple B, Newcomer M, Schwade N, Mabry R. Natural history of allergic fungal rhinosinusitis: a 4- to 10-year follow-up. Otolaryngol. Head Neck Surg. 2002;127:361-6.

302. Melzer JM, Driskill BR, Clenney TL, Gessler EM. Sublingual Immunotherapy for Allergic Fungal Sinusitis. Ann Otol. Rhinol Laryngol. 2015;124:782-7.

303. Greenhaw B, deShazo RD, Arnold J, Wright L. Fungal immunotherapy in patients with allergic fungal sinusitis. Ann Allergy Asthma Immunol 2011;107:432-6.

304. Seiberling K, Wormald PJ. The role of itraconazole in recalcitrant fungal sinusitis. Am J Rhinol Allergy 2009;23:303-6.

305. Rains BM, Mineck CW. Treatment of allergic fungal sinusitis with high-dose itraconazole. Am J Rhinol 2003;17:1-8.

306. Kanda N, Enomoto U, Watanabe S. Anti-mycotics suppress interleukin-4 and interleukin-5 production in anti-CD3 plus anti-CD28-stimulated $T$ cells from patients with atopic dermatitis. J Invest Dermatol 2001;117:1635-46.

307. Verma RK, Patro SK, Francis AA, Panda NK, Chakrabarti A, Singh P. Role of preoperative versus postoperative itraconazole in allergic fungal rhinosinusitis. Medical mycology 2017;55:61423.

308. Rojita M, Samal S, Pradhan P, Venkatachalam VP. Comparison of steroid and itraconazole for prevention of recurrence in allergic fungal rhinosinusitis: a randomized controlled trial. J of clinical and diagnostic research 2017;11:MC01MC3.

309. Khalil Y, Tharwat A, Abdou AG, et al. The role of antifungal therapy in the prevention of recurrent allergic fungal rhinosinusitis after functional endoscopic sinus surgery: a randomized, controlled study. Ear, nose, \& throat journal 2011;90:E1-7.

310. Kennedy DW, Kuhn FA, Hamilos DL, et al. Treatment of chronic rhinosinusitis with high-dose oral terbinafine: a double blind, placebo-controlled study. Laryngoscope. 2005;115:1793-9.

311. Sacks PLt, Harvey RJ, Rimmer J, Gallagher RM, Sacks R. Antifungal therapy in the treatment of chronic rhinosinusitis: a meta-analysis. American J of Rhinology. \& Allergy 2012;26:141-7.

312. Mistry SG, Kumar BN. The value of antifungal therapy in allergic fungal rhinosinusitis. Rhinology. 2014;52:9-18.

313. Liang KL, Su MC, Shiao JY, et al. Amphotericin B irrigation for the treatment of chronic rhinosinusitis without nasal polyps: a randomized, placebo-controlled, double-blind study. Am J Rhinol 2008;22:52-8.

314. Ponikau JU, Sherris DA, Weaver A, Kita H. Treatment of chronic rhinosinusitis with intranasal amphotericin B: a randomized, placebo-controlled, double-blind pilot trial. J of allergy and clinical immunology 2005;115:125-31.

315. Jen A, Kacker A, Huang C, Anand V. Fluconazole nasal spray in the treatment of allergic fungal sinusitis: A pilot study. Ear, Nose, \& Throat J 2004;83:692-5.

316. Schubert MS. Antileukotriene therapy for allergic fungal sinusitis. J Allergy Clin Immunol 2001;108:466-7.

317. Thamboo A, Thamboo A, Philpott C, Javer A, Clark A. Single-blind study of manuka honey in allergic fungal rhinosinusitis. Le J d'oto-rhino-laryngologie et de chirurgie cervico-faciale [J of otolaryngology - head \& neck surgery] 2011;40:238-43.

318. Evans MO, 2nd, Coop CA. Novel treatment of allergic fungal sinusitis using omalizumab. Allergy Rhinol (Providence) 2014;5:172-4.

319. Gan EC, Habib AR, Rajwani A, Javer AR. Omalizumab therapy for refractory allergic fungal rhinosinusitis patients with moderate or severe asthma. Am J Otolaryngol. 2015;36:672-7.

320. Marple B, Newcomer M, Schwade N, Mabry R. Natural history of allergic fungal rhinosinusitis: a 4- to 10-year follow-up. Otolaryngol Head Neck Surg. 2002;127(5):361-6.

321. Loftus PA, Wise SK. Allergic Fungal Rhinosinusitis: The Latest in Diagnosis and Management. Adv Otorhinolaryngol 2016;79:13-20.

322. Marple BF, Mabry RL. Allergic fungal sinusitis: learning from our failures. Am J Rhinol 2000;14:223-6.

323. Tong J, Jefferson N, Chaganti J, et al. Compressive Optic Neuropathy from Allergic Fungal Sinusitis Compressive Optic Neuropathy from Allergic Fungal Sinusitis. 2017;8107.

324. Thakar A, Lal P, Dhiwakar M, Bahadur S. Optic nerve compression in allergic fungal sinusitis. The J of Laryngology \& Otology 2011;125:381-5.

325. Herrmann BW, White FV, Forsen Jr JW. Visual loss in a child due to allergic fungal sinusitis of the sphenoid. Otolaryngology - Head \& Neck Surg. 2006;135:328-9.

326. Illing EA, Dunlap Q, Woodworth BA. Outcomes of pressure-induced cranial neuropathies from allergic fungal rhinosinusitis. Otolaryngol. Head Neck Surg. 2015;152:541-5.

327. Stonebraker AC, Schlosser RJ. Orbital volumetric analysis of allergic fungal sinusitis patients with proptosis before and after endoscopic sinus surgery. American J of Rhinology. 2005;19:302-6.

328. AlQahtani A, Alshaikh N, Alzarei A, et al. Contralateral sinus involvement of surgically treated unilateral allergic fungal rhinosinusitis. Eur Arch Otorhinolaryngol 2017;274:3097-101.

329. Jennette JC, Falk RJ, Bacon PA, et al. 2012 Revised International Chapel Hill Consensus Conference Nomenclature of Vasculitides. Arthritis \& Rheumatism 2013;65:1-11.

330. Waller R, Ahmed A, Patel I, Luqmani R. Update on the classification of vasculitis. Best Practice \& Research Clinical Rheumatology 2013;27:3-17.

331. Mahr A, de Menthon M. Classification and classification criteria for vasculitis: achievements, limitations and prospects. Current opinion in rheumatology 2015;27:1-9.

332. Watts RA. Evolving concepts in classification of systemic vasculitis: where are we and what is the way forward? International journal of rheumatic diseases 2019;22 Suppl 1:21-7.

333. Craven A, Robson J, Ponte C, et al. ACR/EULAR-endorsed study to develop Diagnostic and Classification Criteria for Vasculitis (DCVAS). Clinical and experimental nephrology 2013;17:61921.

334. Gross WE, Aries P, Lamprecht P. Granulomatosis: Wegener's Disease. Encyclopedia of Respiratory Medicine: Elsevier, 2006; 255-61.

335. Cotch MF, Hoffman GS, Yerg DE, Kaufman Gl, Targonski P, Kaslow RA. The epidemiology of Wegener's granulomatosis. Estimates of the five-year period prevalence, annual mortality, and geographic disease distribution from population-based data sources. Arthritis and rheumatism 1996;39:87-92.

336. Jennette JC, Falk RJ. Pathogenesis of antineutrophil cytoplasmic autoantibody-mediated disease. Nature Reviews Rheumatology 2014;10:463-73.

337. Laudien M, Gadola SD, Podschun R, et al. Nasal carriage of Staphylococcus aureus and endonasal activity in Wegener s granulomatosis as compared to rheumatoid arthritis and chronic Rhinosinusitis with nasal polyps. Clin Exp Rheumatol 2010;28:51-5.

338. Stegeman CA, Tervaert JW, Sluiter WJ, Manson WL, de Jong PE, Kallenberg CG. Association of chronic nasal carriage of Staphylococcus aureus and higher relapse rates in Wegener granulomatosis. Annals of internal medicine 1994;120:12-7.

339. Popa ER, Tervaert JW. The relation between Staphylococcus aureus and Wegener's granulomatosis: current knowledge and future 
directions. Intern Med 2003;42:771-80.

340. Stegeman CA, Cohen Tervaert JW, de Jong PE, Kallenberg CGM. Trimethoprim-Sulfamethoxazole (Co-Trimoxazole) for the Prevention of Relapses of Wegener's Granulomatosis. New England J of Medicine 1996;335:16-20.

341. Lyons PA, Rayner TF, Trivedi S, et al. Genetically distinct subsets within ANCA-associated vasculitis. 2012;367.

342. Relle M, Föhr B, Fasola F, Schwarting A. Genetics and pathophysiology of granulomatosis with polyangiitis (GPA) and its main autoantigen proteinase 3 . Molecular and cellular probes 2016;30:366-73.

343. Abdou NI, Kullman GJ, Hoffman GS, et al. Wegener's granulomatosis: survey of 701 patients in North America. Changes in outcome in the 1990s. The J of rheumatology 2002;29:309-16.

344. Srouji IA, Andrews P, Edwards C, Lund VJ. General and rhinosinusitis-related quality of life in patients with Wegener's granulomatosis. Laryngoscope. 2006;116:1621-5.

345. Cannady SB, Batra PS, Koening C, et al. Sinonasal Wegener granulomatosis: A single-institution experience with 120 cases. Laryngoscope. 2009;119:757-61.

346. Krafcik SS, Covin RB, Lynch JP, Sitrin RG. Wegener's granulomatosis in the elderly. Chest 1996;109:430-7.

347. Monti S, Craven A, Klersy C, et al. OP0238 Association between age at diagnosis and clinical presentation and outcomes of anca-associated vasculitis. analysis from the dcvas study. Annals of the Rheumatic Diseases 2018;77:168.

348. Stone JH, Group WsGETR. Limited versus severe Wegener's granulomatosis: Baseline data on patients in the Wegener's granulomatosis etanercept trial. Arthritis \& Rheumatism 2003;48:2299309.

349. de Groot K, Harper L, Jayne DRW, et al. Pulse versus daily oral cyclophosphamide for induction of remission in antineutrophil cytoplasmic antibody-associated vasculitis: a randomized trial. Annals of internal medicine 2009;150:67080.

350. Gottschlich S, Ambrosch P, Kramkowski D, et al. Head and neck manifestations of Wegener's granulomatosis. Rhinology. 2006;44:227-33.

351. Srouji IA, Andrews P, Edwards C, Lund VJ. Patterns of presentation and diagnosis of patients with Wegener's granulomatosis: ENT aspects. The J of laryngology and otology 2007;121:6538.

352. Kühn D, Hospowsky C, Both M, Hey M, Laudien M. Manifestation of granulomatosis with polyangiitis in head and neck. Clinical and experimental rheumatology 2018;36 Suppl 1:78-84.

353. Fasunla JA, Hundt W, Lutz J, Förger F, Thürmel K, Steinbach S. Evaluation of smell and taste in patients with Wegener's granulomatosis. European Archives of Oto-Rhino-Laryngology 2012;269:179-86.

354. Laudien M, Lamprecht P, Hedderich J, Holle J, Ambrosch P. Olfactory dysfunction in Wegener's granulomatosis. Rhinology. journal 2009;47:2549.
355. Proft F, Steinbach S, Dechant C, et al. Gustatory and olfactory function in patients with granulomatosis with polyangiitis (Wegener's). Scandinavian J of Rheumatology 2014;43:512-8.

356. Zycinska K, Straburzynski M, Nitsch-Osuch A, et al. Prevalence of Olfactory Impairment in Granulomatosis with Polyangiitis. Adv Exp Med Biol 2016;878:1-7.

357. Hoare TJ, Jayne D, Rhys Evans P, Croft CB, Howard DJ. Wegener's granulomatosis, subglottic stenosis and antineutrophil cytoplasm antibodies. The J of laryngology and otology 1989;103:1187-91.

358. Tomasson G, Boers M, Walsh M, et al. Assessment of health-related quality of life as an outcome measure in granulomatosis with polyangiitis (Wegener's). Arthritis Care \& Research 2012;64:273-9.

359. Suppiah R, Mukhtyar C, Flossmann O, et al. A cross-sectional study of the Birmingham Vasculitis Activity Score version 3 in systemic vasculitis. Rheumatology 2011;50:899-905.

360. Leavitt RY, Fauci AS, Bloch DA, et al. The American College of Rheumatology 1990 criteria for the classification of Wegener's granulomatosis. Arthritis and rheumatism 1990;33:1101-7.

361. Luqmani RA. Assessing disease activity in the systemic vasculitides. Curr Opin Rheumatol 2002;14:23-8.

362. Felicetti M, Cazzador D, Padoan R, et al. Ear, nose and throat involvement in granulomatosis with polyangiitis: how it presents and how it determines disease severity and long-term outcomes. Clinical rheumatology 2018;37:1075-83.

363. Bossuyt X, Cohen Tervaert J-W, Arimura Y, et al. Position paper: Revised 2017 international consensus on testing of ANCAs in granulomatosis with polyangiitis and microscopic polyangiitis. Nature reviews Rheumatology 2017;13:683-92.

364. Tervaert JW, van der Woude FJ, Fauci AS, et al. Association between active Wegener's granulomatosis and anticytoplasmic antibodies. Arch Intern Med 1989;149:2461-5.

365. Trimarchi M, Bertazzoni G, Bussi M. Cocaine induced midline destructive lesions. Rhinology. 2014;52:104-11.

366. Trimarchi M, Miluzio A, Nicolai P, Morassi ML, Bussi M, Marchisio PC. Massive apoptosis erodes nasal mucosa of cocaine abusers. American J of Rhinology. 2006;20:160-4.

367. Larocque A, Hoffman RS. Levamisole in cocaine: unexpected news from an old acquaintance. Clinical toxicology (Philadelphia, $\mathrm{Pa}$ ) 2012;50:231-41.

368. Peikert T, Finkielman JD, Hummel AM, et al. Functional characterization of antineutrophil cytoplasmic antibodies in patients with cocaine-induced midline destructive lesions. Arthritis and rheumatism 2008;58:1546-51.

369. Lloyd G, Lund VJ, Beale T, Howard D. Rhinologic changes in Wegener's granulomatosis. The J of Laryngology \& Otology 2002;116:565-9.

370. Allen SD, Harvey CJ. Imaging of Wegener's granulomatosis. Br J Radiol 2007;80:757-65.

371. Grindler D, Cannady S, Batra PS. Computed tomography findings in sinonasal Wegener's granulomatosis. American journal of rhinology \& allergy 2009;23:497-501.

372. Zycinska K, Straburzynski M, Nitsch-Osuch A, et al. Lund-Mackay System for Computed Tomography Evaluation of Paranasal Sinuses in Patients with Granulomatosis and Polyangiitis. Adv Exp Med Biol 2016;884:13-9.

373. D'Anza B, Langford CA, Sindwani R. Sinonasal imaging findings in granulomatosis with polyangiitis (Wegener granulomatosis): A systematic review. American journal of rhinology \& allergy 2017;31:16-21.

374. Holme SS, Moen JM, Kilian K, Haukeland H, Molberg O, Eggesbo HB. Development of CT-based methods for longitudinal analyses of paranasal sinus osteitis in granulomatosis with polyangiitis. BMC Med Imaging 2019;19:13.

375. Devaney KO, Travis WD, Hoffman G, Leavitt R, Lebovics R, Fauci AS. Interpretation of head and neck biopsies in Wegener's granulomatosis. A pathologic study of 126 biopsies in 70 patients. Am J Surg. Pathol 1990;14:555-64.

376. Raynaud P, Garrel R, Rigau V, et al. [How can the diagnostic value of head and neck biopsies be increased in Wegener's granulomatosis: a clinicopathologic study of 49 biopsies in 21 patients]. Ann Pathol 2005;25:87-93.

377. Erickson VR, Hwang PH. Wegener's granulomatosis: current trends in diagnosis and management. Current Opinion in Otolaryngology \& Head and Neck Surg. 2007;15:170-6.

378. Beltrán Rodríguez-Cabo O, Reyes E, Rojas-Serrano J, Flores-Suárez LF. Increased histopathological yield for granulomatosis with polyangiitis based on nasal endoscopy of suspected active lesions. European Archives of Oto-Rhino-Laryngology 2018;275:425-9.

379. Borner U, Landis BN, Banz Y, et al. Diagnostic value of biopsies in identifying cytoplasmic antineutrophil cytoplasmic antibody-negative localized Wegener's granulomatosis presenting primarily with sinonasal disease. American journal of rhinology \& allergy 2012;26:475-80.

380. Reinhold-Keller E, Beuge N, Latza U, et al. An interdisciplinary approach to the care of patients with Wegener's granulomatosis: Long-term outcome in 155 patients. Arthritis \& Rheumatism 2000;43:1021.

381. Holle JU, Gross WL, Latza U, et al. Improved outcome in 445 patients with Wegener's granulomatosis in a German vasculitis center over four decades. Arthritis and rheumatism 2011;63:25766.

382. Sproson EL, Jones NS, Al-Deiri M, Lanyon P. Lessons learnt in the management of Wegener's Granulomatosis: long-term follow-up of 60 patients. Rhinology. 2007;45:63-7.

383. Holle JU, Gross WL. Treatment of ANCA-associated vasculitides (AAV). Autoimmunity Reviews 2013;12:483-6.

384. Maritati F, Alberici F, Oliva E, et al. Methotrexate versus cyclophosphamide for remission maintenance in ANCA-associated vasculitis: A randomised trial. PLOS ONE 2017; 12:e0185880.

385. Guerry M-JCJ, Brogan P, Bruce IN, et al. Recommendations for the use of rituximab in anti-neutrophil cytoplasm antibody-associated vasculitis. Rheumatology 2012;51:634-43. 
386. Lutalo PMK, D'Cruz DP. Biological drugs in ANCA-associated vasculitis. International immunopharmacology 2015;27:209-12.

387. Stone JH, Merkel PA, Spiera R, et al. Rituximab versus Cyclophosphamide for ANCA-Associated Vasculitis. New England J of Medicine 2010;363:221-32.

388. Malm I-J, Mener DJ, Kim J, Seo P, Kim YJ. Otolaryngological progression of granulomatosis with polyangiitis after systemic treatment with rituximab. Otolaryngology--head and neck surgery : official journal of American Academy of Otolaryngology-Head and Neck Surg. 2014;150:68-72.

389. Birck R, Schmitt WH, Kaelsch IA, van der Woude FJ. Serial ANCA determinations for monitoring disease activity in patients with ANCA-associated vasculitis: systematic review. American journal of kidney diseases : the official journal of the National Kidney Foundation 2006;47:15-23.

390. Doebbeling BN, Breneman DL, Neu HC, et al. Elimination of Staphylococcus aureus nasal carriage in health care workers: analysis of six clinical trials with calcium mupirocin ointment. The Mupirocin Collaborative Study Group. Clinical infectious diseases : an official publication of the Infectious Diseases Society of America 1993;17:466-74.

391. Congdon D, Sherris DA, Specks U, McDonald T. Long-term follow-up of repair of external nasal deformities in patients with Wegener's granulomatosis. Laryngoscope. 2002;112:731-7.

392. Churg J, Strauss L. Allergic granulomatosis, allergic angiitis, and periarteritis nodosa. The American J of Pathology 1951;27:277-301.

393. Cottin V, Bel E, Bottero P, et al. Revisiting the systemic vasculitis in eosinophilic granulomatosis with polyangiitis (Churg-Strauss). Autoimmunity Reviews 2017;16:1-9.

394. Groh M, Pagnoux C, Baldini C, et al. Eosinophilic granulomatosis with polyangiitis (Churg-Strauss) (EGPA) Consensus Task Force recommendations for evaluation and management. European journal of internal medicine 2015;26:545-53.

395. Keogh KA, Specks U. Churg-Strauss syndrome: clinical presentation, antineutrophil cytoplasmic antibodies, and leukotriene receptor antagonists. The American journal of medicine 2003;115:284-90

396. Wechsler ME, Garpestad E, Flier SR, et al. Pulmonary infiltrates, eosinophilia, and cardiomyopathy following corticosteroid withdrawal in patients with asthma receiving zafirlukast. JAMA 1998;279:455-7.

397. Lane SE, Watts R, Scott DGI. Epidemiology of systemic vasculitis. Current rheumatology reports 2005;7:270-5.

398. Ramentol-Sintas M, Martínez-Valle F, Solans-Laqué R. Churg-Strauss Syndrome: an evolving paradigm. Autoimmunity reviews 2012;12:235-40.

399. Nathani $N$, Little MA, Kunst $H$, Wilson $D$, Thickett DR. Churg-Strauss syndrome and leukotriene antagonist use: a respiratory perspective. Thorax 2008;63:883-8.

400. Hauser T, Mahr A, Metzler C, et al. The leucotriene receptor antagonist montelukast and the risk of Churg-Strauss syndrome: A case-cross- over study. Thorax 2008;63:677-82.

401. Vaglio A, Martorana D, Maggiore U, et al. HLA-DRB4 as a genetic risk factor for ChurgStrauss syndrome. Arthritis and rheumatism 2007:56:3159-66.

402. Wieczorek S, Hellmich B, Gross WL, Epplen JT. Associations of Churg-Strauss syndrome with the HLA-DRB1 locus, and relationship to the genetics of antineutrophil cytoplasmic antibody-associated vasculitides: Comment on the article by Vaglio et al. Arthritis \& Rheumatism 2008;58:329-30.

403. Greco A, Rizzo MI, De Virgilio A, et al. ChurgStrauss syndrome. Autoimmunity Reviews 2015;14:341-8.

404. Vaglio A, StrehI JD, Manger B, et al. IgG4 immune response in Churg-Strauss syndrome. Annals of the Rheumatic Diseases 2012;71:390-3.

405. Lanham JG, Elkon KB, Pusey CD, Hughes GR. Systemic vasculitis with asthma and eosinophilia: a clinical approach to the Churg-Strauss syndrome. Medicine (Baltimore) 1984;63:65-81.

406. Goldfarb JM, Rabinowitz MR, Basnyat S, Nyquist GG, Rosen MR. Head and Neck Manifestations of Eosinophilic Granulomatosis with Polyangiitis: A Systematic Review. Otolaryngology--head and neck surgery : official journal of American Academy of Otolaryngology-Head and Neck Surg. 2016;155:771-8.

407. Piram M, Maldini C, Mahr A. Effect of race/ ethnicity on risk, presentation and course of connective tissue diseases and primary systemic vasculitides. Current opinion in rheumatology 2012;24:193-200.

408. Bacciu A, Bacciu S, Mercante G, et al. Ear, nose and throat manifestations of Churg-Strauss syndrome. Acta Otolaryngol. 2006;126:503-9.

409. Szczeklik W, Jakieła B, Adamek D, Musiał J. Cutting edge issues in the Churg-Strauss syndrome. Clinical reviews in allergy \& immunology 2013;44:39-50.

410. Srouji I, Lund V, Andrews P, Edwards C. Rhinologic symptoms and quality-of-life in patients with Churg-Strauss syndrome vasculitis. American journal of rhinology 2008;22:406-9.

411. Alobid I, Guilemany JM, Mullol J. Nasal manifestations of systemic illnesses. Current allergy and asthma reports 2004;4:208-16.

412. Petersen $H$, Gotz P, Both $M$, et al. Manifestation of eosinophilic granulomatosis with polyangiitis in head and neck. Rhinology. 2015;53:277-85.

413. Moosig F, Bremer JP, Hellmich B, et al. A vasculitis centre based management strategy leads to improved outcome in eosinophilic granulomatosis and polyangiitis (Churg-Strauss, EGPA): monocentric experiences in 150 patients. Annals of the Rheumatic Diseases 2013;72:1011-7.

414. Cottin V, Bel E, Bottero P, et al. Respiratory manifestations of eosinophilic granulomatosis with polyangiitis (Churg-Strauss). European Respiratory J 2016;48:1429-41.

415. Gaffo AL. Diagnostic approach to ANCA-associated vasculitides. Rheumatic diseases clinics of North America 2010;36:491-506.

416. Sinico RA, Di Toma L, Maggiore U, et al. Prevalence and clinical significance of antineutro- phil cytoplasmic antibodies in Churg-Strauss syndrome. Arthritis Rheum 2005;52:2926-35.

417. Comarmond C, Pagnoux C, Khellaf M, et al. Eosinophilic granulomatosis with polyangiitis (Churg-Strauss): clinical characteristics and longterm followup of the 383 patients enrolled in the French Vasculitis Study Group cohort. Arthritis and rheumatism 2013;65:270-81.

418. Healy B, Bibby S, Steele R, Weatherall M, Nelson $\mathrm{H}$, Beasley R. Antineutrophil cytoplasmic autoantibodies and myeloperoxidase autoantibodies in clinical expression of Churg-Strauss syndrome. J of Allergy and Clinical Immunology 2013;131:571-6.e6.

419. Mouthon L, Dunogue B, Guillevin L. Diagnosis and classification of eosinophilic granulomatosis with polyangiitis (formerly named Churg-Strauss syndrome). J of autoimmunity 2014;48-49:99103.

420. Pagnoux C, Guillevin L. Churg-Strauss syndrome: evidence for disease subtypes? Current Opinion in Rheumatology 2010;22:21-8.

421. Dejaco C, Oppl B, Monach P, et al. Serum biomarkers in patients with relapsing eosinophilic granulomatosis with polyangiitis (Churg Strauss). PloS one 2015;10:e0121737.

422. Bacciu A, Buzio C, Giordano D, et al. Nasal polyposis in Churg-Strauss syndrome. Laryngoscope. 2008;118:325-9.

423. Szczeklik W, Sokołowska B, Mastalerz L, et al. Pulmonary findings in Churg-Strauss syndrome in chest $\mathrm{X}$-rays and high resolution computed tomography at the time of initial diagnosis. Clinical Rheumatology 2010;29:1127-34.

424. Seccia V, Baldini C, Latorre M, et al. Focus on the Involvement of the Nose and Paranasal Sinuses in Eosinophilic Granulomatosis with Polyangiitis (Churg-Strauss Syndrome): Nasal Cytology Reveals Infiltration of Eosinophils as a Very Common Feature. International Archives of Allergy and Immunology 2018;175:61-9.

425. Lane SE, Watts RA, Shepstone L, Scott DGI. Primary systemic vasculitis: clinical features and mortality. QJM : monthly journal of the Association of Physicians 2005;98:97-111.

426. Mukhtyar C, Flossmann O, Hellmich B, et al. Outcomes from studies of antineutrophil cytoplasm antibody associated vasculitis: a systematic review by the European League Against Rheumatism systemic vasculitis task force. Annals of the Rheumatic Diseases 2008;67:1004-10.

427. Samson M, Puéchal X, Devilliers $H$, et al. Longterm outcomes of 118 patients with eosinophilic granulomatosis with polyangiitis (Churg-Strauss syndrome) enrolled in two prospective trials. J of autoimmunity 2013;43:60-9.

428. Guillevin L, Lhote F, Amouroux J, Gherardi R, Callard P, Casassus P. Antineutrophil cytoplasmic antibodies, abnormal angiograms and pathological findings in polyarteritis nodosa and Churg-Strauss syndrome: indications for the classification of vasculitides of the polyarteritis Nodosa Group. British journal of rheumatology 1996;35:958-64.

429. Guillevin L, Pagnoux C, Seror R, Mahr A, Mouthon L, Le Toumelin P. The Five-Factor Score revisited: assessment of prognoses of systemic 
necrotizing vasculitides based on the French Vasculitis Study Group (FVSG) cohort. Medicine (Baltimore) 2011;90:19-27.

430. Metzler C, Csernok E, Gross WL, Hellmich B. Interferon-alpha for maintenance of remission in Churg-Strauss syndrome: a long-term observational study. Clinical and experimental rheumatology 2010;28:24-30.

431. Koukoulaki M, Smith KGC, Jayne DRW. Rituximab in Churg-Strauss syndrome. Annals of the rheumatic diseases 2006;65:557-9.

432. Pepper RJ, Fabre MA, Pavesio C, et al. Rituximab is effective in the treatment of refractory Churg-Strauss syndrome and is associated with diminished T-cell interleukin-5 production. Rheumatology (Oxford, England) 2008;47:11045.

433. Jones RB, Ferraro AJ, Chaudhry AN, et al. A multicenter survey of rituximab therapy for refractory antineutrophil cytoplasmic antibody-associated vasculitis. Arthritis \& Rheumatism 2009;60:215668.

434. Cartin-Ceba R, Keogh KA, Specks U, Sethi S, Fervenza FC. Rituximab for the treatment of Churg-Strauss syndrome with renal involvement. Nephrology Dialysis Transplantation 2011;26:2865-71.

435. Kim S, Marigowda G, Oren E, Israel E, Wechsler ME. Mepolizumab as a steroid-sparing treatment option in patients with Churg-Strauss syndrome. The J of allergy and clinical immunology 2010;125:1336-43.

436. Wechsler ME, Akuthota P, Jayne D, et al. Mepolizumab or Placebo for Eosinophilic Granulomatosis with Polyangiitis. New England journal of medicine 2017;376:1921-32.

437. Steinfeld J, Bradford ES, Brown J, et al. Evaluation of clinical benefit from treatment with mepolizumab for patients with eosinophilic granulomatosis with polyangiitis. The J of allergy and clinical immunology 2019;143:2170-7.

438. Giavina-Bianchi P, Giavina-Bianchi M, Agondi R, Kalil J. Administration of Anti-lgE to a ChurgStrauss Syndrome Patient. International Archives of Allergy and Immunology 2007;144:155-8.

439. Wechsler ME, Wong DA, Miller MK, Lawrence-Miyasaki L. Churg-strauss syndrome in patients treated with omalizumab. Chest 2009;136:50718.

440. Miglani A, Divekar RD, Azar A, Rank MA, Lal D. Revision endoscopic sinus surgery rates by chronic rhinosinusitis subtype. Int Forum Allergy Rhinol 2018;8:1047-51.

441. Besnier E. Lupus pernio de la face. Annales de Dermatologie et de Syphiligraphie 2e serie 1889;10:333-6

442. Long CM, Smith TL, Loehrl TA, Komorowski RA, Toohill RJ. Sinonasal disease in patients with sarcoidosis. American journal of rhinology 2001;15:211-5

443. Rybicki BA, Major M, Popovich J, Maliank MJ, Lannuzzi MC. Racial Differences in Sarcoidosis Incidence: A 5-Year Study in a Health Maintenance Organization. American J of Epidemiology 1997;145:234-41.

444. Valeyre D, Prasse A, Nunes $H$, Uzunhan Y, Brillet
PY, Muller-Quernheim J. Sarcoidosis. Lancet 2014;383:1155-67.

445. Newman LS, Rose CS, Bresnitz EA, et al. A Case Control Etiologic Study of Sarcoidosis. American $\mathrm{J}$ of Respiratory and Critical Care Medicine 2004;170:1324-30.

446. Jordan HT, Stellman SD, Prezant D, Teirstein A, Osahan SS, Cone JE. Sarcoidosis diagnosed after September 11, 2001, among adults exposed to the World Trade Center disaster. J of occupational and environmental medicine 2011;53:966-74.

447. Miyoshi S, Hamada H, Kadowaki T, et al. Comparative Evaluation of Serum Markers in Pulmonary Sarcoidosis. Chest 2010;137:1391-7.

448. Facco M, Cabrelle A, Teramo A, et al. Sarcoidosis is a Th1/Th17 multisystem disorder. Thorax 2011;66:144-50.

449. Judson MA, Costabel U, Drent M, et al. The WASOG Sarcoidosis Organ Assessment Instrument: An update of a previous clinical tool. Sarcoidosis, vasculitis, and diffuse lung diseases : official journal of WASOG 2014;31:19-27.

450. Schupp JC, Freitag-Wolf S, Bargagli E, et al. Phenotypes of organ involvement in sarcoidosis. The European respiratory journal 2018;51:1700991.

451. Edriss H, Kelley JS, Demke J, Nugent K. Sinonasal and Laryngeal Sarcoidosis-An Uncommon Presentation and Management Challenge. The American journal of the medical sciences 2019;357:93-102.

452. Alobid I, Mullol J, Cid MC. Rhinitis of granulomatous and vasculitic diseases. Clin Allergy Immunol 2007:19:221-39.

453. Wilson R, Lund V, Sweatman M, Mackay IS, Mitchell DN. Upper respiratory tract involvement in sarcoidosis and its management. The European respiratory journal 1988;1:269-72.

454. Reed J, DeShazo RD, Houle TT, Stringer S, Wright L, Moak JS. Clinical features of sarcoid rhinosinusitis. The American journal of medicine 2010;123:856-62.

455. Sardana K, Goel K. Nasal septal ulceration. Clinics in dermatology 2014;32:817-26.

456. Rottoli P, Bargagli E, Chidichimo C, et al. Sarcoidosis with upper respiratory tract involvement. Respiratory Medicine 2006;100:253-7.

457. Lawson W, Jiang N, Cheng J. Sinonasal sarcoidosis: A new system of classification acting as a guide to diagnosis and treatment. American journal of rhinology \& allergy 2014;28:317-22.

458. Judson MA. The Clinical Features of Sarcoidosis: A Comprehensive Review. Clinical reviews in allergy \& immunology 2015;49:63-78.

459. Schwartbauer H, Tami T. Ear, nose and throat manifestations of sarcoidosis. In: Otolaryngologic Manifestations of systemic diseases. Otolaryngologic Clinics of North America 2003;35:673-84.

460. Braun JJ, Gentine A, Pauli G. Sinonasal sarcoidosis: review and report of fifteen cases. Laryngoscope. 2004;114:1960-3.

461. Aubart FC, Ouayoun M, Brauner M, et al. Sinonasal involvement in sarcoidosis: a case-control study of 20 patients. Medicine 2006;85:365-71.

462. Zeitlin JF, Tami TA, Baughman R, Winget D. Nasal and sinus manifestations of sarcoidosis. American journal of rhinology 2000;14:157-61.
463. Wong JS, Hoffbauer S, Yeh DH, Rotenberg B, Gupta M, Sommer DD. The usefulness of routine histopathology of bilateral nasal polyps - a systematic review, meta-analysis, and cost evaluation. J of Otolaryngology: Head and Neck Surg. 2015;44:46.

464. Joshi R, Zenga J, Getz A, Debnath N. THU0403 Sinonasal sarcoidosis: Review of clinical and imaging features: 7 year experience:. Annals of the Rheumatic Diseases 2013;71:291.3-2.

465. Braun J-J, Imperiale A, Riehm S, Veillon F. Imaging in sinonasal sarcoidosis: CT, MRI, 67Gallium scintigraphy and 18F-FDG PET/CT features. J of Neuroradiology 2010;37:172-81.

466. Silva M, Nunes H, Valeyre D, Sverzellati N. Imaging of Sarcoidosis. Clinical Reviews in Allergy \& Immunology 2015;49:45-53.

467. Bonfils P, Escabasse V, Coste A, et al. Efficacy of tobramycin aerosol in nasal polyposis. European annals of otorhinolaryngology, head and neck diseases 2015;132:119-23.

468. Paramothayan S, Lasserson T. Treatments for pulmonary sarcoidosis. Respiratory Medicine 2008;102:1-9.

469. Ma Y, Gal A, Koss MN. The pathology of pulmonary sarcoidosis: update. Semin Diagn Pathol 2007;24:150-61.

470. Arcasoy SM, Christie JD, Pochettino A, et al. Characteristics and Outcomes of Patients With Sarcoidosis Listed for Lung Transplantation. Chest 2001;120:873-80.

471. Grutters JC, van den Bosch JMM. Corticosteroid treatment in sarcoidosis. European Respiratory J 2006;28:627-36

472. Doty JD, Mazur JE, Judson MA. Treatment of sarcoidosis with infliximab. Chest 2005;127:106471.

473. Ørum M, Hilberg O, Krag S, Bendstrup E. Beneficial effect of infliximab on refractory sarcoidosis. Danish medical journal 2012;59:A4535.

474. Chapelon-Abric C, Saadoun D, Biard L, et al. Long-term outcome of infliximab in severe chronic and refractory systemic sarcoidosis: a report of 16 cases. Clin Exp Rheumatol 2015;33:509-15.

475. Fazzi P, Manni E, Cristofani R, et al. Thalidomide for improving cutaneous and pulmonary sarcoidosis in patients resistant or with contraindications to corticosteroids. Biomedicine \& pharmacotherapy = Biomedecine \& pharmacotherapie 2012;66:300-7.

476. Kirsten A-M, Watz H, Kirsten D. Sarcoidosis with involvement of the paranasal sinuses - a retrospective analysis of 12 biopsy-proven cases. BMC pulmonary medicine 2013;13:59.

477. Marks SC, Goodman RS. Surgical management of nasal and sinus sarcoidosis. Otolaryngol. Head Neck Surg. 1998;118:856-8.

478. Kay DJ, Har-El G. The role of endoscopic sinus surgery in chronic sinonasal sarcoidosis. American journal of rhinology 2001;15:249-54.

479. Gürkov R, Berghaus A. Nasal reconstruction in advanced sinunasal sarcoidosis. Rhinology. 2009;47:327-9.

480. Smith R, Haeney J, Gulraiz Rauf K. Improving cosmesis of lupus pernio by excision and forehead 
flap reconstruction. Clinical and Experimental Dermatology 2009;34:e25-e7.

481. Murphy J, Chauhen S, Stevenson O. Surgical management of cutaneous sarcoid of the nose. European J of Plastic Surg. 2013;36:385-6.

482. Hamizan AW, Christensen JM, Ebenzer J, et al. Middle turbinate edema as a diagnostic marker of inhalant allergy. International Forum of Allergy \& Rhinology. 2017;7:37-42

483. Yacoub M-R, Trimarchi M, Cremona G, et al. Are atopy and eosinophilic bronchial inflammation associated with relapsing forms of chronic rhinosinusitis with nasal polyps? Clinical and molecular allergy : CMA 2015;13:23.

484. Ferguson BJ. Eosinophilic mucin rhinosinusitis: a distinct clinicopathological entity. Laryngoscope. 2000;110:799-813.

485. Manning SC, Holman M. Further evidence for allergic pathophysiology in allergic fungal sinusitis. Laryngoscope. 1998;108:1485-96.

486. Shah A, Panchal N, Agarwal AK. Concomitant allergic bronchopulmonary aspergillosis and allergic Aspergillus sinusitis: a review of an uncommon association*. Clin Exp Allergy 2001;31:1896-905.

487. Ghegan MD, Lee FS, Schlosser RJ. Incidence of skull base and orbital erosion in allergic fungal rhinosinusitis (AFRS) and non-AFRS. Otolaryngology - Head \& Neck Surg. 2006;134:592-5.

488. Granville L, Chirala M, Cernoch P, Ostrowski M,
Truong LD. Fungal sinusitis: histologic spectrum and correlation with culture. Hum Pathol 2004;35:474-81.

489. Hutcheson PS, Schubert MS, Slavin RG. Districtions between allergic fungal rhinosinusitis and chronic rhinosinusitis. American J of Rhinology. and Allergy 2010;24:405-8.

490. Stewart AE, Hunsaker DH. Fungus-specific IgG and $\mathrm{IgE}$ in allergic fungal rhinosinusitis. Otolaryngol. Head Neck Surg. 2002;127:324-32.

491. Collins M, Nair S, Smith W, Kette F, Gillis D, Wormald PJ. Role of local immunoglobulin E production in the pathophysiology of noninvasive fungal sinusitis. Laryngoscope. 2004;114:1242-6.

492. Pratt E, Collins AM, Sewell WA, Harvey RJ. Antigen selection in lgE antibodies from individuals with chronic rhinosinusitis with nasal polyps. Am J Rhinol Allergy 2010;24:416-21.

493. Telmesani LM. Prevalence of allergic fungal sinusitis among patients with nasal polyps. Annals of Saudi medicine 2009;29:212-4.

494. Clark DW, Wenaas A, Luong A, Citardi MJ, Fakhr S. Staphylococcus aureus prevalence in allergic fungal rhinosinusitis vs other subsets of chronic rhinosinusitis with nasal polyps. International forum of allergy \& rhinology 2013;3:89-93.

495. Dutre T, Al Dousary S, Zhang N, Bachert C. Allergic fungal rhinosinusitis-more than a fungal disease? J Allergy Clin Immunol 2013;132:487-9 e1.
496. Pant H, Macardle P. CD8(+) T cells implicated in the pathogenesis of allergic fungal rhinosinusitis. Allergy \& rhinology (Providence, RI) 2014;5:14656.

497. Orlandi RR, Thibeault SL, Ferguson BJ. Microarray analysis of allergic fungal sinusitis and eosinophilic mucin rhinosinusitis. Otolaryngol. Head Neck Surg. 2007;136:707-13.

498. Das S, Maeso PA, Becker AM, Prosser JD, Adam $\mathrm{BL}$, Kountakis SE. Proteomics blood testing to distinguish chronic rhinosinusitis subtypes. Laryngoscope. 2008;118:2231-4.

499. Schubert MS, Hutcheson PS, Graff RJ, Santiago L, Slavin RG. HLA-DQB1*03 in allergic fungal sinusitis and other chronic hypertrophic rhinosinusitis disorders. J of Allergy \& Clinical Immunology 2004;114:1376-83.

500. Nikakhlagh S, Khodadadi A, Kanani M, Karampour LS, Saki N. The effect of the oral itraconazole on the management of allergic fungal sinusitis. Biomed Pharmacol J. 2015;8:85-9.

501. Chan KO, Genoway KA, Javer AR. Effectiveness of itraconazole in the management of refractory allergic fungal rhinosinusitis. J Otolaryngol. Head Neck Surg. 2008;37:870-4. 


\section{Patient participation, prediction, precision medicine and implementation}

\subsection{Patient participation in CRS}

\subsection{Patient participation in CRS}

Patient participation in rhinosinusitis can relate on an individual basis to participation of the patient in the design and/or discussion of the treatment plan, or to participation in the follow-up after medical or surgical treatment. There is limited research on the impact of patient participation on outcomes of treatment in CRS.

Patient involvement, moreover, is recognized as a key component of research and clinical practice guideline development with important implications for guideline implementability ${ }^{(1)}$. For optimal implementation, guideline development must include all key stakeholders, thus including patients from the start and recommendations should be relevant and applicable and address patients' needs. Patient involvement in guidelines is important because it appreciates that patients are experts, empowers and informs consumers in health care decisions, and will lead to the development of more patient-centred and trustworthy guidelines. Therefore, the Agree II instrument requires that guideline developers seek the views of the target population (https://www.agreetrust.org/agree-ii/). In EPOS2020, patients were included in every step of the development from the first questions to the dissemination.

There is limited research on the impact of patient participation on outcomes of treatment in CRS. In this chapter we discuss the data on individual patient participation.

\subsubsection{Areas of patient participation.}

Patient participation in the decision-making process of the therapeutic plan is one of the four cardinal principles of Precision Medicine ${ }^{(2)}$, which are considered to lead to good/ better adherence to treatment, high patient satisfaction and/or cost-savings. Attempts to study the impact of a patient-centred decision on the medical treatment of CRS have not been convincingly showing a major benefit ${ }^{(3)}$. In real-life, patient participation requires efforts from both patients as well as physicians, both physicians' efforts to educate patients on the nature of the disease and the different treatment options, including prediction of success and failure of the different treatment options and patients' willingness to be educated and take part in the decision-making process of any medical or surgical treatment for CRS.

\section{Patient participation in the decision-making process of the therapeutic plan is one of the four cardinal principles of Precision Medicine.}

Patient participation in the follow-up of medical or surgical treatment of CRS is also considered important to achieve optimal outcomes, but needs further study. The current follow-up strategy of CRS patients after initiation of medical and/or surgical care depends on the regional health care system and treating physician, and on the patients' willingness and capabilities of being followed-up on a regular base.

Recent mobile health initiatives to educate patients on CRS, on correct medication use and treatment options have been implemented in certain areas in Europe with success ${ }^{(4)}$. Whilst they allow a more pro-active follow-up of patients with remote monitoring of symptoms by physicians ${ }^{(4,5)}$ the impact of e-health on CRS outcomes still needs to be defined and proven.

\subsubsection{What's important to patients in sinus care?}

Although there is increasing awareness of the need to engage patients both in their own care, and in the design and delivery of trials, there are very few studies that set out to evaluate what issues are most important to patients with rhinosinusitis, and which should be addressed during a consultation. A previous study in allergic rhinitis demonstrated that up to half of patients have no specific opinion on any recommended treatment, but that the other half do have an opinion that should ideally be addressed $^{(6)}$.

Vennik et al. ${ }^{(7)}$ have undertaken structured interviews with 25 patients with CRS, and used thematic analysis to identify key areas of importance;

- Recognising the impact of the disease (symptom severity and the impact on both work and social activities);

- $\quad$ Seeking resolution with treatment (self-management, including dietary changes and complementary therapies and medical treatment options, including how to use medical treatments, concerns regarding potential side effects);

- Surgical intervention (role of surgery, including advice on the optimum time for intervention, the aims of surgery and its anticipated outcome).

Patients in this study also expressed concerns regarding some of their experience with different healthcare interactions, highligh- 
ting that some doctors fail to recognise the impact that sinus disease can have on a patient, a failure to take a holistic approach to both upper and lower airway disease, inadequate time to answer questions fully, and long intervals between reviews. Alanin et al..$^{(8)}$ undertook a qualitative analysis of patients' posts made on the Samter's Society Support Group, with the permission of the members. This is a closed group formed by the Samter's Society, and patient advocacy group for those with $\mathrm{N}$ ERD. Similar themes were identified, including the impact of the disease, and the isolation that can arise from having a condition that can be poorly understood by friends and family members. Patients are keen to explore dietary modifications that can help, as well as find out more about all different treatment options. At a time when there are a number of novel treatments emerging, patients are very keen to share experiences before making decisions regarding whether to try new medications, but also express concerns about the potential for unknown side-effects. Lack of awareness among physicians was a common frustration. Conflicts between different healthcare providers involved in their care was a significant concern, with patients describing a 'tug of war' between surgeons and allergists / pulmonologists with regards to different options, highlighting the need for a multi-disciplinary approach in the setting of upper and lower airway conditions.

Conflicts between different healthcare providers highlights the need for a multi-disciplinary approach in the setting of upper and lower airway conditions.

\subsubsection{Patient compliance}

Intranasal corticosteroids (INCS) form the first line of treatment options for almost all subgroups of CRS. However, a large population based study ${ }^{(9)}$ found that only one in five CRS patients identified on the administrative database had used an INCS in the preceding calendar year, and the mean usage was 2.4 bottles in one year (at recommended dosage that one bottle should last 4-8 weeks). There is, therefore, clear evidence of both failure to use INCS and under-dosage in those that do. This may reflect under-prescribing, lack of patient education regarding the need for ongoing usage, and concerns with regards to costs or potential side effects.

Guo et al. ${ }^{(10)}$ found that postoperative adherence with medical treatment in patients undergoing ESS was poor in patients experiencing their first surgery, with only $42.3 \%$ following their recommended treatment regimen, but was higher in patients undergoing multiple revisions, at $88.9 \%$. Yoo et al. ${ }^{(11)}$ also found compliance with post-operative irrigation to be better in those undergoing revision, but highlighted that language barriers may contribute to non-compliance.

In order to improve compliance, it is important to explain the aim of ongoing usage or any maintenance treatments to both control symptoms and reduce need for recurrent interventions.
Information on the safety of treatment and instructions for use must be provided in all necessary languages. Recent initiatives in e-health make attempts to better educate patients on any chronic condition, hopefully leading to better compliance and outcomes $^{(12)}$. One could speculate that a patient who is properly educated on the disease, on correct use of medication, on the treatment options and alternatives, and the expected outcomes of treatment may have better outcomes than the non-educated patient ${ }^{(13)}$. It can be expected that patients who are conscious of and reminded of the lack of control of CRS despite medical and or surgical treatment are more adherent to therapy and avoid more external triggers like cigarette smoke and/or occupational triggers of CRS. Reminders using digital platforms have been shown to be of benefit in improving compliance, at least in the short term ${ }^{(14)}$.

\section{Recent initiatives in e-health make attempts to better educate patients on any chronic condition, hopefully leading to better compliance and outcomes}

Actively engaging patients in their own care and facilitating self-management using written action plans has been shown to improve compliance and patient satisfaction in asthma care ${ }^{(15)}$, as well as reducing acute exacerbations ${ }^{(16)}$ and improving symptom control and quality of life ${ }^{(17)}$. A CRS action plan has been developed by Nayan et al. ${ }^{(18)}$ which incorporates triggers to alert the patient as to when to step up treatment or seek medical attention.

\subsubsection{Expectation management (see also 9.3.3.)}

While physicians are likely to understand the chronic nature of sinus disease in many patients and the need for ongoing treatment, it is essential to share this with the patient from the outset. The aim of treatment is to achieve adequate control of symptoms with as little need for intervention as possible; for many this will involve ongoing usage of intranasal treatments and in some, repeated need for systemic treatments or surgical interventions. Some patients will remain inadequately controlled despite receiving optimum current evidence-based care. Cure, with an absence of symptoms in the setting of no ongoing medication usage, is unusual with the exception of localized sinus disease where there has been a curable cause, such as an odontogenic source.

Patient advocacy groups and online resources can be valuable to patients and supplement information received during face to face consultations. Sharing personal experiences with other patients may help reduce frustration with limitations in current treatments and also help patients navigate their way through complex healthcare systems.

Patients will often seek out a variety of sources of information. Signposting useful and reliable resources such as the EUFOREA 
site, with both videos and written advice for patients will ensure that they receive correct information (www.euforea.eu/patientplatform).

\subsection{What does EPOS 2020 mean for patients?}

The following section is written by patients involved in the development of the EPOS2020 guidelines to help explain what the guidelines mean for patients.

\section{Q. What does EPOS stand for?}

A. EPOS stands for the European Position Paper on Rhinosinusitis, covering the diagnosis and management of both acute and chronic rhinosinusitis in primary and secondary care.

\section{Q. What are the EPOS2020 guidelines?}

A. The guidelines are a summary of the most up-to-date information regarding the treatment and management of rhinosinusitis and nasal polyposis.

The first EPOS guidelines were developed in 2005 and have subsequently been updated in 2007, 2012 and now 2020. In essence, the guidelines are a summary of the very latest knowledge in rhinosinusitis and nasal polyposis. To develop the guidelines, academic and clinical leaders in this field, along with the help of some patients, looked at all the information available in this area, from as far back as 1960 through to the present day, and summarised the most important elements.

The guide represents what all those experts agree should be recommended practice at this current time.

\section{Q. Why are they needed?}

A. To ensure that everyone who is in any way connected to the treatment or management of rhinosinusitis and nasal polyposis has the best information available.

The field of medicine is constantly changing. The continual emergence of new research and improved practices means it is important from time to time, to take stock of these developments and ensure that current clinical practice reflects the latest thinking.

This process happens in all areas of medicine from heart disease to child health and it is vital to ensure everyone around the world has access to the best information available.

\section{Q. What is the objective of the EPOS2020 guidelines?}

A. It is primarily to provide management strategies for rhinosinusitis based on relevant research.

Beyond this, there are many things that the EPOS2020 guidelines hope to achieve. The guidelines aim to:

- Provide healthcare professionals with a summary of the best methods for diagnosing and managing patients in both the hospital and the wider community setting;
- Inspire researchers to further study acute rhinosinusitis and chronic rhinosinusitis;

- Support the use of standard definitions and measurements of the disease;

- Hopefully reduce unnecessary (antibiotic) treatment;

- Encourage people to form new collaborations to answer unmet research questions.

\section{Q. Who will use the EPOS2020 guidelines?}

A. Many individuals and groups from doctors through to government bodies.

EPOS2020 will provide guidance for relevant government bodies, national and international drug agencies [e.g. the European Medicines Agency (EMA) and the Food and the Drug Administration (FDA) in the USA] and scientific societies, particularly regarding the use of terminology, definitions and classifications for clinical trials. Most importantly it will be used by doctors treating patients with sinus disease to help ensure that all patients are offered evidence-based care.

\section{Q. Which conditions does it cover?}

A. Not just rhinosinusitis and nasal polyposis but other upper airway and related diseases.

The EPOS2020 guidelines apply to children and adult patient populations with acute rhinosinusitis (ARS); viral/common cold, post-viral, bacterial; chronic rhinosinusitis without nasal polyps (CRSsNP) and chronic rhinosinusitis with nasal polyps (CRSwNP). It will also be relevant to patients with associated conditions, such as allergic rhinitis, asthma, bronchiectasis, chronic obstructive pulmonary disease (COPD), severe upper airway disease and rare diseases including primary ciliary dyskinesia (PCD), cystic fibrosis, vasculitis and immune deficiencies. EPOS2020 will also address patients with associated symptoms such as facial pain / pressure, sleep disorders, smell disorders and odontogenic problems, many of which are key symptoms in the diagnosis of rhinosinusitis.

\section{Q. Who writes EPOS?}

A. An international group of experts who are leaders in the field of rhinosinusitis and nasal polyps.

It took approximately 18 months and around 80 people from over 20 different countries to review all the latest information and then write and publish these guidelines.

This group consisted of renowned experts including:

- Ear, Nose and Throat Surgeons

- Allergists

- Pharmacists

- Microbiologists

- General Practitioners

- Paediatricians

- Pulmonologists 
- Neurologists

There were also patients involved in the guidelines to ensure the recommendations were always seen from a patient's perspective.

EPOS2020 is also supported by professional organisations, such as the European Rhinologic Society (ERS) and the European Academy of Allergology and Clinical Immunology (EAACI).

\section{Q. Is this just for use in Europe?}

A. No, EPOS2020 will have relevance around the world. Although EPOS has the word European in its title, and a strong European representation among the people who developed it, it is intended to be used beyond Europe. Individuals from all over the world have contributed to the development of these guidelines and they will have relevance in many countries outside of Europe.

\section{Q. Does a healthcare professional have to follow the guide- lines?}

A. This is intended to be guidance and represents best practice but is just one aspect of what informs the decisions a healthcare professional makes.

Healthcare systems around the world are all different and the healthcare professionals within them will base their decisions on how to manage a patient on many factors. EPOS2020 provides guidance on the latest information that a healthcare professional may use to inform the decisions they make but they are not obliged to follow them.

\section{Q. Where can patients get further information about allergy and airway diseases?}

A. Further information for patients can be found at www.euforea. eu/patient-platform.

The European Forum for Research and Education in Allergy and Airway diseases (EUFOREA) is an international non-profit organization working towards preventing and reducing the burden of chronic respiratory diseases (asthma, rhinitis, and rhinosinusitis). Further information for patients about allergy and airways disease can be found on their website at www.euforea.eu/ patient-platform. The EPOS2020 paper and a patient summary can be found on https://epos2020.eu/

\subsection{Prevention of disease}

\subsubsection{Primary, secondary and tertiary disease prevention in CRS}

Prevention of disease may be thought of in three main dimensions:

Primary prevention aims to reduce incidence of disease by reducing exposure to risk factors or triggers.

Secondary prevention aims to reduce disease prevalence by

\section{Key points | What's new since EPOS 2012}

- Aspects of patient participation are covered for the first time in EPOS2020.

- Relatively few studies have been published on CRS in this area.

- Recent mobile health application initiatives to educate patients on CRS are now available but the impact of e-health on CRS outcomes still needs to be defined and proven.

- Patient involvement is essential in the development of their future care.

early detection and appropriate management, returning a patient to full health and preventing disease persistence. This aims to reduce the severity and impact of disease from the outset. Tertiary prevention aims to reduce the impact of ongoing chronic disease and its complications in order to maintain quality of life and normal functioning as much as possible.

\subsubsection{Primary prevention}

CRS is a heterogeneous disease, where inflammation, mucociliary dysfunction and changes in the microbial community interact with differing influences to cause disease $\mathrm{e}^{(19)}$, the aetiology is likely multifactorial, and opportunities to prevent targeting specific causes will likely vary between subgroups.

\subsubsection{Occupational and environmental factors (see also 5.1.2.9 and 5.1.2.10}

Exposure to toxins, especially tobacco smoke, ozone and particulate air pollutants such as diesel exhaust particles, may exacerbate airway inflammation. The significance of most toxin exposures in the prevalence of CRS is unclear.

A systematic review of risk factors for CRS performed in 2015 highlights the lack of clear definition of CRS used in available studies, reliance on self-reporting of exposure levels, failure to address confounding and almost uniformly poor quality of the available literature in this area ${ }^{(20)}$. Given the poor quality, the conclusion of this study was that it was not possible to make strong conclusions regarding the role of occupation and environmental exposure. Of 41 studies identified, most failed to use a robust definition of CRS. Both Kim et al. and Alexiou et al. ${ }^{(21)}$ classified both environmental and occupational exposure, and adjusted for smoking status, finding increased risk prevalence of nasal polyps for those exposed compared with controls. Woodstove use was associated with higher risk than tobacco or occupational exposure. In a subsequent study, Gao et al. found a significant association between occupational and environmental factors and CRS ${ }^{(22)}$, more specifically exposure to gases, 
fumes, and smoke have been shown to be associated with increased risk of $\mathrm{CRS}^{(23)}$. Hox et al. found occupational exposure to irritants was both a risk factor for disease development, but also for recalcitrance, reflected in higher rates of revision surgery ${ }^{(24)}$. Some studies have looked at specific occupations, with grain farmers ${ }^{(25)}$ and those working in the textile industry ${ }^{(26)}$ being shown to have a higher prevalence of CRSwNP. Regular followup of the first responders following the 9/11 attack on the World Trade Centre have offered a unique opportunity to prospectively study the risk of developing CRS; rates were higher in firefighters involved in digging and rescue work than emergency medical services workers. Those arriving at the site early, with higher levels of exposure to irritants, or those with prolonged exposure had highest rates of CRS, approaching 50\% using an epidemiological definition 10 years after the event ${ }^{(27)}$.

Wolf found no correlation between CRS and outdoor air quality in Cologne ${ }^{(28)}$, similarly there was no difference in CRS prevalence in rural or urban areas of South Korea ${ }^{(29)}$ nor in the Netherlands ${ }^{(30)}$. In contrast weak correlations have been found in US studies $^{(31)}$, where improvements in air quality were also associated with a decreased prevalence of both hay fever and rhinosinusitis. More recent studies have shown a stronger association; Park et al. ${ }^{(32)}$ found a linear association with levels of particulate matter and CRS, although the study sampled only patients with known ENT disease and did not include a control population. Mady et al. ${ }^{(33)}$ found an association between exposure to air pollutants and disease severity in patients with known CRS, but again did not include a control group.

While a causal link cannot be clearly established, evidence is certainly mounting, and it is worth clinical questioning to include environmental and occupational exposure to irritants. There is no available evidence to inform on safe levels of exposure or preventative measures in those exposed. Global action to reduce pollution is clearly beyond the remit of this document, but must be made a priority ${ }^{(34,35)}$ and the effects of global warming closely monitored. Ensuring compliance with international standards for indoor air quality may help at an individual level. There is direct evidence that tobacco smoke diminishes mucociliary clearance and is a potential contributing and/or exacerbating factor in exposed individuals with chronic rhinosinusitis $(36,37)$.

Evidence for both active and passive smoking leading to increased risk of CRS is much stronger. The GA2LEN survey of over 50,000 patients across Europe found that CRS was more common in smokers (OR1.7) compared with non-smokers ${ }^{(38)}$. A 2018 systematic review identified 13 studies evaluating the effect of primary tobacco smoke, with 11 reporting an increased prevalence of CRS in smokers. There was a dose-dependent association found in three studies, with a $1.5 \%$ increase in prevalence for every additional year of smoking ${ }^{(39)}$.

\section{There is direct evidence that tobacco smoke dimi- nishes mucociliary clearance and is a potential contributing and/or exacerbating factor in exposed individuals with chronic rhinosinusitis}

Passive smoking, with exposure, both in childhood and adult life, also seems to have a significant association with $\mathrm{CRS}^{(40-42)}$. Aggressive taxation on tobacco products, plain packaging with warning labels and repeated counselling at every healthcare consultation are important to maintain the declining prevalence of smoking.

\subsubsection{Allergy (see also 5.1.2.1 and 8.1.1)}

It has been proposed that mucosal oedema within the ostiomeatal complex (OMC) in allergic rhinitis may compromise ventilation and even obstruct sinus ostia, leading to mucus retention and infection, however the causal role of allergy in CRS has been long debated ${ }^{(43-46)}$. On one hand, rates of positive skin prick tests are not statistically different between CRS patients or healthy controls, or between CRS sufferers with or without polyps ${ }^{(43)}$ although patients with CRS had a higher number of sensitivities. One recent systematic review evaluated 18 articles examining the relationship between allergy and CRSwNP, 10 articles found an association, seven found no association and one article showed a possible association. Of nine articles which examined the relationship between allergy and CRSsNP, four articles found an association compared with five articles showing no association ${ }^{(47)}$. In a large population based study there was a significantly increased risk of AR prior to a subsequent diagnosis of CRS (OR 2.4 for CRSsNP and 2.6 for CRSwNP) ${ }^{(48)}$. One recent prospective study of a large cohort followed from birth undertook allergy testing at nine months and six years ${ }^{(49)}$. Of patients who developed CRS, three phenotypes were identified; transient childhood rhinosinusitis, early onset adult rhinosinusitis and late onset adult rhinosinusitis. The early onset CRS group had significantly higher rates of inhalant allergy and asthma than other groups. Childhood onset rhinosinusitis symptoms were the biggest risk factor - providing an opportunity to screen children with symptoms of CRS and potentially target immunotherapy in this group; approximately one-third of children with rhinosinusitis in childhood received further investigation or treatment as adults.

No studies have assessed the effectiveness of management of allergic rhinitis on the outcome of established CRS, or on the risk of subsequent development of CRS, and further research in this area is needed before AR management can be recommended as a means of CRS prevention. However, early detection and management of AR has been shown to have a positive impact on the development of lower airway disease ${ }^{(50,51)}$, and is recommended for that aim. 
Although patients often attribute their CRS to food allergies, with the exception of non-steroidal-exacerbated respiratory disease (N-ERD), where there is an increased risk of CRSwNP, there are only limited studies assessing the association between CRS and food allergies. One study showed that milk allergy may be a predisposing factor for $\mathrm{CRS}^{(52)}$, however, overall the evidence is poor ${ }^{(47,53)}$ and certainly there is no evidence to support exclusion diets to prevent CRS. In N-ERD, CRSwNP typically develops after the onset of asthma, but again there are no studies evaluating the effectiveness of desensitization in preventing onset of CRSWNP.

\subsubsection{Asthma (see also 5.1.2.2)}

There is strong evidence to show that asthma and CRS, especially CRSwNP, frequently coexist. Several studies have shown that patients with asthma have a higher likelihood of having $\mathrm{CRS}^{(44,}$ 54-56) and the GA2LEN Survey showed that in all age groups, men and women, and irrespective of smoking behavior, asthma was associated with $\mathrm{CRS}^{(55)}$. The Greisinger Health study ${ }^{(48)}$ found higher rates of premorbid asthma in those developing CRS compared with healthy controlled (OR 2.8 for CRSwNP and 1.7 for CRSsNP). Similarly, higher premorbid rates of bronchitis, pneumonia and bronchiectasis have been reported. Again, a causal relationship has not been confirmed, but it is postulated that acute infective exacerbations may modifiy susceptibility to developing CRS.

\subsubsection{Genetic risk factors (see also 5.2.1)}

A number of genetic disorders, including cystic fibrosis and primary ciliary dyskinesia are associated with a high prevalence of CRS, however these account for only a very small proportion of CRS cases. Currently over 70 genes have been genetically associated with CRS, summarized by a number of recent reviews ${ }^{(57}$, 58). Genes associated with CRS can be broadly categorised into: genes associated with ion channels (e.g. CFTR); immunological genes (HLA, CD, IL); genes involved in tissue remodelling and in arachidonic acid metabolism ${ }^{(57-59)}$, and genes associated with $C F$. Until recently, outside of CFTR, there were no replication studies validating the gene associations with CRS, and no studies demonstrating their biological relevance ${ }^{(59)}$. Henmyr et al. found a significant association in only seven genes of the previous 53 genes associated with $\mathrm{CRS}^{\left({ }^{(60)}\right.}$. One potential target gene, CDHR3, part of the cadherin family expressed primarily in respiratory epithelium, has a number of polymorphisms which determine susceptibility to both rhinovirus infection and adult CRS, suggesting a potential role of rhinovirus as a trigger ${ }^{(61)}$. There has been growing recent interest in the bitter taste receptor T2R38; polymorphisms are associated with an increased risk of CRS, but additionally has been shown to be associated with prognosis, with significantly lower rates of 'super tasters' being found amongst CRS patients undergoing surgery ${ }^{(62-65)}$ compared to expected levels in the normal population. Of course, genetic risk factors may not change, but reduction of environmental exposure in 'at risk' populations, screening of high-risk groups, and identification of genes which are of prognostic value will be of value.

Bitter taste receptor T2R38 polymorphisms are associated with an increased risk of CRS, but additionally has been shown to be associated with prognosis, with significantly lower rates of 'super tasters' being found amongst CRS patients undergoing surgery.

\subsubsection{Microbial exposure (see also 5.1.2.7)}

The natural history of CRS, and the relationship between ARS and CRS development have been very poorly studied. Using the definitions of ARS and CRS based on duration alone, all episodes of CRS must start as ARS. However, what allows persistence of disease, or whether CRS is a completely different pathophysiology from the outset is little known. Tan et al. found that premorbid ARS (OR 2.2 for CRSwNP, OR 3.2 for CRSsNP) and acute upper respiratory tract infections (URTIS) (OR 1.3 for CRSwNP, OR 1.6 for CRSsNP) were more prevalent in patients developing CRS compared to those who did not; however, this data was extracted from electronic health records, and may simply reflect the diagnostic dilemma above. One study followed patients with ARS using repeated aspirates; those that developed chronic symptoms showed transitions from bacteria commonly associated with ARS (Strep. Pneumonia, Moraxella) to a mixed infection which also involved anaerobes ${ }^{(66)}$. However, there is no evidence to suggest that active management of ARS or URTIs may reduce the risk of subsequent development of CRS. Indeed, increased use of antibiotics may cause profound and detrimental effects on the microbiome ${ }^{(67)}$.

One study of children treated for CRS, defined according to

EPOS2012 criteria, and in remission, compared prophylactic use of bacterial lysate for three months to no treatment in an openlabel study and showed significant reduction in subsequent use of antibiotics, nasal obstruction and discharge in the treatment group at one year ${ }^{(68)}$. However, given the lack of blinding further studies are necessary.

The balance of resident microbes is increasingly recognized as important in onset of $\mathrm{CRS}^{(69)}$ and reduced diversity is found in patients with CRS compared to controls ${ }^{(70)}$ and in CRS patients following antimicrobial therapy ${ }^{(71)}$. Given that current evidence suggests that the human microbiome becomes established early in childhood ${ }^{(67)}$, there may be only limited opportunity to influence this later in life.

Unlike many chronic diseases, there is little data suggesting an increased risk of CRS in those with poor socio-economic status, which may be linked to both microbial exposure and higher rates of tobacco exposure. Indeed, one study of CRS in children suggests that when compared to controls, children with CRS were more likely to be white and privately insured ${ }^{(72)}$ this, howe- 
ver, may simply reflect inequity of access to healthcare.

\subsubsection{GORD (see also 5.1 .2 .5 )}

The relationship between gastroesophageal reflux disease and upper and lower airway diseases has been debated in the past $^{(73)}$. However, Wong et al. describe a possible vagal neural reflex existing between the oesophagus and the paranasal sinu$\operatorname{ses}^{(74)}$. A causal relationship between GORD and CRS has yet to be firmly established but GORD does appear to be a risk factor for development ${ }^{(75)}$. Few studies specifically assess whether treatment of reflux has an impact on development of CRS or severity of symptoms, and there is insufficient current evidence to consider anti-reflux therapy for refractory CRS in adults ${ }^{(76)}$. Recent epidemiologic studies using electronic health registries and systematic reviews report that GORD and CRS often coexist.

\subsubsection{Secondary prevention}

Secondary prevention of CRS is concerned with detecting a disease in its earliest stages, intervening to achieve disease and symptom control and preventing future exacerbations. Implicitly, secondary prevention takes place when primary prevention fails. Early diagnosis and selection of the optimal treatment is central to secondary prevention. Recent data suggest that there is still a large portion of the population with CRS not receiving treatment; as above, it is estimated from population studies that CRS affects approximately 5-15\% of the general population both in Europe and the USA in contrast with 2-4\% prevalence of doctor-diagnosed $\mathrm{CRS}^{(77,78)}$. It is unclear whether patients are unable to access care, or effectively self-manage symptoms of CRS.

\subsubsection{Early establishment of diagnosis}

State-of-the-art guidelines like EPOS ${ }^{(78)}$ provide clinicians with evidence-based diagnostic and treatment algorithms for CRS based on symptom duration and severity. However, a symptom based definition alone is likely to over-estimate disease ${ }^{(79)}$ and currently findings on endoscopy or radiological imaging are required to support the diagnosis. It is likely that the diagnostic criteria will be further defined as biomarkers for disease are identified. The Finnish allergy programme ${ }^{(50)}$ has shown the effectiveness of screening for inflammatory airway disease. However, with current financial constraints in healthcare, it is unlikely that such schemes will become widespread. However, symptom-based screening of patients at high risk of secondary CRS, such as those with systemic vasculitides, or those with eosinophilic airway disease and aspirin -exacerbated respiratory disease (N-ERD) is likely to be beneficial.

\subsubsection{Selection of optimal treatment (see also chapter 6)} Currently CRS is broadly categorised into two subgroups: CRS with and without nasal polyps. However, clinical phenotypes do not provide full insight into underlying cellular and molecular pathophysiologic mechanisms of $\mathrm{CRS}^{(80)}$ and further differentiation or "endotyping" will better predict the likely natural course of disease and prognosis in terms of recurrence after surgery and risk of comorbid asthma but also in responsiveness to different treatments, including topical intranasal corticosteroids, surgical interventions, and biological agents. In this regard, novel biological treatments may be given to patients who can be predicted to have an otherwise recalcitrant path, based on their biomarkers, before undergoing ineffective cycles of treatment with corticosteroids and surgery.

Guidelines have traditionally attempted to drive all patients through the same pathway; in reality multiple interconnected pathways are likely to be required to facilitate precision medicine.

\subsubsection{Optimising the outcome of surgical intervention (see chapter 6.2)}

Surgery may be optimized according to two main domains;

\subsection{Timing of surgery}

Once the diagnosis of CRS is made, EPOS advocates surgical treatment when optimal medical management has made no improvement in symptoms after 12 weeks ${ }^{(78)}$.

Current evidence fails to show clear benefit of surgery over medical treatment at first presentation, supporting a role for primary medical treatment ${ }^{(81,82)}$. However, after failed medical therapy, patients who elected to continue with medical therapy achieved poorer outcomes than those choosing surgery ${ }^{(83)}$. Furthermore, delayed surgery in the setting of persistent CRS after failed medical therapy has been shown to be associated with higher ongoing healthcare utilisation postoperatively ${ }^{(84,}$ ${ }^{85)}$ surgical intervention after a trial of medical therapy may also deliver better symptomatic outcomes that are sustained for as long as five years ${ }^{(84)}$.

Appropriate indications for ESS are currently poorly defined and the lack of clear surgical indications for ESS likely contributes to the large geographic variation in surgical rates. A recent study by Rudmik et al. clearly states that ESS can only be indicated after medical treatment has failed with patients still having significant symptoms (SNOT-22 $\geq 20$ ) and at least some abnormalities on a CT scan ${ }^{(86)}$. Pre- and post-operative patient-rated outcome scores such as the SNOT-22 may be used to predict benefit from surgery and identify early failure ${ }^{(84,87)}$. Improved patient selection for surgery is likely to improve outcomes and reduce risk of harm.

\subsection{Extent of surgery}

There is little comparative evidence to direct surgeons as to whether a conservative, aggressive or tailored approach to sinus 


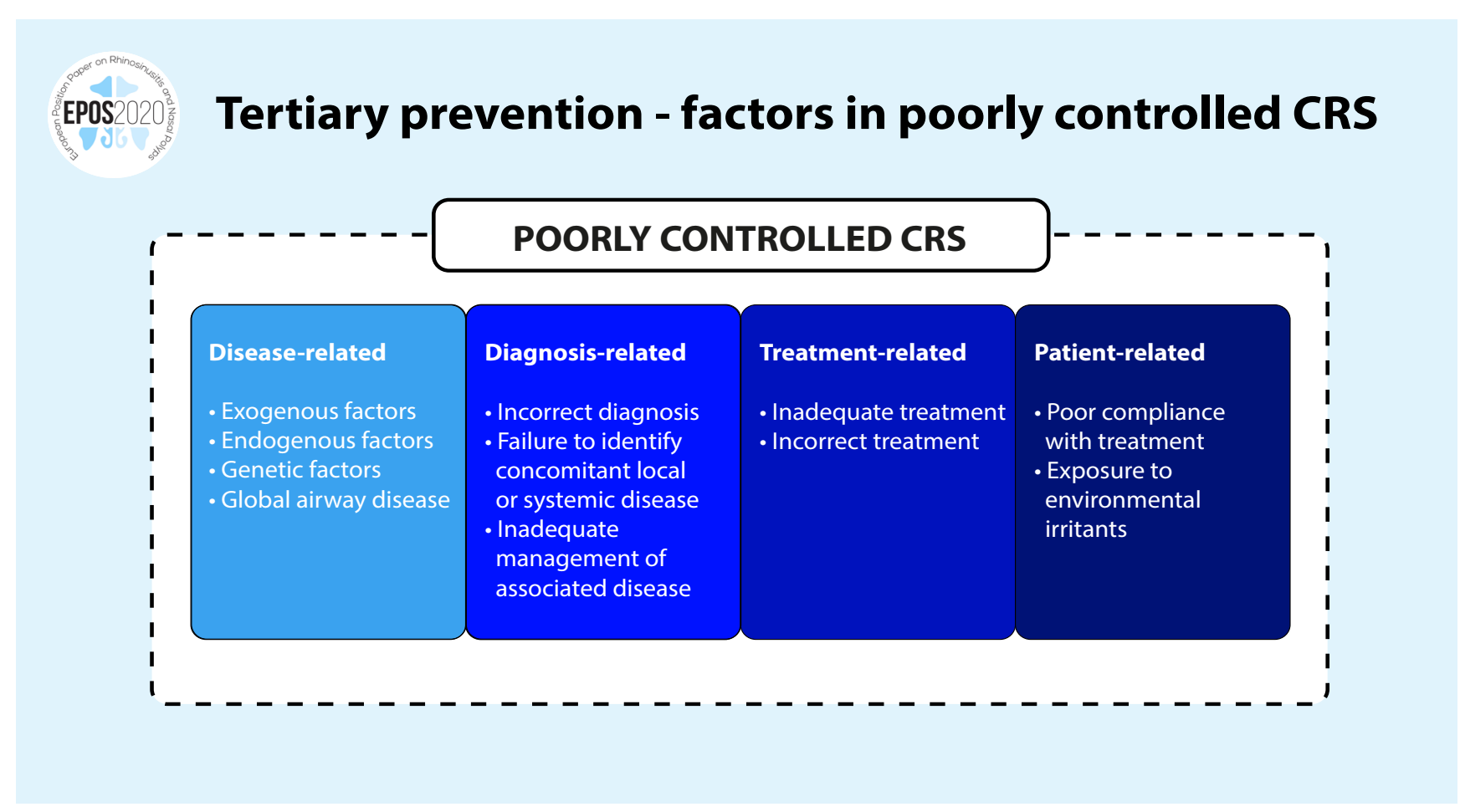

surgery should be taken ${ }^{(88)}$. Data from the UK audit of sinus surgery found no additional benefit of additional sinus surgery over simple polypectomy in terms of symptomatic benefit, and only a small benefit in terms of revision rates ${ }^{(89)}$. However, the additional sinus surgery performed in most cases was very conservative, and less than $2 \%$ of the surgical cohort had complete frontoethmoidectomy and sphenoidotomy. The effectiveness of intranasal steroids has been shown to be increased in the postoperative state, suggesting improved access to topical therapy is an important aspect of the benefits of surgery ${ }^{(90)}$. Furthermore, eosinophilic CRS has been shown not to be associated with ostiomeatal occlusion ${ }^{(91)}$ and therefore simple measures to address the OMC are unlikely to be effective. There is some evidence to support a more extensive approach, particularly in eosinophilic disease or CRSWNP(92,93).

\subsubsection{Minimising risks of treatment}

As a general rule, any treatment should have benefits that always outweigh the risks. When considering medical and surgical interventions, we must consider the risk of harm to the patient. Short courses of oral corticosteroids are widely used, and may result in insomnia, mood and gastrointestinal disturbances ${ }^{(94)}$. There is a paucity of data on the threshold of dose that may expose patients to major complications such as avascular necrosis and osteoporosis, and repeated courses should be used judiciously ${ }^{(95)}$. In contrast, topical corticosteroids are safe and adverse effects are minor. A recent Cochrane review found no difference in the reported side effects between topical corticosteroids and placebo ${ }^{(96)}$, except for an increase in reported epistaxis. Moreover, there seems to be no clinically relevant impact on either ocular pressure, glaucoma, lens opacity or cataract formation ${ }^{(97)}$.

The use of prolonged courses of anti-inflammatory antibiotics needs to be balanced with the potential gastrointestinal disturbances and serious adverse effects of rhabdomyolysis and prolonged $\mathrm{QT}^{\left({ }^{(9)}\right)}$, effects on bacterial resistance and as yet largely unknown consequences to the sinus microbiome. A study in CRS patients receiving macrolide antibiotics found a short-term increase in risk of myocardial infarction, highlighting the need to evaluate cardiac risk factors prior to usage ${ }^{(99)}$.

In comparison with medical therapy, there is more data reporting the rate of surgical complications. The National Sinonasal Audit reported a total adverse event rate of $6.6 \%$, most of which was related to minor bleeding. This rate of major complications from the UK $(0.4 \%)$ compares with a rate of $1.1 \%$ reported in a meta-analysis from 10 years previously of 4691 patients who underwent ESS in the US ${ }^{(100-102)}$. There is conflicting evidence regarding whether use of image guidance may mitigate against the risk of complications ${ }^{(103,104)}$.

\subsubsection{Tertiary prevention}

Tertiary prevention aims to minimise the impact of an ongoing illness or injury that has lasting effects. This is done by helping 
people manage long-term, often-complex health problems and injuries (e.g. chronic rhinosinusitis) in order to improve as much as possible their ability to function, their quality of life and their life expectancy. Ongoing poorly controlled upper airway disease may result from disease-related factors, inadequate treatment, poor compliance or failures in the diagnostic pathway to identify secondary CRS, or perhaps incorrectly diagnosed CRS in the first place $^{(105)}$ (Figure 9.3.1.). A recent study found that at least $40 \%$ of CRS patients would be considered to have uncontrolled disease within 3-5 years of endoscopic sinus surgery ${ }^{(106)}$.

In patients with poor disease control, a careful review of ongoing treatment, technique and compliance with medication should be undertaken. Growth in digital healthcare and patient apps may encourage self-management and increase compliance. The evidence of the impact of ongoing tobacco exposure on quality of life outcomes of CRS treatment is conflicting ${ }^{(107,}$ ${ }^{108)}$, but higher rates of revision surgery are seen in smokers ${ }^{(109)}$, and cessation should therefore be encouraged. Management of individuals with recalcitrant CRS is based on the understanding that this is a chronic condition, and that "cure", as achieved in acute bacterial infections such as tonsillitis, cannot and should not be expected. Caregivers and allied health personnel all share in the task of educating the patient to understand the chronic, ongoing nature of the disorder, and to adjust their expectations accordingly.

\section{Growth in digital healthcare and patient apps may encourage self-management and increase compliance.}

The diagnosis should be reconfirmed, particularly when facial pain is the ongoing primary symptom, and systemic diseases should be considered and excluded when indicated. Conditions such as granulomatosis with polyangiitis (Wegener's) or eosinophilic granulomatosis with polyangiitis (Churg-Strauss) may present with sinonasal disease and the systemic nature of disease may not be apparent in the early stages (section 8.7). Underlying immune deficiency should be considered, particularly where there is a history of concomitant lower airway, ear or skin infections (section 9.2). Up to $10 \%$ patients with refractory CRS were found to have common variable immunodeficiency(CVID), 20\% had decreased IgG, IgA or IgM, and $11-67 \%$ had an inadequate functional response to pneumococcal vaccine ${ }^{(110-112)}$, although all studies were conducted at tertiary institutions, thus it is likely that there is significant selection bias.

Higher levels of anxiety and depression are found in patients with CRS, although it is often undiagnosed ${ }^{(113,114)}$. It is unclear if this is a causative relationship; however, it appears likely that co-existing depression results in both higher rates of symptom reporting and amplifies symptom severity, particularly in
CRSsNP(115). Patients with co-existing depression report poorer disease specific health related quality of life both before and after treatment for CRS ${ }^{(116)}$. Although treatment for CRS has been shown to reduce depression scores, there is no evidence on whether treatment of depression may result in improvement of CRS-related QOL.

Nayan et al. ${ }^{(117)}$ undertook a systematic review of dietary modifications that might have the potential to reduce inflammation in recalcitrant CRS. They did not identify any studies outside of those undertaken in the N-ERD population (reviewed in chapter 6.1.12.4). In acute URTI probiotics are better than placebo in reducing the number of participants experiencing episodes of acute URTI, the mean duration of an episode of acute URTI, antibiotic use and cold-related school absence. This indicates that probiotics may be more beneficial than placebo for preventing acute URTIs. However, the quality of the evidence was low (see chapter 4.6.2.11). Extrapolating from other chronic inflammatory conditions, there is potential benefit in the use of probiotics, and diets promoting healthy gut microbiota, but their role in CRS is yet to be established.

\section{Key points | What's new since EPOS 2012}

- Prevention may be considered as primary, secondary and tertiary.

- Occupational and environmental factors are of increasing importance in primary prevention and the effects of global warming carefully monitored.

- Co-morbidities such as allergy, asthma and GORD should be considered.

- Genetic and microbiological factors will likely become of greater importance.

- Early diagnosis and selection of the optimal treatment is central to secondary prevention.

- Optimising medical treatment and consideration of the timing and extent of surgery can improve outcomes.

- In tertiary prevention, a careful review of ongoing treatment, technique and compliance with medication should be undertaken.

- Growth in digital healthcare and patient apps may encourage self-management and increase compliance.

\subsubsection{Prevention of asthma}

There is extensive literature concerning primary prevention of asthma with respect to allergen avoidance, dietary manipulation and immunotherapy; it is beyond the scope of this document to review these here.

There are a small number of studies using big data sets that 
suggest that endoscopic sinus surgery for CRS reduces the yearly incidence of new asthma diagnoses ${ }^{(84,118)}$. In a cohort free of asthma at enrolment, the preoperative asthma incidence was $4.5 \%$ in patients with allergic rhinitis (AR), and $4.2 \%$ in patients without AR, but fell to $0.4 \%$ post-ESS. Those patients who had later surgery had higher rates of asthma than those who underwent surgery at an earlier timepoint; patients having surgery within two years of CRS diagnosis had an asthma prevalence of $9 \%$ compared to $22 \%$ in patients having surgery $4-5$ years after diagnosis. There is a risk of confounding and further studies are required to establish if this a true consequence of sinus surgery, and to assess if successful medical therapy has the same effect.

\section{Key point | What's new since EPOS 2012}

- Those patients who have later surgery may develop higher rates of asthma than those who undergo surgery at an earlier timepoint.

\subsubsection{Prevention of recurrence}

Patients should be encouraged to continue to use intranasal corticosteroids after surgery, as continued use has been shown to improve post-operative endoscopic scores in all CRS patients ${ }^{(119)}$ and, in those with CRSwNP, reduce risk of recurrence ${ }^{(120)}$. Adherence with prescribed postoperative medications dropped to only $42 \%$ at 12 months after surgery in one study despite regular telephone contact ${ }^{(121)}$ strategies to improve this, such as utilizing digital technology, will likely be important in future. As mucosal eosinophilia has been shown to be highly correlated with risk of polyp recurrence, more so than basic phenotype of CRSwNP, in future this may provide a better indicator for the need for long term INCS use ${ }^{(122)}$. Patients who underwent a single polyp exacerbation after surgery, but were then well controlled were found to have significantly lower adherence to postoperative medications compared to those with no recurren$\mathrm{ce}^{(10)}$, suggesting that patient education regarding the need for ongoing therapy is important and may avoid recurrence in some patients. In contrast, those patients with ongoing recurrence were found to have significantly higher levels of eosinophilia, and had recurrence despite good compliance with post-operative care, suggesting that different strategies may be required to achieve disease control in this group.

Continued use of intranasal corticosteroids after surgery has been shown to improve post-operative endoscopic scores in all CRS patients and, in those with CRSWNP, reduce risk of recurrence.

Saline douching has improves symptoms after surgery ${ }^{(123,124)}$ and may reduce need for nasal cavity debridement. The value of postoperative debridement remains controversial; although systematic reviews have shown benefit in terms of early symptom scores and endoscopic appearances ${ }^{(125)}$ there is no significant impact on long-term outcomes, and current trials have no compared to high-volume saline irrigation alone. Routine use of antibiotics in all patients after ESS is not supported by the literature and increasing bacterial resistance must be considered, but it may be used in selected cases ${ }^{(125)}$.

There are a growing number of drug-eluting stents and topical dressings that may promote early healing and restoration of mucociliary function. A recent systematic review studied steroideluting bioabsorbable intranasal devices, and demonstrated improved objective and subjective outcomes following ESS ${ }^{(126)}$; although their role in preventing recurrence has not been assessed, prevention of adhesions and stenosis may potentially reduce the risk of revision and therefore warrants further study. Currently, costs may restrict widespread usage, although cost-effectiveness may be enhanced by reduced post-operative debridement.

A small number of studies have found that ongoing occupational exposure to irritants may increase risk of recurrence ${ }^{(24,127)}$. Any factors thought to be involved in the underlying aetiology of CRS in each individual patient should be addressed where possible to reduce risk of recurrence.

\subsection{Prevention of patient dissatisfaction after CRS treatment}

In contrast, to the large number of studies evaluating changes in $\mathrm{HRQOL}$ after treatment, few studies have evaluated patient satisfaction with outcomes of treatment, and only following surgical interventions.

A 2003 study found high levels of overall satisfaction after sinus surgery, with $72 \%$ patients being very satisfied. Lower education level and higher severity of disease were found to be associated with lower rates of satisfaction ${ }^{(128)}$.

The UK prospective audit of sinus surgery asked patients to rate change in overall symptoms after surgery and found that this was strongly associated with changes in their SNOT-22 scores ${ }^{(100)}$. Seventy seven percent of patients rated the results of surgery overall as good or excellent. Eighty four percent of patients reported information provided regarding their operation as good or excellent, while only $58 \%$ rated information on post-operative treatment as good or excellent.

Mattos et al. ${ }^{(129)}$ set out to evaluate the association between improvement in patient's symptoms and meeting pre-operative expectations with post-operative satisfaction. Patients were asked to identify if post-operative improvement in specific symptoms was felt to be important; $92 \%$ responded that improvement in nasal obstruction, $77 \%$ need to blow nose and $72 \%$ sense of smell were very important. Eighty two percent of the patients were satisfied overall following surgery; on multivariate logistic regression this was associated with improvement in the symptoms most important to patients, and matching preope- 
rative expectations. Achieving an improvement greater than the MCID was not a predictor of satisfaction; $86 \%$ of patients not achieving the MCID were still satisfied with the outcome of surgery and $87 \%$ would recommend sinus surgery to others. In a similar study, Phillips et al. ${ }^{(130)}$ studied patients who reported overall improvement in their CRS symptoms despite failing to achieve change in SNOT22 scores of greater than the MCID; they found that significant improvement in the nasal subdomain resulted in patients reporting overall improvement, but no other subdomains were associated with overall improvement. Although data is limited, it appears that pre-treatment counselling to ensure that a patient has realistic expectations of treatment outcomes, both with respect to overall improvement and achieving improvement in symptoms deemed to be most important to the patient, as well as optimizing outcomes with respect to nasal symptoms is important to avoid a dissatisfied patient. Provision of information both regarding the likely risks and benefits of treatment and the need for ongoing care may need to be tailored to a patient's educational level.

\subsection{Prediction}

\subsubsection{Prediction of the natural course of disease}

There are no studies evaluating the natural history of untreated CRS. The fact that the prevalence of disease peaks between the ages of 45-54 years, and then declines suggests that remission occurs with later age ${ }^{(54)}$.

De Corso et al. ${ }^{(131)}$ prospectively followed patients with CRSsNP with endoscopy and evaluated the risk of developing nasal polyps. Patients with eosinophilic inflammation demonstrated on nasal cytology were significantly more likely to develop polyps, compared with those with neutrophilic inflammation and controls with vasomotor rhinitis.

\section{Key point | What's new since EPOS 2012}

- There is an urgent need for research to address the natural course of CRS.

\subsubsection{Prediction of 'success' or symptomatic improvement}

There are few studies predicting success of medical therapy response rates for individual treatment options are discussed in chapter 6. A meta-analysis ${ }^{(132)}$ evaluated factors predicting success of low-dose macrolides; patients with CRSsNP achieved greater improvements in symptoms scores, longer courses (24 weeks compared to eight and 12 weeks) and half dose (compared with very low dose) were also more effective. IgE was not assessed, but a randomised trial found that patients with normal IgE achieved greater benefit in symptom scores than patients with elevated $\lg \mathrm{E}^{(133)}$. When used postoperatively, responders were found to have lower tissue and serum eosinophil levels ${ }^{(134)}$. Ebbens et al. ${ }^{(135)}$ showed that CRS patients with higher levels of mucosal eosinophils are less likely to suffer from postoperative recurrent sinonasal disease when treated post-operatively with nasal corticosteroids than patients with lower mucosal eosinophils.

When predicting outcomes following sinus surgery with respect to improvement in symptoms scores measured with PROMs, a number of studies have shown that the preoperative score is the best predictor of outcomes ${ }^{(136,137)}$.Patients with preoperative SNOT-22 scores less than 20 failed to achieve mean improvement greater than the MCID, scores of $>30$ have a $70-75 \%$ chance of achieving improvements greater than the MCID. Patients on average achieve a 45-50\% reduction in SNOT-22 scores ${ }^{(138,139)}$. When predicting outcomes following sinus surgery, a number of studies have shown that the pre-operative symptom score is the best predictor of outcome.

Smith et al. ${ }^{(140)}$ prospectively evaluated 119 adults with CRS to determine other preoperative factors that predicted improvement in endoscopy and quality of life. Smokers, and patients with depression and N-ERD had least benefit in both respects, but still reported improvements in both domains. In a separate study he also demonstrated that patients undergoing primary surgery were more likely to achieve significant improvements in HRQOL than patients undergoing a revision procedure ${ }^{(141)}$. Higher income and better socioeconomic status are associated with greater improvements in quality of life ${ }^{(142,143)}$ it is not clear if this reflects differing access to care.

With respect to olfactory outcomes, Bogdanov et al. ${ }^{(144)}$ demonstrated that for patients with olfactory loss, the response in olfactory function to oral corticosteroids (OCS) predicted the outcome of surgery; improvements after OCS and surgery were significantly correlated and no patient responded to surgery who did not respond to OCS. This would be a useful test to avoid patient dissatisfaction after surgery if hyposmia was their main driver to seek intervention.

\section{Key points | What's new since EPOS 2012}

- When predicting outcomes following sinus surgery, a number of studies have shown that the pre-operative symptom score such as SNOT22 is the best predictor of outcome.

- Primary surgery has better outcomes than revision.

- When loss of smell is a major symptom, response in olfactory function to oral corticosteroids (OCS) predicts the outcome of surgery. 
Figure 9.5.1. Historical approaches to care.

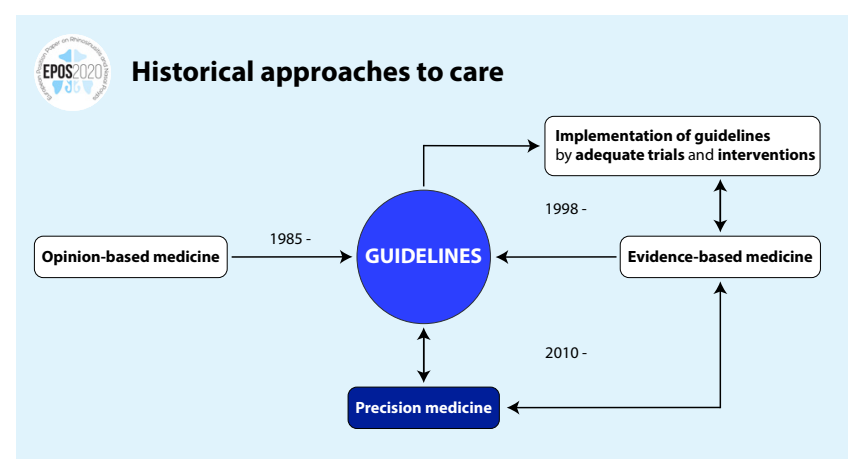

\subsubsection{Prediction of recurrent disease}

There is a paucity of studies evaluating the success rate or relapse rate following appropriate medical therapy of CRS. Young et al. prospectively enrolled 80 patients receiving treatment with systemic steroids and antibiotics in addition to topical steroid sprays and saline irrigation ${ }^{(145)}$. $53 \%$ failed the trial of medical treatment and underwent surgery. Relatively younger age, male gender, European ethnicity, presence of asthma, and positive endoscopic as well as CT findings were factors predictive of a need for surgery. Presence of polyps was not a predictive factor for failure of medical therapy.

A number of long-term studies have reported on the rates of revision surgery. Hopkins et al. reported long-term outcomes from a multi-institutional cohort study, with 1459 patients completing five-year follow-up. 19.1\% patients had undergone revision surgery ( $21 \%$ of CRSwNP, $16 \%$ of CRSsNP). Patients undergoing additional sinus surgery were found to have lower revision rates than those undergoing simple polypectomy for CRSwNP. Improvements in SNOT-22 scores were maintained over a five-year period $^{(146)}$.

Smith et al. ${ }^{(147)}$ prospectively followed 59 adult patients electing endoscopic sinus surgery over a 10 year follow-up period;

$17 \%$ patients ( $25 \%$ of CRSwNP, $7 \%$ of CRSsNP) had undergone revision surgery. Those undergoing subsequent revision had significantly poorer endoscopy scores at their first post-operative visit and were more likely to have developed frank recurrence of polyps by 18 months. Using electronic health records, Smith et al. identified nearly 30,000 patients with a mean follow-up of 9.7 years $^{(143)}$. The long-term revision rate was reported at $15.9 \%$ (29.9\% of CRSwNP, 7\% of CRSsNP), with CRSwNP patients also being more likely to require multiple revision procedures. When examining factors associated with increased risk of revision, female gender, older age at time of first surgery, a history of asthma, allergy and nasal polyps were associated with higher risk of revision, with the presence of polyps having the largest impact. Similarly, Stein et al. ${ }^{(148)}$ looked at a large electronic dataset of 61,000 patients undergoing ESS, of which $6.7 \%$ under-
Figure 9.5.2. Current medical practice versus precision medicine.

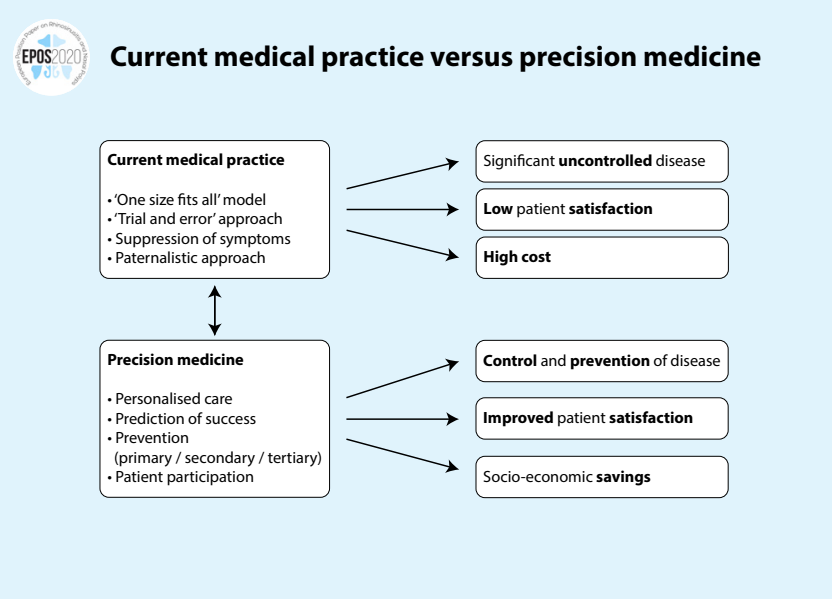

went revision surgery. The presence of nasal polyps and female gender were again shown to be predictive of revision surgery. Revision surgery rates are an imprecise estimate of recurrent disease as they are influenced by both the patient's and the surgeon's willingness to undergo / perform revision surgery and therefore, likely underestimate the rate of recurrent disease. DeConde ${ }^{(149)}$ studied endoscopic recurrence in CRSwNP and found that $38 \%$ patients had recurrent polyps at 12 months after surgery, while $82 \%$ had mucosal oedema. Not all of these would appear to go on to revision but it would be helpful to be able to predict who will develop recurrent disease in order to target treatment. A number of studies have looked at the role of biomarkers to predict disease recurrence. A systematic review ${ }^{(150)}$ identified 11 articles evaluating tissue eosinophilia; $>55$ eosinophils per high powered field had both high sensitivity (0.87) and specificity (0.97) in predicting recurrent disease. Further attempts to avoid the need for tissue biopsy have shown that blood eosinophil levels may be used to identify patients with eosinophilic CRS, but has lower sensitivity; Ho et al. ${ }^{(151)}$ reported that blood eosinophil levels $>0.24 \times 10^{9} / \mathrm{L}$ (sensitivity $71 \%$, specificity $78 \%$ ) or eosinophil ratio of $>4.27 \%$ of total white cell count (sensitivity $64 \%$, specificity $89 \%$ ) could be used to predict eCRS. In a multivariate analysis, eosinophilic CRS were the only significant predictor of recurrence after controlling for other variables, and also showed that the disease-free interval was significantly shorter ${ }^{(152)}$. Both blood and tissue eosinophilia were predictors of poor disease control, alongside more extensive radiological disease (LMS $\geq 15)^{(152)}$. A number of other biomarkers have been evaluated with respect to predicting recurrent disease. CRSwNP patients who undergo revision surgery had significantly higher polyp tissue levels of IgE, ECP and IL-5, while non recurrent patients had a mixed pattern of cytokines with higher levels of IFN-gamma ${ }^{(154)}$.

In another study, CST2, PAPP-A and periostin levels in mucus 
were significantly reduced after surgery, but increased postoperatively in patients who went on to require revision surgery(155). The limitation of many of these studies is the selection of a small number of markers, and the failure to include commonly available markers such as blood or tissue eosinophilia in a regression model to allow comparison of the predictive value. This study also found that increasing levels of all markers was significantly correlated with worsening of the SNOT-22 score at each time point. Indeed, deterioration of the SNOT-22 score of more than the MCID has also been shown to be a good predictor of need for revision surgery.

While the perfect biomarker to predict failure remains elusive, both blood and tissue eosinophil levels can be measured with little additional expense and may be used to help predict risk of recurrence and need for targeted post-operative care. Sequential measurement of symptoms scores using the SNOT-22 may allow remote monitoring of these patients if resources cannot support direct follow-up.

\section{Key points | What's new since EPOS 2012}

- Prediction of recurrent disease involves many factors including age, gender, ethnicity, co-morbidities, and duration of disease.

- Both blood and tissue eosinophil levels can be measured with little additional expense and may be used to help predict risk of recurrence and need for targeted postoperative care.

\subsection{Precision medicine}

In 2015, President Obama launched the precision medicine initiative: "delivering the right treatment at the right time, every time, to the right person". Precision medicine (PM) is an emerging approach for disease treatment and prevention targeted to the needs of individual patient, taking into account individual variability in environment, lifestyle and genes for each person ${ }^{(156,}$ 157). PM represents a novel approach in medicine, embracing four key features: personalized care based on molecular, immunologic and functional endotyping of the disease, with participation of the patient in the decision-making process of therapeutic actions, and taking into account predictive and preventive aspects

Figure 9.5.3. Implementing precision medicine in CRS.

EPOS 2020 Implementing precision medicine in CRS

PRIMARY CARE

Effects of different treatment options on sinonasal and bronchial symptoms and QoL

\section{SECONDARY CARE}

- Step-up or step-down

treatment

- Pharmacotherapy or surgery

\section{Participation}

Prevention strategy
- Mono versus combined

treatment - Treatment of comorbidities

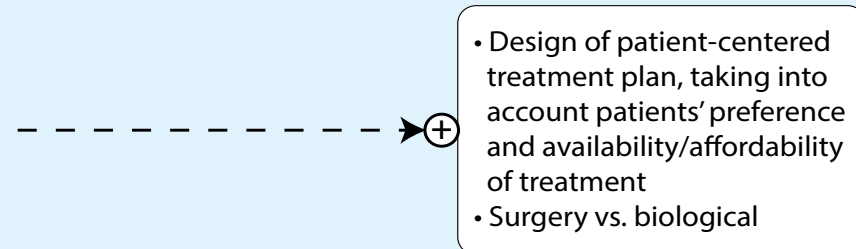

- Secondary prevention: ESS and control of environmental triggers - Tertiary prevention: prevention of organ damage

\section{TERTIARY CARE}

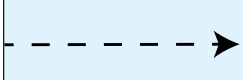

\section{Personalized care}

Endotype-driven treatment 
of the treatment. The major scientific and economic challenges in airway diseases may be addressed through the promising possibilities of PM underpinned by trials and evidence to understand how to use $i^{(157)}$. It has evolved from evidence-based medicine, 'the conscientious, explicit, and judicious use of current best evidence in making decisions about the care of individual patients" which still remains the basis of our clinical practice ${ }^{(158,}$ 159) (Figures 9.5.1. and 9.5.2.).

Based on the knowledge of mechanisms of the disease, PM generally combines diagnosis and treatment to select optimal management ${ }^{(160,161)}$. Precise endotyping of patients using novel methods like proteomics, metabolomics, genomics, diverse cellular assays, biobanks, registries and even mobile health technology ${ }^{(162)}$, allows the design of therapeutic strategies with predictive, preventive, personalized and participatory aspects ${ }^{(163)}$. PM has already gone through its own evolution from personalised medicine to precision medicine and now, 4P medicine. The four Ps constitute the basis of precision medicine (Figure 9.5.3.).

- Personalized care is a medical practise that proposes customization of healthcare, with medical decisions, practises and/or products being tailored to the individual patient.

- Prediction of the natural progress of disease and of the success of treatment allow the medical doctor as well as the patient to decide on the best therapeutic strategy.

- Prevention of disease should be distinguished in primary, secondary and tertiary prevention. Preventive measures should be advised to prevent the disease from occurring (primary), to prevent the disease from becoming symptomatic (secondary) and to prevent from causing damage or disability (tertiary).

- Participation of the patient in the therapeutic plan is crucial for achieving good adherence and hence optimal efficacy of treatment.

PM is not new in other areas of medicine but has been more recently applied to other respiratory diseases such as allergy and asthma and now chronic rhinosinusitis ${ }^{(164,165)}$. This has been promoted by organisations such as EUFOREA, the European Forum for Research and Education in Allergy and Airway disease (www.euforea.eu).

Thus the principles of PM can be implemented within existing adult treatment algorithms for chronic rhinosinusitis (CRS) ${ }^{(165)}$. At the time of diagnosis, prediction of success of the initiated treatment as well as patient participation in the decision of the treatment plan can be implemented. The second level approach ideally involves strategies to prevent progression of disease, in addition to prediction of success of therapy, and patient participation in the long-term therapeutic strategy. At the tertiary level of care, endotype-driven treatment, which is part of a personalized approach, should be positioned, given the high cost, both financial and in effort of implementation, of molecular diagnosis and biological treatment.

Precision medicine allows real-time clinical decision support at the point of care with implementation of harmonized care based on quality criteria and patients to be treated and monitored more precisely and effectively to better meet their individual needs ${ }^{(167)}$. It brings together clinicians from many inter-related specialities, scientists and above all patients in a collaborative effort to provide the most efficient and effective management.

\subsection{Implementation}

The implementation of high-quality guidelines and position papers is essential to improve clinical practice and public health. It is well recognised that clinical guidelines are often ignored, which is perhaps not surprising when so many are published on every conceivable topic ${ }^{(168-170)}$. A clinical practice guideline can be defined as "systematically developed statements to assist practitioners' decisions about appropriate health care for specific clinical circumstances" (171) thereby decreasing the gap between research and current practice and reducing inappropriate variability in practice ${ }^{(172)}$. Their development and implementation is intended to organize and provide the best available evidence to support clinical decision making in order to improve quality of care, patient outcomes and cost effectiveness ${ }^{(173,174)}$. The criteria and prerequisites for developing guidelines are: a highly prevalent disease or frequently used medical procedure, high associated costs and current variations in practice. They are of particular value for a disease such as rhinosinusitis which has significant negative effects on health-related quality of life. However, in medicine in general, it is estimated that about $30 \%-40 \%$ of patients receive treatment that is not based on scientific evidence, and $20 \%-25 \%$ receive treatments that are either not needed or potentially harmful $(168,175,176)$.

The successful introduction of guidelines involves the three steps of: development, dissemination and implementation (177) and the success of implementation depends on determining the barriers and developing strategies tailored to overcome these. Several reviews have considered and categorised the most important barriers and suitable strategies ${ }^{(178,179)}$ based on 76 and 69 articles respectively, of which the latter is the more recent and rigorous systematic analysis.

Cabana et al classified the barriers to implementation into three main categories: barriers related to physicians' knowledge (e.g., lack of awareness and lack of familiarity), barriers that affect physicians' attitudes (e.g., lack of agreement and lack of familiarity), barriers that affect physicians' attitudes (e.g., lack of agreement and lack of motivation) and external barriers (e.g., patient-, guideline- and environment-related factors) ${ }^{(178)}$. 
Table 9.6.1. Barriers and interventions in guideline implementation (adapted from Fisher ${ }^{(179)}$

\begin{tabular}{|c|c|c|}
\hline \multicolumn{2}{|c|}{ Barriers } & Interventions \\
\hline \multicolumn{3}{|c|}{ Guideline related factors } \\
\hline \multirow{2}{*}{\multicolumn{2}{|c|}{ Access to guideline }} & Provide easy access to guideline \\
\hline & & Decision support systems \\
\hline \multirow{2}{*}{\multicolumn{2}{|c|}{ Poor layout }} & Design and development of guideline \\
\hline & & Short and user-friendly versions of guidelines \\
\hline \multirow{4}{*}{\multicolumn{2}{|c|}{ Lack of evidence/plausibility of recommendations }} & Use of methods of evidence-based medicine \\
\hline & & Appraisal of evidence in recommendations \\
\hline & & Regular updates \\
\hline & & Delphi \\
\hline \multicolumn{2}{|l|}{ Lack of applicability } & Sensitivity for differences in healthcare systems \\
\hline \multirow{2}{*}{\multicolumn{2}{|c|}{ Organisational constraints }} & Standardisation of processes and procedure \\
\hline & & Link to quality management \\
\hline \multirow{2}{*}{\multicolumn{2}{|c|}{ Lack of resources (time restrictions, heavy workload, facilitation }} & Financial incentives/compensation \\
\hline & & Providing time for documentation and utilization of guidelines \\
\hline \multicolumn{2}{|c|}{ Social and clinical norms } & Local consensus groups \\
\hline \multicolumn{3}{|c|}{ User factors } \\
\hline \multirow[t]{8}{*}{ Physicians' knowledge } & Lack of awareness & Increased dissemination of guideline \\
\hline & & Use of mass media to increase awareness \\
\hline & & Continuous Medical Education (CME) \\
\hline & Lack of familiarity & Making guideline available with practical instruments \\
\hline & & Education \\
\hline & Lack of agreement & Opinion leaders \\
\hline & & Stakeholder participation in guideline \\
\hline & & Society endorsement of guideline \\
\hline \multirow[t]{6}{*}{ Physicians' attitudes } & Lack of self-efficacy & Interactive learning / group training \\
\hline & & Audit and feedback of individual performance \\
\hline & Lack of skills & CME focusing on skills \\
\hline & Lack of learning culture & Promoting learning organizations \\
\hline & Lack of motivation & Motivational strategies that utilize audit and feedback \\
\hline & & Opinions leaders \\
\hline \multicolumn{3}{|c|}{ External factors } \\
\hline \multirow{3}{*}{\multicolumn{2}{|c|}{ Organisational constraints }} & Standardisation of processes and procedures \\
\hline & & Guideline development needs to consider the care setting \\
\hline & & Link to quality management \\
\hline \multirow{2}{*}{\multicolumn{2}{|c|}{ Lack of resources (time restrictions, heavy workload, facilitation) }} & Financial incentives/compensation \\
\hline & & Providing time for documentation and utilization of guidelines \\
\hline \multicolumn{2}{|l|}{ Lack of collaboration } & $\begin{array}{l}\text { Improving multiprofessional collaboration with other healthcare profes- } \\
\text { sionals }\end{array}$ \\
\hline \multicolumn{2}{|c|}{ Social and clinical norms } & Local consensus groups \\
\hline
\end{tabular}

The systematic review by Fischer et al. included publications from many countries which were both generic and diseasespecific, with several covering asthma but none on rhinologic topics ${ }^{(179)}$. They divided the barriers into guideline-related factors, personal factors, and external factors which are summarised in Table 9.6.1. The scoping review revealed the following aspects as central elements of successful strategies for guideline implementation: dissemination, education and training, social interaction, decision support systems and standing orders. Available evidence indicates that a structured implementation can improve adherence to guidelines (179). 
EPOS is an established 'brand' with a strong legacy from 2005 onwards. However, as it is 7 years since the last edition we have tried to improve EPOS2020 implementation:

- by writing a clear and concise executive summary followed by extensive chapters containing all the supportive evidence.

- by a stronger focus on patient-relevant outcomes and patient involvement during its development.

- by greater involvement of other stakeholders in its development and writing e.g. primary care, pharmacists and neurologists.

- by containing summaries and versions relevant to these other stakeholders to support the implementation process.

- by translating the Executive Summary into a wide range of languages.

- by reaching out to many key opinion leaders all over the world to review and comment on the document and including their suggestions in the final text.

- by widespread presentation, discussion and dissemination at scientific meetings, publications, via websites and developing core sets of slides for presenters to use.

However, we do realize that not all advice in EPOS2020 can be followed in all health care systems and social circumstances. The advises can be adjusted locally depending on the possibilities in the health care system.

EPOS2020 plans a rolling programme of revision and update in response to future research and responses. A full implementation plan for EPOS2020 will be published separately in the near future.

\section{References}

1. Pugin B, Deneyer L, Bachert C, et al. Patient Advisory Board for Chronic Rhinosinusitis - A EUFOREA initiative. Rhinology. 2019 10.4193/Rhin 19.012.

2. Hellings PW. Paving the future of rhinosinusitis care. Rhinology. 2017;55:193-4.

3. Steele TO, Rudmik L, Mace JC, DeConde AS, Alt JA, Smith TL. Patient-centered decision making: the role of the baseline SNOT-22 in predicting outcomes for medical management of chronic rhinosinusitis. Int Forum Allergy Rhinol. 2016;6:590-6.

4. Seys SF, Bousquet J, Bachert C, et al. mySinusitisCoach: patient empowerment in chronic rhinosinusitis using mobile technology. Rhinology. 2018;56:209-15.

5. Khanwalkar AR, Shen J, Kern RC, et al. Utilization of a novel interactive mobile health platform to evaluate functional outcomes and pain following septoplasty and functional endoscopic sinus surgery. Int Forum Allergy Rhinol 2019;9:345-51.

6. Hellings PW, Dobbels F, Denhaerynck K, Piessens M, Ceuppens JL, De Geest S. Explorative study on patient's perceived knowledge level, expectations, preferences and fear of side effects for treatment for allergic rhinitis. Clin Transl Allergy 2012;2:9.

7. Vennik J, Eyles C, Thomas M, et al. Chronic rhinosinusitis: a qualitative study of patient views and experiences of current management in primary and secondary care. BMJ Open 2019;9:e022644.

8. Alanin MC, Laidlaw T, The Samter's Society, Hopkins C. The burden of onn-steroidal anti-inflammatory exacerbated respiratory disease from the patient's perspective - a qualitative analysis of posts from the Samter's Society. Rhinology. 2020 Aug 1;58(4):333-340.

9. Rudmik L, Xu Y, Liu M, Bird C, Kukec E, Quan H. Utilization Patterns of Topical Intranasal Steroid Therapy for Chronic Rhinosinusitis:
A Canadian Population-Based Analysis. JAMA Otolaryngol. Head Neck Surg. 2016;142:1056-62.

10. Guo M, Alasousi F, Okpaleke C, Habib A-R Javer A. Prognosis of Chronic Rhinosinusitis With Nasal Polyps Using Preoperative Eosinophil/Basophil Levels and Treatment Compliance. Am J Rhinol Allergy 2018;32:440-6.

11. Yoo F, Ference EH, Kuan EC, Lee JT, Wang $M B$, Suh JD. Evaluation of patient nasal saline irrigation practices following endoscopic sinus surgery. Int Forum Allergy Rhinol 2018;8:32-40.

12. Matricardi PM, Dramburg S, Alvarez-Perea A, et al. The role of mobile health technologies in allergy care: An EAACl position paper. Allergy 2019, 10.1111/all.13953.

13. Sleurs K, Seys S, Bousquet J, et al. Mobile health tools for the management of chronic respiratory diseases. Allergy 2019, 10.1111/ all.13720.

14. Feng S, Liang Z, Zhang R, et al. Effects of mobile phone WeChat services improve adherence to corticosteroid nasal spray treatment for chronic rhinosinusitis after functional endoscopic sinus surgery: a 3-month follow-up study. Eur Arch Otorhino-laryngol 2017;274:1477-85.

15. Patel MR, Valerio MA, Sanders G, Thomas LJ, Clark NM. Asthma action plans and patient satisfaction among women with asthma. Chest 2012;142:1143-9.

16. Gibson PG, Powell H, Coughlan J, et al. Self-management education and regular practitioner review for adults with asthma. Cochrane Database Syst Rev 2003, 10.1002/14651858.CD001117:CD001117.

17. Gupta S, Wan FT, Hall SE, Straus SE. An asthma action plan created by physician, educator and patient online collaboration with usability and visual design optimization. Respiration 2012;84:406-15.

18. Nayan S, Kilty S, Lloyd HB, Desrosiers M.
Patient and Public Outreach Initiatives in Chronic Rhinosinusitis from the Canadian Sinusitis Working Group: Support for Affected Patients and Extending an Understanding of CRS to the General Public. Curr Allergy Asthma Rep 2017;17:48.

19. Timperley D, Schlosser RJ, Harvey RJ. Chronic rhinosinusitis: an education and treatment model. Otolaryngol. Head Neck Surg. 2010;143:S3-8.

20. Sundaresan AS, Hirsch AG, Storm M, et al. Occupational and environmental risk factors for chronic rhinosinusitis: a systematic review. Int Forum Allergy Rhinol 2015;5:9961003.

21. Alexiou A, Sourtzi P, Dimakopoulou K, Manolis E, Velonakis E. Nasal polyps: heredity, allergies, and environmental and occupational exposure. J Otolaryngol. Head Neck Surg. 2011;40:58-63.

22. Gao WX, Ou CQ, Fang SB, et al. Occupational and environmental risk factors for chronic rhinosinusitis in China: a multicentre crosssectional study. Respir Res 2016;17:54.

23. Thilsing T, Rasmussen J, Lange B, Kjeldsen AD, Al-Kalemji A, Baelum J. Chronic rhinosinusitis and occupational risk factors among 20- to 75-year-old Danes-A GA(2) LENbased study. Am J Ind Med 2012;55:103743.

24. Hox V, Delrue S, Scheers $H$, et al. Negative impact of occupational exposure on surgical outcome in patients with rhinosinusitis. Allergy 2012;67:560-5.

25. Holmstrom M, Thelin A, Kolmodin-Hedman B, Van Hage M. Nasal complaints and signs of disease in farmers--a methodological study. Acta Otolaryngol. 2008;128:193-200.

26. Veloso-Teles R, Cerejeira R, Roque-Farinha $R$, von Buchwald C. Higher prevalence of nasal polyposis among textile workers: an endoscopic based and controlled study. Rhinology. 2018, 10.4193/Rhin 17.228.

27. Ekenga CC, Scheu KE, Cone JE, Stellman 
SD, Farfel MR. 9/11-related experiences and tasks of landfill and barge workers: qualitative analysis from the World Trade Center Health Registry. BMC Public Health 2011;11:321.

28. Wolf C. Urban air pollution and health: an ecological study of chronic rhinosinusitis in Cologne, Germany. Health Place 2002;8:12939.

29. Min YG, Jung HW, Kim HS, Park SK, Yoo KY. Prevalence and risk factors of chronic sinusitis in Korea: results of a nationwide survey. Eur Arch Otorhinolaryngol 1996;253:435-9.

30. Hoffmans R, Wagemakers A, van Drunen C, Hellings P, Fokkens W. Acute and chronic rhinosinusitis and allergic rhinitis in relation to comorbidity, ethnicity and environment. Plos one 2018;13:e0192330.

31. Bhattacharyya N. Air quality influences the prevalence of hay fever and sinusitis. Laryngoscope. 2009;119:429-33. .

32. Park M, Lee JS, Park MK. The Effects of Air Pollutants on the Prevalence of Common Ear, Nose, and Throat Diseases in South Korea: A National Population-Based Study. Clin Exp Otorhinolaryngol 2019;12:294-300.

33. Mady LJ, Schwarzbach HL, Moore JA, et al. Air pollutants may be environmental risk factors in chronic rhinosinusitis disease progression. Int Forum Allergy Rhinol 2018:8:377-84.

34. Hellings PW, Akdis CA, Bachert C, et al. EUFOREA Rhinology. Research Forum 2016: report of the brainstorming sessions on needs and priorities in rhinitis and rhinosinusitis. Rhinology. 2017;55:202-10.

35. Valiulis A, Bousquet J, Veryga A, et al. Vilnius Declaration on chronic respiratory diseases: multisectoral care pathways embedding guided self-management, mHealth and air pollution in chronic respiratory diseases. Clin Transl Allergy 2019;9:7.

36. Cohen NA, Zhang S, Sharp DB, et al. Cigarette smoke condensate inhibits transepithelial chloride transport and ciliary beat frequency. Laryngoscope. 2009;119:2269-74.

37. Tamashiro E, Xiong G, Anselmo-Lima WT, Kreindler JL, Palmer JN, Cohen NA. Cigarette smoke exposure impairs respiratory epithelial ciliogenesis. Am J Rhinol Allergy 2009;23:117-22.

38. Hastan D, Fokkens WJ, Bachert C, et al Chronic rhinosinusitis in Europe--an underestimated disease. A GA(2)LEN study. Allergy 2011;66:1216-23.

39. Lee S-HH, Kim H-JJ, Lee J-WW, Yoon Y-HH, Kim Y-MM, Rha K-SS. Categorization and clinicopathological features of chronic rhinosinusitis with eosinophilic mucin in a korean population. Clin Exp Otorhinolaryngol 2015;8:39-45. Hur K, Liang J, Lin SY. The role of secondhand smoke in sinusitis: a systematic review. Int Forum Allergy Rhinol 2014;4:22-8.

40. Reh DD, Lin SY, Clipp SL, Irani L, Alberg AJ, Navas-Acien A. Secondhand tobacco smoke exposure and chronic rhinosinusitis: a population-based case-control study. Am J Rhinol Allergy 2009;23:562-7.

41. Tammemagi CM, Davis RM, Benninger MS, Holm AL, Krajenta R. Secondhand smoke as a potential cause of chronic rhinosinusitis: a case-control study. Arch Otolaryngol. Head Neck Surg. 2010;136:327-34.

42. Tan BK, Zirkle W, Chandra RK, et al. Atopic profile of patients failing medical therapy for chronic rhinosinusitis. Int Forum Allergy Rhinol 2011;1:88-94

43. Beule A. Epidemiology of chronic rhinosinusitis, selected risk factors, comorbidities, and economic burden. GMS Curr Top Otorhinolaryngol Head Neck Surg. 2015;14:Doc11.

44. Min JY, Tan BK. Risk factors for chronic rhinosinusitis. Curr Opin Allergy Clin Immunol 2015;15:1-13.

45. Tint D, Kubala S, Toskala E. Risk Factors and Comorbidities in Chronic Rhinosinusitis. Curr Allergy Asthma Rep 2016;16:16.

46. Wilson KF, McMains KC, Orlandi RR. The association between allergy and chronic rhinosinusitis with and without nasal polyps: an evidence-based review with recommendations. Int Forum Allergy Rhinol 2014:4:93-103.

47. Tan BK, Chandra RK, Pollak J, et al. Incidence and associated premorbid diagnoses of patients with chronic rhinosinusitis. J Allergy Clin Immunol 2013;131:1350-60.

48. Chang EH, Stern DA, Willis AL, Guerra S, Wright AL, Martinez FD. Early life risk factors for chronic sinusitis: A longitudinal birth cohort study. The J Allergy Clin Immunol 2018;141:1291-7.e2.

49. Haahtela T, Valovirta E, Kauppi P, et al. The Finnish Allergy Programme 2008-2018 - scientific rationale and practical implementation. Asia Pac Allergy 2012;2:275-9.

50. Kauppi P, Linna M, Martikainen J, Makela MJ, Haahtela T. Follow-up of the Finnish Asthma Programme 2000-2010: reduction of hospital burden needs risk group rethinking. Thorax 2013;68:292-3.

51. Lill C, Loader B, Seemann R, et al. Milk allergy is frequent in patients with chronic sinusitis and nasal polyposis. Am J Rhinol Allergy 2011;25:e221-4.

52. Collins MM, Loughran S, Davidson P, Wilson JA. Nasal polyposis: prevalence of positive food and inhalant skin tests. Otolaryngol. Head Neck Surg. 2006;135:680-3

53. Tan BK, Kern RC, Schleimer RP, Schwartz BS. Chronic rhinosinusitis: the unrecognized epidemic. Am J Respir Crit Care Med 2013;188:1275-7.

54. Jarvis D, Newson R, Lotvall J, et al. Asthma in adults and its association with chronic rhinosinusitis: the GA2LEN survey in Europe. Allergy 2012;67:91-8.

55. Frieri M. Asthma linked with rhinosinusitis: An extensive review. Allergy \& rhinology (Providence, RI) 2014;5:41-9.

56. Hsu J, Avila PC, Kern RC, Hayes MG, Schleimer RP, Pinto JM. Genetics of chronic rhinosinusitis: state of the field and direc- tions forward. The J Allergy Clin Immunol 2013;131:975-7.

57. Yoo KH, Ahn HR, Park JK, et al. Burden of Respiratory Disease in Korea: An Observational Study on Allergic Rhinitis, Asthma, COPD, and Rhinosinusitis. Allergy Asthma Immunol Res 2016;8:527-34.

58. Min HJ, Kim SJ, Kim TH, Chung HJ, Yoon $\mathrm{J}-\mathrm{H}$, Kim C-H. Level of secreted HMGB1 correlates with severity of inflammation in chronic rhinosinusitis. Laryngoscope. 2015;125:E225-30.

59. Henmyr V, Vandeplas G, Hallden C, et al. Replication study of genetic variants associated with chronic rhinosinusitis and nasal polyposis. The J Allergy Clin Immunol 2014;133:273-5.

60. Chang EH, Willis AL, McCrary HC, et al. Association between the CDHR3 rs6967330 risk allele and chronic rhinosinusitis. The J Allergy Clin Immunol 2017;139:1990-2.e2.

61. Adappa ND, Zhang Z, Palmer JN, et al. The bitter taste receptor T2R38 is an independent risk factor for chronic rhinosinusitis requiring sinus surgery. Int Forum Allergy Rhinol 2014;4:3-7.

62. Farquhar DR, Kovatch KJ, Palmer JN, Shofer FS, Adappa ND, Cohen NA. Phenylthiocarbamide taste sensitivity is associated with sinonasal symptoms in healthy adults. Int Forum Allergy Rhinol 2015;5:111-8.

63. Lee RJ, Xiong G, Kofonow JM, et al. T2R38 taste receptor polymorphisms underlie susceptibility to upper respiratory infection. J Clin Invest 2012;122:4145-59.

64. Mfuna Endam L, Filali-Mouhim A, Boisvert P, Boulet LP, Bosse Y, Desrosiers M. Genetic variations in taste receptors are associated with chronic rhinosinusitis: a replication study. Int Forum Allergy Rhinol 2014;4:2006.

65. Brook I. Microbiology and management of sinusitis. J Otolaryngol. 1996;25:249-56.

66. Cho I, Blaser MJ. The human microbiome: at the interface of health and disease. Nat Rev Genet 2012;13:260-70.

67. Chen J, Zhou Y, Nie J, et al. Bacterial lysate for the prevention of chronic rhinosinusitis recurrence in children. J of laryngology and otology 2017;131:523-8.

68. Mahdavinia M, Keshavarzian A, Tobin MC, Landay AL, Schleimer RP. A comprehensive review of the nasal microbiome in chronic rhinosinusitis (CRS). Clinical and experimental allergy : journal of the British Society for Allergy and Clinical Immunology 2016;46:21-41.

69. Choi EB, Hong SW, Kim DK, et al. Decreased diversity of nasal microbiota and their secreted extracellular vesicles in patients with chronic rhinosinusitis based on a metagenomic analysis. Allergy 2014;69:51726.

70. Liu CM, Soldanova K, Nordstrom L, et al. Medical therapy reduces microbiota diversity and evenness in surgically recalcitrant chronic rhinosinusitis. Int Forum Allergy 
Rhinol 2013;3:775-81

71. Smith DF, Ishman SL, Tunkel DE, Boss EF. Chronic rhinosinusitis in children: race and socioeconomic status. Otolaryngol. Head Neck Surg. 2013;149:639-44.

72. Sella GCP, Tamashiro E, Anselmo-Lima WT, Valera FCP. Relation between chronic rhinosinusitis and gastroesophageal reflux in adults: systematic review. Revista Brasileira de Otorrinolaringologia 2017;83:356-63.

73. Wong IWY, Rees G, Greiff L, Myers JC, Jamieson GG, Wormald P-J. Gastroesophageal Reflux Disease and Chronic Sinusitis: In Search of an Esophageal-nasal Reflex. Am J Rhinol Allerfy 2010;24:255-9.

74. Lin YH, Chang TS, Yao YC, Li YC. Increased Risk of Chronic Sinusitis in Adults With Gastroesophgeal Reflux Disease: A Nationwide Population-Based Cohort Study. Medicine (Baltimore) 2015;94:e1642.

75. Zelenik K, Formanek M, Matousek P, Kominek P. Chronic rhinosinusitis and extraesophageal reflux: Who is the candidate for antireflux treatment? Am J Rhinol Allergy 2016;30:e5-9.

76. Shashy RG, Moore EJ, Weaver A. Prevalence of the chronic sinusitis diagnosis in Olmsted County, Minnesota. Arch Otolaryngol. Head Neck Surg. 2004;130:320-3.

77. Fokkens WJ, Lund VJ, Mullol J, et al. European Position Paper on Rhinosinusitis and Nasal Polyps 2012. Rhinol Suppl 2012;23:3 p preceding table of contents, 1-298.

78. Bhattacharyya N, Lee LN. Evaluating the diagnosis of chronic rhinosinusitis based on clinical guidelines and endoscopy. Otolaryngology-Head and Neck Surg. 2010;143:147-51.

79. Akdis CA, Bachert C, Cingi $C$, et al. Endotypes and phenotypes of chronic rhinosinusitis: a PRACTALL document of the European Academy of Allergy and Clinical Immunology and the American Academy of Allergy, Asthma \& Immunology. J Allergy Clin Immunol 2013;131:1479-90.

80. Rimmer J, Fokkens W, Chong LY, Hopkins C. Surgical versus medical interventions for chronic rhinosinusitis with nasal polyps. The Cochrane database of systematic reviews 2014;12:CD006991.

81. Ragab SM, Lund VJ, Saleh HA, Scadding G. Nasal nitric oxide in objective evaluation of chronic rhinosinusitis therapy. Allergy 2006:61:717-24

82. Smith KA, Smith TL, Mace JC, Rudmik L. Endoscopic sinus surgery compared to continued medical therapy for patients with refractory chronic rhinosinusitis. Int Forum Allergy Rhinol 2014;4:823-7.

83. Hopkins C, Andrews P, Holy CE. Does time to endoscopic sinus surgery impact outcomes in chronic rhinosinusitis? Retrospective analysis using the UK clinical practice research data. Rhinology. journal 2015:53:18-24.

84. Benninger MS, Sindwani R, Holy CE,
Hopkins C. Early versus delayed endoscopic sinus surgery in patients with chronic rhinosinusitis: impact on health care utilization. Otolaryngol. Head Neck Surg. 2015;152:546-52.

85. Rudmik L, Soler ZM, Hopkins C, et al Defining appropriateness criteria for endoscopic sinus surgery during management of uncomplicated adult chronic rhinosinusitis: a RAND/UCLA appropriateness study. Int Forum Allergy Rhinol 2016;6:557-67.

86. Rudmik L, Soler ZM, Hopkins C. Using postoperative SNOT-22 to help predict the probability of revision sinus surgery. Rhinology. 2016:54:111-6.

87. Sharma R, Lakhani R, Rimmer J, Hopkins C. Surgical interventions for chronic rhinosinusitis with nasal polyps. Cochrane Database of Systematic Reviews 2014:CD006990.

88. Browne JP, Hopkins C, Slack R, et al. Healthrelated quality of life after polypectomy with and without additional surgery. Laryngoscope. 2006;116:297-302.

89. Kalish L, Snidvongs K, Sivasubramaniam R, Cope D, Harvey RJ. Topical steroids for nasal polyps. Cochrane Database Syst Rev 2012;12:CD006549.

90. Snidvongs K, Kalish L, Sacks $R$, Sivasubramaniam R, Cope D, Harvey RJ. Sinus surgery and delivery method influence the effectiveness of topical corticosteroids for chronic rhinosinusitis: systematic review and meta-analysis. Am J Rhinol Allerfy 2013;27:221-33.

91. DeConde AS, Suh JD, Mace JC, Alt JA, Smith TL. Outcomes of complete vs targeted approaches to endoscopic sinus surgery. Int Forum Allergy Rhinol 2015;5:691-700.

92. Chen FH, Deng J, Hong HY, et al. Extensive versus functional endoscopic sinus surgery for chronic rhinosinusitis with nasal polyps and asthma: A 1-year study. Am J Rhinol Allergy 2016;30:143-8.

93. Head K, Chong LY, Hopkins C, Philpott C Schilder AG, Burton MJ. Short-course oral steroids as an adjunct therapy for chronic rhinosinusitis. Cochrane Database of Systematic Reviews 2016;4:CD011992.

94. Poetker DM. Oral corticosteroids in the management of chronic rhinosinusitis with and without nasal polyps: Risks and benefits. Am J Rhinol Allergy 2015;29:339-42.

95. Chong LY, Head K, Hopkins C, Philpott C, Schilder AG, Burton MJ. Intranasal steroids versus placebo or no intervention for chronic rhinosinusitis. Cochrane Database of Systematic Reviews 2016;4:CD011996.

96. Ahmadi N, Snidvongs K, Kalish L, et al. Intranasal corticosteroids do not affect intraocular pressure or lens opacity: a systematic review of controlled trials. Rhinology. 2015;53:290-302.

97. Albert RK, Schuller JL, Network CCR. Macrolide antibiotics and the risk of cardiac arrhythmias. Am J Respir Crit Care Med 2014;189:1173-80

98. Williamson E, Denaxas S, Morris S, et al. Risk of mortality and cardiovascular events following macrolide prescription in chronic rhinosinusitis patients: a cohort study using linked primary care electronic health records. Rhinology. 2019, 10.4193/ Rhin18.237.

99. Hopkins C, Browne JP, Slack R, et al. The national comparative audit of surgery for nasal polyposis and chronic rhinosinusitis. Clinical Otolaryngology 2006;31:390-8.

100. Noon E, Hopkins C. Review article: outcomes in endoscopic sinus surgery. BMC Ear Nose Throat Disord 2016;16:9.

101. May M, Levine HL, Mester SJ, Schaitkin B. Complications of endoscopic sinus surgery: analysis of 2108 patients--incidence and prevention. Laryngoscope. 1994;104:1080-3.

102. Dalgorf DM, Sacks R, Wormald PJ, et al. Image-guided surgery influences perioperative morbidity from endoscopic sinus surgery: A systematic review and metaanalysis. Otolaryngology - Head and Neck Surg. (United States) 2013;149:17-29.

103. Ramakrishnan VR, Orlandi RR, Citardi MJ, Smith TL, Fried MP, Kingdom TT. The use of image-guided surgery in endoscopic sinus surgery: An evidence-based review with recommendations. International Forum of Allergy and Rhinology. 2013;3:236-41.

104. Hellings PW, Fokkens WJ, Akdis C, et al. Uncontrolled allergic rhinitis and chronic rhinosinusitis: where do we stand today? Allergy 2013;68:1-7.

105. van der Veen J, Seys SF, Timmermans M, et al. Real-life study showing uncontrolled rhinosinusitis after sinus surgery in a tertiary referral centre. Allergy 2017;72:282-90.

106. Katotomichelakis M, Simopoulos E, Tripsianis $G$, et al. The effects of smoking on quality of life recovery after surgery for chronic rhinosinusitis. Rhinology. 2014:52:341-7.

107. Rudmik L, Mace JC, Smith TL. Smoking and Endoscopic Sinus Surg: Does smoking volume contribute to clinical outcome? Int Forum Allergy Rhinol 2011;1:145-52.

108. Krzeski A, Galewicz A, Chmielewski R, Kisiel $M$. Influence of cigarette smoking on endoscopic sinus surgery long-term outcomes. Rhinology. 2011:49:577-82.

109. Carr TF, Koterba AP, Chandra R, et al. Characterization of specific antibody deficiency in adults with medically refractory chronic rhinosinusitis. Am J Rhinol Allergy 2011;25:241-4.

110. Chee L, Graham SM, Carothers DG, Ballas ZK. Immune dysfunction in refractory sinusitis in a tertiary care setting. Laryngoscope. 2001;111:233-5.

111. Alqudah M, Graham SM, Ballas ZK. High prevalence of humoral immunodeficiency patients with refractory chronic rhinosinusitis. Am J Rhinol Allergy 2010;24:409-12.

112.Schlosser RJ, Storck K, Cortese BM, Uhde TW, Rudmik L, Soler ZM. Depression in chronic rhinosinusitis: A controlled cohort study. Am J Rhinol Allerfy 2016;30:128-33.

113. Nanayakkara JP, Igwe C, Roberts D, Hopkins 
C. The impact of mental health on chronic rhinosinusitis symptom scores. Eur Arch Otorhinolaryngol 2013;270:1361-4.

114.Davis GE, Yueh B, Walker E, Katon W, Koepsell TD, Weymuller Jr EA. Psychiatric distress amplifies symptoms after surgery for chronic rhinosinusitis. Otolaryngology Head \& Neck Surg. 2005;132:189-96.

115. Adams KN, Schuman TA, Ebert CS, You W, Tomoum MO, Senior BA. Self-reported anxiety and depression unchanged after endoscopic sinus surgery for chronic rhinosinusitis. Rhinology. 2018;56(3):234-40.

116. Nayan S, Maby A, Endam LM, Desrosiers M. Dietary modifications for refractory chronic rhinosinusitis? Manipulating diet for the modulation of inflammation. Am J Rhinol Allerfy 2015;29:e170-e4.

117. Benninger MS, Sindwani R, Holy CE, Hopkins C. Impact of medically recalcitrant chronic rhinosinusitis on incidence of asthma. Int Forum Allergy Rhinol 2016;6:124-9.

118. Pundir V, Pundir J, Lancaster G, et al. Role of corticosteroids in Functional Endoscopic Sinus Surg--a systematic review and metaanalysis. Rhinology. 2016;54:3-19.

119. Rowe-Jones JM, Medcalf M, Durham SR Richards DH, Mackay IS. Functional endoscopic sinus surgery: 5 year follow up and results of a prospective, randomised, stratified, double-blind, placebo controlled study of postoperative fluticasone propionate aqueous nasal spray. Rhinology. 2005;43:2 10.

120.Nabi S, Rotenberg BW, Vukin I, Payton K, Bureau Y. Nasal spray adherence after sinus surgery: problems and predictors. J Otolaryngol. Head Neck Surg. 2012;41 Suppl 1:S49-55

121. Nakayama T, Yoshikawa M, Asaka D, et al. Mucosal eosinophilia and recurrence of nasal polyps - new classification of chronic rhinosinusitis. Rhinology. 2011;49:392-6.

122. Salib RJ, Talpallikar S, Uppal S, Nair SB. A prospective randomised single-blinded clinical trial comparing the efficacy and tolerability of the nasal douching products Sterimar ${ }^{\mathrm{TM}}$ and Sinus Rinse ${ }^{\mathrm{TM}}$ following functional endoscopic sinus surgery. Clinical otolaryngology 2013;38:297-305.

123. Giotakis Al, Karow EM, Scheithauer MO, Weber R, Riechelmann H. Saline irrigations following sinus surgery - a controlled, single blinded, randomized trial. Rhinology. 2016;54:302-10.

124. Rudmik L, Soler ZM, Orlandi RR, et al. Early postoperative care following endoscopic sinus surgery: an evidence-based review with recommendations. Int Forum Allergy Rhinol 2011;1:417-30.

125. Rizan C, Elhassan HA. Post-sinus surgery insertion of steroid-eluting bioabsorbable intranasal devices: A systematic review. Laryngoscope. 2016;126:86-92.

126. Veloso-Teles R, Cerejeira R. Endoscopic sinus surgery for chronic rhinosinusitis with nasal polyps: Clinical outcome and predictive factors of recurrence. Am J Rhinol Allergy
2017;31:56-62.

127. Tai CJ, Chu CC, Liang SC, et al. Use of patient satisfaction data in a continuous quality improvement program for endoscopic sinus surgery. Otolaryngol. Head Neck Surg. 2003;129:210-6.

128. Mattos JL, Rudmik L, Schlosser RJ, et al. Symptom importance, patient expectations, and satisfaction in chronic rhinosinusitis. Int Forum Allergy Rhinol 2019;9:593600

129. Phillips KM, Hoehle LP, Caradonna DS, Gray ST, Sedaghat AR. Determinants of noticeable symptom improvement despite subMCID change in SNOT-22 score after treatment for chronic rhinosinusitis. Int Forum Allergy Rhinol 2019;9:508-13.

130.Corso ED, Lucidi D, Battista $M$, et al. Prognostic value of nasal cytology and clinical factors in nasal polyps development in patients at risk: can the beginning predict the end? Int Forum Allergy Rhinol 2017;7:861-7.

131.Seresirikachorn K, Suwanparin N, Srisunthornphanich C, Chitsuthipakorn W, Kanjanawasee D, Snidvongs K. Factors of success of low-dose macrolides in chronic sinusitis: Systematic review and metaanalysis. Laryngoscope. 2019, https://dx.doi. org/10.1002/lary.27865.

132. Wallwork B, Coman W, Mackay-Sim A, Greiff L, Cervin A. A double-blind, randomized, placebo-controlled trial of macrolide in the treatment of chronic rhinosinusitis. Laryngoscope. 2006;1 16:189-93.

133. Oakley GM, Christensen JM, Sacks R, Earls P, Harvey RJ. Characteristics of macrolide responders in persistent post-surgical rhinosinusitis. Rhinology. 2018;56:111-7.

134. Ebbens FA, Toppila-Salmi S, de Groot EJ, et al. Predictors of post-operative response to treatment: a double blind placebo controlled study in chronic rhinosinusitis patients. Rhinology. 2011;49:413-9.

135. Rudmik L, Hopkins C, Peters A, Smith TL, Schlosser RJ, Soler ZM. Patient-reported outcome measures for adult chronic rhinosinusitis: A systematic review and quality assessment. J Allergy Clin Immunol 2015;136:1532-40.e2.

136. Hopkins C, Rudmik L, Lund VJ. The predictive value of the preoperative Sinonasal Outcome Test-22 score in patients undergoing endoscopic sinus surgery for chronic rhinosinusitis. Laryngoscope. 2015;125:1779-84

137. Kennedy JL, Hubbard MA, Huyett P, Patrie JT, Borish L, Payne SC. Sino-nasal outcome test (SNOT-22): a predictor of postsurgical improvement in patients with chronic sinusitis. Ann Allergy Asthma Immunol 2013;111:246-51 e2.

138. Rudmik L, Soler ZM, Mace JC, DeConde AS, Schlosser RJ, Smith TL. Using preoperative SNOT-22 score to inform patient decision for Endoscopic sinus surgery. Laryngoscope. 2015;125:1517-22.

139.Smith TL, Mendolia-Loffredo S, Loehrl
TA, Sparapani R, Laud PW, Nattinger AB. Predictive factors and outcomes in endoscopic sinus surgery for chronic rhinosinusitis. Laryngoscope. 2005;115:2199-205.

140.Smith TL, Litvack JR, Hwang PH, et al Determinants of outcomes of sinus surgery: a multi-institutional prospective cohort study. Otolaryngol. Head Neck Surg. 2010;142:55-63.

141.Geramas I, Terzakis D, Hatzimanolis E, Georgalas C. Social Factors in the Development of Chronic Rhinosinusitis: a Systematic Review. Current Allergy \& Asthma Reports 2018;18:7.

142. Smith TL, Schlosser RJ, Mace JC, et al. Longterm outcomes of endoscopic sinus surgery in the management of adult chronic rhinosinusitis. Int Forum Allergy Rhinol 2019;9:831-41.

143. Bogdanov V, Walliczek-Dworschak U, Whitcroft KL, Landis BN, Hummel T. Response to Glucocorticosteroids Predicts Olfactory Outcome After ESS in Chronic Rhinosinusitis. Laryngoscope. 2019, 10.1002/lary.28233.

144. Young LC, Stow NW, Zhou L, Douglas RG. Efficacy of medical therapy in treatment of chronic rhinosinusitis. Allergy Rhinol. 2012;3(1):e8-e12.

145. Hopkins C, Slack R, Lund V, Brown P, Copley $\mathrm{L}$, Browne J. Long-term outcomes from the English national comparative audit of surgery for nasal polyposis and chronic rhinosinusitis. Laryngoscope. 2009;1 19:2459-65.

146. Smith KA, Orlandi RR, Oakley G, Meeks H, Curtin K, Alt JA. Long-term revision rates for endoscopic sinus surgery. Int Forum Allergy Rhinol 2019;9:402-8.

147. Stein NR, Jafari A, DeConde AS. Revision rates and time to revision following endoscopic sinus surgery: A large database analysis. Laryngoscope. 2018;128:31-6.

148. DeConde AS, Mace JC, Levy JM, Rudmik L, Alt JA, Smith TL. Prevalence of polyp recurrence after endoscopic sinus surgery for chronic rhinosinusitis with nasal polyposis. Laryngoscope. 2017; 127:550-5.

149. McHugh T, Snidvongs K, Xie M, Banglawala S, Sommer D. High tissue eosinophilia as a marker to predict recurrence for eosinophilic chronic rhinosinusitis: a systematic review and meta-analysis. Int Forum Allergy Rhinol 2018;8:1421-9.

150. Ho J, Hamizan AW, Alvarado R, Rimmer J, Sewell WA, Harvey RJ. Systemic Predictors of Eosinophilic Chronic Rhinosinusitis. Am J Rhinol Allerfy 2018;32:252-7.

151. Brescia G, Pedruzzi B, Barion U, et al. Are neutrophil-, eosinophil-, and basophil-tolymphocyte ratios useful markers for pinpointing patients at higher risk of recurrent sinonasal polyps? American J of Otolaryngology 2016;37:339-45.

152. Tao X, Chen F, Sun Y, et al. Prediction models for postoperative uncontrolled chronic rhinosinusitis in daily practice. Laryngoscope. 2018;128:2673-80.

153.Zele TV, Holtappels G, Gevaert P, Bachert 
C. Differences in initial immunoprofiles between recurrent and nonrecurrent chronic rhinosinusitis with nasal polyps. Am J Rhinol Allergy 2014;28:192-8.

154. Mueller SK, Wendler O, Nocera A, et al. Escalation in mucus cystatin 2, pappalysin$A$, and periostin levels over time predict need for recurrent surgery in chronic rhinosinusitis with nasal polyps. Int Forum Allergy Rhinol 2019;9:1212-9.

155. Jameson JL, Longo DL. Precision medicine-personalized, problematic, and promising. N Engl J Med 2015;372:2229-34.

156. Schork NJ. Personalized medicine: Time for one-person trials. Nature 2015;520:609-11.

157. Sackett DL, Rosenberg WM, Gray JA, Haynes RB, Richardson WS. Evidence based medicine: what it is and what it isn't. BM 1996;312:71-2.

158. Fokkens WJ. Evidence-based and precision medicine two of a kind. Rhinology. 2017;55:1-2.

159. Bousquet J, Jorgensen C, Dauzat M, et al. Systems medicine approaches for the definition of complex phenotypes in chronic diseases and ageing. From concept to implementation and policies. Curr Pharm Des 2014;20:5928-44.

160. Hamburg MA, Collins FS. The path to personalized medicine. N Engl J Med 2010;363:301-4.

161. Seys SF, Bousquet J, Bachert C, et al. mySinusitisCoach: patient empowerment in chronic rhinosinusitis using mobile technology. Rhinology. 2018;56:209-15.

162. Collins FS, Varmus H. A new initiative on precision medicine. $\mathrm{N}$ Engl J Med 2015;372:793-5.
163. Canonica GW, Bachert C, Hellings P, et al. Allergen Immunotherapy (AIT): a prototype of Precision Medicine. World Allergy Organ J 2015:8:31

164. Muraro A, Fokkens WJ, Pietikainen S, et al. European Symposium on Precision Medicine in Allergy and Airways Diseases: Report of the European Union Parliament Symposium (October 14, 2015). Allergy 2016;71:583-7.

165. Hellings PW, Fokkens WJ, Bachert C, et al. Positioning the principles of precision medicine in care pathways for allergic rhinitis and chronic rhinosinusitis - A EUFOREAARIA-EPOS-AIRWAYS ICP statement. Allergy 2017;72:1297-305.

166. Paving the way for personalized medicine, FDA's role in a new era of medical product development. Internet 2013;Available at: http://www.fda.gov/downloads/ ScienceResearch.

167. Grol R. Successes and failures in the implementation of evidence-based guidelines for clinical practice. Med Care. 2001;39(8 Suppl 2):|i46-54.

168. Rycroft-Malone J, Seers K, Crichton N, Chandler J, Hawkes CA, Allen C, et al. A pragmatic cluster randomised trial evaluating three implementation interventions. Implement Sci. 2012;7:80

169. Sheldon TA, Cullum N, Dawson D, Lankshear A, Lowson K, Watt I, et al. What's the evidence that NICE guidance has been implemented? Results from a national evaluation using time series analysis, audit of patients' notes, and interviews. BMJ. 2004;329(7473):999.

170. Field, M.; Lohr, K.N. Guidelines for Clinical
Practice: From Development to Use; National Academy Press: Washington, DC, USA, 1992.

171. Gundersen L. The effect of clinical practice guidelines on variations in care. Ann Intern Med. 2000;133(4):317-8.

172. Alderman MH, Furberg CD, Kostis JB, Laragh JH, Psaty BM, Ruilope LM, et al. Hypertension guidelines: criteria that might make them more clinically useful. Am J Hypertens. 2002;15(10 Pt 1):917-23.

173.van Dulmen SA, Maas M, Staal JB, Rutten G, Kiers H, Nijhuis-van der Sanden M, et al. Effectiveness of peer assessment for implementing a Dutch physical therapy low back pain guideline: cluster randomized controlled trial. Phys Ther. 2014;94(10):1396-409.

174. Doherty SR, Jones PD. Use of an 'evidencebased implementation' strategy to implement evidence-based care of asthma into rural district hospital emergency departments. Rural Remote Health. 2006;6(1):529.

175. Grol R, Grimshaw J. From best evidence to best practice: effective implementation of change in patients' care. Lancet. 2003;362(9391):1225-30.

176. Forrest D, Hoskins A, Hussey R. Clinical guidelines and their implementation. Postgrad Med J. 1996;72(843):19-22.

177. Cabana MD, Rand CS, Powe NR, Wu AW, Wilson MH, Abboud PA, et al. Why don't physicians follow clinical practice guidelines? A framework for improvement. JAMA. 1999;282(15):1458-65

178. Fischer F, Lange K, Klose K, Greiner W, Kraemer A. Barriers and Strategies in Guideline Implementation-A Scoping Review. Healthcare (Basel). 2016;4(3) 


\section{Pharmacist perspective on rhinosinusitis}

\subsection{Differentiating rhinitis and rhinosinusitis in the community pharmacy setting}

Many patients with rhinosinusitis self-manage their condition in the community pharmacy with advice from their local pharmacist. This is particularly true if patients consider their symptoms mild and not bothersome, or that over the counter (OTC) medication is adequate ${ }^{(1,2)}$.

Pharmacists are in an excellent position to identify symptoms of rhinosinusitis and recommend appropriate treatment or referral to a physician ${ }^{(3,4)}$. According to the PGEU (Pharmaceutical Group of the European Union) Annual Report 2018 (https://pgeuannual-report.eu), 58\% of European Union (EU) citizens can reach their nearest community pharmacy within five minutes and $98 \%$ of EU citizens can reach their nearest community pharmacy within 30 minutes, making community pharmacists the most easily accessible healthcare professionals. Thus, community pharmacists can facilitate the development of effective self-management techniques that can have important health and economic ramifications.

Although there are no studies evaluating pharmacist intervention in rhinosinusitis outcomes, results from systematic reviews in other chronic respiratory diseases, namely asthma ${ }^{(5)}$ and $\operatorname{COPD}^{(6)}$, showed that pharmacists have positive impacts on the outcome, scores, severity and control of said diseases. Nevertheless, education programmes are needed to increase awareness and compliance of pharmacists with international guidelines ${ }^{(7)}$.

A cross-sectional observational study of pharmacy customers purchasing OTC medications for nasal symptoms revealed that $69.9 \%$ out of 296 participants self-manage their symptoms with OTC medications. The majority of participants (68\%) experienced allergic rhinitis symptoms, while the most frequent symptom was nasal congestion. The study indicated suboptimal therapeutic choices made by the participants, highlighting the need for pro-active interventions from community pharmacists in the form of patient therapy guidance, including referring to a physician if needed ${ }^{(8)}$. Some patients consulting the pharmacist will have had a previous diagnosis of rhinosinusitis or allergic rhinitis by a physician, others will have made an appropriate self-diagnosis, and some will have no diagnosis at all or may have an incorrect diagnosis ${ }^{(9)}$. General symptoms of several conditions (common cold, postviral ARS, allergic rhinitis, or CRS) are similar and may be confused both by the patients and the pharmacist ${ }^{(10)}$. Patient-reported symptoms and duration should be ascertained (Table 10.1.1.). The presence of nasal congestion, rhinorrhoea, facial pain or pressure and reduction of sense of smell are usually characteristic of rhinosinusitis. In children, the loss of smell component is less important, but cough might be a frequent symptom at presentation. The presence of nasal itching, rhinorrhoea, sneezing and eye symptoms is usually consistent with allergic rhinitis. Patient's relevant demographic information, medical and medication history, along with other non-pharmacological measures that the patient has tried should be taken into consideration when counselling treatment (Table 10.1.1.) $)^{(11)}$.

In some countries, pharmacists may carry out rapid diagnostic tests in order to determine whether infections are bacterial or viral in origin, or to diagnose group A streptococcal infections or influenza (http:www.sante.gouv.fr/IMG/pdf/IASS_ext-int. pdf) ${ }^{(12)}$. Access to point-of-care tests may help to reduce the dispensing of antibiotics among antibiotic-requesting subjects with respiratory tract infections ${ }^{(13)}$.

There is also a significant overlap in symptoms between primary headache disorders and chronic rhinosinusitis ${ }^{(14-16)}$, with up to $42 \%$ of patients with primary headache disorders mistakenly diagnosed with rhinosinusitis ${ }^{(16)}$. The 22 -item Sino-Nasal Outcome Test (SNOT-22) is a validated non-invasive diagnostic tool. This score can be used to aid in patient counselling as different score patterns exist for patients with CRS and headache ${ }^{(17)}$.

Specific guidelines for allergic rhinitis management at community pharmacies were first issued in $2004^{(18)}$, and have recently been updated ${ }^{(19)}$. Computerized decision support systems for rhinosinusitis similar to those proposed for allergic rhinitis might be helpful when counselling patients ${ }^{(20)}$.

\subsection{Dispensing and use of non-prescription antibiotics in URTIs}

ARS subgroups (viral ARS or common cold, post-viral ARS and ABRS) are distinguished based on symptom severity and duration. Thus, common cold / acute viral rhinosinusitis is defined by the duration of symptoms for less than 10 days, while acute post-viral rhinosinusitis is defined as an increase of symptoms after five days or persistent symptoms after 10 days with less than 12 weeks duration. Acute bacterial rhinosinusitis is linked to the presence of at least three symptoms / signs of: discoloured discharge, severe local pain (with unilateral predominance), fever $\left(>38^{\circ} \mathrm{C}\right)$, elevated ESR/CRP and 'double sickening' (i.e. a deterioration after an initial milder phase of illness) ( for details please see chapter 4.1 and 4.3.).

Antibiotics are not effective in the treatment of viral infectious diseases such as the common cold and acute postviral rhinosinusitis.

A systematic review of double-blind placebo-controlled randomized trials on antibiotics in post-viral ARS revealed no 
Table 10.1.1. Questions to assist in managing rhinosinusitis (adapted from Carter et al. 2019(11)).

\section{Questions}

Are your symptoms unilateral?

What symptoms are you experiencing?

What is your main symptom?

Do you have nasal blockage?

Is your nasal discharge clear and watery or mucopurulent?

How long have you had these symptoms?

Do you have the symptoms all the time or do they come and go?

Has a doctor ever diagnosed rhinosinusitis, hay fever or allergic rhinitis?

Are you aware of anything that seems to bring the symptoms on?

Do you have any pain in your face?

Do you have any sneezing, itchy nose, watery or itchy eyes? Have there been any hormonal changes?

Do you use OTC topical nasal decongestants?

Do you use any other medication?

INCS: intranasal corticosteroids; OTC: over the counter benefit of antibiotic prescribing in such patients (for details please see chapters 4.6.3.3. and 4.6.3.4.). Conclusively, careful selection of ABRS patients that could respond to antibiotic therapy is highlighted to avoid injudicious use of antibiotics and related side effects.

Generally, acute rhinosinusitis (ARS) is predominantly viral with a small percentage (0.5-2.0\%) progressing to secondary bacterial infection ${ }^{(21)}$. Antibiotics are effective in a group of adult patients with symptoms and signs strongly suggestive of ABRS (see above). Even though available data are limited, it seems that especially amoxicillin / penicillin (beta-lactams) are effective (for details please see chapter 4.6.3.1.). Very limited data on the effect of antibiotics in ABRS in children revealed no significant difference over placebo while showing a significantly higher percentage of adverse effects (for details please see chapter 4.6.3.2.).

Antibiotics should not be obtained without medical prescription

As for the EP3OS published in 2007, a divergence between European recommendations and antibiotic prescription practice in ARS was reported by Jorgensen and co-workers in $2013^{(22)}$. Thus, effort should be made to implement rational antibiotic prescription following the relevant guidelines in the daily practice of all healthcare providers.

The inappropriate use of antibiotics to treat upper respiratory tract infections (URTIs) is a global public health problem. It has implications regarding the cost of treatment and the development of resistance to antimicrobial agents. Most URTIs or an evidence-based indication.

\section{Observations}

Newly developed unilateral symptoms is a reason for referral

Check for nasal congestion and/or obstruction, rhinorrhoea, facial pain, reduction or loss of smell, cough

Look for the most troublesome symptom for the patient

Check if the nasal blockage is unilateral, alternating or bilateral; alternating or bilateral is usually caused by inflammation

Clear bilateral discharge suggests allergic; purulent discharge suggests rhinosinusitis

Chronic rhinosinusitis symptoms will be present for more than 3 months

Consider being outdoors, pollen seasons, contact with animals, substances handled at work or at home

Facial pain is suggestive of rhinosinusitis if combined with nasal symptoms

This is suggestive of an allergic cause

Pregnancy, menstruation, oral contraception, and hypothyroidism can give rise to rhinitis symptoms Long term use of nasal decongestants can cause nasal rebound congestions (rhinitis medicamentosa) Take special interest in beta-blockers, aspirin or NSAIDs use are viral in origin, self-limiting and resolve in the same amount of time with or without an antibiotic; that is antibiotics do not hasten recovery and do not prevent more serious illness ${ }^{(23-25)}$.

\section{Antibiotics should not be obtained without medical prescription or an evidence-based indication.}

Despite the fact that dispensing of non-prescription antibiotics is prohibited by law and legal regulatory frameworks in many countries, a large amount of antibiotics are dispensed without a prescription all over the world ${ }^{(26-36)}$. This particularly refers to developing countries where the regulation of medicine distribution itself or regulation enforcement is lacking. Community pharmacies have been reported as important sites of non-prescription supply of antibiotics ${ }^{(37-40)}$. Recently, a systematic review with meta-analysis of non-prescription supply of antibiotics in community pharmacy across the world was performed by Auta et al. (2019), taking into consideration relevant studies published from January 2000 to September $2017^{(41)}$. Studies included in the review originated from 24 countries, with 23 of them classifying antibiotics as prescriptiononly medicines. Results revealed the overall pooled proportion of the non-prescription supply of antibiotics to be $62 \%$. Globally, the extent of supply of antibiotics without prescription upon patient request and following community pharmacy staff recommendation was $78 \%$ and $58 \%$, respectively. It was highlighted that antibiotics were commonly supplied in cases of 
acute and self-limiting conditions, including upper respiratory tract infections (URTIs) for which the pooled proportion was reported to be $67 \%$. Penicillins were found to be the most frequently dispensed antibiotics for URTI indications (amoxicillin and amoxicillin-clavulanic acid, followed by macrolide antibiotic azithromycin).

The practice of antibiotic dispensing for URTIs in community pharmacies continues, especially in low- and middle-income countries.

In addition to the irrational sale of non-prescribed antibiotics upon patient request, self-medication also refers to the use of saved / shared antibiotic leftovers ${ }^{(37-39,42-45)}$ leading to a high rate of misuse and incomplete courses of antibiotics. Higher incidence of self-medication with antibiotics is found within low- and middle-income countries ${ }^{(46)}$. A population survey conducted in 2007 in 19 European countries revealed that previous experience with prescribed use of antibiotics for URTIs increased the probability for self-medication with antibiotic leftovers for similar symptoms ${ }^{(43)}$. Therefore, in case of not seeing a doctor, the patient should consult with a pharmacist about suitable therapy for URTI symptoms to avoid inappropriate antibiotic use.

Extensive use of non-prescribed antibiotics augments the risk of injudicious antibiotic therapy of viral infections, acute and self-limiting conditions, and, on the other hand, inadequate antibiotic therapy of bacterial infections, referring to inappropriate antibiotic selection, antibiotic dose and therapy duration. Such practice increases the risk of microbial resistance, particularly in the case of broad-spectrum antibiotics. At the same time, injudicious and inappropriate antibiotic use can result in masking the diagnosis of infectious disease and causing adverse effects such as allergies including anaphylactic reactions, drug interactions and increase in drug therapy costs $^{(24)}$.

Enforcing the laws prohibiting non-prescription dispensing of antibiotics is certainly needed to reduce the extent of irrational antibiotic use. However, it should be pointed out that overthe-counter availability of antibiotics could contribute to timely access to antibiotics in certain circumstances, especially when access to healthcare facilities is limited and community pharmacies present the first (or even the only) point of contact with healthcare professionals. In some developed countries like Canada, UK, New Zealand, pharmacists are authorised to prescribe and dispense antibiotics for certain defined conditions - short-term uncomplicated urinary tract or cutaneous infections - redistributing the workload of GPs ${ }^{(47)}$. Pharmacists are generally well educated in the field of rational antibiotic therapy ${ }^{(48)}$. Nonetheless, updating of pharmacists' training / knowledge in terms of antibiotic stewardship and clear assessment and treatment protocols for URTI diseases should serve as support in ensuring adequate patient counselling and treatment regimen, providing timely access and rational dispensing of antibiotics in the pharmacy setting. A recent cross-sectional survey of community pharmacy staff in Sri Lanka revealed a positive correlation between pharmacists' knowledge on antibiotics (legal aspect of antibiotic use and antibiotic resistance) and antibiotic dispensing practice ${ }^{(26)}$. Any recognition of antibiotic knowledge gaps of pharmacists might serve as a basis for elaborating the education strategies to lower inappropriate antibiotic supply and provide adequate patient counselling. Pharmacists' knowledge and attitudes towards nonprescribed antibiotic dispensing present very important points in antibiotic stewardship, particularly in URTIs which present quite frequent acute problems often related to irrational use of antibiotics ${ }^{(49)}$. In the countries where antibiotics are legally or illegally available over the counter, pharmacists are the key players in restricting antibiotic dispensing only to patients who really need them ${ }^{(49)}$.

The global respiratory infection partnership (GRIP) designed a pentagonal ('five P'; i.e. policy, patient, prevention, pharmacy and prescriber) framework for the non-antibiotic management of upper respiratory tract infections, addressing the problem related to antimicrobial resistance ${ }^{(49)}$. In the mentioned framework the antibiotic stewardship role of pharmacists as community educators on appropriate and rational antibiotic use was pointed out. Pharmacists are ideally positioned for antibiotic stewardship since they have contact with both patients and prescribers. Therefore, pharmacists can promote adherence to guidelines resulting in appropriate prescribing and optimal treatment regimens, as well as advise and educate patients on common / minor ailments and their management ${ }^{(48)}$. Engaging pharmacists to provide minor ailment services represents an innovative strategy reducing patient visits to GPs and allowing for greater overall access to primary health care $^{(50,51)}$. The pharmacist, often being the first point of call, provides full support in the symptomatic management of URTIs considering patient preferences and referring the patient to a physician if needed. Pharmacy staff should inform the patient on the common duration of the disease explaining the possibilities of symptomatic treatment while highlighting the fact that antibiotics cannot reduce the severity or duration of symptoms ${ }^{(49,52)}$. In addition to recommending the dosage regimen, pharmacists should inform the patient on possible side effects of dispensed medicine ${ }^{(28)}$, describe the measures of infection prevention and control including hand and respiratory hygiene ${ }^{(53)}$, and provide education on paths of infection transmission ${ }^{(48)}$.

In cases when pharmacists are authorised and in a position to dispense an antibiotic, apart from consultation related to the symptoms of respiratory disease and past medical and medication history, they should pose questions related to allergy and pregnancy status before antibiotic dispensing, ensuring both effectiveness and safety of antibiotic administration ${ }^{(41)}$. Information on potential drug interactions should also be provided. Whenever dispensing antibiotics, the pharmacist should instruct the patient to complete the antibiotic course promoting compliance with the dosage regimen and to dispose of any antibiotic leftover properly to reduce the chance of subsequent antibiotic misuse (i.e. selfmedication and antibiotic sharing $)^{(48)}$.

Antibiotic prescription and dispensing are influenced by a combination of factors such as the knowledge and attitude of health care providers and patient / parent expectations related to antibiotic therapy ${ }^{(54,55)}$. A decrease in antibiotic dispensing 
Figure 10.1.1. Rhinitis and rhinosinusitis assessment in the community pharmacy.

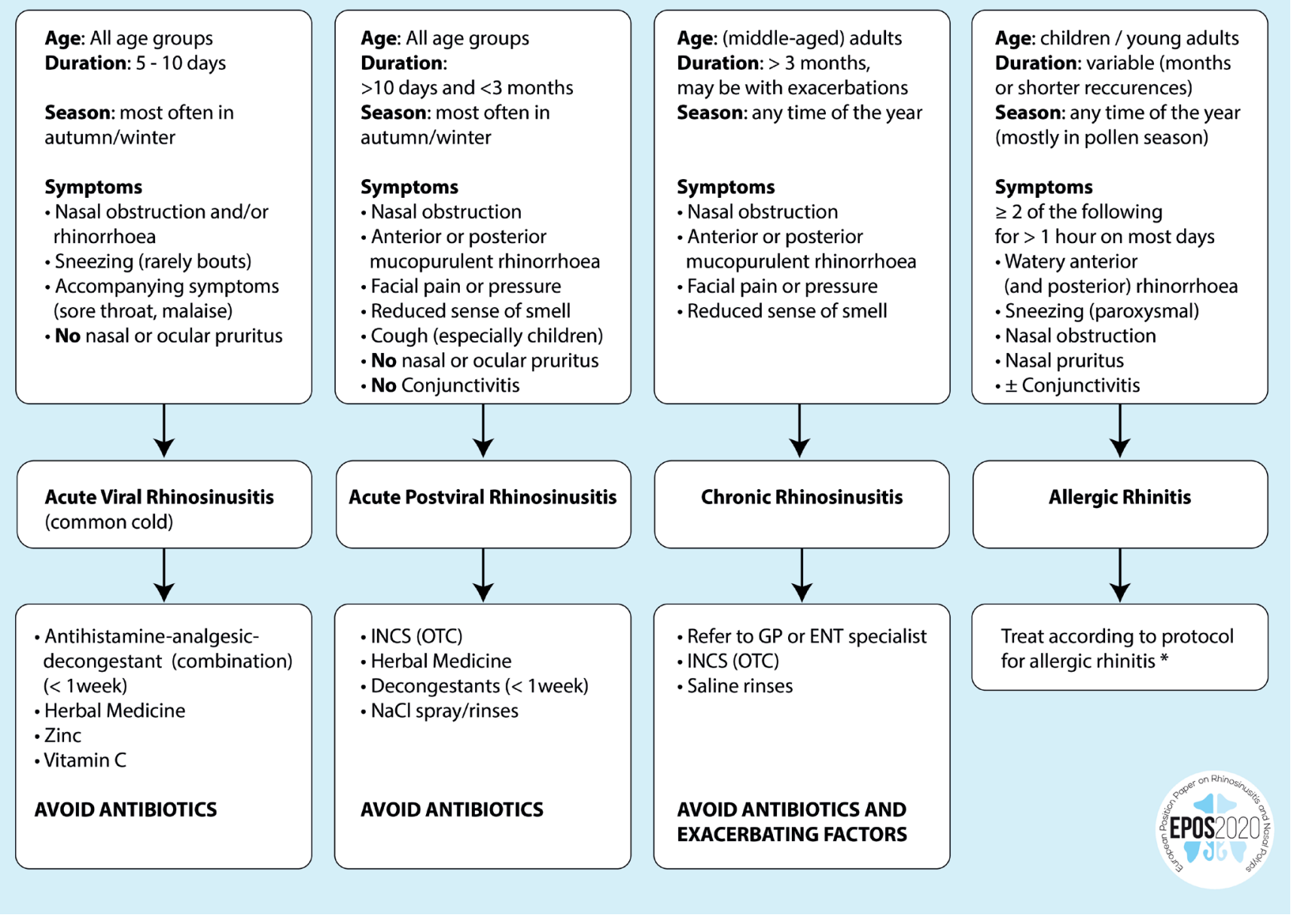

rates owing to implementation of medicine policies aimed at rationalizing antibiotic use, electronic decision support, awareness campaigns, educational interventions or multifaceted interventions have been observed in different parts of the world ${ }^{(28,56-60)}$ indicating the rationale for the continuation of such initiatives. Delayed antibiotic prescribing has also been recognised as a successful approach aimed at reducing the rate of antibiotic use for respiratory tract infections ${ }^{(61-64)}$. Pharmacists are in a position to provide full support to patients when presented with a delayed prescription. Point-of-care tests $^{(13)}$ and antibiotic shared decision-making tools ${ }^{(65)}$ have the potential to help pharmacists to reduce unnecessary antibiotic use. Nonetheless, developed communication skills are of great importance for pharmacists to raise behavioural change in patients by increasing their knowledge and changing their attitude towards antibiotic use ${ }^{(49)}$.

\subsection{Management of rhinitis and rhinosinusitis in the pharmacy}

Viral ARS (common cold) and acute postviral rhinosinusitis can be readily managed in the community pharmacy starting with symptomatic relief (Figure 10.1.1.) with the additional use of topical steroids ${ }^{(66)}$. Only one systematic review that we know of addressed the benefits of increased fluid intake during an acute respiratory infection. This review by Guppy (2011) (67) reported no evidence for or against this advice, implying the need for more studies on this subject. It is important to consider that interests, needs and priorities of professionals might not coincide with those of the patient, and different patients might require different management approaches ${ }^{(68)}$. Patient selfmanagement can be further enhanced through information provision and regular, supportive feedback. Some studies have identified patients' frustration with inadequate treatment and lack of coordinated care ${ }^{(69)}$. Effective shared decision making has shown to improve adherence and lead to better outcomes in respiratory chronic diseases, namely asthma ${ }^{(70)}$ and could also be a valuable strategy in this setting. Moreover, in integrated care centred on the patient, the community pharmacist can act as a bridge between primary (GP) and secondary care (specialist care).

\subsubsection{Over the counter (OTC) medication}

Patients with upper airway symptoms rely on self-medication and/or OTC treatment to deal with the first symptoms of ARS or during acute exacerbation of $\mathrm{CRS}^{(2)}$. When appropriate, selfmedication can be beneficial in terms of significant involvement of the patient and economic benefits ${ }^{(71)}$. However, patients 
with acute exacerbations of CRS should see their physician and should not rely on OTC medication.

OTC medications can be sold directly to patients without a medical prescription and are selected by regulatory agencies to ensure that they are safe and effective when used without a physicians' advice. However, some OTC medications may only be dispensed after an assessment of the patient by a pharmacist, and provision of patient education.

\subsubsection{Intranasal corticosteroids}

In patients with chronic rhinosinusitis symptoms, intranasal corticosteroids are the mainstay and there is a need for longterm adherence to therapy ${ }^{(72)}$. Intranasal corticosteroids may also be recommended to patients with acute post-viral rhinosinusitis if a reduction of symptoms is considered necessary. In contrast, currently, there is no evidence to support the use of intranasal corticosteroids for symptomatic relief from the common cold.

All of the available intranasal corticosteroids are efficacious in controlling symptoms, although mometasone furoate, fluticasone propionate and fluticasone furoate are generally preferred for safety reasons ${ }^{(11)}$ because they have negligible bioavailability and less potential to cause side effects.

Moreover, they only require once daily usage. As such, product differentiation involves factors such as cost, ease of dosage, OTC status, and sensory issues, such as aroma and taste, which can affect patient preferences ${ }^{(73-75)}$. Patients should be aware of the common side effects that they can expect and the serious side effects that they should report to their pharmacist and physician ${ }^{(76)}$. In most developed countries, intranasal medications containing fluticasone are classified as OTC, and can be dispensed with pharmacists' advice (for details please see chapters 4.6.2., 4.6.4. and 6.1.5.).

\subsubsection{Other OTC medication for the treatment of ARS}

The use of other OTC medical treatments such as decongestants, analgesics, antihistamines and herbal medication is commonly reported $^{(77-79)}$ and has been evaluated in chapter 4.4. (ARS). For common cold many OTC medications are available. Antihistamines, analgesics (paracetamol or NDAIDs) and decongestants alone or as combination have some general benefit in adults and older children with common cold. These benefits must be weighed against the risk of adverse effects. There is no evidence of effectiveness in young children.

\subsection{Nasal decongestants}

Nasal decongestants (vasoconstrictors) have been shown to be able to reduce symptoms of nasal blockage. However, the use of decongestants is symptomatic and does in general not influence the course of the disease; moreover they should only be used in the short term ${ }^{(80)}$.

\subsection{Zinc}

Zinc administered as zinc acetate or zinc gluconate lozenges at a dose of $>=75 \mathrm{mg} /$ day and taken within 24 hours of onset of symptoms significantly reduces the duration of common cold. For those considering using zinc it is advised to use it at this dose throughout the cold (for details please see chapter 4.6.2.16.).

\subsection{Herbal medicines (excluding Echinacea)} Some herbal medicines (excluding Echinacea) like BNO1016 (Sinupret) (both common cold and postviral ARS), Cineole (common cold), Andrographis paniculata SHA-10 extract (common cold), Myrtol (post-viral ARS) and pelargonium extracts (post-viral ARS) have significant impact on symptoms of common cold and/or post-viral ARS without important adverse events (for details please see chapter 4.6.2.17.).

\subsection{Echinacea}

Echinacea plant preparations are widely used for the prevention and treatment of common colds. However, a 2014 Cochrane systematic review by Karsch-Völk ${ }^{(81)}$ concluded after analysing 24 double-blind trials with 4631 participants, that most Echinacea products are not effective. Some Echinacea products had some possible weak benefit, however the potential effects were of questionable clinical relevance (for details please see chapter 4.6.2.15.)

\subsection{Vitamin C}

Given the consistent effect of vitamin C on the duration and severity of colds in regular supplementation studies, and the low cost and safety, it may be worthwhile for common cold patients to test on an individual basis whether therapeutic vitamin $C$ is beneficial for them ${ }^{(82)}$ (for details please see chapter 4.6.2.12.).

\subsection{Homeopathic products}

Homoeopathic products have been used for the treatment of acute respiratory tract infections in children and adults. A systematic review by Hawke aimed at assessing the effectiveness and safety of oral homoeopathic medicinal products compared with placebo or conventional therapy to prevent and treat acute respiratory infections in children ${ }^{(83)}$. After analysis of eight RCTs involving 1562 children, the authors reported no significant benefit of homoeopathic products compared to placebo on infection recurrence or cure rates in children ${ }^{(83)}$. No similar study was found for the use of homoeopathic products in adults with respiratory tract infections. We found one study evaluating the effect of homeopathy (Sinfrontal) in acute post-viral rhinosinusitis showing a significant reduction of symptoms and radiographic improvement versus placebo (for details see chapter 4.6.19).

\subsection{Medication with no proven benefits in the treatment of ARS}

Some medication has been shown to be ineffective (e.g. homeopathy, steam inhalation or antibiotics) and sometimes even harmful. Examples are the use of mucolytics (not effective and even harmful in young children) and antibiotics in almost all forms of ARS (not effective and harmful, not only in producing side effects in the individual patient but also pushing antimicrobial resistance).

OTC treatments for the common cold and rhinosinusitis symptoms (analgesic associations, decongestants, 
antihistamines, expectorants and cough suppressants) have not been adequately studied in children, especially children under six years old, and should not be routinely counselled without physician indication ${ }^{(84-88)}$.

\subsubsection{OTC medication for the prevention of ARS}

Some OTC medication and measures have been shown to reduce the incidence of ARS.

The effectiveness and safety of probiotics in the prevention of acute respiratory infections were assessed in a systematic review by Hao in 2015. The authors report that overall probiotics are better than placebo in preventing acute infections ${ }^{(89)}$ however, the quality of the evidence was low. Moreover, one must bear in mind that different strains of probiotics are available and may be administered using different pharmaceutical preparations (for details please see chapter 4.6.2.11).

Regular, moderate-intensity exercise may have an effect on the prevention of the common cold.

The results of individual prophylaxis trials with Echinacea plant preparations consistently show positive (if non-significant) trends, although potential effects are of questionable clinical relevance.

There are no conclusive results to support the use of vaccines for preventing the common cold in healthy people.

Regarding prophylactic zinc supplementation, currently no firm recommendation can be made because of insufficient data.

\subsubsection{Saline irrigation}

Patients with rhinosinusitis commonly use nasal saline solutions to improve symptoms. There are several available options available at the community pharmacy: hypotonic, isotonic or hypertonic; low-volume nebulized, large-volume irrigation; etc. Nasal irrigation works by thinning and removing mucous, clearing proteins that cause inflammation, and flushing out irritants, and bacteria from the nose and sinus cavities. Irrigation with isotonic saline or Ringer's lactate is recommended, but there is insufficient data to show that a large volume is more effective than a nasal spray, or that temperature of the solution influences the outcome.

In ARS, the majority of studies did not find a difference between saline nasal treatment versus control, but one larger trial in children suggests that nasal saline irrigation has benefits for relieving ARS symptoms (for details please see chapter 4.6.2.9). In CRS, nasal saline irrigation is considered to be an important aspect of treatment by improving nasal mucosa function (for details please see chapter 6.1.11.) and should be recommended. In the case of patients with allergic rhinitis, saline irrigation may reduce patient-reported disease severity when compared to no irrigation at up to three months, but no data is available for any outcomes beyond this period ${ }^{(90)}$.

\subsubsection{Adherence}

Medication adherence is defined as active, cooperative and voluntary participation of the patient in following recommendations from a healthcare provider. Personal volition and autonomy, and perceived competence are positively related to optimal health self-management behaviours, such as medication adherence. Especially in chronic rhinosinusitis, patients may be concerned about possible side effects of medication taken long-term.

Adherence in randomized control trials is high but does not reflect the real-life situation ${ }^{(91-93)}$. In real life adherence to treatment is low, especially in mild / moderate disease where patients use "on-demand" instead of continuous treatment ${ }^{(94)}$. Non-adherence to medications is a major obstacle to the effective delivery of health care. Better adherence may reduce the burden of uncontrolled disease and improve clinical outcomes.

Community pharmacists are uniquely positioned to help mitigate the high risk of medication discontinuation and nonadherence to therapy; they improve adherence for patients starting new therapies and continuing existing regimens. In the special case of intranasal steroids, patients must be informed that full benefit may not be evident for two weeks. Patients should be encouraged to continue to use their medication and counselled on the appropriate administration technique for intranasal formulations (especially in avoiding the nasal septum). Special caution is recommended when using nasal decongestants; the pharmacist should emphasize that such medication should only be used in the short term for congestion relief, as prolonged use can lead to rhinitis medicamentosa ${ }^{(80)}$. According to the PGEU (Pharmaceutical Group of the European Union) 2017 and 2018 Annual Reports, 53\% of all responding countries indicated that they provide type 2 medication reviews, which involve a structured, private consultation between the pharmacist and the patient and focus on issues of adherence and the safe, effective and rational use of medicines. Moreover, pharmacists are involved in disease management programs and are the first providers of advice and OTC medication for winter illnesses such as colds, ENT disorders and influenza ${ }^{(7)}$. In addition to giving therapeutic and educational advice at baseline, pharmacists play a crucial role in the follow-up and monitoring of patients ${ }^{(95)}$. Structured community pharmacy services, involving patient assessment, individualized treatment recommendations and follow-up might be of interest in this setting ${ }^{(96)}$. It is important to review the recommended treatment plan at regular intervals and to assess adherence and administration techniques.

Patient information leaflets should be handed out to patients as a supplement to good communication. Leaflets should reinforce recommendations on pharmacological and nonpharmacological treatment, administration techniques and provide contacts in case of any doubts.

Many mobile phone apps and services are available to support people in taking their medications / to improve medication adherence $\mathrm{e}^{(97-100)}$. However, one meta-analysis found that the majority of them did not have many of the desirable features and were of low quality ${ }^{(97)}$. Nevertheless, mobile applications with self-monitoring and medication adherence features can assist with self-management and improve patient control over their illness ${ }^{(99,101,102) \text {. }}$

mySinusitisCoach is an app registered as a class I medical device that enables patients to monitor rhinosinusitis symptoms and their impact on lower airway symptoms, quality of sleep 
and daily life. It also provides longitudinal information on disease control and adherence to therapy for the healthcare professional ${ }^{(103)}$.

MASK is an app for patients with allergic rhinitis to track their nasal, ocular or lung symptoms and share this with their healthcare providers ${ }^{(94)}$.

\subsection{When and how to refer to a physician or to a specialist (ENT)}

Acute viral and post-viral rhinosinusitis can be treated symptomatically and in the case of post-viral rhinosinusitis with INCS as described above. Some signs and symptoms are indicative of an emergency or severe disease and require urgent referral to an ENT physician or to the emergency services (Box 1). Orbital and neurological complications are considered emergencies.

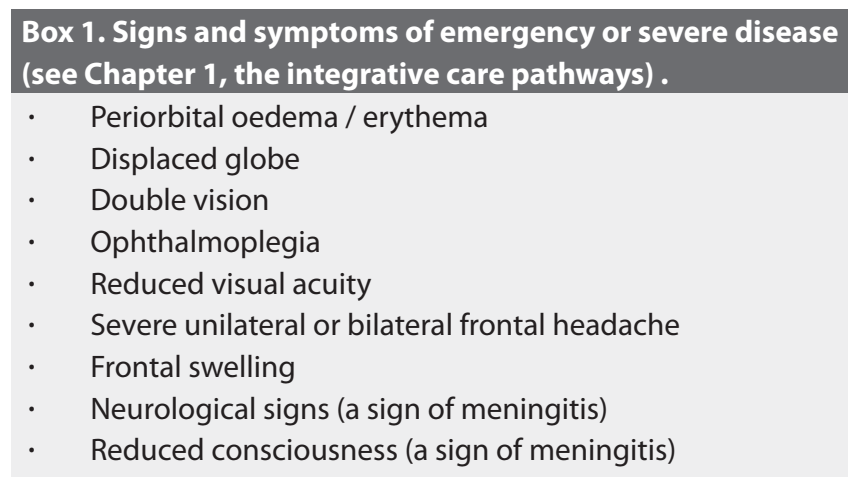

In addition, patients should be referred to a physician if the patient is pregnant, has asthma, shows signs of dyspnoea, is immunocompromised or is on any medication that may be causing the symptoms (aspirin, NSAIDs, angiotensin-converting enzyme inhibitors, alpha-blockers, beta-blockers). Patients who have persistent symptoms of rhinosinusitis despite appropriate treatment, correct technique and adherence should be of special concern $^{(11)}$.

In chronic rhin(osinus)itis referral to a physician should be considered in case of symptoms that are uncontrolled despite treatment with $\operatorname{INCS}^{(19,104)}$.

\subsection{Advice to pharmacists on explaining to patients how to use medication}

\subsubsection{Nasal sprays}

Pharmacists have a very important role in implementing efficient nasal spray use in rhinitis and rhinosinusitis patients, thus contributing to proper treatment, alleviation of symptoms and improvement of patient's quality of life. The role of pharmacists becomes even more pronounced in the era of prescription-to-OTC switches of intranasal corticosteroid sprays $^{(66,68)}$.

All nasal spray products include instructions on their proper use in patient information leaflets. Nonetheless, it is recommended that pharmacists demonstrate to patients how to use a nasal spray properly using a placebo device ${ }^{(19)}$. Namely, teaching patients the proper spray administration technique can improve drug efficacy, reduce the likelihood of side effects and enhance patient adherence / compliance $(7,105,106)$.

Instructions available in patient information leaflets related to the use of nasal sprays differ from each other in terms of recommended head position, nasal spray direction within the nostril, breathing pattern during spraying and closing the free nostril.

\section{The EPOS2020 steering group advises:}

- To prime the bottle before first use.

- To shake the bottle prior to spraying. Since most nasal corticosteroids are formulated in suspensions usually containing thixotropic agents that increase the viscosity. Bottle shaking before use decreases the viscosity of the drug suspension, allowing the creation of fine mist by spraying ${ }^{(107,108)}$, needed to achieve appropriate deposition in the nose.

- To blow the nose prior to using the spray.

- To keep the head in an upright position.

- To hold the spray in the opposite hand in relation to the nostril in which the spray should be applied ${ }^{(109,110)}$. That way the spray is aimed away from the septum reducing the possibility of epistaxis and in exceptional cases even perforation ${ }^{(106,111)}$. In a survey on patients using intranasal corticosteroid spray, it was shown that ipsilateral spray technique resulted in four times higher incidence of epistaxis and three times higher incidence of quitting intranasal steroid spray compared to the contralateral technique(106).

- To breath in gently or slowly during spraying.

- Not to close the opposite nostril to prevent pushing the septum to the side of the spray.

In cases when nasal saline irrigations are recommended, the patient should be advised to perform them prior to topical spray administration so as not to rinse out the medicine from the nose $^{(112)}$.

Special attention should be given to patients with certain physical limitations such as arthritis or Parkinson's disease, and patients unable to coordinate recommended breathing pattern with spray administration, since they may have difficulties using some nasal spray devices ${ }^{(113,114)}$. While data on nasal spray devices are lacking, a systematic review on critical inhaler errors in asthma and COPD patients revealed an increase in handling error frequency in relation to age, comorbidities, education and socioeconomic class ${ }^{(115)}$. A prospective randomised controlled trial in children aged between five and 16 years with moderate to severe intermittent or persistent allergic rhinitis showed significantly higher competency in using intranasal corticosteroid spray in children taught using an animated cartoon, in relation to oral presentation without demonstration ${ }^{(116)}$, indicating the need for special care and approach in such population. The same study suggested repeating the teaching procedure periodically to ensure the long-term correct use of the nasal spray.

In the case of nasal steroid sprays, apart from instructions on appropriate administration technique, the pharmacist should 
provide the patient with explanations related to clinical steroid effectiveness profile including delayed onset of action (12h) and maximal efficacy reached in days to weeks ${ }^{(117)}$. Patients should be instructed to use intranasal corticosteroids regularly in order to maintain symptom control and not to apply a use-as-needed approach $^{(68)}$.

\subsubsection{Nasal drops}

Nasal drops are available in multi-dose and single-dose containers. Like nasal sprays, nasal drops are dispensed with recommendations on their use provided in the patient information leaflets. Most manufacturers recommend administering drops in the head-back position obtained easily by extending the neck. However, research evidence supports administration of nasal drops in the head upsidedown position ${ }^{(118,119)}$, most easily attained by hanging off the edge of a bed (i.e. Mygind's position) ${ }^{(120)}$ or in lying on the side in a head down position (i.e. Ragan position) with drug being administered to the lower nostril ${ }^{(121,122)}$. Mygind's position was shown to enable easier administration of drops, while Ragan position was reported to be more comfortable for the patient ${ }^{(121)}$. Krinsky et al. recommend Mygind's position (i.e. lying on the bed with head tilted back and over the side of the bed), with gently tilting head from side to side, after administration of drops in the nostril ${ }^{(123)}$.

Effective drops delivery to the olfactory epithelium (e.g. for the treatment of anosmia), can be achieved by adopting the Kaiteki position, i.e. lying on one side with the head tilted downwards 20-30 and the chin turned upward $20-40^{\circ}$, with the drops being administered to the upper nostril aiming for the superior margin of the nasal septal mucosa and holding the position for 30 seconds $^{(124)}$.

Because penetration of the sinuses by nasal sprays and drops is minimal, even in patients that have been operated on ${ }^{(125,126)}$ many rhinologists now advise combining nasal drops with saline irrigation. Using a larger volume results in better penetration the sinuses and this combination has been shown to be more effective than nasal spray ${ }^{(127)}$. Whether it is more effective than nasal drops needs further investigation.

To minimize the risk of spreading infections, patients should be advised not to share nasal delivery devices ${ }^{(128)}$.

A proposed technique for the use of nasal sprays and nasal drops provided by the EPOS group is given in Table 10.5.1. However, it should be pointed out that drug deposition pattern within the nasal cavity, apart from administration technique, depends also on nasal formulation properties, the volume of the applied dose, performance of the delivery device, individual anatomical differences and pathological conditions. Therefore, it is difficult to come up with definitive conclusions and identification of a general single-best technique for nasal drug administration $^{(125,126)}$.

Table 10.5.1. EPOS2020 proposed technique for the use of nasal sprays and nasal drops.

\begin{tabular}{|c|c|c|c|c|c|c|}
\hline $\begin{array}{l}\text { Nasal } \\
\text { drug } \\
\text { products }\end{array}$ & $\begin{array}{l}\text { Before spray / } \\
\text { drops } \\
\text { administration a }\end{array}$ & Head position & $\begin{array}{l}\text { Spray / dropper tip } \\
\text { direction }\end{array}$ & Free nostril & $\begin{array}{l}\text { Spray / drops } \\
\text { administration and } \\
\text { breathing pattern } \\
\text { during spray / drops } \\
\text { administration }\end{array}$ & $\begin{array}{l}\text { After spray / drops } \\
\text { administration }\end{array}$ \\
\hline Nasal spray & $\begin{array}{l}\text { 1. Gently blow your } \\
\text { nose. } \\
\text { 2. Shake the nasal } \\
\text { spray vigorously. } \\
\text { 3. Remove the cap. }\end{array}$ & $\begin{array}{l}\text { 4. Keep your } \\
\text { head upright. }\end{array}$ & $\begin{array}{l}\text { 5. Gently insert the } \\
\text { nozzle tip into one } \\
\text { nostril. } \\
\text { 6. Aim the tip of the } \\
\text { product away from } \\
\text { the nasal septum; use } \\
\text { the right hand to spray } \\
\text { the left nostril and vice } \\
\text { versa, to direct the } \\
\text { spray away from the } \\
\text { septum. }\end{array}$ & $\begin{array}{l}\text { 7. DO NOT } \\
\text { close the } \\
\text { nostril not } \\
\text { receiving the } \\
\text { medication. }\end{array}$ & $\begin{array}{l}\text { 8. While slowly } \\
\text { breathing in activate the } \\
\text { spray } \\
\text { 9. Apply the number of } \\
\text { sprays recommended by } \\
\text { the doctor. }\end{array}$ & $\begin{array}{l}\text { 10. Take the nozzle out } \\
\text { and breathe out through } \\
\text { your mouth. } \\
\text { 11. Repeat spray } \\
\text { administration steps in } \\
\text { the other nostril. } \\
\text { 12. The spray nozzle } \\
\text { should be cleaned } \\
\text { according to the product } \\
\text { directions and the cap } \\
\text { replaced. }\end{array}$ \\
\hline $\begin{array}{l}\text { Nasal } \\
\text { drops }\end{array}$ & $\begin{array}{l}\text { 1. Gently blow your } \\
\text { nose. } \\
\text { 2. Shake the drops } \\
\text { container. }\end{array}$ & $\begin{array}{l}\text { 3. Lie down } \\
\text { in a supine } \\
\text { position with } \\
\text { the head just } \\
\text { off the bed } \\
\text { and the chin } \\
\text { being the } \\
\text { highest point } \\
\text { of the head. }\end{array}$ & - & - & $\begin{array}{l}\text { 4. While breathing } \\
\text { normally, instil the drops } \\
\text { in each nostril according } \\
\text { to the product } \\
\text { directions. }\end{array}$ & $\begin{array}{l}\text { 5. Hold the position for } \\
\text { two minutes after the } \\
\text { drop instillation }{ }^{\text {b. }} \\
\text { 6. The dropper should be } \\
\text { cleaned according to the } \\
\text { product directions. }\end{array}$ \\
\hline
\end{tabular}




\section{References}

1. Tan R, Cvetkovski B, Kritikos V, et al. Management of allergic rhinitis in the community pharmacy: identifying the reasons behind medication self-selection. Pharm Pract (Granada) 2018;16:1332.

2. Tan R, Cvetkovski B, Kritikos V, et al. The Burden of Rhinitis and the Impact of Medication Management within the Community Pharmacy Setting. J Allergy Clin Immunol Pract 2018;6:171725.

3. McKeirnan KC, Garrelts MacLean L. Pharmacist, physician, and patient opinions of pharmacist-treated minor ailments and conditions. J Am Pharm Assoc (2003) 2018;58:599-607.

4. Cvetkovski B, Tan R, Kritikos V, et al. A patient-centric analysis to identify key influences in allergic rhinitis management. NPJ Prim Care Respir Med 2018;28:34.

5. Garcia-Cardenas V, Armour C, Benrimoj SI, Martinez-Martinez F, Rotta I, Fernandez-Llimos F. Pharmacists' interventions on clinical asthma outcomes: a systematic review. Eur Respir J 2016;47:1134-43

6. Zhong $\mathrm{H}, \mathrm{Ni}$ XJ, Cui M, Liu XY. Evaluation of pharmacist care for patients with chronic obstructive pulmonary disease: a systematic review and meta-analysis. Int J Clin Pharm 2014;36:1230-40.

7. Arsoy G, Varis A, Saloumi LM, Abdi A, Basgut B. Insights on Allergic Rhinitis Management from a Northern Cyprus Perspective and Evaluation of the Impact of Pharmacist-Led Educational Intervention on Patients' Outcomes. Medicina (Kaunas) 2018;54.

8. Tan R, Cvetkovski B, Kritikos V, et al. Identifying the hidden burden of allergic rhinitis (AR) in community pharmacy: a global phenomenon. Asthma Res Pract 2017;3:8.

9. Mayrhuber EA, Peersman W, van de Kraats N, et al. "With fever it's the real flu I would say": laypersons' perception of common cold and influenza and their differences - a qualitative study in Austria, Belgium and Croatia. BMC Infect Dis 2018;18:647.

10. Topal E, Bakirtas A, Yilmaz O, et al. Predictive factors to differentiate between allergic and nonallergic rhinitis in children. Int Forum Allergy Rhinol 2014;4:447-52.

11. Carter A, Dattani N, Hannan SA. Chronic rhinosinusitis. BMJ 2019;364:1131.

12. Thornley T, Marshall G, Howard P, Wilson AP. A feasibility service evaluation of screening and treatment of group A streptococcal pharyngitis in community pharmacies. J Antimicrob Chemother 2016;71:3293-9.

13. Llor C, Bjerrum L, Munck A, et al. Access to point-of-care tests reduces the prescription of antibiotics among antibiotic-requesting subjects with respiratory tract infections. Respir Care 2014;59:1918-23.

14. Jayawardena ADL, Chandra R. Headaches and facial pain in rhinology. Am J Rhinol Allergy 2018;32:12-5.

15. Charleston Lt, Strabbing R, Cooper W. Is sinus disease the cause of my headaches? An update on sinus disease and headache. Curr Pain Headache Rep 2014;18:418.

16. Eross E, Dodick D, Eross M. The Sinus, Allergy and Migraine Study (SAMS). Headache 2007;47:21324.

17. Wu D, Gray ST, Holbrook EH, BuSaba NY, Bleier BS. SNOT-22 score patterns strongly negatively predict chronic rhinosinusitis in patients with headache. International Forum of Allergy \& Rhinology. 2019;9:9-15.

18. Workshops Mot. ARIA in the pharmacy: management of allergic rhinitis symptoms in the pharmacy. Allergic rhinitis and its impact on asthma. Allergy 2004;59:373-87.

19. Bosnic-Anticevich S, et al. ARIA pharmacy 2018 "Allergic rhinitis care pathways for community pharmacy": AIRWAYS ICPs initiative (European Innovation Partnership on Active and Healthy Ageing, DG CONNECT and DG Sante) POLLAR (Impact of Air POLLution on Asthma and Rhinitis) GARD Demonstration project. Allergy 2018;39.

20. Bertsche T, Nachbar M, Fiederling J, et al. Assessment of a computerised decision support system for allergic rhino-conjunctivitis counselling in German pharmacy. Int J Clin Pharm 2012;34:17-22

21. Fokkens WJ, Lund VJ, Mullol J, et al. European Position Paper on Rhinosinusitis and Nasal Polyps 2012. Rhinol Suppl 2012;23:3 p preceding table of contents, 1-298.

22. Jorgensen LC, Friis Christensen S, Cordoba Currea $\mathrm{G}$, Llor C, Bjerrum L. Antibiotic prescribing in patients with acute rhinosinusitis is not in agreement with European recommendations. Scand J Prim Health Care 2013;31:101-5.

23. Kenealy T, Arroll B. Antibiotics for the common cold and acute purulent rhinitis. Cochrane Database of Systematic Reviews 2013:CD000247.

24. Lemiengre $M B$, van Driel $M L$, Merenstein $D$, Liira $H$, Makela M, De Sutter AI. Antibiotics for acute rhinosinusitis in adults. Cochrane Database of Systematic Reviews 2018;9:CD006089.

25. Alves Galvao MG, Rocha Crispino Santos MA, Alves da Cunha AJ. Antibiotics for preventing suppurative complications from undifferentiated acute respiratory infections in children under five years of age. Cochrane Database Syst Rev 2016;2:CD007880.

26. Zawahir S, Lekamwasam S, Aslani P. A cross-sectional national survey of community pharmacy staff: Knowledge and antibiotic provision. PLoS One 2019;14:e0215484

27. Erku DA, Aberra SY. Non-prescribed sale of antibiotics for acute childhood diarrhea and upper respiratory tract infection in community pharmacies: a 2 phase mixed-methods study. Antimicrob Resist Infect Control 2018;7:92.

28. Markovic-Pekovic V, Grubisa N, Burger J, Bojanic L, Godman B. Initiatives to Reduce Nonprescription Sales and Dispensing of Antibiotics: Findings and Implications. J Res Pharm Pract 2017;6:120-5

29. Yaacoub SG, Lahoud NA, Francis NJ, et al. Antibiotic Prescribing Rate in Lebanese Community Pharmacies: A Nationwide Patient-Simulated
Study of Acute Bacterial Rhinosinusitis. J Epidemiol Glob Health 2019;9:44-9.

30. Roque F, Soares S, Breitenfeld L, Figueiras A, Herdeiro MT. Influence of community pharmacists attitudes on antibiotic dispensing behavior: a cross-sectional study in Portugal. Clin Ther 2015;37:168-77.

31. Zapata-Cachafeiro M, Gonzalez-Gonzalez C, Vaquez-Lago JM, et al. Determinants of antibiotic dispensing without a medical prescription: a cross-sectional study in the north of Spain. J Antimicrob Chemother 2014;69:3156-60.

32. Morgan DJ, Okeke IN, Laxminarayan R, Perencevich EN, Weisenberg S. Non-prescription antimicrobial use worldwide: A systematic review. The Lancet Infectious Diseases 2011;11:692-701.

33. Mohamed Ibrahim Ml, et al.,. Do community pharmacists in Qatar manage acute respiratory conditions rationally? A simulated client study. J of Pharmaceutical Health Services Research 2018;9:33-9.

34. Alhomoud F, Aljamea Z, Almahasnah R, Alkhalifah K, Basalelah L, Alhomoud FK. Self-medication and self-prescription with antibiotics in the Middle East-do they really happen? A systematic review of the prevalence, possible reasons, and outcomes. Int J Infect Dis 2017;57:3-12.

35. Okuyan B, Savan M, Izzettin F, Sancar M. Evaluation of rational antibiotic dispensing in the community pharmacy setting: A simulated patient study. ACTA Pharmaceutica Sciencia 2017;55:7.

36. Kibuule D, Kagoya HR, Godman B. Antibiotic use in acute respiratory infections in under-fives in Uganda: findings and implications. Expert Rev Anti Infect Ther 2016;14:863-72.

37. Berzanskyte A, Valinteliene R, Haaijer-Ruskamp FM, Gurevicius R, Grigoryan L. Self-medication with antibiotics in Lithuania. Int J Occup Med Environ Health 2006;19:246-53.

38. Skliros E, Merkouris P, Papazafiropoulou A, et al. Self-medication with antibiotics in rural population in Greece: a cross-sectional multicenter study. BMC Fam Pract 2010;11:58.

39. Tomas A, Paut Kusturica M, Tomic Z, et al. Self-medication with antibiotics in Serbian households: a case for action? Int J Clin Pharm 2017;39:507-13.

40. Arunkumar, Kumar, Vembu, Abirami. Consumption of antibiotics as self-medication from over-the-counter purchase: an empirical study arunkumar. Asian J Pharmaceutical Clin Res. 2017;10:82.

41. Auta A, Hadi MA, Oga E, et al. Global access to antibiotics without prescription in community pharmacies: A systematic review and meta-analysis. J Infect 2019;78:8-18.

42. Olczak A, Grzesiowski P, Hryniewicz W, Haaijer-Ruskamp FM. [Self-medication with antibiotics in Poland]. Pol Merkur Lekarski 2006;20:151-4.

43. Grigoryan L, Burgerhof JG, Haaijer-Ruskamp $\mathrm{FM}$, et al. Is self-medication with antibiotics in Europe driven by prescribed use? J Antimicrob Chemother 2007;59:152-6.

44. Sawair FA, Baqain ZH, Abu Karaky A, Abu Eid R. Assessment of self-medication of antibiotics in a Jordanian population. Med Princ Pract 
2009;18:21-5.

45. Zoorob R, Grigoryan L, Nash S, Trautner BW. Nonprescription Antimicrobial Use in a Primary Care Population in the United States. Antimicrob Agents Chemother 2016;60:5527-32.

46. Torres NF, Chibi B, Middleton LE, Solomon VP, Mashamba-Thompson TP. Evidence of factors influencing self-medication with antibiotics in low and middle-income countries: a systematic scoping review. Public Health 2019;168:92-101.

47. (FIP) IPF. Fighting Antimicrobial Resistance: The Contribution of Pharmacists. The Hague: International Pharmaceutical Federation 2015.

48. Essack S, Bell J, Shephard A. Community pharmacists-Leaders for antibiotic stewardship in respiratory tract infection. J Clin Pharm Ther 2018;43:302-7.

49. Essack S, Pignatari AC. A framework for the non-antibiotic management of upper respiratory tract infections: towards a global change in antibiotic resistance. Int J Clin Pract Suppl 2013, 10.1111/ijcp.12335:4-9.

50. Aly M, Garcia-Cardenas V, Williams K, Benrimoj SI A review of international pharmacy-based minor ailment services and proposed service design model. Res Social Adm Pharm 2018;14:989-98.

51. Paudyal V, Watson MC, Sach T, et al. Are pharmacy-based minor ailment schemes a substitute for other service providers? A systematic review. $\mathrm{Br} J$ Gen Pract 2013;63:e472-81.

52. Liu HH. Overuse of antimicrobial therapy for upper respiratory infections and acute bronchitis: who, why, and what can be done? Pharmacotherapy 1999;19:371-3.

53. Azor-Martinez E, Cobos-Carrascosa E, Seijas-Vazquez ML, et al. Hand Hygiene Program Decreases School Absenteeism Due to Upper Respiratory Infections. J of school health 2016;86:873-81.

54. Cho HJ, Hong SJ, Park S. Knowledge and beliefs of primary care physicians, pharmacists, and parents on antibiotic use for the pediatric common cold. Soc Sci Med 2004;58:623-9.

55. Pechere JC. Patients' interviews and misuse of antibiotics. Clin Infect Dis 2001;33 Suppl 3:S1703.

56. Holloway KA, Rosella L, Henry D. The Impact of WHO Essential Medicines Policies on Inappropriate Use of Antibiotics. PLoS One 2016;11:e0152020.

57. Wu J, Taylor D, Ovchinikova L, et al. Relationship between antimicrobial-resistance programs and antibiotic dispensing for upper respiratory tract infection: An analysis of Australian data between 2004 and 2015. J Int Med Res 2018;46:1326-38.

58. Dehn Lunn A. Reducing inappropriate antibiotic prescribing in upper respiratory tract infection in a primary care setting in Kolkata, India. BMJ Open Quality 2018;7:e000217.

59. Gulliford MC, Juszczyk D, Prevost AT, et al. Electronically delivered interventions to reduce antibiotic prescribing for respiratory infections in primary care: cluster RCT using electronic health records and cohort study. Health Technol Assess 2019;23:1-70.
60. Ranji SR, Steinman MA, Shojania KG, Gonzales R. Interventions to Reduce Unnecessary Antibiotic Prescribing: A Systematic Review and Quantitative Analysis. Medical Care 2008;46:847-62.

61. Arroll B, Kenealy T, Kerse N. Do delayed prescriptions reduce antibiotic use in respiratory tract infections? A systematic review. British J of General Practice 2003;53:871-7

62. Spurling GK, Del Mar CB, Dooley L, Foxlee R, Farley R. Delayed antibiotic prescriptions for respiratory infections. Cochrane Database of Systematic Reviews 2017;9:CD004417.

63. Arnold SR, Straus SE. Interventions to improve antibiotic prescribing practices in ambulatory care. Cochrane Database Syst Rev 2005, 10.1002/14651858.CD003539.pub2:CD003539.

64. de la Poza Abad M, Mas Dalmau G, Moreno Bakedano $M$, et al. Prescription Strategies in Acute Uncomplicated Respiratory Infections: a Randomized Clinical Trial. JAMA internal medicine 2016;176:21-9.

65. Ngadimon IW, et al. Development of a pharmacists' antibiotic shared decision-making tool for adolescents in upper respiratory tract infections. $\mathrm{J}$ of Public Health (Germany) 2019.

66. Carr WW, Yawn BP. Management of allergic rhinitis in the era of effective over-the-counter treatments. Postgrad Med 2017;129:572-80.

67. Guppy MP, Mickan SM, Del Mar CB, Thorning $S$, Rack A. Advising patients to increase fluid intake for treating acute respiratory infections. Cochrane Database Syst Rev 2011, 10.1002/14651858.CD004419.pub3:Cd004419.

68. Bridgeman MB. Overcoming barriers to intranasal corticosteroid use in patients with uncontrolled allergic rhinitis. Integr Pharm Res Pract 2017;6:109-19.

69. Ta NH, Hopkins C, Vennik J, Philpott C. Optimis ing trial outcomes and patient retention for the MACRO trial for chronic rhinosinusitis. Rhinology. 2019;57:358-66.

70. Blaiss MS, Steven GC, Bender B, Bukstein DA, Meltzer EO, Winders T. Shared decision making for the allergist. Ann Allergy Asthma Immunol 2019;122:463-70.

71. Noone J, Blanchette CM. The value of self-med ication: summary of existing evidence. J Med Econ 2018;21:201-11.

72. Karatzanis A, Chatzidakis A, Milioni A, et al. Contemporary Use of Corticosteroids in Rhinology. Curr Allergy Asthma Rep 2017;17:11.

73. Yanez A, Dimitroff A, Bremner P, et al. A patient preference study that evaluated fluticasone furoate and mometasone furoate nasal sprays for allergic rhinitis. Allergy \& rhinology (Providence, RI) 2016;7:183-92.

74. Sher ER, Ross JA. Intranasal corticosteroids: the role of patient preference and satisfaction. Allergy and asthma proceedings 2014;35:24-33.

75. Bachert C, El-Akkad T. Patient preferences and sensory comparisons of three intranasal corticosteroids for the treatment of allergic rhinitis. Annals of allergy, asthma \& immunology : official publication of the American College of Allergy, Asthma, \& Immunology 2002;89:292-7.
76. Mener DJ, Shargorodsky J, Varadhan R, Lin SY. Topical intranasal corticosteroids and growth velocity in children: a meta-analysis. International forum of allergy \& rhinology 2015;5:95-103.

77. Klimek L, Schumacher H, Schutt T, Grater H, Mueck T, Michel MC. Factors associated with efficacy of an ibuprofen/pseudoephedrine combination drug in pharmacy customers with common cold symptoms. International journal of clinical practice 2017;71.

78. Mehuys E, Gevaert P, Brusselle G, et al. Self-medication in persistent rhinitis: overuse of decongestants in half of the patients. The journal of allergy and clinical immunology In practice 2014;2:313-9.

79. Kushnir NM. The Role of Decongestants, Cromolyn, Guafenesin, Saline Washes, Capsaicin, Leukotriene Antagonists, and Other Treatments on Rhinitis. Immunology and Allergy Clinics of North America 2011;31:601-17.

80. Graf P. Rhinitis medicamentosa: a review of causes and treatment. Treatments in respiratory medicine 2005;4:21-9.

81. Karsch-Völk M, Barrett B, Kiefer D, Bauer R, Ardjomand-Woelkart K, Linde K. Echinacea for preventing and treating the common cold. Cochrane Database of Systematic Reviews 2014.

82. Hemila H, Chalker E. Vitamin C for preventing and treating the common cold. Cochrane Database of Systematic Reviews 2013:CD000980.

83. Hawke K, van Driel ML, Buffington BJ, McGuire TM, King D. Homeopathic medicinal products for preventing and treating acute respiratory tract infections in children. Cochrane Database of Systematic Reviews 2018;4:CD005974.

84. Lowry JA, Leeder JS. Over-the-Counter Medications: Update on Cough and Cold Preparations. Pediatrics in review 2015;36:286-98.

85. Yang M, So T-Y. Revisiting the safety of over-thecounter cough and cold medications in the pediatric population. Clinical pediatrics 2014;53:32630.

86. Schmiedl S, Fischer R, Ibanez L, et al. Utilisation and off-label prescriptions of respiratory drugs in children. PloS one 2014;9:e105110.

87. Rachelefsky G, Farrar JR. Are you comfortable with over-the-counter intranasal steroids for children? A call to action. The journal of allergy and clinical immunology In practice 2014;2:2714.

88. Shaikh N, Wald E. Decongestants, antihistamines and nasal irrigation for acute sinusitis in children. Cochrane Database of Systematic Reviews 2014.

89. Hao Q, Dong B, Wu T. Probiotics for preventing acute upper respiratory tract infections. Cochrane Database of Systematic Reviews 2015.

90. Head K, Snidvongs K, Glew S, et al. Saline irrigation for allergic rhinitis. Cochrane Database of Systematic Reviews 2018.

91. Ocak E, Acar B, Kocaoz D. Medical adherence to intranasal corticosteroids in adult patients Brazilian journal of otorhinolaryngology 2017;83:558-62.

92. Ocak E, Kocaoz D, Acar B. How can we improve medical adherence to intranasal corticosteroids 
in children? International journal of pediatric otorhinolaryngology 2017;100:194-7.

93. Rudmik L, Xu Y, Liu M, Bird C, Kukec E, Quan H Utilization Patterns of Topical Intranasal Steroid Therapy for Chronic Rhinosinusitis: A Canadian Population-Based Analysis. JAMA otolaryngology-- head \& neck surgery 2016;142:1056-62.

94. Menditto E, Costa E, Midao L, et al. Adherence to treatment in allergic rhinitis using mobile technology. The MASK Study. Clin Exp Allergy 2019;49:442-60.

95. Yap JSY, Tang CWQ, Hor HML, Chong JBK, Yap KZ Process Evaluation of the Community Pharmacist-Led Allergic Rhinitis Management (C-PhARM) Service in Singapore. Pharmacy (Basel, Switzerland) $2019 ; 7$

96. Nieuwlaat R, Wilczynski N, Navarro T, et al. Interventions for enhancing medication adherence. Cochrane Database of Systematic Reviews 2014.

97. Santo K, Richtering SS, Chalmers J, Thiagalingam A, Chow CK, Redfern J. Mobile Phone Apps to Improve Medication Adherence: A Systematic Stepwise Process to Identify High-Quality Apps. JMIR Mhealth Uhealth 2016;4:e132.

98. Feng S, Liang Z, Zhang R, et al. Effects of mobile phone WeChat services improve adherence to corticosteroid nasal spray treatment for chronic rhinosinusitis after functional endoscopic sinus surgery: a 3-month follow-up study. European archives of oto-rhino-laryngology 2017;274:147785.

99. Braido F, Baiardini I, Puggioni F, Garuti S, Pawankar R, Walter Canonica G. Rhinitis: adherence to treatment and new technologies. Current opinion in allergy and clinical immunology 2017;17:23-7.

100. Wang K, Wang C, Xi L, et al. A randomized controlled trial to assess adherence to allergic rhinitis treatment following a daily short message service (SMS) via the mobile phone. International archives of allergy and immunology 2014;163:51 8.

101. Zhou AH, Patel VR, Baredes S, Eloy JA, Hsueh WD. Mobile Applications for Allergic Rhinitis. The Annals of otology, rhinology, and laryngology 2018;127:836-40.

102. Thakkar J, Kurup R, Laba TL, et al. Mobile Telephone Text Messaging for Medication Adherence in Chronic Disease: A Meta-analysis. JAMA Intern Med 2016;176:340-9.

103. Seys SF, Bousquet J, Bachert C, et al. mySinusitisCoach: patient empowerment in chronic rhinosinusitis using mobile technology. Rhinology. 2018;56:209-15.

104. Brozek JL, Bousquet J, Agache I, et al. Allergic Rhinitis and its Impact on Asthma (ARIA) guidelines-2016 revision. J Allergy Clin Immunol 2017;140:950-8.

105. Bartle J. Patient education in the effective management of hay fever. Nursing standard (Royal College of Nursing (Great Britain) : 1987) 2016;30:48-53.

106. Ganesh V, Banigo A, McMurran AEL, Shakeel M, Ram B. Does intranasal steroid spray technique affect side effects and compliance? Results of a patient survey. The J of laryngology and otology 2017;131:991-6.

107. Petty DA, Blaiss MS. Intranasal corticosteroids topical characteristics: side effects, formulation and volume. American journal of rhinology \& allergy 2013;27:510-3.

108. Ciprandi G, Varricchio A. The relevance of the mometasone furoate nasal spray in clinical practice. J of Biological Regulators and Homeostatic Agents 2018;32:1051-4.

109. Benninger MS, Hadley JA, Osguthorpe JD, et al. Techniques of intranasal steroid use. Otolaryngol. Head Neck Surg. 2004;130:5-24.

110. Scadding GK, Kariyawasam HH, Scadding G, et al. $\mathrm{BSACl}$ guideline for the diagnosis and management of allergic and non-allergic rhinitis (Revised Edition 2017; First edition 2007). Clinical and Experimental Allergy 2017;47:856-89.

111. Benninger MS. Epistaxis and its relationship to handedness with use of intranasal steroid spray. Ear, Nose, \& Throat J 2008;87:463-5.

112. Stokken JK. Saline Irrigation and Topical Nasal Steroids. JAMA Otolaryngol. Head Neck Surg. 2019, 10.1001/jamaoto.2019.1713.

113. Hoyte FCL, Meltzer EO, Ostrom NK, et al. Recommendations for the pharmacologic management of allergic rhinitis. Allergy and asthma proceedings 2014;35:S20-7.

114. Bukstein D, Luskin AT, Farrar JR. The reality of adherence to rhinitis treatment: identifying and overcoming the barriers. Allergy and asthma proceedings 2011;32:265-71.

115. Usmani OS, Lavorini F, Marshall J, et al. Critical inhaler errors in asthma and COPD: a systematic review of impact on health outcomes. Respir Res 2018;19:10.

116. Indradat S, Jirapongsananuruk O, Visitsunthorn N. Evaluation of animated cartoon-aided teaching of intranasal corticosteroid administration technique among Thai children with allergic rhinitis. Asian Pacific journal of allergy and immunology 2014;32:166-70.

117. van Cauwenberge $P$, Bachert $C$, Passalacqua $G$, et al. Consensus statement on the treatment of allergic rhinitis. European Academy of Allergology and Clinical Immunology. Allergy 2000;55:11634.
118. Moren F, Bjornek K, Klint T, Wagner ZG. A comparative distribution study of two procedures for administration of nose drops. Acta Otolaryngol. 1988;106:286-90.

119. Wilson R, Sykes DA, Chan KL, Cole PJ, Mackay IS. Effect of head position on the efficacy of topical treatment of chronic mucopurulent rhinosinusitis. Thorax 1987;42:631-2.

120. Kubba H. How uncomfortable are the various positions recommended for the instillation of nose drops? J Laryngol. Otol. 1999;113:326-8.

121. Raghavan U, Jones NS. A prospective randomized blinded cross-over trial using nasal drops in patients with nasal polyposis: an evaluation of effectiveness and comfort level of two head positions. American journal of rhinology 2006;20:397-400.

122. Karagama YG, Lancaster JL, Karkanevatos $A$ O'Sullivan G. Delivery of nasal drops to the middle meatus: which is the best head position? Rhinology. 2001;39:226-9.

123. Krinsky DL, Ferreri SP, Hemstreet B, et al. Handbook of Nonprescription Drugs: An Interactive Approach to Self-Care. Washington, DC: American Pharmacists Association, 2017.

124. Mori E, Merkonidis C, Cuevas M, Gudziol V, Matsuwaki Y, Hummel T. The administration of nasal drops in the "Kaiteki" position allows for delivery of the drug to the olfactory cleft: a pilot study in healthy subjects. Eur Arch Otorhinolaryngol 2016;273:939-43.

125. Merkus P, Ebbens FA, Muller B, Fokkens WJ. The 'best method' of topical nasal drug delivery: comparison of seven techniques. Rhinology. 2006;44:102-7.

126. Merkus P, Ebbens FA, Muller B, Fokkens WJ. Influence of anatomy and head position on intranasal drug deposition. Eur Arch Otorhinolaryngol 2006;263:827-32.

127. Harvey RJ, Snidvongs K, Kalish LH, Oakley GM, Sacks R. Corticosteroid nasal irrigations are more effective than simple sprays in a randomized double-blinded placebo-controlled trial for chronic rhinosinusitis after sinus surgery. International forum of allergy \& rhinology 2018;8:46170.

128. Meltzer EO. An overview of current pharmacotherapy in perennial rhinitis. J Allergy Clin Immunol 1995;95:1097-110.

129. Mygind N. Upper airway: structure, function and therapy. In: Moren F, Newhouse MT, and Dolovich MB (eds). Aerosols in Medicine Principles, Diagnoses and Therapy. Amsterdam: Elsevier, 1985; $1-20$ 


\section{Research needs and agenda for the next decade}

\subsection{Introduction}

Although there has been significant progress since EPOS2012, in virtually every area covered by EPOS2020, there is recognition of the need for further high quality studies. The following is a summation of these gaps in our knowledge that require further investigation (Figure 11.1). We placed the research needs in the same order as the chapters in EPOS2020 without any further prioritisation.

\subsection{Classification and definition}

- International agreement on terminology

- There remains the need for clear and widely accepted guidelines on the design of clinical trials which indicate:

- How to define the study population;

- Choice of outcome measurements;

- Choice of instruments to evaluate quality of life (QoL).

- It may also be advantageous to introduce some form of additional aetiological qualification to our classification systems which might be based on International Classification of Diseases (ICD) coding.

\subsection{Burden of acute rhinosinusitis and chronic rhinosinusitis}

- Further studies to assess the impact of acute rhinosinusitis (ARS) on quality of life.

- As most data thus far is produced from the USA, further studies on direct and indirect costs across Europe and globally.

- Create awareness for the government and industry for nonpolyp disease.

- Carefully constructed prospective epidemiological studies with valid diagnoses of ARS to give a clearer picture of the real burden of ARS, in particular the prevalence of acute post-viral and bacterial rhinosinusitis.

- To develop a validated disease-specific QoL questionnaire specific to acute rhinosinusitis.

- Development of patient-reported outcome measure (PROMS) that capture all aspects of CRS not presently covered by established instruments.

- Real life studies evaluating and validating cut off levels for visual analogue scale (VAS) or other measurements of control.

- Studies looking at cost-effectiveness and the socioeconomic impact of implementation of phenotyping and endotyping into care pathways.

\subsection{Acute rhinosinusitis including common cold - and recurrent ARS in adults and children}

\subsubsection{Epidemiology of acute rhinosinusitis}

- To know what factors determine whether ARS patients in the community consult with a doctor, pharmacist or selfmanage without professional support.

- To demonstrate the prevalence of ARS in low, middle and high income countries and consider whether any predisposing factors differ dependant on income.

- Large population studies characterizing co-morbidities in patients with ARS, compared to matched controls to identify significant co-morbidities or risk factors.

\subsubsection{Pathophysiology of ARS}

- Studies to establish how allergic rhinitis increases the predisposition for rhinosinusitis and specifically if it increases the likelihood of S. pneumoniae sinus infection.

- Assuming this is confirmed, studies to establish whether regular antihistamines and/or leukotriene receptor antagonists are effective in reducing ARS episodes in patients with allergic rhinitis.

- The role of gastro-oesophageal reflux disease (GORD) in the pathogenesis of ARS.

- To determine how exposure to cigarette smoke increases the predisposition for ARS, to establish whether exposure to cigarette smoke (active or passive) augments the predisposition for ARS in patients with allergic rhinitis and to show whether smoking cessation improves the frequency of ARS compared to active smokers.

\subsubsection{Diagnosis and differential diagnosis}

- To confirm if there are combinations of symptoms and signs that predict acute bacterial rhinosinusitis in primary and secondary care.

- To show if the relative frequency of different symptoms and signs in ARS predict a differential response to different therapies, such as topical steroids and antibiotics?

- A trial to show if the purulence of nasal discharge is truly an indicator of bacterial infection and can be used as a clinically important response to antibiotics in chronic rhinosinusitis (CRS) and what constitutes a clinically important response to antibiotics in ARS, e.g. change in purulence of nasal discharge.

- To determine biomarkers (e.g. c-reactive protein(CRP), procalcitonoin) that can predict acute bacterial rhinosinusitis or a clinically important response to antibiotics in ARS? 


\subsubsection{Medical management of ARS}

- To confirm whether topical nasal corticosteroids can be the first-line treatment for ARS in primary care and consider whether there are clinically important differences between different topical nasal corticosteroid molecules and dosing regimes.

- Further RCTs in acute bacterial rhinosinusitis on the full range of medications and in particular, topical and oral corticosteroids, antihistamines, decongestants, saline and steam inhalations.

- Large RCTs on the effect of antibiotics on the cure/ improvement of symptoms in ABRS in children and consideration of why there is an apparent difference between the response children and adults.

- To show whether the provision of educational and information materials for patients improve outcomes of ARS and reduce non-essential antibiotic use?

- To demonstrate whether professional education and efficient dissemination of evidence-based guidelines to clinicians improve outcomes of ARS and reduce nonessential antibiotic use?

- Further randomised controlled trials (RCTs) in acute viral rhinosinusitis on prophylactic medications including Vitamin C, vaccines, probiotics, Echinacea and zinc.

- Maxillary puncture for acute rhinosinusitis is currently only being performed in a limited number of countries because there is no evidence to prove that it is effective. Clinicians from those countries should therefore be encouraged to set up studies to evaluate whether puncture reduces pain in the acute phase.

- To show if the clinical and economic outcomes of ARS differ depending on which health professionals (e.g. rhinologists, ENT specialists, GPs, pharmacists?) manage patients.

- Large epidemiological data collection on the true incidence of complications in ARS, determining the role of primary care physicians in the detection and/or prevention of complications and whether complications of ARS relate to access to medical care?

- To establish if early use of therapies in viral URTI prevent bacterial ARS, particularly in those with recurrent ARS or at risk of complications

- A large prospective study on the role of antibiotics in the prevention of acute complications.

- A randomised trial of drainage versus intravenous antibiotics for small abscesses in young children (orbital and intracranial).

- Conduct multicentre trials on endoscopic versus open management of complications of ABRS, both intracranial and orbital.

\subsection{Epidemiology, predisposing factors, pathophysiology, and diagnosis of CRS}

\subsubsection{Epidemiology}

- Studies evaluating the natural history of untreated CRS and the impact of age on disease.

- Epidemiologic studies on the disease process [chronic rhinosinusitis without / with nasal polyps (CRSs/wNP), asthma, N-ERD], prevalence and disposing factors and its impact across the world as there are many areas without much data egMiddle East, Africa, South Americaand compare low, middle and high income countries.

- Create a network with institutions around the world to establish good epidemiologic data and create a unified survey that can be applied globally to collect epidemiologic data on impact of CRS.

- To establish what, if any, childhood events increase chances of developing CRSwNP.

- To establish how smoking increases the risk of CRS and whether the risk is reduced by smoking cessation.

- To show if recognition of and appropriate treatment of allergic rhinitis reduce the incidence of CRSsNP and CRSwNP?

- To consider the role of GORD in CRS.

- To investigate the role of pollution and climate change on disease process of CRS.

- To investigate nutrition effects, including obesity, on CRS.

\subsubsection{Genetics and epigenetics of CRSsNP and CRSwNP}

- Role of genetics using improved technology.

- Understand the environmental factors that alter gene expression which may predispose to CRS which may allow us to recognize disease-causing agents versus diseasemodifiers or exacerbating agents and in turn may allow us to alter behaviour or implement therapies that can counteract any genetic predispositions and reverse/ moderate epigenetic pre-disposition.

- Understand epigenetic regulation of upper airway disease

\subsubsection{Inflammatory mechanisms of CRS}

- Develop a classification of CRS of phenotypes / endotypes based on "hypothesis-free" cluster analyses.

- Understand the regulation of TGF- $ß$ and related molecules in remodelling processes.

- Understand the T regulatory cell deficit and the role of T effector cells in nasal polyp disease.

- Understand the role of dendritic cells in CRS.

- To increase our understanding of the role of ILC and epithelial barrier

- Understand the links between inflammation and remodelling.

- Understand the impact of the microbiome on inflammation. Further research is needed on the impact of bacterial, fungal or other microbial colonization / infection, with clear definition of such impact and we need some standardized methodology for research. For example should measures of minimal undetectable colonization, like PCR, or molecular cultivating techniques or hardly detectable immune response to colonizer be taken into account and if so, when?

- If infection is characterized by invasion, as well as by immune response to the micro-organism, we need to define how this invasion is established at both a local and systemic level.

- There are variations in local anatomic immune response 
GENERAL

Classification and definition

- International agreement

on terminology

- How do we measure

success and failure?
PRECISION MEDICINE

Prevention and prediction

- Can treatment prevent development of disease?

- Can reaction to treatment be predicted?

Patient participation
- Self-management
- Compliance
- Empowerment

- Self-management

- Empowerment

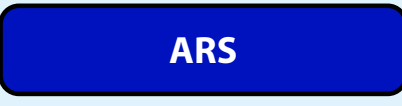

Burden of ARS

- QoL in ARS

- Direct and indirect costs

\section{Management of ARS}

- Predictors for need of antibiotics

- (Early) warning signs of

serious disease

- Treatment of acute

postviral rhinosinusitis

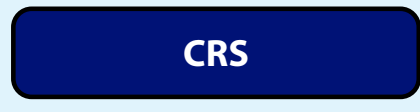

Burden of CRS

- Direct and indirect costs

\section{Management of CRS}

- Better, large RCTs

- Real-life studies combining

surgery + medical treatment

- Place of biologicals

- Treatment of CRSsNP

- Management of

uncontrolled disease

- Impact of extent of surgery

-Which endotypes have

management implications?

\section{Paediatric CRS}

- RCTs for INCS and

other treatments

- Surgery versus natural

course of the disease that are not related to airflow and environmental exposures. Research is needed into variations in immune response of the ethmoid / middle meatus for example, as this is different from the mucosal response of the septum or inferior turbinate.

- There is thus a need for innovative experimental models in CRS. Nearly all of the currently conducted human research is performed in patients who already have established disease or controls who do not. While this is useful in identifying unique contributors to the pathophysiology of CRS and subsequent treatments, it does not identify the actual cause of the disease. Currently available animal models are either allergic models or genetically manipulated animals that artificially generate an inflammatory response and again, do not answer the cause of the disease.

- Differences between CRSwNP in Western patients and elsewhere in the world. What are the key cytokines which mediate Th2 skewing across the epithelial barrier: TSLP vs. IL25 vs. IL-33 vs.? Identification of the key effector cell(s): mast cells vs. esoinophils vs. neutrophils.

- For endotyping and phenotyping, to define the minimal criteria for measuring sinus inflammation, e.g. sampling procedures and expression of data should be unified (ng of cytokine per $\mathrm{ml}$, $\mathrm{mg}$ of tissue or protein content) so that a meta-analysis may be done.

- A long-term study on the natural history of osteitis.

- The role of viruses and microbial dysbiosis in the pathogenesis of acute exacerbations of CRS (AECRS).

- Understand the pathogenesis of 'allergic' fungal rhinosinusitis and N-ERD.

\subsubsection{Diagnosis and differential diagnosis}

- To determine the relative frequency and prognostic significance of different symptoms and signs in CRSsNP and CRSwNP in primary care.

- The correlation of CRS (severity, etc.) and mental health, either directly or indirectly.

- Determine when a mucus sample indicates sinusitis and then determine the criteria to lead to subtypes of CRS and personalized medicine.

- Inclusion criteria for non-ENT control groups.

- To investigate the impact of psychological problems such as depression, stress exposure and anxiety on subjective severity scores and to consider the impact of neurological co-morbidities like chronic fatigue, post-traumatic stress disorder, neurological hyposmia, and measures of other neural-based disorders that play a role in non-allergic rhinitis, which may have an impact on rhinosinusitis scores. 
- To consider neural aspects of facial pain, headache, smell disorders and hypersecretion and investigate better tools for the diagnosis and differential diagnosis of facial pain.

- Comparative studies evaluating symptom prevalence and severity to distinguish CRS, allergic rhinitis and non-allergic rhinitis.

- Studies on sinus computed tomography (CT) to determine which changes eg degree and pattern of mucosal thickening, number of sinuses affected and the status of the ostiomeatal complex are clinically relevant in CRS.

- Development of an objective smell test to assess the presence of olfactory distortions.

- Development of a reliable commercially available test of retronasal olfaction.

- Studies on tissue eosinophilia to determine an internationally agreed value for number of eosinophils / high powered field.

- Studies on specific histopathological and serological markers of inflammation to determine their role as indicators of prognosis or targets for tailored therapy.

- Studies on the role and methodology of microbial sampling in CRS.

\subsection{Management of chronic rhinosinusitis in adults}

- Demonstrate whether the relative frequency of different symptoms and signs in CRSwNP and CRSsNP predict a differential response to different therapies, such as topical steroids and antibiotics.

- Studies to determine the optimum biomarker before we initiate treatment (surgery, steroid or biologic).

- Investigate management recommendations based upon lifestyle factors.

- Improve professional education and efficient dissemination of evidence-based guidelines to optimise outcomes and reduce referral rates to secondary care.

- Conduct multicentre RCTs on surgery versus no treatment for patients with CRS to establish the natural course of disease in the different phenotypes.

- A randomised trial comparing different treatment options for patients with CRS with significant osteitis.

- Test the criteria for concept of control from a data standpoint.

\subsubsection{Medical}

- Develop therapeutic approaches based on endotypes of disease

- Studies regarding what constitutes an adequate trial of medical therapy (both in terms of therapeutic classes, modes of delivery and duration), the importance of patient compliance with treatment and how to determine failure of this approach.

- Trials on medications specifically in AECRS, in particular large, high-quality trials to evaluate the use of short courses of antibiotics in acute exacerbations of CRS.

- High quality RCTs on topical antibiotics in CRS.
- RCTs comparing different delivery methods, dosage and duration of topical intranasal corticosteroids.

- Conduct an RCT on oral steroids versus surgery on the long term outcomes of CRSwNP.

- Conduct an RCT studying the effects of oral corticosteroids on olfactory function in CRSwNP.

- Investigate why there is a lack of response to corticosteroids in some cases of CRS, e.g. smell, QOL, etc.

- Larger long-term studies on efficacy and safety of steroideluting stents.

- Further studies on individual CRS subtypes to determine which patients would benefit from long-term antibiotics and more specifically macrolide antibiotics. To seek better local therapies for immunomodulation.

- Studies evaluating the effect of antihistamines in patients with CRSsNP.

- Studies evaluating the effect of montelukast in CRSwNP patients that failed nasal corticosteroids.

- Studies on saline irrigation to consider if a large volume is more effective than a nasal spray.

- RCTs to evaluate aspirin desensitisation with topical lysine aspirin.

- RCTs to evaluate the effect of probiotics in CRS.

- Further placebo-controlled studies on local furosemide and local verapamil to determine efficacy and appropriate patient selection.

- Larger studies of longer duration on bacterial lysates with proper patient characterization and outcome evaluation.

- Establish whether aspirin desensitization or biologics has better efficacy in patients with N-ERD.

- Studies with larger populations and longer follow-up for all biologics

- Establish a register of patients treated with biologicals.

- Is biological treatment life long?

- Is there a shift based upon age for treatment with biologicals?

- Auto-immunity effect?

- Investigating type 1 CRS patients

- How to choose between different biological treatments.

- How to choose between or combine surgery and biologicals.

\subsubsection{Surgical}

- Studies to agree definitions of surgical procedures.

- Characterisation of CRS endotypes likely to respond best to endoscopic sinus surgery (ESS).

- Studies to show if any pre-operative medication improves surgical field and specifically the role of preoperative antibiotics and oral steroids.

- Studies to show best local anaesthesia and vasocontriction regime to improve surgical field and reduce blood loss.

- Studies/Delphi consensus to consider standardisation of indications for surgery.

- Comparative RCTs of medical versus surgical treatment.

- Further studies on impact of surgery on development of asthma and sleep disturbance.

- Indications for and timing of ESS to see if it alters the course of the disease. 
- RCTs of minimal versus extensive ESS.

- Evidence from RCTs on adjunctive surgery - possibly pragmatic trials comparing different centres

- RCTs for balloon sinuplasty in non-US cohorts with higher disease burden.

- Longer term data on drug eluting stents efficacy, safety and economic benefits.

- Long-term follow up of post-operatively debrided versus non-debrided ESS cases.

- Studies on use of robotic transnasal ESS as this progresses.

- RCTs for novel interventions such as antimicrobial photodynamic therapy

- RCTs for optimal peri-operative medical treatment and use of dressings/packings.

- Pooled multi-centre data on ESS outcomes including complications, revision rates and mucocoele formation.

- Data on extensive surgery vs. limited (bad?)surgery - we have no proof that "bad" surgery equals bad outcomes.

- Evaluation on when to perform revision surgery and to define the goals and extent.

- Large-scale RCTs to answer whether postoperative shortterm antibiotics, oral and topical corticosteroids or saline irrigations after endoscopic sinus surgery are beneficial.

- Training: Studies on new technologies including augmented reality, artificial intelligence $(\mathrm{Al})$ and latest image guidance.

\subsection{Paediatric chronic rhinosinusitis}

- Population studies on prevalence of CRS in children.

- Further studies on gene mutations in paediatric CRS.

- The contribution of viral infection to development of paediatric CRS.

- The importance of allergic disease in the pathogenesis of CRS in children.

- The relationship between CRS and asthma in children.

- The role of adenoids in CRS as a reservoir for pathogenic bacteria or a source of obstruction.

- The role for inflammatory mechanisms in paediatric CRS to facilitate endotyping by better evaluating tissues obtained at the time of surgery for CRS through well organized, multicentre collaborations.

- Develop tools/tests in the context of clinical trials to differentiate the role of chronic adenoiditis from that of chronic rhinosinusitis in children with chronic nasal complaint

- Establish the relevance of CT abnormalities in children with chronic nasal symptoms.

- Improve olfactory testing and reporting in children.

- Definitions of what constitutes a controlled response in the paediatric population.

- Trials on the use of intranasal steroids in children with CRS.

- Randomized, controlled trials to evaluate the use of short and long-term oral antibiotics in children with CRS.

- Better studies on the role and impact of treatment of GORD in children.

- Investigation of best surgical practices for CRS in children required. Prospective, randomized, multicentre trials should be conducted where the severity of disease on CT scans and the symptom questionnaire would be matched preoperatively and the following interventions would be compared: adenoidectomy alone, adenoidectomy with a washout, adenoidectomy with a washout and balloon maxillary sinuplasty, and endoscopic sinus surgery. An additional arm that includes medical therapy should also be included.

\subsection{Concomitant diseases in patients with chronic rhinosinusitis}

\subsubsection{Role of allergy and chronic rhinosinusitis}

- Studies to assess the effectiveness of management of allergic rhinitis on the outcome of established CRS, or on the risk of subsequent development of CRS.

\subsubsection{Immunodeficiencies}

- Large scale studies to determine true incidence and importance of IgG subclass deficiency in general population as a predisposing factor for CRS.

\subsubsection{Lower airway disease including asthma}

- To conduct research on the basic physiology of the nose, including humidification and heat exchange and its effect on pulmonary function.

- To establish whether treatment of CRS affects outcomes of co-morbid lower airways disease [e.g. asthma, chronic obstructive pulmonary disease (COPD)].

- To undertake further RCTs studying the effects of surgery and medical treatment on the lower airways (lung function/ QoL/symptoms) in CRSwNP and concomittant asthma.

- Define predictive factors for the development of asthma in CRS patients, allowing the implementation to preventive strategies.

- Understanding the natural course of disease and mechanisms in N-ERD, with impact of upper on lower airway inflammation and vice versa.

- Further exploration of the involvement of the microbiome and the immune responses to the microbiome in relation to CRS and asthma/COPD development.

- Study the neurogenic pathway involved in CRS and asthma/COPD, with better understanding of mediators and therapeutic options for treatment of nasal and bronchial hyperreactivity.

- Study the relevance and best method for screening of upper and lower airway inflammation in CRS and asthma/COPD patients' outcomes.

- Studies highlighting the impact of personalized follow-up and patient education via e-health tools, leading to early diagnosis and better outcomes of treatment in patients with CRS and asthma/COPD.

\subsubsection{Cystic fibrosis}

- A study to consider correlation between cystic fibrosis (CF) genotype and severity of sinonasal disease. 
- A study to elucidate an association between heterozygote CF patients and CRS.

- A study to determine the best CT staging system in this condition.

- A study to consider whether extent of sinus surgery is important in CF.

\subsubsection{Primary ciliary dyskinesia}

- A study to determine the precise incidence and prevalence of primary ciliary dyskinesia (PCD).

- To establish the prevalence of ARS in the primary ciliary dyskinesia population, to determine whether aggressive treatment of ARS in patients with PCD prevents recurrence of ARS or development of CRS and to establish if aggressive treatment of ARS affects the progression of PCD-related bronchiectatic lung disease.

- Randomized controlled studies are needed to delineate the effects of surgery and adjunctive therapy.

\subsubsection{Fungal rhinosinusitis}

- The factors predisposing individuals to allergic fungal rhinosinusitis (AFRS) need further study.

- Large RCTs to consider dosage and duration of oral and topical corticosteroids in AFRS.

- An RCT to consider the role of immunotherapy, oral and topical antifungals in AFRS.

\subsubsection{Vasculitis, granulomatous diseases}

- A large prospective study considering the use of antineutrophil cytoplasmic antibodies (ANCA) in limited rhinologic cases of granulomatosis with polyangiitis (GPA) and eospinophilic granulomatosis with polyangiitis (EGPA).

- A study of the topical medications used in vasculitis.

- What is the best treatment of limited GPA?

\subsection{Patient participation, prediction, precision medicine and implementation}

- To achieve uniformity in the routine application and interpretation of the existing tools for assessment of CRS control.

- Large-scale studies to confirm the high percentage of uncontrolled patients after surgery shown by earlier reports.
- Prospective studies comparing EPOS control criteria before and after surgery with long follow-upare needed to further evaluate the responsiveness of the EPOS criteria to treatment.

- Explore differences in disease control (based on EPOS criteria) between men and women, patients undergoing primary or revision functional endoscopic sinus surgery (FESS), and between different phenotypes e.g. with or without nasal polyps, allergic rhinitis, asthma, N-ERD, and endotypes based on inflammatory patterns to assist in predicting patients at risk of having uncontrolled disease.

- Further studies are required on patient participation, compliance and self-management.

\subsection{Pharmacist perspective on rhinosinusitis}

- Determining the best way to predict acute bacterial rhinosinusitis

- To show whether the provision of educational and information materials for patients improve outcomes of ARS and reduce non-essential antibiotic use?

- To demonstrate whether professional education and efficient dissemination of evidence-based guidelines to pharmacists improve outcomes of ARS and reduce nonessential antibiotic use?

\subsection{General principles}

- Research on implementation of guidelines.

- Interdisciplinary collaboration is needed.

- Publication of negative as well as positive studies should be stimulated in order to have a better understanding of the efficacy and safety of interventions in AR and CRS.

- Quantitative research in real-life studies evaluating large number of patients should be reinforced.

- Application of health information technology for data collection, real-time analysis and feedback for clinical decision support systems and for education should be available for all stakeholders, including patients, researchers, clinicians, administrators, and politicians.

- Setup of biobanks for biomarker mining and rapid validation of novel candidates. 


\section{Methods used in EPOS2020}

\subsection{Introduction}

Patients with rhinosinusitis, acute and chronic deserve the best possible management of their disease. Our aim is for this next European Position Paper on Rhinosinusitis to promote better-informed decision-making by patients, members of the community, clinicians, and public health policy-makers on the management of rhinosinusitis.

The European Position Paper on Rhinosinusitis (EPOS 2020) is the latest in the EPOS series of guidelines on rhinosinusitis from an international cohort of experts in the field.

The first European Position Paper on Rhinosinusitis and Nasal Polyps (EP3OS) ${ }^{(1)}$ was published in 2005 , and was soon followed by $\mathrm{EP}^{3} \mathrm{OS} 2007^{(2,3)}$ in response to the first position paper's extremely positive reception and the growing body of research in this area.

The guideline development group included all relevant stakeholders including medical specialists of all relevant specialities, microbiologists, primary care physicians, pharmacists and patients were involved. A much larger group of stakeholders and experts from all over the world has been asked to review and comment the document. Their comments have been used to further improve the document.

The development strategy used in EPOS2020 has been published before we started the work ${ }^{(4)}$ and we refer here to that document for details of the methods used. We followed the AGREE II framework ${ }^{(5)}$, a tool which aims to ensure consistency in quality of clinical practice guidelines, provide a methodological strategy for the development of guidelines and clarify what and how information ought to be reported in guidelines. In summary, the EPOS 2020 guideline applies to the adult and paediatric patient population with ARS (viral / common cold, post-viral, bacterial), and all forms of CRS. The EPOS steering committee started to review whether definitions and classifications made in earlier versions of EPOS were still relevant and precise. In ARS the in EPOS2012 proposed classification into acute viral rhinosinusitis (common cold, acute post-viral rhinosinusitis and acute bacterial rhinosinusitis (ABRS)) was considered to still be relevant and now the whole of chapter 4 uses this classification to evaluate the evidence.

Based on new insights developed in the past decade on the different phenotypes and endotypes of CRS it was decided to move away from the phenotypical classification into CRSwNP and CRSsNP and come with a new classification that used anatomical aspects and endotype of disease. We expect that this new classification will be better suited to discuss treatments in the near future.

We used evidence-based medicine (EBM) methodologies to search for evidence, incorporating randomized controlled trials (RCTs) with 'Real World Experience (RWE) - observational studies (surgery) / real-life studies, and using meta-analysis of systematic reviews to combine results from clinical trials. Most surgical evidence is based on observational studies, with recent research showing some justification for incorporating nonrandomized studies ${ }^{(6,7)}$.Some parts of the document did not lend themselves to systematic analysis of the literature (e.g. the parts on pathophysiology). In those (sub)chapters the search used has been indicated. Many questions could not be answered with the existing literature. For that reason, Delphi trials were performed directed to ENT / rhinology specialists / primary practitioners to reach expert consensus on areas including (but not limited to) diagnostics, certain treatments for acute rhinosinusitis, and in CRS, appropriate medical therapy and surgery (and technique to use) in adult and paediatric rhinosinusitis. Two full new chapters focus on patient participation, prevention of disease and the role of the pharmacy in the management of the disease.

\subsection{Search methods to identify RCTs}

\subsubsection{Abstract selection}

An information specialist (J.L.) performed an electronic search in the Cochrane Central Register of Controlled Trials (CENTRAL), OVID MEDLINE and OVID EMBASE using controlled term, such as MeSH-terms, and textwords for chronic or acute rhinosinusitis including nose polyps, Kartagener syndrome, adenoid hypertrophy, common cold and URTI. In MEDLINE and EMBASE the search was combined with a methodological search filter to identify RCTs. No language, date or other restrictions were used. The records identified were downloaded in EndNote and duplicates were removed. The complete search strategies for ARS and CRS are shown at the bottom of this chapter. The search was performed 18/02/2019. For the chapters on management of the disease we decided to only evaluate RCT and systematic reviews (SR). The references (20524 on treatment and 3840 diagnostic) were imported into Rayyan (https://rayyan.qcri.org) and two independent reviewers selected papers based on the abstract. All the papers that had inconclusive selections were discussed.Additional relevant papers found in the SR and newly published in 2019 were added during the writing process. This resulted in 2214 papers of which the full texts were assessed. All SRs were analysed for additional references and 156 SR are referenced in the document. Eventually 26 SRs (on common cold (see below)) and 294 RCTs were used for treatment analyses that are mainly reported in chapters 4,6 and 7. A total of 3182 references were used in the full document, 
Studies that were included/excluded during the abstract selection that needed discussion were:

- Prevention studies of common cold in subpopulations based on age (elderly, day care centres) (included)

- Studies on behavioral changes in prescribing antibiotics (included)

- Studies on wound healing in sinus surgery (included)

- Open randomized studies (excluded)

- $\quad$ Studies in subpopulations of ARS based on other things than age (athletes, swimmers) (excluded)

- Fever not related to ARS (excluded)

- Reaction time and alertness in ARS (excluded)

- Sore throat (excluded)

\subsubsection{Full paper selection}

\subsubsection{Included studies}

In the phase of evaluating full papers we noted that the number of papers on the treatment of URTI/common cold was very large. We decided to only include SRs in our analysis on treatment of common cold. All relevant papers on diagnosis, however, are included in the review.

We only included peer-reviewed papers. Papers were retrieved from international libraries around the world. Occasionally authors were contacted to provide a paper. Papers were translated/extracted from their original language by volunteer specialists who were able to read the language and had sufficient knowledge of EBM to find the relevant data. Especially for Chinese papers, the volunteers were often asked to evaluate whether the paper was an RCT and whether the population evaluated was relevant to the question before full translation of the paper was done.

Papers that evaluated mixed patient populations were included when the relevant data for the relevant patient group (fulfilling the criteria of ARS or CRS as defined by EPOS) were reported separately. The division of ARS into viral ARS (common cold), post-viral ARS and ABRS suggested in EPOS2012 has obviously not been followed in most earlier papers. For papers evaluating ARS, papers were included when it was clear that the majority of the patients fulfilled the EPOS criteria. E.g. for post-viral ARS a study evaluating patients with symptoms for more than three days but with a table indicating that the mean symptom duration at inclusion was 12 days was included. A study where inclusion was more than three days and no indication of symptom duration was excluded. Studies indicating that $70 \%$ of the patients had symptoms for more than 10 days were included.

For ABRS, papers that used either the EPOS criteria or a combination of most of the EPOS criteria for ABRS and radiology and/or positive antral puncture were included.

\section{PRISMA 2009 Flow Diagram}
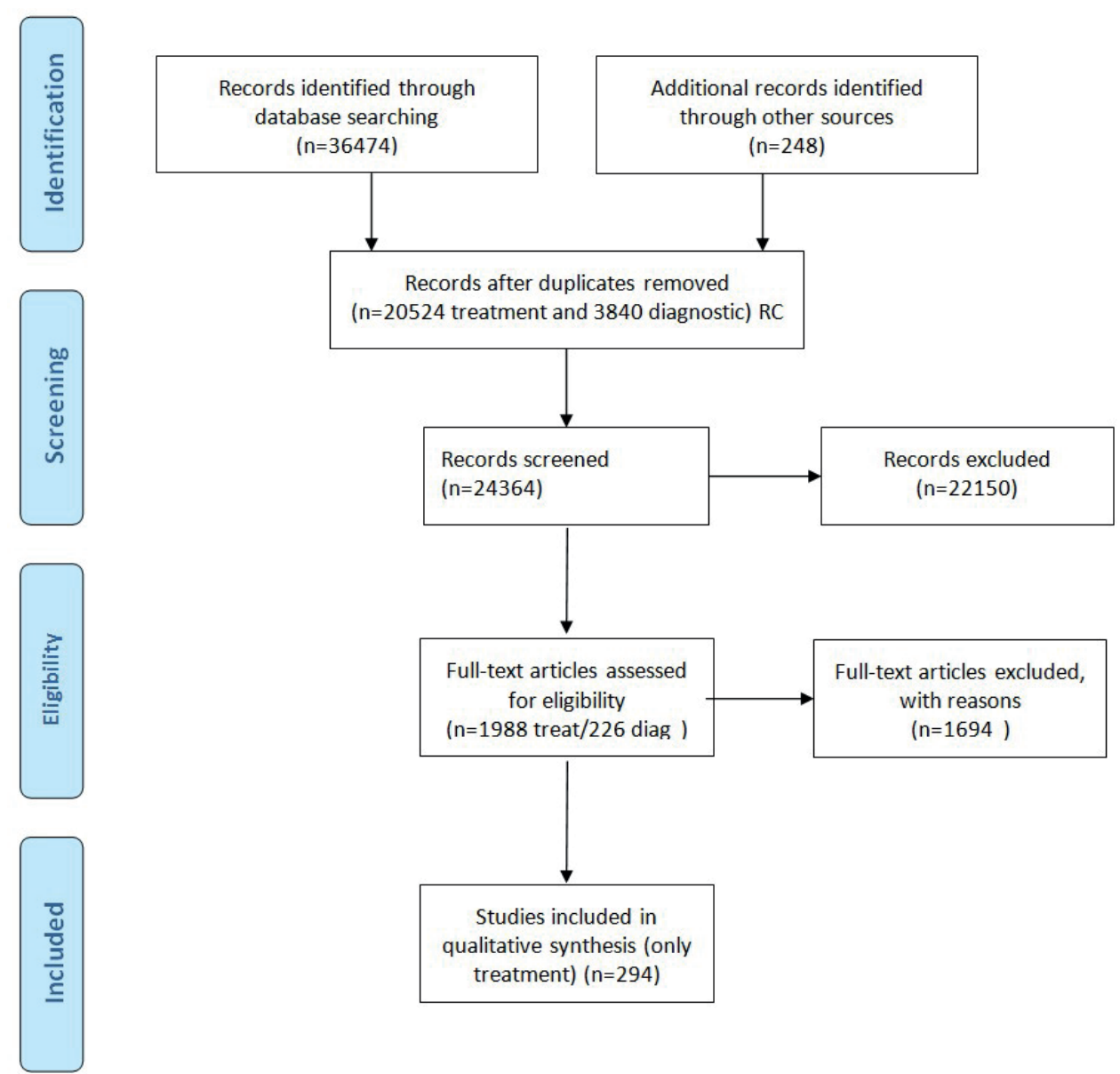
For evaluating safety of effective medication, when possible, systematic reviews on other diseases than CRS were evaluated.

\subsubsection{Excluded studies}

- Comparison of medication in ARS or CRS that was not effective.

- Comparison of antibiotics in the treatment of ARS because either antibiotics were not effective (post-viral ARS) or insufficient RCT were performed (ABRS).

- $\quad$ Evaluation of treatment of the lower airways.

- Studies evaluating safety when efficacy of the medication has not been shown.

- Studies evaluating combination treatment versus single treatment when the single treatment has not been shown to be effective.

- Studies evaluating two treatment options when no comparison with a placebo or with another treatment proven not to be more effective than placebo was made.

- Studies evaluating the treatment of side-effects of treatment (e.g. treatment of osteoporosis in patients with CRSwNP).

- Studies on treatment only evaluating non-clinical outcomes (histology).

\subsubsection{Evaluation of the evidence}

In CRS and ARS, excluding common cold, when enough RCTs with sufficient patient numbers were available, other evidence was not primarily included. We started to evaluate whether DBPCT trials were evaluable. If more than threeDBPCT were available, we did not look for other evidence unless the outcomes of the trials were either not useful or incongruent. When sufficient DBPCTs were not available, other single blind or open RCTs were used to accumulate the evidence. When no RCTs were available,occasionally other forms of evidence such as case control, cohort studies, real-life studies and Delphi rounds were used. Studies were only included when relevant between group data (e.g. mean and s.d. or non-parametric data) could be evaluated either directly from the paper or after recalculation. We accepted either post-treatment comparisons or changes from baseline. Papers only giving statistics for changes from baseline within groups (not between groups) could not be included. If needed statistics were recalculated based on the data reported in the paper, especially in situations where data compared to baseline within groups were reported but statistics of the group comparison after treatment were not reported. If sufficient RCTs were available, we did a metaanalysis. Systematic reviews and meta-analysis from others were used when no new papers appeared after the meta-analysis was done and the relevant questions were evaluated.

Evidence ${ }^{(8)}$ tables were assimilated. GRADE (Grading of Recommendations Assessment, Development and Evaluation, http://www.gradeworkinggroup.org/) was used whenever possible. Harm either for the patient directly or for the population (like costs, or resistance to antibiotics) was consistently part of the evaluation. In surgery in general RCT's were very limited and other evidence is reported. We only used the data that were available in the reported evidence. Finally, the chapters on diagnosis and treatment were combined into integrated care pathways starting at self-management and OTC treatment, via primary and secondary care to specialist management of the disease (see chapter 1).

\subsection{Delphi rounds}

Despite considerable increases in the amount of quality publications in recent years, a large number of practical clinical questions remain. It was agreed that the best way to address these was to conduct a Delphi exercise which is a structured communication technique, originally developed as a systematic, interactive forecasting method which relies on a panel of experts. The EPOS2020 group firstly prioritised the areas for consideration by several rounds of questionnaires and discussion as a result of which we have concentrated on diagnostic issues in the first instance. The final Delphi questions were circulated in several tranches and the respondents were asked to grade their answer from 1-9, where 1 was least and 9 most (essential/clinically relevant/acceptable etc) When the results were analysed, there were three possible results positive, negative or unclear as shown below depending on the percentage of answers from the whole group. The results have been integrated into the respective sections of EPOS2020. It is our intention to develop a rolling Delphi programme under the aegis of EPOS2020 and ERS to achieve expert consensus for other areas of clinical management across the full spectrum of rhinosinusitis in the future.

Positive answer $\geq 70 \%$ Grade $7-9 ; \leq 15 \%$ Grade 1-3

\section{Unclear}

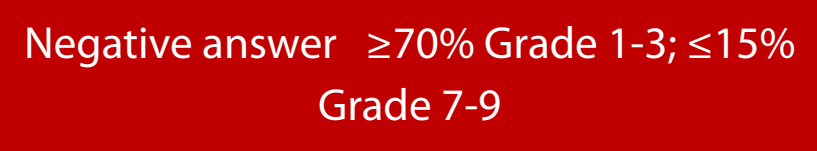

\subsection{Dissemination and future updates}

The number of relevant papers in our field is growing exponentially. This has resulted in a doubling of the number of pages of EPOS2020 compared to EPOS2012. We hope that the executive summary (chapter 1 ) will be used to disseminate the new knowledge. Including everything in all-compassing documents like EPOS2020 becomes more and more challenging. The EPOS group plans to come with yearly smaller updates on the most relevant changes and as already mentioned, a group will be formed to organize frequent Delphi rounds on relevant clinical questions that are difficult to answer with RCTs in the 
near future.

This guideline will again be disseminated in many countries over the world. The executive summary (chapter 1) has a concise format and is easy to read. A website (www.epos2020.com) has been launched and pocket guides for different stakeholder groups including patients will be prepared in the near future. On the website, education material for different stakeholders can be found.

\subsection{Editorial Independence}

This position paper has been funded by the European Rhinologic Society, Journal Rhinology and the Rhinology Foundation. No other funding has been used. All guideline development members have provided their competing interest.

\subsection{Details of search strategies used}

12.6.1. CORE search OVID MEDLINE CRS and ARS 2019-01-30 We advised all authors to use the core searches for CRS and ARS in OVID MEDLINE. We advised them to seek assistance of your local librarian to adapt the search and add the topic(s) of interest. We advised to use a similar approach as below to search for additional concepts, thus controlled terms, including MeSHterms, and textwords (tw,kf).

\subsubsection{CORE ARS-search}

Database(s): Ovid MEDLINE(R) and Epub Ahead of Print, InProcess and Other Non-Indexed Citations and Daily 1946 to January 28, 2019

Search Strategy: 2019-01-30

\begin{tabular}{|c|c|c|}
\hline$\#$ & Searches & Results \\
\hline 1 & exp sinusitis/ & 19569 \\
\hline 2 & rhinitis/ or rhinitis, atrophic/ or rhinitis, vasomotor/ & 13071 \\
\hline 3 & $\begin{array}{l}\text { exp paranasal sinuses/mi or (exp paranasal sinuses/ } \\
\text { and (respiratory tract infections/ or chronic disease/)) }\end{array}$ & 2604 \\
\hline 4 & $\begin{array}{l}\text { (sinusit* or rhinit* or ozena* or rhinosinusit* } \\
\text { or nasosinusit* or pansinusit* or ethmoidit* or } \\
\text { sphenoidit*).tw,kf. }\end{array}$ & 46748 \\
\hline 5 & $\begin{array}{l}\text { ((inflamm* or microb*) adj5 (rhino* or sinus or sinuses } \\
\text { or sinusal)).tw, kf. }\end{array}$ & 2191 \\
\hline 6 & nasal muc?us.tw,kf. & 830 \\
\hline 7 & $\begin{array}{l}\text { (rhinorr* }{ }^{*} t i, o t, k f . \text { not (ci.fs. or (CSF or ((leak } k^{*} \text { or fluid*) } \\
\text { and cerebr*) or safet*).ti,ot, kf.)) or (purulent adj3 } \\
\text { rhinorr*).tw,kf. }\end{array}$ & 586 \\
\hline 8 & or/1-7 [ rhinosinusitus both ARS and CRS] & 56505 \\
\hline 9 & common cold/ & 4131 \\
\hline 10 & ((common or head) adj cold*).tw,kf. & 3819 \\
\hline 11 & coryza.tw,kf. & 619 \\
\hline 12 & $\begin{array}{l}\text { ((upper adj4 (respiratory or airway) adj2 infection*) } \\
\text { or URTI).tw,kf. }\end{array}$ & 8937 \\
\hline 13 & $\begin{array}{l}\text { ARS.tw,kf. and (sinonas* or nasal or naso* or rhin* } \\
\text { or ENT).mp. }\end{array}$ & 166 \\
\hline 14 & or/9-13 [ ARS ] & 15102 \\
\hline 15 & $\begin{array}{l}8 \text { or } 14 \text { [ ARS (incl. umbrella terms for } \\
\text { rhinosinusitis - B) ] }\end{array}$ & 69906 \\
\hline 16 & exp animals/ not exp humans/ & 4541167 \\
\hline 17 & 15 not 16 [ ARS in humans ] & 66289 \\
\hline
\end{tabular}

Notes:

1. The core search for ARS consists of a broad rhinosinusitus search (or/1-7) and a specific search for ARS (or/9-13).

2. You may omit terms that are not relevant for the specific question.

3. If the general part is too broad for the specific topic you may narrow the general terms down by adapting this part conf. Lemiengre, MB et al (instead of or/1-7 given]

1 exp Sinusitis/

2 sinusit*.tw

3 Rhinitis/

4 rhinit*.tw.

5 rhinosinusit*.tw.

6 nasosinusit*.tw.

7 ((suppurative or purulent) adj2 (nasal discharge

or rhinitis or rhinorrhoea or rhinorrhoea)).tw.8 or/1区7

4. italic grey terms are optional (these terms did not result in additional relevant hits in the search aimed to identify SRs). i.e. additional records found with rhinitis are generally about allergic rhinitis

5. row 16 is a search filter meant to safely exclude animal studies. In case of many animal studies, you may want to use a broader filter instead

(exp animals/ not exp humans/) or animal.jw. or (rodent* or rabbit* or mice or mouse or murine or rat or rats or chicken* or (animal* adj3 (experiment* or model*))).ti. [jw=journal word]

\subsubsection{CORE CRS-search}

Database(s): Ovid MEDLINE(R) and Epub Ahead of Print, InProcess \& Other Non-Indexed Citations and Daily 1946 to January 28, 2019

\begin{tabular}{|c|c|c|}
\hline$\#$ & Searches & Results \\
\hline 1 & exp sinusitis/ & 19569 \\
\hline 2 & rhinitis/ or rhinitis, atrophic/ or rhinitis, vasomotor/ & 13071 \\
\hline 3 & $\begin{array}{l}\text { exp paranasal sinuses/mi or (exp paranasal sinuses/ } \\
\text { and (respiratory tract infections/or chronic disease/)) }\end{array}$ & 2604 \\
\hline 4 & $\begin{array}{l}\text { (sinusit }{ }^{*} \text { or rhinit }{ }^{*} \text { or ozena* or rhinosinusit* } \\
\text { or nasosinusit* or pansinusit* or ethmoidit }{ }^{*} \text { or } \\
\text { sphenoidit*).tw,kf. }\end{array}$ & 46748 \\
\hline 5 & $\begin{array}{l}\text { ((inflamm* or microb*) adj5 (rhino* or sinus or sinuses } \\
\text { or sinusal)).tw, kf. }\end{array}$ & 2191 \\
\hline 6 & nasal muc?us.tw,kf. & 830 \\
\hline 7 & $\begin{array}{l}\text { (rhinorr* }{ }^{*} t i, o t, k f . \text { not (ci.fs. or (CSF or ((leak }{ }^{*} \text { or fluid*) } \\
\text { and cerebr*) or safet*).ti,ot,kf.)) or (purulent adj3 } \\
\text { rhinorr* }{ }^{*} . t w, k f .\end{array}$ & 586 \\
\hline 8 & or/1-7 [ rhinosinusitus both ARS and CRS] & 56505 \\
\hline 9 & nasal polyps/ & 6141 \\
\hline 10 & $\begin{array}{l}\text { ((nose or nasal or nasi or rhino* or sinus* or } \\
\text { sinonasal) adj3 (papilloma* or polyp*)).tw,kf. }\end{array}$ & 7987 \\
\hline 11 & (rhinopolyp* or CRSwNP*).tw,kf. & 822 \\
\hline 12 & CRSSNP*.tw,kf. & 373 \\
\hline 13 & $\begin{array}{l}\text { ECRS.tw,kf. or (CRS.tw,kf. and (sinonas* or nasal or } \\
\text { naso* or rhin* or ENT).mp.) }\end{array}$ & 2846 \\
\hline 14 & $\begin{array}{l}\text { (kartagener* syndrom* or primary ciliary } \\
\text { dyskinesi*).mp. not (COPD or thoracic or pulmonary } \\
\text { disease or bronchiectas*).ti,ot. }\end{array}$ & 1939 \\
\hline 15 & adenoids/ab or (adenoids/ and hypertrophy/) & 963 \\
\hline 16 & $\begin{array}{l}\left(\left(\text { adenoid* adj2 (hypertroph* or obstruct* }{ }^{*}\right) \text { or }\right. \\
\text { adenoidit*).tw,kf. }\end{array}$ & 1056 \\
\hline 17 & or/9-16 [ CRS ] & 14635 \\
\hline 18 & $\begin{array}{l}8 \text { or } 17 \text { [ CRS (incl. umbrella terms for } \\
\text { rhinosinusitis -B)] }\end{array}$ & 64185 \\
\hline 19 & exp animals/ not exp humans/ & 4541167 \\
\hline 20 & 18 not 19 [ CRS in humans ] & 60937 \\
\hline 21 & $\begin{array}{l}\text { remove duplicates from } 20 \text { [only possible if \#20 } \\
<6000 \text { hits) }\end{array}$ & \\
\hline
\end{tabular}


Search Strategy: 30-01-2019

Notes:

1. The core search for CRS consists of a broad rhinosinusitis search (or/1-7) and a specific search for CRS (or/9-16).

2. You may omit terms that are not relevant for the specific question, i.e. if adenoids are not relevant you can discard rows 15 and 16

3. If the general part is too broad for the specific topic you may narrow the general terms down by combining these terms with terms for chronic, thus combine or/1-7 with for example (i.e. adapted from Chong et al):

exp chronic disease/ or exp recurrence/ or (chronic* or persis* or recurr* or long-last* or longlast*).tw,kf.

4. italic grey terms are optional (these terms did not result in additional relevant hits in the search aimed to identify SRs)

5. row 19 is a search filter meant to safely exclude animal studies. In case of many animal studies, you may want to use a broader filter instead

(exp animals/ not exp humans/) or animal.jw. or (rodent* or rabbit* or mice or mouse or murine or rat or rats or chicken* or (animal* adj3 (experiment* or model*))).ti. [jw=journal word]

\subsubsection{Searches for RCT on treatment and diagnostics (used} for $\mathrm{CH} 4,6$ and 7)

\section{CENTRAL Date Run: 18/02/2019 18:37:17}

\begin{tabular}{|c|c|c|}
\hline \# & Searches & Results \\
\hline 1 & [mh sinusitis] & 888 \\
\hline 2 & [mh ^rhinitis] or [mh ^"rhinitis, atrophic"] or [mh & 917 \\
\hline 3 & $\begin{array}{l}\text { [mh "paranasal sinuses"/mi] or ([mh "paranasal } \\
\text { sinuses"] and ([mh } \wedge \text { "respiratory tract } \\
\text { infections"] or [mh } \wedge " \text { chronic disease"])) }\end{array}$ & 95 \\
\hline 4 & $\begin{array}{l}\text { (rhinosinusit* or nasosinusit* or } \\
\text { ozena* or pansinusit* or ethmoidit* or } \\
\left.\text { sphenoidit }{ }^{*}\right): t i, a b, k w\end{array}$ & 1008 \\
\hline 5 & sinusit*:ti,ab,kw & 2324 \\
\hline 6 & rhinit $^{*}: t i, a b, k w$ & 8352 \\
\hline 7 & $\begin{array}{l}\text { ((inflamm* or microb*) near } / 5 \text { (rhino* or sinus or } \\
\text { sinuses or sinusal)):ti,ab,kw }\end{array}$ & 172 \\
\hline 8 & (nasal NEXT mucus):ti,ab,kw & 51 \\
\hline 9 & $\begin{array}{l}\text { (rhinorr*:ti,kw) not ([mh /Cl] or (CSF or ((leak* or } \\
\text { fluid*) and cerebr*) or safet*):ti,ab,kw) }\end{array}$ & 344 \\
\hline 10 & [mh "nasal polyps"] & 319 \\
\hline 11 & $\begin{array}{l}\text { ((nose or nasal or nasi or rhino* or sinus* or } \\
\text { sinonasal) near/3 (papilloma* or polyp*)):ti,ab,kw }\end{array}$ & 787 \\
\hline 12 & (rhinopolyp* or CRSwNP* or CRSSNP*):ti,ab,kw & 75 \\
\hline 13 & $\begin{array}{l}\text { ((kartagener* NEXT syndrom*) or (primary NEXT } \\
\text { ciliary NEXT dyskinesi*)):ti,ab,kw not (COPD } \\
\text { or thoracic or (pulmonary NEXT disease) or } \\
\text { bronchiectas*):ti }\end{array}$ & 43 \\
\hline 14 & [mh ^adenoids/AB] or ([mh ^adenoids] and [mh & 57 \\
\hline 15 & $\begin{array}{l}\text { ((adenoid** near/2 (hypertroph* or obstruct*)) or } \\
\text { adenoidit*):ti,ab,kw }\end{array}$ & 125 \\
\hline 16 & [mh ^"common cold"] & 497 \\
\hline 17 & ((common or head) NEXT cold):ti,ab,kw & 1088 \\
\hline 18 & coryza:ti,ab,kw & 36 \\
\hline 19 & 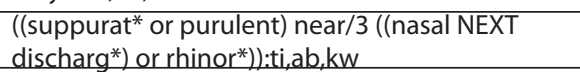 & 37 \\
\hline
\end{tabular}

\begin{tabular}{|c|c|c|}
\hline 20 & $\begin{array}{l}\text { ((upper near/4 (respiratory or airway) near } / 2 \\
\text { infection*) or URTI or (U next R next T next } \\
\text { 1):ti,ab,kw }\end{array}$ & 3814 \\
\hline 21 & $\{O R \# 1-\# 20\}$ & 15326 \\
\hline 22 & \#21 in Trials & 15166 \\
\hline $23^{*}$ & \#22 not Clinicaltrials:so & 14041 \\
\hline $24^{*}$ & \#22 and Clinicaltrials:so & 1125 \\
\hline
\end{tabular}

* do not use $=\# 23$ en \#24 when the trial registers are not kept separate.

Database(s): Ovid MEDLINE(R) and Epub Ahead of Print, InProcess \& Other Non-Indexed Citations and Daily 1946 to February 15, 2019

Search Strategy: 2019-02-18

\begin{tabular}{|c|c|c|}
\hline \# & Searches & Results \\
\hline 1 & exp sinusitis/ & 19603 \\
\hline 2 & rhinitis/ or rhinitis, atrophic/ or rhinitis, vasomotor/ & 13106 \\
\hline 3 & $\begin{array}{l}\text { exp paranasal sinuses/mi or (exp paranasal sinuses/ } \\
\text { and (respiratory tract infections/ or chronic } \\
\text { disease/)) }\end{array}$ & 2607 \\
\hline 4 & 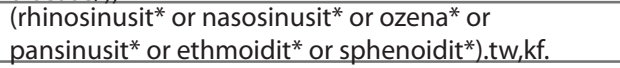 & 9023 \\
\hline 5 & sinusit*.tw,kf. & 15829 \\
\hline 6 & rhinit*.tw,kf. & 26038 \\
\hline 7 & $\begin{array}{l}\text { ((inflamm* or microb*) adj5 (rhino* or sinus or } \\
\text { sinuses or sinusal)).tw,kf. }\end{array}$ & 2180 \\
\hline 8 & nasal muc?us.tw,kf. & 828 \\
\hline 9 & $\begin{array}{l}\text { rhinorr*.ti,ot,kf. not (ci.fs. or (CSF or ((leak* or fluid*) } \\
\left.\text { and cerebr }{ }^{*} \text { ) or safet }{ }^{*}\right) . t i, \text { ot,kf.) }\end{array}$ & 474 \\
\hline 10 & nasal polyps/ & 6150 \\
\hline 11 & $\begin{array}{l}\text { ((nose or nasal or nasi or rhino* or sinus* or } \\
\text { sinonasal) adj3 (papilloma* or polyp*)).tw,kf. }\end{array}$ & 7958 \\
\hline 12 & (rhinopolyp* or CRSwNP* or CRSSNP*).tw,kf. & 869 \\
\hline 13 & $\begin{array}{l}\text { (kartagener* syndrom* or primary ciliary dyskinesi*). } \\
\text { mp. not (COPD or thoracic or pulmonary disease or } \\
\text { bronchiectas*).ti,ot. }\end{array}$ & 1932 \\
\hline 14 & adenoids/ab or (adenoids/ and hypertrophy/) & 964 \\
\hline 15 & $\begin{array}{l}\left(\left(\text { adenoid* adj2 (hypertroph* or obstruct }{ }^{*}\right)\right) \text { or } \\
\text { adenoidit*).tw,kf. }\end{array}$ & 1051 \\
\hline 16 & common cold/ & 4136 \\
\hline 17 & ((common or head) adj cold*).tw,kf. & 3808 \\
\hline 18 & coryza.tw,kf. & 617 \\
\hline 19 & $\begin{array}{l}\text { ((suppurat* or purulent) adj3 (nasal discharg* or } \\
\text { rhinor*)).tw,kf. }\end{array}$ & 279 \\
\hline 20 & $\begin{array}{l}\text { ((upper adj4 (respiratory or airway) adj2 infection*) } \\
\text { or URTI or U-R-T-I).tw,kf. }\end{array}$ & 8909 \\
\hline 21 & or/1-20 [ rhinosinusitis ] & 77127 \\
\hline 22 & $\begin{array}{l}\text { (exp animals/ not exp humans/) or animal.jw. or } \\
\text { (rodent* or rabbit* or mice or mouse or murine or } \\
\text { rat or rats or chicken* or (animal* adj3 (experiment* } \\
\text { or model*))).ti. }\end{array}$ & 4819005 \\
\hline 23 & 21 not 22 [ human rhinosinusitis ] & 72948 \\
\hline 24 & $\begin{array}{l}\text { (controlled clinical trial/ or randomized controlled } \\
\text { trial/ or random allocation/ or double-blind } \\
\text { method/ or single-blind method/ or (randomi?ed or } \\
\text { randomi?ation or placebo* or randomly or (random } \\
\text { adj3 allocat*) or ((random* or controlled) adj2 (study } \\
\text { or trial)) or ((singl* or doubl* or treb* or tripl*) adj } \\
\text { (blind*3 or mask*3))).tw,kf. or trial.ti.) not ((review or } \\
\text { editorial).pt. or review.ti.) [ RCT filter - adapted from } \\
\text { the Cochrane] }\end{array}$ & 1073418 \\
\hline 25 & 23 and 24 [ RCTs on human rhinosinusitis ] & 7258 \\
\hline
\end{tabular}


Database(s): EmbaseClassic+Embase 1947 to 2019 February 15 Search Strategy: 2019-02-18

\begin{tabular}{|c|c|c|}
\hline \# & Searches & Results \\
\hline 1 & exp rhinosinusitis/ & 9352 \\
\hline 2 & exp sinusitis/ & 43919 \\
\hline 3 & rhinitis/ or atrophic rhinitis/ or vasomotor rhinitis/ & 22552 \\
\hline 4 & $\begin{array}{l}\text { (rhinosinusit* or nasosinusit* or ozena* or } \\
\text { pansinusit* or ethmoidit* or sphenoidit*).tw,kw. }\end{array}$ & 11984 \\
\hline 5 & sinusit*.tw,kw. & 23044 \\
\hline 6 & rhinit*.tw,kw. & 40889 \\
\hline 7 & $\begin{array}{l}\text { ((inflamm* or microb*) adj5 (rhino* or sinus or } \\
\text { sinuses or sinusal)).tw,kw. }\end{array}$ & 3184 \\
\hline 8 & nasal muc?us.tw,kw. & 1394 \\
\hline 9 & $\begin{array}{l}{ }^{*}{ }^{r} \text { hinorrhea/ or rhinorr*.ti,ot,kw.) not (si.fs. or (CSF } \\
\text { or ((leak* or fluid*) and cerebr*) or safet*).ti,ot,kw.) }\end{array}$ & 893 \\
\hline 10 & chronic rhinitis/ & 1209 \\
\hline 11 & nose polyp/ & 10758 \\
\hline 12 & $\begin{array}{l}\text { ((nose or nasal or nasi or rhino* or sinus* or } \\
\text { sinonasal) adj3 (papilloma* or polyp*)).tw,kw. }\end{array}$ & 10773 \\
\hline 13 & (rhinopolyp* or CRSwNP* or CRSSNP*).tw,kw. & 1222 \\
\hline 14 & $\begin{array}{l}\text { (kartagener* syndrom* or primary ciliary } \\
\text { dyskinesi*).mp. not (COPD or thoracic or } \\
\text { pulmonary disease or bronchiectas*).ti,ot. }\end{array}$ & 2896 \\
\hline 15 & adenoid hypertrophy/ & 609 \\
\hline 16 & $\begin{array}{l}((\text { adenoid* adj2 (hypertroph* or obstruct*) }) \text { or } \\
\text { adenoidit*).tw,kw. }\end{array}$ & 1440 \\
\hline 17 & common cold/ & 9118 \\
\hline 18 & ((common or head) adj cold*).tw,kw. & 5178 \\
\hline 19 & coryza.tw,kw. & 842 \\
\hline 20 & $\begin{array}{l}\text { ((suppurat* or purulent) adj3 (nasal discharg* or } \\
\left.\text { rhinor }^{*}\right) \text { ).tw,kw. }\end{array}$ & 421 \\
\hline 21 & $\begin{array}{l}\text { ((upper adj4 (respiratory or airway) adj2 } \\
\text { infection*) or URTI or U-R-T-I).tw,kw. }\end{array}$ & 14860 \\
\hline 22 & or/1-21 [ rhinosinusitis ] & 128236 \\
\hline 23 & $\begin{array}{l}\text { ((animal/ or animal experiment/ or exp animal } \\
\text { model/ or nonhuman/ or exp female animal/) not } \\
\text { human/) or exp veterinary medicine/ or animal*. } \\
\text { jw. or (rodent* or rabbit* or mice or mouse or } \\
\text { murine or rat or rats or chicken* or (animal* adj3 } \\
(\text { (experiment* or model*))).ti. }\end{array}$ & 6618264 \\
\hline 24 & 22 not 23 [ rhinosinusitis in humans ] & 122363 \\
\hline 25 & $\begin{array}{l}\text { (exp controlled clinical trial/ or randomization/ } \\
\text { or double blind procedure/ or single blind } \\
\text { procedure/ or (randomi?ed or randomi?ation or } \\
\text { placebo* or randomly or (random adj5 (allocat* or } \\
\text { control*)) or ((random* or controlled) adj2 (study } \text { or trial)) or ((singl* or doubl* or treb* or tripl*) adj } \\
\text { (blind*3 or mask*3))).tw,kw. or trial.ti.) not (review/ } \\
\text { or editorial/ or (review or editorial).pt. or review.ti.) }\end{array}$ & 1503658 \\
\hline 26 & 24 and 25 [ RCTs on rhinosinusitis ] & 14635 \\
\hline
\end{tabular}

\subsubsection{Searches per chapter}

\subsubsection{Introduction}

Here per chapter the searches are given. Often searches were done per subchapter

\subsubsection{Classification, definitions and terminology}

\section{Control}

A literature search was performed in Pubmed using the terms: "chronic rhinosinusitis", "EPOS" and "control". Publication date was set between the third EPOS update in March 2012 and June 2019. This search resulted in 13 articles which were further analyzed. Eventually, we identified 3 studies that evaluated the burden of uncontrolled CRS using the EPOS control criteria.

\subsubsection{Burden of acute and chronic rhinosinusitis}

\subsection{Quality of life}

Main search strategy AND (impact or burden or severity),

\subsection{Costs of rhinosinusitis}

The search utilized for these references was performed in Pubmed with search terms: "sinusitis" "costs" "total" "direct" "indirect" "economic."

12.6.3.4. Acute rhinosinusitis including common cold - and recurrent ARS in adults and children

12.6.3.4.1. Epidemiology of acute rhinosinusitis (ARS) Database(s): Ovid MEDLINE(R) and Epub Ahead of Print, In-Process \& Other Non-Indexed Citations, Daily and Versions(R) 1946 to February 15, 2019 Search Strategy: February 15, 2019

\begin{tabular}{|l|l|l|}
\hline$\#$ & Searches & Results \\
\hline 1 & $\begin{array}{l}\text { (Acute rhinosinusitis or Acute sinusitis) and } \\
\text { prevalence).mp. [mp=title, abstract, original title, name } \\
\text { of substance word, subject heading word, floating } \\
\text { organism supplementary concept word, protocol } \\
\text { supplementary concept word, rare disease } \\
\text { supplementary concept word, unique identifier, } \\
\text { synonyms] }\end{array}$ & 84 \\
\hline 2 & $\begin{array}{l}\text { limit 1 to (abstracts and english language and humans } \\
\text { and yr="2011 -Current") }\end{array}$ & 34 \\
\hline
\end{tabular}

\subsection{Pathophysiology of ARS}

((()(((“"Common Cold"[Mesh]) OR "Sinusitis"[Mesh] OR "Respiratory Tract Infections"[Mesh:NoExp])) OR ((((acute[Title/ Abstract] OR viral[Title/Abstract] OR virus[Title/Abstract]))) AND ((rhinit*[Title/Abstract] OR rhinosinusit*[Title/Abstract] OR sinusit*[Title/Abstract] OR paranasal sinusit*[Title/Abstract] OR nasosinusit*[Title/Abstract])))) OR ((Acute upper respiratory tract infection*[Title/Abstract]) OR viral upper respiratory tract infection*[Title/Abstract] OR respiratory viral infection*[Title/ Abstract] OR respiratory virus infection*[Title/Abstract])) OR ()((rhinit*[Title/Abstract] OR rhinosinusit*[Title/Abstract] OR sinusit*[Title/Abstract] OR paranasal sinusit*[Title/Abstract] OR nasosinusit*[Title/Abstract]))) AND ((pathophysiology[Title/ Abstract] OR pathogenic[Title/Abstract]))))) $=>64,939$ results. Many studies on the pathophysiology of acute rhinosinusitis were published with key words including common cold, viral infection and respiratory tract infection. Therefore, our search strategy had to include these key words in different combinations. The relevant articles were then selected after screening the abstracts.

\subsection{Diagnosis and differential diagnosis of ARS in} adults and children Central search, see above. 
12.6.3.4.4.Medical management of ARS

Central search, see above.

\subsection{Complications of ARS}

No search provided.

\subsubsection{Epidemiology and predisposing factors of CRS}

\subsection{AECRS:}

((((()((((chronic[ti] OR persis*[ti]))) AND ((Acute[tiab] OR exacerbation*[tiab]))) AND (((“Sinusitis"[Mesh] OR "Rhinitis"[Mesh] AND "Chronic Disease"[Mesh]) OR rhinosinusitis[tiab] OR nasosinusitis[tiab] OR sinusitis[tiab] OR pansinusitis[tiab] OR ethmoiditis[tiab] OR ethmoiditis[tiab] OR sphenoiditis[tiab] OR (kartagener* [ti] AND syndrome* [ti])))) AND "last 10 years"[PDat] AND English[lang])) NOT ("case reports"[Publication Type] AND "last 10 years"[PDat] AND English[lang])) AND “last 10 years"[PDat] AND English[lang])) NOT ((animals[mh] NOT humans[mh]))

\subsection{Genetics and epigenetics of CRSsNP and CRSwNP} OVID search history

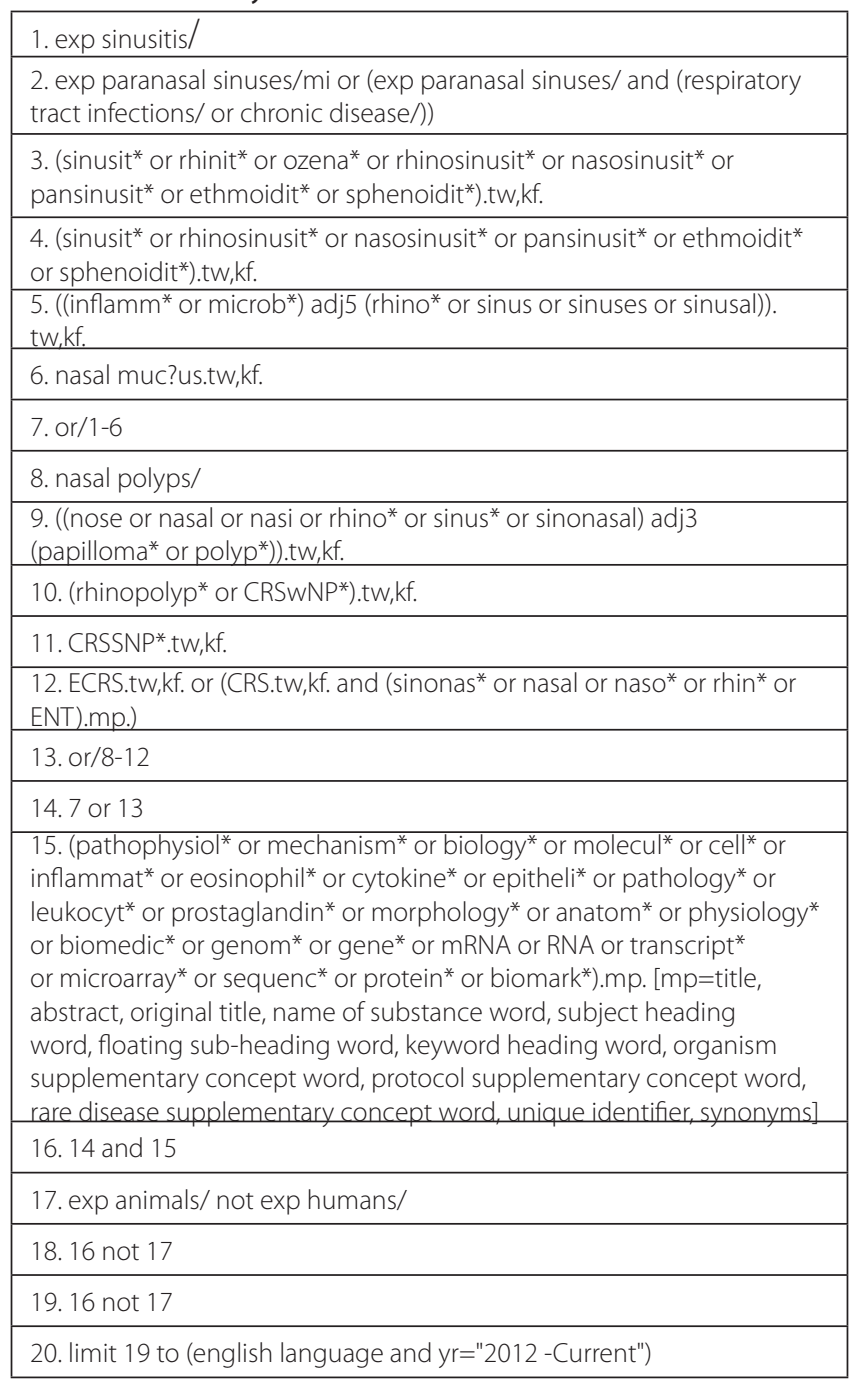

This OVID search produced a total of 9491 publications. Manual curation was performed by reading (STS and HW) all abstracts/ titles and by excluding the publications not meeting with inclusion criteria such as case reports, animal experiments, allergic rhinitis, no English language, no inflammatory mechanisms presented, GWAS, etc. After the manual curation of the search data, the final number of publications was 1202. Of these about 300 have been referred in this EPOS chapter 5.2.2.

\subsection{Diagnosis and differential diagnosis}

Search strategy

The search for CRS and olfaction was performed on November 17th 2019 in OVID Medline. It was combined with "olfactory function or smell" giving 406 hits. These were screened. Exclusion criteria were: keyword coincidences without any information given regarding olfactory measures, children only and wrong subject.

The EPOS 2020 core search for CRS was performed on June 17th 2019 in OVID Medline. It was combined with "(facial pain or headache).ti" giving 330 hits. To exclude case reports all "(case). $\mathrm{ti}^{\prime \prime}$ were removed resulting in a total of 304 records, containing 1 duplicate. These were screened. Exclusion criteria were:. publication before 1996 (101 records), case reports (57 records), children only (12 records) and wrong subject, wrong population or methodological shortcomings (73 records). Thus, 60 records were included. Of these, only 25 (42\%) contained original data. The others were reviews, editorials, letters, comments etc. After a first review by the EPOS steering group, members were asked to supply any missing yet relevant references. This way, another seven papers were included, most of which were not written primarily for facial pain but contained data relevant to this chapter.

\subsubsection{Management of chronic rhinosinusitis in adults Central search, see above.}

\subsubsection{Paediatric CRS}

\subsection{Epidemiology, predisposing factors, and comorbidities}

A PubMed and Scopus (EMBASE) search was performed and relevant data were extracted accordingly. The first search was for the following terms: chronic sinusitis in children or chronic rhinosinusitis in children; paediatric chronic sinusitis or paediatric chronic rhinosinusitis. All of them followed by and:

- Epidemiology or prevalence

- Anatomic variations of the paranasal sinuses

- The effect of environmental exposures

- Occupational and environmental risk factors for

- Risk factors

- The role of pollution, toxins and dust at home

- The role of viral infections in inducing or exacerbating CRS

- The role of viral infections in the onset of CRS in children

- Smoking

- Active and passive tobacco smoke exposure with the prevalence of chronic sinusitis in children

- Allergic rhinitis or allergy

- Asthma

- $\quad$ Role of the Adenoid 
- $\quad$ Relationship between sinusitis and adenoid size in paediatric age group

- Adenoid bacteriology and sinonasal symptoms

- Identification of adenoid biofilms

- Immunological investigation in the adenoid tissues

- Adenoidectomy outcomes

- Gastroesophageal reflux disease

- Humoral immunodeficiency

- Immunologic defects in

- Deficiency of the paediatric immune system

- Immaturity immune system

- Cystic fibrosis

- Diagnosis of cystic fibrosis in newborns

- Primary ciliary dyskinesia

12.6.3.7.2. Pathophysiology of chronic rhinosinusitis with or without nasal polyposis

12.6.3.7.2.1. Genetics

Pubmed

Search words: Sinusitis/Children/genetics

10-year limit

82 hits reviewed and 14 selected for review.

Eliminated irrelevant abstracts and studies that involved adults only.

\subsection{Inflammatory mechanisms}

Pubmed

Search words: Inflammation, Rhinosinusitis

Limits: previous 10 years/Age $<18$ y/Humans

178 hits reviewed and 56 selected for review.

Eliminated adult studies, reviews, and non-English language abstracts.

\subsection{Diagnosis and differential diagnosis}

Given the nature of this chapter (differential diagnosis and use of diagnostic tools, with emphasis on issues specific for the paediatric population) a systematic search on the CRS core set was deemed unfruitful. References were hand-searched. After the first version, the EPOS steering group was asked for input on any missing relevant papers.

\subsection{Management of CRS and co-morbidities} Pubmed

Search words: Chronic Rhinosinusitis and Antibiotics Limits: 10-year, Humans, English, Child: birth-18y 70 hits reviewed and 12 selected for review.

Eliminated irrelevant abstracts, reviews, studies dealing with CF, and studies that involved adults only.

\section{Pubmed}

Search words: Chronic Rhinosinusitis and Saline

Limits: 10-year, Humans, English, Child: birth-18y

26 hits reviewed and 7 selected for review.

Eliminated irrelevant abstracts, reviews, studies dealing with CF, and studies that involved adults only.

Pubmed
Search words: Chronic Rhinosinusitis and Corticosteroids Limits: 10-year, Humans, English, Child: birth-18y

31 hits reviewed and 3 selected for review.

Eliminated irrelevant abstracts, reviews, studies dealing with $C F$, and studies that involved adults only.

\section{Pubmed}

Search words: Chronic Rhinosinusitis and Balloon Limits: 10-year, Humans, English, Child: birth-18y 28 hits reviewed and 12 selected for review.

Eliminated irrelevant abstracts, reviews, studies dealing with CF, and studies that involved adults only.

Pubmed

Search words: Chronic Rhinosinusitis and Adenoidectomy Limits: 10-year, Humans, English, Child: birth-18y

38 hits reviewed and 15 selected for review.

Eliminated irrelevant abstracts, reviews, studies dealing with $C F$, and studies that involved adults only.

Pubmed

Search words: Chronic Rhinosinusitis and Endoscopic Sinus

Surgery

Limits: 10-year, Humans, English, Child: birth-18y

248 hits reviewed and 26 selected for review.

Eliminated irrelevant abstracts, reviews, studies dealing with $C F$, and studies that involved adults only.

Many of the selected articles overlapped.

12.6.3.8. Concomitant diseases in patients with chronic rhinosinusitis

\subsection{Role of allergy and chronic rhinosinusitis} No search provided.

12.6.3.8.2.Immunodeficiencies and their role in CRS Search strategy

\begin{tabular}{l}
\hline 1. Medline (Ovid) \\
- terms: chronic rhinosinusitis immunodeficiency \\
- limits: 2012-current \\
- results: 4671 \\
\hline 2. Medline (Ovid) \\
- terms: primary immunodeficiency \\
- limits: 2012 -current \\
- results: 1713 \\
\hline 3. Medline (Ovid) \\
- terms: secondary immunodeficiency chronic rhinosinusitis \\
- limits: 2012 -current \\
- results: 2811 \\
\hline 4. Medline (Ovid) \\
- terms: secondary immunodeficiency \\
- limits: 2012 -current \\
- results: 120 \\
\hline 5. Google scholar \\
- terms: chronic rhinosinusitis immunodeficiency \\
- limits: 2012 -current \\
- results: 4530 \\
\hline
\end{tabular}


6. Google scholar

- terms: chronic rhinosinusitis secondary immunodeficiency

- limits: 2012-current

- results: 2740

\subsection{Lower airway disease including asthma in relation} to CRS

Pubmed Search:

Chronic Rhinosinutis and asthma: 1489

Chronic Rhinosinusitis and COPD: 159 articles

Chronic Rhinosinusitis and lower airway disease: 199

Selection criteria for inclusion: English language, articles with novel insights and properly conducted trials and/or reviews, preference of recent (last 10 years) over historic reports

\subsection{Cystic fibrosis}

Search strategy

PubMed and Embase were systemically searched for articles addressing chronic rhinosinusitis in patients with cystic fibrosis (CF). The search was last updated on April 21, 2019. The literature search generated 1762 articles of which 40 were included. Nine additional studies were identified through reference lists. Further 17 studies elaborating on the included findings and concerning the CF diagnosis in general were included. The included studies were published between 1991 and 2019.

\subsubsection{Primary ciliary dyskinesia and its role in CRS} Search strategy

PubMed and Embase were systemically searched for articles addressing chronic rhinosinusitis in patients with primary ciliary dyskinesia. The search was last updated on August 19, 2019. After exclusion of duplicates the literature search generated 609 articles of which 37 were included. The included studies were published between 1981 and 2019.

\subsection{Fungal rhinosinusitis}

Search strategy (see pag 460)

(Search for the keywords fungal.mp OR fungi.mp or fungus.mp with the prior search produced $n=372$ articles. Fungal ball was supplemented by the search below and PRISMA, invasive fungal disease includes).

Separate prisma and search was done for fungal ball.

\begin{tabular}{|l|l|l|}
\hline$\#$ & Searches & Results \\
\hline 1. & fungal ball.mp. & 128 \\
\hline 2. & Mycetoma/ & 1153 \\
\hline 3. & 1 or 2 & 1274 \\
\hline 4. & $\begin{array}{l}\text { (rhinosinusitis or \$rhinosinusitis or crs or sinus\$ } \\
\text { or nose or nasal).mp. }\end{array}$ & 219127 \\
\hline 5. & 3 and 4 & 228 \\
\hline 6. & (fungus or fungal or fungi).mp. & 216128 \\
\hline 7. & invasive.mp. & 268714 \\
\hline 8. & (invasive adj3 (fungus or fungal or fungi)).mp. & 4866 \\
\hline 9. & 8 and 4 & 474 \\
\hline
\end{tabular}

\begin{tabular}{|l|l|l|}
\hline 10. & limit 9 to (english language and humans) & 393 \\
\hline
\end{tabular}

12.6.3.8.7. Vasculitis, granulomatous diseases and their role in CRS

12.6.3.8.7.2.Granulomatosis with polyangitis (GPA) Search strategy The EPOS 2020 core search for CRS was performed in June 2019 in OVID Medline. It was combined with "(Wegener).ti" and "(granulomatosis with polyangiitis), giving 421 hits. These were screened. Exclusion criteria were: case reports (125 records), language other than English (30 records), children only (three records) and wrong subject (57 records). A further 102 nonlandmark publications $<1996$ were removed. Thus, 104 records were included. Of these, only 41 (39\%) contained original data. The others were reviews, editorials, letters etc.

\subsection{Eosinophilic granulomatosis with polyangiitis} Search strategy The EPOS 2020 core search for CRS was performed in June 2019 in OVID Medline. It was combined with "(Churg-Strauss). ti" and "(polyangiitis), giving 195 hits. These were screened. Exclusion criteria were: case reports (61 records), language other than English (21 records), children only (four records) and wrong subject (five records). Thus, 104 records were included for review. Of these, only 31 (29\%) contained original data. The others were reviews and editorials.

\subsection{Sarcoidosis}

Search strategy

The EPOS 2020 core search for CRS was performed in June 2019 in OVID Medline. It was combined with "(sarcoidosis).ti" giving 110 hits. These were screened. Exclusion criteria were: publication before 1996 (with two exceptions) (12 records), case reports ( 23 records), language other than English (16 records), children only (one record) and wrong subject (six records). Thus, 52 records were included of which 44 were used. Of these, 22 (50\%) contained original data, the others were reviews.

\subsubsection{Integrated care pathways in CRS and precision medicine}

\subsection{Patient participation in CRS} Pubmed Search:

Participation, (rhino)sinusitis: 51 results Mobile health, (rhino)sinusitis: 20 results Empowerment patients, (rhino)sinusitis: 11 results

\subsubsection{3. and 9.4.Prevention and prediction} Search terms; (prevent* OR predict* OR risk OR success OR failure OR recurrence OR revision) AND full search dataset Search Identified 7599 papers, 269 full papers reviewed

12.6.3.10. Pharmacist perspective on rhinosinusitis 
12.6.3.8.6ungal rhinosinusitis Search strategy

\begin{tabular}{|c|c|c|}
\hline \multicolumn{3}{|l|}{ Ovid MEDLINE(R) 1946 to May 20, 2019} \\
\hline Population/Problem & & Prognostic factor/exposure \\
\hline $\begin{array}{l}\text { 1. Mycetoma/ Fungal terms } \\
\text { 2. Aspergilloma/ } \\
\text { 3. OR/1-2 } \\
\text { 4. Fungal.mp } \\
\text { 5. Fung\$.mp } \\
\text { 6. Mycoses/ } \\
\text { 7. OR/4-6 Ball terms } \\
\text { 8. Ball.mp } \\
\text { 9. Mass.mp } \\
\text { 10. Collection.mp } \\
\text { 11. Concretion.mp } \\
\text { 12. Cyst.mp } \\
\text { 13. OR/8-12 } \\
\text { 14. } 7 \text { ADJ2 } 13 \\
\text { 15. 3OR } 14 \\
\text { 16. Maxillary sinus/ Maxillary sinus } \\
\text { 17. Maxilla.mp } \\
\text { 18. Antrum.mp } \\
\text { 19. Antral.mp } \\
\text { 20. Highmore.mp } \\
\text { 21. Maxillary.mp } \\
\text { 22. Sinus.mp } \\
\text { 23. OR/16-22 } \\
\text { 24. } 23 \text { AND 15 }\end{array}$ & $\begin{array}{l}\text { Maxillary sinusitis } \\
\text { 25. Maxillary sinusitis/ } \\
\text { 26. Sinusitis/ } \\
\text { 27. Rhinosinusitis.mp } \\
\text { 28. Sinusitis.mp } \\
\text { 29. OR/26-28 } \\
\text { 30. } 29 \text { and } 23 \\
\text { 31. } 25 \text { OR } 30 \\
\text { Any fungus } \\
\text { 32. OR/1-6 (any fungus) } \\
\text { Fungal ball maxillary sinus } \\
\text { Fungal ball or fungal sinusitis of the maxillary sinus } \\
\text { 34. } 24 \text { OR } 33\end{array}$ & $\begin{array}{l}\text { 35. Risk factor/ } \\
\text { 36. Risk*.ti } \\
\text { 37. Etiolog*.mp } \\
\text { 38. Aetiology.mp } \\
\text { 39. pathogenesis.mp } \\
\text { 40. Causality/ } \\
\text { 41. Causation.pm } \\
\text { 42. Causes.mp } \\
\text { 43. Predisposing.mp } \\
\text { 44. Origin.mp } \\
\text { 45. Associations/ } \\
\text { 46. Epidemiology/ } \\
\text { 47. OR/35-46 }\end{array}$ \\
\hline $\begin{array}{l}\text { 48. } 34 \text { AND } 47 \\
\text { 49. Limit to English } \\
\text { 50. Limit to conference abstract } \\
\text { 51. } 49 \text { NOT } 50=490\end{array}$ & & \\
\hline
\end{tabular}

12.6.3.8.6. Fungal rhinosinusitis Prisma 2009 flow diagram.

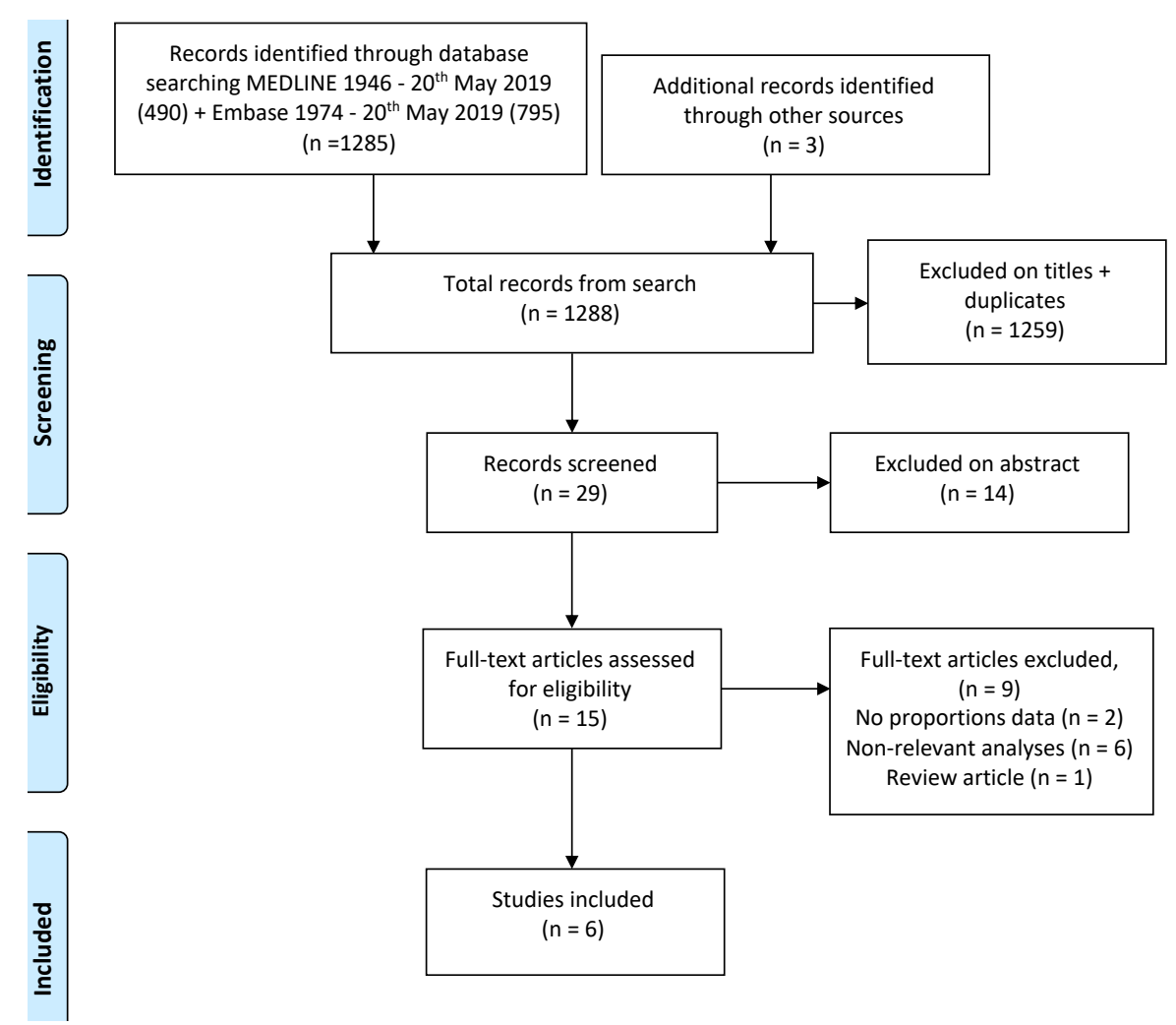


MEDLINE /Ovid, search date: 10-07-2019

\begin{tabular}{|c|c|}
\hline \# & Searches \\
\hline 1. & exp Pharmacy/ \\
\hline 2. & (pharmacy OR pharmacy practice pattern*.tw \\
\hline 3. & Community Pharmacy Services/ \\
\hline 4. & pharmac* service* community.tw \\
\hline 5. & Pharmacists/ \\
\hline 6. & $\begin{array}{l}\text { (pharmacist* OR clinical pharmacist* OR community } \\
\text { pharmacist*).tw }\end{array}$ \\
\hline 7. & Pharmacy Technicians/ \\
\hline 8. & (pharmacy technician* OR pharmacist*' aide*).tw \\
\hline 9. & Pharmacies/ \\
\hline 10. & $\begin{array}{l}\text { (pharmacies OR pharmac* distribution* OR pharmac* } \\
\text { community).tw }\end{array}$ \\
\hline 11. & exp Prescriptions \\
\hline 12. & exp Drug Packaging/ \\
\hline 13. & (packaging drug* OR drug* container*).tw \\
\hline 14. & drug dispensing.tw \\
\hline 15. & exp Drug Utilization/ \\
\hline 16. & drug utilization*.tw \\
\hline 17. & exp Nonprecsription Drugs/ \\
\hline 18. & (drug* non prescription OR OTC drug*).tw \\
\hline 19. & exp Drug Resistance Microbial/ \\
\hline 20. & $\begin{array}{l}\text { (antibiotic drug resistance* OR antibiotic resistance }{ }^{*} \\
\text { OR antimicrobial drug resistance* OR antimicrobial } \\
\text { resistance*).tw }\end{array}$ \\
\hline 21. & exp Drug Information Services \\
\hline 22. & Attitude of Health Personnel/ \\
\hline 23. & exp Interprofessional Relations \\
\hline 24. & exp Patient Compliance/ \\
\hline 25. & $\begin{array}{l}\text { (patient compliance* OR patient adherence OR patient } \\
\text { cooperation OR treatment compliance* OR therapeutic } \\
\text { compliance* OR patient non compliance* OR patient } \\
\text { nonadherence OR medication adherence OR medication } \\
\text { compliance }{ }^{*} \text { OR medication nonadherence OR } \\
\left.\text { medication non compliance }{ }^{*}\right) \text {.tw }\end{array}$ \\
\hline 26. & exp Counseling/ \\
\hline 27. & (counseling OR advice).tw \\
\hline 28. & exp Health Education/ \\
\hline 29. & Health Communication/ \\
\hline
\end{tabular}

Scopus search date: 22-10-2019

SET 1

Field: keywords

1. pharmacy OR "community pharmacy"

2. pharmacist OR"pharmacy technician

3. prescription

4. "drug packaging" OR "drug utilization" OR "non prescription drug"

5. "antibiotic resistance"

6. "drug information"

7. "health personnel attitude"

8. "interpersonal communication" OR "public relations

9. "patient compliance" OR "patient counseling

10. "medical education" OR "patient education" 11."medical information" OR "consumer health information"

Field: abstract

12. pharmacy OR "pharmacy practice pattern*

13. "pharmac* service* community" OR pharmacist* OR "clinical pharmacist*" OR "community pharmacist*

14. "pharmacy technician*" OR "pharmacist* aide*" OR pharmacies OR "pharmac* distribution*" OR "pharmac* community

15. "packaging drug*" OR "drug* container*

16. "drug dispensing" OR "drug utilization*" OR "drug* non prescription" OR "OTC drug*

17. "antibiotic drug resistance ${ }^{* \text { " }} \mathrm{OR}$ "antibiotic resistance*" $O R$ "antimicrobial drug resistance ${ }^{* "} \mathrm{OR}$ "antimicrobial resistance*"

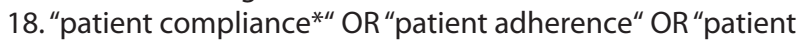
cooperation" OR "treatment compliance*" OR "therapeutic compliance*" OR "patient non compliance* ${ }^{* \text { OR }}$ "patient nonadherence"

19. "medication adherence" OR "medication compliance ${ }^{* \text { " OR }}$ "medication nonadherence" OR

20."medication non compliance*

21. counseling OR advice

22. OR/1-21

23. animal AND NOT human (keywords)

24. 22 AND NOT 23

SET 2

Field: keywords

1.sinusitis OR "paranasal sinus"

2. "paranasal sinuse" and ("respiratory tract infection" or "chronic disease")

3. "common cold" OR "nasal polyps" OR "nose polyp" OR

"adenoid hypertrophy" OR adenoid

Field: abstract

4. rhinitis or "rhinitis atrophic" or "rhinitis vasomotor"

5. sinusit* or rhinit* or ozena* or rhinosinusit* or nasosinusit* or pansinusit* or ethmoidit* or sphenoidit

6. "inflamm* rhino*" or "inflamm* sinus" or "inflamm* sinuses" or "inflamm* sinusal

7. "microb* rhino*" or "microb* sinus" or "microb* sinuses" or "microb* sinusal" or rhinorr* or "purulent rhinor" OR" nasal muc?us

8. "common cold" or "head cold" or coryza or "upper respiratory infection*" or "upper airway infection*" or URTI or "upper respiratory tract infection

9. ARS and (sinonas* or nasal or naso* or rhin* or ENT)

10. "nose papilloma*" or "nasal papilloma*" or "nasi papilloma*" or "rhino papilloma*" or "sinus papilloma*" or "sinonasal papilloma*"

11. rhinopolyp* or CRSwNP* or CRSSNP* or ECRS

12. "nose polyp*"or"nasal polyp*"or"nasi polyp*"or "rhino polyp*" or "sinus polyp*"or "sinonasal polyp*"

13. CRS and (sinonas* or nasal or naso* or rhin* or ENT)

14. ("kartagener* syndrom*" or "primary ciliary dyskinesi*") and not (COPD or thoracic or "pulmonary disease" or bronchiectas*) 15. adenoid* hypertroph* $^{*}$ or adenoid* obstruct* or adenoidit*

16. OR/1-15

17. animal AND NOT human (keywords)

18. 16 AND NOT 17

SET1 AND SET2 


\section{References}

1. Fokkens W, Lund V, Bachert C, et al. European Position Paper on Rhinosinusitis and nasal Polyps. Rhinology 2005;18.

2. Fokkens W, Lund V, Mullol J. European position paper on rhinosinusitis and nasal polyps 2007. Rhinol Suppl 2007;20:1-136.

3. Fokkens W, Lund V, Mullol J. EP3OS 2007: European position paper on rhinosinusitis and nasal polyps 2007. A summary for otorhinolaryngologists.
Rhinology 2007;45:97-101.

4. Fokkens W, Desrosiers M, Harvey R, et al. EPOS2020: development strategy and goals for the latest European Position Paper on Rhinosinusitis. Rhinology 2019;57:162-8.

5. The AGREE II Instrument [Electronic version]. 2017. http://www.agreetrust. org.

6. Higgins JPT, Altman DG, Sterne JAC. Chapter 8: Assessing risk of bias in included studies. In: Higgins JPT, and Green S (eds). Cochrane Handbook for
Systematic Reviews of Interventions Version 510. Oxford, UK: The Cochrane Collaboration, 2011.

7. Reeves BC, Deeks JJ, Higgins JPT, Wells GA. Chapter 13: Including nonrandomized studies. In: Higgins JPT, and Green S (eds). Cochrane Handbook for Systematic Reviews of Interventions Version 510. Oxford, UK: The Cochrane Collaboration, 2011.

8. Shekelle PG, Woolf SH, Eccles M, Grimshaw J. Developing clinical guidelines. West J Med 1999;170:348-51. 


\section{Authors declarations}

Dr. Wytske Fokkens reports that the department of otorhinolaryngology of the Amsterdam University Medical Centres, location AMC received grants from GSK, Sanofi, Novartis Chordate, Mylan, ALK, Allergy diagnostics and ZonMW and that she is a consultant for Sanofi and Lyra Therapeutics.

Dr. Valerie Lund reports that she has received personal fees from Abbott Criteria Fieldwork, Elsevier, Hartington, Medscape, Sprime Health, Kyorin, Quintiles, GSK and Sanofi.

Dr. Claire Hopkins has reported personal fees from Sanofi, Chordate, Olympus, Smith and Nephew and Medtronic.

Dr. Robert Kern has reported grants from NHI and personal fees from Sanofi, Genentech, Lyra and NeurEnt.

Dr. Sietze Reitsma has reported grants from Sanofi, Novartis, Chordate Medical and personal fees from Sanofi.

Dr. Manuel Bernal-Sprekelsen has reported personal fees from Olympus, Hamburg as well as counselling fee from Salvat Pharmaceutical Company, Barcelona, Spain and free provision of dissection sets for coursesnon-financial support from Karl Storz, Germany.

Dr. Joaquim Mullol has reported grants from URIACH Group, MYLAN-MEDA Pharma and personal fees from URIACH Group, MYLANMEDA Pharma, Genetech- Novartis, Regeneron \& Sanofi-Genzyme, Astra Zeneca.

Dr. Isam Alobid has reported grants from MSD and that he received personal fees from Roche, MSD, Mylan, Menarini.

Dr. Claus Bachert has reported that he received personal fees from Sanofi, Novartis, Astra-Zeneca, GSK and ALK.

Dr. Noam Cohen has reported personal fees from Sanofi/Regeneron and Licensing of Patent rights namely GeneOne Life Sciences.

Dr. Ludovic De Gabory has reported personal fees from Zambon, ALK-Abello, Medtronic, Laboratoire de la Mer, Integra Life Sciences, consulting from Zambon, ALK-Abello, Medtronic, Laboratoire de la Mer, Integra Life Sciences, Chiesi, Sanofi-Aventis, funded research from Laboratoire de la Mer and Patent from Integra Life Sciences.

Dr. Martin Desrosiers is on advisory boards and clinical trials of Glaxo Smith Kline, Astra Zeneca, Sanofi/Regeneron. Speaker bureau in Milan. Founder of Probionase Therapies Inc.

Dr. Zuzana Diamant has an academic affiliation with Lund University, Groningen University, Charles University Prague. She works at QPS-NL a phase I unit, which performs phase I/II studies for biotech and pharmaceutical companies. Dr. Diamant has also reported personal fees from ALK, CSL, HAL Allergy, Sanofi Genzyme Regeneron and is doing Medical writing at Acucort, Boehringer Ingelhein and webinars at MSD.

Dr. Philippe Gevaert has reported personal fees from Novartis, Roche, Genentec, Sanofi, Regeneron, Astra Zeneca, 3NT, Stallergens and has been on Advisory board, the speaker and conducted research with financial support of Novartis, Roche, Genentec, Sanofi, Regeneron, Astra Zeneca, 3NT and Stallergens.

Dr. Richard Harvey is a consultant with Medtronic, Stryker, Novartis, Meda, and NeilMed pharmaceuticals. Research grant funding received from Glaxo-Smith-Kline and Stallergenes. He has been on the speakers' bureau for Glaxo-Smith-Kline, Meda Pharmaceuticals and Seqiris.

Dr. Guy Joos has reported grants from GSK,Chiesi, Asta Zeneca and personal fees from Bayer, Eureka VZW, GSK and Teva.

Dr. Janwillem Kocks has reported grants from Astra Zeneca, Boehringer, Ingelheim, Chiesi, Mundi Pharma, TEVA and personal fees from Astra Zeneca, Boehringer, Ingelheim and Chiesi.

Dr. Carl Philpott has reported grants from NIHR, personal fees from Sanofi Genzyme, Olympus, Entellus, Aerin Medical, Acclarent, Navigant and Trustee from Fifth Sense.

Dr. Dermot Ryan has reported grants from Chiessi, personal fees from Mylan, GSK, Novartis, Chiesi, AZ and non-financial support 
from Regeneron Educational activities.

Dr. Rodney Schlosser has reported grants from Sanofi, GSK, Roche, Astra Zeneca, Optinose, Healthy Humming and personal fees from Sanofi, GSK, Optinose and medical director of Healthy Humming.

Dr. Achim Beule has reported in performing studies as part Principal Investigator at Glaxo Smith Kline, Astra Zeneca and Allakos.

Dr. Juan David Bedoya has reported grants from American Academy of OHNS foundation.

Dr. Eliza Brozek-Madry has reported grants from Probios.

Dr. Sean Carrie has reported grans from National Institute Health Research (UK), personal fees from Medtronic and Olympus and travel and accommodation sponsorship from Medtronic.

Dr. Jessica W. Grayson has reported financial support for attendance of the conference in Ghent from Medtronic.

Dr. Kornkiat Snidvongs has reported personal fees from Menarini and MSD.

Dr. Harsha Karyawasam has reported personal fees from Sanofi, Novartis, AZ, MEDA.

Dr. Ana Kjeldsen has reported participation in OSTRO project, randomized controlled study of benralizumab.

Dr. Ludger Klimek has reported grants from Allegopharma, MEDA/Mylan, ALK Abello, LETI Pharma, Stallergenes, Quintiles, Sanofi, Astra Zeneca, GSK, ASIT biotech, Lofarma and personal fees from from Allegopharma, MEDA/Mylan, HAL Allergie, LETI Pharma, Allergy Terapeut., Sanofi.

Dr. Mitsuhiro Okano has reported grants from Tanabe Mitsubishi Pharma Corporation and Taiho Pharmaceutical Co. and personal fees from Sanofi, Tanabe Mitsubishi Pharma Corporation and Taiho Pharmaceutical Co.

Dr. Metin Onerci has reported travel expenses and cadaver courses support from STORZ.

Dr. Mahdi Shkoukani has reported being a member of advisory board of MEDA Pharmaceuticals MEA FZ-LLC, UAE.

Dr. lan Witterick has reported ownership of shares in Proteocyte Diagnostcis Inc. and being on Advisory board at Sanofi Genzyme.

Dr. Retno Wardani reported grants from Abbott Indonesia, speaker fee, accommodation and travel expenses from P\&G, Menarini, Zuellig Pharm and sponsorship from Advance Medica Corpora.

The following authors have not reported any relevant financial relationships:

Dr. Peter Hellings, Dr. Sanna Toppila-Salmi, Dr. Wilma Terezinha Anselmo Lima, Dr. Fuad Baroody, Dr. Christian von Buchwald, Dr. Anders Cervin, Dr. Jannis Constantinidis, Dr. Richard Douglas, Dr. Anita Hafner, Dr. Livije Kalogjera, Dr. Andrew Knill, Dr.Basile Nicolas Landis, Dr. Jacqueline Limpens, Dr. Sarah Lebeer, Dr. Olga Lourenco, Dr. Paolo Maria Matricardi, Dr. Cem Meco, Dr. Liam O'Mahony, Dr. Brent Senior, Dr. Timothy Smith, Dr. Thijs Teeling, Dr. Peter Valentin Tomazic, Dr. De Yun Wang, Dr. Luo Zhang, Dr. Paolo Battaglia, Dr. Cecilia Ahlström-Emanuelsson, Dr. Mohammad Aloulah, Dr. Mohannad Al-Qudah, Dr. Saad Alsaleh, Rashid Alabri, Dr. Saied Alhabash, Dr. Aleksandra Aleksic, Dr. Adrian Agius, Dr.Silvio Albu, Dr. Muad Aziz Baban, Dr.Tomislav Baudoin, Dr. Achim Beule, Dr. Khaled Bofares, Dr. Itzhak Braverman, Dr. Richard Byaruhanga, Dr. Claudio Callejas, Dr. Lisa Caulley, Dr. Caius-Codrut Sarafoleanu, Dr. Desderius Chussi, Dr. Eugenio de Corso, Dr. Lal Devyani, Dr. Usama El Hadi, Dr. Ahmed Elfarouk, Dr. Philippe Eloy, Dr. Shokrollah Farrokhi, Dr. Ferrari Michel, Dr. Giovanni Felisati, Dr.Roman Fishchuk, Dr. Boris Grdinic, Dr. Paulo Goncalves, Prof. Usamah Hadi, Dr. Julio Heinichen, Dr. Salina Husain, Dr. Aneeza Hamizan, Dr. Justinas Ivaska, Dr. Frodita Jakimovska, Dr. Ljiljana Jovancevic, Dr. Reda Kamel, Dr. Hideyuki Kawauchi, Dr. Sergei Karpischenko, Dr. Emily Kakande, Dr. Antoni Krzeski, Dr. Gabriela Kopacheva Barsova, Dr. Yew Kwang Ong, Dr. Devyani Lal, Dr. José Letort, Dr. Andrey Lopatin, Dr. Zhang Luo, Dr. Abdelhak Mahdjoubi, Dr. Alireza Mesbahi, Dr. Jane Netkovski, Dr. Dieudonné Nyenbue Tshipukane, Dr. Andrés Obando, Dr. Muge Ozkan , Dr. Richard Orlandi, Dr. Nobuyoshi Otori, Dr. Kheir Ouennoughy, Dr. Aleksandar Peric , Dr. Jan Plzak, Dr. Emmanuel Prokopakis, Dr. Alkis Psaltis, Dr. Benoit Pugin, Dr. Marco Raftopulos, Dr. Herbert Riechelmann, Dr. Philippe Rombaux, Chae-Seo Rhee, Dr. Kafui Searyoh, Prof. Arthur K. Shukuryan, Dr. Sverre Steinsvag, Dr. David Smyth, Dr. Marian Sicak, Dr.Tanja Soklic Kosak, Dr. Par Stjarne, Dr. Semia Sahtout, Dr. Budi Sutikno, Dr. Kim Sung Wam , Prof Shi Jianbo, Prof. Tang Ing Ping, Dr. Thuy Tran, Prof. Pongsakorn Tantilipikorn, Prof. Sanguansak Thanaviratananich, Dr. Jure Urbancic, Dr. Arunas Valiulis, Dr. Carolina Vasquez de Aparicio, Dr. Dilyana Vicheva, Prof. Richard Voegels, Dr. Martin Wagenmann, Dr. Paula Virkula, Dr. Gil Vicente, Dr. Martin Wagenmann, Dr. Dehui Wang, Dr. Antje Welge-Lussen, Dr. Erin Wright, Dr Dmytro Zabolotniy, Dr. Bella Zsolt. 


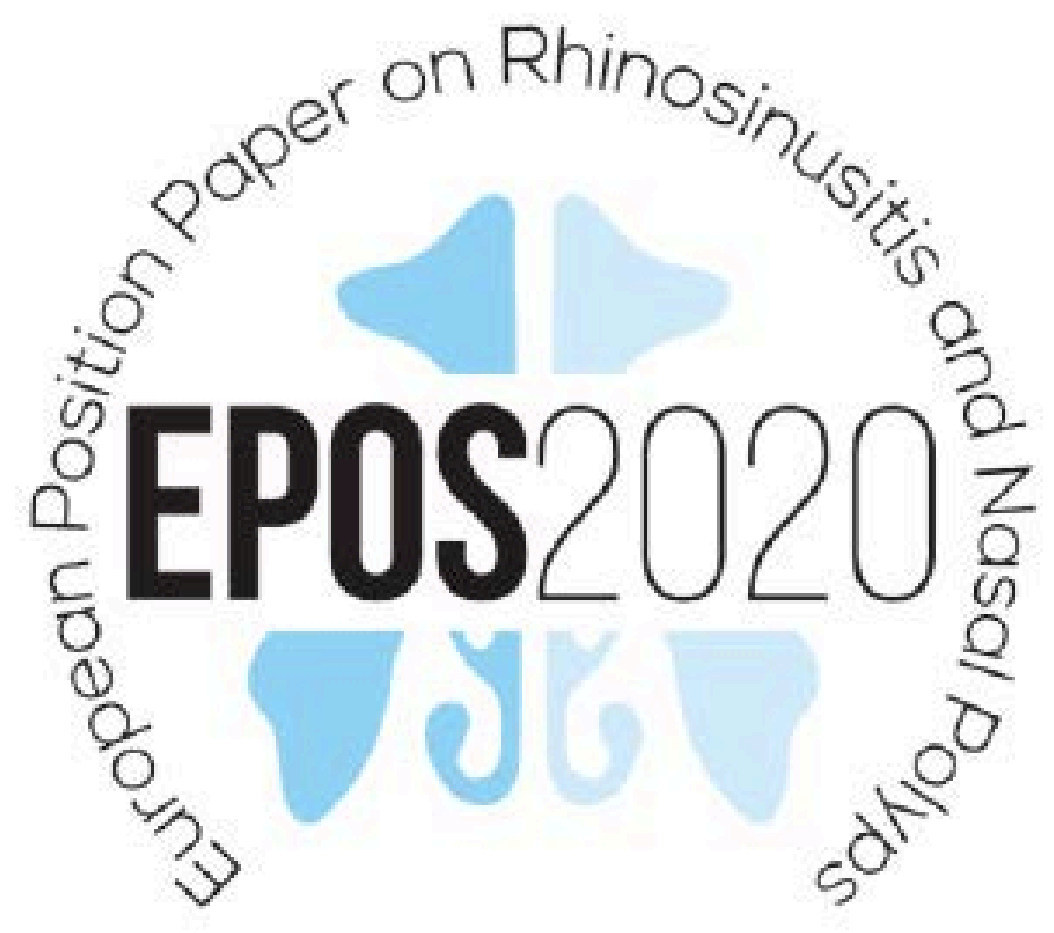




\section{CONTENT}

Position paper Wytske 1. Fokkens, Valerie J. Lund, Claire Hopkins, Peter W. Hellings, Robert Kern, Siet a Reltsma. Sanna Toppila-Salmi, Manuel Bernatsprekelsen, Joaquim Mullol, et al.

European Position Paper on Rhinosinusitis and Nasal Polyps 2020. Rhinology. 2020, Suppl. 29: 1- 464.

1. Buecuthe Summary including management schemes 1

2. Classification definitions and terminology 31

3. Burden of acute and chronic rhinosinusitis 45

4. Acute rhinosinusitis including common cold-and recurrent ARS in adults and children 53

5. Epidemiology, predisposing factors, pathophysiology, and diagnosis of CRS $\quad 115$

6. Management of chronic rhinosinusitis in adults 205

7. Paediatricchronic rhinosinusitis 349

8. Concomitant diseases in patients withchronic rhinosinusitis 369

9. Patient participation, prediction, precision medicine and implementation 413

10. Pharmacist perspective on rhinosinusitis 433

11. Research needs and agenda for the next decade 445

12. Methods used in EPOS2020 451

13. Authors declarations 463

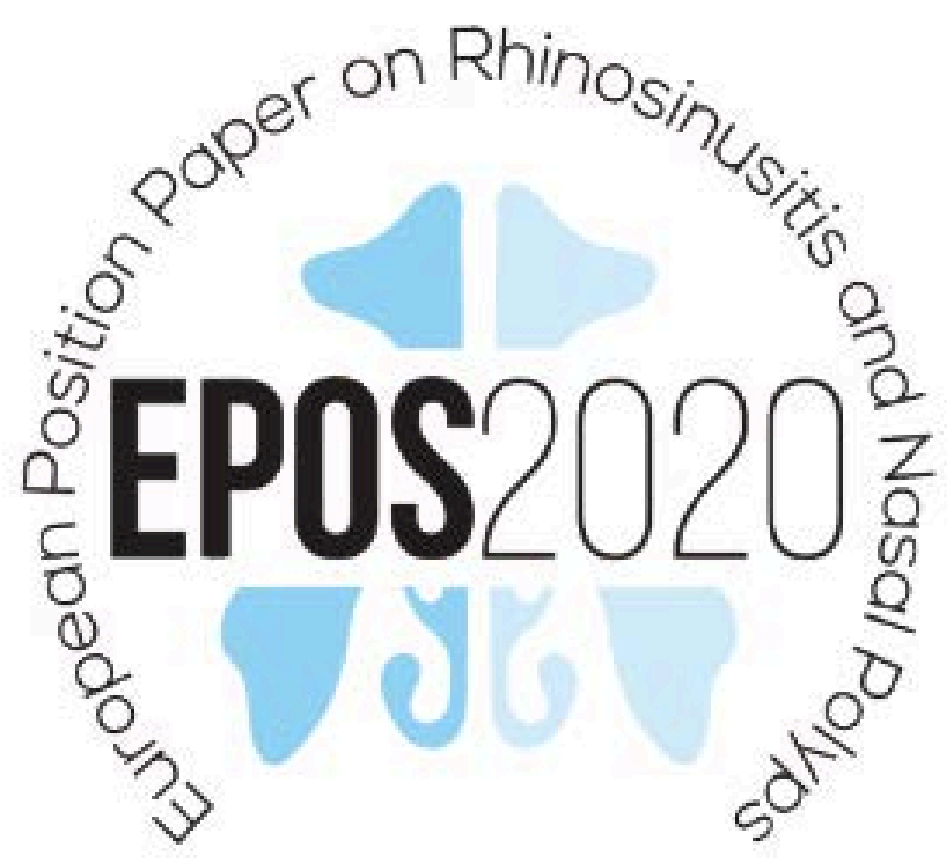

